\title{
The GALEX Ultraviolet Atlas of Nearby Galaxies
}

\author{
Armando Gil de Paz ${ }^{1}$, Samuel Boissier ${ }^{1}$, Barry F. Madore ${ }^{1,2}$, Mark Seibert ${ }^{3}$, Young H. \\ Joe $^{1,4}$, Alessandro Boselli ${ }^{5}$, Ted K. Wyder ${ }^{3}$, David Thilker ${ }^{6}$, Luciana Bianchi ${ }^{6}$, Soo-Chang \\ Rey $^{3}$, R. Michael Rich ${ }^{7}$, Tom A. Barlow ${ }^{3}$, Tim Conrow ${ }^{3}$, Karl Forster ${ }^{3}$, Peter G. \\ Friedman ${ }^{3}$, D. Christopher Martin ${ }^{3}$, Patrick Morrissey ${ }^{3}$, Susan G. Neff ${ }^{8}$, David \\ Schiminovich $^{3}$, Todd Small ${ }^{3}$, José Donas ${ }^{5}$, Timothy M. Heckman ${ }^{9}$, Young-Wook Lee ${ }^{4}$, \\ Bruno Milliard ${ }^{5}$, Alex S. Szalay ${ }^{9}$, Sukyoung $\mathrm{Yi}^{4}$
}

\begin{abstract}
We present images, integrated photometry, surface-brightness and color profiles for a total of 1034 nearby galaxies recently observed by the Galaxy Evolution Explorer (GALEX) satellite in its far-ultraviolet (FUV; $\lambda_{\text {eff }}=1516 \AA$ ) and nearultraviolet $\left(\mathrm{NUV} ; \lambda_{\text {eff }}=2267 \AA\right.$ ) bands. Our catalog of objects is derived primarily from the GALEX Nearby Galaxies Survey (NGS) supplemented by galaxies
\end{abstract}

\footnotetext{
${ }^{1}$ The Observatories, Carnegie Institution of Washington, 813 Santa Barbara Street, Pasadena, CA 91101; agpaz, boissier, young@ociw.edu; AGdP's current address: Departamento de Astrofísica, Universidad Complutense de Madrid, Madrid 28040, Spain; SB's current address: Laboratoire d'Astrophysique de Marseille, BP 8, Traverse du Siphon, 13376 Marseille Cedex 12, France.

${ }^{2}$ NASA/IPAC Extragalactic Database, California Institute of Technology, MS 100-22, Pasadena, CA 91125; barry@ipac.caltech.edu

${ }^{3}$ California Institute of Technology, MC 405-47, 1200 East California Boulevard, Pasadena, CA 91125; mseibert, wyder, screy, tab, tim, krl, friedman, cmartin, patrick, ds, tas@srl.caltech.edu; SCR's current address: Department of Astronomy and Space Sciences, Chungnam National University, 220 Gung-dong, Yuseong-gu, Daejeon 305-764, Korea

${ }^{4}$ Center for Space Astrophysics, Yonsei University, Seoul 120-749, Korea; ywlee@csa.yonsei.ac.kr, yi@astro.ox.ac.uk

${ }^{5}$ Laboratoire d'Astrophysique de Marseille, BP 8, Traverse du Siphon, 13376 Marseille Cedex 12, France; alessandro.boselli, jose.donas, bruno.milliard@oamp.fr

${ }^{6}$ Center for Astrophysical Sciences, The Johns Hopkins University, 3400 N. Charles St., Baltimore, MD 21218; dthilker, bianchi@pha.jhu.edu

${ }^{7}$ Department of Physics and Astronomy, University of California, Los Angeles, CA 90095; rmr@astro.ucla.edu

${ }^{8}$ Laboratory for Astronomy and Solar Physics, NASA Goddard Space Flight Center, Greenbelt, MD 20771; neff@stars.gsfc.nasa.gov

${ }^{9}$ Department of Physics and Astronomy, The Johns Hopkins University, Homewood Campus, Baltimore, MD 21218; heckman, szalay@pha.jhu.edu
} 
larger than 1 arcmin in diameter serendipitously found in these fields and in other GALEX exposures of similar of greater depth. The sample analyzed here adequately describes the distribution and full range of properties (luminosity, color, Star Formation Rate; SFR) of galaxies in the Local Universe.

From the surface brightness profiles obtained we have computed asymptotic magnitudes, colors, and luminosities, along with the concentration indices C31 and C42. We have also morphologically classified the UV surface brightness profiles according to their shape. This data set has been complemented with archival optical, near-infrared, and far-infrared fluxes and colors.

We find that the integrated $(\mathrm{FUV}-K)$ color provides robust discrimination between elliptical and spiral/irregular galaxies and also among spiral galaxies of different sub-types. Elliptical galaxies with brighter $K$-band luminosities (i.e. more massive) are redder in $(\mathrm{NUV}-K)$ color but bluer in (FUV-NUV) (a color sensitive to the presence of a strong UV upturn) than less massive ellipticals. In the case of the spiral/irregular galaxies our analysis shows the presence of a relatively tight correlation between the (FUV-NUV) color (or, equivalently, the slope of the UV spectrum, $\beta$ ) and the total infrared-to-UV ratio. The correlation found between (FUV-NUV) color and $K$-band luminosity (with lower luminosity objects being bluer than more luminous ones) can be explained as due to an increase in the dust content with galaxy luminosity.

The images in this Atlas along with the profiles and integrated properties are publicly available through a dedicated web page at http://nedwww.ipac.caltech.edu/level5/GALEX_Atlas/

Subject headings: galaxies: evolution - galaxies: fundamental parameters galaxies: photometry - ultraviolet: galaxies - atlases

\section{Introduction}

There are several compelling reasons for observing nearby galaxies in the ultraviolet (UV). First of all, massive, young stars emit most of their energy in this part spectrum and at least in star-forming galaxies they outshine the emission from any other stage of the evolution of a composite stellar population (e.g. Bruzual \& Charlot 2003). Therefore, the flux emitted in the UV in spiral and irregular is an excellent measure of the current

Star Formation Rate (SFR; Kennicutt 1998; Donas et al. 1987). In the case of quiescent elliptical galaxies the analysis of the UV upturn (the rising part of the FUV spectrum of these galaxies) promises to provide fundamental clues in our understanding of the evolution 
of low-mass stars on the horizontal branch. Due to its remarkable sensitivity to the physical properties of these stars, the UV upturn could be used, once fully understood, as a powerful diagnostic of old stellar populations (Burstein et al. 1988; O'Connell 1999; Yi et al. 1999; Brown 2004; Rich et al. 2005, 2006, in prep.; Boselli et al. 2005). The UV has also revealed the presence of residual star formation in a non-negligible fraction of low-redshift elliptical galaxies (Yi et al. 2005).

Second, the light emitted in the UV can be very efficiently absorbed by dust and then re-emitted at far-infrared (FIR) wavelengths. Therefore an analysis of the energy budget using a comparison of the infrared and UV emission is a powerful tool to determine the dust attenuation of light at all wavelengths (see Buat et al. 2005 and references therein). In this sense, it is worth emphasizing that dust attenuation is the most vexing problem that one has to face when analyzing the observational properties of composite stellar populations and galaxies.

Finally, the observation of nearby galaxies in the UV is fundamental if we are to understand the evolution of galaxies from the high-redshift Universe (where their properties are commonly derived from rest-frame UV observations) to the present.

There have been many attempts in the past to address some of these issues. Sullivan et al. $(2000,2001,2004)$ studied the star formation histories in a relatively large and complete sample of UV-selected local galaxies, from which Treyer et al. (1998) derived the SFR density of the local Universe. The nature of the UV upturn in elliptical galaxies has been widely studied by several groups, including O’Connell (1999), Brown et al. (2000), Deharveng, Boselli, \& Donas (2002). Studies on the dust attenuation in galaxies based on either photometric or spectroscopic UV studies are numerous, including Calzetti et al. (1994), Heckmann et al. (1995), Meurer et al. (1995, 1999), Buat \& Xu (1996), Gordon et al. (2000, 2003), Buat et al. (2002), Roussel et al. (2005). The analysis of the UV morphology of nearby galaxies as a local benchmark for studies in the optical at high redshift have been also carried out by several authors, including Kuchinski et al. (2000, 2001), Marcum et al. (2001), Windhorst et al. (2002), Lauger, Burgarella, \& Buat (2005).

However, the results of some of these studies were not conclusive mainly due to the small size of the samples used, which were not representative of the overall population of galaxies in the local Universe. This is particularly true for studies on the dust attenuation in star-forming galaxies and on the rest-frame UV morphology in nearby galaxies. In the case of the UV-upturn studies in early-type galaxies this limitation adds to the lack of spatial resolution and depth of previous UV data and, in some cases, to the availability of UV data in only one band, which leads to a loss of sensitivity to the strength of the UV upturn, best traced by the FUV-NUV color (see Gil de Paz et al. 2005 and references therein). 
The availability of deep UV observations with moderately-good spatial resolution for large numbers of well-known nearby galaxies is now possible thanks to the launch of the Galaxy Evolution Explorer (GALEX) on April 28th 2003. The compilation of GALEX UV data carried out as part of this paper will allow us (and other researchers making future use of this dataset) to provide fundamental clues for solving some of the still many open questions regarding the UV properties of galaxies in the local Universe. In particular, we will show how the strength of the UV upturn is function of the stellar mass of the galaxy, with more massive elliptical galaxies showing stronger UV upturns. We will also demonstrate that in a sample like ours, which adequately represents the bulk of the galaxy population in the local Universe, the slope of the UV continuum is well-correlated (although with a significant dispersion) with the infrared-to-UV ratio and, therefore, with the UV extinction, and that the $(\mathrm{FUV}-K)$ color provides and excellent segregation between early-type (ellipticals and lenticulars) and late-type (spirals and irregulars) galaxies.

In this "The GALEX Ultraviolet Atlas of Nearby Galaxies" we present surface photometry in the two GALEX ultraviolet (FUV \& NUV) bands, providing integrated photometry and structural parameters for a total of 1034 nearby galaxies, including extensively-studied objects like M31, M32, M 33, M 51, M 81, M 82, M 83, M 87, M 101, etc. We compare the UV properties of this sample with corollary data in the optical, NIR, and FIR, available for the majority of the galaxies in the Atlas. This comparison allows us to obtain insight into fundamental correlations such as the 'red sequence' found in the color-magnitude diagram of ellipticals and lenticulars, and a better definition of the IRX- $\beta$ relation in normal star-forming galaxies.

In Section 2 we extensively describe the sample of galaxies. Section 3 provides a summary of the GALEX observations. The analysis and results are given in Sections $4 \& 5$, respectively. The conclusions are summarized in Section 6 .

\section{Sample}

\subsection{Selection}

The sample of objects in this Atlas includes galaxies in the GALEX Nearby Galaxies Survey (NGS) (Gil de Paz et al. 2004; Bianchi et al. 2003a, 2003b) plus galaxies serendipitously found in NGS fields or in fields with similar or greater depth obtained as part of other GALEX imaging surveys that have optical diameters at the $\mu_{B}=25 \mathrm{mag} \mathrm{arcsec}^{-2}$ isophote larger than 1 arcmin according to the Third Reference Catalog of Bright Galaxies (RC3 hereafter; de Vaucouleurs et al. 1991). Note also that 1 arcmin is the apparent diameter for 
which the RC3 catalog is reasonably complete (Harold G. Corwin, private communication).

As mentioned above the answers to some of the most fundamental open questions on galaxy evolution in general and on the UV properties of galaxies in particular are largely dependent on the (sometimes very large) corrections for dust extinction that must be applied. With this in mind the NGS survey was constructed to optimally sample the UV as provided by GALEX and the FIR (where most of the UV light absorbed by dust is re-emitted) as seen by the Spitzer Space Telescope that would give us a bolometric view of galaxies in the Local Universe. Thus, we began building the NGS sample using Spitzer's Reserved Observations Catalog (ROC v2.0), which guarantees that both UV and infrared data will be eventually made publicly available to the community for all these galaxies. This includes the Spitzer Infrared Nearby Galaxies Survey legacy project (SINGS; Kennicutt et al. 2003), but also data from Guaranteed Time Observations (GTO) programs like The Mid-IR Hubble Atlas of Galaxies (PI: G. Fazio), Starburst Activity in Nearby Galaxies (PI: G. Rieke), Probing a sample of Interacting and Ultra-luminous Galaxies (PI: G. Fazio), etc. The total number of (targeted) NGS galaxies represents approximately one fourth of the total sample of 1034 galaxies in the present Atlas. The vast majority of the UV images of the galaxies observed as part of NGS have exposure times of 1 GALEX orbit or more ( 1700 s). See section 3 for a detailed description of the GALEX spacecraft and instrument.

In order to cover a wider range of physical properties (see Section 3.1) and taking advantage of the large field-of-view of the GALEX instrument (1.2 degrees in diameter) we added to the Atlas sample all galaxies in the RC3 catalog with D25 diameter larger than 1 arcmin that were serendipitously observed within NGS fields and/or within other GALEX surveys of similar or greater depth that were available to the GALEX team, namely the Medium Deep, Deep, and Ultra-deep Imaging Surveys (MIS, DIS, and UIS, respectively). We also added galaxies that were targeted by GALEX because they had been observed by previous UV missions like UIT or FOCA (as an additional calibration test for GALEX) and galaxies from dedicated observations of the central $12 \mathrm{deg}^{2}$ of the Virgo cluster (Boselli et al. 2005).

Of the original 1136 galaxies compiled 26 were found in images that have failed the quality assurance (QA) test of the image aspect solution that is included as part of the standard GALEX pipeline. A total of 55 additional galaxies were excluded either because were observed in regions of high background, high Galactic extinction, during very short orbits and/or they showed extremely low surface brightness in the UV. After excluding these galaxies and those objects with no published redshift (21) we ended up with a total of 1034 galaxies; 893 of them having both FUV and NUV high-quality observations. 


\subsection{Positions, sizes, morphological types, and distances}

In Table 1 we give some basic parameters for the galaxies included in this Atlas, including their positions, sizes, distances, Galactic color excesses $[\mathrm{E}(B-V)]$, and morphological and spectroscopic types.

The coordinates shown (columns 2 and 3) correspond to those given by NED, which are known to be more accurate than those in the RC3 catalog from which a significant fraction of these galaxies were selected (Harold G. Corwin, private communication). A few objects (29) had NED coordinates that were clearly offset from the position of the galaxy both in the UV and the DSS images. In these cases, the new, correct positions were determined by eye after inspecting the corresponding UV images and the Table 1 corrected accordingly. Finally, for two of the objects in the Atlas (UGC 08650 and UGC 11859) a missing/incomplete World Coordinate System (WCS) solution was recomputed using the positions of nearby stars in the USNO-B catalog (Monet et al. 2003). Sizes are the major (column 4) and minor (column 5 ) axis diameters at the $B$-band 25 mag $\operatorname{arcsec}^{-2}$ isophote (computed from the corresponding D25 and R25 values in the RC3 catalog). For those few objects in our sample not included in the RC3 catalog we used the major and minor axis diameters available in NED. Position angles (PA; column 6) missing or incorrect in the RC3 catalog were determined by eye for a total of 90 of the galaxies in the sample after inspecting the corresponding UV images. Note that the PA is undefined in those galaxies for which the D25 isophote is approximately circular according to the RC3.

The distances (columns 7, 8, and 9) to objects with heliocentric recession velocities larger than $500 \mathrm{~km} \mathrm{~s}^{-1}$ were determined using a Virgo-infall corrected radial velocity and a Hubble constant of $70 \mathrm{~km} \mathrm{~s}^{-1} \mathrm{Mpc}^{-1}$. The correction from heliocentric to Virgo-infall corrected velocity was performed in the same way as in the LEDA database (see also Yahil et al. 1977; Theureau et al. 1998; Sandage \& Tammann et al. 1990). The distances to a total of 801 galaxies were computed in this way. Galaxies with radial velocities less than $500 \mathrm{~km} \mathrm{~s}^{-1}$ had distances computed from a variety of methods, including (in approximate order of preference) the period-luminosity (PL) relation of Cepheids, measurement of the $I$-band magnitude of the Tip of the Red Giant Branch (TRGB), the proper motion of masers, Surface-Brightness Fluctuations (SBF), the Globular Cluster luminosity function, the Tully-Fisher relationship, or the brightest stars method (see Table 1 for individual references on each galaxy). For some galaxies believed to be members of interacting pairs, groups or clusters we adopted the distance to the corresponding pair, group or cluster. Examples of this are NGC 1510 and NGC 1512, NGC 5713 and NGC 5719 as members of interacting pairs; NGC 1546, NGC 1549 and NGC 1553 as part of the Dorado group; ESO 059-G007 and ESO 059-G010 as likely members of the NGC 2442 group; NGC 1316, NGC 1317, NGC 1381, NGC 1387, NGC 1399, 
etc., all members of the Fornax Cluster. For a total of 104 galaxies in the Virgo Cluster area the distances were either derived by adopting a three-dimensional structure of the Virgo Cluster and subdivision into clouds, very similar to that of Gavazzi et al. (1999), or directly from the GOLDMINE database (Gavazzi et al. 2003). In the case of the SINGS galaxies we adopted the distances given by Kennicutt et al. (2003).

Galactic color excesses $\mathrm{E}(B-V)$ (column 10) are those available through NED, which correspond to those given by Schlegel et al. (1998).

Regarding the morphological types (columns 11 and 12), we have adopted those given in the RC3 catalog. Galaxies were broadly binned as elliptical/lenticular galaxies when their morphological type, $\mathrm{T}$, was less than -0.5 , spirals if $\mathrm{T}$ was between -0.5 and 9.5 , and irregulars when T exceeded 9.5 (see de Vaucouleurs et al. 1991). For the Blue Compact Dwarf galaxies NGC 1705, NGC 2537, NGC 3125, NGC 4344, and NGC 4861 (see Gil de Paz et al. 2003, 2005), originally classified in the RC3 catalog as ellipticals or spirals, we changed their morphological type to Compact Irregulars $(\mathrm{T}=11)$. Regarding the morphological classification of mergers we should mention that depending on (1) the stage of evolution of the particular merger, (2) distance and resolution of the images from which the system was classified, and (3) the criterion of the person classifying the object, mergers might appear classified as (1) two galaxies each with its own morphological type (e.g. NGC 4038/4039), (2) one single peculiar (NGC 0520) or even spiral (NGC 6052) object, or (3) they might lack any morphological classification (Mrk 8). Finally, spectroscopic types (column 13) were taken from NED.

In Figure 1a (top panel) we show the distribution of galaxies in the Atlas as a function of the $B$-band apparent magnitude for different morphological types (dark gray histogram for ellipticals, gray for spirals, light gray for irregulars) and for the total sample (solidlined histogram). Figure 1b (top panel) shows the distribution of distances. The shape of this latter distribution appears to be the result of combining the RC3 redshift distribution (broken-line histogram) with a large number of very nearby galaxies with distances closer than $50 \mathrm{Mpc}$ included in this Atlas as part of NGS. It is worth noting here that because our sample is effectively limited in magnitude and (to a lesser extent) in diameter we might be missing a fraction of faint, low-luminosity, low-surface brightness galaxies compared with what we would find in a volume-limited sample of the local Universe. In this sense, objects such as the Antlia Dwarf or the dwarf spheroidal satellites around the Milky Way might be common in the field but they would certainly be underrepresented in either a magnitude or a diameter-limited sample. This limitation should be kept in mind when comparing our results with those obtained from the analysis of a volume-limited sample, such as the 11 HUGS sample (Funes et al. 2005) or the Virgo-cluster sample analyzed by Boselli et al. 
(2005).

\section{GALEX observations}

GALEX is a NASA small explorer class mission that orbits the Earth at an altitude of approximately $700 \mathrm{~km}$. The single instrument onboard consists of a 50-cm-aperture RitcheyChrétien telescope equipped with a dichroic beam splitter that allows simultaneous observation in two separate bands, FUV and NUV, within a circular field of view of 1.2 degrees in diameter. The dichroic also acts as a field-aberration corrector. The UV light is detected using microchannel plates with crossed delay-line anodes. The effective wavelength of the two GALEX bands are 1516 and $2267 \AA$, and their full-width at half-maximum are 269 and $616 \AA$, respectively for the FUV and NUV channels. The observations are carried out only at night-time with a typical total usable time per orbit of $\sim 1700 \mathrm{~s}$. For a more detailed description of the spacecraft and the intrument the reader is refered to Martin et al. (2005) and Morrissey et al. (2005).

The observations for this Atlas were carried out by the GALEX satellite between 7 June 2003 and 29 April 2005. The typical exposure time per field was one orbit (specific exposure times are given in Table 2). During periods of intense solar activity, including the historical solar storm occurred on October-November 2003, or during sporadic overcurrent events the FUV detector was turned off to avoid any damage of the electronics. Although in some occasions the NUV detector had also to be turned off, in most of the cases the scheduled observations were still carried out through the NUV channel. Due to this, a total of 141 galaxies in this Atlas (14 per cent of the sample) were observed only in the NUV band. These galaxies are identified as NUV-only in Table 2. Nineteen galaxies were found to be too faint in our FUV imaging data and were analyzed as NUV-only targets as well. Table 2 also provides information regarding the FUV and NUV background for each of the fields along with the mean standard deviation of the sky and the standard deviation of the mean value of the sky accross different regions in the field (see Section 4.2 for details).

Using the GALEX pipeline the photon lists generated by the detectors for each of the bands were processed to produce the corresponding intensity maps in counts per second. The final output products of the pipeline also include a high-resolution response map, which is the product of the effective exposure time by the flat field at a given position and that was used to estimate the photon noise in our images. Note that the images taken as part of the mosaics of M 31 and of the center of the Virgo cluster were generated using a slightly modified version of the pipeline that, nevertheless, preserve both the image quality and absolute flux calibration generated by the standard GALEX pipeline. 
The point spread function (PSF) of the images was found to vary as a function of the count rate with bright point sources usually leading to a wider PSF than faint sources and as a function of the position of the image. For the average count rates usually obtained from nearby galaxies and for objects located within the central 0.5 degrees of the GALEX field the PSF full-width at half-maximum (FWHM) is in the range 4.0-4.5 arcsec and 5.0-5.5 arcsec, respectively for the FUV and NUV bands.

\subsection{Comparison with a magnitude-limited sample}

Because of the rather arbitrary criteria involved in selecting the objects in this Atlas, a comparison of the properties of these galaxies with the overall population of galaxies in the Local Universe is in order if the conclusions derived from this work are to be applied beyond the limits of this Atlas. We have therefore compared the distribution of properties of the galaxies in the Atlas with those included in the Nearby Field Galaxy Survey (NFGS) of Jansen et al. (2000). The NFGS is composed of a total of 196 galaxies that were selected as a representative sub-sample of the magnitude-limited CfA survey (Huchra et al. 1983).

The $B$-band magnitude distributions obtained for both the GALEX Atlas and the NFGS (see Figure 1a) are a direct consequence of the limits of the surveys from which they are derived. That is, while the faint-end of the distribution for the GALEX Atlas is a result of the effective completeness limit of $\sim 15.5 \mathrm{mag}$ inherent in the RC3 (note again that the completeness of the RC3 is also limited to objects larger than 1 arcmin); galaxies in the NFGS show a sharp cutoff in their apparent magnitudes at the limit of the CfA survey, $B \sim 14.5$ mag.

In Figure $1 \mathrm{~b}$ we compare the distribution of distances of both samples and for the whole RC3 catalog. Both distributions are similar, with most of the galaxies found at distances closer than $100 \mathrm{Mpc}$, with a relatively long tail extending to distances up to $200 \mathrm{Mpc}$ and somewhat beyond. The peak in the NFGS distance distribution is intermediate between the local peak in the Atlas sample associated with NGS and the more distant peak of the (slightly deeper in apparent magnitude) RC3 catalog.

Figure 1c shows the comparison between the D25 major-axis diameter of our sample and the NFGS. The major-axis diameters of galaxies in the NFGS were obtained using the UGC catalog major-axis diameters and the morphological-type-dependent transformation coefficients given by the Table 6 of the RC3 catalog (de Vaucouleurs et al. 1991). We find that a total of 52 galaxies in the NFGS $(\sim 27 \%)$ are smaller than 1 arcmin in D25 major-axis

diameter and would be missed by the size limit imposed to the serendipitous part of the 
Atlas sample. Of these galaxies, approximately half are elliptical/lenticular and half spiral galaxies.

Figure 1 demonstrates that the GALEX Atlas and the NFGS, at least in terms of their apparent magnitudes and redshift distributions, are sampling the same volume of the Universe and represent a similar population of galaxies. It is therefore fair to now carry out a more detail comparison between the intrinsic properties of the galaxies in these two samples, including their luminosities, colors, and SFR. At this point it is also worth noting that the relative numbers of ellipticals/spirals/irregulars $(22 \%, 62 \%, 8 \%)^{1}$ in the GALEX Atlas are similar to those found in the field by the NFGS $(28 \%, 65 \%, 7 \%)$.

In Figure 2a we compare the distribution in $B$-band absolute magnitude of both the GALEX Atlas and the NFGS samples. The distribution of both ellipticals/lenticulars and irregulars is pretty similar between both samples. However, in the case of the spirals, although the range of properties covered is also similar, we find a moderate excess of intrinsically bright spirals $\left(-21<\mathrm{M}_{B}<-20\right)$ and a small paucity of low-luminosity spirals $\left(-19<\mathrm{M}_{B}<-18\right)$ compared with the field for the same number of ellipticals/lenticulars and spirals outside these luminosity bins. Note that thanks to the large number of objects in our sample there are still more than twice more low-luminosity spirals $\left(\mathrm{M}_{B}>-19 \mathrm{mag}\right)$ in this Atlas than in the NFGS sample. Similar behavior is seen when the $(U-B)$ colors of both samples are compared (see Figure $2 \mathrm{~b}$ ). In this case the Atlas sample shows a slight paucity of relatively blue (and probably also faint) spirals. Finally, we use the $60 \mu \mathrm{m}$ and $100 \mu \mathrm{m}$ IRAS fluxes to compute the FIR luminosity using the recipe of Lonsdale et al. (1985). The comparison of the FIR luminosities derived for each sample shows that they both cover the same range of properties with a comparable distribution except for the slight excess (paucity) of high (low) FIR luminosity spirals in the Atlas sample (see Figure 2c).

The origin of this small difference in the luminosity distribution of our sample and that of the NFGS might due in part to the size-limit of 1 arcmin in D25 major-axis diameter imposed to the serendipitous part of the Atlas sample. However, since only $23 \%$ of the spirals in the NFGS are smaller than 1 arcmin this effect only accounts for part of the problem. The other reason for this difference in luminosity is probably intrinsic to the rather heterogeneous selection criteria in the original GALEX NGS, which basically includes all galaxies that were in the Spizter ROC at the time the GALEX surveys were planned. In the GALEX NGS we can find targets from many different Spitzer programs which are in many cases biased to large, bright, nearby galaxies with expectedly bright infra-red emission (i.e. bright, nearby spirals).

\footnotetext{
${ }^{1}$ Note that about $9 \%$ of the galaxies in the Atlas do not have morphological types available in the RC3.
} 


\section{Analysis}

\subsection{Color Images}

The left panels of Figure 3 show false-color RGB maps of the galaxies in our sample. The images used are 'asinh' scaling versions (Lupton et al. 2004) of the 2-pixel-smoothed FUV image (blue), the original NUV image (red), and a linear combination of the two (green). The coefficients used to obtain the green-channel image are 0.2 and 0.8 , respectively for the smoothed FUV and original NUV images. For those galaxies with NUV-only data we give the asinh-scaled NUV image. Shown in green is the RC3 D25 ellipse, which was originally derived from $B$-band photometry.

In most of the cases (891 galaxies) the maps shown correspond to a region 1.5 times the D25 major axis diameter in size. In those cases where the UV emission is comparatively more extended than the optical light this factor was increased up to $5 \times \mathrm{D} 25$ for the most extreme cases. The size of the horizontal tick mark plotted at the bottom of each map corresponds to $2 \mathrm{kpc}$ at the distance of the galaxy, except for the case of the Phoenix Dwarf (ESO 245-G007) where it represents a physical size of $0.2 \mathrm{kpc}$. For comparison purposes the central panels of Figure 3 show DSS-1 images for the same field of view.

\subsection{Surface brightness and color profiles}

Using the central position, ellipticity (derived from the corresponding axial ratio) and position angle of the D25 ellipse given in Table 1 we compute the mean surface brightness within elliptical annuli of fixed center and position angle increasing from 6 arcsec in majoraxis radius to at least 1.5 times the D25 radius. The outermost point where the surface photometry was computed corresponds to the size of the postage stamps shown in Figure 3 and which in turn depends on the extension of the UV emission for each individual galaxy. In some cases a few of the outermost points had to be removed from the profiles shown in Figure 3 because either the mean flux in the isophote was below the level of the sky of the photometry errors were extremely large (see below). Point sources with colors redder

than $(\mathrm{FUV}-\mathrm{NUV})=1$ were automatically identified as foreground stars and masked in the GALEX images. These masks were then visually inspected in order to (1) include blue foreground stars and, in a very few cases, to (2) exclude from this automatically-generated mask the nuclei of some galaxies that had been misclassified as foreground stars.

In order to determine the errors in the surface photometry we used the expressions and methodology described in Gil de Paz \& Madore (2005). Errors in the inner parts of 
the profiles are commonly dominated by photon noise, while in the very outer parts the background subtraction uncertainties dominate. It is important to note here that because the background in these images is very low (see Table 2), especially in the FUV channel, the statistics of the background are highly Poissonian, therefore the common estimators, the mean, median and mode can be very different. In particular, the mode in shallow FUV images can be zero. Thus, since our surface photometry uses the mean as the measure of the flux within each isophote we consistently use the mean of the sky as a consistent estimate of the background, after all Sextractor-detected sources are carefully masked (Bertin \& Arnouts 1996). We further masked all pixels in a $5 \times 5$ pixel box around each detected-source pixel so as to avoid possible contamination from the light in the extended wings of the sources.

The background was computed as the mean of the sky value of a total of 90 different regions of 4000 pixels each located around the source and arranged in two concentric elliptical patterns at a distance never closer than 1.5 times the size of the corresponding D25 ellipse. From a comparison of the mean of the standard deviation within each sky region with the standard deviation of the mean of each region we also determine the impact of low-frequency variations in the background (due, for example, to flat-fielding errors) on the total error budget (see Gil de Paz \& Madore 2005 for details).

In the right panels of Figure 3 we show the FUV and NUV surface-brightness (bottom) and (FUV-NUV) color profiles (top) each corrected for Galactic extinction in units of AB magnitudes per square arcsec along with the corresponding $1 \sigma$ errors. Here we have adopted the Galactic color excesses given by Schlegel et al. (1998) and the parametrization of the Galactic extincion law given by Cardelli et al. (1989) for a total-to-selective extinction ratio of $R_{V}=3.1$. The conversion factors are $\mathrm{A}_{\mathrm{FUV}}=7.9 \times \mathrm{E}(B-V)$ and $\mathrm{A}_{\mathrm{NUV}}=8.0 \times \mathrm{E}(B-V)$. The FUV surface-brightness profile shown in Figure 3 is given in blue, the NUV profile in red, and the (FUV-NUV) color profile is in green. The profiles are plotted against the equivalent radius $(\sqrt{a \times b})$ of the corresponding ellipse (expressed both in arcsec and kiloparsecs). The white error bar at the top-right corner of each diagram represents the $\pm 1 \sigma$ uncertainty on the GALEX zero points, which is estimated to be \pm 0.15 mag for both the FUV and NUV channels. Note that all panels are scaled to the same range in surface brightness and color. Hereafter when we refer to (FUV-NUV) it will be the color corrected for Galactic extinction.

In all cases, except the Antlia Dwarf, the outermost point represented in these plots corresponds to the position beyond which either the intensity in the image falls below the level of the sky, or the error in the surface photometry for the NUV band is larger than $0.8 \mathrm{mag}$, whichever happens first. In the case of the Antlia Dwarf galaxy we limited the radial range to that where the contamination from a diffuse Galactic cirrus, located near the position of the galaxy, was still found to be negligible. 


\subsection{Asymptotic magnitudes, colors, and structural parameters}

Using the surface brightness profiles derived and the area of each elliptical annulus we obtained the asymptotic magnitudes by extrapolating the growth curve to infinity. We first computed the accumulated flux and the gradient in the accumulated flux (i.e. the slope of the growth curve) at each radius and perform an error-weighted linear fit to the accumulated flux versus slope of the growth curve plot. After an appropiate radial range was chosen we took the value of the $y$-intercept of this fit as the asymptotic magnitude of the galaxy. This technique is described in detail in Cairós et al. (2001). These authors also tested the stability of this method against the choice of radial range used for the fit and verified its reliability by comparing their results with those obtained using alternative extrapolation techniques. Note that obtaining growth curves and deriving the corresponding asymptotic magnitudes of galaxies at UV wavelengths have been already done in the past (see Rifatto, Longo, \& Capaccioli 1995).

Asymptotic magnitudes and colors along with their corresponding errors are shown in one of the corners of the panels on the right of Figure 3. These errors are composed of a term derived from the error-weighted fit of the growth curve plus a term (in parentheses) due exclusively to uncertaintines in the GALEX FUV and NUV zero points $( \pm 0.15 \mathrm{mag})$. In Table 3 we give the asymptotic AB magnitudes in both the FUV and NUV bands along with the corresponding asymptotic (FUV-NUV) colors. The asymptotic luminosities (in Watts) and the aperture magnitudes and colors inside the D25 elliptical aperture are also provided in this table. The mean differences obtained between the asymptotic magnitudes and the D25 aperture magnitudes are $-0.19 \pm 0.20$ mag and $-0.23 \pm 0.20$, respectively for the FUV and NUV, with the asymptotic magnitudes being brighter. The errors quoted in Table 3 correspond to the error associated with the fit to the growth curve alone.

From the growth curve obtained we also computed the effective radius as the equivalent radius at which the accumulated flux was equal to the asymptotic magnitude plus $0.7526 \mathrm{mag}$ $[2.5 \log (2)]$. In a similar way we derived the radii containing the $20,25,75$, and 80 per cent of the light $\left(r_{20}, r_{25}, r_{75}\right.$, and $r_{80}$, respectively) that were used to compute the concentration indices C31 (de Vaucouleurs 1977) and C42 (Kent 1985) in both UV bands. These indices are defined as

$$
\begin{array}{r}
\mathrm{C} 31=\frac{r_{75}}{r_{25}} \\
\mathrm{C} 42=5 \log \left(\frac{r_{80}}{r_{20}}\right)
\end{array}
$$




\subsection{Morphological classification of the UV profiles}

We have visually classified the UV surface brightness profiles shown in Figure 3 according to their shape. Since most of the profiles (especially in spiral and irregular galaxies) show two distinct regions, our classification scheme uses two letters: the first letter describing the shape of the outer profile and the second one describing the shape of the inner region. In a few cases where we find an excess or depression associated with the nucleus of the galaxy we add a final suffix $\mathbf{n}$ (nucleated) or $\mathbf{h}$ (hole), respectively, to the corresponding morphological class. Also in galaxies showing obvious extended UV emission (XUV; Thilker et al. 2005; Gil de Paz et al. 2005) the morphological class is preceded by the letter $\mathbf{x}$ (eXtended). The classes assigned are given in column 16 of Table 3. Those galaxies that are barely resolved by our GALEX observations have no such classes assigned. The codes used for the morphological classification of the outer region are: $\mathbf{E}$ for exponential, $\mathbf{V}$ for a de Vaucouleurs profile, or ? if there are not enough points in the outer profile to determine which of the two previous laws works best. In the case of the inner profile we use: $\mathbf{E}$ or $\mathbf{V}$ if the profile is a smooth continuation of the corresponding outer profile or if there is a transition from a $\mathbf{E}$ profile in the outer parts to $\mathbf{V}$ in the inner regions, $\mathbf{F}$ for a flattening of the profile toward the inner regions, $\mathbf{D}$ for a profile falling in brightness toward the center, and finally $\mathbf{R}$ for a profile moderately rising in brightness over what it would be expected from and inward extrapolation of the outer profile law. The letter describing the shape of the inner profile appears in lower case if the radial extension of the inner profile is significantly smaller than that of outer profile. In this scheme galaxies with pure de Vaucouleurs (exponential) profiles would be classified as VV (EE) type. Note that the majority of the galaxies in this Atlas are extracted from GALEX fields of similar depth ( $\sim 1$ orbit) that were obtained as part of the Nearby Galaxies Survey (NGS) or the Medium-deep Imaging Survey (MIS). In the majority of the cases the same classification does apply to both the FUV and NUV profiles. In the few cases where the profiles differ enough to be placed in different classes we give first the FUV and then the NUV morphological class separated by a comma (e.g. NGC1055, NGC1386, NGC1546)

\subsection{Corollary data}

In order to compare the UV properties of the galaxies in this Atlas with those known from previous multi-wavelength surveys we have compiled a large amount of corollary data on this sample (see Table 4). Of the 1034 galaxies in the Atlas a total of 871 (84\%) have asymptotic $B$-band photometry available in the RC3 catalog. We primarily used the $B_{T}$ magnitude and only when $B_{T}$ was unavailable we made use of the $\mathrm{m}_{B}$ magnitude instead. 
A total of 318 (393) galaxies also have asymptotic $U(V)$ magnitudes published in the RC3.

In addition we have also compiled integrated $J H K$ magnitudes from 2MASS. In the first instance we adopted the $J H K_{\text {tot }}$ magnitudes from the 2MASS Large Galaxy Atlas (LGA) of Jarrett et al. (2003). For those objects not in the 2MASS LGA we used the total $J H K_{\text {total }}$ magnitudes given in the Final Release of the 2MASS Extended Source Catalog (XSC). A total of 853 galaxies in the Atlas had $K$-band data available.

The optical and near-infrared magnitudes given in Table 4 are observed values. The corresponding Galactic extinction-corrected magnitudes were derived using the color excesses given in Table 1 and the extinction law of Cardelli et al. (1989) for $R_{V}=3.1$.

Finally, we compiled IRAS photometry using data from (in order of priority) Rice et al. (1988), Knapp (1994, private communication), the IRAS Point Source Catalog (PSC), and Moshir et al. (1990). A total of 459 galaxies had IRAS detections at both 60 and 100 micron. These two bands are required in order to estimate the total infrared emission of the galaxy and from it the total energy budget by means of its comparison with the UV flux (see e.g. Dale et al. 2001).

\section{Results}

\subsection{Global statistical properties}

In Figure 4 we show the frequency histograms of the asymptotic FUV and NUV AB magnitudes, FUV luminosity and (FUV-NUV) (both asymptotic and at the D25 aperture) color. Heckman et al. (2005) have recently shown that galaxies with FUV luminosities brighter than $2 \times 10^{10} \mathrm{~L}_{\odot}\left(7.6 \times 10^{36} \mathrm{~W}\right.$ or $\left.\mathrm{M}_{\mathrm{FUV}}=-19.87\right)$ (also known as ultraviolet-luminous galaxies or UVLGs) are extremely rare in our Local Universe. Their comoving space density is only $\sim 10^{-5} \mathrm{Mpc}^{-3}$, i.e. several hundred times lower than that of their $z=3$ counterparts, the Lyman Break Galaxies (LBG). Indeed, only four galaxies in the Atlas (see Figure 4c) would be classified as UVLGs: two AGN, NGC 7469 and Mrk 501, and two actively starforming interacting systems, the Cartwheel (see e.g. Amram et al. 1998) and UGC 06697 (Gavazzi et al. 2001).

The color distribution of Figure $4 \mathrm{~d}$ shows a pronounced peak at $(\mathrm{FUV}-\mathrm{NUV}) \simeq 0.4 \mathrm{mag}$ and a long tail extending to very red colors. As we will show later, this red tail is, not unexpectedly, mostly populated by elliptical galaxies of intermediate mass that show little recent star formation activity and a weak UV-upturn (see Boselli et al. 2005). This figure also shows the distribution of effective radii both in arcsec (Figure 4e) and in kiloparsecs 
(Figure 4f). The distribution of effective radii is very similar for the FUV and the NUV. Due to the limited spatial resolution of the GALEX data we only computed the effective radius of galaxies for which the semi-major axis of the ellipse including 50 per cent of the light was larger than 6 arcsec in radius. This fact, along with the lower limit in optical diameter ( 1 arcmin) imposed by the completeness of the RC3, results in a paucity of compact galaxies and a relatively narrow distribution in apparent effective radius peaking at $\sim 15$ arcsec. The distribution in physical size (Figure 4f), on the other hand, is significantly wider with a peak around 5-6 kpc.

The distributions of the concentration indices C31 and C42 (Figures 4h \& 4i, respectively) are also very narrow with the galaxies being slightly more concentrated (i.e. larger values of $\mathrm{C} 31$ and $\mathrm{C} 42$ ) in the NUV than in the FUV (see Figures 4j \& 4k for a comparison between the value of these indices in the two bands). This is probably a consequence of the fact that in the NUV a significant fraction of the light in spiral galaxies still arises from within the bulge component, while in the FUV this contribution is in many cases negligible.

\subsection{Properties by morphological type}

The GALEX FUV and NUV observations presented here, along with the corresponding corollary data in the optical, NIR and FIR provides us with an unprecedented set of multiwavelength data for a large population of galaxies in the local Universe. One of the first questions that can be addressed using this sample concerns the relation between the qualitative (optical) morphology of these galaxies and more quantitative properties, such as colors, luminosities, total-infrared-to-UV ratios, etc. In Figure 5 we show the colors of the galaxies as a function of the blue-light morphological type as given by the RC3. Panels $5 \mathrm{a} \& 5 \mathrm{~b}$ show that although late-type spiral and irregular galaxies are somewhat bluer in $(B-V)$ and $(B-K)$ than ellipticals and early-type spirals, these colors are not unique to a given type. In particular, these colors cannot be used to unambiguously discriminate between different kinds of spiral galaxies nor even between elliptical/lenticular galaxies and spirals. As indicated by Roberts \& Haynes (1994), the significant ovelap in $(B-V)$ color between spiral galaxies of different types is mostly due to true variations in the optical colors and star-formation history of galaxies of same morphological type, not to misclassification or observational errors. The equivalent to the Panel 5b for late-type Virgo cluster galaxies was obtained by Boselli et al. (1997). These authors obtained a large overlap in $(B-K)$ color between different morphological types as well.

However, thanks to the extreme sensitivity of the FUV data to the presence of very low levels of recent star formation activity, the use of the (FUV-K) color turns out to be 
a very powerful discriminant between quiescent elliptical and lenticular galaxies, and starforming spirals. In particular, an observed $(\mathrm{FUV}-K)$ color of 8.8 mag provides an excellent discrimination point between these two groups (see Figure $5 \mathrm{c}$ ). In this sense, of all the elliptical/lenticular galaxies in the Atlas with both FUV and $K$-band data available only $23 \%$ of them show a $(\mathrm{FUV}-K)$ color bluer than this threshold. It is worth noting that significant a fraction of these are known to host some residual star formation activity (e.g. NGC 3265, Condon, Cotton, \& Broderick 2002 and NGC 0855, Wiklind, Combes, \& Henkel 1995), or are low-luminosity ellipticals with obvious star formation activity like NGC 1510 (Marlowe, Meurer, \& Heckman 1999). Spiral and irregular galaxies with (FUV-K) colors redder than this value only represent $9 \%$ of the total.

Although with significantly degraded discriminating capabilities compared to the (FUV-K) color, the $(\mathrm{NUV}-K)$ is also well correlated with the morpholotical type (see Figure $5 \mathrm{~d}$ ). The same can be said about the (FUV-NUV) color, where a cut-off at (FUV-NUV) $=0.9 \mathrm{mag}$ provides a relatively clean separation of elliptical/lenticular galaxies from spirals (Figure 5e). The fraction of elliptical/lenticular galaxies with (FUV-NUV) color bluer than 0.9 mag (and both FUV and NUV magnitudes available) is $18 \%$ while the percentage of spiral and irregulars redder than this value is only $12 \%$. Note that in this case the far-left lower corner of the diagram may be populated both by ellipticals with residual star formation and also by elliptical galaxies with a strong UV-upturn (Deharveng, Boselli, \& Donas 2002 and references therein). The best linear fits derived for the correlation of observed colors with the morphological type for spirals and irregulars (types $\mathrm{T}>-0.5$ ) are

$$
\begin{array}{r}
(\mathrm{FUV}-K)=7.97-0.48 \times T \quad ; \quad \sigma=1.36 \mathrm{mag} \\
(\mathrm{NUV}-K)=7.07-0.40 \times T \quad ; \quad \sigma=1.14 \mathrm{mag} \\
(\mathrm{FUV}-\mathrm{NUV})=0.854-0.066 \times T \quad ; \quad \sigma=0.32 \mathrm{mag}
\end{array}
$$

These relations are shown in Figures $5 \mathrm{c}, 5 \mathrm{~d}$, \& 5e. Note that although the r.m.s. of the fit for the $(\mathrm{FUV}-\mathrm{NUV})$ color is smaller than for $(\mathrm{FUV}-K)$ this is purely a consequence of the much smaller dynamic range of the (FUV-NUV) color (1 mag) compared with the (FUV $-K)$ color $(\sim 6 \mathrm{mag})$ (see Figure $5 \mathrm{c}$ ). The corresponding best fits in the type $\mathrm{T}$ versus color diagrams are (only galaxies with types $\mathrm{T}<13$ are considered)

$$
\begin{array}{rrll}
T=11.2-1.28 \times(\mathrm{FUV}-K) & ; & \sigma=2.4 & (\text { in units of T) } \\
T=12.1-1.62 \times(\mathrm{NUV}-K) & ; & \sigma=2.5 & (\text { in units of } \mathrm{T}) \\
T=8.4-8.5 \times(\mathrm{FUV}-\mathrm{NUV}) & ; & \sigma=3.0 & (\text { in units of } \mathrm{T})
\end{array}
$$

These fits are valid only for colors $(\mathrm{FUV}-K)<8.8 \mathrm{mag}$, (NUV $-K)<7.9 \mathrm{mag}$, and (FUVNUV) $<0.9$ mag, respectively. 
Finally, in Figure $5 f$ we compare the total-infrared (TIR hereafter) to FUV ratio with the morphological type of the galaxies in the Atlas. The TIR flux was derived using the parameterization of the TIR-to-FIR ratio given by Dale et al. (2001), where FIR is computed from the 60 and 100 micron IRAS fluxes as in Lonsdale et al. (1985). The flux in the FUV is expressed in units of $\nu \mathrm{F}_{\nu}$ (see Buat et al. 2005). In the case of spiral and irregular galaxies, for which both the UV and infrared emission are ultimately due to young massive stars, this ratio provides a well defined estimator of the dust attenuation in the UV (Buat et al.. 2005; Cortese et al. 2006). Given the sensitivity limits of the IRAS catalog and the low dust content of elliptical and lenticular galaxies the number of these galaxies detected in both the 60 and 100 micron IRAS bands is only 49 out of the 225 ellipticals in the Atlas. Figure $5 f$ shows that late-type spirals and irregulars tend to show, on average, a lower TIR-to-FUV ratio and consequently smaller attenuation in the UV than that derived for early-type spirals.

\subsection{Color-magnitude and color-color diagrams}

Although morphology is certainly related with the way galaxies form and evolve, especially when the properties of elliptical and spiral galaxies are compared, the luminosity and even more the mass (either the luminous or total mass) is thought to be the main driving force of the evolution of galaxies through the history of the Universe. In this sense, the analysis of color-magnitude diagrams (CMD) has traditionally provided a fundamental tool for understanting galaxy evolution.

Figures 6a and 6b show the CMD in $(\mathrm{FUV}-K)-\mathrm{M}_{K}$ and $(\mathrm{NUV}-K)-\mathrm{M}_{K}$. At the top of these diagrams we find the 'red sequence' populated primarily by elliptical and lenticular galaxies (dots). In the case of the $(\mathrm{NUV}-K)-\mathrm{M}_{K} \mathrm{CMD}$ the red sequence shows a clear slope with lower luminosity galaxies showing bluer colors, especially below $\mathrm{M}_{K}>-23 \mathrm{mag}$. A similar behavior is seen when optical or optical-NIR colors are used, both locally and at high redshift (Gladders \& Yee 2005). This is commonly explained in terms of lower metal abundances (thus bluer colors) of the stellar populations in low mass ellipticals as compared to the more massive (higher metallicity) systems (Gladders et al. 1998 and references therein). In the case of the $(\mathrm{FUV}-K)-\mathrm{M}_{K} \mathrm{CMD}$, on the other hand, the distribution of the $(\mathrm{FUV}-K)$ color is rather flat over a range of almost $7 \mathrm{mag}$ in absolute magnitude. The explanation for this different behavior can be found in Figure 6c. Here the (FUV-NUV) gets systematically

redder as we move to lower luminosities. This is opposite to what is seen in any other colors and it is probably a consequence of a weaker UV-upturn in intermediate-mass ellipticals than in the most luminous and massive ones (see Boselli et al. 2005). Note that, due to the stronger UV-upturn towards the centers of elliptical galaxies (Ohl et al. 1998; Rhee et al. 
2006, in preparation), the asymptotic colors do not probably show the full strength of the UV-upturn in the way aperture colors like those obtained from the analysis of IUE spectra do (Burstein et al. 1988).

Dwarf elliptical galaxies have $K$-band absolute magnitudes that are typically fainter than $\mathrm{M}_{K}=-21$ mag. Unfortunately, not many of these more extreme low-luminosity ellipticals are found in the Atlas. This is mainly because dwarf ellipticals in Virgo (where most of the studies on $\mathrm{dE}$ have been carried out to date) are typically smaller than 1 arcmin in size placing them outside the selection limit imposed on the Atlas. Nevertheless, a recent study by Boselli et al. (2005) suggests that residual star formation might play a leading role in the interpretation of the UV emission from dE galaxies, which would explain their behavior in the CMD (i.e. similar to the behavior seen in low mass star-forming galaxies). The tendency for the most luminous ellipticals to show bluer (FUV-NUV) colors is even more clear when the FUV-band absolute magnitude is considered (see Figure 6e). However, if the $B$-band luminosity is used, the (FUV-NUV) color seems to be independent of luminosity.

Regarding the properties of spiral (triangles) and irregular galaxies (asterisks) in these plots we find that the majority of these galaxies are concentrated in a 'blue sequence' with high-luminosity spirals (which also tend to be of earlier types) being redder than lowmass spirals and irregular/compact galaxies. This is true for all the observed (FUV-K), $($ NUV $-K$ ), and (FUV-NUV) colors (Figures 6a, 6b, \& 6c). There are two mechanisms that may lead to the observed behavior. First, low luminosity galaxies are known to have lower metallicities (both in the stars and in the gas) than more luminous ones (Salzer et al. 2005 and references therein). This implies that the amount of dust (and reddening of the colors) in low-luminosity galaxies should be lower than in luminous ones.

The $(\mathrm{FUV}-\mathrm{K})[(\mathrm{NUV}-\mathrm{K})]$ color is found to span a range of $5 \mathrm{mag}[4 \mathrm{mag}]$ in spiral and irregular galaxies of different types and luminosities with a mean value of $5.9 \mathrm{mag}$ [ $5.4 \mathrm{mag}$ ]. The corresponding 1-sigma of the distribution is $1.7 \mathrm{mag}[1.4 \mathrm{mag}]$. On the other hand, the dispersion in the $A_{F U V}\left[A_{N U V}\right]$ derived is only $1.0 \mathrm{mag}[0.8 \mathrm{mag}]$ (see below). Since the $\mathrm{A}_{\mathrm{FUV}} /\left(\mathrm{A}_{\mathrm{FUV}}-\mathrm{A}_{K}\right)\left[\mathrm{A}_{\mathrm{NUV}} /\left(\mathrm{A}_{\mathrm{NUV}}-\mathrm{A}_{K}\right)\right]$ total-to-selective extinction ratio is always between 1.0 and 1.1 for any attenuation law considered, dust extinction alone is not able to explain the dispersion in the observed $(\mathrm{FUV}-\mathrm{K})[(\mathrm{NUV}-\mathrm{K})]$ color neither its dependence on luminosity or morphological type.

It is now widely accepted that the star formation history of galaxies depends strongly on their stellar or total mass. Low mass galaxies show relatively flat star formation histories, while more massive systems have shorter timescales of formation (e.g. Gavazzi et al. 1996, 2002; Gavazzi \& Scodeggio 1996; Boselli et al. 2001). By virtue of this phenomenon, sometimes simplistically referred to as 'down-sizing' (see Cowie et al. 1996), low-mass galaxies 
should be on average bluer in these colors than more massive galaxies. In this sense, we know that the typical stellar mass of a star-forming galaxy in the local Universe is $\sim 1.3 \times 10^{10} \mathrm{M}_{\odot}$ (Pérez-González et al. 2003; Gil de Paz et al. 2000), i.e. more than five times less massive than a L* galaxy in the NIR (Cole et al. 2001; Kauffmann et al. 2003).

Since we have information about the TIR emission for a large fraction of these galaxies we can compute the attenuation in the FUV and NUV from the observed TIR-to-FUV ratio using the recipes published by Buat et al. (2005). The mean and 1-sigma FUV [NUV] attenuation of the sample of spiral and irregular galaxies in the Atlas is $1.8 \pm 1.0 \mathrm{mag}$ [ $1.3 \pm 0.8 \mathrm{mag}]$. The extinction-corrected (FUV-NUV) color is plotted in Figure $6 \mathrm{~d}$ as a function of the $K$-band absolute magnitude. The solid (dashed) line shown in this plot represents the best weighted (non-weighted) fit to the data

$$
\begin{array}{r}
(\mathrm{FUV}-\mathrm{NUV})_{0}=0.1083+0.00371 \times \mathrm{M}_{K} \quad ; \quad \sigma=0.054 \mathrm{mag} \text { (weighted) } \\
(\mathrm{FUV}-\mathrm{NUV})_{0}=0.0942+0.00299 \times \mathrm{M}_{K} \quad ; \quad \sigma=0.055 \mathrm{mag} \quad \text { (non - weighted) }
\end{array}
$$

Although there is a small tendency for the galaxies to show redder UV colors at lower luminosities and later types, we do not exclude the possibility that the intrinsic (FUV-NUV) color derived in this way is independent of luminosity with an average value of (FUV-NUV) $)_{0}=$ $0.025 \pm 0.049 \mathrm{mag}$ (i.e. $\beta_{\mathrm{GLX}, 0}=-1.94 \pm 0.11$; see Kong et al. 2004). We should note here that the measurements of the extinction in the FUV and NUV from which this intrinsic (FUV-NUV) color is derived are not fully independent since both are obtained by comparing the corresponding observed FUV and NUV flux with the same total-infrared emission (Buat et al. 2005). Consequently, there might be some additional weak dependency of the intrinsic (FUV-NUV) color with the luminosity that could be identified by analyzing both the detailed star formation history and dust properties (composition, geometry, temperature distribution) of individual galaxies.

Figure 6e shows that the most luminous galaxies in the FUV are spirals (both earlyand late-type ones). In the optical (Figure 6f) and NIR (Figure 6c), on the other hand, the bright end of the luminosity function is populated by both elliptical and spiral galaxies. It is also worth noting that the galaxies in the bright end of the FUV luminosity function show a very narrow dispersion in the observed (FUV-NUV) color, that results in a very similar shape for the bright end of the FUV and the NUV local luminosity functions (Wyder et al. 2005).

In Figure 7a we analyze the (FUV-NUV)-(NUV-B) color-color diagram of the galaxies in the Atlas. It is remarkable the relatively narrow strip of this diagram where the galaxies are located. In the case of the spiral galaxies this is due in part to the well-known degeneracy in these colors between dust extinction and star formation history (see e.g. Gil de Paz \& 
Madore 2002). The ellipticals show a very narrow range in (NUV-B) color but a wide range of (FUV-NUV) colors, probably due to differences in the strength of the UV-upturn from galaxy to galaxy. In the (FUV-NUV)-(NUV-K) color diagram (Figure $7 \mathrm{~b}$ ) we find that ellipticals with redder (NUV-K) color tend to show bluer (FUV-NUV) colors. This is again a consequence of the weaker UV-upturn present in optically blue, intermediate-mass ellipticals. The combination of the (FUV-NUV) color with either the (NUV-B) or the (NUV-K) color clearly improves the discrimination between elliptical/lenticular galaxies and spirals (see broken lines in Figures 7a \& 7b). In the case of the (FUV-NUV)-(NUV-B) color-color diagram the origin $\{$ destination $\}$ of the cut-off line is $[(\mathrm{FUV}-\mathrm{NUV}),(\mathrm{NUV}-B)]=[2.0,2.0]$ $\{5.0,0.0\}$. For the (FUV-NUV)-(NUV-K) color-color diagram the corresponding origin $\{$ destination $\}$ of the cut-off line is $[(\mathrm{FUV}-\mathrm{NUV}),(\mathrm{NUV}-K)]=[5.0,1.7]\{9.5,0.4\}$.

\subsection{Dust extinction and the IRX- $\beta$ relation}

The relation found by Heckman et al. (1995) and Meurer et al. $(1995,1999)$ between the TIR-to-FUV ratio and the slope of the UV spectrum in starburst galaxies (IRX- $\beta$ relationship; see also Seibert et al. 2005) can be used in principle to estimate the dust extinction in galaxies even if FIR data are not available. Some recent works have claimed that this relationship is valid only when applied to UV-selected starburst galaxies but not in the case of infrared-bright objects like the luminous/ultra-luminous infrared galaxies (LIRGs/ULIRGs; Goldader et al. 2002) or even for normal spiral or irregular galaxies (see Bell et al. 2002 for results on the LMC). In Figure 8a we compare the TIR-to-FUV ratio with the observed (FUV-NUV) color, which is equivalent to the slope of the UV continuum (see Kong et al. 2004). Here we have only plotted galaxies with observed (FUV-NUV) color bluer than 0.9 mag. This criterion guarantees that the vast majority of the objects considered are either spiral or irregular galaxies. The dotted line represents the IRX- $\beta$ relation given by Meurer et al. (1999). This figure demonstrates that the slope of the UV is indeed well correlated with the TIR-to-FUV and can be used to estimate (at least in a statistical way) the dust extinction in nearby galaxies. Similar results are found by Cortese et al. (2006) using a volume-limited optically-selected sample of galaxies in nearby clusters.

The solid line in Figure 8a represents the best linear fit to the data. The dashed line is the same but excluding objects with luminosities below $0.1 \times \mathrm{L}^{*}\left(\mathrm{M}_{\mathrm{FUV}}^{*}=-18.12\right.$; Wyder et al. 2005), for which the relation begins to depart from linearity. The results of these fits are

$$
\begin{array}{r}
\log (\mathrm{TIR} / \mathrm{FUV})=-0.18+2.05 \times(\mathrm{FUV}-\mathrm{NUV}) \quad ; \quad \sigma=0.36 \mathrm{dex} \\
\log (\mathrm{TIR} / \mathrm{FUV})=-0.15+2.00 \times(\mathrm{FUV}-\mathrm{NUV}) \quad ; \quad \sigma=0.36 \mathrm{dex} \quad\left(\text { for } \mathrm{L}>\frac{\mathrm{L}^{*}}{10}\right)
\end{array}
$$


Note that our sample suffers of a small deficiency of low-luminosity spirals. This fact might have an impact on the best-fit IRX- $\beta$ relationship derived above. Cortese et al. (2006) have recently proposed a set of recipes that can be used to estimate the TIR-to-FUV ratio in star-forming galaxies using not only the (FUV-NUV) color but other parameters such as the oxygen abundance, the luminosity, the mean surface brightness, etc.

The majority of the objects in Figure 8a are found below the relationship defined for starburst galaxies. It is worth noting that objects with higher UV luminosity, some of them starburst galaxies, seem to fall closer on average [at least in the region with $($ FUV $-N U V)<0.6 \mathrm{mag}]$ to Meurer et al.'s relation than lower luminosity galaxies. According to Kong et al. (2004) the offset between normal galaxies and starbursts is primarily due to a lower ratio of present to past-averaged SFR in normal galaxies. However, the results obtained by Seibert et al. (2005) and Cortese et al. (2006) using GALEX data of nearby galaxies do not support this idea. These recent studies suggest that this offset might be due instead to a different geometry of the dust in normal galaxies compared with starbursts or, alternatively, to aperture effects present in the IUE dataset used by Meurer et al. (1999).

The fact that we find such a good correlation between the TIR-to-FUV ratio and the (FUV-NUV) color and that the intrinsic (FUV-NUV) color seems to be rather constant for spiral and irregular galaxies suggests that the attenuation law in the UV for these galaxies is different from a pure Galactic extinction law. In the case of the Milky Way the extinction law shows a bump at $2175 \AA$ that would result in a similar extinction in both bands, $\mathrm{A}_{\mathrm{FUV}}=7.9 \times \mathrm{E}(B-V)$ and $\mathrm{A}_{\mathrm{NUV}}=8.0 \times \mathrm{E}(B-V)$ (Bianchi et al. 2005). Thus, the observed trend in the (FUV-NUV) color with the TIR-to-FUV ratio is most probably due to a different extinction law since scattering, either for a shell or clumpy dust geometry, would result in an even lower FUV attenuation (compared with the NUV) than that expected from the Galactic extinction law alone (see e.g. Roussel et al. 2005). The SMC Bar or 30 Doradus extinction laws and the attenuation law proposed by Calzetti et al. (1994) all show a weak $2175 \AA$ feature and, especially in the case of the SMC Bar extinction law, a relatively steep FUV rise. In this sense, despite of including scattering, the FUV rise of the Calzetti law is apparently too modest to reproduce the dependence between $A_{F U V}$ and $A_{F U V}-A_{N U V}$ followed by the majority of the galaxies in our sample (see Figure $8 b)^{2}$. Thus, although the Calzetti law, originally built for UV-bright starburst galaxies, still provides an adequate approximation to the relation between $A_{F U V}$ and $A_{F U V}-A_{N U V}$ for galaxies with UV luminosities above $L^{*}$, an attenuation law based on the SMC-Bar extinction law is favored for

\footnotetext{
${ }^{2}$ We have adopted $R_{V}=3.1$ for the Milky Way and LMC 30 Doradus extinction laws (Cardelli et al. 1989), $R_{V}=4.05$ for the Calzetti law (Calzetti et al. 2000), $R_{V}=2.87$ for the SMC Bar law (star AzV 398; Gordon \& Clayton 1998), and $R_{V}=2.66$ for the SMC Wing law (star AzV 456; Gordon \& Clayton 1998)
} 
the bulk of the galaxies in this Atlas.

We cannot exclude, however, that the FUV emission might be arising from young stars more deeply embedded in their parent molecular clouds than those responsible for the NUV emission. If that is the case, the differential extinction between the FUV and NUV emitting sources would lead to an artificial FUV rise in the global attenuation law even if the extinction law is rather flat in the UV.

In this same sense, it is worth noting that here we are referring to the attenuation law of the dust associated with the regions responsible for the UV emission, which could be quite different from the law we would obtain from regions dominating the emission at other wavelengths and also different from the extinction law that would be derived from line-of-sight absorption studies of individual stars.

\subsection{Structural properties and UV morphology}

Concentration indices have been commonly used in the past to infer the morphological types of barely resolved intermediate redshift galaxies found in HST images (see e.g. Abraham et al. 1996). One of the problems associated with these studies is the fact that in many cases the concentration indices derived for the high-redshift galaxies are measured in the restframe UV while the local reference samples are usually observed in the optical (Bershady, Jangren, \& Conselice 2000). In this sense, it is important to know the structural parameters in the UV of a sample of well-known nearby galaxies, like the one collected for this Atlas. Concentration indices C31 and C42 are provided in Table 3. The number of objects with these indices is small because we only computed the C31 (C42) concentration index for those galaxies whose radius containing $25 \%(20 \%)$ of the light was larger than 6 arcsec. The same criterion applies to the effective radius, where the radius containing $50 \%$ of the light was imposed to be larger than 6 arcsec in order for it to be measured.

In Figure 9 we compare the concentration index $\mathrm{C} 42$ with the $(\mathrm{FUV}-K)$ color. As we commented in Section 5.2 this color discriminates very well between elliptical and spiral galaxies and also between spiral galaxies of different types (see Section 5.2). This figure shows that the C42 index improves the discrimination between ellipticals (dots) and lenticulars (open circles) and also between these and early-type spirals (open triangles). Joe et al. (2006, in preparation) have recently carried out a more detail study of the structural properties (including both concentration and asymmetry parameters) of nearby galaxies in the UV using the same sample presented in this Atlas.

Regarding the morphological classification of the UV surface-brightness profiles we first 
notice a large variety of morphologies even within each of the classes defined in Section 4.4. This is partly a consequence of the high sensitivity of the UV to the recent star formation which results in the presence of structures having relatively short evolutionary time-scales that might dominate the UV profiles but that are not as obvious in the optical or NIR profiles. There is also the difficulty of dealing with degeneracies between some morphological classes. In this sense, some of the Blue Compact Dwarf galaxies in the sample could be easily classified as having ER or EV profiles. Also, some of the profiles inspected could be either classified as EEh or Ed. Despite of these issues we successfully classify the profiles of 970 of the 1034 galaxy in the Atlas. Moreover, we find that most of the galaxies (615 out of 970) have UV profiles that can be grouped in three main classes: (1) profiles that can be reproduced entirely by a de Vaucouleurs law (class VV), (2) pure exponential profiles (class EE), (3) profiles with an exponential component in the outer region and significant flattening in the inner region (EF and Ef classes). Only 19 galaxies were classified as EV class, despite being the dominant morphology in the optical and near-infrared profiles of spiral galaxies.

This paucity of EV profiles seems to be due, at least in the case of late type spirals, to the fact that even in the central regions the bulge is much fainter than the disk, which results in these galaxias being classified as having type EE or EF/Ef profiles (e.g. NGC 0628, M 33, NGC 1042, NGC 2403). In early-type spirals, like the Sb galaxies NGC 0986, M 31, M 81, M 95, the bulge is dominant only in the nucleus of the galaxy where is also commonly found associated with a flattening or decrease in the surface brightness of the disk toward the center. Because of the small spatial extension of these bulges in the UV surface brightness profiles Sb galaxies get usually classified as EFn, VFn, or EDn. Only lenticulars (e.g. NGC 1387, NGC 1546, NGC 4310, NGC 4477, NGC 6945, NGC 7252, M 86), intermediate S0/a (NGC 2681, NGC 3816, NGC 3885, IC 0796), or very early-type spirals like the Sa galaxies NGC 1022, NGC 2798, NGC 4314, or NGC 4491, are sometimes best classified as having EV-type UV profiles. This is true for both UV bands although it is more frequent in the case of the NUV profile.

In Figure 10 we plot the distribution of galaxies classified within each of these groups: de Vaucouleurs profiles (v), pure exponential profiles (e), and flattened exponential profiles $(\mathbf{f})$; in the $(\mathrm{FUV}-K)$ versus morphological type diagram. Again, the morphological types used are those published in the RC3. In the light of this figure it is fair to say that the majority of the elliptical galaxies in the Atlas follow a de Vaucouleurs profile in the UV, like is the case of the optical and NIR profiles of luminous elliptical galaxies. Note that because of our selection limits a small number of dwarf elliptical galaxies (which commonly show exponential light profiles in the optical) is expected to be found in this Atlas. A few v-type galaxies classified morphologically as late T-type objects are found to be well-known Blue Compact Dwarf (BCD) galaxies: NGC 1569, NGC 3125, NGC 5253, NGC 6789, UGC 05720 
(Haro 2). See Doublier et al. $(1997,1999)$ for some other examples of BCD galaxies with $\mathrm{R}^{1 / 4}$ profiles in the optical.

Regarding the distribution of the other two types of profiles we point out that while galaxies with pure exponential profiles (a total of 173) are widely distributed in morphological type and color, galaxies with flattened exponential profiles (269) have, in the majority of the cases, morphological types $\mathrm{T}$ in the range $2<\mathrm{T}<8$, i.e. they are truly spiral galaxies. In order to explain this behavior is necessary to understand first what is the mechanism(s) behind the flattening of the UV profiles.

In the spectro-photometric models of the evolution of disk galaxies of Boissier \& Prantzos (2000; see also Boissier 2000), a similar flattening in blue bands is obtained. The main reason for it is that the rate the stars formed (i.e. SFR) in the inner disk has been higher than the infall of gas, leading to a progressive consumption of the gas in these regions. In the outer parts, however, star formation is less efficient and infall proceeds on longer timescales. As a result, the gas reservoir of the outer disk is not exhausted, and the shape of the exponential profile is preserved (in adition, an extinction gradient could enhance the difference between the inner regions, metal and dust rich, and outer regions suffering low metallicity and low extinction).

The dependence of the degree of flattening with the morphological type found, with most galaxies showing flattened-exponential profiles having types Sab-Sdm, is probably a consequence of the fact that (1) early-type galaxies have already consumed the majority of their gas at all radii, due to a high global star formation efficiency and low current infall, and (2) late-type spirals, because of their current large supply of gas and infall, still have enough gas to prevent its consumption at all radii. Note also that in some very early type spirals (S0/a and Sa types) the presence or a relatively bright bulge might also difficult the detection of any flattening in the inner-disk profile.

The models referred above use as parameters the circular velocity (i.e. total mass), and the spin parameter (i.e. angular momentum). For a fixed spin parameter, the degree of flatenning should depend on mass since e.g. the infall time-scale depends on the mass. Indeed, at very low mass a modest flattening occurs, a more visible one at intermediate mass, and no flatenning again in very massive galaxies (where the gas has been consumed over the whole galaxy) (Boissier 2000). However, using the K-band absolute magnitude as a tracer of the total mass of the system we found no difference between the distribution of galaxies with or without flattening in their profiles. This disagreement with the naive expectation from the models could be linked to the existence of the second parameter (at fixed velocity, the flattening of the star formation rate is more noticeable for smaller spin parameters), or more fundamental differences between EF/Ef and EE galaxies, not yet included in models. 
A more direct measure of the total mass and spin parameter, or detailed modeling of these galaxies (or a sub-sample of them) could help us to understand what makes the EF/Ef galaxies different from the EE ones.

\section{Conclusions}

We have presented an imaging Atlas of 1034 galaxies observed in two UV bands by the GALEX satellite. From these we have derived surface brightness and color profiles in the FUV \& NUV GALEX bands. Asymptotic magnitudes and colors along with concentration indices have also been obtained. A morphological classification of the profiles is also carried out. Despite a small but non-negligible excess of high-luminosity and paucity of lowluminosity spiral galaxies (compared with the luminosity distribution of ellipticals both in our and the NFGS samples) it is shown that this sample adequately matches the distribution and full range of properties of galaxies in the local Universe. We have augmented this data set with corollary data from the optical (RC3), NIR (2MASS), and far-infrared (IRAS). We emphasize here the special caution should be observed when comparing these results with those derived from a volume-limited sample. From a broad-based initial analysis of the UV properties of this sample we conclude:

- The value of the integrated $(\mathrm{FUV}-K)$ color of galaxies provides an excellent criterion with which to discriminate elliptical/lenticular galaxies from spirals and irregulars. The best discrimination between these two classes of galaxies (quiescent vs. star-forming) is achieved if a cut-off color $(\mathrm{FUV}-K)=8.8 \mathrm{mag}$ is adopted. A reasonably good separation is also obtained by using a (FUV-NUV) cut-off color at 0.9 mag. These colors also allow for a continuous distinction (although with a significant dispersion) of spiral galaxies of different types.

- Elliptical/lenticular galaxies with brighter FUV and $K$-band luminosities show bluer (FUV-NUV) colors than ellipticals with fainter luminosities but redder (NUV-K) colors. This is true for ellipticals galaxies specifically within the range of absolute magnitudes covered by this Atlas (i.e. $\mathrm{M}_{K}<-21 \mathrm{mag}$ ). This behavior is probably a consequence of luminous elliptical galaxies having stronger UV upturns than their intermediate-mass counterparts (see Boselli et al. 2005).

- We do not find a large dispersion in the intrinsic (corrected for internal extinction) (FUV-NUV) colors of the spiral/irregular galaxies in the Atlas $\left(\sigma_{(\mathrm{FUV}-\mathrm{NUV})_{0}}=0.05 \mathrm{mag}\right)$ neither a strong dependence of it with the galaxy luminosity. Consequently, the variations in the observed (FUV-NUV) colors with the luminosity or morphological type 
of the spiral and irregular galaxies in the sample are plausibly due to variations in the dust content (due for example to changes in metallicity) with these magnitudes. In the case of the $(\mathrm{FUV}-K)$ color the star formation history necessarily contributes to its dependence on luminosity and morphological type.

- The change in the observed (FUV-NUV) color with the TIR-to-FUV ratio also suggests that the attenuation law in these galaxies differs from a pure Milky-Way extinction law. In particular, attenuation laws with relatively steep FUV rise and no $2175 \AA$ bump, like those based on a SMC Bar extinction law or the Calzetti law in the case of the most luminous objects, are favored.

- A significant fraction (28\%) of the UV profiles show some degree of flattening in the inner regions. The galaxies showing this kind of profiles belong to a relatively small range of optical morphological types (compared with the pure-exponential profiles), $2<\mathrm{T}<8$, i.e. they are all truly spiral galaxies. We interpret this as a consequence of the high past SFR but comparatively low current gas infall rate in the inner disks of spiral galaxies, leading to an efficient consumption of the gas in these regions and, consequently, to a flattening of the UV profiles compared with the outer disks, where the gas supply is still abundant. This is, indeed, expected to be particularly important in intermediate-type spirals.

The GALEX and corollary photometry data along with the profiles and UV images of galaxies in the sample can be accessed through a dedicated web page at http://nedwww.ipac.caltech.edu/level5/GALEX_Atlas/.

GALEX (Galaxy Evolution Explorer) is a NASA Small Explorer, launched in April 2003. We gratefully acknowledge NASA's support for construction, operation, and science analysis for the GALEX mission, developed in cooperation with the Centre National d'Etudes Spatiales of France and the Korean Ministry of Science and Technology. AGdP is partially financed by the MAGPOP EU Marie Curie Research Training Network and the Spanish Programa Nacional de Astronomía y Astrofísica under grant AYA2003-01676. We thank Cren Frayer and Olga Pevunova for preparing the online version of the Atlas. We are also thankful to the referee for his/her valuable comments which helped to improve the paper.

Facilities: GALEX 


\section{REFERENCES}

Abraham, R.G., Tanvir, N.R., Santiago, B.X., Ellis, R.S., Glazebrook, K., \& van den Bergh, S., 1996, MNRAS, 279, L47

Amram, P., Mendes de Oliveira, C., Boulesteix, J., \& Balkowski, C., 1998, A\&A, 330, 881

Bell, E.F., Gordon, K.D., Kennicutt, R.C.Jr., \& Zaritsky, D., 2002, ApJ, 565, 994

Bershady, M.A., Jangren, A., \& Conselice, C.J., 2002, AJ, 119, 2645

Bertin, E., \& Arnouts, S., 1996, A\&AS, 117, 393

Bianchi, L., et al., 2003a, BAAS, 203, \#91.12

Bianchi, L., et al., 2005, ApJ, 619, L71

Bianchi, L., Madore, B., Thilker, D., \& Gil de Paz, A., 2003b, in "The Local Group as an Astrophysical Laboratory", STScI Publications (M. Livio and T. Brown eds.), p. 10

Bland-Hawthorn, J., Gallimore, J.F., Tacconi, L.J., Brinks, E., Baum, S.A., Antonucci, R.R.J., \& Cecil, G.N., 1997, Ap\&SS, 248, 9

Boissier, S., 2000, Ph.D. thesis, Université Paris 7

Boissier, S., \& Prantzos, N., 2000, MNRAS, 312, 398

Boselli, A., et al., 2005, ApJ, 629, L29

Boselli, A., Gavazzi, G., Donas, J., \& Scodeggio, M., 2001, AJ, 121, 753

Boselli, A., Tuffs, R.J., Gavazzi, G., Hippelein, H., \& Pierini, D., 1997, A\&AS, 121, 507

Boselli, A., et al., 2005, ApJ, 629, L29

Bottinelli, L., Gouguenheim, L., Paturel, G., \& de Vaucouleurs, G., 1984, A\&AS, 56, 381

Brown, T.M., 2004, Ap\&SS, 291, 215

Brown, T.M., Bowers, C.W., Kimble, R.A., Sweigart, A.V., \& Ferguson, H.C., 2000, ApJ, 532,308

Bruzual, G., \& Charlot, S., 2003, MNRAS, 344, 1000

Buat, V., Boselli, A., Gavazzi, G., \& Bonfanti, C., 2002, A\&A, 383, 801 
Buat, V., et al., 2005, ApJ, 619, L51

Buat, V., \& Xu, K., 1996, A\&A, 306, 61

Burstein, D., Bertola, F., Buson, L.M., Faber, S.M., \& Lauer, T.R., 1988, ApJ, 328, 440

Cairós, L.M., Caon, N., Vúlchez, J.M., González-Pérez, J.N., \& Muñoz-Tuñón, C., 2001, ApJS, 136, 393

Calzetti, D., Armus, L., Bohlin, R.C., Kinney, A.L., Koornneef, J., \& Storchi-Bergmann, T., 2000, ApJ, 533, 682

Calzetti, D., Kinney, A.L., \& Storchi-Bergmann, T., 1994, ApJ, 429, 582

Cardelli, J.A., Clayton, G.C., \& Mathis, J.S., 1989, ApJ, 345, 245

Cole, S., et al., 2001, MNRAS, 326, 255

Condon, J.J., Cotton, W.D., \& Broderick, J.J., 2002, AJ, 124, 675

Cortese, L., et al., 2006, ApJ, 637, 242

Cowie, L.L., Songaila, A., Hu, E.M., \& Cohen, J.G., 1996, AJ, 112, 839

Dale, D.A., Helou, G., Contursi, A., Silbermann, N.A., \& Kolhatkar, S., 2001, ApJ, 549, 215

Davidge, T.J., 2003, AJ, 125, 3046

de Vaucouleurs, G., 1977, Evolution of galaxies and stellar populations, ed. R. B. Larson, \& B. M., Tynsley, Yale Univ. Obs., New Haven, 43

de Vaucouleurs, G., de Vaucouleurs, A., Corwin, H.G., Buta, R.J., Paturel, G., \& Fouqué, P., 1991, Third Reference Catalogue of Bright Galaxies (RC3) (Springer-Verlag)

Deharveng, J.-M., Boselli, A., \& Donas, J., 2002, A\&A, 393, 843

Donas, J., Deharveng, J.M., Laget, M., Milliard, B., \& Huguenin, D., 1987, A\&A, 180, 12

Doublier, V., Caulet, A., \& Comte, G., 1999, A\&AS, 138, 213

Doublier, V., Comte, G., Petrosian, A., Surace, C., \& Turatto, M., 1997, A\&AS, 124, 405

Drozdovsky, I.O., \& Karachentsev, I.D., 2000, A\&AS, 142, 425

Feldmeier, J.J., Ciardullo, R., \& Jacoby, G.H., 1997, ApJ, 479, 231 
Freedman, W.L., et al., 1994, ApJ, 427, 628

Freedman, W.L., et al., 2001, ApJ, 553, 47

Freedman, W.L., Wilson, C.D., \& Madore, B.F., 1991, ApJ, 372, 455

Freedman, W.L., \& Madore, B.F., 1990, ApJ, 365, 186

Funes, J., Kennicutt, R.C.Jr., Lee, J.C., Sakai, S., Tremonti, C.A., \& van Zee, L., 2005, in "Island Universes: The Structure and Evolution of Disk Galaxies", in press.

Gallart, C., Aparicio, A., Freedman, W.L., Madore, B.F., Martínez-Delgado, D., \& Stetson, P.B., 2004, AJ, 127, 1486

Gavazzi, G., Bonfanti, C., Sanvito, G., Boselli, A., \& Scodeggio, M., 2002, ApJ, 576, 135

Gavazzi, G., Boselli, A., Donati, A., Franzetti, P., \& Scodeggio, M., 2003, A\&A, 400, 451

Gavazzi, G., Boselli, A., Scodeggio, M., Pierini, D., \& Belsole, E., 1999, MNRAS, 304, 595

Gavazzi, G., Marcelin, M., Boselli, A., Amram, P., Vílchez, J.M., Iglesias-Páramo, J., \& Tarenghi, M., 2001, A\&A, 377, 745

Gavazzi, G., Pierini, D., \& Boselli, A., 1996, A\&A, 312, 397

Gavazzi, G., \& Scodeggio, M., 1996, A\&A, 312, L29

Gil de Paz, A., Aragón-Salamanca, A., Gallego, J., Alonso-Herrero, A., Zamorano, J., \& Kauffmann, G., 2000, MNRAS, 316, 357

Gil de Paz, A., et al., 2004, BAAS, 205, \#42.01

Gil de Paz, A., et al., 2005, ApJ, 619, L115

Gil de Paz, A., et al., 2005, ApJ, 627, L29

Gil de Paz, A., Madore, B.F., \& Pevunova, O., 2003, ApJS, 147, 29

Gil de Paz, A., Zamorano, J., \& Gallego, J., 2000, A\&A, 361, 465

Gil de Paz, A., \& Madore, B.F., 2002, AJ, 123, 1864

Gil de Paz, A., \& Madore, B.F., 2005, ApJS, 156, 345

Gladders, M.D., López-Cruz, O., Yee, H.K.C., \& Kodama, T., 1998, ApJ, 501, 571 
Gladders, M.D., \& Yee, H.K.C., 2005, ApJS, 157, 1

Goldader, J.D., et al., 2002, ApJ, 568, 651

Gordon, K.D., \& Clayton, G.C., 1998, ApJ, 500, 816

Gordon, K. D., Clayton, G. C., Witt, A. N., \& Misselt, K. A., 2000, ApJ, 533, 236

Gordon, K. D., Clayton, G. C., Misselt, K. A., Landolt, A. U., \& Wolff, M. J., 2003, ApJ, 594,279

Heckman, T.M., et al., 1995, ApJ, 452, 549

Heckman, T.M., et al., 2005, ApJ, 619, L35

Herrnstein, J.R., et al., 1999, Nature, 400, 539

Huchra, J., Davis, M., Latham, D., \& Tonry, J., 1983, ApJS, 52, 89

Jansen, R.A., Fabricant, D., Franx, M., \& Caldwell, N., 2000, ApJS, 126, 331

Jarrett, T.H., Chester, T., Cutri, R., Schneider, S.E., \& Huchra, J.P., 2003, AJ, 125, 525

Jensen, J.B., et al., 2003, ApJ, 583, 712

Johnson, H.L., \& Morgan, W.W., 1953, ApJ, 117, 313

Karachentsev, I.D. \& Drozdovsky, I.O., 1998, A\&AS, 131, 1

Karachentsev, I.D., et al., 2002, A\&A, 383, 125

Karachentsev, I.D., et al., 2003, A\&A, 398, 479

Karachentsev, I.D., et al., 2003, A\&A, 404, 93

Karachentsev, I.D., Karachentseva, V.E., Huchtmeier, W.K., \& Makarov, D.I., 2004, AJ, 127,2031

Karachentsev, I.D., Musella, I., \& Grimaldi, A., 1996, A\&A, 310, 722

Kauffmann, G., et al., 2003, MNRAS, 341, 33

Kelson, D.D., et al., 1996, ApJ, 463, 26

Kennicutt, R.C.Jr., 1998, ARA\&A, 36, 189

Kennicutt, R.C.Jr., et al., 2003, PASP, 115, 928 
Kent, S.M., 1985, ApJS, 59, 115

Kong, X., Charlot, S., Brinchmann, J., \& Fall, S.M., 2004, MNRAS, 349, 769

Kuchinski, L.E., et al., 2000, ApJS, 131, 441

Kuchinski, L.E., Madore, B.F., Freedman, W.L., \& Trewhella, M., 2001, AJ, 122, 729

Lauger, S., Burgarella, D., \& Buat, V., 2005, A\&A, 434, 77

Lonsdale, C., Helou, Good, J.C., \& Rice, W., 1985, Catalogued Galaxies and Quasars Observed in the IRAS Survey (Pasadena: Jet Propulsion Laboratory)

Lupton, R., et al., 2004, PASP, 116, 133

Macri, L.M., et al., 2001, ApJ, 559, 243

Madore, B.F., et al., 1998, Nature, 395, 47

Makarova, L., Karachentsev, I.D., Takalo, L.O., Heinaemaeki, P., \& Valtonen, M., 1998, A\&AS, 128, 459

Makarova, L.N., \& Karachentsev, I.D., 2003, Ap, 46, 144

Marcum, P.M., et al., 2001, ApJS, 132, 129

Marlowe, A.T., Meurer, G.R., \& Heckman, T.M., 1999, ApJ, 522, 183

Martin, D.C., et al., 2005, ApJ, 619, L1

Meurer, G.R., Heckman, T.M., Leitherer, C., Kinney, A., Robert, C., \& Garnett, D.R., 1995, AJ, 110, 2665

Meurer, G.R., Heckman, T.M., \& Calzetti, D., 1999, ApJ, 521, 64

Monet, D.G., et al., 2003, AJ, 125, 984

Morrissey, P., et al., 2005, ApJ, 619, L7

Moshir, M., et al., 1990, Infrared Astronomical Satellite Catalogs, The Faint Source Catalog

O’Connell, R.W., 1999, ARA\&A, 37, 603

Ohl, R., et al., 1998, ApJ, 505, L11 
Paturel, G., Teerikorpi, P., Theureau, G., Fouqué, P., Musella, I., \& Terry, J.N., 2002, A\&A, 389,19

Perrett, K.M., Hanes, D.A., Butterworth, S.T., Kavelaars, J., Geisler, D., \& Harris, W.E., 1997, AJ, 113, 895

Pérez-González, P.G., Gil de Paz, A., Zamorano, J., Gallego, J., Alonso-Herrero, A., \& Aragón-Salamanca, A., 2003, MNRAS, 338, 525

Rejkuba, M., 2004, A\&A, 413, 903

Rejkuba, M., Minniti, D., Gregg, M.D., Zijlstra, A.A., Alonso, M.V., \& Goudfrooij, P.., 2000, AJ, 120, 801

Rice, W., et al., 1988, ApJS, 68, 91

Rich, R.M., et al., 2005, ApJ, 619, L107

Rifatto, A., Longo, G., \& Capaccioli, M., 1995, A\&AS, 114, 527

Roberts, M.S., \& Haynes, M.P., 1994, ARA\&A, 32, 115

Roussel, H., Gil de Paz, A., Seibert, M., Helou, G., Madore, B.F., \& Martin, C., 2005, ApJ, 632,227

Sakai, S., Madore, B.F., \& Freedman, W.L., 1997, AJ, 480, 589

Sakai, S., \& Madore, B.F., 1999, ApJ, 526, 599

Salzer, J.J., Lee, J.C., Melbourne, J., Hinz, J.L., Alonso-Herrero, A., \& Jangren, A., 2005, ApJ, 624, 661

Sandage, A., \& Tammann, G.A., 1990, ApJ, 365, 1

Schlegel, D.J., Finkbeiner, D.P., \& Davis, M., 1998, ApJ, 500, 525

Schulte-Ladbeck, R.E., Hopp, U., Crone, M.M., \& Greggio, L., 1999, ApJ, 525, 709

Seibert, M., et al., 2005, ApJ, 619, L55

Sersic, J.L., \& Donzelli, C., 1993, A\&AS, 98, 21

Sharina, M.E., Karachentsev, I.D., \& Tikhonov, N.A., 1999, Astronomy Letters, 25, 322

Silbermann, N.A., et al., 1996, ApJ, 470, 1 
Sullivan, M., Mobasher, B., Chan, B., Cram, L., Ellis, R.S., Treyer, M.A., \& Hopkins, A., 2001, ApJ, 558, 72

Sullivan, M., Treyer, M.A., Ellis, R.S., Bridges, T.J., Milliard, B., \& Donas, J., 2000, MNRAS, 312, 442

Sullivan, M., Treyer, M.A., Ellis, R.S., \& Mobasher, B., 2004, MNRAS, 350, 21

Theureau, G., Rauzy, S., Bottinelli, L., \& Gouguenheim, L., 1998, A\&A, 340, 21

Thilker, D.A., et al., 2005, ApJ, 619, L79

Thim, F., Tammann, G.A., Saha, A., Dolphin, A., Sandage, A., Tolstoy, E., \& Labhardt, L., 2003, ApJ, 590, 256

Tikhonov, N.A., \& Galazutdinova, O.A., 2002, A\&A, 394, 33

Tolstoy, E., Saha, A., Hoessel, J.G., \& McQuade, K., 1995, AJ, 110, 1640

Tonry, J.L., et al., 2001, ApJ, 546, 681

Tosi, M., Sabbi, E., Bellazzini, M., Aloisi, A., Greggio, L., Leitherer, C., \& Montegriffo, P. 2001, AJ, 122, 1271

Treyer, M.A., Ellis, R.S., Milliard, B., Donas, J., \& Bridges, T.J., 1998, MNRAS, 300, 303

Tully, R.B., 1988, Nearby Galaxies Catalogue

van de Steene, G.C., Jacoby, G.H., Praet, C., Ciardullo, R., \& Dejonghe, H., 2004, in "Planetary Nebulae beyond the Milky Way", Garching (Germany) (astro-ph/0407348)

van den Bergh, S., 2000, Galaxies of the Local Group (Cambridge: Cambridge Univ. Press)

Verdes-Montenegro, L., Bosma, A., \& Athanassoula, E., 2000, A\&A, 356, 827

Wiklind, T., Combes, F., \& Henkel, C., 1995, A\&A, 297, 643

Windhorst, R.A., et al., 2002, ApJS, 143, 113

Wyder, T.K., et al., 2005, ApJ, 619, L15

Yahil, A., Tammann, G.A., \& Sandage, A., 1977, ApJ, 217, 903

Yi, S.K., et al., 2005, ApJ, 619, L111 
Yi, S.K., Lee, Y.-W., Woo, J.-H., Park, J.-H., Demarque, P., \& Oemler, A.Jr., 1999, ApJ, 513,128

Östlin, G., 2000, ApJ, 535, L99

This preprint was prepared with the AAS IATEX macros v5.2. 
Table 1. GALEX Atlas sample

\begin{tabular}{|c|c|c|c|c|c|c|c|c|c|c|c|c|}
\hline $\begin{array}{c}\text { Object Name } \\
\text { (1) }\end{array}$ & $\begin{array}{c}\mathrm{RA}_{2000} \\
(\mathrm{~h}: \mathrm{m}: \mathrm{s}) \\
(2)\end{array}$ & $\begin{array}{c}\mathrm{DEC}_{2000} \\
(\mathrm{~d}: \mathrm{m}: \mathrm{s}) \\
(3)\end{array}$ & $\begin{array}{c}2 \times \mathrm{A} \\
(\operatorname{arcmin}) \\
(4)\end{array}$ & $\begin{array}{c}2 \times \mathrm{B} \\
(\operatorname{arcmin}) \\
(5)\end{array}$ & $\begin{array}{c}\text { PA } \\
(\operatorname{deg}) \\
(6)\end{array}$ & $\begin{array}{c}(\mathrm{Mpc}) \\
(7)\end{array}$ & $\begin{array}{l}\text { istance } \\
\text { DM } \\
(8)\end{array}$ & $\begin{array}{l}\text { ref. } \\
(9)\end{array}$ & $\begin{array}{c}\mathrm{E}(B-V) \\
\quad(\mathrm{mag}) \\
(10)\end{array}$ & $\begin{array}{l}\text { Morphological } \\
\text { Type } \\
\text { (11) }\end{array}$ & $\begin{array}{c}\mathrm{T} \\
\text { Type } \\
(12)\end{array}$ & $\begin{array}{c}\text { Spectral } \\
\text { Type } \\
(13)\end{array}$ \\
\hline WLM & 000158.2 & -152739.3 & 11.5 & 4.0 & 4 & 0.98 & 24.96 & 2 & 0.04 & $\mathrm{IB}(\mathrm{s}) \mathrm{m}$ & $10.0 \pm 0.3$ & \\
\hline NGC 7808 & 000332.1 & -104440.8 & 1.3 & 1.3 & $\ldots$ & 124 & 35.48 & 1 & 0.04 & $\left(\mathrm{R}^{\prime}\right) \mathrm{SA} 0 * 0 *$ : & $-2.0 \pm 1.2$ & Sy1? \\
\hline UGC 00017 & 000343.3 & +151306.0 & 2.5 & 1.7 & 160 & 13 & 30.62 & 1 & 0.05 & Sm: & $9.3 \pm 0.6$ & \\
\hline PGC 00282 & 000401.5 & -111027.3 & 1.1 & 0.9 & 0 & 161 & 36.04 & 1 & 0.04 & $\mathrm{SB}(\mathrm{rs}) \mathrm{c}:$ & $5.0 \pm 1.0$ & \\
\hline NGC 0024 & 000956.5 & -245747.3 & 5.8 & 1.3 & 46 & 8.2 & 29.57 & 3 & 0.02 & $\mathrm{SA}(\mathrm{s}) \mathrm{c}$ & $5.0 \pm 0.3$ & \\
\hline UGC 00128 & 001350.9 & +355939.0 & 2.1 & 1.7 & 65 & 67 & 34.13 & 1 & 0.06 & $\mathrm{Sdm}$ & $8.0 \pm 0.7$ & \\
\hline NGC 0055 & 001453.6 & -39 1147.9 & 32.4 & 5.6 & 108 & 2.0 & 26.51 & 4 & 0.01 & $\mathrm{SB}(\mathrm{s}) \mathrm{m}: \mathrm{sp}$ & $9.0 \pm 0.7$ & \\
\hline ARP 256 NED02 & 001850.1 & -102141.8 & 1.1 & 0.8 & 0 & 116 & 35.32 & 1 & 0.04 & $\mathrm{SB}(\mathrm{s}) \mathrm{c}$ pec & $4.5 \pm 0.6$ & HII \\
\hline ARP 256 NED01 & 001850.9 & -102236.6 & 1.1 & 0.6 & 0 & 115 & 35.30 & 1 & 0.04 & $\mathrm{SB}(\mathrm{s}) \mathrm{b}$ pec? & $3.0 \pm 1.3$ & HII \\
\hline UGC 00226 & 002348.2 & +144102.8 & 1.1 & 0.6 & 4 & 77 & 34.43 & 1 & 0.06 & $\mathrm{Sb}$ & $\ldots$ & \\
\hline NGC 0099 & 002359.4 & +154613.5 & 1.4 & 1.3 & 0 & 77 & 34.43 & 1 & 0.06 & Scd: & $6.0 \pm 1.1$ & \\
\hline UGC 00247 & 002559.0 & +142052.6 & 1.0 & 0.2 & 19 & 160 & 36.02 & 1 & 0.07 & Scd: & $6.0 \pm 1.4$ & \\
\hline UGC 00249 & 002610.2 & +133914.2 & 1.4 & 1.0 & 90 & 76 & 34.40 & 1 & 0.08 & $\mathrm{~S} ?$ & $8.0 \pm 1.2$ & \\
\hline NGC 0115 & 002646.6 & -334036.1 & 1.9 & 0.9 & 127 & 23 & 31.85 & 1 & 0.01 & $\mathrm{SB}(\mathrm{s}) \mathrm{bc}:$ & $4.0 \pm 0.6$ & \\
\hline NGC 0131 & 002938.5 & -331535.1 & 1.9 & 0.6 & 63 & 18 & 31.22 & 1 & 0.02 & $\mathrm{SB}(\mathrm{s}) \mathrm{b}: \mathrm{sp}$ & $3.0 \pm 0.4$ & \\
\hline PGC 01862 & 003029.8 & -084659.9 & 1.2 & 0.4 & 140 & 76 & 34.40 & 1 & 0.04 & & $3.0 \pm 1.4$ & \\
\hline UGC 00316 & 003135.8 & +143646.6 & 1.3 & 0.2 & 40 & 164 & 36.07 & 1 & 0.06 & $\mathrm{Sc}$ & $6.0 \pm 1.4$ & \\
\hline ESO $473-G 025$ & 003149.4 & $-2643 \quad 13.9$ & 2.6 & 0.3 & 83 & 99 & 34.99 & 1 & 0.02 & $\mathrm{Sb}$ & $4.7 \pm 0.7$ & \\
\hline IC 1554 & 003307.4 & $-32 \quad 1530.1$ & 1.4 & 0.8 & 24 & 22 & 31.72 & 1 & 0.01 & $\mathrm{SB}:(\mathrm{r}:) 0 / \mathrm{a}$ & $-0.5 \pm 0.5$ & \\
\hline UGC 00330 & 003341.8 & +39 3241.2 & 1.4 & 0.5 & 140 & 86 & 34.67 & 1 & 0.05 & So & $-2.0 \pm 0.9$ & \\
\hline NGC 0151 & 003402.8 & $-0942 \quad 19.2$ & 3.7 & 1.7 & 75 & 52 & 33.60 & 1 & 0.03 & $\mathrm{SB}(\mathrm{r}) \mathrm{bc}$ & $4.0 \pm 0.3$ & \\
\hline NGC 0155 & 003440.1 & -104559.4 & 1.7 & 1.3 & 0 & 88 & 34.71 & 1 & 0.03 & $\mathrm{~S} 0 * 0^{*}$ pec & $-2.0 \pm 0.8$ & \\
\hline UGC 00344 & 003451.0 & +393242.4 & 1.0 & 0.7 & 110 & 85 & 34.64 & 1 & 0.05 & $\mathrm{SAB}(\mathrm{s}) \mathrm{cd}$ & $6.0 \pm 0.9$ & \\
\hline NGC 0163 & 003559.8 & -100718.1 & 1.5 & 1.2 & 0 & 84 & 34.63 & 1 & 0.03 & E0 & $-5.0 \pm 0.4$ & \\
\hline VV 548 & 003602.0 & -09 5318.7 & 1.2 & 1.0 & 0 & 69 & 34.20 & 1 & 0.04 & SB(s)c pec: & $5.0 \pm 1.2$ & \\
\hline NGC 0165 & 003628.9 & -100622.2 & 1.5 & 1.3 & 0 & 83 & 34.59 & 1 & 0.03 & $\mathrm{SB}(\mathrm{rs}) \mathrm{bc}$ & $4.0 \pm 0.4$ & \\
\hline UGC 00372 & 003727.9 & +425408.1 & 1.5 & 1.4 & 0 & 80 & 34.51 & 1 & 0.06 & $\mathrm{SAm}$ & $9.0 \pm 0.7$ & \\
\hline Cartwheel & 003740.4 & -334259.5 & 1.1 & 0.9 & 128 & 125 & 35.48 & 1 & 0.01 & S pec (Ring) & $\ldots$ & \\
\hline PGC 02269 & 003757.5 & -09 1509.3 & 1.4 & 1.2 & 0 & 74 & 34.36 & 1 & 0.04 & $\mathrm{SAB}(\mathrm{s}) \mathrm{cd}$ & $6.0 \pm 0.8$ & \\
\hline UGC 00394 & 003843.4 & +415949.7 & 2.0 & 0.8 & 5 & 82 & 34.58 & 1 & 0.06 & SABdm & $8.0 \pm 0.8$ & \\
\hline NGC 0195 & 003935.8 & -09 1140.3 & 1.1 & 0.8 & 90 & 69 & 34.19 & 1 & 0.03 & (R)SB(r)a: & $1.0 \pm 0.7$ & HII \\
\hline NGC 0205 & 004022.1 & +414107.1 & 21.9 & 11.0 & 170 & 0.81 & 24.54 & 5 & 0.06 & E5 pec & $-5.0 \pm 0.3$ & \\
\hline NGC 0213 & 004110.0 & +162809.8 & 1.7 & 1.4 & 90 & 79 & 34.47 & 1 & 0.04 & $\mathrm{SB}(\mathrm{rs}) \mathrm{a}$ & $1.0 \pm 0.8$ & \\
\hline
\end{tabular}


Table 1-Continued

\begin{tabular}{|c|c|c|c|c|c|c|c|c|c|c|c|c|}
\hline $\begin{array}{c}\text { Object Name } \\
\text { (1) }\end{array}$ & $\begin{array}{c}\mathrm{RA}_{2000} \\
(\mathrm{~h}: \mathrm{m}: \mathrm{s}) \\
(2)\end{array}$ & $\begin{array}{c}\mathrm{DEC}_{2000} \\
(\mathrm{~d}: \mathrm{m}: \mathrm{s}) \\
(3)\end{array}$ & $\begin{array}{c}2 \times \mathrm{A} \\
(\operatorname{arcmin}) \\
(4)\end{array}$ & $\begin{array}{c}2 \times \mathrm{B} \\
(\operatorname{arcmin}) \\
(5)\end{array}$ & $\begin{array}{c}\text { PA } \\
(\mathrm{deg}) \\
(6)\end{array}$ & $\begin{array}{c}(\mathrm{Mpc}) \\
(7)\end{array}$ & $\begin{array}{l}\text { stance } \\
\text { DM } \\
(8)\end{array}$ & $\begin{array}{l}\text { ref. } \\
(9)\end{array}$ & $\begin{array}{c}\mathrm{E}(B-V) \\
(\mathrm{mag}) \\
(10)\end{array}$ & $\begin{array}{c}\text { Morphological } \\
\text { Type } \\
(11)\end{array}$ & $\begin{array}{c}\mathrm{T} \\
\text { Type } \\
(12)\end{array}$ & $\begin{array}{c}\text { Spectral } \\
\text { Type } \\
(13)\end{array}$ \\
\hline NGC 0223 & 004215.9 & +00 5043.7 & 1.3 & 0.9 & 62 & 76 & 34.40 & 1 & 0.02 & $\left(\mathrm{R}^{\prime}\right) \mathrm{SB}(\mathrm{r}) 0 / \mathrm{a}$ : & $0.0 \pm 0.9$ & \\
\hline MESSIER 032 & 004241.8 & +405154.6 & 8.7 & 6.5 & 170 & 0.78 & 24.46 & 6 & 0.06 & $\mathrm{cE} 2$ & $-6.0 \pm 0.3$ & \\
\hline MESSIER 031 & 004244.3 & +411608.5 & 190.0 & 60.0 & 35 & 0.78 & 24.46 & 6 & 0.06 & $\mathrm{SA}(\mathrm{s}) \mathrm{b}$ & $3.0 \pm 0.3$ & LINER \\
\hline UGC 00484 & 004656.0 & +324031.2 & 2.4 & 0.8 & 25 & 72 & 34.29 & 1 & 0.09 & $\left(\mathrm{R}^{\prime}\right) \mathrm{SB}(\mathrm{s}) \mathrm{b}$ & $3.0 \pm 0.8$ & \\
\hline NGC 0247 & 004708.6 & -204537.4 & 21.4 & 6.9 & 174 & 3.1 & 27.45 & 7 & 0.02 & $\mathrm{SAB}(\mathrm{s}) \mathrm{d}$ & $7.0 \pm 0.3$ & \\
\hline NGC 0247B & 004735.1 & -202543.3 & 1.0 & 0.4 & 15 & 86 & 34.66 & 1 & 0.02 & $\mathrm{SB}(\mathrm{s}) \mathrm{b}$ pec? & $3.0 \pm 0.9$ & \\
\hline ESO 540-G025 & 004737.9 & -203110.2 & 1.0 & 0.9 & 0 & 89 & 34.74 & 1 & 0.02 & SB(s)c pec: & $4.5 \pm 0.6$ & \\
\hline NGC 0262 & 004847.1 & +315725.1 & 1.1 & 1.1 & $\ldots$ & 66 & 34.10 & 1 & 0.07 & $\mathrm{SA}(\mathrm{s}) 0 / \mathrm{a}:$ & $0.0 \pm 0.4$ & Sy2 \\
\hline UGC 00507 & 004935.7 & +010658.1 & 1.9 & 0.2 & 128 & 74 & 34.36 & 1 & 0.02 & Scd & $5.5 \pm 0.9$ & \\
\hline NGC 0266 & 004947.8 & +321639.8 & 3.0 & 2.9 & 0 & 68 & 34.17 & 1 & 0.07 & $\mathrm{SB}(\mathrm{rs}) \mathrm{ab}$ & $2.0 \pm 0.7$ & LINER \\
\hline NGC 0270 & 005032.5 & -083905.9 & 1.7 & 1.5 & 15 & 53 & 33.61 & 1 & 0.05 & $\mathrm{~S} 0+$ & $-1.0 \pm 0.8$ & \\
\hline ESO 351-G011 & 005104.3 & -322521.1 & 1.2 & 0.4 & 163 & 137 & 35.68 & 1 & 0.02 & $\mathrm{~S}(\mathrm{r}) 0 / \mathrm{a}$ & $-0.1 \pm 0.9$ & \\
\hline NGC 0277 & 005117.2 & -083548.6 & 1.4 & 1.2 & 0 & 60 & 33.89 & 1 & 0.04 & SO- & $-3.0 \pm 0.9$ & \\
\hline PGC 03004 & 005122.7 & -083106.3 & 1.3 & 0.7 & 60 & 59 & 33.86 & 1 & 0.04 & $\mathrm{SB}(\mathrm{rs}) \mathrm{d}$ & $7.0 \pm 0.9$ & \\
\hline NGC 0291 & 005329.9 & $-0846 \quad 04.1$ & 1.1 & 0.5 & 75 & 80 & 34.52 & 1 & 0.04 & (R')SB(r)a: & $1.0 \pm 0.9$ & Sy2 \\
\hline NGC 0300 & 005453.5 & -374103.8 & 21.9 & 15.5 & 111 & 2.0 & 26.51 & 9 & 0.01 & $\mathrm{SA}(\mathrm{s}) \mathrm{d}$ & $7.0 \pm 0.3$ & \\
\hline UGC 00590 & 005705.2 & +145431.2 & 1.0 & 0.6 & 145 & 213 & 36.65 & 1 & 0.05 & $\mathrm{~S} ?$ & $\ldots$ & \\
\hline NGC 0311 & 005732.7 & +301650.7 & 1.5 & 0.8 & 120 & 74 & 34.34 & 1 & 0.07 & So & $-2.0 \pm 0.8$ & \\
\hline NGC 0315 & 005748.9 & +302108.8 & 3.2 & 2.0 & 40 & 72 & 34.29 & 1 & 0.06 & E+:;LINER;Sy3b & $-4.0 \pm 0.5$ & Sy1 \\
\hline ESO 351-G028 & 005929.4 & $-3611 \quad 11.8$ & 1.4 & 0.6 & 80 & 47 & 33.36 & 1 & 0.02 & $\mathrm{SB}(\mathrm{s}) \mathrm{c}:$ pec & $4.7 \pm 0.7$ & \\
\hline UGC 00619 & 005948.7 & +144324.7 & 1.2 & 0.1 & 5 & 175 & 36.21 & 1 & 0.08 & $\mathrm{Sd}$ & $6.0 \pm 1.4$ & NLAGN \\
\hline NGC 0337 & 005950.1 & -073440.7 & 2.9 & 1.8 & 130 & 25 & 31.96 & 3 & 0.11 & $\mathrm{SB}(\mathrm{s}) \mathrm{d}$ & $7.0 \pm 0.3$ & \\
\hline PGC 03613 & 010038.1 & -075851.8 & 1.3 & 1.2 & 0 & 82 & 34.58 & 1 & 0.10 & $\mathrm{SAB}(\mathrm{rs}) \mathrm{c}$ & $5.0 \pm 0.8$ & \\
\hline UGC 00627 & 010100.5 & +132806.9 & 1.1 & 0.8 & 115 & 169 & 36.14 & 1 & 0.05 & $\mathrm{~S} ?$ & $\ldots$ & \\
\hline NGC 0337A & 010133.9 & -073517.7 & 5.9 & 4.5 & 0 & 14 & 30.77 & 1 & 0.10 & $\mathrm{SAB}(\mathrm{s}) \mathrm{dm}$ & $8.0 \pm 0.4$ & \\
\hline UGC 00652 & 010350.4 & +220140.0 & 1.3 & 0.7 & 35 & 82 & 34.57 & 1 & 0.04 & SBcd? & $6.0 \pm 1.8$ & \\
\hline ESO 352-G002 & 010430.4 & -333916.1 & 1.3 & 0.6 & 168 & 140 & 35.73 & 1 & 0.03 & $\mathrm{Sc}$ & $\ldots$ & \\
\hline IC 1613 & 010447.8 & +020704.0 & 16.2 & 14.5 & 50 & 0.81 & 24.54 & 10 & 0.03 & $\mathrm{IB}(\mathrm{s}) \mathrm{m}$ & $10.0 \pm 0.3$ & \\
\hline IC 1616 & 010456.2 & -272545.7 & 1.6 & 1.4 & 0 & 78 & 34.47 & 1 & 0.02 & $\mathrm{SB}(\mathrm{rs}) \mathrm{bc}$ & $4.3 \pm 0.4$ & \\
\hline ESO $352-G 007$ & $\begin{array}{lll}01 & 07 & 36.0\end{array}$ & -333816.4 & 1.6 & 0.8 & 61 & 142 & 35.76 & 1 & 0.02 & $\left(\mathrm{R}^{\prime}\right) \mathrm{SB}(\mathrm{rs}) \mathrm{a}$ & $1.0 \pm 0.8$ & \\
\hline NGC 0392 & 010823.5 & +330801.0 & 1.2 & 0.9 & 50 & 69 & 34.19 & 1 & 0.06 & SO-: & $-3.0 \pm 1.2$ & \\
\hline
\end{tabular}


Table 1-Continued

\begin{tabular}{|c|c|c|c|c|c|c|c|c|c|c|c|c|}
\hline $\begin{array}{c}\text { Object Name } \\
\text { (1) }\end{array}$ & $\begin{array}{c}\mathrm{RA}_{2000} \\
(\mathrm{~h}: \mathrm{m}: \mathrm{s}) \\
(2)\end{array}$ & $\begin{array}{c}\mathrm{DEC}_{2000} \\
(\mathrm{~d}: \mathrm{m}: \mathrm{s}) \\
(3)\end{array}$ & $\begin{array}{c}2 \times \mathrm{A} \\
(\operatorname{arcmin}) \\
(4)\end{array}$ & $\begin{array}{c}2 \times \mathrm{B} \\
(\operatorname{arcmin}) \\
(5)\end{array}$ & $\begin{array}{c}\text { PA } \\
(\mathrm{deg}) \\
(6)\end{array}$ & $\begin{array}{c}(\mathrm{Mpc}) \\
(7)\end{array}$ & $\begin{array}{c}\text { istance } \\
\text { DM } \\
(8)\end{array}$ & $\begin{array}{l}\text { ref. } \\
(9)\end{array}$ & $\begin{array}{c}\mathrm{E}(B-V) \\
(\mathrm{mag}) \\
(10)\end{array}$ & $\begin{array}{c}\text { Morphological } \\
\text { Type } \\
(11)\end{array}$ & $\begin{array}{c}\mathrm{T} \\
\text { Type } \\
(12)\end{array}$ & $\begin{array}{c}\text { Spectral } \\
\text { Type } \\
(13)\end{array}$ \\
\hline ESO 243-G041 & 010851.8 & -454957.6 & 1.0 & 0.3 & 93 & 100 & 34.99 & 1 & 0.01 & $\mathrm{~S} 0^{*} 0^{*}:$ pec & $-2.0 \pm 0.9$ & \\
\hline ESO 296-G002 & 010902.6 & -371720.0 & 1.2 & 1.0 & 165 & 91 & 34.80 & 1 & 0.01 & $\left(\mathrm{R}^{\prime}-1\right) \mathrm{SB}(\mathrm{r}) \mathrm{ab}$ & $1.1 \pm 0.5$ & \\
\hline ESO 243-G045 & 010904.5 & -454624.7 & 1.4 & 1.2 & 0 & 107 & 35.15 & 1 & 0.01 & S0- & $-3.0 \pm 0.7$ & \\
\hline NGC 0403 & 010914.2 & +324507.7 & 1.9 & 0.6 & 86 & 74 & 34.36 & 1 & 0.06 & S0/a: & $0.3 \pm 0.7$ & \\
\hline IC 1633 & 010955.6 & -455552.3 & 2.9 & 2.4 & 120 & 101 & 35.01 & 1 & 0.01 & $\mathrm{cD} ; \mathrm{E}+1$ & $-4.0 \pm 0.5$ & \\
\hline UGC 00726 & 010957.6 & -014458.9 & 1.6 & 0.9 & 144 & 54 & 33.68 & 1 & 0.05 & $\mathrm{SB}(\mathrm{s}) \mathrm{d} ?$ & $7.3 \pm 0.7$ & \\
\hline NGC 0407 & 011036.6 & +330735.4 & 1.7 & 0.4 & 0 & 81 & 34.55 & 1 & 0.06 & S0/a: sp & $0.2 \pm 0.8$ & \\
\hline UGC 00732 & 011044.3 & +333330.2 & 1.0 & 0.6 & 80 & 79 & 34.50 & 1 & 0.05 & $\mathrm{SA}(\mathrm{r}) \mathrm{d}$ & $7.0 \pm 0.8$ & \\
\hline UGC 00736 & 011049.0 & -014524.1 & 1.3 & 0.9 & 125 & 72 & 34.29 & 1 & 0.05 & Scd: & $6.0 \pm 1.2$ & \\
\hline NGC 0410 & 011058.9 & +330908.3 & 2.4 & 1.3 & 30 & 77 & 34.44 & 1 & 0.06 & E+:;LINER & $-4.0 \pm 0.6$ & HII \\
\hline ESO 243-G051 & 011119.3 & -455555.6 & 1.0 & 0.6 & 51 & 89 & 34.76 & 1 & 0.01 & $\mathrm{Sb}$ & $1.0 \pm 0.6$ & \\
\hline ESO 243-G052 & 011127.6 & -455615.6 & 1.3 & 0.2 & 106 & 113 & 35.27 & 1 & 0.01 & $\mathrm{~S} 0 * 0 *: \mathrm{sp}$ & $-1.5 \pm 1.0$ & \\
\hline PGC 04663 & 011748.4 & -083626.9 & 1.6 & 1.1 & 110 & 57 & 33.78 & 1 & 0.04 & $\mathrm{SB}(\mathrm{s}) \mathrm{m}$ & $9.0 \pm 0.8$ & \\
\hline NGC 0467 & 011910.1 & +031803.0 & 1.7 & 1.7 & $\ldots$ & 78 & 34.45 & 1 & 0.04 & $\mathrm{SA}(\mathrm{s}) 0^{*} 0 *$ pec? & $-2.0 \pm 0.3$ & \\
\hline NGC 0470 & 011944.8 & +032435.8 & 2.8 & 1.7 & 155 & 34 & 32.63 & 1 & 0.04 & $\mathrm{SA}(\mathrm{rs}) \mathrm{b}$ & $3.0 \pm 0.3$ & HII \\
\hline NGC 0474 & 012006.7 & +032455.0 & 7.1 & 6.3 & 75 & 33 & 32.61 & 1 & 0.03 & $\left(\mathrm{R}^{\prime}\right) \mathrm{SA}(\mathrm{s}) 0^{*} 0^{*}$ & $-2.0 \pm 0.3$ & \\
\hline ESO 352-G047 & 012034.0 & -340719.5 & 1.4 & 0.9 & 73 & 52 & 33.57 & 1 & 0.03 & $\mathrm{IB}(\mathrm{s}) \mathrm{m}$ & $10.0 \pm 0.8$ & \\
\hline UGC 00885 & 012102.4 & +025736.0 & 1.0 & 0.4 & 138 & 76 & 34.40 & 1 & 0.03 & Scd: & $6.0 \pm 1.3$ & \\
\hline ESO 352-G050 & $\begin{array}{lll}01 & 21 & 07.3\end{array}$ & $\begin{array}{lll}-35 & 12 & 07.1\end{array}$ & 1.5 & 0.3 & 145 & 81 & 34.55 & 1 & 0.03 & Sbc? sp & $4.0 \pm 1.8$ & \\
\hline NGC 0479 & $\begin{array}{lll}01 & 21 & 15.7\end{array}$ & +035144.2 & 1.1 & 0.9 & 0 & 74 & 34.36 & 1 & 0.04 & $\mathrm{SB}(\mathrm{rs}) \mathrm{bc}:$ & $3.7 \pm 0.7$ & \\
\hline NGC 0491 & 012120.4 & -340347.8 & 1.4 & 1.0 & 93 & 52 & 33.60 & 1 & 0.03 & $\mathrm{SB}(\mathrm{rs}) \mathrm{b}:$ & $3.0 \pm 0.6$ & \\
\hline UGC 00910 & 012158.3 & +154742.5 & 1.0 & 0.9 & 0 & 91 & 34.80 & 1 & 0.07 & $\mathrm{SA}(\mathrm{s}) \mathrm{c}$ & $5.0 \pm 0.8$ & \\
\hline ESO 352-G057 & 012202.4 & -341148.4 & 1.4 & 0.4 & 12 & 76 & 34.42 & 1 & 0.03 & $\mathrm{SB}(\mathrm{s}) 0^{*} 0^{*}$ pec & $-2.0 \pm 0.9$ & \\
\hline ESO 352-G062 & 012306.9 & -344403.8 & 1.6 & 0.2 & 53 & 134 & 35.63 & 1 & 0.03 & $\mathrm{Sbc}$ & $4.7 \pm 0.7$ & \\
\hline ESO 352-G064 & 012334.9 & -345609.1 & 1.2 & 0.3 & 63 & 83 & 34.61 & 1 & 0.02 & S0-: sp & $-3.0 \pm 1.2$ & \\
\hline NGC 0527 & 012358.1 & -350654.4 & 1.7 & 0.4 & 14 & 80 & 34.51 & 1 & 0.03 & $\mathrm{SB}(\mathrm{r}) 0 / \mathrm{a} ?$ & $0.0 \pm 0.8$ & \\
\hline NGC 0514 & 012403.9 & +125502.6 & 3.5 & 2.8 & 110 & 36 & 32.75 & 1 & 0.04 & $\mathrm{SAB}(\mathrm{rs}) \mathrm{c}$ & $5.0 \pm 0.3$ & \\
\hline ESO 352-G069 & 012414.1 & -344334.7 & 1.5 & 1.2 & 85 & 84 & 34.61 & 1 & 0.02 & $\mathrm{SB}(\mathrm{s}) \mathrm{ab} ?$ pec & $2.2 \pm 0.7$ & \\
\hline UGC 00957 & 012424.4 & +035256.0 & 1.2 & 0.9 & 0 & 30 & 32.41 & 1 & 0.03 & Im: & $10.0 \pm 1.2$ & \\
\hline NGC 0520 & 012435.1 & +034732.7 & 4.5 & 1.8 & 135 & 31 & 32.48 & 1 & 0.03 & & $99.0 \pm 0.0$ & \\
\hline NGC 0530 & 012441.7 & -013513.5 & 1.5 & 0.4 & 134 & 71 & 34.25 & 1 & 0.04 & SB0+ & $-0.7 \pm 0.5$ & \\
\hline IC 0107 & 012513.3 & +145220.4 & 1.1 & 0.9 & 100 & 91 & 34.81 & 1 & 0.05 & $\mathrm{~S} ?$ & $-5.0 \pm 0.7$ & \\
\hline UGC 00984 & 012517.9 & -013102.8 & 1.2 & 0.4 & 130 & 69 & 34.18 & 1 & 0.04 & So & $-2.0 \pm 0.9$ & \\
\hline
\end{tabular}


Table 1-Continued

\begin{tabular}{|c|c|c|c|c|c|c|c|c|c|c|c|c|}
\hline $\begin{array}{c}\text { Object Name } \\
\text { (1) }\end{array}$ & $\begin{array}{c}\mathrm{RA}_{2000} \\
(\mathrm{~h}: \mathrm{m}: \mathrm{s}) \\
(2)\end{array}$ & $\begin{array}{c}\mathrm{DEC}_{2000} \\
(\mathrm{~d}: \mathrm{m}: \mathrm{s}) \\
(3)\end{array}$ & $\begin{array}{c}2 \times \mathrm{A} \\
(\operatorname{arcmin}) \\
(4)\end{array}$ & $\begin{array}{c}2 \times \mathrm{B} \\
(\operatorname{arcmin}) \\
(5)\end{array}$ & $\begin{array}{c}\text { PA } \\
(\mathrm{deg}) \\
(6)\end{array}$ & $\begin{array}{c}(\mathrm{Mpc}) \\
(7)\end{array}$ & $\begin{array}{c}\text { istance } \\
\text { DM } \\
(8)\end{array}$ & $\begin{array}{l}\text { ref. } \\
(9)\end{array}$ & $\begin{array}{c}\mathrm{E}(B-V) \\
(\mathrm{mag}) \\
(10)\end{array}$ & $\begin{array}{c}\text { Morphological } \\
\text { Type } \\
\text { (11) }\end{array}$ & $\begin{array}{c}\mathrm{T} \\
\text { Type } \\
(12)\end{array}$ & $\begin{array}{c}\text { Spectral } \\
\text { Type } \\
(13)\end{array}$ \\
\hline IC 1698 & 012522.1 & +145018.9 & 1.8 & 0.7 & 120 & 93 & 34.85 & 1 & 0.05 & So & $-2.0 \pm 0.8$ & \\
\hline UGC 00985 & 012523.7 & +1431 11.9 & 1.5 & 0.4 & 19 & 159 & 36.00 & 1 & 0.04 & $\mathrm{Sb}$ & $3.0 \pm 0.9$ & \\
\hline IC 1700 & 012524.6 & +145152.4 & 1.4 & 1.4 & $\ldots$ & 91 & 34.80 & 1 & 0.05 & $\mathrm{E}$ & $-5.0 \pm 0.7$ & \\
\hline NGC 0538 & 012526.0 & -013302.3 & 1.0 & 0.5 & 40 & 77 & 34.44 & 1 & 0.04 & SB(s)ab: & $1.6 \pm 0.7$ & \\
\hline NGC 0535 & 012531.1 & -012429.3 & 1.0 & 0.3 & 58 & 69 & 34.20 & 1 & 0.05 & S0+ & $-0.8 \pm 0.6$ & \\
\hline UGC 00999 & 012534.6 & +145831.7 & 1.0 & 0.3 & 62 & 225 & 36.77 & 1 & 0.04 & Scd: & $6.0 \pm 1.3$ & \\
\hline UGC 01003 & 012544.3 & -012724.1 & 1.0 & 0.3 & 130 & 74 & 34.35 & 1 & 0.04 & So & $-2.0 \pm 0.9$ & \\
\hline NGC 0541 & 012544.3 & -012246.1 & 1.8 & 1.7 & 0 & 76 & 34.41 & 1 & 0.05 & $\mathrm{cD} ; \mathrm{SO}-:$ & $-3.0 \pm 0.4$ & \\
\hline NGC 0545 & 012559.1 & -012024.8 & 2.4 & 1.6 & 55 & 76 & 34.39 & 1 & 0.04 & SA0- & $-3.0 \pm 0.3$ & \\
\hline NGC 0547 & 012600.6 & -012042.6 & 1.3 & 1.3 & $\cdots$ & 77 & 34.44 & 1 & 0.04 & $\mathrm{E} 1$ & $-5.0 \pm 0.4$ & \\
\hline NGC 0557 & 012625.1 & -013819.4 & 1.4 & 0.8 & 45 & 78 & 34.47 & 1 & 0.05 & $\mathrm{SB}(\mathrm{rs}) 0+$ pec: & $-0.8 \pm 0.5$ & \\
\hline ESO 353-G002 & 012705.4 & -355121.7 & 1.0 & 0.5 & 80 & 83 & 34.61 & 1 & 0.02 & $\mathrm{~S} ?$ & $\cdots$ & \\
\hline UGC 01026 & 012713.1 & +133608.3 & 1.8 & 1.4 & 115 & 65 & 34.05 & 1 & 0.05 & Sm: & $9.0 \pm 1.1$ & NLAGN \\
\hline UGC 01040 & 012735.9 & -010618.5 & 1.1 & 0.2 & 34 & 63 & 34.01 & 1 & 0.03 & $\mathrm{~S} 0 / \mathrm{a}$ & $0.0 \pm 1.0$ & \\
\hline NGC 0568 & 012757.0 & -354303.7 & 2.2 & 1.4 & 137 & 78 & 34.47 & 1 & 0.02 & SA0- pec: & $-3.0 \pm 0.5$ & \\
\hline UGC 01057 & 012853.3 & +134737.7 & 1.5 & 0.5 & 153 & 91 & 34.80 & 1 & 0.05 & Sbc & $4.0 \pm 0.9$ & NLAGN \\
\hline NGC 0574 & 012903.1 & -353556.1 & 1.1 & 0.7 & 2 & 79 & 34.48 & 1 & 0.02 & $\left(\mathrm{R}^{\prime}-1\right) \mathrm{SB}(\mathrm{rl}) \mathrm{b}$ & $2.5 \pm 0.6$ & \\
\hline IC 0127 & 012947.6 & -065848.2 & 1.8 & 0.5 & 120 & 27 & 32.18 & 1 & 0.04 & Sb: sp & $3.0 \pm 0.7$ & \\
\hline NGC 0584 & $\begin{array}{lll}01 & 31 & 20.7\end{array}$ & -065205.0 & 4.2 & 2.3 & 55 & 28 & 32.20 & 3 & 0.04 & $\mathrm{E} 4$ & $-5.0 \pm 0.3$ & \\
\hline NGC 0586 & 013136.9 & -065337.5 & 1.6 & 0.8 & 0 & 27 & 32.18 & 1 & 0.04 & SA(s)a:? & $1.0 \pm 0.9$ & \\
\hline MESSIER 033 & 013350.9 & +303935.8 & 70.8 & 41.7 & 23 & 0.84 & 24.62 & 11 & 0.04 & $\mathrm{SA}(\mathrm{s}) \mathrm{cd}$ & $6.0 \pm 0.3$ & HII \\
\hline NGC 0628 & 013641.8 & +154700.5 & 10.5 & 9.5 & 25 & 11 & 30.24 & 12 & 0.07 & $\mathrm{SA}(\mathrm{s}) \mathrm{c}$ & $5.0 \pm 0.3$ & \\
\hline UGC 01181 & 014030.0 & +143122.4 & 1.0 & 0.2 & 133 & 116 & 35.33 & 1 & 0.07 & $\mathrm{Sc}$ & $6.0 \pm 1.4$ & \\
\hline IC 0148 & 014227.0 & +135837.3 & 3.4 & 1.1 & 50 & 11 & 30.26 & 1 & 0.07 & Im: & $10.0 \pm 1.1$ & \\
\hline UGC 01200 & 014248.3 & +130921.5 & 2.0 & 1.4 & 170 & 12 & 30.34 & 1 & 0.05 & IBm: & $10.0 \pm 0.8$ & \\
\hline NGC 0660 & 014302.4 & +133842.2 & 8.3 & 3.2 & 170 & 12 & 30.46 & 1 & 0.06 & SB(s)a pec;HII & $1.0 \pm 0.3$ & LINER \\
\hline UGC 01211 & 014355.1 & +134824.7 & 2.3 & 1.9 & 0 & 35 & 32.70 & 1 & 0.06 & $\operatorname{Im}:$ & $10.0 \pm 1.0$ & \\
\hline IC 0159 & 014625.1 & -083811.9 & 1.4 & 0.7 & 45 & 55 & 33.68 & 1 & 0.03 & SB(rs)b pec: & $3.0 \pm 1.2$ & \\
\hline PGC 06504 & 014638.2 & -084043.1 & 1.2 & 0.7 & 92 & 55 & 33.68 & 13 & 0.03 & $\mathrm{IB}(\mathrm{s}) \mathrm{m}$ & $10.0 \pm 0.9$ & \\
\hline NGC 0671 & $\begin{array}{lll}01 & 46 & 59.1\end{array}$ & +130730.5 & 1.5 & 0.5 & 55 & 79 & 34.49 & 1 & 0.05 & $\mathrm{~S} ?$ & $\ldots$ & NLAGN \\
\hline UGC 01261 & 014833.5 & +132550.0 & 1.4 & 0.3 & 92 & 72 & 34.28 & 1 & 0.06 & Scd: & $6.0 \pm 1.3$ & \\
\hline UGC 01262 & 014834.4 & +134204.0 & 1.3 & 0.7 & 170 & 73 & 34.31 & 1 & 0.05 & $\operatorname{Im}$ & $10.0 \pm 0.9$ & \\
\hline UGC 01264 & 014837.9 & +134609.4 & 1.1 & 0.3 & 75 & 69 & 34.18 & 1 & 0.05 & Scd: & $6.0 \pm 1.3$ & \\
\hline
\end{tabular}


Table 1-Continued

\begin{tabular}{|c|c|c|c|c|c|c|c|c|c|c|c|c|}
\hline $\begin{array}{c}\text { Object Name } \\
\text { (1) }\end{array}$ & $\begin{array}{c}\mathrm{RA}_{2000} \\
(\mathrm{~h}: \mathrm{m}: \mathrm{s}) \\
(2)\end{array}$ & $\begin{array}{c}\mathrm{DEC}_{2000} \\
(\mathrm{~d}: \mathrm{m}: \mathrm{s}) \\
(3)\end{array}$ & $\begin{array}{c}2 \times \mathrm{A} \\
(\operatorname{arcmin}) \\
(4)\end{array}$ & $\begin{array}{c}2 \times \mathrm{B} \\
(\operatorname{arcmin}) \\
(5)\end{array}$ & $\begin{array}{c}\text { PA } \\
(\mathrm{deg}) \\
(6)\end{array}$ & $\begin{array}{c}(\mathrm{Mpc}) \\
(7)\end{array}$ & $\begin{array}{c}\text { istance } \\
\text { DM } \\
(8)\end{array}$ & $\begin{array}{l}\text { ref. } \\
(9)\end{array}$ & $\begin{array}{c}\mathrm{E}(B-V) \\
(\mathrm{mag}) \\
(10)\end{array}$ & $\begin{array}{c}\text { Morphological } \\
\text { Type } \\
\text { (11) }\end{array}$ & $\begin{array}{c}\mathrm{T} \\
\text { Type } \\
(12)\end{array}$ & $\begin{array}{c}\text { Spectral } \\
\text { Type } \\
(13)\end{array}$ \\
\hline NGC 0676 & 014857.3 & +05 5427.1 & 4.0 & 1.2 & 172 & 21 & 31.63 & 1 & 0.06 & S0/a: sp & $0.0 \pm 0.4$ & Sy2 \\
\hline UGC 01271 & 014900.0 & +13 1240.1 & 1.7 & 1.1 & 95 & 73 & 34.31 & 1 & 0.07 & SB0 & $-2.0 \pm 0.8$ & \\
\hline UGC 01274 & 014911.6 & +125111.7 & 1.4 & 0.5 & 108 & 113 & 35.26 & 1 & 0.08 & Sa: & $1.0 \pm 1.2$ & \\
\hline UGC 01278 & 014921.2 & +124254.2 & 1.0 & 0.5 & 105 & 143 & 35.78 & 1 & 0.08 & Scd: & $6.0 \pm 1.3$ & \\
\hline NGC 0693 & 015030.9 & +060842.8 & 2.1 & 1.0 & 106 & 22 & 31.71 & 1 & 0.05 & $\mathrm{~S} 0 / \mathrm{a} ?$ & $0.0 \pm 1.5$ & \\
\hline UGC 01312 & 015102.1 & +131747.5 & 1.4 & 0.4 & 96 & 74 & 34.35 & 1 & 0.08 & $\mathrm{~S} ?$ & $\ldots$ & \\
\hline ESO 245-G007 & 015106.3 & -442640.9 & 4.9 & 4.1 & 90 & 0.40 & 23.01 & 14 & 0.02 & IAm & $10.0 \pm 0.6$ & \\
\hline NGC 0707 & 015127.1 & -08 3019.4 & 1.3 & 0.8 & 55 & 77 & 34.43 & 1 & 0.02 & $\left(\mathrm{R}^{\prime}\right) \mathrm{SAB}(\mathrm{s}) 0-:$ & $-3.4 \pm 0.7$ & \\
\hline NGC 0706 & 015150.5 & +06 1748.8 & 1.9 & 1.4 & 150 & 71 & 34.25 & 1 & 0.06 & Sbc? & $4.0 \pm 0.9$ & \\
\hline UGC 01364 & 015403.7 & +145434.2 & 1.4 & 0.8 & 30 & 73 & 34.32 & 1 & 0.05 & SBcd: & $6.0 \pm 1.2$ & \\
\hline PGC 07064 & 015418.1 & -094248.5 & 2.1 & 1.2 & 100 & 222 & 36.73 & 1 & 0.02 & SB0-: & $-2.5 \pm 0.6$ & \\
\hline PGC 07210 & 015551.3 & -0958 00.5 & 1.3 & 1.0 & 115 & 115 & 35.30 & 1 & 0.02 & $\mathrm{SB}(\mathrm{r}) \mathrm{c}$ pec & $4.5 \pm 0.6$ & \\
\hline UGC 01408 & 015615.3 & $\begin{array}{r}+130957.0 \\
\end{array}$ & 1.0 & 0.5 & 90 & 110 & 35.20 & 1 & 0.06 & $\mathrm{Sd}$ & $7.0 \pm 0.9$ & \\
\hline IC 1755 & 015709.8 & +143259.6 & 1.4 & 0.3 & 154 & 114 & 35.28 & 1 & 0.05 & $\mathrm{Sa}$ & $1.0 \pm 0.9$ & \\
\hline UGC 01448 & 015808.6 & +020352.2 & 1.3 & 0.3 & 110 & 89 & 34.75 & 1 & 0.03 & Scd: & $6.0 \pm 1.3$ & \\
\hline KUG 0156-084 & 015851.9 & -080944.8 & 1.2 & 1.0 & 52 & 67 & 34.11 & 1 & 0.03 & $\mathrm{SB}(\mathrm{r}) \mathrm{c}$ pec & $4.6 \pm 0.6$ & \\
\hline NGC 0770 & 015913.6 & +185716.8 & 1.2 & 0.9 & 15 & 36 & 32.76 & 1 & 0.07 & E3: & $-5.0 \pm 0.6$ & \\
\hline NGC 0772 & 015919.6 & +190027.1 & 7.2 & 4.3 & 130 & 36 & 32.76 & 1 & 0.07 & $\mathrm{SA}(\mathrm{s}) \mathrm{b}$ & $3.0 \pm 0.3$ & \\
\hline UGC 01468 & 015931.0 & +135637.6 & 1.5 & 0.9 & 145 & 66 & 34.10 & 1 & 0.06 & $\mathrm{Sd}$ & $7.0 \pm 0.8$ & \\
\hline NGC 0774 & 015934.7 & +140029.6 & 1.5 & 1.2 & 165 & 66 & 34.09 & 1 & 0.06 & So & $-2.0 \pm 0.8$ & \\
\hline NGC 0777 & 020014.9 & +312545.8 & 2.5 & 2.0 & 155 & 73 & 34.32 & 1 & 0.05 & $\mathrm{E} 1$ & $-5.0 \pm 0.3$ & Sy2 \\
\hline NGC 0778 & 020019.4 & +311846.9 & 1.1 & 0.5 & 150 & 79 & 34.49 & 1 & 0.05 & S0: & $-2.0 \pm 1.3$ & \\
\hline NGC 0787 & 020048.6 & -0900 09.3 & 2.5 & 1.9 & 90 & 67 & 34.12 & 1 & 0.03 & (R)SA(rs)b: & $3.0 \pm 0.7$ & \\
\hline PGC 07654 & 020055.1 & -085027.9 & 1.7 & 1.1 & 35 & 22 & 31.67 & 1 & 0.03 & $\mathrm{IB}(\mathrm{s}) \mathrm{m}:$ & $10.0 \pm 1.2$ & \\
\hline NGC 0783 & 020106.6 & +315256.9 & 1.6 & 1.4 & 35 & 76 & 34.39 & 1 & 0.06 & $\mathrm{SBc}$ & $5.0 \pm 0.8$ & \\
\hline UGCA 023 & $\begin{array}{lll}02 & 03 & 02.2\end{array}$ & -09 3919.5 & 2.8 & 0.2 & 37 & 54 & 33.65 & 1 & 0.03 & $\mathrm{Sd}$ & $6.8 \pm 0.8$ & \\
\hline NGC 0809 & $\begin{array}{lll}02 & 04 & 19.0\end{array}$ & -084407.1 & 1.5 & 1.0 & 175 & 75 & 34.38 & 1 & 0.02 & (R)S0+: & $-1.0 \pm 1.2$ & \\
\hline UGC 01584 & $\begin{array}{lll}02 & 0526.8\end{array}$ & +131937.5 & 1.5 & 0.7 & 82 & 108 & 35.16 & 1 & 0.10 & Scd: & $6.0 \pm 1.2$ & \\
\hline NGC 0810 & 020528.9 & +131505.0 & 1.7 & 1.3 & 25 & 110 & 35.20 & 1 & 0.10 & & $-5.0 \pm 1.6$ & \\
\hline UGC 01593 & 020606.0 & +131706.8 & 1.0 & 0.8 & 0 & 106 & 35.13 & 1 & 0.09 & $\mathrm{SAB}(\mathrm{rs}) \mathrm{c}$ & $5.0 \pm 0.8$ & \\
\hline UGC 01603 & 020642.5 & -005137.7 & 1.2 & 1.0 & 0 & 84 & 34.62 & 1 & 0.04 & SAdm: & $8.0 \pm 1.1$ & NLAGN \\
\hline NGC 0830 & 020858.7 & -074600.5 & 1.4 & 0.9 & 60 & 54 & 33.65 & 1 & 0.02 & SB0-? & $-3.0 \pm 1.3$ & \\
\hline NGC 0842 & 020950.8 & -074544.8 & 1.2 & 0.9 & 142 & 54 & 33.65 & 1 & 0.02 & $\mathrm{SB} ?(\mathrm{r}:) 0^{*} 0^{*}$ & $\ldots$ & \\
\hline
\end{tabular}


Table 1-Continued

\begin{tabular}{|c|c|c|c|c|c|c|c|c|c|c|c|c|}
\hline $\begin{array}{c}\text { Object Name } \\
\text { (1) }\end{array}$ & $\begin{array}{c}\mathrm{RA}_{2000} \\
(\mathrm{~h}: \mathrm{m}: \mathrm{s}) \\
(2)\end{array}$ & $\begin{array}{c}\mathrm{DEC}_{2000} \\
(\mathrm{~d}: \mathrm{m}: \mathrm{s}) \\
(3)\end{array}$ & $\begin{array}{c}2 \times \mathrm{A} \\
(\operatorname{arcmin}) \\
(4)\end{array}$ & $\begin{array}{c}2 \times \mathrm{B} \\
(\operatorname{arcmin}) \\
(5)\end{array}$ & $\begin{array}{c}\text { PA } \\
(\mathrm{deg}) \\
(6)\end{array}$ & $\begin{array}{c}(\mathrm{Mpc}) \\
(7)\end{array}$ & $\begin{array}{l}\text { stance } \\
\text { DM } \\
(8)\end{array}$ & $\begin{array}{l}\text { ref. } \\
(9)\end{array}$ & $\begin{array}{c}\mathrm{E}(B-V) \\
(\mathrm{mag}) \\
(10)\end{array}$ & $\begin{array}{c}\text { Morphological } \\
\text { Type } \\
\text { (11) }\end{array}$ & $\begin{array}{c}\mathrm{T} \\
\text { Type } \\
(12)\end{array}$ & $\begin{array}{c}\text { Spectral } \\
\text { Type } \\
(13)\end{array}$ \\
\hline NGC 0814 & 021037.6 & -154624.9 & 1.3 & 0.5 & 0 & 21 & 31.63 & 1 & 0.03 & SB0*0* pec: & $-2.0 \pm 1.3$ & HII \\
\hline KUG 0210-078 & 021315.8 & -073942.8 & 1.7 & 1.3 & 125 & 67 & 34.13 & 1 & 0.03 & $\left(\mathrm{R}^{\prime}\right) \mathrm{SB}(\mathrm{rs}) \mathrm{a}$ & $1.0 \pm 0.8$ & \\
\hline NGC 0855 & 021403.6 & +275237.8 & 2.6 & 1.0 & 60 & 9.7 & 29.94 & 15 & 0.07 & $\mathrm{E}$ & $-5.0 \pm 0.7$ & \\
\hline ESO 415-G011 & 021415.6 & -320312.1 & 1.1 & 0.3 & 132 & 47 & 33.36 & 1 & 0.02 & $\mathrm{SB}(\mathrm{s}) \mathrm{b}$ pec? & $3.0 \pm 1.8$ & \\
\hline KUG 0211-075 & 021426.7 & -072159.3 & 2.3 & 0.4 & 60 & 70 & 34.21 & 1 & 0.03 & Sb pec sp & $3.0 \pm 0.8$ & \\
\hline NGC 0871 & $\begin{array}{lll}02 & 17 & 10.7\end{array}$ & +143252.2 & 1.2 & 0.5 & 4 & 54 & 33.64 & 1 & 0.10 & $\mathrm{SB}(\mathrm{s}) \mathrm{c}:$ & $5.0 \pm 0.7$ & \\
\hline KUG 0214-057 & $02 \quad 17 \quad 19.4$ & -052850.4 & 1.3 & 0.8 & 140 & 75 & 34.38 & 1 & 0.02 & SB? & $\ldots$ & \\
\hline UGC 01761 & $\begin{array}{lll}02 & 17 & 26.3\end{array}$ & $\begin{array}{r}+143448.7 \\
\end{array}$ & 1.1 & 1.1 & $\ldots$ & 57 & 33.80 & 1 & 0.11 & $\mathrm{Im}$ & $10.0 \pm 0.8$ & \\
\hline NGC 0881 & 021845.3 & -063820.7 & 2.2 & 1.5 & 140 & 74 & 34.34 & 1 & 0.03 & $\mathrm{SAB}(\mathrm{r}) \mathrm{c}$ & $5.0 \pm 0.6$ & \\
\hline NGC 0895 & 022136.5 & -053117.0 & 3.6 & 2.6 & 115 & 31 & 32.48 & 1 & 0.03 & $\mathrm{SA}(\mathrm{s}) \mathrm{cd}$ & $6.0 \pm 0.3$ & \\
\hline NGC 0891 & 022233.4 & +422056.9 & 13.5 & 2.5 & 22 & 9.6 & 29.91 & 12 & 0.06 & $\mathrm{SA}(\mathrm{s}) \mathrm{b} ? \mathrm{sp}$ & $3.0 \pm 0.3$ & HII \\
\hline NGC 0898 & 022320.4 & +415705.1 & 1.9 & 0.5 & 170 & 81 & 34.53 & 1 & 0.07 & $\mathrm{Sab}$ & $2.0 \pm 0.6$ & \\
\hline UGC 01859 & 022444.4 & +423723.7 & 1.7 & 1.1 & 47 & 87 & 34.69 & 1 & 0.06 & E? & $\ldots$ & \\
\hline NGC 0906 & $02 \quad 2516.3$ & +420523.6 & 1.8 & 1.6 & 0 & 69 & 34.19 & 1 & 0.07 & SBab & $2.0 \pm 0.8$ & \\
\hline NGC 0925 & $\begin{array}{lll}02 & 27 & 16.9\end{array}$ & +333445.0 & 10.5 & 5.9 & 102 & 9.3 & 29.84 & 16 & 0.08 & $\mathrm{SAB}(\mathrm{s}) \mathrm{d}$ & $7.0 \pm 0.3$ & HII \\
\hline PGC 09333 & $0227 \quad 17.6$ & -035358.2 & 1.1 & 0.9 & 0 & 186 & 36.34 & 1 & 0.03 & $\mathrm{SB}(\mathrm{s}) \mathrm{dm}$ & $8.0 \pm 0.9$ & \\
\hline NGC 0934 & 022732.9 & -001440.3 & 1.3 & 0.9 & 130 & 90 & 34.77 & 1 & 0.03 & SAB0- & $-2.5 \pm 0.5$ & \\
\hline UGC 01949 & $\begin{array}{lll}02 & 28 & 18.9\end{array}$ & -003629.5 & 1.2 & 0.9 & 55 & 20 & 31.50 & 1 & 0.03 & $\mathrm{IAB}(\mathrm{s}) \mathrm{m}$ : & $10.0 \pm 0.7$ & \\
\hline UGC 01976 & 023021.5 & +351933.4 & 1.6 & 0.8 & 113 & 132 & 35.60 & 1 & 0.07 & $\mathrm{Sb}$ & $3.0 \pm 0.8$ & \\
\hline NGC 0955 & 023033.1 & -010630.5 & 2.8 & 0.7 & 19 & 20 & 31.53 & 1 & 0.04 & Sab: sp & $1.8 \pm 0.6$ & \\
\hline UGC 02010 & 023207.1 & -012143.7 & 1.3 & 0.8 & 85 & 160 & 36.02 & 1 & 0.04 & $\mathrm{SB}(\mathrm{rs}) \mathrm{bc}$ & $4.0 \pm 0.6$ & \\
\hline NGC 0959 & 023223.9 & +352940.7 & 2.3 & 1.4 & 65 & 11 & 30.29 & 17 & 0.07 & Sdm: & $8.0 \pm 1.1$ & \\
\hline NGC 0986A & 023241.5 & -391746.4 & 1.8 & 0.7 & 72 & 17 & 31.11 & 1 & 0.02 & $\mathrm{SB}(\mathrm{s}) \mathrm{dm}$ & $9.7 \pm 0.7$ & \\
\hline NGC 0986 & 023334.3 & $\begin{array}{lll}-39 & 02 & 42.2\end{array}$ & 3.9 & 3.0 & 150 & 25 & 31.98 & 1 & 0.02 & $\left(\mathrm{R}^{\prime}-1\right) \mathrm{SB}(\mathrm{rs}) \mathrm{b}$ & $2.0 \pm 0.3$ & HII \\
\hline KUG 0232-079 & 023448.4 & -074100.9 & 1.1 & 1.0 & 0 & 93 & 34.84 & 1 & 0.03 & $\mathrm{SB}(\mathrm{s}) \mathrm{c}$ & $5.0 \pm 0.9$ & \\
\hline NGC 0991 & 023532.7 & -070916.0 & 2.7 & 2.4 & 75 & 20 & 31.55 & 1 & 0.03 & $\mathrm{SAB}(\mathrm{rs}) \mathrm{c}$ & $5.0 \pm 0.3$ & \\
\hline IC 0243 & $\begin{array}{lll}02 & 38 & 32.2\end{array}$ & -065407.7 & 1.2 & 0.8 & 33 & 102 & 35.04 & 1 & 0.03 & $\left(\mathrm{R}^{\prime}\right) \mathrm{SB}(\mathrm{rs}) 0 / \mathrm{a}$ & $0.0 \pm 0.9$ & \\
\hline NGC 1022 & 023832.7 & -064038.7 & 2.4 & 2.0 & 115 & 19 & 31.43 & 1 & 0.03 & (R')SB(s)a;HII & $1.0 \pm 0.3$ & Sbrst \\
\hline NGC 1035 & 023929.1 & -080758.6 & 2.2 & 0.7 & 150 & 16 & 31.05 & 1 & 0.03 & $\mathrm{SA}(\mathrm{s}) \mathrm{c} ?$ & $5.0 \pm 0.8$ & \\
\hline NGC 1033 & $\begin{array}{lll}02 & 40 & 16.1\end{array}$ & -084637.1 & 1.3 & 1.1 & 0 & 103 & 35.06 & 1 & 0.03 & $\mathrm{SA}(\mathrm{s}) \mathrm{c}:$ & $5.0 \pm 0.8$ & \\
\hline NGC 1042 & 024024.0 & -08 2600.8 & 4.7 & 3.6 & 10 & 18 & 31.28 & 1 & 0.03 & $\mathrm{SAB}(\mathrm{rs}) \mathrm{cd}$ & $6.0 \pm 0.3$ & NLAGN \\
\hline NGC 1023 & 024024.0 & +390347.7 & 8.7 & 3.0 & 87 & 11 & 30.29 & 15 & 0.06 & $\mathrm{SB}(\mathrm{rs}) 0-$ & $-3.0 \pm 0.3$ & \\
\hline NGC 1047 & 024032.8 & -080851.6 & 1.3 & 0.6 & 90 & 18 & 31.23 & 1 & 0.03 & $\mathrm{~S} 0+: \mathrm{sp}$ & $-0.5 \pm 0.9$ & \\
\hline
\end{tabular}


Table 1-Continued

\begin{tabular}{|c|c|c|c|c|c|c|c|c|c|c|c|c|}
\hline $\begin{array}{c}\text { Object Name } \\
\text { (1) }\end{array}$ & $\begin{array}{c}\mathrm{RA}_{2000} \\
(\mathrm{~h}: \mathrm{m}: \mathrm{s}) \\
(2)\end{array}$ & $\begin{array}{c}\mathrm{DEC}_{2000} \\
(\mathrm{~d}: \mathrm{m}: \mathrm{s}) \\
(3)\end{array}$ & $\begin{array}{c}2 \times \mathrm{A} \\
(\operatorname{arcmin}) \\
(4)\end{array}$ & $\begin{array}{c}2 \times \mathrm{B} \\
(\operatorname{arcmin}) \\
(5)\end{array}$ & $\begin{array}{c}\text { PA } \\
(\mathrm{deg}) \\
(6)\end{array}$ & $\begin{array}{c}(\mathrm{Mpc}) \\
\quad(7)\end{array}$ & $\begin{array}{l}\text { istance } \\
\text { DM } \\
(8)\end{array}$ & $\begin{array}{l}\text { ref. } \\
(9)\end{array}$ & $\begin{array}{c}\mathrm{E}(B-V) \\
(\mathrm{mag}) \\
(10)\end{array}$ & $\begin{array}{c}\text { Morphological } \\
\text { Type } \\
\text { (11) }\end{array}$ & $\begin{array}{c}\mathrm{T} \\
\text { Type } \\
(12)\end{array}$ & $\begin{array}{c}\text { Spectral } \\
\text { Type } \\
(13)\end{array}$ \\
\hline NGC 1023A & 024037.7 & +390327.0 & 1.3 & 0.6 & 50 & 12 & 30.47 & 1 & 0.06 & IB? & . & \\
\hline NGC 0961 & 024102.5 & -065609.1 & 2.1 & 1.5 & 50 & 17 & 31.16 & 1 & 0.03 & $\mathrm{SB}(\mathrm{rs}) \mathrm{m}$ pec & $8.5 \pm 0.5$ & \\
\hline NGC 1052 & 024104.8 & -081520.8 & 3.0 & 2.1 & 120 & 19 & 31.44 & 1 & 0.03 & E4;LINER & $-5.0 \pm 0.3$ & Sy2 \\
\hline NGC 1055 & 024145.2 & +002635.4 & 7.6 & 2.7 & 105 & 13 & 30.61 & 1 & 0.03 & SBb: sp & $3.0 \pm 0.4$ & LINER2 \\
\hline PGC 10213 & 024150.0 & -075530.0 & 1.3 & 1.3 & $\ldots$ & 18 & 31.28 & 1 & 0.03 & $\mathrm{SB}(\mathrm{s}) \mathrm{m}$ & $9.0 \pm 0.9$ & \\
\hline UGC 02174 & 024205.8 & +322244.0 & 2.1 & 2.1 & $\cdots$ & 75 & 34.36 & 1 & 0.13 & $\mathrm{SAB}(\mathrm{s}) \mathrm{c}$ & $5.0 \pm 0.7$ & \\
\hline NGC 1068 & 024240.7 & -000047.8 & 7.1 & 6.0 & 70 & 14 & 30.79 & 18 & 0.03 & (R)SA(rs)b;Sy1 & $3.0 \pm 0.3$ & Sy2 \\
\hline UGC 02182 & 024249.6 & +324108.1 & 1.3 & 0.4 & 161 & 77 & 34.42 & 1 & 0.14 & $\mathrm{Im}$ & $10.0 \pm 0.9$ & \\
\hline NGC 1069 & 024259.8 & -081722.2 & 1.4 & 0.9 & 145 & 134 & 35.64 & 1 & 0.02 & $\mathrm{SAB}(\mathrm{s}) \mathrm{c}$ & $5.0 \pm 0.5$ & \\
\hline NGC 1060 & $0243 \quad 15.1$ & +322529.9 & 2.3 & 1.7 & 75 & 75 & 34.38 & 1 & 0.21 & S0-: & $-3.0 \pm 1.0$ & \\
\hline NGC 1072 & 024331.3 & +00 1824.6 & 1.5 & 0.5 & 11 & 114 & 35.28 & 1 & 0.04 & $\mathrm{SB}(\mathrm{rs}) \mathrm{b}:$ & $2.7 \pm 0.5$ & \\
\hline PGC 10334 & 024342.8 & -063905.0 & 1.5 & 0.9 & 15 & 19 & 31.36 & 1 & 0.03 & $\mathrm{IB}(\mathrm{s}) \mathrm{m}$ & $10.0 \pm 0.6$ & \\
\hline UGC 02201 & 024344.2 & +322945.3 & 1.4 & 0.3 & 101 & 60 & 33.90 & 1 & 0.19 & $\mathrm{Sc}$ & $7.0 \pm 0.9$ & \\
\hline NGC 1066 & 024349.9 & +322830.0 & 1.7 & 1.6 & 0 & 63 & 34.01 & 1 & 0.22 & $\mathrm{E}:$ & $-5.0 \pm 1.1$ & \\
\hline NGC 1067 & 024350.5 & +323042.8 & 1.0 & 1.0 & $\ldots$ & 66 & 34.10 & 1 & 0.19 & $\mathrm{SAB}(\mathrm{s}) \mathrm{c}$ & $5.0 \pm 0.8$ & \\
\hline NGC 1084 & 024559.9 & -073443.1 & 3.2 & 1.8 & 35 & 19 & 31.34 & 1 & 0.03 & $\mathrm{SA}(\mathrm{s}) \mathrm{c}$ & $5.0 \pm 0.3$ & \\
\hline NGC 1097 & 024619.1 & $\begin{array}{lll}-30 & 16 & 29.7\end{array}$ & 9.3 & 6.3 & 130 & 15 & 30.92 & 1 & 0.03 & $\left(\mathrm{R}^{\prime}-1:\right) \mathrm{SB}\left(\mathrm{r}^{\prime} \mathrm{l}\right) \mathrm{b}$ & $3.0 \pm 0.3$ & Sy1 \\
\hline PGC 10766 & 025017.8 & -083549.5 & 2.2 & 0.3 & 95 & 74 & 34.36 & 1 & 0.03 & $\mathrm{Sbc}$ & $5.0 \pm 0.6$ & \\
\hline PGC 10794 & 025044.2 & -064445.3 & 1.3 & 0.6 & 10 & 98 & 34.97 & 1 & 0.04 & Sb pec? & $3.0 \pm 1.8$ & \\
\hline PGC 10875 & 025223.4 & -083037.5 & 1.4 & 0.2 & 96 & 70 & 34.23 & 1 & 0.05 & $\mathrm{Sb}$ & $0.5 \pm 1.3$ & \\
\hline NGC 1140 & 025433.6 & -100139.9 & 1.7 & 0.9 & 10 & 18 & 31.30 & 19 & 0.04 & IBm pec:;HII & $10.0 \pm 0.4$ & Sy2 \\
\hline NGC 1148 & 025704.4 & -074108.4 & 1.4 & 0.7 & 70 & 73 & 34.30 & 1 & 0.05 & $\mathrm{SB}(\mathrm{rs}) \mathrm{d}$ pec? & $7.0 \pm 1.7$ & \\
\hline UGC 02442 & 025835.6 & +251648.3 & 1.1 & 1.0 & 150 & 150 & 35.88 & 1 & 0.15 & $\mathrm{~S} ?$ & $\ldots$ & \\
\hline NGC 1156 & 025942.2 & +251414.2 & 3.3 & 2.5 & 25 & 7.8 & 29.46 & 20 & 0.22 & $\mathrm{IB}(\mathrm{s}) \mathrm{m}$ & $10.0 \pm 0.3$ & \\
\hline PGC 11767 & 030848.3 & -070226.2 & 1.3 & 0.4 & 10 & 123 & 35.44 & 1 & 0.07 & $\mathrm{SB}(\mathrm{rs}) \mathrm{cd} ?$ & $6.0 \pm 0.9$ & \\
\hline UGC 02519 & 030919.9 & +800754.8 & 1.2 & 0.7 & 80 & 38 & 32.89 & 1 & 0.26 & Scd? & $6.0 \pm 1.7$ & \\
\hline NGC 1241 & 031114.6 & -085519.7 & 2.8 & 1.7 & 140 & 56 & 33.73 & 1 & 0.11 & $\mathrm{SB}(\mathrm{rs}) \mathrm{b}$ & $3.0 \pm 0.3$ & Sy2 \\
\hline NGC 1242 & 031119.3 & -085408.7 & 1.2 & 0.7 & 130 & 55 & 33.70 & 1 & 0.11 & $\mathrm{SB}(\mathrm{rs}) \mathrm{c}:$ & $5.4 \pm 0.5$ & \\
\hline NGC 1266 & 031600.8 & -022538.5 & 1.5 & 1.0 & 110 & 31 & 32.48 & 3 & 0.10 & $\left(\mathrm{R}^{\prime}\right) \mathrm{SB}(\mathrm{rs}) 0$ pec & $-2.0 \pm 0.7$ & LINER \\
\hline NGC 1291 & 031718.6 & -410629.1 & 9.8 & 8.1 & 165 & 9.7 & 29.93 & 3 & 0.01 & $(\mathrm{R}-1) \mathrm{SB}(\mathrm{l}) 0 / \mathrm{a}$ & $0.0 \pm 0.3$ & \\
\hline NGC 1285 & 031753.4 & -071752.1 & 1.5 & 1.1 & 10 & 73 & 34.32 & 1 & 0.05 & $\left(\mathrm{R}^{\prime}\right) \mathrm{SB}(\mathrm{r}) \mathrm{b}$ pec & $3.0 \pm 0.8$ & \\
\hline NGC 1299 & 032009.7 & -061543.0 & 1.1 & 0.6 & 40 & 32 & 32.50 & 1 & 0.05 & $\mathrm{SB}(\mathrm{rs}) \mathrm{b} ?$ & $3.0 \pm 1.3$ & \\
\hline NGC 1310 & 032103.4 & -370606.1 & 2.0 & 1.5 & 95 & 22 & 31.76 & 1 & 0.02 & $\mathrm{SB}(\mathrm{rs}) \mathrm{cd}$ & $5.0 \pm 0.5$ & HII \\
\hline
\end{tabular}


Table 1-Continued

\begin{tabular}{|c|c|c|c|c|c|c|c|c|c|c|c|c|}
\hline $\begin{array}{c}\text { Object Name } \\
\text { (1) }\end{array}$ & $\begin{array}{c}\mathrm{RA}_{2000} \\
(\mathrm{~h}: \mathrm{m}: \mathrm{s}) \\
(2)\end{array}$ & $\begin{array}{c}\mathrm{DEC}_{2000} \\
(\mathrm{~d}: \mathrm{m}: \mathrm{s}) \\
(3)\end{array}$ & $\begin{array}{c}2 \times \mathrm{A} \\
(\operatorname{arcmin}) \\
(4)\end{array}$ & $\begin{array}{c}2 \times \mathrm{B} \\
(\operatorname{arcmin}) \\
(5)\end{array}$ & $\begin{array}{c}\text { PA } \\
(\mathrm{deg}) \\
(6)\end{array}$ & $\begin{array}{c}(\mathrm{Mpc}) \\
(7)\end{array}$ & $\begin{array}{c}\text { istance } \\
\text { DM } \\
(8)\end{array}$ & $\begin{array}{l}\text { ref. } \\
(9)\end{array}$ & $\begin{array}{c}\mathrm{E}(B-V) \\
(\mathrm{mag}) \\
(10)\end{array}$ & $\begin{array}{c}\text { Morphological } \\
\text { Type } \\
(11)\end{array}$ & $\begin{array}{c}\mathrm{T} \\
\text { Type } \\
(12)\end{array}$ & $\begin{array}{c}\text { Spectral } \\
\text { Type } \\
(13)\end{array}$ \\
\hline KUG 0319-072 & 032217.5 & -070526.5 & 1.1 & 1.0 & 0 & 37 & 32.86 & 1 & 0.07 & SA(r)ab pec: & $2.0 \pm 1.2$ & \\
\hline NGC 1316 & 032241.7 & -371229.6 & 12.0 & 8.5 & 50 & 19 & 31.35 & 21 & 0.02 & $\left(\mathrm{R}^{\prime}\right) \mathrm{SAB}(\mathrm{s}) 0^{*} 0$ & $-2.0 \pm 0.3$ & LINER \\
\hline NGC 1317 & 032244.3 & -370613.6 & 2.8 & 2.4 & 78 & 19 & 31.35 & 21 & 0.02 & $\left(\mathrm{R}^{\prime}\right) \mathrm{SAB}(\mathrm{rl}) 0 / \mathrm{a}$ & $1.0 \pm 0.3$ & \\
\hline ESO 357-G025 & 032337.3 & -354642.2 & 1.3 & 0.4 & 25 & 23 & 31.79 & 1 & 0.02 & SAB $0 * 0 *:$ & $-2.5 \pm 0.6$ & \\
\hline PGC 12706 & 032354.3 & -373024.4 & 1.4 & 1.3 & 0 & 17 & 31.12 & 1 & 0.02 & $\mathrm{SB} ?(\mathrm{r}) \mathrm{cd} ? \mathrm{p}$ & $10.0 \pm 0.7$ & \\
\hline NGC 1326 & 032356.4 & -362752.8 & 3.9 & 2.9 & 77 & 16 & 31.04 & 1 & 0.02 & $(\mathrm{R}-1) \mathrm{SB}(\mathrm{rl}) 0 / \mathrm{a}$ & $-1.0 \pm 0.3$ & LINER \\
\hline PGC 13005 & 033009.5 & -053117.9 & 1.2 & 0.3 & 155 & 58 & 33.82 & 1 & 0.05 & Sa pec sp & $0.5 \pm 1.3$ & \\
\hline NGC 1346 & 033013.3 & -053235.9 & 1.0 & 0.7 & 75 & 56 & 33.74 & 1 & 0.05 & Sb pec? & $3.0 \pm 1.3$ & \\
\hline PGC 13058 & 033108.3 & -361724.5 & 1.0 & 0.6 & 160 & 23 & 31.77 & 1 & 0.01 & SA0-: & $-3.0 \pm 0.9$ & \\
\hline ESO 418-G008 & 033130.7 & -301248.0 & 1.2 & 0.8 & 149 & 14 & 30.74 & 1 & 0.01 & $\mathrm{SB}(\mathrm{r}) \mathrm{d}$ & $8.0 \pm 0.5$ & \\
\hline NGC 1365 & 033336.4 & -360825.5 & 11.2 & 6.2 & 32 & 20 & 31.51 & 1 & 0.02 & $\left(\mathrm{R}^{\prime}\right) \mathrm{SBb}(\mathrm{s}) \mathrm{b}$ & $3.0 \pm 0.3$ & Sy1.8 \\
\hline PGC 13186 & 033343.4 & -355133.0 & 1.3 & 1.0 & 45 & 19 & 31.36 & 1 & 0.02 & E4: & $-5.0 \pm 0.8$ & \\
\hline NGC 1361 & 033417.7 & -061554.0 & 1.6 & 1.4 & 0 & 74 & 34.34 & 1 & 0.04 & E+ pec: & $-4.0 \pm 1.2$ & \\
\hline PGC 13230 & 033429.5 & -353247.0 & 1.3 & 1.0 & 0 & 14 & 30.72 & 1 & 0.02 & $\mathrm{SAB}(\mathrm{rs}) 0 * 0 *$ & $-2.0 \pm 0.8$ & \\
\hline NGC 1373 & 033459.2 & -351016.0 & 1.1 & 0.9 & 131 & 16 & 30.99 & 1 & 0.01 & $\mathrm{E}+:$ & $-4.3 \pm 0.6$ & \\
\hline NGC 1374 & 033516.6 & -351334.5 & 2.5 & 2.3 & 0 & 15 & 30.91 & 1 & 0.01 & E3 & $-4.5 \pm 0.4$ & \\
\hline NGC 1375 & 033516.8 & -351556.4 & 2.2 & 0.9 & 91 & 7.3 & 29.32 & 1 & 0.01 & $\mathrm{SAB} 0^{*} 0^{*}: \mathrm{sp}$ & $-2.0 \pm 0.5$ & \\
\hline NGC 1379 & $03 \quad 3603.9$ & -352628.3 & 2.4 & 2.3 & 0 & 16 & 30.97 & 1 & 0.01 & E3 & $-5.0 \pm 0.4$ & \\
\hline UGCA 080 & 033621.3 & -064253.7 & 1.6 & 1.0 & 165 & 42 & 33.14 & 1 & 0.04 & $\mathrm{SB}(\mathrm{s}) \mathrm{m}$ & $9.0 \pm 0.6$ & \\
\hline NGC 1380 & 033627.6 & -345833.6 & 4.8 & 2.3 & 7 & 24 & 31.86 & 1 & 0.02 & SAO & $-2.0 \pm 0.6$ & \\
\hline NGC 1381 & 033631.7 & -351742.7 & 2.7 & 0.7 & 139 & 19 & 31.35 & 21 & 0.01 & SA0: sp & $-1.6 \pm 0.5$ & \\
\hline NGC 1386 & 033646.2 & -355957.3 & 3.4 & 1.3 & 25 & 9.1 & 29.80 & 1 & 0.01 & $\mathrm{SB}(\mathrm{s}) 0+$ & $-0.6 \pm 0.5$ & Sy2 \\
\hline NGC $1380 \mathrm{~A}$ & 033647.5 & -344422.6 & 2.4 & 0.7 & 179 & 19 & 31.40 & 1 & 0.01 & $\mathrm{~S} 0^{*} 0^{*}: \mathrm{sp}$ & $-2.0 \pm 0.8$ & \\
\hline PGC 13343 & 033654.3 & -352229.0 & 1.1 & 1.1 & $\cdots$ & 20 & 31.55 & 1 & 0.01 & SA0-: & $-3.0 \pm 1.2$ & \\
\hline NGC 1387 & 033657.1 & -353023.9 & 2.8 & 2.8 & $\ldots$ & 19 & 31.35 & 21 & 0.01 & $\mathrm{SAB}(\mathrm{s}) 0-$ & $-3.0 \pm 0.3$ & \\
\hline NGC 1380B & 033709.0 & -351142.1 & 1.5 & 1.3 & 0 & 22 & 31.67 & 1 & 0.02 & $\mathrm{SAB}(\mathrm{s}) 0-:$ & $-2.7 \pm 0.6$ & \\
\hline NGC 1389 & 033711.7 & -354446.0 & 2.3 & 1.4 & 30 & 19 & 31.35 & 21 & 0.01 & $\mathrm{SAB}(\mathrm{s}) 0-\mathrm{:}$ & $-3.3 \pm 0.4$ & \\
\hline NGC 1385 & 033728.3 & -243004.7 & 3.4 & 2.0 & 165 & 19 & 31.36 & 1 & 0.02 & $\mathrm{SB}(\mathrm{s}) \mathrm{cd}$ & $6.0 \pm 0.3$ & \\
\hline NGC 1383 & 033739.2 & -182022.1 & 1.9 & 0.9 & 91 & 25 & 32.03 & 1 & 0.07 & $\operatorname{SAB}(\mathrm{s}) 0^{*} 0^{*}$ & $-2.0 \pm 0.6$ & \\
\hline NGC 1396 & 033806.5 & -352624.4 & 1.0 & 0.9 & 0 & 8.3 & 29.59 & 1 & 0.01 & SABO-: & $-3.0 \pm 1.2$ & \\
\hline ESO 358-G042 & $03 \quad 3809.2$ & -343106.7 & 1.2 & 0.5 & 135 & 13 & 30.57 & 1 & 0.01 & SB $0 * 0 *$ : & $-2.0 \pm 0.8$ & \\
\hline NGC 1399 & 033829.1 & -352702.7 & 6.9 & 6.5 & 0 & 19 & 31.35 & 21 & 0.01 & $\mathrm{cD} ; \mathrm{E} 1 \mathrm{pec}$ & $-5.0 \pm 0.3$ & \\
\hline NGC 1393 & 033838.6 & -182540.7 & 1.9 & 1.3 & 170 & 28 & 32.24 & 1 & 0.07 & $\mathrm{SA}(\mathrm{rl}) 0 * 0$ & $-1.9 \pm 0.4$ & \\
\hline
\end{tabular}


Table 1-Continued

\begin{tabular}{|c|c|c|c|c|c|c|c|c|c|c|c|c|}
\hline $\begin{array}{c}\text { Object Name } \\
\text { (1) }\end{array}$ & $\begin{array}{c}\mathrm{RA}_{2000} \\
(\mathrm{~h}: \mathrm{m}: \mathrm{s}) \\
(2)\end{array}$ & $\begin{array}{c}\mathrm{DEC}_{2000} \\
(\mathrm{~d}: \mathrm{m}: \mathrm{s}) \\
(3)\end{array}$ & $\begin{array}{c}2 \times \mathrm{A} \\
(\operatorname{arcmin}) \\
(4)\end{array}$ & $\begin{array}{c}2 \times \mathrm{B} \\
(\operatorname{arcmin}) \\
(5)\end{array}$ & $\begin{array}{c}\text { PA } \\
(\text { deg }) \\
(6)\end{array}$ & $\begin{array}{c}(\mathrm{Mpc}) \\
(7)\end{array}$ & $\begin{array}{c}\text { istance } \\
\text { DM } \\
(8)\end{array}$ & $\begin{array}{l}\text { ref. } \\
(9)\end{array}$ & $\begin{array}{c}\mathrm{E}(B-V) \\
(\mathrm{mag}) \\
(10)\end{array}$ & $\begin{array}{c}\text { Morphological } \\
\text { Type } \\
(11)\end{array}$ & $\begin{array}{c}\mathrm{T} \\
\text { Type } \\
(12)\end{array}$ & $\begin{array}{c}\text { Spectral } \\
\text { Type } \\
(13)\end{array}$ \\
\hline NGC 1404 & 033851.9 & -353539.8 & 3.3 & 3.0 & 0 & 19 & 31.35 & 21 & 0.01 & E1 & $-5.0 \pm 0.3$ & \\
\hline NGC 1391 & 033853.0 & -182114.8 & 1.1 & 0.5 & 65 & 59 & 33.87 & 1 & 0.07 & $\mathrm{SB}(\mathrm{s}) 0^{*} 0^{*}$ & $-1.5 \pm 0.6$ & \\
\hline NGC 1394 & 033906.9 & -181732.2 & 1.3 & 0.4 & 5 & 58 & 33.83 & 1 & 0.07 & $\mathrm{~S} 0^{*} 0^{*}: \mathrm{sp}$ & $-2.0 \pm 0.8$ & \\
\hline AM 0337-355 & 033919.6 & -354335.0 & 1.1 & 1.1 & $\cdots$ & 9.6 & 29.91 & 1 & 0.01 & E0: & $-5.0 \pm 1.2$ & \\
\hline NGC 1400 & 033930.8 & $-1841 \quad 17.4$ & 2.3 & 2.0 & 40 & 25 & 32.02 & 22 & 0.06 & SA0- & $-3.0 \pm 0.3$ & \\
\hline IC 0343 & 034007.1 & -182636.5 & 1.6 & 0.8 & 118 & 24 & 31.90 & 1 & 0.07 & $\mathrm{SB}(\mathrm{rs}) 0+:$ & $-1.0 \pm 0.5$ & \\
\hline NGC $1427 \mathrm{~A}$ & 034009.3 & -353728.0 & 2.3 & 1.5 & 76 & 19 & 31.35 & 21 & 0.01 & $\mathrm{IB}(\mathrm{s}) \mathrm{m}$ & $10.0 \pm 0.6$ & \\
\hline NGC 1407 & 034011.9 & -183449.4 & 4.6 & 4.3 & 35 & 18 & 31.23 & 22 & 0.07 & E0 & $-5.0 \pm 0.3$ & \\
\hline ESO 548-G068 & 034019.2 & -185553.4 & 1.4 & 0.7 & 133 & 22 & 31.69 & 1 & 0.07 & $\mathrm{SB}(\mathrm{s}) 0-: \mathrm{sp}$ & $-2.5 \pm 0.8$ & \\
\hline PGC 13515 & 034023.6 & -351636.4 & 1.2 & 1.0 & 0 & 26 & 32.07 & 1 & 0.01 & E0 & $-5.0 \pm 0.8$ & \\
\hline PGC 13535 & 034043.0 & -062454.6 & 1.9 & 1.5 & 15 & 73 & 34.33 & 1 & 0.05 & $\mathrm{SAB}(\mathrm{r}) \mathrm{cd}$ & $6.0 \pm 0.8$ & \\
\hline PGC 13600 & 034210.3 & -064555.2 & 1.8 & 1.4 & 115 & 73 & 34.30 & 1 & 0.07 & $\left(\mathrm{R}^{\prime}\right) \mathrm{SAB}(\mathrm{rs}) 0 / \mathrm{a}:$ & $0.0 \pm 0.8$ & \\
\hline IC 0334 & $03 \quad 4517.1$ & +763817.9 & 2.5 & 1.9 & 80 & 40 & 33.00 & 1 & 0.24 & $\mathrm{~S} ?$ & $\ldots$ & \\
\hline PGC 13820 & 034635.9 & -042701.5 & 2.3 & 0.3 & 25 & 53 & 33.61 & 1 & 0.07 & $\mathrm{Sc}$ & $5.0 \pm 0.9$ & \\
\hline NGC 1481 & 035429.0 & -202537.8 & 1.0 & 0.7 & 133 & 22 & 31.74 & 1 & 0.04 & SA0-: & $-3.3 \pm 0.6$ & \\
\hline NGC 1482 & 035438.9 & -203008.8 & 2.5 & 1.4 & 103 & 25 & 31.98 & 1 & 0.04 & $\mathrm{SA} 0+$ pec sp & $-0.8 \pm 0.5$ & HII \\
\hline PGC 14100 & 035504.8 & -061316.9 & 1.4 & 0.9 & 120 & 69 & 34.18 & 1 & 0.09 & $\mathrm{SAB}(\mathrm{rs}) \mathrm{dm}:$ & $8.3 \pm 0.7$ & \\
\hline NGC 1510 & 040332.6 & -432400.4 & 1.3 & 0.7 & 90 & 10 & 30.09 & 23 & 0.01 & SA $0 * 0^{*}$ pec?;HII & $-2.3 \pm 0.7$ & $\mathrm{BCDG}$ \\
\hline NGC 1512 & 040354.3 & -432055.9 & 8.9 & 5.6 & 90 & 10 & 30.09 & 3 & 0.01 & $\mathrm{SB}(\mathrm{r}) \mathrm{ab}$ & $1.0 \pm 0.5$ & \\
\hline UGC 02955 & 040829.3 & +694021.1 & 1.2 & 0.4 & 98 & 18 & 31.25 & 1 & 0.43 & $\mathrm{~S} ?$ & $\ldots$ & \\
\hline NGC 1546 & 041436.5 & -560338.9 & 3.0 & 1.7 & 147 & 17 & 31.18 & 24 & 0.01 & $\mathrm{SA} 0+?$ & $-1.3 \pm 0.6$ & \\
\hline NGC 1549 & 041545.1 & -553532.1 & 4.9 & 4.1 & 135 & 17 & 31.18 & 24 & 0.01 & $\mathrm{E} 0-1$ & $-5.0 \pm 0.6$ & \\
\hline NGC 1553 & 041610.5 & -554648.5 & 4.5 & 2.8 & 150 & 17 & 31.18 & 25 & 0.01 & $\mathrm{SA}(\mathrm{rl}) 0 * 0$ & $-2.0 \pm 0.3$ & LINER \\
\hline IC 2058 & 041754.3 & -555558.4 & 3.0 & 0.4 & 18 & 15 & 30.94 & 1 & 0.02 & $\mathrm{Sc}$ & $6.6 \pm 0.7$ & \\
\hline NGC 1566 & 042000.4 & -545616.1 & 8.3 & 6.6 & 60 & 17 & 31.21 & 1 & 0.01 & $\left(\mathrm{R}^{\prime}-1\right) \mathrm{SAB}(\mathrm{rs}) \mathrm{bc}$ & $4.0 \pm 0.6$ & Sy1 \\
\hline NGC 1569 & 043049.1 & +645052.6 & 3.6 & 1.8 & 120 & 2.0 & 26.45 & 26 & 0.70 & IBm;Sbrst & $10.0 \pm 0.4$ & Sy1 \\
\hline NGC 1672 & 044542.5 & -591450.2 & 6.6 & 5.5 & 170 & 15 & 30.90 & 1 & 0.02 & $\left(\mathrm{R}^{\prime}-1:\right) \mathrm{SB}(\mathrm{r}) \mathrm{bc}$ & $3.0 \pm 0.6$ & Sy2 \\
\hline NGC 1705 & 045413.5 & $\begin{array}{lll}-532139.8\end{array}$ & 1.9 & 1.4 & 50 & 5.1 & 28.54 & 27 & 0.01 & SA0- pec:;HII & $11.0 \pm 0.6$ & $\mathrm{BCDG}$ \\
\hline ESO 422-G027 & 050507.1 & -314703.1 & 1.4 & 0.9 & 9 & 53 & 33.63 & 1 & 0.02 & $\mathrm{SAB}(\mathrm{r}) \mathrm{c}$ & $5.0 \pm 0.8$ & \\
\hline NGC 1800 & 050625.7 & -315715.2 & 2.0 & 1.1 & 113 & 8.3 & 29.59 & 1 & 0.01 & $\mathrm{IB}(\mathrm{s}) \mathrm{m}$ & $10.0 \pm 0.6$ & HII \\
\hline NGC 1808 & 050742.3 & -373047.0 & 6.5 & 3.9 & 133 & 11 & 30.18 & 1 & 0.03 & $\left(\mathrm{R}^{\prime}-1\right) \mathrm{SAB}(\mathrm{s}:) \mathrm{b}$ & $1.0 \pm 0.3$ & Sy2 \\
\hline IC 0411 & 052018.6 & -251928.2 & 1.2 & 0.9 & 141 & 134 & 35.63 & 1 & 0.03 & S0? & $\ldots$ & \\
\hline ESO 204-G006 & 052450.4 & -482716.4 & 1.1 & 0.5 & 65 & 190 & 36.40 & 1 & 0.03 & (R)SA(r)a & $1.0 \pm 0.6$ & \\
\hline
\end{tabular}


Table 1-Continued

\begin{tabular}{|c|c|c|c|c|c|c|c|c|c|c|c|c|}
\hline $\begin{array}{c}\text { Object Name } \\
\text { (1) }\end{array}$ & $\begin{array}{c}\mathrm{RA}_{2000} \\
(\mathrm{~h}: \mathrm{m}: \mathrm{s}) \\
(2)\end{array}$ & $\begin{array}{c}\mathrm{DEC}_{2000} \\
(\mathrm{~d}: \mathrm{m}: \mathrm{s}) \\
(3)\end{array}$ & $\begin{array}{c}2 \times \mathrm{A} \\
(\operatorname{arcmin}) \\
(4)\end{array}$ & $\begin{array}{c}2 \times \mathrm{B} \\
(\operatorname{arcmin}) \\
(5)\end{array}$ & $\begin{array}{c}\text { PA } \\
(\mathrm{deg}) \\
(6)\end{array}$ & $\begin{array}{c}(\mathrm{Mpc}) \\
(7)\end{array}$ & $\begin{array}{c}\text { istance } \\
\text { DM } \\
(8)\end{array}$ & $\begin{array}{l}\text { ref. } \\
(9)\end{array}$ & $\begin{array}{c}\mathrm{E}(B-V) \\
(\mathrm{mag}) \\
(10)\end{array}$ & $\begin{array}{c}\text { Morphological } \\
\text { Type } \\
\text { (11) }\end{array}$ & $\begin{array}{c}\mathrm{T} \\
\text { Type } \\
(12)\end{array}$ & $\begin{array}{c}\text { Spectral } \\
\text { Type } \\
(13)\end{array}$ \\
\hline ESO 204-G007 & 052506.0 & -483512.2 & 1.3 & 0.2 & 94 & 102 & 35.04 & 1 & 0.04 & $\mathrm{SB}(\mathrm{s}) \mathrm{b} ? \mathrm{sp}$ & $3.0 \pm 1.3$ & \\
\hline ESO 033-G022 & 053140.4 & -734450.5 & 2.0 & 0.1 & 170 & 58 & 33.83 & 1 & 0.08 & Scd & $7.0 \pm 1.0$ & \\
\hline NGC 1964 & 053321.8 & -215644.8 & 5.6 & 2.1 & 32 & 21 & 31.62 & 1 & 0.04 & $\mathrm{SAB}(\mathrm{s}) \mathrm{b}$ & $3.0 \pm 0.3$ & \\
\hline NGC 1961 & 054204.8 & +692243.3 & 4.6 & 3.0 & 85 & 60 & 33.87 & 1 & 0.12 & $\mathrm{SAB}(\mathrm{rs}) \mathrm{c}$ & $5.0 \pm 0.3$ & LINER \\
\hline UGC 03342 & 054429.7 & +69 1756.3 & 1.7 & 0.4 & 42 & 60 & 33.90 & 1 & 0.11 & Scd: & $6.0 \pm 1.3$ & \\
\hline UGC 03344 & 054456.6 & +690933.5 & 2.5 & 1.5 & 25 & 65 & 34.05 & 1 & 0.12 & SABbc & $4.0 \pm 0.7$ & \\
\hline NGC 2090 & 054701.9 & $-34 \quad 1502.2$ & 4.9 & 2.4 & 13 & 11 & 30.27 & 9 & 0.04 & $\mathrm{SA}:(\mathrm{rs}) \mathrm{b}$ & $5.0 \pm 0.3$ & \\
\hline UGC 03403 & 061032.9 & +712245.0 & 2.3 & 0.7 & 27 & 22 & 31.66 & 1 & 0.23 & SBcd? & $6.0 \pm 1.1$ & \\
\hline UGC 03422 & 061508.1 & +710812.1 & 2.0 & 1.6 & 43 & 61 & 33.94 & 1 & 0.19 & $\mathrm{SAB}(\mathrm{rs}) \mathrm{b}$ & $3.0 \pm 0.8$ & \\
\hline Mrk 3 & 061536.4 & +710215.1 & 1.8 & 1.6 & 20 & 61 & 33.92 & 1 & 0.19 & S0: & $-2.0 \pm 1.1$ & Sy2 \\
\hline NGC 2207 & $\begin{array}{lll}06 & 16 & 22.0\end{array}$ & -212221.6 & 4.3 & 2.8 & 141 & 37 & 32.82 & 1 & 0.09 & $\mathrm{SAB}(\mathrm{rs}) \mathrm{bc}$ pec & $4.0 \pm 0.3$ & \\
\hline IC 2163 & $\begin{array}{lll}06 & 1628.0\end{array}$ & -212233.1 & 3.0 & 1.2 & 98 & 36 & 32.78 & 1 & 0.09 & $\mathrm{SB}(\mathrm{rs}) \mathrm{c}$ pec & $5.0 \pm 0.5$ & \\
\hline UGC 03423 & 061742.5 & +784918.4 & 1.1 & 0.2 & 150 & 65 & 34.05 & 1 & 0.11 & $\mathrm{Sdm}$ & $8.0 \pm 1.3$ & \\
\hline ESO 556-G012 & $\begin{array}{lll}06 & 17 & 49.2\end{array}$ & -210338.0 & 1.6 & 0.9 & 126 & 34 & 32.67 & 1 & 0.09 & $\mathrm{SB}(\mathrm{s}) \mathrm{m}$ & $8.7 \pm 0.5$ & \\
\hline NGC 2146 & $\begin{array}{lll}06 & 18 & 37.7\end{array}$ & +782125.3 & 6.0 & 3.4 & 124 & 16 & 31.08 & 1 & 0.10 & $\mathrm{SB}(\mathrm{s}) \mathrm{ab}$ pec & $2.0 \pm 0.3$ & HII \\
\hline NGC $2146 \mathrm{~A}$ & 062355.2 & +783148.4 & 3.0 & 1.1 & 30 & 25 & 32.00 & 1 & 0.10 & $\mathrm{SAB}(\mathrm{s}) \mathrm{c}:$ & $5.3 \pm 0.6$ & \\
\hline AM $0644-741$ & 064304.4 & -741431.4 & 1.5 & 0.9 & 5 & 94 & 34.87 & 1 & 0.14 & & $\ldots$ & \\
\hline PGC 19480 & 064306.0 & -741255.2 & 1.6 & 0.9 & 150 & 87 & 34.71 & 1 & 0.14 & S0- & $-3.0 \pm 0.9$ & \\
\hline PGC 19481 & 064306.0 & -741410.5 & 1.5 & 0.9 & 5 & 89 & 34.74 & 1 & 0.14 & Ring & $10.0 \pm 0.8$ & \\
\hline ESO 034-G013 & 064347.4 & -734031.4 & 1.3 & 0.5 & 45 & 86 & 34.68 & 1 & 0.14 & $\mathrm{SA}(\mathrm{rs}) \mathrm{c}$ & $4.5 \pm 0.9$ & \\
\hline NGC 2310 & 065340.0 & -405037.0 & 4.4 & 0.8 & 47 & 13 & 30.59 & 1 & 0.11 & So & $-2.0 \pm 0.8$ & \\
\hline NGC 2366 & 072854.7 & +691256.8 & 8.1 & 3.3 & 25 & 3.4 & 27.68 & 28 & 0.04 & $\mathrm{IB}(\mathrm{s}) \mathrm{m}$ & $10.0 \pm 0.3$ & \\
\hline Mrk 8 & 072925.4 & +720744.0 & 0.8 & 0.6 & 0 & 55 & 33.69 & 1 & 0.03 & & $\ldots$ & \\
\hline UGC 03864 & 073056.6 & +723103.5 & 1.2 & 0.8 & 20 & 40 & 33.02 & 1 & 0.03 & & $\ldots$ & \\
\hline ESO 059-G006 & 073451.1 & -694649.5 & 1.3 & 0.7 & 113 & 15 & 30.89 & 1 & 0.20 & $\mathrm{IAB}(\mathrm{s}) \mathrm{m} ?$ & $9.7 \pm 0.7$ & \\
\hline NGC 2434 & 073451.2 & -691702.9 & 2.5 & 2.3 & 0 & 16 & 30.98 & 1 & 0.25 & E0-1 & $-5.0 \pm 0.7$ & \\
\hline ESO 059-G007 & $\begin{array}{lll}07 & 36 & 12.3\end{array}$ & -694746.6 & 1.2 & 0.6 & 104 & 14 & 30.73 & 29 & 0.19 & $\mathrm{SAB}(\mathrm{rs}) 0 / \mathrm{a}$ & $-0.3 \pm 0.8$ & \\
\hline NGC 2442 & 073623.8 & -693151.0 & 5.5 & 4.9 & 0 & 14 & 30.73 & 30 & 0.20 & $\mathrm{SAB}(\mathrm{s}) \mathrm{bc}$ pec & $3.7 \pm 0.3$ & \\
\hline NGC 2403 & 073651.4 & +653609.2 & 21.9 & 12.3 & 127 & 3.2 & 27.51 & 31 & 0.04 & $\mathrm{SAB}(\mathrm{s}) \mathrm{cd}$ & $6.0 \pm 0.3$ & HII \\
\hline ESO 059-G010 & 073736.7 & -694513.2 & 1.0 & 0.5 & 136 & 14 & 30.73 & 29 & 0.18 & $\mathrm{SA}(\mathrm{rs}) \mathrm{cd}$ & $6.0 \pm 0.9$ & \\
\hline UGC 03942 & 073747.1 & +270211.3 & 1.1 & 0.2 & 151 & 115 & 35.30 & 1 & 0.04 & $\mathrm{Sb}$ & $3.0 \pm 1.0$ & \\
\hline ESO 059-G011 & 073812.0 & -692831.5 & 1.9 & 1.0 & 163 & 18 & 31.24 & 1 & 0.18 & $\mathrm{SB}(\mathrm{s}) 0 / \mathrm{a}$ & $-0.5 \pm 0.6$ & \\
\hline UGC 03995 & $07 \quad 4409.3$ & +291448.0 & 2.5 & 1.1 & 85 & 69 & 34.19 & 1 & 0.03 & & $\ldots$ & \\
\hline
\end{tabular}


Table 1-Continued

\begin{tabular}{|c|c|c|c|c|c|c|c|c|c|c|c|c|}
\hline $\begin{array}{c}\text { Object Name } \\
\text { (1) }\end{array}$ & $\begin{array}{c}\mathrm{RA}_{2000} \\
(\mathrm{~h}: \mathrm{m}: \mathrm{s}) \\
(2)\end{array}$ & $\begin{array}{c}\mathrm{DEC}_{2000} \\
(\mathrm{~d}: \mathrm{m}: \mathrm{s}) \\
(3)\end{array}$ & $\begin{array}{c}2 \times \mathrm{A} \\
(\operatorname{arcmin}) \\
(4)\end{array}$ & $\begin{array}{c}2 \times \mathrm{B} \\
(\operatorname{arcmin}) \\
(5)\end{array}$ & $\begin{array}{c}\text { PA } \\
(\operatorname{deg}) \\
(6)\end{array}$ & $\begin{array}{c}(\mathrm{Mpc}) \\
(7)\end{array}$ & $\begin{array}{c}\text { stance } \\
\text { DM } \\
(8)\end{array}$ & $\begin{array}{l}\text { ref. } \\
(9)\end{array}$ & $\begin{array}{c}\mathrm{E}(B-V) \\
(\mathrm{mag}) \\
(10)\end{array}$ & $\begin{array}{c}\text { Morphological } \\
\text { Type } \\
(11)\end{array}$ & $\begin{array}{c}\mathrm{T} \\
\text { Type } \\
(12)\end{array}$ & $\begin{array}{c}\text { Spectral } \\
\text { Type } \\
(13)\end{array}$ \\
\hline UGC 03997 & 074438.7 & +402158.9 & 1.2 & 1.2 & $\ldots$ & 86 & 34.68 & 1 & 0.05 & $\operatorname{Im} ?$ & $10.0 \pm 1.6$ & \\
\hline UGC 04056 & 075135.6 & +425248.3 & 1.1 & 0.7 & 30 & 140 & 35.72 & 1 & 0.05 & $\mathrm{SAB}(\mathrm{s}) \mathrm{c}$ & $5.0 \pm 0.9$ & \\
\hline UGC 04136 & 075954.4 & +472447.2 & 1.4 & 0.3 & 142 & 98 & 34.96 & 1 & 0.04 & $\mathrm{Sa}$ & $1.0 \pm 0.9$ & \\
\hline UGC 04148 & 080023.8 & +421137.5 & 2.5 & 0.3 & 10 & 13 & 30.50 & 1 & 0.04 & $\mathrm{Sm}$ & $7.0 \pm 1.2$ & \\
\hline NGC 2500 & 080153.2 & +504413.6 & 2.9 & 2.6 & 0 & 9.9 & 29.98 & 1 & 0.04 & $\mathrm{SB}(\mathrm{rs}) \mathrm{d}$ & $7.0 \pm 0.3$ & \\
\hline UGC 04176 & $08 \quad 0243.2$ & +404044.1 & 1.8 & 0.5 & 112 & 46 & 33.32 & 1 & 0.05 & SBd & $7.0 \pm 0.9$ & \\
\hline UGC 04188 & $\begin{array}{lll}08 & 03 & 24.1\end{array}$ & +415453.4 & 1.3 & 0.6 & 115 & 140 & 35.74 & 1 & 0.05 & So & $-2.0 \pm 0.8$ & \\
\hline NGC 2538 & 081123.1 & +033759.1 & 1.4 & 1.2 & 25 & 57 & 33.76 & 1 & 0.02 & $\left(\mathrm{R}^{\prime}\right) \mathrm{SBa}$ & $1.0 \pm 0.8$ & \\
\hline NGC 2543 & $08 \quad 1257.9$ & +361516.7 & 2.3 & 1.3 & 45 & 37 & 32.84 & 1 & 0.07 & $\mathrm{SB}(\mathrm{s}) \mathrm{b}$ & $3.0 \pm 0.4$ & \\
\hline NGC 2537 & 081314.6 & +455923.3 & 1.7 & 1.5 & 0 & 6.9 & 29.19 & 32 & 0.05 & $\mathrm{SB}(\mathrm{s}) \mathrm{m} \mathrm{pec}$ & $11.0 \pm 0.4$ & \\
\hline UGC4278 & 081358.9 & +454431.7 & 4.7 & 0.5 & 172 & 14 & 30.72 & 33 & 0.05 & $\mathrm{SB}(\mathrm{s}) \mathrm{d}: \mathrm{sp}$ & $7.0 \pm 0.4$ & \\
\hline NGC 2541 & $08 \quad 1440.1$ & +490341.2 & 6.3 & 3.2 & 165 & 10 & 30.07 & 1 & 0.05 & $\mathrm{SA}(\mathrm{s}) \mathrm{cd}$ & $6.0 \pm 0.3$ & LINER \\
\hline NGC $2523 \mathrm{C}$ & 081744.3 & +731903.4 & 1.5 & 0.8 & 95 & 55 & 33.71 & 1 & 0.03 & $\mathrm{E} ?$ & $-5.0 \pm 1.0$ & \\
\hline UGC 04311 & $\begin{array}{lll}08 & 18 & 06.9\end{array}$ & +372317.5 & 1.0 & 0.2 & 135 & 181 & 36.28 & 1 & 0.06 & Sb: & $3.0 \pm 1.4$ & \\
\hline Holmberg II & 081905.0 & +704312.1 & 7.9 & 6.3 & 15 & 3.4 & 27.65 & 34 & 0.03 & $\operatorname{Im}$ & $\cdots$ & \\
\hline NGC 2552 & 081920.5 & +500034.7 & 3.5 & 2.3 & 45 & 10 & 30.01 & 1 & 0.05 & $\mathrm{SA}(\mathrm{s}) \mathrm{m} ?$ & $9.0 \pm 0.3$ & \\
\hline UGC 04387 & 082444.9 & +465425.9 & 1.3 & 0.2 & 97 & 174 & 36.20 & 1 & 0.04 & $\mathrm{Sc}$ & $6.0 \pm 1.4$ & \\
\hline NGC 2551 & 082450.3 & +732443.3 & 1.7 & 1.1 & 55 & 37 & 32.85 & 1 & 0.03 & $\mathrm{SA}(\mathrm{s}) 0 / \mathrm{a}$ & $0.2 \pm 0.4$ & \\
\hline HS $0822+3542$ & 082555.4 & +353231.9 & 0.2 & 0.2 & $\ldots$ & 10 & 30.02 & 35 & 0.05 & $\mathrm{BCG}$ & $\ldots$ & \\
\hline UGC 04393 & $\begin{array}{lll}08 & 26 & 04.4\end{array}$ & +455803.5 & 2.2 & 1.6 & 45 & 33 & 32.57 & 1 & 0.04 & $\mathrm{SBc} ?$ & $\ldots$ & HII \\
\hline UGC 04401 & 082644.1 & +484831.5 & 1.3 & 1.3 & $\cdots$ & 47 & 33.34 & 1 & 0.04 & $\operatorname{Im}:$ & $10.0 \pm 1.2$ & \\
\hline UGC 04390 & 082751.6 & +733101.0 & 1.9 & 1.6 & 50 & 35 & 32.70 & 1 & 0.03 & $\mathrm{SBd}$ & $7.0 \pm 0.7$ & \\
\hline NGC $2550 \mathrm{~A}$ & 082839.9 & +734452.8 & 1.6 & 1.4 & 0 & 56 & 33.73 & 1 & 0.03 & $\mathrm{Sc}$ & $5.0 \pm 0.7$ & \\
\hline UGC 04436 & 082950.3 & +484651.9 & 1.3 & 0.3 & 42 & 106 & 35.12 & 1 & 0.04 & $\mathrm{Sbc}$ & $4.0 \pm 0.9$ & \\
\hline UGC 04461 & 083322.7 & +523156.1 & 1.7 & 0.5 & 43 & 74 & 34.35 & 1 & 0.04 & $\mathrm{Sbc}$ & $4.0 \pm 0.9$ & \\
\hline DDO 053 & 083407.2 & +661054.0 & 1.5 & 1.3 & 120 & 3.6 & 27.76 & 34 & 0.04 & $\operatorname{Im}$ & $10.0 \pm 0.8$ & \\
\hline NGC 2600 & 083445.1 & +524256.5 & 1.2 & 0.4 & 78 & 195 & 36.45 & 1 & 0.04 & $\mathrm{Sb}$ & $3.0 \pm 0.9$ & \\
\hline UGC 04499 & 083741.5 & +513908.4 & 2.6 & 1.9 & 140 & 13 & 30.50 & 1 & 0.04 & SABdm & $8.0 \pm 0.7$ & \\
\hline NGC 2623 & 083824.1 & +254516.9 & 2.4 & 0.7 & 60 & 80 & 34.52 & 1 & 0.04 & & $99.0 \pm 0.0$ & \\
\hline UGC 04514 & 083937.7 & +532723.4 & 2.1 & 0.9 & 70 & 13 & 30.52 & 1 & 0.04 & SBcd? & $6.0 \pm 1.6$ & HII \\
\hline UGC 04515 & 084009.5 & +522721.8 & 1.5 & 0.6 & 175 & 74 & 34.34 & 1 & 0.04 & $\mathrm{SB}(\mathrm{r}) \mathrm{b}:$ & $3.0 \pm 0.8$ & \\
\hline UGC 04525 & 084132.1 & +511446.8 & 1.1 & 0.7 & 88 & 75 & 34.39 & 1 & 0.02 & $\mathrm{SB}(\mathrm{s}) \mathrm{b}$ & $3.0 \pm 0.9$ & \\
\hline UGC 04529 & 084137.8 & +464736.6 & 1.1 & 0.2 & 123 & 161 & 36.04 & 1 & 0.03 & $\mathrm{Sbc}$ & $4.0 \pm 1.0$ & \\
\hline
\end{tabular}


Table 1-Continued

\begin{tabular}{|c|c|c|c|c|c|c|c|c|c|c|c|c|}
\hline $\begin{array}{c}\text { Object Name } \\
\text { (1) }\end{array}$ & $\begin{array}{c}\mathrm{RA}_{2000} \\
(\mathrm{~h}: \mathrm{m}: \mathrm{s}) \\
(2)\end{array}$ & $\begin{array}{c}\mathrm{DEC}_{2000} \\
(\mathrm{~d}: \mathrm{m}: \mathrm{s}) \\
(3)\end{array}$ & $\begin{array}{c}2 \times \mathrm{A} \\
(\operatorname{arcmin}) \\
(4)\end{array}$ & $\begin{array}{c}2 \times \mathrm{B} \\
(\operatorname{arcmin}) \\
(5)\end{array}$ & $\begin{array}{c}\text { PA } \\
(\mathrm{deg}) \\
(6)\end{array}$ & $\begin{array}{c}\text { (Mpc) } \\
(7)\end{array}$ & $\begin{array}{l}\text { istance } \\
\text { DM } \\
(8)\end{array}$ & $\begin{array}{l}\text { ref. } \\
(9)\end{array}$ & $\begin{array}{c}\mathrm{E}(B-V) \\
(\mathrm{mag}) \\
(10)\end{array}$ & $\begin{array}{c}\text { Morphological } \\
\text { Type } \\
(11)\end{array}$ & $\begin{array}{c}\mathrm{T} \\
\text { Type } \\
(12)\end{array}$ & $\begin{array}{c}\text { Spectral } \\
\text { Type } \\
(13)\end{array}$ \\
\hline NGC 2639 & 084338.1 & +501220.0 & 1.8 & 1.1 & 140 & 50 & 33.51 & 1 & 0.02 & $(\mathrm{R}) \mathrm{SA}(\mathrm{r}) \mathrm{a}: ?$ & $1.0 \pm 0.6$ & Sy1.9 \\
\hline UGC 04546 & 084351.3 & +515928.3 & 1.3 & 0.2 & 25 & 77 & 34.44 & 1 & 0.03 & $\mathrm{Sa}$ & $1.0 \pm 1.0$ & \\
\hline UGC 04551 & 084405.9 & +494738.1 & 2.0 & 0.7 & 113 & 28 & 32.20 & 1 & 0.02 & S0? & $-2.0 \pm 1.7$ & \\
\hline UGC 04562 & 084455.2 & +474444.9 & 1.2 & 0.8 & 127 & 127 & 35.51 & 1 & 0.03 & $\mathrm{SB}(\mathrm{s}) \mathrm{c}$ & $5.0 \pm 0.9$ & \\
\hline UGC 04560 & 084455.5 & +522902.9 & 1.2 & 0.3 & 122 & 177 & 36.25 & 1 & 0.03 & $\mathrm{Sab}$ & $2.0 \pm 0.9$ & \\
\hline VV 703 & 084753.1 & +535234.3 & 1.3 & 1.3 & $\ldots$ & 197 & 36.47 & 1 & 0.03 & & $-3.0 \pm 0.8$ & \\
\hline UGC 04628 & 085140.7 & +510707.3 & 1.7 & 0.3 & 74 & 14 & 30.73 & 1 & 0.03 & Scd: & $6.0 \pm 1.3$ & \\
\hline NGC 2675 & 085205.0 & +533702.3 & 1.5 & 1.1 & 80 & 135 & 35.65 & 1 & 0.02 & $\mathrm{E}$ & $-5.0 \pm 0.8$ & \\
\hline NGC 2681 & $0853 \quad 32.7$ & +511849.3 & 3.6 & 3.3 & 0 & 13 & 30.50 & 1 & 0.02 & $\left(\mathrm{R}^{\prime}\right) \mathrm{SAB}(\mathrm{rs}) 0 / \mathrm{a}$ & $0.0 \pm 0.3$ & Sy3 \\
\hline IC 0522 & 085434.9 & +571000.2 & 1.0 & 0.8 & 165 & 76 & 34.39 & 1 & 0.05 & So & $-2.0 \pm 0.9$ & \\
\hline VV 761 & 085541.1 & +573422.7 & 1.0 & 0.2 & 170 & 173 & 36.19 & 1 & 0.05 & & $\ldots$ & \\
\hline UGC 04668 & 085557.6 & +574040.8 & 1.1 & 0.2 & 84 & 64 & 34.02 & 1 & 0.05 & $\mathrm{Sbc}$ & $4.0 \pm 1.0$ & \\
\hline UGC 04684 & 085640.7 & +0022 29.8 & 1.4 & 1.1 & 175 & 35 & 32.74 & 1 & 0.04 & $\mathrm{SA}(\mathrm{rs}) \mathrm{dm}:$ & $8.0 \pm 0.6$ & \\
\hline UGC 04671 & 085642.7 & +520619.4 & 1.3 & 1.1 & 69 & 61 & 33.91 & 1 & 0.02 & $\mathrm{~S} ?$ & $\cdots$ & \\
\hline NGC 2692 & 085658.0 & +520357.4 & 1.3 & 0.5 & 165 & 60 & 33.90 & 1 & 0.02 & SBab: & $1.7 \pm 0.7$ & \\
\hline NGC 2693 & 085659.2 & +512051.0 & 2.6 & 1.8 & 160 & 73 & 34.33 & 1 & 0.02 & E3: & $-5.0 \pm 0.5$ & \\
\hline UGC 04676 & 085705.7 & +514850.7 & 1.1 & 0.4 & 55 & 77 & 34.43 & 1 & 0.02 & $\mathrm{Sd}$ & $7.0 \pm 0.9$ & \\
\hline UGC 04679 & 085708.2 & +512817.6 & 1.3 & 0.2 & 91 & 70 & 34.22 & 1 & 0.02 & $\mathrm{Sbc}$ & $6.0 \pm 1.4$ & \\
\hline UGC 04690 & $\begin{array}{lll}08 & 58 & 10.8\end{array}$ & +521058.3 & 1.2 & 0.6 & 120 & 137 & 35.69 & 1 & 0.02 & $\mathrm{Sab}$ & $2.0 \pm 0.9$ & \\
\hline UGC 04702 & 085851.2 & +384834.2 & 1.5 & 1.4 & 0 & 123 & 35.44 & 1 & 0.03 & $\mathrm{~S} 0 ?$ & $-2.0 \pm 1.6$ & \\
\hline UGC 04704 & 085900.3 & +391235.7 & 4.1 & 0.4 & 115 & 11 & 30.12 & 1 & 0.03 & $\mathrm{Sdm}$ & $8.0 \pm 1.2$ & \\
\hline NGC 2710 & 085948.4 & +554223.0 & 2.0 & 1.0 & 125 & 39 & 32.96 & 1 & 0.02 & $\mathrm{SB}(\mathrm{rs}) \mathrm{b}$ & $3.0 \pm 0.8$ & \\
\hline UGC 04800 & 090919.4 & +545443.1 & 1.6 & 0.5 & 120 & 38 & 32.89 & 1 & 0.02 & $\mathrm{SB}(\mathrm{s}) \mathrm{cd} ?$ & $6.0 \pm 1.8$ & \\
\hline UGC 04807 & 091005.5 & +543449.1 & 1.0 & 1.0 & $\ldots$ & 60 & 33.87 & 1 & 0.02 & $\mathrm{SA}(\mathrm{rs}) \mathrm{cd}$ : & $6.0 \pm 0.9$ & \\
\hline NGC 2768 & 091137.5 & +600214.0 & 8.1 & 4.3 & 95 & 23 & 31.79 & 1 & 0.04 & S0-1/2- & $-5.0 \pm 0.3$ & LINER \\
\hline NGC 2784 & 091219.5 & -241021.4 & 5.5 & 2.2 & 73 & 7.7 & 29.42 & 1 & 0.21 & $\mathrm{SA}(\mathrm{s}) 0^{*} 0^{*}:$ & $-2.0 \pm 0.3$ & \\
\hline UGC 04844 & 091302.2 & +493822.1 & 1.4 & 1.1 & 170 & 60 & 33.88 & 1 & 0.02 & SABbc: & $4.0 \pm 0.8$ & \\
\hline UGC 04851 & 091325.9 & +525852.8 & 1.1 & 0.8 & 145 & 112 & 35.24 & 1 & 0.01 & S0-: & $-3.0 \pm 1.3$ & \\
\hline NGC 2782 & $\begin{array}{llll}09 & 14 & 05.1\end{array}$ & +400649.2 & 3.5 & 2.6 & 0 & 39 & 32.94 & 1 & 0.02 & $\mathrm{SAB}(\mathrm{rs}) \mathrm{a} ; \mathrm{Sy} 1$ & $1.0 \pm 0.3$ & Sbrst \\
\hline UGC 04872 & $09 \quad 1501.5$ & +400211.4 & 1.9 & 0.2 & 12 & 119 & 35.39 & 1 & 0.02 & $\mathrm{SBb}$ & $3.0 \pm 0.9$ & \\
\hline NGC 2798 & 091722.9 & +415959.0 & 2.6 & 1.0 & 160 & 27 & 32.15 & 1 & 0.02 & $\mathrm{SB}(\mathrm{s})$ a pec & $1.0 \pm 0.4$ & \\
\hline UGC 04915 & 091729.0 & -003714.1 & 1.0 & 0.2 & 119 & 70 & 34.22 & 1 & 0.04 & $\mathrm{Sbc}$ & $4.0 \pm 0.9$ & \\
\hline NGC 2799 & 091731.0 & +415938.7 & 1.9 & 0.5 & 125 & 26 & 32.09 & 1 & 0.02 & $\mathrm{SB}(\mathrm{s}) \mathrm{m} ?$ & $9.0 \pm 0.9$ & \\
\hline
\end{tabular}


Table 1-Continued

\begin{tabular}{|c|c|c|c|c|c|c|c|c|c|c|c|c|}
\hline $\begin{array}{c}\text { Object Name } \\
\text { (1) }\end{array}$ & $\begin{array}{c}\mathrm{RA}_{2000} \\
(\mathrm{~h}: \mathrm{m}: \mathrm{s}) \\
(2)\end{array}$ & $\begin{array}{c}\mathrm{DEC}_{2000} \\
(\mathrm{~d}: \mathrm{m}: \mathrm{s}) \\
(3)\end{array}$ & $\begin{array}{c}2 \times \mathrm{A} \\
(\operatorname{arcmin}) \\
(4)\end{array}$ & $\begin{array}{c}2 \times \mathrm{B} \\
(\operatorname{arcmin}) \\
(5)\end{array}$ & $\begin{array}{c}\mathrm{PA} \\
(\mathrm{deg}) \\
(6)\end{array}$ & $\begin{array}{c}(\mathrm{Mpc}) \\
(7)\end{array}$ & $\begin{array}{l}\text { stance } \\
\text { DM } \\
(8)\end{array}$ & $\begin{array}{l}\text { ref. } \\
(9)\end{array}$ & $\begin{array}{c}\mathrm{E}(B-V) \\
(\mathrm{mag}) \\
(10)\end{array}$ & $\begin{array}{c}\text { Morphological } \\
\text { Type } \\
\text { (11) }\end{array}$ & $\begin{array}{c}\mathrm{T} \\
\text { Type } \\
(12)\end{array}$ & $\begin{array}{c}\text { Spectral } \\
\text { Type } \\
(13)\end{array}$ \\
\hline IC 0531 & 091750.8 & -001642.5 & 1.7 & 0.5 & 60 & 74 & 34.35 & 1 & 0.03 & $\left(\mathrm{R}^{\prime}\right) \mathrm{SB}(\mathrm{rs}) \mathrm{ab} ?$ & $1.7 \pm 0.7$ & \\
\hline UGC 04921 & 091831.3 & +493243.6 & 1.1 & 0.2 & 26 & 72 & 34.27 & 1 & 0.02 & Scd & $6.0 \pm 1.4$ & \\
\hline NGC 2841 & 092202.6 & +505835.5 & 8.1 & 3.5 & 147 & 14 & 30.75 & 36 & 0.02 & SA(r)b:;LINER & $3.0 \pm 0.3$ & Sy1 \\
\hline NGC 2854 & 092403.2 & +4912 15.6 & 1.7 & 0.6 & 50 & 42 & 33.13 & 1 & 0.02 & $\mathrm{SB}(\mathrm{s}) \mathrm{b}$ & $3.0 \pm 0.9$ & \\
\hline NGC 2856 & 092416.0 & +491456.8 & 1.1 & 0.5 & 134 & 40 & 33.03 & 1 & 0.02 & $\mathrm{~S} ?$ & $\ldots$ & \\
\hline NGC 2857 & 092437.7 & +492125.4 & 2.2 & 2.0 & 0 & 73 & 34.30 & 1 & 0.02 & $\mathrm{SA}(\mathrm{s}) \mathrm{c}$ & $5.0 \pm 0.3$ & \\
\hline NGC 2915 & 092611.5 & -763734.8 & 1.9 & 1.0 & 129 & 3.8 & 27.89 & 37 & 0.28 & I0 & $90.0 \pm 0.0$ & \\
\hline UGC 05013 & 092616.8 & +612254.2 & 1.6 & 0.9 & 120 & 164 & 36.07 & 1 & 0.03 & $\mathrm{Sb}$ & $3.0 \pm 0.8$ & \\
\hline UGC 05027 & 092617.3 & +030805.5 & 1.0 & 0.2 & 173 & 59 & 33.86 & 1 & 0.04 & $\mathrm{Sbc}$ & $4.0 \pm 1.0$ & \\
\hline NGC 2870 & 092753.7 & +572231.8 & 2.5 & 0.6 & 123 & 49 & 33.45 & 1 & 0.04 & $\mathrm{Sbc}$ & $4.0 \pm 0.8$ & \\
\hline UGC 05053 & 093007.5 & +600822.6 & 1.1 & 0.5 & 67 & 49 & 33.46 & 1 & 0.03 & Sdm: & $8.0 \pm 1.3$ & \\
\hline NGC 2903 & 093210.1 & +213003.0 & 12.6 & 6.0 & 17 & 8.9 & 29.75 & 38 & 0.03 & $\mathrm{SB}(\mathrm{s}) \mathrm{d}$ & $4.0 \pm 0.3$ & HII \\
\hline UGC 05077 & 093250.7 & +594441.3 & 1.7 & 0.6 & 77 & 174 & 36.21 & 1 & 0.03 & $\mathrm{SBb}$ & $3.0 \pm 0.8$ & NLAGN \\
\hline I $\mathrm{Zw} 18$ & 093402.0 & +551428.1 & 2.0 & 2.0 & $\ldots$ & 13 & 30.50 & 39 & 0.03 & & $11.0 \pm 1.0$ & \\
\hline NGC 2916 & 093457.6 & +214218.9 & 2.5 & 1.7 & 20 & 54 & 33.67 & 1 & 0.03 & $\mathrm{SA}(\mathrm{rs}) \mathrm{b} ?$ & $3.0 \pm 1.5$ & \\
\hline UGC 05107 & 093507.5 & +050712.3 & 1.8 & 0.5 & 47 & 28 & 32.27 & 1 & 0.04 & $\mathrm{SBd}$ & $7.0 \pm 0.8$ & \\
\hline UGC 05101 & 093551.7 & +612111.3 & 1.1 & 0.7 & 87 & 172 & 36.18 & 1 & 0.03 & S?;LINER & $\ldots$ & Sy1.5 \\
\hline NGC 2936 & 093744.1 & +024539.3 & 1.3 & 1.1 & 45 & 100 & 35.01 & 1 & 0.03 & $\mathrm{I} ?$ & $\ldots$ & \\
\hline NGC 2937 & 093745.0 & +024450.5 & 2.1 & 0.7 & 10 & 97 & 34.93 & 1 & 0.03 & $\mathrm{E}$ & $-5.0 \pm 0.9$ & \\
\hline UGC 05147 & 093927.0 & +382547.9 & 1.1 & 0.1 & 147 & 86 & 34.67 & 1 & 0.02 & Scd & $6.0 \pm 1.5$ & \\
\hline UGC 05114 & 094003.2 & +820617.0 & 1.7 & 0.7 & 140 & 27 & 32.15 & 1 & 0.02 & IBm: & $10.0 \pm 0.8$ & \\
\hline Holmberg I & 094032.3 & +711056.0 & 3.6 & 3.0 & 0 & 3.8 & 27.92 & 34 & 0.05 & $\operatorname{IAB}(\mathrm{s}) \mathrm{m}$ & $\ldots$ & \\
\hline UGC 05201 & 094435.0 & +554546.1 & 1.5 & 1.0 & 45 & 112 & 35.25 & 1 & 0.01 & $\mathrm{SAB}(\mathrm{s}) \mathrm{c}$ & $5.0 \pm 0.8$ & \\
\hline NGC 2992 & 094542.1 & -141935.0 & 3.5 & 1.1 & 25 & 32 & 32.50 & 1 & 0.06 & Sa pec;Sy1 & $1.0 \pm 0.3$ & Sy2 \\
\hline NGC 2993 & 094548.3 & -142205.9 & 1.3 & 0.9 & 90 & 32 & 32.50 & 1 & 0.06 & Sa pec & $1.0 \pm 0.4$ & HII \\
\hline NGC 2976 & 094715.5 & +675459.0 & 5.9 & 2.7 & 143 & 3.6 & 27.76 & 34 & 0.07 & SAc pec & $5.0 \pm 0.3$ & HII \\
\hline UGC 05237 & 094720.2 & +463636.2 & 1.5 & 0.7 & 155 & 70 & 34.21 & 1 & 0.01 & Scd: & $6.0 \pm 1.2$ & \\
\hline NGC 3018 & 094941.5 & +003716.5 & 1.2 & 0.7 & 27 & 26 & 32.09 & 1 & 0.07 & $\mathrm{SB}(\mathrm{s}) \mathrm{b}$ pec? & $3.0 \pm 1.7$ & \\
\hline NGC 3023 & 094952.6 & +003705.4 & 2.9 & 1.4 & 70 & 26 & 32.11 & 1 & 0.06 & SAB(s)c pec: & $5.0 \pm 0.5$ & \\
\hline UGC 05268 & 095056.2 & +621108.5 & 1.3 & 0.4 & 134 & 109 & 35.18 & 1 & 0.03 & Sab & $2.0 \pm 0.9$ & \\
\hline UGC 05314 & 095351.0 & +085241.5 & 1.0 & 0.1 & 168 & 92 & 34.81 & 1 & 0.04 & Scd & $6.0 \pm 1.5$ & \\
\hline NGC 3049 & 095449.6 & +09 1617.9 & 2.2 & 1.4 & 25 & 22 & 31.67 & 1 & 0.04 & SB(rs)ab;HII & $2.0 \pm 0.8$ & Sbrst \\
\hline MESSIER 081 & 095533.2 & +690355.1 & 26.9 & 14.1 & 157 & 3.6 & 27.80 & 40 & 0.08 & SA(s)ab;LINER & $2.0 \pm 0.3$ & Sy1.8 \\
\hline
\end{tabular}


Table 1-Continued

\begin{tabular}{|c|c|c|c|c|c|c|c|c|c|c|c|c|}
\hline $\begin{array}{l}\text { Object Name } \\
\text { (1) }\end{array}$ & $\begin{array}{c}\mathrm{RA}_{2000} \\
(\mathrm{~h}: \mathrm{m}: \mathrm{s}) \\
(2)\end{array}$ & $\begin{array}{c}\mathrm{DEC}_{2000} \\
(\mathrm{~d}: \mathrm{m}: \mathrm{s}) \\
(3)\end{array}$ & $\begin{array}{c}2 \times \mathrm{A} \\
(\operatorname{arcmin}) \\
(4)\end{array}$ & $\begin{array}{c}2 \times \mathrm{B} \\
(\operatorname{arcmin}) \\
(5)\end{array}$ & $\begin{array}{c}\text { PA } \\
(\mathrm{deg}) \\
(6)\end{array}$ & $\begin{array}{c}(\mathrm{Mpc}) \\
(7)\end{array}$ & $\begin{array}{c}\text { istance } \\
\text { DM } \\
(8)\end{array}$ & $\begin{array}{l}\text { ref. } \\
(9)\end{array}$ & $\begin{array}{c}\mathrm{E}(B-V) \\
\quad(\mathrm{mag}) \\
(10)\end{array}$ & $\begin{array}{c}\text { Morphological } \\
\text { Type } \\
(11)\end{array}$ & $\begin{array}{c}\mathrm{T} \\
\text { Type } \\
(12)\end{array}$ & $\begin{array}{c}\text { Spectral } \\
\text { Type } \\
(13)\end{array}$ \\
\hline MESSIER 082 & 095552.2 & +694046.9 & 11.2 & 4.3 & 65 & 3.9 & 27.96 & 41 & 0.16 & I0;Sbrst & $90.0 \pm 0.0$ & HII \\
\hline Holmberg IX & 095732.0 & +690245.0 & 2.5 & 2.0 & 40 & 3.6 & 27.80 & 42 & 0.08 & $\operatorname{Im}$ & $\cdots$ & \\
\hline ESO 435-G014 & 095748.4 & -283023.6 & 2.6 & 0.3 & 53 & 36 & 32.77 & 1 & 0.09 & Sc: sp & $5.0 \pm 1.3$ & \\
\hline ESO 435-G016 & 095846.2 & -283718.8 & 1.7 & 1.1 & 100 & 12 & 30.35 & 1 & 0.10 & I0? pec & $90.0 \pm 0.0$ & \\
\hline Tol 2 & 095921.2 & -280800.3 & 0.8 & 0.6 & 100 & 7.1 & 29.26 & 35 & 0.09 & Merger? & $\cdots$ & HII \\
\hline NGC 3089 & 095936.7 & $-28 \quad 1952.9$ & 1.8 & 1.0 & 139 & 36 & 32.81 & 1 & 0.10 & $\mathrm{SAB}(\mathrm{rs}) \mathrm{b}$ & $3.0 \pm 0.4$ & \\
\hline NGC 3073 & 100052.1 & +553707.8 & 1.3 & 1.2 & 0 & 20 & 31.46 & 1 & 0.01 & SABO- & $-2.5 \pm 0.6$ & \\
\hline NGC 3079 & 100157.8 & +554047.1 & 7.9 & 1.4 & 165 & 19 & 31.40 & 1 & 0.01 & SB(s)c;LINER & $7.0 \pm 0.4$ & Sy2 \\
\hline NGC 3109 & 100306.9 & -260934.5 & 19.1 & 3.7 & 93 & 1.1 & 25.21 & 43 & 0.07 & $\mathrm{SB}(\mathrm{s}) \mathrm{m}$ & $9.0 \pm 0.3$ & \\
\hline UGCA 196 & 100341.8 & -270140.1 & 3.2 & 1.3 & 53 & 12 & 30.31 & 1 & 0.08 & $\left(\mathrm{R}^{\prime}\right) \mathrm{SA}(\mathrm{s}) \mathrm{b}$ & $7.0 \pm 0.6$ & \\
\hline IC 2537 & 100351.9 & -273415.1 & 2.6 & 1.7 & 26 & 38 & 32.88 & 1 & 0.10 & $\mathrm{SAB}(\mathrm{rs}) \mathrm{c}$ & $5.0 \pm 0.4$ & \\
\hline UGC 05406 & 100355.6 & +633511.3 & 1.0 & 0.4 & 62 & 105 & 35.11 & 1 & 0.04 & Scd: & $6.0 \pm 1.3$ & \\
\hline Antlia Dwarf & 100403.9 & -271955.0 & 2.0 & 1.5 & 160 & 1.1 & 25.21 & 44 & 0.08 & dE3.5 & $\cdots$ & \\
\hline M81 Dwarf B & 100530.6 & +702152.0 & 0.9 & 0.6 & 140 & 5.3 & 28.62 & 32 & 0.08 & $\operatorname{Im}$ & $10.0 \pm 0.8$ & \\
\hline NGC 3125 & 100633.3 & -295606.6 & 1.1 & 0.7 & 114 & 9.3 & 29.84 & 35 & 0.08 & $\mathrm{~S}$ & $11.0 \pm 1.7$ & $\mathrm{BCDG}$ \\
\hline UGC 05455 & 100850.3 & +703801.0 & 1.8 & 1.8 & $\ldots$ & 22 & 31.73 & 1 & 0.14 & $\operatorname{Im}$ & $10.0 \pm 0.8$ & \\
\hline Sextans A & 101100.8 & -044134.0 & 5.9 & 4.9 & 0 & 1.4 & 25.76 & 43 & 0.04 & $\mathrm{IBm}$ & $\ldots$ & \\
\hline UGC 05493 & $1011 \quad 17.9$ & +002632.6 & 1.6 & 1.3 & 15 & 52 & 33.57 & 1 & 0.04 & $\mathrm{SAB}(\mathrm{rs}) \mathrm{c}$ & $4.5 \pm 0.6$ & \\
\hline UGC 05515 & 101338.3 & -005531.8 & 1.5 & 1.3 & 90 & 190 & 36.39 & 1 & 0.04 & E+ pec: & $-4.3 \pm 0.6$ & \\
\hline UGC 05528 & 101439.6 & -004951.2 & 1.1 & 0.8 & 150 & 210 & 36.61 & 1 & 0.04 & SAB(r)a pec: & $1.0 \pm 1.2$ & BLAGN \\
\hline NGC 3147 & 101653.7 & +732402.7 & 3.9 & 3.5 & 155 & 44 & 33.22 & 1 & 0.02 & $\mathrm{SA}(\mathrm{rs}) \mathrm{bc}$ & $4.0 \pm 0.3$ & $\mathrm{Sy} 2$ \\
\hline NGC 3185 & 101738.6 & +214117.7 & 2.3 & 1.6 & 130 & 17 & 31.20 & 45 & 0.03 & (R)SB(r)a & $1.0 \pm 0.4$ & Sy2 \\
\hline NGC 3187 & 101747.8 & +215223.8 & 3.0 & 1.3 & 50 & 17 & 31.20 & 45 & 0.03 & $\mathrm{SB}(\mathrm{s}) \mathrm{c}$ pec & $5.0 \pm 0.3$ & HII \\
\hline NGC 3190 & 101805.6 & +214955.0 & 4.4 & 1.5 & 125 & 17 & 31.20 & 3 & 0.03 & $\mathrm{SA}(\mathrm{s}) \mathrm{a}$ pec sp & $1.0 \pm 0.3$ & LINER \\
\hline UGC 05558 & 101822.7 & +455716.8 & 1.1 & 0.2 & 12 & 111 & 35.22 & 1 & 0.01 & $\mathrm{Sa}$ & $1.0 \pm 1.0$ & \\
\hline NGC 3193 & 101824.9 & +215338.3 & 3.0 & 2.7 & 0 & 17 & 31.20 & 45 & 0.03 & $\mathrm{E} 2$ & $-5.0 \pm 0.3$ & \\
\hline NGC 3198 & 101954.9 & +453259.0 & 8.5 & 3.3 & 35 & 17 & 31.11 & 43 & 0.01 & $\mathrm{SB}(\mathrm{rs}) \mathrm{c}$ & $5.0 \pm 0.3$ & \\
\hline UGC 05570 & 102047.0 & +731703.3 & 1.1 & 0.1 & 160 & 43 & 33.15 & 1 & 0.05 & $\mathrm{Sbc}$ & $4.0 \pm 1.0$ & \\
\hline NGC 3183 & 102149.0 & +741036.7 & 2.3 & 1.4 & 170 & 48 & 33.40 & 1 & 0.04 & $\mathrm{SB}(\mathrm{s}) \mathrm{bc}:$ & $3.5 \pm 0.5$ & \\
\hline ESO 317-G019 & 102302.3 & -390959.6 & 1.1 & 0.9 & 63 & 38 & 32.88 & 1 & 0.10 & $\left(\mathrm{R}^{\prime}-1\right) \mathrm{SAB}(\mathrm{rl}) \mathrm{a}$ & $1.0 \pm 0.8$ & \\
\hline ESO 317-G023 & 102442.5 & -391821.2 & 1.9 & 0.8 & 14 & 38 & 32.90 & 1 & 0.10 & $\left(\mathrm{R}^{\prime}-1\right) \mathrm{SB}(\mathrm{rs}) \mathrm{a}$ & $1.0 \pm 0.6$ & \\
\hline ESO 263-G033 & 102447.5 & -435751.6 & 1.3 & 1.1 & 0 & 38 & 32.90 & 1 & 0.13 & (R')SA(s)0-: & $-3.0 \pm 0.8$ & \\
\hline NGC 3225 & 102509.9 & +580900.0 & 2.0 & 1.0 & 155 & 34 & 32.64 & 1 & 0.01 & Scd: & $6.0 \pm 1.1$ & \\
\hline
\end{tabular}


Table 1-Continued

\begin{tabular}{|c|c|c|c|c|c|c|c|c|c|c|c|c|}
\hline $\begin{array}{c}\text { Object Name } \\
\text { (1) }\end{array}$ & $\begin{array}{c}\mathrm{RA}_{2000} \\
(\mathrm{~h}: \mathrm{m}: \mathrm{s}) \\
(2)\end{array}$ & $\begin{array}{c}\mathrm{DEC}_{2000} \\
(\mathrm{~d}: \mathrm{m}: \mathrm{s}) \\
(3)\end{array}$ & $\begin{array}{c}2 \times \mathrm{A} \\
(\operatorname{arcmin}) \\
(4)\end{array}$ & $\begin{array}{c}2 \times \mathrm{B} \\
(\operatorname{arcmin}) \\
(5)\end{array}$ & $\begin{array}{c}\text { PA } \\
(\mathrm{deg}) \\
(6)\end{array}$ & $\begin{array}{c}(\mathrm{Mpc}) \\
(7)\end{array}$ & $\begin{array}{l}\text { istance } \\
\text { DM } \\
(8)\end{array}$ & $\begin{array}{l}\text { ref. } \\
(9)\end{array}$ & $\begin{array}{c}\mathrm{E}(B-V) \\
\quad(\mathrm{mag}) \\
(10)\end{array}$ & $\begin{array}{c}\text { Morphological } \\
\text { Type } \\
\text { (11) }\end{array}$ & $\begin{array}{c}\mathrm{T} \\
\text { Type } \\
(12)\end{array}$ & $\begin{array}{c}\text { Spectral } \\
\text { Type } \\
(13)\end{array}$ \\
\hline NGC 3244 & 102528.8 & -394939.2 & 2.0 & 1.5 & 170 & 37 & 32.82 & 1 & 0.10 & $\mathrm{SA}(\mathrm{rs}) \mathrm{cd}$ & $6.0 \pm 0.5$ & \\
\hline NGC 3256A & 102551.0 & -434453.2 & 1.3 & 0.6 & 85 & 38 & 32.88 & 1 & 0.12 & SB(s)m pec: & $9.0 \pm 0.8$ & \\
\hline NGC 3238 & 102643.0 & +571334.8 & 1.4 & 1.3 & 0 & 109 & 35.18 & 1 & 0.01 & $\mathrm{SA}(\mathrm{r}) 0^{*} 0 *$ : & $-2.3 \pm 0.7$ & \\
\hline IC 2574 & 102823.5 & +682443.7 & 13.2 & 5.4 & 50 & 4.0 & 28.02 & 34 & 0.04 & $\mathrm{SAB}(\mathrm{s}) \mathrm{m}$ & $9.0 \pm 0.3$ & \\
\hline NGC 3265 & 103106.8 & +284747.0 & 1.3 & 1.0 & 73 & 20 & 31.51 & 3 & 0.02 & $\mathrm{E}:$ & $-5.0 \pm 0.7$ & HII \\
\hline UGC 05715 & 103135.2 & +00 2831.4 & 1.1 & 0.7 & 155 & 122 & 35.44 & 1 & 0.06 & $\mathrm{Sbc}$ & $4.0 \pm 0.9$ & \\
\hline UGC 05720 & 103231.9 & +542403.7 & 1.0 & 0.9 & 0 & 24 & 31.86 & 1 & 0.01 & Im pec & $10.0 \pm 0.5$ & HII \\
\hline NGC 3277 & 103255.5 & +283042.2 & 1.9 & 1.7 & 0 & 22 & 31.70 & 1 & 0.03 & $\mathrm{SA}(\mathrm{r}) \mathrm{ab}$ & $2.0 \pm 0.4$ & HII \\
\hline NGC 3288 & 103625.7 & +583322.3 & 1.1 & 0.9 & 175 & 120 & 35.39 & 1 & 0.01 & SABbc: & $3.7 \pm 0.7$ & \\
\hline UGC 05772 & 103657.4 & +001347.0 & 1.0 & 0.6 & 88 & 126 & 35.50 & 1 & 0.07 & Scd & $\ldots$ & NLAGN \\
\hline NGC 3319 & 103909.5 & +414112.7 & 6.2 & 3.4 & 37 & 15 & 30.83 & 43 & 0.01 & $\mathrm{SB}(\mathrm{rs}) \mathrm{cd}$ & $6.0 \pm 0.3$ & HII: \\
\hline UGC 05818 & 104115.7 & +062140.6 & 1.2 & 0.8 & 137 & 90 & 34.76 & 1 & 0.03 & Scd: & $6.0 \pm 1.2$ & \\
\hline UGC 05823 & 104153.4 & +004735.4 & 1.0 & 0.8 & 165 & 80 & 34.52 & 1 & 0.05 & Im: & $10.0 \pm 1.3$ & \\
\hline NGC 3344 & 104331.2 & +245520.0 & 7.1 & 6.5 & 0 & 6.9 & 29.19 & 46 & 0.03 & $(\mathrm{R}) \mathrm{SAB}(\mathrm{r}) \mathrm{bc}$ & $4.0 \pm 0.3$ & HII \\
\hline MESSIER 095 & 104357.7 & +114213.0 & 7.4 & 5.0 & 13 & 12 & 30.36 & 1 & 0.03 & SB(r)b;HII & $3.0 \pm 0.3$ & Sbrst \\
\hline UGC 05848 & 104422.8 & +562514.0 & 2.1 & 1.0 & 115 & 15 & 30.88 & 1 & 0.01 & Sm: & $9.0 \pm 1.1$ & \\
\hline UGC 05853 & 104445.2 & +582717.8 & 1.3 & 0.4 & 38 & 138 & 35.69 & 1 & 0.01 & Scd: & $6.0 \pm 1.4$ & \\
\hline NGC 3353 & 104522.4 & +555737.4 & 1.3 & 1.0 & 45 & 17 & 31.12 & 1 & 0.01 & $\mathrm{BCD} / \mathrm{Irr}$ & $3.0 \pm 1.7$ & HII \\
\hline UGC 05869 & 104542.7 & +112039.0 & 1.4 & 0.8 & 95 & 95 & 34.88 & 1 & 0.03 & $\mathrm{SAB}(\mathrm{rs}) \mathrm{b}:$ & $3.0 \pm 1.2$ & \\
\hline NGC 3367 & 104635.0 & +134502.8 & 2.5 & 2.2 & 0 & 44 & 33.23 & 1 & 0.03 & $\mathrm{SB}(\mathrm{rs}) \mathrm{c} ; \mathrm{LINER}$ & $5.0 \pm 0.3$ & Sy \\
\hline UGC 05876 & 104636.4 & +520858.4 & 1.3 & 0.8 & 103 & 97 & 34.93 & 1 & 0.01 & Scd: & $6.0 \pm 1.2$ & \\
\hline NGC 3359 & 104636.8 & +631325.1 & 7.2 & 4.4 & 170 & 18 & 31.28 & 1 & 0.01 & $\mathrm{SB}(\mathrm{rs}) \mathrm{c}$ & $5.0 \pm 0.3$ & HII \\
\hline MESSIER 096 & 104645.7 & +114911.8 & 7.6 & 5.2 & 5 & 14 & 30.65 & 1 & 0.03 & $\mathrm{SAB}(\mathrm{rs}) \mathrm{ab} ; \mathrm{Sy}$ & $2.0 \pm 0.3$ & LINER \\
\hline UGC 05886 & 104651.5 & -012329.4 & 1.0 & 0.7 & 115 & 162 & 36.05 & 1 & 0.04 & $\mathrm{SBc}$ & $\cdots$ & \\
\hline NGC 3377A & 104722.3 & +140410.0 & 2.2 & 2.1 & 0 & 9.1 & 29.78 & 1 & 0.03 & $\mathrm{SAB}(\mathrm{s}) \mathrm{m}$ & $9.0 \pm 0.4$ & \\
\hline UGC 05896 & 104730.5 & -012933.5 & 1.3 & 0.5 & 132 & 163 & 36.06 & 1 & 0.04 & Sab & $2.0 \pm 0.9$ & \\
\hline NGC 3377 & 104742.4 & +135908.3 & 5.2 & 3.0 & 35 & 10 & 30.08 & 1 & 0.03 & E5-6 & $-5.0 \pm 0.3$ & \\
\hline UGC 05888 & 104745.8 & +560528.1 & 1.2 & 1.2 & $\cdots$ & 21 & 31.61 & 1 & 0.01 & $\operatorname{Im}$ & $10.0 \pm 0.8$ & \\
\hline UGC 05904 & 104837.9 & +662143.2 & 2.0 & 0.3 & 152 & 98 & 34.95 & 1 & 0.01 & $\mathrm{Sb}$ & $3.0 \pm 0.9$ & NLAGN \\
\hline UGC 05907 & 104858.3 & +660540.8 & 1.7 & 1.3 & 165 & 51 & 33.52 & 1 & 0.01 & Im: & $10.0 \pm 1.1$ & \\
\hline UGC 05922 & 104901.5 & -003825.5 & 1.0 & 0.7 & 10 & 26 & 32.09 & 1 & 0.04 & Scd & $\ldots$ & \\
\hline UGC 05929 & 104928.4 & +044757.8 & 1.1 & 0.5 & 68 & 112 & 35.25 & 1 & 0.03 & $\mathrm{Sbc}$ & $4.0 \pm 0.9$ & \\
\hline UGC 05928 & 104947.1 & +515338.8 & 1.0 & 1.0 & $\ldots$ & 109 & 35.18 & 1 & 0.01 & S0-: & $-3.0 \pm 1.2$ & \\
\hline
\end{tabular}


Table 1-Continued

\begin{tabular}{|c|c|c|c|c|c|c|c|c|c|c|c|c|}
\hline $\begin{array}{l}\text { Object Name } \\
\text { (1) }\end{array}$ & $\begin{array}{c}\mathrm{RA}_{2000} \\
(\mathrm{~h}: \mathrm{m}: \mathrm{s}) \\
(2)\end{array}$ & $\begin{array}{c}\mathrm{DEC}_{2000} \\
(\mathrm{~d}: \mathrm{m}: \mathrm{s}) \\
(3)\end{array}$ & $\begin{array}{c}2 \times \mathrm{A} \\
(\operatorname{arcmin}) \\
(4)\end{array}$ & $\begin{array}{c}2 \times \mathrm{B} \\
(\operatorname{arcmin}) \\
(5)\end{array}$ & $\begin{array}{c}\text { PA } \\
(\operatorname{deg}) \\
(6)\end{array}$ & $\begin{array}{c}(\mathrm{Mpc}) \\
(7)\end{array}$ & $\begin{array}{l}\text { istance } \\
\text { DM } \\
(8)\end{array}$ & $\begin{array}{l}\text { ref. } \\
(9)\end{array}$ & $\begin{array}{c}\mathrm{E}(B-V) \\
\quad(\mathrm{mag}) \\
(10)\end{array}$ & $\begin{array}{c}\text { Morphological } \\
\text { Type } \\
(11)\end{array}$ & $\begin{array}{c}\mathrm{T} \\
\text { Type } \\
(12)\end{array}$ & $\begin{array}{l}\text { Spectral } \\
\text { Type } \\
(13)\end{array}$ \\
\hline UGC 05943 & 105013.5 & $\begin{array}{lll}-01 & 17 & 24.7\end{array}$ & 1.1 & 0.9 & 155 & 65 & 34.06 & 1 & 0.05 & $\mathrm{SAB}(\mathrm{r}) \mathrm{c}$ & $4.5 \pm 0.6$ & \\
\hline NGC 3394 & 105039.8 & +654338.0 & 1.9 & 1.4 & 35 & 52 & 33.59 & 1 & 0.01 & $\mathrm{SA}(\mathrm{rs}) \mathrm{c}$ & $5.0 \pm 0.8$ & \\
\hline NGC 3412 & 105053.3 & +132443.7 & 3.6 & 2.0 & 155 & 13 & 30.54 & 1 & 0.03 & $\mathrm{SB}(\mathrm{s}) 0^{*} 0^{*}$ & $-2.0 \pm 0.3$ & \\
\hline NGC 3419 & 105117.7 & +135645.6 & 1.2 & 1.0 & 115 & 44 & 33.23 & 1 & 0.04 & $(\mathrm{R}) \mathrm{SAB}(\mathrm{r}) 0+$ & $-1.0 \pm 0.5$ & \\
\hline UGC 05974 & 105135.2 & +043459.0 & 2.0 & 0.8 & 130 & 15 & 30.89 & 1 & 0.03 & Scd: & $6.0 \pm 1.2$ & \\
\hline IC 0653 & 105206.8 & -003338.9 & 1.9 & 0.9 & 55 & 79 & 34.49 & 1 & 0.06 & S0/a: & $0.0 \pm 0.6$ & \\
\hline UGC 05971 & 105211.3 & +664717.5 & 1.0 & 0.3 & 7 & 184 & 36.32 & 1 & 0.02 & Sab & $2.0 \pm 0.9$ & \\
\hline UGC 06011 & 105320.8 & -003623.0 & 1.4 & 0.4 & 143 & 79 & 34.49 & 1 & 0.05 & Sdm: & $8.0 \pm 1.3$ & \\
\hline NGC 3440 & 105349.5 & +570707.5 & 2.1 & 0.5 & 48 & 31 & 32.44 & 1 & 0.01 & $\mathrm{SBb} ? \mathrm{sp}$ & $3.0 \pm 1.7$ & \\
\hline NGC 3445 & 105435.5 & +565926.6 & 1.6 & 1.5 & 115 & 32 & 32.54 & 1 & 0.01 & $\mathrm{SAB}(\mathrm{s}) \mathrm{m}$ & $9.0 \pm 0.4$ & \\
\hline NGC 3458 & 105601.5 & +570701.1 & 1.4 & 0.9 & 5 & 29 & 32.34 & 1 & 0.01 & SAB: & $-2.0 \pm 0.4$ & \\
\hline UGC 06039 & 105620.9 & +564534.4 & 1.4 & 0.5 & 25 & 30 & 32.41 & 1 & 0.01 & Sd: & $7.0 \pm 1.2$ & \\
\hline NGC 3475 & 105825.2 & $\begin{array}{r}+241335.0 \\
\end{array}$ & 1.7 & 1.1 & 65 & 93 & 34.85 & 1 & 0.02 & $\mathrm{Sa}$ & $1.0 \pm 1.1$ & \\
\hline NGC 3470 & 105844.9 & $\begin{array}{r}+593038.5 \\
\end{array}$ & 1.4 & 1.2 & 170 & 97 & 34.93 & 1 & 0.01 & $\mathrm{SA}(\mathrm{r}) \mathrm{ab}:$ & $2.0 \pm 0.6$ & \\
\hline NGC 3489 & 110018.6 & +135404.4 & 3.5 & 2.0 & 70 & 12 & 30.41 & 15 & 0.02 & $\mathrm{SAB}(\mathrm{rs}) 0+$ & $-1.0 \pm 0.3$ & Sy2 \\
\hline NGC 3486 & 110024.0 & +285829.3 & 7.1 & 5.2 & 80 & 12 & 30.33 & 1 & 0.02 & $\mathrm{SAB}(\mathrm{r}) \mathrm{c}$ & $5.0 \pm 0.3$ & Sy2 \\
\hline UGC 06102 & $\begin{array}{lll}11 & 0148.4\end{array}$ & +284121.2 & 1.0 & 0.8 & 140 & 12 & 30.38 & 1 & 0.03 & $\mathrm{Im}$ & $10.0 \pm 0.9$ & \\
\hline NGC 3521 & 110548.6 & -000209.1 & 11.0 & 5.1 & 163 & 9.0 & 29.77 & 3 & 0.06 & $\mathrm{SAB}(\mathrm{rs}) \mathrm{bc}$ & $4.0 \pm 0.3$ & LINER \\
\hline UGC 06151 & 110556.3 & +194931.0 & 1.9 & 1.3 & 0 & 20 & 31.54 & 1 & 0.03 & Sm: & $9.0 \pm 1.1$ & \\
\hline NGC 3522 & 110640.5 & +200508.1 & 1.2 & 0.7 & 117 & 19 & 31.37 & 1 & 0.02 & $\mathrm{E}$ & $-5.0 \pm 0.9$ & \\
\hline IC 0671 & 110731.6 & +004659.2 & 1.3 & 1.2 & 22 & 169 & 36.14 & 1 & 0.04 & $\mathrm{SAB}(\mathrm{r}) \mathrm{bc}:$ & $4.2 \pm 0.5$ & \\
\hline UGC 06181 & 110746.7 & +193257.4 & 1.0 & 1.0 & $\ldots$ & 18 & 31.28 & 1 & 0.02 & Im: & $10.0 \pm 1.2$ & \\
\hline NGC 3539 & 110908.8 & +284019.0 & 1.1 & 0.2 & 10 & 141 & 35.74 & 1 & 0.03 & So/a & $\ldots$ & \\
\hline IC 0673 & $\begin{array}{lll}11 & 09 & 25.3\end{array}$ & -000551.8 & 1.7 & 0.7 & 165 & 55 & 33.71 & 1 & 0.04 & $\left(\mathrm{R}^{\prime}\right) \mathrm{SAB}(\mathrm{rs}) \mathrm{c}$ & $5.0 \pm 0.8$ & NLAGN \\
\hline PGC 33931 & 111038.4 & +281900.6 & 1.2 & 1.2 & $\cdots$ & 149 & 35.87 & 1 & 0.02 & So & $\ldots$ & \\
\hline NGC 3550 & 111038.7 & +284606.0 & 1.0 & 1.0 & $\cdots$ & 150 & 35.89 & 1 & 0.03 & & $99.0 \pm 0.0$ & \\
\hline NGC 3620 & 111604.7 & -761258.7 & 2.8 & 1.1 & 78 & 20 & 31.51 & 1 & 0.42 & $\left(\mathrm{R}^{\prime}-1\right) \mathrm{SB}(\mathrm{s}) \mathrm{ab}$ & $1.7 \pm 0.4$ & \\
\hline NGC 3621 & 111816.5 & -324850.6 & 12.3 & 7.1 & 159 & 8.3 & 29.59 & 1 & 0.08 & $\mathrm{SA}(\mathrm{s}) \mathrm{d}$ & $7.0 \pm 0.3$ & \\
\hline UGC 06329 & 111856.2 & +00 1034.0 & 1.1 & 1.0 & 0 & 107 & 35.14 & 1 & 0.04 & $\mathrm{SAB}(\mathrm{rs}) \mathrm{c}:$ & $5.3 \pm 0.7$ & \\
\hline UGC 06331 & 111907.6 & +031351.9 & 1.2 & 0.3 & 57 & 86 & 34.68 & 1 & 0.06 & Sdm? sp & $8.0 \pm 1.1$ & \\
\hline NGC 3627 & 112015.0 & +125929.6 & 9.1 & 4.2 & 173 & 9.1 & 29.79 & 43 & 0.03 & SAB(s)b;LINER & $3.0 \pm 0.3$ & $\mathrm{Sy} 2$ \\
\hline NGC 3630 & 112017.0 & +025751.0 & 4.6 & 3.0 & 37 & 22 & 31.67 & 1 & 0.04 & So & $-2.0 \pm 0.4$ & \\
\hline NGC 3628 & 112017.0 & +133522.2 & 14.8 & 3.0 & 104 & 13 & 30.58 & 1 & 0.03 & SAb pec sp & $3.0 \pm 0.3$ & LINER \\
\hline
\end{tabular}


Table 1-Continued

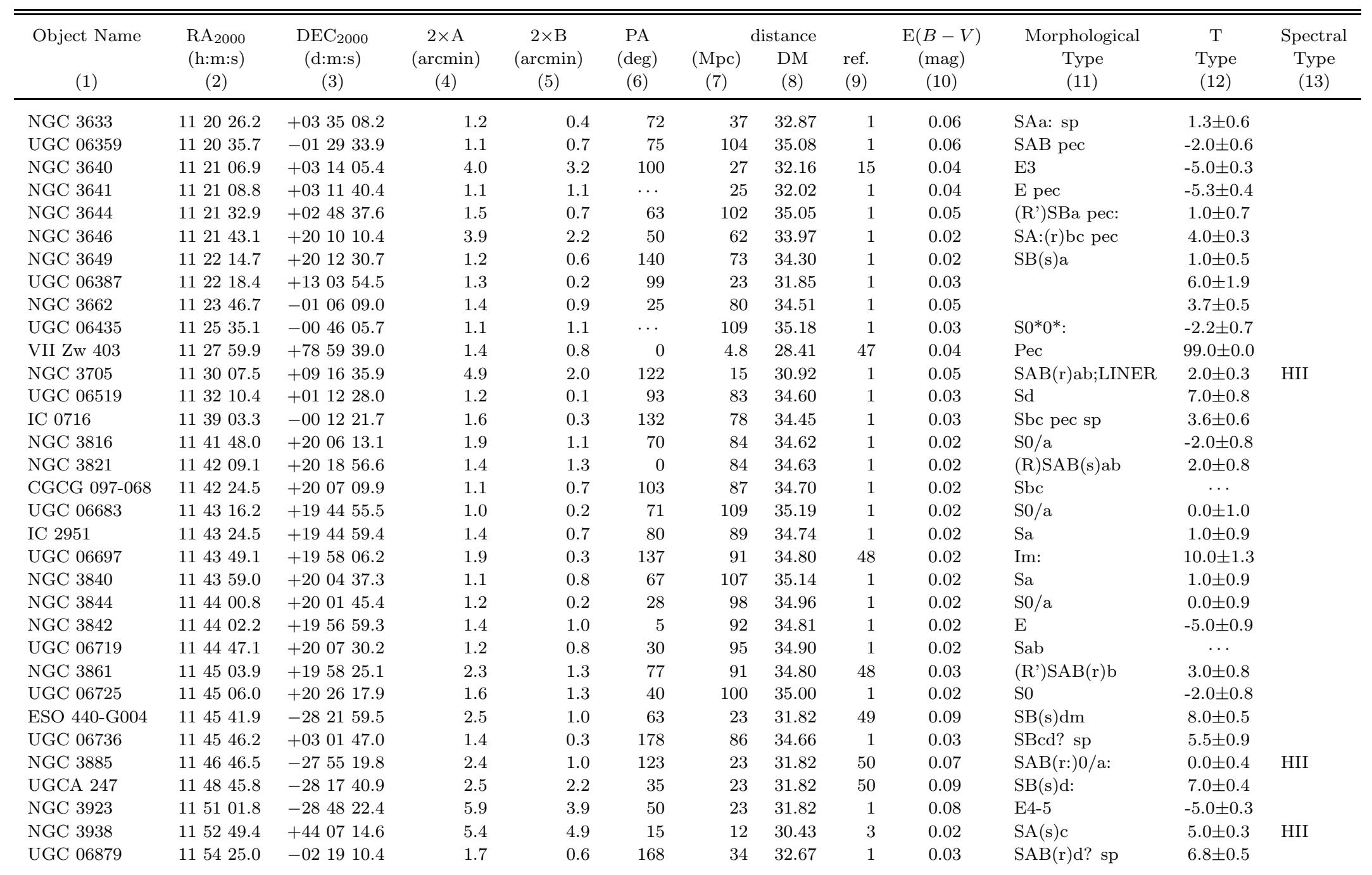


Table 1-Continued

\begin{tabular}{|c|c|c|c|c|c|c|c|c|c|c|c|c|}
\hline $\begin{array}{l}\text { Object Name } \\
\text { (1) }\end{array}$ & $\begin{array}{c}\mathrm{RA}_{2000} \\
(\mathrm{~h}: \mathrm{m}: \mathrm{s}) \\
(2)\end{array}$ & $\begin{array}{c}\mathrm{DEC}_{2000} \\
(\mathrm{~d}: \mathrm{m}: \mathrm{s}) \\
(3)\end{array}$ & $\begin{array}{c}2 \times \mathrm{A} \\
(\operatorname{arcmin}) \\
(4)\end{array}$ & $\begin{array}{c}2 \times \mathrm{B} \\
(\operatorname{arcmin}) \\
(5)\end{array}$ & $\begin{array}{c}\text { PA } \\
(\mathrm{deg}) \\
(6)\end{array}$ & $\begin{array}{c}(\mathrm{Mpc}) \\
(7)\end{array}$ & $\begin{array}{c}\text { istance } \\
\text { DM } \\
(8)\end{array}$ & $\begin{array}{l}\text { ref. } \\
(9)\end{array}$ & $\begin{array}{c}\mathrm{E}(B-V) \\
(\mathrm{mag}) \\
(10)\end{array}$ & $\begin{array}{c}\text { Morphological } \\
\text { Type } \\
(11)\end{array}$ & $\begin{array}{c}\mathrm{T} \\
\text { Type } \\
(12)\end{array}$ & $\begin{array}{c}\text { Spectral } \\
\text { Type } \\
(13)\end{array}$ \\
\hline UGC 06934 & 115731.8 & -011510.6 & 1.8 & 0.3 & 141 & 79 & 34.50 & 1 & 0.03 & $\mathrm{SA}(\mathrm{r}) \mathrm{cd}: \mathrm{sp}$ & $5.8 \pm 0.7$ & \\
\hline UGC 06970 & 115845.7 & -012741.2 & 1.5 & 0.8 & 75 & 21 & 31.65 & 1 & 0.03 & $\mathrm{SB}(\mathrm{s}) \mathrm{m}$ & $9.2 \pm 0.5$ & \\
\hline IC 0754 & 115923.6 & -013916.5 & 1.1 & 1.0 & 40 & 87 & 34.69 & 1 & 0.03 & $\mathrm{E}+$ & $-4.3 \pm 0.4$ & \\
\hline NGC 4030 & 120023.6 & -010600.0 & 4.2 & 3.0 & 27 & 21 & 31.62 & 1 & 0.03 & $\mathrm{SA}(\mathrm{s}) \mathrm{bc}$ & $4.0 \pm 0.3$ & \\
\hline UGC 07000 & 120111.2 & -011745.1 & 1.2 & 1.0 & 50 & 22 & 31.66 & 1 & 0.04 & $\mathrm{IB}(\mathrm{s}) \mathrm{m}$ & $10.0 \pm 0.5$ & \\
\hline NGC 4038 & 120153.0 & -185209.9 & 5.2 & 3.1 & 80 & 22 & 31.73 & 51 & 0.05 & $\mathrm{SB}(\mathrm{s}) \mathrm{m}$ pec & $9.0 \pm 0.4$ & \\
\hline NGC 4039 & 120153.6 & -185310.8 & 3.1 & 1.6 & 50 & 22 & 31.73 & 51 & 0.05 & $\mathrm{SA}(\mathrm{s}) \mathrm{m}$ pec & $9.0 \pm 0.4$ & \\
\hline UGC 07011 & 120155.6 & -010409.5 & 1.1 & 0.2 & 40 & 88 & 34.72 & 1 & 0.03 & $\mathrm{SA}(\mathrm{r}) \mathrm{bc}: \mathrm{sp}$ & $3.7 \pm 0.8$ & \\
\hline NGC $4108 \mathrm{~A}$ & 120549.7 & +671507.5 & 1.4 & 0.5 & 7 & 36 & 32.79 & 1 & 0.02 & SBbc: & $3.5 \pm 0.6$ & \\
\hline UGC 07089 & 120558.1 & +430843.0 & 3.2 & 0.7 & 36 & 14 & 30.73 & 1 & 0.01 & Sdm: & $8.0 \pm 1.1$ & \\
\hline NGC 4108 & 120644.6 & +670947.5 & 1.7 & 1.4 & 105 & 40 & 33.00 & 1 & 0.02 & (R')SAc: & $5.3 \pm 0.6$ & \\
\hline NGC 4109 & 120651.1 & +425944.1 & 1.0 & 0.9 & 0 & 104 & 35.08 & 1 & 0.01 & $\mathrm{Sa} ?$ & $1.0 \pm 1.8$ & \\
\hline NGC 4111 & 120703.1 & +430355.4 & 4.6 & 1.0 & 150 & 15 & 30.81 & 1 & 0.01 & $\mathrm{SA}(\mathrm{r}) 0+: \mathrm{sp} ; \mathrm{HII}$ & $-1.0 \pm 0.5$ & LINER \\
\hline NGC 4108B & 120711.6 & +671406.6 & 1.3 & 1.1 & 125 & 42 & 33.12 & 1 & 0.02 & $\mathrm{SAB}(\mathrm{s}) \mathrm{d}$ pec? & $6.8 \pm 0.7$ & \\
\hline NGC 4116 & 120737.1 & +024125.8 & 3.8 & 2.2 & 155 & 17 & 31.15 & 48 & 0.02 & $\mathrm{SB}(\mathrm{rs}) \mathrm{dm}$ & $8.0 \pm 0.3$ & \\
\hline NGC 4117 & 120746.1 & +430735.0 & 1.8 & 0.9 & 18 & 16 & 31.06 & 1 & 0.01 & $\mathrm{~S} 0 * 0^{*}:$ & $-2.3 \pm 0.6$ & Sy2 \\
\hline NGC 4125 & 120806.0 & +651026.9 & 5.8 & 3.2 & 95 & 21 & 31.65 & 3 & 0.02 & E6 pec & $-5.0 \pm 0.3$ & LINER \\
\hline NGC 4136 & 120917.7 & +295539.4 & 4.0 & 3.7 & 0 & 11 & 30.20 & 1 & 0.02 & $\mathrm{SAB}(\mathrm{r}) \mathrm{c}$ & $5.0 \pm 0.5$ & HII \\
\hline NGC 4138 & 120929.8 & +434107.1 & 2.6 & 1.7 & 150 & 16 & 30.98 & 1 & 0.01 & $\mathrm{SA}(\mathrm{r}) 0+$ & $-1.0 \pm 0.3$ & Sy1.9 \\
\hline UGC 07148 & 120953.9 & +005542.8 & 1.2 & 0.2 & 57 & 84 & 34.63 & 1 & 0.03 & (R)SB(r)a? sp & $1.0 \pm 0.9$ & NLAGN \\
\hline NGC 4150 & 121033.7 & +302405.5 & 2.3 & 1.6 & 147 & 14 & 30.69 & 15 & 0.02 & $\mathrm{SA}(\mathrm{r}) 0^{*} 0^{*} ?$ & $-2.0 \pm 0.4$ & \\
\hline VII Zw 173 & 121041.8 & +13 1952.4 & 1.3 & 0.3 & 30 & 32 & 32.53 & 52 & 0.04 & & $\ldots$ & \\
\hline UGC 07176 & 121055.9 & +501718.1 & 1.4 & 0.5 & 80 & 16 & 31.02 & 1 & 0.02 & Im: & $10.0 \pm 0.7$ & \\
\hline UGC 07178 & 121103.5 & +020016.4 & 1.4 & 1.1 & 75 & 20 & 31.46 & 1 & 0.03 & $\mathrm{IAB}(\mathrm{rs}) \mathrm{m}:$ & $9.6 \pm 0.5$ & \\
\hline NGC 4157 & 121104.4 & +502904.8 & 6.8 & 1.3 & 66 & 14 & 30.79 & 1 & 0.02 & $\mathrm{SAB}(\mathrm{s}) \mathrm{b} ? \mathrm{sp}$ & $3.0 \pm 0.5$ & HII \\
\hline IC 3033 & 121110.0 & +133514.8 & 1.1 & 0.7 & 2 & 32 & 32.53 & 52 & 0.03 & $\mathrm{Sdm} ?$ & $8.0 \pm 1.7$ & \\
\hline UGC 07184 & 121119.9 & +012932.1 & 1.5 & 0.7 & 148 & 31 & 32.44 & 1 & 0.03 & $\mathrm{SB}(\mathrm{rs}) \mathrm{d}$ pec & $7.4 \pm 0.5$ & \\
\hline UGC 07196 & 121159.4 & +152404.7 & 1.3 & 0.3 & 57 & 95 & 34.88 & 53 & 0.04 & Sbc (on edge) & $\ldots$ & \\
\hline NGC 4165 & 121211.8 & +131447.3 & 1.3 & 0.9 & 160 & 32 & 32.53 & 52 & 0.04 & $\mathrm{SAB}(\mathrm{r}) \mathrm{a}: ?$ & $1.0 \pm 0.9$ & \\
\hline NGC 4168 & 121217.3 & +131218.7 & 2.8 & 2.3 & 125 & 32 & 32.53 & 52 & 0.04 & $\mathrm{E} 2$ & $-5.0 \pm 0.3$ & Sy1.9 \\
\hline IC 3046 & 121307.9 & +125505.5 & 1.3 & 0.3 & 130 & 108 & 35.17 & 53 & 0.03 & $\mathrm{~S} ?$ & $\ldots$ & \\
\hline NGC 4192A & 121326.2 & +144620.1 & 1.3 & 1.2 & 0 & 32 & 32.53 & 52 & 0.03 & SAdm & $8.0 \pm 0.8$ & \\
\hline NGC 4187 & 121329.3 & +504429.3 & 1.3 & 1.0 & 145 & 134 & 35.63 & 1 & 0.02 & $\mathrm{E}$ & $-5.0 \pm 0.8$ & \\
\hline
\end{tabular}


Table 1-Continued

\begin{tabular}{|c|c|c|c|c|c|c|c|c|c|c|c|c|}
\hline $\begin{array}{c}\text { Object Name } \\
\text { (1) }\end{array}$ & $\begin{array}{c}\mathrm{RA}_{2000} \\
(\mathrm{~h}: \mathrm{m}: \mathrm{s}) \\
(2)\end{array}$ & $\begin{array}{c}\mathrm{DEC}_{2000} \\
(\mathrm{~d}: \mathrm{m}: \mathrm{s}) \\
(3)\end{array}$ & $\begin{array}{c}2 \times \mathrm{A} \\
(\operatorname{arcmin}) \\
(4)\end{array}$ & $\begin{array}{c}2 \times \mathrm{B} \\
(\operatorname{arcmin}) \\
(5)\end{array}$ & $\begin{array}{c}\text { PA } \\
(\mathrm{deg}) \\
(6)\end{array}$ & $\begin{array}{c}(\mathrm{Mpc}) \\
(7)\end{array}$ & $\begin{array}{l}\text { stance } \\
\text { DM } \\
(8)\end{array}$ & $\begin{array}{l}\text { ref. } \\
(9)\end{array}$ & $\begin{array}{c}\mathrm{E}(B-V) \\
(\mathrm{mag}) \\
(10)\end{array}$ & $\begin{array}{c}\text { Morphological } \\
\text { Type } \\
(11)\end{array}$ & $\begin{array}{c}\mathrm{T} \\
\text { Type } \\
(12)\end{array}$ & $\begin{array}{c}\text { Spectral } \\
\text { Type } \\
(13)\end{array}$ \\
\hline NGC 4189 & 121347.3 & +132529.2 & 2.4 & 1.7 & 85 & 32 & 32.53 & 48 & 0.03 & $\mathrm{SAB}(\mathrm{rs}) \mathrm{cd} ?$ & $6.0 \pm 0.3$ & \\
\hline MESSIER 098 & 121348.3 & +145401.2 & 9.8 & 2.8 & 155 & 17 & 31.15 & 54 & 0.04 & $\mathrm{SAB}(\mathrm{s}) \mathrm{ab} ; \mathrm{HII}$ & $2.0 \pm 0.3$ & Sy \\
\hline NGC 4193 & 121353.6 & +131022.4 & 2.0 & 1.0 & 93 & 32 & 32.53 & 48 & 0.03 & $\mathrm{SAB}(\mathrm{s}) \mathrm{c}: ?$ & $5.0 \pm 0.5$ & \\
\hline NGC 4186 & 121406.5 & +144332.7 & 1.1 & 0.9 & 60 & 105 & 35.11 & 53 & 0.04 & SA(s)ab: & $1.5 \pm 0.6$ & \\
\hline UGC 07242 & 121408.4 & +660541.0 & 1.9 & 0.8 & 172 & 4.3 & 28.17 & 55 & 0.02 & Scd: & $6.0 \pm 1.2$ & \\
\hline IC 3059 & 121455.0 & +132737.9 & 1.7 & 1.3 & 0 & 17 & 31.15 & 54 & 0.03 & Im: & $10.0 \pm 0.6$ & \\
\hline VCC 0132 & 121503.9 & +130155.3 & 1.2 & 0.9 & 0 & 17 & 31.15 & 54 & 0.03 & SB? & $\cdots$ & \\
\hline IC 3066 & 121516.1 & +132829.3 & 1.0 & 0.2 & 138 & 17 & 31.15 & 54 & 0.03 & $\mathrm{~S} ?$ & $\cdots$ & \\
\hline NGC 4206 & 121516.9 & +130125.8 & 6.2 & 1.2 & 0 & 17 & 31.15 & 48 & 0.03 & $\mathrm{SA}(\mathrm{s}) \mathrm{bc}:$ & $4.0 \pm 0.3$ & \\
\hline IC 3073 & 121535.7 & +133711.0 & 1.0 & 0.6 & 95 & 17 & 31.15 & 54 & 0.03 & Im: & $10.0 \pm 1.3$ & \\
\hline NGC 4216 & 121554.4 & +130857.8 & 8.1 & 1.8 & 19 & 17 & 31.15 & 48 & 0.03 & SAB(s)b:;HII & $3.0 \pm 0.3$ & LINER \\
\hline NGC 4222 & 121622.5 & +131825.4 & 3.3 & 0.5 & 56 & 17 & 31.15 & 48 & 0.03 & $\mathrm{Sc}$ & $7.0 \pm 0.6$ & \\
\hline NGC 4226 & 121626.3 & +470131.3 & 1.3 & 0.6 & 127 & 107 & 35.14 & 1 & 0.02 & Sa pec? & $1.0 \pm 0.9$ & \\
\hline NGC 4236 & 121642.1 & +692745.3 & 21.9 & 7.2 & 162 & 4.4 & 28.24 & 34 & 0.01 & $\mathrm{SB}(\mathrm{s}) \mathrm{dm}$ & $8.0 \pm 0.3$ & \\
\hline NGC 4231 & 121648.9 & +472726.9 & 1.2 & 1.1 & 0 & 109 & 35.19 & 1 & 0.02 & SA0+ pec? & $-1.0 \pm 1.2$ & \\
\hline NGC 4232 & 121649.0 & +472619.8 & 1.4 & 0.7 & 155 & 107 & 35.15 & 1 & 0.02 & SBb pec: & $3.0 \pm 0.6$ & \\
\hline UGC 07325 & 121728.7 & +464938.9 & 1.3 & 0.5 & 145 & 104 & 35.08 & 1 & 0.02 & $\mathrm{Sab}$ & $2.0 \pm 0.9$ & \\
\hline NGC 4242 & 121730.1 & +453707.5 & 5.0 & 3.8 & 25 & 10 & 30.08 & 1 & 0.01 & $\mathrm{SAB}(\mathrm{s}) \mathrm{dm}$ & $8.0 \pm 0.3$ & \\
\hline NGC 4248 & 121749.9 & +472433.0 & 3.0 & 1.1 & 108 & 7.2 & 29.29 & 56 & 0.02 & $\mathrm{I} 0 ? \mathrm{sp}$ & $90.0 \pm 0.0$ & \\
\hline MESSIER 099 & 121849.6 & +142459.4 & 5.4 & 4.7 & 0 & 17 & 31.15 & 54 & 0.04 & $\mathrm{SA}(\mathrm{s}) \mathrm{c}$ & $5.0 \pm 0.3$ & \\
\hline MESSIER 106 & 121857.5 & +471814.2 & 18.6 & 7.2 & 150 & 7.2 & 29.29 & 57 & 0.02 & SAB(s)bc;LINER & $4.0 \pm 0.3$ & Sy1.9 \\
\hline NGC 4262 & 121930.6 & +145239.7 & 1.9 & 1.7 & 0 & 17 & 31.15 & 54 & 0.04 & $\mathrm{SB}(\mathrm{s}) 0-?$ & $-3.0 \pm 0.3$ & \\
\hline NGC 4274 & 121950.6 & +293652.1 & 6.8 & 2.5 & 102 & 16 & 31.03 & 58 & 0.02 & (R)SB(r)ab & $2.0 \pm 0.3$ & LINER \\
\hline NGC 4278 & 122006.8 & +291650.7 & 4.1 & 3.8 & 40 & 16 & 31.03 & 15 & 0.03 & E1-2;LINER & $-5.0 \pm 0.3$ & Sy1 \\
\hline UGC 07387 & 122017.4 & +041206.0 & 1.8 & 0.2 & 16 & 17 & 31.15 & 59 & 0.02 & $\mathrm{Sd}$ & $6.5 \pm 0.9$ & \\
\hline NGC 4283 & 122020.8 & +29 1839.2 & 1.5 & 1.5 & $\cdots$ & 16 & 31.07 & 1 & 0.03 & E0 & $-5.0 \pm 0.4$ & \\
\hline NGC 4286 & 122042.1 & +292045.4 & 1.6 & 1.0 & 150 & 12 & 30.31 & 1 & 0.02 & $\mathrm{SA}(\mathrm{r}) 0 / \mathrm{a}:$ & $0.0 \pm 0.4$ & \\
\hline NGC 4292 & 122116.5 & +043544.2 & 1.7 & 1.1 & 7 & 17 & 31.15 & 59 & 0.02 & $(\mathrm{R}) \mathrm{SB}(\mathrm{r}) 0^{*} 0 *$ & $-1.7 \pm 0.5$ & \\
\hline NGC 4298 & 122132.8 & +143621.8 & 3.2 & 1.8 & 140 & 17 & 31.15 & 60 & 0.04 & $\mathrm{SA}(\mathrm{rs}) \mathrm{c}$ & $5.0 \pm 0.3$ & \\
\hline UGC 07411 & 122134.1 & +044646.1 & 1.4 & 0.5 & 132 & 17 & 31.15 & 59 & 0.02 & $\mathrm{SA}(\mathrm{r}) 0 / \mathrm{a}: \mathrm{sp}$ & $-0.3 \pm 0.5$ & \\
\hline IC 0783 & 122138.8 & +154442.7 & 1.3 & 1.2 & 0 & 17 & 31.15 & 60 & 0.02 & $\mathrm{SAB}(\mathrm{rs}) 0 / \mathrm{a} ?$ & $0.0 \pm 1.6$ & \\
\hline
\end{tabular}


Table 1-Continued

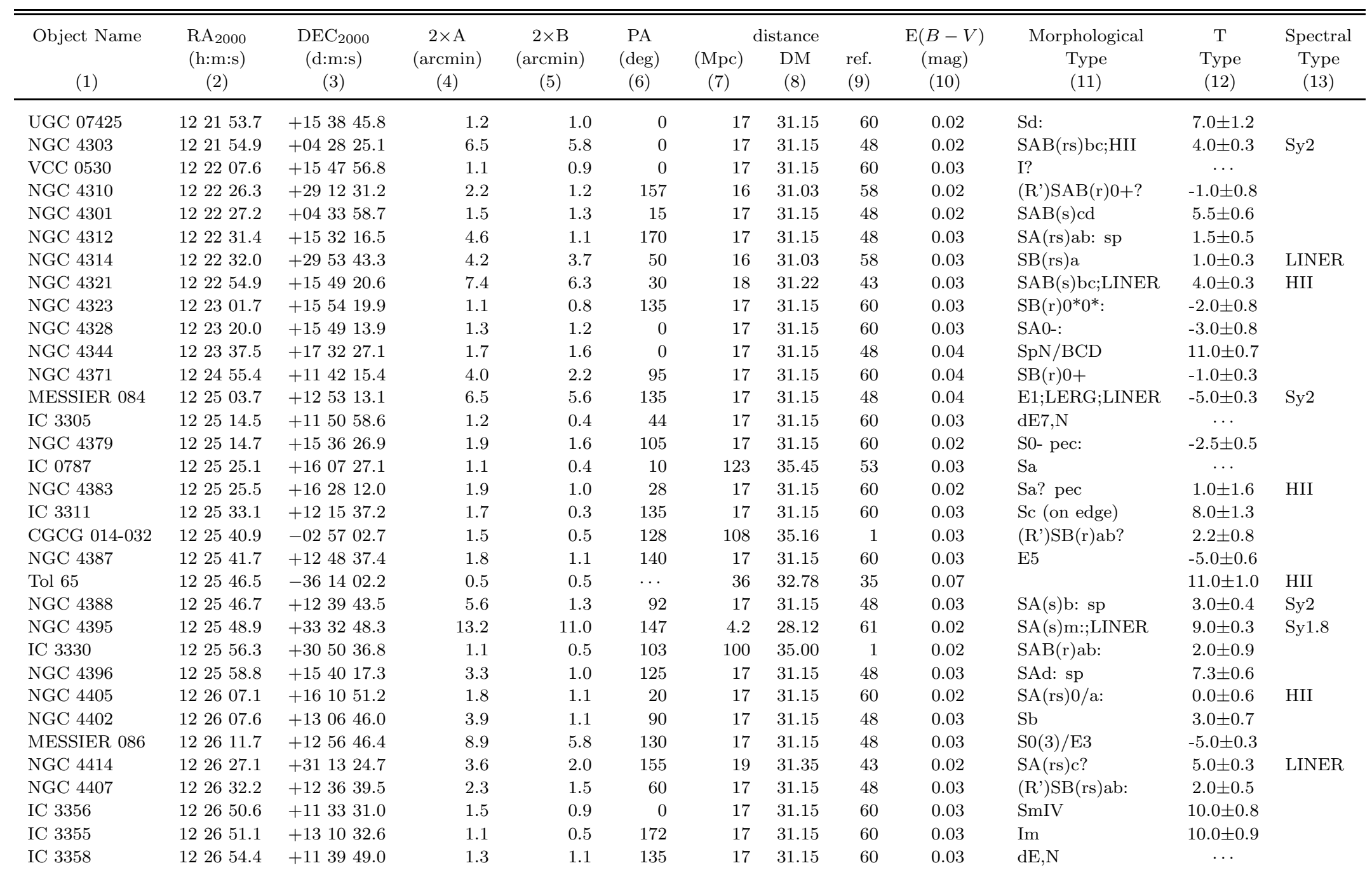


Table 1-Continued

\begin{tabular}{|c|c|c|c|c|c|c|c|c|c|c|c|c|}
\hline $\begin{array}{c}\text { Object Name } \\
\text { (1) }\end{array}$ & $\begin{array}{c}\mathrm{RA}_{2000} \\
(\mathrm{~h}: \mathrm{m}: \mathrm{s}) \\
(2)\end{array}$ & $\begin{array}{c}\mathrm{DEC}_{2000} \\
(\mathrm{~d}: \mathrm{m}: \mathrm{s}) \\
(3)\end{array}$ & $\begin{array}{c}2 \times \mathrm{A} \\
(\operatorname{arcmin}) \\
(4)\end{array}$ & $\begin{array}{c}2 \times \mathrm{B} \\
(\operatorname{arcmin}) \\
(5)\end{array}$ & $\begin{array}{c}\text { PA } \\
(\mathrm{deg}) \\
(6)\end{array}$ & $\begin{array}{c}(\mathrm{Mpc}) \\
(7)\end{array}$ & $\begin{array}{l}\text { istance } \\
\text { DM } \\
(8)\end{array}$ & $\begin{array}{l}\text { ref. } \\
(9)\end{array}$ & $\begin{array}{c}\mathrm{E}(B-V) \\
(\mathrm{mag}) \\
(10)\end{array}$ & $\begin{array}{c}\text { Morphological } \\
\text { Type } \\
(11)\end{array}$ & $\begin{array}{c}\mathrm{T} \\
\text { Type } \\
(12)\end{array}$ & $\begin{array}{c}\text { Spectral } \\
\text { Type } \\
(13)\end{array}$ \\
\hline ESO 380-G029 & 122654.4 & -362527.2 & 1.8 & 0.6 & 77 & 56 & 33.74 & 1 & 0.08 & $\mathrm{SB}(\mathrm{s}) \mathrm{m}:$ & $9.0 \pm 1.2$ & \\
\hline NGC 4419 & 122656.4 & +150250.7 & 3.3 & 1.1 & 133 & 17 & 31.15 & 48 & 0.03 & SB(s)a;LINER & $1.0 \pm 0.4$ & HII \\
\hline NGC 4421 & 122702.6 & +152740.9 & 2.7 & 2.0 & 20 & 17 & 31.15 & 48 & 0.02 & $\mathrm{SB}(\mathrm{s}) 0 / \mathrm{a}$ & $0.0 \pm 0.5$ & \\
\hline IC 3363 & 122703.3 & +123337.8 & 1.3 & 0.4 & 128 & 17 & 31.15 & 60 & 0.03 & $\mathrm{dE} 7$ & $\ldots$ & \\
\hline UGC 07553 & 122703.7 & -013057.5 & 1.0 & 0.1 & 60 & 126 & 35.51 & 1 & 0.03 & Sd pec sp & $7.3 \pm 0.8$ & \\
\hline IC 0792 & 122708.8 & +16 1931.4 & 1.6 & 0.6 & 59 & 83 & 34.59 & 53 & 0.03 & $\mathrm{Sc}(\mathrm{s}) \mathrm{II}$ & $3.0 \pm 0.9$ & \\
\hline IC 3365 & 122711.5 & +155351.5 & 2.1 & 1.0 & 72 & 17 & 31.15 & 48 & 0.03 & $\mathrm{Im}$ & $10.0 \pm 0.8$ & \\
\hline NGC 4425 & 122713.3 & +124405.0 & 3.0 & 1.0 & 27 & 17 & 31.15 & 48 & 0.03 & SB0+: sp & $-1.0 \pm 0.5$ & \\
\hline NGC 4431 & 122727.4 & +121725.2 & 1.7 & 1.1 & 177 & 17 & 31.15 & 60 & 0.03 & $\mathrm{SA}(\mathrm{r}) 0$ & $-2.0 \pm 0.6$ & \\
\hline NGC 4435 & 122740.5 & +130444.2 & 2.8 & 2.0 & 13 & 17 & 31.15 & 48 & 0.03 & $\mathrm{SB}(\mathrm{s}) 0^{*} 0^{*} ; \mathrm{LINER}$ & $-2.0 \pm 0.3$ & HII \\
\hline NGC 4436 & 122741.2 & +121857.4 & 1.5 & 0.7 & 118 & 17 & 31.15 & 60 & 0.03 & $\mathrm{dE6} / \mathrm{dS} 0, \mathrm{~N}$ & $-2.0 \pm 0.4$ & \\
\hline NGC 4438 & 122745.6 & +130031.8 & 8.5 & 3.2 & 27 & 17 & 31.15 & 48 & 0.03 & $\mathrm{SA}(\mathrm{s}) 0 / \mathrm{a}$ pec: & $0.0 \pm 0.3$ & LINER \\
\hline NGC 4440 & 122753.6 & +121735.6 & 1.9 & 1.5 & 0 & 17 & 31.15 & 60 & 0.03 & $\mathrm{SB}(\mathrm{rs}) \mathrm{a}$ & $1.0 \pm 0.4$ & \\
\hline IC 0794 & 122808.6 & +120535.7 & 1.3 & 1.0 & 110 & 17 & 31.15 & 60 & 0.03 & $\mathrm{dE} 3$ & $-4.0 \pm 0.6$ & \\
\hline IC 3381 & 122814.9 & +114723.6 & 1.2 & 0.9 & 110 & 17 & 31.15 & 60 & 0.03 & $\mathrm{dE}, \mathrm{N}$ & $-4.0 \pm 0.6$ & \\
\hline NGC 4450 & 122829.6 & +170505.8 & 5.2 & 3.9 & 175 & 17 & 31.15 & 48 & 0.03 & SA(s)ab;LINER & $2.0 \pm 0.3$ & Sy3 \\
\hline UGC 07604 & 122836.2 & +312855.2 & 1.1 & 0.1 & 12 & 101 & 35.02 & 1 & 0.01 & Scd & $6.0 \pm 1.5$ & \\
\hline IC 3393 & 122841.7 & +125457.0 & 1.3 & 0.4 & 127 & 17 & 31.15 & 60 & 0.02 & $\mathrm{dE}, \mathrm{N}$ & $-1.0 \pm 1.3$ & \\
\hline NGC 4452 & 122843.6 & +114526.1 & 2.8 & 0.6 & 32 & 17 & 31.15 & 60 & 0.03 & $\mathrm{SO}(9)$ & $-2.0 \pm 1.7$ & \\
\hline NGC 4454 & 122850.7 & -015621.0 & 2.0 & 1.7 & 100 & 35 & 32.70 & 1 & 0.03 & $(\mathrm{R}) \mathrm{SB}(\mathrm{r}) 0 / \mathrm{a}$ & $0.0 \pm 0.4$ & \\
\hline NGC 4458 & 122857.6 & +131430.8 & 1.7 & 1.6 & 0 & 17 & 31.15 & 60 & 0.02 & E0-1 & $-5.0 \pm 0.4$ & \\
\hline NGC 4461 & 122903.0 & +131101.5 & 3.5 & 1.4 & 9 & 17 & 31.15 & 48 & 0.02 & $\mathrm{SB}(\mathrm{s}) 0+:$ & $-1.0 \pm 0.5$ & \\
\hline IC 0796 & 122926.3 & +162417.2 & 1.3 & 0.6 & 145 & 17 & 31.15 & 60 & 0.03 & $\mathrm{~S} 0 / \mathrm{a}$ & $0.0 \pm 0.9$ & \\
\hline IC 3418 & 122943.8 & +112409.3 & 1.5 & 1.0 & 45 & 17 & 31.15 & 62 & 0.03 & IBm: & $10.0 \pm 0.6$ & \\
\hline NGC 4473 & 122948.9 & +132545.7 & 4.5 & 2.5 & 100 & 16 & 30.98 & 15 & 0.03 & E5 & $-5.0 \pm 0.3$ & \\
\hline NGC 4476 & 122959.1 & +122055.2 & 1.7 & 1.2 & 25 & 17 & 31.15 & 60 & 0.03 & $\mathrm{SA}(\mathrm{r}) 0-:$ & $-3.0 \pm 0.4$ & \\
\hline NGC 4477 & 123002.2 & +133811.3 & 3.8 & 3.5 & 15 & 17 & 31.15 & 48 & 0.03 & $\mathrm{SB}(\mathrm{s}) 0: ?$ & $-2.0 \pm 0.4$ & Sy2 \\
\hline NGC 4478 & 123017.4 & +121942.8 & 1.9 & 1.6 & 140 & 17 & 31.15 & 60 & 0.03 & E2 & $-5.0 \pm 0.4$ & \\
\hline NGC 4479 & 123018.4 & +133439.0 & 1.5 & 1.3 & 13 & 17 & 31.15 & 60 & 0.03 & $\mathrm{SB}(\mathrm{s}) 0^{*} 0^{*}: ?$ & $-2.0 \pm 0.6$ & \\
\hline NGC 4485 & 123031.1 & +414204.2 & 2.3 & 1.6 & 15 & 11 & 30.22 & 63 & 0.02 & $\mathrm{IB}(\mathrm{s}) \mathrm{m} \mathrm{pec}$ & $10.0 \pm 0.4$ & HII \\
\hline NGC 4490 & 123036.4 & +413837.1 & 6.3 & 3.1 & 125 & 11 & 30.22 & 1 & 0.02 & $\mathrm{SB}(\mathrm{s}) \mathrm{d}$ pec & $7.0 \pm 0.3$ & \\
\hline MESSIER 087 & 123049.4 & +122328.0 & 8.3 & 6.6 & 160 & 17 & 31.15 & 60 & 0.02 & E+0-1 pec;NLRG & $-4.0 \pm 0.3$ & Sy \\
\hline NGC 4491 & 123057.1 & +112900.8 & 1.7 & 0.9 & 148 & 17 & 31.15 & 48 & 0.04 & SB(s)a: & $1.0 \pm 0.8$ & \\
\hline
\end{tabular}


Table 1-Continued

\begin{tabular}{|c|c|c|c|c|c|c|c|c|c|c|c|c|}
\hline $\begin{array}{c}\text { Object Name } \\
\text { (1) }\end{array}$ & $\begin{array}{c}\mathrm{RA}_{2000} \\
(\mathrm{~h}: \mathrm{m}: \mathrm{s}) \\
(2)\end{array}$ & $\begin{array}{c}\mathrm{DEC}_{2000} \\
(\mathrm{~d}: \mathrm{m}: \mathrm{s}) \\
(3)\end{array}$ & $\begin{array}{c}2 \times \mathrm{A} \\
(\operatorname{arcmin}) \\
(4)\end{array}$ & $\begin{array}{c}2 \times \mathrm{B} \\
(\operatorname{arcmin}) \\
(5)\end{array}$ & $\begin{array}{c}\text { PA } \\
(\mathrm{deg}) \\
(6)\end{array}$ & $\begin{array}{c}(\mathrm{Mpc}) \\
(7)\end{array}$ & $\begin{array}{l}\text { stance } \\
\text { DM } \\
(8)\end{array}$ & $\begin{array}{c}\text { ref. } \\
(9)\end{array}$ & $\begin{array}{c}\mathrm{E}(B-V) \\
(\mathrm{mag}) \\
(10)\end{array}$ & $\begin{array}{c}\text { Morphological } \\
\text { Type } \\
(11)\end{array}$ & $\begin{array}{c}\mathrm{T} \\
\text { Type } \\
(12)\end{array}$ & $\begin{array}{c}\text { Spectral } \\
\text { Type } \\
(13)\end{array}$ \\
\hline CGCG 014-054 & 123103.8 & +014032.5 & 1.2 & 0.7 & 80 & 16 & 31.07 & 1 & 0.02 & $\mathrm{IAB}(\mathrm{s}) \mathrm{m}$ pec: & $9.5 \pm 0.6$ & \\
\hline IC 3446 & 123122.9 & +112934.0 & 0.6 & 0.3 & 170 & 17 & 31.15 & 48 & 0.05 & SmIII/BCD & $\ldots$ & \\
\hline NGC 4497 & 123132.5 & +113729.1 & 2.0 & 0.9 & 65 & 17 & 31.15 & 48 & 0.04 & $\mathrm{SAB}(\mathrm{s}) 0+:$ & $-1.0 \pm 0.6$ & \\
\hline IC 3457 & 123151.4 & +123926.0 & 1.2 & 0.9 & 150 & 17 & 31.15 & 60 & 0.03 & E3: & $-5.0 \pm 1.2$ & \\
\hline IC 3459 & 123156.0 & +121025.9 & 1.1 & 0.9 & 0 & 17 & 31.15 & 60 & 0.03 & $\mathrm{dSB} 0$ & $10.0 \pm 1.2$ & \\
\hline NGC 4503 & 123206.2 & +111035.1 & 3.5 & 1.7 & 12 & 17 & 31.15 & 48 & 0.05 & SB0-: & $-3.0 \pm 0.4$ & \\
\hline NGC 4506 & 123210.5 & +132510.7 & 1.6 & 1.1 & 110 & 17 & 31.15 & 60 & 0.03 & Sa pec? & $1.0 \pm 0.8$ & \\
\hline IC 3467 & 123224.6 & +114716.3 & 1.1 & 0.2 & 72 & 17 & 31.15 & 48 & 0.03 & Scd: & $6.0 \pm 1.3$ & \\
\hline UGC 07710 & 123345.4 & -023855.1 & 1.4 & 1.3 & 105 & 36 & 32.77 & 1 & 0.04 & $\mathrm{IAB}(\mathrm{s}) \mathrm{m}$ pec & $9.7 \pm 0.5$ & \\
\hline NGC 4528 & 123406.1 & +11 1916.5 & 1.7 & 1.0 & 5 & 17 & 31.15 & 60 & 0.05 & $\mathrm{~S} 0 * 0 *:$ & $-2.3 \pm 0.7$ & \\
\hline NGC 4531 & 123415.9 & +130431.8 & 3.1 & 2.0 & 155 & 17 & 31.15 & 60 & 0.04 & SB0+: & $-0.5 \pm 0.5$ & \\
\hline NGC 4536 & 123427.1 & +021116.4 & 7.6 & 3.2 & 130 & 15 & 30.93 & 43 & 0.02 & $\mathrm{SAB}(\mathrm{rs}) \mathrm{bc}$ & $4.0 \pm 0.3$ & HII \\
\hline UGC 07748 & 123438.4 & +682025.1 & 1.1 & 0.6 & 35 & 10 & 30.10 & 1 & 0.02 & Sdm: & $8.0 \pm 1.2$ & \\
\hline NGC 4546 & 123529.5 & -034735.5 & 3.3 & 1.4 & 78 & 15 & 30.91 & 1 & 0.03 & $\mathrm{SB}(\mathrm{s}) 0-:$ & $-3.0 \pm 0.3$ & \\
\hline NGC 4550 & 123530.6 & +12 1315.3 & 3.3 & 0.9 & 178 & 16 & 31.00 & 15 & 0.04 & SB0*0*: sp & $-1.5 \pm 0.5$ & LINER \\
\hline NGC 4551 & 123538.0 & +121550.4 & 1.8 & 1.4 & 70 & 17 & 31.15 & 60 & 0.04 & $\mathrm{E}:$ & $-5.0 \pm 0.6$ & \\
\hline MESSIER 089 & 123539.8 & +123322.8 & 5.1 & 4.7 & 0 & 15 & 30.93 & 15 & 0.04 & E;LINER;HII & $-5.0 \pm 0.4$ & Sy2 \\
\hline NGC 4559 & 123557.7 & +275735.1 & 10.7 & 4.4 & 150 & 17 & 31.15 & 48 & 0.02 & $\mathrm{SAB}(\mathrm{rs}) \mathrm{cd}$ & $6.0 \pm 0.3$ & HII \\
\hline PGC 42042 & 123621.9 & -035518.1 & 1.4 & 0.6 & 135 & 102 & 35.05 & 1 & 0.03 & $\mathrm{SB}(\mathrm{s}) 0+$ pec? & $-1.2 \pm 0.8$ & \\
\hline NGC 4564 & 123627.0 & +112621.5 & 3.5 & 1.5 & 47 & 17 & 31.15 & 60 & 0.04 & $\mathrm{E} 6$ & $-5.0 \pm 0.5$ & \\
\hline NGC 4567 & 123632.7 & +111528.3 & 3.0 & 2.0 & 85 & 17 & 31.15 & 60 & 0.03 & $\mathrm{SA}(\mathrm{rs}) \mathrm{bc}$ & $4.0 \pm 0.3$ & \\
\hline IC 3583 & 123643.5 & +131533.6 & 2.2 & 1.1 & 0 & 17 & 31.15 & 48 & 0.04 & SmIII & $10.0 \pm 0.4$ & \\
\hline IC 3587 & 123648.4 & +273254.9 & 1.4 & 0.2 & 122 & 107 & 35.15 & 1 & 0.02 & Scd & $6.0 \pm 1.4$ & \\
\hline NGC 4569 & 123649.8 & +130946.3 & 9.5 & 4.4 & 23 & 17 & 31.15 & 48 & 0.05 & SAB(rs)ab;LINER & $2.0 \pm 0.3$ & Sy \\
\hline NGC $4559 A$ & 123653.4 & +275143.7 & 1.0 & 0.5 & 126 & 110 & 35.20 & 1 & 0.02 & Sa? & $1.0 \pm 1.8$ & \\
\hline IC 3598 & 123721.1 & +281229.6 & 1.5 & 0.4 & 140 & 112 & 35.25 & 1 & 0.02 & $\mathrm{SA}(\mathrm{r}) \mathrm{ab}:$ & $1.5 \pm 0.6$ & \\
\hline MESSIER 058 & 123743.6 & +114905.1 & 5.9 & 4.7 & 95 & 17 & 31.15 & 60 & 0.04 & SAB(rs)b;LINER & $3.0 \pm 0.3$ & Sy1.9 \\
\hline NGC 4584 & 123817.9 & +130635.5 & 1.4 & 1.0 & 5 & 17 & 31.15 & 60 & 0.04 & SAB(s)a? & $1.0 \pm 0.8$ & \\
\hline NGC 4594 & 123959.4 & -113723.0 & 8.7 & 3.5 & 90 & 9.1 & 29.79 & 25 & 0.05 & SA(s)a;LINER & $1.0 \pm 0.3$ & Sy1.9 \\
\hline NGC 4612 & 124132.7 & +071853.4 & 2.5 & 1.9 & 145 & 17 & 31.15 & 59 & 0.03 & $(\mathrm{R}) \mathrm{SAB} 0 * 0 *$ & $-2.0 \pm 0.4$ & \\
\hline NGC 4618 & 124132.8 & +410841.2 & 4.2 & 3.4 & 25 & 9.5 & 29.89 & 64 & 0.02 & $\mathrm{SB}(\mathrm{rs}) \mathrm{m}$ & $9.0 \pm 0.3$ & HII \\
\hline NGC 4625 & 124152.7 & +411625.4 & 2.2 & 1.9 & 150 & 9.5 & 29.89 & 3 & 0.02 & $\mathrm{SAB}(\mathrm{rs}) \mathrm{m}$ pec & $9.0 \pm 0.3$ & \\
\hline NGC 4627 & 124159.7 & +323424.8 & 2.6 & 1.8 & 10 & 9.0 & 29.77 & 65 & 0.02 & $\mathrm{E} 4 \mathrm{pec}$ & $-5.0 \pm 0.3$ & \\
\hline
\end{tabular}


Table 1-Continued

\begin{tabular}{|c|c|c|c|c|c|c|c|c|c|c|c|c|}
\hline $\begin{array}{c}\text { Object Name } \\
\text { (1) }\end{array}$ & $\begin{array}{c}\mathrm{RA}_{2000} \\
(\mathrm{~h}: \mathrm{m}: \mathrm{s}) \\
(2)\end{array}$ & $\begin{array}{c}\mathrm{DEC}_{2000} \\
(\mathrm{~d}: \mathrm{m}: \mathrm{s}) \\
(3)\end{array}$ & $\begin{array}{c}2 \times \mathrm{A} \\
(\operatorname{arcmin}) \\
(4)\end{array}$ & $\begin{array}{c}2 \times \mathrm{B} \\
(\operatorname{arcmin}) \\
(5)\end{array}$ & $\begin{array}{c}\text { PA } \\
(\mathrm{deg}) \\
(6)\end{array}$ & $\begin{array}{c}(\mathrm{Mpc}) \\
\quad(7)\end{array}$ & $\begin{array}{l}\text { istance } \\
\text { DM } \\
(8)\end{array}$ & $\begin{array}{l}\text { ref. } \\
(9)\end{array}$ & $\begin{array}{c}\mathrm{E}(B-V) \\
(\mathrm{mag}) \\
(10)\end{array}$ & $\begin{array}{c}\text { Morphological } \\
\text { Type } \\
(11)\end{array}$ & $\begin{array}{c}\mathrm{T} \\
\text { Type } \\
(12)\end{array}$ & $\begin{array}{c}\text { Spectral } \\
\text { Type } \\
(13)\end{array}$ \\
\hline NGC 4631 & 124208.0 & +323229.4 & 15.5 & 2.7 & 86 & 9.0 & 29.77 & 3 & 0.02 & $\mathrm{SB}(\mathrm{s}) \mathrm{d}$ & $7.0 \pm 0.3$ & \\
\hline NGC 4623 & 124210.7 & +074037.0 & 2.2 & 0.7 & 176 & 17 & 31.15 & 59 & 0.02 & $\mathrm{SB} 0+: \mathrm{sp}$ & $-0.5 \pm 0.6$ & \\
\hline NGC 4656 & 124357.7 & +321005.3 & 15.1 & 3.0 & 33 & 9.0 & 29.77 & 65 & 0.01 & & $9.0 \pm 0.3$ & \\
\hline NGC 4665 & 124506.0 & +030320.6 & 3.8 & 3.2 & 95 & 17 & 31.15 & 48 & 0.02 & $\mathrm{SB}(\mathrm{s}) 0 / \mathrm{a}$ & $0.0 \pm 0.3$ & \\
\hline NGC 4691 & 124813.6 & -031957.8 & 2.8 & 2.3 & 85 & 16 & 31.04 & 1 & 0.03 & (R) $\mathrm{SB}(\mathrm{s}) 0 / \mathrm{a}$ pec & $0.0 \pm 0.3$ & HII \\
\hline DDO 149 & 124918.3 & -040058.7 & 1.6 & 0.7 & 142 & 22 & 31.69 & 1 & 0.03 & $\mathrm{IB}(\mathrm{s}) \mathrm{m}$ & $10.0 \pm 0.6$ & \\
\hline UGC 07982 & 124950.2 & +025110.4 & 3.4 & 0.7 & 0 & 17 & 31.18 & 1 & 0.03 & $\mathrm{~S}$ & $\cdots$ & \\
\hline UGC 07991 & 125039.0 & +012752.3 & 1.7 & 0.2 & 170 & 19 & 31.37 & 1 & 0.03 & $\mathrm{Sd}$ & $6.7 \pm 0.8$ & \\
\hline NGC 4736 & 125053.1 & +410713.6 & 11.2 & 9.1 & 105 & 5.2 & 28.58 & 15 & 0.02 & (R)SA(r)ab;Sy2 & $2.0 \pm 0.3$ & LINER \\
\hline NGC 4753 & 125222.1 & -011158.9 & 6.0 & 2.8 & 80 & 24 & 31.86 & 15 & 0.03 & I0 & $90.0 \pm 0.0$ & \\
\hline UGC 08013 & 125236.3 & +264459.5 & 1.4 & 0.4 & 97 & 115 & 35.30 & 1 & 0.01 & $\mathrm{Sb}$ & $\cdots$ & \\
\hline NGC 4771 & 125321.2 & +011608.7 & 3.9 & 0.8 & 133 & 17 & 31.15 & 48 & 0.02 & SAd? sp & $7.0 \pm 0.7$ & NLAGN \\
\hline NGC 4772 & 125329.2 & +021006.2 & 3.4 & 1.7 & 147 & 17 & 31.15 & 48 & 0.03 & $\mathrm{SA}(\mathrm{s}) \mathrm{a}$ & $1.0 \pm 0.3$ & LINER \\
\hline DDO 154 & 125405.3 & +270858.7 & 3.0 & 2.2 & 35 & 4.3 & 28.17 & 66 & 0.01 & $\mathrm{IB}(\mathrm{s}) \mathrm{m} \mathrm{IV}-\mathrm{V}$ & $10.0 \pm 0.5$ & \\
\hline NGC 4787 & 125405.5 & +270407.1 & 1.1 & 0.3 & 2 & 111 & 35.22 & 1 & 0.01 & $\mathrm{~S} 0 / \mathrm{a}$ & $0.0 \pm 1.0$ & \\
\hline NGC 4789 & 125419.0 & +270405.0 & 1.9 & 1.5 & 0 & 122 & 35.43 & 1 & 0.01 & SA0: & $-2.3 \pm 0.7$ & \\
\hline NGC 4809 & 125451.1 & +023914.8 & 1.7 & 0.7 & 65 & 14 & 30.70 & 1 & 0.03 & Im pec & $10.0 \pm 0.4$ & HII \\
\hline NGC 4797 & 125455.2 & +272445.7 & 1.2 & 0.9 & 30 & 115 & 35.30 & 1 & 0.01 & S0-: & $-3.0 \pm 1.2$ & \\
\hline NGC 4799 & 125515.5 & +025347.9 & 1.3 & 0.6 & 91 & 41 & 33.05 & 1 & 0.03 & $\mathrm{~S} ?$ & $\cdots$ & \\
\hline NGC 4807 & 125529.1 & +273116.9 & 1.0 & 0.8 & 30 & 102 & 35.05 & 1 & 0.01 & SAB0- pec: & $-3.0 \pm 0.9$ & \\
\hline NGC 4816 & 125612.1 & +274443.9 & 1.3 & 1.1 & 88 & 101 & 35.03 & 1 & 0.01 & S0-: & $-3.0 \pm 1.1$ & \\
\hline NGC 4819 & 125627.8 & +265914.9 & 1.2 & 0.9 & 160 & 95 & 34.88 & 1 & 0.01 & $\left(\mathrm{R}^{\prime}\right) \mathrm{SAB}(\mathrm{r}) \mathrm{a}:$ & $1.0 \pm 0.6$ & \\
\hline NGC 4827 & 125643.5 & +271043.8 & 1.4 & 1.3 & 0 & 111 & 35.23 & 1 & 0.01 & $\mathrm{~S} 0-$ & $-2.5 \pm 0.6$ & \\
\hline MESSIER 064 & 125643.8 & +214051.9 & 10.0 & 5.4 & 115 & 17 & 31.15 & 48 & 0.04 & (R)SA(rs)ab & $2.0 \pm 0.3$ & Sy2 \\
\hline NGC 4839 & 125724.3 & +272951.8 & 4.0 & 1.9 & 65 & 96 & 34.91 & 48 & 0.01 & $\mathrm{cD} ; \mathrm{SAO}$ & $-4.0 \pm 0.5$ & \\
\hline IC 3949 & 125855.8 & +274959.5 & 1.0 & 0.2 & 73 & 111 & 35.22 & 1 & 0.01 & SA0 pec sp & $-2.0 \pm 0.6$ & \\
\hline NGC 4861 & 125902.3 & +345134.0 & 4.0 & 1.5 & 15 & 13 & 30.50 & 35 & 0.01 & $\mathrm{SB}(\mathrm{s}) \mathrm{m}:$ & $11.0 \pm 0.4$ & Sbrst \\
\hline IC 0842 & 130039.7 & +2901 10.0 & 1.2 & 0.6 & 57 & 107 & 35.14 & 1 & 0.01 & $\mathrm{Sb}$ & $\ldots$ & \\
\hline UGC 08127 & 130103.7 & -015712.2 & 1.5 & 0.5 & 17 & 21 & 31.65 & 1 & 0.02 & $\mathrm{IB}(\mathrm{s}) \mathrm{m}:$ & $9.5 \pm 0.9$ & \\
\hline NGC 4922 & 130124.9 & +291840.0 & 1.8 & 1.3 & 0 & 104 & 35.07 & 1 & 0.01 & & $90.0 \pm 0.0$ & \\
\hline IC 0843 & 130133.6 & +290750.0 & 1.1 & 0.6 & 137 & 108 & 35.17 & 1 & 0.01 & So & $-2.0 \pm 0.9$ & \\
\hline IC 4088 & 130143.4 & +290240.3 & 1.5 & 0.5 & 89 & 104 & 35.08 & 1 & 0.02 & $\mathrm{Sab}$ & $2.0 \pm 0.9$ & Sbrst \\
\hline NGC 4952 & 130458.4 & +290720.3 & 1.8 & 1.1 & 23 & 88 & 34.72 & 1 & 0.01 & $\mathrm{E}$ & $-5.0 \pm 0.6$ & \\
\hline
\end{tabular}


Table 1-Continued

\begin{tabular}{|c|c|c|c|c|c|c|c|c|c|c|c|c|}
\hline $\begin{array}{l}\text { Object Name } \\
\text { (1) }\end{array}$ & $\begin{array}{c}\mathrm{RA}_{2000} \\
(\mathrm{~h}: \mathrm{m}: \mathrm{s}) \\
(2)\end{array}$ & $\begin{array}{c}\mathrm{DEC}_{2000} \\
(\mathrm{~d}: \mathrm{m}: \mathrm{s}) \\
(3)\end{array}$ & $\begin{array}{c}2 \times \mathrm{A} \\
(\operatorname{arcmin}) \\
(4)\end{array}$ & $\begin{array}{c}2 \times \mathrm{B} \\
(\operatorname{arcmin}) \\
(5)\end{array}$ & $\begin{array}{c}\text { PA } \\
(\mathrm{deg}) \\
(6)\end{array}$ & $\begin{array}{c}(\mathrm{Mpc}) \\
\quad(7)\end{array}$ & $\begin{array}{l}\text { stance } \\
\text { DM } \\
(8)\end{array}$ & $\begin{array}{l}\text { ref. } \\
(9)\end{array}$ & $\begin{array}{c}\mathrm{E}(B-V) \\
(\mathrm{mag}) \\
(10)\end{array}$ & $\begin{array}{c}\text { Morphological } \\
\text { Type } \\
(11)\end{array}$ & $\begin{array}{c}\mathrm{T} \\
\text { Type } \\
(12)\end{array}$ & $\begin{array}{c}\text { Spectral } \\
\text { Type } \\
(13)\end{array}$ \\
\hline UGC 08195 & 130622.2 & +293926.5 & 1.2 & 0.1 & 88 & 103 & 35.07 & 1 & 0.01 & $\mathrm{Sd}$ & $8.0 \pm 1.4$ & Sbrst \\
\hline DDO 165 & 130624.9 & +674225.0 & 3.5 & 1.9 & 90 & 4.6 & 28.30 & 34 & 0.02 & $\operatorname{Im}$ & $10.0 \pm 0.7$ & \\
\hline NGC 5004 & 131101.6 & +293812.0 & 1.4 & 1.1 & 170 & 103 & 35.07 & 1 & 0.01 & S0 & $-2.0 \pm 0.8$ & \\
\hline NGC 5004C & 131101.7 & +293441.8 & 1.4 & 0.8 & 172 & 106 & 35.13 & 1 & 0.01 & SBab & $2.0 \pm 0.8$ & Sbrst \\
\hline UGC 08313 & 131353.9 & +421231.0 & 1.7 & 0.4 & 35 & 12 & 30.42 & 1 & 0.01 & $\mathrm{SB}(\mathrm{s}) \mathrm{c} ? \mathrm{sp}$ & $5.0 \pm 0.9$ & \\
\hline UGCA 342 & 131506.7 & +420005.0 & 1.6 & 0.6 & 95 & 8.2 & 29.57 & 67 & 0.02 & $\operatorname{Im}$ & $10.0 \pm 0.9$ & \\
\hline NGC 5055 & 131549.3 & +420145.4 & 12.6 & 7.2 & 105 & 8.2 & 29.57 & 3 & 0.02 & $\mathrm{SA}(\mathrm{rs}) \mathrm{bc} ; \mathrm{HII}$ & $4.0 \pm 0.3$ & LINER \\
\hline UGC 08340 & 131635.5 & -020528.8 & 1.0 & 1.0 & $\ldots$ & 81 & 34.55 & 1 & 0.03 & Scd? & $6.0 \pm 1.7$ & \\
\hline IC 4218 & 131703.4 & $-02 \quad 1540.6$ & 1.3 & 0.3 & 158 & 84 & 34.61 & 1 & 0.03 & $\mathrm{Sa}$ & $\cdots$ & Sy1 \\
\hline UGC 08365 & 131845.2 & +415659.0 & 2.3 & 1.4 & 115 & 21 & 31.56 & 1 & 0.01 & $\mathrm{SB}(\mathrm{s}) \mathrm{d}$ & $7.0 \pm 0.8$ & \\
\hline IC 4229 & 132226.1 & -022505.8 & 1.0 & 0.7 & 115 & 100 & 35.00 & 1 & 0.03 & $\left(\mathrm{R}^{\prime}\right) \mathrm{SB}(\mathrm{r}) \mathrm{b}$ pec: & $3.0 \pm 1.2$ & \\
\hline Centaurus A & 132527.6 & -430108.8 & 25.7 & 20.0 & 25 & 3.8 & 27.92 & 68 & 0.12 & So pec & $\ldots$ & Sy2 \\
\hline NGC 5169 & 132810.0 & +464019.6 & 2.3 & 0.9 & 103 & 38 & 32.91 & 1 & 0.04 & $\mathrm{SB}(\mathrm{rs}) \mathrm{b}:$ & $3.0 \pm 0.6$ & \\
\hline NGC 5173 & 132825.3 & +463529.6 & 1.8 & 1.7 & 0 & 38 & 32.90 & 1 & 0.03 & E0: & $-5.0 \pm 0.4$ & \\
\hline IC 4263 & 132833.3 & +465536.2 & 2.0 & 0.4 & 105 & 38 & 32.91 & 69 & 0.03 & $\mathrm{SB}(\mathrm{s}) \mathrm{d}: \mathrm{sp}$ & $6.7 \pm 0.7$ & \\
\hline MESSIER 051a & 132952.7 & +471142.6 & 11.2 & 6.9 & 163 & 8.4 & 29.62 & 70 & 0.04 & SA(s)bc pec;HII & $4.0 \pm 0.3$ & Sy2.5 \\
\hline MESSIER 051b & 132959.6 & +471558.1 & 5.8 & 4.6 & 79 & 8.4 & 29.62 & 71 & 0.04 & SB0-1 pec & $90.0 \pm 0.0$ & LINER \\
\hline NGC 5231 & 133548.2 & +025956.1 & 1.1 & 1.0 & 0 & 94 & 34.87 & 1 & 0.02 & $\mathrm{SBa}$ & $1.0 \pm 0.8$ & \\
\hline ESO 444-G077 & 133551.9 & -302548.3 & 1.1 & 0.9 & 0 & 54 & 33.65 & 1 & 0.05 & $\mathrm{~S} ?$ & $\ldots$ & \\
\hline MESSIER 083 & 133700.9 & -295156.7 & 12.9 & 11.5 & 0 & 4.5 & 28.27 & 72 & 0.07 & $\mathrm{SAB}(\mathrm{s}) \mathrm{c} ; \mathrm{HII}$ & $5.0 \pm 0.3$ & Sbrst \\
\hline ESO 444-G087 & 133812.0 & $\begin{array}{lll}-31 & 25 & 01.1\end{array}$ & 1.2 & 0.9 & 102 & 61 & 33.92 & 1 & 0.06 & $\left(\mathrm{R}^{\prime}\right) \mathrm{SB}(\mathrm{r}) \mathrm{ab}$ & $1.0 \pm 0.9$ & \\
\hline NGC 5253 & 133956.0 & -313824.4 & 5.0 & 1.9 & 45 & 3.1 & 27.48 & 43 & 0.06 & Im pec;HII & $10.0 \pm 0.7$ & Sbrst \\
\hline UGC 08650 & 134012.6 & +022848.4 & 1.9 & 0.3 & 151 & 100 & 34.99 & 1 & 0.03 & $\mathrm{Sb}$ pec sp & $3.0 \pm 0.8$ & \\
\hline ESO 445-G007 & 134021.9 & -314204.4 & 1.3 & 0.7 & 0 & 22 & 31.74 & 1 & 0.05 & $\mathrm{IB}(\mathrm{s}) \mathrm{m}$ & $10.0 \pm 0.9$ & \\
\hline NGC 5329 & 135210.1 & +021930.2 & 1.3 & 1.3 & $\ldots$ & 103 & 35.05 & 1 & 0.03 & $\mathrm{E}:$ & $-4.7 \pm 0.6$ & \\
\hline UGC 08787 & 135250.1 & +021549.2 & 1.7 & 0.5 & 146 & 63 & 34.00 & 1 & 0.03 & Sbc: sp & $4.0 \pm 0.7$ & \\
\hline IC 0952 & 135341.9 & +032238.6 & 1.3 & 0.4 & 93 & 67 & 34.14 & 1 & 0.03 & $\mathrm{SBbc}$ & $4.0 \pm 0.9$ & \\
\hline UGC 08816 & 135403.8 & +035704.9 & 1.1 & 0.5 & 25 & 67 & 34.13 & 1 & 0.03 & Scd: & $6.0 \pm 1.3$ & \\
\hline NGC 5398 & 140121.6 & -330349.6 & 2.8 & 1.7 & 172 & 16 & 31.01 & 1 & 0.07 & $\mathrm{SB}(\mathrm{rs}) \mathrm{dm}$ & $8.1 \pm 0.3$ & \\
\hline MESSIER 101 & 140312.6 & +542056.7 & 28.8 & 26.9 & 90 & 7.5 & 29.38 & 73 & 0.01 & $\mathrm{SAB}(\mathrm{rs}) \mathrm{cd}$ & $6.0 \pm 0.3$ & \\
\hline ESO 446-G002 & 140342.7 & -324300.7 & 1.6 & 0.3 & 155 & 54 & 33.67 & 1 & 0.07 & Sab: sp & $2.0 \pm 1.3$ & \\
\hline UGC 08986 & 140415.9 & +040643.3 & 1.4 & 1.4 & $\ldots$ & 19 & 31.36 & 1 & 0.03 & S0? & $-2.0 \pm 1.6$ & \\
\hline NGC 5474 & $\begin{array}{lll}14 & 05 & 01.6\end{array}$ & +533944.0 & 4.8 & 4.3 & 0 & 6.8 & 29.16 & 38 & 0.01 & $\mathrm{SA}(\mathrm{s}) \mathrm{cd}$ pec & $6.0 \pm 0.3$ & HII \\
\hline
\end{tabular}


Table 1-Continued

\begin{tabular}{|c|c|c|c|c|c|c|c|c|c|c|c|c|}
\hline $\begin{array}{l}\text { Object Name } \\
\text { (1) }\end{array}$ & $\begin{array}{c}\mathrm{RA}_{2000} \\
(\mathrm{~h}: \mathrm{m}: \mathrm{s}) \\
(2)\end{array}$ & $\begin{array}{c}\mathrm{DEC}_{2000} \\
(\mathrm{~d}: \mathrm{m}: \mathrm{s}) \\
(3)\end{array}$ & $\begin{array}{c}2 \times \mathrm{A} \\
(\operatorname{arcmin}) \\
(4)\end{array}$ & $\begin{array}{c}2 \times \mathrm{B} \\
(\operatorname{arcmin}) \\
(5)\end{array}$ & $\begin{array}{c}\text { PA } \\
(\text { deg }) \\
(6)\end{array}$ & $\begin{array}{c}(\mathrm{Mpc}) \\
(7)\end{array}$ & $\begin{array}{c}\text { istance } \\
\text { DM } \\
(8)\end{array}$ & $\begin{array}{l}\text { ref. } \\
(9)\end{array}$ & $\begin{array}{c}\mathrm{E}(B-V) \\
(\mathrm{mag}) \\
(10)\end{array}$ & $\begin{array}{c}\text { Morphological } \\
\text { Type } \\
(11)\end{array}$ & $\begin{array}{c}\mathrm{T} \\
\text { Type } \\
(12)\end{array}$ & $\begin{array}{c}\text { Spectral } \\
\text { Type } \\
(13)\end{array}$ \\
\hline NGC 5477 & 140533.2 & +542738.7 & 1.7 & 1.3 & 95 & 7.7 & 29.43 & 55 & 0.01 & $\mathrm{SA}(\mathrm{s}) \mathrm{m}$ & $9.0 \pm 0.4$ & \\
\hline UGC 09120 & 141512.3 & +044926.9 & 1.1 & 0.8 & 0 & 83 & 34.60 & 1 & 0.03 & Scd: & $6.0 \pm 1.2$ & \\
\hline UGC 09140 & 141721.1 & +010752.4 & 1.0 & 0.2 & 107 & 231 & 36.82 & 1 & 0.04 & Scd: & $6.0 \pm 1.4$ & NLAGN \\
\hline NGC 5560 & 142005.4 & +03 5928.4 & 3.7 & 0.7 & 115 & 26 & 32.07 & 1 & 0.03 & $\mathrm{SB}(\mathrm{s}) \mathrm{b}$ pec & $3.0 \pm 0.4$ & \\
\hline NGC 5566 & 142020.0 & +035600.9 & 6.6 & 2.2 & 35 & 23 & 31.79 & 1 & 0.03 & $\mathrm{SB}(\mathrm{r}) \mathrm{ab}$ & $2.0 \pm 0.3$ & LINER \\
\hline NGC 5569 & 142032.1 & +035859.6 & 1.7 & 1.4 & 85 & 27 & 32.12 & 1 & 0.03 & $\mathrm{SAB}(\mathrm{rs}) \mathrm{cd}$ : & $6.0 \pm 0.5$ & \\
\hline NGC 5574 & 142056.0 & +031416.8 & 1.6 & 1.0 & 63 & 25 & 31.98 & 1 & 0.03 & SB0-? sp & $-3.0 \pm 0.9$ & \\
\hline NGC 5576 & 142103.7 & +031615.6 & 3.5 & 2.2 & 95 & 22 & 31.75 & 1 & 0.03 & E3 & $-5.0 \pm 0.3$ & \\
\hline NGC 5577 & 142113.1 & +032608.8 & 3.4 & 1.0 & 56 & 22 & 31.75 & 1 & 0.04 & $\mathrm{SA}(\mathrm{rs}) \mathrm{bc}:$ & $4.0 \pm 0.5$ & \\
\hline UGC 09215 & 142327.1 & +014334.7 & 2.2 & 1.3 & 165 & 21 & 31.60 & 1 & 0.03 & $\mathrm{SB}(\mathrm{s}) \mathrm{d}$ & $6.5 \pm 0.5$ & \\
\hline NGC 5619 & 142718.2 & +044810.2 & 2.2 & 1.2 & 8 & 121 & 35.41 & 1 & 0.03 & $\mathrm{SAB}(\mathrm{rs}) \mathrm{b}$ & $3.0 \pm 0.8$ & \\
\hline UGC 09277 & 142833.3 & +031543.2 & 1.4 & 0.2 & 45 & 124 & 35.47 & 1 & 0.03 & $\mathrm{Sb}$ & $3.0 \pm 0.9$ & \\
\hline UGC 09285 & 142903.9 & +030856.5 & 1.3 & 0.3 & 87 & 28 & 32.23 & 1 & 0.03 & Scd? & $6.0 \pm 1.9$ & \\
\hline NGC 5646 & 142934.1 & +352742.2 & 1.5 & 0.4 & 81 & 126 & 35.50 & 1 & 0.02 & SBb: & $3.0 \pm 0.9$ & \\
\hline NGC 5636 & 142939.0 & +031558.7 & 1.9 & 1.4 & 40 & 26 & 32.08 & 1 & 0.03 & $\mathrm{SAB}(\mathrm{r}) 0+$ & $-1.0 \pm 0.4$ & \\
\hline NGC 5638 & 142940.4 & +031400.2 & 2.7 & 2.4 & 150 & 26 & 32.10 & 15 & 0.03 & $\mathrm{E} 1$ & $-5.0 \pm 0.3$ & \\
\hline UGC 09305 & 142940.5 & +040344.5 & 1.0 & 0.2 & 164 & 35 & 32.72 & 1 & 0.03 & Sdm: & $8.0 \pm 1.4$ & \\
\hline UGC 09310 & 143001.1 & +031314.0 & 2.0 & 0.6 & 163 & 28 & 32.20 & 1 & 0.03 & SBdm & $8.0 \pm 0.8$ & \\
\hline IC 1022 & 143001.8 & +034622.3 & 1.1 & 0.4 & 161 & 26 & 32.06 & 1 & 0.03 & $\mathrm{~S} ?$ & $\ldots$ & \\
\hline NGC 5656 & 143025.4 & +351914.6 & 1.9 & 1.5 & 50 & 48 & 33.42 & 1 & 0.01 & SAab & $2.0 \pm 0.8$ & LINER \\
\hline UGC 09338 & 143111.7 & +051826.4 & 1.0 & 0.4 & 49 & 119 & 35.38 & 1 & 0.04 & $\mathrm{Sb}$ & $3.0 \pm 0.9$ & \\
\hline IC 1024 & 143127.2 & +030032.7 & 1.6 & 0.6 & 30 & 22 & 31.71 & 1 & 0.03 & $\mathrm{~S} 0 ?$ & $-2.0 \pm 1.7$ & \\
\hline UGC 09380 & 143439.2 & +041546.0 & 1.9 & 1.0 & 0 & 25 & 32.03 & 1 & 0.03 & LSBG;Im & $10.0 \pm 0.8$ & HII \\
\hline UGC 09382 & 143505.4 & +033306.3 & 1.2 & 0.2 & 166 & 126 & 35.50 & 1 & 0.04 & $\mathrm{Sab}$ & $2.0 \pm 1.0$ & \\
\hline UGC 09432 & 143904.3 & +025657.0 & 1.2 & 0.8 & 45 & 24 & 31.87 & 1 & 0.03 & $\mathrm{Im}:$ & $10.0 \pm 1.1$ & \\
\hline NGC 5701 & 143911.1 & +052148.8 & 4.3 & 4.1 & 85 & 23 & 31.80 & 1 & 0.04 & (R)SB(rs)0/a & $0.0 \pm 0.3$ & LINER \\
\hline NGC 5705 & 143949.7 & -004306.4 & 2.9 & 1.7 & 75 & 27 & 32.15 & 74 & 0.04 & $\mathrm{SB}(\mathrm{rs}) \mathrm{d}$ & $7.3 \pm 0.4$ & \\
\hline NGC 5713 & 144011.5 & -00 1721.2 & 2.8 & 2.5 & 10 & 27 & 32.15 & 75 & 0.04 & $\mathrm{SAB}(\mathrm{rs}) \mathrm{bc}$ pec & $4.0 \pm 0.3$ & HII \\
\hline NGC 5727 & 144026.2 & +335920.9 & 2.2 & 1.2 & 135 & 24 & 31.93 & 1 & 0.01 & SABdm & $8.0 \pm 0.8$ & \\
\hline NGC 5719 & 144056.4 & -00 1905.4 & 3.2 & 1.2 & 107 & 27 & 32.15 & 75 & 0.04 & $\mathrm{SAB}(\mathrm{s}) \mathrm{ab}$ pec & $2.0 \pm 0.3$ & NLAGN \\
\hline UGC 09463 & 144059.3 & +0308 13.5 & 1.0 & 0.2 & 42 & 115 & 35.31 & 1 & 0.03 & $\mathrm{~S} 0 / \mathrm{a}$ & $0.0 \pm 1.0$ & \\
\hline UGC 09479 & 144232.6 & +042549.6 & 1.5 & 0.4 & 27 & 118 & 35.36 & 1 & 0.03 & $\mathrm{Sb}$ & $3.0 \pm 0.9$ & \\
\hline UGC 09491 & 144414.7 & +041306.5 & 1.3 & 0.9 & 50 & 111 & 35.23 & 1 & 0.03 & $\mathrm{~S} ?$ & $\ldots$ & \\
\hline
\end{tabular}


Table 1-Continued

\begin{tabular}{|c|c|c|c|c|c|c|c|c|c|c|c|c|}
\hline $\begin{array}{l}\text { Object Name } \\
\text { (1) }\end{array}$ & $\begin{array}{c}\mathrm{RA}_{2000} \\
(\mathrm{~h}: \mathrm{m}: \mathrm{s}) \\
(2)\end{array}$ & $\begin{array}{c}\mathrm{DEC}_{2000} \\
(\mathrm{~d}: \mathrm{m}: \mathrm{s}) \\
(3)\end{array}$ & $\begin{array}{c}2 \times \mathrm{A} \\
(\operatorname{arcmin}) \\
(4)\end{array}$ & $\begin{array}{c}2 \times \mathrm{B} \\
(\operatorname{arcmin}) \\
(5)\end{array}$ & $\begin{array}{c}\text { PA } \\
(\mathrm{deg}) \\
(6)\end{array}$ & $\begin{array}{c}\text { (Mpc) } \\
(7)\end{array}$ & $\begin{array}{l}\text { stance } \\
\text { DM } \\
(8)\end{array}$ & $\begin{array}{l}\text { ref. } \\
(9)\end{array}$ & $\begin{array}{c}\mathrm{E}(B-V) \\
(\mathrm{mag}) \\
(10)\end{array}$ & $\begin{array}{c}\text { Morphological } \\
\text { Type } \\
\text { (11) }\end{array}$ & $\begin{array}{c}\mathrm{T} \\
\text { Type } \\
(12)\end{array}$ & $\begin{array}{c}\text { Spectral } \\
\text { Type } \\
(13)\end{array}$ \\
\hline IC 1063 & 145211.0 & +044055.4 & 1.3 & 1.1 & 162 & 201 & 36.51 & 1 & 0.04 & $\mathrm{SBb}$ & $3.0 \pm 0.9$ & \\
\hline NGC 5770 & 145315.0 & +035735.0 & 1.7 & 1.3 & 0 & 23 & 31.77 & 1 & 0.04 & SB0 & $-2.0 \pm 0.8$ & \\
\hline IC 1071 & 145412.5 & +044500.1 & 1.0 & 0.8 & 150 & 120 & 35.40 & 1 & 0.04 & So & $-2.0 \pm 0.9$ & \\
\hline UGC 09584 & 145415.3 & +043051.3 & 1.0 & 0.4 & 142 & 122 & 35.43 & 1 & 0.04 & $\mathrm{Sb}$ & $3.0 \pm 0.9$ & \\
\hline NGC 5832 & 145745.7 & +714056.4 & 3.7 & 2.2 & 45 & 11 & 30.13 & 1 & 0.03 & $\mathrm{SB}(\mathrm{rs}) \mathrm{b} ?$ & $3.0 \pm 0.6$ & \\
\hline NGC 5806 & 150000.4 & +015328.7 & 3.1 & 1.6 & 170 & 21 & 31.57 & 1 & 0.05 & $\mathrm{SAB}(\mathrm{s}) \mathrm{b}$ & $3.0 \pm 0.3$ & NLAGN \\
\hline NGC 5813 & 150111.3 & +014207.1 & 4.2 & 3.0 & 145 & 29 & 32.31 & 1 & 0.06 & E1-2 & $-5.0 \pm 0.3$ & \\
\hline UGC 09661 & 150203.5 & +015028.2 & 1.4 & 0.5 & 65 & 19 & 31.38 & 1 & 0.06 & $\mathrm{SB}(\mathrm{rs}) \mathrm{dm}$ & $8.0 \pm 0.6$ & \\
\hline NGC 5866 & 150629.6 & +554547.9 & 4.7 & 1.9 & 128 & 15 & 30.93 & 15 & 0.01 & S0-3;HII & $-1.0 \pm 0.3$ & LINER \\
\hline NGC 5826 & 150633.8 & +552844.8 & 1.2 & 0.9 & 25 & 16 & 30.98 & 1 & 0.01 & S0? & $-2.0 \pm 1.7$ & \\
\hline IC 1102 & 151104.9 & +041737.9 & 1.1 & 0.6 & 25 & 181 & 36.29 & 1 & 0.04 & Sb: & $3.0 \pm 1.3$ & Sy1 \\
\hline NGC 5894 & 151141.0 & +594832.1 & 3.0 & 0.4 & 13 & 39 & 32.98 & 1 & 0.01 & SBdm? & $8.0 \pm 1.2$ & \\
\hline IRAS $15250+3609$ & 152659.4 & +355837.5 & 0.6 & 0.5 & 0 & 239 & 36.89 & 1 & 0.02 & Ring galaxy & $\cdots$ & LINER \\
\hline UGC 09912 & 153510.5 & +163258.0 & 1.7 & 1.7 & $\cdots$ & 17 & 31.15 & 1 & 0.05 & SBdm & $8.0 \pm 0.8$ & \\
\hline NGC 5962 & 153631.7 & +163628.3 & 3.0 & 2.1 & 110 & 30 & 32.40 & 1 & 0.05 & $\mathrm{SA}(\mathrm{r}) \mathrm{c}$ & $5.0 \pm 0.3$ & HII \\
\hline UGC 09925 & 153632.0 & +162623.0 & 1.4 & 0.8 & 10 & 30 & 32.36 & 1 & 0.05 & Scd: & $6.0 \pm 1.2$ & \\
\hline NGC 5972 & 153854.1 & +170134.4 & 1.0 & 0.7 & 5 & 130 & 35.56 & 1 & 0.04 & $\mathrm{~S} 0 / \mathrm{a}$ & $0.0 \pm 0.9$ & Sy2 \\
\hline UGC 09953 & 153939.4 & +031156.5 & 1.2 & 0.8 & 150 & 146 & 35.82 & 1 & 0.06 & SBcd: & $6.0 \pm 1.2$ & \\
\hline UGC 10043 & 154841.2 & +215209.8 & 2.4 & 0.4 & 151 & 33 & 32.62 & 1 & 0.06 & $\mathrm{Sbc}$ & $4.0 \pm 0.9$ & \\
\hline UGC 10109 & 155721.0 & +470959.3 & 1.4 & 0.7 & 145 & 86 & 34.68 & 1 & 0.02 & $\mathrm{SAB}(\mathrm{r}) \mathrm{b}$ & $3.0 \pm 0.9$ & \\
\hline UGC 10153 & 160319.5 & +203812.8 & 1.2 & 0.9 & 125 & 178 & 36.25 & 1 & 0.07 & $\mathrm{SB}(\mathrm{r}) \mathrm{b}$ & $3.0 \pm 0.9$ & \\
\hline NGC 6036 & 160430.7 & +035206.7 & 1.1 & 0.4 & 146 & 80 & 34.52 & 1 & 0.11 & So/a & $0.0 \pm 0.9$ & \\
\hline NGC 6052 & 160512.9 & +203232.0 & 0.9 & 0.7 & 0 & 70 & 34.22 & 1 & 0.08 & & $5.0 \pm 0.5$ & \\
\hline UGC 10197 & 160604.4 & +204805.4 & 1.2 & 0.5 & 42 & 71 & 34.25 & 1 & 0.15 & SAd & $7.0 \pm 0.9$ & \\
\hline UGC 10198 & 160605.9 & +204703.3 & 1.1 & 0.6 & 172 & 69 & 34.18 & 1 & 0.15 & $\mathrm{Sdm}$ & $8.0 \pm 0.9$ & \\
\hline UGC 10245 & 160951.3 & +491000.3 & 1.1 & 0.2 & 74 & 194 & 36.44 & 1 & 0.02 & $\mathrm{Sbc}$ & $4.0 \pm 1.0$ & \\
\hline CGCG 023-019 & 161014.6 & +010320.5 & 1.5 & 1.0 & 155 & 121 & 35.41 & 1 & 0.13 & $\mathrm{SAB}(\mathrm{s}) \mathrm{dm}:$ & $8.0 \pm 1.2$ & \\
\hline UGC 10261 & 161104.0 & +522701.0 & 1.1 & 0.8 & 170 & 276 & 37.20 & 1 & 0.02 & S0-: & $-3.0 \pm 1.2$ & \\
\hline NGC 6090 & 161140.7 & +522724.0 & 1.7 & 0.7 & 0 & 129 & 35.56 & 1 & 0.02 & & $\ldots$ & \\
\hline UGC 10278 & $16 \quad 12 \quad 18.9$ & +492353.5 & 1.0 & 0.5 & 170 & 59 & 33.86 & 1 & 0.02 & $\mathrm{Sab}$ & $2.0 \pm 0.9$ & \\
\hline NGC 6100 & 161652.4 & +005028.7 & 1.9 & 1.1 & 120 & 71 & 34.24 & 1 & 0.09 & (R)SAB(r)a: & $0.7 \pm 0.7$ & \\
\hline IC 4595 & 162044.4 & -700833.4 & 2.7 & 0.5 & 60 & 45 & 33.29 & 1 & 0.11 & Sc: $\mathrm{sp}$ & $5.0 \pm 1.2$ & \\
\hline NGC 6154 & 162530.5 & +495024.9 & 2.1 & 2.0 & 0 & 90 & 34.77 & 1 & 0.02 & $\mathrm{SB}(\mathrm{r}) \mathrm{a}$ & $1.0 \pm 0.7$ & \\
\hline
\end{tabular}


Table 1-Continued

\begin{tabular}{|c|c|c|c|c|c|c|c|c|c|c|c|c|}
\hline $\begin{array}{c}\text { Object Name } \\
\text { (1) }\end{array}$ & $\begin{array}{c}\mathrm{RA}_{2000} \\
(\mathrm{~h}: \mathrm{m}: \mathrm{s}) \\
(2)\end{array}$ & $\begin{array}{c}\mathrm{DEC}_{2000} \\
(\mathrm{~d}: \mathrm{m}: \mathrm{s}) \\
(3)\end{array}$ & $\begin{array}{c}2 \times \mathrm{A} \\
(\operatorname{arcmin}) \\
(4)\end{array}$ & $\begin{array}{c}2 \times \mathrm{B} \\
(\operatorname{arcmin}) \\
(5)\end{array}$ & $\begin{array}{c}\text { PA } \\
(\operatorname{deg}) \\
(6)\end{array}$ & $\begin{array}{c}(\mathrm{Mpc}) \\
(7)\end{array}$ & $\begin{array}{c}\text { istance } \\
\text { DM } \\
(8)\end{array}$ & $\begin{array}{l}\text { ref. } \\
(9)\end{array}$ & $\begin{array}{c}\mathrm{E}(B-V) \\
(\mathrm{mag}) \\
(10)\end{array}$ & $\begin{array}{c}\text { Morphological } \\
\text { Type } \\
(11)\end{array}$ & $\begin{array}{c}\mathrm{T} \\
\text { Type } \\
(12)\end{array}$ & $\begin{array}{c}\text { Spectral } \\
\text { Type } \\
(13)\end{array}$ \\
\hline NGC 6155 & 162608.3 & +482200.5 & 1.3 & 0.9 & 145 & 38 & 32.92 & 1 & 0.01 & $\mathrm{~S} ?$ & $\ldots$ & \\
\hline UGC 10404 & 162811.6 & +394918.8 & 1.4 & 1.1 & 160 & 119 & 35.37 & 1 & 0.01 & $\mathrm{SB} ?$ & $\ldots$ & \\
\hline NGC 6166 & 162838.5 & +393305.6 & 1.9 & 1.4 & 35 & 137 & 35.68 & 1 & 0.01 & $\mathrm{cD} ; \mathrm{E}$ & $-4.0 \pm 0.4$ & NLRG \\
\hline UGC 10420 & 162951.0 & +394559.4 & 1.7 & 1.2 & 155 & 138 & 35.69 & 1 & 0.01 & $\mathrm{SB}(\mathrm{r}) \mathrm{b}$ & $3.0 \pm 0.8$ & \\
\hline UGC 10445 & 163347.6 & +285905.7 & 2.8 & 1.7 & 145 & 17 & 31.12 & 1 & 0.03 & $\mathrm{SBc}$ & $6.0 \pm 1.5$ & Sbrst \\
\hline IC 1221 & 163441.6 & +462331.8 & 1.3 & 1.1 & 0 & 81 & 34.55 & 1 & 0.02 & $\mathrm{Sd}$ & $7.0 \pm 0.8$ & \\
\hline IC 1222 & 163509.1 & +461251.0 & 1.7 & 1.3 & 50 & 136 & 35.66 & 1 & 0.02 & $\mathrm{SAB}(\mathrm{s}) \mathrm{c}$ & $5.0 \pm 0.4$ & \\
\hline UGC 10468 & 163606.8 & +443609.9 & 1.0 & 0.1 & 176 & 134 & 35.64 & 1 & 0.01 & Scd: & $6.0 \pm 1.4$ & NLAGN \\
\hline UGC 10491 & 163814.0 & +415619.9 & 1.1 & 0.4 & 135 & 120 & 35.39 & 1 & 0.02 & & $10.0 \pm 0.3$ & \\
\hline NGC 6239 & 165005.0 & +424422.9 & 2.6 & 1.1 & 118 & 17 & 31.14 & 1 & 0.02 & $\mathrm{SB}(\mathrm{s}) \mathrm{b}$ pec? & $3.0 \pm 0.7$ & HII \\
\hline Mrk 501 & 165352.2 & +394536.6 & 1.2 & 1.0 & 160 & 148 & 35.85 & 1 & 0.02 & $\mathrm{E} ?$ & $\ldots$ & BLLAC \\
\hline UGC 10600 & 165420.8 & +360049.1 & 1.0 & 0.1 & 50 & 139 & 35.72 & 1 & 0.02 & $\mathrm{~S} ?$ & $\ldots$ & \\
\hline NGC 6255 & 165448.0 & +363004.0 & 3.6 & 1.5 & 85 & 17 & 31.09 & 1 & 0.02 & SBcd: & $6.0 \pm 1.0$ & \\
\hline UGC 10651 & 165944.1 & +423222.4 & 1.1 & 0.9 & 0 & 121 & 35.42 & 1 & 0.03 & $\mathrm{~S} ?$ & $\ldots$ & \\
\hline UGC 10687 & 170327.4 & +594332.0 & 1.0 & 0.7 & 35 & 79 & 34.50 & 1 & 0.02 & $\mathrm{SBd}$ & $7.0 \pm 0.9$ & \\
\hline UGC 10713 & 170433.9 & +722647.5 & 1.8 & 0.3 & 8 & 20 & 31.46 & 1 & 0.04 & $\mathrm{Sb}$ & $3.0 \pm 0.9$ & HII \\
\hline NGC 6306 & 170736.9 & +604343.3 & 1.0 & 0.3 & 166 & 47 & 33.34 & 1 & 0.02 & SB(s)ab pec: & $2.0 \pm 0.7$ & \\
\hline NGC 6307 & 170740.5 & +604503.0 & 1.3 & 1.0 & 145 & 48 & 33.40 & 1 & 0.02 & $\left(\mathrm{R}^{\prime}\right) \mathrm{SB}(\mathrm{s}) 0 / \mathrm{a}$ & $0.0 \pm 0.4$ & \\
\hline UGC 10729 & 170917.0 & +313659.4 & 1.3 & 0.3 & 128 & 134 & 35.63 & 1 & 0.04 & Sdm: & $8.0 \pm 1.3$ & \\
\hline IC 1251 & 171013.2 & +722438.5 & 1.4 & 1.0 & 70 & 21 & 31.65 & 76 & 0.05 & Scd: & $6.0 \pm 1.2$ & \\
\hline NGC 6340 & 171024.9 & +721815.8 & 3.2 & 3.0 & 120 & 21 & 31.65 & 1 & 0.05 & $\mathrm{SA}(\mathrm{s}) 0 / \mathrm{a}$ & $0.0 \pm 0.3$ & LINER \\
\hline IC 1254 & 171133.4 & +722407.2 & 1.6 & 0.7 & 32 & 23 & 31.77 & 1 & 0.05 & $\mathrm{Sb} ?$ pec & $3.0 \pm 1.7$ & \\
\hline IC 1248 & 171140.2 & +595944.2 & 1.3 & 1.2 & 0 & 76 & 34.40 & 1 & 0.02 & $\mathrm{SB}(\mathrm{r}) \mathrm{c}$ & $5.0 \pm 0.6$ & \\
\hline UGC 10770 & $\begin{array}{lll}17 & 13 & 07.0\end{array}$ & +591924.0 & 1.2 & 0.6 & 15 & 20 & 31.50 & 1 & 0.02 & & $10.0 \pm 0.5$ & \\
\hline UGC 10791 & 171438.7 & +722356.0 & 1.5 & 1.2 & 100 & 23 & 31.83 & 1 & 0.05 & Sm: & $9.0 \pm 1.0$ & \\
\hline NGC 6330 & 171544.4 & +292415.5 & 1.4 & 0.5 & 160 & 127 & 35.52 & 1 & 0.05 & $\mathrm{SBb}$ & $3.0 \pm 0.9$ & \\
\hline UGC 10783 & 171629.3 & +292646.5 & 1.2 & 0.5 & 130 & 133 & 35.61 & 1 & 0.05 & $\mathrm{~S} ?$ & $\ldots$ & \\
\hline UGC 10796 & $1716 \quad 47.7$ & +615512.4 & 1.6 & 1.2 & 20 & 48 & 33.41 & 1 & 0.02 & $\mathrm{SB}(\mathrm{s}) \mathrm{b}$ & $3.0 \pm 0.8$ & \\
\hline NGC 6359 & 171753.0 & +614650.8 & 1.2 & 0.9 & 145 & 46 & 33.34 & 1 & 0.02 & SA0-: & $-2.5 \pm 0.6$ & \\
\hline UGC 10795 & 171805.6 & +305528.0 & 1.4 & 0.8 & 70 & 67 & 34.12 & 1 & 0.04 & Im: & $10.0 \pm 1.2$ & \\
\hline NGC 6361 & 171841.1 & +603629.2 & 2.2 & 0.6 & 54 & 59 & 33.84 & 1 & 0.03 & SAb: sp & $3.0 \pm 0.4$ & NLAGN \\
\hline UGC 10811 & 171843.7 & +580806.5 & 1.6 & 0.5 & 93 & 129 & 35.55 & 1 & 0.03 & SBab? & $2.0 \pm 0.9$ & \\
\hline NGC 6373 & 172408.1 & +585942.3 & 1.3 & 1.0 & 90 & 52 & 33.56 & 1 & 0.03 & $\mathrm{SAB}(\mathrm{s}) \mathrm{c}$ & $5.0 \pm 0.8$ & \\
\hline
\end{tabular}


Table 1-Continued

\begin{tabular}{|c|c|c|c|c|c|c|c|c|c|c|c|c|}
\hline $\begin{array}{l}\text { Object Name } \\
\text { (1) }\end{array}$ & $\begin{array}{c}\mathrm{RA}_{2000} \\
(\mathrm{~h}: \mathrm{m}: \mathrm{s}) \\
(2)\end{array}$ & $\begin{array}{c}\mathrm{DEC}_{2000} \\
(\mathrm{~d}: \mathrm{m}: \mathrm{s}) \\
(3)\end{array}$ & $\begin{array}{c}2 \times \mathrm{A} \\
(\operatorname{arcmin}) \\
(4)\end{array}$ & $\begin{array}{c}2 \times \mathrm{B} \\
(\operatorname{arcmin}) \\
(5)\end{array}$ & $\begin{array}{c}\text { PA } \\
(\operatorname{deg}) \\
(6)\end{array}$ & $\begin{array}{c}(\mathrm{Mpc}) \\
(7)\end{array}$ & $\begin{array}{l}\text { istance } \\
\text { DM } \\
(8)\end{array}$ & $\begin{array}{l}\text { ref. } \\
(9)\end{array}$ & $\begin{array}{c}\mathrm{E}(B-V) \\
(\mathrm{mag}) \\
(10)\end{array}$ & $\begin{array}{l}\text { Morphological } \\
\text { Type } \\
\text { (11) }\end{array}$ & $\begin{array}{c}\mathrm{T} \\
\text { Type } \\
(12)\end{array}$ & $\begin{array}{c}\text { Spectral } \\
\text { Type } \\
(13)\end{array}$ \\
\hline NGC 6364 & 172427.3 & +292324.6 & 1.5 & 1.2 & 5 & 101 & 35.02 & 1 & 0.04 & So & $-2.0 \pm 0.8$ & \\
\hline UGC 10842 & 172452.1 & +280441.8 & 1.1 & 0.5 & 40 & 208 & 36.59 & 1 & 0.05 & $\mathrm{SBb}$ & $3.0 \pm 0.9$ & \\
\hline UGC 10872 & 172718.4 & +611039.9 & 1.2 & 0.1 & 58 & 48 & 33.39 & 1 & 0.03 & & $8.0 \pm 1.5$ & \\
\hline UGC 10888 & 172959.3 & +602101.0 & 1.1 & 0.7 & 150 & 92 & 34.82 & 1 & 0.04 & $\left(\mathrm{R}^{\prime}\right) \mathrm{SB}(\mathrm{r}) \mathrm{b}$ & $3.0 \pm 0.9$ & NLAGN \\
\hline NGC 6394 & 173021.4 & +593823.7 & 1.3 & 0.4 & 42 & 125 & 35.49 & 1 & 0.03 & $\mathrm{SBb}$ & $3.0 \pm 0.9$ & Sy2 \\
\hline UGC 10895 & 173202.3 & +592705.7 & 1.0 & 0.2 & 145 & 126 & 35.50 & 1 & 0.03 & SBbc: & $4.0 \pm 1.0$ & \\
\hline UGC 10935 & 173843.3 & +571421.1 & 1.1 & 0.5 & 116 & 130 & 35.57 & 1 & 0.05 & So & $-2.0 \pm 0.9$ & \\
\hline UGC 10971 & 174449.5 & +552058.8 & 1.1 & 0.4 & 173 & 129 & 35.55 & 1 & 0.07 & $\mathrm{Sa}$ & $1.0 \pm 0.9$ & \\
\hline NGC 6482 & 175148.8 & +230419.0 & 2.0 & 1.7 & 70 & 59 & 33.85 & 1 & 0.10 & E: & $-5.0 \pm 0.6$ & LINER \\
\hline IC 4836 & 191617.9 & -601201.2 & 1.5 & 1.3 & 0 & 56 & 33.73 & 1 & 0.05 & $\mathrm{SA}(\mathrm{s}) \mathrm{c}$ & $4.3 \pm 0.5$ & \\
\hline NGC 6789 & 191641.6 & +635817.2 & 1.3 & 1.0 & 70 & 3.6 & 27.78 & 35 & 0.07 & $\operatorname{Im}$ & $10.0 \pm 0.8$ & \\
\hline NGC 6769 & 191822.7 & -603003.9 & 2.3 & 1.5 & 123 & 51 & 33.55 & 1 & 0.06 & $\left(\mathrm{R}^{\prime}\right) \mathrm{SA}(\mathrm{r}) \mathrm{b}$ & $3.0 \pm 0.4$ & \\
\hline NGC 6770 & 191837.3 & -60 2947.3 & 2.3 & 1.7 & 20 & 52 & 33.57 & 1 & 0.06 & $\mathrm{SB}(\mathrm{rs}) \mathrm{b}$ & $3.0 \pm 0.4$ & \\
\hline NGC 6771 & 191839.5 & -603245.6 & 2.3 & 0.5 & 118 & 57 & 33.77 & 1 & 0.06 & $\mathrm{~S}^{*}+\mathrm{p}$ & $-1.0 \pm 0.5$ & \\
\hline IC 4842 & 191924.5 & -603839.3 & 1.9 & 0.9 & 20 & 55 & 33.70 & 1 & 0.06 & $\mathrm{E}:$ & $-5.0 \pm 0.5$ & \\
\hline IC 4845 & 192022.5 & -602321.0 & 1.8 & 1.5 & 87 & 53 & 33.64 & 1 & 0.06 & $\mathrm{SAB}(\mathrm{rs}) \mathrm{ab}$ & $2.9 \pm 0.4$ & \\
\hline NGC 6782 & 192357.9 & -595520.9 & 2.2 & 1.4 & 45 & 50 & 33.51 & 1 & 0.06 & $\left(\mathrm{R}^{\prime}-1\right) \mathrm{SB}(\mathrm{r}) 0 / \mathrm{a}$ & $0.8 \pm 0.4$ & \\
\hline Superantena & 193121.4 & -723918.0 & 1.0 & 1.0 & $\ldots$ & 261 & 37.08 & 1 & 0.09 & & $\ldots$ & \\
\hline NGC $6845 \mathrm{~A}$ & 200058.4 & -470412.9 & 3.7 & 1.7 & 40 & 90 & 34.76 & 1 & 0.05 & $\mathrm{SB}(\mathrm{s}) \mathrm{b}:$ pec & $3.0 \pm 1.0$ & \\
\hline ESO 284-G009 & 200120.9 & -464001.2 & 1.3 & 0.6 & 152 & 91 & 34.79 & 1 & 0.05 & E5: & $-5.0 \pm 1.2$ & \\
\hline NGC 6902B & 202307.1 & -435207.0 & 1.5 & 1.3 & 0 & 40 & 33.02 & 1 & 0.05 & $\mathrm{SA}(\mathrm{s}) \mathrm{c}$ & $5.6 \pm 0.5$ & \\
\hline IC 4946 & 202358.1 & -435943.0 & 2.5 & 1.0 & 68 & 39 & 32.97 & 1 & 0.04 & $\mathrm{SB}(\mathrm{rl}) 0^{*} 0$ & $-0.1 \pm 0.4$ & \\
\hline NGC 6902 & 202428.1 & -433912.7 & 5.6 & 3.9 & 153 & 38 & 32.88 & 1 & 0.04 & $\mathrm{SA}(\mathrm{r}) \mathrm{b}$ & $3.1 \pm 0.3$ & LINER \\
\hline ESO 285-G009 & 202511.9 & -431524.6 & 1.3 & 0.5 & 118 & 42 & 33.11 & 1 & 0.04 & $\mathrm{SB}(\mathrm{s}) \mathrm{d}$ & $7.0 \pm 0.9$ & \\
\hline PGC 65022 & 203523.8 & -061440.7 & 2.0 & 0.3 & 105 & 83 & 34.60 & 1 & 0.05 & Scd & $5.0 \pm 1.2$ & \\
\hline NGC 6941 & 203623.5 & -043707.5 & 2.0 & 1.4 & 115 & 89 & 34.76 & 1 & 0.06 & $\mathrm{SAB}(\mathrm{rs}) \mathrm{b}$ & $3.0 \pm 0.8$ & \\
\hline NGC 6951 & 203714.1 & +660620.3 & 3.9 & 3.2 & 170 & 24 & 31.93 & 1 & 0.37 & $\mathrm{SAB}(\mathrm{rs}) \mathrm{bc} ; \mathrm{LINER}$ & $4.0 \pm 0.3$ & Sy2 \\
\hline NGC 6945 & 203900.6 & -045821.3 & 1.6 & 0.9 & 120 & 55 & 33.69 & 1 & 0.05 & S0- & $-3.0 \pm 0.9$ & \\
\hline PGC 65158 & 204006.2 & -042007.0 & 1.8 & 0.4 & 60 & 57 & 33.80 & 1 & 0.06 & $\mathrm{Sd} ?$ & $7.0 \pm 1.8$ & \\
\hline UGC 11612 & 204052.1 & +003910.0 & 1.4 & 0.2 & 45 & 116 & 35.32 & 1 & 0.08 & $\mathrm{Sbc}$ & $4.5 \pm 0.7$ & \\
\hline PGC 65328 & 204541.2 & -045700.4 & 1.3 & 1.3 & $\cdots$ & 123 & 35.45 & 1 & 0.05 & $\left(\mathrm{R}^{\prime}\right) \mathrm{Sab}$ & $2.0 \pm 0.8$ & \\
\hline ESO 341-G013 & 204708.8 & -380518.7 & 1.1 & 1.0 & 2 & 97 & 34.93 & 1 & 0.04 & S0? & $\cdots$ & \\
\hline NGC 6962 & $2047 \quad 19.1$ & +001914.9 & 2.9 & 2.3 & 75 & 61 & 33.92 & 1 & 0.10 & $\mathrm{SAB}(\mathrm{r}) \mathrm{ab}$ & $2.0 \pm 0.3$ & \\
\hline
\end{tabular}


Table 1-Continued

\begin{tabular}{|c|c|c|c|c|c|c|c|c|c|c|c|c|}
\hline $\begin{array}{c}\text { Object Name } \\
\text { (1) }\end{array}$ & $\begin{array}{c}\mathrm{RA}_{2000} \\
(\mathrm{~h}: \mathrm{m}: \mathrm{s}) \\
(2)\end{array}$ & $\begin{array}{c}\mathrm{DEC}_{2000} \\
(\mathrm{~d}: \mathrm{m}: \mathrm{s}) \\
(3)\end{array}$ & $\begin{array}{c}2 \times \mathrm{A} \\
(\operatorname{arcmin}) \\
(4)\end{array}$ & $\begin{array}{c}2 \times \mathrm{B} \\
(\operatorname{arcmin}) \\
(5)\end{array}$ & $\begin{array}{c}\text { PA } \\
(\mathrm{deg}) \\
(6)\end{array}$ & $\begin{array}{c}(\mathrm{Mpc}) \\
(7)\end{array}$ & $\begin{array}{l}\text { stance } \\
\text { DM } \\
(8)\end{array}$ & $\begin{array}{l}\text { ref. } \\
(9)\end{array}$ & $\begin{array}{c}\mathrm{E}(B-V) \\
(\mathrm{mag}) \\
(10)\end{array}$ & $\begin{array}{c}\text { Morphological } \\
\text { Type } \\
\text { (11) }\end{array}$ & $\begin{array}{c}\mathrm{T} \\
\text { Type } \\
(12)\end{array}$ & $\begin{array}{c}\text { Spectral } \\
\text { Type } \\
(13)\end{array}$ \\
\hline NGC 6964 & 204724.3 & +001803.0 & 1.7 & 1.3 & 168 & 55 & 33.70 & 1 & 0.09 & E+ pec: & $-4.0 \pm 0.5$ & \\
\hline PGC 65420 & 204813.5 & -054741.8 & 1.3 & 0.7 & 0 & 87 & 34.70 & 1 & 0.05 & SB? & $\ldots$ & \\
\hline NGC 6958 & 204842.6 & -375950.7 & 2.1 & 1.7 & 107 & 37 & 32.82 & 1 & 0.05 & $\mathrm{E}+$ & $-3.8 \pm 0.3$ & \\
\hline UGC 11646 & 205404.4 & +004638.8 & 1.1 & 0.4 & 167 & 125 & 35.48 & 1 & 0.09 & Sbc pec? & $4.0 \pm 1.8$ & \\
\hline PGC 66559 & 211943.0 & -073312.5 & 1.3 & 1.2 & 0 & 39 & 32.95 & 1 & 0.20 & $\mathrm{SB}(\mathrm{s}) \mathrm{dm}$ pec & $8.0 \pm 0.8$ & \\
\hline NGC 7080 & 213002.0 & +264304.1 & 1.8 & 1.7 & 90 & 71 & 34.27 & 1 & 0.14 & $\mathrm{SB}(\mathrm{r}) \mathrm{b}$ & $3.0 \pm 0.8$ & \\
\hline UGC 11776 & 213622.8 & +121410.7 & 1.1 & 0.2 & 172 & 125 & 35.49 & 1 & 0.12 & Scd: & $6.0 \pm 1.4$ & \\
\hline PGC 67153 & 214020.9 & +122117.3 & 2.3 & 0.5 & 100 & 86 & 34.66 & 1 & 0.12 & $\mathrm{Sb}$ & $\ldots$ & \\
\hline UGC 11789 & 214100.1 & +012006.2 & 1.0 & 0.6 & 108 & 128 & 35.53 & 1 & 0.05 & $\mathrm{Sb}$ & $3.0 \pm 0.9$ & \\
\hline Tol 2138-405 & 214121.8 & -401906.2 & 0.3 & 0.2 & 20 & 238 & 36.88 & 1 & 0.02 & HII & $\ldots$ & \\
\hline ESO 343-G018 & 214128.1 & -394553.7 & 1.5 & 0.3 & 137 & 67 & 34.14 & 1 & 0.03 & Sc: sp & $5.0 \pm 1.3$ & \\
\hline UGC 11790 & 214130.0 & +005340.7 & 1.5 & 1.1 & 160 & 65 & 34.08 & 1 & 0.07 & $\mathrm{SA}(\mathrm{rs}) \mathrm{d}:$ & $6.5 \pm 0.6$ & \\
\hline UGC 11794 & $2142 \quad 22.9$ & +122953.7 & 1.2 & 0.4 & 81 & 85 & 34.64 & 1 & 0.10 & Sab & $2.0 \pm 0.9$ & \\
\hline ESO 466-G001 & $\begin{array}{lll}21 & 42 & 32.7\end{array}$ & -292202.7 & 1.4 & 0.3 & 104 & 100 & 34.99 & 1 & 0.05 & $\mathrm{Sab}$ & $2.0 \pm 0.9$ & \\
\hline ESO 466-G005 & 214532.3 & -290424.4 & 1.0 & 0.6 & 120 & 83 & 34.59 & 1 & 0.04 & $\mathrm{Sb}$ & $\ldots$ & \\
\hline UGC 11816 & 214907.3 & +002650.4 & 1.5 & 1.4 & 85 & 68 & 34.17 & 1 & 0.13 & $\mathrm{SB}(\mathrm{rs}) \mathrm{c}:$ & $4.7 \pm 0.6$ & \\
\hline NGC 7152 & 215359.0 & -291720.7 & 1.2 & 0.6 & 17 & 92 & 34.81 & 1 & 0.03 & $\mathrm{SB}(\mathrm{rs}) \mathrm{b} ?$ pec & $3.0 \pm 0.9$ & \\
\hline ESO 466-G014 & 215422.9 & -315809.1 & 1.3 & 0.2 & 50 & 33 & 32.57 & 1 & 0.03 & Sb: & $\ldots$ & \\
\hline UGC 11859 & 215807.4 & +010032.3 & 3.1 & 0.2 & 63 & 43 & 33.19 & 1 & 0.05 & $\mathrm{Sc}$ & $4.0 \pm 0.7$ & \\
\hline ESO 404-G015 & 220029.4 & -332217.8 & 1.3 & 0.3 & 147 & 62 & 33.96 & 1 & 0.02 & $\mathrm{~S} ?$ & $\ldots$ & \\
\hline NGC 7167 & 220030.6 & -243757.4 & 1.7 & 1.3 & 125 & 35 & 32.74 & 1 & 0.04 & $\mathrm{SB}(\mathrm{s}) \mathrm{c}:$ & $5.0 \pm 0.6$ & HII \\
\hline ESO 404-G023 & 220241.6 & $-3348 \quad 18.2$ & 1.7 & 1.1 & 10 & 62 & 33.96 & 1 & 0.02 & $\mathrm{SB}(\mathrm{rs}) \mathrm{bc}$ & $4.0 \pm 0.8$ & \\
\hline IC 5156 & 220314.9 & -335018.4 & 2.2 & 0.8 & 175 & 37 & 32.87 & 1 & 0.03 & $\mathrm{SB}(\mathrm{rs}) \mathrm{ab}$ & $1.6 \pm 0.4$ & \\
\hline NGC 7215 & 220834.5 & +003042.1 & 1.0 & 0.4 & 100 & 58 & 33.82 & 1 & 0.05 & $\mathrm{~S} 0 * 0 * ?$ & $-2.0 \pm 1.9$ & \\
\hline NGC 7221 & $22 \quad 11 \quad 15.2$ & -303347.4 & 2.0 & 1.6 & 10 & 60 & 33.91 & 1 & 0.02 & $\mathrm{SB}(\mathrm{rs}) \mathrm{b}$ & $3.6 \pm 0.6$ & \\
\hline CGCG 377-039 & 221152.9 & +000631.4 & 1.0 & 0.7 & 35 & 143 & 35.78 & 1 & 0.06 & $\mathrm{SB}(\mathrm{rs}) \mathrm{bc}$ & $3.5 \pm 0.6$ & \\
\hline NGC 7248 & 221652.6 & +403016.6 & 1.7 & 0.9 & 133 & 65 & 34.08 & 1 & 0.16 & SA0-: & $-2.5 \pm 0.6$ & \\
\hline NGC 7250 & $22 \quad 18 \quad 17.8$ & +403344.6 & 1.7 & 0.8 & 157 & 19 & 31.45 & 1 & 0.15 & Sdm? & $8.0 \pm 1.8$ & Sbrst \\
\hline NGC 7252 & 222044.8 & -244041.8 & 1.9 & 1.6 & 150 & 66 & 34.08 & 1 & 0.03 & $(\mathrm{R}) \mathrm{SA}(\mathrm{r}) 0^{*} 0^{*}$ : & $-2.0 \pm 0.5$ & \\
\hline ESO 467-G058 & 222605.8 & -305204.6 & 1.3 & 0.4 & 75 & 119 & 35.38 & 1 & 0.02 & SA0/a: pec & $0.0 \pm 0.6$ & \\
\hline ESO 345-G011 & 222616.0 & -372222.8 & 1.2 & 0.6 & 175 & 134 & 35.63 & 1 & 0.02 & $\mathrm{~S}$ pec sp & $-1.0 \pm 1.2$ & \\
\hline NGC 7279 & $22 \quad 2712.7$ & -350825.6 & 1.2 & 0.8 & 68 & 127 & 35.52 & 1 & 0.01 & $\mathrm{SB}(\mathrm{rs}) \mathrm{c}$ & $4.9 \pm 0.5$ & \\
\hline PKS 2225-308 & $22 \quad 2754.4$ & -303431.8 & 2.5 & 0.6 & 145 & 247 & 36.96 & 1 & 0.01 & $\mathrm{E}+3$ & $-4.0 \pm 0.8$ & \\
\hline
\end{tabular}


Table 1-Continued

\begin{tabular}{|c|c|c|c|c|c|c|c|c|c|c|c|c|}
\hline $\begin{array}{c}\text { Object Name } \\
\text { (1) }\end{array}$ & $\begin{array}{c}\mathrm{RA}_{2000} \\
(\mathrm{~h}: \mathrm{m}: \mathrm{s}) \\
(2)\end{array}$ & $\begin{array}{c}\mathrm{DEC}_{2000} \\
(\mathrm{~d}: \mathrm{m}: \mathrm{s}) \\
(3)\end{array}$ & $\begin{array}{c}2 \times \mathrm{A} \\
(\operatorname{arcmin}) \\
(4)\end{array}$ & $\begin{array}{c}2 \times \mathrm{B} \\
(\operatorname{arcmin}) \\
(5)\end{array}$ & $\begin{array}{c}\text { PA } \\
(\mathrm{deg}) \\
(6)\end{array}$ & $\begin{array}{c}(\mathrm{Mpc}) \\
(7)\end{array}$ & $\begin{array}{l}\text { istance } \\
\text { DM } \\
(8)\end{array}$ & $\begin{array}{l}\text { ref. } \\
(9)\end{array}$ & $\begin{array}{c}\mathrm{E}(B-V) \\
\quad(\mathrm{mag}) \\
(10)\end{array}$ & $\begin{array}{c}\text { Morphological } \\
\text { Type } \\
(11)\end{array}$ & $\begin{array}{c}\mathrm{T} \\
\text { Type } \\
(12)\end{array}$ & $\begin{array}{c}\text { Spectral } \\
\text { Type } \\
(13)\end{array}$ \\
\hline NGC 7289 & 222920.3 & -352819.1 & 1.4 & 1.1 & 145 & 119 & 35.37 & 1 & 0.01 & $\mathrm{SA}(\mathrm{r}) 0^{*} 0^{*}:$ & $-2.4 \pm 0.7$ & \\
\hline ESO 468-G006 & 223118.2 & -282359.3 & 1.3 & 1.1 & 0 & 36 & 32.81 & 1 & 0.02 & $\mathrm{SB}(\mathrm{s}) \mathrm{d}$ pec & $7.0 \pm 0.8$ & \\
\hline NGC 7317 & 223551.9 & +335641.6 & 1.1 & 1.1 & $\ldots$ & 97 & 34.93 & 1 & 0.08 & $\mathrm{E} 4$ & $-5.0 \pm 0.5$ & \\
\hline NGC 7320 & 223603.4 & +335653.2 & 2.2 & 1.1 & 132 & 14 & 30.65 & 1 & 0.08 & $\mathrm{SA}(\mathrm{s}) \mathrm{d}$ & $7.0 \pm 0.4$ & HII \\
\hline UGC 12110 & 223655.1 & +142444.3 & 1.0 & 0.2 & 137 & 158 & 36.00 & 1 & 0.07 & $\mathrm{~S} ?$ & $\ldots$ & \\
\hline NGC 7331 & 223704.1 & +342456.3 & 10.5 & 3.7 & 171 & 15 & 30.86 & 43 & 0.09 & $\mathrm{SA}(\mathrm{s}) \mathrm{b}$ & $3.0 \pm 0.3$ & LINER \\
\hline NGC 7335 & 223719.4 & +342651.9 & 1.3 & 0.6 & 151 & 93 & 34.83 & 1 & 0.09 & $\mathrm{SA}(\mathrm{rs}) 0+$ & $-1.0 \pm 0.5$ & \\
\hline NGC 7337 & 223726.6 & +342227.5 & 1.1 & 0.9 & 0 & 96 & 34.92 & 1 & 0.08 & $\mathrm{SB}(\mathrm{rs}) \mathrm{b}$ & $3.0 \pm 0.5$ & \\
\hline NGC 7343 & 223837.9 & +340417.2 & 1.1 & 0.9 & 160 & 109 & 35.19 & 1 & 0.07 & $\left(\mathrm{R}^{\prime}\right) \mathrm{SB}(\mathrm{s}) \mathrm{bc}:$ & $3.5 \pm 0.6$ & LINER \\
\hline UGC 12134 & 223937.5 & +114611.6 & 1.8 & 0.6 & 145 & 106 & 35.13 & 1 & 0.05 & $\mathrm{Sbc}$ & $4.0 \pm 0.8$ & \\
\hline NGC 7348 & 224036.3 & +115422.4 & 1.1 & 0.7 & 12 & 105 & 35.11 & 1 & 0.05 & Scd: & $6.0 \pm 0.9$ & \\
\hline IRAS 22491-1808 & 225149.3 & -175223.5 & 0.4 & 0.4 & $\ldots$ & 331 & 37.60 & 1 & 0.04 & & .. & \\
\hline NGC 7396 & 225222.6 & +010533.1 & 1.9 & 1.1 & 103 & 71 & 34.26 & 1 & 0.09 & Sa pec sp & $0.5 \pm 0.6$ & \\
\hline ESO 346-G006 & 225239.3 & -401949.3 & 1.0 & 0.9 & 130 & 137 & 35.69 & 1 & 0.01 & Sc: pec & $5.0 \pm 1.2$ & \\
\hline NGC 7398 & 225249.3 & +011204.0 & 1.2 & 0.8 & 75 & 67 & 34.15 & 1 & 0.07 & $\mathrm{SA}(\mathrm{r}) \mathrm{a}$ : & $0.5 \pm 0.6$ & \\
\hline UGC 12250 & 225535.9 & +124725.1 & 1.6 & 1.0 & 13 & 105 & 35.10 & 1 & 0.05 & $\mathrm{SBb}$ & $3.0 \pm 0.8$ & \\
\hline UGC 12253 & 225601.8 & +124559.9 & 1.7 & 0.2 & 145 & 112 & 35.25 & 1 & 0.05 & $\mathrm{Sbc}$ & $3.0 \pm 1.0$ & \\
\hline NGC 7418 & 225636.2 & -370148.3 & 3.5 & 2.6 & 139 & 18 & 31.31 & 1 & 0.02 & $\mathrm{SAB}(\mathrm{rs}) \mathrm{cd}$ & $6.0 \pm 0.3$ & \\
\hline NGC $7418 \mathrm{~A}$ & 225641.2 & -364621.2 & 3.7 & 1.8 & 83 & 28 & 32.21 & 1 & 0.02 & $\mathrm{SA}(\mathrm{rs}) \mathrm{d}:$ & $7.0 \pm 0.7$ & \\
\hline ESO 534-G032 & 225644.9 & -245709.1 & 1.4 & 0.6 & 170 & 129 & 35.55 & 1 & 0.03 & $\mathrm{SAB}(\mathrm{s}) \mathrm{c}:$ & $5.0 \pm 0.6$ & \\
\hline IC 5264 & 225653.0 & -363315.0 & 2.5 & 0.5 & 82 & 26 & 32.04 & 1 & 0.02 & Sab pec sp & $2.3 \pm 0.6$ & \\
\hline NGC 7421 & 225654.3 & -372050.1 & 2.0 & 1.8 & 85 & 24 & 31.88 & 1 & 0.01 & $\mathrm{SB}(\mathrm{r}) \mathrm{bc}$ & $4.0 \pm 0.4$ & \\
\hline NGC 7432 & 225801.9 & +130804.2 & 1.5 & 1.2 & 40 & 110 & 35.20 & 1 & 0.08 & $\mathrm{E}$ & $-5.0 \pm 0.8$ & \\
\hline ARP 314 NED01 & 225802.2 & -034610.9 & 1.1 & 0.9 & 0 & 52 & 33.60 & 1 & 0.09 & $\left(\mathrm{R}^{\prime}\right) \mathrm{SA}(\mathrm{s}) \mathrm{bc}: \mathrm{pec}$ & $4.0 \pm 0.4$ & \\
\hline ARP 314 NED03 & $2258 \quad 07.3$ & -034838.4 & 1.1 & 0.9 & 0 & 53 & 33.61 & 1 & 0.08 & $\left(\mathrm{R}^{\prime}\right) \mathrm{SB}(\mathrm{s}) \mathrm{dm}$ pec & $8.0 \pm 0.4$ & \\
\hline ARP 314 NED02 & 225807.5 & -034719.6 & 1.3 & 1.1 & 0 & 52 & 33.59 & 1 & 0.09 & SB(rs)cd: pec & $6.0 \pm 0.4$ & \\
\hline UGC 12285 & 225926.7 & $\begin{array}{r}+124254.4\end{array}$ & $\begin{array}{l}1.0 \\
1.0\end{array}$ & $\begin{array}{l}1.1 \\
0.5\end{array}$ & 10 & 208 & 36.59 & 1 & 0.16 & S? & $\begin{array}{l}\ldots .0 .0 \\
\ldots\end{array}$ & \\
\hline ESO $406-$ G042 & $\begin{array}{lll}23 & 02 & 14.2\end{array}$ & -370501.4 & 1.7 & 1.2 & 66 & 17 & 31.18 & 1 & 0.02 & $\mathrm{SAB}(\mathrm{s}) \mathrm{m}:$ & $8.7 \pm 0.5$ & \\
\hline NGC 7469 & $2303 \quad 15.6$ & +085226.4 & 1.5 & 1.1 & 125 & 71 & 34.25 & 1 & 0.07 & $\left(\mathrm{R}^{\prime}\right) \mathrm{SAB}(\mathrm{rs}) \mathrm{a}$ & $1.0 \pm 0.4$ & Sy1.2 \\
\hline NGC 7479 & 230456.7 & +121922.4 & 4.1 & 3.1 & 25 & 35 & 32.71 & 1 & 0.11 & SB(s)c;LINER & $5.0 \pm 0.3$ & Sy 2 \\
\hline UGC 12346 & 230512.6 & +00 5003.9 & 1.4 & 1.3 & 0 & 108 & 35.17 & 1 & 0.05 & $\left(\mathrm{R}^{\prime}\right) \mathrm{SB}(\mathrm{rs}) \mathrm{bc} ?$ & $4.3 \pm 0.9$ & \\
\hline UGC 12354 & 230556.5 & +142127.6 & 1.0 & 0.4 & 159 & 57 & 33.77 & 1 & 0.24 & Scd: & $6.0 \pm 1.3$ & \\
\hline ESO 469-G012 & 230706.8 & -283643.7 & 1.1 & 0.5 & 64 & 137 & 35.68 & 1 & 0.03 & $\mathrm{~S} ?$ & $\ldots$ & \\
\hline
\end{tabular}


Table 1-Continued

\begin{tabular}{|c|c|c|c|c|c|c|c|c|c|c|c|c|}
\hline $\begin{array}{c}\text { Object Name } \\
\text { (1) }\end{array}$ & $\begin{array}{c}\mathrm{RA}_{2000} \\
(\mathrm{~h}: \mathrm{m}: \mathrm{s}) \\
(2)\end{array}$ & $\begin{array}{c}\mathrm{DEC}_{2000} \\
(\mathrm{~d}: \mathrm{m}: \mathrm{s}) \\
(3)\end{array}$ & $\begin{array}{c}2 \times \mathrm{A} \\
(\operatorname{arcmin}) \\
(4)\end{array}$ & $\begin{array}{c}2 \times \mathrm{B} \\
(\operatorname{arcmin}) \\
(5)\end{array}$ & $\begin{array}{c}\text { PA } \\
(\mathrm{deg}) \\
(6)\end{array}$ & $\begin{array}{c}(\mathrm{Mpc}) \\
(7)\end{array}$ & $\begin{array}{c}\text { istance } \\
\text { DM } \\
(8)\end{array}$ & $\begin{array}{l}\text { ref. } \\
(9)\end{array}$ & $\begin{array}{c}\mathrm{E}(B-V) \\
(\mathrm{mag}) \\
(10)\end{array}$ & $\begin{array}{c}\text { Morphological } \\
\text { Type } \\
\text { (11) }\end{array}$ & $\begin{array}{c}\mathrm{T} \\
\text { Type } \\
(12)\end{array}$ & $\begin{array}{c}\text { Spectral } \\
\text { Type } \\
(13)\end{array}$ \\
\hline ESO 469-G015 & 230855.6 & -305128.5 & 2.0 & 0.2 & 149 & 21 & 31.63 & 1 & 0.03 & Sb: sp & $3.0 \pm 1.2$ & \\
\hline IC 5287 & 230920.3 & +004523.3 & 1.1 & 1.0 & 155 & 139 & 35.72 & 1 & 0.04 & $\left(\mathrm{R}^{\prime}\right) \mathrm{SB}(\mathrm{r}) \mathrm{b}$ & $3.0 \pm 0.6$ & Sy1.2 \\
\hline ESO 407-G007 & 230939.4 & -362512.3 & 2.8 & 0.4 & 170 & 21 & 31.62 & 1 & 0.01 & $\mathrm{SAb} ? \mathrm{sp}$ & $3.0 \pm 1.7$ & \\
\hline NGC 7496 & 230947.3 & -432540.6 & 3.3 & 3.0 & 0 & 21 & 31.59 & 1 & 0.01 & $\left(\mathrm{R}^{\prime}:\right) \mathrm{SB}(\mathrm{rs}) \mathrm{bc}$ & $3.0 \pm 0.3$ & Sy2 \\
\hline ESO 291-G005 & 231156.7 & $-43 \quad 43 \quad 17.2$ & 1.0 & 0.7 & 35 & 146 & 35.82 & 1 & 0.01 & $(\mathrm{R}-1) \mathrm{SB}(\mathrm{l}) 0 / \mathrm{a}$ & $1.0 \pm 0.8$ & \\
\hline ESO 291-G006 & $23 \quad 12 \quad 22.7$ & -435223.2 & 1.3 & 0.5 & 167 & 354 & 37.74 & 1 & 0.01 & SA0-: & $-3.0 \pm 0.8$ & \\
\hline NGC $7496 \mathrm{~A}$ & $23 \quad 1223.3$ & -434641.5 & 1.5 & 1.2 & 85 & 21 & 31.59 & 77 & 0.01 & $\mathrm{SB}(\mathrm{s}) \mathrm{m}$ & $9.0 \pm 0.5$ & \\
\hline NGC 7511 & 231226.3 & +134335.7 & 1.1 & 0.5 & 133 & 71 & 34.25 & 1 & 0.06 & $\mathrm{~S} ?$ & $\ldots$ & \\
\hline ESO 407-G009 & 231244.9 & $-37 \quad 1228.5$ & 1.9 & 0.7 & 30 & 20 & 31.50 & 1 & 0.02 & $\mathrm{SB}(\mathrm{s}) \mathrm{d}:$ & $7.0 \pm 0.5$ & \\
\hline ESO 291-G009 & 231358.6 & -424339.2 & 1.3 & 0.8 & 55 & 239 & 36.89 & 1 & 0.01 & $\mathrm{SA} 0^{*}$ & $-4.0 \pm 0.6$ & \\
\hline UGC 12434 & 231405.4 & +131040.2 & 1.0 & 0.2 & 156 & 39 & 32.96 & 1 & 0.05 & $\mathrm{Sa} ?$ & $1.0 \pm 1.9$ & \\
\hline NGC 7535 & 231412.8 & +133454.8 & 1.5 & 1.5 & $\ldots$ & 67 & 34.12 & 1 & 0.07 & $\mathrm{Sd}$ & $7.0 \pm 0.8$ & \\
\hline NGC 7536 & 231413.2 & +132535.0 & 1.9 & 0.7 & 56 & 68 & 34.16 & 1 & 0.06 & $\mathrm{SBbc}$ & $4.0 \pm 0.8$ & \\
\hline NGC $7559 B$ & 231546.6 & +131725.0 & 1.1 & 1.0 & 75 & 67 & 34.12 & 1 & 0.05 & & $\ldots$ & \\
\hline NGC 7563 & 231555.9 & +131146.0 & 1.9 & 1.0 & 155 & 61 & 33.91 & 1 & 0.06 & $\mathrm{SBa}$ & $1.0 \pm 0.8$ & \\
\hline UGC 12479 & 231725.7 & -013510.2 & 1.0 & 0.3 & 33 & 60 & 33.89 & 1 & 0.04 & $\mathrm{Sa}$ & $1.0 \pm 0.9$ & \\
\hline ESO 407-G014 & 231739.5 & -344726.9 & 1.9 & 1.1 & 40 & 37 & 32.84 & 1 & 0.02 & $\mathrm{SB}(\mathrm{s}) \mathrm{c} ?$ & $5.0 \pm 1.1$ & HII \\
\hline NGC 7589 & 231815.7 & +00 1540.2 & 1.1 & 0.7 & 105 & 128 & 35.53 & 1 & 0.04 & $\mathrm{SAB}(\mathrm{rs}) \mathrm{a}:$ & $1.0 \pm 1.3$ & BLAGN \\
\hline NGC 7582 & 231823.5 & -422214.0 & 5.0 & 2.1 & 157 & 22 & 31.74 & 78 & 0.01 & $\left(\mathrm{R}^{\prime}-1\right) \mathrm{SB}(\mathrm{s}) \mathrm{ab}$ & $2.0 \pm 0.3$ & Sy2 \\
\hline PGC 71025 & 231838.4 & -102429.4 & 1.3 & 0.2 & 15 & 130 & 35.57 & 1 & 0.03 & $\mathrm{Sb}$ pec sp & $3.0 \pm 1.3$ & \\
\hline IC 5304 & 231852.6 & $-10 \quad 1533.4$ & 1.4 & 0.9 & 18 & 137 & 35.68 & 1 & 0.03 & SAB0- pec: & $-3.0 \pm 0.9$ & \\
\hline NGC 7645 & 232347.3 & -292316.9 & 1.4 & 1.2 & 0 & 97 & 34.93 & 1 & 0.03 & $\mathrm{SB}(\mathrm{r}) \mathrm{c}$ & $5.0 \pm 0.8$ & \\
\hline UGC 12578 & 232423.1 & -000629.0 & 1.6 & 1.1 & 100 & 39 & 32.93 & 1 & 0.05 & $\mathrm{SB}(\mathrm{s}) \mathrm{m}$ pec & $9.0 \pm 0.8$ & \\
\hline UGC 12589 & 232501.6 & +000000.7 & 1.4 & 0.5 & 57 & 145 & 35.80 & 1 & 0.04 & & $9.3 \pm 0.7$ & \\
\hline CGCG 406-109 & 232654.2 & +084701.0 & 1.1 & 0.2 & 130 & 88 & 34.72 & 1 & 0.07 & & $\ldots$ & \\
\hline NGC 7673 & 232741.1 & +233520.2 & 1.3 & 1.2 & 0 & 50 & 33.50 & 1 & 0.04 & (R')SAc?pec;HII & $5.0 \pm 1.1$ & Sbrst \\
\hline NGC 7674 & 232756.7 & +084644.5 & 1.1 & 1.0 & 0 & 124 & 35.47 & 1 & 0.06 & SA(r)bc pec;HII & $4.0 \pm 0.5$ & Sy2 \\
\hline NGC 7677 & 232806.2 & +233153.2 & 1.6 & 1.0 & 35 & 52 & 33.59 & 1 & 0.04 & SAB(r)bc: & $3.5 \pm 0.6$ & Sbrst \\
\hline IC 5325 & 232843.4 & -412000.5 & 2.8 & 2.5 & 0 & 19 & 31.36 & 1 & 0.02 & $\mathrm{SAB}(\mathrm{rs}) \mathrm{bc}$ & $4.0 \pm 0.7$ & \\
\hline UGC 12635 & 233025.7 & +000924.1 & 1.3 & 1.2 & 0 & 74 & 34.36 & 1 & 0.05 & $\mathrm{SA}(\mathrm{rs}) \mathrm{d}:$ & $6.5 \pm 0.9$ & \\
\hline NGC 7684 & 233032.0 & +000451.7 & 1.4 & 0.4 & 21 & 73 & 34.32 & 1 & 0.05 & $\mathrm{~S} 0+$ & $-0.5 \pm 0.6$ & \\
\hline
\end{tabular}


Table 1-Continued

\begin{tabular}{|c|c|c|c|c|c|c|c|c|c|c|c|c|}
\hline $\begin{array}{c}\text { Object Name } \\
\text { (1) }\end{array}$ & $\begin{array}{c}\mathrm{RA}_{2000} \\
(\mathrm{~h}: \mathrm{m}: \mathrm{s}) \\
(2)\end{array}$ & $\begin{array}{c}\mathrm{DEC}_{2000} \\
(\mathrm{~d}: \mathrm{m}: \mathrm{s}) \\
(3)\end{array}$ & $\begin{array}{c}2 \times \mathrm{A} \\
(\operatorname{arcmin}) \\
(4)\end{array}$ & $\begin{array}{c}2 \times \mathrm{B} \\
(\operatorname{arcmin}) \\
(5)\end{array}$ & $\begin{array}{c}\text { PA } \\
(\operatorname{deg}) \\
(6)\end{array}$ & $\begin{array}{l}(\mathrm{Mpc}) \\
(7)\end{array}$ & $\begin{array}{l}\text { stance } \\
\text { DM } \\
(8)\end{array}$ & $\begin{array}{l}\text { ref. } \\
(9)\end{array}$ & $\begin{array}{c}\mathrm{E}(B-V) \\
\quad(\mathrm{mag}) \\
(10)\end{array}$ & $\begin{array}{c}\text { Morphological } \\
\text { Type } \\
(11)\end{array}$ & $\begin{array}{c}\mathrm{T} \\
\text { Type } \\
(12)\end{array}$ & $\begin{array}{c}\text { Spectral } \\
\text { Type } \\
(13)\end{array}$ \\
\hline UGC 12685 & 233513.8 & +00 0232.8 & 1.2 & 1.1 & 135 & 75 & 34.39 & 1 & 0.04 & SB(s)d pec: & $7.0 \pm 0.8$ & \\
\hline IRAS $23365+3604$ & 233901.3 & +362108.7 & 0.5 & 0.3 & 160 & 278 & 37.22 & 1 & 0.11 & S?Ba? pec & $\cdots$ & LINER \\
\hline ARP 295A & 234147.3 & -034002.0 & 1.9 & 0.3 & 38 & 94 & 34.86 & 1 & 0.04 & $\mathrm{Sc}$ & $5.0 \pm 1.1$ & \\
\hline NGC 7735 & 234217.3 & +261354.3 & 1.3 & 0.9 & 90 & 139 & 35.71 & 1 & 0.08 & $\mathrm{E}$ & $-5.0 \pm 0.8$ & \\
\hline NGC 7741 & 234354.4 & +260432.2 & 4.4 & 3.0 & 170 & 12 & 30.46 & 1 & 0.08 & $\mathrm{SB}(\mathrm{s}) \mathrm{cd}$ & $6.0 \pm 0.3$ & \\
\hline NGC 7771 & 235124.9 & +200642.6 & 2.5 & 1.0 & 68 & 63 & 33.98 & 1 & 0.07 & $\mathrm{SB}(\mathrm{s}) \mathrm{a}$ & $1.0 \pm 0.4$ & HII \\
\hline CGCG 432-040 & 235516.1 & +142231.7 & 1.1 & 0.3 & 168 & 155 & 35.95 & 1 & 0.04 & $\mathrm{~S} ?$ & $\cdots$ & \\
\hline NGC 7793 & 235749.8 & -323527.7 & 9.3 & 6.3 & 98 & 2.0 & 26.51 & 79 & 0.02 & $\mathrm{SA}(\mathrm{s}) \mathrm{d}$ & $7.0 \pm 0.3$ & HII \\
\hline ESO 349-G014 & 235827.9 & -325639.3 & 1.4 & 0.1 & 7 & 174 & 36.20 & 1 & 0.01 & $\mathrm{Sc}$ & $\ldots$ & \\
\hline NGC 7798 & 235925.5 & +204459.5 & 1.4 & 1.3 & 90 & 36 & 32.75 & 1 & 0.07 & $\mathrm{SBc}$ & $\ldots$ & Sbrst \\
\hline
\end{tabular}

Note. - GALEX Atlas sample. Col. (1): Galaxy name. Col. (2): RA(J2000) of the galaxy center. Col. (3): Dec(J2000) of the galaxy center. Col. (4): Major-axis diameter of the D25 ellipse. Col. (5): Minor-axis diameter of the D25 ellipse. Col. (6): Position angle (PA) of the D25 ellipse. In those cases where the D25 ellipse is approximately circular the PA is undefined. Col. (7): Distance to the galaxy in Mpc. Col. (8): Distance modulus. Col. (9): Reference from which the distance to the galaxy was taken (see below). Col. (10): Galactic color excess from Schlegel et al. (1998). Col. (11): Morphological type from NED. Col. (12): Morphological type T from the RC3 catalog when available. Col. (13): Spectral type from NED. References for determining the distances: (1) from the Virgo-infall corrected radial velocity adopting $\mathrm{H}_{0}=70 \mathrm{~km} \mathrm{~s}^{-1} \mathrm{Mpc}^{-1}$ (2) Rejkuba et al., 2000, AJ, 120, 801 (3) Kennicutt et al., 2003, PASP 115, 928 (4) Van de Steene et al., 2004, in "Planetary Nebulae beyond the Milky Way", Garching (Germany) (5) van den Bergh, 2000, Galaxies of the Local Group (Cambridge: Cambridge Univ. Press) (6) Freedman \& Madore, 1990, ApJ 365, 186 (7) LEDA's Tully-Fisher relationship value (8) Karachentsev et al., 2003, A\&A 404, 93 (9) Freedman et al., 2001, ApJ 553, 47 (10) Tikhonov \& Galazutdinova 2002, A\&A 394, 33 (11) Freedman, Wilson, \& Madore, 1991, ApJ 372, 455 (12) Tully, 1988, Nearby Galaxies Catalogue (13) assumed to be at the same distance as IC 0159 (14) Gallart et al., 2004, AJ, 127, 1486 (15) Tonry et al., 2001, ApJ 546, 681 (16) Silbermann et al., 1996, ApJ 470, 1 (17) assumed to be at the same distance as NGC 1023 (18) Bland-Hawthorn et al., 1997, Ap\&SS 248, 9 (19) Bottinelli et al., 1984, A\&AS 56, 381 (20) Karachentsev, Musella \& Grimaldi, 1996, A\&A 310, 722 (21) Madore et al., 1998, Nature 395:3, 47 (fornax cluster) (22) Perrett et al., 1997, AJ, 113, 895 (23) assumed to be at the same distance as NGC 1512 (24) assumed to be at the same distance as NGC 1553 (Dorado group) (25) Jensen et al., (2003, ApJ 583, 712) (26) Makarova \& Karachentsev, 2003, Ap, 46, 144 (27) Tosi et al., 2001, AJ, 122, 1271 (28) Tolstoy et al., 1995, AJ, 110, 1640 (29) assumed to be in the NGC 2442 group (30) Sersic \& Donzelli, 1993, A\&AS, 98, 21 (31) Davidge, 2003, AJ 125, 3046 (32) Sharina, Karachentsev, \& Tikhonov, 1999, Astronomy Letters 25, 322 (33) Gil de Paz, Zamorano, \& Gallego, 2000, A\&A 361, 465 (34) Karachentsev et al., 2002, A\&A 383, 125 (35) Gil de Paz, Madore, \& Pevunova, 2003, ApJS 147, 29 (36) Macri et al., 2001, ApJ 559, 243 (37) Karachentsev et al., 2003, A\&A 398, 479 (38) Drozdovsky \& Karachentsev, 2000, A\&AS 142, 425 (39) Östlin, 2000, ApJL 535, 99 (40) Freedman et al., 1994, ApJ 427, 628 (41) Sakai \& Madore, 1999, ApJ 526, 599 (42) assumed to be at the same distance as M 81 (43) Paturel et al., 2002, A\&A 389, 19 (44) assumed to be at the same distance as NGC 3109 (45) assumed to be at the same distance as NGC 3190 (46) Verdes-Montenegro, Bosma, \& Athanassoula, 2000, A\&A 356, 827 (47) Schulte-Ladbeck et al., 1999, ApJ 525, 709 (48) GOLDMINE database (Gavazzi et al. 2003) (49) assumed to be at the same distance of NGC 3923 (50) assumed to be at the same 
distance as NGC 3923 (51) using the average of NGC4038/NGC4039 (52) Gavazzi et al., 1999, MNRAS 304, 595 (Virgo M) (53) GOLDMINE database (Gavazzi et al. 2003) (Virgo Bckg) (54) Gavazzi et al., 1999, MNRAS 304, 595 (Virgo N) (55) Karachentsev et al., 2004, AJ, 127, 2031 (56) assumed to be at the same distance as MESSIER 106 (57) Herrnstein et al., 1999, Nature, 400, 539 (58) assumed to be at the same distance as NGC 4278 (59) Gavazzi et al., 1999, MNRAS 304, 595 (Virgo S+E) (60) Gavazzi et al., 1999, MNRAS 304, 595 (Virgo A) (61) Karachentsev \& Drozdovsky 1998, A\&AS 131, 1 (62) GOLDMINE database (Gavazzi et al. 2003) (Virgo A) (63) assumed to be at the same distance as NGC 4490 (64) assumed to be at the same distance as NGC 4625 (65) assumed to be at the same distance as NGC 4631 (66) Makarova et al., 1998, A\&AS 128, 459 (67) assumed to be at the same distance as NGC 5055 (68) Rejkuba, 2004, A\&A 413, 903 (69) assumed to be at the same distance as NGC 5169 (70) Feldmeier, Ciardullo, \& Jacoby, 1997, ApJ 479, 231 (71) assumed to be at the same distance as M 51a (72) Thim et al., 2003, ApJ 590, 256 (73) Kelson et al., 1996, ApJ 463, 26 (74) assumed to be at the same distance as NGC 5713/NGC 5719 (75) using the average of NGC 5713/NGC 5719 (76) assumed to be at the same distance as NGC 6340 (77) assumed to be at the same distance as NGC 7496 (78) assumed to be at the same distance as NGC 7552 (79) assumed to be at the same distance as NGC 0300 
Table 2. GALEX observations

\begin{tabular}{|c|c|c|c|c|c|c|c|c|c|c|}
\hline \multirow{2}{*}{$\begin{array}{c}\text { Object Name } \\
\text { (1) }\end{array}$} & \multirow{2}{*}{$\begin{array}{c}\text { Date } \\
\text { Observed } \\
(2) \\
\end{array}$} & \multirow{2}{*}{$\begin{array}{c}\text { Exposure } \\
\quad(\mathrm{sec}) \\
(3)\end{array}$} & \multirow{2}{*}{$\begin{array}{l}\text { Tile } \\
(4)\end{array}$} & \multicolumn{3}{|c|}{ FUV sky background } & \multicolumn{3}{|c|}{ NUV sky background } & \multirow{2}{*}{$\begin{array}{l}\text { notes } \\
\text { (11) }\end{array}$} \\
\hline & & & & $\begin{array}{c}\text { mean } \\
(\text { counts/s) } \\
(5)\end{array}$ & $\begin{array}{c}<\sigma> \\
(\text { counts } / \mathrm{s}) \\
(6)\end{array}$ & $\begin{array}{c}\sigma(\text { mean }) \\
(\text { counts/s) } \\
(7)\end{array}$ & $\begin{array}{c}\text { mean } \\
(\text { counts/s) } \\
(8)\end{array}$ & $\begin{array}{c}<\sigma> \\
(\text { counts } / \mathrm{s}) \\
(9)\end{array}$ & $\begin{array}{c}\sigma(\text { mean }) \\
(\text { counts } / \mathrm{s}) \\
(10)\end{array}$ & \\
\hline WLM & $2003-10-23$ & 1442 & NGA-WLM & $2.657 \mathrm{E}-04$ & $4.595 \mathrm{E}-04$ & $1.133 \mathrm{E}-05$ & $2.968 \mathrm{E}-03$ & $1.681 \mathrm{E}-03$ & $8.973 \mathrm{E}-06$ & \\
\hline NGC 7808 & 2003-09-17 & 592 & MISDR1-29571-0650 & $2.403 \mathrm{E}-04$ & $6.984 \mathrm{E}-04$ & $3.168 \mathrm{E}-05$ & $3.119 \mathrm{E}-03$ & $2.688 \mathrm{E}-03$ & $1.062 \mathrm{E}-05$ & \\
\hline UGC 00017 & $2004-08-26$ & 1705 & MISDR2-28657-0750 & $4.114 \mathrm{E}-04$ & $4.988 \mathrm{E}-04$ & $1.217 \mathrm{E}-05$ & $3.018 \mathrm{E}-03$ & $1.500 \mathrm{E}-03$ & $9.886 \mathrm{E}-06$ & (1) \\
\hline PGC 00282 & 2003-09-17 & 592 & MISDR1-29571-0650 & $2.228 \mathrm{E}-04$ & 7.111E-04 & $2.995 \mathrm{E}-06$ & $3.116 \mathrm{E}-03$ & $2.730 \mathrm{E}-03$ & $1.126 \mathrm{E}-04$ & \\
\hline NGC 0024 & 2003-09-16 & 1602 & NGA-NGC0024 & $2.434 \mathrm{E}-04$ & $4.232 \mathrm{E}-04$ & $3.274 \mathrm{E}-05$ & $2.789 \mathrm{E}-03$ & $1.537 \mathrm{E}-03$ & $8.181 \mathrm{E}-05$ & \\
\hline UGC 00128 & 2003-09-09 & 1405 & NGA-UGC0128 & $5.992 \mathrm{E}-04$ & $8.596 \mathrm{E}-04$ & $1.123 \mathrm{E}-05$ & $3.526 \mathrm{E}-03$ & $1.794 \mathrm{E}-03$ & $4.927 \mathrm{E}-05$ & \\
\hline NGC 0055 & 2003-09-14 & 1511 & NGA-NGC0055 & $2.564 \mathrm{E}-04$ & $4.457 \mathrm{E}-04$ & $5.672 \mathrm{E}-05$ & $2.503 \mathrm{E}-03$ & $1.512 \mathrm{E}-03$ & $1.213 \mathrm{E}-04$ & \\
\hline ARP 256 NED02 & 2003-09-19 & 1429 & MISDR1-29523-0652 & $2.269 \mathrm{E}-04$ & $4.122 \mathrm{E}-04$ & $7.915 \mathrm{E}-06$ & $3.016 \mathrm{E}-03$ & $1.627 \mathrm{E}-03$ & $2.816 \mathrm{E}-06$ & \\
\hline ARP 256 NED01 & 2003-09-19 & 1429 & MISDR1-29523-0652 & $2.272 \mathrm{E}-04$ & $4.123 \mathrm{E}-04$ & $1.762 \mathrm{E}-06$ & $3.011 \mathrm{E}-03$ & $1.627 \mathrm{E}-03$ & $8.552 \mathrm{E}-06$ & \\
\hline UGC 00226 & 2003-08-29 & 3537 & MISDR1-28652-0417 & $5.036 \mathrm{E}-04$ & $4.411 \mathrm{E}-04$ & $7.978 \mathrm{E}-06$ & $3.283 \mathrm{E}-03$ & $1.148 \mathrm{E}-03$ & $6.477 \mathrm{E}-05$ & \\
\hline NGC 0099 & 2004-08-19 & 4871 & MISDR2-28639-0753 & $4.486 \mathrm{E}-04$ & $5.825 \mathrm{E}-04$ & $2.883 \mathrm{E}-05$ & 3.333E-03 & $9.613 \mathrm{E}-04$ & $3.805 \mathrm{E}-05$ & \\
\hline UGC 00247 & 2003-08-29 & 3537 & MISDR1-28652-0417 & $4.579 \mathrm{E}-04$ & $3.666 \mathrm{E}-04$ & $1.514 \mathrm{E}-05$ & $3.328 \mathrm{E}-03$ & $1.095 \mathrm{E}-03$ & $2.795 \mathrm{E}-05$ & \\
\hline UGC 00249 & 2003-08-31 & 2989 & MISDR1-28666-0417 & $4.844 \mathrm{E}-04$ & $4.192 \mathrm{E}-04$ & $2.574 \mathrm{E}-05$ & $3.304 \mathrm{E}-03$ & $1.189 \mathrm{E}-03$ & $4.856 \mathrm{E}-06$ & \\
\hline NGC 0115 & 2004-10-05 & 1634 & MIS2DFSGP-41281-0499 & $1.461 \mathrm{E}-04$ & $3.041 \mathrm{E}-04$ & $6.002 \mathrm{E}-06$ & $2.186 \mathrm{E}-03$ & $1.295 \mathrm{E}-03$ & $4.421 \mathrm{E}-05$ & \\
\hline NGC 0131 & 2004-10-05 & 1634 & MIS2DFSGP-41281-0499 & $1.733 \mathrm{E}-04$ & $4.039 \mathrm{E}-04$ & $1.890 \mathrm{E}-06$ & $2.241 \mathrm{E}-03$ & $1.335 \mathrm{E}-03$ & $3.214 \mathrm{E}-05$ & \\
\hline PGC 01862 & 2003-09-19 & 1621 & MISDR1-29477-0654 & $2.242 \mathrm{E}-04$ & $3.987 \mathrm{E}-04$ & $1.876 \mathrm{E}-05$ & $2.955 \mathrm{E}-03$ & $1.499 \mathrm{E}-03$ & $2.084 \mathrm{E}-05$ & (1) \\
\hline UGC 00316 & $2003-08-31$ & 3173 & MISDR1-28651-0417 & $4.568 \mathrm{E}-04$ & $4.203 \mathrm{E}-04$ & $1.529 \mathrm{E}-05$ & $3.189 \mathrm{E}-03$ & $1.138 \mathrm{E}-03$ & $6.290 \mathrm{E}-05$ & $(2)$ \\
\hline ESO 473-G025 & 2004-12-09 & 1480 & MIS2DFSGP-30536-0140 & $2.634 \mathrm{E}-04$ & $4.327 \mathrm{E}-04$ & $6.181 \mathrm{E}-06$ & 2.903E-03 & $1.598 \mathrm{E}-03$ & $9.710 \mathrm{E}-05$ & \\
\hline IC 1554 & 2004-10-05 & 1631 & MIS2DFSGP-41321-0440 & $1.946 \mathrm{E}-04$ & $3.900 \mathrm{E}-04$ & $1.986 \mathrm{E}-06$ & $2.315 \mathrm{E}-03$ & $1.397 \mathrm{E}-03$ & $7.427 \mathrm{E}-06$ & \\
\hline UGC 00330 & $2003-10-08$ & 1641 & NGA-M31-MOS5 & $5.923 \mathrm{E}-04$ & $6.384 \mathrm{E}-04$ & $5.842 \mathrm{E}-05$ & $4.020 \mathrm{E}-03$ & $1.846 \mathrm{E}-03$ & $1.798 \mathrm{E}-04$ & \\
\hline NGC 0151 & 2003-09-20 & 1628 & MISDR1-29519-0654 & $2.101 \mathrm{E}-04$ & $3.924 \mathrm{E}-04$ & $5.902 \mathrm{E}-06$ & $2.891 \mathrm{E}-03$ & $1.558 \mathrm{E}-03$ & $5.519 \mathrm{E}-05$ & \\
\hline NGC 0155 & 2003-09-20 & 1618 & MISDR1-29563-0654 & $2.060 \mathrm{E}-04$ & $4.740 \mathrm{E}-04$ & $4.365 \mathrm{E}-05$ & $2.871 \mathrm{E}-03$ & $1.563 \mathrm{E}-03$ & $9.605 \mathrm{E}-06$ & \\
\hline UGC 00344 & 2003-10-08 & 1641 & NGA-M31-MOS5 & $5.920 \mathrm{E}-04$ & $6.107 \mathrm{E}-04$ & $1.228 \mathrm{E}-05$ & $4.073 \mathrm{E}-03$ & $1.813 \mathrm{E}-03$ & $5.551 \mathrm{E}-05$ & $(2)$ \\
\hline NGC 0163 & 2003-09-20 & 1618 & MISDR1-29563-0654 & $2.244 \mathrm{E}-04$ & $4.792 \mathrm{E}-04$ & $2.046 \mathrm{E}-05$ & $2.882 \mathrm{E}-03$ & $1.495 \mathrm{E}-03$ & $2.629 \mathrm{E}-05$ & \\
\hline VV 548 & 2003-09-20 & 1628 & MISDR1-29519-0654 & $2.216 \mathrm{E}-04$ & $4.469 \mathrm{E}-04$ & $1.170 \mathrm{E}-05$ & $2.888 \mathrm{E}-03$ & $1.507 \mathrm{E}-03$ & $4.359 \mathrm{E}-06$ & \\
\hline NGC 0165 & 2003-09-20 & 1618 & MISDR1-29563-0654 & $2.219 \mathrm{E}-04$ & $4.721 \mathrm{E}-04$ & $1.964 \mathrm{E}-06$ & $2.877 \mathrm{E}-03$ & $1.493 \mathrm{E}-03$ & $5.123 \mathrm{E}-05$ & \\
\hline UGC 00372 & $2003-10-08$ & 3267 & NGA-M31-MOS9 & $7.478 \mathrm{E}-04$ & $5.547 \mathrm{E}-04$ & $5.869 \mathrm{E}-05$ & $4.379 \mathrm{E}-03$ & $1.405 \mathrm{E}-03$ & $2.243 \mathrm{E}-05$ & \\
\hline Cartwheel & $2003-10-10$ & 1632 & NGA-Cartwheel & $1.799 \mathrm{E}-04$ & $3.440 \mathrm{E}-04$ & $1.470 \mathrm{E}-06$ & $2.482 \mathrm{E}-03$ & $1.420 \mathrm{E}-03$ & $8.808 \mathrm{E}-06$ & (2) \\
\hline PGC 02269 & 2003-09-20 & 1630 & MISDR1-29518-0655 & $2.075 \mathrm{E}-04$ & $3.688 \mathrm{E}-04$ & $1.426 \mathrm{E}-05$ & $2.911 \mathrm{E}-03$ & $1.492 \mathrm{E}-03$ & $3.736 \mathrm{E}-05$ & \\
\hline UGC 00394 & 2003-10-08 & 1636 & NGA-M31-MOS2 & $7.364 \mathrm{E}-04$ & 7.773E-04 & $1.849 \mathrm{E}-05$ & $4.507 \mathrm{E}-03$ & $2.045 \mathrm{E}-03$ & $9.780 \mathrm{E}-05$ & \\
\hline NGC 0195 & 2003-09-20 & 1630 & MISDR1-29518-0655 & $2.011 \mathrm{E}-04$ & $3.579 \mathrm{E}-04$ & $1.111 \mathrm{E}-06$ & $2.900 \mathrm{E}-03$ & $1.459 \mathrm{E}-03$ & $2.324 \mathrm{E}-06$ & (1) \\
\hline NGC 0205 & 2003-10-08 & 1636 & NGA-M31-MOS2 & $7.295 \mathrm{E}-04$ & $6.905 \mathrm{E}-04$ & $6.067 \mathrm{E}-05$ & $5.001 \mathrm{E}-03$ & $2.074 \mathrm{E}-03$ & $2.828 \mathrm{E}-04$ & \\
\hline
\end{tabular}


Table 2-Continued

\begin{tabular}{|c|c|c|c|c|c|c|c|c|c|c|}
\hline \multirow[b]{2}{*}{$\begin{array}{l}\text { Object Name } \\
\text { (1) }\end{array}$} & \multirow[b]{2}{*}{$\begin{array}{c}\text { Date } \\
\text { Observed } \\
(2)\end{array}$} & \multirow[b]{2}{*}{$\begin{array}{l}\text { Exposure } \\
\quad(\mathrm{sec}) \\
(3)\end{array}$} & \multirow[b]{2}{*}{$\begin{array}{l}\text { Tile } \\
\text { (4) }\end{array}$} & \multicolumn{3}{|c|}{ FUV sky background } & \multicolumn{3}{|c|}{ NUV sky background } & \multirow[b]{2}{*}{$\begin{array}{l}\text { notes } \\
(11)\end{array}$} \\
\hline & & & & $\begin{array}{c}\text { mean } \\
(\text { counts/s) } \\
(5)\end{array}$ & $\begin{array}{c}<\sigma> \\
\text { (counts/s) } \\
(6)\end{array}$ & $\begin{array}{c}\sigma(\text { mean }) \\
(\text { counts/s) } \\
(7)\end{array}$ & $\begin{array}{c}\text { mean } \\
(\text { counts/s) } \\
(8)\end{array}$ & $\begin{array}{c}<\sigma> \\
(\text { counts/s) } \\
(9)\end{array}$ & $\begin{array}{c}\sigma(\text { mean }) \\
(\text { counts/s) } \\
(10)\end{array}$ & \\
\hline NGC 0213 & 2003-08-29 & 1631 & MISDR1-16778-0418 & $4.106 \mathrm{E}-04$ & $6.537 \mathrm{E}-04$ & $1.899 \mathrm{E}-05$ & $3.406 \mathrm{E}-03$ & $1.719 \mathrm{E}-03$ & $3.790 \mathrm{E}-05$ & (1) \\
\hline NGC 0223 & 2003-09-16 & 1602 & MISDR1-29030-0393 & $1.707 \mathrm{E}-04$ & $3.462 \mathrm{E}-04$ & $6.668 \mathrm{E}-06$ & $2.972 \mathrm{E}-03$ & $1.530 \mathrm{E}-03$ & $3.422 \mathrm{E}-05$ & \\
\hline MESSIER 032 & 2003-09-05 & 758 & NGA-M31-F1 & $1.286 \mathrm{E}-03$ & $1.374 \mathrm{E}-03$ & $6.005 \mathrm{E}-05$ & $8.369 \mathrm{E}-03$ & $4.200 \mathrm{E}-03$ & $2.474 \mathrm{E}-03$ & \\
\hline MESSIER 031 & 2003-09-05 & 800 & NGA-M31-MOSAIC & $9.290 \mathrm{E}-04$ & $5.240 \mathrm{E}-04$ & $1.790 \mathrm{E}-04$ & $4.712 \mathrm{E}-03$ & $1.529 \mathrm{E}-03$ & $7.000 \mathrm{E}-04$ & (3) \\
\hline UGC 00484 & $2003-10-07$ & 1161 & NGA-NGC0266 & $5.820 \mathrm{E}-04$ & $8.908 \mathrm{E}-04$ & $4.978 \mathrm{E}-05$ & $3.557 \mathrm{E}-03$ & $2.075 \mathrm{E}-03$ & $2.767 \mathrm{E}-05$ & \\
\hline NGC 0247 & 2003-09-18 & 2116 & NGA-NGC0247 & $2.106 \mathrm{E}-04$ & $3.372 \mathrm{E}-04$ & $1.629 \mathrm{E}-05$ & $2.764 \mathrm{E}-03$ & $1.343 \mathrm{E}-03$ & $1.018 \mathrm{E}-05$ & \\
\hline NGC 0253 & $2003-10-13$ & 3289 & NGA-NGC0253 & $1.983 \mathrm{E}-04$ & $2.691 \mathrm{E}-04$ & $3.676 \mathrm{E}-05$ & $2.602 \mathrm{E}-03$ & $1.031 \mathrm{E}-03$ & $2.742 \mathrm{E}-04$ & \\
\hline NGC 0247B & 2003-09-18 & 2116 & NGA-NGC0247 & $2.118 \mathrm{E}-04$ & $3.625 \mathrm{E}-04$ & $9.052 \mathrm{E}-05$ & $2.810 \mathrm{E}-03$ & $1.364 \mathrm{E}-03$ & $1.950 \mathrm{E}-04$ & (1) \\
\hline ESO 540-G025 & 2003-09-18 & 2116 & NGA-NGC0247 & $2.100 \mathrm{E}-04$ & $3.482 \mathrm{E}-04$ & $1.891 \mathrm{E}-06$ & $2.803 \mathrm{E}-03$ & $1.369 \mathrm{E}-03$ & $1.178 \mathrm{E}-05$ & \\
\hline NGC 0262 & $2003-10-07$ & 1161 & NGA-NGC0266 & $5.030 \mathrm{E}-04$ & $6.711 \mathrm{E}-04$ & $1.613 \mathrm{E}-05$ & $3.560 \mathrm{E}-03$ & $1.986 \mathrm{E}-03$ & $8.130 \mathrm{E}-05$ & \\
\hline UGC 00507 & $2003-10-13$ & 1611 & MISDR1-29028-0394 & $1.977 \mathrm{E}-04$ & $4.256 \mathrm{E}-04$ & $1.489 \mathrm{E}-05$ & $3.140 \mathrm{E}-03$ & $1.649 \mathrm{E}-03$ & $4.024 \mathrm{E}-05$ & $(2)$ \\
\hline NGC 0266 & $2003-10-07$ & 1161 & NGA-NGC0266 & $4.851 \mathrm{E}-04$ & $6.537 \mathrm{E}-04$ & $2.528 \mathrm{E}-06$ & $3.489 \mathrm{E}-03$ & $1.997 \mathrm{E}-03$ & $4.926 \mathrm{E}-05$ & \\
\hline NGC 0270 & 2003-09-21 & 1641 & MISDR1-29472-0656 & $2.840 \mathrm{E}-04$ & $4.324 \mathrm{E}-04$ & $5.085 \mathrm{E}-05$ & $3.005 \mathrm{E}-03$ & $1.515 \mathrm{E}-03$ & $5.745 \mathrm{E}-05$ & \\
\hline ESO 351-G011 & 2004-12-06 & 594 & MIS2DFSGP-41495-0443 & $2.738 \mathrm{E}-04$ & $8.301 \mathrm{E}-04$ & $1.881 \mathrm{E}-05$ & $2.636 \mathrm{E}-03$ & $2.430 \mathrm{E}-03$ & $8.605 \mathrm{E}-05$ & \\
\hline NGC 0277 & 2003-09-16 & 1602 & MISDR1-29471-0657 & $\ldots$ & $\ldots$ & $\ldots$ & $3.060 \mathrm{E}-03$ & $1.647 \mathrm{E}-03$ & $1.747 \mathrm{E}-05$ & $(4)(5)$ \\
\hline PGC 03004 & 2003-09-16 & 1602 & MISDR1-29471-0657 & $2.973 \mathrm{E}-04$ & $4.999 \mathrm{E}-04$ & $9.516 \mathrm{E}-06$ & 3.051E-03 & $1.649 \mathrm{E}-03$ & $3.901 \mathrm{E}-05$ & \\
\hline UGC 00533 & 2003-09-25 & 1685 & MISDR1-16846-0420 & $3.643 \mathrm{E}-04$ & $5.554 \mathrm{E}-04$ & $3.663 \mathrm{E}-05$ & $3.224 \mathrm{E}-03$ & $1.650 \mathrm{E}-03$ & 3.307E-05 & \\
\hline NGC 0291 & 2003-09-16 & 1602 & MISDR1-29471-0657 & $3.162 \mathrm{E}-04$ & $4.562 \mathrm{E}-04$ & $8.001 \mathrm{E}-06$ & $3.026 \mathrm{E}-03$ & $1.563 \mathrm{E}-03$ & $4.862 \mathrm{E}-05$ & \\
\hline NGC 0300 & $2003-10-10$ & 1632 & NGA-NGC0300 & $2.288 \mathrm{E}-04$ & $4.094 \mathrm{E}-04$ & $3.045 \mathrm{E}-06$ & $2.519 \mathrm{E}-03$ & $1.463 \mathrm{E}-03$ & $7.375 \mathrm{E}-05$ & \\
\hline UGC 00590 & 2003-10-06 & 1065 & MISDR1-16865-0420 & $2.965 \mathrm{E}-04$ & $5.534 \mathrm{E}-04$ & $1.350 \mathrm{E}-05$ & $3.196 \mathrm{E}-03$ & $2.003 \mathrm{E}-03$ & $2.684 \mathrm{E}-05$ & \\
\hline NGC 0311 & 2003-08-29 & 267 & NGA-NGC0315 & $\cdots$ & $\ldots$ & $\ldots$ & 3.553E-03 & $4.318 \mathrm{E}-03$ & $4.395 \mathrm{E}-05$ & $(4)(5)$ \\
\hline NGC 0315 & $2003-08-29$ & 267 & NGA-NGC0315 & $5.529 \mathrm{E}-04$ & $1.608 \mathrm{E}-03$ & $3.913 \mathrm{E}-05$ & $3.548 \mathrm{E}-03$ & $4.344 \mathrm{E}-03$ & $1.007 \mathrm{E}-04$ & \\
\hline ESO 351-G028 & $2004-09-29$ & 1610 & MIS2DFSGP-41641-0617 & $1.965 \mathrm{E}-04$ & $4.474 \mathrm{E}-04$ & $3.639 \mathrm{E}-06$ & $2.250 \mathrm{E}-03$ & $1.322 \mathrm{E}-03$ & $1.021 \mathrm{E}-04$ & \\
\hline UGC 00619 & $2003-10-05$ & 1317 & MISDR1-16886-0420 & $3.324 \mathrm{E}-04$ & $6.049 \mathrm{E}-04$ & $1.286 \mathrm{E}-05$ & $3.283 \mathrm{E}-03$ & $1.812 \mathrm{E}-03$ & $2.417 \mathrm{E}-05$ & \\
\hline NGC 0337 & 2003-09-25 & 438 & NGA-NGC0337 & 3.999E-04 & $1.022 \mathrm{E}-03$ & 4.113E-05 & $3.152 \mathrm{E}-03$ & $3.152 \mathrm{E}-03$ & $6.360 \mathrm{E}-05$ & \\
\hline PGC 03613 & 2003-09-25 & 438 & NGA-NGC0337 & $4.415 \mathrm{E}-04$ & $1.195 \mathrm{E}-03$ & $4.950 \mathrm{E}-05$ & $3.182 \mathrm{E}-03$ & $3.172 \mathrm{E}-03$ & $7.072 \mathrm{E}-05$ & \\
\hline UGC 00627 & $2003-10-06$ & 1936 & MISDR1-16908-0421 & $2.796 \mathrm{E}-04$ & $4.140 \mathrm{E}-04$ & $2.345 \mathrm{E}-06$ & $3.181 \mathrm{E}-03$ & $1.514 \mathrm{E}-03$ & $2.337 \mathrm{E}-05$ & (2) \\
\hline NGC 0337A & 2003-09-25 & 438 & NGA-NGC0337 & $4.106 \mathrm{E}-04$ & $1.000 \mathrm{E}-03$ & $2.508 \mathrm{E}-05$ & $3.127 \mathrm{E}-03$ & $3.031 \mathrm{E}-03$ & $3.265 \mathrm{E}-05$ & \\
\hline UGC 00652 & $2003-10-22$ & 1703 & NGA-LGS3 & $4.234 \mathrm{E}-04$ & $5.507 \mathrm{E}-04$ & $2.378 \mathrm{E}-05$ & $3.699 \mathrm{E}-03$ & $1.767 \mathrm{E}-03$ & $4.872 \mathrm{E}-05$ & \\
\hline ESO 352-G002 & 2004-09-29 & 1494 & MIS2DFSGP-41687-0504 & $2.263 \mathrm{E}-04$ & $4.279 \mathrm{E}-04$ & $4.254 \mathrm{E}-06$ & $2.409 \mathrm{E}-03$ & $1.471 \mathrm{E}-03$ & $1.711 \mathrm{E}-05$ & \\
\hline IC 1613 & $2003-10-01$ & 1700 & NGA-IC1613 & $2.111 \mathrm{E}-04$ & $3.826 \mathrm{E}-04$ & $6.559 \mathrm{E}-06$ & $3.258 \mathrm{E}-03$ & $1.627 \mathrm{E}-03$ & $2.224 \mathrm{E}-05$ & \\
\hline IC 1616 & $2004-12-04$ & 1653 & MIS2DFSGP-30656-0213 & $2.690 \mathrm{E}-04$ & $4.094 \mathrm{E}-04$ & $3.127 \mathrm{E}-05$ & $2.589 \mathrm{E}-03$ & $1.380 \mathrm{E}-03$ & $6.570 \mathrm{E}-05$ & \\
\hline
\end{tabular}


Table 2-Continued

\begin{tabular}{|c|c|c|c|c|c|c|c|c|c|c|}
\hline \multirow{2}{*}{$\begin{array}{l}\text { Object Name } \\
\text { (1) }\end{array}$} & \multirow{2}{*}{$\begin{array}{c}\text { Date } \\
\text { Observed } \\
(2)\end{array}$} & \multirow{2}{*}{$\begin{array}{l}\text { Exposure } \\
\quad(\mathrm{sec}) \\
(3)\end{array}$} & \multirow{2}{*}{$\begin{array}{l}\text { Tile } \\
(4)\end{array}$} & \multicolumn{3}{|c|}{ FUV sky background } & \multicolumn{3}{|c|}{ NUV sky background } & \multirow{2}{*}{$\begin{array}{r}\text { notes } \\
(11)\end{array}$} \\
\hline & & & & $\begin{array}{c}\text { mean } \\
(\text { counts/s) } \\
(5)\end{array}$ & $\begin{array}{c}<\sigma> \\
(\text { counts } / \mathrm{s}) \\
(6)\end{array}$ & $\begin{array}{c}\sigma(\text { mean }) \\
(\text { counts/s) } \\
(7)\end{array}$ & $\begin{array}{c}\text { mean } \\
\left(\begin{array}{c}\text { counts/s) } \\
(8)\end{array}\right.\end{array}$ & $\begin{array}{c}<\sigma> \\
(\text { counts/s) } \\
(9)\end{array}$ & $\begin{array}{c}\sigma(\text { mean }) \\
(\text { counts/s) } \\
(10)\end{array}$ & \\
\hline ESO 352-G007 & 2004-09-29 & 1494 & MIS2DFSGP-41687-0504 & $1.952 \mathrm{E}-04$ & $3.910 \mathrm{E}-04$ & 4.032E-06 & $2.313 \mathrm{E}-03$ & $1.393 \mathrm{E}-03$ & $1.200 \mathrm{E}-05$ & \\
\hline NGC 0392 & $2003-08-29$ & 1179 & NGA-NGC0410 & $6.098 \mathrm{E}-04$ & $9.925 \mathrm{E}-04$ & $6.763 \mathrm{E}-05$ & $3.697 \mathrm{E}-03$ & $2.115 \mathrm{E}-03$ & $1.078 \mathrm{E}-04$ & \\
\hline ESO 243-G041 & $2003-12-18$ & 10009 & PHOENIX-00 & $2.365 \mathrm{E}-04$ & $2.320 \mathrm{E}-04$ & $1.075 \mathrm{E}-06$ & $2.374 \mathrm{E}-03$ & $5.590 \mathrm{E}-04$ & $6.236 \mathrm{E}-05$ & \\
\hline ESO 296-G002 & $2003-10-10$ & 1631 & MIS2DFSGP-41792-0620 & $1.868 \mathrm{E}-04$ & $3.449 \mathrm{E}-04$ & $7.422 \mathrm{E}-06$ & $2.349 \mathrm{E}-03$ & $1.342 \mathrm{E}-03$ & $1.978 \mathrm{E}-06$ & \\
\hline ESO 243-G045 & $2003-12-18$ & 10009 & PHOENIX-00 & $2.381 \mathrm{E}-04$ & $2.379 \mathrm{E}-04$ & $1.375 \mathrm{E}-05$ & $2.361 \mathrm{E}-03$ & $5.601 \mathrm{E}-04$ & $7.933 \mathrm{E}-05$ & \\
\hline NGC 0403 & 2003-08-29 & 1179 & NGA-NGC0410 & $5.781 \mathrm{E}-04$ & $7.507 \mathrm{E}-04$ & $4.435 \mathrm{E}-05$ & $3.807 \mathrm{E}-03$ & $2.081 \mathrm{E}-03$ & 1.373E-04 & \\
\hline IC 1633 & $2003-12-18$ & 10009 & PHOENIX-00 & $2.341 \mathrm{E}-04$ & $2.276 \mathrm{E}-04$ & $4.850 \mathrm{E}-06$ & $2.372 \mathrm{E}-03$ & $5.577 \mathrm{E}-04$ & $4.274 \mathrm{E}-06$ & \\
\hline UGC 00726 & $2003-10-06$ & 1652 & MISDR1-29155-0397 & $3.315 \mathrm{E}-04$ & 4.699E-04 & $3.773 \mathrm{E}-05$ & $3.180 \mathrm{E}-03$ & $1.560 \mathrm{E}-03$ & $1.976 \mathrm{E}-06$ & \\
\hline NGC 0407 & 2003-08-29 & 1179 & NGA-NGC0410 & $5.477 \mathrm{E}-04$ & $7.588 \mathrm{E}-04$ & 5.403E-06 & $3.802 \mathrm{E}-03$ & $2.153 \mathrm{E}-03$ & 4.287E-06 & \\
\hline UGC 00732 & $2003-08-29$ & 1179 & NGA-NGC0410 & $5.477 \mathrm{E}-04$ & $8.569 \mathrm{E}-04$ & $9.718 \mathrm{E}-06$ & $3.651 \mathrm{E}-03$ & $2.056 \mathrm{E}-03$ & $8.684 \mathrm{E}-06$ & \\
\hline UGC 00736 & $2003-10-06$ & 1652 & MISDR1-29155-0397 & $3.646 \mathrm{E}-04$ & $5.323 \mathrm{E}-04$ & $5.586 \mathrm{E}-06$ & $3.176 \mathrm{E}-03$ & $1.564 \mathrm{E}-03$ & $1.726 \mathrm{E}-05$ & \\
\hline NGC 0410 & $2003-08-29$ & 1179 & NGA-NGC0410 & $5.386 \mathrm{E}-04$ & $7.239 \mathrm{E}-04$ & $5.367 \mathrm{E}-06$ & $3.830 \mathrm{E}-03$ & $2.155 \mathrm{E}-03$ & $9.494 \mathrm{E}-05$ & \\
\hline ESO 243-G051 & $2003-12-18$ & 10009 & PHOENIX-00 & $2.231 \mathrm{E}-04$ & $2.258 \mathrm{E}-04$ & $9.126 \mathrm{E}-06$ & $2.342 \mathrm{E}-03$ & $5.514 \mathrm{E}-04$ & $1.808 \mathrm{E}-05$ & \\
\hline ESO 243-G052 & $2003-12-18$ & 10009 & PHOENIX-00 & $2.252 \mathrm{E}-04$ & 2.329E-04 & $1.490 \mathrm{E}-05$ & $2.356 \mathrm{E}-03$ & $5.518 \mathrm{E}-04$ & $3.627 \mathrm{E}-05$ & \\
\hline PGC 04663 & $2003-10-05$ & 1665 & MISDR1-29508-0660 & $2.474 \mathrm{E}-04$ & $4.022 \mathrm{E}-04$ & $1.023 \mathrm{E}-05$ & $2.912 \mathrm{E}-03$ & $1.512 \mathrm{E}-03$ & $2.213 \mathrm{E}-05$ & (1) \\
\hline NGC 0467 & 2003-10-05 & 1668 & NGRG-A227 & $2.529 \mathrm{E}-04$ & 4.091E-04 & $9.976 \mathrm{E}-06$ & $3.429 \mathrm{E}-03$ & $1.650 \mathrm{E}-03$ & 1.369E-04 & \\
\hline NGC 0470 & $2003-10-05$ & 1668 & NGRG-A227 & $2.653 \mathrm{E}-04$ & $4.381 \mathrm{E}-04$ & $9.383 \mathrm{E}-06$ & $3.465 \mathrm{E}-03$ & $1.699 \mathrm{E}-03$ & $6.259 \mathrm{E}-05$ & \\
\hline NGC 0474 & $2003-10-05$ & 1668 & NGRG-A227 & $2.577 \mathrm{E}-04$ & $4.297 \mathrm{E}-04$ & 7.319E-06 & $3.413 \mathrm{E}-03$ & $1.691 \mathrm{E}-03$ & $5.027 \mathrm{E}-05$ & \\
\hline ESO 352-G047 & 2003-10-11 & 1631 & MIS2DFSGP-41894-0507 & $2.273 \mathrm{E}-04$ & $3.780 \mathrm{E}-04$ & $4.798 \mathrm{E}-06$ & $2.473 \mathrm{E}-03$ & $1.372 \mathrm{E}-03$ & $5.104 \mathrm{E}-05$ & \\
\hline UGC 00885 & $2003-10-05$ & 1668 & NGRG-A227 & $2.205 \mathrm{E}-04$ & $3.705 \mathrm{E}-04$ & $1.629 \mathrm{E}-05$ & $3.268 \mathrm{E}-03$ & $1.568 \mathrm{E}-03$ & $2.044 \mathrm{E}-05$ & \\
\hline ESO 352-G050 & $2003-10-10$ & 1632 & MIS2DFSGP-41895-0565 & $2.075 \mathrm{E}-04$ & $3.626 \mathrm{E}-04$ & $1.133 \mathrm{E}-05$ & $2.406 \mathrm{E}-03$ & $1.349 \mathrm{E}-03$ & $2.029 \mathrm{E}-05$ & \\
\hline NGC 0479 & $2003-10-05$ & 1668 & NGRG-A227 & $2.543 \mathrm{E}-04$ & $4.845 \mathrm{E}-04$ & $9.059 \mathrm{E}-06$ & $3.297 \mathrm{E}-03$ & $1.642 \mathrm{E}-03$ & $1.949 \mathrm{E}-05$ & \\
\hline NGC 0491 & $2003-10-11$ & 1631 & MIS2DFSGP-41894-0507 & $2.333 \mathrm{E}-04$ & $3.898 \mathrm{E}-04$ & $1.498 \mathrm{E}-05$ & $2.473 \mathrm{E}-03$ & $1.387 \mathrm{E}-03$ & $2.727 \mathrm{E}-05$ & \\
\hline UGC 00910 & $2003-10-16$ & 1662 & MISDR1-17027-0424 & $3.134 \mathrm{E}-04$ & $5.618 \mathrm{E}-04$ & $1.471 \mathrm{E}-05$ & $3.325 \mathrm{E}-03$ & $1.680 \mathrm{E}-03$ & $2.073 \mathrm{E}-04$ & (6) \\
\hline ESO 352-G057 & $2003-10-11$ & 1631 & MIS2DFSGP-41894-0507 & $2.331 \mathrm{E}-04$ & $3.851 \mathrm{E}-04$ & $2.150 \mathrm{E}-06$ & $2.481 \mathrm{E}-03$ & $1.376 \mathrm{E}-03$ & $2.062 \mathrm{E}-05$ & \\
\hline ESO 352-G062 & $2003-10-10$ & 1632 & MIS2DFSGP-41895-0565 & $2.211 \mathrm{E}-04$ & $4.108 \mathrm{E}-04$ & $1.586 \mathrm{E}-05$ & $2.439 \mathrm{E}-03$ & $1.398 \mathrm{E}-03$ & $1.034 \mathrm{E}-04$ & \\
\hline ESO 352-G064 & $2003-10-10$ & 1632 & MIS2DFSGP-41895-0565 & $2.166 \mathrm{E}-04$ & $3.913 \mathrm{E}-04$ & $2.625 \mathrm{E}-05$ & $2.433 \mathrm{E}-03$ & $1.374 \mathrm{E}-03$ & $2.888 \mathrm{E}-05$ & \\
\hline NGC 0527 & $2003-10-10$ & 1632 & MIS2DFSGP-41895-0565 & $2.217 \mathrm{E}-04$ & $3.987 \mathrm{E}-04$ & $2.500 \mathrm{E}-06$ & $2.446 \mathrm{E}-03$ & $1.359 \mathrm{E}-03$ & $9.073 \mathrm{E}-05$ & \\
\hline NGC 0514 & $2003-10-21$ & 1705 & NGA-NGC0514 & $2.662 \mathrm{E}-04$ & $4.536 \mathrm{E}-04$ & $3.309 \mathrm{E}-06$ & $3.343 \mathrm{E}-03$ & $1.669 \mathrm{E}-03$ & $6.682 \mathrm{E}-06$ & \\
\hline ESO 352-G069 & $2003-10-10$ & 1632 & MIS2DFSGP-41895-0565 & $2.294 \mathrm{E}-04$ & $4.549 \mathrm{E}-04$ & $1.540 \mathrm{E}-05$ & $2.412 \mathrm{E}-03$ & $1.360 \mathrm{E}-03$ & $2.525 \mathrm{E}-06$ & (1) \\
\hline UGC 00957 & $2003-10-22$ & 1703 & NGA-NGC0520 & $2.513 \mathrm{E}-04$ & 4.103E-04 & $5.039 \mathrm{E}-06$ & $3.404 \mathrm{E}-03$ & $1.643 \mathrm{E}-03$ & $2.389 \mathrm{E}-06$ & \\
\hline NGC 0520 & $2003-10-22$ & 1703 & NGA-NGC0520 & $2.614 \mathrm{E}-04$ & $4.146 \mathrm{E}-04$ & $2.289 \mathrm{E}-05$ & $3.397 \mathrm{E}-03$ & $1.624 \mathrm{E}-03$ & $3.589 \mathrm{E}-06$ & \\
\hline
\end{tabular}


Table 2-Continued

\begin{tabular}{|c|c|c|c|c|c|c|c|c|c|c|}
\hline \multirow{2}{*}{$\begin{array}{l}\text { Object Name } \\
\text { (1) }\end{array}$} & \multirow{2}{*}{$\begin{array}{c}\text { Date } \\
\text { Observed } \\
(2)\end{array}$} & \multirow{2}{*}{$\begin{array}{l}\text { Exposure } \\
\quad(\mathrm{sec}) \\
(3)\end{array}$} & \multirow{2}{*}{$\begin{array}{l}\text { Tile } \\
\text { (4) }\end{array}$} & \multicolumn{3}{|c|}{ FUV sky background } & \multicolumn{3}{|c|}{ NUV sky background } & \multirow{2}{*}{$\begin{array}{r}\text { notes } \\
(11)\end{array}$} \\
\hline & & & & $\begin{array}{c}\text { mean } \\
(\text { counts/s) } \\
(5)\end{array}$ & $\begin{array}{c}<\sigma> \\
(\text { counts } / \mathrm{s}) \\
(6)\end{array}$ & $\begin{array}{c}\sigma(\text { mean }) \\
(\text { counts/s) } \\
(7)\end{array}$ & $\begin{array}{c}\text { mean } \\
(\text { counts/s) } \\
(8)\end{array}$ & $\begin{array}{c}<\sigma> \\
(\text { counts/s) } \\
(9)\end{array}$ & $\begin{array}{c}\sigma(\text { mean }) \\
(\text { counts/s) } \\
(10)\end{array}$ & \\
\hline NGC 0530 & $2003-10-23$ & 1699 & NGA-NGC0541 & $3.109 \mathrm{E}-04$ & $4.359 \mathrm{E}-04$ & $3.959 \mathrm{E}-05$ & $3.038 \mathrm{E}-03$ & $1.515 \mathrm{E}-03$ & $6.670 \mathrm{E}-05$ & \\
\hline IC 0107 & $2003-10-16$ & 1674 & MISDR1-17055-0424 & $3.108 \mathrm{E}-04$ & $5.259 \mathrm{E}-04$ & $7.248 \mathrm{E}-06$ & $3.299 \mathrm{E}-03$ & $1.671 \mathrm{E}-03$ & $1.927 \mathrm{E}-05$ & \\
\hline UGC 00984 & $2003-10-23$ & 1699 & NGA-NGC0541 & $3.041 \mathrm{E}-04$ & $4.329 \mathrm{E}-04$ & $3.867 \mathrm{E}-05$ & $3.045 \mathrm{E}-03$ & $1.535 \mathrm{E}-03$ & $4.460 \mathrm{E}-05$ & \\
\hline IC 1698 & $2003-10-16$ & 1674 & MISDR1-17055-0424 & $3.087 \mathrm{E}-04$ & $5.187 \mathrm{E}-04$ & $1.388 \mathrm{E}-05$ & 3.307E-03 & $1.673 \mathrm{E}-03$ & $6.936 \mathrm{E}-05$ & \\
\hline UGC 00985 & $2003-10-16$ & 1674 & MISDR1-17055-0424 & $2.768 \mathrm{E}-04$ & $4.602 \mathrm{E}-04$ & $2.171 \mathrm{E}-05$ & $3.249 \mathrm{E}-03$ & $1.648 \mathrm{E}-03$ & $1.060 \mathrm{E}-05$ & \\
\hline IC 1700 & $2003-10-16$ & 1674 & MISDR1-17055-0424 & $3.102 \mathrm{E}-04$ & $5.267 \mathrm{E}-04$ & $1.141 \mathrm{E}-05$ & $3.299 \mathrm{E}-03$ & $1.663 \mathrm{E}-03$ & $3.479 \mathrm{E}-05$ & \\
\hline NGC 0538 & $2003-10-23$ & 1699 & NGA-NGC0541 & $2.912 \mathrm{E}-04$ & $4.242 \mathrm{E}-04$ & $2.716 \mathrm{E}-05$ & $3.056 \mathrm{E}-03$ & $1.546 \mathrm{E}-03$ & $3.739 \mathrm{E}-05$ & \\
\hline NGC 0535 & $2003-10-23$ & 1699 & NGA-NGC0541 & $3.016 \mathrm{E}-04$ & $4.387 \mathrm{E}-04$ & $5.778 \mathrm{E}-06$ & 3.093E-03 & $1.572 \mathrm{E}-03$ & $5.830 \mathrm{E}-05$ & \\
\hline UGC 00999 & $2003-10-16$ & 1674 & MISDR1-17055-0424 & $3.077 \mathrm{E}-04$ & $5.471 \mathrm{E}-04$ & $6.672 \mathrm{E}-06$ & $3.276 \mathrm{E}-03$ & $1.651 \mathrm{E}-03$ & $3.521 \mathrm{E}-05$ & \\
\hline UGC 01003 & $2003-10-23$ & 1699 & NGA-NGC0541 & $2.998 \mathrm{E}-04$ & $4.446 \mathrm{E}-04$ & $4.016 \mathrm{E}-05$ & $3.088 \mathrm{E}-03$ & $1.573 \mathrm{E}-03$ & $2.585 \mathrm{E}-06$ & (1) \\
\hline NGC 0541 & $2003-10-23$ & 1699 & NGA-NGC0541 & $3.004 \mathrm{E}-04$ & $4.456 \mathrm{E}-04$ & $8.066 \mathrm{E}-06$ & $3.104 \mathrm{E}-03$ & $1.591 \mathrm{E}-03$ & $5.073 \mathrm{E}-05$ & \\
\hline NGC 0545 & $2003-10-23$ & 1699 & NGA-NGC0541 & $2.973 \mathrm{E}-04$ & $4.540 \mathrm{E}-04$ & $7.952 \mathrm{E}-06$ & $3.102 \mathrm{E}-03$ & $1.599 \mathrm{E}-03$ & $4.191 \mathrm{E}-05$ & \\
\hline NGC 0547 & $2003-10-23$ & 1699 & NGA-NGC0541 & $2.977 \mathrm{E}-04$ & $4.565 \mathrm{E}-04$ & $2.027 \mathrm{E}-05$ & $3.100 \mathrm{E}-03$ & $1.600 \mathrm{E}-03$ & $1.737 \mathrm{E}-05$ & \\
\hline NGC 0557 & $2003-10-23$ & 1699 & NGA-NGC0541 & 3.367E-04 & $5.219 \mathrm{E}-04$ & $8.541 \mathrm{E}-07$ & 3.051E-03 & $1.546 \mathrm{E}-03$ & 7.263E-05 & \\
\hline ESO 353-G002 & $2004-09-26$ & 1640 & MIS2DFSGP-41950-0566 & $2.029 \mathrm{E}-04$ & $3.747 \mathrm{E}-04$ & $1.277 \mathrm{E}-05$ & $2.263 \mathrm{E}-03$ & $1.314 \mathrm{E}-03$ & $2.686 \mathrm{E}-05$ & \\
\hline UGC 01026 & $2003-10-16$ & 1676 & MISDR1-17084-0424 & $2.810 \mathrm{E}-04$ & $4.346 \mathrm{E}-04$ & $1.246 \mathrm{E}-05$ & 3.273E-03 & $1.636 \mathrm{E}-03$ & 7.110E-06 & \\
\hline UGC 01040 & $2003-10-23$ & 1699 & NGA-NGC0541 & $2.669 \mathrm{E}-04$ & $4.846 \mathrm{E}-04$ & $2.965 \mathrm{E}-05$ & $3.080 \mathrm{E}-03$ & $1.586 \mathrm{E}-03$ & $1.681 \mathrm{E}-05$ & \\
\hline NGC 0568 & $2004-09-26$ & 1640 & MIS2DFSGP-41950-0566 & $2.059 \mathrm{E}-04$ & $4.165 \mathrm{E}-04$ & $3.386 \mathrm{E}-06$ & $2.258 \mathrm{E}-03$ & $1.312 \mathrm{E}-03$ & $2.601 \mathrm{E}-05$ & \\
\hline UGC 01057 & $2003-10-16$ & 1676 & MISDR1-17084-0424 & $2.978 \mathrm{E}-04$ & $5.476 \mathrm{E}-04$ & $3.395 \mathrm{E}-05$ & $3.264 \mathrm{E}-03$ & $1.563 \mathrm{E}-03$ & $2.356 \mathrm{E}-05$ & \\
\hline NGC 0574 & 2004-09-26 & 1637 & MIS2DFSGP-42005-0567 & $1.884 \mathrm{E}-04$ & $3.455 \mathrm{E}-04$ & $5.810 \mathrm{E}-06$ & $2.204 \mathrm{E}-03$ & $1.264 \mathrm{E}-03$ & $3.113 \mathrm{E}-05$ & \\
\hline IC 0127 & $2003-10-20$ & 1706 & NGA-NGC0584 & $2.532 \mathrm{E}-04$ & $4.149 \mathrm{E}-04$ & $1.756 \mathrm{E}-05$ & $2.886 \mathrm{E}-03$ & $1.498 \mathrm{E}-03$ & $7.970 \mathrm{E}-05$ & (1) \\
\hline NGC 0584 & $2003-10-20$ & 1706 & NGA-NGC0584 & $2.672 \mathrm{E}-04$ & $4.183 \mathrm{E}-04$ & $1.355 \mathrm{E}-05$ & $2.887 \mathrm{E}-03$ & $1.504 \mathrm{E}-03$ & $7.203 \mathrm{E}-05$ & \\
\hline NGC 0586 & $2003-10-20$ & 1706 & NGA-NGC0584 & $2.623 \mathrm{E}-04$ & $4.164 \mathrm{E}-04$ & $1.266 \mathrm{E}-05$ & $2.892 \mathrm{E}-03$ & $1.500 \mathrm{E}-03$ & $1.590 \mathrm{E}-05$ & \\
\hline MESSIER 033 & $2003-11-25$ & 3400 & NGA-M33-MOS0 & $5.157 \mathrm{E}-04$ & $4.610 \mathrm{E}-04$ & $2.139 \mathrm{E}-05$ & $3.874 \mathrm{E}-03$ & $1.417 \mathrm{E}-03$ & $2.233 \mathrm{E}-04$ & \\
\hline NGC 0628 & $2003-10-07$ & 1644 & NGA-NGC0628 & $3.679 \mathrm{E}-04$ & $5.107 \mathrm{E}-04$ & $2.243 \mathrm{E}-06$ & $3.531 \mathrm{E}-03$ & $1.727 \mathrm{E}-03$ & $7.508 \mathrm{E}-05$ & \\
\hline UGC 01181 & $2004-11-15$ & 2965 & MISDR2-17173-0426 & $3.321 \mathrm{E}-04$ & $3.404 \mathrm{E}-04$ & $9.313 \mathrm{E}-06$ & $3.222 \mathrm{E}-03$ & $1.174 \mathrm{E}-03$ & $1.920 \mathrm{E}-05$ & \\
\hline IC 0148 & 2004-10-04 & 1629 & MISDR2-17206-0426 & $3.103 \mathrm{E}-04$ & $4.597 \mathrm{E}-04$ & $9.707 \mathrm{E}-06$ & $3.193 \mathrm{E}-03$ & $1.625 \mathrm{E}-03$ & $1.785 \mathrm{E}-06$ & \\
\hline UGC 01200 & $2004-10-03$ & 1616 & MISDR2-17207-0426 & $2.723 \mathrm{E}-04$ & $4.182 \mathrm{E}-04$ & $1.023 \mathrm{E}-05$ & $3.145 \mathrm{E}-03$ & $1.560 \mathrm{E}-03$ & $5.994 \mathrm{E}-05$ & \\
\hline NGC 0660 & 2004-10-04 & 1629 & MISDR2-17206-0426 & $3.017 \mathrm{E}-04$ & $4.396 \mathrm{E}-04$ & $1.287 \mathrm{E}-05$ & $3.205 \mathrm{E}-03$ & $1.585 \mathrm{E}-03$ & $8.168 \mathrm{E}-06$ & \\
\hline UGC 01211 & $2004-10-04$ & 1629 & MISDR2-17206-0426 & $3.351 \mathrm{E}-04$ & $5.004 \mathrm{E}-04$ & $1.509 \mathrm{E}-05$ & $3.248 \mathrm{E}-03$ & $1.622 \mathrm{E}-03$ & $1.206 \mathrm{E}-05$ & \\
\hline IC 0159 & $2003-10-14$ & 1292 & MISDR1-17833-0664 & $2.096 \mathrm{E}-04$ & $4.091 \mathrm{E}-04$ & $3.251 \mathrm{E}-07$ & $2.758 \mathrm{E}-03$ & $1.664 \mathrm{E}-03$ & $1.116 \mathrm{E}-04$ & (1) \\
\hline PGC 06504 & $2003-10-14$ & 1292 & MISDR1-17833-0664 & $2.087 \mathrm{E}-04$ & $4.059 \mathrm{E}-04$ & $2.280 \mathrm{E}-07$ & $2.745 \mathrm{E}-03$ & $1.657 \mathrm{E}-03$ & $2.411 \mathrm{E}-05$ & (1) \\
\hline
\end{tabular}


Table 2-Continued

\begin{tabular}{|c|c|c|c|c|c|c|c|c|c|c|}
\hline \multirow{2}{*}{$\begin{array}{l}\text { Object Name } \\
\text { (1) }\end{array}$} & \multirow{2}{*}{$\begin{array}{c}\text { Date } \\
\text { Observed } \\
(2)\end{array}$} & \multirow{2}{*}{$\begin{array}{l}\text { Exposure } \\
\quad(\mathrm{sec}) \\
(3)\end{array}$} & \multirow{2}{*}{$\begin{array}{l}\text { Tile } \\
(4) \\
\end{array}$} & \multicolumn{3}{|c|}{ FUV sky background } & \multicolumn{3}{|c|}{ NUV sky background } & \multirow{2}{*}{$\begin{array}{l}\text { notes } \\
\text { (11) }\end{array}$} \\
\hline & & & & $\begin{array}{c}\text { mean } \\
(\text { counts/s) } \\
(5)\end{array}$ & $\begin{array}{c}<\sigma> \\
(\text { counts/s) } \\
(6)\end{array}$ & $\begin{array}{c}\sigma(\text { mean }) \\
(\text { counts/s) } \\
(7)\end{array}$ & $\begin{array}{c}\text { mean } \\
(\text { counts/s) } \\
(8)\end{array}$ & $\begin{array}{c}<\sigma> \\
(\text { counts/s) } \\
(9)\end{array}$ & $\begin{array}{c}\sigma(\text { mean }) \\
(\text { counts/s }) \\
(10)\end{array}$ & \\
\hline NGC 0671 & $2003-10-20$ & 1095 & MISDR1-17240-0429 & $2.726 \mathrm{E}-04$ & $5.086 \mathrm{E}-04$ & $9.548 \mathrm{E}-06$ & $3.433 \mathrm{E}-03$ & $1.985 \mathrm{E}-03$ & $6.826 \mathrm{E}-05$ & \\
\hline UGC 01261 & 2003-10-07 & 1644 & MISDR1-17274-0429 & $3.531 \mathrm{E}-04$ & $5.394 \mathrm{E}-04$ & $1.733 \mathrm{E}-05$ & $3.446 \mathrm{E}-03$ & $1.734 \mathrm{E}-03$ & $9.789 \mathrm{E}-05$ & \\
\hline UGC 01262 & 2003-10-07 & 1644 & MISDR1-17274-0429 & $3.473 \mathrm{E}-04$ & $5.706 \mathrm{E}-04$ & $9.009 \mathrm{E}-06$ & $3.366 \mathrm{E}-03$ & $1.673 \mathrm{E}-03$ & $7.038 \mathrm{E}-06$ & (1) \\
\hline UGC 01264 & 2003-10-07 & 1638 & MISDR1-17239-0429 & $3.278 \mathrm{E}-04$ & $4.639 \mathrm{E}-04$ & $8.572 \mathrm{E}-06$ & $3.345 \mathrm{E}-03$ & $1.607 \mathrm{E}-03$ & $9.515 \mathrm{E}-05$ & $(1)(2)$ \\
\hline NGC 0676 & $2003-10-23$ & 1721 & NGA-NGC0693 & $3.601 \mathrm{E}-04$ & $4.992 \mathrm{E}-04$ & $2.258 \mathrm{E}-08$ & $3.494 \mathrm{E}-03$ & $1.640 \mathrm{E}-03$ & $6.030 \mathrm{E}-05$ & (6) \\
\hline UGC 01271 & 2003-10-07 & 1644 & MISDR1-17274-0429 & $3.458 \mathrm{E}-04$ & $4.941 \mathrm{E}-04$ & $6.162 \mathrm{E}-06$ & $3.478 \mathrm{E}-03$ & $1.721 \mathrm{E}-03$ & $7.717 \mathrm{E}-05$ & \\
\hline UGC 01274 & $2003-10-07$ & 1644 & MISDR1-17274-0429 & $\cdots$ & $\cdots$ & $\cdots$ & $3.446 \mathrm{E}-03$ & $1.644 \mathrm{E}-03$ & $8.124 \mathrm{E}-05$ & $(4)(5)$ \\
\hline UGC 01278 & 2003-10-07 & 1644 & MISDR1-17274-0429 & $3.278 \mathrm{E}-04$ & $4.542 \mathrm{E}-04$ & $2.557 \mathrm{E}-05$ & $3.427 \mathrm{E}-03$ & $1.604 \mathrm{E}-03$ & $1.136 \mathrm{E}-05$ & \\
\hline NGC 0693 & $2003-10-23$ & 1721 & NGA-NGC0693 & $3.166 \mathrm{E}-04$ & $4.532 \mathrm{E}-04$ & $3.828 \mathrm{E}-06$ & $3.444 \mathrm{E}-03$ & $1.670 \mathrm{E}-03$ & $1.383 \mathrm{E}-05$ & \\
\hline UGC 01312 & 2003-10-07 & 1644 & MISDR1-17274-0429 & 3.639E-04 & $5.362 \mathrm{E}-04$ & $9.145 \mathrm{E}-06$ & $3.442 \mathrm{E}-03$ & $1.654 \mathrm{E}-03$ & $1.355 \mathrm{E}-05$ & \\
\hline ESO 245-G007 & 2004-09-18 & 1706 & NGA-Phoenix & $2.449 \mathrm{E}-04$ & $4.040 \mathrm{E}-04$ & $1.220 \mathrm{E}-05$ & $2.289 \mathrm{E}-03$ & $1.346 \mathrm{E}-03$ & $1.043 \mathrm{E}-05$ & \\
\hline NGC 0707 & 2003-10-03 & 1599 & MISDR1-17881-0664 & $1.905 \mathrm{E}-04$ & $3.598 \mathrm{E}-04$ & $8.780 \mathrm{E}-06$ & $2.687 \mathrm{E}-03$ & $1.504 \mathrm{E}-03$ & $1.106 \mathrm{E}-05$ & (1) \\
\hline NGC 0706 & $2003-10-23$ & 1721 & NGA-NGC0693 & $3.518 \mathrm{E}-04$ & $5.054 \mathrm{E}-04$ & $2.186 \mathrm{E}-05$ & $3.466 \mathrm{E}-03$ & $1.667 \mathrm{E}-03$ & $5.337 \mathrm{E}-06$ & \\
\hline UGC 01364 & 2003-10-16 & 1662 & MISDR1-17272-0430 & $2.669 \mathrm{E}-04$ & $4.085 \mathrm{E}-04$ & $1.630 \mathrm{E}-05$ & $3.280 \mathrm{E}-03$ & $1.616 \mathrm{E}-03$ & $2.309 \mathrm{E}-05$ & \\
\hline PGC 07064 & 2003-09-27 & 1702 & MISDR1-17982-0665 & $2.174 \mathrm{E}-04$ & $4.511 \mathrm{E}-04$ & $1.381 \mathrm{E}-05$ & $2.730 \mathrm{E}-03$ & $1.435 \mathrm{E}-03$ & $5.281 \mathrm{E}-05$ & \\
\hline PGC 07210 & 2003-09-27 & 1702 & MISDR1-17982-0665 & $1.927 \mathrm{E}-04$ & $3.436 \mathrm{E}-04$ & $2.788 \mathrm{E}-06$ & $2.679 \mathrm{E}-03$ & $1.436 \mathrm{E}-03$ & $6.108 \mathrm{E}-05$ & (1) \\
\hline UGC 01408 & $2003-10-15$ & 1661 & MISDR1-17345-0427 & 3.103E-04 & $4.898 \mathrm{E}-04$ & $7.060 \mathrm{E}-06$ & $3.489 \mathrm{E}-03$ & $1.729 \mathrm{E}-03$ & $9.269 \mathrm{E}-06$ & \\
\hline IC 1755 & $2003-10-17$ & 1668 & MISDR1-17307-0430 & $2.929 \mathrm{E}-04$ & $4.260 \mathrm{E}-04$ & $1.149 \mathrm{E}-05$ & $3.364 \mathrm{E}-03$ & $1.594 \mathrm{E}-03$ & $8.886 \mathrm{E}-06$ & \\
\hline UGC 01448 & 2004-12-14 & 1161 & NGA-UGC1449 & $3.271 \mathrm{E}-04$ & $6.678 \mathrm{E}-04$ & $2.730 \mathrm{E}-06$ & $3.511 \mathrm{E}-03$ & $1.957 \mathrm{E}-03$ & $5.344 \mathrm{E}-05$ & \\
\hline KUG 0156-084 & 2003-10-14 & 520 & MISDR1-17980-0665 & $2.103 \mathrm{E}-04$ & $8.166 \mathrm{E}-04$ & $2.083 \mathrm{E}-05$ & $2.739 \mathrm{E}-03$ & $2.707 \mathrm{E}-03$ & $3.546 \mathrm{E}-05$ & (1) (2) \\
\hline NGC 0770 & $2003-10-21$ & 1705 & NGA-NGC0772 & 4.645E-04 & $5.644 \mathrm{E}-04$ & $3.189 \mathrm{E}-05$ & $4.740 \mathrm{E}-03$ & $2.008 \mathrm{E}-03$ & $2.402 \mathrm{E}-04$ & \\
\hline NGC 0772 & 2003-10-21 & 1705 & NGA-NGC0772 & 4.692E-04 & $5.608 \mathrm{E}-04$ & $8.427 \mathrm{E}-05$ & $4.778 \mathrm{E}-03$ & $2.013 \mathrm{E}-03$ & $4.666 \mathrm{E}-05$ & \\
\hline UGC 01468 & 2003-10-15 & 1664 & MISDR1-17344-0427 & $3.315 \mathrm{E}-04$ & $4.970 \mathrm{E}-04$ & $1.720 \mathrm{E}-05$ & $3.459 \mathrm{E}-03$ & $1.719 \mathrm{E}-03$ & $5.885 \mathrm{E}-05$ & \\
\hline NGC 0774 & 2003-10-15 & 1664 & MISDR1-17344-0427 & $3.271 \mathrm{E}-04$ & $4.933 \mathrm{E}-04$ & $1.681 \mathrm{E}-05$ & $3.441 \mathrm{E}-03$ & $1.711 \mathrm{E}-03$ & $5.824 \mathrm{E}-06$ & \\
\hline NGC 0777 & $2003-10-22$ & 1989 & NGA-NGC0777 & $4.227 \mathrm{E}-04$ & $4.907 \mathrm{E}-04$ & $2.146 \mathrm{E}-05$ & $3.630 \mathrm{E}-03$ & $1.619 \mathrm{E}-03$ & $4.638 \mathrm{E}-05$ & \\
\hline NGC 0778 & $2003-10-22$ & 1989 & NGA-NGC0777 & 4.174E-04 & $4.866 \mathrm{E}-04$ & $7.388 \mathrm{E}-06$ & $3.620 \mathrm{E}-03$ & $1.603 \mathrm{E}-03$ & $1.254 \mathrm{E}-05$ & \\
\hline NGC 0787 & 2003-10-01 & 3347 & MISDR1-18032-0666 & $2.044 \mathrm{E}-04$ & $3.076 \mathrm{E}-04$ & $1.364 \mathrm{E}-06$ & $2.686 \mathrm{E}-03$ & $1.041 \mathrm{E}-03$ & $3.119 \mathrm{E}-06$ & \\
\hline PGC 07654 & 2003-10-14 & 520 & MISDR1-17980-0665 & $1.809 \mathrm{E}-04$ & $5.991 \mathrm{E}-04$ & $1.386 \mathrm{E}-06$ & $2.657 \mathrm{E}-03$ & $2.534 \mathrm{E}-03$ & $9.652 \mathrm{E}-06$ & (1) \\
\hline NGC 0783 & $2003-10-22$ & 1989 & NGA-NGC0777 & $5.276 \mathrm{E}-04$ & $6.410 \mathrm{E}-04$ & $2.047 \mathrm{E}-05$ & $3.748 \mathrm{E}-03$ & $1.617 \mathrm{E}-03$ & $4.502 \mathrm{E}-05$ & \\
\hline UGCA 023 & 2003-10-01 & 3347 & MISDR1-18032-0666 & $1.974 \mathrm{E}-04$ & $2.467 \mathrm{E}-04$ & $8.726 \mathrm{E}-06$ & $2.679 \mathrm{E}-03$ & $1.009 \mathrm{E}-03$ & $9.768 \mathrm{E}-06$ & (1) \\
\hline NGC 0809 & 2003-10-14 & 1201 & MISDR1-18031-0666 & $1.732 \mathrm{E}-04$ & $3.853 \mathrm{E}-04$ & $6.413 \mathrm{E}-06$ & $2.672 \mathrm{E}-03$ & $1.699 \mathrm{E}-03$ & $4.510 \mathrm{E}-06$ & (1) \\
\hline UGC 01584 & $2003-10-15$ & 1663 & MISDR1-17420-0427 & 3.635E-04 & $4.883 \mathrm{E}-04$ & $1.215 \mathrm{E}-05$ & $3.566 \mathrm{E}-03$ & $1.722 \mathrm{E}-03$ & $3.858 \mathrm{E}-05$ & \\
\hline
\end{tabular}


Table 2-Continued

\begin{tabular}{|c|c|c|c|c|c|c|c|c|c|c|}
\hline \multirow[b]{2}{*}{$\begin{array}{l}\text { Object Name } \\
\text { (1) }\end{array}$} & \multirow[b]{2}{*}{$\begin{array}{c}\text { Date } \\
\text { Observed } \\
(2)\end{array}$} & \multirow[b]{2}{*}{$\begin{array}{l}\text { Exposure } \\
\quad(\mathrm{sec}) \\
(3)\end{array}$} & \multirow[b]{2}{*}{$\begin{array}{l}\text { Tile } \\
\text { (4) }\end{array}$} & \multicolumn{3}{|c|}{ FUV sky background } & \multicolumn{3}{|c|}{ NUV sky background } & \multirow{2}{*}{$\begin{array}{c}\text { notes } \\
\text { (11) }\end{array}$} \\
\hline & & & & $\begin{array}{c}\text { mean } \\
(\text { counts/s) } \\
(5)\end{array}$ & $\begin{array}{c}<\sigma> \\
(\text { counts/s) } \\
(6)\end{array}$ & $\begin{array}{c}\sigma(\text { mean }) \\
(\text { counts/s) } \\
(7)\end{array}$ & $\begin{array}{c}\text { mean } \\
(\text { counts/s) } \\
(8)\end{array}$ & $\begin{array}{c}<\sigma> \\
(\text { counts } / \mathrm{s}) \\
(9)\end{array}$ & $\begin{array}{c}\sigma(\text { mean }) \\
(\text { counts/s) } \\
(10)\end{array}$ & \\
\hline NGC 0810 & $2003-10-15$ & 1663 & MISDR1-17420-0427 & $3.581 \mathrm{E}-04$ & $4.793 \mathrm{E}-04$ & $1.950 \mathrm{E}-05$ & $3.555 \mathrm{E}-03$ & $1.709 \mathrm{E}-03$ & $5.889 \mathrm{E}-05$ & \\
\hline UGC 01593 & $2003-10-15$ & 1663 & MISDR1-17420-0427 & $3.676 \mathrm{E}-04$ & $5.212 \mathrm{E}-04$ & $2.383 \mathrm{E}-05$ & $3.564 \mathrm{E}-03$ & $1.733 \mathrm{E}-03$ & $4.632 \mathrm{E}-05$ & \\
\hline UGC 01603 & $2003-10-15$ & 1665 & MISDR1-17824-0404 & $2.523 \mathrm{E}-04$ & $4.364 \mathrm{E}-04$ & $4.468 \mathrm{E}-06$ & $3.012 \mathrm{E}-03$ & $1.589 \mathrm{E}-03$ & $6.410 \mathrm{E}-05$ & \\
\hline NGC 0830 & $2003-10-17$ & 1685 & MISDR1-18082-0667 & $1.824 \mathrm{E}-04$ & $3.331 \mathrm{E}-04$ & $9.239 \mathrm{E}-07$ & $2.697 \mathrm{E}-03$ & $1.387 \mathrm{E}-03$ & $3.194 \mathrm{E}-06$ & \\
\hline NGC 0842 & $2003-10-17$ & 1685 & MISDR1-18082-0667 & $1.835 \mathrm{E}-04$ & $3.366 \mathrm{E}-04$ & 3.910E-06 & $2.671 \mathrm{E}-03$ & $1.414 \mathrm{E}-03$ & $9.336 \mathrm{E}-06$ & $(1)(7)$ \\
\hline NGC 0814 & $2004-11-12$ & 1162 & NGA-NGC0814 & $\cdots$ & $\cdots$ & $\cdots$ & $2.558 \mathrm{E}-03$ & $1.674 \mathrm{E}-03$ & $4.731 \mathrm{E}-05$ & (4) \\
\hline KUG 0210-078 & $2003-10-17$ & 1685 & MISDR1-18082-0667 & $2.092 \mathrm{E}-04$ & $4.458 \mathrm{E}-04$ & $9.858 \mathrm{E}-06$ & $2.668 \mathrm{E}-03$ & $1.428 \mathrm{E}-03$ & $3.090 \mathrm{E}-05$ & $(1)(2)$ \\
\hline NGC 0855 & $2003-10-17$ & 1500 & NGA-NGC0855 & $4.738 \mathrm{E}-04$ & $6.046 \mathrm{E}-04$ & $1.014 \mathrm{E}-05$ & $3.841 \mathrm{E}-03$ & $1.910 \mathrm{E}-03$ & $4.048 \mathrm{E}-05$ & \\
\hline ESO 415-G011 & $2004-12-04$ & 1655 & MIS2DFSGP-42609-0461 & $2.377 \mathrm{E}-04$ & $3.970 \mathrm{E}-04$ & $3.961 \mathrm{E}-06$ & $2.409 \mathrm{E}-03$ & $1.359 \mathrm{E}-03$ & $3.773 \mathrm{E}-05$ & \\
\hline KUG 0211-075 & 2004-11-04 & 1560 & MISDR1-18135-0668 & $2.837 \mathrm{E}-04$ & $5.272 \mathrm{E}-04$ & $4.533 \mathrm{E}-05$ & $2.813 \mathrm{E}-03$ & $1.578 \mathrm{E}-03$ & $2.438 \mathrm{E}-05$ & $(1)(2)$ \\
\hline NGC 0871 & $2003-10-18$ & 1694 & MISDR1-17498-0428 & $4.394 \mathrm{E}-04$ & $5.359 \mathrm{E}-04$ & $2.433 \mathrm{E}-05$ & $3.730 \mathrm{E}-03$ & $1.694 \mathrm{E}-03$ & $3.574 \mathrm{E}-05$ & \\
\hline KUG 0214-057 & $2004-10-30$ & 6823 & XMMLSS-07 & $2.118 \mathrm{E}-04$ & $1.816 \mathrm{E}-04$ & $1.502 \mathrm{E}-05$ & $2.782 \mathrm{E}-03$ & $7.148 \mathrm{E}-04$ & $4.719 \mathrm{E}-05$ & (1) \\
\hline UGC 01761 & $2003-10-18$ & 1694 & MISDR1-17498-0428 & $4.514 \mathrm{E}-04$ & $5.463 \mathrm{E}-04$ & $2.654 \mathrm{E}-05$ & $3.757 \mathrm{E}-03$ & $1.690 \mathrm{E}-03$ & $4.562 \mathrm{E}-05$ & \\
\hline NGC 0881 & $2004-10-20$ & 5502 & XMMLSS-08 & $2.272 \mathrm{E}-04$ & $2.072 \mathrm{E}-04$ & $3.280 \mathrm{E}-07$ & $2.827 \mathrm{E}-03$ & $7.996 \mathrm{E}-04$ & $9.111 \mathrm{E}-06$ & \\
\hline NGC 0895 & 2004-11-14 & 4860 & XMMLSS-03 & $2.861 \mathrm{E}-04$ & $2.556 \mathrm{E}-04$ & $9.391 \mathrm{E}-06$ & $2.983 \mathrm{E}-03$ & $9.120 \mathrm{E}-04$ & $1.286 \mathrm{E}-05$ & (1) \\
\hline NGC 0891 & $2003-10-22$ & 1704 & NGA-NGC0891 & $7.854 \mathrm{E}-04$ & 7.301E-04 & $5.974 \mathrm{E}-05$ & $4.942 \mathrm{E}-03$ & $2.071 \mathrm{E}-03$ & $9.690 \mathrm{E}-05$ & \\
\hline NGC 0898 & $2003-10-22$ & 1704 & NGA-NGC0891 & $8.443 \mathrm{E}-04$ & $8.160 \mathrm{E}-04$ & $7.974 \mathrm{E}-05$ & $4.889 \mathrm{E}-03$ & $2.092 \mathrm{E}-03$ & $3.205 \mathrm{E}-04$ & \\
\hline UGC 01859 & $2003-10-22$ & 1704 & NGA-NGC0891 & $7.231 \mathrm{E}-04$ & $6.688 \mathrm{E}-04$ & $1.164 \mathrm{E}-05$ & $4.640 \mathrm{E}-03$ & $1.909 \mathrm{E}-03$ & $4.477 \mathrm{E}-05$ & \\
\hline NGC 0906 & $2003-10-22$ & 1704 & NGA-NGC0891 & $7.703 \mathrm{E}-04$ & $7.028 \mathrm{E}-04$ & $4.124 \mathrm{E}-05$ & $4.839 \mathrm{E}-03$ & $1.999 \mathrm{E}-03$ & $7.725 \mathrm{E}-05$ & \\
\hline NGC 0925 & $2003-10-16$ & 1670 & NGA-NGC0925 & $6.422 \mathrm{E}-04$ & $6.829 \mathrm{E}-04$ & $2.755 \mathrm{E}-05$ & $4.135 \mathrm{E}-03$ & $1.898 \mathrm{E}-03$ & $4.112 \mathrm{E}-05$ & \\
\hline PGC 09333 & $2003-11-23$ & 5463 & XMMLSS-05 & $2.819 \mathrm{E}-04$ & $2.843 \mathrm{E}-04$ & $5.440 \mathrm{E}-06$ & $3.016 \mathrm{E}-03$ & $8.530 \mathrm{E}-04$ & $1.094 \mathrm{E}-04$ & \\
\hline NGC 0934 & $2004-10-23$ & 27972 & DEEP2H & $2.782 \mathrm{E}-04$ & $1.216 \mathrm{E}-04$ & $1.570 \mathrm{E}-05$ & $3.048 \mathrm{E}-03$ & $3.817 \mathrm{E}-04$ & $7.429 \mathrm{E}-06$ & \\
\hline UGC 01949 & $2004-10-23$ & 27972 & DEEP2H & $2.819 \mathrm{E}-04$ & $1.228 \mathrm{E}-04$ & $5.085 \mathrm{E}-06$ & $3.036 \mathrm{E}-03$ & $3.790 \mathrm{E}-04$ & $4.611 \mathrm{E}-05$ & \\
\hline UGC 01976 & $2003-10-21$ & 1705 & NGA-NGC0959 & $5.927 \mathrm{E}-04$ & $6.532 \mathrm{E}-04$ & $1.541 \mathrm{E}-05$ & $4.175 \mathrm{E}-03$ & $1.915 \mathrm{E}-03$ & $9.986 \mathrm{E}-06$ & \\
\hline NGC 0955 & $2003-10-27$ & 1636 & MISDR1-18127-0407 & $3.290 \mathrm{E}-04$ & $4.573 \mathrm{E}-04$ & $5.727 \mathrm{E}-06$ & $3.444 \mathrm{E}-03$ & $1.635 \mathrm{E}-03$ & $3.088 \mathrm{E}-05$ & \\
\hline UGC 02010 & $2003-10-27$ & 1636 & MISDR1-18127-0407 & $3.488 \mathrm{E}-04$ & $4.822 \mathrm{E}-04$ & $2.638 \mathrm{E}-06$ & $3.588 \mathrm{E}-03$ & $1.722 \mathrm{E}-03$ & $8.229 \mathrm{E}-05$ & \\
\hline NGC 0959 & $2003-10-21$ & 1705 & NGA-NGC0959 & $6.131 \mathrm{E}-04$ & $6.099 \mathrm{E}-04$ & $4.138 \mathrm{E}-05$ & $4.248 \mathrm{E}-03$ & $1.838 \mathrm{E}-03$ & $6.774 \mathrm{E}-05$ & \\
\hline NGC 0986A & $2004-12-12$ & 1282 & NGA-NGC0986 & $3.025 \mathrm{E}-04$ & $5.708 \mathrm{E}-04$ & $6.712 \mathrm{E}-06$ & $2.578 \mathrm{E}-03$ & $1.674 \mathrm{E}-03$ & $5.927 \mathrm{E}-05$ & \\
\hline NGC 0986 & $2004-12-12$ & 1282 & NGA-NGC0986 & $2.950 \mathrm{E}-04$ & $5.124 \mathrm{E}-04$ & $2.626 \mathrm{E}-05$ & $2.597 \mathrm{E}-03$ & $1.668 \mathrm{E}-03$ & $9.528 \mathrm{E}-06$ & \\
\hline KUG 0232-079 & $2003-11-24$ & 1705 & MISDR1-18415-0455 & $2.815 \mathrm{E}-04$ & $4.142 \mathrm{E}-04$ & $1.803 \mathrm{E}-05$ & $2.915 \mathrm{E}-03$ & $1.475 \mathrm{E}-03$ & $3.760 \mathrm{E}-05$ & \\
\hline NGC 0991 & $2003-11-24$ & 1697 & MISDR1-18356-0455 & $2.372 \mathrm{E}-04$ & $4.077 \mathrm{E}-04$ & $1.805 \mathrm{E}-05$ & $2.764 \mathrm{E}-03$ & $1.462 \mathrm{E}-03$ & $6.260 \mathrm{E}-05$ & (2) \\
\hline IC 0243 & $2004-11-17$ & 1555 & NGA-NGC1022 & $1.811 \mathrm{E}-04$ & $3.469 \mathrm{E}-04$ & $1.621 \mathrm{E}-05$ & $2.495 \mathrm{E}-03$ & $1.435 \mathrm{E}-03$ & $2.892 \mathrm{E}-07$ & (1) \\
\hline
\end{tabular}


Table 2-Continued

\begin{tabular}{|c|c|c|c|c|c|c|c|c|c|c|}
\hline \multirow{2}{*}{$\begin{array}{c}\text { Object Name } \\
\text { (1) }\end{array}$} & \multirow{2}{*}{$\begin{array}{c}\text { Date } \\
\text { Observed } \\
\\
(2)\end{array}$} & \multirow{2}{*}{$\begin{array}{l}\text { Exposure } \\
\quad(\mathrm{sec}) \\
(3)\end{array}$} & \multirow{2}{*}{$\begin{array}{l}\text { Tile } \\
(4)\end{array}$} & \multicolumn{3}{|c|}{ FUV sky background } & \multicolumn{3}{|c|}{ NUV sky background } & \multirow{2}{*}{$\begin{array}{c}\text { notes } \\
(11)\end{array}$} \\
\hline & & & & $\begin{array}{c}\text { mean } \\
(\text { counts/s) } \\
(5)\end{array}$ & $\begin{array}{c}<\sigma> \\
(\text { counts } / \mathrm{s}) \\
(6)\end{array}$ & $\begin{array}{c}\sigma(\text { mean }) \\
(\text { counts/s) } \\
(7)\end{array}$ & $\begin{array}{c}\text { mean } \\
(\text { counts/s) } \\
(8)\end{array}$ & $\begin{array}{c}<\sigma> \\
(\text { counts } / s) \\
(9)\end{array}$ & $\begin{array}{c}\sigma(\text { mean }) \\
(\text { counts/s) } \\
(10)\end{array}$ & \\
\hline NGC 1022 & $2004-11-17$ & 1555 & NGA-NGC1022 & $1.984 \mathrm{E}-04$ & $3.790 \mathrm{E}-04$ & $9.981 \mathrm{E}-06$ & $2.560 \mathrm{E}-03$ & $1.492 \mathrm{E}-03$ & $1.704 \mathrm{E}-05$ & \\
\hline NGC 1035 & 2004-11-21 & 2667 & NGA-NGC1052 & $2.554 \mathrm{E}-04$ & $3.409 \mathrm{E}-04$ & $6.748 \mathrm{E}-06$ & $2.775 \mathrm{E}-03$ & $1.150 \mathrm{E}-03$ & $3.399 \mathrm{E}-05$ & \\
\hline NGC 1033 & 2003-11-24 & 1704 & MISDR1-18475-0455 & $2.634 \mathrm{E}-04$ & $4.801 \mathrm{E}-04$ & $3.269 \mathrm{E}-06$ & $2.904 \mathrm{E}-03$ & $1.531 \mathrm{E}-03$ & $5.895 \mathrm{E}-05$ & \\
\hline NGC 1042 & $2004-11-21$ & 2667 & NGA-NGC1052 & $2.614 \mathrm{E}-04$ & $3.388 \mathrm{E}-04$ & $1.174 \mathrm{E}-05$ & $2.760 \mathrm{E}-03$ & $1.169 \mathrm{E}-03$ & $3.784 \mathrm{E}-05$ & \\
\hline NGC 1023 & $2003-10-18$ & 1665 & NGA-NGC1023 & $7.760 \mathrm{E}-04$ & $8.252 \mathrm{E}-04$ & $1.150 \mathrm{E}-04$ & $4.683 \mathrm{E}-03$ & $2.075 \mathrm{E}-03$ & $2.462 \mathrm{E}-04$ & \\
\hline NGC 1047 & $2004-11-21$ & 2667 & NGA-NGC1052 & $2.508 \mathrm{E}-04$ & $3.272 \mathrm{E}-04$ & $2.454 \mathrm{E}-05$ & $2.794 \mathrm{E}-03$ & $1.186 \mathrm{E}-03$ & $2.412 \mathrm{E}-05$ & (1) \\
\hline NGC 1023A & $2003-10-18$ & 1665 & NGA-NGC1023 & $7.732 \mathrm{E}-04$ & $8.229 \mathrm{E}-04$ & $9.649 \mathrm{E}-06$ & $4.681 \mathrm{E}-03$ & $2.076 \mathrm{E}-03$ & 1.910E-04 & \\
\hline NGC 0961 & $2003-11-26$ & 1699 & MISDR1-18473-0456 & $2.162 \mathrm{E}-04$ & $3.668 \mathrm{E}-04$ & $4.112 \mathrm{E}-07$ & $2.771 \mathrm{E}-03$ & $1.408 \mathrm{E}-03$ & $4.830 \mathrm{E}-06$ & \\
\hline NGC 1052 & $2004-11-21$ & 2667 & NGA-NGC1052 & $2.540 \mathrm{E}-04$ & $3.272 \mathrm{E}-04$ & $6.452 \mathrm{E}-06$ & $2.814 \mathrm{E}-03$ & $1.201 \mathrm{E}-03$ & $6.024 \mathrm{E}-06$ & \\
\hline NGC 1055 & $2003-10-27$ & 1626 & NGA-NGC1068 & $1.564 \mathrm{E}-03$ & $1.252 \mathrm{E}-03$ & $1.005 \mathrm{E}-03$ & $7.938 \mathrm{E}-03$ & $2.676 \mathrm{E}-03$ & $7.774 \mathrm{E}-04$ & \\
\hline PGC 10213 & $2004-11-21$ & 2667 & NGA-NGC1052 & $2.475 \mathrm{E}-04$ & $3.319 \mathrm{E}-04$ & $5.640 \mathrm{E}-06$ & $2.762 \mathrm{E}-03$ & $1.172 \mathrm{E}-03$ & 4.157E-05 & \\
\hline UGC 02174 & $2003-10-23$ & 1152 & NGA-NGC1057 & $7.884 \mathrm{E}-04$ & $1.011 \mathrm{E}-03$ & $7.752 \mathrm{E}-06$ & $4.617 \mathrm{E}-03$ & $2.430 \mathrm{E}-03$ & $6.251 \mathrm{E}-06$ & \\
\hline NGC 1068 & $2003-10-27$ & 1626 & NGA-NGC1068 & $1.118 \mathrm{E}-03$ & $8.972 \mathrm{E}-04$ & $2.045 \mathrm{E}-04$ & $7.310 \mathrm{E}-03$ & $2.648 \mathrm{E}-03$ & 4.342E-04 & \\
\hline UGC 02182 & $2003-10-23$ & 1152 & NGA-NGC1057 & $8.050 \mathrm{E}-04$ & $9.755 \mathrm{E}-04$ & $3.825 \mathrm{E}-05$ & $4.699 \mathrm{E}-03$ & $2.437 \mathrm{E}-03$ & $1.199 \mathrm{E}-04$ & \\
\hline NGC 1069 & $2004-11-21$ & 2667 & NGA-NGC1052 & $2.531 \mathrm{E}-04$ & $3.587 \mathrm{E}-04$ & $1.189 \mathrm{E}-05$ & $2.707 \mathrm{E}-03$ & $1.141 \mathrm{E}-03$ & $3.612 \mathrm{E}-05$ & (1) \\
\hline NGC 1060 & $2003-10-23$ & 1152 & NGA-NGC1057 & $8.404 \mathrm{E}-04$ & $9.719 \mathrm{E}-04$ & $2.808 \mathrm{E}-05$ & $4.886 \mathrm{E}-03$ & $2.525 \mathrm{E}-03$ & $2.082 \mathrm{E}-04$ & \\
\hline NGC 1072 & $2003-10-27$ & 1626 & NGA-NGC1068 & $1.002 \mathrm{E}-03$ & $9.394 \mathrm{E}-04$ & $5.345 \mathrm{E}-05$ & $6.694 \mathrm{E}-03$ & $2.456 \mathrm{E}-03$ & $3.487 \mathrm{E}-05$ & \\
\hline PGC 10334 & $2003-11-26$ & 1699 & MISDR1-18473-0456 & $2.467 \mathrm{E}-04$ & $4.232 \mathrm{E}-04$ & $1.464 \mathrm{E}-05$ & $2.877 \mathrm{E}-03$ & $1.536 \mathrm{E}-03$ & $3.908 \mathrm{E}-05$ & (1) \\
\hline UGC 02201 & $2003-10-23$ & 1152 & NGA-NGC1057 & $8.458 \mathrm{E}-04$ & $9.540 \mathrm{E}-04$ & $7.526 \mathrm{E}-05$ & $4.904 \mathrm{E}-03$ & $2.504 \mathrm{E}-03$ & $1.757 \mathrm{E}-04$ & \\
\hline NGC 1066 & $2003-10-23$ & 1152 & NGA-NGC1057 & $8.539 \mathrm{E}-04$ & $9.513 \mathrm{E}-04$ & $2.760 \mathrm{E}-05$ & $4.951 \mathrm{E}-03$ & $2.516 \mathrm{E}-03$ & $1.370 \mathrm{E}-04$ & \\
\hline NGC 1067 & $2003-10-23$ & 1152 & NGA-NGC1057 & $8.459 \mathrm{E}-04$ & $9.371 \mathrm{E}-04$ & $3.219 \mathrm{E}-05$ & $4.901 \mathrm{E}-03$ & $2.501 \mathrm{E}-03$ & $3.120 \mathrm{E}-04$ & \\
\hline NGC 1084 & $2003-11-26$ & 1703 & MISDR1-18534-0456 & $2.434 \mathrm{E}-04$ & $4.206 \mathrm{E}-04$ & $1.302 \mathrm{E}-05$ & $2.848 \mathrm{E}-03$ & $1.532 \mathrm{E}-03$ & $2.168 \mathrm{E}-04$ & \\
\hline NGC 1097 & $2004-10-25$ & 2061 & NGA-NGC1097 & $2.704 \mathrm{E}-04$ & $3.879 \mathrm{E}-04$ & $2.506 \mathrm{E}-05$ & $2.652 \mathrm{E}-03$ & $1.326 \mathrm{E}-03$ & $3.880 \mathrm{E}-05$ & \\
\hline PGC 10766 & $2003-11-26$ & 1704 & MISDR1-18658-0457 & $3.547 \mathrm{E}-04$ & $5.886 \mathrm{E}-04$ & $2.568 \mathrm{E}-05$ & $2.884 \mathrm{E}-03$ & $1.437 \mathrm{E}-03$ & $5.284 \mathrm{E}-05$ & \\
\hline PGC 10794 & $2003-11-26$ & 1636 & MISDR1-18594-0457 & $2.891 \mathrm{E}-04$ & $4.284 \mathrm{E}-04$ & $2.116 \mathrm{E}-05$ & $2.819 \mathrm{E}-03$ & $1.487 \mathrm{E}-03$ & $3.244 \mathrm{E}-05$ & (1) \\
\hline PGC 10875 & $2003-11-26$ & 1704 & MISDR1-18658-0457 & $3.924 \mathrm{E}-04$ & $5.178 \mathrm{E}-04$ & $5.830 \mathrm{E}-05$ & $2.994 \mathrm{E}-03$ & $1.549 \mathrm{E}-03$ & $2.693 \mathrm{E}-05$ & (1) \\
\hline NGC 1140 & $2003-11-28$ & 1698 & NGA-NGC1140 & $3.070 \mathrm{E}-04$ & $4.953 \mathrm{E}-04$ & $2.026 \mathrm{E}-06$ & $2.904 \mathrm{E}-03$ & $1.493 \mathrm{E}-03$ & $3.128 \mathrm{E}-05$ & \\
\hline NGC 1148 & $2003-11-27$ & 1701 & MISDR1-18720-0457 & $3.326 \mathrm{E}-04$ & $5.050 \mathrm{E}-04$ & $4.771 \mathrm{E}-05$ & $3.079 \mathrm{E}-03$ & $1.530 \mathrm{E}-03$ & $3.310 \mathrm{E}-05$ & (1) \\
\hline UGC 02442 & $2003-10-21$ & 1544 & NGA-NGC1156 & $8.421 \mathrm{E}-04$ & $7.605 \mathrm{E}-04$ & $4.545 \mathrm{E}-05$ & $5.160 \mathrm{E}-03$ & $2.170 \mathrm{E}-03$ & $1.671 \mathrm{E}-04$ & \\
\hline NGC 1156 & $2003-10-21$ & 1544 & NGA-NGC1156 & $9.871 \mathrm{E}-04$ & $8.552 \mathrm{E}-04$ & $7.410 \mathrm{E}-05$ & $5.553 \mathrm{E}-03$ & $2.322 \mathrm{E}-03$ & $1.968 \mathrm{E}-04$ & \\
\hline PGC 11767 & $2003-11-28$ & 1700 & MISDR1-18848-0459 & $5.454 \mathrm{E}-04$ & $5.799 \mathrm{E}-04$ & $1.982 \mathrm{E}-05$ & $3.564 \mathrm{E}-03$ & $1.675 \mathrm{E}-03$ & $1.394 \mathrm{E}-04$ & (1) \\
\hline UGC 02519 & $2004-01-20$ & 1694 & NGA-UGC2519 & $7.229 \mathrm{E}-04$ & $6.636 \mathrm{E}-04$ & $2.494 \mathrm{E}-05$ & $4.211 \mathrm{E}-03$ & $1.779 \mathrm{E}-03$ & $1.732 \mathrm{E}-05$ & \\
\hline
\end{tabular}


Table 2-Continued

\begin{tabular}{|c|c|c|c|c|c|c|c|c|c|c|}
\hline \multirow{2}{*}{$\begin{array}{c}\text { Object Name } \\
\text { (1) }\end{array}$} & \multirow{2}{*}{$\begin{array}{c}\text { Date } \\
\text { Observed } \\
\\
(2)\end{array}$} & \multirow{2}{*}{$\begin{array}{l}\text { Exposure } \\
\quad(\mathrm{sec}) \\
\\
(3)\end{array}$} & \multirow{2}{*}{$\begin{array}{l}\text { Tile } \\
(4)\end{array}$} & \multicolumn{3}{|c|}{ FUV sky background } & \multicolumn{3}{|c|}{ NUV sky background } & \multirow{2}{*}{$\begin{array}{c}\text { notes } \\
(11)\end{array}$} \\
\hline & & & & $\begin{array}{c}\text { mean } \\
(\text { counts/s) } \\
(5)\end{array}$ & $\begin{array}{c}<\sigma> \\
(\text { counts } / \mathrm{s}) \\
(6)\end{array}$ & $\begin{array}{c}\sigma(\text { mean }) \\
(\text { counts/s) } \\
(7)\end{array}$ & $\begin{array}{c}\text { mean } \\
(\text { counts/s) } \\
(8)\end{array}$ & $\begin{array}{c}<\sigma> \\
(\text { counts } / \mathrm{s}) \\
(9)\end{array}$ & $\begin{array}{c}\sigma(\text { mean }) \\
(\text { counts/s) } \\
(10)\end{array}$ & \\
\hline NGC 1241 & 2003-11-29 & 1698 & MISDR1-18916-0459 & $6.629 \mathrm{E}-04$ & 7.067E-04 & $5.218 \mathrm{E}-05$ & $3.512 \mathrm{E}-03$ & $1.624 \mathrm{E}-03$ & $1.876 \mathrm{E}-04$ & \\
\hline NGC 1242 & $2003-11-29$ & 1698 & MISDR1-18916-0459 & $6.641 \mathrm{E}-04$ & $7.133 \mathrm{E}-04$ & $6.755 \mathrm{E}-05$ & $3.501 \mathrm{E}-03$ & $1.621 \mathrm{E}-03$ & $1.860 \mathrm{E}-04$ & \\
\hline NGC 1266 & $2003-11-27$ & 1701 & NGA-NGC1266 & $8.461 \mathrm{E}-04$ & $7.515 \mathrm{E}-04$ & $2.768 \mathrm{E}-05$ & $4.667 \mathrm{E}-03$ & $1.987 \mathrm{E}-03$ & $5.922 \mathrm{E}-05$ & \\
\hline NGC 1291 & $2003-12-06$ & 1705 & NGA-NGC1291 & $2.564 \mathrm{E}-04$ & 4.165E-04 & $2.302 \mathrm{E}-05$ & $2.368 \mathrm{E}-03$ & $1.371 \mathrm{E}-03$ & $7.670 \mathrm{E}-05$ & $(2)$ \\
\hline NGC 1285 & $2003-11-28$ & 1699 & MISDR1-28516-0459 & $9.844 \mathrm{E}-04$ & 8.927E-04 & $2.132 \mathrm{E}-04$ & $4.954 \mathrm{E}-03$ & $2.070 \mathrm{E}-03$ & $6.343 \mathrm{E}-04$ & \\
\hline NGC 1299 & $2003-11-27$ & 1700 & MISDR1-28517-0460 & $5.755 \mathrm{E}-04$ & $6.035 \mathrm{E}-04$ & $2.493 \mathrm{E}-04$ & $3.930 \mathrm{E}-03$ & $1.798 \mathrm{E}-03$ & $6.955 \mathrm{E}-04$ & \\
\hline NGC 1310 & $2003-12-06$ & 1699 & NGA-NGC1316 & $2.829 \mathrm{E}-04$ & 4.370E-04 & 4.034E-06 & $2.483 \mathrm{E}-03$ & $1.384 \mathrm{E}-03$ & $9.541 \mathrm{E}-05$ & \\
\hline KUG 0319-072 & 2003-11-29 & 1697 & MISDR1-27125-0460 & $7.578 \mathrm{E}-04$ & $6.802 \mathrm{E}-04$ & $8.202 \mathrm{E}-05$ & $4.259 \mathrm{E}-03$ & $1.854 \mathrm{E}-03$ & $1.830 \mathrm{E}-04$ & \\
\hline NGC 1316 & $2003-12-06$ & 1699 & NGA-NGC1316 & $2.931 \mathrm{E}-04$ & $5.043 \mathrm{E}-04$ & $1.364 \mathrm{E}-06$ & $2.543 \mathrm{E}-03$ & $1.436 \mathrm{E}-03$ & $9.236 \mathrm{E}-06$ & \\
\hline NGC 1317 & $2003-12-06$ & 1699 & NGA-NGC1316 & $3.199 \mathrm{E}-04$ & $5.339 \mathrm{E}-04$ & $5.252 \mathrm{E}-05$ & $2.643 \mathrm{E}-03$ & $1.462 \mathrm{E}-03$ & $9.196 \mathrm{E}-05$ & \\
\hline ESO 357-G025 & $2004-11-10$ & 2910 & NGA-NGC1326 & $2.695 \mathrm{E}-04$ & $4.763 \mathrm{E}-04$ & $4.254 \mathrm{E}-06$ & $2.448 \mathrm{E}-03$ & $1.064 \mathrm{E}-03$ & $5.505 \mathrm{E}-05$ & \\
\hline PGC 12706 & $2003-12-06$ & 1699 & NGA-NGC1316 & $2.591 \mathrm{E}-04$ & $4.834 \mathrm{E}-04$ & $1.317 \mathrm{E}-05$ & $2.343 \mathrm{E}-03$ & $1.352 \mathrm{E}-03$ & $1.437 \mathrm{E}-04$ & \\
\hline NGC 1326 & $2004-11-10$ & 2910 & NGA-NGC1326 & $2.730 \mathrm{E}-04$ & $4.801 \mathrm{E}-04$ & $1.639 \mathrm{E}-06$ & $2.433 \mathrm{E}-03$ & $1.015 \mathrm{E}-03$ & $2.892 \mathrm{E}-05$ & \\
\hline PGC 13005 & $2003-11-30$ & 1523 & MISDR1-27039-0461 & 7.727E-04 & $8.058 \mathrm{E}-04$ & $1.158 \mathrm{E}-04$ & $4.321 \mathrm{E}-03$ & $2.006 \mathrm{E}-03$ & $3.148 \mathrm{E}-04$ & (1) \\
\hline NGC 1346 & 2003-11-30 & 1523 & MISDR1-27039-0461 & $7.548 \mathrm{E}-04$ & 7.957E-04 & $1.104 \mathrm{E}-04$ & $4.239 \mathrm{E}-03$ & $1.983 \mathrm{E}-03$ & $3.573 \mathrm{E}-04$ & (1) \\
\hline PGC 13058 & $2004-11-11$ & 1009 & NGA-NGC1365 & $\ldots$ & $\ldots$ & $\ldots$ & $2.247 \mathrm{E}-03$ & $1.658 \mathrm{E}-03$ & $8.413 \mathrm{E}-08$ & $(4)(1)$ \\
\hline ESO 418-G008 & $2004-11-18$ & 2119 & NGA-ESO418-G008 & $2.484 \mathrm{E}-04$ & $3.628 \mathrm{E}-04$ & $1.797 \mathrm{E}-05$ & $2.342 \mathrm{E}-03$ & $1.228 \mathrm{E}-03$ & $1.108 \mathrm{E}-05$ & \\
\hline NGC 1365 & $2004-11-11$ & 1009 & NGA-NGC1365 & $\ldots$ & $\ldots$ & $\ldots$ & $2.330 \mathrm{E}-03$ & $1.771 \mathrm{E}-03$ & $4.219 \mathrm{E}-05$ & (4) \\
\hline PGC 13186 & 2004-11-11 & 1009 & NGA-NGC1365 & $\ldots$ & $\ldots$ & $\ldots$ & $2.282 \mathrm{E}-03$ & $1.694 \mathrm{E}-03$ & $4.127 \mathrm{E}-05$ & $(4)(1)$ \\
\hline NGC 1361 & 2003-11-30 & 1697 & MISDR1-27038-0461 & $4.890 \mathrm{E}-04$ & $6.051 \mathrm{E}-04$ & $5.263 \mathrm{E}-05$ & $3.505 \mathrm{E}-03$ & $1.719 \mathrm{E}-03$ & $1.387 \mathrm{E}-04$ & \\
\hline PGC 13230 & 2004-11-13 & 14226 & UVE-FORNAX & $2.501 \mathrm{E}-04$ & $1.374 \mathrm{E}-04$ & $8.612 \mathrm{E}-06$ & $2.220 \mathrm{E}-03$ & $4.423 \mathrm{E}-04$ & $3.693 \mathrm{E}-05$ & \\
\hline NGC 1373 & $2004-11-13$ & 14226 & UVE-FORNAX & $2.523 \mathrm{E}-04$ & $1.479 \mathrm{E}-04$ & $3.826 \mathrm{E}-06$ & $2.256 \mathrm{E}-03$ & $4.569 \mathrm{E}-04$ & $6.374 \mathrm{E}-06$ & \\
\hline NGC 1374 & $2004-11-13$ & 14226 & UVE-FORNAX & $2.509 \mathrm{E}-04$ & $1.467 \mathrm{E}-04$ & $8.405 \mathrm{E}-08$ & $2.253 \mathrm{E}-03$ & $4.581 \mathrm{E}-04$ & $1.387 \mathrm{E}-05$ & \\
\hline NGC 1375 & $2004-11-13$ & 14226 & UVE-FORNAX & $2.500 \mathrm{E}-04$ & $1.447 \mathrm{E}-04$ & $9.537 \mathrm{E}-06$ & $2.259 \mathrm{E}-03$ & $4.593 \mathrm{E}-04$ & $6.777 \mathrm{E}-05$ & \\
\hline NGC 1379 & $2004-11-13$ & 14226 & UVE-FORNAX & $2.434 \mathrm{E}-04$ & $1.429 \mathrm{E}-04$ & $1.532 \mathrm{E}-05$ & $2.260 \mathrm{E}-03$ & $4.629 \mathrm{E}-04$ & $4.788 \mathrm{E}-05$ & \\
\hline UGCA 080 & $2004-11-06$ & 1625 & MISDR1-27079-0461 & $4.116 \mathrm{E}-04$ & $5.509 \mathrm{E}-04$ & 4.355E-05 & $3.267 \mathrm{E}-03$ & $1.630 \mathrm{E}-03$ & $3.063 \mathrm{E}-04$ & (1) \\
\hline NGC 1380 & $2004-11-13$ & 2671 & NGA-NGC1380 & $2.787 \mathrm{E}-04$ & 4.623E-04 & $9.042 \mathrm{E}-06$ & $2.372 \mathrm{E}-03$ & $1.106 \mathrm{E}-03$ & $5.465 \mathrm{E}-05$ & \\
\hline NGC 1381 & $2003-12-06$ & 1704 & NGA-NGC1399 & $2.555 \mathrm{E}-04$ & $3.935 \mathrm{E}-04$ & $7.789 \mathrm{E}-06$ & $2.415 \mathrm{E}-03$ & $1.339 \mathrm{E}-03$ & $4.730 \mathrm{E}-06$ & \\
\hline NGC 1386 & 2004-11-13 & 14226 & UVE-FORNAX & $2.346 \mathrm{E}-04$ & $1.417 \mathrm{E}-04$ & $1.037 \mathrm{E}-05$ & $2.181 \mathrm{E}-03$ & $4.387 \mathrm{E}-04$ & $6.243 \mathrm{E}-05$ & \\
\hline NGC $1380 A$ & 2004-11-13 & 2671 & NGA-NGC1380 & $2.767 \mathrm{E}-04$ & $4.840 \mathrm{E}-04$ & $1.294 \mathrm{E}-05$ & $2.361 \mathrm{E}-03$ & $1.103 \mathrm{E}-03$ & $9.248 \mathrm{E}-05$ & \\
\hline PGC 13343 & $2003-12-06$ & 1704 & NGA-NGC1399 & $\ldots$ & $\ldots$ & $\ldots$ & $2.410 \mathrm{E}-03$ & $1.344 \mathrm{E}-03$ & $2.130 \mathrm{E}-05$ & $(4)(5)$ \\
\hline NGC 1387 & $2003-12-06$ & 1704 & NGA-NGC1399 & $2.484 \mathrm{E}-04$ & $3.885 \mathrm{E}-04$ & $1.744 \mathrm{E}-06$ & $2.408 \mathrm{E}-03$ & $1.338 \mathrm{E}-03$ & $3.137 \mathrm{E}-05$ & \\
\hline
\end{tabular}


Table 2-Continued

\begin{tabular}{|c|c|c|c|c|c|c|c|c|c|c|}
\hline \multirow{2}{*}{$\begin{array}{l}\text { Object Name } \\
\text { (1) }\end{array}$} & \multirow{2}{*}{$\begin{array}{c}\text { Date } \\
\text { Observed } \\
(2)\end{array}$} & \multirow{2}{*}{$\begin{array}{l}\text { Exposure } \\
\quad(\mathrm{sec}) \\
(3)\end{array}$} & \multirow{2}{*}{$\begin{array}{l}\text { Tile } \\
(4) \\
\end{array}$} & \multicolumn{3}{|c|}{ FUV sky background } & \multicolumn{3}{|c|}{ NUV sky background } & \multirow{2}{*}{$\begin{array}{l}\text { notes } \\
(11)\end{array}$} \\
\hline & & & & $\begin{array}{c}\text { mean } \\
(\text { counts/s) } \\
(5)\end{array}$ & $\begin{array}{c}<\sigma> \\
(\text { counts/s) } \\
(6)\end{array}$ & $\begin{array}{c}\sigma(\text { mean }) \\
(\text { counts } / \mathrm{s}) \\
(7)\end{array}$ & $\begin{array}{c}\text { mean } \\
(\text { counts/s) } \\
(8)\end{array}$ & $\begin{array}{c}<\sigma> \\
(\text { counts } / \mathrm{s}) \\
(9)\end{array}$ & $\begin{array}{c}\sigma(\text { mean }) \\
(\text { counts/s }) \\
(10)\end{array}$ & \\
\hline NGC 1380B & $2003-12-06$ & 1704 & NGA-NGC1399 & $2.604 \mathrm{E}-04$ & $4.095 \mathrm{E}-04$ & $1.255 \mathrm{E}-05$ & $2.418 \mathrm{E}-03$ & $1.357 \mathrm{E}-03$ & $2.901 \mathrm{E}-06$ & \\
\hline NGC 1389 & $2003-12-06$ & 1704 & NGA-NGC1399 & $2.433 \mathrm{E}-04$ & $3.825 \mathrm{E}-04$ & $5.437 \mathrm{E}-06$ & $2.361 \mathrm{E}-03$ & $1.320 \mathrm{E}-03$ & $6.569 \mathrm{E}-06$ & \\
\hline NGC 1385 & 2004-11-18 & 1694 & NGA-NGC1385 & $2.747 \mathrm{E}-04$ & $4.282 \mathrm{E}-04$ & $8.575 \mathrm{E}-06$ & $2.494 \mathrm{E}-03$ & $1.418 \mathrm{E}-03$ & $3.788 \mathrm{E}-05$ & \\
\hline NGC 1383 & $2004-11-17$ & 1575 & NGA-NGC1407 & $5.263 \mathrm{E}-04$ & $6.011 \mathrm{E}-04$ & $3.576 \mathrm{E}-05$ & $3.625 \mathrm{E}-03$ & $1.740 \mathrm{E}-03$ & $1.876 \mathrm{E}-05$ & \\
\hline NGC 1396 & $2003-12-06$ & 1704 & NGA-NGC1399 & $\cdots$ & $\cdots$ & $\cdots$ & $2.596 \mathrm{E}-03$ & $1.433 \mathrm{E}-03$ & $7.997 \mathrm{E}-05$ & $(4)(5)$ \\
\hline ESO 358-G042 & 2004-11-13 & 2671 & NGA-NGC1380 & $\cdots$ & $\cdots$ & $\cdots$ & $2.262 \mathrm{E}-03$ & $1.039 \mathrm{E}-03$ & $1.469 \mathrm{E}-05$ & $(4)(5)$ \\
\hline NGC 1399 & $2003-12-06$ & 1704 & NGA-NGC1399 & $2.754 \mathrm{E}-04$ & $4.345 \mathrm{E}-04$ & $1.242 \mathrm{E}-05$ & $2.574 \mathrm{E}-03$ & $1.439 \mathrm{E}-03$ & $8.576 \mathrm{E}-05$ & \\
\hline NGC 1393 & $2004-11-17$ & 1575 & NGA-NGC1407 & $5.190 \mathrm{E}-04$ & $6.283 \mathrm{E}-04$ & $3.297 \mathrm{E}-05$ & 3.663E-03 & $1.787 \mathrm{E}-03$ & $9.928 \mathrm{E}-05$ & \\
\hline NGC 1404 & $2003-12-06$ & 1704 & NGA-NGC1399 & $2.711 \mathrm{E}-04$ & $4.283 \mathrm{E}-04$ & $2.003 \mathrm{E}-05$ & $2.534 \mathrm{E}-03$ & $1.422 \mathrm{E}-03$ & $1.290 \mathrm{E}-04$ & \\
\hline NGC 1391 & 2004-11-17 & 1575 & NGA-NGC1407 & $5.391 \mathrm{E}-04$ & $6.445 \mathrm{E}-04$ & $2.333 \mathrm{E}-05$ & $3.671 \mathrm{E}-03$ & $1.804 \mathrm{E}-03$ & $2.374 \mathrm{E}-05$ & \\
\hline NGC 1394 & $2004-11-17$ & 1575 & NGA-NGC1407 & $5.609 \mathrm{E}-04$ & $6.780 \mathrm{E}-04$ & $7.802 \mathrm{E}-05$ & $3.665 \mathrm{E}-03$ & $1.805 \mathrm{E}-03$ & $5.569 \mathrm{E}-05$ & \\
\hline AM 0337-355 & 2003-12-06 & 1704 & NGA-NGC1399 & $2.608 \mathrm{E}-04$ & $4.479 \mathrm{E}-04$ & $2.983 \mathrm{E}-07$ & $2.400 \mathrm{E}-03$ & $1.367 \mathrm{E}-03$ & $4.221 \mathrm{E}-05$ & \\
\hline NGC 1400 & $2004-11-17$ & 1575 & NGA-NGC1407 & $4.906 \mathrm{E}-04$ & $5.804 \mathrm{E}-04$ & $2.434 \mathrm{E}-05$ & $3.782 \mathrm{E}-03$ & $1.813 \mathrm{E}-03$ & $2.222 \mathrm{E}-05$ & \\
\hline IC 0343 & 2004-11-17 & 1575 & NGA-NGC1407 & $\cdots$ & $\cdots$ & $\cdots$ & $3.730 \mathrm{E}-03$ & $1.840 \mathrm{E}-03$ & $7.086 \mathrm{E}-05$ & $(4)(5)$ \\
\hline NGC $1427 \mathrm{~A}$ & 2003-12-06 & 1704 & NGA-NGC1399 & $2.637 \mathrm{E}-04$ & $4.818 \mathrm{E}-04$ & $4.812 \mathrm{E}-07$ & $2.363 \mathrm{E}-03$ & $1.353 \mathrm{E}-03$ & $4.137 \mathrm{E}-06$ & \\
\hline NGC 1407 & 2004-11-17 & 1575 & NGA-NGC1407 & $5.321 \mathrm{E}-04$ & $6.567 \mathrm{E}-04$ & $3.654 \mathrm{E}-05$ & $3.785 \mathrm{E}-03$ & $1.843 \mathrm{E}-03$ & $1.123 \mathrm{E}-04$ & \\
\hline ESO 548-G068 & 2004-11-17 & 1575 & NGA-NGC1407 & $5.119 \mathrm{E}-04$ & $6.543 \mathrm{E}-04$ & $3.582 \mathrm{E}-05$ & $3.702 \mathrm{E}-03$ & $1.754 \mathrm{E}-03$ & $1.965 \mathrm{E}-05$ & \\
\hline PGC 13515 & $2003-12-06$ & 1704 & NGA-NGC1399 & $\ldots$ & $\ldots$ & $\ldots$ & $2.400 \mathrm{E}-03$ & $1.389 \mathrm{E}-03$ & $9.211 \mathrm{E}-06$ & $(4)(5)$ \\
\hline PGC 13535 & 2004-11-02 & 1028 & MISDR1-27036-0462 & $7.468 \mathrm{E}-04$ & $1.082 \mathrm{E}-03$ & $2.376 \mathrm{E}-05$ & $4.304 \mathrm{E}-03$ & $2.440 \mathrm{E}-03$ & $6.572 \mathrm{E}-05$ & \\
\hline PGC 13600 & 2004-11-02 & 1028 & MISDR1-27036-0462 & $6.959 \mathrm{E}-04$ & 8.609E-04 & $4.666 \mathrm{E}-06$ & $4.494 \mathrm{E}-03$ & $2.429 \mathrm{E}-03$ & $1.760 \mathrm{E}-04$ & \\
\hline IC 0334 & 2004-03-09 & 1618 & LGAL-IC334 & $\cdots$ & $\ldots$ & $\cdots$ & $3.971 \mathrm{E}-03$ & $1.788 \mathrm{E}-03$ & $5.503 \mathrm{E}-05$ & \\
\hline PGC 13820 & 2004-11-05 & 1918 & MISDR1-26955-0463 & $6.469 \mathrm{E}-04$ & $6.789 \mathrm{E}-04$ & $5.156 \mathrm{E}-05$ & $3.917 \mathrm{E}-03$ & $1.710 \mathrm{E}-03$ & $1.780 \mathrm{E}-05$ & $(1)(2)$ \\
\hline NGC 1481 & 2004-11-05 & 1588 & NGA-NGC1482 & $4.686 \mathrm{E}-04$ & $5.978 \mathrm{E}-04$ & $4.388 \mathrm{E}-05$ & $3.141 \mathrm{E}-03$ & $1.679 \mathrm{E}-03$ & $1.394 \mathrm{E}-04$ & \\
\hline NGC 1482 & 2004-11-05 & 1588 & NGA-NGC1482 & $4.496 \mathrm{E}-04$ & $5.723 \mathrm{E}-04$ & $4.378 \mathrm{E}-05$ & $3.122 \mathrm{E}-03$ & $1.666 \mathrm{E}-03$ & $2.404 \mathrm{E}-05$ & \\
\hline PGC 14100 & 2004-11-06 & 1222 & MISDR1-26992-0464 & $9.070 \mathrm{E}-04$ & $9.934 \mathrm{E}-04$ & $7.257 \mathrm{E}-05$ & $4.579 \mathrm{E}-03$ & $2.292 \mathrm{E}-03$ & $8.247 \mathrm{E}-06$ & (1) \\
\hline NGC 1510 & 2003-12-29 & 2380 & NGA-NGC1512 & $4.045 \mathrm{E}-04$ & $4.453 \mathrm{E}-04$ & $1.005 \mathrm{E}-05$ & $2.845 \mathrm{E}-03$ & $1.286 \mathrm{E}-03$ & $4.173 \mathrm{E}-05$ & \\
\hline NGC 1512 & 2003-12-29 & 2380 & NGA-NGC1512 & $3.875 \mathrm{E}-04$ & $4.332 \mathrm{E}-04$ & $1.965 \mathrm{E}-05$ & $2.813 \mathrm{E}-03$ & $1.273 \mathrm{E}-03$ & $5.169 \mathrm{E}-05$ & \\
\hline UGC 02955 & $2003-10-18$ & 1269 & NGRG-A213 & $7.401 \mathrm{E}-04$ & $5.541 \mathrm{E}-04$ & $2.202 \mathrm{E}-06$ & 4.031E-03 & $1.396 \mathrm{E}-03$ & $1.280 \mathrm{E}-04$ & \\
\hline NGC 1546 & 2003-12-29 & 2018 & NGA-NGC1546 & $3.800 \mathrm{E}-04$ & $4.600 \mathrm{E}-04$ & $2.177 \mathrm{E}-07$ & $2.884 \mathrm{E}-03$ & $1.405 \mathrm{E}-03$ & $1.245 \mathrm{E}-04$ & \\
\hline NGC 1549 & $2003-12-29$ & 2018 & NGA-NGC1546 & $4.136 \mathrm{E}-04$ & $5.458 \mathrm{E}-04$ & $2.515 \mathrm{E}-05$ & $2.878 \mathrm{E}-03$ & $1.402 \mathrm{E}-03$ & $3.610 \mathrm{E}-05$ & \\
\hline NGC 1553 & 2003-12-29 & 2018 & NGA-NGC1546 & $3.896 \mathrm{E}-04$ & $5.026 \mathrm{E}-04$ & $5.231 \mathrm{E}-06$ & $2.882 \mathrm{E}-03$ & $1.419 \mathrm{E}-03$ & $1.270 \mathrm{E}-04$ & \\
\hline IC 2058 & 2003-12-29 & 2018 & NGA-NGC1546 & $4.066 \mathrm{E}-04$ & $5.603 \mathrm{E}-04$ & $2.334 \mathrm{E}-05$ & $2.700 \mathrm{E}-03$ & $1.342 \mathrm{E}-03$ & $3.891 \mathrm{E}-07$ & \\
\hline
\end{tabular}


Table 2-Continued

\begin{tabular}{|c|c|c|c|c|c|c|c|c|c|c|}
\hline \multirow{2}{*}{$\begin{array}{c}\text { Object Name } \\
\text { (1) }\end{array}$} & \multirow{2}{*}{$\begin{array}{c}\text { Date } \\
\text { Observed } \\
(2)\end{array}$} & \multirow{2}{*}{$\begin{array}{l}\text { Exposure } \\
\quad(\mathrm{sec}) \\
(3)\end{array}$} & \multirow{2}{*}{$\begin{array}{l}\text { Tile } \\
(4)\end{array}$} & \multicolumn{3}{|c|}{ FUV sky background } & \multicolumn{3}{|c|}{ NUV sky background } & \multirow{2}{*}{$\begin{array}{l}\text { notes } \\
\text { (11) }\end{array}$} \\
\hline & & & & $\begin{array}{c}\text { mean } \\
(\text { counts/s) } \\
(5)\end{array}$ & $\begin{array}{c}<\sigma> \\
(\text { counts/s) } \\
(6)\end{array}$ & $\begin{array}{c}\sigma(\text { mean }) \\
(\text { counts/s) } \\
(7)\end{array}$ & $\begin{array}{c}\text { mean } \\
(\text { counts/s) } \\
(8)\end{array}$ & $\begin{array}{c}<\sigma> \\
(\text { counts/s) } \\
(9)\end{array}$ & $\begin{array}{c}\sigma(\text { mean }) \\
(\text { counts/s }) \\
(10)\end{array}$ & \\
\hline NGC 1566 & $2003-12-29$ & 3232 & NGA-NGC1566 & $4.122 \mathrm{E}-04$ & $3.844 \mathrm{E}-04$ & $8.508 \mathrm{E}-07$ & 2.929E-03 & $1.126 \mathrm{E}-03$ & $1.358 \mathrm{E}-05$ & \\
\hline NGC 1569 & $2003-10-18$ & 2375 & NGA-NGC1569 & 8.129E-04 & $6.267 \mathrm{E}-04$ & $7.930 \mathrm{E}-05$ & 4.490E-03 & $1.667 \mathrm{E}-03$ & $2.616 \mathrm{E}-04$ & \\
\hline NGC 1672 & 2004-02-06 & 867 & NGA-NGC1672 & $\cdots$ & $\cdots$ & $\cdots$ & $3.074 \mathrm{E}-03$ & $2.200 \mathrm{E}-03$ & $7.771 \mathrm{E}-05$ & (4) \\
\hline NGC 1705 & 2004-02-08 & 713 & NGA-NGC1705 & $\cdots$ & $\cdots$ & $\cdots$ & $2.898 \mathrm{E}-03$ & $2.289 \mathrm{E}-03$ & $1.183 \mathrm{E}-04$ & (4) \\
\hline ESO 422-G027 & $2003-11-27$ & 4593 & NGA-NGC1800 & $3.369 \mathrm{E}-04$ & $4.731 \mathrm{E}-04$ & $2.984 \mathrm{E}-05$ & 2.669E-03 & $8.706 \mathrm{E}-04$ & $4.883 \mathrm{E}-05$ & \\
\hline NGC 1800 & $2003-11-27$ & 4593 & NGA-NGC1800 & $3.185 \mathrm{E}-04$ & $4.586 \mathrm{E}-04$ & $1.031 \mathrm{E}-06$ & $2.637 \mathrm{E}-03$ & $8.874 \mathrm{E}-04$ & $2.444 \mathrm{E}-05$ & \\
\hline NGC 1808 & 2004-02-06 & 1577 & LGAL-NGC1808 & $\cdots$ & $\cdots$ & $\cdots$ & $2.885 \mathrm{E}-03$ & $1.572 \mathrm{E}-03$ & $5.606 \mathrm{E}-05$ & (4) \\
\hline IC 0411 & $2003-11-26$ & 3211 & NGA-IRAS05189 & $5.786 \mathrm{E}-04$ & $7.245 \mathrm{E}-04$ & $6.053 \mathrm{E}-06$ & $3.640 \mathrm{E}-03$ & $1.244 \mathrm{E}-03$ & $1.377 \mathrm{E}-04$ & \\
\hline ESO 204-G006 & 2005-01-30 & 1453 & DLENSS-01 & $5.086 \mathrm{E}-04$ & $2.640 \mathrm{E}-04$ & $4.272 \mathrm{E}-05$ & $3.072 \mathrm{E}-03$ & $6.723 \mathrm{E}-04$ & $7.688 \mathrm{E}-05$ & \\
\hline ESO 204-G007 & 2005-01-30 & 1453 & DLENSS-01 & $5.204 \mathrm{E}-04$ & $2.719 \mathrm{E}-04$ & $3.741 \mathrm{E}-06$ & $3.057 \mathrm{E}-03$ & $6.701 \mathrm{E}-04$ & $9.212 \mathrm{E}-05$ & \\
\hline ESO 033-G022 & 2003-09-21 & 3022 & NGA-ESO033-G022 & $9.650 \mathrm{E}-04$ & $6.196 \mathrm{E}-04$ & $5.075 \mathrm{E}-05$ & $5.282 \mathrm{E}-03$ & $1.601 \mathrm{E}-03$ & $1.608 \mathrm{E}-04$ & \\
\hline NGC 1964 & 2004-02-06 & 1561 & LGAL-NGC1964 & $\cdots$ & $\cdots$ & $\cdots$ & $4.548 \mathrm{E}-03$ & $2.035 \mathrm{E}-03$ & $1.365 \mathrm{E}-04$ & (4) \\
\hline NGC 1961 & 2004-01-19 & 1695 & NGA-NGC1961 & $5.920 \mathrm{E}-04$ & $6.330 \mathrm{E}-04$ & $1.590 \mathrm{E}-05$ & $3.814 \mathrm{E}-03$ & $1.813 \mathrm{E}-03$ & $4.144 \mathrm{E}-06$ & \\
\hline UGC 03342 & 2004-01-19 & 1695 & NGA-NGC1961 & $6.402 \mathrm{E}-04$ & $6.938 \mathrm{E}-04$ & $1.258 \mathrm{E}-05$ & $3.865 \mathrm{E}-03$ & $1.841 \mathrm{E}-03$ & $3.291 \mathrm{E}-05$ & \\
\hline UGC 03344 & 2004-01-19 & 1695 & NGA-NGC1961 & $6.662 \mathrm{E}-04$ & 7.269E-04 & 8.917E-06 & $3.898 \mathrm{E}-03$ & $1.858 \mathrm{E}-03$ & $4.138 \mathrm{E}-05$ & \\
\hline NGC 2090 & 2004-02-05 & 3875 & NGA-NGC2090 & $9.337 \mathrm{E}-04$ & $6.864 \mathrm{E}-04$ & $4.234 \mathrm{E}-07$ & $6.001 \mathrm{E}-03$ & $1.529 \mathrm{E}-03$ & $1.860 \mathrm{E}-04$ & \\
\hline UGC 03403 & 2004-03-05 & 1674 & NGA-Mrk3 & $\cdots$ & $\cdots$ & $\cdots$ & $3.672 \mathrm{E}-03$ & $1.668 \mathrm{E}-03$ & $2.135 \mathrm{E}-05$ & (4) \\
\hline UGC 03422 & 2004-03-05 & 1674 & NGA-Mrk3 & $\cdots$ & $\cdots$ & $\cdots$ & $3.772 \mathrm{E}-03$ & $1.766 \mathrm{E}-03$ & $1.377 \mathrm{E}-04$ & (4) \\
\hline Mrk 3 & 2004-03-05 & 1674 & NGA-Mrk3 & $\cdots$ & $\cdots$ & $\cdots$ & $3.819 \mathrm{E}-03$ & $1.806 \mathrm{E}-03$ & $2.936 \mathrm{E}-04$ & (4) \\
\hline NGC 2207 & 2004-02-06 & 2009 & NGA-NGC2207 & $\cdots$ & $\cdots$ & $\cdots$ & 7.015E-03 & $2.332 \mathrm{E}-03$ & $3.900 \mathrm{E}-05$ & (4) \\
\hline IC 2163 & 2004-02-06 & 2009 & NGA-NGC2207 & $\cdots$ & $\cdots$ & $\cdots$ & 7.047E-03 & $2.343 \mathrm{E}-03$ & $9.893 \mathrm{E}-05$ & (4) \\
\hline UGC 03423 & 2004-10-03 & 1619 & NGA-NGC2146 & $6.387 \mathrm{E}-04$ & $7.750 \mathrm{E}-04$ & $2.618 \mathrm{E}-05$ & $3.368 \mathrm{E}-03$ & $1.679 \mathrm{E}-03$ & $1.366 \mathrm{E}-04$ & (1) \\
\hline ESO 556-G012 & 2004-02-06 & 2009 & NGA-NGC2207 & $\cdots$ & $\cdots$ & $\cdots$ & 7.480E-03 & $2.378 \mathrm{E}-03$ & $1.667 \mathrm{E}-04$ & (4) \\
\hline NGC 2146 & 2004-10-03 & 1619 & NGA-NGC2146 & $5.949 \mathrm{E}-04$ & $6.515 \mathrm{E}-04$ & $3.471 \mathrm{E}-05$ & $3.367 \mathrm{E}-03$ & $1.706 \mathrm{E}-03$ & $2.690 \mathrm{E}-05$ & (1) \\
\hline NGC 2146A & 2004-10-03 & 1619 & NGA-NGC2146 & $5.934 \mathrm{E}-04$ & $6.783 \mathrm{E}-04$ & $3.306 \mathrm{E}-05$ & $3.285 \mathrm{E}-03$ & $1.637 \mathrm{E}-03$ & $1.563 \mathrm{E}-05$ & \\
\hline AM 0644-741 & 2003-09-20 & 2513 & NGA-AM0644m741 & $1.146 \mathrm{E}-03$ & $7.245 \mathrm{E}-04$ & $8.419 \mathrm{E}-05$ & $5.330 \mathrm{E}-03$ & $1.786 \mathrm{E}-03$ & $2.306 \mathrm{E}-04$ & \\
\hline PGC 19480 & 2003-09-20 & 2513 & NGA-AM0644m741 & $\ldots$ & $\ldots$ & $\ldots$ & $5.341 \mathrm{E}-03$ & $1.789 \mathrm{E}-03$ & $1.810 \mathrm{E}-04$ & $(4)(1)(5)$ \\
\hline PGC 19481 & 2003-09-20 & 2513 & NGA-AM0644m741 & $1.148 \mathrm{E}-03$ & $7.253 \mathrm{E}-04$ & $6.923 \mathrm{E}-05$ & 5.333E-03 & $1.787 \mathrm{E}-03$ & $2.361 \mathrm{E}-04$ & \\
\hline ESO 034-G013 & 2003-09-20 & 2513 & NGA-AM0644m741 & $1.269 \mathrm{E}-03$ & $8.410 \mathrm{E}-04$ & $3.341 \mathrm{E}-05$ & $5.502 \mathrm{E}-03$ & $1.738 \mathrm{E}-03$ & $5.151 \mathrm{E}-05$ & \\
\hline NGC 2310 & $2004-12-26$ & 586 & LGAL-NGC2310 & $\ldots$ & $\ldots$ & $\ldots$ & $1.263 \mathrm{E}-02$ & $6.164 \mathrm{E}-03$ & $6.548 \mathrm{E}-04$ & $(4)(2)$ \\
\hline NGC 2366 & 2004-01-16 & 1702 & NGA-NGC2366 & $3.802 \mathrm{E}-04$ & $5.022 \mathrm{E}-04$ & $1.699 \mathrm{E}-07$ & $2.855 \mathrm{E}-03$ & $1.523 \mathrm{E}-03$ & $3.620 \mathrm{E}-05$ & \\
\hline Mrk 8 & $2003-10-17$ & 1400 & NGA-Mrk8 & $3.424 \mathrm{E}-04$ & $5.077 \mathrm{E}-04$ & $1.322 \mathrm{E}-05$ & 2.643E-03 & $1.587 \mathrm{E}-03$ & $1.602 \mathrm{E}-05$ & \\
\hline
\end{tabular}


Table 2-Continued

\begin{tabular}{|c|c|c|c|c|c|c|c|c|c|c|}
\hline \multirow{2}{*}{$\begin{array}{l}\text { Object Name } \\
\text { (1) }\end{array}$} & \multirow{2}{*}{$\begin{array}{c}\text { Date } \\
\text { Observed } \\
\\
(2)\end{array}$} & \multirow{2}{*}{$\begin{array}{l}\text { Exposure } \\
\quad(\mathrm{sec}) \\
(3)\end{array}$} & \multirow{2}{*}{$\begin{array}{l}\text { Tile } \\
\text { (4) }\end{array}$} & \multicolumn{3}{|c|}{ FUV sky background } & \multicolumn{3}{|c|}{ NUV sky background } & \multirow{2}{*}{$\begin{array}{c}\text { notes } \\
(11)\end{array}$} \\
\hline & & & & $\begin{array}{c}\text { mean } \\
(\text { counts/s) } \\
(5)\end{array}$ & $\begin{array}{c}<\sigma> \\
(\text { counts/s) } \\
(6)\end{array}$ & $\begin{array}{c}\sigma(\text { mean }) \\
(\text { counts/s) } \\
(7)\end{array}$ & $\begin{array}{c}\text { mean } \\
(\text { counts/s) } \\
(8)\end{array}$ & $\begin{array}{c}<\sigma> \\
(\text { counts } / s) \\
(9)\end{array}$ & $\begin{array}{c}\sigma(\text { mean }) \\
(\text { counts/s) } \\
(10)\end{array}$ & \\
\hline UGC 03864 & $2003-10-17$ & 1400 & NGA-Mrk8 & $3.829 \mathrm{E}-04$ & $6.421 \mathrm{E}-04$ & $2.289 \mathrm{E}-05$ & $2.622 \mathrm{E}-03$ & $1.564 \mathrm{E}-03$ & $4.370 \mathrm{E}-06$ & (1) \\
\hline ESO 059-G006 & 2004-04-03 & 690 & LGAL-NGC2442 & $1.691 \mathrm{E}-03$ & $1.773 \mathrm{E}-03$ & $4.107 \mathrm{E}-05$ & $6.932 \mathrm{E}-03$ & $3.923 \mathrm{E}-03$ & $5.260 \mathrm{E}-05$ & \\
\hline NGC 2434 & 2004-04-03 & 690 & LGAL-NGC2442 & $2.036 \mathrm{E}-03$ & $1.864 \mathrm{E}-03$ & $1.752 \mathrm{E}-04$ & 7.062E-03 & $3.691 \mathrm{E}-03$ & $3.192 \mathrm{E}-04$ & \\
\hline ESO 059-G007 & 2004-04-03 & 690 & LGAL-NGC2442 & $1.665 \mathrm{E}-03$ & $1.809 \mathrm{E}-03$ & $1.501 \mathrm{E}-05$ & $6.832 \mathrm{E}-03$ & $3.898 \mathrm{E}-03$ & $1.417 \mathrm{E}-04$ & \\
\hline NGC 2442 & 2004-04-03 & 690 & LGAL-NGC2442 & $1.874 \mathrm{E}-03$ & $1.934 \mathrm{E}-03$ & $8.665 \mathrm{E}-05$ & $6.721 \mathrm{E}-03$ & $3.756 \mathrm{E}-03$ & $1.409 \mathrm{E}-04$ & \\
\hline NGC 2403 & $2003-12-05$ & 1704 & NGA-NGC2403 & $3.732 \mathrm{E}-04$ & $5.100 \mathrm{E}-04$ & $4.784 \mathrm{E}-05$ & $2.901 \mathrm{E}-03$ & $1.552 \mathrm{E}-03$ & $1.241 \mathrm{E}-04$ & \\
\hline ESO 059-G010 & 2004-04-03 & 690 & LGAL-NGC2442 & $1.641 \mathrm{E}-03$ & $1.818 \mathrm{E}-03$ & $2.045 \mathrm{E}-04$ & $6.476 \mathrm{E}-03$ & $3.761 \mathrm{E}-03$ & $2.639 \mathrm{E}-04$ & \\
\hline UGC 03942 & 2005-01-15 & 1509 & MISDR2-15145-0888 & $\ldots$ & $\ldots$ & $\ldots$ & $3.908 \mathrm{E}-03$ & $1.859 \mathrm{E}-03$ & $4.123 \mathrm{E}-05$ & (4) \\
\hline ESO 059-G011 & 2004-04-03 & 690 & LGAL-NGC2442 & $\ldots$ & $\ldots$ & $\ldots$ & $6.430 \mathrm{E}-03$ & $3.628 \mathrm{E}-03$ & $6.788 \mathrm{E}-04$ & $(4)(5)$ \\
\hline UGC 03995 & $2005-01-28$ & 1535 & MISDR2-15142-0754 & $3.073 \mathrm{E}-04$ & 4.627E-04 & $3.118 \mathrm{E}-05$ & $3.505 \mathrm{E}-03$ & $1.734 \mathrm{E}-03$ & $4.743 \mathrm{E}-05$ & \\
\hline UGC 03997 & $2004-01-12$ & 4706 & MISDR1-04055-0432 & $4.049 \mathrm{E}-04$ & $4.253 \mathrm{E}-04$ & $7.448 \mathrm{E}-05$ & $3.362 \mathrm{E}-03$ & $9.927 \mathrm{E}-04$ & $2.137 \mathrm{E}-05$ & \\
\hline UGC 04056 & $2005-01-10$ & 1976 & MISDR1-03940-0434 & $3.469 \mathrm{E}-04$ & $1.117 \mathrm{E}-03$ & $5.314 \mathrm{E}-05$ & $3.119 \mathrm{E}-03$ & $1.448 \mathrm{E}-03$ & $6.515 \mathrm{E}-05$ & \\
\hline UGC 04136 & $2004-01-12$ & 1702 & MISDR1-03621-0438 & $3.304 \mathrm{E}-04$ & $5.442 \mathrm{E}-04$ & $1.165 \mathrm{E}-05$ & $2.909 \mathrm{E}-03$ & $1.533 \mathrm{E}-03$ & $7.513 \mathrm{E}-05$ & \\
\hline UGC 04148 & $2004-01-11$ & 1433 & MISDR1-03938-0437 & $3.153 \mathrm{E}-04$ & $5.578 \mathrm{E}-04$ & $3.404 \mathrm{E}-06$ & $3.311 \mathrm{E}-03$ & $1.819 \mathrm{E}-03$ & $4.026 \mathrm{E}-06$ & \\
\hline NGC 2500 & $2005-02-23$ & 1696 & NGA-NGC2500 & 3.337E-04 & 4.733E-04 & $4.249 \mathrm{E}-05$ & $2.998 \mathrm{E}-03$ & $1.568 \mathrm{E}-03$ & $2.058 \mathrm{E}-05$ & \\
\hline UGC 04176 & $2004-01-11$ & 1696 & MISDR1-04052-0435 & $3.251 \mathrm{E}-04$ & $5.388 \mathrm{E}-04$ & $2.103 \mathrm{E}-06$ & $3.210 \mathrm{E}-03$ & $1.565 \mathrm{E}-03$ & $2.119 \mathrm{E}-05$ & \\
\hline UGC 04188 & 2004-01-11 & 1433 & MISDR1-03938-0437 & $3.175 \mathrm{E}-04$ & 4.834E-04 & $2.461 \mathrm{E}-05$ & $3.243 \mathrm{E}-03$ & $1.710 \mathrm{E}-03$ & $4.032 \mathrm{E}-05$ & \\
\hline NGC 2538 & 2005-01-01 & 1675 & MISDR3-16262-1184 & $\ldots$ & $\ldots$ & $\ldots$ & $3.128 \mathrm{E}-03$ & $1.554 \mathrm{E}-03$ & $1.068 \mathrm{E}-04$ & (4) \\
\hline NGC 2543 & 2005-03-03 & 1632 & MISDR2-04288-0826 & $4.767 \mathrm{E}-04$ & $5.490 \mathrm{E}-04$ & $2.414 \mathrm{E}-05$ & $3.386 \mathrm{E}-03$ & $1.613 \mathrm{E}-03$ & $5.940 \mathrm{E}-05$ & \\
\hline NGC 2537 & 2004-01-13 & 1704 & NGA-NGC2537 & $4.035 \mathrm{E}-04$ & $5.161 \mathrm{E}-04$ & $2.318 \mathrm{E}-05$ & $3.017 \mathrm{E}-03$ & $1.571 \mathrm{E}-03$ & $1.049 \mathrm{E}-04$ & \\
\hline UGC4278 & 2004-01-13 & 1704 & NGA-NGC2537 & $3.947 \mathrm{E}-04$ & $4.987 \mathrm{E}-04$ & $1.411 \mathrm{E}-05$ & $2.997 \mathrm{E}-03$ & $1.531 \mathrm{E}-03$ & $2.034 \mathrm{E}-05$ & \\
\hline NGC 2541 & $2005-02-14$ & 2898 & NGA-NGC2541 & $3.430 \mathrm{E}-04$ & $4.886 \mathrm{E}-04$ & $1.057 \mathrm{E}-05$ & $2.945 \mathrm{E}-03$ & $1.188 \mathrm{E}-03$ & $2.292 \mathrm{E}-05$ & \\
\hline NGC $2523 \mathrm{C}$ & $2003-10-16$ & 1657 & NGA-NGC2551 & $3.784 \mathrm{E}-04$ & $5.310 \mathrm{E}-04$ & $2.654 \mathrm{E}-05$ & $2.677 \mathrm{E}-03$ & $1.478 \mathrm{E}-03$ & $6.809 \mathrm{E}-05$ & \\
\hline UGC 04311 & 2005-03-03 & 1632 & MISDR3-04226-0893 & $3.359 \mathrm{E}-04$ & $4.715 \mathrm{E}-04$ & $2.123 \mathrm{E}-05$ & $3.042 \mathrm{E}-03$ & $1.583 \mathrm{E}-03$ & $6.963 \mathrm{E}-05$ & \\
\hline Holmberg II & $2003-12-05$ & 1704 & NGA-HolmbergII & $3.341 \mathrm{E}-04$ & $5.052 \mathrm{E}-04$ & $6.710 \mathrm{E}-06$ & $2.607 \mathrm{E}-03$ & $1.478 \mathrm{E}-03$ & $2.690 \mathrm{E}-05$ & \\
\hline NGC 2552 & $2005-01-08$ & 545 & MISDR1-03473-0440 & $\begin{array}{c}0.0710-01 \\
\ldots\end{array}$ & $\begin{array}{l}0.0020-07 \\
\ldots\end{array}$ & $\ldots$ & $2.939 \mathrm{E}-03$ & $2.688 \mathrm{E}-03$ & $8.810 \mathrm{E}-05$ & (4) \\
\hline UGC 04387 & 2005-01-09 & 1687 & MISDR1-03617-0441 & $\ldots$ & $\ldots$ & $\ldots$ & $2.751 \mathrm{E}-03$ & $1.488 \mathrm{E}-03$ & $5.837 \mathrm{E}-05$ & (4) \\
\hline NGC 2551 & $2003-10-16$ & 1657 & NGA-NGC2551 & $3.597 \mathrm{E}-04$ & 4.973E-04 & $1.878 \mathrm{E}-05$ & $2.675 \mathrm{E}-03$ & $1.503 \mathrm{E}-03$ & $1.137 \mathrm{E}-04$ & \\
\hline HS $0822+3542$ & $2005-02-12$ & 1120 & NGA-HS0822p3542 & $3.377 \mathrm{E}-04$ & $5.713 \mathrm{E}-04$ & $5.509 \mathrm{E}-06$ & $3.466 \mathrm{E}-03$ & $2.070 \mathrm{E}-03$ & $5.419 \mathrm{E}-05$ & \\
\hline UGC 04393 & 2005-01-11 & 1691 & MISDR1-03667-0441 & $\ldots$ & $\ldots$ & $\ldots$ & $2.786 \mathrm{E}-03$ & $1.521 \mathrm{E}-03$ & $2.908 \mathrm{E}-07$ & (4) \\
\hline UGC 04401 & $2005-01-08$ & 1695 & MISDR1-03519-0443 & $\ldots$ & $\ldots$ & $\ldots$ & $2.730 \mathrm{E}-03$ & $1.513 \mathrm{E}-03$ & $3.292 \mathrm{E}-05$ & (4) \\
\hline UGC 04390 & $2003-10-16$ & 1657 & NGA-NGC2551 & $3.862 \mathrm{E}-04$ & $5.425 \mathrm{E}-04$ & $1.832 \mathrm{E}-05$ & $2.651 \mathrm{E}-03$ & $1.477 \mathrm{E}-03$ & $7.472 \mathrm{E}-05$ & \\
\hline
\end{tabular}


Table 2-Continued

\begin{tabular}{|c|c|c|c|c|c|c|c|c|c|c|}
\hline \multirow{2}{*}{$\begin{array}{l}\text { Object Name } \\
\text { (1) }\end{array}$} & \multirow{2}{*}{$\begin{array}{c}\text { Date } \\
\text { Observed } \\
(2)\end{array}$} & \multirow{2}{*}{$\begin{array}{l}\text { Exposure } \\
\text { (sec) } \\
(3)\end{array}$} & \multirow{2}{*}{$\begin{array}{l}\text { Tile } \\
(4)\end{array}$} & \multicolumn{3}{|c|}{ FUV sky background } & \multicolumn{3}{|c|}{ NUV sky background } & \multirow{2}{*}{$\begin{array}{l}\text { notes } \\
\text { (11) }\end{array}$} \\
\hline & & & & $\begin{array}{c}\text { mean } \\
(\text { counts/s) } \\
(5)\end{array}$ & $\begin{array}{c}<\sigma> \\
(\text { counts } / \mathrm{s}) \\
(6)\end{array}$ & $\begin{array}{c}\sigma(\text { mean }) \\
(\text { counts/s) } \\
(7)\end{array}$ & $\begin{array}{c}\text { mean } \\
(\text { counts/s) } \\
(8)\end{array}$ & $\begin{array}{c}<\sigma> \\
(\text { counts } / \mathrm{s}) \\
(9)\end{array}$ & $\begin{array}{c}\sigma(\text { mean }) \\
(\text { counts/s) } \\
(10)\end{array}$ & \\
\hline NGC $2550 A$ & $2003-10-16$ & 1657 & NGA-NGC2551 & $3.804 \mathrm{E}-04$ & $6.087 \mathrm{E}-04$ & $1.509 \mathrm{E}-05$ & $2.549 \mathrm{E}-03$ & $1.415 \mathrm{E}-03$ & $7.249 \mathrm{E}-06$ & \\
\hline UGC 04436 & 2005-01-08 & 1695 & MISDR1-03519-0443 & $\ldots$ & $\ldots$ & $\cdots$ & $2.668 \mathrm{E}-03$ & $1.440 \mathrm{E}-03$ & $3.496 \mathrm{E}-05$ & (4) \\
\hline UGC 04461 & 2004-01-23 & 1686 & MISDR1-03292-0444 & $2.753 \mathrm{E}-04$ & $4.706 \mathrm{E}-04$ & $1.696 \mathrm{E}-05$ & $2.880 \mathrm{E}-03$ & $1.547 \mathrm{E}-03$ & $7.004 \mathrm{E}-05$ & \\
\hline DDO 053 & 2004-01-17 & 1699 & NGA-DDO053 & $3.125 \mathrm{E}-04$ & $4.579 \mathrm{E}-04$ & $2.232 \mathrm{E}-05$ & $2.717 \mathrm{E}-03$ & $1.499 \mathrm{E}-03$ & $9.860 \mathrm{E}-05$ & \\
\hline NGC 2600 & 2004-01-23 & 1686 & MISDR1-03292-0444 & 2.693E-04 & $4.354 \mathrm{E}-04$ & $3.184 \mathrm{E}-07$ & $2.890 \mathrm{E}-03$ & $1.526 \mathrm{E}-03$ & $3.827 \mathrm{E}-05$ & \\
\hline UGC 04499 & 2005-01-09 & 93 & MISDR1-03334-0445 & $\ldots$ & $\cdots$ & $\cdots$ & $2.614 \mathrm{E}-03$ & $5.860 \mathrm{E}-03$ & $6.532 \mathrm{E}-05$ & (4) \\
\hline NGC 2623 & $2004-02-08$ & 2328 & NGA-NGC2623 & $\ldots$ & $\cdots$ & $\ldots$ & $3.565 \mathrm{E}-03$ & $1.443 \mathrm{E}-03$ & $5.081 \mathrm{E}-08$ & (4) \\
\hline UGC 04514 & 2004-01-23 & 1169 & MISDR1-03249-0444 & $2.882 \mathrm{E}-04$ & $5.739 \mathrm{E}-04$ & $6.931 \mathrm{E}-06$ & $2.802 \mathrm{E}-03$ & $1.798 \mathrm{E}-03$ & $6.537 \mathrm{E}-05$ & \\
\hline UGC 04515 & 2005-01-09 & 1695 & MISDR1-03291-0444 & $\ldots$ & $\ldots$ & $\ldots$ & $2.504 \mathrm{E}-03$ & $1.381 \mathrm{E}-03$ & $1.476 \mathrm{E}-05$ & (4) \\
\hline UGC 04525 & 2005-01-09 & 93 & MISDR1-03334-0445 & $\ldots$ & $\ldots$ & $\ldots$ & $2.511 \mathrm{E}-03$ & $5.785 \mathrm{E}-03$ & $4.516 \mathrm{E}-05$ & (4) \\
\hline UGC 04529 & $2004-01-15$ & 3404 & MISDR1-03565-0549 & $2.176 \mathrm{E}-04$ & $2.697 \mathrm{E}-04$ & $1.579 \mathrm{E}-05$ & $2.724 \mathrm{E}-03$ & $1.030 \mathrm{E}-03$ & $2.148 \mathrm{E}-06$ & \\
\hline NGC 2639 & 2005-01-09 & 3231 & MISDR1-03423-0445 & $2.004 \mathrm{E}-04$ & $4.165 \mathrm{E}-04$ & $2.421 \mathrm{E}-06$ & $2.473 \mathrm{E}-03$ & $1.002 \mathrm{E}-03$ & $2.525 \mathrm{E}-05$ & \\
\hline UGC 04546 & 2004-01-16 & 3242 & MISDR1-03290-0447 & $2.301 \mathrm{E}-04$ & $2.927 \mathrm{E}-04$ & $1.413 \mathrm{E}-06$ & $2.585 \mathrm{E}-03$ & $1.020 \mathrm{E}-03$ & $3.475 \mathrm{E}-05$ & \\
\hline UGC 04551 & 2005-01-09 & 3231 & MISDR1-03423-0445 & $1.868 \mathrm{E}-04$ & $3.555 \mathrm{E}-04$ & $2.502 \mathrm{E}-06$ & $2.471 \mathrm{E}-03$ & $1.003 \mathrm{E}-03$ & $5.527 \mathrm{E}-05$ & \\
\hline UGC 04562 & 2005-01-10 & 179 & MISDR1-03516-0550 & $\ldots$ & $\ldots$ & $\ldots$ & $2.740 \mathrm{E}-03$ & $4.487 \mathrm{E}-03$ & $3.592 \mathrm{E}-05$ & (4) \\
\hline UGC 04560 & 2004-01-16 & 3242 & MISDR1-03290-0447 & $2.604 \mathrm{E}-04$ & $3.106 \mathrm{E}-04$ & $3.125 \mathrm{E}-06$ & $2.653 \mathrm{E}-03$ & $1.050 \mathrm{E}-03$ & $4.011 \mathrm{E}-05$ & \\
\hline VV 703 & 2004-01-17 & 1700 & MISDR1-03206-0446 & $2.484 \mathrm{E}-04$ & $4.628 \mathrm{E}-04$ & $6.548 \mathrm{E}-06$ & $2.689 \mathrm{E}-03$ & $1.476 \mathrm{E}-03$ & $1.840 \mathrm{E}-05$ & (2) \\
\hline UGC 04628 & $2005-02-24$ & 1212 & NGA-NGC2681 & $2.157 \mathrm{E}-04$ & 4.966E-04 & $2.610 \mathrm{E}-06$ & $2.506 \mathrm{E}-03$ & $1.688 \mathrm{E}-03$ & $4.783 \mathrm{E}-05$ & \\
\hline NGC 2675 & 2004-01-17 & 1700 & MISDR1-03206-0446 & $2.133 \mathrm{E}-04$ & $3.751 \mathrm{E}-04$ & $1.215 \mathrm{E}-06$ & $2.536 \mathrm{E}-03$ & $1.397 \mathrm{E}-03$ & $2.809 \mathrm{E}-05$ & \\
\hline NGC 2681 & $2005-02-24$ & 1212 & NGA-NGC2681 & $2.051 \mathrm{E}-04$ & $4.380 \mathrm{E}-04$ & $1.579 \mathrm{E}-05$ & $2.513 \mathrm{E}-03$ & $1.682 \mathrm{E}-03$ & $6.759 \mathrm{E}-05$ & \\
\hline IC 0522 & 2004-01-17 & 1699 & MISDR1-03051-0448 & $3.004 \mathrm{E}-04$ & $4.364 \mathrm{E}-04$ & $1.815 \mathrm{E}-05$ & $2.680 \mathrm{E}-03$ & $1.437 \mathrm{E}-03$ & $2.797 \mathrm{E}-05$ & \\
\hline VV 761 & $2004-01-17$ & 1699 & MISDR1-03051-0448 & $3.216 \mathrm{E}-04$ & $4.460 \mathrm{E}-04$ & $6.409 \mathrm{E}-05$ & $2.674 \mathrm{E}-03$ & $1.408 \mathrm{E}-03$ & $4.522 \mathrm{E}-05$ & $(1)(2)$ \\
\hline UGC 04668 & 2004-01-17 & 1699 & MISDR1-03051-0448 & $3.419 \mathrm{E}-04$ & $4.683 \mathrm{E}-04$ & $2.115 \mathrm{E}-06$ & $2.706 \mathrm{E}-03$ & $1.428 \mathrm{E}-03$ & $5.819 \mathrm{E}-06$ & \\
\hline UGC 04684 & 2004-01-16 & 3399 & MISDR1-24321-0468 & $3.289 \mathrm{E}-04$ & $3.227 \mathrm{E}-04$ & $1.586 \mathrm{E}-05$ & $3.290 \mathrm{E}-03$ & $1.153 \mathrm{E}-03$ & $3.930 \mathrm{E}-05$ & \\
\hline UGC 04671 & $2004-01-17$ & 1699 & MISDR1-03288-0551 & $2.173 \mathrm{E}-04$ & $3.968 \mathrm{E}-04$ & $7.286 \mathrm{E}-06$ & $2.630 \mathrm{E}-03$ & $1.457 \mathrm{E}-03$ & $3.800 \mathrm{E}-05$ & \\
\hline NGC 2692 & $2004-01-17$ & 1699 & MISDR1-03288-0551 & $2.180 \mathrm{E}-04$ & 4.007E-04 & $2.684 \mathrm{E}-05$ & $2.629 \mathrm{E}-03$ & $1.457 \mathrm{E}-03$ & $9.978 \mathrm{E}-05$ & \\
\hline NGC 2693 & $2005-02-24$ & 1212 & NGA-NGC2681 & $1.939 \mathrm{E}-04$ & $4.068 \mathrm{E}-04$ & $1.893 \mathrm{E}-05$ & $2.448 \mathrm{E}-03$ & $1.555 \mathrm{E}-03$ & $3.016 \mathrm{E}-05$ & \\
\hline UGC 04676 & 2004-01-17 & 1699 & MISDR1-03288-0551 & $2.086 \mathrm{E}-04$ & $3.724 \mathrm{E}-04$ & $6.028 \mathrm{E}-06$ & $2.609 \mathrm{E}-03$ & $1.427 \mathrm{E}-03$ & $7.716 \mathrm{E}-05$ & \\
\hline UGC 04679 & 2004-01-17 & 1699 & MISDR1-03288-0551 & $1.989 \mathrm{E}-04$ & $3.474 \mathrm{E}-04$ & $1.302 \mathrm{E}-05$ & $2.571 \mathrm{E}-03$ & $1.368 \mathrm{E}-03$ & $4.239 \mathrm{E}-06$ & \\
\hline UGC 04690 & 2004-01-17 & 1699 & MISDR1-03288-0551 & $2.135 \mathrm{E}-04$ & $4.072 \mathrm{E}-04$ & $4.380 \mathrm{E}-07$ & $2.631 \mathrm{E}-03$ & $1.479 \mathrm{E}-03$ & $3.499 \mathrm{E}-05$ & \\
\hline UGC 04702 & $2005-02-13$ & 1035 & NGA-IRAS08572 & $\ldots$ & $\ldots$ & $\ldots$ & $2.887 \mathrm{E}-03$ & $1.973 \mathrm{E}-03$ & $6.235 \mathrm{E}-05$ & (4) \\
\hline UGC 04704 & $2005-02-13$ & 1035 & NGA-IRAS08572 & $\ldots$ & $\cdots$ & $\ldots$ & $2.850 \mathrm{E}-03$ & $1.912 \mathrm{E}-03$ & $9.897 \mathrm{E}-05$ & (4) \\
\hline
\end{tabular}


Table 2-Continued

\begin{tabular}{|c|c|c|c|c|c|c|c|c|c|c|}
\hline \multirow{2}{*}{$\begin{array}{l}\text { Object Name } \\
\text { (1) }\end{array}$} & \multirow{2}{*}{$\begin{array}{c}\text { Date } \\
\text { Observed } \\
(2)\end{array}$} & \multirow{2}{*}{$\begin{array}{l}\text { Exposure } \\
\quad(\mathrm{sec}) \\
(3)\end{array}$} & \multirow{2}{*}{$\begin{array}{l}\text { Tile } \\
\text { (4) }\end{array}$} & \multicolumn{3}{|c|}{ FUV sky background } & \multicolumn{3}{|c|}{ NUV sky background } & \multirow{2}{*}{$\begin{array}{l}\text { notes } \\
(11)\end{array}$} \\
\hline & & & & $\begin{array}{c}\text { mean } \\
(\text { counts } / \mathrm{s}) \\
(5)\end{array}$ & $\begin{array}{c}<\sigma> \\
(\text { counts/s) } \\
(6)\end{array}$ & $\begin{array}{c}\sigma(\text { mean }) \\
(\text { counts/s) } \\
(7)\end{array}$ & $\begin{array}{c}\text { mean } \\
(\text { counts/s) } \\
(8)\end{array}$ & $\begin{array}{c}<\sigma> \\
(\text { counts/s) } \\
(9)\end{array}$ & $\begin{array}{c}\sigma(\text { mean }) \\
(\text { counts/s) } \\
(10)\end{array}$ & \\
\hline NGC 2710 & 2004-01-18 & 1696 & MISDR1-03087-0448 & $2.033 \mathrm{E}-04$ & $3.537 \mathrm{E}-04$ & $1.946 \mathrm{E}-06$ & $2.464 \mathrm{E}-03$ & $1.325 \mathrm{E}-03$ & $1.124 \mathrm{E}-05$ & \\
\hline UGC 04800 & 2004-01-28 & 2353 & MISDR1-03124-0450 & $3.085 \mathrm{E}-04$ & $5.096 \mathrm{E}-04$ & $4.119 \mathrm{E}-06$ & $2.912 \mathrm{E}-03$ & $1.310 \mathrm{E}-03$ & $2.716 \mathrm{E}-06$ & \\
\hline UGC 04807 & 2004-01-28 & 2353 & MISDR1-03124-0450 & $3.534 \mathrm{E}-04$ & $6.153 \mathrm{E}-04$ & $8.636 \mathrm{E}-06$ & $2.869 \mathrm{E}-03$ & $1.300 \mathrm{E}-03$ & $9.331 \mathrm{E}-05$ & \\
\hline NGC 2768 & $2005-02-23$ & 182 & NGA-NGC2768 & $2.983 \mathrm{E}-04$ & $1.377 \mathrm{E}-03$ & $3.592 \mathrm{E}-05$ & $2.619 \mathrm{E}-03$ & $4.424 \mathrm{E}-03$ & $2.697 \mathrm{E}-05$ & \\
\hline NGC 2784 & 2004-01-13 & 1704 & NGA-NGC2784 & $1.591 \mathrm{E}-03$ & $1.049 \mathrm{E}-03$ & $9.355 \mathrm{E}-05$ & $6.514 \mathrm{E}-03$ & $2.417 \mathrm{E}-03$ & $1.365 \mathrm{E}-04$ & \\
\hline UGC 04844 & 2005-01-11 & 1692 & MISDR2-03373-0765 & $\cdots$ & $\cdots$ & $\cdots$ & $2.588 \mathrm{E}-03$ & $1.426 \mathrm{E}-03$ & $1.611 \mathrm{E}-05$ & (4) \\
\hline UGC 04851 & 2004-04-24 & 1562 & MISDR1-03203-0553 & $3.356 \mathrm{E}-04$ & $6.035 \mathrm{E}-04$ & $1.289 \mathrm{E}-05$ & 3.093E-03 & $1.683 \mathrm{E}-03$ & $2.122 \mathrm{E}-05$ & \\
\hline NGC 2782 & 2005-02-15 & 722 & NGA-NGC2782 & $1.896 \mathrm{E}-04$ & $5.503 \mathrm{E}-04$ & $3.825 \mathrm{E}-06$ & $2.740 \mathrm{E}-03$ & $2.289 \mathrm{E}-03$ & $2.467 \mathrm{E}-05$ & \\
\hline UGC 04872 & $2005-02-15$ & 722 & NGA-NGC2782 & $1.887 \mathrm{E}-04$ & $5.684 \mathrm{E}-04$ & $3.478 \mathrm{E}-06$ & $2.715 \mathrm{E}-03$ & $2.266 \mathrm{E}-03$ & $6.362 \mathrm{E}-05$ & \\
\hline NGC 2798 & 2004-02-08 & 3001 & NGA-NGC2798 & $2.060 \mathrm{E}-04$ & $3.969 \mathrm{E}-04$ & $1.009 \mathrm{E}-05$ & $2.917 \mathrm{E}-03$ & $1.157 \mathrm{E}-03$ & $1.479 \mathrm{E}-04$ & \\
\hline UGC 04915 & $2004-01-26$ & 3357 & MISDR1-24349-0472 & $2.984 \mathrm{E}-04$ & $3.258 \mathrm{E}-04$ & $2.898 \mathrm{E}-05$ & $2.986 \mathrm{E}-03$ & $1.080 \mathrm{E}-03$ & $9.869 \mathrm{E}-06$ & \\
\hline NGC 2799 & 2004-02-08 & 3001 & NGA-NGC2798 & $2.026 \mathrm{E}-04$ & $3.893 \mathrm{E}-04$ & $2.873 \mathrm{E}-05$ & $2.913 \mathrm{E}-03$ & $1.151 \mathrm{E}-03$ & $1.192 \mathrm{E}-04$ & \\
\hline IC 0531 & 2004-02-09 & 1471 & MISDR1-24348-0474 & $3.021 \mathrm{E}-04$ & $5.484 \mathrm{E}-04$ & $2.115 \mathrm{E}-05$ & $3.200 \mathrm{E}-03$ & $1.667 \mathrm{E}-03$ & $6.589 \mathrm{E}-05$ & \\
\hline UGC 04921 & 2005-01-11 & 1692 & MISDR2-03372-0766 & $\ldots$ & $\ldots$ & $\ldots$ & $2.429 \mathrm{E}-03$ & $1.386 \mathrm{E}-03$ & $2.525 \mathrm{E}-06$ & (4) \\
\hline NGC 2841 & 2004-01-18 & 1821 & NGA-NGC2841 & 3.033E-04 & $4.502 \mathrm{E}-04$ & $1.346 \mathrm{E}-05$ & $2.959 \mathrm{E}-03$ & $1.453 \mathrm{E}-03$ & 7.167E-06 & \\
\hline NGC 2854 & 2005-04-21 & 813 & MISDR3-03371-0900 & $\cdots$ & $\cdots$ & $\cdots$ & $2.920 \mathrm{E}-03$ & $2.238 \mathrm{E}-03$ & $6.298 \mathrm{E}-06$ & (4) \\
\hline NGC 2856 & 2005-04-21 & 813 & MISDR3-03371-0900 & $\cdots$ & $\cdots$ & $\cdots$ & $2.918 \mathrm{E}-03$ & $2.243 \mathrm{E}-03$ & $6.453 \mathrm{E}-05$ & (4) \\
\hline NGC 2857 & 2005-04-21 & 813 & MISDR3-03371-0900 & $\cdots$ & $\ldots$ & $\cdots$ & $2.906 \mathrm{E}-03$ & $2.228 \mathrm{E}-03$ & $3.498 \mathrm{E}-05$ & (4) \\
\hline NGC 2915 & 2004-04-01 & 1652 & NGA-NGC2915 & $2.016 \mathrm{E}-03$ & $7.070 \mathrm{E}-04$ & $2.146 \mathrm{E}-04$ & 7.197E-03 & $1.529 \mathrm{E}-03$ & $1.930 \mathrm{E}-04$ & \\
\hline UGC 05013 & 2004-01-21 & 1592 & MISDR1-02845-0485 & $3.068 \mathrm{E}-04$ & $5.240 \mathrm{E}-04$ & $1.144 \mathrm{E}-05$ & $2.592 \mathrm{E}-03$ & $1.429 \mathrm{E}-03$ & $2.548 \mathrm{E}-05$ & \\
\hline UGC 05027 & $2005-03-15$ & 1307 & MISDR1-24222-0475 & $\ldots$ & $\ldots$ & $\ldots$ & $2.969 \mathrm{E}-03$ & $1.707 \mathrm{E}-03$ & $7.684 \mathrm{E}-06$ & (4) \\
\hline NGC 2870 & 2004-04-25 & 1171 & MISDR1-02975-0451 & $3.600 \mathrm{E}-04$ & $5.847 \mathrm{E}-04$ & $2.752 \mathrm{E}-06$ & $2.980 \mathrm{E}-03$ & $1.858 \mathrm{E}-03$ & $3.429 \mathrm{E}-05$ & (2) \\
\hline UGC 05053 & 2004-01-21 & 1688 & MISDR1-02876-0485 & $2.748 \mathrm{E}-04$ & $4.079 \mathrm{E}-04$ & $2.008 \mathrm{E}-06$ & $2.559 \mathrm{E}-03$ & $1.391 \mathrm{E}-03$ & $1.126 \mathrm{E}-05$ & \\
\hline NGC 2903 & $2005-02-16$ & 454 & NGA-NGC2903 & $2.776 \mathrm{E}-04$ & $8.532 \mathrm{E}-04$ & $4.582 \mathrm{E}-05$ & $3.322 \mathrm{E}-03$ & $3.187 \mathrm{E}-03$ & $5.041 \mathrm{E}-05$ & \\
\hline UGC 05077 & 2004-03-05 & 1671 & MISDR1-04758-0453 & $\cdots$ & $\cdots$ & $\cdots$ & $2.421 \mathrm{E}-03$ & $1.370 \mathrm{E}-03$ & $2.085 \mathrm{E}-05$ & (4) \\
\hline I Zw 18 & 2004-01-19 & 1695 & NGA-IZw18 & $2.902 \mathrm{E}-04$ & $4.471 \mathrm{E}-04$ & $3.198 \mathrm{E}-06$ & $2.599 \mathrm{E}-03$ & $1.408 \mathrm{E}-03$ & $6.093 \mathrm{E}-06$ & \\
\hline NGC 2916 & $2005-02-16$ & 454 & NGA-NGC2903 & $2.620 \mathrm{E}-04$ & $9.765 \mathrm{E}-04$ & $5.121 \mathrm{E}-05$ & $3.249 \mathrm{E}-03$ & $3.029 \mathrm{E}-03$ & $2.009 \mathrm{E}-05$ & \\
\hline UGC 05107 & 2005-03-18 & 1684 & MISDR2-24164-0569 & $\cdots$ & $\cdots$ & $\cdots$ & 2.973E-03 & $1.470 \mathrm{E}-03$ & 3.397E-05 & (4) \\
\hline UGC 05101 & 2005-02-24 & 1665 & NGA-UGC5101 & $2.755 \mathrm{E}-04$ & $4.476 \mathrm{E}-04$ & $2.873 \mathrm{E}-05$ & $2.446 \mathrm{E}-03$ & $1.409 \mathrm{E}-03$ & $4.505 \mathrm{E}-06$ & \\
\hline NGC 2936 & 2004-03-02 & 1102 & MISDR1-24220-0477 & $2.681 \mathrm{E}-04$ & $6.274 \mathrm{E}-04$ & 7.393E-07 & $2.950 \mathrm{E}-03$ & $1.839 \mathrm{E}-03$ & $1.938 \mathrm{E}-06$ & \\
\hline NGC 2937 & 2004-03-02 & 1102 & MISDR1-24220-0477 & $2.664 \mathrm{E}-04$ & $6.291 \mathrm{E}-04$ & $3.213 \mathrm{E}-05$ & $2.947 \mathrm{E}-03$ & $1.836 \mathrm{E}-03$ & $4.366 \mathrm{E}-06$ & (1) \\
\hline UGC 05147 & 2005-04-14 & 691 & MISDR3-01595-1215 & $\cdots$ & $\cdots$ & $\cdots$ & $2.775 \mathrm{E}-03$ & $2.290 \mathrm{E}-03$ & $5.340 \mathrm{E}-05$ & (4) \\
\hline
\end{tabular}


Table 2-Continued

\begin{tabular}{|c|c|c|c|c|c|c|c|c|c|c|}
\hline \multirow{2}{*}{$\begin{array}{l}\text { Object Name } \\
\text { (1) }\end{array}$} & \multirow{2}{*}{$\begin{array}{c}\text { Date } \\
\text { Observed } \\
(2)\end{array}$} & \multirow{2}{*}{$\begin{array}{l}\text { Exposure } \\
\quad(\mathrm{sec}) \\
(3)\end{array}$} & \multirow{2}{*}{$\begin{array}{l}\text { Tile } \\
(4)\end{array}$} & \multicolumn{3}{|c|}{ FUV sky background } & \multicolumn{3}{|c|}{ NUV sky background } & \multirow{2}{*}{$\begin{array}{l}\text { notes } \\
\text { (11) }\end{array}$} \\
\hline & & & & $\begin{array}{c}\text { mean } \\
(\text { counts/s) } \\
(5)\end{array}$ & $\begin{array}{c}<\sigma> \\
(\text { counts } / \mathrm{s}) \\
(6)\end{array}$ & $\begin{array}{c}\sigma(\text { mean }) \\
(\text { counts/s) } \\
(7)\end{array}$ & $\begin{array}{c}\text { mean } \\
(\text { counts/s) } \\
(8)\end{array}$ & $\begin{array}{c}<\sigma> \\
(\text { counts } / \mathrm{s}) \\
(9)\end{array}$ & $\begin{array}{c}\sigma(\text { mean }) \\
(\text { counts/s) } \\
(10)\end{array}$ & \\
\hline UGC 05114 & 2004-03-13 & 1611 & WDST-AGK-+81d266 & $3.574 \mathrm{E}-04$ & $4.824 \mathrm{E}-04$ & $1.984 \mathrm{E}-05$ & $2.748 \mathrm{E}-03$ & $1.450 \mathrm{E}-03$ & $2.147 \mathrm{E}-04$ & \\
\hline Holmberg I & $2003-12-06$ & 1704 & NGA-HolmbergI & $3.173 \mathrm{E}-04$ & $4.592 \mathrm{E}-04$ & $3.773 \mathrm{E}-06$ & $2.426 \mathrm{E}-03$ & $1.395 \mathrm{E}-03$ & $6.051 \mathrm{E}-05$ & \\
\hline UGC 05201 & 2005-01-13 & 1693 & MISDR1-00702-0556 & $\cdots$ & $\cdots$ & $\cdots$ & $2.246 \mathrm{E}-03$ & 1.302E-03 & $2.637 \mathrm{E}-05$ & (4) \\
\hline NGC 2992 & 2004-01-08 & 2710 & NGA-Arp245 & $4.980 \mathrm{E}-04$ & $7.371 \mathrm{E}-04$ & $5.984 \mathrm{E}-05$ & $4.008 \mathrm{E}-03$ & $1.452 \mathrm{E}-03$ & $9.459 \mathrm{E}-05$ & \\
\hline NGC 2993 & 2004-01-08 & 2710 & NGA-Arp245 & $5.139 \mathrm{E}-04$ & $7.494 \mathrm{E}-04$ & $2.310 \mathrm{E}-05$ & $4.036 \mathrm{E}-03$ & $1.459 \mathrm{E}-03$ & $8.290 \mathrm{E}-06$ & (1) \\
\hline NGC 2976 & 2005-01-11 & 1692 & NGA-NGC2976 & $\cdots$ & $\cdots$ & $\cdots$ & $2.447 \mathrm{E}-03$ & $1.395 \mathrm{E}-03$ & $1.153 \mathrm{E}-04$ & (4) \\
\hline UGC 05237 & $2005-04-21$ & 1402 & MISDR3-01126-1005 & $\cdots$ & $\ldots$ & $\ldots$ & $2.634 \mathrm{E}-03$ & $1.540 \mathrm{E}-03$ & $6.025 \mathrm{E}-05$ & (4) \\
\hline NGC 3018 & 2004-03-19 & 872 & MISDR1-24308-0267 & 4.009E-04 & 7.023E-04 & $2.878 \mathrm{E}-05$ & $3.180 \mathrm{E}-03$ & $2.196 \mathrm{E}-03$ & $1.329 \mathrm{E}-04$ & \\
\hline NGC 3023 & 2004-03-19 & 872 & MISDR1-24308-0267 & $3.926 \mathrm{E}-04$ & $6.899 \mathrm{E}-04$ & $1.287 \mathrm{E}-05$ & $3.156 \mathrm{E}-03$ & $2.186 \mathrm{E}-03$ & $7.670 \mathrm{E}-05$ & \\
\hline UGC 05268 & 2005-01-12 & 1693 & MISDR1-00463-0487 & $\ldots$ & $\ldots$ & $\ldots$ & $2.283 \mathrm{E}-03$ & $1.358 \mathrm{E}-03$ & $8.868 \mathrm{E}-05$ & (4) \\
\hline UGC 05314 & $2005-03-17$ & 1634 & NGA-NGC3049 & $\ldots$ & $\ldots$ & $\ldots$ & $3.203 \mathrm{E}-03$ & $1.665 \mathrm{E}-03$ & $1.068 \mathrm{E}-05$ & (4) \\
\hline NGC 3049 & $2005-03-17$ & 1634 & NGA-NGC3049 & $\ldots$ & $\ldots$ & $\ldots$ & $3.151 \mathrm{E}-03$ & $1.629 \mathrm{E}-03$ & $3.670 \mathrm{E}-05$ & (4) \\
\hline MESSIER 081 & $2003-12-08$ & 3089 & NGA-M81andM82 & $3.977 \mathrm{E}-04$ & $3.842 \mathrm{E}-04$ & $2.977 \mathrm{E}-05$ & $2.641 \mathrm{E}-03$ & $1.075 \mathrm{E}-03$ & $5.032 \mathrm{E}-05$ & \\
\hline MESSIER 082 & $2003-12-08$ & 3089 & NGA-M81andM82 & $3.895 \mathrm{E}-04$ & $4.298 \mathrm{E}-04$ & $1.751 \mathrm{E}-05$ & $2.568 \mathrm{E}-03$ & $1.066 \mathrm{E}-03$ & $5.821 \mathrm{E}-05$ & \\
\hline Holmberg IX & $2003-12-08$ & 3089 & NGA-M81andM82 & $4.750 \mathrm{E}-04$ & $3.999 \mathrm{E}-04$ & $4.880 \mathrm{E}-05$ & $2.804 \mathrm{E}-03$ & $1.084 \mathrm{E}-03$ & $2.217 \mathrm{E}-04$ & \\
\hline ESO 435-G014 & 2004-03-31 & 1291 & NGA-Tol2 & $1.326 \mathrm{E}-03$ & $1.100 \mathrm{E}-03$ & $2.011 \mathrm{E}-04$ & $6.515 \mathrm{E}-03$ & 2.697E-03 & 7.363E-04 & (2) \\
\hline ESO 435-G016 & 2004-03-31 & 1291 & NGA-Tol2 & $1.460 \mathrm{E}-03$ & $1.297 \mathrm{E}-03$ & $2.265 \mathrm{E}-05$ & $6.699 \mathrm{E}-03$ & $2.695 \mathrm{E}-03$ & $9.753 \mathrm{E}-05$ & (1) \\
\hline Tol 2 & $2004-03-31$ & 1291 & NGA-Tol2 & $1.134 \mathrm{E}-03$ & $1.001 \mathrm{E}-03$ & $2.371 \mathrm{E}-05$ & $5.912 \mathrm{E}-03$ & $2.644 \mathrm{E}-03$ & $4.059 \mathrm{E}-05$ & \\
\hline NGC 3089 & 2004-03-31 & 1291 & NGA-Tol2 & $1.268 \mathrm{E}-03$ & $1.126 \mathrm{E}-03$ & $1.028 \mathrm{E}-04$ & $6.220 \mathrm{E}-03$ & $2.696 \mathrm{E}-03$ & $1.154 \mathrm{E}-04$ & \\
\hline NGC 3073 & $2005-02-24$ & 1690 & NGA-NGC3079 & $1.770 \mathrm{E}-04$ & $3.542 \mathrm{E}-04$ & $1.136 \mathrm{E}-05$ & $2.278 \mathrm{E}-03$ & $1.362 \mathrm{E}-03$ & $8.037 \mathrm{E}-05$ & \\
\hline NGC 3079 & $2005-02-24$ & 1690 & NGA-NGC3079 & $1.759 \mathrm{E}-04$ & $3.406 \mathrm{E}-04$ & $3.501 \mathrm{E}-06$ & $2.279 \mathrm{E}-03$ & $1.331 \mathrm{E}-03$ & $2.716 \mathrm{E}-05$ & \\
\hline NGC 3109 & $2004-01-07$ & 6590 & NGA-NGC3109 & $9.284 \mathrm{E}-04$ & $4.347 \mathrm{E}-04$ & $1.193 \mathrm{E}-04$ & $4.998 \mathrm{E}-03$ & $1.054 \mathrm{E}-03$ & $2.418 \mathrm{E}-04$ & \\
\hline UGCA 196 & 2004-01-08 & 7525 & NGA-Antlia-Dw & $1.040 \mathrm{E}-03$ & $4.389 \mathrm{E}-04$ & $7.609 \mathrm{E}-05$ & $5.713 \mathrm{E}-03$ & $1.044 \mathrm{E}-03$ & $2.905 \mathrm{E}-04$ & \\
\hline IC 2537 & 2004-01-08 & 7525 & NGA-Antlia-Dw & $1.115 \mathrm{E}-03$ & $5.016 \mathrm{E}-04$ & 7.601E-05 & $5.660 \mathrm{E}-03$ & $1.058 \mathrm{E}-03$ & $1.586 \mathrm{E}-04$ & \\
\hline UGC 05406 & 2004-01-20 & 1694 & MISDR1-00432-0487 & $2.891 \mathrm{E}-04$ & $4.213 \mathrm{E}-04$ & $4.382 \mathrm{E}-06$ & $2.496 \mathrm{E}-03$ & $1.380 \mathrm{E}-03$ & $1.070 \mathrm{E}-05$ & \\
\hline Antlia Dwarf & $2004-01-08$ & 7525 & NGA-Antlia-Dw & $1.070 \mathrm{E}-03$ & $4.579 \mathrm{E}-04$ & $6.445 \mathrm{E}-05$ & $5.708 \mathrm{E}-03$ & $1.070 \mathrm{E}-03$ & $1.521 \mathrm{E}-04$ & \\
\hline M81 Dwarf B & $2003-12-07$ & 1705 & NGA-M81DwB & $4.016 \mathrm{E}-04$ & $5.161 \mathrm{E}-04$ & $4.247 \mathrm{E}-05$ & $2.652 \mathrm{E}-03$ & $1.469 \mathrm{E}-03$ & $4.341 \mathrm{E}-05$ & \\
\hline NGC 3125 & 2004-01-08 & 3754 & NGA-NGC3125 & $1.046 \mathrm{E}-03$ & $7.370 \mathrm{E}-04$ & $5.141 \mathrm{E}-06$ & $5.367 \mathrm{E}-03$ & $1.475 \mathrm{E}-03$ & $1.083 \mathrm{E}-04$ & \\
\hline UGC 05455 & $2003-12-07$ & 1705 & NGA-M81DwB & $4.309 \mathrm{E}-04$ & $6.169 \mathrm{E}-04$ & $1.349 \mathrm{E}-06$ & $2.691 \mathrm{E}-03$ & $1.437 \mathrm{E}-03$ & $6.253 \mathrm{E}-05$ & \\
\hline Sextans A & $2004-03-28$ & 1689 & NGA-SextansA & $3.380 \mathrm{E}-04$ & $4.779 \mathrm{E}-04$ & $8.716 \mathrm{E}-06$ & $2.976 \mathrm{E}-03$ & $1.564 \mathrm{E}-03$ & $3.464 \mathrm{E}-05$ & \\
\hline UGC 05493 & $2004-03-25$ & 1218 & MISDR1-24303-0270 & $2.804 \mathrm{E}-04$ & $5.014 \mathrm{E}-04$ & $2.993 \mathrm{E}-05$ & $3.341 \mathrm{E}-03$ & $1.944 \mathrm{E}-03$ & $5.429 \mathrm{E}-06$ & \\
\hline UGC 05515 & $2004-03-24$ & 451 & MISDR1-24368-0271 & $3.382 \mathrm{E}-04$ & $9.371 \mathrm{E}-04$ & $3.863 \mathrm{E}-05$ & $3.153 \mathrm{E}-03$ & $2.988 \mathrm{E}-03$ & $9.082 \mathrm{E}-05$ & \\
\hline
\end{tabular}


Table 2-Continued

\begin{tabular}{|c|c|c|c|c|c|c|c|c|c|c|}
\hline \multirow{2}{*}{$\begin{array}{c}\text { Object Name } \\
\text { (1) }\end{array}$} & \multirow{2}{*}{$\begin{array}{c}\text { Date } \\
\text { Observed } \\
\\
(2)\end{array}$} & \multirow{2}{*}{$\begin{array}{l}\text { Exposure } \\
\quad(\mathrm{sec}) \\
(3)\end{array}$} & \multirow{2}{*}{$\begin{array}{l}\text { Tile } \\
(4)\end{array}$} & \multicolumn{3}{|c|}{ FUV sky background } & \multicolumn{3}{|c|}{ NUV sky background } & \multirow{2}{*}{$\begin{array}{r}\text { notes } \\
(11)\end{array}$} \\
\hline & & & & $\begin{array}{c}\text { mean } \\
(\text { counts/s) } \\
(5)\end{array}$ & $\begin{array}{c}<\sigma> \\
(\text { counts/s) } \\
(6)\end{array}$ & $\begin{array}{c}\sigma(\text { mean }) \\
(\text { counts/s) } \\
(7)\end{array}$ & $\begin{array}{c}\text { mean } \\
(\text { counts/s) } \\
(8)\end{array}$ & $\begin{array}{c}<\sigma> \\
(\text { counts } / \mathrm{s}) \\
(9)\end{array}$ & $\begin{array}{c}\sigma(\text { mean }) \\
(\text { counts/s) } \\
(10)\end{array}$ & \\
\hline UGC 05528 & 2004-03-24 & 451 & MISDR1-24368-0271 & $3.123 \mathrm{E}-04$ & $8.454 \mathrm{E}-04$ & $3.196 \mathrm{E}-05$ & $3.113 \mathrm{E}-03$ & $2.963 \mathrm{E}-03$ & $7.727 \mathrm{E}-05$ & \\
\hline NGC 3147 & $2004-01-21$ & 1335 & NGA-NGC3147 & $3.421 \mathrm{E}-04$ & $5.969 \mathrm{E}-04$ & $2.243 \mathrm{E}-05$ & $2.651 \mathrm{E}-03$ & $1.638 \mathrm{E}-03$ & $5.775 \mathrm{E}-05$ & \\
\hline NGC 3185 & $2005-02-19$ & 1396 & NGA-NGC3190 & $2.297 \mathrm{E}-04$ & $4.553 \mathrm{E}-04$ & $4.051 \mathrm{E}-06$ & $3.076 \mathrm{E}-03$ & $1.767 \mathrm{E}-03$ & $7.327 \mathrm{E}-06$ & \\
\hline NGC 3187 & $2005-02-19$ & 1396 & NGA-NGC3190 & $2.307 \mathrm{E}-04$ & $4.287 \mathrm{E}-04$ & $1.484 \mathrm{E}-05$ & $3.059 \mathrm{E}-03$ & $1.736 \mathrm{E}-03$ & $5.990 \mathrm{E}-05$ & \\
\hline NGC 3190 & $2005-02-19$ & 1396 & NGA-NGC3190 & 2.303E-04 & $4.332 \mathrm{E}-04$ & $1.001 \mathrm{E}-05$ & $3.072 \mathrm{E}-03$ & $1.740 \mathrm{E}-03$ & $7.540 \mathrm{E}-05$ & \\
\hline UGC 05558 & 2004-02-09 & 1182 & NGA-NGC3198 & $1.865 \mathrm{E}-04$ & $5.032 \mathrm{E}-04$ & $1.909 \mathrm{E}-05$ & $2.484 \mathrm{E}-03$ & $1.648 \mathrm{E}-03$ & $1.681 \mathrm{E}-05$ & \\
\hline NGC 3193 & $2005-02-19$ & 1396 & NGA-NGC3190 & $2.291 \mathrm{E}-04$ & $4.158 \mathrm{E}-04$ & $1.315 \mathrm{E}-05$ & $3.045 \mathrm{E}-03$ & $1.707 \mathrm{E}-03$ & $4.007 \mathrm{E}-05$ & \\
\hline NGC 3198 & 2004-02-09 & 1182 & NGA-NGC3198 & $1.933 \mathrm{E}-04$ & $4.324 \mathrm{E}-04$ & $7.973 \mathrm{E}-06$ & $2.592 \mathrm{E}-03$ & $1.735 \mathrm{E}-03$ & $8.424 \mathrm{E}-05$ & \\
\hline UGC 05570 & $2004-01-21$ & 1335 & NGA-NGC3147 & $3.448 \mathrm{E}-04$ & $5.436 \mathrm{E}-04$ & $1.963 \mathrm{E}-05$ & $2.685 \mathrm{E}-03$ & $1.653 \mathrm{E}-03$ & $9.971 \mathrm{E}-06$ & \\
\hline NGC 3183 & 2004-01-21 & 1335 & NGA-NGC3147 & $4.222 \mathrm{E}-04$ & $6.995 \mathrm{E}-04$ & $1.915 \mathrm{E}-05$ & $2.704 \mathrm{E}-03$ & $1.636 \mathrm{E}-03$ & $4.395 \mathrm{E}-05$ & \\
\hline ESO 317-G019 & 2004-01-07 & 6070 & NGA-ESO317-G023 & $1.275 \mathrm{E}-03$ & $6.769 \mathrm{E}-04$ & $9.029 \mathrm{E}-05$ & $6.263 \mathrm{E}-03$ & $1.220 \mathrm{E}-03$ & $3.650 \mathrm{E}-05$ & \\
\hline ESO $317-G 023$ & 2004-01-07 & 6070 & NGA-ESO317-G023 & $1.289 \mathrm{E}-03$ & $6.043 \mathrm{E}-04$ & $1.408 \mathrm{E}-04$ & $6.461 \mathrm{E}-03$ & $1.280 \mathrm{E}-03$ & $2.192 \mathrm{E}-04$ & \\
\hline ESO 263-G033 & 2004-01-08 & 1662 & NGA-NGC3256 & $\ldots$ & $\ldots$ & $\ldots$ & $7.348 \mathrm{E}-03$ & $2.579 \mathrm{E}-03$ & $4.259 \mathrm{E}-04$ & (4) \\
\hline NGC 3225 & 2005-01-14 & 1696 & MISDR2-00659-0559 & $\ldots$ & $\ldots$ & $\ldots$ & $2.165 \mathrm{E}-03$ & $1.308 \mathrm{E}-03$ & $5.440 \mathrm{E}-05$ & (4) \\
\hline NGC 3244 & 2004-01-07 & 6070 & NGA-ESO317-G023 & $1.339 \mathrm{E}-03$ & $7.122 \mathrm{E}-04$ & $1.582 \mathrm{E}-05$ & $6.388 \mathrm{E}-03$ & $1.261 \mathrm{E}-03$ & $1.854 \mathrm{E}-04$ & \\
\hline NGC $3256 \mathrm{~A}$ & 2004-01-08 & 1662 & NGA-NGC3256 & $\ldots$ & $\ldots$ & & $7.238 \mathrm{E}-03$ & $2.616 \mathrm{E}-03$ & $5.863 \mathrm{E}-05$ & (4) \\
\hline NGC 3238 & $2005-02-25$ & 1681 & LOCK-13 & $1.925 \mathrm{E}-04$ & $1.868 \mathrm{E}-04$ & $2.760 \mathrm{E}-06$ & $2.181 \mathrm{E}-03$ & $5.840 \mathrm{E}-04$ & $5.369 \mathrm{E}-05$ & \\
\hline IC 2574 & $2004-01-21$ & 271 & NGA-IC2574 & $3.926 \mathrm{E}-04$ & $1.302 \mathrm{E}-03$ & $6.530 \mathrm{E}-06$ & $2.824 \mathrm{E}-03$ & $3.772 \mathrm{E}-03$ & $4.385 \mathrm{E}-05$ & \\
\hline NGC 3265 & 2004-04-19 & 1575 & NGA-NGC3265 & $2.794 \mathrm{E}-04$ & $4.464 \mathrm{E}-04$ & $4.549 \mathrm{E}-06$ & $2.896 \mathrm{E}-03$ & $1.592 \mathrm{E}-03$ & $3.001 \mathrm{E}-05$ & \\
\hline UGC 05715 & $2004-03-27$ & 1681 & MISDR1-24298-0273 & $4.510 \mathrm{E}-04$ & $5.367 \mathrm{E}-04$ & $4.894 \mathrm{E}-06$ & $3.363 \mathrm{E}-03$ & $1.611 \mathrm{E}-03$ & $2.201 \mathrm{E}-05$ & \\
\hline UGC 05720 & $2005-02-25$ & 1682 & NGA-Mrk33 & $1.933 \mathrm{E}-04$ & $3.598 \mathrm{E}-04$ & $1.778 \mathrm{E}-05$ & $2.198 \mathrm{E}-03$ & $1.329 \mathrm{E}-03$ & $5.294 \mathrm{E}-05$ & \\
\hline NGC 3277 & 2004-04-19 & 1575 & NGA-NGC3265 & $3.273 \mathrm{E}-04$ & $5.940 \mathrm{E}-04$ & $5.873 \mathrm{E}-05$ & $2.965 \mathrm{E}-03$ & $1.606 \mathrm{E}-03$ & $2.584 \mathrm{E}-06$ & \\
\hline NGC 3288 & $2005-02-25$ & 1677 & LOCK-06 & $1.926 \mathrm{E}-04$ & $1.533 \mathrm{E}-04$ & $1.219 \mathrm{E}-05$ & $2.241 \mathrm{E}-03$ & $5.329 \mathrm{E}-04$ & $6.962 \mathrm{E}-05$ & \\
\hline UGC 05772 & 2004-03-29 & 1680 & MISDR1-24329-0274 & 4.964E-04 & $6.427 \mathrm{E}-04$ & $1.448 \mathrm{E}-05$ & $3.465 \mathrm{E}-03$ & $1.638 \mathrm{E}-03$ & $5.517 \mathrm{E}-05$ & \\
\hline NGC 3319 & 2004-04-16 & 989 & NGA-NGC3319 & $2.324 \mathrm{E}-04$ & $5.169 \mathrm{E}-04$ & $2.756 \mathrm{E}-06$ & $2.503 \mathrm{E}-03$ & $1.860 \mathrm{E}-03$ & $7.477 \mathrm{E}-05$ & \\
\hline UGC 05818 & $2005-04-16$ & 177 & QSOGRP-03 & $\begin{array}{c}2.0240-07 \\
\ldots\end{array}$ & $\begin{array}{l}\ldots .1000 \\
\ldots\end{array}$ & $\ldots$ & $3.176 \mathrm{E}-03$ & $4.792 \mathrm{E}-03$ & $1.419 \mathrm{E}-04$ & (4) \\
\hline UGC 05823 & $2004-03-29$ & 1382 & MISDR1-24296-0274 & $4.226 \mathrm{E}-04$ & $6.711 \mathrm{E}-04$ & $3.269 \mathrm{E}-05$ & $3.309 \mathrm{E}-03$ & $1.814 \mathrm{E}-03$ & $3.438 \mathrm{E}-06$ & \\
\hline NGC 3344 & 2004-04-19 & 1429 & NGA-NGC3344 & $2.911 \mathrm{E}-04$ & $4.821 \mathrm{E}-04$ & $5.966 \mathrm{E}-06$ & $2.973 \mathrm{E}-03$ & $1.694 \mathrm{E}-03$ & $3.660 \mathrm{E}-05$ & \\
\hline MESSIER 095 & $2005-02-20$ & 1703 & NGA-NGC3351 & $2.311 \mathrm{E}-04$ & $3.918 \mathrm{E}-04$ & $1.327 \mathrm{E}-05$ & $3.244 \mathrm{E}-03$ & $1.623 \mathrm{E}-03$ & $4.737 \mathrm{E}-05$ & (6) \\
\hline UGC 05848 & $2005-02-25$ & 1409 & LOCK-10 & $1.944 \mathrm{E}-04$ & $1.753 \mathrm{E}-04$ & $2.057 \mathrm{E}-06$ & $2.304 \mathrm{E}-03$ & $6.097 \mathrm{E}-04$ & $3.645 \mathrm{E}-05$ & \\
\hline UGC 05853 & $2005-02-25$ & 1607 & LOCK-01 & $2.037 \mathrm{E}-04$ & $1.621 \mathrm{E}-04$ & $1.741 \mathrm{E}-05$ & $2.237 \mathrm{E}-03$ & $5.711 \mathrm{E}-04$ & $3.564 \mathrm{E}-06$ & \\
\hline NGC 3353 & $2005-02-25$ & 1409 & LOCK-10 & $1.921 \mathrm{E}-04$ & $1.648 \mathrm{E}-04$ & $8.006 \mathrm{E}-06$ & $2.415 \mathrm{E}-03$ & $6.379 \mathrm{E}-04$ & $2.316 \mathrm{E}-05$ & (1) \\
\hline
\end{tabular}


Table 2-Continued

\begin{tabular}{|c|c|c|c|c|c|c|c|c|c|c|}
\hline \multirow{2}{*}{$\begin{array}{l}\text { Object Name } \\
\text { (1) }\end{array}$} & \multirow{2}{*}{$\begin{array}{c}\text { Date } \\
\text { Observed } \\
(2)\end{array}$} & \multirow{2}{*}{$\begin{array}{l}\text { Exposure } \\
\quad(\mathrm{sec}) \\
(3)\end{array}$} & \multirow{2}{*}{$\begin{array}{l}\text { Tile } \\
(4) \\
\end{array}$} & \multicolumn{3}{|c|}{ FUV sky background } & \multicolumn{3}{|c|}{ NUV sky background } & \multirow{2}{*}{$\begin{array}{l}\text { notes } \\
\text { (11) }\end{array}$} \\
\hline & & & & $\begin{array}{c}\text { mean } \\
(\text { counts/s) } \\
(5)\end{array}$ & $\begin{array}{c}<\sigma> \\
(\text { counts/s) } \\
(6)\end{array}$ & $\begin{array}{c}\sigma(\text { mean }) \\
(\text { counts/s) } \\
(7)\end{array}$ & $\begin{array}{c}\text { mean } \\
(\text { counts/s) } \\
(8)\end{array}$ & $\begin{array}{c}<\sigma> \\
(\text { counts/s) } \\
(9)\end{array}$ & $\begin{array}{c}\sigma(\text { mean }) \\
(\text { counts/s }) \\
(10)\end{array}$ & \\
\hline UGC 05869 & $2005-02-20$ & 1704 & NGA-NGC3368 & $2.356 \mathrm{E}-04$ & $4.057 \mathrm{E}-04$ & $7.239 \mathrm{E}-06$ & $3.302 \mathrm{E}-03$ & $1.583 \mathrm{E}-03$ & $4.204 \mathrm{E}-05$ & \\
\hline NGC 3367 & 2005-04-06 & 143 & NGA-NGC3377 & $\ldots$ & $\ldots$ & $\ldots$ & $3.113 \mathrm{E}-03$ & $2.498 \mathrm{E}-03$ & $5.577 \mathrm{E}-06$ & (4) \\
\hline UGC 05876 & 2005-04-11 & 913 & NGA-Mrk153 & $\cdots$ & $\cdots$ & $\cdots$ & $2.333 \mathrm{E}-03$ & $1.857 \mathrm{E}-03$ & $4.718 \mathrm{E}-05$ & (4) \\
\hline NGC 3359 & 2005-01-14 & 1694 & MISDR2-00488-0773 & $\cdots$ & $\cdots$ & $\cdots$ & $2.065 \mathrm{E}-03$ & $1.240 \mathrm{E}-03$ & $3.206 \mathrm{E}-05$ & (4) \\
\hline MESSIER 096 & $2005-02-20$ & 1704 & NGA-NGC3368 & $2.247 \mathrm{E}-04$ & $3.888 \mathrm{E}-04$ & $5.927 \mathrm{E}-07$ & $3.323 \mathrm{E}-03$ & $1.644 \mathrm{E}-03$ & $2.185 \mathrm{E}-05$ & \\
\hline UGC 05886 & $2004-03-28$ & 1686 & MISDR1-24395-0275 & $2.932 \mathrm{E}-04$ & $4.549 \mathrm{E}-04$ & $1.232 \mathrm{E}-05$ & $3.112 \mathrm{E}-03$ & $1.588 \mathrm{E}-03$ & $3.781 \mathrm{E}-05$ & \\
\hline NGC 3377A & 2005-04-06 & 143 & NGA-NGC3377 & $\cdots$ & $\cdots$ & $\cdots$ & $3.176 \mathrm{E}-03$ & $2.480 \mathrm{E}-03$ & $5.265 \mathrm{E}-05$ & (4) \\
\hline UGC 05896 & $2004-03-28$ & 1686 & MISDR1-24395-0275 & $3.066 \mathrm{E}-04$ & $5.165 \mathrm{E}-04$ & $2.091 \mathrm{E}-05$ & 3.091E-03 & $1.558 \mathrm{E}-03$ & $3.219 \mathrm{E}-05$ & \\
\hline NGC 3377 & 2005-04-06 & 143 & NGA-NGC3377 & $\cdots$ & $\cdots$ & $\cdots$ & $3.158 \mathrm{E}-03$ & $2.506 \mathrm{E}-03$ & $1.746 \mathrm{E}-05$ & (4) \\
\hline UGC 05888 & $2005-02-25$ & 1409 & LOCK-10 & $1.904 \mathrm{E}-04$ & $1.700 \mathrm{E}-04$ & $1.223 \mathrm{E}-05$ & $2.326 \mathrm{E}-03$ & $6.175 \mathrm{E}-04$ & $6.810 \mathrm{E}-05$ & \\
\hline UGC 05904 & 2004-01-22 & 1694 & MISDR1-00398-0490 & $2.530 \mathrm{E}-04$ & $4.561 \mathrm{E}-04$ & $5.044 \mathrm{E}-06$ & $2.505 \mathrm{E}-03$ & $1.440 \mathrm{E}-03$ & $7.626 \mathrm{E}-05$ & \\
\hline UGC 05907 & 2004-01-22 & 1694 & MISDR1-00398-0490 & $2.495 \mathrm{E}-04$ & 4.306E-04 & $5.402 \mathrm{E}-07$ & $2.514 \mathrm{E}-03$ & $1.456 \mathrm{E}-03$ & $1.225 \mathrm{E}-05$ & (1) \\
\hline UGC 05922 & $2004-03-28$ & 680 & MISDR1-14245-0275 & $3.452 \mathrm{E}-04$ & 8.015E-04 & $9.484 \mathrm{E}-06$ & $3.225 \mathrm{E}-03$ & $2.593 \mathrm{E}-03$ & $4.449 \mathrm{E}-05$ & \\
\hline UGC 05929 & 2005-04-01 & 1636 & QSOGRP-06 & $\cdots$ & $\cdots$ & $\cdots$ & $2.987 \mathrm{E}-03$ & $1.557 \mathrm{E}-03$ & $2.869 \mathrm{E}-05$ & (4) \\
\hline UGC 05928 & 2005-04-11 & 913 & NGA-Mrk153 & $\cdots$ & $\cdots$ & $\ldots$ & $2.325 \mathrm{E}-03$ & $1.862 \mathrm{E}-03$ & $5.091 \mathrm{E}-05$ & (4) \\
\hline UGC 05943 & 2004-03-29 & 1931 & MISDR1-14244-0276 & $3.283 \mathrm{E}-04$ & $4.398 \mathrm{E}-04$ & $2.754 \mathrm{E}-05$ & $3.205 \mathrm{E}-03$ & $1.488 \mathrm{E}-03$ & $6.003 \mathrm{E}-05$ & \\
\hline NGC 3394 & 2004-01-22 & 1694 & MISDR1-00398-0490 & $2.455 \mathrm{E}-04$ & $3.976 \mathrm{E}-04$ & $4.916 \mathrm{E}-06$ & $2.516 \mathrm{E}-03$ & $1.399 \mathrm{E}-03$ & $6.320 \mathrm{E}-05$ & \\
\hline NGC 3412 & 2005-04-07 & 1177 & NGA-NGC3412 & $\ldots$ & $\cdots$ & $\cdots$ & $2.998 \mathrm{E}-03$ & $1.885 \mathrm{E}-03$ & $5.578 \mathrm{E}-06$ & (4) \\
\hline NGC 3419 & 2005-04-07 & 1177 & NGA-NGC3412 & $\cdots$ & $\cdots$ & $\cdots$ & $3.023 \mathrm{E}-03$ & $1.818 \mathrm{E}-03$ & $1.031 \mathrm{E}-05$ & (4) \\
\hline UGC 05974 & 2005-04-01 & 1636 & QSOGRP-06 & $\ldots$ & $\cdots$ & $\ldots$ & $2.942 \mathrm{E}-03$ & $1.561 \mathrm{E}-03$ & $3.325 \mathrm{E}-05$ & (4) \\
\hline IC 0653 & 2004-03-29 & 2056 & MISDR1-12529-0276 & $4.170 \mathrm{E}-04$ & $5.410 \mathrm{E}-04$ & $1.577 \mathrm{E}-05$ & $3.469 \mathrm{E}-03$ & $1.493 \mathrm{E}-03$ & $8.571 \mathrm{E}-05$ & \\
\hline UGC 05971 & 2004-01-22 & 1695 & MISDR1-00370-0490 & $2.670 \mathrm{E}-04$ & $4.444 \mathrm{E}-04$ & $3.667 \mathrm{E}-06$ & $2.451 \mathrm{E}-03$ & $1.407 \mathrm{E}-03$ & $1.024 \mathrm{E}-04$ & \\
\hline UGC 06011 & 2004-03-29 & 2056 & MISDR1-12529-0276 & $3.529 \mathrm{E}-04$ & $4.878 \mathrm{E}-04$ & $2.118 \mathrm{E}-05$ & $3.284 \mathrm{E}-03$ & $1.495 \mathrm{E}-03$ & $1.207 \mathrm{E}-04$ & \\
\hline NGC 3440 & 2004-01-31 & 55433 & LOCK-05 & $2.899 \mathrm{E}-04$ & $1.177 \mathrm{E}-04$ & $6.374 \mathrm{E}-06$ & $4.254 \mathrm{E}-03$ & $3.256 \mathrm{E}-04$ & $1.889 \mathrm{E}-04$ & \\
\hline NGC 3445 & 2004-01-31 & 55433 & LOCK-05 & $3.154 \mathrm{E}-04$ & $1.276 \mathrm{E}-04$ & $3.170 \mathrm{E}-06$ & $4.010 \mathrm{E}-03$ & $3.152 \mathrm{E}-04$ & $1.875 \mathrm{E}-04$ & \\
\hline NGC 3458 & 2004-01-31 & 55433 & LOCK-05 & $3.041 \mathrm{E}-04$ & $1.234 \mathrm{E}-04$ & $1.729 \mathrm{E}-05$ & $3.514 \mathrm{E}-03$ & $2.891 \mathrm{E}-04$ & $1.791 \mathrm{E}-04$ & \\
\hline UGC 06039 & 2004-01-31 & 55433 & LOCK-05 & $3.471 \mathrm{E}-04$ & $1.416 \mathrm{E}-04$ & $3.799 \mathrm{E}-05$ & $3.261 \mathrm{E}-03$ & $2.775 \mathrm{E}-04$ & $1.050 \mathrm{E}-04$ & \\
\hline NGC 3475 & 2005-04-07 & 825 & NGA-IRAS10565 & $\cdots$ & $\cdots$ & $\cdots$ & $2.814 \mathrm{E}-03$ & $2.145 \mathrm{E}-03$ & $1.803 \mathrm{E}-04$ & (4) \\
\hline NGC 3470 & $2005-02-28$ & 1461 & LOCK-11 & $1.890 \mathrm{E}-04$ & $2.076 \mathrm{E}-04$ & $9.791 \mathrm{E}-07$ & $2.081 \mathrm{E}-03$ & $6.468 \mathrm{E}-04$ & $1.081 \mathrm{E}-05$ & \\
\hline NGC 3489 & $2004-04-17$ & 186 & NGA-NGC3489 & $2.449 \mathrm{E}-04$ & $1.219 \mathrm{E}-03$ & $2.228 \mathrm{E}-05$ & $3.075 \mathrm{E}-03$ & $4.758 \mathrm{E}-03$ & $1.787 \mathrm{E}-05$ & \\
\hline NGC 3486 & $2004-04-15$ & 541 & NGA-NGC3486 & $2.428 \mathrm{E}-04$ & 7.172E-04 & $2.383 \mathrm{E}-05$ & $2.640 \mathrm{E}-03$ & $2.593 \mathrm{E}-03$ & $9.583 \mathrm{E}-06$ & \\
\hline UGC 06102 & 2004-04-15 & 541 & NGA-NGC3486 & $2.506 \mathrm{E}-04$ & 7.861E-04 & $2.933 \mathrm{E}-05$ & $2.666 \mathrm{E}-03$ & $2.602 \mathrm{E}-03$ & $6.633 \mathrm{E}-05$ & \\
\hline
\end{tabular}


Table 2-Continued

\begin{tabular}{|c|c|c|c|c|c|c|c|c|c|c|}
\hline \multirow{2}{*}{$\begin{array}{l}\text { Object Name } \\
\text { (1) }\end{array}$} & \multirow[b]{2}{*}{$\begin{array}{c}\text { Date } \\
\text { Observed } \\
(2)\end{array}$} & \multirow[b]{2}{*}{$\begin{array}{l}\text { Exposure } \\
\quad(\mathrm{sec}) \\
(3)\end{array}$} & \multirow[b]{2}{*}{$\begin{array}{l}\text { Tile } \\
(4)\end{array}$} & \multicolumn{3}{|c|}{ FUV sky background } & \multicolumn{3}{|c|}{ NUV sky background } & \multirow[b]{2}{*}{$\begin{array}{l}\text { notes } \\
\text { (11) }\end{array}$} \\
\hline & & & & $\begin{array}{c}\text { mean } \\
(\text { counts } / \mathrm{s}) \\
(5)\end{array}$ & $\begin{array}{c}<\sigma> \\
(\text { counts } / \mathrm{s}) \\
(6)\end{array}$ & $\begin{array}{c}\sigma(\text { mean }) \\
(\text { counts } / \mathrm{s}) \\
(7)\end{array}$ & $\begin{array}{c}\text { mean } \\
(\text { counts/s) } \\
(8)\end{array}$ & $\begin{array}{c}<\sigma> \\
(\text { counts } / \mathrm{s}) \\
(9)\end{array}$ & $\begin{array}{c}\sigma(\text { mean }) \\
(\text { counts/s) } \\
(10)\end{array}$ & \\
\hline NGC 3521 & 2004-03-02 & 1157 & NGA-NGC3521 & $3.042 \mathrm{E}-04$ & 4.402E-04 & $3.789 \mathrm{E}-05$ & $3.309 \mathrm{E}-03$ & $1.587 \mathrm{E}-03$ & $1.433 \mathrm{E}-04$ & \\
\hline UGC 06151 & 2005-04-07 & 1700 & NGA-UGC6151 & $\ldots$ & $\cdots$ & $\ldots$ & 3.040E-03 & $1.553 \mathrm{E}-03$ & $2.014 \mathrm{E}-05$ & (4) \\
\hline NGC 3522 & 2005-04-07 & 1700 & NGA-UGC6151 & $\cdots$ & $\cdots$ & $\cdots$ & $2.744 \mathrm{E}-03$ & $1.430 \mathrm{E}-03$ & $2.100 \mathrm{E}-05$ & (4) \\
\hline IC 0671 & 2004-03-30 & 1743 & MISDR1-12636-0278 & $2.942 \mathrm{E}-04$ & 4.823E-04 & $1.322 \mathrm{E}-05$ & $3.007 \mathrm{E}-03$ & $1.525 \mathrm{E}-03$ & 7.987E-05 & \\
\hline UGC 06181 & 2005-04-07 & 1700 & NGA-UGC6151 & $\ldots$ & $\ldots$ & $\ldots$ & $3.026 \mathrm{E}-03$ & $1.524 \mathrm{E}-03$ & $8.983 \mathrm{E}-05$ & (4) \\
\hline NGC 3539 & 2005-04-08 & 936 & NGA-Arp105 & $\cdots$ & $\cdots$ & $\cdots$ & $2.436 \mathrm{E}-03$ & $1.789 \mathrm{E}-03$ & $1.678 \mathrm{E}-05$ & $(4)(1)$ \\
\hline IC 0673 & 2004-03-30 & 1584 & MISDR1-12675-0278 & $3.038 \mathrm{E}-04$ & $5.233 \mathrm{E}-04$ & $4.274 \mathrm{E}-05$ & 3.047E-03 & $1.616 \mathrm{E}-03$ & $1.065 \mathrm{E}-04$ & \\
\hline PGC 33931 & 2005-04-08 & 936 & NGA-Arp105 & $\cdots$ & $\cdots$ & $\cdots$ & $2.479 \mathrm{E}-03$ & $1.867 \mathrm{E}-03$ & $4.741 \mathrm{E}-05$ & (4) \\
\hline NGC 3550 & 2005-04-08 & 936 & NGA-Arp105 & $\ldots$ & $\cdots$ & $\ldots$ & $2.522 \mathrm{E}-03$ & $1.869 \mathrm{E}-03$ & $2.605 \mathrm{E}-05$ & (4) \\
\hline NGC 3620 & 2004-04-03 & 2680 & NGA-NGC3620 & $2.230 \mathrm{E}-03$ & $9.914 \mathrm{E}-04$ & $1.875 \mathrm{E}-04$ & $8.438 \mathrm{E}-03$ & $2.238 \mathrm{E}-03$ & $8.216 \mathrm{E}-05$ & \\
\hline NGC 3621 & $2005-02-21$ & 4582 & NGA-NGC3621 & $7.258 \mathrm{E}-04$ & 4.294E-04 & $8.169 \mathrm{E}-05$ & $4.149 \mathrm{E}-03$ & $1.138 \mathrm{E}-03$ & $1.348 \mathrm{E}-04$ & \\
\hline UGC 06329 & 2004-04-07 & 1396 & MISDR1-12754-0279 & $3.349 \mathrm{E}-04$ & $6.346 \mathrm{E}-04$ & $4.952 \mathrm{E}-06$ & $3.259 \mathrm{E}-03$ & $1.817 \mathrm{E}-03$ & $3.243 \mathrm{E}-05$ & \\
\hline UGC 06331 & 2004-04-08 & 2051 & NGA-NGC3640 & $3.982 \mathrm{E}-04$ & $4.555 \mathrm{E}-04$ & $7.178 \mathrm{E}-05$ & $3.281 \mathrm{E}-03$ & $1.441 \mathrm{E}-03$ & $1.113 \mathrm{E}-05$ & \\
\hline NGC 3627 & 2004-04-11 & 3069 & NGA-NGC3627 & $2.476 \mathrm{E}-04$ & $4.096 \mathrm{E}-04$ & $1.362 \mathrm{E}-05$ & $2.921 \mathrm{E}-03$ & $1.125 \mathrm{E}-03$ & $1.562 \mathrm{E}-05$ & \\
\hline NGC 3630 & 2004-04-08 & 2051 & NGA-NGC3640 & $3.031 \mathrm{E}-04$ & $4.148 \mathrm{E}-04$ & $1.321 \mathrm{E}-06$ & $3.141 \mathrm{E}-03$ & $1.406 \mathrm{E}-03$ & $4.030 \mathrm{E}-05$ & \\
\hline NGC 3628 & 2005-04-08 & 277 & NGA-NGC3628 & & $\cdots$ & $\cdots$ & $3.002 \mathrm{E}-03$ & $3.636 \mathrm{E}-03$ & 7.116E-05 & (4) \\
\hline NGC 3633 & 2004-04-08 & 2051 & NGA-NGC3640 & 3.807E-04 & $4.640 \mathrm{E}-04$ & $4.648 \mathrm{E}-05$ & $3.270 \mathrm{E}-03$ & $1.464 \mathrm{E}-03$ & 4.673E-06 & \\
\hline UGC 06359 & 2004-04-07 & 682 & MISDR1-12839-0280 & $\ldots$ & $\ldots$ & $\ldots$ & $3.162 \mathrm{E}-03$ & $2.426 \mathrm{E}-03$ & $6.918 \mathrm{E}-05$ & $(4)(5)$ \\
\hline NGC 3640 & 2004-04-08 & 2051 & NGA-NGC3640 & $3.547 \mathrm{E}-04$ & $4.416 \mathrm{E}-04$ & $2.517 \mathrm{E}-05$ & $3.283 \mathrm{E}-03$ & $1.493 \mathrm{E}-03$ & $4.972 \mathrm{E}-06$ & \\
\hline NGC 3641 & 2004-04-08 & 2051 & NGA-NGC3640 & 3.317E-04 & $4.248 \mathrm{E}-04$ & $5.460 \mathrm{E}-05$ & $3.268 \mathrm{E}-03$ & $1.487 \mathrm{E}-03$ & $1.316 \mathrm{E}-04$ & \\
\hline NGC 3644 & 2004-04-08 & 2051 & NGA-NGC3640 & $3.316 \mathrm{E}-04$ & $4.634 \mathrm{E}-04$ & $2.566 \mathrm{E}-05$ & $3.166 \mathrm{E}-03$ & $1.402 \mathrm{E}-03$ & $4.355 \mathrm{E}-06$ & \\
\hline NGC 3646 & $2004-04-13$ & 1116 & NGA-NGC3646 & $\ldots$ & $\ldots$ & $\ldots$ & $2.775 \mathrm{E}-03$ & $1.842 \mathrm{E}-03$ & $2.878 \mathrm{E}-05$ & (4) \\
\hline NGC 3649 & 2004-04-13 & 1116 & NGA-NGC3646 & .. & $\ldots$ & $\cdots$ & $2.760 \mathrm{E}-03$ & $1.814 \mathrm{E}-03$ & $9.472 \mathrm{E}-05$ & (4) \\
\hline UGC 06387 & 2004-04-11 & 3069 & NGA-NGC3627 & $2.461 \mathrm{E}-04$ & $4.535 \mathrm{E}-04$ & $2.614 \mathrm{E}-05$ & $2.946 \mathrm{E}-03$ & $1.136 \mathrm{E}-03$ & $1.074 \mathrm{E}-04$ & (2) \\
\hline NGC 3662 & 2004-04-07 & 682 & MISDR1-12839-0280 & $3.115 \mathrm{E}-04$ & $6.897 \mathrm{E}-04$ & $3.188 \mathrm{E}-05$ & $3.131 \mathrm{E}-03$ & $2.423 \mathrm{E}-03$ & $2.553 \mathrm{E}-05$ & \\
\hline UGC 06435 & 2004-04-07 & 1472 & MISDR1-12838-0280 & $\ldots$ & $\ldots$ & $\ldots$ & $2.980 \mathrm{E}-03$ & $1.615 \mathrm{E}-03$ & $1.291 \mathrm{E}-05$ & $(4)(5)$ \\
\hline VII Zw 403 & $2003-10-13$ & 1645 & NGA-VIIZw403 & $3.953 \mathrm{E}-04$ & $5.205 \mathrm{E}-04$ & $1.768 \mathrm{E}-05$ & $2.659 \mathrm{E}-03$ & $1.486 \mathrm{E}-03$ & 7.351E-05 & \\
\hline NGC 3705 & 2005-04-08 & 1102 & NGA-NGC3705 & $\ldots$ & $\cdots$ & $\ldots$ & $2.947 \mathrm{E}-03$ & $1.895 \mathrm{E}-03$ & $1.024 \mathrm{E}-04$ & (4) \\
\hline UGC 06519 & 2004-04-07 & 1078 & MISDR1-12879-0282 & $2.458 \mathrm{E}-04$ & $5.438 \mathrm{E}-04$ & $8.828 \mathrm{E}-06$ & $2.987 \mathrm{E}-03$ & $1.933 \mathrm{E}-03$ & $1.459 \mathrm{E}-05$ & (2) \\
\hline IC 0716 & $2005-02-20$ & 1508 & NGA-UM448 & $2.551 \mathrm{E}-04$ & $5.415 \mathrm{E}-04$ & $3.569 \mathrm{E}-05$ & $2.876 \mathrm{E}-03$ & $1.591 \mathrm{E}-03$ & $6.591 \mathrm{E}-05$ & \\
\hline NGC 3816 & $2004-04-20$ & 1675 & A1367-SPEC-A & $2.073 \mathrm{E}-04$ & $4.409 \mathrm{E}-04$ & $2.708 \mathrm{E}-05$ & $2.579 \mathrm{E}-03$ & $1.361 \mathrm{E}-03$ & $1.393 \mathrm{E}-04$ & \\
\hline NGC 3821 & $2004-04-20$ & 1675 & A1367-SPEC-A & $2.078 \mathrm{E}-04$ & $4.309 \mathrm{E}-04$ & $3.606 \mathrm{E}-06$ & $2.624 \mathrm{E}-03$ & $1.375 \mathrm{E}-03$ & $3.446 \mathrm{E}-05$ & \\
\hline
\end{tabular}


Table 2-Continued

\begin{tabular}{|c|c|c|c|c|c|c|c|c|c|c|}
\hline \multirow{2}{*}{$\begin{array}{c}\text { Object Name } \\
\text { (1) }\end{array}$} & \multirow{2}{*}{$\begin{array}{c}\text { Date } \\
\text { Observed } \\
(2)\end{array}$} & \multirow{2}{*}{$\begin{array}{l}\text { Exposure } \\
\quad(\mathrm{sec}) \\
(3)\end{array}$} & \multirow{2}{*}{$\begin{array}{l}\text { Tile } \\
(4) \\
\end{array}$} & \multicolumn{3}{|c|}{ FUV sky background } & \multicolumn{3}{|c|}{ NUV sky background } & \multirow{2}{*}{$\begin{array}{l}\text { notes } \\
\text { (11) }\end{array}$} \\
\hline & & & & $\begin{array}{c}\text { mean } \\
(\text { counts/s) } \\
(5)\end{array}$ & $\begin{array}{c}<\sigma> \\
(\text { counts/s) } \\
(6)\end{array}$ & $\begin{array}{c}\sigma(\text { mean }) \\
(\text { counts } / \mathrm{s}) \\
(7)\end{array}$ & $\begin{array}{c}\text { mean } \\
(\text { counts/s) } \\
(8)\end{array}$ & $\begin{array}{c}<\sigma> \\
(\text { counts/s) } \\
(9)\end{array}$ & $\begin{array}{c}\sigma(\text { mean }) \\
(\text { counts } / \mathrm{s}) \\
(10)\end{array}$ & \\
\hline CGCG 097-068 & 2004-04-20 & 1675 & A1367-SPEC-A & $2.124 \mathrm{E}-04$ & $4.282 \mathrm{E}-04$ & $9.446 \mathrm{E}-06$ & $2.652 \mathrm{E}-03$ & $1.420 \mathrm{E}-03$ & $4.426 \mathrm{E}-05$ & (1) \\
\hline UGC 06683 & 2004-04-20 & 1675 & A1367-SPEC-A & $2.332 \mathrm{E}-04$ & $4.617 \mathrm{E}-04$ & $5.059 \mathrm{E}-05$ & $2.670 \mathrm{E}-03$ & 1.433E-03 & $4.355 \mathrm{E}-05$ & \\
\hline IC 2951 & 2004-04-20 & 1675 & A1367-SPEC-A & $2.317 \mathrm{E}-04$ & $4.728 \mathrm{E}-04$ & $3.733 \mathrm{E}-05$ & $2.663 \mathrm{E}-03$ & $1.431 \mathrm{E}-03$ & $1.902 \mathrm{E}-04$ & \\
\hline UGC 06697 & 2004-04-20 & 1675 & A1367-SPEC-A & $2.366 \mathrm{E}-04$ & $4.305 \mathrm{E}-04$ & $3.489 \mathrm{E}-05$ & $2.759 \mathrm{E}-03$ & $1.477 \mathrm{E}-03$ & $3.499 \mathrm{E}-06$ & (2) \\
\hline NGC 3840 & 2004-04-20 & 1675 & A1367-SPEC-A & $2.504 \mathrm{E}-04$ & $4.427 \mathrm{E}-04$ & $2.712 \mathrm{E}-05$ & $2.788 \mathrm{E}-03$ & $1.484 \mathrm{E}-03$ & $4.194 \mathrm{E}-05$ & \\
\hline NGC 3844 & 2004-04-20 & 1675 & A1367-SPEC-A & $2.492 \mathrm{E}-04$ & 4.407E-04 & $2.945 \mathrm{E}-06$ & $2.776 \mathrm{E}-03$ & $1.470 \mathrm{E}-03$ & $7.948 \mathrm{E}-05$ & \\
\hline NGC 3842 & 2004-04-20 & 1675 & A1367-SPEC-A & $2.504 \mathrm{E}-04$ & 4.494E-04 & $2.085 \mathrm{E}-05$ & $2.771 \mathrm{E}-03$ & $1.477 \mathrm{E}-03$ & $6.441 \mathrm{E}-05$ & \\
\hline UGC 06719 & 2004-04-20 & 1675 & A1367-SPEC-A & $2.450 \mathrm{E}-04$ & $4.254 \mathrm{E}-04$ & $7.779 \mathrm{E}-08$ & $2.681 \mathrm{E}-03$ & $1.410 \mathrm{E}-03$ & $3.575 \mathrm{E}-05$ & \\
\hline NGC 3861 & 2004-04-20 & 1675 & A1367-SPEC-A & $2.563 \mathrm{E}-04$ & $4.335 \mathrm{E}-04$ & $2.173 \mathrm{E}-05$ & $2.646 \mathrm{E}-03$ & $1.390 \mathrm{E}-03$ & $1.944 \mathrm{E}-05$ & \\
\hline UGC 06725 & 2004-04-20 & 1675 & A1367-SPEC-A & $2.167 \mathrm{E}-04$ & $3.800 \mathrm{E}-04$ & $3.603 \mathrm{E}-06$ & $2.666 \mathrm{E}-03$ & $1.357 \mathrm{E}-03$ & $4.731 \mathrm{E}-05$ & \\
\hline ESO 440-G004 & 2004-04-07 & 1310 & NGA-NGC3885 & $5.381 \mathrm{E}-04$ & $6.723 \mathrm{E}-04$ & $2.828 \mathrm{E}-05$ & $3.630 \mathrm{E}-03$ & $1.899 \mathrm{E}-03$ & $3.241 \mathrm{E}-05$ & \\
\hline UGC 06736 & 2004-04-08 & 1563 & MISDR1-12966-0514 & $2.650 \mathrm{E}-04$ & $4.378 \mathrm{E}-04$ & $2.326 \mathrm{E}-05$ & 3.012E-03 & $1.628 \mathrm{E}-03$ & $1.702 \mathrm{E}-05$ & \\
\hline NGC 3885 & 2004-04-07 & 1310 & NGA-NGC3885 & $4.990 \mathrm{E}-04$ & $6.574 \mathrm{E}-04$ & $3.680 \mathrm{E}-05$ & $3.578 \mathrm{E}-03$ & $1.968 \mathrm{E}-03$ & $5.726 \mathrm{E}-05$ & \\
\hline UGCA 247 & 2004-04-07 & 1310 & NGA-NGC3885 & $5.920 \mathrm{E}-04$ & $8.848 \mathrm{E}-04$ & $2.187 \mathrm{E}-05$ & $3.676 \mathrm{E}-03$ & $1.889 \mathrm{E}-03$ & $1.232 \mathrm{E}-04$ & \\
\hline NGC 3923 & 2004-04-07 & 3027 & NGA-NGC3923 & $5.438 \mathrm{E}-04$ & $5.199 \mathrm{E}-04$ & $1.120 \mathrm{E}-05$ & $3.620 \mathrm{E}-03$ & $1.295 \mathrm{E}-03$ & $1.457 \mathrm{E}-04$ & \\
\hline NGC 3938 & 2004-04-10 & 1662 & NGA-NGC3938 & $\cdots$ & $\cdots$ & $\cdots$ & $2.328 \mathrm{E}-03$ & $1.378 \mathrm{E}-03$ & $4.522 \mathrm{E}-05$ & (4) \\
\hline UGC 06879 & 2005-02-19 & 353 & NGA-UGC6879 & $2.506 \mathrm{E}-04$ & $9.112 \mathrm{E}-04$ & $8.499 \mathrm{E}-07$ & 3.013E-03 & $3.423 \mathrm{E}-03$ & $5.501 \mathrm{E}-05$ & \\
\hline UGC 06934 & 2004-04-20 & 664 & MISDR1-13211-0285 & $2.558 \mathrm{E}-04$ & $6.944 \mathrm{E}-04$ & $1.832 \mathrm{E}-05$ & 3.013E-03 & 2.491E-03 & $7.776 \mathrm{E}-05$ & \\
\hline UGC 06970 & 2004-04-13 & 123 & MISDR1-13262-0285 & $\ldots$ & $\ldots$ & $\ldots$ & $3.058 \mathrm{E}-03$ & $5.581 \mathrm{E}-03$ & $4.764 \mathrm{E}-05$ & (4) \\
\hline IC 0754 & 2004-04-13 & 123 & MISDR1-13262-0285 & $\cdots$ & $\cdots$ & $\cdots$ & $3.122 \mathrm{E}-03$ & $5.630 \mathrm{E}-03$ & $1.392 \mathrm{E}-04$ & (4) \\
\hline NGC 4030 & 2004-04-13 & 123 & MISDR1-13262-0285 & $\cdots$ & $\cdots$ & $\cdots$ & $3.119 \mathrm{E}-03$ & $5.992 \mathrm{E}-03$ & $1.942 \mathrm{E}-04$ & (4) \\
\hline UGC 07000 & 2004-04-13 & 123 & MISDR1-13262-0285 & $\cdots$ & $\cdots$ & $\cdots$ & $3.137 \mathrm{E}-03$ & $5.850 \mathrm{E}-03$ & $7.831 \mathrm{E}-05$ & (4) \\
\hline NGC 4038 & $2004-02-22$ & 400 & NGA-Antennae & $6.104 \mathrm{E}-04$ & $5.600 \mathrm{E}-04$ & $1.726 \mathrm{E}-05$ & $4.326 \mathrm{E}-03$ & $1.677 \mathrm{E}-03$ & $8.628 \mathrm{E}-05$ & \\
\hline NGC 4039 & 2004-02-22 & 400 & NGA-Antennae & $6.214 \mathrm{E}-04$ & $5.612 \mathrm{E}-04$ & $1.148 \mathrm{E}-05$ & $4.322 \mathrm{E}-03$ & $1.669 \mathrm{E}-03$ & $4.728 \mathrm{E}-05$ & \\
\hline UGC 07011 & 2004-04-13 & 123 & MISDR1-13262-0285 & $\cdots$ & $\cdots$ & $\cdots$ & $3.115 \mathrm{E}-03$ & $5.744 \mathrm{E}-03$ & $2.682 \mathrm{E}-04$ & (4) \\
\hline NGC 4108A & 2004-01-24 & 1604 & MISDR1-00391-0493 & $2.585 \mathrm{E}-04$ & $4.341 \mathrm{E}-04$ & $1.886 \mathrm{E}-05$ & $2.306 \mathrm{E}-03$ & $1.387 \mathrm{E}-03$ & $2.148 \mathrm{E}-05$ & \\
\hline UGC 07089 & $2005-03-17$ & 1062 & NGA-NGC4111 & $\ldots$ & $\ldots$ & $\ldots$ & $2.233 \mathrm{E}-03$ & $1.658 \mathrm{E}-03$ & $1.143 \mathrm{E}-04$ & (4) \\
\hline NGC 4108 & 2004-01-24 & 1604 & MISDR1-00391-0493 & $2.553 \mathrm{E}-04$ & 4.110E-04 & $1.184 \mathrm{E}-05$ & $2.297 \mathrm{E}-03$ & $1.366 \mathrm{E}-03$ & $3.499 \mathrm{E}-05$ & \\
\hline NGC 4109 & $2005-03-17$ & 1062 & NGA-NGC4111 & $\ldots$ & $\ldots$ & $\ldots$ & $2.255 \mathrm{E}-03$ & $1.690 \mathrm{E}-03$ & $3.882 \mathrm{E}-05$ & (4) \\
\hline NGC 4111 & $2005-03-17$ & 1062 & NGA-NGC4111 & $\cdots$ & $\cdots$ & $\cdots$ & $2.270 \mathrm{E}-03$ & $1.705 \mathrm{E}-03$ & $5.094 \mathrm{E}-05$ & (4) \\
\hline NGC 4108B & 2004-01-24 & 1604 & MISDR1-00391-0493 & $2.616 \mathrm{E}-04$ & $4.274 \mathrm{E}-04$ & $3.007 \mathrm{E}-07$ & $2.291 \mathrm{E}-03$ & $1.367 \mathrm{E}-03$ & $4.437 \mathrm{E}-05$ & \\
\hline NGC 4116 & 2004-04-11 & 1562 & MISDR1-13206-0516 & $\cdots$ & $\cdots$ & $\cdots$ & $2.823 \mathrm{E}-03$ & $1.509 \mathrm{E}-03$ & $2.156 \mathrm{E}-05$ & (4) \\
\hline
\end{tabular}


Table 2-Continued

\begin{tabular}{|c|c|c|c|c|c|c|c|c|c|c|}
\hline \multirow[b]{2}{*}{$\begin{array}{l}\text { Object Name } \\
\qquad(1)\end{array}$} & \multirow[b]{2}{*}{$\begin{array}{c}\text { Date } \\
\text { Observed } \\
(2)\end{array}$} & \multirow[b]{2}{*}{$\begin{array}{l}\text { Exposure } \\
\quad(\mathrm{sec}) \\
(3)\end{array}$} & \multirow[b]{2}{*}{$\begin{array}{l}\text { Tile } \\
(4)\end{array}$} & \multicolumn{3}{|c|}{ FUV sky background } & \multicolumn{3}{|c|}{ NUV sky background } & \multirow[b]{2}{*}{$\begin{array}{l}\text { notes } \\
\text { (11) }\end{array}$} \\
\hline & & & & $\begin{array}{c}\text { mean } \\
(\text { counts/s) } \\
(5)\end{array}$ & $\begin{array}{c}<\sigma> \\
(\text { counts } / \mathrm{s}) \\
(6)\end{array}$ & $\begin{array}{c}\sigma(\text { mean }) \\
(\text { counts/s) } \\
(7)\end{array}$ & $\begin{array}{c}\text { mean } \\
(\text { counts/s) } \\
(8)\end{array}$ & $\begin{array}{c}<\sigma> \\
(\text { counts } / \mathrm{s}) \\
(9)\end{array}$ & $\begin{array}{c}\sigma(\text { mean }) \\
(\text { counts/s) } \\
(10)\end{array}$ & \\
\hline NGC 4117 & 2005-03-17 & 1062 & NGA-NGC4111 & $\cdots$ & $\ldots$ & $\ldots$ & $2.275 \mathrm{E}-03$ & $1.724 \mathrm{E}-03$ & $8.560 \mathrm{E}-06$ & (4) \\
\hline NGC 4125 & $2004-01-27$ & 1707 & NGA-NGC4125 & $\cdots$ & $\cdots$ & $\cdots$ & 2.301E-03 & $1.352 \mathrm{E}-03$ & $8.424 \mathrm{E}-06$ & (4) \\
\hline NGC 4136 & 2004-04-14 & 886 & NGA-NGC4136 & $1.986 \mathrm{E}-04$ & $5.341 \mathrm{E}-04$ & $1.343 \mathrm{E}-05$ & $2.365 \mathrm{E}-03$ & $1.928 \mathrm{E}-03$ & $5.993 \mathrm{E}-05$ & \\
\hline NGC 4138 & 2005-03-17 & 1062 & NGA-NGC4111 & $\cdots$ & $\ldots$ & $\ldots$ & $2.198 \mathrm{E}-03$ & $1.597 \mathrm{E}-03$ & $4.218 \mathrm{E}-06$ & (4) \\
\hline NGC 4150 & 2004-04-14 & 886 & NGA-NGC4136 & $1.801 \mathrm{E}-04$ & $4.559 \mathrm{E}-04$ & $1.767 \mathrm{E}-06$ & $2.337 \mathrm{E}-03$ & $1.813 \mathrm{E}-03$ & $1.601 \mathrm{E}-05$ & \\
\hline VII Zw 173 & 2005-04-11 & 1394 & NGA-NGC4168 & $\cdots$ & $\ldots$ & $\ldots$ & $2.631 \mathrm{E}-03$ & $1.551 \mathrm{E}-03$ & $1.847 \mathrm{E}-05$ & $(4)(1)(7)$ \\
\hline UGC 07176 & $2005-04-27$ & 1401 & NGA-NGC4157 & $\cdots$ & $\cdots$ & $\cdots$ & $2.423 \mathrm{E}-03$ & $1.556 \mathrm{E}-03$ & $3.340 \mathrm{E}-05$ & (4) \\
\hline UGC 07178 & 2004-04-12 & 1421 & MISDR1-13310-0517 & $\cdots$ & $\ldots$ & $\ldots$ & $2.848 \mathrm{E}-03$ & $1.641 \mathrm{E}-03$ & $2.189 \mathrm{E}-05$ & (4) \\
\hline NGC 4157 & $2005-04-27$ & 1401 & NGA-NGC4157 & $\ldots$ & $\ldots$ & $\ldots$ & $2.400 \mathrm{E}-03$ & $1.523 \mathrm{E}-03$ & $7.116 \mathrm{E}-05$ & (4) \\
\hline IC 3033 & 2005-04-11 & 1394 & NGA-NGC4168 & $\ldots$ & $\ldots$ & $\ldots$ & $2.624 \mathrm{E}-03$ & $1.536 \mathrm{E}-03$ & $1.435 \mathrm{E}-05$ & (4) \\
\hline UGC 07184 & $2004-04-12$ & 1683 & MISDR1-13311-0287 & $\ldots$ & $\ldots$ & $\ldots$ & $2.883 \mathrm{E}-03$ & $1.473 \mathrm{E}-03$ & $2.262 \mathrm{E}-05$ & (4) \\
\hline UGC 07196 & $2005-04-11$ & 1231 & NGA-NGC4192 & $\cdots$ & $\cdots$ & $\cdots$ & $2.722 \mathrm{E}-03$ & $1.646 \mathrm{E}-03$ & $1.164 \mathrm{E}-05$ & (4) \\
\hline NGC 4165 & 2005-04-11 & 1394 & NGA-NGC4168 & $\cdots$ & $\cdots$ & $\cdots$ & $2.684 \mathrm{E}-03$ & $1.624 \mathrm{E}-03$ & $7.041 \mathrm{E}-05$ & (4) \\
\hline NGC $4192 \mathrm{~A}$ & 2005-04-11 & 1231 & NGA-NGC4192 & $\cdots$ & $\ldots$ & $\cdots$ & $2.805 \mathrm{E}-03$ & $1.749 \mathrm{E}-03$ & $2.674 \mathrm{E}-05$ & (4) \\
\hline NGC 4187 & $2005-04-27$ & 1401 & NGA-NGC4157 & $\cdots$ & $\ldots$ & $\ldots$ & $2.356 \mathrm{E}-03$ & $1.428 \mathrm{E}-03$ & $1.584 \mathrm{E}-05$ & (4) \\
\hline NGC 4189 & 2005-04-11 & 1394 & NGA-NGC4168 & $\ldots$ & $\ldots$ & $\ldots$ & $2.625 \mathrm{E}-03$ & $1.525 \mathrm{E}-03$ & $1.467 \mathrm{E}-05$ & (4) \\
\hline MESSIER 098 & 2005-04-11 & 1231 & NGA-NGC4192 & $\cdots$ & $\ldots$ & $\cdots$ & $2.821 \mathrm{E}-03$ & $1.714 \mathrm{E}-03$ & $7.903 \mathrm{E}-05$ & (4) \\
\hline NGC 4193 & 2005-04-11 & 1394 & NGA-NGC4168 & $\cdots$ & $\ldots$ & $\cdots$ & $2.638 \mathrm{E}-03$ & $1.559 \mathrm{E}-03$ & $4.122 \mathrm{E}-05$ & (4) \\
\hline NGC 4186 & $2005-04-11$ & 1231 & NGA-NGC4192 & $\ldots$ & $\ldots$ & $\ldots$ & $2.853 \mathrm{E}-03$ & $1.734 \mathrm{E}-03$ & $1.768 \mathrm{E}-05$ & (4) \\
\hline UGC 07242 & 2004-01-26 & 1386 & MISDR1-00419-0493 & $2.481 \mathrm{E}-04$ & $4.336 \mathrm{E}-04$ & $2.732 \mathrm{E}-05$ & $2.261 \mathrm{E}-03$ & $1.410 \mathrm{E}-03$ & $4.437 \mathrm{E}-05$ & \\
\hline UGC 07249 & 2004-04-20 & 1672 & VIRGO-SPEC-1 & $2.470 \mathrm{E}-04$ & $4.532 \mathrm{E}-04$ & $2.472 \mathrm{E}-05$ & $2.654 \mathrm{E}-03$ & $1.448 \mathrm{E}-03$ & $2.367 \mathrm{E}-05$ & \\
\hline IC 3059 & 2004-04-20 & 1672 & VIRGO-SPEC-1 & $2.449 \mathrm{E}-04$ & 4.135E-04 & $1.704 \mathrm{E}-05$ & $2.698 \mathrm{E}-03$ & $1.404 \mathrm{E}-03$ & $3.160 \mathrm{E}-05$ & \\
\hline VCC 0132 & 2004-04-20 & 1672 & VIRGO-SPEC-1 & $2.486 \mathrm{E}-04$ & $4.309 \mathrm{E}-04$ & $7.552 \mathrm{E}-06$ & $2.762 \mathrm{E}-03$ & $1.496 \mathrm{E}-03$ & $3.931 \mathrm{E}-05$ & \\
\hline IC 3066 & 2004-04-20 & 1672 & VIRGO-SPEC-1 & $2.472 \mathrm{E}-04$ & $4.076 \mathrm{E}-04$ & $4.685 \mathrm{E}-06$ & $2.681 \mathrm{E}-03$ & $1.391 \mathrm{E}-03$ & $8.874 \mathrm{E}-05$ & \\
\hline NGC 4206 & 2004-04-20 & 1672 & VIRGO-SPEC-1 & $2.505 \mathrm{E}-04$ & $4.295 \mathrm{E}-04$ & $2.102 \mathrm{E}-05$ & $2.739 \mathrm{E}-03$ & $1.476 \mathrm{E}-03$ & $7.269 \mathrm{E}-05$ & \\
\hline IC 3073 & 2004-04-20 & 1672 & VIRGO-SPEC-1 & $2.395 \mathrm{E}-04$ & $4.009 \mathrm{E}-04$ & $7.704 \mathrm{E}-06$ & $2.689 \mathrm{E}-03$ & $1.372 \mathrm{E}-03$ & $4.819 \mathrm{E}-05$ & (1) \\
\hline NGC 4216 & 2004-04-20 & 1672 & VIRGO-SPEC-1 & $2.576 \mathrm{E}-04$ & $4.284 \mathrm{E}-04$ & $4.835 \mathrm{E}-06$ & $2.689 \mathrm{E}-03$ & $1.420 \mathrm{E}-03$ & $2.493 \mathrm{E}-05$ & \\
\hline NGC 4222 & 2004-04-20 & 1672 & VIRGO-SPEC-1 & $2.468 \mathrm{E}-04$ & $4.032 \mathrm{E}-04$ & $1.319 \mathrm{E}-05$ & $2.731 \mathrm{E}-03$ & $1.410 \mathrm{E}-03$ & $1.337 \mathrm{E}-04$ & \\
\hline NGC 4226 & $2005-03-18$ & 1687 & NGA-NGC4258 & $\ldots$ & $\ldots$ & $\ldots$ & $2.265 \mathrm{E}-03$ & $1.356 \mathrm{E}-03$ & $4.203 \mathrm{E}-05$ & (4) \\
\hline
\end{tabular}


Table 2-Continued

\begin{tabular}{|c|c|c|c|c|c|c|c|c|c|c|}
\hline \multirow{2}{*}{$\begin{array}{l}\text { Object Name } \\
\text { (1) }\end{array}$} & \multirow{2}{*}{$\begin{array}{c}\text { Date } \\
\text { Observed } \\
(2)\end{array}$} & \multirow{2}{*}{$\begin{array}{l}\text { Exposure } \\
\quad(\mathrm{sec}) \\
(3)\end{array}$} & \multirow{2}{*}{$\begin{array}{l}\text { Tile } \\
\text { (4) }\end{array}$} & \multicolumn{3}{|c|}{ FUV sky background } & \multicolumn{3}{|c|}{ NUV sky background } & \multirow{2}{*}{$\begin{array}{l}\text { notes } \\
\text { (11) }\end{array}$} \\
\hline & & & & $\begin{array}{c}\text { mean } \\
(\text { counts/s) } \\
(5)\end{array}$ & $\begin{array}{c}<\sigma> \\
(\text { counts/s) } \\
(6)\end{array}$ & $\begin{array}{c}\sigma(\text { mean }) \\
(\text { counts/s) } \\
(7)\end{array}$ & $\begin{array}{c}\text { mean } \\
(\text { counts/s) } \\
(8)\end{array}$ & $\begin{array}{c}<\sigma> \\
(\text { counts/s) } \\
(9)\end{array}$ & $\begin{array}{c}\sigma(\text { mean }) \\
(\text { counts/s) } \\
(10)\end{array}$ & \\
\hline NGC 4236 & 2004-01-24 & 1686 & NGA-NGC4236 & $3.519 \mathrm{E}-04$ & $5.007 \mathrm{E}-04$ & $4.308 \mathrm{E}-06$ & $2.718 \mathrm{E}-03$ & $1.479 \mathrm{E}-03$ & $1.247 \mathrm{E}-05$ & \\
\hline UGC 07301 & 2005-03-18 & 1686 & NGA-NGC4242 & $\ldots$ & $\ldots$ & $\ldots$ & $2.150 \mathrm{E}-03$ & $1.236 \mathrm{E}-03$ & $7.961 \mathrm{E}-05$ & (4) \\
\hline NGC 4231 & 2005-03-18 & 1687 & NGA-NGC4258 & $\ldots$ & $\ldots$ & $\ldots$ & $2.195 \mathrm{E}-03$ & $1.315 \mathrm{E}-03$ & $1.376 \mathrm{E}-05$ & (4) \\
\hline NGC 4232 & 2005-03-18 & 1687 & NGA-NGC4258 & $\ldots$ & $\ldots$ & $\ldots$ & $2.192 \mathrm{E}-03$ & $1.316 \mathrm{E}-03$ & $8.320 \mathrm{E}-05$ & (4) \\
\hline UGC 07325 & 2005-03-18 & 1687 & NGA-NGC4258 & $\ldots$ & $\ldots$ & $\ldots$ & $2.239 \mathrm{E}-03$ & $1.338 \mathrm{E}-03$ & $4.354 \mathrm{E}-05$ & (4) \\
\hline NGC 4242 & 2005-03-18 & 1686 & NGA-NGC4242 & $\ldots$ & $\ldots$ & $\ldots$ & $2.132 \mathrm{E}-03$ & $1.302 \mathrm{E}-03$ & 3.637E-05 & (4) \\
\hline NGC 4248 & 2005-03-18 & 1687 & NGA-NGC4258 & $\ldots$ & $\ldots$ & $\ldots$ & $2.283 \mathrm{E}-03$ & $1.357 \mathrm{E}-03$ & $1.561 \mathrm{E}-04$ & (4) \\
\hline MESSIER 099 & 2005-03-28 & 1489 & VIRGOHI21 & $\ldots$ & $\ldots$ & $\ldots$ & $2.841 \mathrm{E}-03$ & $4.784 \mathrm{E}-04$ & $4.438 \mathrm{E}-05$ & (4) \\
\hline MESSIER 106 & 2005-03-18 & 1687 & NGA-NGC4258 & $\ldots$ & $\ldots$ & $\ldots$ & $2.241 \mathrm{E}-03$ & $1.338 \mathrm{E}-03$ & $3.269 \mathrm{E}-05$ & (4) \\
\hline NGC 4262 & 2005-03-28 & 1489 & VIRGOHI21 & $\ldots$ & $\ldots$ & $\ldots$ & $2.737 \mathrm{E}-03$ & $4.525 \mathrm{E}-04$ & 8.771E-05 & (4) \\
\hline NGC 4274 & 2004-04-06 & 1511 & NGA-NGC4278 & $1.990 \mathrm{E}-04$ & $4.320 \mathrm{E}-04$ & $2.470 \mathrm{E}-06$ & $2.565 \mathrm{E}-03$ & $1.501 \mathrm{E}-03$ & $4.454 \mathrm{E}-05$ & \\
\hline NGC 4278 & 2004-04-06 & 1511 & NGA-NGC4278 & $2.147 \mathrm{E}-04$ & $4.516 \mathrm{E}-04$ & $6.491 \mathrm{E}-05$ & $2.615 \mathrm{E}-03$ & $1.545 \mathrm{E}-03$ & $5.739 \mathrm{E}-05$ & \\
\hline UGC 07387 & 2004-04-13 & 1574 & NGA-NGC4303 & $1.849 \mathrm{E}-04$ & $5.419 \mathrm{E}-04$ & $5.048 \mathrm{E}-06$ & $2.845 \mathrm{E}-03$ & $1.571 \mathrm{E}-03$ & $1.120 \mathrm{E}-04$ & \\
\hline NGC 4283 & 2004-04-06 & 1511 & NGA-NGC4278 & 2.103E-04 & $4.341 \mathrm{E}-04$ & $1.030 \mathrm{E}-05$ & $2.629 \mathrm{E}-03$ & $1.555 \mathrm{E}-03$ & $2.908 \mathrm{E}-05$ & \\
\hline NGC 4286 & 2004-04-06 & 1511 & NGA-NGC4278 & $2.004 \mathrm{E}-04$ & $4.127 \mathrm{E}-04$ & $3.809 \mathrm{E}-06$ & $2.607 \mathrm{E}-03$ & $1.552 \mathrm{E}-03$ & $4.308 \mathrm{E}-05$ & \\
\hline NGC 4292 & 2004-04-13 & 1574 & NGA-NGC4303 & $1.820 \mathrm{E}-04$ & 4.883E-04 & $1.003 \mathrm{E}-05$ & $2.837 \mathrm{E}-03$ & $1.570 \mathrm{E}-03$ & $5.268 \mathrm{E}-05$ & \\
\hline NGC 4298 & 2005-03-28 & 1489 & VIRGOHI21 & $\ldots$ & $\ldots$ & $\ldots$ & $2.708 \mathrm{E}-03$ & $4.338 \mathrm{E}-04$ & $1.066 \mathrm{E}-05$ & (4) \\
\hline UGC 07411 & 2004-04-13 & 1574 & NGA-NGC4303 & $\ldots$ & $\ldots$ & $\ldots$ & $2.805 \mathrm{E}-03$ & $1.521 \mathrm{E}-03$ & $6.551 \mathrm{E}-05$ & $(4)(5)$ \\
\hline IC 0783 & 2004-04-11 & 1183 & NGA-NGC4321 & $\ldots$ & $\ldots$ & $\ldots$ & $2.594 \mathrm{E}-03$ & $1.704 \mathrm{E}-03$ & $2.868 \mathrm{E}-05$ & (4) \\
\hline UGC 07425 & 2004-04-11 & 1183 & NGA-NGC4321 & $\ldots$ & $\ldots$ & $\ldots$ & $2.610 \mathrm{E}-03$ & $1.726 \mathrm{E}-03$ & $3.251 \mathrm{E}-05$ & (4) \\
\hline NGC 4303 & 2004-04-13 & 1574 & NGA-NGC4303 & $2.091 \mathrm{E}-04$ & $5.015 \mathrm{E}-04$ & $8.796 \mathrm{E}-06$ & $2.887 \mathrm{E}-03$ & $1.591 \mathrm{E}-03$ & $2.562 \mathrm{E}-05$ & \\
\hline VCC 0530 & 2004-04-11 & 1183 & NGA-NGC4321 & $\ldots$ & $\ldots$ & $\ldots$ & $2.635 \mathrm{E}-03$ & $1.737 \mathrm{E}-03$ & $2.397 \mathrm{E}-05$ & (4) \\
\hline NGC 4310 & 2004-04-06 & 1511 & NGA-NGC4278 & $2.031 \mathrm{E}-04$ & $4.043 \mathrm{E}-04$ & $2.008 \mathrm{E}-05$ & $2.530 \mathrm{E}-03$ & $1.487 \mathrm{E}-03$ & $2.081 \mathrm{E}-05$ & (1) \\
\hline NGC 4301 & 2004-04-13 & 1574 & NGA-NGC4303 & $2.017 \mathrm{E}-04$ & $4.686 \mathrm{E}-04$ & $1.321 \mathrm{E}-05$ & $2.863 \mathrm{E}-03$ & $1.548 \mathrm{E}-03$ & $2.300 \mathrm{E}-05$ & \\
\hline NGC 4312 & 2004-04-11 & 1183 & NGA-NGC4321 & $\ldots$ & $\cdots$ & $\cdots$ & $2.638 \mathrm{E}-03$ & $1.762 \mathrm{E}-03$ & 3.909E-05 & (4) \\
\hline NGC 4314 & 2004-04-06 & 1511 & NGA-NGC4278 & $1.907 \mathrm{E}-04$ & $3.609 \mathrm{E}-04$ & $1.916 \mathrm{E}-05$ & $2.519 \mathrm{E}-03$ & $1.425 \mathrm{E}-03$ & $5.489 \mathrm{E}-05$ & \\
\hline NGC 4321 & 2004-04-11 & 1183 & NGA-NGC4321 & $\ldots$ & $\ldots$ & $\ldots$ & $2.655 \mathrm{E}-03$ & $1.751 \mathrm{E}-03$ & $4.509 \mathrm{E}-06$ & (4) \\
\hline NGC 4323 & 2004-04-11 & 1183 & NGA-NGC4321 & $\cdots$ & $\cdots$ & $\cdots$ & $2.660 \mathrm{E}-03$ & $1.735 \mathrm{E}-03$ & $6.400 \mathrm{E}-05$ & $(4)(1)$ \\
\hline NGC 4328 & 2004-04-11 & 1183 & NGA-NGC4321 & $\cdots$ & $\cdots$ & $\cdots$ & $2.721 \mathrm{E}-03$ & $1.767 \mathrm{E}-03$ & 8.919E-05 & (4) \\
\hline NGC 4344 & 2004-03-22 & 1633 & NGA-NGC4344 & $1.902 \mathrm{E}-04$ & $3.616 \mathrm{E}-04$ & $3.627 \mathrm{E}-06$ & $2.770 \mathrm{E}-03$ & $1.520 \mathrm{E}-03$ & $5.020 \mathrm{E}-05$ & \\
\hline NGC 4371 & 2004-03-11 & 1611 & NGA-Virgo-MOS08-0001 & $2.632 \mathrm{E}-04$ & $4.612 \mathrm{E}-04$ & $1.522 \mathrm{E}-05$ & $2.950 \mathrm{E}-03$ & $1.566 \mathrm{E}-03$ & $6.620 \mathrm{E}-05$ & (3) \\
\hline MESSIER 084 & 2004-03-11 & 1612 & NGA-Virgo-MOS10-0001 & $2.772 \mathrm{E}-04$ & $4.735 \mathrm{E}-04$ & $1.557 \mathrm{E}-06$ & $3.128 \mathrm{E}-03$ & $1.656 \mathrm{E}-03$ & $7.371 \mathrm{E}-05$ & (3) \\
\hline
\end{tabular}


Table 2-Continued

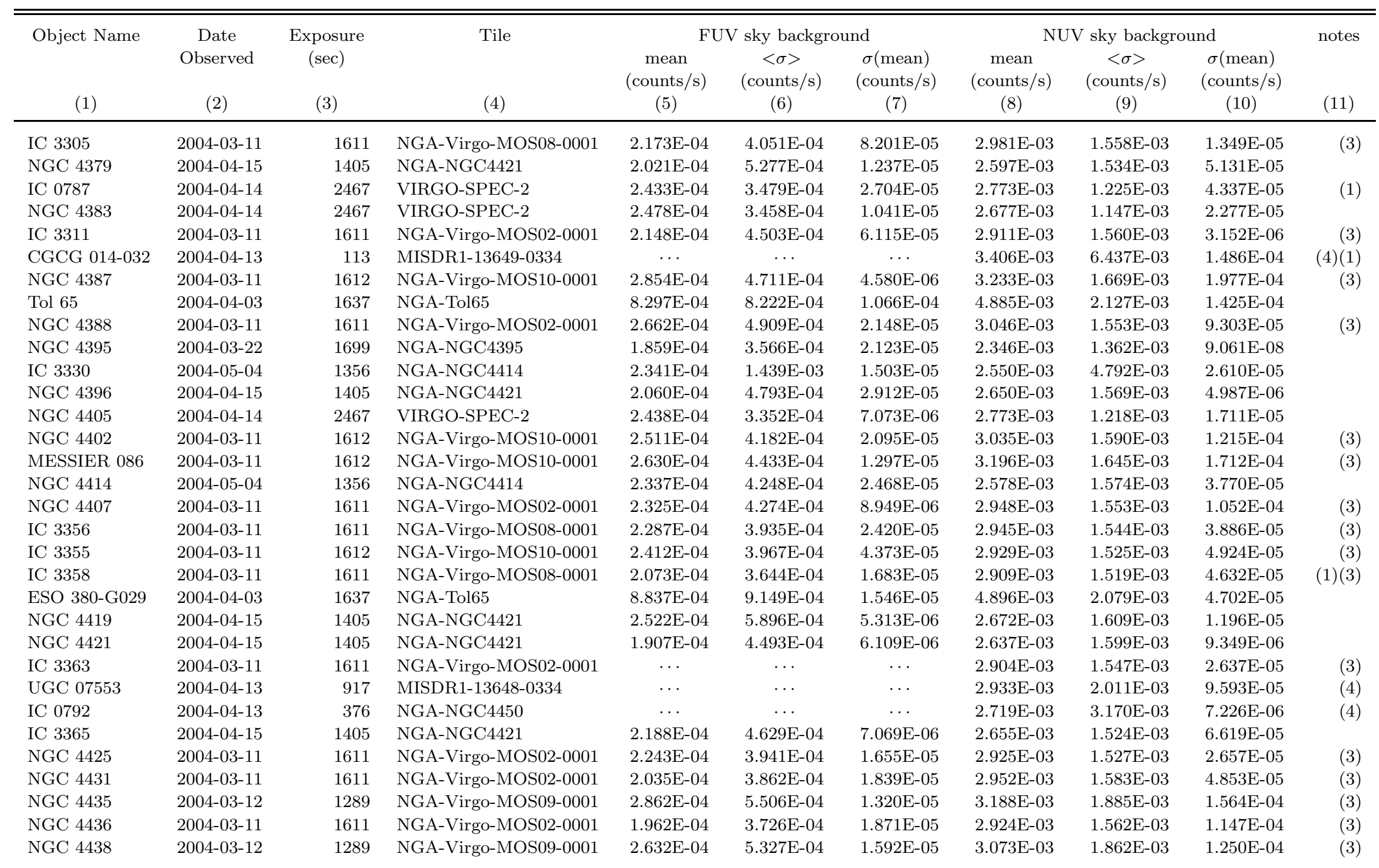


Table 2-Continued

\begin{tabular}{|c|c|c|c|c|c|c|c|c|c|c|}
\hline \multirow[b]{2}{*}{$\begin{array}{c}\text { Object Name } \\
\text { (1) }\end{array}$} & \multirow[b]{2}{*}{$\begin{array}{c}\text { Date } \\
\text { Observed } \\
(2)\end{array}$} & \multirow[b]{2}{*}{$\begin{array}{l}\text { Exposure } \\
\quad(\mathrm{sec}) \\
(3)\end{array}$} & \multirow[b]{2}{*}{$\begin{array}{l}\text { Tile } \\
\text { (4) }\end{array}$} & \multicolumn{3}{|c|}{ FUV sky background } & \multicolumn{3}{|c|}{ NUV sky background } & \multirow{2}{*}{$\begin{array}{l}\text { notes } \\
\text { (11) }\end{array}$} \\
\hline & & & & $\begin{array}{c}\text { mean } \\
(\text { counts/s) } \\
(5)\end{array}$ & $\begin{array}{c}<\sigma> \\
(\text { counts } / \mathrm{s}) \\
(6)\end{array}$ & $\begin{array}{c}\sigma(\text { mean }) \\
(\text { counts/s) } \\
(7)\end{array}$ & $\begin{array}{c}\text { mean } \\
(\text { counts/s) } \\
(8)\end{array}$ & $\begin{array}{c}<\sigma> \\
(\text { counts } / \mathrm{s}) \\
(9)\end{array}$ & $\begin{array}{c}\sigma(\text { mean }) \\
(\text { counts/s) } \\
(10)\end{array}$ & \\
\hline NGC 4440 & 2004-03-11 & 1611 & NGA-Virgo-MOS02-0001 & $2.038 \mathrm{E}-04$ & $3.817 \mathrm{E}-04$ & $9.566 \mathrm{E}-06$ & $2.944 \mathrm{E}-03$ & $1.563 \mathrm{E}-03$ & $2.975 \mathrm{E}-06$ & (3) \\
\hline IC 0794 & $004-03-11$ & 1611 & NGA-Virgo-MOS02-0001 & $2.020 \mathrm{E}-04$ & $3.832 \mathrm{E}-04$ & $3.208 \mathrm{E}-05$ & $2.937 \mathrm{E}-03$ & $1.568 \mathrm{E}-03$ & $1.758 \mathrm{E}-04$ & (3) \\
\hline IC 3381 & 2004-03-11 & 1611 & NGA-Virgo-MOS06-0001 & $2.175 \mathrm{E}-04$ & $4.515 \mathrm{E}-04$ & $1.302 \mathrm{E}-05$ & $2.963 \mathrm{E}-03$ & $1.576 \mathrm{E}-03$ & $1.489 \mathrm{E}-04$ & (3) \\
\hline NGC 4450 & 2004-04-13 & 376 & NGA-NGC4450 & $\cdots$ & $\ldots$ & $\cdots$ & $2.751 \mathrm{E}-03$ & $3.009 \mathrm{E}-03$ & $2.384 \mathrm{E}-05$ & (4) \\
\hline UGC 07604 & 2004-05-04 & 1356 & NGA-NGC4414 & $1.970 \mathrm{E}-04$ & $1.150 \mathrm{E}-03$ & $1.350 \mathrm{E}-05$ & $2.501 \mathrm{E}-03$ & 4.436E-03 & $1.519 \mathrm{E}-04$ & \\
\hline IC 3393 & 2004-03-12 & 1289 & NGA-Virgo-MOS09-0001 & $2.160 \mathrm{E}-04$ & $4.554 \mathrm{E}-04$ & $2.735 \mathrm{E}-05$ & $2.954 \mathrm{E}-03$ & $1.788 \mathrm{E}-03$ & $8.463 \mathrm{E}-05$ & $(1)(3)$ \\
\hline NGC 4452 & 2004-03-11 & 1611 & NGA-Virgo-MOS06-0001 & $2.204 \mathrm{E}-04$ & $4.375 \mathrm{E}-04$ & $1.184 \mathrm{E}-05$ & $2.956 \mathrm{E}-03$ & $1.589 \mathrm{E}-03$ & $1.865 \mathrm{E}-05$ & (3) \\
\hline NGC 4454 & $2004-04-13$ & 917 & MISDR1-13648-0334 & $\cdots$ & $\cdots$ & $\cdots$ & $2.903 \mathrm{E}-03$ & $2.004 \mathrm{E}-03$ & $4.566 \mathrm{E}-05$ & (4) \\
\hline NGC 4458 & 2004-03-12 & 1289 & NGA-Virgo-MOS09-0001 & $2.278 \mathrm{E}-04$ & $4.525 \mathrm{E}-04$ & $5.719 \mathrm{E}-06$ & 3.017E-03 & $1.794 \mathrm{E}-03$ & $9.688 \mathrm{E}-05$ & (3) \\
\hline NGC 4461 & 2004-03-12 & 1289 & NGA-Virgo-MOS09-0001 & $2.246 \mathrm{E}-04$ & $4.460 \mathrm{E}-04$ & $7.770 \mathrm{E}-06$ & $3.026 \mathrm{E}-03$ & $1.791 \mathrm{E}-03$ & $2.713 \mathrm{E}-05$ & (3) \\
\hline IC 0796 & 2004-04-13 & 376 & NGA-NGC4450 & $\ldots$ & $\ldots$ & $\ldots$ & $2.699 \mathrm{E}-03$ & $3.003 \mathrm{E}-03$ & $2.586 \mathrm{E}-05$ & (4) \\
\hline IC 3418 & $2004-03-11$ & 1611 & NGA-Virgo-MOS06-0001 & $2.524 \mathrm{E}-04$ & 4.239E-04 & $2.849 \mathrm{E}-06$ & $3.059 \mathrm{E}-03$ & $1.601 \mathrm{E}-03$ & $1.012 \mathrm{E}-04$ & $(1)(3)$ \\
\hline NGC 4473 & $2004-03-12$ & 1289 & NGA-Virgo-MOS09-0001 & $2.540 \mathrm{E}-04$ & 4.895E-04 & $2.212 \mathrm{E}-05$ & 3.077E-03 & $1.764 \mathrm{E}-03$ & 8.339E-05 & (3) \\
\hline NGC 4476 & $2004-03-12$ & 1585 & NGA-Virgo-MOS01-0001 & $2.201 \mathrm{E}-04$ & 4.150E-04 & $5.381 \mathrm{E}-06$ & 3.083E-03 & $1.647 \mathrm{E}-03$ & $1.608 \mathrm{E}-04$ & (3) \\
\hline NGC 4477 & $2004-03-12$ & 1289 & NGA-Virgo-MOS09-0001 & $2.672 \mathrm{E}-04$ & $5.523 \mathrm{E}-04$ & $6.876 \mathrm{E}-06$ & $3.022 \mathrm{E}-03$ & $1.707 \mathrm{E}-03$ & $1.364 \mathrm{E}-04$ & (3) \\
\hline NGC 4478 & $2004-03-12$ & 1585 & NGA-Virgo-MOS01-0001 & $2.424 \mathrm{E}-04$ & $4.289 \mathrm{E}-04$ & $2.276 \mathrm{E}-05$ & $3.288 \mathrm{E}-03$ & $1.692 \mathrm{E}-03$ & $2.308 \mathrm{E}-04$ & (3) \\
\hline NGC 4479 & 2004-03-12 & 1289 & NGA-Virgo-MOS09-0001 & $2.649 \mathrm{E}-04$ & 5.302E-04 & $2.480 \mathrm{E}-06$ & $3.089 \mathrm{E}-03$ & $1.725 \mathrm{E}-03$ & 3.743E-05 & $(1)(3)$ \\
\hline NGC 4485 & $2005-03-26$ & 1218 & NGA-NGC4490 & $\ldots$ & $\ldots$ & $\ldots$ & $2.449 \mathrm{E}-03$ & $1.656 \mathrm{E}-03$ & $5.500 \mathrm{E}-05$ & (4) \\
\hline NGC 4490 & $2005-03-26$ & 1218 & NGA-NGC4490 & $\ldots$ & $\ldots$ & $\ldots$ & $2.445 \mathrm{E}-03$ & $1.652 \mathrm{E}-03$ & $1.167 \mathrm{E}-05$ & (4) \\
\hline MESSIER 087 & 2004-03-12 & 1585 & NGA-Virgo-MOS01-0001 & $2.333 \mathrm{E}-04$ & $4.155 \mathrm{E}-04$ & $2.589 \mathrm{E}-06$ & $3.264 \mathrm{E}-03$ & $1.676 \mathrm{E}-03$ & $5.321 \mathrm{E}-05$ & $(1)(3)$ \\
\hline NGC 4491 & 2004-03-11 & 1611 & NGA-Virgo-MOS06-0001 & $2.538 \mathrm{E}-04$ & 4.013E-04 & $2.370 \mathrm{E}-05$ & $2.961 \mathrm{E}-03$ & $1.520 \mathrm{E}-03$ & 3.507E-07 & (3) \\
\hline CGCG 014-054 & 2004-04-14 & & NGA-NGC4536 & $2.187 \mathrm{E}-04$ & $5.412 \mathrm{E}-04$ & $7.000 \mathrm{E}-06$ & $2.979 \mathrm{E}-03$ & $1.519 \mathrm{E}-03$ & $7.635 \mathrm{E}-05$ & \\
\hline IC 3446 & $2004-03-12$ & & NGA-Virgo-MOS05-00 & $2.865 \mathrm{E}-04$ & $5.045 \mathrm{E}-04$ & $3.854 \mathrm{E}-05$ & $3.021 \mathrm{E}-03$ & $1.625 \mathrm{E}-03$ & $1.084 \mathrm{E}-04$ & (3) \\
\hline NGC 4497 & 2004-03-12 & 1606 & NGA-Virgo-MOS05-0001 & $2.642 \mathrm{E}-04$ & $4.833 \mathrm{E}-04$ & $2.515 \mathrm{E}-05$ & $3.024 \mathrm{E}-03$ & $1.619 \mathrm{E}-03$ & $7.585 \mathrm{E}-05$ & (3) \\
\hline IC 3457 & $2004-03-12$ & 1585 & NGA-Virgo-MOS01-0001 & $2.114 \mathrm{E}-04$ & $3.725 \mathrm{E}-04$ & $2.605 \mathrm{E}-06$ & $2.965 \mathrm{E}-03$ & $1.565 \mathrm{E}-03$ & $4.714 \mathrm{E}-06$ & $(1)(3)$ \\
\hline IC 3459 & $2004-03-12$ & 1585 & NGA-Virgo-MOS01-0001 & $\ldots$ & $\ldots$ & $\ldots$ & $3.193 \mathrm{E}-03$ & $1.600 \mathrm{E}-03$ & $2.998 \mathrm{E}-04$ & $(4)(5)(3)$ \\
\hline NGC 4503 & 2004-03-12 & 1606 & NGA-Virgo-MOS05-0001 & $2.918 \mathrm{E}-04$ & $4.845 \mathrm{E}-04$ & $3.519 \mathrm{E}-05$ & $2.995 \mathrm{E}-03$ & $1.590 \mathrm{E}-03$ & $2.315 \mathrm{E}-05$ & (3) \\
\hline NGC 4506 & 2004-03-12 & 1582 & NGA-Virgo-MOS11-0001 & $2.279 \mathrm{E}-04$ & $4.294 \mathrm{E}-04$ & $1.075 \mathrm{E}-05$ & $2.922 \mathrm{E}-03$ & $1.610 \mathrm{E}-03$ & $8.680 \mathrm{E}-05$ & (3) \\
\hline IC 3467 & 2004-03-12 & 1606 & NGA-Virgo-MOS05-0001 & $2.520 \mathrm{E}-04$ & $4.631 \mathrm{E}-04$ & $1.698 \mathrm{E}-05$ & $3.136 \mathrm{E}-03$ & $1.624 \mathrm{E}-03$ & $1.607 \mathrm{E}-04$ & (3) \\
\hline UGC 07710 & 2004-04-14 & 1461 & MISDR1-13768-0334 & $2.697 \mathrm{E}-04$ & $4.738 \mathrm{E}-04$ & $6.226 \mathrm{E}-06$ & $3.109 \mathrm{E}-03$ & $1.726 \mathrm{E}-03$ & $5.542 \mathrm{E}-05$ & \\
\hline NGC 4528 & 2004-03-12 & 1606 & NGA-Virgo-MOS05-0001 & $2.462 \mathrm{E}-04$ & $3.975 \mathrm{E}-04$ & $3.397 \mathrm{E}-05$ & $2.999 \mathrm{E}-03$ & $1.532 \mathrm{E}-03$ & $1.051 \mathrm{E}-05$ & (3) \\
\hline NGC 4531 & 2004-03-12 & 1582 & NGA-Virgo-MOS11-0001 & $2.435 \mathrm{E}-04$ & 4.007E-04 & $2.865 \mathrm{E}-05$ & $2.923 \mathrm{E}-03$ & $1.502 \mathrm{E}-03$ & $1.261 \mathrm{E}-04$ & (3) \\
\hline
\end{tabular}


Table 2-Continued

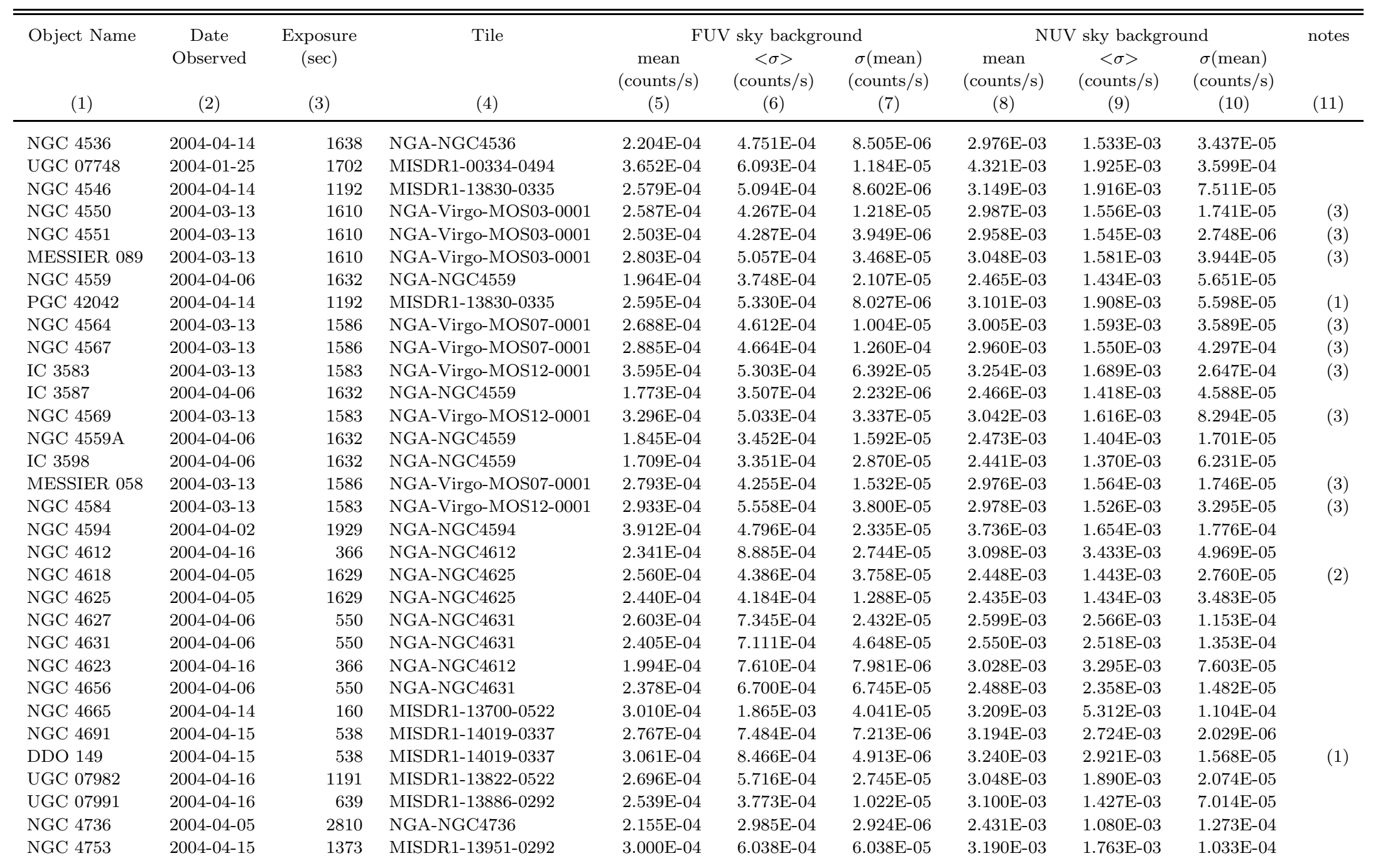


Table 2-Continued

\begin{tabular}{|c|c|c|c|c|c|c|c|c|c|c|}
\hline \multirow[b]{2}{*}{$\begin{array}{c}\text { Object Name } \\
\text { (1) }\end{array}$} & \multirow[b]{2}{*}{$\begin{array}{c}\text { Date } \\
\text { Observed } \\
(2)\end{array}$} & \multirow[b]{2}{*}{$\begin{array}{c}\text { Exposure } \\
(\mathrm{sec}) \\
(3)\end{array}$} & \multirow[b]{2}{*}{$\begin{array}{l}\text { Tile } \\
(4)\end{array}$} & \multicolumn{3}{|c|}{ FUV sky background } & \multicolumn{3}{|c|}{ NUV sky background } & \multirow{2}{*}{$\begin{array}{c}\text { notes } \\
\text { (11) }\end{array}$} \\
\hline & & & & $\begin{array}{c}\text { mean } \\
(\text { counts/s) } \\
(5)\end{array}$ & $\begin{array}{c}<\sigma> \\
(\text { counts } / \mathrm{s}) \\
(6)\end{array}$ & $\begin{array}{c}\sigma(\text { mean }) \\
(\text { counts } / \mathrm{s}) \\
(7)\end{array}$ & $\begin{array}{c}\text { mean } \\
(\text { counts/s) } \\
(8)\end{array}$ & $\begin{array}{c}<\sigma> \\
(\text { counts/s) } \\
(9)\end{array}$ & $\begin{array}{c}\sigma(\text { mean }) \\
(\text { counts } / \mathrm{s}) \\
(10)\end{array}$ & \\
\hline UGC 08013 & 2004-06-14 & 1451 & NGA-DDO154 & $2.776 \mathrm{E}-04$ & 4.431E-04 & $1.825 \mathrm{E}-06$ & $2.794 \mathrm{E}-03$ & $1.549 \mathrm{E}-03$ & $7.120 \mathrm{E}-05$ & \\
\hline NGC 4771 & 2004-04-16 & 1245 & MISDR1-13885-0523 & $1.981 \mathrm{E}-04$ & 4.077E-04 & $1.231 \mathrm{E}-06$ & $2.852 \mathrm{E}-03$ & $1.684 \mathrm{E}-03$ & $5.541 \mathrm{E}-05$ & \\
\hline NGC 4772 & 2004-04-16 & 1245 & MISDR1-13885-0523-0002 & $2.170 \mathrm{E}-04$ & $5.114 \mathrm{E}-04$ & $5.439 \mathrm{E}-06$ & $2.913 \mathrm{E}-03$ & $1.815 \mathrm{E}-03$ & $4.682 \mathrm{E}-05$ & \\
\hline DDO 154 & 2004-06-14 & 1451 & NGA-DDO154 & $2.976 \mathrm{E}-04$ & $5.018 \mathrm{E}-04$ & $1.410 \mathrm{E}-06$ & $2.793 \mathrm{E}-03$ & $1.630 \mathrm{E}-03$ & $1.104 \mathrm{E}-04$ & \\
\hline NGC 4787 & 2004-06-14 & 1451 & NGA-DDO154 & $2.945 \mathrm{E}-04$ & $4.898 \mathrm{E}-04$ & $1.188 \mathrm{E}-05$ & $2.800 \mathrm{E}-03$ & $1.626 \mathrm{E}-03$ & $3.628 \mathrm{E}-05$ & \\
\hline NGC 4789 & 2004-06-14 & 1451 & NGA-DDO154 & $2.953 \mathrm{E}-04$ & $4.978 \mathrm{E}-04$ & $2.153 \mathrm{E}-05$ & $2.776 \mathrm{E}-03$ & $1.628 \mathrm{E}-03$ & $1.121 \mathrm{E}-05$ & \\
\hline NGC 4809 & 2004-04-17 & 2537 & MISDR1-13884-0523 & $2.671 \mathrm{E}-04$ & $3.435 \mathrm{E}-04$ & $3.129 \mathrm{E}-05$ & $3.036 \mathrm{E}-03$ & $1.267 \mathrm{E}-03$ & $8.459 \mathrm{E}-05$ & (1) \\
\hline NGC 4797 & 2004-06-14 & 1451 & NGA-DDO154 & $3.183 \mathrm{E}-04$ & $5.786 \mathrm{E}-04$ & $2.862 \mathrm{E}-05$ & $2.797 \mathrm{E}-03$ & $1.627 \mathrm{E}-03$ & $1.890 \mathrm{E}-05$ & \\
\hline NGC 4799 & 2004-04-17 & 2537 & MISDR1-13884-0523 & $2.558 \mathrm{E}-04$ & 3.379E-04 & $1.553 \mathrm{E}-05$ & $3.036 \mathrm{E}-03$ & $1.267 \mathrm{E}-03$ & $2.643 \mathrm{E}-05$ & \\
\hline NGC 4807 & 2004-05-04 & 1350 & COMA-SPEC-A & $2.502 \mathrm{E}-04$ & $4.600 \mathrm{E}-04$ & $3.738 \mathrm{E}-06$ & $2.496 \mathrm{E}-03$ & $1.473 \mathrm{E}-03$ & $1.347 \mathrm{E}-06$ & $(1)(2)$ \\
\hline NGC 4816 & $2004-05-04$ & 1350 & COMA-SPEC-A & $2.937 \mathrm{E}-04$ & $5.512 \mathrm{E}-04$ & $2.586 \mathrm{E}-05$ & $2.543 \mathrm{E}-03$ & $1.533 \mathrm{E}-03$ & $1.245 \mathrm{E}-04$ & (1) \\
\hline NGC 4819 & 2004-05-04 & 1350 & COMA-SPEC-A & $2.280 \mathrm{E}-04$ & 4.357E-04 & $6.425 \mathrm{E}-06$ & $2.551 \mathrm{E}-03$ & $1.468 \mathrm{E}-03$ & $1.358 \mathrm{E}-04$ & \\
\hline NGC 4827 & 2004-05-04 & 1350 & COMA-SPEC-A & $2.298 \mathrm{E}-04$ & $4.320 \mathrm{E}-04$ & $1.300 \mathrm{E}-06$ & $2.521 \mathrm{E}-03$ & $1.493 \mathrm{E}-03$ & $1.011 \mathrm{E}-04$ & \\
\hline MESSIER 064 & 2004-05-04 & 1340 & WDST-GD-153 & $3.031 \mathrm{E}-04$ & $5.782 \mathrm{E}-04$ & $1.282 \mathrm{E}-05$ & $2.888 \mathrm{E}-03$ & $1.678 \mathrm{E}-03$ & $7.150 \mathrm{E}-05$ & \\
\hline NGC 4861 & 2004-04-05 & 102 & NGA-NGC4861 & $2.310 \mathrm{E}-04$ & $1.615 \mathrm{E}-03$ & $3.394 \mathrm{E}-05$ & $2.604 \mathrm{E}-03$ & $5.879 \mathrm{E}-03$ & $1.523 \mathrm{E}-04$ & \\
\hline IC 0842 & 2004-04-08 & 1976 & SA57-01 & $2.001 \mathrm{E}-04$ & $4.034 \mathrm{E}-04$ & $8.831 \mathrm{E}-06$ & $2.348 \mathrm{E}-03$ & $1.221 \mathrm{E}-03$ & $4.269 \mathrm{E}-06$ & \\
\hline UGC 08127 & 2004-04-17 & 1703 & MISDR1-14148-0338 & $2.528 \mathrm{E}-04$ & $4.213 \mathrm{E}-04$ & $7.253 \mathrm{E}-06$ & $3.257 \mathrm{E}-03$ & $1.563 \mathrm{E}-03$ & $3.721 \mathrm{E}-05$ & \\
\hline NGC 4922 & 2004-04-08 & 1976 & SA57-01 & $1.959 \mathrm{E}-04$ & $3.695 \mathrm{E}-04$ & $6.863 \mathrm{E}-06$ & $2.365 \mathrm{E}-03$ & $1.223 \mathrm{E}-03$ & $4.372 \mathrm{E}-05$ & \\
\hline IC 0843 & 2004-04-08 & 1976 & SA57-01 & $1.958 \mathrm{E}-04$ & $3.620 \mathrm{E}-04$ & $9.084 \mathrm{E}-06$ & $2.389 \mathrm{E}-03$ & $1.258 \mathrm{E}-03$ & $1.911 \mathrm{E}-05$ & \\
\hline IC 4088 & 2004-04-08 & 1976 & SA57-01 & $1.951 \mathrm{E}-04$ & $3.580 \mathrm{E}-04$ & $1.827 \mathrm{E}-05$ & $2.383 \mathrm{E}-03$ & $1.263 \mathrm{E}-03$ & $1.892 \mathrm{E}-05$ & \\
\hline NGC 4952 & 2004-04-08 & 1976 & SA57-01 & $1.882 \mathrm{E}-04$ & $3.121 \mathrm{E}-04$ & $1.338 \mathrm{E}-05$ & $2.348 \mathrm{E}-03$ & $1.205 \mathrm{E}-03$ & $6.227 \mathrm{E}-05$ & \\
\hline UGC 08195 & 2004-04-01 & 3071 & SA57-00 & $1.686 \mathrm{E}-04$ & $2.692 \mathrm{E}-04$ & $3.289 \mathrm{E}-06$ & $2.254 \mathrm{E}-03$ & $9.900 \mathrm{E}-04$ & $4.208 \mathrm{E}-05$ & \\
\hline DDO 165 & 2004-01-26 & 1704 & NGA-DDO165 & $3.168 \mathrm{E}-04$ & 4.961E-04 & 3.437E-05 & $2.457 \mathrm{E}-03$ & $1.422 \mathrm{E}-03$ & $1.052 \mathrm{E}-04$ & \\
\hline NGC 5004 & $2004-03-31$ & 5449 & SA57-02 & $1.421 \mathrm{E}-04$ & $1.652 \mathrm{E}-04$ & $1.793 \mathrm{E}-06$ & $2.262 \mathrm{E}-03$ & $7.043 \mathrm{E}-04$ & $5.649 \mathrm{E}-05$ & \\
\hline NGC $5004 \mathrm{C}$ & $2004-03-31$ & 5449 & SA57-02 & $1.416 \mathrm{E}-04$ & $1.648 \mathrm{E}-04$ & $2.053 \mathrm{E}-08$ & $2.256 \mathrm{E}-03$ & $7.010 \mathrm{E}-04$ & $5.145 \mathrm{E}-05$ & \\
\hline UGC 08313 & 2004-03-31 & 1660 & NGA-NGC5055 & $2.160 \mathrm{E}-04$ & $4.462 \mathrm{E}-04$ & 7.665E-06 & $2.225 \mathrm{E}-03$ & $1.332 \mathrm{E}-03$ & $2.684 \mathrm{E}-06$ & \\
\hline UGCA 342 & 2004-03-31 & 1660 & NGA-NGC5055 & $2.428 \mathrm{E}-04$ & $4.199 \mathrm{E}-04$ & $1.855 \mathrm{E}-05$ & $2.418 \mathrm{E}-03$ & $1.412 \mathrm{E}-03$ & $1.588 \mathrm{E}-04$ & \\
\hline NGC 5055 & 2004-03-31 & 1660 & NGA-NGC5055 & $2.242 \mathrm{E}-04$ & $3.953 \mathrm{E}-04$ & $4.674 \mathrm{E}-06$ & $2.379 \mathrm{E}-03$ & $1.387 \mathrm{E}-03$ & $3.401 \mathrm{E}-05$ & \\
\hline UGC 08340 & 2004-05-08 & 1509 & MISDR1-33984-0340 & $3.413 \mathrm{E}-04$ & $5.258 \mathrm{E}-04$ & $1.200 \mathrm{E}-05$ & 3.363E-03 & $1.759 \mathrm{E}-03$ & $1.413 \mathrm{E}-04$ & \\
\hline IC 4218 & 2004-05-08 & 1509 & MISDR1-33984-0340 & $3.403 \mathrm{E}-04$ & $5.497 \mathrm{E}-04$ & $3.273 \mathrm{E}-06$ & $3.299 \mathrm{E}-03$ & $1.756 \mathrm{E}-03$ & $5.499 \mathrm{E}-05$ & \\
\hline
\end{tabular}


Table 2-Continued

\begin{tabular}{|c|c|c|c|c|c|c|c|c|c|c|}
\hline \multirow{2}{*}{$\begin{array}{l}\text { Object Name } \\
\text { (1) }\end{array}$} & \multirow{2}{*}{$\begin{array}{c}\text { Date } \\
\text { Observed } \\
(2)\end{array}$} & \multirow{2}{*}{$\begin{array}{l}\text { Exposure } \\
\quad(\mathrm{sec}) \\
(3)\end{array}$} & \multirow{2}{*}{$\begin{array}{l}\text { Tile } \\
(4)\end{array}$} & \multicolumn{3}{|c|}{ FUV sky background } & \multicolumn{3}{|c|}{ NUV sky background } & \multirow{2}{*}{$\begin{array}{l}\text { notes } \\
\text { (11) }\end{array}$} \\
\hline & & & & $\begin{array}{c}\text { mean } \\
(\text { counts/s) } \\
(5)\end{array}$ & $\begin{array}{c}<\sigma> \\
(\text { counts/s) } \\
(6)\end{array}$ & $\begin{array}{c}\sigma(\text { mean }) \\
(\text { counts/s) } \\
(7)\end{array}$ & $\begin{array}{c}\text { mean } \\
(\text { counts/s) } \\
(8)\end{array}$ & $\begin{array}{c}<\sigma> \\
(\text { counts } / \mathrm{s}) \\
(9)\end{array}$ & $\begin{array}{c}\sigma(\text { mean }) \\
(\text { counts/s) } \\
(10)\end{array}$ & \\
\hline UGC 08365 & 2004-03-31 & 1660 & NGA-NGC5055 & $1.865 \mathrm{E}-04$ & $3.419 \mathrm{E}-04$ & $1.253 \mathrm{E}-06$ & $2.224 \mathrm{E}-03$ & $1.263 \mathrm{E}-03$ & $3.877 \mathrm{E}-05$ & \\
\hline IC 4229 & 2004-05-09 & 1544 & MISDR1-33982-0341 & $3.515 \mathrm{E}-04$ & $6.105 \mathrm{E}-04$ & $2.859 \mathrm{E}-05$ & $3.284 \mathrm{E}-03$ & $1.656 \mathrm{E}-03$ & $5.598 \mathrm{E}-05$ & \\
\hline Centaurus A & 2003-06-07 & 937 & NGA-Centaurus-A-581-F5-19-158 & $1.510 \mathrm{E}-03$ & $1.397 \mathrm{E}-03$ & $2.584 \mathrm{E}-05$ & $7.045 \mathrm{E}-03$ & $3.446 \mathrm{E}-03$ & $1.364 \mathrm{E}-04$ & \\
\hline NGC 5169 & 2003-06-19 & 1412 & NGA-M51 & $4.750 \mathrm{E}-04$ & $7.289 \mathrm{E}-04$ & $3.517 \mathrm{E}-05$ & $2.847 \mathrm{E}-03$ & $1.635 \mathrm{E}-03$ & $4.885 \mathrm{E}-05$ & \\
\hline NGC 5173 & 2003-06-19 & 1412 & NGA-M51 & $4.588 \mathrm{E}-04$ & $7.298 \mathrm{E}-04$ & $3.048 \mathrm{E}-05$ & $2.830 \mathrm{E}-03$ & $1.618 \mathrm{E}-03$ & $1.658 \mathrm{E}-06$ & \\
\hline IC 4263 & 2003-06-19 & 1412 & NGA-M51 & $4.155 \mathrm{E}-04$ & $6.172 \mathrm{E}-04$ & $3.080 \mathrm{E}-05$ & $2.776 \mathrm{E}-03$ & $1.676 \mathrm{E}-03$ & $4.180 \mathrm{E}-05$ & \\
\hline MESSIER 051a & 2003-06-19 & 1412 & NGA-M51 & $4.698 \mathrm{E}-04$ & $6.765 \mathrm{E}-04$ & $6.871 \mathrm{E}-05$ & $2.895 \mathrm{E}-03$ & $1.692 \mathrm{E}-03$ & $2.478 \mathrm{E}-05$ & \\
\hline MESSIER 051b & 2003-06-19 & 1412 & NGA-M51 & $4.976 \mathrm{E}-04$ & $6.905 \mathrm{E}-04$ & $4.683 \mathrm{E}-05$ & $2.947 \mathrm{E}-03$ & $1.700 \mathrm{E}-03$ & $1.841 \mathrm{E}-04$ & \\
\hline NGC 5231 & 2004-04-02 & 1640 & MISDR1-33751-0528 & $2.065 \mathrm{E}-04$ & $4.449 \mathrm{E}-04$ & $2.466 \mathrm{E}-06$ & $3.043 \mathrm{E}-03$ & $1.573 \mathrm{E}-03$ & $1.256 \mathrm{E}-04$ & \\
\hline ESO 444-G077 & 2003-06-07 & 1352 & NGA-M83-582-F5-19-158 & $6.477 \mathrm{E}-04$ & $8.029 \mathrm{E}-04$ & $8.970 \mathrm{E}-07$ & $4.236 \mathrm{E}-03$ & $2.198 \mathrm{E}-03$ & $1.883 \mathrm{E}-05$ & \\
\hline MESSIER 083 & $2003-06-07$ & 1352 & NGA-M83-582-F5-19-158 & $7.012 \mathrm{E}-04$ & $7.408 \mathrm{E}-04$ & $3.214 \mathrm{E}-05$ & $4.418 \mathrm{E}-03$ & $2.124 \mathrm{E}-03$ & $2.552 \mathrm{E}-05$ & \\
\hline ESO $444-G 087$ & $2004-05-22$ & 538 & NGA-NGC5253 & $7.355 \mathrm{E}-04$ & $1.473 \mathrm{E}-03$ & $4.730 \mathrm{E}-05$ & $4.336 \mathrm{E}-03$ & $3.304 \mathrm{E}-03$ & $1.038 \mathrm{E}-04$ & \\
\hline NGC 5253 & $2004-05-22$ & 538 & NGA-NGC5253 & 7.469E-04 & $1.262 \mathrm{E}-03$ & $1.000 \mathrm{E}-05$ & 4.469E-03 & $3.482 \mathrm{E}-03$ & $1.035 \mathrm{E}-04$ & \\
\hline UGC 08650 & 2004-05-10 & 1498 & MISDR1-33780-0528 & $2.439 \mathrm{E}-04$ & $4.502 \mathrm{E}-04$ & $2.250 \mathrm{E}-06$ & $2.851 \mathrm{E}-03$ & $1.635 \mathrm{E}-03$ & $1.996 \mathrm{E}-05$ & (2) \\
\hline ESO 445-G007 & $2004-05-22$ & 538 & NGA-NGC5253 & $7.599 \mathrm{E}-04$ & $1.295 \mathrm{E}-03$ & $7.415 \mathrm{E}-06$ & $4.478 \mathrm{E}-03$ & $3.480 \mathrm{E}-03$ & $6.549 \mathrm{E}-05$ & (2) \\
\hline NGC 5329 & 2004-05-13 & 3020 & MISDR1-33777-0530 & $2.887 \mathrm{E}-04$ & 4.653E-04 & $1.176 \mathrm{E}-05$ & $2.974 \mathrm{E}-03$ & $1.130 \mathrm{E}-03$ & $1.987 \mathrm{E}-05$ & \\
\hline UGC 08787 & 2004-05-13 & 3020 & MISDR1-33777-0530 & $3.152 \mathrm{E}-04$ & $5.153 \mathrm{E}-04$ & $2.254 \mathrm{E}-05$ & 2.993E-03 & $1.147 \mathrm{E}-03$ & $5.899 \mathrm{E}-05$ & \\
\hline IC 0952 & 2004-04-02 & 1635 & MISDR1-33719-0530 & $1.972 \mathrm{E}-04$ & $3.785 \mathrm{E}-04$ & $1.504 \mathrm{E}-05$ & $2.832 \mathrm{E}-03$ & $1.490 \mathrm{E}-03$ & $5.801 \mathrm{E}-05$ & \\
\hline UGC 08816 & 2004-04-02 & 1635 & MISDR1-33719-0530 & 2.131E-04 & $4.609 \mathrm{E}-04$ & $1.690 \mathrm{E}-05$ & $2.829 \mathrm{E}-03$ & $1.491 \mathrm{E}-03$ & $9.180 \mathrm{E}-05$ & \\
\hline NGC 5398 & 2003-06-07 & 553 & NGA-NGC5398-580-F5-19-158 & $1.079 \mathrm{E}-03$ & $1.712 \mathrm{E}-03$ & $6.581 \mathrm{E}-06$ & $5.638 \mathrm{E}-03$ & $3.979 \mathrm{E}-03$ & $1.117 \mathrm{E}-04$ & \\
\hline MESSIER 101 & $2003-06-20$ & 1039 & NGA-M101 & $3.893 \mathrm{E}-04$ & $6.859 \mathrm{E}-04$ & $6.998 \mathrm{E}-06$ & $2.789 \mathrm{E}-03$ & $1.950 \mathrm{E}-03$ & $1.346 \mathrm{E}-05$ & \\
\hline ESO $446-G 002$ & $2003-06-07$ & 553 & NGA-NGC5398-580-F5-19-158 & $1.042 \mathrm{E}-03$ & $1.431 \mathrm{E}-03$ & $5.716 \mathrm{E}-05$ & $5.825 \mathrm{E}-03$ & $3.881 \mathrm{E}-03$ & $1.866 \mathrm{E}-06$ & \\
\hline UGC 08986 & $2004-05-15$ & 1684 & MISDR1-33716-0582 & $2.314 \mathrm{E}-04$ & $3.753 \mathrm{E}-04$ & $5.367 \mathrm{E}-06$ & $2.850 \mathrm{E}-03$ & $1.457 \mathrm{E}-03$ & $3.257 \mathrm{E}-05$ & \\
\hline NGC 5474 & 2003-06-19 & 1610 & NGA-NGC5474 & $3.336 \mathrm{E}-04$ & $4.863 \mathrm{E}-04$ & $6.514 \mathrm{E}-06$ & $2.628 \mathrm{E}-03$ & $1.501 \mathrm{E}-03$ & $1.499 \mathrm{E}-04$ & \\
\hline NGC 5477 & $2003-06-20$ & 1039 & NGA-M101 & $4.421 \mathrm{E}-04$ & $6.804 \mathrm{E}-04$ & 3.607E-04 & $2.955 \mathrm{E}-03$ & $1.978 \mathrm{E}-03$ & $1.173 \mathrm{E}-03$ & \\
\hline UGC 09120 & $2004-06-07$ & 2557 & MISDR1-33686-0583 & $2.886 \mathrm{E}-04$ & $3.496 \mathrm{E}-04$ & $3.972 \mathrm{E}-06$ & $2.901 \mathrm{E}-03$ & $1.214 \mathrm{E}-03$ & $1.115 \mathrm{E}-04$ & \\
\hline UGC 09140 & 2004-05-19 & 662 & MISDR1-33802-0303 & $3.185 \mathrm{E}-04$ & $8.073 \mathrm{E}-04$ & $5.004 \mathrm{E}-06$ & $3.126 \mathrm{E}-03$ & $2.577 \mathrm{E}-03$ & $3.973 \mathrm{E}-05$ & \\
\hline NGC 5560 & 2004-06-08 & 2515 & MISDR1-33712-0584 & $3.035 \mathrm{E}-04$ & $3.718 \mathrm{E}-04$ & $2.347 \mathrm{E}-05$ & $2.948 \mathrm{E}-03$ & $1.249 \mathrm{E}-03$ & $2.026 \mathrm{E}-05$ & \\
\hline NGC 5566 & 2004-06-08 & 2515 & MISDR1-33712-0584 & $2.905 \mathrm{E}-04$ & $3.553 \mathrm{E}-04$ & $1.107 \mathrm{E}-05$ & $2.927 \mathrm{E}-03$ & $1.233 \mathrm{E}-03$ & $8.838 \mathrm{E}-06$ & \\
\hline NGC 5569 & 2004-06-08 & 2515 & MISDR1-33712-0584 & $2.965 \mathrm{E}-04$ & $3.665 \mathrm{E}-04$ & $6.986 \mathrm{E}-06$ & $2.934 \mathrm{E}-03$ & $1.244 \mathrm{E}-03$ & $9.248 \mathrm{E}-06$ & \\
\hline NGC 5574 & $2004-05-20$ & 1706 & MISDR1-33741-0533 & $2.663 \mathrm{E}-04$ & $4.050 \mathrm{E}-04$ & $8.856 \mathrm{E}-06$ & $2.911 \mathrm{E}-03$ & $1.458 \mathrm{E}-03$ & $5.825 \mathrm{E}-05$ & \\
\hline NGC 5576 & $2004-05-20$ & 1706 & MISDR1-33741-0533 & $2.654 \mathrm{E}-04$ & $4.068 \mathrm{E}-04$ & $1.595 \mathrm{E}-05$ & $2.912 \mathrm{E}-03$ & $1.451 \mathrm{E}-03$ & $2.523 \mathrm{E}-05$ & \\
\hline
\end{tabular}


Table 2-Continued

\begin{tabular}{|c|c|c|c|c|c|c|c|c|c|c|}
\hline \multirow{2}{*}{$\begin{array}{l}\text { Object Name } \\
\text { (1) }\end{array}$} & \multirow{2}{*}{$\begin{array}{c}\text { Date } \\
\text { Observed } \\
(2)\end{array}$} & \multirow{2}{*}{$\begin{array}{l}\text { Exposure } \\
\quad(\mathrm{sec}) \\
(3)\end{array}$} & \multirow{2}{*}{$\begin{array}{l}\text { Tile } \\
\text { (4) }\end{array}$} & \multicolumn{3}{|c|}{ FUV sky background } & \multicolumn{3}{|c|}{ NUV sky background } & \multirow{2}{*}{$\begin{array}{l}\text { notes } \\
\text { (11) }\end{array}$} \\
\hline & & & & $\begin{array}{c}\text { mean } \\
(\text { counts/s) } \\
(5)\end{array}$ & $\begin{array}{c}<\sigma> \\
(\text { counts } / \mathrm{s}) \\
(6)\end{array}$ & $\begin{array}{c}\sigma(\text { mean }) \\
(\text { counts/s) } \\
(7)\end{array}$ & $\begin{array}{c}\text { mean } \\
(\text { counts/s) } \\
(8)\end{array}$ & $\begin{array}{c}<\sigma> \\
(\text { counts } / \mathrm{s}) \\
(9)\end{array}$ & $\begin{array}{c}\sigma(\text { mean }) \\
(\text { counts/s) } \\
(10)\end{array}$ & \\
\hline NGC 5577 & 2004-06-08 & 2515 & MISDR1-33712-0584 & $3.036 \mathrm{E}-04$ & $3.518 \mathrm{E}-04$ & $1.627 \mathrm{E}-05$ & $2.997 \mathrm{E}-03$ & $1.228 \mathrm{E}-03$ & $1.626 \mathrm{E}-04$ & \\
\hline UGC 09215 & 2004-06-03 & 1704 & MISDR1-33770-0534 & $3.249 \mathrm{E}-04$ & $5.502 \mathrm{E}-04$ & $1.942 \mathrm{E}-05$ & $3.029 \mathrm{E}-03$ & $1.536 \mathrm{E}-03$ & $5.557 \mathrm{E}-06$ & \\
\hline NGC 5619 & 2004-06-11 & 1581 & MISDR1-33683-0584 & $2.804 \mathrm{E}-04$ & $4.717 \mathrm{E}-04$ & $7.765 \mathrm{E}-06$ & $2.838 \mathrm{E}-03$ & $1.578 \mathrm{E}-03$ & $1.995 \mathrm{E}-05$ & \\
\hline UGC 09277 & 2004-05-22 & 1704 & MISDR1-33739-0535 & $2.466 \mathrm{E}-04$ & $4.006 \mathrm{E}-04$ & $1.417 \mathrm{E}-05$ & $2.807 \mathrm{E}-03$ & $1.471 \mathrm{E}-03$ & $5.484 \mathrm{E}-05$ & \\
\hline UGC 09285 & 2004-05-22 & 1704 & MISDR1-33739-0535 & $2.468 \mathrm{E}-04$ & $3.876 \mathrm{E}-04$ & 3.663E-06 & $2.822 \mathrm{E}-03$ & $1.453 \mathrm{E}-03$ & $2.620 \mathrm{E}-05$ & \\
\hline NGC 5646 & 2004-03-26 & 8654 & NGPDWS-01 & $2.742 \mathrm{E}-04$ & $1.989 \mathrm{E}-04$ & $3.075 \mathrm{E}-06$ & $2.448 \mathrm{E}-03$ & $6.046 \mathrm{E}-04$ & $1.164 \mathrm{E}-05$ & \\
\hline NGC 5636 & 2004-05-22 & 1703 & MISDR1-33710-0585 & $2.764 \mathrm{E}-04$ & $5.157 \mathrm{E}-04$ & $3.969 \mathrm{E}-06$ & $2.827 \mathrm{E}-03$ & $1.439 \mathrm{E}-03$ & $3.987 \mathrm{E}-05$ & \\
\hline NGC 5638 & $2004-05-22$ & 1704 & MISDR1-33739-0535 & $2.531 \mathrm{E}-04$ & $3.952 \mathrm{E}-04$ & $1.432 \mathrm{E}-05$ & $2.839 \mathrm{E}-03$ & $1.429 \mathrm{E}-03$ & $5.738 \mathrm{E}-05$ & \\
\hline UGC 09305 & 2004-05-22 & 1703 & MISDR1-33710-0585 & $2.358 \mathrm{E}-04$ & $3.796 \mathrm{E}-04$ & $1.044 \mathrm{E}-05$ & $2.716 \mathrm{E}-03$ & $1.430 \mathrm{E}-03$ & $4.711 \mathrm{E}-05$ & \\
\hline UGC 09310 & 2004-05-22 & 1703 & MISDR1-33710-0585 & $2.791 \mathrm{E}-04$ & $5.120 \mathrm{E}-04$ & $3.552 \mathrm{E}-05$ & $2.827 \mathrm{E}-03$ & $1.447 \mathrm{E}-03$ & $1.214 \mathrm{E}-04$ & \\
\hline IC 1022 & $2004-05-22$ & 1703 & MISDR1-33710-0585 & $2.418 \mathrm{E}-04$ & $3.923 \mathrm{E}-04$ & $1.689 \mathrm{E}-05$ & $2.750 \mathrm{E}-03$ & $1.472 \mathrm{E}-03$ & $6.704 \mathrm{E}-05$ & \\
\hline NGC 5656 & $2004-03-26$ & 8654 & NGPDWS-01 & $2.746 \mathrm{E}-04$ & $1.935 \mathrm{E}-04$ & $6.683 \mathrm{E}-06$ & $2.456 \mathrm{E}-03$ & $6.150 \mathrm{E}-04$ & $9.844 \mathrm{E}-06$ & \\
\hline UGC 09338 & 2004-06-11 & 1577 & MISDR1-33656-0585 & $2.841 \mathrm{E}-04$ & $4.965 \mathrm{E}-04$ & $1.021 \mathrm{E}-05$ & $2.776 \mathrm{E}-03$ & $1.552 \mathrm{E}-03$ & $5.114 \mathrm{E}-07$ & \\
\hline IC 1024 & 2004-05-23 & 1701 & MISDR1-33738-0535 & $2.550 \mathrm{E}-04$ & $4.302 \mathrm{E}-04$ & $7.820 \mathrm{E}-06$ & $2.779 \mathrm{E}-03$ & $1.475 \mathrm{E}-03$ & $1.308 \mathrm{E}-06$ & \\
\hline UGC 09380 & 2004-05-24 & 1451 & MISDR1-33681-0586 & $2.686 \mathrm{E}-04$ & $5.565 \mathrm{E}-04$ & $1.088 \mathrm{E}-06$ & $2.724 \mathrm{E}-03$ & $1.530 \mathrm{E}-03$ & $2.847 \mathrm{E}-05$ & \\
\hline UGC 09382 & $2004-05-23$ & 1701 & MISDR1-33709-0585 & $2.849 \mathrm{E}-04$ & $4.477 \mathrm{E}-04$ & $1.874 \mathrm{E}-05$ & $2.846 \mathrm{E}-03$ & $1.520 \mathrm{E}-03$ & $7.165 \mathrm{E}-05$ & \\
\hline UGC 09432 & 2004-06-06 & 2322 & MISDR1-33736-0536 & 3.099E-04 & $4.011 \mathrm{E}-04$ & $1.549 \mathrm{E}-05$ & 3.037E-03 & $1.296 \mathrm{E}-03$ & $5.669 \mathrm{E}-05$ & (1) \\
\hline NGC 5701 & 2004-05-24 & 1700 & MISDR1-33654-0586 & $3.033 \mathrm{E}-04$ & $4.599 \mathrm{E}-04$ & $3.446 \mathrm{E}-05$ & $2.789 \mathrm{E}-03$ & $1.498 \mathrm{E}-03$ & $1.316 \mathrm{E}-05$ & \\
\hline NGC 5705 & 2003-06-21 & 844 & NGA-NGC5713 & $3.682 \mathrm{E}-04$ & $6.774 \mathrm{E}-04$ & $2.860 \mathrm{E}-05$ & $3.350 \mathrm{E}-03$ & $2.228 \mathrm{E}-03$ & $7.089 \mathrm{E}-05$ & \\
\hline NGC 5713 & 2003-06-21 & 844 & NGA-NGC5713 & $3.734 \mathrm{E}-04$ & $7.044 \mathrm{E}-04$ & $2.567 \mathrm{E}-05$ & $3.354 \mathrm{E}-03$ & $2.355 \mathrm{E}-03$ & $5.281 \mathrm{E}-05$ & \\
\hline NGC 5727 & 2004-06-01 & 961 & NGPDWS-11 & $2.160 \mathrm{E}-04$ & $4.921 \mathrm{E}-04$ & $9.563 \mathrm{E}-06$ & $2.117 \mathrm{E}-03$ & $1.660 \mathrm{E}-03$ & $3.631 \mathrm{E}-06$ & \\
\hline NGC 5719 & $2003-06-21$ & 844 & NGA-NGC5713 & $3.898 \mathrm{E}-04$ & $7.549 \mathrm{E}-04$ & $1.989 \mathrm{E}-06$ & $3.385 \mathrm{E}-03$ & $2.348 \mathrm{E}-03$ & $1.685 \mathrm{E}-05$ & \\
\hline UGC 09463 & 2004-06-06 & 2322 & MISDR1-33736-0536 & $3.092 \mathrm{E}-04$ & 3.709E-04 & $1.470 \mathrm{E}-06$ & $3.029 \mathrm{E}-03$ & $1.294 \mathrm{E}-03$ & $6.873 \mathrm{E}-05$ & \\
\hline UGC 09479 & $2004-05-25$ & 1700 & MISDR1-33680-0586 & $2.878 \mathrm{E}-04$ & $5.232 \mathrm{E}-04$ & $4.256 \mathrm{E}-06$ & $2.762 \mathrm{E}-03$ & $1.502 \mathrm{E}-03$ & $6.361 \mathrm{E}-06$ & \\
\hline UGC 09491 & 2004-05-26 & 1317 & MISDR1-33679-0587 & $2.619 \mathrm{E}-04$ & $5.139 \mathrm{E}-04$ & $2.568 \mathrm{E}-05$ & $2.767 \mathrm{E}-03$ & $1.677 \mathrm{E}-03$ & $9.235 \mathrm{E}-05$ & \\
\hline IC 1063 & $2004-05-28$ & 3668 & MISDR1-33677-0588 & $3.247 \mathrm{E}-04$ & $3.734 \mathrm{E}-04$ & $5.402 \mathrm{E}-06$ & $2.876 \mathrm{E}-03$ & $9.962 \mathrm{E}-04$ & $4.208 \mathrm{E}-06$ & \\
\hline NGC 5770 & $2004-05-28$ & 3668 & MISDR1-33677-0588 & $3.543 \mathrm{E}-04$ & $4.821 \mathrm{E}-04$ & $2.223 \mathrm{E}-05$ & $2.929 \mathrm{E}-03$ & $1.021 \mathrm{E}-03$ & 5.313E-05 & \\
\hline IC 1071 & 2004-05-28 & 3668 & MISDR1-33677-0588 & 3.313E-04 & 3.963E-04 & $1.610 \mathrm{E}-05$ & $2.888 \mathrm{E}-03$ & $1.019 \mathrm{E}-03$ & $4.248 \mathrm{E}-05$ & \\
\hline UGC 09584 & $2004-05-28$ & 3668 & MISDR1-33677-0588 & $3.480 \mathrm{E}-04$ & $4.226 \mathrm{E}-04$ & $1.547 \mathrm{E}-06$ & $2.925 \mathrm{E}-03$ & $1.044 \mathrm{E}-03$ & $2.554 \mathrm{E}-05$ & \\
\hline NGC 5832 & 2005-04-29 & 1246 & SA-P041C & $\cdots$ & $\cdots$ & $\cdots$ & $2.429 \mathrm{E}-03$ & $1.579 \mathrm{E}-03$ & $5.211 \mathrm{E}-06$ & (4) \\
\hline NGC 5806 & $2004-05-26$ & 695 & NGA-NGC5813 & $3.340 \mathrm{E}-04$ & 7.073E-04 & $2.074 \mathrm{E}-06$ & $3.059 \mathrm{E}-03$ & $2.379 \mathrm{E}-03$ & $5.195 \mathrm{E}-07$ & \\
\hline NGC 5813 & $2004-05-26$ & 695 & NGA-NGC5813 & $3.606 \mathrm{E}-04$ & $7.654 \mathrm{E}-04$ & $3.357 \mathrm{E}-05$ & $3.147 \mathrm{E}-03$ & $2.509 \mathrm{E}-03$ & $1.069 \mathrm{E}-04$ & \\
\hline
\end{tabular}


Table 2-Continued

\begin{tabular}{|c|c|c|c|c|c|c|c|c|c|c|}
\hline \multirow{2}{*}{$\begin{array}{c}\text { Object Name } \\
\text { (1) }\end{array}$} & \multirow{2}{*}{$\begin{array}{c}\text { Date } \\
\text { Observed } \\
(2)\end{array}$} & \multirow{2}{*}{$\begin{array}{l}\text { Exposure } \\
\quad(\mathrm{sec}) \\
(3)\end{array}$} & \multirow{2}{*}{$\begin{array}{l}\text { Tile } \\
\text { (4) }\end{array}$} & \multicolumn{3}{|c|}{ FUV sky background } & \multicolumn{3}{|c|}{ NUV sky background } & \multirow{2}{*}{$\begin{array}{l}\text { notes } \\
(11)\end{array}$} \\
\hline & & & & $\begin{array}{c}\text { mean } \\
(\text { counts/s) } \\
(5)\end{array}$ & $\begin{array}{c}<\sigma> \\
(\text { counts/s) } \\
(6)\end{array}$ & $\begin{array}{c}\sigma(\text { mean }) \\
(\text { counts/s) } \\
(7)\end{array}$ & $\begin{array}{c}\text { mean } \\
(\text { counts/s) } \\
(8)\end{array}$ & $\begin{array}{c}<\sigma> \\
(\text { counts } / \mathrm{s}) \\
(9)\end{array}$ & $\begin{array}{c}\sigma(\text { mean }) \\
(\text { counts/s) } \\
(10)\end{array}$ & \\
\hline UGC 09661 & 2004-05-26 & 695 & NGA-NGC5813 & $3.604 \mathrm{E}-04$ & $7.897 \mathrm{E}-04$ & $1.566 \mathrm{E}-05$ & $3.079 \mathrm{E}-03$ & $2.470 \mathrm{E}-03$ & $1.759 \mathrm{E}-04$ & (1) \\
\hline NGC 5866 & 2003-06-21 & 1515 & NGA-NGC5866 & $3.301 \mathrm{E}-04$ & $4.977 \mathrm{E}-04$ & $1.112 \mathrm{E}-05$ & $2.637 \mathrm{E}-03$ & $1.542 \mathrm{E}-03$ & $3.967 \mathrm{E}-05$ & \\
\hline NGC 5826 & 2003-06-21 & 1515 & NGA-NGC5866 & $3.535 \mathrm{E}-04$ & $5.677 \mathrm{E}-04$ & $1.484 \mathrm{E}-06$ & $2.611 \mathrm{E}-03$ & $1.555 \mathrm{E}-03$ & $1.378 \mathrm{E}-05$ & \\
\hline IC 1102 & $2004-05-27$ & 539 & MISDR1-33673-0590 & $3.497 \mathrm{E}-04$ & $9.453 \mathrm{E}-04$ & $2.792 \mathrm{E}-05$ & $2.872 \mathrm{E}-03$ & $2.743 \mathrm{E}-03$ & $1.162 \mathrm{E}-06$ & \\
\hline NGC 5894 & 2004-06-24 & 1165 & MISDR1-10109-0611 & 3.663E-04 & $6.660 \mathrm{E}-04$ & $1.832 \mathrm{E}-05$ & $2.663 \mathrm{E}-03$ & $1.772 \mathrm{E}-03$ & $5.113 \mathrm{E}-05$ & \\
\hline IRAS $15250+3609$ & 2004-05-29 & 1590 & NGA-IRAS15250 & $\cdots$ & $\cdots$ & $\cdots$ & $2.209 \mathrm{E}-03$ & $1.369 \mathrm{E}-03$ & $5.694 \mathrm{E}-05$ & (4) \\
\hline UGC 09912 & 2004-05-29 & 1696 & NGA-NGC5962 & $\cdots$ & $\ldots$ & $\ldots$ & $2.805 \mathrm{E}-03$ & $1.513 \mathrm{E}-03$ & $1.228 \mathrm{E}-05$ & (4) \\
\hline NGC 5962 & 2004-05-29 & 1696 & NGA-NGC5962 & $\cdots$ & $\cdots$ & $\cdots$ & $2.890 \mathrm{E}-03$ & $1.545 \mathrm{E}-03$ & $4.960 \mathrm{E}-05$ & (4) \\
\hline UGC 09925 & 2004-05-29 & 1696 & NGA-NGC5962 & $\cdots$ & $\ldots$ & $\ldots$ & 2.903E-03 & $1.537 \mathrm{E}-03$ & $1.036 \mathrm{E}-05$ & (4) \\
\hline NGC 5972 & 2004-05-29 & 1696 & NGA-NGC5962 & $\ldots$ & $\ldots$ & $\ldots$ & $2.809 \mathrm{E}-03$ & $1.412 \mathrm{E}-03$ & $7.670 \mathrm{E}-05$ & (4) \\
\hline UGC 09953 & 2004-06-13 & 1501 & MISDR1-21946-0593 & $5.712 \mathrm{E}-04$ & $8.057 \mathrm{E}-04$ & $1.614 \mathrm{E}-05$ & $3.389 \mathrm{E}-03$ & $1.776 \mathrm{E}-03$ & $7.618 \mathrm{E}-05$ & \\
\hline UGC 10043 & $2004-05-29$ & 2801 & NGA-UGC10043 & $\ldots$ & $\ldots$ & $\ldots$ & $2.608 \mathrm{E}-03$ & $1.119 \mathrm{E}-03$ & $2.529 \mathrm{E}-05$ & $(4)$ \\
\hline UGC 10109 & 2005-04-29 & 1501 & SA-P177D & $\cdots$ & $\cdots$ & $\cdots$ & $2.354 \mathrm{E}-03$ & $1.447 \mathrm{E}-03$ & $3.425 \mathrm{E}-05$ & (4) \\
\hline UGC 10153 & 2004-05-29 & 1791 & NGA-NGC6052 & $\cdots$ & $\ldots$ & $\cdots$ & $2.695 \mathrm{E}-03$ & $1.384 \mathrm{E}-03$ & $5.773 \mathrm{E}-05$ & (4) \\
\hline UGC 10197 & 2004-05-29 & 1791 & NGA-NGC6052 & $\ldots$ & .. & $\ldots$ & $3.031 \mathrm{E}-03$ & $1.458 \mathrm{E}-03$ & $1.442 \mathrm{E}-04$ & (4) \\
\hline UGC 10198 & 2004-05-29 & 1791 & NGA-NGC6052 & $\cdots$ & $\ldots$ & $\cdots$ & 3.047E-03 & $1.467 \mathrm{E}-03$ & $3.893 \mathrm{E}-05$ & (4) \\
\hline UGC 10245 & 2004-08-06 & 1432 & MISDR1-10726-0622 & $3.291 \mathrm{E}-04$ & $5.028 \mathrm{E}-04$ & $1.054 \mathrm{E}-05$ & $2.389 \mathrm{E}-03$ & $1.466 \mathrm{E}-03$ & $3.607 \mathrm{E}-05$ & \\
\hline CGCG 023-019 & 2003-07-02 & 2216 & MISDR1-22273-0345 & $9.707 \mathrm{E}-04$ & $7.378 \mathrm{E}-04$ & $6.692 \mathrm{E}-05$ & $4.412 \mathrm{E}-03$ & $1.644 \mathrm{E}-03$ & $6.365 \mathrm{E}-05$ & \\
\hline UGC 10261 & $2004-07-31$ & 1632 & NGA-NGC6090 & $\ldots$ & $\ldots$ & $\ldots$ & $2.326 \mathrm{E}-03$ & $1.377 \mathrm{E}-03$ & $3.192 \mathrm{E}-06$ & $(4)(5)$ \\
\hline NGC 6090 & $2004-07-31$ & 1632 & NGA-NGC6090 & $3.454 \mathrm{E}-04$ & $4.907 \mathrm{E}-04$ & $8.602 \mathrm{E}-06$ & $2.377 \mathrm{E}-03$ & $1.407 \mathrm{E}-03$ & $2.647 \mathrm{E}-04$ & \\
\hline UGC 10278 & 2004-08-06 & 1430 & MISDR1-10677-0622 & $3.100 \mathrm{E}-04$ & $4.924 \mathrm{E}-04$ & $2.695 \mathrm{E}-05$ & $2.321 \mathrm{E}-03$ & $1.426 \mathrm{E}-03$ & $3.865 \mathrm{E}-06$ & \\
\hline NGC 6100 & 2004-05-29 & 4704 & MISDR1-22356-0346 & $7.000 \mathrm{E}-04$ & $6.721 \mathrm{E}-04$ & $8.469 \mathrm{E}-06$ & $3.826 \mathrm{E}-03$ & $1.061 \mathrm{E}-03$ & $9.210 \mathrm{E}-05$ & \\
\hline IC 4595 & 2004-05-31 & 1886 & NGA-IC4595 & $\ldots$ & $\ldots$ & $\cdots$ & 7.627E-03 & $2.612 \mathrm{E}-03$ & 4.463E-04 & (4) \\
\hline NGC 6154 & 2004-08-07 & 37 & MISDR2-10675-0625 & $3.566 \mathrm{E}-04$ & $5.288 \mathrm{E}-04$ & $2.558 \mathrm{E}-05$ & $2.913 \mathrm{E}-03$ & $1.655 \mathrm{E}-03$ & $1.852 \mathrm{E}-04$ & \\
\hline NGC 6155 & $2004-07-30$ & 3305 & MISDR2-10773-0625 & $3.084 \mathrm{E}-04$ & $3.090 \mathrm{E}-04$ & $1.268 \mathrm{E}-05$ & $2.328 \mathrm{E}-03$ & $9.495 \mathrm{E}-04$ & $6.728 \mathrm{E}-05$ & \\
\hline UGC 10404 & 2004-08-06 & 1437 & NGA-NGC6166 & $2.946 \mathrm{E}-04$ & $4.817 \mathrm{E}-04$ & $1.188 \mathrm{E}-05$ & $2.331 \mathrm{E}-03$ & $1.463 \mathrm{E}-03$ & $3.809 \mathrm{E}-05$ & \\
\hline NGC 6166 & 2004-08-06 & 1437 & NGA-NGC6166 & $2.928 \mathrm{E}-04$ & $4.788 \mathrm{E}-04$ & $1.648 \mathrm{E}-05$ & $2.356 \mathrm{E}-03$ & $1.496 \mathrm{E}-03$ & $7.870 \mathrm{E}-05$ & \\
\hline UGC 10420 & 2004-08-06 & 1437 & NGA-NGC6166 & $3.054 \mathrm{E}-04$ & $5.152 \mathrm{E}-04$ & $5.018 \mathrm{E}-06$ & $2.328 \mathrm{E}-03$ & $1.506 \mathrm{E}-03$ & $4.064 \mathrm{E}-05$ & \\
\hline UGC 10445 & 2004-06-19 & 4309 & NGA-UGC10445 & $3.753 \mathrm{E}-04$ & $3.153 \mathrm{E}-04$ & 8.413E-06 & $2.779 \mathrm{E}-03$ & $9.429 \mathrm{E}-04$ & 8.435E-05 & \\
\hline IC 1221 & 2004-08-07 & 2825 & MISDR2-10874-0627 & $3.936 \mathrm{E}-04$ & $4.183 \mathrm{E}-04$ & $1.273 \mathrm{E}-05$ & $2.622 \mathrm{E}-03$ & $1.139 \mathrm{E}-03$ & $2.956 \mathrm{E}-06$ & \\
\hline
\end{tabular}


Table 2-Continued

\begin{tabular}{|c|c|c|c|c|c|c|c|c|c|c|}
\hline \multirow{2}{*}{$\begin{array}{l}\text { Object Name } \\
\text { (1) }\end{array}$} & \multirow{2}{*}{$\begin{array}{c}\text { Date } \\
\text { Observed } \\
(2)\end{array}$} & \multirow{2}{*}{$\begin{array}{l}\text { Exposure } \\
\quad(\mathrm{sec}) \\
(3)\end{array}$} & \multirow{2}{*}{$\begin{array}{l}\text { Tile } \\
(4)\end{array}$} & \multicolumn{3}{|c|}{ FUV sky background } & \multicolumn{3}{|c|}{ NUV sky background } & \multirow{2}{*}{$\begin{array}{l}\text { notes } \\
\text { (11) }\end{array}$} \\
\hline & & & & $\begin{array}{c}\text { mean } \\
(\text { counts/s) } \\
(5)\end{array}$ & $\begin{array}{c}<\sigma> \\
(\text { counts } / \mathrm{s}) \\
(6)\end{array}$ & $\begin{array}{c}\sigma(\text { mean }) \\
(\text { counts } / \mathrm{s}) \\
(7)\end{array}$ & $\begin{array}{c}\text { mean } \\
(\text { counts/s) } \\
(8)\end{array}$ & $\begin{array}{c}<\sigma> \\
(\text { counts } / \mathrm{s}) \\
(9)\end{array}$ & $\begin{array}{c}\sigma(\text { mean }) \\
(\text { counts/s) } \\
(10)\end{array}$ & \\
\hline IC 1222 & 2004-08-07 & 2825 & MISDR2-10874-0627 & $3.915 \mathrm{E}-04$ & $4.348 \mathrm{E}-04$ & $1.042 \mathrm{E}-05$ & $2.598 \mathrm{E}-03$ & $1.133 \mathrm{E}-03$ & $2.639 \mathrm{E}-05$ & \\
\hline UGC 10468 & $2004-05-15$ & 1597 & MISDR1-10980-0626 & $2.843 \mathrm{E}-04$ & 4.609E-04 & $9.667 \mathrm{E}-06$ & $2.420 \mathrm{E}-03$ & $1.416 \mathrm{E}-03$ & $3.647 \mathrm{E}-05$ & \\
\hline UGC 10491 & $2004-05-23$ & 1702 & MISDR1-11146-0628 & $2.930 \mathrm{E}-04$ & $4.478 \mathrm{E}-04$ & $2.056 \mathrm{E}-05$ & $2.652 \mathrm{E}-03$ & $1.457 \mathrm{E}-03$ & 8.091E-05 & $(1)(2)$ \\
\hline NGC 6239 & 2004-05-19 & 1704 & MISDR1-11087-0631 & $3.052 \mathrm{E}-04$ & 4.705E-04 & $1.385 \mathrm{E}-05$ & $2.340 \mathrm{E}-03$ & $1.375 \mathrm{E}-03$ & $5.693 \mathrm{E}-05$ & \\
\hline Mrk 501 & $2004-05-12$ & 32647 & UVE-A2235 & $3.135 \mathrm{E}-04$ & $1.253 \mathrm{E}-04$ & $1.631 \mathrm{E}-07$ & $2.392 \mathrm{E}-03$ & $3.078 \mathrm{E}-04$ & $8.859 \mathrm{E}-06$ & (6) \\
\hline UGC 10600 & $2004-07-30$ & 2254 & MISDR2-11566-0819 & $2.907 \mathrm{E}-04$ & $3.806 \mathrm{E}-04$ & $2.774 \mathrm{E}-05$ & $2.189 \mathrm{E}-03$ & $1.106 \mathrm{E}-03$ & 2.363E-05 & \\
\hline NGC 6255 & $2004-07-29$ & 667 & MISDR2-11503-0819 & $3.332 \mathrm{E}-04$ & $7.841 \mathrm{E}-04$ & $1.519 \mathrm{E}-05$ & $2.274 \mathrm{E}-03$ & $2.137 \mathrm{E}-03$ & $1.912 \mathrm{E}-05$ & \\
\hline UGC 10651 & $2003-08-10$ & 1456 & MISDR1-11142-0631 & $3.820 \mathrm{E}-04$ & $5.237 \mathrm{E}-04$ & $2.727 \mathrm{E}-05$ & $2.592 \mathrm{E}-03$ & $1.465 \mathrm{E}-03$ & $2.422 \mathrm{E}-05$ & \\
\hline UGC 10687 & 2004-08-09 & 2681 & MISDR1-10202-0353 & $4.025 \mathrm{E}-04$ & $3.990 \mathrm{E}-04$ & $6.142 \mathrm{E}-05$ & $2.760 \mathrm{E}-03$ & $1.159 \mathrm{E}-03$ & $1.150 \mathrm{E}-04$ & \\
\hline UGC 10713 & $2003-10-07$ & 1640 & NGA-NGC6340 & $5.164 \mathrm{E}-04$ & $5.762 \mathrm{E}-04$ & $1.257 \mathrm{E}-05$ & $3.092 \mathrm{E}-03$ & $1.522 \mathrm{E}-03$ & $6.856 \mathrm{E}-05$ & \\
\hline NGC 6306 & 2004-08-09 & 1339 & MISDR1-10165-0351 & $4.119 \mathrm{E}-04$ & 4.033E-04 & $1.728 \mathrm{E}-06$ & $2.840 \mathrm{E}-03$ & $1.160 \mathrm{E}-03$ & $1.828 \mathrm{E}-05$ & \\
\hline NGC 6307 & 2004-08-09 & 1339 & MISDR1-10165-0351 & $4.123 \mathrm{E}-04$ & $4.052 \mathrm{E}-04$ & $1.145 \mathrm{E}-06$ & $2.847 \mathrm{E}-03$ & $1.152 \mathrm{E}-03$ & $2.167 \mathrm{E}-05$ & \\
\hline UGC 10729 & $2004-06-28$ & 1398 & MISDR2-11826-0975 & $5.159 \mathrm{E}-04$ & $7.074 \mathrm{E}-04$ & $6.350 \mathrm{E}-05$ & $3.201 \mathrm{E}-03$ & $1.769 \mathrm{E}-03$ & $2.771 \mathrm{E}-04$ & \\
\hline IC 1251 & $2003-10-07$ & 1640 & NGA-NGC6340 & $5.352 \mathrm{E}-04$ & $6.132 \mathrm{E}-04$ & $5.075 \mathrm{E}-05$ & $3.169 \mathrm{E}-03$ & $1.631 \mathrm{E}-03$ & $5.726 \mathrm{E}-05$ & \\
\hline NGC 6340 & $2003-10-07$ & 1640 & NGA-NGC6340 & $5.284 \mathrm{E}-04$ & $6.017 \mathrm{E}-04$ & $1.911 \mathrm{E}-05$ & $3.155 \mathrm{E}-03$ & $1.642 \mathrm{E}-03$ & $5.747 \mathrm{E}-05$ & \\
\hline IC 1254 & $2003-10-07$ & 1640 & NGA-NGC6340 & $5.480 \mathrm{E}-04$ & $6.355 \mathrm{E}-04$ & $4.870 \mathrm{E}-05$ & $3.165 \mathrm{E}-03$ & $1.650 \mathrm{E}-03$ & 7.460E-05 & \\
\hline IC 1248 & 2004-08-09 & 1339 & MISDR1-10165-0351 & $4.676 \mathrm{E}-04$ & 4.962E-04 & $1.354 \mathrm{E}-04$ & $2.842 \mathrm{E}-03$ & $1.206 \mathrm{E}-03$ & $4.256 \mathrm{E}-05$ & \\
\hline UGC 10770 & 2004-08-09 & 1334 & MISDR1-10201-0353 & $4.575 \mathrm{E}-04$ & $6.632 \mathrm{E}-04$ & $2.657 \mathrm{E}-05$ & $2.848 \mathrm{E}-03$ & $1.727 \mathrm{E}-03$ & $4.328 \mathrm{E}-05$ & $(1)(2)$ \\
\hline UGC 10791 & $2003-10-07$ & 1640 & NGA-NGC6340 & $5.654 \mathrm{E}-04$ & $6.693 \mathrm{E}-04$ & $2.021 \mathrm{E}-05$ & $3.157 \mathrm{E}-03$ & $1.669 \mathrm{E}-03$ & $1.268 \mathrm{E}-05$ & (1) \\
\hline NGC 6330 & $2004-06-28$ & 1374 & MISDR2-22241-0977 & $4.977 \mathrm{E}-04$ & $6.477 \mathrm{E}-04$ & $6.083 \mathrm{E}-05$ & $2.866 \mathrm{E}-03$ & $1.678 \mathrm{E}-03$ & $1.033 \mathrm{E}-04$ & \\
\hline UGC 10783 & $2004-06-28$ & 1374 & MISDR2-22241-0977 & $4.422 \mathrm{E}-04$ & 5.837E-04 & $9.663 \mathrm{E}-06$ & $2.805 \mathrm{E}-03$ & $1.621 \mathrm{E}-03$ & $4.201 \mathrm{E}-05$ & \\
\hline UGC 10796 & $2004-08-10$ & 2658 & MISDR1-10094-0352 & $4.441 \mathrm{E}-04$ & $4.226 \mathrm{E}-04$ & $9.649 \mathrm{E}-06$ & $2.990 \mathrm{E}-03$ & $1.227 \mathrm{E}-03$ & $7.583 \mathrm{E}-05$ & \\
\hline NGC 6359 & $2004-08-10$ & 2658 & MISDR1-10094-0352 & $4.520 \mathrm{E}-04$ & $4.344 \mathrm{E}-04$ & $7.219 \mathrm{E}-06$ & $3.038 \mathrm{E}-03$ & $1.251 \mathrm{E}-03$ & $1.235 \mathrm{E}-05$ & \\
\hline UGC 10795 & $2004-08-08$ & 1372 & MISDR2-22240-0978 & $5.028 \mathrm{E}-04$ & 7.472E-04 & $4.220 \mathrm{E}-06$ & $2.876 \mathrm{E}-03$ & $1.717 \mathrm{E}-03$ & $7.524 \mathrm{E}-06$ & (1) \\
\hline NGC 6361 & 2004-05-03 & 2667 & SIRTFFL-05 & $4.803 \mathrm{E}-04$ & 4.930E-04 & $2.154 \mathrm{E}-05$ & $3.025 \mathrm{E}-03$ & $1.259 \mathrm{E}-03$ & $1.472 \mathrm{E}-04$ & \\
\hline UGC 10811 & $2003-08-18$ & 2803 & SIRTFFL-02 & $5.378 \mathrm{E}-04$ & $4.769 \mathrm{E}-04$ & $1.624 \mathrm{E}-05$ & $3.390 \mathrm{E}-03$ & $1.260 \mathrm{E}-03$ & $1.503 \mathrm{E}-04$ & \\
\hline NGC 6373 & $2003-07-04$ & 23591 & SIRTFFL-01 & $5.616 \mathrm{E}-04$ & $1.883 \mathrm{E}-04$ & $1.958 \mathrm{E}-05$ & $3.111 \mathrm{E}-03$ & $4.311 \mathrm{E}-04$ & $5.285 \mathrm{E}-06$ & \\
\hline NGC 6364 & $2004-06-28$ & 1440 & MISDR2-22324-0980 & $4.317 \mathrm{E}-04$ & $6.608 \mathrm{E}-04$ & $2.159 \mathrm{E}-05$ & $2.845 \mathrm{E}-03$ & $1.675 \mathrm{E}-03$ & $1.326 \mathrm{E}-04$ & \\
\hline UGC 10842 & $2004-06-29$ & 1450 & MISDR2-22368-0980 & $4.894 \mathrm{E}-04$ & $6.954 \mathrm{E}-04$ & $2.329 \mathrm{E}-04$ & $3.094 \mathrm{E}-03$ & $1.751 \mathrm{E}-03$ & $2.312 \mathrm{E}-05$ & \\
\hline UGC 10872 & $2004-06-27$ & 1285 & MISDR1-10127-0354 & $4.232 \mathrm{E}-04$ & $5.885 \mathrm{E}-04$ & $1.099 \mathrm{E}-05$ & $2.879 \mathrm{E}-03$ & $1.652 \mathrm{E}-03$ & $3.457 \mathrm{E}-05$ & \\
\hline UGC 10888 & $2004-06-27$ & 1285 & MISDR1-10127-0354 & $4.736 \mathrm{E}-04$ & $7.716 \mathrm{E}-04$ & $1.711 \mathrm{E}-05$ & $2.904 \mathrm{E}-03$ & $1.721 \mathrm{E}-03$ & $8.684 \mathrm{E}-05$ & \\
\hline NGC 6394 & $2004-06-27$ & 1350 & MISDR1-10162-0354 & $5.125 \mathrm{E}-04$ & $7.196 \mathrm{E}-04$ & $3.512 \mathrm{E}-06$ & $2.942 \mathrm{E}-03$ & $1.692 \mathrm{E}-03$ & $6.997 \mathrm{E}-05$ & \\
\hline
\end{tabular}


Table 2-Continued

\begin{tabular}{|c|c|c|c|c|c|c|c|c|c|c|}
\hline \multirow{2}{*}{$\begin{array}{c}\text { Object Name } \\
\text { (1) }\end{array}$} & \multirow{2}{*}{$\begin{array}{c}\text { Date } \\
\text { Observed } \\
(2)\end{array}$} & \multirow{2}{*}{$\begin{array}{l}\text { Exposure } \\
\quad(\mathrm{sec}) \\
(3)\end{array}$} & \multirow{2}{*}{$\begin{array}{l}\text { Tile } \\
(4)\end{array}$} & \multicolumn{3}{|c|}{ FUV sky background } & \multicolumn{3}{|c|}{ NUV sky background } & \multirow{2}{*}{$\begin{array}{l}\text { notes } \\
(11)\end{array}$} \\
\hline & & & & $\begin{array}{c}\text { mean } \\
(\text { counts/s) } \\
(5)\end{array}$ & $\begin{array}{c}<\sigma> \\
(\text { counts } / \mathrm{s}) \\
(6)\end{array}$ & $\begin{array}{c}\sigma(\text { mean }) \\
(\text { counts/s) } \\
(7)\end{array}$ & $\begin{array}{c}\text { mean } \\
(\text { counts/s) } \\
(8)\end{array}$ & $\begin{array}{c}<\sigma> \\
(\text { counts } / \mathrm{s}) \\
(9)\end{array}$ & $\begin{array}{c}\sigma(\text { mean }) \\
(\text { counts/s) } \\
(10)\end{array}$ & \\
\hline UGC 10895 & $2004-06-27$ & 1350 & MISDR1-10162-0354 & $5.088 \mathrm{E}-04$ & $7.760 \mathrm{E}-04$ & $2.787 \mathrm{E}-05$ & $2.895 \mathrm{E}-03$ & $1.662 \mathrm{E}-03$ & $1.192 \mathrm{E}-04$ & \\
\hline UGC 10935 & $2003-08-11$ & 1615 & MISDR1-10273-0358 & $\cdots$ & $\cdots$ & $\cdots$ & $3.103 \mathrm{E}-03$ & $1.604 \mathrm{E}-03$ & $2.361 \mathrm{E}-05$ & $(4)(5)$ \\
\hline UGC 10971 & $2004-08-10$ & 2651 & MISDR1-10352-0360 & $6.542 \mathrm{E}-04$ & $5.244 \mathrm{E}-04$ & $1.889 \mathrm{E}-05$ & $3.542 \mathrm{E}-03$ & $1.365 \mathrm{E}-03$ & 8.583E-06 & \\
\hline NGC 6482 & 2004-07-02 & 2526 & NGA-NGC6482 & $6.845 \mathrm{E}-04$ & $5.550 \mathrm{E}-04$ & $2.577 \mathrm{E}-05$ & $4.055 \mathrm{E}-03$ & $1.524 \mathrm{E}-03$ & $1.509 \mathrm{E}-04$ & (6) \\
\hline IC 4836 & $2004-07-08$ & 4907 & UVE-NGC6769 & $7.186 \mathrm{E}-04$ & $7.543 \mathrm{E}-04$ & $6.122 \mathrm{E}-06$ & $4.054 \mathrm{E}-03$ & $1.078 \mathrm{E}-03$ & $9.399 \mathrm{E}-05$ & \\
\hline NGC 6789 & $2003-08-22$ & 1338 & NGA-NGC6789 & $8.456 \mathrm{E}-04$ & $8.389 \mathrm{E}-04$ & $2.973 \mathrm{E}-05$ & $4.408 \mathrm{E}-03$ & $2.168 \mathrm{E}-03$ & $1.356 \mathrm{E}-04$ & (2) \\
\hline NGC 6769 & 2004-07-08 & 4907 & UVE-NGC6769 & $6.568 \mathrm{E}-04$ & $6.625 \mathrm{E}-04$ & $6.006 \mathrm{E}-05$ & $4.026 \mathrm{E}-03$ & $1.090 \mathrm{E}-03$ & $2.055 \mathrm{E}-04$ & \\
\hline NGC 6770 & $2004-07-08$ & 4907 & UVE-NGC6769 & $6.511 \mathrm{E}-04$ & $6.542 \mathrm{E}-04$ & $1.649 \mathrm{E}-05$ & 4.011E-03 & $1.087 \mathrm{E}-03$ & $7.866 \mathrm{E}-05$ & \\
\hline NGC 6771 & 2004-07-08 & 4907 & UVE-NGC6769 & $6.495 \mathrm{E}-04$ & $6.578 \mathrm{E}-04$ & $8.697 \mathrm{E}-05$ & $3.990 \mathrm{E}-03$ & $1.077 \mathrm{E}-03$ & $1.495 \mathrm{E}-04$ & \\
\hline IC 4842 & $2004-07-08$ & 4907 & UVE-NGC6769 & $6.447 \mathrm{E}-04$ & $6.618 \mathrm{E}-04$ & $1.545 \mathrm{E}-05$ & $3.913 \mathrm{E}-03$ & $1.061 \mathrm{E}-03$ & $9.000 \mathrm{E}-05$ & \\
\hline IC 4845 & $2004-07-08$ & 4907 & UVE-NGC6769 & $5.985 \mathrm{E}-04$ & $6.003 \mathrm{E}-04$ & $3.063 \mathrm{E}-05$ & $3.876 \mathrm{E}-03$ & $1.058 \mathrm{E}-03$ & $2.121 \mathrm{E}-04$ & \\
\hline NGC 6782 & $2003-09-08$ & 1892 & NGA-NGC6782 & $7.152 \mathrm{E}-04$ & $6.965 \mathrm{E}-04$ & $1.649 \mathrm{E}-05$ & $4.339 \mathrm{E}-03$ & $1.862 \mathrm{E}-03$ & $3.278 \mathrm{E}-05$ & \\
\hline Superantena & 2003-09-09 & 1668 & NGA-Superantenna & $7.148 \mathrm{E}-04$ & $6.723 \mathrm{E}-04$ & $4.641 \mathrm{E}-05$ & $4.320 \mathrm{E}-03$ & $1.892 \mathrm{E}-03$ & $1.448 \mathrm{E}-04$ & \\
\hline NGC $6845 \mathrm{~A}$ & 2004-07-05 & 8460 & UVE-NGC6845 & $5.216 \mathrm{E}-04$ & $3.990 \mathrm{E}-04$ & $2.512 \mathrm{E}-05$ & $3.767 \mathrm{E}-03$ & 7.596E-04 & $2.827 \mathrm{E}-06$ & \\
\hline ESO 284-G009 & 2004-07-05 & 8460 & UVE-NGC6845 & $5.081 \mathrm{E}-04$ & $3.995 \mathrm{E}-04$ & $4.218 \mathrm{E}-06$ & $3.838 \mathrm{E}-03$ & $7.938 \mathrm{E}-04$ & $1.019 \mathrm{E}-04$ & (6) \\
\hline NGC 6902B & $2004-07-08$ & 3845 & UVE-NGC6902 & $4.915 \mathrm{E}-04$ & $5.544 \mathrm{E}-04$ & $4.724 \mathrm{E}-06$ & $3.587 \mathrm{E}-03$ & $1.140 \mathrm{E}-03$ & $3.404 \mathrm{E}-05$ & \\
\hline IC 4946 & 2004-07-08 & 3845 & UVE-NGC6902 & $4.632 \mathrm{E}-04$ & $5.254 \mathrm{E}-04$ & $1.734 \mathrm{E}-05$ & $3.543 \mathrm{E}-03$ & $1.131 \mathrm{E}-03$ & $1.516 \mathrm{E}-04$ & \\
\hline NGC 6902 & $2004-07-08$ & 3845 & UVE-NGC6902 & $4.650 \mathrm{E}-04$ & $4.986 \mathrm{E}-04$ & $3.646 \mathrm{E}-05$ & $3.572 \mathrm{E}-03$ & $1.154 \mathrm{E}-03$ & $6.521 \mathrm{E}-05$ & \\
\hline ESO 285-G009 & 2004-07-08 & 3845 & UVE-NGC6902 & 4.507E-04 & $5.059 \mathrm{E}-04$ & $2.003 \mathrm{E}-05$ & $3.504 \mathrm{E}-03$ & $1.119 \mathrm{E}-03$ & $2.700 \mathrm{E}-06$ & \\
\hline PGC 65022 & 2004-07-07 & 2647 & MISDR1-31801-0634 & $\ldots$ & $\ldots$ & $\ldots$ & $3.616 \mathrm{E}-03$ & $1.398 \mathrm{E}-03$ & $8.866 \mathrm{E}-05$ & (4) \\
\hline NGC 6941 & 2004-07-07 & 5253 & MISDR1-21379-0634 & 4.457E-04 & $5.047 \mathrm{E}-04$ & $1.684 \mathrm{E}-05$ & $3.588 \mathrm{E}-03$ & $9.784 \mathrm{E}-04$ & $5.385 \mathrm{E}-05$ & \\
\hline NGC 6951 & $2003-08-22$ & 521 & NGA-NGC6951 & $1.104 \mathrm{E}-03$ & $1.510 \mathrm{E}-03$ & $4.159 \mathrm{E}-05$ & $5.219 \mathrm{E}-03$ & $3.796 \mathrm{E}-03$ & $3.915 \mathrm{E}-05$ & \\
\hline NGC 6945 & $2004-07-07$ & 7029 & MISDR1-19900-0634 & $4.206 \mathrm{E}-04$ & $6.274 \mathrm{E}-04$ & $2.249 \mathrm{E}-05$ & $3.441 \mathrm{E}-03$ & $8.166 \mathrm{E}-04$ & $8.787 \mathrm{E}-06$ & (1) \\
\hline PGC 65158 & $2004-07-07$ & 7029 & MISDR1-19900-0634 & $5.465 \mathrm{E}-04$ & $8.363 \mathrm{E}-04$ & $3.982 \mathrm{E}-06$ & $3.630 \mathrm{E}-03$ & $8.430 \mathrm{E}-04$ & $3.194 \mathrm{E}-05$ & (1) \\
\hline UGC 11612 & $2004-07-11$ & 3032 & MISDR2-19739-0981 & $6.163 \mathrm{E}-04$ & $7.312 \mathrm{E}-04$ & $5.383 \mathrm{E}-06$ & $4.106 \mathrm{E}-03$ & $1.377 \mathrm{E}-03$ & $2.342 \mathrm{E}-06$ & \\
\hline PGC 65328 & 2003-08-03 & 5798 & MISDR1-19941-0635 & $4.768 \mathrm{E}-04$ & $4.976 \mathrm{E}-04$ & $1.515 \mathrm{E}-05$ & $3.628 \mathrm{E}-03$ & $9.216 \mathrm{E}-04$ & $3.583 \mathrm{E}-05$ & \\
\hline ESO 341-G013 & $2003-08-29$ & 615 & NGA-NGC6958 & $\ldots$ & $\ldots$ & $\ldots$ & $3.646 \mathrm{E}-03$ & $2.823 \mathrm{E}-03$ & $3.799 \mathrm{E}-05$ & $(4)(5)$ \\
\hline NGC 6962 & 2004-07-11 & 3012 & MISDR2-19815-0981 & $6.614 \mathrm{E}-04$ & $8.201 \mathrm{E}-04$ & $5.449 \mathrm{E}-05$ & $4.070 \mathrm{E}-03$ & $1.386 \mathrm{E}-03$ & $1.084 \mathrm{E}-04$ & \\
\hline NGC 6964 & $2004-07-11$ & 3012 & MISDR2-19815-0981 & $6.606 \mathrm{E}-04$ & $8.275 \mathrm{E}-04$ & $8.878 \mathrm{E}-06$ & $4.065 \mathrm{E}-03$ & $1.383 \mathrm{E}-03$ & $1.848 \mathrm{E}-05$ & \\
\hline PGC 65420 & 2004-07-09 & 2023 & MISDR1-20028-0635 & $5.116 \mathrm{E}-04$ & $1.562 \mathrm{E}-03$ & $3.209 \mathrm{E}-05$ & $3.739 \mathrm{E}-03$ & $1.585 \mathrm{E}-03$ & $3.554 \mathrm{E}-04$ & \\
\hline NGC 6958 & $2003-08-29$ & 615 & NGA-NGC6958 & $3.864 \mathrm{E}-04$ & $8.399 \mathrm{E}-04$ & $3.614 \mathrm{E}-06$ & $3.875 \mathrm{E}-03$ & $2.999 \mathrm{E}-03$ & $7.124 \mathrm{E}-05$ & \\
\hline UGC 11646 & $2004-07-10$ & 4259 & MISDR2-19894-0983 & $5.631 \mathrm{E}-04$ & $4.867 \mathrm{E}-04$ & $3.660 \mathrm{E}-06$ & $3.915 \mathrm{E}-03$ & $1.122 \mathrm{E}-03$ & $3.228 \mathrm{E}-05$ & \\
\hline
\end{tabular}


Table 2-Continued

\begin{tabular}{|c|c|c|c|c|c|c|c|c|c|c|}
\hline \multirow{2}{*}{$\begin{array}{c}\text { Object Name } \\
\text { (1) }\end{array}$} & \multirow{2}{*}{$\begin{array}{c}\text { Date } \\
\text { Observed } \\
\\
(2)\end{array}$} & \multirow{2}{*}{$\begin{array}{l}\text { Exposure } \\
\quad(\mathrm{sec}) \\
(3)\end{array}$} & \multirow{2}{*}{$\begin{array}{l}\text { Tile } \\
\text { (4) }\end{array}$} & \multicolumn{3}{|c|}{ FUV sky background } & \multicolumn{3}{|c|}{ NUV sky background } & \multirow{2}{*}{$\begin{array}{c}\text { notes } \\
(11)\end{array}$} \\
\hline & & & & $\begin{array}{c}\text { mean } \\
(\text { counts/s) } \\
(5)\end{array}$ & $\begin{array}{c}<\sigma> \\
(\text { counts } / \mathrm{s}) \\
(6)\end{array}$ & $\begin{array}{c}\sigma(\text { mean }) \\
(\text { counts/s) } \\
(7)\end{array}$ & $\begin{array}{c}\text { mean } \\
\text { (counts/s) } \\
(8)\end{array}$ & $\begin{array}{c}<\sigma> \\
(\text { counts } / \mathrm{s}) \\
(9)\end{array}$ & $\begin{array}{c}\sigma(\text { mean }) \\
(\text { counts/s) } \\
(10)\end{array}$ & \\
\hline PGC 66559 & $2003-08-06$ & 353 & MISDR1-20410-0639 & $6.184 \mathrm{E}-04$ & $1.343 \mathrm{E}-03$ & $1.126 \mathrm{E}$ & $4.157 \mathrm{E}-03$ & $3.986 \mathrm{E}-03$ & 8.987E-05 & \\
\hline NGC 7080 & $2004-07-03$ & 9247 & NGA-NGC7080 & $8.176 \mathrm{E}-04$ & $3.185 \mathrm{E}-04$ & $1.711 \mathrm{E}-05$ & $4.482 \mathrm{E}-03$ & $8.447 \mathrm{E}-04$ & & \\
\hline UGC 11776 & $2004-07-01$ & 1376 & MISDR2-20006-0731 & $6.615 \mathrm{E}-04$ & $7.098 \mathrm{E}-04$ & $2.732 \mathrm{E}-05$ & $4.097 \mathrm{E}-03$ & $1.994 \mathrm{E}-03$ & $8.200 \mathrm{H}$ & \\
\hline PGC 67153 & $2004-06-29$ & 4573 & MISDR2-20050-0732 & $6.145 \mathrm{E}-04$ & $3.787 \mathrm{E}-04$ & $4.945 \mathrm{E}-06$ & $3.667 \mathrm{E}-03$ & $1.043 \mathrm{E}-03$ & $4.164 \mathrm{E}-05$ & \\
\hline UGC 11789 & $2004-08-23$ & 1684 & MISDR2-20399-0990 & $3.646 \mathrm{E}-04$ & $4.698 \mathrm{E}-04$ & $9.938 \mathrm{E}-07$ & $3.261 \mathrm{E}-03$ & $1.597 \mathrm{E}-03$ & $4.878 \mathrm{E}-05$ & \\
\hline Tol 2138-405 & $2003-08-05$ & 774 & NGA-Tol2138m405 & $2.200 \mathrm{E}-04$ & $5.677 \mathrm{E}-04$ & $6.050 \mathrm{E}-07$ & $2.683 \mathrm{E}-03$ & $2.182 \mathrm{E}-03$ & $6.459 \mathrm{E}-05$ & (7) \\
\hline ESO 343-G018 & $2003-08-05$ & 774 & NGA-Tol2138m405 & $2.521 \mathrm{E}-04$ & $7.309 \mathrm{E}-04$ & $1.980 \mathrm{E}-06$ & $2.692 \mathrm{E}-03$ & $2.096 \mathrm{E}-03$ & $4.690 \mathrm{E}-05$ & \\
\hline UGC 11790 & $2004-08-23$ & 1684 & MISDR2-20399-0990 & $4.462 \mathrm{E}-04$ & $6.088 \mathrm{E}-04$ & $1.785 \mathrm{E}-05$ & $3.345 \mathrm{E}-03$ & $1.596 \mathrm{E}-03$ & $1.830 \mathrm{E}-05$ & \\
\hline UGC 11794 & $2004-08-24$ & 1950 & MISDR2-20095-0732 & $5.499 \mathrm{E}-04$ & $5.410 \mathrm{E}-04$ & $3.669 \mathrm{E}-05$ & $3.569 \mathrm{E}-03$ & $1.529 \mathrm{E}-03$ & $1.020 \mathrm{E}-04$ & \\
\hline ESO 466-G001 & 2003-09-19 & 1268 & MIS2DFSGP-40464-0247 & $4.117 \mathrm{E}-04$ & $7.280 \mathrm{E}-04$ & $2.406 \mathrm{E}-05$ & $3.273 \mathrm{E}-03$ & $1.857 \mathrm{E}-03$ & $6.220 \mathrm{E}-06$ & \\
\hline ESO 466-G005 & 2003-09-19 & 1268 & MIS2DFSGP-40464-0247 & $2.984 \mathrm{E}-04$ & $5.300 \mathrm{E}-04$ & $1.129 \mathrm{E}-05$ & $3.134 \mathrm{E}-03$ & $1.838 \mathrm{E}-03$ & $6.580 \mathrm{E}-05$ & \\
\hline UGC 11816 & $2004-08-23$ & 1682 & MISDR2-20505-0371 & $5.527 \mathrm{E}-04$ & $6.686 \mathrm{E}-04$ & $2.550 \mathrm{E}-05$ & $3.689 \mathrm{E}-03$ & $1.763 \mathrm{E}-03$ & $9.746 \mathrm{E}-05$ & \\
\hline NGC 7152 & $2004-07-23$ & 2643 & MIS2DFSGP-40471-0249 & $2.415 \mathrm{E}-04$ & $3.735 \mathrm{E}-04$ & $9.843 \mathrm{E}-06$ & $2.885 \mathrm{E}-03$ & $1.201 \mathrm{E}-03$ & $7.644 \mathrm{E}-06$ & \\
\hline ESO 466-G014 & $2004-07-22$ & 1624 & MIS2DFSGP-40492-0406 & $2.241 \mathrm{E}-04$ & $3.775 \mathrm{E}-04$ & $3.997 \mathrm{E}-06$ & $2.801 \mathrm{E}-03$ & $1.478 \mathrm{E}-03$ & 7.030E-05 & \\
\hline UGC 11859 & $2004-08-23$ & 404 & MISDR2-20613-0372 & & $1.109 \mathrm{E}-03$ & $1.647 \mathrm{E}-05$ & $3.211 \mathrm{E}-03$ & $3.339 \mathrm{E}-03$ & $2.299 \mathrm{E}-05$ & $(2)$ \\
\hline ESO 404-G015 & $2004-07-23$ & 901 & MIS2DFSGP-40520-0478 & $2.356 \mathrm{E}-04$ & $5.896 \mathrm{E}-04$ & $6.715 \mathrm{E}-05$ & $2.931 \mathrm{E}-03$ & $2.111 \mathrm{E}-03$ & $2.186 \mathrm{E}-05$ & \\
\hline NGC 7167 & $2003-09-21$ & 319 & MIS2DFSGP-30636-0060 & $3.675 \mathrm{E}-04$ & $1.115 \mathrm{E}-03$ & $2.779 \mathrm{E}-05$ & $3.449 \mathrm{E}-03$ & $3.883 \mathrm{E}-03$ & $2.668 \mathrm{E}-05$ & (1) \\
\hline ESO 404-G023 & $2004-07-23$ & 901 & MIS2DFSGP-40520-0478 & $2.115 \mathrm{E}-04$ & $5.262 \mathrm{E}-04$ & $9.995 \mathrm{E}-06$ & $2.905 \mathrm{E}-03$ & $2.060 \mathrm{E}-03$ & $7.611 \mathrm{E}-06$ & \\
\hline IC 5156 & $2004-07-23$ & 901 & MIS2DFSGP-40520-0478 & $2.144 \mathrm{E}-04$ & $5.440 \mathrm{E}-04$ & $3.463 \mathrm{E}-06$ & $2.886 \mathrm{E}-03$ & $2.020 \mathrm{E}-03$ & $5.248 \mathrm{E}-06$ & \\
\hline NGC 7215 & $2004-08-23$ & 989 & MISDR2-20785-0373 & $4.149 \mathrm{E}-04$ & $7.215 \mathrm{E}-04$ & $3.111 \mathrm{E}-05$ & $3.290 \mathrm{E}-03$ & $2.159 \mathrm{E}-03$ & 4.093E-05 & \\
\hline NGC 7221 & $2004-07-25$ & 689 & MIS2DFSGP-40497-0334 & $1.998 \mathrm{E}-04$ & $5.834 \mathrm{E}-04$ & $3.339 \mathrm{E}-06$ & $2.765 \mathrm{E}-03$ & $2.313 \mathrm{E}-03$ & $2.486 \mathrm{E}-05$ & \\
\hline CGCG 377-039 & $2004-08-24$ & 581 & MISDR2-20844-0374 & $4.770 \mathrm{E}-04$ & $1.116 \mathrm{E}-03$ & $1.402 \mathrm{E}-05$ & $3.371 \mathrm{E}-03$ & $2.873 \mathrm{E}-03$ & $4.919 \mathrm{E}-05$ & (1) \\
\hline NGC 7248 & $2004-09-26$ & 3044 & UVE-NGC7250 & $1.523 \mathrm{E}-03$ & $7.524 \mathrm{E}-04$ & $1.669 \mathrm{E}-06$ & $6.423 \mathrm{E}-03$ & $1.780 \mathrm{E}-03$ & $3.991 \mathrm{E}-05$ & (2) \\
\hline NGC 7250 & $2004-09-26$ & 3044 & UVE-NGC7250 & $1.586 \mathrm{E}-03$ & $7.799 \mathrm{E}-04$ & $1.195 \mathrm{E}-05$ & $6.523 \mathrm{E}-03$ & $1.833 \mathrm{E}-03$ & $1.639 \mathrm{E}-05$ & \\
\hline NGC 7252 & $2004-10-16$ & 562 & MIS2DFSGP-30568-0C & $2.457 \mathrm{E}-04$ & $6.802 \mathrm{E}-04$ & $6.340 \mathrm{E}-06$ & $3.046 \mathrm{E}-03$ & $2.675 \mathrm{E}-03$ & $1.097 \mathrm{E}-04$ & (1) \\
\hline ESO $467-G 058$ & $2003-10-24$ & 1684 & MIS2DFSGP-40526-0: & $2.401 \mathrm{E}-04$ & $3.885 \mathrm{E}-04$ & $1.788 \mathrm{E}-06$ & $3.034 \mathrm{E}-03$ & $1.520 \mathrm{E}-03$ & $3.236 \mathrm{E}-05$ & \\
\hline ESO 345-G011 & $2003-10-23$ & 1694 & MIS2DFSGP-40620-0590 & $2.753 \mathrm{E}-04$ & $4.849 \mathrm{E}-04$ & $3.795 \mathrm{E}-06$ & $2.839 \mathrm{E}-03$ & $1.463 \mathrm{E}-03$ & $4.677 \mathrm{E}-05$ & \\
\hline NGC 7279 & $2003-10-24$ & 682 & MIS2DFSGP-40601-0532 & $2.302 \mathrm{E}-04$ & $5.909 \mathrm{E}-04$ & $2.003 \mathrm{E}-06$ & $2.863 \mathrm{E}-03$ & $2.283 \mathrm{E}-03$ & $3.316 \mathrm{E}-05$ & \\
\hline PKS 2225-308 & 2003-10-24 & 1684 & MIS2DFSGP-40526-0338 & $2.494 \mathrm{E}-04$ & $4.281 \mathrm{E}-04$ & $1.892 \mathrm{E}-05$ & $3.113 \mathrm{E}-03$ & $1.608 \mathrm{E}-03$ & $1.119 \mathrm{E}-04$ & (1) \\
\hline NGC 7289 & $2003-10-24$ & 682 & MIS2DFSGP-40601-0532 & $2.520 \mathrm{E}-04$ & $6.839 \mathrm{E}-04$ & $8.678 \mathrm{E}-06$ & $2.890 \mathrm{E}-03$ & $2.416 \mathrm{E}-03$ & $1.088 \mathrm{E}-04$ & (1) \\
\hline ESO 468-G006 & $2003-10-24$ & 1620 & MIS2DFSGP-30759-0180 & $2.694 \mathrm{E}-04$ & $5.158 \mathrm{E}-04$ & 4.070E-06 & $3.107 \mathrm{E}-03$ & $1.554 \mathrm{E}-03$ & $5.678 \mathrm{E}-05$ & \\
\hline NGC 7317 & $2003-08-23$ & 3322 & NGA-HCG092 & $9.787 \mathrm{E}-04$ & $5.970 \mathrm{E}-04$ & $2.301 \mathrm{E}-05$ & $4.731 \mathrm{E}-03$ & $1.440 \mathrm{E}-03$ & $2.661 \mathrm{E}-05$ & \\
\hline
\end{tabular}


Table 2-Continued

\begin{tabular}{|c|c|c|c|c|c|c|c|c|c|c|}
\hline \multirow{2}{*}{$\begin{array}{c}\text { Object Name } \\
\text { (1) }\end{array}$} & \multirow{2}{*}{$\begin{array}{c}\text { Date } \\
\text { Observed } \\
(2)\end{array}$} & \multirow{2}{*}{$\begin{array}{l}\text { Exposure } \\
\quad(\mathrm{sec}) \\
(3)\end{array}$} & \multirow{2}{*}{$\begin{array}{l}\text { Tile } \\
\text { (4) }\end{array}$} & \multicolumn{3}{|c|}{ FUV sky background } & \multicolumn{3}{|c|}{ NUV sky background } & \multirow{2}{*}{$\begin{array}{l}\text { notes } \\
\text { (11) }\end{array}$} \\
\hline & & & & $\begin{array}{c}\text { mean } \\
(\text { counts/s) } \\
(5)\end{array}$ & $\begin{array}{c}<\sigma> \\
(\text { counts/s) } \\
(6)\end{array}$ & $\begin{array}{c}\sigma(\text { mean }) \\
(\text { counts/s) } \\
(7)\end{array}$ & $\begin{array}{c}\text { mean } \\
(\text { counts/s) } \\
(8)\end{array}$ & $\begin{array}{c}<\sigma> \\
(\text { counts/s) } \\
(9)\end{array}$ & $\begin{array}{c}\sigma(\text { mean }) \\
(\text { counts/s) } \\
(10)\end{array}$ & \\
\hline NGC 7320 & 2003-08-23 & 3322 & NGA-HCG092 & $9.744 \mathrm{E}-04$ & $5.939 \mathrm{E}-04$ & $1.423 \mathrm{E}-05$ & $4.716 \mathrm{E}-03$ & $1.439 \mathrm{E}-03$ & $2.675 \mathrm{E}-05$ & \\
\hline UGC 12110 & $2003-08-23$ & 27976 & CCS-Q2233 & $4.355 \mathrm{E}-04$ & $1.420 \mathrm{E}-04$ & $9.848 \mathrm{E}-06$ & $3.104 \mathrm{E}-03$ & $3.791 \mathrm{E}-04$ & $3.065 \mathrm{E}-05$ & \\
\hline NGC 7331 & 2003-08-23 & 3322 & NGA-HCG092 & 9.907E-04 & $5.919 \mathrm{E}-04$ & $3.806 \mathrm{E}-07$ & $4.784 \mathrm{E}-03$ & $1.437 \mathrm{E}-03$ & $9.051 \mathrm{E}-05$ & \\
\hline NGC 7335 & 2003-08-23 & 3322 & NGA-HCG092 & $9.841 \mathrm{E}-04$ & $5.938 \mathrm{E}-04$ & $3.417 \mathrm{E}-05$ & $4.743 \mathrm{E}-03$ & $1.425 \mathrm{E}-03$ & $2.152 \mathrm{E}-05$ & \\
\hline NGC 7337 & 2003-08-23 & 3322 & NGA-HCG092 & $9.708 \mathrm{E}-04$ & $5.824 \mathrm{E}-04$ & $9.029 \mathrm{E}-05$ & $4.736 \mathrm{E}-03$ & $1.426 \mathrm{E}-03$ & $1.545 \mathrm{E}-04$ & \\
\hline NGC 7343 & 2003-08-23 & 3322 & NGA-HCG092 & 8.783E-04 & $5.578 \mathrm{E}-04$ & $4.654 \mathrm{E}-06$ & $4.490 \mathrm{E}-03$ & $1.361 \mathrm{E}-03$ & $4.618 \mathrm{E}-05$ & \\
\hline UGC 12134 & 2003-08-24 & 24199 & CCS-DSF2237 & $4.937 \mathrm{E}-04$ & $1.542 \mathrm{E}-04$ & $3.636 \mathrm{E}-05$ & $4.478 \mathrm{E}-03$ & $5.140 \mathrm{E}-04$ & $3.137 \mathrm{E}-05$ & \\
\hline NGC 7348 & 2003-08-24 & 24199 & CCS-DSF2237 & $4.876 \mathrm{E}-04$ & $1.523 \mathrm{E}-04$ & $4.439 \mathrm{E}-05$ & $4.514 \mathrm{E}-03$ & $5.147 \mathrm{E}-04$ & $9.379 \mathrm{E}-05$ & \\
\hline IRAS 22491-1808 & $2003-10-23$ & 1696 & NGA-IRAS22491 & $2.660 \mathrm{E}-04$ & $4.201 \mathrm{E}-04$ & $2.684 \mathrm{E}-06$ & $3.219 \mathrm{E}-03$ & $1.628 \mathrm{E}-03$ & $2.922 \mathrm{E}-05$ & \\
\hline NGC 7396 & $2004-08-27$ & 1705 & MISDR2-21350-0379 & $4.129 \mathrm{E}-04$ & $5.041 \mathrm{E}-04$ & $9.548 \mathrm{E}-06$ & $3.300 \mathrm{E}-03$ & $1.572 \mathrm{E}-03$ & $3.710 \mathrm{E}-05$ & \\
\hline ESO 346-G006 & 2003-08-03 & 24289 & EISD1AB & $2.035 \mathrm{E}-04$ & $9.905 \mathrm{E}-05$ & $9.129 \mathrm{E}-06$ & $2.414 \mathrm{E}-03$ & $3.651 \mathrm{E}-04$ & $4.567 \mathrm{E}-05$ & \\
\hline NGC 7398 & $2004-08-27$ & 1705 & MISDR2-21350-0379 & $4.031 \mathrm{E}-04$ & $4.945 \mathrm{E}-04$ & $1.985 \mathrm{E}-05$ & $3.271 \mathrm{E}-03$ & $1.543 \mathrm{E}-03$ & $1.533 \mathrm{E}-05$ & \\
\hline UGC 12250 & $2004-08-27$ & 2922 & MISDR2-21011-0741 & $3.269 \mathrm{E}-04$ & $4.104 \mathrm{E}-04$ & $3.120 \mathrm{E}-05$ & $2.966 \mathrm{E}-03$ & $1.167 \mathrm{E}-03$ & $8.105 \mathrm{E}-05$ & \\
\hline UGC 12253 & $2004-08-27$ & 2922 & MISDR2-21011-0741 & $3.248 \mathrm{E}-04$ & $4.064 \mathrm{E}-04$ & $2.314 \mathrm{E}-05$ & $2.980 \mathrm{E}-03$ & $1.172 \mathrm{E}-03$ & $3.289 \mathrm{E}-05$ & \\
\hline NGC 7418 & $2003-10-21$ & 1679 & NGA-NGC7418 & $2.278 \mathrm{E}-04$ & $3.901 \mathrm{E}-04$ & $1.926 \mathrm{E}-06$ & $2.631 \mathrm{E}-03$ & $1.470 \mathrm{E}-03$ & $2.942 \mathrm{E}-05$ & \\
\hline NGC 7418A & $2003-10-21$ & 1679 & NGA-NGC7418 & 2.363E-04 & $4.136 \mathrm{E}-04$ & $1.632 \mathrm{E}-05$ & $2.664 \mathrm{E}-03$ & $1.478 \mathrm{E}-03$ & $7.422 \mathrm{E}-05$ & \\
\hline ESO 534-G032 & 2004-10-07 & 1666 & MIS2DFSGP-30497-0069 & $2.247 \mathrm{E}-04$ & $4.186 \mathrm{E}-04$ & $2.039 \mathrm{E}-06$ & $2.726 \mathrm{E}-03$ & $1.512 \mathrm{E}-03$ & $1.585 \mathrm{E}-06$ & \\
\hline IC 5264 & $2003-10-21$ & 1679 & NGA-NGC7418 & $2.388 \mathrm{E}-04$ & $4.205 \mathrm{E}-04$ & $1.872 \mathrm{E}-05$ & $2.690 \mathrm{E}-03$ & $1.457 \mathrm{E}-03$ & $1.893 \mathrm{E}-05$ & \\
\hline NGC 7421 & $2003-10-21$ & 1679 & NGA-NGC7418 & $2.332 \mathrm{E}-04$ & $4.368 \mathrm{E}-04$ & $2.560 \mathrm{E}-06$ & $2.587 \mathrm{E}-03$ & $1.424 \mathrm{E}-03$ & $1.252 \mathrm{E}-04$ & \\
\hline NGC 7432 & $2004-08-27$ & 2922 & MISDR2-21011-0741 & $3.789 \mathrm{E}-04$ & $4.542 \mathrm{E}-04$ & $5.737 \mathrm{E}-06$ & $3.080 \mathrm{E}-03$ & $1.173 \mathrm{E}-03$ & $4.528 \mathrm{E}-05$ & \\
\hline ARP 314 NED01 & $2003-10-25$ & 1638 & NGRG-A314 & $4.405 \mathrm{E}-04$ & $5.274 \mathrm{E}-04$ & $1.105 \mathrm{E}-05$ & $3.644 \mathrm{E}-03$ & $1.716 \mathrm{E}-03$ & $5.876 \mathrm{E}-05$ & \\
\hline ARP 314 NED03 & $2003-10-25$ & 1638 & NGRG-A314 & $4.405 \mathrm{E}-04$ & $5.279 \mathrm{E}-04$ & $4.927 \mathrm{E}-06$ & $3.644 \mathrm{E}-03$ & $1.723 \mathrm{E}-03$ & $1.201 \mathrm{E}-05$ & (8) \\
\hline ARP 314 NED02 & $2003-10-25$ & 1638 & NGRG-A314 & 4.409E-04 & $5.276 \mathrm{E}-04$ & $4.450 \mathrm{E}-06$ & $3.645 \mathrm{E}-03$ & $1.720 \mathrm{E}-03$ & $3.162 \mathrm{E}-05$ & (2) \\
\hline UGC 12285 & $2004-08-27$ & 2922 & MISDR2-21011-0741 & 3.893E-04 & $4.790 \mathrm{E}-04$ & $1.397 \mathrm{E}-05$ & $3.223 \mathrm{E}-03$ & $1.156 \mathrm{E}-03$ & $1.741 \mathrm{E}-05$ & \\
\hline ESO 406-G042 & 2004-10-07 & 1666 & MIS2DFSGP-40741-0598 & $1.877 \mathrm{E}-04$ & $4.172 \mathrm{E}-04$ & $1.891 \mathrm{E}-06$ & $2.337 \mathrm{E}-03$ & $1.315 \mathrm{E}-03$ & $3.424 \mathrm{E}-05$ & \\
\hline NGC 7469 & 2003-09-04 & 3770 & NGA-NGC7469 & $3.906 \mathrm{E}-04$ & $3.416 \mathrm{E}-04$ & $1.450 \mathrm{E}-06$ & $3.318 \mathrm{E}-03$ & $1.120 \mathrm{E}-03$ & $8.729 \mathrm{E}-05$ & \\
\hline NGC 7479 & $2004-10-02$ & 1606 & NGA-NGC7479 & $3.768 \mathrm{E}-04$ & $5.136 \mathrm{E}-04$ & $1.398 \mathrm{E}-05$ & $3.143 \mathrm{E}-03$ & $1.646 \mathrm{E}-03$ & $1.335 \mathrm{E}-04$ & \\
\hline UGC 12346 & 2004-09-10 & 1575 & MISDR2-29085-0380 & $\cdots$ & $\ldots$ & $\cdots$ & $3.331 \mathrm{E}-03$ & $1.635 \mathrm{E}-03$ & $3.142 \mathrm{E}-05$ & (4) \\
\hline UGC 12354 & $2004-08-27$ & 1705 & MISDR2-21072-0742 & $5.179 \mathrm{E}-04$ & $6.345 \mathrm{E}-04$ & $8.238 \mathrm{E}-05$ & $4.798 \mathrm{E}-03$ & $2.041 \mathrm{E}-03$ & $3.493 \mathrm{E}-04$ & \\
\hline ESO 469-G012 & 2004-10-06 & 1650 & MIS2DFSGP-30685-0187 & $2.375 \mathrm{E}-04$ & $4.221 \mathrm{E}-04$ & $1.063 \mathrm{E}-05$ & $2.685 \mathrm{E}-03$ & $1.405 \mathrm{E}-03$ & $5.732 \mathrm{E}-05$ & \\
\hline ESO 469-G015 & $2004-10-06$ & 1651 & MIS2DFSGP-42827-0346 & $2.231 \mathrm{E}-04$ & 4.199E-04 & $2.027 \mathrm{E}-05$ & $2.637 \mathrm{E}-03$ & $1.488 \mathrm{E}-03$ & $5.193 \mathrm{E}-05$ & \\
\hline IC 5287 & $2003-08-24$ & 3181 & MISDR1-29084-0381 & $3.465 \mathrm{E}-04$ & $3.936 \mathrm{E}-04$ & $2.111 \mathrm{E}-05$ & $3.288 \mathrm{E}-03$ & $1.210 \mathrm{E}-03$ & $4.127 \mathrm{E}-06$ & \\
\hline
\end{tabular}


Table 2-Continued

\begin{tabular}{|c|c|c|c|c|c|c|c|c|c|c|}
\hline \multirow{2}{*}{$\begin{array}{c}\text { Object Name } \\
\text { (1) }\end{array}$} & \multirow{2}{*}{$\begin{array}{c}\text { Date } \\
\text { Observed } \\
(2)\end{array}$} & \multirow{2}{*}{$\begin{array}{c}\text { Exposure } \\
\quad(\mathrm{sec}) \\
(3)\end{array}$} & \multirow{2}{*}{$\begin{array}{l}\text { Tile } \\
(4) \\
\end{array}$} & \multicolumn{3}{|c|}{ FUV sky background } & \multicolumn{3}{|c|}{ NUV sky background } & \multirow{2}{*}{$\begin{array}{l}\text { notes } \\
(11)\end{array}$} \\
\hline & & & & $\begin{array}{c}\text { mean } \\
(\text { counts/s) } \\
(5)\end{array}$ & $\begin{array}{c}<\sigma> \\
(\text { counts/s) } \\
(6)\end{array}$ & $\begin{array}{c}\sigma(\text { mean }) \\
(\text { counts/s) } \\
(7)\end{array}$ & $\begin{array}{c}\text { mean } \\
(\text { counts/s) } \\
(8)\end{array}$ & $\begin{array}{c}<\sigma> \\
(\text { counts/s) } \\
(9)\end{array}$ & $\begin{array}{c}\sigma(\text { mean }) \\
(\text { counts/s }) \\
(10)\end{array}$ & \\
\hline ESO 407-G007 & 2004-10-07 & 1663 & MIS2DFSGP-40790-0599 & $1.759 \mathrm{E}-04$ & $3.370 \mathrm{E}-04$ & $7.090 \mathrm{E}-06$ & $2.354 \mathrm{E}-03$ & $1.344 \mathrm{E}-03$ & $4.604 \mathrm{E}-05$ & \\
\hline NGC 7496 & $2003-10-22$ & 1099 & NGA-NGC7496 & $2.243 \mathrm{E}-04$ & $4.600 \mathrm{E}-04$ & $2.692 \mathrm{E}-05$ & $2.707 \mathrm{E}-03$ & $1.775 \mathrm{E}-03$ & $3.760 \mathrm{E}-05$ & \\
\hline ESO 291-G005 & $2003-10-22$ & 1099 & NGA-NGC7496 & $2.378 \mathrm{E}-04$ & $5.369 \mathrm{E}-04$ & $7.543 \mathrm{E}-06$ & $2.718 \mathrm{E}-03$ & $1.851 \mathrm{E}-03$ & $1.114 \mathrm{E}-04$ & \\
\hline ESO 291-G006 & $2003-10-22$ & 1099 & NGA-NGC7496 & $2.407 \mathrm{E}-04$ & $5.796 \mathrm{E}-04$ & $1.805 \mathrm{E}-05$ & $2.683 \mathrm{E}-03$ & $1.795 \mathrm{E}-03$ & $4.240 \mathrm{E}-05$ & \\
\hline NGC 7511 & 2004-09-25 & 1657 & MISDR2-21201-0743 & $3.696 \mathrm{E}-04$ & $5.243 \mathrm{E}-04$ & $2.689 \mathrm{E}-05$ & $3.027 \mathrm{E}-03$ & $1.600 \mathrm{E}-03$ & $2.538 \mathrm{E}-05$ & \\
\hline ESO 407-G009 & 2004-10-07 & 1663 & MIS2DFSGP-40790-0599 & $2.086 \mathrm{E}-04$ & 4.601E-04 & $1.237 \mathrm{E}-05$ & $2.375 \mathrm{E}-03$ & $1.320 \mathrm{E}-03$ & $5.574 \mathrm{E}-06$ & \\
\hline ESO 291-G009 & 2003-08-04 & 3400 & NGA-NGC7552 & $1.911 \mathrm{E}-04$ & $2.573 \mathrm{E}-04$ & $8.004 \mathrm{E}-07$ & $2.357 \mathrm{E}-03$ & $9.530 \mathrm{E}-04$ & $6.277 \mathrm{~F}$ & \\
\hline UGC 12434 & 2004-09-25 & 1657 & MISDR2-21201-0743 & $3.438 \mathrm{E}-04$ & 4.643E-04 & $4.963 \mathrm{E}-06$ & $2.978 \mathrm{E}-03$ & $1.509 \mathrm{E}-03$ & $1.443 \mathrm{E}-05$ & \\
\hline NGC 7535 & 2004-09-25 & 1657 & MISDR2-21201-0743 & $3.740 \mathrm{E}-04$ & $5.150 \mathrm{E}-04$ & $1.106 \mathrm{E}-05$ & $3.058 \mathrm{E}-03$ & $1.568 \mathrm{E}-03$ & $4.430 \mathrm{E}-06$ & \\
\hline NGC 7536 & 2004-09-25 & 1657 & MISDR2-21201-0743 & $3.615 \mathrm{E}-04$ & $4.922 \mathrm{E}-04$ & $1.394 \mathrm{E}-05$ & $3.022 \mathrm{E}-03$ & $1.539 \mathrm{E}-03$ & $4.292 \mathrm{E}-05$ & \\
\hline NGC $7559 \mathrm{~B}$ & 2004-09-19 & 1705 & MISDR2-21267-0744 & $\cdots$ & $\cdots$ & $\cdots$ & $2.982 \mathrm{E}-03$ & $1.508 \mathrm{E}-03$ & $1.635 \mathrm{E}-05$ & (5) \\
\hline NGC 7563 & 2004-09-19 & 1705 & MISDR2-21267-0744 & $3.548 \mathrm{E}-04$ & $5.525 \mathrm{E}-04$ & $2.482 \mathrm{E}-05$ & $2.980 \mathrm{E}-03$ & $1.509 \mathrm{E}-03$ & $1.554 \mathrm{E}-05$ & \\
\hline NGC 7552 & 2003-08-04 & 3400 & NGA-NGC7552 & $1.978 \mathrm{E}-04$ & $2.561 \mathrm{E}-04$ & $7.912 \mathrm{E}-06$ & $2.361 \mathrm{E}-03$ & $9.738 \mathrm{E}-04$ & $2.630 \mathrm{E}-05$ & \\
\hline UGC 12479 & 2003-08-24 & 1380 & MISDR1-29148-0382 & $3.405 \mathrm{E}-04$ & $5.243 \mathrm{E}-04$ & $1.198 \mathrm{E}-05$ & $3.344 \mathrm{E}-03$ & $1.761 \mathrm{E}-03$ & $6.381 \mathrm{E}-05$ & \\
\hline ESO 407-G014 & 2004-10-07 & 1668 & MIS2DFSGP-40788-0540 & $2.067 \mathrm{E}-04$ & 4.070E-04 & $1.340 \mathrm{E}-06$ & $2.419 \mathrm{E}-03$ & $1.419 \mathrm{E}-03$ & $1.672 \mathrm{E}-05$ & \\
\hline NGC 7589 & $2003-08-25$ & 1494 & MISDR1-29082-0382 & $3.306 \mathrm{E}-04$ & $4.782 \mathrm{E}-04$ & $7.241 \mathrm{E}-06$ & $3.289 \mathrm{E}-03$ & $1.676 \mathrm{E}-03$ & $8.273 \mathrm{E}-05$ & (1) \\
\hline NGC 7582 & 2003-08-04 & 3400 & NGA-NGC7552 & $2.244 \mathrm{E}-04$ & $3.121 \mathrm{E}-04$ & $1.273 \mathrm{E}-06$ & $2.388 \mathrm{E}-03$ & $9.565 \mathrm{E}-04$ & $1.422 \mathrm{E}-05$ & \\
\hline PGC 71025 & $2003-08-24$ & 1458 & MISDR1-29582-0645 & $4.100 \mathrm{E}-04$ & $6.206 \mathrm{E}-04$ & $6.545 \mathrm{E}-05$ & $4.267 \mathrm{E}-03$ & $2.037 \mathrm{E}-03$ & $1.964 \mathrm{E}-04$ & (1) \\
\hline IC 5304 & 2003-08-24 & 1458 & MISDR1-29582-0645 & $4.264 \mathrm{E}-04$ & $6.364 \mathrm{E}-04$ & $2.698 \mathrm{E}-05$ & $4.128 \mathrm{E}-03$ & $1.981 \mathrm{E}-03$ & $3.268 \mathrm{E}-04$ & (1) \\
\hline NGC 7645 & 2004-10-05 & 1641 & MIS2DFSGP-30746-0269 & $2.755 \mathrm{E}-04$ & $4.159 \mathrm{E}-04$ & $2.693 \mathrm{E}-05$ & $2.526 \mathrm{E}-03$ & $1.393 \mathrm{E}-03$ & $8.039 \mathrm{E}-06$ & \\
\hline UGC 12578 & $2003-08-25$ & 1512 & MISDR1-29113-0383 & $3.789 \mathrm{E}-04$ & $6.368 \mathrm{E}-04$ & $4.057 \mathrm{E}-06$ & $3.319 \mathrm{E}-03$ & $1.670 \mathrm{E}-03$ & $3.008 \mathrm{E}-06$ & \\
\hline UGC 12589 & $2003-08-25$ & 1514 & MISDR1-29080-0383 & $3.553 \mathrm{E}-04$ & $5.213 \mathrm{E}-04$ & $1.901 \mathrm{E}-05$ & $3.431 \mathrm{E}-03$ & $1.708 \mathrm{E}-03$ & $1.554 \mathrm{E}-06$ & \\
\hline CGCG 406-109 & 2003-10-05 & 1666 & NGRG-HCG96 & $3.198 \mathrm{E}-04$ & $4.663 \mathrm{E}-04$ & $3.226 \mathrm{E}-05$ & $3.078 \mathrm{E}-03$ & $1.570 \mathrm{E}-03$ & $6.758 \mathrm{E}-05$ & $(1)(2)$ \\
\hline NGC 7673 & 2004-08-29 & 1539 & NGA-NGC7673 & $3.546 \mathrm{E}-04$ & $5.369 \mathrm{E}-04$ & $9.948 \mathrm{E}-06$ & $2.955 \mathrm{E}-03$ & $1.651 \mathrm{E}-03$ & $6.425 \mathrm{E}-05$ & \\
\hline NGC 7674 & 2003-10-05 & 1666 & NGRG-HCG96 & $3.182 \mathrm{E}-04$ & $4.641 \mathrm{E}-04$ & $1.439 \mathrm{E}-06$ & $3.113 \mathrm{E}-03$ & $1.607 \mathrm{E}-03$ & $1.487 \mathrm{E}-04$ & \\
\hline NGC 7677 & 2004-08-29 & 1539 & NGA-NGC7673 & $3.469 \mathrm{E}-04$ & $5.221 \mathrm{E}-04$ & $5.495 \mathrm{E}-06$ & $2.946 \mathrm{E}-03$ & $1.633 \mathrm{E}-03$ & $3.757 \mathrm{E}-05$ & \\
\hline IC 5325 & $2003-10-22$ & 1346 & NGA-IC5325 & $2.841 \mathrm{E}-04$ & 4.856E-04 & $4.164 \mathrm{E}-05$ & $2.680 \mathrm{E}-03$ & $1.648 \mathrm{E}-03$ & $4.909 \mathrm{E}-05$ & \\
\hline UGC 12635 & $2003-08-25$ & 3047 & MISDR1-29079-0383 & $3.792 \mathrm{E}-04$ & $4.444 \mathrm{E}-04$ & $8.522 \mathrm{E}-06$ & $3.388 \mathrm{E}-03$ & $1.199 \mathrm{E}-03$ & $6.612 \mathrm{E}-05$ & \\
\hline NGC 7684 & $2003-08-25$ & 3047 & MISDR1-29079-0383 & $3.702 \mathrm{E}-04$ & $4.543 \mathrm{E}-04$ & $2.829 \mathrm{E}-06$ & $3.358 \mathrm{E}-03$ & $1.177 \mathrm{E}-03$ & $4.492 \mathrm{E}-05$ & \\
\hline UGC 12685 & $2004-10-17$ & 17334 & DEEP23H-02 & $2.928 \mathrm{E}-04$ & $1.556 \mathrm{E}-04$ & $1.169 \mathrm{E}-05$ & $3.049 \mathrm{E}-03$ & $4.842 \mathrm{E}-04$ & $1.511 \mathrm{E}-05$ & 8) \\
\hline
\end{tabular}


Table 2-Continued

\begin{tabular}{|c|c|c|c|c|c|c|c|c|c|c|}
\hline \multirow{2}{*}{$\begin{array}{c}\text { Object Name } \\
\text { (1) }\end{array}$} & \multirow{2}{*}{$\begin{array}{c}\text { Date } \\
\text { Observed } \\
(2)\end{array}$} & \multirow{2}{*}{$\begin{array}{l}\text { Exposure } \\
\quad(\mathrm{sec}) \\
(3)\end{array}$} & \multirow{2}{*}{$\begin{array}{l}\text { Tile } \\
\text { (4) }\end{array}$} & \multicolumn{3}{|c|}{ FUV sky background } & \multicolumn{3}{|c|}{ NUV sky background } & \multirow{2}{*}{$\begin{array}{r}\text { notes } \\
(11)\end{array}$} \\
\hline & & & & $\begin{array}{c}\text { mean } \\
(\text { counts/s) } \\
(5)\end{array}$ & $\begin{array}{c}<\sigma> \\
(\text { counts/s) } \\
(6)\end{array}$ & $\begin{array}{c}\sigma(\text { mean }) \\
(\text { counts/s) } \\
(7)\end{array}$ & $\begin{array}{c}\text { mean } \\
(\text { counts } / \mathrm{s}) \\
(8)\end{array}$ & $\begin{array}{c}<\sigma> \\
(\text { counts } / \mathrm{s}) \\
(9)\end{array}$ & $\begin{array}{c}\sigma(\text { mean }) \\
(\text { counts/s) } \\
(10)\end{array}$ & \\
\hline IRAS $23365+3604$ & 2003-09-04 & 4426 & NGA-IRAS23365 & $7.482 \mathrm{E}-04$ & $4.192 \mathrm{E}-04$ & $1.082 \mathrm{E}-05$ & $4.185 \mathrm{E}-03$ & $1.129 \mathrm{E}-03$ & $2.738 \mathrm{E}-05$ & \\
\hline ARP 295A & 2003-10-05 & 1233 & NGRG-A295 & $2.568 \mathrm{E}-04$ & $4.841 \mathrm{E}-04$ & $7.229 \mathrm{E}-06$ & $3.191 \mathrm{E}-03$ & $1.882 \mathrm{E}-03$ & $2.342 \mathrm{E}-05$ & (1) \\
\hline NGC 7735 & 2003-10-15 & 1639 & NGA-NGC7741 & $4.533 \mathrm{E}-04$ & $6.486 \mathrm{E}-04$ & $1.849 \mathrm{E}-05$ & $3.114 \mathrm{E}-03$ & $1.568 \mathrm{E}-03$ & $9.595 \mathrm{E}-05$ & \\
\hline NGC 7741 & $2003-10-15$ & 1639 & NGA-NGC7741 & $4.158 \mathrm{E}-04$ & $5.376 \mathrm{E}-04$ & $9.426 \mathrm{E}-06$ & $3.088 \mathrm{E}-03$ & $1.617 \mathrm{E}-03$ & 7.362E-05 & \\
\hline NGC 7771 & $2003-10-20$ & 1663 & NGA-NGC7771 & 4.113E-04 & $5.314 \mathrm{E}-04$ & $1.929 \mathrm{E}-06$ & $3.180 \mathrm{E}-03$ & $1.627 \mathrm{E}-03$ & $1.070 \mathrm{E}-06$ & \\
\hline CGCG 432-040 & 2004-08-27 & 1705 & MISDR2-28673-0749 & $2.911 \mathrm{E}-04$ & $4.427 \mathrm{E}-04$ & $1.343 \mathrm{E}-06$ & $2.752 \mathrm{E}-03$ & $1.477 \mathrm{E}-03$ & $1.601 \mathrm{E}-05$ & (1) \\
\hline NGC 7793 & 2003-10-14 & 1490 & NGA-NGC7793 & $2.645 \mathrm{E}-04$ & $4.516 \mathrm{E}-04$ & $2.662 \mathrm{E}-05$ & $2.724 \mathrm{E}-03$ & $1.597 \mathrm{E}-03$ & $1.677 \mathrm{E}-05$ & \\
\hline ESO 349-G014 & 2003-10-14 & 1490 & NGA-NGC7793 & $2.132 \mathrm{E}-04$ & $4.106 \mathrm{E}-04$ & $1.753 \mathrm{E}-05$ & $2.618 \mathrm{E}-03$ & $1.544 \mathrm{E}-03$ & $5.794 \mathrm{E}-05$ & \\
\hline NGC 7798 & 2003-09-09 & 1668 & NGA-NGC7798 & $4.155 \mathrm{E}-04$ & $6.076 \mathrm{E}-04$ & $9.496 \mathrm{E}-05$ & $3.143 \mathrm{E}-03$ & $1.640 \mathrm{E}-03$ & $1.143 \mathrm{E}-04$ & \\
\hline
\end{tabular}

Note. - Log of GALEX observations. Col. (1): Galaxy name. Col. (2): Date the galaxy was observed. Col. (3): Total exposure time in seconds for each of the GALEX bands. Col. (4): Tile name assigned by the GALEX mission. It includes the name of the survey as part of which the galaxy was observed and the name of the targetted field. Col. (5): Mean sky background of the FUV image (in counts per second). Col. (6): Mean standard deviation of the sky in the FUV image measured by averaging the standard deviation within several regions around the position of the object. Col. (7): Standard deviation of the mean values of the sky measured in these regions. Cols. $(8,9,10)$ : The same for the NUV image. Col. (11): Notes for individual objects: (1) Nominal galaxy position angle changed, (2) nominal galaxy coordinates changed, (3) special pipeline processing, (4) NUV-only, (5) very shallow FUV image, (6) very bright star near or on top of the galaxy, (7) nominal galaxy size changed, (8) crowded field, uncertain photometry. 
Table 3. UV properties

\begin{tabular}{|c|c|c|c|c|c|c|c|c|c|c|c|c|c|c|c|}
\hline \multirow{2}{*}{$\begin{array}{c}\text { Object Name } \\
(1) \\
\end{array}$} & \multicolumn{3}{|c|}{ Asymptotic magnitudes and color } & \multicolumn{3}{|c|}{ D25 magnitudes and color } & \multicolumn{2}{|c|}{$\log L$} & \multicolumn{2}{|c|}{ Effective Radii } & \multicolumn{2}{|c|}{$\mathrm{C} 31$} & \multicolumn{2}{|c|}{$\mathrm{C} 42$} & \multirow{2}{*}{$\begin{array}{r}\text { UV } \\
\text { profile } \\
(16)\end{array}$} \\
\hline & $\begin{array}{c}\text { FUV } \\
(\mathrm{mag}) \\
(2)\end{array}$ & $\begin{array}{c}\text { NUV } \\
(\mathrm{mag}) \\
(3)\end{array}$ & $\begin{array}{l}\text { FUV-NUV } \\
(\mathrm{mag}) \\
(4)\end{array}$ & $\begin{array}{c}\text { FUV } \\
(\mathrm{mag}) \\
(5)\end{array}$ & $\begin{array}{c}\text { NUV } \\
(\mathrm{mag}) \\
(6)\end{array}$ & $\begin{array}{c}\text { FUV-NUV } \\
\text { (mag) } \\
(7)\end{array}$ & $\begin{array}{c}\text { FUV } \\
(\mathrm{W}) \\
(8)\end{array}$ & $\begin{array}{l}\text { NUV } \\
(\mathrm{W}) \\
(9)\end{array}$ & $\begin{array}{c}\text { FUV } \\
(\operatorname{arcsec}) \\
(10)\end{array}$ & $\begin{array}{c}\text { NUV } \\
(\operatorname{arcsec}) \\
(11)\end{array}$ & $\begin{array}{l}\text { FUV } \\
(12)\end{array}$ & $\begin{array}{l}\text { NUV } \\
(13)\end{array}$ & $\begin{array}{l}\text { FUV } \\
(14)\end{array}$ & $\begin{array}{l}\text { NUV } \\
(15)\end{array}$ & \\
\hline WLM & $12.47 \pm 0.01$ & $12.34 \pm 0.01$ & $0.13 \pm 0.01$ & $12.54 \pm 0.01$ & $12.42 \pm 0.01$ & $0.12 \pm 0.01$ & 33.93 & 33.80 & 90.89 & 92.77 & 2.11 & 2.15 & 1.97 & 2.08 & $\mathrm{EF}$ \\
\hline NGC 7808 & $17.49 \pm 0.03$ & $16.77 \pm 0.03$ & $0.72 \pm 0.04$ & $17.52 \pm 0.05$ & $16.88 \pm 0.02$ & $0.64 \pm 0.05$ & 36.13 & 36.24 & $\ldots$ & $\ldots$ & $\ldots$ & $\ldots$ & $\ldots$ & $\ldots$ & $\mathrm{EE}$ \\
\hline UGC 00017 & $16.86 \pm 0.03$ & $16.47 \pm 0.01$ & $0.39 \pm 0.03$ & $17.01 \pm 0.02$ & $16.65 \pm 0.01$ & $0.35 \pm 0.03$ & 34.44 & 34.42 & 31.81 & 32.48 & 2.32 & 2.52 & 2.37 & 2.61 & $\mathrm{EF}$ \\
\hline PGC 00282 & $16.29 \pm 0.04$ & $16.03 \pm 0.02$ & $0.26 \pm 0.04$ & $16.36 \pm 0.02$ & $16.11 \pm 0.01$ & $0.25 \pm 0.03$ & 36.83 & 36.76 & 14.55 & 14.43 & $\cdots$ & $\cdots$ & $\cdots$ & $\cdots$ & ED \\
\hline NGC 0024 & $14.04 \pm 0.01$ & $13.75 \pm 0.01$ & $0.29 \pm 0.01$ & $14.16 \pm 0.01$ & $13.87 \pm 0.01$ & $0.29 \pm 0.01$ & 35.15 & 35.09 & 35.98 & 36.16 & 2.74 & 2.73 & 2.71 & 2.71 & Ef \\
\hline UGC 00128 & $16.51 \pm 0.04$ & $16.33 \pm 0.02$ & $0.18 \pm 0.04$ & $16.65 \pm 0.03$ & $16.53 \pm 0.02$ & $0.12 \pm 0.03$ & 35.98 & 35.88 & 32.48 & 32.78 & 2.29 & 2.40 & 2.27 & 2.41 & $\mathrm{EFn}$ \\
\hline NGC 0055 & $10.20 \pm 0.01$ & $9.91 \pm 0.01$ & $0.29 \pm 0.01$ & $10.26 \pm 0.01$ & $9.97 \pm 0.01$ & $0.29 \pm 0.01$ & 35.46 & 35.40 & 155.54 & 149.95 & 2.80 & 2.87 & 2.79 & 2.79 & $\mathrm{EE}$ \\
\hline ARP 256 NED02 & $15.83 \pm 0.01$ & $15.39 \pm 0.01$ & $0.44 \pm 0.02$ & $16.09 \pm 0.01$ & $15.63 \pm 0.01$ & $0.46 \pm 0.02$ & 36.73 & 36.73 & 11.48 & 11.36 & $\cdots$ & $\cdots$ & $\cdots$ & $\cdots$ & $\mathrm{Er}$ \\
\hline ARP 256 NED01 & $15.85 \pm 0.03$ & $15.43 \pm 0.04$ & $0.43 \pm 0.05$ & $15.94 \pm 0.01$ & $15.54 \pm 0.01$ & $0.40 \pm 0.02$ & 36.72 & 36.71 & $\cdots$ & $\cdots$ & $\cdots$ & $\cdots$ & $\cdots$ & $\cdots$ & ER \\
\hline UGC 00226 & $16.65 \pm 0.02$ & $16.32 \pm 0.01$ & $0.33 \pm 0.03$ & $16.74 \pm 0.02$ & $16.41 \pm 0.01$ & $0.33 \pm 0.02$ & 36.05 & 36.00 & $\ldots$ & $\ldots$ & $\cdots$ & $\ldots$ & $\ldots$ & $\ldots$ & $\ldots$ \\
\hline NGC 0099 & $15.13 \pm 0.01$ & $14.90 \pm 0.01$ & $0.23 \pm 0.01$ & $15.22 \pm 0.01$ & $14.98 \pm 0.01$ & $0.24 \pm 0.01$ & 36.65 & 36.57 & 17.60 & 16.70 & $\cdots$ & $\ldots$ & $\ldots$ & $\ldots$ & $\mathrm{EF}$ \\
\hline UGC 00247 & $18.23 \pm 0.01$ & $17.63 \pm 0.02$ & $0.61 \pm 0.02$ & $18.54 \pm 0.03$ & $18.01 \pm 0.01$ & $0.53 \pm 0.03$ & 36.05 & 36.12 & 8.03 & 8.80 & $\ldots$ & $\ldots$ & $\ldots$ & $\ldots$ & $? \mathrm{~F}$ \\
\hline UGC 00249 & $16.30 \pm 0.02$ & $15.85 \pm 0.01$ & $0.45 \pm 0.02$ & $16.39 \pm 0.02$ & $15.96 \pm 0.01$ & $0.43 \pm 0.02$ & 36.17 & 36.18 & 14.45 & 14.02 & $\ldots$ & $\ldots$ & $\ldots$ & $\ldots$ & Vfn \\
\hline NGC 0115 & $15.24 \pm 0.01$ & $14.93 \pm 0.01$ & $0.31 \pm 0.01$ & $15.36 \pm 0.01$ & $15.05 \pm 0.01$ & $0.31 \pm 0.01$ & 35.58 & 35.53 & 19.09 & 19.12 & 2.47 & 2.53 & 2.44 & 2.52 & $\mathrm{EF}$ \\
\hline NGC 0131 & $15.94 \pm 0.03$ & $15.50 \pm 0.01$ & $0.44 \pm 0.03$ & $16.10 \pm 0.01$ & $15.63 \pm 0.01$ & $0.47 \pm 0.02$ & 35.05 & 35.05 & 13.62 & 13.61 & 2.26 & 2.31 & 2.33 & 2.38 & VF \\
\hline PGC 01862 & $16.85 \pm 0.02$ & $16.26 \pm 0.01$ & $0.59 \pm 0.02$ & $16.94 \pm 0.02$ & $16.45 \pm 0.01$ & $0.49 \pm 0.02$ & 35.96 & 36.02 & 10.21 & 11.32 & $\ldots$ & $\ldots$ & $\ldots$ & $\ldots$ & $? \mathrm{~F}$ \\
\hline UGC 00316 & $18.91 \pm 0.07$ & $18.25 \pm 0.04$ & $0.66 \pm 0.08$ & $19.28 \pm 0.05$ & $18.69 \pm 0.02$ & $0.59 \pm 0.05$ & 35.80 & 35.89 & 10.01 & 10.81 & 2.67 & 2.52 & $\ldots$ & 2.56 & $? \mathrm{~F}$ \\
\hline ESO $473-G 025$ & $20.00 \pm 0.21$ & $18.29 \pm 0.02$ & $1.71 \pm 0.21$ & $20.15 \pm 0.10$ & $18.65 \pm 0.03$ & $1.50 \pm 0.10$ & 34.93 & 35.44 & 15.21 & 18.20 & 2.08 & 2.32 & 1.89 & 2.31 & $\ldots$ \\
\hline IC 1554 & $16.27 \pm 0.01$ & $15.72 \pm 0.01$ & $0.55 \pm 0.01$ & $16.36 \pm 0.02$ & $15.82 \pm 0.01$ & $0.54 \pm 0.02$ & 35.12 & 35.16 & & $\ldots$ & $\ldots$ & $\ldots$ & $\ldots$ & $\ldots$ & ER \\
\hline UGC 00330 & $20.15 \pm 0.10$ & $18.87 \pm 0.06$ & $1.28 \pm 0.11$ & $\ldots$ & $18.89 \pm 0.06$ & $\ldots$ & 34.74 & 35.08 & 7.40 & 7.37 & $\ldots$ & $\ldots$ & $\ldots$ & $\ldots$ & $\ldots$ \\
\hline NGC 0151 & $14.56 \pm 0.01$ & $14.21 \pm 0.01$ & $0.35 \pm 0.01$ & $14.61 \pm 0.01$ & $14.26 \pm 0.01$ & $0.35 \pm 0.01$ & 36.55 & 36.51 & 41.25 & 39.66 & 1.85 & 1.92 & 1.63 & 1.74 & $? \mathrm{~F}$ \\
\hline NGC 0155 & $18.49 \pm 0.27$ & $17.59 \pm 0.10$ & $0.90 \pm 0.29$ & $19.08 \pm 0.11$ & $17.99 \pm 0.03$ & $1.09 \pm 0.11$ & 35.42 & 35.61 & 30.71 & 22.44 & 4.31 & $\ldots$ & $\ldots$ & $\ldots$ & $\ldots$ \\
\hline UGC 00344 & $16.71 \pm 0.01$ & $16.33 \pm 0.01$ & $0.38 \pm 0.02$ & $16.87 \pm 0.02$ & $16.51 \pm 0.01$ & $0.36 \pm 0.02$ & 36.11 & 36.08 & 13.44 & 13.46 & $\ldots$ & $\ldots$ & $\ldots$ & $\ldots$ & $\mathrm{EF}$ \\
\hline NGC 0163 & $19.12 \pm 0.16$ & $17.63 \pm 0.10$ & $1.49 \pm 0.19$ & $19.34 \pm 0.10$ & $18.19 \pm 0.03$ & $1.15 \pm 0.10$ & 35.14 & 35.56 & 12.36 & 26.49 & $\ldots$ & $\ldots$ & $\ldots$ & $\ldots$ & $\ldots$ \\
\hline VV 548 & $15.67 \pm 0.01$ & $15.41 \pm 0.01$ & $0.27 \pm 0.02$ & $15.75 \pm 0.01$ & $15.48 \pm 0.01$ & $0.27 \pm 0.02$ & 36.35 & 36.28 & 14.97 & 14.70 & $\ldots$ & $\ldots$ & $\ldots$ & $\ldots$ & $\mathrm{EF}$ \\
\hline NGC 0165 & $16.60 \pm 0.01$ & $16.04 \pm 0.01$ & $0.56 \pm 0.01$ & $16.71 \pm 0.02$ & $16.17 \pm 0.01$ & $0.54 \pm 0.02$ & 36.13 & 36.18 & 19.99 & 19.24 & 2.21 & $\ldots$ & $\ldots$ & $\ldots$ & $\mathrm{EF}$ \\
\hline UGC 00372 & $16.95 \pm 0.04$ & $16.73 \pm 0.01$ & $0.22 \pm 0.04$ & $17.01 \pm 0.03$ & $16.80 \pm 0.02$ & $0.22 \pm 0.03$ & 35.96 & 35.87 & 16.93 & 16.86 & $\ldots$ & $\ldots$ & $\ldots$ & $\ldots$ & $? \mathrm{~F}$ \\
\hline Cartwheel & $14.92 \pm 0.01$ & $14.73 \pm 0.01$ & $0.19 \pm 0.01$ & $15.90 \pm 0.02$ & $15.57 \pm 0.01$ & $0.33 \pm 0.02$ & 37.16 & 37.06 & 28.65 & 27.88 & 1.26 & 1.32 & 0.62 & 0.75 & ERh \\
\hline PGC 02269 & $15.64 \pm 0.02$ & $15.28 \pm 0.01$ & $0.36 \pm 0.02$ & $15.74 \pm 0.01$ & $15.38 \pm 0.01$ & $0.37 \pm 0.01$ & 36.42 & 36.39 & 18.56 & 17.79 & 2.35 & $\ldots$ & $\ldots$ & $\ldots$ & $\mathrm{EF}$ \\
\hline UGC 00394 & $16.74 \pm 0.03$ & $16.47 \pm 0.03$ & $0.27 \pm 0.04$ & $16.80 \pm 0.02$ & $16.56 \pm 0.01$ & $0.24 \pm 0.03$ & 36.07 & 36.00 & 17.31 & 17.03 & 2.34 & 2.40 & 2.33 & 2.43 & $? \mathrm{~F}$ \\
\hline NGC 0195 & $17.64 \pm 0.07$ & $16.85 \pm 0.02$ & $0.79 \pm 0.08$ & $17.86 \pm 0.03$ & $17.08 \pm 0.01$ & $0.78 \pm 0.04$ & 35.55 & 35.69 & 10.44 & $\ldots$ & $\ldots$ & $\ldots$ & $\ldots$ & $\ldots$ & $? \mathrm{R}$ \\
\hline NGC 0205 & $14.05 \pm 0.01$ & $11.97 \pm 0.01$ & $2.08 \pm 0.01$ & $\ldots$ & $12.00 \pm 0.01$ & $\ldots$ & 33.13 & 33.79 & 57.74 & 141.86 & 9.50 & 4.07 & 5.64 & 3.98 & Er \\
\hline
\end{tabular}


Table 3-Continued

\begin{tabular}{|c|c|c|c|c|c|c|c|c|c|c|c|c|c|c|c|}
\hline \multirow{2}{*}{$\begin{array}{l}\text { Object Name } \\
\text { (1) }\end{array}$} & \multicolumn{3}{|c|}{ Asymptotic magnitudes and color } & \multicolumn{3}{|c|}{ D25 magnitudes and color } & \multicolumn{2}{|c|}{$\log L$} & \multicolumn{2}{|c|}{ Effective Radii } & \multicolumn{2}{|c|}{$\mathrm{C} 31$} & \multicolumn{2}{|c|}{$\mathrm{C} 42$} & \multirow{2}{*}{$\begin{array}{c}\text { UV } \\
\text { profile } \\
(16)\end{array}$} \\
\hline & $\begin{array}{c}\text { FUV } \\
(\mathrm{mag}) \\
(2)\end{array}$ & $\begin{array}{c}\text { NUV } \\
(\mathrm{mag}) \\
(3)\end{array}$ & $\begin{array}{l}\text { FUV-NUV } \\
\text { (mag) } \\
(4)\end{array}$ & $\begin{array}{c}\text { FUV } \\
(\mathrm{mag}) \\
(5)\end{array}$ & $\begin{array}{c}\text { NUV } \\
(\mathrm{mag}) \\
(6)\end{array}$ & $\begin{array}{l}\text { FUV-NUV } \\
\text { (mag) } \\
(7)\end{array}$ & $\begin{array}{c}\text { FUV } \\
(\mathrm{W}) \\
(8)\end{array}$ & $\begin{array}{l}\text { NUV } \\
(\mathrm{W}) \\
(9)\end{array}$ & $\begin{array}{c}\text { FUV } \\
(\operatorname{arcsec}) \\
(10)\end{array}$ & $\begin{array}{c}\text { NUV } \\
(\operatorname{arcsec}) \\
(11)\end{array}$ & $\begin{array}{l}\text { FUV } \\
(12)\end{array}$ & $\begin{array}{l}\text { NUV } \\
(13)\end{array}$ & $\begin{array}{l}\text { FUV } \\
(14)\end{array}$ & $\begin{array}{l}\text { NUV } \\
(15)\end{array}$ & \\
\hline NGC 0213 & $16.33 \pm 0.03$ & $16.17 \pm 0.01$ & $0.17 \pm 0.04$ & $16.44 \pm 0.02$ & $16.24 \pm 0.01$ & $0.21 \pm 0.02$ & 36.19 & 36.08 & 25.36 & 22.98 & 2.07 & 2.31 & 2.07 & 2.32 & $? \mathrm{~F}$ \\
\hline NGC 0223 & $17.43 \pm 0.02$ & $16.79 \pm 0.01$ & $0.64 \pm 0.02$ & $17.56 \pm 0.03$ & $16.92 \pm 0.01$ & $0.64 \pm 0.03$ & 35.72 & 35.80 & $\cdots$ & $\cdots$ & $\cdots$ & $\cdots$ & $\ldots$ & $\cdots$ & $\mathrm{ERh}$ \\
\hline MESSIER 032 & $15.35 \pm 0.03$ & $13.36 \pm 0.01$ & $1.99 \pm 0.03$ & $\cdots$ & $\cdots$ & $\cdots$ & 32.58 & 33.20 & 32.83 & 29.17 & 4.81 & 5.43 & $\cdots$ & $\cdots$ & VV \\
\hline MESSIER 031 & $8.34 \pm 0.01$ & $7.50 \pm 0.01$ & $0.85 \pm 0.01$ & . & $\ldots$ & $\cdots$ & 35.38 & 35.54 & 1462.20 & 1430.04 & 1.60 & 2.00 & 1.34 & 2.15 & EFn \\
\hline NGC 0247 & $11.42 \pm 0.01$ & $11.20 \pm 0.01$ & $0.22 \pm 0.01$ & $11.48 \pm 0.01$ & $11.25 \pm 0.01$ & $0.22 \pm 0.01$ & 35.35 & 35.26 & 187.88 & 180.84 & 2.10 & 2.15 & 1.98 & 2.04 & EFn \\
\hline NGC 0253 & $11.32 \pm 0.01$ & $10.79 \pm 0.01$ & $0.54 \pm 0.01$ & $11.38 \pm 0.01$ & $10.83 \pm 0.01$ & $0.55 \pm 0.01$ & 35.60 & 35.63 & 203.48 & 196.21 & 1.74 & 1.84 & 1.55 & 1.76 & VFh \\
\hline NGC 0247B & $16.09 \pm 0.01$ & $15.71 \pm 0.01$ & $0.38 \pm 0.01$ & $16.24 \pm 0.01$ & $15.88 \pm 0.01$ & $0.36 \pm$ & 36.36 & 36.34 & & $\ldots$ & $\ldots$ & $\cdots$ & $\ldots$ & $\ldots$ & $\mathrm{EE}$ \\
\hline ESO 540-G025 & $15.91 \pm 0.02$ & $15.65 \pm 0.02$ & $0.26 \pm 0.03$ & $16.03 \pm 0.01$ & $15.79 \pm 0.01$ & $0.23 \pm 0.02$ & 36.47 & 36.40 & $\cdots$ & $\cdots$ & $\cdots$ & $\cdots$ & $\cdots$ & $\ldots$ & $\mathrm{EE}$ \\
\hline NGC 0262 & $16.17 \pm 0.01$ & $15.63 \pm 0.01$ & \pm 0.02 & $17.12 \pm 0.03$ & $16.63 \pm 0.01$ & $0.49 \pm 0.03$ & 36.11 & 36.15 & 41.04 & 43.25 & $\ldots$ & $\ldots$ & $\ldots$ & $\cdots$ & $\mathrm{xEr}$ \\
\hline UGC 00507 & $17.64 \pm 0.02$ & $17.16 \pm 0.01$ & 02 & $17.92 \pm 0.02$ & $17.56 \pm 0.01$ & $6 \pm($ & 35.62 & 35.64 & 10 & 12.48 & 3.09 & 2.80 & 3.06 & 2.86 & $\mathrm{EE}$ \\
\hline NGC 0266 & $73 \pm 0.01$ & $15.11 \exists$ & $2 \pm 0.01$ & $15.76 \pm 0.01$ & $15.17 \pm 0.01$ & $0.59 \pm 0.02$ & 36.31 & 36.38 & 45.71 & 44.90 & 1.86 & 1.92 & 1.59 & 1.69 & EFn \\
\hline NGC 0270 & $17.74 \pm 0.07$ & $16.87 \pm 0.03$ & $0.86 \pm 0.07$ & $18.05 \pm 0.05$ & $17.08 \pm 0.02$ & $0.97 \pm 0.05$ & 35.28 & 35.46 & 13.16 & 14.21 & $\ldots$ & $\ldots$ & $\ldots$ & $\ldots$ & ER \\
\hline ESO 351-G011 & $18.06 \pm 0.12$ & $17.58 \pm 0.06$ & $0.48 \pm 0.13$ & $18.40 \pm 0.06$ & $17.82 \pm 0.03$ & $0.58 \pm 0.07$ & 35.98 & 36.00 & 12.00 & 10.82 & 2.55 & $\cdots$ & $\cdots$ & $\cdots$ & $? \mathrm{~F}$ \\
\hline PGC 03004 & $17.24 \pm 0.01$ & $16.98 \pm 0.01$ & $0.26 \pm 0.01$ & $17.34 \pm 0.03$ & $17.06 \pm 0.01$ & $0.28 \pm 0.03$ & 35.58 & 35.51 & 12.17 & 11.73 & $\ldots$ & $\ldots$ & $\ldots$ & $\ldots$ & Efn \\
\hline UGC 00533 & $17.48 \pm 0.11$ & $17.05 \pm 0.02$ & $0.42 \pm 0$ & $17.54 \pm 0.03$ & $17.16 \pm 0.02$ & $0.38 \pm 0.03$ & 35.73 & 35.73 & 11.76 & 12.81 & $\cdots$ & 2.35 & $\ldots$ & $\cdots$ & $\mathrm{VF}$ \\
\hline NGC 0291 & $17.39 \pm 0.07$ & $16.78 \pm 0.11$ & $0.61 \pm 0.13$ & $17.67 \pm 0.03$ & $17.06 \pm 0.01$ & $0.61 \pm 0.03$ & 35.79 & 35.86 & & $\ldots$ & $\ldots$ & $\ldots$ & $\ldots$ & $\ldots$ & ER \\
\hline NGC 0300 & $10.21 \pm 0.01$ & $10.04 \pm 0.01$ & $0.17 \pm 0.01$ & $10.34 \pm 0.01$ & $10.18 \pm 0.01$ & $0.16 \pm 0.01$ & 35.45 & 35.34 & 293.40 & 289.70 & 2.39 & 2.43 & 2.26 & 2.31 & $\mathrm{EF}$ \\
\hline UGC 00590 & $18.07 \pm 0.07$ & $17.67 \pm 0.01$ & $0.40 \pm 0.07$ & $18.13 \pm 0.07$ & $17.74 \pm 0.03$ & $0.40 \pm 0.08$ & 36.36 & & $\ldots$ & $\ldots$ & $\ldots$ & $\ldots$ & $\ldots$ & $\ldots$ & $? \mathrm{~F}$ \\
\hline NGC 0311 & & $18.39 \pm 0.07$ & $\cdots$ & .. & $\cdots$ & $\cdots$ & 0.00 & 35.14 & $\cdots$ & $\ldots$ & $\cdots$ & $\cdots$ & $\ldots$ & $\cdots$ & $\cdots$ \\
\hline NGC 0315 & $17.47 \pm 0.12$ & $16.32 \pm 0.04$ & $1.14 \pm 0.13$ & $17.55 \pm 0.13$ & $16.56 \pm 0.05$ & $0.99 \pm 0.14$ & 35.66 & 35.95 & 18.07 & 29.65 & $\cdots$ & 6.57 & $\ldots$ & $\cdots$ & $\mathrm{Er}$ \\
\hline ESO 351-G028 & $15.89 \pm 0.01$ & $15.62 \pm 0.01$ & $0.28 \pm 0.01$ & $15.98 \pm 0.01$ & $15.70 \pm 0.01$ & $0.28 \pm 0.02$ & 35.92 & 35.85 & 11.44 & 11.36 & $\ldots$ & $\ldots$ & $\ldots$ & $\cdots$ & Ef \\
\hline UGC 00619 & $18.50 \pm 0.12$ & $18.30 \pm 0.05$ & $0.20 \pm 0.13$ & $19.48 \pm 0.06$ & $19.26 \pm 0.03$ & $0.23 \pm 0.06$ & 36.02 & 35.92 & 11.24 & 10.88 & $\ldots$ & $\ldots$ & $\ldots$ & $\ldots$ & Er,Ef \\
\hline NGC 0337 & $13.85 \pm 0.01$ & $13.22 \pm 0.01$ & $0.63 \pm 0.01$ & $13.88 \pm 0.01$ & $13.25 \pm 0.01$ & $0.63 \pm 0.01$ & 36.18 & 36.26 & 26.68 & 26.18 & 2.16 & 2.24 & 2.19 & 2.32 & Efn \\
\hline PGC 03613 & $17.24 \pm 0.07$ & $16.32 \pm 0.05$ & $0.91 \pm 0.08$ & $17.34 \pm 0.07$ & $16.47 \pm 0.03$ & $0.87 \pm 0.07$ & 35.87 & 36.06 & 20.05 & 19.72 & 1.94 & 2.17 & $\ldots$ & $\ldots$ & $\cdots$ \\
\hline UGC 00627 & $17.95 \pm 0.07$ & $17.45 \pm 0.01$ & $0.50 \pm 0.07$ & $18.01 \pm 0.05$ & $17.58 \pm 0.02$ & $0.44 \pm 0.05$ & 36.21 & 36.24 & $\cdots$ & $\ldots$ & $\ldots$ & $\ldots$ & $\ldots$ & $\ldots$ & $? \mathrm{~F}$ \\
\hline NGC 0337A & $14.12 \pm 0.04$ & $13.62 \pm 0.03$ & $0.50 \pm 0.05$ & $14.39 \pm 0.01$ & $13.84 \pm 0.01$ & $0.54 \pm 0.02$ & 35.59 & 35.62 & 107.84 & 100.61 & 2.25 & 2.39 & 2.27 & 2.36 & $? \mathrm{~F}$ \\
\hline UGC 00652 & $17.19 \pm 0.02$ & $17.06 \pm 0.01$ & $0.13 \pm 0.02$ & $17.45 \pm 0.03$ & $17.31 \pm 0.02$ & $0.14 \pm 0.04$ & 35.89 & 35.76 & 16.34 & 15.20 & $\ldots$ & $\ldots$ & $\ldots$ & $\ldots$ & $\cdots$ \\
\hline ESO 352-G002 & $16.65 \pm 0.01$ & $16.19 \pm 0.01$ & $0.47 \pm 0.01$ & $16.71 \pm 0.02$ & $16.26 \pm 0.01$ & $0.45 \pm 0.02$ & 36.57 & 36.58 & 9.24 & 8.79 & $\ldots$ & $\ldots$ & $\ldots$ & $\ldots$ & $\mathrm{EE}$ \\
\hline IC 1613 & $11.46 \pm 0.01$ & $11.38 \pm 0.01$ & $0.08 \pm 0.01$ & $11.51 \pm 0.01$ & $11.45 \pm 0.01$ & $0.06 \pm 0.01$ & 34.17 & 34.02 & 234.20 & 236.21 & 1.77 & 1.87 & 1.67 & 1.82 & $\mathrm{EF}$ \\
\hline IC 1616 & $15.85 \pm 0.01$ & $15.41 \pm 0.01$ & $0.45 \pm 0.01$ & $15.94 \pm 0.01$ & $15.49 \pm 0.01$ & $0.45 \pm 0.01$ & 36.38 & 36.38 & 24.62 & 23.17 & 2.10 & 2.25 & 1.95 & 2.16 & $\mathrm{EFh}$ \\
\hline
\end{tabular}


Table 3-Continued

\begin{tabular}{|c|c|c|c|c|c|c|c|c|c|c|c|c|c|c|c|}
\hline \multirow{2}{*}{$\begin{array}{l}\text { Object Name } \\
\text { (1) }\end{array}$} & \multicolumn{3}{|c|}{ Asymptotic magnitudes and color } & \multicolumn{3}{|c|}{ D25 magnitudes and color } & \multicolumn{2}{|c|}{$\log L$} & \multicolumn{2}{|c|}{ Effective Radii } & \multicolumn{2}{|c|}{ C31 } & \multicolumn{2}{|c|}{$\mathrm{C} 42$} & \multirow{2}{*}{$\begin{array}{c}\text { UV } \\
\text { profile } \\
(16)\end{array}$} \\
\hline & $\begin{array}{c}\text { FUV } \\
(\mathrm{mag}) \\
(2)\end{array}$ & $\begin{array}{c}\text { NUV } \\
(\mathrm{mag}) \\
(3)\end{array}$ & $\begin{array}{l}\text { FUV-NUV } \\
\text { (mag) } \\
(4)\end{array}$ & $\begin{array}{c}\text { FUV } \\
(\mathrm{mag}) \\
(5)\end{array}$ & $\begin{array}{c}\text { NUV } \\
(\mathrm{mag}) \\
(6)\end{array}$ & $\begin{array}{l}\text { FUV-NUV } \\
(\operatorname{mag}) \\
(7)\end{array}$ & $\begin{array}{c}\text { FUV } \\
(W) \\
(8)\end{array}$ & $\begin{array}{l}\text { NUV } \\
(\mathrm{W}) \\
(9)\end{array}$ & $\begin{array}{c}\text { FUV } \\
(\operatorname{arcsec}) \\
(10)\end{array}$ & $\begin{array}{c}\text { NUV } \\
(\operatorname{arcsec}) \\
(11)\end{array}$ & $\begin{array}{l}\text { FUV } \\
(12)\end{array}$ & $\begin{array}{l}\text { NUV } \\
\text { (13) }\end{array}$ & $\begin{array}{l}\text { FUV } \\
(14)\end{array}$ & $\begin{array}{l}\text { NUV } \\
(15)\end{array}$ & \\
\hline ESO 352-G007 & $.36 \pm 0.01$ & $84+0.01$ & 1 & $.50 \pm 0.02$ & $16.98 \pm$ & $0.52 \pm$ & 36.29 & 36.32 & 17.14 & 3 & 2.70 & $\ldots$ & $\ldots$ & $\ldots$ & $? \mathrm{Fn}$ \\
\hline NGC 0392 & $27 \pm 0.16$ & $17.53 \pm 0.04$ & & $02 \pm 0.13$ & $17.98 \pm 0.04$ & 1.0 & 34.90 & & . & .08 & $\ldots$ & $\ldots$ & $\ldots$ & $\ldots$ & $\ldots$ \\
\hline ESO 243-G041 & $19.91 \pm 0.28$ & $18.89 \pm 0.04$ & $1.03 \pm 0.28$ & $.27 \pm 0.06$ & $19.22 \pm 0.02$ & $1.05 \pm 0.06$ & 34.97 & 35.20 & 8.12 & 8.02 & $\cdots$ & $\cdots$ & $\cdots$ & $\cdots$ & $? \mathrm{R}$ \\
\hline ESO 296-G002 & $16.79 \pm 0.02$ & $16.32 \pm 0.02$ & $0.47 \pm 0.03$ & $.94 \pm 0.02$ & $16.46 \pm 0.01$ & $0.49 \pm 0.02$ & 36.14 & 36.15 & 14.44 & 13.25 & $\cdots$ & $\cdots$ & $\cdots$ & $\cdots$ & $\cdots$ \\
\hline NGC 0403 & $19.00 \pm 0.16$ & $17.74 \pm 0.04$ & & $.21 \pm 0.12$ & $17.96 \pm 0.04$ & $1.25 \pm 0$ & 35.08 & 35.41 & & & $\cdots$ & $\cdots$ & $\cdots$ & $\cdots$ & VV \\
\hline IC 1633 & $17.51\rfloor$ & & & 3 & & & & & & & & & $\cdots$ & & VV \\
\hline UGC & $92 \pm$ & 16.26 & 0.6 & $.98 \pm$ & $16.34 \pm$ & & 35.64 & & & & 2.05 & 3 & 1.99 & 2.10 & EFn \\
\hline NGC 0407 & 知 & $18.06 \pm$ & & $.38 \pm 0$ & $18.32 \pm 0$ & 1. & 34.97 & 35.35 & 8 & 11.98 & $\ldots$ & & $\ldots$ & $\ldots$ & $\ldots$ \\
\hline UG & $90 \pm$ & 16.4 & & $34 \pm 0$ & 1 & 4 & 35.97 & 35.98 & & & 2.31 & $\ldots$ & $\ldots$ & $\ldots$ & ?Fn \\
\hline 36 & 3 & & & $97 \pm 0$ & 16.58 & $\pm 0 \pm 0$ & 87 & 3 & & & $\cdots$ & $\cdots$ & $\cdots$ & $\cdots$ & ?Fn \\
\hline 0410 & 2 & 16.87 & & $\ldots$ & 17.11 & & 35.54 & 35.79 & 10.25 & 21.90 & $\cdots$ & $\ldots$ & $\ldots$ & $\cdots$ & VV \\
\hline ESO 243-G051 & $17.68 \pm 0$ & 16.85 & 3 & $17.73 \pm 0.03$ & $16.95=$ & 3 & 35.76 & 35.92 & $\ldots$ & & $\ldots$ & $\ldots$ & $\cdots$ & $\cdots$ & $\mathrm{EF}$ \\
\hline ESO 243- & 20.08 & 18.2 & & $20.29 \pm$ & 18.75 & 1.53 & 35.01 & & 7.09 & & $\cdots$ & 3.04 & $\cdots$ & 3.05 & $\mathrm{EE}$ \\
\hline & 19.24 & 17.15 & & $\ldots$ & $17.43 \exists$ & 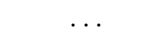 & 35.02 & 35.68 & 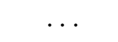 & & $\ldots$ & $\ldots$ & $\ldots$ & $\ldots$ & Er \\
\hline NGC 0470 & $14.66 \pm 0.01$ & $14.27 \pm$ & 01 & $71 \pm 0$ & $14.32 \pm$ & $0.39 \pm 0$ & 36.12 & 36.10 & 31.81 & 3 & 1.67 & 1.75 & 1.41 & 1.56 & EFn \\
\hline NGC 0474 & $17.00 \pm 0.11$ & $15.65 \pm 0.04$ & $1.36 \pm 0.12$ & $7.50 \pm 0.07$ & $15.88 \pm 0.02$ & $1.62 \pm 0$ & 35.18 & 35.54 & 140.90 & 8 & 4.80 & 6.23 & 4.24 & 4.76 & VV \\
\hline ESO $352-G$ & $16.85 \pm 0.04$ & $16.50 \pm 0.02$ & 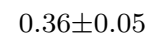 & $.97 \pm 0.02$ & $16.66 \pm 0.01$ & $0.30 \pm 0.02$ & 35.62 & & 13.69 & & $\ldots$ & & $\ldots$ & $\ldots$ & $\mathrm{EE}$ \\
\hline & 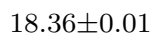 & & & & & & & & & & $\ldots$ & & $\ldots$ & $\ldots$ & $\ldots$ \\
\hline ESO 352-G050 & 17.45 & 16.97 & & $17.68 \pm 0$ & 17.2 & $0.45 \pm$ & 35.78 & 35.79 & 12.08 & & 2.42 & 2.57 & 2.41 & 2.61 & $\mathrm{EF}$ \\
\hline & 16.4 & $16.0^{\prime}$ & 0.03 & $85 \pm$ & 16. & 0.4 & 36.08 & 36.07 & 1 & & $\cdots$ & $\ldots$ & $\cdots$ & $\cdots$ & Er \\
\hline NG & 15.78 & 15.07 & 01 & $15.82 \pm 0.01$ & 15.12 & $0.70 \pm$ & 36.06 & 36.17 & 12.65 & 11 & $\cdots$ & $\cdots$ & $\cdots$ & $\cdots$ & VF \\
\hline UGC 00910 & $16.34 \pm 0.02$ & $15.95 \pm 0.01$ & $0.40 \pm 0.03$ & $16.47 \pm 0.02$ & $16.10 \pm 0.01$ & $0.37 \pm 0.03$ & 36.32 & 36.30 & 14.87 & 14.38 & $\cdots$ & $\ldots$ & $\ldots$ & $\cdots$ & $\mathrm{EF}$ \\
\hline ESO 352-G057 & $20.53 \pm 0.36$ & $18.61 \pm 0.08$ & $1.92 \pm 0.37$ & $\cdots$ & $18.86 \pm 0.03$ & $\cdots$ & 34.49 & 35.08 & 7.41 & 9.18 & $\cdots$ & $\cdots$ & $\cdots$ & $\cdots$ & ?R \\
\hline ESO 352-G062 & $18.31 \pm 0.01$ & $17.87 \pm 0.03$ & $0.44 \pm 0.03$ & $18.67 \pm 0.03$ & $18.34 \pm 0.02$ & $0.33 \pm 0.03$ & 35.86 & 35.86 & 12.00 & 12.56 & 2.01 & 2.47 & 1.92 & 2.50 & $\mathrm{EDn}, \mathrm{EF}$ \\
\hline ESO 352-G064 & $20.82 \pm 0.06$ & $18.45 \pm 0.02$ & $2.38 \pm 0.06$ & $\cdots$ & $18.58 \pm 0.02$ & $\ldots$ & 34.45 & 35.22 & $\cdots$ & 6.51 & $\ldots$ & $\ldots$ & $\cdots$ & $\ldots$ & $\mathrm{EE}$ \\
\hline NGC 0527 & $19.67 \pm 0.05$ & $18.04 \pm 0.19$ & $1.64 \pm 0.19$ & $19.69 \pm 0.07$ & $18.23 \pm 0.02$ & $1.45 \pm 0$. & 34.87 & 35.35 & 8.38 & 11.15 & $\cdots$ & 3.11 & $\cdots$ & $\cdots$ & ER \\
\hline NGC 0514 & $14.59 \pm 0.01$ & $14.21 \pm 0.01$ & $0.38 \pm 0.01$ & $14.64 \pm 0.01$ & $14.26 \pm 0.01$ & $0.38 \pm 0.01$ & 36.20 & 36.18 & 42.16 & & 2.43 & 2.47 & 2.34 & 2.42 & $\mathrm{EF}$ \\
\hline ESO 352-G069 & & $15.89 \pm 0.01$ & & $16.54 \pm 0.02$ & $16.03 \pm 0.01$ & $0.50 \pm 0.02$ & 36.24 & 36.25 & & & $\ldots$ & $\ldots$ & $\ldots$ & $\ldots$ & $\mathrm{EF}$ \\
\hline & & $18.56 \pm 0.05$ & & $19.26 \pm 0.09$ & $18.70=$ & & 34.23 & 34.30 & 13.62 & & $\cdots$ & $\cdots$ & $\cdots$ & $\ldots$ & $\mathrm{EE}$ \\
\hline NGC 0520 & $16.04 \pm 0.01$ & $15.03 \pm 0.02$ & $1.01 \pm 0.02$ & $16.19 \pm 0.01$ & $15.21 \pm 0.01$ & $0.97 \pm 0.02$ & 35.51 & 35.74 & 29.29 & 31.43 & 2.93 & 3.12 & 3.05 & 3.21 & $\mathrm{EE}$ \\
\hline
\end{tabular}


Table 3-Continued

\begin{tabular}{|c|c|c|c|c|c|c|c|c|c|c|c|c|c|c|c|}
\hline \multirow{2}{*}{$\begin{array}{l}\text { Object Name } \\
\text { (1) }\end{array}$} & \multicolumn{3}{|c|}{ Asymptotic magnitudes and color } & \multicolumn{3}{|c|}{ D25 magnitudes and color } & \multicolumn{2}{|c|}{$\log L$} & \multicolumn{2}{|c|}{ Effective Radii } & \multicolumn{2}{|c|}{$\mathrm{C} 31$} & \multicolumn{2}{|c|}{$\mathrm{C} 42$} & \multirow{2}{*}{$\begin{array}{c}\text { UV } \\
\text { profile } \\
(16)\end{array}$} \\
\hline & $\begin{array}{c}\text { FUV } \\
(\mathrm{mag}) \\
(2)\end{array}$ & $\begin{array}{c}\text { NUV } \\
\text { (mag) } \\
(3)\end{array}$ & $\begin{array}{c}\text { FUV-NUV } \\
\text { (mag) } \\
(4)\end{array}$ & $\begin{array}{c}\text { FUV } \\
(\mathrm{mag}) \\
(5)\end{array}$ & $\begin{array}{c}\text { NUV } \\
(\mathrm{mag}) \\
(6)\end{array}$ & $\begin{array}{l}\text { FUV-NUV } \\
\text { (mag) } \\
(7)\end{array}$ & $\begin{array}{c}\text { FUV } \\
(\mathrm{W}) \\
(8)\end{array}$ & $\begin{array}{l}\text { NUV } \\
(\mathrm{W}) \\
(9)\end{array}$ & $\begin{array}{c}\text { FUV } \\
(\operatorname{arcsec}) \\
(10)\end{array}$ & $\begin{array}{c}\text { NUV } \\
(\operatorname{arcsec}) \\
(11)\end{array}$ & $\begin{array}{l}\text { FUV } \\
(12)\end{array}$ & $\begin{array}{l}\text { NUV } \\
(13)\end{array}$ & $\begin{array}{l}\text { FUV } \\
(14)\end{array}$ & $\begin{array}{l}\text { NUV } \\
(15)\end{array}$ & \\
\hline NGC 0530 & $.89 \pm 0.11$ & $.27 \pm 0.02$ & $1.62 \pm 0.12$ & & $18.38 \pm 0.02$ & & 34.68 & 35.15 & & 8.40 & $\ldots$ & 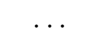 & $\ldots$ & 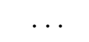 & $\mathrm{VF}$ \\
\hline IC 0107 & $16.94 \pm 0.01$ & $16.59 \pm 0.01$ & $0.36 \pm 0.02$ & $16.99 \pm 0.03$ & $16.66 \pm 0.01$ & $0.33 \pm 0.03$ & 36.08 & 36.04 & 12.28 & 12.52 & $\cdots$ & $\cdots$ & $\cdots$ & $\cdots$ & $? \mathrm{~F}$ \\
\hline UGC 00984 & $21.01 \pm 0.34$ & $18.94 \pm 0.04$ & $2.07 \pm 0.34$ & & $19.03 \pm 0.04$ & & 34.20 & 34.85 & & $\ldots$ & $\cdots$ & .. & $\ldots$ & .. & $\ldots$ \\
\hline IC 1698 & $17.70 \pm 0.06$ & $17.19 \pm 0.02$ & $0.50 \pm 0.07$ & $17.78 \pm 0.03$ & $17.40 \pm 0.02$ & $0.38 \pm 0.04$ & 35.79 & 35.82 & 13.55 & 14.16 & $\ldots$ & $\ldots$ & $\ldots$ & $\ldots$ & $\mathrm{EE}$ \\
\hline IC 1700 & $19.94 \pm 0.09$ & $17.86 \pm 0.04$ & $2.08 \pm 0.10$ & $19.93 \pm 0.22$ & $18.16 \pm 0.04$ & $1.77 \pm 0.22$ & 34.88 & 35.53 & ... & 16.70 & $\cdots$ & $\cdots$ & $\ldots$ & $\cdots$ & $\ldots$ \\
\hline NGC 0538 & $19.77 \pm 0.01$ & $18.74 \pm 0.08$ & $1.03 \pm 0.09$ & $\ldots$ & $18.97 \pm 0.05$ & $\ldots$ & 34.80 & 35.04 & $\ldots$ & $\ldots$ & $\ldots$ & $\ldots$ & $\ldots$ & $\ldots$ & $\ldots$ \\
\hline NGC 0535 & $20.10 \pm 0.05$ & $18.66 \pm 0.03$ & $1.44 \pm 0.06$ & $\ldots$ & $18.85 \pm 0.03$ & $\ldots$ & 34.58 & 34.98 & $\ldots$ & 7.36 & $\ldots$ & $\ldots$ & $\ldots$ & $\ldots$ & $\ldots$ \\
\hline UGC 00999 & $18.36 \pm 0.01$ & $17.85 \pm 0.01$ & $0.51 \pm 0.02$ & $18.53 \pm 0.05$ & $18.04 \pm 0.02$ & $0.49 \pm 0.05$ & 36.30 & 36.32 & 7.89 & 8.33 & $\ldots$ & $\ldots$ & $\ldots$ & $\ldots$ & $\mathrm{EF}$ \\
\hline UGC 01003 & $20.98 \pm 0.10$ & $19.30 \pm 0.01$ & $1.68 \pm 0.10$ & & $19.43 \pm 0.05$ & & 34.28 & 34.78 & & & $\ldots$ & & $\ldots$ & $\ldots$ & $\ldots$ \\
\hline NGC 0541 & $.74 \pm 0.07$ & $17.44 \pm 0.05$ & $1.31 \pm 0.09$ & $\ldots$ & $17.74 \pm 0.04$ & . & 35.20 & 35.55 & & 22.11 & $\ldots$ & $\ldots$ & $\ldots$ & $\ldots$ & VV \\
\hline NGC 0545 & $18.65 \pm 0.10$ & $16.95 \pm 0.04$ & $1.70 \pm 0.10$ & $18.67 \pm 0.11$ & $17.31 \pm 0.03$ & $1.35 \pm 0.12$ & 35.23 & 35.74 & 9.85 & 30.41 & $\ldots$ & 5.00 & $\ldots$ & $\ldots$ & VV \\
\hline NGC 0547 & $18.56 \pm 0.11$ & $16.61 \pm 0.22$ & $1.95 \pm 0.25$ & $18.87 \pm 0.08$ & $17.69 \pm 0.03$ & $1.18 \pm 0.09$ & 35.29 & 35.89 & $\ldots$ & $\ldots$ & $\ldots$ & $\ldots$ & $\ldots$ & $\ldots$ & ER \\
\hline NGC 0557 & $19.03 \pm 0.16$ & $17.92 \pm 0.02$ & $1.11 \pm 0.16$ & & $18.07 \pm 0.03$ & $\ldots$ & 35.11 & 35.38 & 13.78 & 12.83 & $\ldots$ & $\ldots$ & $\ldots$ & $\ldots$ & $\mathrm{EE}$ \\
\hline UGC 01026 & $17.75 \pm 0.03$ & $17.47 \pm 0.01$ & $0.27 \pm 0.03$ & $17.77 \pm 0.04$ & $17.50 \pm 0.02$ & $0.27 \pm 0.04$ & 35.46 & 35.39 & 19.06 & 19.39 & 2.22 & 2.25 & $\ldots$ & $\ldots$ & $? \mathrm{~F}$ \\
\hline UGC 01040 & $20.78 \pm 0.18$ & $18.78 \pm 0.02$ & $2.00 \pm 0.18$ & $20.89 \pm 0.13$ & $19.01 \pm 0.03$ & $1.88 \pm 0.13$ & 34.23 & 34.85 & 5.44 & 7.29 & $\ldots$ & $\ldots$ & $\ldots$ & $\ldots$ & $? \mathrm{~F}$ \\
\hline NGC 0568 & $19.11 \pm 0.17$ & $17.57 \pm 0.04$ & $1.54 \pm 0.17$ & $19.15 \pm 0.08$ & $17.93 \pm 0.03$ & $1.23 \pm 0.09$ & 35.08 & 35.52 & 11.84 & 24.37 & $\ldots$ & $\ldots$ & $\ldots$ & $\ldots$ & ER \\
\hline UGC 01057 & $16.62 \pm 0.02$ & $16.32 \pm 0.01$ & $0.29 \pm 0.02$ & $16.74 \pm 0.02$ & $16.42 \pm 0.01$ & $0.32 \pm 0.02$ & 36.20 & 36.15 & 13.25 & 12.64 & 2.16 & 2.26 & 2.12 & $\ldots$ & $\mathrm{EF}$ \\
\hline NGC 0574 & $16.15 \pm 0.01$ & $15.52 \pm 0.01$ & $0.63 \pm 0.02$ & $16.29 \pm 0.01$ & $15.64 \pm 0.01$ & $0.64 \pm 0.02$ & 36.27 & 36.34 & 10.55 & $\ldots$ & $\ldots$ & $\ldots$ & $\ldots$ & $\ldots$ & $\mathrm{EE}$ \\
\hline IC 0127 & $19.05 \pm 0.06$ & $17.76 \pm 0.16$ & $1.29 \pm 0.17$ & $19.18 \pm 0.07$ & $17.98 \pm 0.02$ & $1.19 \pm 0.07$ & 34.19 & 34.53 & 11.32 & 13.62 & $\ldots$ & 2.93 & $\ldots$ & $\ldots$ & Ef \\
\hline NGC 05 & $17.47 \pm 0.04$ & $15.65 \pm 0.01$ & $1.82 \pm 0.04$ & $17.65 \pm 0.04$ & $15.80 \pm 0.01$ & $1.86 \pm 0.04$ & 34.83 & 35.38 & 28.82 & 27.02 & 7.04 & 5.72 & $\ldots$ & $\ldots$ & VV \\
\hline NGC 0586 & $18.31 \pm 0.04$ & $17.27 \pm 0.02$ & $1.03 \pm 0.05$ & $18.33 \pm 0.05$ & $17.37 \pm 0.02$ & $0.96 \pm 0.05$ & 34.48 & 34.72 & 9.01 & 10.63 & $\cdots$ & $\cdots$ & $\ldots$ & $\cdots$ & $\ldots$ \\
\hline MESSIER 033 & $7.99 \pm 0.01$ & $7.78 \pm 0.02$ & $0.21 \pm 0.02$ & $7.98 \pm 0.01$ & $\ldots$ & $\ldots$ & 35.59 & 35.50 & 623.82 & 577.54 & 2.44 & 2.38 & 2.36 & 2.30 & EFn \\
\hline NGC 0628 & $11.70 \pm 0.01$ & $11.41 \pm 0.01$ & $0.29 \pm 0.01$ & $11.81 \pm 0.01$ & $11.50 \pm 0.01$ & $0.31 \pm 0.01$ & 36.35 & 36.29 & 150.00 & 141.70 & 2.24 & 2.31 & 2.17 & 2.30 & $\mathrm{EFn}$ \\
\hline UGC 01181 & $18.94 \pm 0.05$ & $18.22 \pm 0.02$ & $0.71 \pm 0.05$ & $19.07 \pm 0.05$ & $18.41 \pm 0.02$ & $0.66 \pm 0.05$ & 35.49 & 35.60 & 5.94 & 6.68 & $\ldots$ & $\ldots$ & $\ldots$ & $\ldots$ & $\ldots$ \\
\hline IC 0148 & $14.98 \pm 0.01$ & $14.66 \pm 0.01$ & $0.32 \pm 0.01$ & $15.03 \pm 0.01$ & $14.71 \pm 0.01$ & $0.32 \pm 0.01$ & 35.05 & 35.00 & 20.69 & 19.98 & 3.23 & 3.30 & 3.14 & 3.18 & $\mathrm{EE}$ \\
\hline UGC 01200 & $15.51 \pm 0.01$ & $15.20 \pm 0.01$ & $0.32 \pm 0.01$ & $15.55 \pm 0.01$ & $15.23 \pm 0.01$ & $0.32 \pm 0.01$ & 34.87 & 34.82 & 12.99 & 13.21 & $\ldots$ & $\ldots$ & $\ldots$ & $\ldots$ & Ef \\
\hline NGC 0660 & $14.69 \pm 0.01$ & $14.28 \pm 0.01$ & $0.41 \pm 0.01$ & $14.81 \pm 0.01$ & $14.38 \pm 0.01$ & $0.43 \pm 0.01$ & 35.24 & 35.23 & 86.35 & 82.80 & 2.20 & 2.26 & 2.16 & 2.24 & $? \mathrm{~F}$ \\
\hline UGC 01211 & $17.37 \pm 0.05$ & $17.03 \pm 0.01$ & $0.34 \pm 0.05$ & $\ldots$ & $17.05 \pm 0.02$ & $\ldots$ & 35.06 & 35.02 & 23.79 & 22.35 & 2.48 & 2.64 & $\ldots$ & $\ldots$ & $\ldots$ \\
\hline IC 0159 & $15.72 \pm 0.01$ & $15.30 \pm 0.01$ & $0.42 \pm 0.01$ & $15.77 \pm 0.01$ & $15.38 \pm 0.01$ & $0.39 \pm 0.01$ & 36.12 & 36.11 & 12.62 & 12.89 & $\ldots$ & $\ldots$ & $\ldots$ & $\ldots$ & $? \mathrm{~F}$ \\
\hline PGC 06504 & $17.27 \pm 0.03$ & $17.05 \pm 0.03$ & $0.22 \pm 0.04$ & $17.35 \pm 0.03$ & $17.21 \pm 0.02$ & $0.14 \pm 0.03$ & 35.50 & 35.41 & $\ldots$ & 10.56 & $\ldots$ & $\ldots$ & $\ldots$ & $\ldots$ & $\ldots$ \\
\hline
\end{tabular}


Table 3-Continued

\begin{tabular}{|c|c|c|c|c|c|c|c|c|c|c|c|c|c|c|c|}
\hline \multirow{2}{*}{$\begin{array}{l}\text { Object Name } \\
\qquad(1)\end{array}$} & \multicolumn{3}{|c|}{ Asymptotic magnitudes and color } & \multicolumn{3}{|c|}{ D25 magnitudes and color } & \multicolumn{2}{|c|}{$\log L$} & \multicolumn{2}{|c|}{ Effective Radii } & \multicolumn{2}{|c|}{ C31 } & \multicolumn{2}{|c|}{$\mathrm{C} 42$} & \multirow{2}{*}{$\begin{array}{c}\text { UV } \\
\text { profile } \\
(16)\end{array}$} \\
\hline & $\begin{array}{c}\text { FUV } \\
(\mathrm{mag}) \\
(2)\end{array}$ & $\begin{array}{c}\text { NUV } \\
(\mathrm{mag}) \\
(3)\end{array}$ & $\begin{array}{l}\text { FUV-NUV } \\
\text { (mag) } \\
(4)\end{array}$ & $\begin{array}{l}\text { FUV } \\
(\mathrm{mag}) \\
(5)\end{array}$ & $\begin{array}{l}\text { NUV } \\
(\mathrm{mag}) \\
(6)\end{array}$ & $\begin{array}{l}\text { FUV-NUV } \\
\text { (mag) } \\
(7)\end{array}$ & $\begin{array}{c}\text { FUV } \\
(W) \\
(8)\end{array}$ & $\begin{array}{l}\text { NUV } \\
(\mathrm{W}) \\
(9)\end{array}$ & $\begin{array}{c}\text { FUV } \\
(\operatorname{arcsec}) \\
(10)\end{array}$ & $\begin{array}{c}\text { NUV } \\
(\operatorname{arcsec}) \\
(11)\end{array}$ & $\begin{array}{l}\text { FUV } \\
(12)\end{array}$ & $\begin{array}{l}\text { NUV } \\
(13)\end{array}$ & $\begin{array}{l}\text { FUV } \\
(14)\end{array}$ & $\begin{array}{l}\text { NUV } \\
(15)\end{array}$ & \\
\hline NGC 0671 & 1 & 1 & $0.60 \pm 0.01$ & 2 & 02 & 0 & 35 & & & 9 . & & & & & $\mathrm{EE}$ \\
\hline UGC 01261 & & & & & & & & & & & & 2.57 & 2.46 & & $\mathrm{EF}$ \\
\hline UGC 01262 & $17.37 \pm 0.01$ & $17.16 \pm 0.01$ & $0.21 \pm 0.01$ & $.42 \pm 0.04$ & $17.21 \pm 0.02$ & $0.21 \pm 0.04$ & 35.71 & 35.62 & 9.07 & 9.73 & & & $\ldots$ & $\ldots$ & EFn \\
\hline UGC 01264 & $.76 \pm 0.04$ & $18.19 \pm 0.02$ & $0.57 \pm 0.04$ & $.90 \pm 0.05$ & $18.32 \pm 0.02$ & $0.58 \pm 0.06$ & 35.10 & 35.16 & 7.07 & 7.41 & $\ldots$ & $\ldots$ & $\ldots$ & $\ldots$ & $\mathrm{EF}$ \\
\hline NGC 0676 & $16.77 \pm 0.01$ & $15.19 \pm 0.01$ & $1.58 \pm 0.02$ & $.82 \pm 0.02$ & $15.23 \pm 0.01$ & $1.59 \pm 0.02$ & 34.88 & 35.33 & 24.09 & 10.14 & 2.12 & $\ldots$ & 2.12 & $\ldots$ & Ef \\
\hline UGC 01271 & $20.08 \pm 0.33$ & $18.87 \pm 0.05$ & $1.21 \pm 0.33$ & $\ldots$ & $\ldots$ & $\ldots$ & 34.63 & 34.93 & 12.21 & $\ldots$ & $\ldots$ & $\ldots$ & $\ldots$ & $\ldots$ & ?Fn,?Rn \\
\hline UGC 01274 & $\ldots$ & $19.30 \pm 0.17$ & & $\ldots$ & $19.35 \pm 0.08$ & $\ldots$ & 0.00 & 35.14 & & 8.01 & $\ldots$ & $\ldots$ & $\ldots$ & $\ldots$ & $\ldots$ \\
\hline UGC 01278 & $17.85 \pm 0.10$ & $17.45 \pm 0.03$ & $40 \pm 0.11$ & $.01 \pm 0.04$ & $17.51 \pm 0.02$ & $0.50 \pm 0$ & 36.11 & 36.09 & 10.08 & 9.22 & $\ldots$ & $\ldots$ & $\ldots$ & $\ldots$ & $? \mathrm{~F}$ \\
\hline NGC 0693 & $.30 \pm 0.01$ & $15.72 \pm 0.01$ & $0.58 \pm 0.02$ & $16.40 \pm 0.02$ & $15.83 \pm 0.01$ & $0.57 \pm 0.02$ & 35.10 & 35.15 & 10.82 & 11.78 & $\ldots$ & $\ldots$ & $\ldots$ & $\ldots$ & ER \\
\hline UGC 01312 & $32 \pm 0.12$ & 16.76 & $0.56 \pm 0.12$ & $.44 \pm 0.03$ & $16.94 \pm 0$ & $0.51 \pm c$ & 35.75 & 35.79 & & 0 & $\ldots$ & $\ldots$ & $\ldots$ & $\ldots$ & $? \mathrm{~F}$ \\
\hline ESO 245-G007 & $16.22 \pm 0.01$ & $15.43 \pm 0.02$ & $0.79 \pm 0.02$ & $16.24 \pm 0.02$ & $15.57 \pm 0.01$ & $0.67 \pm 0.02$ & 31.65 & 31.79 & 48.29 & 35 & 2.03 & 2.45 & 1.90 & 2.51 & $\mathrm{EFn}$ \\
\hline NGC 0707 & $19.17 \pm 0.09$ & $18.07 \pm 0.06$ & $1.10 \pm 0.11$ & $19.30 \pm 0.07$ & $18.22 \pm 0.03$ & $1.08 \pm 0.08$ & 35.04 & 35.30 & 13 & 6 & $\ldots$ & $\ldots$ & $\ldots$ & $\ldots$ & $\mathrm{EE}$ \\
\hline NGC 0706 & $.52 \pm 0.01$ & $14.93 \pm 0.01$ & $0.60 \pm 0.01$ & $.54 \pm 0.01$ & $14.95 \pm 0.01$ & $0.59 \pm 0.01$ & 36.43 & 36.49 & & & 1.98 & $\ldots$ & $\ldots$ & $\ldots$ & $\mathrm{VF}$ \\
\hline UGC 01364 & $17.28 \pm 0.02$ & $17.01 \pm 0.01$ & $0.27 \pm 0.03$ & $17.47 \pm 0.03$ & $17.24 \pm 0.02$ & $0.24 \pm 0.03$ & 35.75 & 35.68 & 15.93 & 16.08 & $\ldots$ & $\ldots$ & $\ldots$ & $\ldots$ & $\mathrm{EE}$ \\
\hline $\mathrm{PG}$ & $20.21 \pm 0$ & 18.5 & 013 & $\ldots$ & $18.88 \pm 0.06$ & $\ldots$ & 35.54 & 36.03 & $\ldots$ & 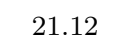 & $\ldots$ & $\ldots$ & $\ldots$ & $\cdots$ & $\ldots$ \\
\hline PG & $83 \pm$ & 15.58 & $0.25=$ & $.06 \pm 0.01$ & $15.79 \pm$ & $0.27 \pm 0$ & 36.72 & 36.64 & 15. & 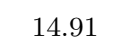 & $\ldots$ & $\ldots$ & $\ldots$ & $\ldots$ & $\mathrm{EE}$ \\
\hline UGC 01408 & $96 \pm 0.05$ & 17.57 & $0.39=$ & $18.12 \pm 0.04$ & $17.71 \pm 0.02$ & $0.41 \pm 0.05$ & 35.83 & 35.81 & 11.02 & 0 & $\ldots$ & $\ldots$ & $\ldots$ & $\ldots$ & $? \mathrm{~F}$ \\
\hline IC 1755 & $18.61 \pm 0.02$ & $18.07 \pm 0.02$ & $0.54 \pm 0.03$ & $18.90 \pm 0.04$ & $18.28 \pm 0.02$ & $0.62 \pm 0.05$ & 35.60 & 35.64 & 13.47 & 5 & 1.86 & 2.10 & 1.69 & 2.05 & $? \mathrm{~F}$ \\
\hline UGC 01448 & $17.79 \pm 0.01$ & $17.14 \pm 0.02$ & 0.65 & $17.89 \pm 0.03$ & $17.33 \pm 0.01$ & $0.55 \pm 0.04$ & 35.72 & 35.80 & 10.11 & 10.82 & 1.98 & 2.05 & 1.88 & 2.01 & $? \mathrm{D}$ \\
\hline KUG 0156-084 & $15.78 \pm 0.02$ & $15.45 \pm 0.01$ & $0.33 \pm 0.02$ & $15.84 \pm 0.02$ & $15.52 \pm 0.01$ & $0.32 \pm 0.02$ & 36.27 & 36.22 & 16.21 & 15.99 & 1.97 & $\ldots$ & $\ldots$ & $\ldots$ & $? \mathrm{D}$ \\
\hline NGC 0770 & $20.15 \pm 0.68$ & $18.36 \pm 0.06$ & $1.79 \pm 0.68$ & $\ldots$ & $18.48 \pm 0.06$ & $\cdots$ & 33.98 & 34.52 & $\cdots$ & $\cdots$ & $\ldots$ & $\cdots$ & $\ldots$ & $\ldots$ & $\cdots$ \\
\hline NGC 0772 & $14.00 \pm 0.02$ & $13.54 \pm 0.01$ & $0.46 \pm 0.02$ & $14.09 \pm 0.01$ & $13.63 \pm 0.01$ & $0.45 \pm 0.01$ & 36.44 & 36.45 & 69.69 & 64.57 & 2.76 & 3.08 & 2.94 & 3.15 & ER \\
\hline UGC 01468 & $17.54 \pm 0.05$ & $17.15 \pm 0.02$ & $0.39 \pm 0.05$ & $17.60 \pm 0.03$ & $17.27 \pm 0.02$ & $0.33 \pm 0.04$ & 35.56 & & 14.22 & 14.56 & $\ldots$ & $\ldots$ & $\ldots$ & $\ldots$ & $\ldots$ \\
\hline NGC 0774 & $72 \pm 0$ & 17.8 & & $.71 \pm 0.11$ & $17.92 \pm 0$ & & & & $\ldots$ & & $\ldots$ & $\ldots$ & $\ldots$ & $\ldots$ & $\ldots$ \\
\hline NGC 0777 & & & & & & & & & & & $\ldots$ & $\ldots$ & $\ldots$ & $\ldots$ & VV \\
\hline & 18.21 & 17.3 & & $18.24 \pm 0.04$ & 17.48 & & 35.45 & 35.60 & $\cdots$ & 9. & $\ldots$ & $\ldots$ & $\ldots$ & $\ldots$ & $? \mathrm{~d}$ \\
\hline NGC 0787 & $16.65 \pm 0.01$ & $16.12 \pm 0.01$ & $0.53 \pm 0.01$ & $16.67 \pm 0.02$ & $16.17 \pm 0.01$ & $0.50 \pm 0.02$ & 35.92 & 35.96 & 19.53 & 19.66 & 2.02 & 2.39 & 2.12 & $\ldots$ & Vfn \\
\hline PGC 07654 & $16.45 \pm 0.01$ & $16.16 \pm 0.03$ & $0.28 \pm 0.03$ & $16.62 \pm 0.02$ & $16.34 \pm 0.01$ & $0.29 \pm 0.03$ & 35.02 & 34.96 & 18.02 & 18.20 & 2.78 & 2.76 & $\ldots$ & $\ldots$ & Ef \\
\hline NGC 0783 & $15.25 \pm 0.01$ & $14.80 \pm 0.01$ & $0.46 \pm 0.01$ & $15.33 \pm 0.01$ & $14.87 \pm 0.01$ & $0.46 \pm 0.01$ & 36.59 & 36.60 & 20.89 & 20.05 & 2.05 & 2.34 & 2.11 & $\ldots$ & EDn \\
\hline UGCA 023 & $16.99 \pm 0.03$ & $16.24 \pm 0.02$ & $0.75 \pm 0.04$ & $17.35 \pm 0.01$ & $16.71 \pm 0.01$ & $0.65 \pm 0.01$ & 35.60 & 35.72 & 15.53 & 17.13 & 2.54 & 2.48 & 2.55 & 2.51 & Ef \\
\hline NGC 0809 & $19.74 \pm 1.01$ & $18.03 \pm 0.09$ & $1.71 \pm 1.01$ & $\ldots$ & $18.22 \pm 0.03$ & $\ldots$ & 34.79 & 35.30 & 15.28 & 17.49 & $\ldots$ & $\ldots$ & $\ldots$ & $\ldots$ & $\ldots$ \\
\hline UGC 01584 & $17.38 \pm 0.04$ & $17.02 \pm 0.02$ & $0.36 \pm 0.05$ & $17.51 \pm 0.04$ & $17.14 \pm 0.02$ & $0.37 \pm 0.04$ & 36.05 & 36.01 & 13.32 & 13.38 & $\ldots$ & $\ldots$ & $\ldots$ & $\ldots$ & $\mathrm{EE}$ \\
\hline
\end{tabular}


Table 3-Continued

\begin{tabular}{|c|c|c|c|c|c|c|c|c|c|c|c|c|c|c|c|}
\hline \multirow{2}{*}{$\begin{array}{l}\text { Object Name } \\
\text { (1) }\end{array}$} & \multicolumn{3}{|c|}{ Asymptotic magnitudes and color } & \multicolumn{3}{|c|}{ D25 magnitudes and color } & \multicolumn{2}{|c|}{$\log L$} & \multicolumn{2}{|c|}{ Effective Radii } & \multicolumn{2}{|c|}{ C31 } & \multicolumn{2}{|c|}{$\mathrm{C} 42$} & \multirow{2}{*}{$\begin{array}{c}\text { UV } \\
\text { profile } \\
(16)\end{array}$} \\
\hline & $\begin{array}{c}\text { FUV } \\
(\mathrm{mag}) \\
(2)\end{array}$ & $\begin{array}{c}\text { NUV } \\
(\mathrm{mag}) \\
(3)\end{array}$ & $\begin{array}{c}\text { FUV-NUV } \\
(\mathrm{mag}) \\
(4)\end{array}$ & $\begin{array}{c}\text { FUV } \\
(\mathrm{mag}) \\
(5)\end{array}$ & $\begin{array}{c}\text { NUV } \\
(\mathrm{mag}) \\
(6)\end{array}$ & $\begin{array}{l}\text { FUV-NUV } \\
(\mathrm{mag}) \\
(7)\end{array}$ & $\begin{array}{c}\text { FUV } \\
(\mathrm{W}) \\
(8)\end{array}$ & $\begin{array}{l}\text { NUV } \\
(\mathrm{W}) \\
(9)\end{array}$ & $\begin{array}{c}\text { FUV } \\
(\operatorname{arcsec}) \\
(10)\end{array}$ & $\begin{array}{c}\text { NUV } \\
\text { (arcsec) } \\
(11)\end{array}$ & $\begin{array}{l}\text { FUV } \\
(12)\end{array}$ & $\begin{array}{l}\text { NUV } \\
(13)\end{array}$ & $\begin{array}{l}\text { FUV } \\
(14)\end{array}$ & $\begin{array}{l}\text { NUV } \\
(15)\end{array}$ & \\
\hline NGC 0810 & $.05 \pm 0$. & $76 \pm 0$ & $1.29 \pm 0$ & \pm 0.19 & 1 & $1.02 \perp$ & & & & & & & . & $\cdots$ & . \\
\hline UGC 01593 & $16.49 \pm 0.01$ & $16.33 \pm 0.01$ & $.17 \pm 0.01$ & $97 \pm 0.03$ & $16.73 \pm 0.02$ & $23 \pm 0$ & 36.39 & 36.28 & 16.32 & 14.75 & $\ldots$ & $\ldots$ & $\ldots$ & $\ldots$ & $? \mathrm{R}$ \\
\hline UGC 01603 & $16.76 \pm 0.05$ & $16.42 \pm 0.05$ & $0.35 \pm 0.07$ & $17.04 \pm 0.02$ & $16.71 \pm 0.01$ & $0.34 \pm 0.03$ & 36.08 & 36.04 & 17.29 & 17.00 & $\cdots$ & $\ldots$ & $\cdots$ & $\ldots$ & $\mathrm{EE}$ \\
\hline NGC 0830 & $47 \pm 0.10$ & $17.86 \pm 0.04$ & $1.60 \pm 0.11$ & $19.85 \pm 0.11$ & $18.11 \pm 0.03$ & $1.74 \pm 0.12$ & 34.61 & 35.07 & 15.59 & 13.14 & $\ldots$ & $\ldots$ & $\ldots$ & $\ldots$ & VV \\
\hline NGC 0842 & $19.76 \pm 0.51$ & $17.78 \pm 0.03$ & $1.99 \pm 0.51$ & $19.71 \pm 0.10$ & $18.07 \pm 0.02$ & $1.64 \pm 0.10$ & 34.49 & 35.11 & $\ldots$ & 11.50 & $\ldots$ & $\ldots$ & $\ldots$ & $\ldots$ & VV \\
\hline NGC 0814 & $\ldots$ & $16.65 \pm 0.01$ & $\cdots$ & 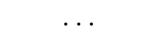 & $16.80 \pm 0.01$ & $\ldots$ & 0.00 & 34.75 & $\ldots$ & $\ldots$ & $\cdots$ & $\ldots$ & $\cdots$ & $\cdots$ & VV \\
\hline KUG 0210-078 & $16.21 \pm 0.01$ & $15.90 \pm 0.01$ & $0.30 \pm 0$. & $38 \pm 0.02$ & $16.07 \pm 0.01$ & $31 \pm 0.02$ & 36.10 & 36.05 & 30.43 & 28.54 & 1.98 & 2.40 & 2.11 & 2.41 & $\mathrm{EF}$ \\
\hline NGC 0855 & $15.75 \pm 0$ & $15.11 \pm$ & $0.64 \pm 0$ & $15.79 \pm 0.01$ & $15.22 \pm$ & $0.57 \pm$ & 34.61 & 34 & $\cdots$ & & $\ldots$ & $\cdots$ & $\ldots$ & $\cdots$ & VV \\
\hline ESO 415-G011 & $.02 \pm 0.01$ & $17.44 \pm 0.01$ & $0.58 \pm 0.01$ & $18.14 \pm 0.03$ & $17.64 \pm 0.01$ & $0.51 \pm 0$ & 35.07 & 35.13 & 8.15 & 8.37 & $\ldots$ & $\ldots$ & $\ldots$ & $\ldots$ & $\mathrm{EF}$ \\
\hline KUG 0211-075 & $.44 \pm 0.04$ & $16.18 \pm 0.02$ & $0.25 \pm 0$ & $16.73 \pm 0.02$ & $16.45 \pm 0$ & 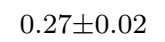 & 36.04 & 35 & 13.33 & 8 & 3.86 & 3.36 & 3.71 & 3.27 & $\mathrm{ER}$ \\
\hline NGC 0871 & $.29 \pm 0.02$ & $14.74 \pm 0$. & 0 . & $15.37 \pm 0.01$ & $14.8^{:}$ & $2 \pm$ & 36.28 & & & & & & $\ldots$ & $\ldots$ & $\mathrm{EE}$ \\
\hline KUG 0214- & $60 \pm 0.01$ & $16.23 \pm 0.01$ & $0.37 \pm 0.02$ & $16.76 \pm 0.01$ & $16.41 \pm 0.01$ & $0.35 \pm 0.01$ & 36.05 & 36.02 & 15 & 7 & 2.33 & 2.45 & $\ldots$ & $\ldots$ & $\mathrm{EF}$ \\
\hline UGC 01761 & $40 \pm 0.02$ & $16 \pm$ & $0.24 \pm 0.02$ & $16.45 \pm 0.02$ & $16.24 \pm 0$ & 03 & 35.89 & 35.81 & 16.69 & 6. & $\ldots$ & $\ldots$ & $\ldots$ & $\ldots$ & $\mathrm{EF}$ \\
\hline NGC 0881 & $16.44 \pm 0.01$ & $15.70 \pm 0.01$ & $0.74 \pm 0.01$ & $16.49 \pm 0.01$ & $15.76 \pm 0.01$ & $0.73 \pm 0$ & 36.09 & 36.21 & 24.07 & ) & 2.57 & 2.82 & 2.56 & 2.88 & $? \mathrm{~F}$ \\
\hline NGC 0895 & $14.11 \pm 0.01$ & $13.79 \pm 0.01$ & $0.32 \pm 0.01$ & $14.18 \pm 0.01$ & $13.86 \pm$ & $0.33 \pm 0$ & 36.28 & 36.24 & 49.41 & 47.06 & 2.12 & 2.25 & 2.14 & 2.30 & $\mathrm{VF}$ \\
\hline NGC 0891 & $14.74 \pm 0.01$ & $13.83 \pm 0.01$ & $0.90=$ & $14.87 \pm 0.01$ & $14.02 \pm 0.01$ & $0.85 \pm 0.02$ & 35.00 & 35.19 & 87.24 & 4 & 2.51 & 2.51 & 2.47 & 2.48 & $\mathrm{EFh}$ \\
\hline NGC 0898 & $19.04 \pm 0.17$ & $17.72 \pm 0.03$ & $1.32 \pm 0.17$ & & $17.87 \pm 0.03$ & $\ldots$ & 35.13 & 35.48 & 11.31 & 11.89 & 2.43 & 2.95 & $\ldots$ & $\ldots$ & $\mathrm{EE}$ \\
\hline UGC 01859 & $19.89 \pm 0.12$ & $18.45 \pm 0.06$ & $1.44 \pm 0.14$ & $19.65 \pm 0.17$ & $\ldots$ & $\ldots$ & 34.85 & 35.25 & $\ldots$ & $\ldots$ & $\ldots$ & $\ldots$ & $\ldots$ & $\ldots$ & $\ldots$ \\
\hline NGC 0906 & $16.68 \pm 0.01$ & $16.05 \pm 0.01$ & $0.63 \pm 0.02$ & $16.78 \pm 0.03$ & $16.13 \pm 0.01$ & $0.65 \pm 0.03$ & 35.94 & 36.01 & 25.82 & 24.52 & 2.12 & 2.26 & 2.02 & 2.27 & ?Fn \\
\hline NGC 0925 & $12.13 \pm 0.01$ & $11.91 \pm 0.01$ & $0.23 \pm 0.01$ & $12.19 \pm 0.01$ & $11.96 \pm 0.01$ & $0.23 \pm 0.01$ & 36.02 & 35.93 & 113.72 & 110.82 & 2.47 & 2.57 & 2.57 & 2.66 & EFn \\
\hline PGC 09333 & $17.72 \pm 0.02$ & $17.39 \pm 0.01$ & $0.33 \pm 0.03$ & $17.83 \pm 0.02$ & $17.49 \pm 0.01$ & $0.33 \pm 0.03$ & 36.38 & 36.34 & $\ldots$ & $\ldots$ & $\ldots$ & $\ldots$ & $\ldots$ & $\ldots$ & $\mathrm{EFn}$ \\
\hline NGC 0934 & $27 \pm 0.02$ & $17.57 \pm 0.01$ & & $19.47 \pm 0.04$ & & $0.96 \pm 0.04$ & 35.53 & & & & 1.82 & 3.38 & 1.84 & $\ldots$ & EDn \\
\hline UGC & & & & $18.44 \pm 0$ & & $0.21 \pm$ & 34.17 & & 13.8 & 13. & $\ldots$ & $\ldots$ & $\ldots$ & $\ldots$ & $\mathrm{EF}$ \\
\hline UGC 01976 & $17.31 \pm 0.09$ & $16.82 \pm 0.02$ & $0.49 \pm 0.09$ & $17.39 \pm 0.03$ & $16.93 \pm 0.02$ & $0.47 \pm 0.04$ & 36.25 & 36.27 & 17.44 & 17.24 & 2.12 & 2.38 & 2.07 & $\ldots$ & $? \mathrm{~F}$ \\
\hline NGC 0955 & $16.84 \pm 0.01$ & $16.09 \pm 0.01$ & $0.75 \pm 0.02$ & $17.03 \pm 0.02$ & $16.27 \pm 0.01$ & $0.76 \pm 0.02$ & 34.81 & 34.93 & 22.36 & 19.59 & 2.74 & 3.27 & 2.67 & 3.16 & $\mathrm{E}$ E,ER \\
\hline UGC 02010 & $16.89 \pm 0.05$ & $16.46 \pm 0.02$ & $0.42 \pm 0.05$ & $17.11 \pm 0.02$ & $16.67 \pm 0.01$ & $0.44 \pm 0.03$ & 36.59 & 36.58 & 17.26 & 16.81 & 2.34 & 2.43 & $\ldots$ & $\ldots$ & $\mathrm{EF}$ \\
\hline NGC 0959 & $14.57 \pm 0.01$ & $14.19 \pm 0.01$ & $0.37 \pm 0.01$ & $14.60 \pm 0.01$ & $14.23 \pm 0.01$ & $0.37 \pm 0.01$ & 35.22 & 35.20 & 19.21 & 19.83 & 2.16 & 2.15 & 2.11 & 2.14 & $\mathrm{VF}$ \\
\hline NGC 0986A & $16.28 \pm 0.01$ & $16.02 \pm 0.01$ & $0.26 \pm 0.02$ & $16.34 \pm 0.02$ & $16.11 \pm 0.01$ & $0.23 \pm 0.02$ & 34.87 & 34.80 & 12.08 & 13.43 & $\ldots$ & $\ldots$ & $\ldots$ & $\ldots$ & $? \mathrm{~F}$ \\
\hline NGC 0986 & $14.81 \pm 0.01$ & $14.02 \pm 0.01$ & $0.79 \pm 0.01$ & $14.85 \pm 0.01$ & $14.06 \pm 0.01$ & $0.79 \pm 0.01$ & 35.80 & 35.94 & 52.89 & 44.41 & 1.89 & 3.86 & 1.84 & $\ldots$ & $\mathrm{EFn}$ \\
\hline KUG 0232-079 & $15.72 \pm 0.01$ & $15.25 \pm 0.01$ & $0.47 \pm 0.01$ & $15.90 \pm 0.01$ & $15.41 \pm 0.01$ & $0.49 \pm 0.02$ & 36.58 & 36.59 & 16.01 & 14.27 & $\ldots$ & $\cdots$ & $\cdots$ & $\ldots$ & VFn \\
\hline NGC 0991 & $14.35 \pm 0.01$ & $14.05 \pm 0.01$ & $0.30 \pm 0.01$ & $14.43 \pm 0.01$ & $14.12 \pm 0.01$ & $0.30 \pm 0.01$ & 35.82 & 35.76 & 41.89 & 40.63 & 1.98 & 2.03 & 1.81 & 1.90 & VF \\
\hline IC 0243 & $18.24 \pm 0.01$ & $17.48 \pm 0.04$ & $0.77 \pm 0.04$ & $18.31 \pm 0.03$ & $17.65 \pm 0.02$ & $0.66 \pm 0.04$ & 35.65 & 35.78 & 11.59 & 12.48 & $\ldots$ & $\ldots$ & $\ldots$ & $\ldots$ & . \\
\hline
\end{tabular}


Table 3-Continued

\begin{tabular}{|c|c|c|c|c|c|c|c|c|c|c|c|c|c|c|c|}
\hline \multirow{2}{*}{$\begin{array}{l}\text { Object Name } \\
\text { (1) }\end{array}$} & \multicolumn{3}{|c|}{ Asymptotic magnitudes and color } & \multicolumn{3}{|c|}{ D25 magnitudes and color } & \multicolumn{2}{|c|}{$\log L$} & \multicolumn{2}{|c|}{ Effective Radii } & \multicolumn{2}{|c|}{ C31 } & \multicolumn{2}{|c|}{$\mathrm{C} 42$} & \multirow{2}{*}{$\begin{array}{c}\text { UV } \\
\text { profile } \\
(16)\end{array}$} \\
\hline & $\begin{array}{c}\text { FUV } \\
(\mathrm{mag}) \\
(2)\end{array}$ & $\begin{array}{c}\text { NUV } \\
(\mathrm{mag}) \\
(3)\end{array}$ & $\begin{array}{l}\text { FUV-NUV } \\
(\mathrm{mag}) \\
(4)\end{array}$ & $\begin{array}{l}\text { FUV } \\
(\mathrm{mag}) \\
(5)\end{array}$ & $\begin{array}{c}\text { NUV } \\
(\mathrm{mag}) \\
(6)\end{array}$ & $\begin{array}{l}\text { FUV-NUV } \\
(\mathrm{mag}) \\
(7)\end{array}$ & $\begin{array}{c}\text { FUV } \\
(\mathrm{W}) \\
(8)\end{array}$ & $\begin{array}{l}\text { NUV } \\
(\mathrm{W}) \\
(9)\end{array}$ & $\begin{array}{c}\text { FUV } \\
(\operatorname{arcsec}) \\
(10)\end{array}$ & $\begin{array}{c}\text { NUV } \\
\text { (arcsec) } \\
(11)\end{array}$ & $\begin{array}{l}\text { FUV } \\
(12)\end{array}$ & $\begin{array}{l}\text { NUV } \\
(13)\end{array}$ & $\begin{array}{l}\text { FUV } \\
\text { (14) }\end{array}$ & $\begin{array}{l}\text { NUV } \\
(15)\end{array}$ & \\
\hline NGC 1022 & 1 & $35 \pm 0.01$ & $6 \pm 0$ & $01 \pm 0.02$ & $51 \pm$ & $1.50 \pm$ & 34.74 & 35 & & 18.42 & & & & & EV \\
\hline NGC 1035 & $90 \pm 0.01$ & $15.24 \pm 0.01$ & $0.67 \pm 0$ & $00 \pm 0.01$ & $15.35 \pm 0.01$ & $0.65 \pm 0.01$ & 34.99 & & 16.87 & 17.34 & 2.02 & 2.19 & 2.03 & 2.21 & ?Fn \\
\hline NGC 1033 & $16.86 \pm 0.03$ & $16.44 \pm 0.01$ & $0.42 \pm 0.03$ & $16.93 \pm 0.02$ & $16.53 \pm 0.01$ & $0.40 \pm 0.02$ & 36.21 & 36.21 & 17.26 & 16.74 & $\ldots$ & 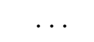 & $\ldots$ & 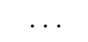 & $\mathrm{EFn}$ \\
\hline NGC 1042 & $13.46 \pm 0.01$ & $13.19 \pm 0.01$ & $0.27 \pm 0.01$ & $13.66 \pm 0.01$ & $13.37 \pm 0.01$ & $0.29 \pm 0.01$ & 36.06 & 36.00 & 73.44 & 72.23 & 2.02 & 2.08 & 2.04 & 2.09 & EFn \\
\hline NGC 1023 & $16.11 \pm 0.04$ & $14.50 \pm 0.01$ & $1.61 \pm 0.05$ & $16.08 \pm 0.05$ & $14.68 \pm 0.01$ & $1.40 \pm 0.05$ & 34.61 & 35.07 & 32.44 & 48.46 & 5.28 & 5.85 & 4.48 & 4.74 & VV \\
\hline NGC 1047 & $19.68 \pm 0.33$ & $17.33 \pm 0.11$ & $2.34 \pm 0.35$ & $19.84 \pm 0.09$ & $17.98 \pm 0.02$ & $1.86 \pm 0.09$ & 33.55 & 34.32 & $\ldots$ & 20.17 & $\ldots$ & $\ldots$ & $\ldots$ & $\ldots$ & $\ldots$ \\
\hline NGC $1023 \mathrm{~A}$ & $17.44 \pm 0.09$ & $16.32 \pm 0.02$ & $1.12 \pm 0.10$ & $18.09 \pm 0.06$ & $17.17 \pm 0.02$ & $0.92 \pm 0.06$ & 34.14 & 34.42 & 22.76 & 26.50 & 2.69 & $\ldots$ & $\ldots$ & $\ldots$ & $\mathrm{EE}$ \\
\hline NGC 0961 & $14.96 \pm 0.01$ & $14.69 \pm 0.01$ & $0.27 \pm 0.01$ & $15.16 \pm 0.01$ & $14.88 \pm 0.01$ & $0.28 \pm 0.01$ & 35.42 & 35.35 & 25.55 & 25.27 & 2.71 & 2.76 & 2.66 & 2.71 & $\mathrm{Ed}$ \\
\hline NGC 1052 & $16.80 \pm 0.02$ & $15.65 \pm 0.02$ & $1.15 \pm 0.03$ & $17.01 \pm 0.02$ & $15.98 \pm 0.01$ & $1.03 \pm 0.02$ & 34.79 & 35.08 & 19.10 & 31.32 & $\ldots$ & 7.32 & $\ldots$ & $\ldots$ & VV \\
\hline NGC 1055 & $14.68 \pm 0.03$ & $14.30 \pm 0.01$ & $0.38 \pm 0.03$ & $14.94 \pm 0.01$ & $14.45 \pm 0.01$ & $0.49 \pm 0.02$ & 35.31 & 35.28 & 68.31 & 60.96 & 3.02 & 2.79 & 2.96 & 2.77 & Ed,Ef \\
\hline PGC 10213 & $17.36 \pm 0.01$ & $16.90 \pm 0.03$ & $0.46 \pm 0.03$ & $17.44 \pm 0.03$ & $17.04 \pm 0.01$ & $0.40 \pm 0.03$ & 34.50 & 34.51 & 17.03 & 17.76 & & $\ldots$ & $\ldots$ & $\ldots$ & $\mathrm{EF}$ \\
\hline UGC 02174 & $15.84 \pm 0.03$ & $15.53 \pm 0.02$ & $0.31 \pm 0.04$ & $15.93 \pm 0.03$ & $15.62 \pm 0.02$ & $0.30 \pm 0.03$ & 36.34 & 36.29 & 34.59 & 34.88 & 2.16 & 2.19 & 2.11 & 2.16 & $? \mathrm{~F}$ \\
\hline NGC 1068 & $12.52 \pm 0.01$ & $11.95 \pm 0.01$ & $0.57 \pm 0.01$ & $12.52 \pm 0.01$ & $11.94 \pm 0.01$ & $0.57 \pm 0$ & 36.24 & 36.30 & 17.24 & 3 & $\ldots$ & $\ldots$ & $\ldots$ & $\ldots$ & ERn \\
\hline UGC 02182 & $17.64 \pm 0.13$ & $17.51 \pm 0.05$ & $0.13 \pm 0.14$ & $17.91 \pm 0.06$ & $17.73 \pm 0.04$ & $0.18 \pm 0.08$ & 35.65 & 35.52 & 11.40 & 11.16 & $\ldots$ & $\ldots$ & $\ldots$ & $\ldots$ & $\mathrm{EE}$ \\
\hline NGC 1069 & $16.38 \pm 0.01$ & $15.89 \pm 0.01$ & $0.49 \pm 0.02$ & $16.52 \pm 0.01$ & $16.04 \pm 0.01$ & $0.49 \pm 0.02$ & 36.64 & 36.66 & 17.18 & 16.21 & 2.38 & $\ldots$ & $\cdots$ & $\ldots$ & $\mathrm{EF}$ \\
\hline NGC 1060 & $18.04 \pm 0.17$ & $16.33 \pm 0.07$ & $1.72 \pm 0.18$ & $\ldots$ & $16.66 \pm 0.06$ & & 35.47 & 35.98 & $\ldots$ & 28.77 & $\ldots$ & $\ldots$ & $\ldots$ & $\ldots$ & $\ldots$ \\
\hline NGC 1072 & $16.88 \pm 0.04$ & $16.12 \pm 0.09$ & $0.76 \pm 0.10$ & $17.12 \pm 0.02$ & $16.63 \pm 0.01$ & $0.49 \pm 0.03$ & 36.29 & 36.42 & 15.54 & 18.22 & 2.23 & $\ldots$ & 2.26 & $\ldots$ & $\mathrm{EF}$ \\
\hline PGC 10334 & $16.49 \pm 0.02$ & $16.19 \pm 0.03$ & $0.31 \pm 0.03$ & $16.77 \pm 0.02$ & $16.49 \pm 0.01$ & $0.28 \pm 0.02$ & 34.88 & 34.83 & 19.17 & 20.04 & 2.42 & 2.53 & 2.53 & 2.60 & $? \mathrm{~F}$ \\
\hline UGC 02201 & $17.52 \pm 0.06$ & $17.21 \pm 0.05$ & $0.30 \pm 0.07$ & $17.88 \pm 0.08$ & $17.61 \pm 0.04$ & $0.27 \pm 0.09$ & 35.49 & 35.44 & 13.30 & 13.20 & 2.08 & 2.41 & 2.05 & 2.35 & ?d,?f \\
\hline NGC 1066 & $16.89 \pm 0.56$ & $16.76 \pm 0.37$ & $0.13 \pm 0.67$ & $17.73 \pm 0.17$ & $17.25 \pm 0.09$ & $0.48 \pm 0.19$ & 35.78 & 35.66 & 49.53 & 33.96 & 2.48 & 4.25 & $\ldots$ & $\ldots$ & Er \\
\hline NGC 1067 & $16.14 \pm 0.03$ & $15.68 \pm 0.02$ & $0.47 \pm 0.04$ & $16.33 \pm 0.03$ & $15.86 \pm 0.02$ & $0.46 \pm 0.04$ & 36.12 & 36.13 & 17.11 & 16.54 & $\ldots$ & $\ldots$ & $\ldots$ & $\ldots$ & $\cdots$ \\
\hline NGC 1084 & $13.50 \pm 0.01$ & $12.97 \pm 0.01$ & $0.53 \pm 0.01$ & $13.56 \pm 0.01$ & $13.01 \pm 0.01$ & $0.55 \pm 0.01$ & 36.07 & 36.11 & 27.29 & 25.57 & 1.97 & 2.01 & 1.92 & 1.98 & $\mathrm{VF}$ \\
\hline NGC 1097 & $12.50 \pm 0.04$ & $12.13 \pm 0.03$ & $0.37 \pm 0.05$ & $12.60 \pm 0.01$ & $12.21 \pm 0.01$ & $0.39 \pm 0.01$ & 36.30 & 36.27 & 129.37 & 102.90 & 2.73 & 3.89 & 2.74 & 4.01 & EFn \\
\hline PGC 10766 & $18.43 \pm 0.08$ & $17.88 \pm 0.02$ & $0.56 \pm 0$. & $18.80 \pm 0.05$ & $18.16 \pm 0.02$ & $0.65 \pm 0$ & 35.31 & & & & 2.77 & 2.57 & 2.69 & 2.53 & $\mathrm{Ef}$ \\
\hline PGC 10794 & 1 & $15.95 \pm 0$ & & & & & & & & & $\ldots$ & $\ldots$ & $\ldots$ & $\ldots$ & $? \mathrm{~F}$ \\
\hline PGC 10875 & $19.02 \pm 0.02$ & $18.15 \pm 0.03$ & $0.87 \pm 0.03$ & $19.37 \pm 0.05$ & $18.64 \pm 0.02$ & $0.73 \pm 0.06$ & 35.02 & 35.19 & 10.38 & 11.73 & 2.44 & 2.69 & 2.46 & 2.70 & $\mathrm{EE}$ \\
\hline NGC 1140 & $13.73 \pm 0.01$ & $13.54 \pm 0.01$ & $0.19 \pm 0.01$ & $13.86 \pm 0.01$ & $13.65 \pm 0.01$ & $0.21 \pm 0.01$ & 35.96 & 35.86 & $\ldots$ & $\ldots$ & $\ldots$ & $\ldots$ & $\ldots$ & $\ldots$ & VFn \\
\hline NGC 1148 & $16.20 \pm 0.02$ & $15.82 \pm 0.01$ & $0.38 \pm 0.02$ & $16.35 \pm 0.02$ & $15.97 \pm 0.01$ & $0.38 \pm 0.02$ & 36.18 & 36.15 & 15.83 & 15.71 & 1.93 & 1.98 & 1.84 & 1.91 & $\mathrm{ED}$ \\
\hline UGC 02442 & $16.77 \pm 0.07$ & $16.33 \pm 0.06$ & $0.44 \pm 0.10$ & $17.09 \pm 0.05$ & $16.65 \pm 0.02$ & $0.44 \pm 0.06$ & 36.58 & 36.58 & 18.35 & 18.05 & $\ldots$ & $\ldots$ & $\ldots$ & $\ldots$ & $\mathrm{EE}$ \\
\hline NGC 1156 & $12.69 \pm 0.01$ & $12.48 \pm 0.01$ & $0.21 \pm 0.01$ & $12.73 \pm 0.01$ & $12.50 \pm 0.01$ & $0.23 \pm 0.01$ & 35.64 & 35.55 & 25.00 & 25.51 & 3.35 & 3.21 & 3.34 & 3.19 & $\mathrm{EE}$ \\
\hline PGC 11767 & $17.04 \pm 0.04$ & $16.55 \pm 0.02$ & $0.49 \pm 0.04$ & $17.34 \pm 0.02$ & $16.83 \pm 0.01$ & $0.51 \pm 0.03$ & 36.30 & 36.32 & 13.94 & 13.88 & 2.14 & 2.18 & 2.12 & 2.16 & $\mathrm{EF}$ \\
\hline UGC 02519 & $15.70 \pm 0.02$ & $14.96 \pm 0.01$ & $0.73 \pm 0.02$ & $15.81 \pm 0.03$ & $15.09 \pm 0.01$ & $0.73 \pm 0.04$ & 35.81 & 35.93 & 14.03 & 13.53 & $\ldots$ & $\ldots$ & .. & .. & $\mathrm{EF}$ \\
\hline
\end{tabular}


Table 3-Continued

\begin{tabular}{|c|c|c|c|c|c|c|c|c|c|c|c|c|c|c|c|}
\hline \multirow{2}{*}{$\begin{array}{l}\text { Object Name } \\
\text { (1) }\end{array}$} & \multicolumn{3}{|c|}{ Asymptotic magnitudes and color } & \multicolumn{3}{|c|}{ D25 magnitudes and color } & \multicolumn{2}{|c|}{$\log L$} & \multicolumn{2}{|c|}{ Effective Radii } & \multicolumn{2}{|c|}{$\mathrm{C} 31$} & \multicolumn{2}{|c|}{$\mathrm{C} 42$} & \multirow{2}{*}{$\begin{array}{c}\text { UV } \\
\text { profile } \\
(16)\end{array}$} \\
\hline & $\begin{array}{l}\text { FUV } \\
(\mathrm{mag}) \\
(2)\end{array}$ & $\begin{array}{c}\text { NUV } \\
(\mathrm{mag}) \\
(3)\end{array}$ & $\begin{array}{c}\text { FUV-NUV } \\
\text { (mag) } \\
(4)\end{array}$ & $\begin{array}{c}\text { FUV } \\
(\mathrm{mag}) \\
(5)\end{array}$ & $\begin{array}{c}\text { NUV } \\
(\mathrm{mag}) \\
(6)\end{array}$ & $\begin{array}{l}\text { FUV-NUV } \\
(\mathrm{mag}) \\
(7)\end{array}$ & $\begin{array}{c}\text { FUV } \\
(\mathrm{W}) \\
(8)\end{array}$ & $\begin{array}{c}\text { NUV } \\
(\mathrm{W}) \\
(9)\end{array}$ & $\begin{array}{c}\text { FUV } \\
(\operatorname{arcsec}) \\
(10)\end{array}$ & $\begin{array}{c}\text { NUV } \\
(\operatorname{arcsec}) \\
(11)\end{array}$ & $\begin{array}{l}\text { FUV } \\
(12)\end{array}$ & $\begin{array}{l}\text { NUV } \\
(13)\end{array}$ & $\begin{array}{l}\text { FUV } \\
(14)\end{array}$ & $\begin{array}{l}\text { NUV } \\
(15)\end{array}$ & \\
\hline NGC 1241 & .01 & 0.01 & 02 & $.88 \pm 0.01$ & $14.56 \pm 0$ & $0.32 \pm$ & 36.56 & 36.48 & 31.88 & 27.89 & 2.43 & 2.29 & 2.39 & 2.20 & Edn \\
\hline NGC 1242 & 1 & 15.52 & $0.34 \pm 0.03$ & $.92 \pm 0.02$ & $15.62 \pm 0.01$ & $0.30 \pm 0$ & 36.07 & & & & $\ldots$ & $\ldots$ & $\ldots$ & $\ldots$ & $? \mathrm{~F}$ \\
\hline NGC 1266 & $19.67 \pm 0.01$ & $17.74 \pm 0.03$ & $1.93 \pm 0.03$ & $\ldots$ & $17.82 \pm 0.04$ & ... & 34.06 & 34.65 & 10.51 & 13.32 & $\ldots$ & $\ldots$ & $\ldots$ & $\ldots$ & $\mathrm{EE}$ \\
\hline NGC 1291 & $14.23 \pm 0.01$ & $13.37 \pm 0.01$ & $0.86 \pm 0.01$ & $14.83 \pm 0.01$ & $13.81 \pm 0.01$ & $1.03 \pm 0.01$ & 35.22 & 35.38 & 252.51 & 211.55 & 1.92 & 4.14 & 2.62 & 3.91 & EDn \\
\hline NGC 1285 & $15.02 \pm 0.01$ & $14.59 \pm 0.01$ & $0.43 \pm 0.01$ & $15.12 \pm 0.01$ & $14.69 \pm 0.01$ & $0.43 \pm 0.01$ & 36.66 & 36.65 & 16.99 & 16.27 & $\ldots$ & $\ldots$ & $\ldots$ & $\ldots$ & Ef \\
\hline NGC 1299 & $15.52 \pm 0.02$ & $15.07 \pm 0.01$ & $0.45 \pm 0.02$ & $15.62 \pm 0.01$ & $15.20 \pm 0.01$ & $0.42 \pm 0.02$ & 35.73 & 35.73 & 10.11 & 10.04 & $\ldots$ & $\ldots$ & $\ldots$ & $\ldots$ & Ef \\
\hline NGC 1310 & $15.07 \pm 0.01$ & $14.64 \pm 0.01$ & $0.43 \pm 0.01$ & $15.12 \pm 0.01$ & $14.70 \pm 0.01$ & $0.42 \pm 0.01$ & 35.61 & 35.61 & 20.67 & 20.69 & 2.37 & 2.42 & 2.36 & 2.44 & ?f \\
\hline KUG 0319-072 & $15.61 \pm 0.01$ & $15.29 \pm 0.01$ & $0.31 \pm 0.01$ & $15.74 \pm 0.01$ & $15.43 \pm 0.01$ & $0.31 \pm 0.01$ & 35.83 & 35.79 & 15.84 & 15.78 & $\ldots$ & $\ldots$ & $\ldots$ & $\ldots$ & $? \mathrm{~F}$ \\
\hline NGC 1316 & $15.16 \pm 0.02$ & $13.35 \pm 0.01$ & $1.81 \pm 0.02$ & $15.30 \pm 0.01$ & $13.53 \pm 0.01$ & $1.77 \pm 0.02$ & 35.41 & 35.96 & 89.24 & 107.26 & 5.81 & 5.26 & 4.74 & 4.47 & $\mathrm{VV}$ \\
\hline NGC 1317 & $15.38 \pm 0.01$ & $14.78 \pm 0.01$ & $0.59 \pm 0.02$ & $15.39 \pm 0.01$ & $14.86 \pm 0.01$ & $0.53 \pm 0.02$ & 35.32 & 35.39 & $\ldots$ & $\ldots$ & $\ldots$ & $\ldots$ & $\ldots$ & $\ldots$ & ?Rh \\
\hline ESO 357-G025 & $17.34 \pm 0.01$ & $16.75 \pm 0.03$ & $0.59 \pm 0.03$ & $17.35 \pm 0.02$ & $16.89 \pm 0.01$ & $0.47 \pm 0.02$ & 34.71 & 34.77 & $\ldots$ & $\ldots$ & $\ldots$ & $\ldots$ & $\ldots$ & $\ldots$ & $\ldots$ \\
\hline PGC 12706 & $16.14 \pm 0.01$ & $15.68 \pm 0.01$ & $0.46 \pm 0.01$ & $16.17 \pm 0.02$ & $15.78 \pm 0.01$ & $0.39 \pm 0.02$ & 34.93 & 34.93 & 14.92 & 15.76 & $\ldots$ & $\ldots$ & $\ldots$ & $\ldots$ & ?Fh \\
\hline NGC 1326 & $14.60 \pm 0.07$ & $14.02 \pm 0.04$ & $0.58 \pm 0.08$ & $14.67 \pm 0.01$ & $14.15 \pm 0.01$ & $0.52 \pm 0.01$ & 35.51 & 35.57 & $\ldots$ & 11.70 & $\ldots$ & $\ldots$ & $\ldots$ & $\ldots$ & EFn \\
\hline PGC 13005 & $19.99 \pm 0.23$ & $18.56 \pm 0.04$ & $1.43 \pm 0.23$ & $\ldots$ & $18.92 \pm 0.04$ & ... & 34.47 & 34.86 & 8.52 & 11.09 & $\ldots$ & 2.84 & $\ldots$ & $\ldots$ & $\mathrm{EE}$ \\
\hline ESO $418-G 008$ & $15.18 \pm 0.01$ & $15.00 \pm 0.01$ & $0.18 \pm 0.02$ & $15.27 \pm 0.01$ & $15.11 \pm 0.01$ & $0.16 \pm 0.01$ & 35.16 & 35.06 & 13.20 & 13.29 & $\ldots$ & $\ldots$ & $\ldots$ & $\ldots$ & $? \mathrm{~F}$ \\
\hline NGC 1365 & $\ldots$ & $11.83 \pm 0.01$ & $\ldots$ & $\ldots$ & $11.90 \pm 0.01$ & $\ldots$ & 0.00 & 36.63 & $\ldots$ & 129.82 & $\ldots$ & 2.00 & $\ldots$ & 2.07 & EFn \\
\hline PGC 13186 & $\ldots$ & $19.73 \pm 0.31$ & $\ldots$ & $\ldots$ & $19.85 \pm 0.12$ & .. & 0.00 & 33.41 & $\ldots$ & 15.44 & $\ldots$ & $\ldots$ & $\ldots$ & $\ldots$ & $\mathrm{EE}$ \\
\hline NGC 1361 & $19.39 \pm 0.25$ & $18.14 \pm 0.07$ & $1.25 \pm 0.26$ & $19.45 \pm 0.15$ & $18.31 \pm 0.05$ & $1.15 \pm 0.15$ & 34.91 & 35.24 & $\ldots$ & 15.70 & $\ldots$ & $\ldots$ & $\ldots$ & $\ldots$ & $\mathrm{VV}$ \\
\hline PGC 13230 & $21.48 \pm 0.23$ & $18.53 \pm 0.04$ & $2.95 \pm 0.24$ & $21.38 \pm 0.32$ & $18.87 \pm 0.02$ & $2.51 \pm 0.32$ & 32.63 & 33.64 & 10.82 & 17.99 & $\ldots$ & $\ldots$ & $\ldots$ & $\ldots$ & VV \\
\hline NGC 1373 & $19.84 \pm 0.09$ & $17.94 \pm 0.03$ & $1.90 \pm 0.10$ & $20.08 \pm 0.10$ & $18.25 \pm 0.02$ & $1.84 \pm 0.10$ & 33.40 & 33.98 & 12.46 & 12.91 & $\ldots$ & $\ldots$ & $\ldots$ & $\ldots$ & VV \\
\hline NGC 1374 & $17.54 \pm 0.02$ & $16.02 \pm 0.02$ & $1.52 \pm 0.03$ & $17.77 \pm 0.02$ & $16.33 \pm 0.01$ & $1.44 \pm 0.02$ & 34.28 & 34.72 & 24.19 & 31.15 & $\ldots$ & 5.90 & $\ldots$ & $\ldots$ & VV \\
\hline NGC 1375 & $18.34 \pm 0.04$ & $16.63 \pm 0.02$ & $1.71 \pm 0.05$ & $18.51 \pm 0.03$ & $16.92 \pm 0.01$ & $1.59 \pm 0.03$ & 33.33 & & $\ldots$ & & $\ldots$ & $\ldots$ & $\ldots$ & $\ldots$ & VV \\
\hline NGC 1379 & $17.89 \pm$ & 16. & & $18.05 \pm 0$ & & $1.62 \pm$ & & & & & $\ldots$ & $\ldots$ & $\ldots$ & $\ldots$ & $\mathrm{VV}$ \\
\hline UGCA 080 & $16.24 \pm 0.02$ & $16.05 \pm 0.01$ & $0.19 \pm 0.02$ & $16.44 \pm 0.02$ & $16.27 \pm 0.01$ & $0.17 \pm 0.02$ & 35.69 & 35.59 & 24.58 & 23.59 & 2.79 & 2.94 & $\ldots$ & $\ldots$ & ?Fn \\
\hline NGC 1380 & $16.81 \pm 0.02$ & $15.20 \pm 0.01$ & $1.62 \pm 0.02$ & $16.93 \pm 0.02$ & $15.38 \pm 0.01$ & $1.55 \pm 0.03$ & 34.96 & 35.42 & 27.71 & 35.87 & 5.97 & 4.97 & $\ldots$ & 4.32 & $\mathrm{VV}$ \\
\hline NGC 1381 & $17.83 \pm 0.05$ & $16.38 \pm 0.01$ & $1.45 \pm 0.05$ & $18.04 \pm 0.03$ & $16.54 \pm 0.01$ & $1.50 \pm 0.03$ & 34.34 & 34.75 & 14.66 & 14.30 & 4.70 & 3.97 & $\ldots$ & $\ldots$ & ?R \\
\hline NGC 1386 & $16.62 \pm 0.01$ & $15.46 \pm 0.01$ & $1.16 \pm 0.01$ & $16.67 \pm 0.01$ & $15.51 \pm 0.01$ & $1.15 \pm 0.01$ & 34.21 & 34.49 & 9.78 & 13.44 & $\ldots$ & $\ldots$ & $\ldots$ & $\ldots$ & VF,ER \\
\hline NGC $1380 \mathrm{~A}$ & $19.24 \pm 0.08$ & $17.09 \pm 0.01$ & $2.15 \pm 0.08$ & $\ldots$ & $17.30 \pm 0.01$ & $\ldots$ & 33.80 & 34.48 & 9.93 & 16.76 & $\ldots$ & 3.71 & $\ldots$ & 3.60 & VV \\
\hline PGC 13343 & $\ldots$ & $18.57 \pm 0.10$ & $\ldots$ & $\ldots$ & $18.82 \pm 0.04$ & .. & 0.00 & 33.95 & $\ldots$ & 12.91 & $\ldots$ & $\ldots$ & $\ldots$ & $\ldots$ & VV \\
\hline NGC 1387 & $16.48 \pm 0.01$ & $15.48 \pm 0.01$ & $1.00 \pm 0.02$ & $16.56 \pm 0.02$ & $15.61 \pm 0.01$ & $0.96 \pm 0.02$ & 34.88 & 35.11 & $\ldots$ & $\ldots$ & $\ldots$ & $\ldots$ & $\ldots$ & $\ldots$ & $\mathrm{EV}$ \\
\hline
\end{tabular}


Table 3-Continued

\begin{tabular}{|c|c|c|c|c|c|c|c|c|c|c|c|c|c|c|c|}
\hline \multirow{2}{*}{$\begin{array}{l}\text { Object Name } \\
\text { (1) }\end{array}$} & \multicolumn{3}{|c|}{ Asymptotic magnitudes and color } & \multicolumn{3}{|c|}{ D25 magnitudes and color } & \multicolumn{2}{|c|}{$\log L$} & \multicolumn{2}{|c|}{ Effective Radii } & \multicolumn{2}{|c|}{$\mathrm{C} 31$} & \multicolumn{2}{|c|}{$\mathrm{C} 42$} & \multirow{2}{*}{$\begin{array}{c}\text { UV } \\
\text { profile } \\
(16)\end{array}$} \\
\hline & $\begin{array}{l}\text { FUV } \\
(\mathrm{mag}) \\
(2)\end{array}$ & $\begin{array}{c}\text { NUV } \\
(\mathrm{mag}) \\
(3)\end{array}$ & $\begin{array}{c}\text { FUV-NUV } \\
\text { (mag) } \\
(4)\end{array}$ & $\begin{array}{c}\text { FUV } \\
(\mathrm{mag}) \\
(5)\end{array}$ & $\begin{array}{c}\text { NUV } \\
(\mathrm{mag}) \\
(6)\end{array}$ & $\begin{array}{l}\text { FUV-NUV } \\
(\mathrm{mag}) \\
(7)\end{array}$ & $\begin{array}{c}\text { FUV } \\
(\mathrm{W}) \\
(8)\end{array}$ & $\begin{array}{c}\text { NUV } \\
(\mathrm{W}) \\
(9)\end{array}$ & $\begin{array}{c}\text { FUV } \\
(\operatorname{arcsec}) \\
(10)\end{array}$ & $\begin{array}{c}\text { NUV } \\
(\operatorname{arcsec}) \\
(11)\end{array}$ & $\begin{array}{l}\text { FUV } \\
(12)\end{array}$ & $\begin{array}{l}\text { NUV } \\
(13)\end{array}$ & $\begin{array}{l}\text { FUV } \\
(14)\end{array}$ & $\begin{array}{l}\text { NUV } \\
(15)\end{array}$ & \\
\hline NGC $1380 B$ & 10 & 0.01 & 0 & 8 & $17.74 \pm 0$ & $1.53 \pm$ & 34.06 & 34 & 53 & 1 & & & & & $\mathrm{VV}$ \\
\hline NGC 1389 & & & & $71 \pm 0.06$ & 16.7 & & & & & & $\ldots$ & $\ldots$ & $\ldots$ & $\ldots$ & $\mathrm{VV}$ \\
\hline NGC 1385 & $23 \pm 0.01$ & $12.87 \pm 0.01$ & & $13.25 \pm 0.01$ & $12.89 \pm 0$ & & 36.19 & 36.15 & 25.94 & 25.33 & 3.12 & 3.20 & 2.94 & 3.03 & VFn \\
\hline NGC 1383 & $26 \pm 0.94$ & $17.60 \pm 0.05$ & $0.66 \pm 0.94$ & $9.24 \pm 0.13$ & $17.72 \pm 0.03$ & $1.52 \pm 0.13$ & 34.44 & 34.53 & $\ldots$ & 11.30 & $\ldots$ & $\ldots$ & $\ldots$ & $\ldots$ & VV \\
\hline NGC 1396 & $\ldots$ & $18.29 \pm 0.19$ & $\ldots$ & $\ldots$ & $18.98 \pm 0.05$ & $\ldots$ & 0.00 & 33.28 & $\ldots$ & 22.99 & $\ldots$ & $\ldots$ & $\ldots$ & $\cdots$ & VV \\
\hline ESO 358-G042 & $\ldots$ & $18.97 \pm 0.05$ & $\ldots$ & $\ldots$ & $19.36 \pm 0.04$ & $\ldots$ & 0.00 & 33.40 & $\ldots$ & 14.15 & $\ldots$ & $\ldots$ & $\ldots$ & $\ldots$ & $\ldots$ \\
\hline NGC 1399 & $15.01 \pm 0.01$ & $14.34 \pm 0.02$ & $0.67 \pm 0.02$ & $14.96 \pm 0.01$ & $14.38 \pm 0.01$ & $0.58 \pm 0.01$ & 35.47 & 35.56 & 17.71 & 42.94 & $\ldots$ & $\ldots$ & $\ldots$ & $\ldots$ & VV \\
\hline NGC 1393 & $18.53 \pm 0.10$ & $17.27 \pm 0.05$ & $1.26 \pm 0.11$ & $18.61 \pm 0.09$ & $17.41 \pm 0.03$ & $1.20 \pm 0.10$ & 34.42 & 34.75 & 12.77 & 14.26 & $\ldots$ & $\ldots$ & $\ldots$ & $\ldots$ & $\mathrm{VV}$ \\
\hline NGC 1404 & $16.44 \pm 0.01$ & $15.30 \pm 0.01$ & $1.14 \pm 0.02$ & $16.50 \pm 0.02$ & $15.42 \pm 0.01$ & $1.07 \pm 0.02$ & 34.90 & 35.18 & $\ldots$ & 22.58 & $\ldots$ & $\ldots$ & $\ldots$ & $\ldots$ & VV \\
\hline NGC 1391 & $19.74 \pm 0.07$ & $18.41 \pm 0.05$ & $1.33 \pm 0.09$ & $\ldots$ & $18.56 \pm 0.04$ & $\ldots$ & 34.59 & 34.94 & $\ldots$ & $\ldots$ & $\ldots$ & $\ldots$ & $\ldots$ & $\ldots$ & VV \\
\hline NGC 1394 & $18.91 \pm 0.08$ & $17.54 \pm 0.07$ & $1.37 \pm 0.11$ & $\ldots$ & $17.75 \pm 0.02$ & . & 34.90 & 35.27 & $\ldots$ & 7.14 & $\ldots$ & $\ldots$ & $\ldots$ & $\ldots$ & VV \\
\hline AM 0337-355 & $21.16 \pm 0.82$ & $19.21 \pm 0.16$ & $1.95 \pm 0.84$ & $21.29 \pm 0.47$ & $19.59 \pm 0.08$ & $1.70 \pm 0.47$ & 32.44 & 33.04 & 13.83 & 17 & $\ldots$ & $\ldots$ & $\ldots$ & $\ldots$ & $\ldots$ \\
\hline NGC 1400 & $16.64 \pm 0.03$ & $15.71 \pm 0.02$ & $0.93 \pm 0.03$ & $16.77 \pm 0.03$ & $15.91 \pm 0.01$ & $0.86 \pm 0.03$ & 35.09 & 35.28 & & & $\ldots$ & $\ldots$ & $\ldots$ & $\ldots$ & VV \\
\hline IC 0343 & & $17.83 \pm 0.13$ & & $\ldots$ & $18.20 \pm 0.04$ & & 0.00 & 34.38 & $\ldots$ & & $\ldots$ & $\ldots$ & $\ldots$ & $\ldots$ & VV \\
\hline ESO 548-G068 & $19.46 \pm 0.76$ & $17.77 \pm 0.03$ & $1.68 \pm 0.76$ & $19.82 \pm 0.21$ & $18.10 \pm 0.04$ & $1.73 \pm 0.22$ & 33.83 & 34.33 & 20.10 & 14.94 & 3.31 & $\ldots$ & $\ldots$ & $\ldots$ & VV \\
\hline PGC 13515 & $\ldots$ & $19.80 \pm 0.42$ & $\ldots$ & $\ldots$ & $20.05 \pm 0.13$ & & 0.00 & 33.67 & $\ldots$ & 16.32 & $\ldots$ & $\ldots$ & $\ldots$ & $\ldots$ & VV \\
\hline PGC 13535 & $15.88 \pm 0.03$ & $15.67 \pm 0.01$ & $0.21 \pm 0.03$ & $15.97 \pm 0.02$ & $15.76 \pm 0.01$ & $0.20 \pm 0.02$ & 36.31 & 36.22 & 25.27 & 24.88 & 2.23 & 2.39 & 2.26 & 2.45 & ?Fn \\
\hline PGC 13600 & $17.49 \pm 0.32$ & $16.92 \pm 0.07$ & $0.57 \pm 0.33$ & $17.62 \pm 0.05$ & $17.16 \pm 0.03$ & $0.46 \pm 0.06$ & 35.66 & 35.71 & 20.02 & 21.71 & 2.42 & 3.06 & 2.59 & 3.07 & Ef \\
\hline IC 0334 & $\ldots$ & $15.34 \pm 0.10$ & $\ldots$ & $\ldots$ & $15.94 \pm 0.04$ & $\ldots$ & 0.00 & 35.82 & $\ldots$ & 53.46 & $\ldots$ & 3.26 & $\ldots$ & 3.14 & Er \\
\hline PGC 13820 & $16.73 \pm 0.09$ & $16.37 \pm 0.04$ & $0.36 \pm 0.10$ & $17.85 \pm 0.03$ & $17.19 \pm 0.01$ & $0.67 \pm 0.03$ & 35.69 & 35.65 & 31.28 & 25.03 & $\ldots$ & $\ldots$ & $\ldots$ & $\ldots$ & $\mathrm{EE}$ \\
\hline NGC 1481 & $16.61 \pm 0.01$ & $16.05 \pm 0.02$ & $0.55 \pm 0.02$ & $16.65 \pm 0.02$ & $16.19 \pm 0.01$ & $0.46 \pm 0.02$ & 34.99 & 35.03 & $\ldots$ & $\ldots$ & $\ldots$ & $\ldots$ & $\ldots$ & $\ldots$ & ER \\
\hline NGC 1482 & $17.38 \pm 0.08$ & $16.01 \pm 0.05$ & $1.37 \pm 0.10$ & $17.46 \pm 0.03$ & $16.42 \pm 0.01$ & $1.04 \pm 0.04$ & 34.77 & & 18.98 & & $\ldots$ & 5.10 & $\ldots$ & 4.35 & VV \\
\hline PGC 14100 & & $15.91=$ & & $16.28 \pm 0$ & 16.1 & & & & 19.39 & 17.61 & 2.76 & $\ldots$ & $\ldots$ & $\ldots$ & $? \mathrm{~F}$ \\
\hline NGC 1 & & & $0.27 \pm 0$ & $15.09 \pm$ & & & & & $\ldots$ & $\ldots$ & $\ldots$ & $\ldots$ & $\ldots$ & $\ldots$ & VV \\
\hline NGC 1512 & $13.46 \pm 0.02$ & $13.15 \pm 0.02$ & $0.31 \pm 0.03$ & $13.89 \pm 0.01$ & $13.58 \pm 0.01$ & $0.32 \pm 0.01$ & 35.58 & 35.53 & 136.35 & 118.18 & 3.97 & 4.36 & 3.55 & 3.82 & $\mathrm{xErn}$ \\
\hline UGC 02955 & $16.91 \pm 0.62$ & $16.50 \pm 0.08$ & $0.41 \pm 0.63$ & $17.71 \pm 0.33$ & $16.82 \pm 0.07$ & $0.89 \pm 0.34$ & 34.67 & 34.66 & 19.71 & 11.88 & $\ldots$ & $\ldots$ & $\ldots$ & $\ldots$ & $\ldots$ \\
\hline NGC 1546 & $17.07 \pm 0.01$ & $15.75 \pm 0.02$ & $1.32 \pm 0.02$ & $17.17 \pm 0.02$ & $15.99 \pm 0.01$ & $1.18 \pm 0.02$ & 34.58 & 34.93 & 17.97 & 23.57 & $\ldots$ & 5.95 & $\ldots$ & $\ldots$ & $\mathrm{VV}, \mathrm{EV}$ \\
\hline NGC 1549 & $16.65 \pm 0.01$ & $14.89 \pm 0.01$ & $1.76 \pm 0.02$ & $16.70 \pm 0.03$ & $15.04 \pm 0.01$ & $1.66 \pm 0.03$ & 34.75 & 35.27 & 31.00 & 44.76 & $\ldots$ & 5.22 & $\ldots$ & 4.51 & VV \\
\hline NGC 1553 & $16.21 \pm 0.02$ & $14.45 \pm 0.01$ & $1.76 \pm 0.02$ & $16.42 \pm 0.02$ & $14.76 \pm 0.01$ & $1.66 \pm 0.02$ & 34.92 & 35.45 & 37.44 & 50.04 & 5.97 & 4.76 & 4.78 & 4.18 & VV \\
\hline IC 2058 & $16.11 \pm 0.02$ & $15.63 \pm 0.01$ & $0.48 \pm 0.02$ & $16.25 \pm 0.01$ & $15.84 \pm 0.01$ & $0.41 \pm 0.01$ & 34.87 & 34.88 & 13.46 & 15.80 & 3.19 & 3.18 & 3.15 & 3.11 & $\mathrm{EE}$ \\
\hline
\end{tabular}


Table 3-Continued

\begin{tabular}{|c|c|c|c|c|c|c|c|c|c|c|c|c|c|c|c|}
\hline \multirow{2}{*}{$\begin{array}{l}\text { Object Name } \\
\qquad(1)\end{array}$} & \multicolumn{3}{|c|}{ Asymptotic magnitudes and color } & \multicolumn{3}{|c|}{ D25 magnitudes and color } & \multicolumn{2}{|c|}{$\log L$} & \multicolumn{2}{|c|}{ Effective Radii } & \multicolumn{2}{|c|}{$\mathrm{C} 31$} & \multicolumn{2}{|c|}{$\mathrm{C} 42$} & \multirow{2}{*}{$\begin{array}{c}\text { UV } \\
\text { profile } \\
(16)\end{array}$} \\
\hline & $\begin{array}{c}\text { FUV } \\
(\mathrm{mag}) \\
(2)\end{array}$ & $\begin{array}{c}\text { NUV } \\
(\mathrm{mag}) \\
(3)\end{array}$ & $\begin{array}{c}\text { FUV-NUV } \\
\text { (mag) } \\
(4)\end{array}$ & $\begin{array}{c}\text { FUV } \\
(\mathrm{mag}) \\
(5)\end{array}$ & $\begin{array}{c}\text { NUV } \\
(\mathrm{mag}) \\
(6)\end{array}$ & $\begin{array}{l}\text { FUV-NUV } \\
\text { (mag) } \\
(7)\end{array}$ & $\begin{array}{c}\text { FUV } \\
(\mathrm{W}) \\
(8)\end{array}$ & $\begin{array}{l}\text { NUV } \\
(\mathrm{W}) \\
(9)\end{array}$ & $\begin{array}{c}\text { FUV } \\
(\operatorname{arcsec}) \\
(10)\end{array}$ & $\begin{array}{c}\text { NUV } \\
(\operatorname{arcsec}) \\
(11)\end{array}$ & $\begin{array}{l}\text { FUV } \\
(12)\end{array}$ & $\begin{array}{l}\text { NUV } \\
(13)\end{array}$ & $\begin{array}{l}\text { FUV } \\
(14)\end{array}$ & $\begin{array}{l}\text { NUV } \\
(15)\end{array}$ & \\
\hline NGC 1566 & $12.06 \pm 0.02$ & $11.86 \pm 0.01$ & $0.20 \pm 0.02$ & $12.18 \pm 0.01$ & $11.96 \pm 0.01$ & $0.22 \pm 0.01$ & 36.60 & 36.50 & 84.26 & 77.95 & 3.20 & 3.21 & 3.22 & 3.29 & Ern \\
\hline NGC 1569 & $85 \pm 0.01$ & $9.22 \pm 0.01$ & $0.63 \pm 0.01$ & $9.93 \pm 0.01$ & $9.28 \pm 0.01$ & $0.65 \pm 0.01$ & 35.57 & 35.65 & 9.35 & 11.11 & $\cdots$ & $\cdots$ & $\ldots$ & . & VV \\
\hline NGC 1672 & $\cdots$ & $11.98 \pm 0.01$ & $\cdots$ & $\cdots$ & $12.11 \pm 0.01$ & $\cdots$ & 0.00 & 36.33 & $\cdots$ & 81.20 & $\cdots$ & 2.89 & $\cdots$ & 3.04 & Ern \\
\hline NGC 1705 & $\ldots$ & $13.34 \pm 0.01$ & $\ldots$ & .. & $13.37 \pm 0.01$ & $\cdots$ & 0.00 & 34.84 & $\cdots$ & $\ldots$ & $\cdots$ & $\ldots$ & $\ldots$ & $\ldots$ & ER \\
\hline NGC 1800 & $14.86 \pm 0.01$ & $14.65 \pm 0.01$ & $0.22 \pm 0.01$ & $14.90 \pm 0.01$ & $14.69 \pm 0.01$ & $0.20 \pm 0.01$ & 34.83 & 34.73 & $\cdots$ & 9.17 & $\cdots$ & $\cdots$ & $\cdots$ & $\cdots$ & ER \\
\hline NGC 1808 & $\ldots$ & $13.51 \pm 0.02$ & $\ldots$ & $\cdots$ & $13.60 \pm 0.01$ & $\ldots$ & 0.00 & 35.43 & $\ldots$ & 52.79 & $\ldots$ & 2.23 & $\ldots$ & 2.41 & ERn \\
\hline IC 0411 & $19.94 \pm 1.17$ & $18.27 \pm 0.08$ & $1.67 \pm 1.17$ & $19.86 \pm 0.18$ & $18.73 \pm 0.04$ & $1.13 \pm 0.19$ & 35.21 & 35.70 & $\ldots$ & 13 & $\ldots$ & $\cdots$ & $\ldots$ & $\ldots$ & $\ldots$ \\
\hline ESO 204-G006 & $18.86 \pm 0.04$ & $18.18 \pm 0.02$ & $0.68 \pm 0.04$ & $18.90 \pm 0.04$ & $18.31 \pm 0.02$ & $0.59 \pm 0.05$ & 35.95 & 36.05 & 10.95 & & $\cdots$ & $\cdots$ & $\ldots$ & $\cdots$ & $? \mathrm{~F}$ \\
\hline ESO 204-G007 & $19.13 \pm 0.05$ & $18.30 \pm 0.02$ & $0.83 \pm 0.05$ & $19.45 \pm 0.03$ & $18.74 \pm 0.01$ & $0.71 \pm 0.03$ & 35.30 & 35.45 & 10.16 & 11.10 & 2.23 & 2.35 & 2.24 & 2.33 & $? \mathrm{~F}$ \\
\hline ESO 033-G022 & $18.08 \pm 0.02$ & $17.51 \pm 0.06$ & $0.58 \pm 0.06$ & $18.88 \pm 0.03$ & $18.55 \pm 0.02$ & $0.33 \pm 0.03$ & 35.23 & 35.29 & 13.14 & 0 & $\cdots$ & $\cdots$ & $\cdots$ & $\cdots$ & $\mathrm{EF}$ \\
\hline NGC 1964 & $\cdots$ & $13.50 \pm 0.01$ & $\cdots$ & $\ldots$ & $13.68 \pm 0.01$ & $\cdots$ & 0.00 & 36.01 & $\ldots$ & 51.98 & $\cdots$ & 2.19 & $\ldots$ & 2.54 & EEn \\
\hline NGC 1961 & $14.27 \pm 0.01$ & $13.68 \pm 0.01$ & $0.59 \pm 0.01$ & $14.35 \pm 0.01$ & $13.78 \pm 0.01$ & $0.57 \pm 0.01$ & 36.78 & 36.84 & 59.28 & 58.19 & 2.21 & 2.38 & 2.12 & 2.31 & $\mathrm{EF}$ \\
\hline UGC 03342 & $17.32 \pm 0.05$ & $16.80 \pm 0.01$ & $0.51 \pm 0.05$ & $17.52 \pm 0.03$ & $16.97 \pm 0.02$ & $0.55 \pm 0.04$ & 35.56 & 35.60 & 14.42 & 14.22 & 2.19 & 2.17 & 2.13 & 2.12 & $? \mathrm{~F}$ \\
\hline NGC 2090 & $13.45 \pm 0.01$ & $13.08 \pm 0.01$ & $0.37 \pm 0.01$ & $14.05 \pm 0.01$ & $13.63 \pm 0.01$ & $0.42 \pm 0.01$ & 35.66 & 35.63 & 89.98 & 84.26 & 2.71 & 3.59 & 2.97 & 3.54 & xErh \\
\hline UGC 03403 & $\ldots$ & $16.27 \pm 0.05$ & $\cdots$ & $\ldots$ & $16.50 \pm 0.03$ & $\ldots$ & 0.00 & 34.92 & $\cdots$ & 23.18 & $\ldots$ & 2.38 & $\ldots$ & 2.39 & $\mathrm{EE}$ \\
\hline UGC 03422 & $\ldots$ & $15.07 \pm 0.07$ & $\ldots$ & $\ldots$ & $15.44 \pm 0.01$ & $\ldots$ & 0.00 & 36.31 & $\ldots$ & 38.50 & $\ldots$ & 1.95 & $\ldots$ & 1.95 & ?D \\
\hline Mrk 3 & $\ldots$ & $15.99 \pm 0.06$ & $\ldots$ & ... & $16.19 \pm 0.02$ & $\ldots$ & 0.00 & 35.93 & $\ldots$ & $\ldots$ & $\ldots$ & $\ldots$ & $\ldots$ & $\ldots$ & VV \\
\hline NGC 2207 & $\ldots$ & $12.66 \pm 0.02$ & $\ldots$ & $\ldots$ & $12.73 \pm 0.01$ & $\ldots$ & 0.00 & & $\ldots$ & & $\ldots$ & 2.09 & $\ldots$ & 2.08 & $? \mathrm{~F}$ \\
\hline IC 2163 & $\ldots$ & $13.89 \pm 0.03$ & $\ldots$ & ... & $14.07 \pm 0.01$ & $\ldots$ & 0.00 & 36.32 & $\ldots$ & 27.02 & $\ldots$ & 2.39 & $\ldots$ & 2.48 & ?F \\
\hline UGC 03423 & $17.11 \pm 0.06$ & $16.76 \pm 0.03$ & $0.35 \pm 0.06$ & $17.64 \pm 0.03$ & $17.26 \pm 0.01$ & $0.38 \pm 0.04$ & 35.71 & 35.68 & 10.21 & 10.20 & 2.82 & 2.61 & $\ldots$ & $\ldots$ & $\mathrm{EE}, \mathrm{Ef}$ \\
\hline ESO 556-G012 & $\cdots$ & $15.65 \pm 0.09$ & $\cdots$ & . & $16.28 \pm 0.01$ & $\cdots$ & 0.00 & 35.57 & & 29.45 & $\cdots$ & 3.16 & $\cdots$ & . & $\mathrm{EE}$ \\
\hline NGC 2146 & $14.82 \pm 0.01$ & $13.95 \pm 0.01$ & $0.87 \pm 0.01$ & $14.93 \pm 0.01$ & $14.04 \pm 0.01$ & $0.89 \pm 0.02$ & 35.44 & 35.61 & 70.51 & 66.39 & 2.48 & 2.56 & 2.57 & 2.59 & Ef \\
\hline NGC $2146 \mathrm{~A}$ & $15.24 \pm 0.02$ & $14.84 \pm 0.01$ & $0.40 \pm 0.02$ & $15.39 \pm 0.01$ & $15.01 \pm 0.01$ & $0.38 \pm 0.02$ & 35.64 & 35.62 & 30.71 & 30.72 & 2.24 & 2.40 & 2.37 & 2.48 & $\mathrm{EE}$ \\
\hline AM 0644-741 & $15.04 \pm 0.01$ & $14.69 \pm 0.01$ & $0.35 \pm 0.01$ & $15.60 \pm 0.02$ & $15.23 \pm 0.01$ & $0.37 \pm 0.02$ & 36.87 & 36.83 & 31.62 & 31.31 & 1.27 & 1.31 & 0.67 & 0.75 & ED \\
\hline PGC 19480 & $\ldots$ & $19.34 \pm 0.28$ & $\ldots$ & $\ldots$ & $\cdots$ & $\ldots$ & 0.00 & 34.91 & $\cdots$ & $\ldots$ & $\ldots$ & $\ldots$ & $\ldots$ & $\ldots$ & $\ldots$ \\
\hline PGC 19481 & $17.44 \pm 0.29$ & $16.41 \pm 0.06$ & $1.02 \pm 0.29$ & $17.92 \pm 0.22$ & $17.08 \pm 0.08$ & $0.84 \pm 0.24$ & 35.85 & 36.09 & 28.12 & 30.64 & 2.16 & 2.65 & 2.27 & 2.68 & $\mathrm{EE}$ \\
\hline ESO 034-G013 & $15.75 \pm 0.02$ & $15.34 \pm 0.01$ & $0.41 \pm 0.02$ & $16.03 \pm 0.02$ & $15.60 \pm 0.01$ & $0.43 \pm 0.02$ & 36.51 & 36.49 & 13.95 & 13.67 & 2.50 & 2.73 & $\ldots$ & $\ldots$ & Ef \\
\hline NGC 2310 & $\ldots$ & $16.25 \pm 0.19$ & $\ldots$ & $\ldots$ & $16.30 \pm 0.05$ & $\ldots$ & 0.00 & 34.49 & $\ldots$ & 19.78 & $\ldots$ & 2.87 & $\ldots$ & 2.89 & Er \\
\hline NGC 2366 & $12.50 \pm 0.04$ & $12.42 \pm 0.03$ & $0.08 \pm 0.05$ & $12.67 \pm 0.01$ & $12.61 \pm 0.01$ & $0.06 \pm 0.01$ & 35.01 & 34.86 & 75.14 & 75.36 & 2.32 & 2.37 & 2.45 & 2.48 & $\mathrm{EE}$ \\
\hline Mrk 8 & $15.55 \pm 0.01$ & $15.20 \pm 0.01$ & $0.35 \pm 0.01$ & $15.77 \pm 0.02$ & $15.43 \pm 0.01$ & $0.33 \pm 0.02$ & 36.19 & 36.16 & $\ldots$ & $\cdots$ & $\ldots$ & $\ldots$ & $\ldots$ & $\ldots$ & VV \\
\hline
\end{tabular}


Table 3-Continued

\begin{tabular}{|c|c|c|c|c|c|c|c|c|c|c|c|c|c|c|c|}
\hline \multirow{2}{*}{$\begin{array}{l}\text { Object Name } \\
\text { (1) }\end{array}$} & \multicolumn{3}{|c|}{ Asymptotic magnitudes and color } & \multicolumn{3}{|c|}{ D25 magnitudes and color } & \multicolumn{2}{|c|}{$\log L$} & \multicolumn{2}{|c|}{ Effective Radii } & \multicolumn{2}{|c|}{$\mathrm{C} 31$} & \multicolumn{2}{|c|}{$\mathrm{C} 42$} & \multirow{2}{*}{$\begin{array}{l}\text { UV } \\
\text { profile } \\
(16)\end{array}$} \\
\hline & $\begin{array}{l}\text { FUV } \\
(\mathrm{mag}) \\
(2)\end{array}$ & $\begin{array}{c}\text { NUV } \\
(\mathrm{mag}) \\
(3)\end{array}$ & $\begin{array}{c}\text { FUV-NUV } \\
\text { (mag) } \\
(4)\end{array}$ & $\begin{array}{c}\text { FUV } \\
(\mathrm{mag}) \\
(5)\end{array}$ & $\begin{array}{c}\text { NUV } \\
(\mathrm{mag}) \\
(6)\end{array}$ & $\begin{array}{l}\text { FUV-NUV } \\
(\mathrm{mag}) \\
(7)\end{array}$ & $\begin{array}{c}\text { FUV } \\
(\mathrm{W}) \\
(8)\end{array}$ & $\begin{array}{c}\text { NUV } \\
(\mathrm{W}) \\
(9)\end{array}$ & $\begin{array}{c}\text { FUV } \\
(\operatorname{arcsec}) \\
(10)\end{array}$ & $\begin{array}{c}\text { NUV } \\
(\operatorname{arcsec}) \\
(11)\end{array}$ & $\begin{array}{l}\text { FUV } \\
(12)\end{array}$ & $\begin{array}{l}\text { NUV } \\
(13)\end{array}$ & $\begin{array}{l}\text { FUV } \\
(14)\end{array}$ & $\begin{array}{l}\text { NUV } \\
(15)\end{array}$ & \\
\hline 64 & 1 & 1 & $z$ & 1 & 1 & 0 & & & & & & 2 & & & ?Fn \\
\hline 006 & & & & & & & & & & & & & & & \\
\hline NGC 2434 & & $.84 \pm$ & $2.01 \pm 0$ & $80 \pm 0$ & $15.24 \pm 0.04$ & $1.56 \pm$ & 34.59 & 35.22 & 21.98 & & $\ldots$ & 4.65 & $\ldots$ & 4.08 & $\mathrm{VV}$ \\
\hline ESO 059-G007 & $18.20 \pm 1.08$ & $18.39 \pm 0.27$ & $-0.19 \pm 1.11$ & $\ldots$ & $18.49 \pm 0.21$ & & 33.95 & 33.69 & 15.68 & 10.89 & $\ldots$ & $\ldots$ & $\ldots$ & 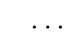 & $\ldots$ \\
\hline NGC 2442 & $.59 \pm 0.02$ & $.24 \pm 0.01$ & $0.36 \pm 0.02$ & $12.74 \pm 0.01$ & $12.36 \pm 0.01$ & $0.38 \pm 0.01$ & 36.19 & 36.15 & 81.38 & 80.91 & 2.10 & 2.13 & 2.08 & 2.12 & ?Fh \\
\hline NGC 2403 & $.37 \pm 0.01$ & $10.18 \pm 0.01$ & $0.19 \pm 0.01$ & $10.37 \pm 0.01$ & $10.18 \pm 0.01$ & $0.20 \pm 0.01$ & 35.79 & 35.69 & 161.07 & 152.57 & 2.67 & 2.69 & 2.68 & 2.71 & $\mathrm{EE}$ \\
\hline ESO 059-G010 & $16.72 \pm 0.08$ & $16.74 \pm 0.08$ & $-0.02 \pm 0.11$ & $17.25 \pm 0.09$ & $17.06 \pm 0.05$ & $0.19 \pm 0.10$ & 34.54 & 34.35 & 15.22 & 12.94 & 2.54 & $\ldots$ & $\ldots$ & $\ldots$ & $? \mathrm{~F}$ \\
\hline UGC 03942 & $\ldots$ & $18.60 \pm 0.06$ & $\ldots$ & $\ldots$ & $18.99 \pm 0.03$ & $\ldots$ & 0.00 & 35.44 & $\ldots$ & 9.10 & $\ldots$ & $\ldots$ & $\ldots$ & $\ldots$ & $? \mathrm{~F}$ \\
\hline ESO 059-G0 & $\ldots$ & $16.98 \pm 0.15$ & $\ldots$ & $\ldots$ & $17.06 \pm 0.09$ & $\ldots$ & 0.00 & 34.46 & $\ldots$ & & $\ldots$ & $\ldots$ & $\ldots$ & $\ldots$ & $\mathrm{VV}$ \\
\hline UGC 03995 & $16.09 \pm 0.01$ & 15.58 & $0.51 \pm 0.01$ & $17+0$ & 15.6 & $0.51 \pm 0.02$ & 36.18 & 36.20 & 28.05 & & 2.06 & 2.09 & 2.00 & 2.03 & ?Fn \\
\hline UGC 03997 & $16.74 \pm 0.01$ & $16.55=$ & $0.19 \pm$ & $16.87 \pm$ & $16.6 \mathrm{~s}$ & $0.19 \pm$ & 36.11 & 36.01 & 5 & & $\ldots$ & $\ldots$ & $\ldots$ & $\ldots$ & $? \mathrm{~F}$ \\
\hline UGC 04056 & $16.86 \pm 0.09$ & $16.53 \pm 0.02$ & $0.33 \pm 0.09$ & $17.05 \pm 0.05$ & $16.69 \pm 0$ & $0.36 \pm$ & 36.48 & 36 & & & .. & $\ldots$ & $\ldots$ & $\ldots$ & ?Fn \\
\hline UGC 04136 & & $18.74 \exists$ & & 0 & & $0.95 \pm$ & 34.97 & & & & $\ldots$ & 2.62 & $\ldots$ & $\ldots$ & ?Fn \\
\hline UGC 04148 & & 15.9 & & $6.41 \pm 0.01$ & 16.2 & $5 \pm 0$. & & & & & 2.19 & 4 & 2.17 & 2.36 & $? \mathrm{~F}$ \\
\hline 1188 & $19.48 \pm 0.07$ & 18.58 & $0.90 \pm 0.08$ & $19.80 \pm 0.12$ & 18.75 & $1.04 \pm 0.13$ & 35.44 & 35.62 & 14.95 & 41 & 2.96 & $\ldots$ & $\ldots$ & $\ldots$ & $\ldots$ \\
\hline NGC 2538 & $\ldots$ & $16.37 \pm 0.01$ & $\ldots$ & $\ldots$ & $16.43 \pm 0.01$ & $\ldots$ & 0.00 & 35.71 & $\ldots$ & & $\ldots$ & $\ldots$ & $\ldots$ & $\ldots$ & $\mathrm{EE}$ \\
\hline NGC 2543 & $.12 \pm 0.01$ & 14.67 & $0.45 \pm 0$ & $.25 \pm 0.01$ & $14.7 \mathrm{~S}$ & $0.46 \pm 0$ & 36.02 & 36.03 & 30.59 & 7 & 1.63 & 1.73 & 1.37 & 1.54 & EDn \\
\hline NGC 2537 & $89 \pm 0.01$ & 13.73 & $0.17 \pm 0$ & $3.97 \pm 0.01$ & $13.79 \pm 0.01$ & $0.18 \pm 0.01$ & 35.06 & 34.94 & 19.05 & 21 & 1.72 & 1.83 & 1.60 & 1.75 & ERn \\
\hline UGC4278 & $87 \pm 0.01$ & $14.50 \pm 0.01$ & $0.38 \pm 0.01$ & $15.02 \pm 0.01$ & $14.67 \pm 0.01$ & $0.35 \pm 0.01$ & 35.27 & 35.25 & 22.62 & 23.43 & 2.86 & 2.72 & 2.78 & 2.71 & Ef \\
\hline NGC 2541 & $13.41 \pm 0.01$ & $13.32 \pm 0.01$ & $0.10 \pm 0.01$ & $13.50 \pm 0.01$ & $13.39 \pm 0.01$ & $0.11 \pm 0.01$ & 35.60 & 35.46 & 67.70 & & 2.14 & 2.22 & 2.13 & 2.21 & EFn \\
\hline NGC 25230 & $20.68 \pm 0.39$ & $18.16 \pm 0.04$ & $2.52 \pm 0.40$ & $\ldots$ & $18.34 \pm 0.03$ & $\ldots$ & 34.15 & 34.98 & & & $\ldots$ & $\ldots$ & $\ldots$ & $\ldots$ & $\mathrm{VV}$ \\
\hline & 1 & & 0 & $72 \pm 0$ & & & & & & & 2.13 & 2.34 & $\ldots$ & $\ldots$ & $? \mathrm{~F}$ \\
\hline & $12.20 \pm 0$ & & $0.01 \pm 0.01$ & $12.29 \pm 0.01$ & & $0.02 \pm 0$. & & & 119.43 & & 2.47 & & 2.50 & & $\mathrm{Ef}$ \\
\hline & $\begin{array}{c}12.20+0 \\
\ldots\end{array}$ & & $\ldots$ & $\ldots$ & & $\ldots$ & & & $\ldots$ & & $\ldots$ & $2.1+2=0$ & $\ldots$ & & $\mathrm{EF}$ \\
\hline UGC 04387 & $\ldots$ & $19.03 \pm 0.08$ & $\ldots$ & $\ldots$ & $19.55 \pm 0.04$ & $\ldots$ & 0.00 & 35.63 & $\ldots$ & 11.64 & $\ldots$ & 2.44 & $\ldots$ & 2.42 & $\mathrm{EE}$ \\
\hline NGC 2551 & $15.96 \pm 0.01$ & $15.69 \pm 0.01$ & $0.28 \pm 0.01$ & $16.07 \pm 0.01$ & $15.81 \pm 0.01$ & $0.25 \pm 0.01$ & 35.69 & 35.62 & 21.59 & 21.30 & 1.83 & 2.00 & 1.65 & 1.92 & $\mathrm{Fh}, \mathrm{EFr}$ \\
\hline HS $0822+3542$ & $18.26 \pm 0.13$ & $18.15 \pm 0.09$ & $0.11 \pm 0.16$ & $\ldots$ & $\ldots$ & $\ldots$ & 33.64 & 33.51 & $\ldots$ & $\ldots$ & $\ldots$ & $\ldots$ & $\ldots$ & $\ldots$ & $\ldots$ \\
\hline UGC 04393 & $\ldots$ & $14.86 \pm 0.02$ & $\ldots$ & $\ldots$ & $14.95 \pm 0.01$ & $\ldots$ & 0.00 & 35.84 & $\ldots$ & 18.62 & $\ldots$ & $\ldots$ & $\ldots$ & $\ldots$ & Er \\
\hline UGC 04401 & $\ldots$ & $16.74 \pm 0.02$ & $\ldots$ & $\ldots$ & $16.98 \pm 0.02$ & $\ldots$ & 0.00 & 35.40 & $\ldots$ & 21.92 & $\ldots$ & 2.65 & $\ldots$ & $\ldots$ & $? \mathrm{~F}$ \\
\hline UGC 04390 & $15.67 \pm 0.01$ & $15.43 \pm 0.01$ & $0.25 \pm 0.01$ & $16.11 \pm 0.01$ & $15.82 \pm 0.01$ & $0.29 \pm 0.02$ & 35.75 & 35.67 & 37.28 & 35.45 & 2.49 & 2.58 & 2.47 & 2.59 & ?Fn \\
\hline
\end{tabular}


Table 3-Continued

\begin{tabular}{|c|c|c|c|c|c|c|c|c|c|c|c|c|c|c|c|}
\hline \multirow{2}{*}{$\begin{array}{l}\text { Object Name } \\
\text { (1) }\end{array}$} & \multicolumn{3}{|c|}{ Asymptotic magnitudes and color } & \multicolumn{3}{|c|}{ D25 magnitudes and color } & \multicolumn{2}{|c|}{$\log L$} & \multicolumn{2}{|c|}{ Effective Radii } & \multicolumn{2}{|c|}{$\mathrm{C} 31$} & \multicolumn{2}{|c|}{$\mathrm{C} 42$} & \multirow{2}{*}{$\begin{array}{c}\text { UV } \\
\text { profile } \\
(16)\end{array}$} \\
\hline & $\begin{array}{c}\text { FUV } \\
(\mathrm{mag}) \\
(2)\end{array}$ & $\begin{array}{c}\text { NUV } \\
(\mathrm{mag}) \\
(3)\end{array}$ & $\begin{array}{l}\text { FUV-NUV } \\
\text { (mag) } \\
(4)\end{array}$ & $\begin{array}{l}\text { FUV } \\
(\mathrm{mag}) \\
(5)\end{array}$ & $\begin{array}{c}\text { NUV } \\
(\mathrm{mag}) \\
(6)\end{array}$ & $\begin{array}{l}\text { FUV-NUV } \\
\text { (mag) } \\
(7)\end{array}$ & $\begin{array}{l}\text { FUV } \\
(\mathrm{W}) \\
(8)\end{array}$ & $\begin{array}{c}\text { NUV } \\
(\mathrm{W}) \\
(9)\end{array}$ & $\begin{array}{c}\text { FUV } \\
(\operatorname{arcsec}) \\
(10)\end{array}$ & $\begin{array}{c}\text { NUV } \\
(\operatorname{arcsec}) \\
(11)\end{array}$ & $\begin{array}{l}\text { FUV } \\
(12)\end{array}$ & $\begin{array}{l}\text { NUV } \\
(13)\end{array}$ & $\begin{array}{l}\text { FUV } \\
(14)\end{array}$ & $\begin{array}{l}\text { NUV } \\
(15)\end{array}$ & \\
\hline NGC 2550A & $15.29 \pm 0.01$ & $14.94 \pm 0.01$ & $0.35 \pm 0.01$ & $15.34 \pm 0.01$ & $14.99 \pm 0.01$ & $0.36 \pm 0.01$ & 36.31 & 36.27 & 20.50 & 19.68 & 2.21 & 2.34 & $\cdots$ & $\cdots$ & $? \mathrm{Fh}$ \\
\hline UGC 04436 & $\ldots$ & $17.12 \pm 0.02$ & $\cdots$ & $\ldots$ & $17.35 \pm 0.01$ & $\cdots$ & 0.00 & 35.96 & $\cdots$ & 10.74 & $\ldots$ & 2.54 & $\cdots$ & $\cdots$ & $? \mathrm{~F}$ \\
\hline UGC 04461 & $16.25 \pm 0.03$ & $15.81 \pm 0.02$ & $0.44 \pm 0.03$ & $16.38 \pm 0.01$ & $15.96 \pm 0.01$ & $0.42 \pm 0.02$ & 36.17 & 36.17 & 14.66 & 14.49 & 2.22 & 2.31 & 2.18 & 2.30 & $\mathrm{EF}$ \\
\hline DDO 053 & $15.35 \pm 0.01$ & $15.38 \pm 0.01$ & $-0.03 \pm 0.01$ & $15.56 \pm 0.01$ & $15.65 \pm 0.01$ & $-0.09 \pm 0.01$ & 33.90 & 33.71 & 22.90 & 24.64 & $\cdots$ & $\cdots$ & $\ldots$ & $\ldots$ & ?Fn \\
\hline UGC 04499 & $\cdots$ & $14.48 \pm 0.01$ & $\cdots$ & $\cdots$ & $14.63 \pm 0.01$ & $\cdots$ & 0.00 & 35.17 & $\ldots$ & 35.03 & $\ldots$ & 2.43 & $\cdots$ & 2.57 & $\mathrm{EE}$ \\
\hline NGC 2623 & $\ldots$ & $15.77 \pm 0.01$ & $\cdots$ & $\ldots$ & $15.83 \pm 0.01$ & $\ldots$ & 0.00 & 36.26 & $\ldots$ & 16.12 & $\ldots$ & 3.09 & $\ldots$ & $\ldots$ & $\mathrm{VF}$ \\
\hline UGC 04514 & $15.38 \pm 0.02$ & $15.19 \pm 0.01$ & $0.19 \pm 0.03$ & $15.79 \pm 0.01$ & $15.54 \pm 0.01$ & $0.25 \pm 0.02$ & 34.99 & 34.89 & 25.35 & 23.49 & 3.55 & 3.46 & 3.51 & 3.40 & Er \\
\hline UGC 04515 & $\cdots$ & $16.64 \pm 0.02$ & $\cdots$ & $\ldots$ & $16.94 \pm 0.01$ & $\cdots$ & 0.00 & 35.84 & $\ldots$ & 16.83 & $\ldots$ & 2.39 & $\ldots$ & 2.28 & $\mathrm{Ed}$ \\
\hline UGC 04525 & $\ldots$ & $16.17 \pm 0.03$ & $\cdots$ & $\ldots$ & $16.51 \pm 0.03$ & $\cdots$ & 0.00 & 36.05 & $\ldots$ & 15.39 & $\ldots$ & $\ldots$ & $\ldots$ & $\ldots$ & $\mathrm{Ef}$ \\
\hline UGC 04529 & $18.65 \pm 0.04$ & $18.27 \pm 0.02$ & $0.38 \pm 0.04$ & $19.03 \pm 0.03$ & $18.69 \pm 0.02$ & $0.33 \pm 0.04$ & 35.89 & 35.87 & 9.70 & 9.69 & 2.25 & 2.56 & 2.29 & $\ldots$ & $\mathrm{EF}$ \\
\hline NGC 2639 & $16.95 \pm 0.02$ & $15.87 \pm 0.01$ & $1.08 \pm 0.02$ & $17.01 \pm 0.02$ & $16.01 \pm 0.01$ & $1.00 \pm 0.02$ & 35.56 & 35.81 & 13.40 & 13.76 & $\ldots$ & $\ldots$ & $\ldots$ & $\ldots$ & ER \\
\hline UGC 04546 & $19.62 \pm 0.01$ & $18.51 \pm 0.02$ & $1.11 \pm 0.02$ & $19.83 \pm 0.05$ & $18.86 \pm 0.02$ & $0.97 \pm 0.05$ & 34.86 & 35.13 & 9.09 & 9.33 & 2.21 & 2.78 & 2.23 & $\ldots$ & $\mathrm{EF}$ \\
\hline UGC 04551 & $18.78 \pm 0.05$ & $17.51 \pm 0.02$ & $1.27 \pm 0.06$ & $18.94 \pm 0.05$ & $17.62 \pm 0.01$ & $1.32 \pm 0.05$ & 34.30 & 34.64 & 9.65 & 9.17 & $\ldots$ & $\cdots$ & $\ldots$ & $\cdots$ & EV \\
\hline UGC 04560 & $19.22 \pm 0.06$ & $18.46 \pm 0.03$ & $0.76 \pm 0.07$ & $19.38 \pm 0.05$ & $18.67 \pm 0.02$ & $0.70 \pm 0.05$ & 35.74 & 35.87 & 10.03 & 10.19 & 2.06 & 2.37 & $\cdots$ & $\cdots$ & $? \mathrm{~F}$ \\
\hline VV 703 & $20.26 \pm 0.24$ & $18.56 \pm 0.16$ & $1.70 \pm 0.29$ & $\ldots$ & $18.70 \pm 0.04$ & $\ldots$ & 35.42 & 35.92 & $\ldots$ & $\ldots$ & $\ldots$ & $\cdots$ & $\cdots$ & $\ldots$ & $\cdots$ \\
\hline UGC 04628 & $17.16 \pm 0.02$ & $16.63 \pm 0.03$ & $0.53 \pm 0.04$ & $17.30 \pm 0.02$ & $16.91 \pm 0.01$ & $0.39 \pm 0.02$ & 34.36 & 34.40 & 9.62 & 11.99 & 2.69 & 2.76 & $\ldots$ & 2.77 & $? \mathrm{~F}$ \\
\hline NGC 2675 & $20.16 \pm 0.15$ & $18.35 \pm 0.09$ & $1.81 \pm 0.18$ & $20.25 \pm 0.18$ & $18.75 \pm 0.05$ & $1.51 \pm 0.19$ & 35.13 & 35.68 & 12.95 & 20.81 & $\ldots$ & $\ldots$ & $\ldots$ & $\ldots$ & VV \\
\hline NGC 2681 & $16.54 \pm 0.01$ & $14.89 \pm 0.01$ & $1.65 \pm 0.01$ & $16.58 \pm 0.02$ & $14.99 \pm 0.01$ & $1.59 \pm 0.02$ & 34.52 & 35.00 & 13.19 & 22 . & $\ldots$ & $\ldots$ & $\ldots$ & $\ldots$ & $\mathrm{EV}$ \\
\hline IC 0522 & $18.27 \pm 0.05$ & $17.54 \pm 0.01$ & $0.73 \pm 0.05$ & $18.58 \pm 0.05$ & $17.81 \pm 0.02$ & $0.77 \pm 0.06$ & 35.38 & 35.50 & 17.71 & 15.67 & 1.83 & $\ldots$ & 1.66 & $\cdots$ & $? \mathrm{~F}$ \\
\hline VV 761 & $19.65 \pm 0.44$ & $18.74 \pm 0.02$ & $0.91 \pm 0.44$ & $20.11 \pm 0.08$ & $19.22 \pm 0.03$ & $0.89 \pm 0.09$ & 35.55 & 35.74 & 8.20 & 8.58 & $\ldots$ & $\ldots$ & $\ldots$ & $\ldots$ & $\mathrm{EE}$ \\
\hline UGC 04668 & $18.99 \pm 0.07$ & $18.11 \pm 0.04$ & $0.89 \pm 0.08$ & $19.25 \pm 0.05$ & $18.62 \pm 0.02$ & $0.63 \pm 0.05$ & 34.95 & 35.12 & 8.66 & 10.57 & 2.29 & 2.59 & $\cdots$ & $\cdots$ & $? \mathrm{~F}$ \\
\hline UGC 04684 & $15.63 \pm 0.01$ & $15.31 \pm 0.01$ & $0.33 \pm 0.01$ & $15.75 \pm 0.01$ & $15.43 \pm 0.01$ & $0.31 \pm 0.01$ & 35.78 & 35.73 & 18.72 & 18.05 & $\ldots$ & $\ldots$ & $\ldots$ & $\ldots$ & $\mathrm{EF}$ \\
\hline UGC 04671 & $15.93 \pm 0.02$ & $15.38 \pm 0.01$ & $0.54 \pm 0.02$ & $16.04 \pm 0.01$ & $15.50 \pm 0.01$ & $0.55 \pm 0.02$ & 36.13 & 36.17 & 12.48 & $\cdots$ & $\cdots$ & $\ldots$ & $\cdots$ & $\cdots$ & EFn \\
\hline NGC 2692 & $19.51 \pm 0.17$ & $18.03 \pm 0.10$ & $1.48 \pm 0.20$ & $19.59 \pm 0.08$ & $18.28 \pm 0.02$ & $1.30 \pm 0.08$ & 34.69 & 35.11 & $\ldots$ & 10.94 & $\ldots$ & $\ldots$ & $\cdots$ & $\ldots$ & VV \\
\hline NGC 2693 & $17.95 \pm 0.05$ & $16.93 \pm 0.05$ & $1.02 \pm 0.07$ & $18.06 \pm 0.04$ & $17.12 \pm 0.02$ & $0.93 \pm 0.05$ & 35.49 & 35.72 & $\ldots$ & 19.46 & $\ldots$ & $\ldots$ & $\ldots$ & $\ldots$ & VV \\
\hline UGC 04676 & $17.37 \pm 0.06$ & $17.03 \pm 0.04$ & $0.34 \pm 0.07$ & $17.77 \pm 0.02$ & $17.42 \pm 0.01$ & $0.35 \pm 0.03$ & 35.76 & 35.72 & 12.92 & 12.62 & $\cdots$ & $\ldots$ & $\cdots$ & $\ldots$ & $\mathrm{EE}$ \\
\hline UGC 04679 & $20.53 \pm 0.14$ & $19.61 \pm 0.08$ & $0.92 \pm 0.16$ & $20.74 \pm 0.09$ & $20.00 \pm 0.05$ & $0.74 \pm 0.10$ & 34.41 & 34.60 & 8.55 & 10.76 & 2.41 & 2.34 & $\ldots$ & 2.35 & $\mathrm{EF}$ \\
\hline UGC 04690 & $19.05 \pm 0.37$ & $18.47 \pm 0.07$ & $0.58 \pm 0.38$ & .. & $18.64 \pm 0.03$ & $\ldots$ & 35.59 & 35.65 & 15.97 & 10.89 & $\ldots$ & $\ldots$ & $\ldots$ & $\ldots$ & $\cdots$ \\
\hline UGC 04702 & $\ldots$ & $18.32 \pm 0.15$ & $\ldots$ & $\ldots$ & $18.73 \pm 0.07$ & $\cdots$ & 0.00 & 35.61 & $\ldots$ & 26.63 & $\ldots$ & $\ldots$ & $\cdots$ & $\ldots$ & $\mathrm{Er}$ \\
\hline UGC 04704 & $\ldots$ & $15.15 \pm 0.01$ & $\cdots$ & $\ldots$ & $15.43 \pm 0.01$ & $\cdots$ & 0.00 & 34.74 & $\ldots$ & 19.72 & $\ldots$ & 3.22 & $\ldots$ & 3.22 & Ef \\
\hline
\end{tabular}


Table 3-Continued

\begin{tabular}{|c|c|c|c|c|c|c|c|c|c|c|c|c|c|c|c|}
\hline \multirow{2}{*}{$\begin{array}{l}\text { Object Name } \\
\text { (1) }\end{array}$} & \multicolumn{3}{|c|}{ Asymptotic magnitudes and color } & \multicolumn{3}{|c|}{ D25 magnitudes and color } & \multicolumn{2}{|c|}{$\log L$} & \multicolumn{2}{|c|}{ Effective Radii } & \multicolumn{2}{|c|}{ C31 } & \multicolumn{2}{|c|}{$\mathrm{C} 42$} & \multirow{2}{*}{$\begin{array}{c}\text { UV } \\
\text { profile } \\
(16)\end{array}$} \\
\hline & $\begin{array}{c}\text { FUV } \\
(\mathrm{mag}) \\
(2)\end{array}$ & $\begin{array}{c}\text { NUV } \\
(\mathrm{mag}) \\
(3)\end{array}$ & $\begin{array}{l}\text { FUV-NUV } \\
(\mathrm{mag}) \\
(4)\end{array}$ & $\begin{array}{l}\text { FUV } \\
(\mathrm{mag}) \\
(5)\end{array}$ & $\begin{array}{c}\text { NUV } \\
(\mathrm{mag}) \\
(6)\end{array}$ & $\begin{array}{l}\text { FUV-NUV } \\
(\mathrm{mag}) \\
(7)\end{array}$ & $\begin{array}{c}\text { FUV } \\
(\mathrm{W}) \\
(8)\end{array}$ & $\begin{array}{l}\text { NUV } \\
(\mathrm{W}) \\
(9)\end{array}$ & $\begin{array}{c}\text { FUV } \\
(\operatorname{arcsec}) \\
(10)\end{array}$ & $\begin{array}{c}\text { NUV } \\
\text { (arcsec) } \\
(11)\end{array}$ & $\begin{array}{l}\text { FUV } \\
(12)\end{array}$ & $\begin{array}{l}\text { NUV } \\
(13)\end{array}$ & $\begin{array}{l}\text { FUV } \\
\text { (14) }\end{array}$ & $\begin{array}{l}\text { NUV } \\
(15)\end{array}$ & \\
\hline NGC 2710 & $.69 \pm 0.04$ & $15.29 \pm 0.03$ & $0.41 \pm 0.04$ & $.86 \pm 0.01$ & $15.45 \pm 0.01$ & $0.42 \pm 0$ & 35.84 & 35.83 & 19.80 & 19.42 & 2.65 & 2.73 & 2.73 & 2.81 & $? \mathrm{~F}$ \\
\hline UGC 04800 & $16.61 \pm 0.02$ & $16.33 \pm 0.01$ & $0.29 \pm 0.02$ & $16.81 \pm 0.02$ & $16.53 \pm 0.01$ & $0.28 \pm 0.02$ & 35.45 & 35.38 & 15.81 & 15.47 & 2.28 & 2.31 & 2.20 & 2.26 & $? \mathrm{~F}$ \\
\hline UGC 04807 & $16.24 \pm 0.01$ & $15.97 \pm 0.01$ & $0.26 \pm 0.01$ & $16.40 \pm 0.02$ & $16.14 \pm 0.01$ & $0.26 \pm 0.02$ & 35.99 & 35.92 & 15.30 & 14.98 & $\ldots$ & $\ldots$ & $\ldots$ & $\ldots$ & $\ldots$ \\
\hline NGC 2768 & $17.26 \pm 0.07$ & $15.24 \pm 0.03$ & $2.02 \pm 0.08$ & $\ldots$ & $15.41 \pm 0.03$ & $\ldots$ & 34.75 & 35.38 & 35.02 & 65.33 & 4.61 & 4.92 & 4.03 & 4.28 & $\mathrm{VV}$ \\
\hline NGC 2784 & $16.57 \pm 0.16$ & $14.75 \pm 0.03$ & $1.82 \pm 0.17$ & $16.33 \pm 0.10$ & $14.94 \pm 0.02$ & $1.39 \pm 0.11$ & 34.08 & 34.63 & 22.76 & 34.03 & $\ldots$ & 6.17 & $\ldots$ & 4.76 & $\mathrm{VV}$ \\
\hline UGC 04844 & $\ldots$ & $15.19 \pm 0.01$ & $\ldots$ & $\ldots$ & $15.42 \pm 0.01$ & $\ldots$ & 0.00 & 36.24 & $\ldots$ & 15.59 & $\ldots$ & $\ldots$ & $\ldots$ & $\ldots$ & $\mathrm{ER}$ \\
\hline UGC 04851 & $19.19 \pm 0.12$ & $18.29 \pm 0.03$ & $0.90 \pm 0.12$ & $19.32 \pm 0.09$ & $18.49 \pm 0.03$ & $0.83 \pm 0.10$ & 35.35 & 35.54 & 12.64 & 12.04 & $\ldots$ & $\ldots$ & $\ldots$ & $\ldots$ & $\mathrm{EE}$ \\
\hline NGC 2782 & $14.69 \pm 0.01$ & $14.25 \pm 0.01$ & $0.44 \pm 0.01$ & $14.73 \pm 0.01$ & $14.29 \pm 0.01$ & $0.44 \pm 0.01$ & 36.24 & 36.24 & 11.54 & 14.78 & $\ldots$ & $\ldots$ & $\ldots$ & $\ldots$ & Ern \\
\hline UGC 04872 & $17.59 \pm 0.04$ & $17.16 \pm 0.02$ & $0.43 \pm 0.04$ & $18.16 \pm 0.03$ & $17.76 \pm 0.02$ & $0.40 \pm 0.04$ & 36.05 & 36.05 & 15.51 & 15.76 & 2.13 & 2.24 & 2.07 & 2.24 & $? \mathrm{~F}$ \\
\hline NGC 2798 & $16.28 \pm 0.01$ & $15.48 \pm 0.01$ & $0.80 \pm 0.01$ & $16.33 \pm 0.01$ & $15.54 \pm 0.01$ & $0.80 \pm 0.02$ & 35.28 & 35.43 & 7.80 & 7.65 & $\ldots$ & $\ldots$ & $\ldots$ & $\ldots$ & $\mathrm{EV}$ \\
\hline UGC 04915 & $17.64 \pm 0.02$ & $17.04 \pm 0.01$ & $0.60 \pm 0.02$ & $17.93 \pm 0.02$ & $17.40 \pm 0.01$ & $0.53 \pm 0.02$ & 35.57 & 35.63 & 7.90 & 8.50 & $\ldots$ & $\ldots$ & $\ldots$ & $\ldots$ & $? \mathrm{~F}$ \\
\hline NGC 2799 & $16.33 \pm 0.02$ & $15.91 \pm 0.02$ & $0.41 \pm 0.02$ & $16.49 \pm 0.01$ & $16.14 \pm 0.01$ & $0.35 \pm 0.02$ & 35.24 & 35.23 & 7.78 & 9.59 & $\ldots$ & $\ldots$ & $\ldots$ & $\ldots$ & VV \\
\hline IC 0531 & $17.59 \pm 0.03$ & $16.92 \pm 0.01$ & $0.67 \pm 0.04$ & $17.66 \pm 0.03$ & $17.05 \pm 0.01$ & $0.61 \pm 0.03$ & 35.64 & 35.73 & 13.29 & 13.31 & 2.70 & 2.71 & $\ldots$ & $\ldots$ & $? \mathrm{~F}$ \\
\hline UGC 04921 & $\ldots$ & $17.62 \pm 0.01$ & $\ldots$ & $\ldots$ & $17.88 \pm 0.01$ & & 0.00 & 35.42 & $\ldots$ & 8.05 & $\ldots$ & $\ldots$ & $\ldots$ & $\ldots$ & $? \mathrm{~F}$ \\
\hline NGC 2841 & $13.61 \pm 0.01$ & $13.11 \pm 0.01$ & $0.50 \pm 0.01$ & $13.73 \pm 0.01$ & $13.20 \pm 0.01$ & $0.53 \pm 0.01$ & 35.79 & 35.81 & 74.19 & 68.81 & 2.08 & 2.21 & 2.12 & 2.24 & ERn \\
\hline NGC 2854 & $\ldots$ & $15.67 \pm 0.01$ & $\ldots$ & $\ldots$ & $15.77 \pm 0.01$ & $\ldots$ & 0.00 & 35.74 & $\ldots$ & 15.02 & $\ldots$ & 2.29 & $\ldots$ & 2.30 & $? \mathrm{~F}$ \\
\hline NGC 2856 & $\ldots$ & $16.36 \pm 0.01$ & $\ldots$ & $\ldots$ & $16.52 \pm 0.01$ & $\ldots$ & 0.00 & 35.43 & $\ldots$ & 9.15 & $\ldots$ & $\ldots$ & $\ldots$ & $\ldots$ & $? \mathrm{~F}$ \\
\hline NGC 2857 & $\ldots$ & $14.95 \pm 0.02$ & $\ldots$ & $\ldots$ & $15.10 \pm 0.01$ & $\ldots$ & 0.00 & 36.50 & $\ldots$ & 33.69 & $\ldots$ & 2.20 & $\ldots$ & 2.12 & $? \mathrm{~F}$ \\
\hline NGC 2915 & $13.38 \pm 0.01$ & $13.36 \pm 0.01$ & $0.02 \pm 0.01$ & $13.41 \pm 0.01$ & $13.42 \pm 0.01$ & $0.00 \pm 0.01$ & 34.74 & 34.57 & $\ldots$ & $\ldots$ & $\ldots$ & $\ldots$ & $\ldots$ & $\ldots$ & $\mathrm{VV}$ \\
\hline UGC 05013 & $17.28 \pm 0.05$ & $16.93 \pm 0.01$ & $0.35 \pm 0.06$ & $17.42 \pm 0.03$ & $17.06 \pm 0.01$ & $0.37 \pm 0.03$ & 36.45 & 36.42 & 19.45 & 19.24 & 2.31 & 2.46 & 2.20 & 2.49 & $? \mathrm{~F}$ \\
\hline UGC 05027 & $\ldots$ & $16.99 \pm 0.03$ & $\ldots$ & $\ldots$ & $17.35 \pm 0.01$ & $\ldots$ & 0.00 & 35.51 & $\cdots$ & 7.71 & $\ldots$ & $\ldots$ & $\ldots$ & $\ldots$ & $? \mathrm{~F}$ \\
\hline NGC 2870 & $16.55 \pm 0.02$ & $15.99 \pm 0.01$ & $0.56 \pm 0.02$ & $16.70 \pm 0.02$ & $16.15 \pm 0.01$ & $0.55 \pm 0.02$ & 35.70 & 35.74 & 21.58 & 21.08 & 2.03 & 2.22 & 2.02 & 2.24 & $\mathrm{EF}$ \\
\hline UGC 05053 & $17.94 \pm 0.04$ & $17.81 \pm 0.02$ & $0.13 \pm 0.05$ & $18.13 \pm 0.04$ & $18.04 \pm 0.02$ & $0.10 \pm 0.04$ & 35.14 & 35.02 & 10.94 & 10.80 & $\ldots$ & $\ldots$ & $\ldots$ & $\ldots$ & $? \mathrm{~F}$ \\
\hline NGC 2903 & $12.12 \pm 0.01$ & $11.69 \pm 0.01$ & $0.43 \pm 0.01$ & $12.19 \pm 0.01$ & $11.73 \pm 0.01$ & $0.46 \pm 0.01$ & 35.99 & 35.98 & 83.14 & 71.73 & 2.79 & 2.86 & 2.97 & 3.12 & Ern \\
\hline UGC 05077 & & $17.14 \pm 0.01$ & $\ldots$ & $\ldots$ & $17.29 \pm 0.01$ & $\cdots$ & 0.00 & & $\ldots$ & 13.61 & $\ldots$ & $\ldots$ & $\ldots$ & $\ldots$ & Er \\
\hline I Zw 18 & $15.82 \pm 0.01$ & $15.86 \pm 0.01$ & $-0.05 \pm 0.01$ & $15.82 \pm 0.01$ & $15.87 \pm 0.01$ & $-0.05 \pm 0.02$ & 34.81 & 34.62 & $\ldots$ & $\ldots$ & $\ldots$ & $\ldots$ & $\ldots$ & $\ldots$ & VV \\
\hline NGC 2916 & $15.07 \pm 0.01$ & $14.66 \pm 0.01$ & $0.41 \pm 0.01$ & $15.12 \pm 0.01$ & $14.71 \pm 0.01$ & $0.41 \pm 0.02$ & 36.38 & 36.36 & 27.76 & 27.04 & 1.74 & 1.86 & 1.58 & 1.75 & $\mathrm{EFh}$ \\
\hline UGC 05107 & $\ldots$ & $16.42 \pm 0.01$ & $\ldots$ & $\ldots$ & $16.62 \pm 0.01$ & $\ldots$ & 0.00 & 35.10 & $\ldots$ & 15.59 & $\ldots$ & 2.55 & $\ldots$ & 2.54 & $? \mathrm{~F}$ \\
\hline UGC 05101 & $19.10 \pm 0.04$ & $17.78 \pm 0.04$ & $1.32 \pm 0.06$ & $19.30 \pm 0.09$ & $18.13 \pm 0.03$ & $1.17 \pm 0.09$ & 35.77 & 36.12 & 10.11 & 11.89 & $\ldots$ & $\ldots$ & $\ldots$ & $\ldots$ & ?R \\
\hline NGC 2936 & $16.13 \pm 0.01$ & $15.60 \pm 0.01$ & $0.53 \pm 0.01$ & $16.40 \pm 0.03$ & $15.87 \pm 0.01$ & $0.54 \pm 0.03$ & 36.49 & 36.52 & 20.67 & 19.47 & $\ldots$ & $\ldots$ & $\ldots$ & $\ldots$ & Er \\
\hline NGC 2937 & $19.28 \pm 0.10$ & $17.99 \pm 0.42$ & $1.29 \pm 0.43$ & $19.24 \pm 0.15$ & $18.23 \pm 0.06$ & $1.01 \pm 0.16$ & 35.20 & 35.54 & 8.57 & 15.43 & $\ldots$ & $\ldots$ & $\ldots$ & $\ldots$ & $\mathrm{VV}$ \\
\hline UGC 05147 & $\ldots$ & $17.22 \pm 0.02$ & $\ldots$ & $\ldots$ & $18.35 \pm 0.02$ & ... & 0.00 & 35.74 & $\ldots$ & 12.04 & $\ldots$ & 2.71 & .. & 2.66 & $? \mathrm{~F}$ \\
\hline
\end{tabular}


Table 3-Continued

\begin{tabular}{|c|c|c|c|c|c|c|c|c|c|c|c|c|c|c|c|}
\hline \multirow{2}{*}{$\begin{array}{c}\text { Object Name } \\
\text { (1) }\end{array}$} & \multicolumn{3}{|c|}{ Asymptotic magnitudes and color } & \multicolumn{3}{|c|}{ D25 magnitudes and color } & \multicolumn{2}{|c|}{$\log L$} & \multicolumn{2}{|c|}{ Effective Radii } & \multicolumn{2}{|c|}{$\mathrm{C} 31$} & \multicolumn{2}{|c|}{$\mathrm{C} 42$} & \multirow{2}{*}{$\begin{array}{c}\text { UV } \\
\text { profile } \\
(16)\end{array}$} \\
\hline & $\begin{array}{l}\text { FUV } \\
(\mathrm{mag}) \\
(2)\end{array}$ & $\begin{array}{c}\text { NUV } \\
(\mathrm{mag}) \\
(3)\end{array}$ & $\begin{array}{c}\text { FUV-NUV } \\
\text { (mag) } \\
(4)\end{array}$ & $\begin{array}{c}\text { FUV } \\
(\mathrm{mag}) \\
(5)\end{array}$ & $\begin{array}{c}\text { NUV } \\
(\mathrm{mag}) \\
(6)\end{array}$ & $\begin{array}{c}\text { FUV-NUV } \\
(\mathrm{mag}) \\
(7)\end{array}$ & $\begin{array}{c}\text { FUV } \\
(\mathrm{W}) \\
(8)\end{array}$ & $\begin{array}{l}\text { NUV } \\
(\mathrm{W}) \\
(9)\end{array}$ & $\begin{array}{c}\text { FUV } \\
(\operatorname{arcsec}) \\
(10)\end{array}$ & $\begin{array}{c}\text { NUV } \\
(\operatorname{arcsec}) \\
(11)\end{array}$ & $\begin{array}{l}\text { FUV } \\
(12)\end{array}$ & $\begin{array}{l}\text { NUV } \\
(13)\end{array}$ & $\begin{array}{l}\text { FUV } \\
(14)\end{array}$ & $\begin{array}{l}\text { NUV } \\
(15)\end{array}$ & \\
\hline UGC 05114 & $.95 \pm 0.01$ & $.70 \pm 0.01$ & $2 U \perp 0$ & $0 \pm 0.01$ & $15.77 \pm 0$ & $0.23 \pm$ & & & & & & & & & Ef \\
\hline Holmberg I & $.61 \pm 0.01$ & $14.55 \pm 0.01$ & $0.06 \pm 0.02$ & $.84 \pm 0.01$ & $14.86 \pm 0.01$ & $-0.02 \pm 0.01$ & 34.26 & 34.11 & 59.87 & 64.79 & 1.93 & 2.11 & 1.84 & 2.03 & $\mathrm{EF}$ \\
\hline UGC 05201 & $\ldots$ & $16.66 \pm 0.01$ & $\ldots$ & $\ldots$ & $16.71 \pm 0.01$ & & 0.00 & 36.19 & $\ldots$ & 18.63 & $\ldots$ & 2.27 & $\ldots$ & $\ldots$ & $? \mathrm{~F}$ \\
\hline NGC 2992 & $16.00 \pm 0.05$ & $15.39 \pm 0.05$ & $0.61 \pm 0.06$ & $16.22 \pm 0.02$ & $15.67 \pm 0.01$ & $0.55 \pm 0.02$ & 35.54 & 35.60 & 22.59 & 25.60 & 3.63 & 4.04 & 3.55 & 3.70 & $\mathrm{VF}$ \\
\hline NGC 2976 & $\ldots$ & $12.77 \pm 0.01$ & $\ldots$ & $\ldots$ & $12.80 \pm 0.01$ & $\ldots$ & 0.00 & 34.75 & $\ldots$ & 44.86 & $\ldots$ & 1.95 & $\ldots$ & 1.90 & $\mathrm{VF}$ \\
\hline UGC 05237 & $\ldots$ & $16.32 \pm 0.01$ & $\ldots$ & $\ldots$ & $16.43 \pm 0.01$ & $\ldots$ & 0.00 & 35.92 & $\ldots$ & 15.61 & $\ldots$ & 2.56 & $\ldots$ & $\ldots$ & $? \mathrm{~F}$ \\
\hline NGC 3018 & $15.39 \pm 0.02$ & $14.94 \pm 0.01$ & $0.45 \pm 0.02$ & $15.52 \pm 0.01$ & $15.08 \pm 0.01$ & $0.44 \pm 0.02$ & 35.62 & 35.62 & $\ldots$ & $\ldots$ & $\ldots$ & $\ldots$ & $\ldots$ & $\ldots$ & Er \\
\hline NGC 3023 & $14.27 \pm 0.01$ & $13.93 \pm 0.01$ & $0.34 \pm 0.01$ & $14.31 \pm 0.01$ & $13.97 \pm 0.01$ & $0.34 \pm 0.01$ & 36.07 & 36.03 & 23.09 & 23.00 & 2.44 & 2.56 & 2.55 & 2.64 & $\mathrm{EF}$ \\
\hline UGC 05268 & $\ldots$ & $16.83 \pm 0.01$ & $\ldots$ & $\ldots$ & $17.07 \pm 0.01$ & 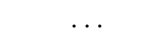 & 0.00 & 36.10 & & 12.47 & $\ldots$ & 2.58 & $\ldots$ & $\ldots$ & $? \mathrm{~F}$ \\
\hline UGC 05314 & $\ldots$ & $17.68 \pm 0.01$ & & $\ldots$ & $18.82 \pm 0$ & & 0.00 & & & & $\ldots$ & 2.52 & $\ldots$ & 2.57 & Ef \\
\hline NGC 3049 & 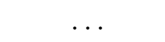 & $14.77 \pm 0.01$ & & $\ldots$ & $14.85 \pm 0$ & $\ldots$ & 0.00 & & $\ldots$ & & $\ldots$ & $\ldots$ & $\ldots$ & & EFn \\
\hline MESSIER 081 & $10.77 \pm 0.01$ & $10.38 \pm 0.01$ & $0.39 \pm 0.01$ & $10.79 \pm 0.01$ & $10.40 \pm 0.01$ & $0.39 \pm 0.01$ & 35.75 & & 323.94 & 30 & 1.60 & 1.78 & 1.29 & 1.55 & VFn \\
\hline MESSIER 082 & $12.13 \pm 0.01$ & $11.32 \pm 0.01$ & $0.81 \pm 0.02$ & $12.66 \pm 0.01$ & $11.53 \pm 0.01$ & $1.13 \pm 0.01$ & 35.26 & 35.41 & 159.44 & 91 & 3.63 & 3.27 & 3.36 & 3.18 & $\ldots$ \\
\hline ESO 435-G016 & $15.48 \pm 0.01$ & $14.84 \pm 0.01$ & $0.64 \pm 0.01$ & $15.58 \pm 0.02$ & $14.98 \pm 0.01$ & $0.61 \pm 0.02$ & 34.88 & 34.96 & 18.92 & 18.38 & $\ldots$ & $\ldots$ & $\ldots$ & $\ldots$ & Er \\
\hline Tol 2 & $14.93 \pm 0.01$ & $14.59 \pm 0.01$ & $0.34 \pm 0.01$ & $14.99 \pm 0.01$ & $14.69 \pm 0.01$ & $0.31 \pm 0.02$ & 34.67 & 34.62 & $\ldots$ & $\ldots$ & $\ldots$ & $\ldots$ & $\ldots$ & $\ldots$ & $\mathrm{ER}$ \\
\hline NGC 3089 & $15.45 \pm 0.01$ & $14.77 \pm 0.01$ & $0.68 \pm 0.01$ & $15.48 \pm 0.02$ & $14.82 \pm 0.01$ & $0.66 \pm 0.02$ & 35.88 & 35.97 & 17.37 & 17.06 & 1.95 & 2.23 & 1.95 & $\ldots$ & ?Fn \\
\hline NGC 3073 & $17.51 \pm 0.01$ & $16.61 \pm 0.02$ & $0.89 \pm 0.02$ & $17.54 \pm 0.02$ & $16.77 \pm 0.01$ & $0.77 \pm 0.03$ & 34.52 & 34.70 & $\ldots$ & $\ldots$ & $\ldots$ & $\ldots$ & $\ldots$ & $\ldots$ & VV \\
\hline NGC 3079 & $13.93 \pm 0.01$ & $13.50 \pm 0.01$ & $0.42 \pm 0.02$ & $14.09 \pm 0.01$ & $13.65 \pm 0.01$ & $0.44 \pm 0.01$ & 35.92 & 35.92 & 52.78 & 51.44 & 2.25 & 2.24 & 2.13 & 2.23 & $\mathrm{EE}$ \\
\hline NGC 3109 & $11.42 \pm 0.01$ & $11.18 \pm 0.01$ & $0.23 \pm 0.01$ & $11.51 \pm 0.01$ & $11.28 \pm 0.01$ & $0.23 \pm 0.01$ & 34.45 & & 107.93 & 108.47 & 2.33 & 2.36 & 2.40 & 2.41 & EEh \\
\hline UGCA 196 & $14.49 \pm 0$ & 14.0 & $0.42 \pm 0$ & $14.83 \pm 0.01$ & 14.3 & $0.44 \pm 0$ & 35.26 & & 38.20 & & 2.28 & 6 & 2.30 & 2.44 & EEn \\
\hline IC 2537 & $14.84 \pm 0.01$ & $14.30 \pm 0.01$ & $0.54 \pm 0.01$ & $14.92 \pm 0.01$ & $14.37 \pm 0.01$ & $0.55 \pm 0.01$ & 36.15 & 36.19 & 36.70 & 34.26 & 2.00 & 2.16 & 1.89 & 2.12 & $? \mathrm{Fn}$ \\
\hline UGC 05406 & $18.27 \pm 0.06$ & $18.08 \pm 0.03$ & $0.19 \pm 0.06$ & $18.53 \pm 0.04$ & $18.27 \pm 0.02$ & $0.26 \pm 0.05$ & 35.67 & 35.57 & 10.11 & 9.10 & $\ldots$ & $\ldots$ & $\cdots$ & $\ldots$ & $? \mathrm{~F}$ \\
\hline Antlia Dwarf & $17.57 \pm 0.04$ & $16.89 \pm 0.02$ & $0.68 \pm 0.05$ & $17.76 \pm 0.04$ & $17.13 \pm 0.02$ & $0.63 \pm 0.05$ & 31.99 & 32.09 & 29.93 & 30.71 & 2.24 & 2.33 & 2.21 & 2.33 & $? \mathrm{~F}$ \\
\hline M81 Dwarf B & $16.73 \pm 0.01$ & $16.50 \pm 0.02$ & $0.22 \pm 0.02$ & $16.82 \pm 0.02$ & $16.73 \pm 0.01$ & $0.10 \pm 0.03$ & 33.69 & 33.61 & $\ldots$ & 10.36 & $\ldots$ & $\ldots$ & $\ldots$ & $\ldots$ & $\mathrm{ER}$ \\
\hline NGC 3125 & $14.46 \pm 0.01$ & $14.10 \pm 0.01$ & $0.36 \pm 0.01$ & $14.55 \pm 0.01$ & $14.20 \pm 0.01$ & $0.35 \pm 0.01$ & 35.09 & 35.06 & $\ldots$ & $\ldots$ & $\ldots$ & $\ldots$ & $\ldots$ & $\ldots$ & VV,ER \\
\hline UGC 05455 & $16.99 \pm 0.20$ & $16.71 \pm 0.14$ & $0.28 \pm 0.24$ & $17.10 \pm 0.05$ & $16.93 \pm 0.03$ & $0.17 \pm 0.06$ & 34.83 & 34.77 & 19.60 & 23.19 & $\ldots$ & $\ldots$ & $\ldots$ & $\ldots$ & ?R \\
\hline Sextans A & $12.58 \pm 0.01$ & $12.54 \pm 0.01$ & $0.04 \pm 0.01$ & $12.63 \pm 0.01$ & $12.59 \pm 0.01$ & $0.04 \pm 0.01$ & 34.21 & 34.05 & 91.35 & 90.29 & 1.63 & 1.66 & 1.36 & 1.42 & ED \\
\hline UGC 05493 & $15.95 \pm 0.01$ & $15.68 \pm 0.01$ & $0.27 \pm 0.01$ & $15.99 \pm 0.01$ & $15.73 \pm 0.01$ & $0.27 \pm 0.02$ & 35.98 & 35.91 & 20.06 & 19.49 & 2.35 & 2.50 & $\ldots$ & $\ldots$ & $? \mathrm{~F}$ \\
\hline UGC 05515 & $20.25 \pm 0.42$ & $17.97 \pm 0.24$ & $2.28 \pm 0.49$ & $\ldots$ & $18.46 \pm 0.09$ & $\ldots$ & 35.39 & 36.13 & $\ldots$ & 26.95 & $\ldots$ & $\ldots$ & $\ldots$ & $\ldots$ & $\ldots$ \\
\hline
\end{tabular}


Table 3-Continued

\begin{tabular}{|c|c|c|c|c|c|c|c|c|c|c|c|c|c|c|c|}
\hline \multirow{2}{*}{$\begin{array}{l}\text { Object Name } \\
\text { (1) }\end{array}$} & \multicolumn{3}{|c|}{ Asymptotic magnitudes and color } & \multicolumn{3}{|c|}{ D25 magnitudes and color } & \multicolumn{2}{|c|}{$\log L$} & \multicolumn{2}{|c|}{ Effective Radii } & \multicolumn{2}{|c|}{$\mathrm{C} 31$} & \multicolumn{2}{|c|}{$\mathrm{C} 42$} & \multirow{2}{*}{$\begin{array}{c}\text { UV } \\
\text { profile } \\
(16)\end{array}$} \\
\hline & $\begin{array}{c}\text { FUV } \\
(\mathrm{mag}) \\
(2)\end{array}$ & $\begin{array}{c}\text { NUV } \\
(\mathrm{mag}) \\
(3)\end{array}$ & $\begin{array}{c}\text { FUV-NUV } \\
(\mathrm{mag}) \\
(4)\end{array}$ & $\begin{array}{c}\text { FUV } \\
(\mathrm{mag}) \\
(5)\end{array}$ & $\begin{array}{c}\text { NUV } \\
(\mathrm{mag}) \\
(6)\end{array}$ & $\begin{array}{l}\text { FUV-NUV } \\
\text { (mag) } \\
(7)\end{array}$ & $\begin{array}{c}\text { FUV } \\
(\mathrm{W}) \\
(8)\end{array}$ & $\begin{array}{l}\text { NUV } \\
(\mathrm{W}) \\
(9)\end{array}$ & $\begin{array}{c}\text { FUV } \\
(\operatorname{arcsec}) \\
(10)\end{array}$ & $\begin{array}{c}\text { NUV } \\
(\operatorname{arcsec}) \\
(11)\end{array}$ & $\begin{array}{l}\text { FUV } \\
(12)\end{array}$ & $\begin{array}{l}\text { NUV } \\
(13)\end{array}$ & $\begin{array}{l}\text { FUV } \\
(14)\end{array}$ & $\begin{array}{l}\text { NUV } \\
(15)\end{array}$ & \\
\hline UGC 05528 & $18.63 \pm 0.08$ & $17.83 \pm 0.01$ & $0.81 \pm 0.08$ & & $17.89 \pm 0.04$ & & 36.13 & 36.27 & $\ldots$ & 11.96 & . & & . & & $? \mathrm{D}$ \\
\hline NGC 3147 & $14.14 \pm 0.01$ & $13.62 \pm 0.01$ & $0.52 \pm 0.01$ & $14.21 \pm 0.01$ & $13.67 \pm 0.01$ & $0.54 \pm 0.01$ & 36.57 & 36.60 & 44.75 & 39.63 & 2.53 & 2.81 & 2.61 & 2.79 & EEh \\
\hline NGC 3185 & $16.12 \pm 0.03$ & $15.60 \pm 0.01$ & $0.52 \pm 0.03$ & $16.23 \pm 0.01$ & $15.68 \pm 0.01$ & $0.54 \pm 0.02$ & 34.97 & 35.00 & 28.19 & 26.94 & 1.56 & 1.73 & 1.31 & 1.62 & $? \mathrm{Rn}$ \\
\hline NGC 3187 & $14.84 \pm 0.01$ & $14.57 \pm 0.01$ & $0.27 \pm 0.01$ & $14.97 \pm 0.01$ & $14.71 \pm 0.01$ & $0.26 \pm 0.01$ & 35.48 & 35.41 & 30.82 & 30.77 & 2.86 & 2.83 & 2.90 & 2.84 & $\mathrm{EE}$ \\
\hline NGC 3190 & $17.39 \pm 0.02$ & $15.76 \pm 0.01$ & $1.63 \pm 0.02$ & $17.45 \pm 0.03$ & $15.86 \pm 0.01$ & $1.60 \pm 0.03$ & 34.46 & 34.94 & 29.21 & 28.38 & 3.51 & 3.86 & 3.33 & 3.64 & VV \\
\hline UGC 05558 & $18.96 \pm 0.07$ & $18.01 \pm 0.04$ & $0.95 \pm 0.08$ & $19.32 \pm 0.06$ & $18.57 \pm 0.02$ & $0.75 \pm 0.06$ & 35.44 & 35.64 & 9.56 & 10.81 & 2.36 & 2.75 & $\cdots$ & 2.84 & $\mathrm{EE}$ \\
\hline NGC 3193 & $17.43 \pm 0.05$ & $15.93 \pm 0.02$ & $1.50 \pm 0.05$ & $17.65 \pm 0.04$ & $16.17 \pm 0.01$ & $1.47 \pm 0.04$ & 34.44 & 34.87 & 25.10 & & $\cdots$ & & $\ldots$ & $\cdots$ & VV \\
\hline NGC 3198 & $.97 \pm 0$ & $12.77 \pm 0.01$ & $0.19 \pm 0$ & $13.13 \pm 0.01$ & $12.91 \pm 0.01$ & $0.22 \pm$ & 36.19 & 36 & 83 & 77.93 & 2.18 & 2.20 & 2.20 & 2.15 & $\mathrm{ERh}$ \\
\hline UGC 05570 & $17.95 \pm 0.06$ & $17.20 \pm 0.12$ & $0.75 \pm 0.13$ & $19.24 \pm 0.04$ & $18.62 \pm 0.02$ & $0.62 \pm 0.04$ & 35.02 & 35.14 & 13.13 & $\cdots$ & $\cdots$ & .. & $\cdots$ & . & $\mathrm{EE}$ \\
\hline NGC 3183 & $15.16 \pm 0.01$ & $14.79 \pm 0.01$ & $0.37 \pm 0.01$ & $15.27 \pm 0.01$ & $14.88 \pm 0.01$ & $0.39 \pm 0.01$ & 36.23 & 36.20 & 32.06 & 28.94 & 2.30 & 2.49 & 2.25 & 2.46 & $? \mathrm{Fh}$ \\
\hline ESO 317-G019 & $16.92 \pm 0.03$ & $16.09 \pm 0.02$ & $0.83 \pm 0.03$ & $17.04 \pm 0.03$ & $16.29 \pm 0.01$ & $0.75 \pm 0.03$ & 35.32 & 35.48 & $\cdots$ & $\cdots$ & $\cdots$ & $\cdots$ & $\cdots$ & $\cdots$ & $? \mathrm{Fn}$ \\
\hline ESO 317-G023 & $17.60 \pm 0.02$ & $16.65 \pm 0.01$ & $0.95 \pm 0.02$ & $17.68 \pm 0.04$ & $16.77 \pm 0.01$ & $0.91 \pm 0.04$ & 35.05 & 35.26 & 12.89 & 14.11 & 2.69 & 3.04 & $\cdots$ & $\cdots$ & Ef \\
\hline ESO 263-G033 & $\cdots$ & $17.40 \pm 0.04$ & $\cdots$ & $\cdots$ & $17.72 \pm 0.06$ & $\ldots$ & 0.00 & 34.96 & $\cdots$ & 16.35 & $\ldots$ & $\cdots$ & $\cdots$ & $\cdots$ & VV \\
\hline NGC 3225 & $\cdots$ & $15.01 \pm 0.01$ & $\cdots$ & $\cdots$ & $15.15 \pm 0.01$ & $\cdots$ & 0.00 & 35.81 & & 20.40 & $\cdots$ & 2.64 & $\cdots$ & 2.58 & $? \mathrm{~F}$ \\
\hline NGC 3244 & $14.58 \pm 0.02$ & $14.23 \pm 0.01$ & $0.35 \pm 0.02$ & $14.91 \pm 0.01$ & $14.43 \pm 0.01$ & $0.48 \pm 0.01$ & 36.23 & 36.19 & 30.34 & 26.48 & 2.85 & 2.79 & 2.84 & 2.76 & Ef \\
\hline NGC $3256 \mathrm{~A}$ & $\cdots$ & $16.48 \pm 0.01$ & $\cdots$ & $\cdots$ & $16.62 \pm 0.02$ & $\cdots$ & 0.00 & 35.32 & $\cdots$ & 14.95 & $\ldots$ & 2.17 & $\cdots$ & $\ldots$ & $? \mathrm{~F}$ \\
\hline NGC 3238 & $19.99 \pm 0.04$ & $18.17 \pm 0.02$ & $1.82 \pm 0.04$ & $\ldots$ & $18.43 \pm 0.02$ & $\cdots$ & 35.01 & 35.56 & $\cdots$ & 15.90 & $\ldots$ & $\cdots$ & $\ldots$ & $\cdots$ & Er \\
\hline IC 2574 & $12.23 \pm 0.01$ & $12.19 \pm 0.01$ & $0.04 \pm 0.01$ & $12.38 \pm 0.01$ & $12.34 \pm 0.01$ & $0.04 \pm 0$ & 35.25 & 35.09 & 188.28 & 181.08 & 1.77 & 1.90 & 1.59 & 1.84 & $\mathrm{EE}$ \\
\hline NGC 3265 & $17.02 \pm 0.01$ & $16.44 \pm 0.01$ & $0.59 \pm 0.01$ & $17.03 \pm 0.02$ & $16.47 \pm 0.01$ & $0.56 \pm 0$ & 34.73 & 34.78 & $\ldots$ & & $\ldots$ & $\ldots$ & $\ldots$ & $\ldots$ & ER \\
\hline UGC 05715 & $16.76 \pm 0$ & $16.16 \pm 0.01$ & & $16.88 \pm 0.02$ & $16.27 \pm 0.01$ & $0.61 \pm 0$ & 36.40 & & 14.15 & 13.77 & $\ldots$ & $\cdots$ & $\ldots$ & $\cdots$ & $? \mathrm{~F}$ \\
\hline UGC 05720 & $14.86 \pm 0.01$ & $14.61 \pm 0.01$ & $0.25 \pm 0.01$ & $14.92 \pm 0.01$ & $14.68 \pm 0.01$ & $0.24 \pm 0.01$ & 35.74 & 35.66 & $\cdots$ & $\cdots$ & $\cdots$ & $\cdots$ & $\cdots$ & $\cdots$ & VV \\
\hline NGC 3277 & $15.78 \pm 0.01$ & $15.15 \pm 0.01$ & $0.63 \pm 0.02$ & $15.83 \pm 0.01$ & $15.27 \pm 0.01$ & $0.56 \pm 0.02$ & 35.30 & 35.38 & 16.61 & 16.76 & $\ldots$ & $\ldots$ & $\ldots$ & $\cdots$ & VFn \\
\hline NGC 3288 & $17.61 \pm 0.01$ & $17.10 \pm 0.01$ & $0.51 \pm 0.01$ & $17.67 \pm 0.02$ & $17.17 \pm 0.01$ & $0.49 \pm 0.03$ & 36.05 & 36.07 & 11.26 & 11.09 & $\cdots$ & $\cdots$ & $\cdots$ & $\cdots$ & ?D \\
\hline UGC 05772 & $17.16 \pm 0.04$ & $16.59 \pm 0.02$ & $0.57 \pm 0.04$ & $17.36 \pm 0.04$ & $16.76 \pm 0.01$ & $0.60 \pm 0.04$ & 36.27 & 36.32 & 12.81 & 12.03 & $\cdots$ & $\cdots$ & $\cdots$ & $\cdots$ & $? \mathrm{D}$ \\
\hline NGC 3319 & $13.50 \pm 0.01$ & $13.32 \pm 0.01$ & $0.18 \pm 0.01$ & $13.58 \pm 0.01$ & $13.40 \pm 0.01$ & $0.18 \pm 0.01$ & 35.87 & 35.76 & 86.15 & 84.50 & 1.78 & 1.88 & 1.63 & 1.80 & ?Fn \\
\hline UGC 05818 & $\cdots$ & $16.33 \pm 0.05$ & $\cdots$ & $\cdots$ & $16.67 \pm 0.03$ & $\cdots$ & 0.00 & 36.13 & $\cdots$ & 18.15 & $\cdots$ & 2.50 & $\cdots$ & $\cdots$ & $\mathrm{EE}$ \\
\hline UGC 05823 & $16.55 \pm 0.02$ & $16.16 \pm 0.01$ & $0.39 \pm 0.02$ & $16.70 \pm 0.02$ & $16.32 \pm 0.01$ & $0.37 \pm 0.03$ & 36.12 & 36.10 & 12.68 & 12.87 & $\cdots$ & $\cdots$ & $\cdots$ & $\cdots$ & $? \mathrm{~F}$ \\
\hline NGC 3344 & $12.43 \pm 0.01$ & $12.12 \pm 0.01$ & $0.31 \pm 0.02$ & $12.55 \pm 0.01$ & $12.21 \pm 0.01$ & $0.34 \pm 0.01$ & 35.64 & 35.59 & 92.57 & & 2.50 & 2.46 & 2.51 & 2.54 & Efn \\
\hline MESSIER 095 & $13.28 \pm 0.01$ & $12.75 \pm 0.01$ & $0.53 \pm 0.01$ & $13.36 \pm 0.01$ & $12.82 \pm 0.01$ & $0.54 \pm 0.01$ & 35.77 & 35.80 & 84.54 & 72.26 & 2.46 & 4.41 & 2.46 & $\ldots$ & EFn \\
\hline UGC 05848 & $16.23 \pm 0.01$ & $15.95 \pm 0.01$ & $0.28 \pm 0.01$ & $16.45 \pm 0.01$ & $16.18 \pm 0.01$ & $0.27 \pm 0.01$ & 34.80 & 34.73 & 24.11 & 24.37 & 2.47 & 2.51 & 2.52 & 2.58 & ?Fn \\
\hline UGC 05853 & $18.44 \pm 0.01$ & $17.88 \pm 0.01$ & $0.56 \pm 0.01$ & $18.53 \pm 0.02$ & $18.01 \pm 0.01$ & $0.51 \pm 0.02$ & 35.84 & 35.88 & 9.57 & 9.78 & $\ldots$ & $\ldots$ & $\ldots$ & $\ldots$ & $? \mathrm{~F}$ \\
\hline NGC 3353 & $14.69 \pm 0.01$ & $14.44 \pm 0.01$ & $0.24 \pm 0.01$ & $14.72 \pm 0.01$ & $14.49 \pm 0.01$ & $0.23 \pm 0.01$ & 35.51 & 35.43 & $\cdots$ & $\cdots$ & $\cdots$ & $\ldots$ & $\ldots$ & . & VV \\
\hline
\end{tabular}


Table 3-Continued

\begin{tabular}{|c|c|c|c|c|c|c|c|c|c|c|c|c|c|c|c|}
\hline \multirow{2}{*}{$\begin{array}{l}\text { Object Name } \\
\text { (1) }\end{array}$} & \multicolumn{3}{|c|}{ Asymptotic magnitudes and color } & \multicolumn{3}{|c|}{ D25 magnitudes and color } & \multicolumn{2}{|c|}{$\log L$} & \multicolumn{2}{|c|}{ Effective Radii } & \multicolumn{2}{|c|}{ C31 } & \multicolumn{2}{|c|}{$\mathrm{C} 42$} & \multirow{2}{*}{$\begin{array}{c}\text { UV } \\
\text { profile } \\
(16)\end{array}$} \\
\hline & $\begin{array}{c}\text { FUV } \\
(\mathrm{mag}) \\
(2)\end{array}$ & $\begin{array}{c}\text { NUV } \\
(\mathrm{mag}) \\
(3)\end{array}$ & $\begin{array}{l}\text { FUV-NUV } \\
(\text { mag) } \\
(4)\end{array}$ & $\begin{array}{c}\text { FUV } \\
(\mathrm{mag}) \\
(5)\end{array}$ & $\begin{array}{c}\text { NUV } \\
(\mathrm{mag}) \\
(6)\end{array}$ & $\begin{array}{c}\text { FUV-NUV } \\
(\mathrm{mag}) \\
(7)\end{array}$ & $\begin{array}{c}\text { FUV } \\
(\mathrm{W}) \\
(8)\end{array}$ & $\begin{array}{c}\text { NUV } \\
(\mathrm{W}) \\
(9)\end{array}$ & $\begin{array}{c}\text { FUV } \\
(\operatorname{arcsec}) \\
(10)\end{array}$ & $\begin{array}{c}\text { NUV } \\
(\operatorname{arcsec}) \\
(11)\end{array}$ & $\begin{array}{l}\text { FUV } \\
(12)\end{array}$ & $\begin{array}{l}\text { NUV } \\
(13)\end{array}$ & $\begin{array}{l}\text { FUV } \\
(14)\end{array}$ & $\begin{array}{l}\text { NUV } \\
(15)\end{array}$ & \\
\hline GC 05869 & $33 \pm 0.02$ & $.04 \pm 0.02$ & $8 \pm 0.03$ & $.82 \pm 0.02$ & $16.52 \pm 0.01$ & $30 \pm 0$ & 36.35 & 36.29 & 23.28 & 22.32 & 2.49 & 2.76 & 2.55 & 2.77 & $? \mathrm{fh}, ? \mathrm{f}$ \\
\hline NGC 3367 & $\ldots$ & $13.38 \pm 0.01$ & $\ldots$ & $\ldots$ & $13.40 \pm 0.01$ & $\ldots$ & 0.00 & & $\cdots$ & 31.61 & $\ldots$ & 2.31 & $\cdots$ & 2.38 & VFn \\
\hline UGC 05876 & $\cdots$ & $16.43 \pm 0.02$ & $\cdots$ & $\cdots$ & $16.51 \pm 0.01$ & $\cdots$ & 0.00 & & $\cdots$ & 14.38 & $\cdots$ & $\cdots$ & $\cdots$ & $\cdots$ & ?F \\
\hline NGC 3359 & $\cdots$ & $12.67 \pm 0.01$ & $\cdots$ & $\cdots$ & $12.71 \pm 0.01$ & $\cdots$ & 0.00 & 36.20 & $\ldots$ & 67.93 & $\ldots$ & 2.56 & $\ldots$ & 2.48 & EEn \\
\hline UGC 05886 & $.32 \pm 0.02$ & $.82 \pm 0.01$ & $.50 \pm 0.02$ & $6.41 \pm 0.02$ & $.91 \pm$ & $0.50 \pm 0.02$ & 36.83 & 36.85 & 10.82 & $\ldots$ & $\cdots$ & $\ldots$ & $\ldots$ & $\cdots$ & $? \mathrm{~F}$ \\
\hline NGC $3377 \mathrm{~A}$ & $\ldots$ & $.89 \pm 0.01$ & $\ldots$ & $\ldots$ & $15.96 \pm 0.01$ & & 0.00 & 34.32 & & 29.00 & $\ldots$ & 2.24 & $\ldots$ & 2.29 & $? \mathrm{~F}$ \\
\hline UGC 05896 & $3.09 \pm 0.05$ & $17.71 \pm 0.02$ & $0.38 \pm 0.06$ & $8.19 \pm 0.04$ & $17.82 \pm($ & $0.38 \pm 0.04$ & 36.12 & 36 & 13.01 & & 2.08 & 2.26 & $\ldots$ & $\ldots$ & $? \mathrm{~F}$ \\
\hline NGC 3377 & $\cdots$ & $1487+0$ & $\ldots$ & $\cdots$ & 15.14 & $\cdots$ & 0.00 & 34.84 & $\ldots$ & 9 & $\ldots$ & 5.59 & $\ldots$ & 4.64 & $\mathrm{VV}$ \\
\hline UGC 0 & $.96 \pm 0.01$ & 01 & $17 \pm 0.01$ & $12 \pm 0.01$ & 0.01 & $17 \pm 0$. & 5.19 & 35 & 15.58 & & $\ldots$ & $\ldots$ & $\ldots$ & $\ldots$ & Efn \\
\hline & $15 \pm 0$ & 0 & $\delta 0 \pm 0$ & $8.77 \pm 0.04$ & 18.18 & $.59 \pm 0$ & 35.54 & & 20 & & 2.58 & 2.92 & 2.59 & $\ldots$ & Ef \\
\hline $\mathrm{U}$ & $76 \pm 0.16$ & 02 & $.21 \pm 0.16$ & $\ldots$ & 02 & $\ldots$ & 35.24 & 35 & 15.65 & & $\ldots$ & $\ldots$ & $\ldots$ & $\ldots$ & $\mathrm{EE}$ \\
\hline UGC 0 & $16.27 \pm 0.06$ & $16.00 \pm$ & $0.27 \pm 0.06$ & $16.50 \pm 0.02$ & $16.17 \pm 0.01$ & $0.33 \pm 0.03$ & 35.26 & 35.20 & 12.68 & 9 & $\ldots$ & $\ldots$ & $\ldots$ & $\ldots$ & $\mathrm{Ef}$ \\
\hline UGC 05929 & $\ldots$ & $16.58 \pm 0.01$ & $\ldots$ & $\cdots$ & $16.76 \pm 0.01$ & $\cdots$ & 0.00 & 36.23 & $\ldots$ & & $\ldots$ & $\ldots$ & $\ldots$ & $\cdots$ & $\mathrm{Ef}$ \\
\hline UGC 05943 & $5.26 \pm 0.01$ & 0.01 & $.51 \pm 0.02$ & $16.32 \pm 0.01$ & & $0.45 \pm 0.02$ & 36.05 & 36.08 & 14.12 & & $\ldots$ & $\ldots$ & $\ldots$ & $\ldots$ & $? \mathrm{~F}$ \\
\hline NGC 3394 & $15.79 \pm 0.01$ & $5.33 \pm 0.01$ & $0.46 \pm 0.01$ & $15.83 \pm 0.01$ & $15.37 \pm 0.01$ & $0.46 \pm 0.01$ & 36.06 & 36.06 & 24.43 & 23.69 & 2.18 & 2.34 & 2.12 & 2.31 & $\mathrm{EF}$ \\
\hline NGC 3412 & $\ldots$ & $15.75 \pm 0.01$ & $\ldots$ & $\ldots$ & $15.83 \pm 0.01$ & $\ldots$ & 0.00 & 34.68 & $\ldots$ & 24.39 & $\ldots$ & 4.76 & $\ldots$ & $\ldots$ & $\mathrm{Er}$ \\
\hline NGC 3419 & $\ldots$ & $17.11 \pm 0.01$ & $\ldots$ & $\ldots$ & & & 0.00 & 35.21 & $\ldots$ & & $\ldots$ & $\ldots$ & $\ldots$ & $\ldots$ & $\mathrm{VV}$ \\
\hline UGC 059 & $\ldots$ & $15.44 \pm 0.01$ & $\ldots$ & $\ldots$ & & $\ldots$ & & & $\ldots$ & & $\ldots$ & 3.19 & $\ldots$ & $\ldots$ & $\mathrm{EE}$ \\
\hline IC 0653 & $96 \pm 0.15$ & $17.13=$ & $0.83 \pm 0$ & $18.12 \pm 0.06$ & & $73 \pm 0.06$ & 35.55 & 35.70 & 14.18 & & $\ldots$ & $\ldots$ & $\ldots$ & $\ldots$ & Ef \\
\hline & \pm 0.13 & 12 & $14+0$ & $21.14 \pm 0.19$ & & 1. & 35.16 & 35 & 9.06 & 10 & $\ldots$ & $\ldots$ & $\ldots$ & $\ldots$ & $\mathrm{EE}$ \\
\hline 6011 & $20 \pm 0.02$ & .02 & $0.33 \pm 0.03$ & $17.34 \pm 0.02$ & 17. & $0.30 \pm 0.02$ & 35.85 & 35.81 & 10.96 & 10 & $\ldots$ & $\ldots$ & $\ldots$ & $\ldots$ & $\mathrm{EE}$ \\
\hline NGC 3440 & $15.55 \pm 0.01$ & $.14 \pm 0.01$ & $0.41 \pm 0.01$ & $15.64 \pm 0.01$ & $15.27 \pm 0.01$ & $0.37 \pm 0.01$ & 35.69 & 35.68 & 11.58 & 12.05 & 2.62 & 2.88 & $\ldots$ & $\ldots$ & $\mathrm{EE}$ \\
\hline NGC 3445 & $14.13 \pm 0.01$ & $3.96 \pm 0.01$ & $0.17 \pm 0.01$ & $14.18 \pm 0.01$ & $14.01 \pm 0.01$ & $0.18 \pm 0.01$ & 36.30 & 36.19 & 16.87 & 16.94 & $\cdots$ & $\cdots$ & $\cdots$ & $\ldots$ & $\mathrm{Vf}$ \\
\hline NGC 3458 & $19.43 \pm 0.02$ & $.65 \pm 0.02$ & $1.78 \pm 0.02$ & $19.52 \pm 0.05$ & $17.85 \pm 0.01$ & $1.67 \pm 0.05$ & 34.10 & 34.63 & $\ldots$ & 10.37 & $\ldots$ & $\ldots$ & $\ldots$ & $\ldots$ & $\mathrm{VV}$ \\
\hline UGC 06039 & $18.20 \pm 0.01$ & $17.73 \pm 0.01$ & $0.47 \pm 0.01$ & $18.28 \pm 0.02$ & $17.88 \pm 0.01$ & $0.40 \pm 0.02$ & 34.62 & 34.63 & 11.60 & 12.52 & $\ldots$ & 2.57 & $\ldots$ & $\ldots$ & $? \mathrm{~F}$ \\
\hline NGC 3475 & $\cdots$ & $16.71 \pm 0.01$ & $\ldots$ & $\ldots$ & $16.88 \pm 0.02$ & $\cdots$ & 0.00 & 36.02 & $\ldots$ & 18.03 & $\ldots$ & $\ldots$ & $\ldots$ & $\ldots$ & Er \\
\hline NGC 3470 & $.38 \pm 0.01$ & $16.09 \pm 0.01$ & $0.29 \pm 0$ & $16.74 \pm 0.02$ & $16.40 \pm 0.01$ & $0.35 \pm 0.02$ & 36.36 & 36.30 & 22.20 & 20.25 & 3.37 & $\ldots$ & $\ldots$ & $\ldots$ & $\mathrm{EE}$ \\
\hline & $.64 \pm 0.02$ & $14.97 \pm 0.01$ & $1.66 \pm 0$ & $16.67 \pm 0.04$ & $15.05 \pm 0.01$ & $1.62 \pm$ & 34.44 & & & & $\ldots$ & $\ldots$ & $\ldots$ & $\ldots$ & $\mathrm{VV}$ \\
\hline & $.52 \pm 0.01$ & $12.37 \pm 0.01$ & $0.15 \pm 0.01$ & $12.56 \pm 0.01$ & & $0.16 \pm 0.01$ & 36.06 & 35.94 & 76.19 & 70.04 & 3.25 & 3.39 & 3.09 & 3.19 & EEh,VF \\
\hline UGC 06102 & $16.56 \pm 0.01$ & $16.40 \pm 0.01$ & $0.17 \pm 0.01$ & $16.72 \pm 0.03$ & $16.60 \pm 0.02$ & $0.12 \pm 0.03$ & 34.46 & 34.35 & 11.92 & 13.11 & $\ldots$ & $\ldots$ & $\ldots$ & $\ldots$ & ?f \\
\hline
\end{tabular}


Table 3-Continued

\begin{tabular}{|c|c|c|c|c|c|c|c|c|c|c|c|c|c|c|c|}
\hline \multirow{2}{*}{$\begin{array}{c}\text { Object Name } \\
\text { (1) }\end{array}$} & \multicolumn{3}{|c|}{ Asymptotic magnitudes and color } & \multicolumn{3}{|c|}{ D25 magnitudes and color } & \multicolumn{2}{|c|}{$\log L$} & \multicolumn{2}{|c|}{ Effective Radii } & \multicolumn{2}{|c|}{ C31 } & \multicolumn{2}{|c|}{$\mathrm{C} 42$} & \multirow{2}{*}{$\begin{array}{r}\text { UV } \\
\text { profile } \\
(16)\end{array}$} \\
\hline & $\begin{array}{c}\text { FUV } \\
(\mathrm{mag}) \\
(2)\end{array}$ & $\begin{array}{c}\text { NUV } \\
(\mathrm{mag}) \\
(3)\end{array}$ & $\begin{array}{c}\text { FUV-NUV } \\
(\mathrm{mag}) \\
(4)\end{array}$ & $\begin{array}{c}\text { FUV } \\
(\mathrm{mag}) \\
(5)\end{array}$ & $\begin{array}{c}\text { NUV } \\
(\mathrm{mag}) \\
(6)\end{array}$ & $\begin{array}{l}\text { FUV-NUV } \\
(\mathrm{mag}) \\
(7)\end{array}$ & $\begin{array}{c}\text { FUV } \\
(\mathrm{W}) \\
(8)\end{array}$ & $\begin{array}{c}\text { NUV } \\
(\mathrm{W}) \\
(9)\end{array}$ & $\begin{array}{c}\text { FUV } \\
(\operatorname{arcsec}) \\
(10)\end{array}$ & $\begin{array}{c}\text { NUV } \\
(\operatorname{arcsec}) \\
(11)\end{array}$ & $\begin{array}{l}\text { FUV } \\
(12)\end{array}$ & $\begin{array}{l}\text { NUV } \\
(13)\end{array}$ & $\begin{array}{l}\text { FUV } \\
(14)\end{array}$ & $\begin{array}{l}\text { NUV } \\
(15)\end{array}$ & \\
\hline NGC 3521 & $3.03 \pm 0.01$ & $12.27 \pm 0.01$ & $0.76 \pm 0.01$ & $3.09 \pm 0.01$ & $12.33 \pm 0.01$ & $0.75 \pm 0.01$ & 35.63 & 35.76 & 89.55 & 73.55 & 2.51 & 2.88 & 2.40 & 2.78 & ER \\
\hline UGC 06151 & $\cdots$ & $15.87 \pm 0.02$ & $\cdots$ & $\cdots$ & $16.21 \pm 0.01$ & $\cdots$ & 0.00 & 35.03 & $\cdots$ & 30.19 & $\cdots$ & 2.61 & $\cdots$ & 2.75 & $\mathrm{EE}$ \\
\hline NGC 3522 & $\cdots$ & $17.40 \pm 0.06$ & $\cdots$ & $\cdots$ & $17.77 \pm 0.02$ & $\cdots$ & 0.00 & 34.35 & $\cdots$ & 13.43 & $\cdots$ & $\cdots$ & $\cdots$ & $\cdots$ & Er \\
\hline IC 0671 & $17.22 \pm 0.02$ & $16.79 \pm 0.01$ & $0.42 \pm 0.02$ & $17.38 \pm 0.03$ & $16.94 \pm 0.01$ & $0.45 \pm 0.03$ & 36.50 & 36.50 & 21.31 & 19.65 & 2.05 & 2.20 & 2.05 & $\ldots$ & Ed \\
\hline UGC 06181 & $\ldots$ & $15.92 \pm 0.01$ & $\ldots$ & $\ldots$ & $16.16 \pm 0.01$ & $\ldots$ & 0.00 & 34.90 & $\ldots$ & 16.00 & $\ldots$ & $\ldots$ & $\ldots$ & $\ldots$ & $? \mathrm{~F}$ \\
\hline NGC 3539 & $\ldots$ & $17.89 \pm 0.08$ & $\ldots$ & $\ldots$ & $19.39 \pm 0.04$ & $\ldots$ & 0.00 & 35.90 & $\ldots$ & 25.51 & $\ldots$ & 3.43 & $\ldots$ & 3.25 & Er \\
\hline IC 0673 & $16.25 \pm 0.02$ & $15.79 \pm 0.01$ & $0.46 \pm 0.02$ & $17.00 \pm 0.02$ & $16.49 \pm 0.01$ & $0.51 \pm 0.03$ & 35.92 & 35.93 & 30.75 & 28.82 & 3.87 & 4.57 & 3.58 & 4.03 & $\mathrm{Er}$ \\
\hline PGC 33931 & $\cdots$ & $18.81 \pm 0.07$ & $\ldots$ & $\ldots$ & $19.18 \pm 0.07$ & $\ldots$ & 0.00 & 35.58 & $\ldots$ & 15.86 & $\ldots$ & $\ldots$ & $\ldots$ & $\ldots$ & VV \\
\hline NGC 3550 & $\ldots$ & $18.11 \pm 0.07$ & $\ldots$ & $\ldots$ & $18.57 \pm 0.05$ & $\ldots$ & 0.00 & 35.87 & $\ldots$ & 16.68 & $\ldots$ & $\ldots$ & $\ldots$ & $\ldots$ & VV \\
\hline NGC 3620 & $16.05 \pm 0.29$ & $14.32 \pm 0.11$ & $1.73 \pm 0.31$ & $16.19 \pm 0.20$ & $14.90 \pm 0.05$ & $1.29 \pm 0.20$ & 35.12 & 35.64 & 24.93 & 40 & 2.43 & 3.14 & 2.20 & $\ldots$ & $\mathrm{EE}$ \\
\hline NGC 3621 & $.69 \pm 0.05$ & $11.30 \pm 0.04$ & $0.39 \pm 0.06$ & $11.97 \pm 0.01$ & $11.51 \pm 0.01$ & $0.46 \pm 0.01$ & 36.09 & 36.07 & 124.64 & 107.82 & 3.77 & 3.88 & 3.43 & 3.55 & $\mathrm{EE}$ \\
\hline UGC 06329 & $.38 \pm 0.02$ & $16.18 \pm 0.01$ & $0.19 \pm 0.02$ & $16.54 \pm 0.02$ & $16.33 \pm 0.01$ & $0.21 \pm 0.02$ & 36.44 & 36.34 & 16.68 & 15.97 & $\ldots$ & $\ldots$ & $\ldots$ & $\ldots$ & $? \mathrm{~F}$ \\
\hline UGC 06331 & $.65 \pm 0.04$ & $16.96 \pm 0.05$ & $0.70 \pm 0.07$ & $18.22 \pm 0.03$ & $17.57 \pm 0.01$ & $0.65 \pm 0.03$ & 35.75 & 35.85 & 13.96 & 14.43 & 2.74 & 2.81 & 2.74 & $\ldots$ & $\mathrm{EE}$ \\
\hline NGC 3627 & $12.70 \pm 0.01$ & $11.94 \pm 0.01$ & $0.76 \pm 0.01$ & $12.72 \pm 0.01$ & $11.96 \pm 0.01$ & $0.76 \pm 0.01$ & 35.77 & 35.90 & 55.98 & 54.27 & 2.13 & 2.24 & 1.97 & 2.12 & VFh \\
\hline NGC 3630 & $18.75 \pm 0.04$ & $17.07 \pm 0.01$ & $1.68 \pm 0.04$ & $\ldots$ & $\ldots$ & $\ldots$ & 34.10 & 34.60 & 12.13 & 12.02 & $\ldots$ & $\ldots$ & $\ldots$ & $\ldots$ & $\mathrm{VV}$ \\
\hline NGC 3628 & $\ldots$ & $13.41 \pm 0.01$ & $\ldots$ & $\ldots$ & $13.67 \pm 0.01$ & $\ldots$ & 0.00 & 35.63 & $\ldots$ & 120.23 & $\ldots$ & 2.72 & $\ldots$ & 2.71 & EEh \\
\hline NGC 3633 & $17.75 \pm 0.03$ & $16.83 \pm 0.01$ & $0.91 \pm 0.03$ & $17.99 \pm 0.03$ & $17.10 \pm 0.01$ & $0.89 \pm 0.04$ & 34.98 & 35.17 & 10.33 & 10.20 & $\ldots$ & $\ldots$ & $\ldots$ & $\ldots$ & Ef \\
\hline UGC 06359 & $\ldots$ & $18.51 \pm 0.36$ & $\ldots$ & $\ldots$ & $18.98 \pm 0.09$ & $\ldots$ & 0.00 & 35.39 & $\ldots$ & 13.44 & $\ldots$ & $\ldots$ & $\ldots$ & $\ldots$ & VV \\
\hline NGC 3640 & $17.96 \pm 0.04$ & $15.52 \pm 0.02$ & $2.44 \pm 0.04$ & $\ldots$ & $15.79 \pm 0.01$ & $\ldots$ & 34.61 & 35.41 & 15.74 & 42.08 & $\ldots$ & 6.71 & $\ldots$ & $\cdots$ & VV \\
\hline NGC 3641 & $19.42 \pm 0.09$ & $17.51 \pm 0.06$ & $1.91 \pm 0.11$ & $\ldots$ & $18.05 \pm 0.03$ & $\ldots$ & 33.97 & 34.56 & $\ldots$ & 21.09 & $\ldots$ & $\ldots$ & $\ldots$ & $\ldots$ & VV \\
\hline NGC 3644 & $17.39 \pm 0.01$ & $16.78 \pm 0.01$ & $0.61 \pm 0.02$ & $17.48 \pm 0.04$ & $16.91 \pm 0.01$ & $0.57 \pm 0.04$ & 36.00 & 36.07 & 13.74 & 13.35 & $\ldots$ & $\ldots$ & $\ldots$ & $\ldots$ & $\mathrm{EE}$ \\
\hline NGC 3646 & $\ldots$ & $13.76 \pm 0.01$ & $\ldots$ & $\ldots$ & $13.81 \pm 0.01$ & $\ldots$ & 0.00 & 36.84 & $\ldots$ & 47.75 & $\ldots$ & 1.65 & $\ldots$ & 1.44 & $\mathrm{EF}$ \\
\hline NGC 3649 & $\ldots$ & $17.17 \pm 0.03$ & $\ldots$ & $\ldots$ & $17.27 \pm 0.02$ & $\ldots$ & 0.00 & 35.61 & $\ldots$ & $\ldots$ & $\ldots$ & $\ldots$ & $\ldots$ & $\ldots$ & VV \\
\hline UGC 06387 & $17.06 \pm 0.01$ & $16.45 \pm 0.01$ & $0.61 \pm 0.02$ & $17.21 \pm 0.01$ & $16.74 \pm 0.01$ & $0.47 \pm 0.02$ & 34.85 & 34.92 & 6.66 & 8.34 & $\ldots$ & $\ldots$ & $\ldots$ & $\ldots$ & $? \mathrm{~F}$ \\
\hline NGC 3662 & $16.28 \pm 0.02$ & $15.49 \pm 0.01$ & $0.79 \pm 0.02$ & $16.40 \pm 0.02$ & $15.60 \pm 0.01$ & $0.80 \pm 0.03$ & 36.23 & 36.37 & 16.66 & 15.76 & 1.74 & 1.87 & 1.54 & 1.74 & ED \\
\hline UGC 06435 & $\ldots$ & $18.27 \pm 0.08$ & $\ldots$ & $\ldots$ & $18.61 \pm 0.04$ & $\ldots$ & 0.00 & 35.52 & $\ldots$ & 15.62 & $\ldots$ & $\ldots$ & $\ldots$ & $\ldots$ & VV \\
\hline VII Zw 403 & $14.96 \pm 0.01$ & $15.00 \pm 0.01$ & $-0.04 \pm 0.02$ & $15.00 \pm 0.01$ & $15.06 \pm 0.01$ & $-0.07 \pm 0.01$ & 34.31 & 34.12 & $\ldots$ & $\ldots$ & $\ldots$ & $\ldots$ & $\ldots$ & $\ldots$ & $\mathrm{VF}$ \\
\hline NGC 3705 & $\ldots$ & $13.76 \pm 0.01$ & $\ldots$ & $\ldots$ & $13.87 \pm 0.01$ & $\ldots$ & 0.00 & 35.62 & $\ldots$ & 45.02 & $\ldots$ & 2.61 & $\ldots$ & 2.55 & $\mathrm{EE}$ \\
\hline UGC 06519 & $18.28 \pm 0.01$ & $17.57 \pm 0.03$ & $0.71 \pm 0.03$ & $19.09 \pm 0.04$ & $18.94 \pm 0.02$ & $0.15 \pm 0.04$ & 35.46 & 35.57 & 9.96 & 14.72 & 2.80 & 2.95 & 2.79 & 2.98 & $? \mathrm{~F}$ \\
\hline IC 0716 & $18.28 \pm 0.06$ & $17.69 \pm 0.01$ & $0.59 \pm 0.06$ & $18.54 \pm 0.04$ & $17.99 \pm 0.02$ & $0.56 \pm 0.04$ & 35.40 & 35.46 & 12.21 & 12.60 & 2.51 & 2.48 & 2.47 & 2.46 & $? \mathrm{~F}$ \\
\hline NGC 3816 & $20.17 \pm 0.15$ & $17.89 \pm 0.02$ & $2.28 \pm 0.15$ & $\ldots$ & $18.09 \pm 0.03$ & $\ldots$ & 34.71 & 35.45 & $\ldots$ & 16.85 & $\ldots$ & $\ldots$ & $\ldots$ & $\ldots$ & EV \\
\hline NGC 3821 & $17.91 \pm 0.37$ & $17.30 \pm 0.30$ & $0.61 \pm 0.47$ & $18.12 \pm 0.04$ & $17.59 \pm 0.02$ & $0.53 \pm 0.04$ & 35.62 & 35.69 & 29.77 & 29.19 & 2.16 & 2.52 & 2.36 & $\ldots$ & $\ldots$ \\
\hline
\end{tabular}


Table 3-Continued

\begin{tabular}{|c|c|c|c|c|c|c|c|c|c|c|c|c|c|c|c|}
\hline \multirow{2}{*}{$\begin{array}{c}\text { Object Name } \\
\text { (1) }\end{array}$} & \multicolumn{3}{|c|}{ Asymptotic magnitudes and color } & \multicolumn{3}{|c|}{ D25 magnitudes and color } & \multicolumn{2}{|c|}{$\log L$} & \multicolumn{2}{|c|}{ Effective Radii } & \multicolumn{2}{|c|}{ C31 } & \multicolumn{2}{|c|}{$\mathrm{C} 42$} & \multirow{2}{*}{$\begin{array}{r}\text { UV } \\
\text { profile } \\
(16)\end{array}$} \\
\hline & $\begin{array}{l}\text { FUV } \\
\text { (mag) } \\
(2)\end{array}$ & $\begin{array}{c}\text { NUV } \\
(\mathrm{mag}) \\
(3)\end{array}$ & $\begin{array}{c}\text { FUV-NUV } \\
\text { (mag) } \\
(4)\end{array}$ & $\begin{array}{c}\text { FUV } \\
(\mathrm{mag}) \\
(5)\end{array}$ & $\begin{array}{c}\text { NUV } \\
(\mathrm{mag}) \\
(6)\end{array}$ & $\begin{array}{c}\text { FUV-NUV } \\
\text { (mag) } \\
(7)\end{array}$ & $\begin{array}{c}\text { FUV } \\
(\mathrm{W}) \\
(8)\end{array}$ & $\begin{array}{l}\text { NUV } \\
(\mathrm{W}) \\
(9)\end{array}$ & $\begin{array}{c}\text { FUV } \\
\text { (arcsec) } \\
(10)\end{array}$ & $\begin{array}{c}\text { NUV } \\
(\operatorname{arcsec}) \\
(11)\end{array}$ & $\begin{array}{l}\text { FUV } \\
(12)\end{array}$ & $\begin{array}{l}\text { NUV } \\
(13)\end{array}$ & $\begin{array}{l}\text { FUV } \\
(14)\end{array}$ & $\begin{array}{l}\text { NUV } \\
(15)\end{array}$ & \\
\hline CGCG 097-068 & $16.67 \pm 0.01$ & $16.43 \pm 0.01$ & $0.24 \pm 0.02$ & $17.09 \pm 0.02$ & $16.77 \pm 0.01$ & $0.31 \pm 0.03$ & 36.14 & 36.06 & 18.11 & 15.75 & 2.55 & & & 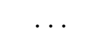 & $\mathrm{EE}$ \\
\hline UGC 06683 & $19.86 \pm 0.14$ & $19.23 \pm 0.06$ & $0.63 \pm 0.15$ & $20.31 \pm 0.08$ & $19.55 \pm 0.03$ & $0.76 \pm 0.09$ & 35.07 & 35.14 & 8.41 & 7.40 & $\cdots$ & $\cdots$ & $\cdots$ & $\cdots$ & $\mathrm{EE}$ \\
\hline IC 2951 & $20.07 \pm 0.08$ & $18.89 \pm 0.04$ & $1.18 \pm 0.09$ & $19.97 \pm 0.13$ & $19.00 \pm 0.05$ & $0.98 \pm 0.14$ & 34.80 & 35.10 & $\cdots$ & 10.95 & $\cdots$ & $\cdots$ & $\cdots$ & $\cdots$ & VV \\
\hline UGC 06697 & $14.92 \pm 0.01$ & $14.92 \pm 0.01$ & $0.00 \pm 0.01$ & $15.21 \pm 0.01$ & $15.19 \pm 0.01$ & $0.02 \pm 0.01$ & 36.89 & 36.71 & 12.53 & 12.32 & 3.13 & 3.00 & 3.04 & 2.94 & $\mathrm{EE}$ \\
\hline NGC 3844 & $20.27 \pm 0.13$ & $18.81 \pm 0.02$ & $1.46 \pm 0.13$ & $20.37 \pm 0.09$ & $19.05 \pm 0.02$ & $1.32 \pm 0.09$ & 34.81 & 35.22 & 5.90 & 7.32 & $\cdots$ & $\cdots$ & $\cdots$ & $\cdots$ & $\mathrm{EE}$ \\
\hline NGC 3842 & $17.97 \pm 0.08$ & $17.32 \pm 0.07$ & $0.64 \pm 0.11$ & $18.25 \pm 0.04$ & $17.71 \pm 0.02$ & $0.53 \pm 0.05$ & 35.67 & 35.76 & 10.71 & 18.41 & $\ldots$ & $\ldots$ & $\ldots$ & $\ldots$ & VV \\
\hline UGC 06719 & $17.23 \pm 0.05$ & $16.92 \pm 0.03$ & $0.31 \pm 0.06$ & $17.45 \pm 0.03$ & $17.13 \pm 0.01$ & $0.31 \pm 0.03$ & 36.00 & 35.95 & 15.60 & 9 & $\ldots$ & $\ldots$ & $\ldots$ & $\ldots$ & $\mathrm{EF}$ \\
\hline NGC 3861 & $15.72 \pm 0.01$ & $15.44 \pm 0.01$ & $0.28 \pm 0.01$ & $15.86 \pm 0.01$ & $15.58 \pm 0.01$ & $0.28 \pm 0.01$ & 36.57 & 36.50 & 31.84 & 31.17 & 2.23 & 2.30 & 2.10 & 2.19 & $\mathrm{EFh}$ \\
\hline UGC 06725 & $18.67 \pm 0.08$ & $17.37 \pm 0.03$ & $1.30 \pm 0.08$ & $\cdots$ & $17.46 \pm 0.02$ & $\ldots$ & 35.47 & 35.81 & $\ldots$ & $\ldots$ & $\ldots$ & $\ldots$ & $\ldots$ & $\ldots$ & $\mathrm{EV}$ \\
\hline ESO 440-G004 & $.25 \pm 0.02$ & $14.89 \pm 0.01$ & $0.36 \pm 0.03$ & $15.72 \pm 0.01$ & $15.31 \pm 0.01$ & $0.41 \pm 0.02$ & 35.56 & 35.53 & 33.41 & 31.90 & 3.09 & 3.03 & 3.17 & 3.04 & $\mathrm{EE}$ \\
\hline UGC 06736 & $16.54 \pm 0.02$ & $16.28 \pm 0.01$ & $0.26 \pm 0.02$ & $16.80 \pm 0.01$ & $16.54 \pm 0.01$ & $0.26 \pm 0.02$ & 36.18 & 36.11 & 11.66 & 11.96 & 2.42 & 2.35 & 2.48 & 2.40 & $? \mathrm{~F}$ \\
\hline NGC 3885 & $17.01 \pm 0.05$ & $15.94 \pm 0.01$ & $1.07 \pm 0.06$ & $17.17 \pm 0.03$ & $16.08 \pm 0.01$ & $1.09 \pm 0.03$ & 34.86 & 35.11 & 10.58 & 11.29 & $\ldots$ & $\ldots$ & $\ldots$ & $\ldots$ & $\mathrm{EV}$ \\
\hline UGCA 247 & $14.42 \pm 0.02$ & $14.06 \pm 0.01$ & $0.36 \pm 0.02$ & $14.73 \pm 0.01$ & $14.35 \pm 0.01$ & $0.38 \pm 0.01$ & 35.90 & 35.86 & 46.28 & 44.82 & 2.42 & 2.50 & 2.50 & 2.53 & $\mathrm{EE}$ \\
\hline NGC 3938 & $\ldots$ & $12.50 \pm 0.01$ & $\cdots$ & $\ldots$ & $12.56 \pm 0.01$ & $\ldots$ & 0.00 & 35.93 & $\ldots$ & 70.22 & $\ldots$ & 2.64 & $\ldots$ & 2.61 & $\mathrm{EE}$ \\
\hline UGC 06879 & $16.42 \pm 0.01$ & $16.13 \pm 0.01$ & $0.28 \pm 0.02$ & $16.53 \pm 0.03$ & $16.27 \pm 0.02$ & $0.26 \pm 0.03$ & 35.43 & 35.37 & 15.78 & 15.57 & 2.17 & 2.33 & 2.12 & 2.30 & $? \mathrm{~F}$ \\
\hline UGC 06934 & $17.51 \pm 0.05$ & $17.10 \pm 0.03$ & $0.41 \pm 0.06$ & $17.69 \pm 0.03$ & $17.31 \pm 0.02$ & $0.38 \pm 0.04$ & 35.73 & 35.72 & 11.88 & 11.91 & 2.26 & 2.47 & 2.23 & 2.44 & $\mathrm{EF}$ \\
\hline UGC 06970 & $\ldots$ & $15.73 \pm 0.01$ & $\ldots$ & $\ldots$ & $15.81 \pm 0.02$ & $\ldots$ & 0.00 & 35.13 & $\ldots$ & 17.33 & $\ldots$ & 2.08 & $\ldots$ & 2.11 & EFn \\
\hline IC 0754 & $\ldots$ & $17.94 \pm 0.12$ & $\ldots$ & $\ldots$ & $18.05 \pm 0.10$ & $\ldots$ & 0.00 & 35.46 & $\ldots$ & $\ldots$ & $\ldots$ & $\ldots$ & $\ldots$ & $\ldots$ & $\ldots$ \\
\hline NGC 4030 & $\ldots$ & $12.95 \pm 0.01$ & $\ldots$ & $\ldots$ & $12.98 \pm 0.01$ & $\ldots$ & 0.00 & 36.23 & $\ldots$ & 37.82 & $\ldots$ & 3.11 & $\ldots$ & 3.02 & $\mathrm{EE}$ \\
\hline UGC 07000 & $\ldots$ & $15.16 \pm 0.01$ & $\ldots$ & $\ldots$ & $15.23 \pm 0.02$ & $\ldots$ & 0.00 & 35.36 & $\ldots$ & 13.64 & $\ldots$ & $\ldots$ & $\ldots$ & $\ldots$ & $\mathrm{EF}$ \\
\hline NGC 4038 & $12.82 \pm 0.01$ & $12.47 \pm 0.01$ & $0.35 \pm 0.01$ & $12.83 \pm 0.01$ & $12.47 \pm 0.01$ & $0.35 \pm 0.01$ & 36.50 & 36.46 & 31.16 & 30.91 & 1.63 & 1.68 & 1.34 & 1.46 & ERn \\
\hline NGC 4039 & $14.46 \pm 0.01$ & $13.99 \pm 0.01$ & $0.47 \pm 0.01$ & $14.61 \pm 0.01$ & $14.12 \pm 0.01$ & $0.50 \pm 0.01$ & 35.84 & 35.86 & 26.36 & 25.88 & 2.99 & 3.20 & 3.15 & 3.20 & ERn \\
\hline UGC 07011 & $\cdots$ & $17.69 \pm 0.09$ & $\cdots$ & $\cdots$ & $18.19 \pm 0.06$ & $\ldots$ & 0.00 & 35.57 & $\ldots$ & 10.62 & $\cdots$ & 2.53 & $\cdots$ & $\ldots$ & $\ldots$ \\
\hline NGC $4108 \mathrm{~A}$ & $16.14 \pm 0.01$ & $15.74 \pm 0.01$ & $0.40 \pm 0.02$ & $16.24 \pm 0.01$ & $15.87 \pm 0.01$ & $0.38 \pm 0.01$ & 35.59 & 35.58 & 11.36 & 11.63 & $\cdots$ & $\ldots$ & $\ldots$ & $\ldots$ & $\mathrm{Ef}$ \\
\hline UGC 07089 & $\ldots$ & $15.53 \pm 0.01$ & $\ldots$ & $\cdots$ & $15.66 \pm 0.01$ & $\ldots$ & 0.00 & 34.84 & $\ldots$ & 22.29 & $\ldots$ & 2.27 & $\ldots$ & 2.30 & $\mathrm{EF}$ \\
\hline NGC 4108 & $14.89 \pm 0.01$ & $14.51 \pm 0.01$ & $0.38 \pm 0.01$ & $14.94 \pm 0.01$ & $14.54 \pm 0.01$ & $0.40 \pm 0.01$ & 36.18 & 36.15 & 15.78 & 14.39 & $\cdots$ & $\ldots$ & $\ldots$ & $\ldots$ & $\mathrm{EE}$ \\
\hline NGC 4109 & $\ldots$ & $16.68 \pm 0.01$ & $\cdots$ & $\ldots$ & $16.72 \pm 0.01$ & $\ldots$ & 0.00 & 36.12 & $\cdots$ & $\ldots$ & $\ldots$ & $\ldots$ & $\ldots$ & $\ldots$ & $\ldots$ \\
\hline NGC 4111 & $\ldots$ & $15.43 \pm 0.01$ & $\ldots$ & $\ldots$ & $15.51 \pm 0.01$ & $\ldots$ & 0.00 & 34.91 & $\ldots$ & 13.96 & $\ldots$ & 3.83 & $\ldots$ & 3.67 & ER \\
\hline NGC 4108B & $15.48 \pm 0.01$ & $15.22 \pm 0.01$ & $0.26 \pm 0.02$ & $15.59 \pm 0.01$ & $15.35 \pm 0.01$ & $0.24 \pm 0.01$ & 35.99 & 35.92 & 14.54 & 14.88 & $\cdots$ & $\ldots$ & $\ldots$ & $\ldots$ & $\mathrm{EE}$ \\
\hline NGC 4116 & $\ldots$ & $13.62 \pm 0.01$ & $\cdots$ & $\cdots$ & $13.73 \pm 0.01$ & $\ldots$ & 0.00 & 35.77 & $\ldots$ & 42.50 & $\ldots$ & 2.29 & $\ldots$ & 2.36 & EEn \\
\hline
\end{tabular}


Table 3-Continued

\begin{tabular}{|c|c|c|c|c|c|c|c|c|c|c|c|c|c|c|c|}
\hline \multirow{2}{*}{$\begin{array}{l}\text { Object Name } \\
\text { (1) }\end{array}$} & \multicolumn{3}{|c|}{ Asymptotic magnitudes and color } & \multicolumn{3}{|c|}{ D25 magnitudes and color } & \multicolumn{2}{|c|}{$\log L$} & \multicolumn{2}{|c|}{ Effective Radii } & \multicolumn{2}{|c|}{$\mathrm{C} 31$} & \multicolumn{2}{|c|}{$\mathrm{C} 42$} & \multirow{2}{*}{$\begin{array}{r}\text { UV } \\
\text { profile } \\
(16)\end{array}$} \\
\hline & $\begin{array}{c}\text { FUV } \\
(\mathrm{mag}) \\
(2)\end{array}$ & $\begin{array}{c}\text { NUV } \\
(\mathrm{mag}) \\
(3)\end{array}$ & $\begin{array}{l}\text { FUV-NUV } \\
\text { (mag) } \\
(4)\end{array}$ & $\begin{array}{l}\text { FUV } \\
(\mathrm{mag}) \\
(5)\end{array}$ & $\begin{array}{c}\text { NUV } \\
(\mathrm{mag}) \\
(6)\end{array}$ & $\begin{array}{c}\text { FUV-NUV } \\
(\mathrm{mag}) \\
(7)\end{array}$ & $\begin{array}{c}\text { FUV } \\
(\mathrm{W}) \\
(8)\end{array}$ & $\begin{array}{l}\text { NUV } \\
(\mathrm{W}) \\
(9)\end{array}$ & $\begin{array}{c}\text { FUV } \\
(\operatorname{arcsec}) \\
(10)\end{array}$ & $\begin{array}{c}\text { NUV } \\
(\operatorname{arcsec}) \\
(11)\end{array}$ & $\begin{array}{l}\text { FUV } \\
(12)\end{array}$ & $\begin{array}{l}\text { NUV } \\
(13)\end{array}$ & $\begin{array}{l}\text { FUV } \\
(14)\end{array}$ & $\begin{array}{l}\text { NUV } \\
(15)\end{array}$ & \\
\hline NGC 4117 & $\cdots$ & $17.30 \pm 0.02$ & $\cdots$ & $\cdots$ & $17.48 \pm 0.02$ & $\cdots$ & 0.00 & 34.26 & $\cdots$ & 13.31 & $\cdots$ & $\cdots$ & $\cdots$ & $\cdots$ & ER \\
\hline NGC 4125 & $\ldots$ & $15.06 \pm 0.01$ & $\ldots$ & $\ldots$ & $15.39 \pm 0.01$ & $\ldots$ & 0.00 & 35.40 & $\ldots$ & 59.57 & $\ldots$ & 5.99 & $\ldots$ & 4.77 & $\mathrm{VV}$ \\
\hline NGC 4136 & $13.61 \pm 0.01$ & $13.35 \pm 0.01$ & $0.26 \pm 0.01$ & $13.69 \pm 0.01$ & $13.42 \pm 0.01$ & $0.27 \pm 0.01$ & 35.57 & 35.50 & 52.27 & 50.32 & 2.32 & 2.32 & 2.30 & 2.31 & $\mathrm{EFh}$ \\
\hline NGC 4138 & $\ldots$ & $14.92 \pm 0.01$ & $\ldots$ & $\cdots$ & $14.95 \pm 0.01$ & $\cdots$ & 0.00 & 35.18 & $\cdots$ & 16.60 & $\cdots$ & 2.06 & $\cdots$ & 2.10 & Ef \\
\hline NGC 4150 & $17.82 \pm 0.03$ & $16.09 \pm 0.01$ & $1.72 \pm 0.03$ & $17.88 \pm 0.04$ & $16.18 \pm 0.01$ & $1.70 \pm 0.04$ & 34.08 & 34.60 & $\ldots$ & 13.66 & $\cdots$ & $\ldots$ & $\ldots$ & $\ldots$ & EEn \\
\hline VII Zw 173 & $\cdots$ & $15.99 \pm 0.01$ & $\ldots$ & $\ldots$ & $16.42 \pm 0.01$ & $\ldots$ & 0.00 & 35.37 & $\cdots$ & 13.40 & $\ldots$ & 2.34 & $\ldots$ & 2.31 & ?f \\
\hline UGC 07176 & $\ldots$ & $17.54 \pm 0.04$ & $\ldots$ & $\ldots$ & $17.80 \pm 0.02$ & $\ldots$ & 0.00 & 34.15 & $\cdots$ & 14.25 & $\ldots$ & 2.48 & $\ldots$ & 2.57 & ?F \\
\hline UGC 07178 & $\ldots$ & $16.25 \pm 0.01$ & $\ldots$ & $\ldots$ & $16.99 \pm 0.01$ & $\ldots$ & 0.00 & 34.84 & $\ldots$ & 33.80 & $\ldots$ & 2.96 & $\ldots$ & 2.93 & EEh \\
\hline NGC 4157 & $\ldots$ & $14.61 \pm 0.01$ & $\ldots$ & $\ldots$ & $14.74 \pm 0.01$ & $\ldots$ & 0.00 & 35.23 & $\ldots$ & 47.55 & $\ldots$ & 1.97 & $\ldots$ & 1.93 & $\mathrm{EF}$ \\
\hline IC 3033 & $\ldots$ & $16.54 \pm 0.02$ & $\ldots$ & $\ldots$ & $16.79 \pm 0.01$ & $\ldots$ & 0.00 & 35.15 & $\ldots$ & 15.89 & $\ldots$ & $\ldots$ & $\ldots$ & $\ldots$ & $\mathrm{EF}$ \\
\hline UGC 07184 & $\ldots$ & $16.13 \pm 0.01$ & $\ldots$ & $\ldots$ & $16.23 \pm 0.01$ & $\ldots$ & 0.00 & 35.28 & $\ldots$ & 14.00 & $\ldots$ & 2.33 & $\ldots$ & $\ldots$ & $\mathrm{EF}$ \\
\hline UGC 07196 & $\ldots$ & $18.19 \pm 0.10$ & $\ldots$ & $\ldots$ & $18.59 \pm 0.03$ & $\ldots$ & 0.00 & 35.43 & $\ldots$ & 11.92 & $\ldots$ & 3.01 & $\ldots$ & $\ldots$ & $\mathrm{Ef}$ \\
\hline NGC 4165 & $\ldots$ & $16.72 \pm 0.02$ & $\ldots$ & $\ldots$ & $16.81 \pm 0.01$ & $\ldots$ & 0.00 & 35.08 & $\ldots$ & 14.17 & $\ldots$ & 2.00 & $\ldots$ & $\ldots$ & $\mathrm{ED}$ \\
\hline NGC $4192 \mathrm{~A}$ & $\ldots$ & $16.28 \pm 0.01$ & $\ldots$ & $\ldots$ & $16.63 \pm 0.01$ & $\ldots$ & 0.00 & 35.26 & $\ldots$ & 23.32 & $\ldots$ & 2.43 & $\ldots$ & 2.45 & $\mathrm{EF}$ \\
\hline NGC 4187 & $\ldots$ & $18.02 \pm 0.27$ & $\ldots$ & $\ldots$ & $18.65 \pm 0.04$ & $\ldots$ & 0.00 & 35.80 & $\ldots$ & 25.70 & $\ldots$ & $\ldots$ & $\ldots$ & $\ldots$ & ER \\
\hline NGC 4189 & $\ldots$ & $14.29 \pm 0.01$ & $\ldots$ & $\ldots$ & $14.33 \pm 0.01$ & $\ldots$ & 0.00 & 36.05 & $\ldots$ & 31.36 & $\ldots$ & 1.84 & $\ldots$ & 1.71 & $? \mathrm{Fn}$ \\
\hline MESSIER 098 & $\ldots$ & $13.45 \pm 0.01$ & $\ldots$ & $\ldots$ & $13.47 \pm 0.01$ & $\ldots$ & 0.00 & 35.84 & $\ldots$ & 70.70 & $\ldots$ & 2.07 & $\ldots$ & 1.91 & EFn \\
\hline NGC 4193 & $\ldots$ & $15.27 \pm 0.03$ & $\ldots$ & $\ldots$ & $15.51 \pm 0.01$ & $\ldots$ & 0.00 & 35.66 & $\ldots$ & 23.83 & $\ldots$ & 2.75 & $\ldots$ & 2.71 & $\mathrm{EF}$ \\
\hline NGC 4186 & $\ldots$ & $17.08 \pm 0.01$ & $\ldots$ & $\ldots$ & $17.20 \pm 0.02$ & $\ldots$ & 0.00 & 35.97 & $\ldots$ & 14.66 & $\ldots$ & $\ldots$ & $\ldots$ & $\ldots$ & $\mathrm{ED}$ \\
\hline UGC 07242 & $15.91 \pm 0.01$ & $15.45 \pm 0.01$ & $0.46 \pm 0.02$ & $15.99 \pm 0.01$ & $15.60 \pm 0.01$ & $0.38 \pm 0.01$ & 33.84 & 33.85 & 15.90 & 17.14 & 2.35 & 2.57 & $\ldots$ & 2.71 & $\mathrm{EF}$ \\
\hline UGC 07249 & $17.02 \pm 0.02$ & $16.78 \pm 0.02$ & $0.24 \pm 0.03$ & $17.21 \pm 0.02$ & $16.99 \pm 0.01$ & $0.23 \pm 0.02$ & 34.59 & 34.51 & 15.74 & 15.39 & 2.21 & 2.39 & 2.24 & 2.43 & $\mathrm{EF}$ \\
\hline IC 3059 & $16.90 \pm 0.01$ & $16.53 \pm 0.01$ & $0.38 \pm 0.01$ & $16.95 \pm 0.02$ & $16.61 \pm 0.01$ & $0.34 \pm 0.02$ & 34.64 & 34.61 & 22.52 & 23.76 & 2.09 & 2.10 & 1.98 & 2.03 & $\mathrm{EF}$ \\
\hline VCC 0132 & $17.80 \pm 0.05$ & $17.68 \pm 0.04$ & $0.11 \pm 0.06$ & $18.19 \pm 0.04$ & $18.17 \pm 0.03$ & $0.02 \pm 0.05$ & 34.28 & 34.15 & 19.48 & 20.75 & 2.73 & 3.04 & $\ldots$ & $\ldots$ & $\mathrm{EE}$ \\
\hline IC 3066 & $18.10 \pm 0.04$ & $17.56 \pm 0.02$ & $0.54 \pm 0.04$ & $18.33 \pm 0.03$ & $17.90 \pm 0.01$ & $0.42 \pm 0.03$ & 34.16 & 34.20 & 7.02 & 8.01 & $\ldots$ & $\ldots$ & $\ldots$ & $\ldots$ & $\mathrm{Ef}$ \\
\hline NGC 4206 & $15.20 \pm 0.01$ & $14.85 \pm 0.01$ & $0.35 \pm 0.01$ & $15.24 \pm 0.01$ & $14.89 \pm 0.01$ & $0.36 \pm 0.01$ & 35.32 & 35.28 & 33.97 & 33.80 & 1.89 & 2.02 & 1.79 & 1.98 & $\mathrm{EFh}$ \\
\hline IC 3073 & $20.27 \pm 0.32$ & $18.16 \pm 0.04$ & $2.11 \pm 0.32$ & $20.84 \pm 0.27$ & $18.79 \pm 0.04$ & $2.05 \pm 0.27$ & 33.29 & 33.96 & 17.05 & 18.28 & $\ldots$ & $\ldots$ & $\ldots$ & $\ldots$ & Er \\
\hline NGC 4216 & $15.71 \pm 0.01$ & $14.73 \pm 0.01$ & $0.98 \pm 0.01$ & $15.74 \pm 0.01$ & $14.80 \pm 0.01$ & $0.94 \pm 0.01$ & 35.11 & 35.33 & 50.03 & 50.37 & 2.15 & 2.47 & 2.07 & 2.45 & $\mathrm{EF}$ \\
\hline NGC 4222 & $16.75 \pm 0.01$ & $16.23 \pm 0.01$ & $0.52 \pm 0.01$ & $16.92 \pm 0.01$ & $16.44 \pm 0.01$ & $0.48 \pm 0.02$ & 34.70 & 34.73 & 17.81 & 19.53 & 2.96 & 2.90 & 2.89 & 2.90 & $\mathrm{Ef}$ \\
\hline NGC 4226 & $\ldots$ & $16.87 \pm 0.01$ & $\ldots$ & $\ldots$ & $16.97 \pm 0.01$ & $\ldots$ & 0.00 & 36.07 & $\ldots$ & 9.83 & $\ldots$ & $\ldots$ & $\ldots$ & $\ldots$ & ?f \\
\hline
\end{tabular}


Table 3-Continued

\begin{tabular}{|c|c|c|c|c|c|c|c|c|c|c|c|c|c|c|c|}
\hline \multirow{2}{*}{$\begin{array}{l}\text { Object Name } \\
\text { (1) }\end{array}$} & \multicolumn{3}{|c|}{ Asymptotic magnitudes and color } & \multicolumn{3}{|c|}{ D25 magnitudes and color } & \multicolumn{2}{|c|}{$\log L$} & \multicolumn{2}{|c|}{ Effective Radii } & \multicolumn{2}{|c|}{ C31 } & \multicolumn{2}{|c|}{$\mathrm{C} 42$} & \multirow{2}{*}{$\begin{array}{l}\mathrm{UV} \\
\text { profile } \\
(16)\end{array}$} \\
\hline & $\begin{array}{c}\text { FUV } \\
(\mathrm{mag}) \\
(2)\end{array}$ & $\begin{array}{c}\text { NUV } \\
(\mathrm{mag}) \\
(3)\end{array}$ & $\begin{array}{l}\text { FUV-NUV } \\
(\mathrm{mag}) \\
(4)\end{array}$ & $\begin{array}{c}\text { FUV } \\
(\mathrm{mag}) \\
(5)\end{array}$ & $\begin{array}{l}\mathrm{NUV} \\
(\mathrm{mag}) \\
(6)\end{array}$ & $\begin{array}{l}\text { FUV-NUV } \\
(\mathrm{mag}) \\
(7)\end{array}$ & $\begin{array}{l}\text { FUV } \\
(\mathrm{W}) \\
(8)\end{array}$ & $\begin{array}{c}\text { NUV } \\
(\mathrm{W}) \\
(9)\end{array}$ & $\begin{array}{c}\text { FUV } \\
\text { (arcsec) } \\
(10)\end{array}$ & $\begin{array}{c}\text { NUV } \\
\text { (arcsec) } \\
(11)\end{array}$ & $\begin{array}{l}\text { FUV } \\
(12)\end{array}$ & $\begin{array}{l}\text { NUV } \\
(13)\end{array}$ & $\begin{array}{l}\text { FUV } \\
(14)\end{array}$ & $\begin{array}{l}\text { NUV } \\
\text { (15) }\end{array}$ & \\
\hline NGC 4236 & $11.89 \pm 0.01$ & $11.69 \pm 0.01$ & $0.20 \pm 0.01$ & $11.92 \pm 0.01$ & $11.72 \pm 0.01$ & $0.20 \pm 0.01$ & 35.48 & 35.38 & 169.27 & 166.62 & 2.04 & 2.09 & 2.05 & 2.11 & $\mathrm{EF}$ \\
\hline UGC 07301 & $\ldots$ & $16.63 \pm 0.02$ & $\ldots$ & $\ldots$ & $17.27 \pm 0.01$ & $\ldots$ & 0.00 & 34.36 & $\ldots$ & 15.32 & $\ldots$ & 2.80 & $\ldots$ & 2.84 & Ef \\
\hline NGC 4231 & $\ldots$ & $16.02 \pm 0.01$ & $\ldots$ & $\ldots$ & $16.13 \pm 0.01$ & $\ldots$ & 0.00 & 36.42 & $\cdots$ & 11.66 & $\ldots$ & $\ldots$ & $\ldots$ & $\ldots$ & $\mathrm{EE}$ \\
\hline NGC 4232 & $\ldots$ & $16.44 \pm 0.01$ & $\ldots$ & $\ldots$ & $16.52 \pm 0.01$ & $\ldots$ & 0.00 & 36.24 & $\ldots$ & 13.46 & $\ldots$ & $\ldots$ & $\ldots$ & $\ldots$ & $? \mathrm{~F}$ \\
\hline UGC 07325 & $\cdots$ & $17.53 \pm 0.01$ & $\ldots$ & $\ldots$ & $17.76 \pm 0.02$ & $\ldots$ & 0.00 & 35.78 & $\cdots$ & 14.24 & $\cdots$ & 2.27 & $\cdots$ & 2.30 & $\mathrm{EF}$ \\
\hline NGC 4242 & $\ldots$ & $13.60 \pm 0.01$ & $\ldots$ & $\ldots$ & $13.66 \pm 0.01$ & $\ldots$ & 0.00 & 35.35 & $\ldots$ & 68.28 & $\ldots$ & 2.00 & $\ldots$ & 2.00 & $\mathrm{VF}$ \\
\hline NGC 4248 & .. & $15.97 \pm 0.01$ & $\ldots$ & $\ldots$ & $16.17 \pm 0.01$ & $\ldots$ & 0.00 & 34.09 & $\ldots$ & 20.27 & $\cdots$ & 4.61 & $\ldots$ & 4.10 & ER \\
\hline MESSIER 099 & $\cdots$ & $11.94 \pm 0.01$ & $\ldots$ & $\ldots$ & $11.98 \pm 0.01$ & $\ldots$ & 0.00 & 36.44 & $\cdots$ & 63.24 & $\cdots$ & 2.62 & $\ldots$ & 2.58 & $\mathrm{EF}$ \\
\hline MESSIER 106 & $\ldots$ & $11.45 \pm 0.01$ & $\ldots$ & $\ldots$ & $11.54 \pm 0.01$ & $\ldots$ & 0.00 & 35.89 & $\ldots$ & 125.52 & $\ldots$ & 3.73 & $\ldots$ & 3.53 & EEn \\
\hline NGC 4262 & $\ldots$ & $16.04 \pm 0.02$ & $\ldots$ & $\ldots$ & $16.53 \pm 0.01$ & $\ldots$ & 0.00 & 34.80 & $\ldots$ & 28 & $\ldots$ & $\ldots$ & $\ldots$ & $\ldots$ & $\mathrm{EV}$ \\
\hline NGC 4274 & $15.48 \pm 0.01$ & $14.64 \pm 0.01$ & $0.83 \pm 0.01$ & $15.52 \pm 0.01$ & $14.69 \pm 0.01$ & $0.83 \pm 0.01$ & 35.15 & 35.31 & 50.43 & 47 & 1.97 & 2.27 & 2.01 & 2.68 & EDn \\
\hline NGC 4278 & $15.87 \pm 0.02$ & $14.86 \pm 0.03$ & $1.02 \pm 0.03$ & $16.11 \pm 0.02$ & $15.18 \pm 0.01$ & $0.92 \pm 0.02$ & 35.00 & 35.23 & 30.88 & 47.59 & $\ldots$ & 8.98 & $\ldots$ & $\ldots$ & $\mathrm{VV}$ \\
\hline UGC 07387 & $17.91 \pm 0.03$ & $17.19 \pm 0.01$ & $0.72 \pm 0.04$ & $18.48 \pm 0.04$ & $17.87 \pm 0.01$ & $0.60 \pm 0.04$ & 34.23 & 34.34 & 14.75 & 16.09 & 2.23 & 2.25 & 2.16 & 2.22 & $\mathrm{EF}$ \\
\hline NGC 4283 & $18.82 \pm 0.14$ & $17.11 \pm 0.07$ & $1.71 \pm 0.16$ & $19.03 \pm 0.08$ & $17.47 \pm 0.02$ & $1.56 \pm 0.08$ & 33.83 & 34.34 & 12.23 & 18.77 & $\ldots$ & $\ldots$ & $\ldots$ & $\ldots$ & VV \\
\hline NGC 4286 & $17.23 \pm 0.01$ & $16.57 \pm 0.02$ & $0.66 \pm 0.03$ & $17.26 \pm 0.03$ & $16.77 \pm 0.01$ & $0.49 \pm 0.03$ & 34.16 & 34.25 & 11.84 & 14.33 & $\ldots$ & $\ldots$ & $\ldots$ & $\ldots$ & $? \mathrm{~F}$ \\
\hline NGC 4292 & $18.58 \pm 0.05$ & $17.05 \pm 0.03$ & $1.53 \pm 0.06$ & $\ldots$ & $17.25 \pm 0.02$ & $\ldots$ & 33.96 & 34.40 & $\ldots$ & 16.44 & $\ldots$ & $\ldots$ & $\ldots$ & $\ldots$ & VV \\
\hline NGC 4298 & $\ldots$ & $14.30 \pm 0.01$ & $\ldots$ & $\ldots$ & $14.35 \pm 0.01$ & $\ldots$ & 0.00 & 35.50 & $\ldots$ & 31.76 & $\ldots$ & 2.20 & $\ldots$ & 2.18 & ERn \\
\hline UGC 07411 & $\ldots$ & $18.72 \pm 0.10$ & $\ldots$ & $\ldots$ & $18.78 \pm 0.03$ & $\ldots$ & 0.00 & 33.73 & $\ldots$ & 9.10 & $\ldots$ & $\ldots$ & $\ldots$ & $\ldots$ & $\mathrm{EE}$ \\
\hline IC 0783 & $\ldots$ & $17.67 \pm 0.08$ & $\ldots$ & $\ldots$ & $18.19 \pm 0.03$ & $\ldots$ & 0.00 & 34.15 & $\ldots$ & 25.72 & $\ldots$ & 3.86 & $\ldots$ & $\ldots$ & $\mathrm{ER}$ \\
\hline UGC 07425 & $\ldots$ & $18.99 \pm 0.12$ & $\ldots$ & $\ldots$ & $19.33 \pm 0.08$ & $\ldots$ & 0.00 & 33.62 & $\ldots$ & 20.42 & $\ldots$ & 2.73 & $\ldots$ & $\ldots$ & $\mathrm{EE}$ \\
\hline NGC 4303 & $12.08 \pm 0.01$ & $11.75 \pm 0.01$ & $0.33 \pm 0.01$ & $12.13 \pm 0.01$ & $11.78 \pm 0.01$ & $0.34 \pm 0.01$ & 36.56 & 36.52 & 62.09 & 58.05 & 2.32 & 2.14 & 2.33 & 2.18 & Edn \\
\hline VCC 0530 & $\ldots$ & $17.60 \pm 0.02$ & $\ldots$ & $\ldots$ & $18.17 \pm 0.03$ & $\ldots$ & 0.00 & 34.18 & $\ldots$ & 21.23 & $\ldots$ & $\ldots$ & $\ldots$ & $\ldots$ & $\mathrm{ER}$ \\
\hline NGC 4310 & $17.68 \pm 0.02$ & $16.55 \pm 0.01$ & $1.13 \pm 0.02$ & $17.74 \pm 0.03$ & $16.67 \pm 0.01$ & $1.07 \pm 0.04$ & 34.27 & 34.55 & $\ldots$ & 10.05 & $\ldots$ & $\ldots$ & $\ldots$ & $\ldots$ & $\mathrm{EV}$ \\
\hline NGC 4301 & $14.55 \pm 0.01$ & $14.37 \pm 0.01$ & $0.18 \pm 0.01$ & $14.85 \pm 0.01$ & $14.64 \pm 0.01$ & $0.21 \pm 0.01$ & 35.58 & 35.47 & 22.17 & 22.00 & 2.34 & 2.40 & 2.44 & 2.51 & Edn \\
\hline NGC 4312 & $\ldots$ & $15.84 \pm 0.01$ & $\ldots$ & $\ldots$ & $16.05 \pm 0.01$ & $\ldots$ & 0.00 & 34.88 & $\ldots$ & 31.13 & $\ldots$ & 3.23 & $\ldots$ & 3.22 & $\mathrm{EE}$ \\
\hline NGC 4314 & $15.49 \pm 0.01$ & $14.56 \pm 0.01$ & $0.93 \pm 0.01$ & $15.52 \pm 0.02$ & $14.60 \pm 0.01$ & $0.92 \pm 0.02$ & 35.15 & 35.35 & $\ldots$ & $\ldots$ & $\ldots$ & $\ldots$ & $\ldots$ & $\ldots$ & $\mathrm{EV}$ \\
\hline NGC 4321 & $\ldots$ & $12.07 \pm 0.01$ & $\ldots$ & $\ldots$ & $12.10 \pm 0.01$ & $\ldots$ & 0.00 & 36.42 & $\ldots$ & 83.29 & $\ldots$ & 2.42 & $\ldots$ & 2.51 & VFn \\
\hline NGC 4323 & $\ldots$ & $17.72 \pm 0.02$ & $\ldots$ & $\ldots$ & $18.54 \pm 0.04$ & $\ldots$ & 0.00 & 34.13 & $\ldots$ & 28.06 & $\ldots$ & $\ldots$ & $\ldots$ & $\ldots$ & ER \\
\hline NGC 4328 & $\cdots$ & $17.62 \pm 0.05$ & $\ldots$ & $\ldots$ & $18.12 \pm 0.03$ & $\ldots$ & 0.00 & 34.17 & $\ldots$ & 24.96 & $\ldots$ & $\cdots$ & $\ldots$ & $\cdots$ & ER \\
\hline NGC 4344 & $16.39 \pm 0.01$ & $15.72 \pm 0.01$ & $0.67 \pm 0.01$ & $16.43 \pm 0.03$ & $15.80 \pm 0.01$ & $0.63 \pm 0.04$ & 34.84 & 34.93 & $\cdots$ & $\ldots$ & $\ldots$ & $\ldots$ & $\ldots$ & $\ldots$ & $\mathrm{ER}$ \\
\hline NGC 4371 & $17.46 \pm 0.04$ & $16.00 \pm 0.01$ & $1.46 \pm 0.04$ & $17.53 \pm 0.04$ & $16.13 \pm 0.01$ & $1.40 \pm 0.04$ & 34.41 & 34.82 & 21.06 & 26.87 & $\ldots$ & 5.52 & $\ldots$ & $\ldots$ & $\mathrm{VV}$ \\
\hline MESSIER 084 & $15.60 \pm 0.02$ & $14.32 \pm 0.01$ & $1.28 \pm 0.02$ & $15.74 \pm 0.02$ & $14.54 \pm 0.01$ & $1.20 \pm 0.02$ & 35.16 & 35.49 & 41.79 & 64.14 & 9.59 & 6.80 & $\ldots$ & 5.17 & VV \\
\hline
\end{tabular}


Table 3-Continued

\begin{tabular}{|c|c|c|c|c|c|c|c|c|c|c|c|c|c|c|c|}
\hline \multirow{2}{*}{$\begin{array}{l}\text { Object Name } \\
\text { (1) }\end{array}$} & \multicolumn{3}{|c|}{ Asymptotic magnitudes and color } & \multicolumn{3}{|c|}{ D25 magnitudes and color } & \multicolumn{2}{|c|}{$\log L$} & \multicolumn{2}{|c|}{ Effective Radii } & \multicolumn{2}{|c|}{ C31 } & \multicolumn{2}{|c|}{$\mathrm{C} 42$} & \multirow{2}{*}{$\begin{array}{c}\text { UV } \\
\text { profile } \\
(16)\end{array}$} \\
\hline & $\begin{array}{c}\text { FUV } \\
(\mathrm{mag}) \\
(2)\end{array}$ & $\begin{array}{c}\text { NUV } \\
(\mathrm{mag}) \\
(3)\end{array}$ & $\begin{array}{c}\text { FUV-NUV } \\
(\mathrm{mag}) \\
(4)\end{array}$ & $\begin{array}{c}\text { FUV } \\
(\mathrm{mag}) \\
(5)\end{array}$ & $\begin{array}{c}\text { NUV } \\
(\mathrm{mag}) \\
(6)\end{array}$ & $\begin{array}{c}\text { FUV-NUV } \\
(\mathrm{mag}) \\
(7)\end{array}$ & $\begin{array}{c}\text { FUV } \\
(\mathrm{W}) \\
(8)\end{array}$ & $\begin{array}{c}\text { NUV } \\
(\mathrm{W}) \\
(9)\end{array}$ & $\begin{array}{c}\text { FUV } \\
\text { (arcsec) } \\
(10)\end{array}$ & $\begin{array}{c}\text { NUV } \\
\text { (arcsec) } \\
(11)\end{array}$ & $\begin{array}{l}\text { FUV } \\
(12)\end{array}$ & $\begin{array}{l}\text { NUV } \\
(13)\end{array}$ & $\begin{array}{l}\text { FUV } \\
(14)\end{array}$ & $\begin{array}{l}\text { NUV } \\
(15)\end{array}$ & \\
\hline IC 3305 & $21.19 \pm 0.18$ & $18.30 \pm 0.02$ & $2.90 \pm 0.18$ & & $18.71 \pm 0.03$ & & 32.92 & 33.90 & 10.14 & 13.62 & $\cdots$ & 2.76 & & . & $\mathrm{EE}$ \\
\hline NGC 4379 & $.31 \pm 0.04$ & $16.78 \pm 0.01$ & $1.53 \pm 0.04$ & $8.34 \pm 0.06$ & $16.84 \pm 0.01$ & $1.50 \pm 0.06$ & 34.07 & 34.51 & $\cdots$ & 13.46 & $\cdots$ & $\cdots$ & $\cdots$ & $\cdots$ & $\mathrm{VV}$ \\
\hline IC 0787 & $.48 \pm 0.10$ & $18.81 \pm 0.05$ & $0.67 \pm 0.11$ & $19.77 \pm 0.08$ & $19.12 \pm 0.03$ & $0.65 \pm 0.09$ & 35.32 & 35.41 & 10.55 & 10.90 & $\cdots$ & $\cdots$ & $\cdots$ & $\cdots$ & $\mathrm{EE}$ \\
\hline NGC 4383 & $.17 \pm 0.01$ & $13.94 \pm 0.01$ & $0.24 \pm 0.01$ & $4.33 \pm 0.01$ & $14.06 \pm 0.01$ & $0.27 \pm 0.01$ & 35.73 & 35.64 & $\cdots$ & $\cdots$ & $\cdots$ & $\cdots$ & $\cdots$ & $\cdots$ & VV \\
\hline CGCG 01 & $\cdots$ & $16.71 \pm 0.08$ & $\cdots$ & $\cdots$ & $17.06 \pm 0.04$ & $\cdots$ & 0.00 & 36.14 & $\cdots$ & 16.53 & $\cdots$ & 2.44 & $\cdots$ & 2.48 & EFn \\
\hline NGC 4387 & $.07 \pm 0.05$ & $17.48 \pm 0.01$ & $1.59 \pm 0.05$ & $\cdots$ & $17.51 \pm 0.02$ & 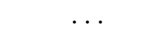 & 33.77 & 34.23 & $\cdots$ & $\ldots$ & $\cdots$ & $\ldots$ & $\ldots$ & $\ldots$ & $\mathrm{EE}$ \\
\hline Tol 65 & $.48 \pm$ & $17.36 \pm 0$ & $0.12 \pm$ & $17.50 \pm 0.03$ & 2 & $0.10 \pm 0.03$ & 35.06 & 34 & $\ldots$ & & $\ldots$ & $\ldots$ & $\ldots$ & $\cdots$ & $\ldots$ \\
\hline NGC 4388 & $14.68 \pm 0.01$ & $14.01 \pm 0.01$ & $0.67 \pm 0.01$ & $14.73 \pm 0.01$ & $14.14 \pm 0.01$ & $0.59 \pm 0.01$ & 35.52 & 35.62 & 13.81 & 20.74 & 3.54 & 4.59 & 3.46 & 4.03 & ER \\
\hline NGC 439 & $11.67 \pm 0.01$ & $11.54 \pm 0.01$ & $0.13 \pm 0.01$ & $.71 \pm 0.01$ & $.59 \pm 0.01$ & $.12 \pm 0$ & 35.51 & 35.39 & 148.72 & 153.14 & 1.98 & 2.11 & 1.91 & 2.00 & EFn \\
\hline IC 3 & $.02 \pm 0.03$ & $16.59 \pm 0$ & $0.43 \pm$ & $.17 \pm 0.03$ & $.78 \pm 0.01$ & $0.39 \pm 0$ & 36.13 & 36.12 & 12.69 & 12.20 & $\ldots$ & $\ldots$ & $\ldots$ & $\ldots$ & $\mathrm{EF}$ \\
\hline NGC 4 & $.99 \pm 0.01$ & $14.58 \pm 0.01$ & $0.40 \pm 0.01$ & $.09 \pm 0.01$ & .01 & $0.38 \pm 0$ & 35.40 & 35.39 & 26.06 & 26.77 & 2.61 & 2.68 & 2.57 & 2.66 & Ef \\
\hline NGC 4405 & $.53 \pm 0.01$ & $15.66 \pm 0.01$ & $0.87 \pm 0.01$ & $16.57 \pm 0.01$ & $15.74 \pm 0.01$ & $0.83 \pm 0.02$ & 34.78 & 34.96 & $\ldots$ & 9.99 & $\ldots$ & $\ldots$ & $\ldots$ & $\ldots$ & ER \\
\hline NGC 4402 & $16.54 \pm 0.01$ & $15.60 \pm 0.01$ & $0.94 \pm 0.01$ & $16.63 \pm 0.01$ & $15.76 \pm 0.01$ & $0.87 \pm 0.02$ & 34.78 & 34.98 & 25.22 & 29.78 & 2.05 & 2.39 & 2.00 & 2.35 & ED \\
\hline NGC 4414 & $14.08 \pm 0.01$ & $13.39 \pm 0.01$ & $0.69 \pm 0.02$ & $14.26 \pm 0.01$ & $13.51 \pm 0.01$ & $0.75 \pm 0.01$ & 35.84 & 35.94 & 42.48 & 37.76 & 2.00 & 2.39 & 2.02 & 2.51 & $\mathrm{EF}, \mathrm{EE}$ \\
\hline NGC 4407 & $15.56 \pm 0.01$ & $15.04 \pm 0.01$ & $0.52 \pm 0.01$ & $15.58 \pm 0.01$ & $15.08 \pm 0.01$ & $0.51 \pm 0.01$ & 35.17 & 35.20 & 14.83 & 15 & $\cdots$ & $\cdots$ & $\cdots$ & $\cdots$ & ERn \\
\hline IC 3356 & $16.50 \pm 0.01$ & $16.33 \pm 0.01$ & $0.17 \pm 0.02$ & $17.18 \pm 0.02$ & $16.98 \pm 0.01$ & $0.20 \pm 0.03$ & 34.80 & 34.69 & 30.70 & 2 & 3.19 & 3.19 & 3.25 & 3.21 & Er \\
\hline IC 3355 & $62 \pm 0.01$ & $16.29 \pm 0.01$ & $0.33 \pm 0.01$ & $16.91 \pm 0.02$ & $16.63 \pm 0.01$ & $0.28 \pm 0.02$ & 34.75 & 34 & 12.53 & & $\cdots$ & $\cdots$ & $\ldots$ & $\cdots$ & $\mathrm{EE}$ \\
\hline IC 33 & $.35 \pm 0.11$ & 17. & $2.00=$ & $\cdots$ & $17.66 \pm 0$ & $\cdots$ & 33.66 & & & & $\cdots$ & $\cdots$ & $\cdots$ & $\cdots$ & VV \\
\hline ESO 380-G029 & $.36 \pm 0.01$ & $15.83 \pm$ & $0.53 \pm 0.01$ & $16.58 \pm 0.02$ & $16.06=$ & $0.52 \pm 0.02$ & 35.89 & 35.92 & 20.99 & 20 & 2.23 & 2.32 & 2.28 & 2.33 & EFn \\
\hline NGC 4419 & $.85 \pm 0.01$ & $15.56 \pm 0.01$ & $1.29 \pm 0.02$ & $16.91 \pm 0.03$ & $15.65 \pm 0.01$ & $1.26 \pm 0.03$ & 34.66 & 35.00 & 17.34 & 18.96 & 2.35 & 3.07 & 2.48 & 3.12 & $\mathrm{EF}, \mathrm{EE}$ \\
\hline NGC 4421 & $18.75 \pm 0.08$ & $16.57 \pm 0.02$ & $2.17 \pm 0.09$ & $18.95 \pm 0.10$ & $16.83 \pm 0.02$ & $2.12 \pm 0.10$ & 33.90 & 34.59 & 30.94 & 32.92 & $\cdots$ & 4.17 & $\cdots$ & 3.97 & VV \\
\hline IC 3363 & $\cdots$ & $18.98 \pm 0.04$ & $\cdots$ & $\cdots$ & $19.35 \pm 0.05$ & $\cdots$ & 0.00 & 33.63 & $\cdots$ & 13.53 & $\cdots$ & 2.88 & $\cdots$ & $\cdots$ & $\mathrm{EE}$ \\
\hline UGC 07553 & $\cdots$ & $17.95 \pm 0.04$ & $\cdots$ & $\cdots$ & $19.22 \pm 0.03$ & $\cdots$ & 0.00 & 35.78 & $\cdots$ & 12.12 & $\cdots$ & 2.37 & $\ldots$ & 2.35 & $\mathrm{EE}$ \\
\hline IC 0792 & $\cdots$ & $16.55 \pm 0.02$ & $\cdots$ & $\cdots$ & $16.65 \pm 0.02$ & $\cdots$ & 0.00 & 35.98 & $\cdots$ & 13.87 & $\cdots$ & 2.63 & $\ldots$ & $\ldots$ & $\mathrm{EF}$ \\
\hline IC 3365 & $53 \pm 0.01$ & $15.21 \pm 0.01$ & $0.32 \pm 0.01$ & $15.63 \pm 0.01$ & $15.32 \pm 0.01$ & $0.31 \pm 0.01$ & 35.18 & 35.14 & 19.16 & 18.75 & 2.54 & 2.64 & 2.59 & 2.68 & Ef \\
\hline NGC 4425 & $.01 \pm 0.10$ & $16.79 \pm 0.01$ & $2.23 \pm 0.10$ & $\cdots$ & $16.90 \pm 0.01$ & $\ldots$ & 33.79 & 34.50 & 11.48 & 18.03 & $\cdots$ & 3.63 & $\cdots$ & 3.57 & VV \\
\hline NGC 4431 & $.30 \pm 0.15$ & $17.80 \pm 0.02$ & $1.50 \pm 0.15$ & $\ldots$ & $17.97 \pm 0.03$ & $\ldots$ & 33.68 & & 21.27 & & $\ldots$ & $\ldots$ & $\cdots$ & $\cdots$ & $\mathrm{VV}$ \\
\hline NGC 4435 & $17.56 \pm 0.24$ & $15.71 \pm 0.02$ & $1.85 \pm 0.24$ & $17.85 \pm 0.05$ & $15.98 \pm 0.01$ & $1.87 \pm 0.05$ & 34.37 & 34.94 & 27.58 & 26.32 & $\cdots$ & $\cdots$ & $\cdots$ & $\cdots$ & VV \\
\hline NGC 4436 & $20.51 \pm 0.49$ & $17.88 \pm 0.03$ & $2.63 \pm 0.49$ & $\cdots$ & $18.16 \pm 0.03$ & $\ldots$ & 33.19 & 34.07 & 12.98 & 14.90 & $\ldots$ & $\cdots$ & $\cdots$ & $\cdots$ & VV \\
\hline NGC 4438 & $15.06 \pm 0.01$ & $14.20 \pm 0.01$ & $0.86 \pm 0.01$ & $15.18 \pm 0.01$ & $14.41 \pm 0.01$ & $0.78 \pm 0.01$ & 35.37 & 35.54 & 77.56 & 79.34 & 2.49 & 3.21 & 2.51 & 3.21 & $\mathrm{EF}, \mathrm{EE}$ \\
\hline
\end{tabular}


Table 3-Continued

\begin{tabular}{|c|c|c|c|c|c|c|c|c|c|c|c|c|c|c|c|}
\hline \multirow{2}{*}{$\begin{array}{c}\text { Object Name } \\
\text { (1) }\end{array}$} & \multicolumn{3}{|c|}{ Asymptotic magnitudes and color } & \multicolumn{3}{|c|}{ D25 magnitudes and color } & \multicolumn{2}{|c|}{$\log L$} & \multicolumn{2}{|c|}{ Effective Radii } & \multicolumn{2}{|c|}{ C31 } & \multicolumn{2}{|c|}{$\mathrm{C} 42$} & \multirow{2}{*}{$\begin{array}{r}\text { UV } \\
\text { profile } \\
(16)\end{array}$} \\
\hline & $\begin{array}{c}\text { FUV } \\
(\mathrm{mag}) \\
(2)\end{array}$ & $\begin{array}{c}\text { NUV } \\
(\mathrm{mag}) \\
(3)\end{array}$ & $\begin{array}{l}\text { FUV-NUV } \\
\text { (mag) } \\
(4)\end{array}$ & $\begin{array}{c}\text { FUV } \\
(\mathrm{mag}) \\
(5)\end{array}$ & $\begin{array}{c}\text { NUV } \\
(\mathrm{mag}) \\
(6)\end{array}$ & $\begin{array}{c}\text { FUV-NUV } \\
(\mathrm{mag}) \\
(7)\end{array}$ & $\begin{array}{c}\text { FUV } \\
(W) \\
(8)\end{array}$ & $\begin{array}{c}\text { NUV } \\
(\mathrm{W}) \\
(9)\end{array}$ & $\begin{array}{c}\text { FUV } \\
(\operatorname{arcsec}) \\
(10)\end{array}$ & $\begin{array}{c}\text { NUV } \\
(\operatorname{arcsec}) \\
(11)\end{array}$ & $\begin{array}{l}\text { FUV } \\
(12)\end{array}$ & $\begin{array}{l}\text { NUV } \\
(13)\end{array}$ & $\begin{array}{l}\text { FUV } \\
(14)\end{array}$ & $\begin{array}{l}\text { NUV } \\
(15)\end{array}$ & \\
\hline NGC 4440 & $18.92 \pm 0.08$ & $17.05 \pm 0.02$ & $1.87 \pm 0.08$ & $18.98 \pm 0.08$ & $17.14 \pm 0.02$ & $1.85 \pm 0.08$ & 33.83 & 34.40 & 10.83 & 15.94 & $\cdots$ & 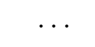 & & & $\mathrm{VV}$ \\
\hline IC 0794 & $.05 \pm 0.16$ & $18.30 \pm 0.04$ & $1.75 \pm 0.17$ & $20.24 \pm 0.19$ & $18.56 \pm 0.04$ & $1.68 \pm 0.19$ & 33.38 & 33.90 & 13.23 & 16.58 & $\cdots$ & $\cdots$ & $\cdots$ & $\cdots$ & Er \\
\hline IC 3381 & $20.29 \pm 1.78$ & $18.08 \pm 0.04$ & $2.22 \pm 1.78$ & $\cdots$ & $18.36 \pm 0.03$ & $\cdots$ & 33.28 & 33.99 & $\cdots$ & 16.51 & $\cdots$ & $\cdots$ & $\cdots$ & $\cdots$ & $\mathrm{EE}$ \\
\hline NGC 4450 & & $14.57 \pm 0.01$ & $\cdots$ & $\cdots$ & $14.63 \pm 0.01$ & $\cdots$ & 0.00 & 35.39 & $\cdots$ & 52.35 & $\ldots$ & 2.63 & $\ldots$ & 2.62 & EEn \\
\hline IC 3393 & $20.82 \pm 0.32$ & $18.36 \pm 0.03$ & $2.46 \pm 0.32$ & $\ldots$ & $18.63 \pm 0.03$ & $\cdots$ & 33.07 & 33.88 & $\cdots$ & 11.40 & $\ldots$ & $\ldots$ & $\cdots$ & $\ldots$ & $\mathrm{EE}$ \\
\hline NGC 4452 & $18.89 \pm 0.07$ & $16.79 \pm 0.04$ & $2.10 \pm 0.08$ & $18.90 \pm 0.06$ & $17.08 \pm 0.01$ & $1.82 \pm 0.06$ & 33.84 & 34.50 & 12.74 & 19.34 & 2.91 & 3.67 & 2.94 & 3.52 & $\mathrm{Er}$ \\
\hline NGC 4454 & & $16.19 \pm 0.02$ & $\ldots$ & $\ldots$ & $16.35 \pm 0.01$ & $\ldots$ & 0.00 & 35.36 & $\ldots$ & 26.47 & $\ldots$ & 1.84 & $\ldots$ & 1.83 & $\mathrm{EF}$ \\
\hline NGC 4458 & $19.52 \pm 0.25$ & $17.28 \pm 0.03$ & $2.24 \pm 0.25$ & $\ldots$ & $17.41 \pm 0.02$ & $\cdots$ & 33.59 & 34.31 & & 8 & $\ldots$ & $\ldots$ & $\ldots$ & $\ldots$ & VV \\
\hline NGC 4461 & $.80 \pm 0.04$ & $16.49 \pm 0.01$ & $1.31 \pm 0.04$ & $17.84 \pm 0.04$ & $16.54 \pm 0.01$ & $1.30 \pm 0.04$ & 34.28 & 34.62 & 11.09 & & $\ldots$ & $\ldots$ & $\ldots$ & $\ldots$ & VV \\
\hline IC 0796 & $\cdots$ & $16.69 \pm 0.03$ & $\cdots$ & $\ldots$ & $16.89 \pm 0.02$ & $\cdots$ & 0.00 & 34.54 & $\cdots$ & $\ldots$ & $\ldots$ & $\ldots$ & $\cdots$ & $\cdots$ & EV \\
\hline IC 3418 & $17.55 \pm 0.09$ & $16.69 \pm 0.03$ & $0.85 \pm 0.10$ & $18.16 \pm 0.05$ & $17.33 \pm 0.02$ & $0.83 \pm 0.05$ & 34.38 & 34.54 & 29.19 & 30.65 & 3.95 & 3.39 & 3.74 & 3.33 & $\mathrm{EE}$ \\
\hline NGC 4473 & $17.02 \pm 0.02$ & $15.47 \pm 0.01$ & $1.55 \pm 0.02$ & $17.11 \pm 0.03$ & $15.60 \pm 0.01$ & $1.51 \pm 0.03$ & 34.52 & 34.96 & 21.87 & 28.67 & $\ldots$ & 6.07 & $\ldots$ & $\ldots$ & VV \\
\hline NGC 4476 & $18.18 \pm 0.02$ & $16.36 \pm 0.02$ & $1.82 \pm 0.03$ & $18.23 \pm 0.04$ & $16.57 \pm 0.01$ & $1.66 \pm 0.05$ & 34.12 & 34.68 & $\ldots$ & 11.25 & $\ldots$ & $\ldots$ & $\ldots$ & $\ldots$ & VV \\
\hline NGC 4478 & $18.20 \pm 0.05$ & $16.59 \pm 0.01$ & $1.61 \pm 0.05$ & $18.21 \pm 0.05$ & $16.65 \pm 0.01$ & $1.56 \pm 0.05$ & 34.12 & 34.58 & $\ldots$ & 14.04 & $\ldots$ & $\ldots$ & $\ldots$ & $\ldots$ & VV \\
\hline NGC 4479 & $18.86 \pm 0.15$ & $17.53 \pm 0.01$ & $1.32 \pm 0.15$ & $19.20 \pm 0.11$ & $17.67 \pm 0.02$ & $1.52 \pm 0.11$ & 33.85 & 34.21 & 16.49 & 14.42 & $\cdots$ & $\ldots$ & $\cdots$ & $\ldots$ & VV \\
\hline NGC 4485 & & $13.35 \pm 0.01$ & $\ldots$ & $\ldots$ & $13.40 \pm 0.01$ & $\ldots$ & 0.00 & 35.51 & $\ldots$ & 22.25 & $\ldots$ & 2.73 & $\cdots$ & 2.77 & $\mathrm{EE}$ \\
\hline NGC 4490 & $\ldots$ & $11.54 \pm 0.01$ & $\ldots$ & $\ldots$ & $11.56 \pm 0.01$ & $\ldots$ & 0.00 & 36.23 & $\ldots$ & 48.30 & $\ldots$ & 2.59 & $\ldots$ & 2.62 & VF \\
\hline MESSIER 087 & $.40 \pm 0.01$ & $13.80 \pm 0.01$ & $0.60 \pm 0.01$ & $14.50 \pm 0.01$ & $13.92 \pm 0.01$ & $0.58 \pm 0$ & 35.64 & 35.70 & 44.08 & 60.05 & 8.92 & 6.76 & $\ldots$ & 5.19 & VV \\
\hline NGC 4491 & $17.52 \pm 0.02$ & $16.30 \pm 0.01$ & $1.23 \pm 0.02$ & $17.56 \pm 0.03$ & $16.42 \pm 0.01$ & $1.14 \pm 0.03$ & 34.39 & 34.70 & $\ldots$ & $\ldots$ & $\ldots$ & $\ldots$ & $\cdots$ & $\cdots$ & EV \\
\hline CGCG 014-054 & $17.27 \pm 0.03$ & $16.94 \pm 0.01$ & $0.32 \pm 0.03$ & $17.32 \pm 0.03$ & $17.06 \pm 0.01$ & $0.26 \pm 0.03$ & 34.45 & 34.41 & 10.65 & 11.86 & $\cdots$ & $\cdots$ & $\cdots$ & $\cdots$ & $\mathrm{EE}$ \\
\hline IC 3446 & $16.58 \pm 0.02$ & $16.37 \pm 0.01$ & $0.21 \pm 0.02$ & $16.80 \pm 0.02$ & $16.63 \pm 0.01$ & $0.17 \pm 0.02$ & 34.76 & 34.67 & 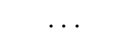 & $\ldots$ & $\ldots$ & $\ldots$ & $\ldots$ & $\ldots$ & VV \\
\hline NGC 4497 & $19.62 \pm 0.36$ & $17.12 \pm 0.03$ & $2.50 \pm 0.36$ & & $17.34 \pm 0.02$ & $\ldots$ & 33.55 & 34.37 & 18.67 & 19.15 & 4.17 & 3.35 & $\ldots$ & $\ldots$ & VV \\
\hline IC 3457 & $20.67 \pm 0.73$ & $18.53 \pm 0.10$ & $2.14 \pm 0.73$ & $20.86 \pm 0.29$ & $19.00 \pm 0.05$ & $1.86 \pm 0.29$ & 33.13 & 33.81 & 17.75 & 21.06 & $\ldots$ & 3.33 & $\cdots$ & $\cdots$ & Er \\
\hline IC 3459 & & $18.82 \pm 0.08$ & $\ldots$ & $\ldots$ & $19.33 \pm 0.08$ & $\ldots$ & 0.00 & 33.69 & $\ldots$ & 20.98 & $\ldots$ & 3.15 & $\ldots$ & $\ldots$ & Er \\
\hline NGC 4503 & $18.10 \pm 0.04$ & $16.40 \pm 0.01$ & $1.70 \pm 0.04$ & $\ldots$ & $16.49 \pm 0.01$ & $\ldots$ & 34.16 & 34.66 & 13.28 & 21.46 & $\ldots$ & 5.02 & $\ldots$ & $\ldots$ & VV \\
\hline NGC 4506 & $18.05 \pm 0.03$ & $16.94 \pm 0.04$ & $1.11 \pm 0.05$ & $18.10 \pm 0.04$ & $17.11 \pm 0.02$ & $0.99 \pm 0.05$ & 34.18 & 34.44 & $\ldots$ & $\ldots$ & $\ldots$ & $\cdots$ & $\ldots$ & $\cdots$ & VV \\
\hline IC 3467 & $17.25 \pm 0.01$ & $16.85 \pm 0.02$ & $0.39 \pm 0.02$ & $17.46 \pm 0.02$ & $17.18 \pm 0.01$ & $0.28 \pm 0.02$ & 34.50 & 34.48 & 7.44 & 8.48 & $\ldots$ & $\ldots$ & $\ldots$ & $\ldots$ & $\mathrm{Ef}$ \\
\hline UGC 07710 & $16.47 \pm 0.01$ & $16.16 \pm 0.01$ & $0.31 \pm 0.01$ & $17.07 \pm 0.03$ & $16.78 \pm 0.01$ & $0.29 \pm 0.03$ & 35.45 & 35.40 & 34.03 & 34.26 & 2.29 & 2.48 & 2.44 & 2.53 & $\mathrm{EF}$ \\
\hline NGC 4528 & $18.63 \pm 0.12$ & $17.03 \pm 0.01$ & $1.59 \pm 0.12$ & $19.07 \pm 0.08$ & $17.13 \pm 0.02$ & $1.94 \pm 0.08$ & 33.94 & 34.41 & 20.26 & $\ldots$ & $\ldots$ & $\ldots$ & $\ldots$ & $\ldots$ & VV \\
\hline NGC 4531 & $16.86 \pm 0.02$ & $15.68 \pm 0.01$ & $1.19 \pm 0.02$ & $16.92 \pm 0.02$ & $15.76 \pm 0.01$ & $1.16 \pm 0.03$ & 34.65 & 34.95 & 13.54 & 16.90 & $\ldots$ & 3.50 & $\ldots$ & $\cdots$ & Erh \\
\hline
\end{tabular}


Table 3-Continued

\begin{tabular}{|c|c|c|c|c|c|c|c|c|c|c|c|c|c|c|c|}
\hline \multirow{2}{*}{$\begin{array}{l}\text { Object Name } \\
\text { (1) }\end{array}$} & \multicolumn{3}{|c|}{ Asymptotic magnitudes and color } & \multicolumn{3}{|c|}{ D25 magnitudes and color } & \multicolumn{2}{|c|}{$\log L$} & \multicolumn{2}{|c|}{ Effective Radii } & \multicolumn{2}{|c|}{$\mathrm{C} 31$} & \multicolumn{2}{|c|}{$\mathrm{C} 42$} & \multirow{2}{*}{$\begin{array}{c}\text { UV } \\
\text { profile } \\
(16)\end{array}$} \\
\hline & $\begin{array}{c}\text { FUV } \\
(\mathrm{mag}) \\
(2)\end{array}$ & $\begin{array}{c}\text { NUV } \\
(\mathrm{mag}) \\
(3)\end{array}$ & $\begin{array}{l}\text { FUV-NUV } \\
\text { (mag) } \\
(4)\end{array}$ & $\begin{array}{c}\text { FUV } \\
(\mathrm{mag}) \\
(5)\end{array}$ & $\begin{array}{l}\text { NUV } \\
(\mathrm{mag}) \\
(6)\end{array}$ & $\begin{array}{l}\text { FUV-NUV } \\
(\mathrm{mag}) \\
(7)\end{array}$ & $\begin{array}{c}\text { FUV } \\
(\mathrm{W}) \\
(8)\end{array}$ & $\begin{array}{c}\text { NUV } \\
(\mathrm{W}) \\
(9)\end{array}$ & $\begin{array}{c}\text { FUV } \\
\text { (arcsec) } \\
(10)\end{array}$ & $\begin{array}{c}\text { NUV } \\
\text { (arcsec) } \\
(11)\end{array}$ & $\begin{array}{l}\text { FUV } \\
(12)\end{array}$ & $\begin{array}{l}\text { NUV } \\
(13)\end{array}$ & $\begin{array}{l}\text { FUV } \\
(14)\end{array}$ & $\begin{array}{l}\text { NUV } \\
(15)\end{array}$ & \\
\hline NGS 40 & 01 & 1 & I & 1 & 1 & $\perp$ & & & $r s$ & 76 & & & & 2.09 & ?Fn \\
\hline $\mathrm{UGC} 0$ & & & & & & & & & & & & & 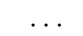 & & VV \\
\hline NGC 4546 & & $48 \pm 0.02$ & & $17.51 \pm 0.03$ & & & 34.36 & & 20.03 & 26.86 & $\ldots$ & 5.12 & $\ldots$ & 4.45 & VV \\
\hline NGC 4550 & $57 \pm 0.01$ & $16.02 \pm 0.01$ & $1.55 \pm 0.01$ & $17.59 \pm 0.03$ & $16.08 \pm 0.01$ & $1.51 \pm 0.03$ & 34.31 & 34.75 & 8.66 & 14.36 & $\ldots$ & 4.16 & $\ldots$ & $\ldots$ & VV \\
\hline NGC 4551 & $95 \pm 0.09$ & $17.30 \pm 0.01$ & $1.65 \pm 0.09$ & $\ldots$ & $17.36 \pm 0.02$ & $\ldots$ & 33.82 & 34.30 & 12.21 & 12.33 & $\ldots$ & $\ldots$ & $\ldots$ & $\ldots$ & VV \\
\hline MESSIER 089 & $.71 \pm 0.02$ & $14.73 \pm 0.01$ & $.98 \pm 0.02$ & $5.81 \pm 0.02$ & $14.97 \pm 0.01$ & $0.85 \pm 0.02$ & 35.02 & 35.24 & 13.34 & 39.85 & $\ldots$ & $\ldots$ & $\ldots$ & $\ldots$ & VV \\
\hline NGC 4559 & $12.07 \pm 0.01$ & $11.87 \pm 0.01$ & $0.20 \pm 0.01$ & $2.20 \pm 0.01$ & $11.97 \pm 0.01$ & $0.23 \pm 0.01$ & 36.57 & 36.47 & 87.14 & 80.79 & 2.89 & 2.79 & 2.90 & 2.82 & EEn \\
\hline PGC 42042 & $18.29 \pm 0.02$ & $17.25 \pm 0.03$ & $1.04 \pm 0.04$ & $18.30 \pm 0.04$ & $17.31 \pm 0.02$ & $0.99 \pm 0.04$ & 35.64 & 35.88 & $\ldots$ & $\ldots$ & $\ldots$ & $\ldots$ & $\ldots$ & $\ldots$ & VV \\
\hline NGC 4564 & $17.52 \pm 0.04$ & $16.14 \pm 0.01$ & $1.38 \pm 0.04$ & $\ldots$ & $16.20 \pm 0.01$ & $\ldots$ & 34.39 & 34.76 & $\ldots$ & 14.91 & $\ldots$ & $\ldots$ & $\ldots$ & $\ldots$ & VV \\
\hline NGC 4567 & 01 & $.18 \pm 0.01$ & $085+1-2-1-1$ & $15.07 \pm 0.01$ & $14.25 \pm 0.01$ & $0.82 \pm 0.01$ & 35.38 & 35.55 & 27.11 & 26.58 & 1.94 & 2.28 & 1.88 & 2.34 & ?Fn \\
\hline IC 3583 & $00 \pm 0.01$ & $14.70 \pm 0.01$ & $0.31 \pm 0$ & $15.10 \pm 0.01$ & $14.80 \pm 0.01$ & $0.30 \pm$ & 35.40 & 35.34 & 17.35 & 6 & 2.68 & 2.53 & $\ldots$ & 2.70 & $? \mathrm{fn}$ \\
\hline IC 3587 & $40 \pm 0.04$ & $17.77 \pm 0.02$ & $0.64 \pm 0.05$ & $18.78 \pm 0.03$ & $18.25 \pm 0.01$ & $0.53 \pm 0.03$ & 35.63 & 35.71 & 9.37 & 63 & 3.17 & 2.71 & $\ldots$ & 2.73 & $\mathrm{EE}$ \\
\hline NGC 456 & $46 \pm 0.01$ & $.17 \pm 0.01$ & 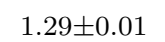 & $14.54 \pm 0.01$ & $13.24 \pm 0.01$ & $1.29 \pm 0$ & 35.61 & & 59.22 & 65 & 5.36 & 5.29 & 4.63 & 4.82 & EEn \\
\hline NGC $4559 A$ & & & 2 & $18.11 \pm 0.03$ & $17.38 \pm 0.01$ & & 35.86 & & & $\ldots$ & $\ldots$ & & $\ldots$ & & VV \\
\hline IC 3 & & & & 3 & & & & & & & 2.47 & 2.81 & $\ldots$ & & Ef \\
\hline $\mathrm{ME}$ & 1 & 01 & 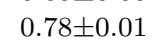 & $14.49 \pm 0.01$ & 13.71 & 0.7 & 35.60 & 35.74 & 54.47 & 54.14 & 1.83 & 1.95 & 1.58 & 1.82 & ?Fn,Edn \\
\hline NGC 4 & 03 & 01 & 1.1 & $7.86 \pm 0.04$ & $16.81=$ & 04 & 34.26 & 34.56 & $\ldots$ & $\ldots$ & $\ldots$ & 等 & $\ldots$ & $\ldots$ & Er \\
\hline NGC 4594 & $.54 \pm 0.01$ & 02 & 1.26 & $14.73 \pm 0.01$ & $13.60 \pm 0.01$ & $1.13 \pm 0.01$ & 35.03 & 35.36 & 60.96 & 81.40 & 5.50 & 4.65 & 4.76 & 4.23 & VV \\
\hline NGC 4612 & $18.81 \pm 0.05$ & 16.31 & $2.50 \pm 0.06$ & $\ldots$ & $16.39 \pm 0.03$ & $\ldots$ & 33.87 & 34.70 & $\ldots$ & 18.71 & $\ldots$ & $\ldots$ & $\ldots$ & $\ldots$ & VV \\
\hline NGC 4618 & $12.78 \pm 0.01$ & $12.53 \pm 0.01$ & $0.24 \pm 0.01$ & $12.80 \pm 0.01$ & $12.55 \pm 0.01$ & $0.25 \pm 0.01$ & 35.78 & 35.70 & 45.19 & 44.57 & 2.01 & 2.08 & 1.92 & 2.00 & VFh \\
\hline NGC 4625 & $14.45 \pm 0.01$ & $14.15 \pm 0.01$ & $0.30 \pm 0.02$ & $14.93 \pm 0.01$ & $14.57 \pm 0.01$ & $0.36 \pm 0.01$ & 35.11 & 35.05 & 28.08 & 27.18 & 7.53 & 6.91 & $\ldots$ & $\ldots$ & $\mathrm{x} ? \mathrm{Fn}$ \\
\hline NGC 4627 & $16.12 \pm 0.14$ & $15.53 \pm 0.02$ & $0.59 \pm 0.14$ & $16.84 \pm 0.04$ & $15.85 \pm 0.01$ & $1.00 \pm 0.04$ & 34.40 & 34.45 & 60.84 & 33.28 & 3.02 & 4.41 & $\ldots$ & 3.99 & VV \\
\hline NGC 4631 & $11.63 \pm 0.01$ & $11.35 \pm 0.01$ & $0.28 \pm 0.01$ & $11.72 \pm 0.01$ & $11.42 \pm 0.01$ & $0.30 \pm 0.01$ & 36.19 & 36.13 & 8425 & & 2.30 & 2.24 & 2.30 & 2.24 & $\mathrm{EE}$ \\
\hline NGC 4623 & & $17.12 \pm 0$ & & $9.24 \pm 0$ & $17.40 \pm 0$ & & 33.70 & & & & $\ldots$ & 4 & $\ldots$ & 3.69 & VV \\
\hline NGC 4656 & & $11.46 \pm$ & & & & & & & & & & 9 & 3.46 & 3.62 & $\mathrm{Er}$ \\
\hline & & & & & & & & & 47.71 & 45.23 & 4.52 & 4.96 & 4.15 & 4.36 & VV \\
\hline NGC 4691 & $13.66 \pm 0.01$ & $13.22 \pm 0.01$ & $0.44 \pm 0.01$ & $13.67 \pm 0.01$ & $13.24 \pm 0.01$ & $0.43 \pm 0.01$ & 35.89 & 35.89 & $\ldots$ & $\cdots$ & $\ldots$ & $\ldots$ & $\ldots$ & $\ldots$ & VV \\
\hline DDO 149 & $15.70 \pm 0.01$ & $15.48 \pm 0.01$ & $0.22 \pm 0.01$ & $16.37 \pm 0.02$ & $16.11 \pm 0.01$ & $0.26 \pm 0.03$ & 35.33 & 35.24 & 27.50 & 26.73 & 2.61 & 2.56 & 2.62 & 2.61 & Efn \\
\hline UGC 07982 & $17.51 \pm 0.05$ & $16.60 \pm 0.01$ & $0.91 \pm 0.05$ & $17.57 \pm 0.03$ & $16.71 \pm 0.01$ & $0.86 \pm 0.03$ & 34.40 & 34.59 & 17.87 & 19.27 & 2.54 & 2.98 & 2.57 & 2.99 & $\mathrm{EE}$ \\
\hline UGC 07991 & $18.18 \pm 0.01$ & $17.24 \pm 0.02$ & $0.94 \pm 0.03$ & $18.58 \pm 0.03$ & $17.90 \pm 0.01$ & $0.68 \pm 0.03$ & 34.21 & 34.41 & 11.95 & 15.25 & 2.54 & 2.66 & 2.59 & $\ldots$ & $\mathrm{Ef}$ \\
\hline NGC 4736 & $11.83 \pm 0.01$ & $11.49 \pm 0.01$ & $0.34 \pm 0.01$ & $11.86 \pm 0.01$ & $11.50 \pm 0.01$ & $0.36 \pm 0.01$ & 35.63 & 35.59 & 40.72 & 39.64 & 1.73 & 1.81 & 2.05 & 2.07 & ?Rh \\
\hline NGC 4753 & $17.15 \pm 0.03$ & $14.70 \pm 0.02$ & $2.45 \pm 0.03$ & $17.29 \pm 0.05$ & $15.00 \pm 0.01$ & $2.29 \pm 0.05$ & 34.82 & 35.62 & 50.18 & 66.63 & 3.70 & 3.62 & 3.48 & 3.43 & VV \\
\hline
\end{tabular}


Table 3-Continued

\begin{tabular}{|c|c|c|c|c|c|c|c|c|c|c|c|c|c|c|c|}
\hline \multirow{2}{*}{$\begin{array}{l}\text { Object Name } \\
\text { (1) }\end{array}$} & \multicolumn{3}{|c|}{ Asymptotic magnitudes and color } & \multicolumn{3}{|c|}{ D25 magnitudes and color } & \multicolumn{2}{|c|}{$\log L$} & \multicolumn{2}{|c|}{ Effective Radii } & \multicolumn{2}{|c|}{$\mathrm{C} 31$} & \multicolumn{2}{|c|}{$\mathrm{C} 42$} & \multirow{2}{*}{$\begin{array}{c}\text { UV } \\
\text { profile } \\
(16)\end{array}$} \\
\hline & $\begin{array}{c}\text { FUV } \\
(\mathrm{mag}) \\
(2)\end{array}$ & $\begin{array}{c}\text { NUV } \\
(\mathrm{mag}) \\
(3)\end{array}$ & $\begin{array}{c}\text { FUV-NUV } \\
\text { (mag) } \\
(4)\end{array}$ & $\begin{array}{c}\text { FUV } \\
(\mathrm{mag}) \\
(5)\end{array}$ & $\begin{array}{c}\text { NUV } \\
(\mathrm{mag}) \\
(6)\end{array}$ & $\begin{array}{c}\text { FUV-NUV } \\
(\mathrm{mag}) \\
(7)\end{array}$ & $\begin{array}{c}\text { FUV } \\
(\mathrm{W}) \\
(8)\end{array}$ & $\begin{array}{c}\text { NUV } \\
(\mathrm{W}) \\
(9)\end{array}$ & $\begin{array}{c}\text { FUV } \\
(\operatorname{arcsec}) \\
(10)\end{array}$ & $\begin{array}{c}\text { NUV } \\
\text { (arcsec) } \\
(11)\end{array}$ & $\begin{array}{l}\text { FUV } \\
(12)\end{array}$ & $\begin{array}{l}\text { NUV } \\
(13)\end{array}$ & $\begin{array}{l}\text { FUV } \\
(14)\end{array}$ & $\begin{array}{l}\text { NUV } \\
(15)\end{array}$ & \\
\hline UGC 08013 & $89 \pm 0.03$ & $.43 \pm 0.02$ & $0.46=$ & \pm 0.03 & $17.72=$ & 3 & 35.90 & & 9 & 1 & 2.21 & & 2.16 & & $\mathrm{EF}$ \\
\hline NGC 4771 & & 01 & 0.70 & \pm 0.01 & & & & & & & 2.22 & & 2.16 & & Ef \\
\hline NGC 4772 & $.58 \pm 0.02$ & $15.52 \pm 0.02$ & $1.06 \pm 0.03$ & $16.68 \pm 0.02$ & $15.75 \pm 0.01$ & $0.93 \pm 0.02$ & 34.76 & 35.01 & 34.56 & 37.71 & 2.00 & 2.81 & 1.96 & 2.99 & Er \\
\hline DDO 154 & $.76 \pm 0.01$ & $14.79 \pm 0.01$ & $-0.04 \pm 0.01$ & $4.93 \pm 0.01$ & $14.96 \pm 0.01$ & $-0.03 \pm 0.01$ & 34.30 & 34.11 & 33.16 & 32.88 & 2.83 & 2.81 & 2.78 & 2.78 & $\mathrm{Ef}$ \\
\hline NGC 4787 & $.12 \pm 0.08$ & $19.50 \pm 0.03$ & $1.62 \pm 0.08$ & $1.22 \pm 0.19$ & $19.63 \pm 0.05$ & $1.59 \pm 0.19$ & 34.57 & 35.05 & $\ldots$ & 7.12 & $\ldots$ & $\ldots$ & $\ldots$ & $\ldots$ & $\mathrm{EE}$ \\
\hline NGC 4789 & $19.30 \pm 0.16$ & $17.66 \pm 0.11$ & $1.65 \pm 0.20$ & $19.39 \pm 0.11$ & $18.03 \pm 0.03$ & $1.36 \pm 0.12$ & 35.39 & 35.87 & $\ldots$ & 25.03 & $\ldots$ & $\ldots$ & $\ldots$ & $\ldots$ & VV \\
\hline NGC 4809 & $15.10 \pm 0.01$ & $14.91 \pm 0.01$ & $0.20 \pm 0.01$ & $15.20 \pm 0.01$ & $15.01 \pm 0.01$ & $0.19 \pm 0.01$ & 35.17 & 35.07 & 16.14 & & $\ldots$ & 3.01 & $\ldots$ & $\ldots$ & EFn \\
\hline NGC 4797 & $.70 \pm 0.10$ & $18.29 \pm 0.04$ & $1.41 \pm 0.11$ & $\ldots$ & $18.64 \pm 0.04$ & $\ldots$ & 35.17 & 35.56 & $\ldots$ & 15.75 & $\ldots$ & $\ldots$ & $\ldots$ & $\ldots$ & Er \\
\hline NGC 4799 & $.87 \pm 0.07$ & $16.12 \pm 0.03$ & $0.75 \pm 0.08$ & $17.16 \pm 0.02$ & $16.41 \pm 0.01$ & $0.74 \pm 0.02$ & 35.41 & 35.53 & 10.81 & 10.42 & $\ldots$ & $\ldots$ & $\ldots$ & $\ldots$ & VV \\
\hline NGC 4807 & $.22 \pm 0.28$ & $3.94 \pm 0.08$ & $1.28 \pm 0.29$ & $20.35 \pm 0.17$ & $19.17 \pm 0.05$ & $1.18 \pm$ & 34.87 & 35.20 & $\ldots$ & $\ldots$ & $\ldots$ & $\ldots$ & $\ldots$ & $\ldots$ & $\mathrm{VV}$ \\
\hline NGC 4816 & $19.05 \pm 0.08$ & $17.93 \pm 0.08$ & $1.11 \pm 0.11$ & $19.30 \pm 0.10$ & $18.46 \pm 0.04$ & $0.84 \pm 0.11$ & 35.32 & 35.60 & 15.25 & 25.12 & $\ldots$ & $\ldots$ & $\ldots$ & $\ldots$ & VV,EEn \\
\hline NGC 4819 & $.30 \pm 0.01$ & $17.93 \pm 0.02$ & $1.37 \pm 0.02$ & $\ldots$ & $17.99 \pm 0.02$ & & 35.17 & 35.54 & $\ldots$ & & $\ldots$ & $\ldots$ & $\ldots$ & $\ldots$ & $\mathrm{EE}$ \\
\hline NGC 4827 & $.58 \pm 0.04$ & $18.44 \pm 0.02$ & $1.13 \pm 0$ & $\ldots$ & $18.57 \pm 0.04$ & & 35.20 & & $\ldots$ & & $\ldots$ & $\ldots$ & $\ldots$ & $\ldots$ & $\mathrm{VV}$ \\
\hline MESSIER 064 & $.50 \pm 0.01$ & $12.47 \pm 0.01$ & $1.03 \pm 0$ & $13.50 \pm 0.01$ & $12.49 \pm 0.01$ & $1.01 \pm 0$ & 36.00 & 36.23 & 21 & & 1.96 & 2.64 & 2.06 & 2.82 & Erh \\
\hline NGC 4861 & $13.54 \pm 0.01$ & $13.57 \pm 0.01$ & $-0.03 \pm 0.01$ & $13.62 \pm 0.01$ & $13.64 \pm 0.01$ & $-0.03 \pm 0.02$ & 35.72 & 35.53 & 34.62 & 35.60 & 1.38 & 1.46 & 1.07 & 1.25 & $\mathrm{EE}$ \\
\hline IC 0842 & $17.56 \pm 0.03$ & $17.05 \pm 0.01$ & $0.51 \pm 0.03$ & $17.62 \pm 0.03$ & $17.15 \pm 0.01$ & $0.47 \pm 0.03$ & 35.97 & 35.99 & 12.91 & 29 & 1.92 & $\ldots$ & $\ldots$ & $\ldots$ & $\mathrm{EF}$ \\
\hline UGC 08127 & $16.92 \pm 0.02$ & $16.64 \pm 0.02$ & $0.28 \pm 0.03$ & $17.10 \pm 0.02$ & $16.83 \pm 0.01$ & $0.28 \pm 0.02$ & 34.83 & 34.76 & 12.63 & 12.50 & 2.66 & 2.69 & $\ldots$ & $\ldots$ & Ef \\
\hline NGC 4922 & $18.48 \pm 0.03$ & $17.40 \pm 0.03$ & $1.08 \pm 0.05$ & $18.52 \pm 0.06$ & $17.47 \pm 0.02$ & $1.05 \pm 0.06$ & 35.57 & 35.83 & 11.60 & 13.43 & $\ldots$ & $\ldots$ & $\ldots$ & $\ldots$ & ?f \\
\hline IC 0843 & $20.30 \pm 0.05$ & $19.06 \pm 0.03$ & $1.24 \pm 0.06$ & $\ldots$ & $19.25 \pm 0.05$ & $\ldots$ & 34.88 & 35.20 & $\ldots$ & $\ldots$ & $\ldots$ & $\ldots$ & $\ldots$ & $\ldots$ & VV \\
\hline IC 4088 & $17.51 \pm 0.01$ & $17.06 \pm 0.01$ & $0.45 \pm 0.01$ & $17.64 \pm 0.02$ & $17.22 \pm 0.01$ & $0.42 \pm 0.02$ & 35.96 & 35.97 & 13.63 & 13.90 & 2.20 & 2.46 & 2.17 & $\ldots$ & $? \mathrm{~F}$ \\
\hline NGC 4952 & $.35 \pm 0.15$ & $17.90 \pm 0.05$ & $1.46 \pm 0.16$ & $19.51 \pm 0.08$ & $18.24 \pm 0.03$ & $1.27 \pm 0.09$ & 35.08 & 35.48 & $\ldots$ & & $\ldots$ & $\ldots$ & $\ldots$ & $\ldots$ & ER \\
\hline UGC 08195 & $64 \pm 0.01$ & $17.09 \pm 0.02$ & $0.55 \pm$ & $18.65 \pm 0.02$ & $18.22 \pm 0.01$ & $0.43 \pm 0.02$ & 35.90 & & $11.4^{7}$ & & 2.43 & 2.68 & 2.41 & 2.69 & $\mathrm{Ef}$ \\
\hline DDO 165 & & & & $14.42 \pm 0.01$ & & & & & 41.72 & & 1.93 & 1.99 & 1.81 & 1.89 & $\mathrm{EF}$ \\
\hline NGC 5004 & 19.81 & & $1.73=$ & $19.92 \pm 0.08$ & $18.42 \pm$ & $1.51 \pm$ & 35.04 & 35.56 & $\ldots$ & 15.92 & $\ldots$ & $\ldots$ & $\ldots$ & $\ldots$ & VV \\
\hline NGC $5004 \mathrm{C}$ & $18.36 \pm 0.06$ & $17.48 \pm 0.02$ & $0.88 \pm 0.06$ & $18.61 \pm 0.03$ & $17.88 \pm 0.01$ & $0.73 \pm 0.03$ & 35.64 & 35.82 & 19.10 & 20.75 & 1.83 & 2.44 & 1.70 & 2.62 & $? \mathrm{fn}$ \\
\hline UGC 08313 & $16.33 \pm 0.01$ & $15.88 \pm 0.01$ & $0.45 \pm 0.01$ & $16.48 \pm 0.01$ & $16.12 \pm 0.01$ & $0.36 \pm 0.02$ & 34.57 & 34.57 & 9.87 & 11.44 & $\ldots$ & 3.27 & $\ldots$ & $\ldots$ & $\mathrm{Ef}$ \\
\hline UGCA 342 & $16.90 \pm 0.11$ & $16.90 \pm 0.18$ & $0.01 \pm 0.21$ & $17.72 \pm 0.03$ & $17.70 \pm 0.02$ & $0.03 \pm 0.03$ & 34.00 & 33.83 & 30.83 & 29.19 & 4.35 & 4.03 & $\ldots$ & 3.63 & ER \\
\hline NGC 5055 & $12.41 \pm 0.01$ & $11.89 \pm 0.01$ & $0.53 \pm 0.01$ & $12.50 \pm 0.01$ & $11.96 \pm 0.01$ & $0.54 \pm 0.01$ & 35.80 & 35.83 & 108.47 & 96.85 & 2.62 & 3.04 & 2.67 & 3.10 & ER \\
\hline UGC 08340 & $16.47 \pm 0.01$ & $16.10 \pm 0.01$ & $0.37 \pm 0.01$ & $16.70 \pm 0.03$ & $16.30 \pm 0.01$ & $0.40 \pm 0.03$ & 36.17 & 36.14 & 15.45 & 14.40 & $\ldots$ & $\ldots$ & $\ldots$ & $\ldots$ & Ef \\
\hline IC 4218 & $16.54 \pm 0.01$ & $16.09 \pm 0.01$ & $0.45 \pm 0.02$ & $16.71 \pm 0.01$ & $16.29 \pm 0.01$ & $0.42 \pm 0.02$ & 36.16 & 36.17 & 7.11 & 8.31 & $\ldots$ & $\ldots$ & $\ldots$ & $\ldots$ & Er \\
\hline
\end{tabular}


Table 3-Continued

\begin{tabular}{|c|c|c|c|c|c|c|c|c|c|c|c|c|c|c|c|}
\hline \multirow{2}{*}{$\begin{array}{c}\text { Object Name } \\
\text { (1) }\end{array}$} & \multicolumn{3}{|c|}{ Asymptotic magnitudes and color } & \multicolumn{3}{|c|}{ D25 magnitudes and color } & \multicolumn{2}{|c|}{$\log L$} & \multicolumn{2}{|c|}{ Effective Radii } & \multicolumn{2}{|c|}{ C31 } & \multicolumn{2}{|c|}{$\mathrm{C} 42$} & \multirow{2}{*}{$\begin{array}{c}\mathrm{UV} \\
\text { profile } \\
(16)\end{array}$} \\
\hline & $\begin{array}{c}\text { FUV } \\
(\mathrm{mag}) \\
(2)\end{array}$ & $\begin{array}{c}\text { NUV } \\
(\mathrm{mag}) \\
(3)\end{array}$ & $\begin{array}{l}\text { FUV-NUV } \\
\text { (mag) } \\
(4)\end{array}$ & $\begin{array}{c}\text { FUV } \\
(\mathrm{mag}) \\
(5)\end{array}$ & $\begin{array}{c}\text { NUV } \\
(\mathrm{mag}) \\
(6)\end{array}$ & $\begin{array}{l}\text { FUV-NUV } \\
(\mathrm{mag}) \\
(7)\end{array}$ & $\begin{array}{c}\text { FUV } \\
(\mathrm{W}) \\
(8)\end{array}$ & $\begin{array}{l}\text { NUV } \\
(\mathrm{W}) \\
(9)\end{array}$ & $\begin{array}{c}\text { FUV } \\
(\operatorname{arcsec}) \\
(10)\end{array}$ & $\begin{array}{c}\text { NUV } \\
\text { (arcsec) } \\
(11)\end{array}$ & $\begin{array}{l}\text { FUV } \\
(12)\end{array}$ & $\begin{array}{l}\text { NUV } \\
(13)\end{array}$ & $\begin{array}{l}\text { FUV } \\
(14)\end{array}$ & $\begin{array}{l}\text { NUV } \\
(15)\end{array}$ & \\
\hline (1) & . & 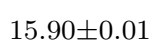 & & 1 & & & & & & & 1 & 5 & 77 & 9 & LL \\
\hline 4229 & & $.67 \pm 0.01$ & & $42 \pm 0.02$ & 1 & 2 & 36.46 & 36.49 & & & & & & & $\mathrm{EE}$ \\
\hline entaurus A & $67 \pm 0.01$ & $.61 \pm 0.01$ & $6 \pm 0.01$ & $.69 \pm 0.01$ & $.57 \pm 0.01$ & $1.12 \pm 0.01$ & 35.44 & 35.68 & 105.23 & 58 & 3.32 & 3.90 & 2.96 & 10 & $\mathrm{ERh}$ \\
\hline NGC 5169 & $64 \pm 0.01$ & $.27 \pm 0.01$ & & $.66 \pm 0.01$ & $31 \pm 0.01$ & & 35.84 & 35.81 & 17.67 & & 2.39 & .52 & 2.39 & 2.57 & EFn \\
\hline IC 4263 & 1 & & & $.89 \pm 0.02$ & 1 & 0 & 35.39 & 35.35 & 15.72 & & 2.05 & 2.07 & 1.98 & 1.98 & $\mathrm{EF}$ \\
\hline MESSIEI & & & & & & & & & & & 1.82 & & 1.81 & & EFn \\
\hline [ESSIEF & 4 & & & & & & & & 110.40 & 3 & 3.06 & 8 & 2.93 & 3.18 & EEn \\
\hline NGC 5231 & $170-$ & 1 & & & & & & & & & & & & $\ldots$ & VV,ER \\
\hline FSO 444 & & & & & & & & & & & & & 2.55 & & $\mathrm{EE}$ \\
\hline MESSIEI & & & & & & & & & & & 81 & & 88 & & $\mathrm{xEFn}$ \\
\hline & & & & & & & & & & & 2.06 & 3 & 1.94 & 1.95 & $\mathrm{ED}, \mathrm{EFn}$ \\
\hline & & & & & & & & & & & $\cdots$ & & $\ldots$ & $\ldots$ & $\mathrm{VV}$ \\
\hline UG & 2 & & & 3 & & & 35.84 & 35 . & & & 1.82 & 2.12 & 1.68 & 2.13 & $? \mathrm{Fn}$ \\
\hline $\mathrm{UGC} 0$ & & & & 2 & & & 35.82 & 35.81 & & & 2.52 & 2.70 & 2.55 & 2.70 & Ef \\
\hline & .02 & & & $.91 \pm$ & & & 35.91 & 35.93 & & & 1.93 & 2.13 & 1.75 & 2.07 & $\mathrm{ED}, \mathrm{EF}$ \\
\hline UGC 088 & $62 \pm 0.02$ & $16.43=$ & 2 & $.80 \pm 0.02$ & $.59 \pm$ & $0.21 \pm$ & 35.94 & 35.84 & 12 & 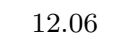 & $\ldots$ & $\ldots$ & $\ldots$ & $\ldots$ & $\mathrm{EF}$ \\
\hline NGC 5398 & $14.20 \pm 0.01$ & $13.76 \pm 0.01$ & & $4.25 \pm 0.01$ & $13.83 \pm 0.01$ & $0.42 \pm 0.01$ & 35.66 & 35.66 & & & 1.64 & 1.81 & 1.43 & 1.67 & $\mathrm{EF}$ \\
\hline MESSIEI & & & & 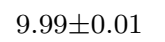 & & & & & & & 2.34 & 2.43 & 2.35 & & Efn \\
\hline & & & & 0.25 & & $1.66 \pm 0.26$ & & & 13.12 & & $\ldots$ & 2.79 & $\ldots$ & 2.78 & $\mathrm{EE}$ \\
\hline & & & & & & & 33.19 & 34. & & & $\ldots$ & $\ldots$ & $\ldots$ & $\ldots$ & Er \\
\hline & . & 1 & 1 & $16+0$ & 1 & 1 & 35.43 & 35.30 & 84.30 & 8 & 2.55 & 2.57 & 2.63 & 2.68 & $\mathrm{EE}$ \\
\hline $\mathrm{N}$ & 1 & $14.80 \pm 0.01$ & 01 & $01 \pm 0.01$ & $91 \pm$ & 0.1 & 34.75 & 34.61 & 20.2 & 1 & 2.71 & 2.75 & $\ldots$ & $\ldots$ & Efn \\
\hline UGC 09120 & $77 \pm 0.01$ & $15.38 \pm 0.01$ & $0.38 \pm 0.01$ & $.84 \pm 0.01$ & $15.47 \pm 0.01$ & $0.37 \pm 0.01$ & 36.47 & 36.45 & 14.37 & 14.20 & $\ldots$ & $\ldots$ & $\ldots$ & $\ldots$ & $\mathrm{ED}$ \\
\hline UGC 09140 & $.99 \pm 0.23$ & $17.55 \pm 0.10$ & $0.44 \pm 0.25$ & $19.01 \pm 0.07$ & $18.42 \pm 0.03$ & $0.59 \pm 0.07$ & 36.47 & 36.47 & 15.72 & 13.24 & $\ldots$ & 2.64 & $\ldots$ & 2.57 & $\mathrm{Ef}$ \\
\hline NGC 5560 & $.07 \pm 0.01$ & $15.39 \pm 0.01$ & & $.18 \pm 0.01$ & $5.57 \pm 0.01$ & $0.61 \pm 0.01$ & 35.33 & 35.43 & & & 2.24 & 2.55 & 2.29 & 2.61 & VD \\
\hline C 556 & -8 & $14.73 \pm 0.01$ & & $.65 \pm 0.01$ & $14.82 \pm 0$ & $0.83 \pm 0.01$ & 35.42 & 35.58 & & & 2.57 & 3.03 & 2.52 & 3.05 & EEn \\
\hline$a a_{-}$ & $71 \pm 0.01$ & $15.36 \pm 0.01$ & $0.35 \pm$ & $15.92 \pm 0.01$ & $15.59 \pm 0.01$ & $0.33 \pm 0.01$ & 35.50 & 35.46 & 25.24 & & 2.50 & 2.59 & 2.53 & 2.64 & Ef \\
\hline & $6 \pm 0.07$ & $17.02 \pm 0.01$ & & $\ldots$ & $17.18 \pm 0.02$ & 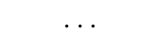 & & & . & & $\ldots$ & $\ldots$ & $\ldots$ & $\ldots$ & VV \\
\hline NGC 5576 & $17.50 \pm 0.08$ & $15.42 \pm 0.02$ & $2.08 \pm 0.08$ & $17.74 \pm 0.04$ & $15.81 \pm 0.01$ & $1.93 \pm 0.04$ & 34.63 & 35.29 & 34.53 & 42.65 & $\ldots$ & 7.31 & $\ldots$ & $\ldots$ & VV \\
\hline
\end{tabular}


Table 3-Continued

\begin{tabular}{|c|c|c|c|c|c|c|c|c|c|c|c|c|c|c|c|}
\hline \multirow{2}{*}{$\begin{array}{l}\text { Object Name } \\
\text { (1) }\end{array}$} & \multicolumn{3}{|c|}{ Asymptotic magnitudes and color } & \multicolumn{3}{|c|}{ D25 magnitudes and color } & \multicolumn{2}{|c|}{$\log L$} & \multicolumn{2}{|c|}{ Effective Radii } & \multicolumn{2}{|c|}{ C31 } & \multicolumn{2}{|c|}{$\mathrm{C} 42$} & \multirow{2}{*}{$\begin{array}{c}\text { UV } \\
\text { profile } \\
(16)\end{array}$} \\
\hline & $\begin{array}{c}\text { FUV } \\
(\mathrm{mag}) \\
(2)\end{array}$ & $\begin{array}{c}\text { NUV } \\
(\mathrm{mag}) \\
(3)\end{array}$ & $\begin{array}{l}\text { FUV-NUV } \\
(\mathrm{mag}) \\
(4)\end{array}$ & $\begin{array}{l}\text { FUV } \\
(\mathrm{mag}) \\
(5)\end{array}$ & $\begin{array}{c}\text { NUV } \\
(\mathrm{mag}) \\
(6)\end{array}$ & $\begin{array}{l}\text { FUV-NUV } \\
(\mathrm{mag}) \\
(7)\end{array}$ & $\begin{array}{c}\text { FUV } \\
(\mathrm{W}) \\
(8)\end{array}$ & $\begin{array}{l}\text { NUV } \\
(\mathrm{W}) \\
(9)\end{array}$ & $\begin{array}{c}\text { FUV } \\
(\operatorname{arcsec}) \\
(10)\end{array}$ & $\begin{array}{c}\text { NUV } \\
\text { (arcsec) } \\
(11)\end{array}$ & $\begin{array}{l}\text { FUV } \\
(12)\end{array}$ & $\begin{array}{l}\text { NUV } \\
(13)\end{array}$ & $\begin{array}{l}\text { FUV } \\
\text { (14) }\end{array}$ & $\begin{array}{l}\text { NUV } \\
(15)\end{array}$ & \\
\hline NGC 5577 & $62 \pm 0.01$ & $15.06 \pm 0.01$ & $0.56 \pm 0.01$ & $65 \pm 0.01$ & $15.10 \pm 0.01$ & $0.55 \pm 0$ & 35.39 & 35.43 & 23.31 & 23.62 & 2.17 & 2.29 & 2.16 & 2.28 & VFh \\
\hline UGC 09215 & $33 \pm 0.01$ & $14.08 \pm 0.01$ & $0.25 \pm 0.01$ & $14.45 \pm 0.01$ & $14.19 \pm 0.01$ & $0.26 \pm 0.01$ & 35.84 & 35.77 & 21.86 & & 2.88 & 2.87 & 2.96 & 2.98 & $\mathrm{EE}$ \\
\hline NGC 5619 & $16.67 \pm 0.02$ & $16.13 \pm 0.01$ & $0.55 \pm 0.02$ & $16.71 \pm 0.02$ & $16.21 \pm 0.01$ & $0.51 \pm 0.02$ & 36.43 & 36.47 & 26.62 & 26.39 & 2.05 & 2.10 & 1.80 & 1.89 & $? \mathrm{Fh}$ \\
\hline UGC 09277 & $18.46 \pm 0.05$ & $17.71 \pm 0.03$ & $0.74 \pm 0.06$ & $19.02 \pm 0.03$ & $18.29 \pm 0.02$ & $0.72 \pm 0.04$ & 35.74 & 35.86 & 13.07 & 13.12 & 2.01 & 2.21 & 1.93 & 2.22 & $\mathrm{EF}$ \\
\hline UGC 09285 & $18.02 \pm 0.04$ & $17.16 \pm 0.03$ & $0.86 \pm 0.05$ & $18.15 \pm 0.03$ & $17.51 \pm 0.01$ & $0.64 \pm 0.03$ & 34.62 & 34.79 & 7.35 & 10.86 & $\ldots$ & 3.22 & $\ldots$ & $\ldots$ & $\mathrm{Ef}$ \\
\hline NGC 5646 & $17.36 \pm 0.01$ & $16.80 \pm 0.01$ & $0.56 \pm 0.02$ & $17.58 \pm 0.01$ & $17.05 \pm 0.01$ & $0.53 \pm 0.02$ & 36.19 & 36.24 & 14.01 & 14.03 & 2.05 & 2.19 & 1.95 & 2.12 & $? \mathrm{~F}$ \\
\hline NGC 5636 & $17.54 \pm 0.02$ & $16.53 \pm 0.02$ & $1.01 \pm 0.03$ & $17.56 \pm 0.03$ & $16.72 \pm 0.01$ & $0.85 \pm 0.03$ & 34.75 & 34.98 & 16.73 & 18.74 & 1.98 & 2.75 & $\ldots$ & $\ldots$ & ERn \\
\hline NGC 5638 & $17.99 \pm 0.08$ & $16.13 \pm 0.04$ & $1.86 \pm 0.09$ & $18.03 \pm 0.05$ & $16.46 \pm 0.01$ & $1.57 \pm 0.05$ & 34.58 & 35.15 & 22.43 & 35.31 & $\ldots$ & 5.68 & $\ldots$ & $\ldots$ & VV \\
\hline UGC 09305 & $17.12 \pm 0.01$ & $16.89 \pm 0.01$ & $0.23 \pm 0.01$ & $17.53 \pm 0.02$ & $17.27 \pm 0.01$ & $0.27 \pm 0.02$ & 35.18 & 35.09 & 8.62 & 8.05 & $\ldots$ & $\ldots$ & $\ldots$ & $\ldots$ & $\mathrm{EE}$ \\
\hline UGC 09310 & $16.73 \pm 0.03$ & $16.20 \pm 0.01$ & $0.53 \pm 0.04$ & $16.86 \pm 0.02$ & $16.38 \pm 0.01$ & $0.48 \pm 0.02$ & 35.12 & 35.16 & 15.06 & 15.77 & 2.57 & 2.65 & 2.68 & 2.69 & Ef \\
\hline IC 1022 & $16.74 \pm 0.01$ & $16.38 \pm 0.01$ & $0.36 \pm 0.02$ & $16.99 \pm 0.02$ & $16.69 \pm 0.01$ & $0.30 \pm 0.02$ & 35.06 & 35.03 & 9.22 & 9.95 & $\ldots$ & $\ldots$ & $\ldots$ & $\ldots$ & $\mathrm{EE}$ \\
\hline NGC 5656 & $15.57 \pm 0.01$ & $15.03 \pm 0.01$ & $0.54 \pm 0.01$ & $15.63 \pm 0.01$ & $15.10 \pm 0.01$ & $0.54 \pm 0.01$ & 36.07 & 36.11 & 17.01 & 15.60 & $\ldots$ & $\ldots$ & $\ldots$ & $\ldots$ & $\mathrm{ER}$ \\
\hline UGC 09338 & $17.38 \pm 0.05$ & $16.97 \pm 0.04$ & $0.41 \pm 0.06$ & $17.81 \pm 0.03$ & $17.46 \pm 0.01$ & $0.35 \pm 0.03$ & 36.13 & 36.12 & 13 & 13. & 2.33 & 2.64 & $\ldots$ & $\ldots$ & $\mathrm{EF}$ \\
\hline IC 1024 & $16.48 \pm 0.05$ & $15.95 \pm 0.02$ & $0.53 \pm 0.06$ & $16.63 \pm 0.02$ & $16.12 \pm 0.01$ & $0.50 \pm 0.02$ & 35.03 & 35.06 & 9.64 & 10.33 & $\ldots$ & $\ldots$ & $\ldots$ & $\ldots$ & $\mathrm{ER}$ \\
\hline UGC 09380 & $16.09 \pm 0.02$ & $15.93 \pm 0.02$ & $0.16 \pm 0.03$ & $16.28 \pm 0.02$ & $16.13 \pm 0.01$ & $0.15 \pm 0.02$ & 35.31 & 35.20 & 24.70 & 24.23 & 2.88 & 2.81 & 3.00 & 2.93 & ?Fn \\
\hline UGC 09382 & $19.34 \pm 0.10$ & $18.47 \pm 0.05$ & $0.86 \pm 0.11$ & $19.67 \pm 0.06$ & $18.78 \pm 0.02$ & $0.89 \pm 0.07$ & 35.40 & 35.57 & 9.23 & 8.36 & 2.55 & $\ldots$ & $\ldots$ & $\ldots$ & $\mathrm{Ef}$ \\
\hline UGC 09432 & $16.28 \pm 0.03$ & $16.11 \pm 0.02$ & $0.18 \pm 0.04$ & $16.70 \pm 0.02$ & $16.52 \pm 0.01$ & $0.18 \pm 0.02$ & 35.17 & 35.06 & 19.98 & 19.58 & 2.41 & 2.56 & 2.55 & 2.72 & $\mathrm{EF}$ \\
\hline NGC 5701 & $14.98 \pm 0.01$ & $14.61 \pm 0.01$ & $0.37 \pm 0.01$ & $15.15 \pm 0.01$ & $14.76 \pm 0.01$ & $0.39 \pm 0.01$ & 35.66 & 35.63 & 98.27 & 95.00 & 1.38 & 1.56 & 0.91 & 1.48 & $? \mathrm{Fn}$ \\
\hline NGC 5705 & $14.78 \pm 0.01$ & $14.44 \pm 0.01$ & $0.34 \pm 0.01$ & $14.87 \pm 0.01$ & $14.52 \pm 0.01$ & $0.35 \pm 0.01$ & 35.88 & 35.84 & 40.81 & 39.65 & 2.09 & 2.20 & 2.04 & 2.23 & $? \mathrm{Fn}$ \\
\hline NGC 5713 & $14.62 \pm 0.01$ & $13.90 \pm 0.01$ & $0.72 \pm 0.01$ & $14.64 \pm 0.01$ & $13.92 \pm 0.01$ & $0.72 \pm 0.01$ & 35.95 & 36.06 & 15.04 & 15.93 & $\ldots$ & $\ldots$ & $\ldots$ & $\ldots$ & ER \\
\hline NGC 5727 & $15.22 \pm 0.01$ & $14.99 \pm 0.01$ & $0.23 \pm 0.01$ & $15.31 \pm 0.01$ & $15.06 \pm 0.01$ & $0.25 \pm 0.01$ & 35.62 & 35.54 & 20.56 & 20.52 & 2.67 & 2.64 & 2.64 & 2.62 & $\mathrm{EF}$ \\
\hline NGC 5719 & $16.66 \pm 0.03$ & $15.77 \pm 0.03$ & $0.88 \pm 0.04$ & $17.46 \pm 0.04$ & $16.52 \pm 0.02$ & $0.94 \pm 0.05$ & 35.13 & 35.31 & 59.05 & 56.42 & 2.85 & 3.73 & 2.97 & 3.71 & $\mathrm{xVV}$ \\
\hline UGC 09463 & $20.44 \pm 0.01$ & $18.79 \pm 0.02$ & $1.65 \pm 0.03$ & $\ldots$ & $19.06 \pm 0.02$ & $\ldots$ & 34.88 & 35.37 & $\ldots$ & & $\ldots$ & $\ldots$ & $\ldots$ & $\ldots$ & Ef \\
\hline UGC 09479 & $17.27 \pm 0.01$ & $16.83 \pm 0.01$ & $0.44 \pm 0.01$ & $17.44 \pm 0.02$ & $17.02 \pm 0.01$ & $0.42 \pm 0$ & 36.17 & & 14.10 & & 2.07 & 2.20 & 1.95 & 2.18 & $\mathrm{EF}$ \\
\hline UGC 09491 & $17.27 \pm 0$ & $16.87 \pm 0$ & $0.40 \pm$ & $18.09 \pm 0.04$ & $17.65 \pm$ & $0.45 \pm 0.04$ & 36.12 & & 31.14 & 30.46 & 1.95 & 2.22 & 1.80 & 2.09 & EDn \\
\hline IC 1063 & $16.99 \pm 0.03$ & $16.35 \pm 0.01$ & $0.65 \pm 0.03$ & $17.04 \pm 0.02$ & $16.40 \pm 0.01$ & $0.64 \pm 0.02$ & 36.74 & 36.82 & 13.08 & 12.46 & $\ldots$ & $\ldots$ & $\ldots$ & $\ldots$ & Ef \\
\hline NGC 5770 & $19.45 \pm 0.36$ & $16.95 \pm 0.02$ & $2.50 \pm 0.36$ & $\ldots$ & $17.08 \pm 0.01$ & $\ldots$ & 33.86 & 34.69 & $\ldots$ & 13.64 & $\ldots$ & $\ldots$ & $\cdots$ & $\ldots$ & $\mathrm{EV}$ \\
\hline IC 1071 & $19.45 \pm 0.08$ & $18.21 \pm 0.05$ & $1.24 \pm 0.09$ & $19.78 \pm 0.11$ & $18.60 \pm 0.03$ & $1.18 \pm 0.12$ & 35.31 & 35.63 & $\ldots$ & 13.95 & $\ldots$ & $\ldots$ & $\ldots$ & $\ldots$ & $\mathrm{VV}$ \\
\hline UGC 09584 & $17.60 \pm 0.03$ & $17.14 \pm 0.01$ & $0.47 \pm 0.03$ & $17.84 \pm 0.03$ & $17.37 \pm 0.01$ & $0.46 \pm 0.03$ & 36.06 & 36.07 & 10.93 & 10.26 & $\ldots$ & $\ldots$ & $\ldots$ & $\ldots$ & $\mathrm{EF}$ \\
\hline NGC 5832 & $\ldots$ & $14.16 \pm 0.01$ & $\ldots$ & $\ldots$ & $14.27 \pm 0.01$ & $\ldots$ & 0.00 & 35.15 & $\ldots$ & 44.36 & $\ldots$ & 2.27 & $\ldots$ & 2.21 & $\mathrm{EF}$ \\
\hline NGC 5806 & $15.37 \pm 0.01$ & $14.68 \pm 0.01$ & $0.70 \pm 0.01$ & $15.43 \pm 0.01$ & $14.74 \pm 0.01$ & $0.70 \pm 0.02$ & 35.41 & 35.51 & 27.87 & 26.40 & 1.95 & 2.15 & 1.96 & 2.25 & EDn \\
\hline NGC 5813 & $16.86 \pm 0.07$ & $15.56 \pm 0.04$ & $1.29 \pm 0.08$ & $17.08 \pm 0.05$ & $15.93 \pm 0.02$ & $1.15 \pm 0.06$ & 35.11 & 35.46 & 39.42 & 54.95 & 6.89 & 5.35 & .. & 4.52 & $\mathrm{VV}$ \\
\hline
\end{tabular}


Table 3-Continued

\begin{tabular}{|c|c|c|c|c|c|c|c|c|c|c|c|c|c|c|c|}
\hline \multirow{2}{*}{$\begin{array}{l}\text { Object Name } \\
\text { (1) }\end{array}$} & \multicolumn{3}{|c|}{ Asymptotic magnitudes and color } & \multicolumn{3}{|c|}{ D25 magnitudes and color } & \multicolumn{2}{|c|}{$\log L$} & \multicolumn{2}{|c|}{ Effective Radii } & \multicolumn{2}{|c|}{ C31 } & \multicolumn{2}{|c|}{$\mathrm{C} 42$} & \multirow{2}{*}{$\begin{array}{l}\text { UV } \\
\text { profile } \\
(16)\end{array}$} \\
\hline & $\begin{array}{c}\text { FUV } \\
(\mathrm{mag}) \\
(2)\end{array}$ & $\begin{array}{c}\text { NUV } \\
(\mathrm{mag}) \\
(3)\end{array}$ & $\begin{array}{l}\text { FUV-NUV } \\
\text { (mag) } \\
(4)\end{array}$ & $\begin{array}{c}\text { FUV } \\
(\mathrm{mag}) \\
(5)\end{array}$ & $\begin{array}{l}\text { NUV } \\
(\mathrm{mag}) \\
(6)\end{array}$ & $\begin{array}{l}\text { FUV-NUV } \\
(\mathrm{mag}) \\
(7)\end{array}$ & $\begin{array}{c}\text { FUV } \\
(\mathrm{W}) \\
(8)\end{array}$ & $\begin{array}{c}\text { NUV } \\
(\mathrm{W}) \\
(9)\end{array}$ & $\begin{array}{c}\text { FUV } \\
\text { (arcsec) } \\
(10)\end{array}$ & $\begin{array}{c}\text { NUV } \\
(\operatorname{arcsec}) \\
(11)\end{array}$ & $\begin{array}{l}\text { FUV } \\
(12)\end{array}$ & $\begin{array}{l}\text { NUV } \\
(13)\end{array}$ & $\begin{array}{l}\text { FUV } \\
(14)\end{array}$ & $\begin{array}{l}\text { NUV } \\
(15)\end{array}$ & \\
\hline UGC 09661 & $.57 \pm 0.02$ & $15.27 \pm 0.01$ & $0.29 \pm 0.02$ & $15.94 \pm 0.02$ & $15.67 \pm 0.01$ & $0.26 \pm 0$ & 35.26 & 35.20 & 16.37 & 16.72 & 2.60 & 2.64 & 2.62 & 2.66 & $\mathrm{EE}$ \\
\hline NGC 5866 & $88 \pm 0.02$ & $14.86 \pm 0.01$ & $2.02 \pm 0.03$ & $17.05 \pm 0.03$ & $15.15 \pm 0.01$ & $1.90 \pm 0.03$ & 34.55 & 35.19 & 26.26 & 47 & 4.43 & 4.66 & 4.10 & 4.09 & VV \\
\hline NGC 5826 & $19.63 \pm 0.37$ & $17.93 \pm 0.05$ & $1.71 \pm 0.38$ & $\ldots$ & $18.18 \pm 0.03$ & $\ldots$ & 33.47 & 33.98 & 15.96 & 14.61 & $\ldots$ & $\ldots$ & $\ldots$ & $\ldots$ & VV \\
\hline IC 1102 & $18.29 \pm 0.30$ & $17.60 \pm 0.05$ & $0.69 \pm 0.30$ & $18.52 \pm 0.08$ & $17.96 \pm 0.04$ & $0.56 \pm 0.09$ & 36.13 & 36.23 & 16.81 & 16.53 & 1.84 & 2.30 & 1.65 & $\ldots$ & $\ldots$ \\
\hline NGC 5894 & $16.94 \pm 0.02$ & $16.19 \pm 0.02$ & $0.76 \pm 0.03$ & $17.17 \pm 0.02$ & $16.47 \pm 0.01$ & $0.71 \pm 0.02$ & 35.35 & 35.47 & 21.53 & 20.60 & 1.94 & 2.32 & 1.87 & 2.33 & EFn \\
\hline IRAS $15250+3609$ & $\ldots$ & $17.51 \pm 0.06$ & $\ldots$ & $\ldots$ & $17.79 \pm 0.02$ & $\ldots$ & 0.00 & 36.51 & $\ldots$ & $\ldots$ & $\ldots$ & $\ldots$ & $\ldots$ & $\ldots$ & $\ldots$ \\
\hline UGC 09912 & $\cdots$ & $15.38 \pm 0.01$ & $\ldots$ & $\ldots$ & $15.47 \pm 0.01$ & $\ldots$ & 0.00 & 35.07 & $\ldots$ & 23.17 & $\ldots$ & 2.32 & $\ldots$ & $\ldots$ & EFn \\
\hline NGC 5962 & $\ldots$ & $13.59 \pm 0.01$ & $\ldots$ & $\ldots$ & $13.64 \pm 0.01$ & $\ldots$ & 0.00 & 36.28 & $\ldots$ & 27.51 & $\ldots$ & 2.69 & $\ldots$ & 2.75 & $\mathrm{EE}$ \\
\hline UGC 09925 & $\cdots$ & $17.08 \pm 0.02$ & $\ldots$ & $\ldots$ & $17.14 \pm 0.02$ & $\ldots$ & 0.00 & 34.87 & $\ldots$ & 12.58 & $\ldots$ & $\ldots$ & $\ldots$ & $\ldots$ & $\mathrm{EF}$ \\
\hline NGC 5972 & $\ldots$ & $17.82 \pm 0.03$ & $\ldots$ & $\ldots$ & $18.04 \pm 0.02$ & $\ldots$ & 0.00 & 35.86 & $\ldots$ & $\ldots$ & $\ldots$ & $\ldots$ & $\ldots$ & $\ldots$ & $\mathrm{ER}$ \\
\hline UGC 09953 & $16.51 \pm 0.01$ & $16.26 \pm 0.01$ & $0.25 \pm 0.02$ & $16.80 \pm 0.03$ & $16.52 \pm 0.01$ & $0.28 \pm 0.03$ & 36.66 & 36.58 & 18.20 & 17.32 & 2.16 & 2.28 & 2.15 & $\ldots$ & ED \\
\hline UGC 10043 & $\ldots$ & $17.17 \pm 0.03$ & $\ldots$ & $\ldots$ & $17.46 \pm 0.01$ & $\ldots$ & 0.00 & 34.94 & $\ldots$ & 18.32 & $\ldots$ & 2.48 & $\ldots$ & 2.50 & $\mathrm{EE}$ \\
\hline UGC 10109 & $\ldots$ & $16.33 \pm 0.02$ & $\ldots$ & $\ldots$ & $16.71 \pm 0.01$ & $\ldots$ & 0.00 & 36.10 & $\ldots$ & 8 & $\ldots$ & 2.30 & $\ldots$ & 2.47 & $\mathrm{EE}$ \\
\hline UGC 10153 & $\ldots$ & $16.69 \pm 0.02$ & $\ldots$ & $\ldots$ & $16.83 \pm 0.01$ & $\ldots$ & 0.00 & 36.58 & $\ldots$ & 14.35 & $\ldots$ & $\ldots$ & $\ldots$ & $\ldots$ & $\mathrm{EF}$ \\
\hline NGC 6036 & $18.48 \pm 0.10$ & $17.31 \pm 0.02$ & $1.17 \pm 0.10$ & $18.54 \pm 0.10$ & $17.46 \pm 0.03$ & $1.08 \pm 0.11$ & 35.35 & 35.64 & 9.04 & 9.40 & $\ldots$ & $\ldots$ & $\ldots$ & $\ldots$ & $\mathrm{EE}$ \\
\hline NGC 6052 & $\ldots$ & $14.26 \pm 0.01$ & $\ldots$ & $\ldots$ & $14.33 \pm 0.01$ & $\ldots$ & 0.00 & 36.74 & $\ldots$ & $\ldots$ & $\ldots$ & $\ldots$ & $\ldots$ & ... & $\mathrm{ER}$ \\
\hline UGC 10197 & $\ldots$ & $16.93 \pm 0.03$ & $\ldots$ & $\ldots$ & $17.21 \pm 0.02$ & $\ldots$ & 0.00 & 35.69 & $\ldots$ & 13.80 & $\ldots$ & 2.38 & $\ldots$ & $\ldots$ & $\mathrm{EF}$ \\
\hline UGC 10198 & $\ldots$ & $16.28 \pm 0.02$ & $\ldots$ & $\ldots$ & $16.42 \pm 0.01$ & $\ldots$ & 0.00 & 35.92 & $\ldots$ & 12.82 & $\ldots$ & $\ldots$ & $\ldots$ & $\ldots$ & $\mathrm{EF}$ \\
\hline UGC 10245 & $19.18 \pm 0.04$ & $18.31 \pm 0.09$ & $0.87 \pm 0.10$ & $19.88 \pm 0.08$ & $19.25 \pm 0.03$ & $0.63 \pm 0.08$ & 35.84 & 36.01 & 12.32 & 14.90 & 2.34 & $\ldots$ & 2.27 & $\ldots$ & $\mathrm{ED}, \mathrm{EF}$ \\
\hline CGCG 023-019 & $16.53 \pm 0.06$ & $16.19 \pm 0.01$ & $0.33 \pm 0.06$ & $16.87 \pm 0.04$ & $16.43 \pm 0.02$ & $0.44 \pm 0.04$ & 36.49 & 36.45 & 23.51 & 20.97 & 2.38 & 2.31 & 2.42 & 2.35 & $\mathrm{EF}$ \\
\hline UGC 10261 & $\ldots$ & $19.41 \pm 0.41$ & $\ldots$ & $\ldots$ & $19.78 \pm 0.10$ & $\ldots$ & 0.00 & 35.88 & $\ldots$ & 12.87 & $\ldots$ & $\ldots$ & $\ldots$ & $\ldots$ & VV \\
\hline NGC 6090 & $15.81 \pm 0.01$ & $15.21 \pm 0.01$ & $0.60 \pm 0.01$ & $15.91 \pm 0.01$ & $15.34 \pm 0.01$ & $0.58 \pm 0.01$ & 36.83 & 36.90 & $\ldots$ & $\ldots$ & $\ldots$ & $\ldots$ & $\ldots$ & $\ldots$ & VV \\
\hline UGC 10278 & $16.70 \pm 0.01$ & $16.17 \pm 0.01$ & $0.52 \pm 0.01$ & $16.77 \pm 0.02$ & $16.28 \pm 0.01$ & $0.49 \pm 0.02$ & 35.80 & 35.83 & $\ldots$ & $\ldots$ & $\ldots$ & $\ldots$ & $\ldots$ & $\ldots$ & VF \\
\hline NGC 6100 & $16.26 \pm 0.03$ & $15.92 \pm 0.01$ & $0.34 \pm 0.03$ & $16.58 \pm 0.02$ & $16.24 \pm 0.01$ & $0.34 \pm 0.02$ & 36.13 & 36.09 & 31.32 & 30.90 & 1.72 & 1.84 & 1.51 & 1.72 & $? \mathrm{~F}$ \\
\hline IC 4595 & $\ldots$ & $16.10 \pm 0.03$ & $\ldots$ & $\ldots$ & $16.43 \pm 0.02$ & $\ldots$ & 0.00 & 35.63 & $\ldots$ & 22.94 & $\ldots$ & 2.23 & $\ldots$ & 2.19 & $\mathrm{EF}$ \\
\hline NGC 6154 & $16.65 \pm 0.03$ & $16.34 \pm 0.01$ & $0.31 \pm 0.03$ & $16.71 \pm 0.02$ & $16.38 \pm 0.01$ & $0.32 \pm 0.02$ & 36.18 & 36.13 & 25.48 & 25.19 & 2.00 & 1.95 & 1.87 & 1.88 & EFn \\
\hline NGC 6155 & $15.59 \pm 0.01$ & $15.00 \pm 0.01$ & $0.58 \pm 0.01$ & $15.68 \pm 0.01$ & $15.10 \pm 0.01$ & $0.58 \pm 0.01$ & 35.87 & 35.93 & 13.39 & 13.18 & $\ldots$ & $\ldots$ & $\ldots$ & $\ldots$ & $\mathrm{VF}$ \\
\hline UGC 10404 & $18.76 \pm 0.06$ & $17.74 \pm 0.03$ & $1.02 \pm 0.07$ & $\ldots$ & $17.96 \pm 0.02$ & $\ldots$ & 35.58 & 35.81 & $\ldots$ & 12.13 & $\ldots$ & $\ldots$ & $\ldots$ & $\ldots$ & Er \\
\hline NGC 6166 & $18.09 \pm 0.09$ & $17.15 \pm 0.04$ & $0.95 \pm 0.10$ & $18.47 \pm 0.06$ & $17.65 \pm 0.02$ & $0.83 \pm 0.06$ & 35.97 & 36.17 & 21.18 & 33.04 & $\ldots$ & 5.38 & $\ldots$ & $\ldots$ & Er \\
\hline UGC 10420 & $16.02 \pm 0.01$ & $15.87 \pm 0.01$ & $0.15 \pm 0.01$ & $16.22 \pm 0.01$ & $16.08 \pm 0.01$ & $0.14 \pm 0.02$ & 36.80 & 36.69 & 26.19 & 25.83 & 1.87 & 1.97 & 1.79 & 1.95 & $? \mathrm{D}$ \\
\hline UGC 10445 & $14.85 \pm 0.01$ & $14.64 \pm 0.01$ & $0.21 \pm 0.01$ & $14.99 \pm 0.01$ & $14.78 \pm 0.01$ & $0.21 \pm 0.01$ & 35.44 & 35.35 & 32.42 & 32.05 & 1.97 & 2.01 & 1.89 & 1.98 & EDn \\
\hline IC 1221 & $15.85 \pm 0.01$ & $15.59 \pm 0.01$ & $0.25 \pm 0.02$ & $15.96 \pm 0.01$ & $15.71 \pm 0.01$ & $0.25 \pm 0.01$ & 36.42 & 36.34 & 18.84 & 18.27 & 2.41 & $\ldots$ & $\ldots$ & $\ldots$ & $? \mathrm{~F}$ \\
\hline
\end{tabular}


Table 3-Continued

\begin{tabular}{|c|c|c|c|c|c|c|c|c|c|c|c|c|c|c|c|}
\hline \multirow{2}{*}{$\begin{array}{l}\text { Object Name } \\
\text { (1) }\end{array}$} & \multicolumn{3}{|c|}{ Asymptotic magnitudes and color } & \multicolumn{3}{|c|}{ D25 magnitudes and color } & \multicolumn{2}{|c|}{$\log L$} & \multicolumn{2}{|c|}{ Effective Radii } & \multicolumn{2}{|c|}{ C31 } & \multicolumn{2}{|c|}{$\mathrm{C} 42$} & \multirow{2}{*}{$\begin{array}{c}\text { UV } \\
\text { profile } \\
(16)\end{array}$} \\
\hline & $\begin{array}{l}\text { FUV } \\
(\mathrm{mag}) \\
(2)\end{array}$ & $\begin{array}{c}\text { NUV } \\
(\mathrm{mag}) \\
(3)\end{array}$ & $\begin{array}{l}\text { FUV-NUV } \\
\text { (mag) } \\
(4)\end{array}$ & $\begin{array}{l}\text { FUV } \\
(\mathrm{mag}) \\
(5)\end{array}$ & $\begin{array}{c}\text { NUV } \\
(\mathrm{mag}) \\
(6)\end{array}$ & $\begin{array}{l}\text { FUV-NUV } \\
\text { (mag) } \\
(7)\end{array}$ & $\begin{array}{l}\text { FUV } \\
(\mathrm{W}) \\
(8)\end{array}$ & $\begin{array}{c}\text { NUV } \\
(\mathrm{W}) \\
(9)\end{array}$ & $\begin{array}{c}\text { FUV } \\
(\operatorname{arcsec}) \\
(10)\end{array}$ & $\begin{array}{c}\text { NUV } \\
\text { (arcsec) } \\
(11)\end{array}$ & $\begin{array}{l}\text { FUV } \\
(12)\end{array}$ & $\begin{array}{l}\text { NUV } \\
(13)\end{array}$ & $\begin{array}{l}\text { FUV } \\
(14)\end{array}$ & $\begin{array}{l}\text { NUV } \\
(15)\end{array}$ & \\
\hline IC 1222 & $.17 \pm 0.01$ & $15.72 \pm 0.01$ & $0.45 \pm 0.01$ & $21 \pm 0.01$ & $15.76 \pm 0.01$ & 2 & 36.73 & 36.73 & 18 & 18.04 & & $\cdots$ & $\cdots$ & $\cdots$ & $? \mathrm{~F}$ \\
\hline UGC 10468 & $.55 \pm 0.07$ & $17.78 \pm 0.08$ & $0.76 \pm 0.11$ & $.37 \pm 0.03$ & $18.81 \pm 0.01$ & $0.55 \pm 0.03$ & 35.77 & 35.90 & 8.97 & 10.53 & 2.36 & $\ldots$ & $\ldots$ & $\ldots$ & $\mathrm{EE}$ \\
\hline UGC 10491 & $17.56 \pm 0.02$ & $16.92 \pm 0.01$ & $0.64 \pm 0.02$ & $17.82 \pm 0.03$ & $17.23 \pm 0.01$ & $0.59 \pm 0.03$ & 36.07 & 36.15 & 8.62 & 9.12 & 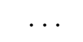 & $\cdots$ & $\cdots$ & $\cdots$ & $\mathrm{ER}$ \\
\hline NGC 6239 & $14.50 \pm 0.01$ & $14.18 \pm 0.01$ & $0.32 \pm 0.01$ & $14.64 \pm 0.01$ & $14.30 \pm 0.01$ & $0.34 \pm 0.01$ & 35.59 & 35.54 & 15.57 & 15.55 & $\ldots$ & $\ldots$ & $\ldots$ & $\ldots$ & VV \\
\hline Mrk 501 & $15.51 \pm 0.01$ & $15.06 \pm 0.01$ & $0.45 \pm 0.01$ & $15.57 \pm 0.01$ & $15.12 \pm 0.01$ & $0.45 \pm 0.01$ & 37.07 & 37.07 & $\ldots$ & $\ldots$ & $\ldots$ & $\ldots$ & $\ldots$ & $\ldots$ & VV \\
\hline UGC 10600 & $18.80 \pm 0.05$ & $18.00 \pm 0.05$ & $0.79 \pm 0.07$ & $20.21 \pm 0.06$ & $19.42 \pm 0.02$ & $0.79 \pm 0.07$ & 35.70 & 35.85 & 13.46 & 14.29 & 2.70 & $\ldots$ & $\ldots$ & $\ldots$ & $\mathrm{EF}$ \\
\hline NGC 6255 & $14.35 \pm 0.01$ & $14.17 \pm 0.01$ & $0.17 \pm 0.01$ & $14.42 \pm 0.01$ & $14.24 \pm 0.01$ & $0.18 \pm 0.01$ & 35.63 & 35.53 & 41.39 & 40.42 & 1.66 & 1.80 & 1.59 & 1.79 & ?Fn \\
\hline UGC 10651 & $16.60 \pm 0.02$ & $16.21 \pm 0.01$ & $0.40 \pm 0.02$ & $17.24 \pm 0.02$ & $16.80 \pm 0.01$ & $0.43 \pm 0.03$ & 36.46 & 36.44 & 23.93 & 22.73 & 3.04 & 3.14 & 2.96 & 3.11 & $\mathrm{EE}$ \\
\hline UGC 10687 & $16.56 \pm 0.01$ & $16.19 \pm 0.01$ & $0.36 \pm 0.02$ & $16.65 \pm 0.02$ & $16.33 \pm 0.01$ & $0.32 \pm 0.02$ & 36.11 & 36.08 & 12.06 & 12.78 & & $\ldots$ & $\ldots$ & $\ldots$ & $\mathrm{EF}$ \\
\hline UGC 10713 & $16.85 \pm 0.02$ & $15.96 \pm 0.02$ & $0.89 \pm 0.03$ & $17.12 \pm 0.02$ & $16.34 \pm 0.01$ & $0.78 \pm 0$ & 34.78 & 34 & 12.26 & 14.04 & 2.75 & 2.90 & 2.74 & 2.93 & $\mathrm{EF}$ \\
\hline NGC 6306 & $16.48 \pm 0.01$ & $15.99 \pm 0.01$ & $0.49 \pm 0$ & $16.55 \pm 0.01$ & $16.10 \pm 0.01$ & $0.44 \pm 0$ & 35.68 & & & & $\ldots$ & & $\ldots$ & $\ldots$ & $\mathrm{Ef}$ \\
\hline NGC 6307 & $19.22 \pm 0.13$ & $17.56 \pm 0.01$ & $1.65 \pm 0.13$ & $19.45 \pm 0.09$ & $17.80 \pm 0.02$ & $1.65 \pm 0.09$ & 34.61 & 35.09 & 16.66 & 16.86 & $\ldots$ & $\ldots$ & $\ldots$ & $\ldots$ & Er \\
\hline UGC 10729 & $17.48 \pm 0.01$ & $16.84 \pm 0.01$ & $0.64 \pm 0.01$ & $17.64 \pm 0.03$ & $17.09 \pm 0.01$ & $0.55 \pm 0.03$ & 36.20 & 36.28 & 7.80 & 9.02 & $\cdots$ & $\ldots$ & $\cdots$ & $\ldots$ & $\mathrm{EE}$ \\
\hline IC 1251 & $15.77 \pm 0.01$ & $15.36 \pm 0.01$ & $0.41 \pm 0.01$ & $15.86 \pm 0.01$ & $15.48 \pm 0.01$ & $0.38 \pm 0.02$ & 35.29 & 35.27 & 14.70 & 15.35 & $\ldots$ & $\ldots$ & $\ldots$ & $\ldots$ & $\mathrm{EF}$ \\
\hline NGC 6340 & $17.39 \pm 0.01$ & $15.83 \pm 0.01$ & $1.56 \pm 0.02$ & $\ldots$ & $15.92 \pm 0.01$ & & 34.64 & 35.09 & 35.48 & 8.01 & 3.28 & 3.24 & 3.23 & 3.24 & $\mathrm{EE}$ \\
\hline IC 1254 & $18.38 \pm 0.07$ & $17.08 \pm 0.03$ & $1.29 \pm 0.08$ & $18.70 \pm 0.07$ & $17.56 \pm 0.02$ & $1.14 \pm 0.08$ & 34.29 & 34.63 & 21.75 & 21.83 & 2.03 & 3.28 & 2.07 & 3.31 & $\mathrm{EE}$ \\
\hline IC 1248 & $16.11 \pm 0.01$ & $15.79 \pm 0.01$ & $0.32 \pm 0.01$ & $16.42 \pm 0.01$ & $16.06 \pm 0.01$ & $0.35 \pm 0.02$ & 36.25 & 36.20 & 20.76 & 19.01 & $\ldots$ & $\ldots$ & $\ldots$ & $\ldots$ & $\mathrm{EE}$ \\
\hline UGC 10770 & $15.12 \pm 0.01$ & $15.08 \pm 0.01$ & $0.04 \pm 0.01$ & $15.48 \pm 0.01$ & $15.48 \pm 0.01$ & $0.00 \pm 0.01$ & 35.49 & 35.33 & 15.53 & 15.95 & 2.85 & 2.99 & $\ldots$ & $\ldots$ & $\mathrm{EE}$ \\
\hline UGC 10791 & $16.99 \pm 0.05$ & $16.53 \pm 0.01$ & $0.46 \pm 0.05$ & $17.39 \pm 0.03$ & $16.89 \pm 0.01$ & $0.51 \pm 0.03$ & 34.87 & 34.88 & 26.52 & 25.96 & 2.55 & 2.45 & 2.51 & 2.41 & EFn \\
\hline NGC 6330 & $17.79 \pm 0.05$ & $17.36 \pm 0.02$ & $0.43 \pm 0.06$ & $17.99 \pm 0.04$ & $17.60 \pm 0.02$ & $0.39 \pm 0.05$ & 36.03 & 36.02 & 17.05 & 16.42 & 1.74 & 2.22 & 1.58 & 2.18 & $? \mathrm{~F}$ \\
\hline UGC 10783 & $17.60 \pm 0.11$ & $17.11 \pm 0.01$ & $0.50 \pm 0.11$ & $17.85 \pm 0.03$ & $17.33 \pm 0.02$ & $0.52 \pm 0.04$ & 36.14 & 36.16 & 11.55 & 1146 & $\ldots$ & $\ldots$ & $\ldots$ & $\ldots$ & $? \mathrm{~F}$ \\
\hline UGC 10796 & $.16 \pm 0.01$ & $15.85 \pm 0.01$ & $0.31 \pm 0.01$ & $16.21 \pm 0.01$ & $15.92 \pm 0.01$ & $0.29 \pm$ & 35.84 & & 12.59 & 7 & $\ldots$ & $\ldots$ & $\ldots$ & $\ldots$ & EFn \\
\hline NGC 6359 & $19.03 \pm 0.11$ & $17.55 \pm 0.06$ & $1.48 \pm 0.12$ & $19.13 \pm 0.06$ & $17.88 \pm 0.02$ & $1.25 \pm 0.07$ & 34.66 & 35.07 & $\ldots$ & 11.19 & $\ldots$ & $\ldots$ & $\ldots$ & $\ldots$ & VV \\
\hline UGC 10795 & $17.09 \pm 0.03$ & $16.83 \pm 0.01$ & $0.26 \pm 0.03$ & $17.36 \pm 0.03$ & $17.11 \pm 0.02$ & $0.25 \pm 0.04$ & 35.75 & 35.67 & 23.22 & 22.41 & 2.14 & 2.31 & 2.08 & 2.32 & $? \mathrm{~F}$ \\
\hline NGC 6361 & $17.32 \pm 0.05$ & $16.69 \pm 0.03$ & $0.63 \pm 0.06$ & $17.69 \pm 0.02$ & $16.99 \pm 0.01$ & $0.70 \pm 0.03$ & 35.54 & 35.62 & 23.65 & 21.83 & 2.35 & 2.44 & 2.33 & 2.45 & $\mathrm{ED}, \mathrm{EF}$ \\
\hline UGC 10811 & $17.61 \pm 0.02$ & $17.20 \pm 0.01$ & $0.42 \pm 0.02$ & $17.73 \pm 0.02$ & $17.31 \pm 0.01$ & $0.42 \pm 0.02$ & 36.11 & 36.10 & 15.76 & 14.99 & 1.76 & 1.97 & 1.63 & 1.86 & ED \\
\hline NGC 6373 & $15.85 \pm 0.01$ & $15.58 \pm 0.01$ & $0.27 \pm 0.01$ & $16.02 \pm 0.01$ & $15.76 \pm 0.01$ & $0.25 \pm 0.01$ & 36.02 & 35.95 & 18.21 & 18.31 & 2.32 & 2.41 & $\ldots$ & $\ldots$ & $\mathrm{Ef}$ \\
\hline NGC 6364 & $19.68 \pm 0.25$ & $18.08 \pm 0.04$ & $1.60 \pm 0.25$ & $\ldots$ & $18.25 \pm 0.05$ & $\ldots$ & 35.07 & 35.54 & $\ldots$ & 14.15 & $\ldots$ & $\ldots$ & $\ldots$ & $\ldots$ & VV \\
\hline UGC 10842 & $17.88 \pm 0.18$ & $17.37 \pm 0.06$ & $0.51 \pm 0.19$ & $18.53 \pm 0.06$ & $17.94 \pm 0.02$ & $0.59 \pm 0.06$ & 36.42 & 36.45 & 18.55 & 16.70 & $\ldots$ & 3.43 & $\ldots$ & $\ldots$ & $\mathrm{EE}$ \\
\hline UGC 10872 & $18.67 \pm 0.13$ & $17.66 \pm 0.06$ & $1.01 \pm 0.14$ & $19.61 \pm 0.04$ & $18.89 \pm 0.02$ & $0.72 \pm 0.04$ & 34.82 & 35.05 & 11.26 & 13.56 & $\ldots$ & $\ldots$ & $\ldots$ & $\ldots$ & Ef \\
\hline UGC 10888 & $16.97 \pm 0.03$ & $16.45 \pm 0.01$ & $0.52 \pm 0.03$ & $17.06 \pm 0.03$ & $16.52 \pm 0.01$ & $0.53 \pm 0.03$ & 36.07 & 36.11 & 13.68 & 12.60 & $\ldots$ & $\ldots$ & $\ldots$ & $\ldots$ & $? \mathrm{~F}$ \\
\hline NGC 6394 & $18.12 \pm 0.02$ & $17.39 \pm 0.01$ & $0.73 \pm 0.02$ & $18.21 \pm 0.04$ & $17.52 \pm 0.02$ & $0.69 \pm 0.04$ & 35.88 & 36.00 & 8.39 & 9.28 & $\ldots$ & $\ldots$ & $\ldots$ & $\ldots$ & Ef \\
\hline
\end{tabular}


Table 3-Continued

\begin{tabular}{|c|c|c|c|c|c|c|c|c|c|c|c|c|c|c|c|}
\hline \multirow{2}{*}{$\begin{array}{l}\text { Object Name } \\
\text { (1) }\end{array}$} & \multicolumn{3}{|c|}{ Asymptotic magnitudes and color } & \multicolumn{3}{|c|}{ D25 magnitudes and color } & \multicolumn{2}{|c|}{$\log L$} & \multicolumn{2}{|c|}{ Effective Radii } & \multicolumn{2}{|c|}{$\mathrm{C} 31$} & \multicolumn{2}{|c|}{$\mathrm{C} 42$} & \multirow{2}{*}{$\begin{array}{c}\mathrm{UV} \\
\text { profile } \\
(16)\end{array}$} \\
\hline & $\begin{array}{l}\text { FUV } \\
(\mathrm{mag}) \\
(2)\end{array}$ & $\begin{array}{c}\text { NUV } \\
(\mathrm{mag}) \\
(3)\end{array}$ & $\begin{array}{c}\text { FUV-NUV } \\
\text { (mag) } \\
(4)\end{array}$ & $\begin{array}{c}\text { FUV } \\
(\mathrm{mag}) \\
(5)\end{array}$ & $\begin{array}{c}\text { NUV } \\
(\mathrm{mag}) \\
(6)\end{array}$ & $\begin{array}{l}\text { FUV-NUV } \\
(\mathrm{mag}) \\
(7)\end{array}$ & $\begin{array}{c}\text { FUV } \\
(\mathrm{W}) \\
(8)\end{array}$ & $\begin{array}{c}\text { NUV } \\
(\mathrm{W}) \\
(9)\end{array}$ & $\begin{array}{c}\text { FUV } \\
(\operatorname{arcsec}) \\
(10)\end{array}$ & $\begin{array}{c}\text { NUV } \\
(\operatorname{arcsec}) \\
(11)\end{array}$ & $\begin{array}{l}\text { FUV } \\
(12)\end{array}$ & $\begin{array}{l}\text { NUV } \\
(13)\end{array}$ & $\begin{array}{l}\text { FUV } \\
(14)\end{array}$ & $\begin{array}{l}\text { NUV } \\
(15)\end{array}$ & \\
\hline UGC 10895 & 2 & 17.44 & $3 \pm 0$. & $52 \pm 0$ & $17.90 \pm 0$ & $2 \pm 0$ & 35.87 & & 0.02 & 9 & & & & & Ef \\
\hline UGC 10935 & & $18.77 \pm 0.30$ & & & $19.18 \pm 0.06$ & & & & & & $\ldots$ & & $\ldots$ & & $\mathrm{EE}$ \\
\hline UGC 10971 & $18.54 \pm 0.19$ & $17.94 \pm 0.05$ & $0.60 \pm 0.20$ & $18.78 \pm 0.06$ & $18.09 \pm 0.02$ & $0.69 \pm 0.07$ & 35.74 & 35.80 & 11.46 & 9.96 & $\ldots$ & $\ldots$ & $\ldots$ & $\ldots$ & $? \mathrm{~F}$ \\
\hline NGC 6482 & $18.45 \pm 0.09$ & $16.19 \pm 0.02$ & $2.27 \pm 0.10$ & $18.55 \pm 0.11$ & $16.30 \pm 0.02$ & $2.25 \pm 0.11$ & 35.09 & 35.82 & $\ldots$ & 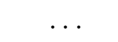 & $\ldots$ & $\ldots$ & $\ldots$ & $\ldots$ & $\mathrm{VV}$ \\
\hline IC 4836 & $15.47 \pm 0.01$ & $14.93 \pm 0.01$ & $0.54 \pm 0.02$ & $15.51 \pm 0.01$ & $14.96 \pm 0.01$ & $0.55 \pm 0.02$ & 36.24 & 36.28 & 16.63 & 16.50 & 1.75 & 1.88 & $\ldots$ & $\ldots$ & $\mathrm{VF}$ \\
\hline NGC 6789 & $16.22 \pm 0.01$ & $15.61 \pm 0.01$ & $0.61 \pm 0.01$ & $16.28 \pm 0.02$ & $15.72 \pm 0.01$ & $0.57 \pm 0.03$ & 33.56 & 33.63 & $\ldots$ & $\ldots$ & $\ldots$ & $\ldots$ & $\ldots$ & $\ldots$ & $\mathrm{VV}$ \\
\hline NGC 6769 & $15.34 \pm 0.01$ & $14.80 \pm 0.01$ & $0.54 \pm 0.01$ & $15.37 \pm 0.01$ & $14.84 \pm 0.01$ & $0.53 \pm 0.01$ & 36.22 & 36.26 & 31.38 & 30.52 & 1.53 & 1.71 & 1.19 & 1.51 & ?Fn \\
\hline NGC 6770 & $15.48 \pm 0.04$ & $15.00 \pm 0.04$ & $0.48 \pm 0.06$ & $15.61 \pm 0.01$ & $15.20 \pm 0.01$ & $0.42 \pm 0.01$ & 36.17 & 36.18 & 36.58 & 37.51 & 1.79 & 1.85 & 1.53 & 1.64 & ?Fn \\
\hline NGC 6771 & $19.00 \pm 0.53$ & $17.42 \pm 0.02$ & $1.58 \pm 0.53$ & ... & $17.71 \pm 0.02$ & .. & 34.84 & 35.30 & 12.95 & 16.60 & 3.81 & 3.74 & $\ldots$ & 3.50 & VV \\
\hline IC 4842 & $43 \pm 0.17$ & $17.45=$ & $1.98 \pm 0$ & $19.41 \pm 0.15$ & $17.84 \pm 0.02$ & $1.57 \pm 0.15$ & 34.64 & 35.26 & $\ldots$ & 21.24 & $\ldots$ & $\ldots$ & $\ldots$ & $\ldots$ & VV \\
\hline IC 4845 & $15.64 \pm 0.01$ & $15.08 \pm 0.01$ & $0.56 \pm 0.01$ & $15.88 \pm 0.01$ & $15.31 \pm 0.01$ & $0.57 \pm 0.02$ & 36.13 & 36.18 & 23.18 & 21.83 & 3.30 & 3.50 & $\ldots$ & $\ldots$ & $\mathrm{EEh}, \mathrm{EE}$ \\
\hline NGC 6782 & $15.57 \pm 0.25$ & $14.95 \pm 0.17$ & $0.62 \pm 0.30$ & $15.91 \pm 0.02$ & $15.21 \pm 0.01$ & $0.71 \pm 0.02$ & 36.11 & 36.18 & 22.46 & 19.28 & $\ldots$ & $\ldots$ & $\ldots$ & $\ldots$ & ?Fn \\
\hline Superanten & $28 \pm 0.13$ & $18.46 \pm 0.10$ & & $19.29 \pm 0.17$ & $18.49 \pm 0$ & $0.80 \pm 0$ & 36.05 & & & $\ldots$ & $\ldots$ & $\ldots$ & $\ldots$ & $\ldots$ & $\ldots$ \\
\hline NGC 6845A & $45 \pm 0.01$ & $15.00 \pm 0.01$ & & $15.50 \pm 0.01$ & $15.03 \pm 0.01$ & & 36.66 & & 20.65 & 19.68 & 2.93 & 2.90 & 2.86 & 2.82 & EEh \\
\hline IC 4946 & $18.80 \pm 0.20$ & $16.42 \pm 0.01$ & $2.38 \pm$ & $18.86 \pm 0.09$ & $16.63 \pm 0.01$ & $2.23 \pm 0.09$ & 34.60 & 35.38 & 13.17 & 19.04 & $\ldots$ & 4.24 & $\ldots$ & $\ldots$ & $\mathrm{EF}$ \\
\hline NGC 6902 & $14.04 \pm 0.01$ & $13.66 \pm 0.01$ & $0.38 \pm 0.01$ & $14.32 \pm 0.01$ & $13.99 \pm 0.01$ & $0.34 \pm 0.01$ & 36.47 & 36.45 & 89.34 & 87.91 & 2.63 & 3.33 & 3.03 & 3.99 & $\ldots$ \\
\hline ESO 285-G009 & $16.47 \pm 0.01$ & $16.18 \pm 0.01$ & $0.29 \pm 0.01$ & $16.60 \pm 0.01$ & $16.36 \pm 0.01$ & $0.24 \pm 0.02$ & 35.59 & 35.53 & 13.45 & 14.06 & 2.14 & 2.22 & $\ldots$ & $\ldots$ & VV \\
\hline PGC 65022 & & $17.77 \pm 0.03$ & $\ldots$ & ... & $18.23 \pm 0.02$ & & 0.00 & 35.49 & $\ldots$ & 17.02 & $\ldots$ & 2.78 & $\ldots$ & 2.75 & $\mathrm{EF}$ \\
\hline NGC 6941 & $15.85 \pm 0.03$ & $15.49 \pm 0.01$ & $0.36 \pm 0.03$ & $16.10 \pm 0.01$ & $15.69 \pm 0.01$ & $0.41 \pm 0.02$ & 36.50 & 36.46 & 30.60 & 28.57 & 2.21 & 2.29 & 2.13 & 2.28 & $\mathrm{EE}$ \\
\hline NGC 6951 & $14.62 \pm 0.18$ & $13.43 \pm 0.11$ & $1.19 \pm 0.21$ & $\cdots$ & & & 35.86 & 36.16 & 44.62 & 47.71 & 1.93 & 2.32 & 1.83 & 2.45 & EFn \\
\hline NGC 6945 & $19.05 \pm 0.36$ & $17.49 \pm 0.02$ & $1.56 \pm 0.36$ & $.53 \pm 0$ & $17.73 \pm 0$ & $1.79 \pm 0$ & 34.79 & & & & 4.39 & $\ldots$ & $\ldots$ & $\ldots$ & $\mathrm{EV}$ \\
\hline PGC 65158 & $17.06 \pm 0.02$ & $16.64 \pm 0$ & $0.41 \pm$ & $17.38 \pm 0$ & $16.98 \pm 0$ & & 35.63 & & & & 2.32 & 2.41 & 2.28 & 2.40 & $? \mathrm{~F}$ \\
\hline UGC 116 & & 18.04 & & $19.57 \pm($ & & & 35.42 & & & & 2.51 & 2.32 & $\ldots$ & 2.36 & $\mathrm{EE}$ \\
\hline PGC 65328 & $16.74 \pm 0$ & $16.38 \pm 0.01$ & $0.36 \pm 0.03$ & $16.82 \pm 0.03$ & $16.47 \pm 0.01$ & $0.35 \pm 0.04$ & 36.42 & 36.39 & 19.30 & & $\ldots$ & $\ldots$ & $\ldots$ & $\ldots$ & EFn \\
\hline ESO $341-G 0$ & $\ldots$ & $18.22 \pm 0.07$ & $\ldots$ & $\cdots$ & $18.53 \pm 0.06$ & $\ldots$ & 0.00 & 35.44 & $\ldots$ & 14.09 & $\ldots$ & $\ldots$ & $\ldots$ & $\ldots$ & $\mathrm{VV}$ \\
\hline NGC 6962 & $15.69 \pm 0.01$ & $15.12 \pm 0.01$ & $0.57 \pm 0.02$ & $15.80 \pm 0.02$ & $15.28 \pm 0.01$ & $0.52 \pm 0.02$ & 36.23 & 36.28 & 56.60 & 55.74 & 1.52 & 1.69 & 1.23 & 1.54 & $\ldots$ \\
\hline NGC 6964 & $19.05 \pm 0.16$ & $17.53 \pm 0.05$ & $1.52 \pm 0.16$ & $19.17 \pm 0.24$ & $17.78 \pm 0.04$ & $1.40 \pm 0.24$ & 34.80 & 35.23 & 19.09 & 19.50 & $\ldots$ & $\ldots$ & $\ldots$ & $\ldots$ & VV \\
\hline PGC 65420 & $16.35 \pm 0.04$ & $16.23 \pm 0.01$ & $0.12 \pm 0.04$ & $16.41 \pm 0.04$ & $16.27 \pm 0.01$ & $0.13 \pm 0.04$ & 36.28 & 36.15 & 12.41 & 11.83 & $\ldots$ & $\ldots$ & $\ldots$ & $\ldots$ & $\mathrm{EF}$ \\
\hline NGC 6958 & $18.58 \pm 0.11$ & $16.46 \pm 0.04$ & $2.12 \pm 0.12$ & $\ldots$ & $16.56 \pm 0.02$ & $\ldots$ & 34.63 & 35.30 & $\ldots$ & 17.88 & $\ldots$ & $\ldots$ & $\ldots$ & $\ldots$ & VV \\
\hline UGC 11646 & $17.73 \pm 0.03$ & $17.02 \pm 0.02$ & $0.70 \pm 0.04$ & $18.07 \pm 0.04$ & $17.39 \pm 0.01$ & $0.68 \pm 0.04$ & 36.03 & 36.14 & 12.99 & 12.74 & 2.28 & 2.55 & $\ldots$ & $\ldots$ & $\mathrm{Ef}$ \\
\hline
\end{tabular}


Table 3-Continued

\begin{tabular}{|c|c|c|c|c|c|c|c|c|c|c|c|c|c|c|c|}
\hline \multirow{2}{*}{$\begin{array}{l}\text { Object Name } \\
\text { (1) }\end{array}$} & \multicolumn{3}{|c|}{ Asymptotic magnitudes and color } & \multicolumn{3}{|c|}{ D25 magnitudes and color } & \multicolumn{2}{|c|}{$\log L$} & \multicolumn{2}{|c|}{ Effective Radii } & \multicolumn{2}{|c|}{ C31 } & \multicolumn{2}{|c|}{$\mathrm{C} 42$} & \multirow{2}{*}{$\begin{array}{r}\text { UV } \\
\text { profile } \\
(16)\end{array}$} \\
\hline & $\begin{array}{c}\text { FUV } \\
(\mathrm{mag}) \\
(2)\end{array}$ & $\begin{array}{c}\text { NUV } \\
(\mathrm{mag}) \\
(3)\end{array}$ & $\begin{array}{c}\text { FUV-NUV } \\
\text { (mag) } \\
(4)\end{array}$ & $\begin{array}{c}\text { FUV } \\
(\mathrm{mag}) \\
(5)\end{array}$ & $\begin{array}{c}\text { NUV } \\
(\mathrm{mag}) \\
(6)\end{array}$ & $\begin{array}{c}\text { FUV-NUV } \\
(\mathrm{mag}) \\
(7)\end{array}$ & $\begin{array}{c}\text { FUV } \\
(\mathrm{W}) \\
(8)\end{array}$ & $\begin{array}{c}\text { NUV } \\
(\mathrm{W}) \\
(9)\end{array}$ & $\begin{array}{c}\text { FUV } \\
\text { (arcsec) } \\
(10)\end{array}$ & $\begin{array}{c}\text { NUV } \\
\text { (arcsec) } \\
(11)\end{array}$ & $\begin{array}{l}\text { FUV } \\
(12)\end{array}$ & $\begin{array}{l}\text { NUV } \\
(13)\end{array}$ & $\begin{array}{l}\text { FUV } \\
(14)\end{array}$ & $\begin{array}{l}\text { NUV } \\
(15)\end{array}$ & \\
\hline PGC 66559 & .06 & 0.04 & I & $73 \pm 0.04$ & $15.42=$ & 0 & 35.90 & & 7 & 1 & 2.28 & & & & $\mathrm{EF}$ \\
\hline NGC 7080 & & .42 & & $.39 \pm 0.01$ & $15.48=$ & $0.92 \pm 0.02$ & & & & & 2.46 & 2.69 & 2.44 & & Efn \\
\hline UGC 11776 & $18.43 \pm 0.10$ & $17.59 \pm 0.03$ & $0.85 \pm 0.11$ & $18.99 \pm 0.08$ & $18.18 \pm 0.03$ & $0.81 \pm 0.09$ & 35.76 & 35.92 & 11.56 & 11.69 & 2.11 & 2.23 & 2.03 & 2.20 & $\mathrm{EE}$ \\
\hline PGC 67153 & $18.78 \pm 0.05$ & $17.93 \pm 0.02$ & $0.85 \pm 0.06$ & $\ldots$ & $17.99 \pm 0.03$ & $\cdots$ & 35.29 & 35.45 & 7.95 & 8.98 & $\cdots$ & $\ldots$ & $\cdots$ & $\cdots$ & $\mathrm{Ef}$ \\
\hline UGC 11789 & $17.35 \pm 0.03$ & $16.81 \pm 0.01$ & $0.54 \pm 0.03$ & $7.43 \pm 0.03$ & $16.94 \pm 0.01$ & $0.49 \pm 0.03$ & 36.21 & 36.25 & 9.80 & 10.93 & $\ldots$ & $\ldots$ & $\ldots$ & $\ldots$ & Ef \\
\hline Tol 2138-405 & $18.06 \pm 0.02$ & $18.04 \pm 0.03$ & $0.03 \pm 0.04$ & $\ldots$ & $\ldots$ & $\ldots$ & 36.46 & 36.29 & $\ldots$ & $\ldots$ & $\ldots$ & $\ldots$ & $\ldots$ & $\ldots$ & $\ldots$ \\
\hline ESO 343-G018 & $16.83 \pm 0.04$ & $16.37 \pm 0.02$ & $0.46 \pm 0.04$ & $17.03 \pm 0.02$ & $16.60 \pm 0.01$ & $0.42 \pm 0$ & 35.86 & 35.87 & 11.24 & 11.73 & 2.44 & 2.44 & 2.48 & 2.46 & $\mathrm{EF}$ \\
\hline UGC 11790 & $16.84 \pm 0.03$ & $16.25 \pm 0.01$ & $0.59 \pm 0.03$ & $16.95 \pm 0.03$ & $16.39 \pm 0.01$ & $0.56 \pm 0.03$ & 35.83 & 35.89 & 20.75 & 20.51 & 2.05 & 2.10 & 1.94 & 2.02 & ED \\
\hline UGC 11794 & $18.09 \pm 0.05$ & $17.39 \pm 0.03$ & $0.71 \pm 0.05$ & $18.37 \pm 0.05$ & $17.53 \pm 0.02$ & $0.83 \pm 0.05$ & 35.56 & 35.66 & 10.64 & 9.61 & $\ldots$ & $\ldots$ & $\ldots$ & $\ldots$ & $\mathrm{EE}$ \\
\hline ESO 466-G001 & $19.59 \pm 0.24$ & $18.37 \pm 0.14$ & $1.21 \pm 0.28$ & $19.93 \pm 0.14$ & $18.84 \pm 0.04$ & $1.09 \pm 0.14$ & 35.10 & 35.41 & 10.56 & 13.27 & 2.81 & 2.88 & 2.89 & 2.89 & $\mathrm{EF}$ \\
\hline ESO 466-G005 & $16.99 \pm 0.01$ & $16.53 \pm 0.01$ & $0.46 \pm 0.02$ & $17.22 \pm 0.03$ & $16.81 \pm 0.01$ & $0.41 \pm 0.03$ & 35.98 & 35.98 & 11.23 & 11.58 & $\ldots$ & $\ldots$ & $\ldots$ & $\ldots$ & $\mathrm{EE}$ \\
\hline UGC 11816 & $15.96 \pm 0.04$ & $15.65 \pm 0.02$ & $0.31 \pm 0.05$ & $16.16 \pm 0.02$ & $15.81 \pm 0.01$ & $0.35 \pm 0.02$ & 36.22 & 36.17 & 24.19 & 67 & 2.33 & 2.46 & 2.30 & 2.50 & $\mathrm{Ed}$ \\
\hline NGC 7152 & $16.79 \pm 0.02$ & $.34 \pm 0.01$ & $0.45 \pm 0.02$ & $16.88 \pm 0.02$ & $16.45 \pm 0.01$ & $0.44 \pm 0$ & 36.14 & 36.15 & 50 & & 1.67 & 1.82 & 1.52 & $\ldots$ & $? \mathrm{D}$ \\
\hline ESO 466-G014 & $19.29 \pm 0.10$ & $18.14 \pm 0.01$ & $1.16 \pm 0.10$ & $19.64 \pm 0.05$ & $18.70 \pm 0.02$ & $0.94 \pm 0.05$ & 34.25 & 34.53 & 9.46 & 11 & 2.57 & 2.72 & 2.55 & 2.71 & $\mathrm{EE}$ \\
\hline UGC 11859 & $69 \pm 0.12$ & $.09 \pm 0.02$ & $0.61 \pm 0.12$ & $18.17 \pm 0.06$ & $17.66 \pm 0.03$ & $0.51 \pm 0.06$ & 35.13 & 35.20 & 16.22 & 19.80 & 3.66 & 2.42 & 3.50 & 2.37 & $\mathrm{EE}$ \\
\hline ESO 404-G015 & $01 \pm 0.02$ & $.53 \pm 0.01$ & $0.48 \pm 0.02$ & $17.18 \pm 0.02$ & $16.70 \pm 0.01$ & $0.48 \pm 0.02$ & 35.71 & 35.73 & 9.16 & 8.79 & $\ldots$ & & & & $\mathrm{Ef}$ \\
\hline NGC 7167 & $14.84 \pm 0.01$ & $14.54 \pm 0.01$ & $0.29 \pm 0.02$ & $14.99 \pm 0.01$ & $14.69 \pm 0.01$ & $0.30 \pm 0.02$ & 36.10 & 36.04 & 23.02 & 22.37 & 2.28 & 2.43 & 2.30 & 2.48 & $\mathrm{EF}$ \\
\hline ESO 404-G023 & $15.96 \pm 0.07$ & $15.62 \pm 0.04$ & $0.34 \pm 0.08$ & $16.38 \pm 0.02$ & $15.98 \pm 0.01$ & $0.39 \pm 0.02$ & 36.14 & 36.10 & 28.75 & 05 & 2.41 & 2.54 & 2.38 & 2.52 & $\mathrm{EE}$ \\
\hline IC 5156 & $16.15 \pm 0.02$ & $15.46 \pm 0.01$ & $0.68 \pm 0.02$ & $16.24 \pm 0.02$ & $15.57 \pm 0.01$ & $0.67 \pm 0.02$ & 35.62 & 35.72 & 18.90 & 17.37 & 2.11 & 2.42 & 2.04 & 2.39 & $? \mathrm{D}$ \\
\hline NGC 7215 & $18.47 \pm 0.07$ & $17.61 \pm 0.02$ & $0.85 \pm 0.07$ & $\ldots$ & $17.65 \pm 0.02$ & $\ldots$ & 35.07 & 35.24 & $\ldots$ & $\ldots$ & $\ldots$ & $\ldots$ & $\ldots$ & $\ldots$ & $\mathrm{VV}$ \\
\hline NGC 7221 & $15.28 \pm 0.01$ & $14.97 \pm 0.01$ & $0.31 \pm 0.01$ & $15.54 \pm 0.01$ & $15.21 \pm 0.01$ & $0.33 \pm 0.02$ & 36.39 & 36.33 & 30.08 & 28.96 & 2.34 & 2.36 & 2.35 & 2.36 & $\mathrm{Ed}$ \\
\hline CGCG 377-039 & $17.67 \pm 0.02$ & $17.26 \pm 0.03$ & $0.41 \pm 0.04$ & $17.78 \pm 0.08$ & $17.36 \pm 0.03$ & $0.42 \pm 0.09$ & 36.18 & 36.17 & 13.00 & 12.75 & $\ldots$ & $\ldots$ & $\ldots$ & $\ldots$ & $\mathrm{EF}$ \\
\hline NGC 7248 & $19.15 \pm 0.46$ & $17.27 \pm 0.04$ & $1.89 \pm 0.46$ & $\ldots$ & $17.66 \pm 0.05$ & $\ldots$ & 34.91 & & 13.42 & 21.43 & $\ldots$ & $\ldots$ & $\ldots$ & $\ldots$ & $\mathrm{VV}$ \\
\hline NGC 7250 & $14.36 \pm 0.01$ & $13.95 \pm 0$ & $0.40=$ & $14.41 \pm 0$ & $14.06 \pm 0.01$ & $0.34 \pm 0$ & & & $\ldots$ & & $\ldots$ & $\ldots$ & $\ldots$ & $\ldots$ & $\mathrm{VV}$ \\
\hline NGC 7252 & & & & $16.48 \pm 0$ & $15.29 \pm 0$ & $19 \pm 0.02$ & 36.01 & 36.34 & $\ldots$ & $\ldots$ & $\ldots$ & $\ldots$ & $\ldots$ & $\ldots$ & $\mathrm{EV}$ \\
\hline ESO 467-G058 & $20.63 \pm 0.14$ & $19.13 \pm 0.04$ & $1.50 \pm 0.14$ & $\ldots$ & $19.34 \pm 0.04$ & $\ldots$ & 34.83 & 35.26 & 7.42 & 10.01 & $\ldots$ & $\ldots$ & $\ldots$ & $\ldots$ & $\mathrm{EE}$ \\
\hline ESO 345-G011 & $20.71 \pm 0.22$ & $19.14 \pm 0.04$ & $1.57 \pm 0.22$ & $20.83 \pm 0.22$ & $19.26 \pm 0.05$ & $1.56 \pm 0.23$ & 34.90 & 35.36 & $\ldots$ & 9.02 & $\ldots$ & $\ldots$ & $\ldots$ & $\ldots$ & $\mathrm{VV}$ \\
\hline NGC 7279 & $16.37 \pm 0.02$ & $15.90 \pm 0.01$ & $0.48 \pm 0.02$ & $16.49 \pm 0.02$ & $16.01 \pm 0.01$ & $0.48 \pm 0.02$ & 36.59 & 36.60 & 14.03 & 13.15 & $\ldots$ & $\ldots$ & $\ldots$ & $\ldots$ & $\mathrm{EF}$ \\
\hline PKS 2225-308 & $18.62 \pm 0.05$ & $17.77 \pm 0.10$ & $0.84 \pm 0.11$ & $18.73 \pm 0.06$ & $17.97 \pm 0.03$ & $0.76 \pm 0.07$ & 36.27 & 36.44 & 9.32 & 13.16 & $\ldots$ & 3.77 & $\ldots$ & $\ldots$ & ER \\
\hline NGC 7289 & $19.52 \pm 0.27$ & $18.08 \pm 0.10$ & $1.45 \pm 0.29$ & $19.67 \pm 0.16$ & $18.64 \pm 0.06$ & $1.04 \pm 0.17$ & 35.28 & 35.68 & 11.97 & 25.60 & $\ldots$ & $\ldots$ & $\ldots$ & $\ldots$ & VV \\
\hline ESO 468-G006 & $16.31 \pm 0.01$ & $16.06 \pm 0.01$ & $0.25 \pm 0.02$ & $16.52 \pm 0.02$ & $16.32 \pm 0.01$ & $0.20 \pm 0.02$ & 35.53 & 35.46 & 15.99 & 17.54 & $\ldots$ & $\ldots$ & $\ldots$ & $\ldots$ & EEh \\
\hline NGC 7317 & $19.00 \pm 0.77$ & $18.36 \pm 0.12$ & $0.64 \pm 0.78$ & $19.71 \pm 0.21$ & $18.97 \pm 0.08$ & $0.74 \pm 0.22$ & 35.31 & 35.39 & 28.68 & 23.36 & 3.35 & $\ldots$ & $\ldots$ & $\ldots$ & $\ldots$ \\
\hline
\end{tabular}


Table 3-Continued

\begin{tabular}{|c|c|c|c|c|c|c|c|c|c|c|c|c|c|c|c|}
\hline \multirow{2}{*}{$\begin{array}{c}\text { Object Name } \\
\text { (1) }\end{array}$} & \multicolumn{3}{|c|}{ Asymptotic magnitudes and color } & \multicolumn{3}{|c|}{ D25 magnitudes and color } & \multicolumn{2}{|c|}{$\log L$} & \multicolumn{2}{|c|}{ Effective Radii } & \multicolumn{2}{|c|}{$\mathrm{C} 31$} & \multicolumn{2}{|c|}{$\mathrm{C} 42$} & \multirow{2}{*}{$\begin{array}{r}\text { UV } \\
\text { profile } \\
(16)\end{array}$} \\
\hline & $\begin{array}{c}\text { FUV } \\
(\mathrm{mag}) \\
(2)\end{array}$ & $\begin{array}{c}\text { NUV } \\
(\mathrm{mag}) \\
(3)\end{array}$ & $\begin{array}{l}\text { FUV-NUV } \\
\text { (mag) } \\
(4)\end{array}$ & $\begin{array}{l}\text { FUV } \\
(\mathrm{mag}) \\
(5)\end{array}$ & $\begin{array}{l}\text { NUV } \\
(\mathrm{mag}) \\
(6)\end{array}$ & $\begin{array}{l}\text { FUV-NUV } \\
\text { (mag) } \\
(7)\end{array}$ & $\begin{array}{c}\text { FUV } \\
(\mathrm{W}) \\
(8)\end{array}$ & $\begin{array}{c}\text { NUV } \\
(\mathrm{W}) \\
(9)\end{array}$ & $\begin{array}{c}\text { FUV } \\
(\operatorname{arcsec}) \\
(10)\end{array}$ & $\begin{array}{c}\text { NUV } \\
(\operatorname{arcsec}) \\
(11)\end{array}$ & $\begin{array}{l}\text { FUV } \\
(12)\end{array}$ & $\begin{array}{l}\text { NUV } \\
(13)\end{array}$ & $\begin{array}{l}\text { FUV } \\
(14)\end{array}$ & $\begin{array}{l}\text { NUV } \\
(15)\end{array}$ & \\
\hline NGC 7320 & $15.03 \pm 0.01$ & $14.68 \pm 0.01$ & $0.36 \pm 0.01$ & $15.09 \pm 0.01$ & $14.74 \pm 0.01$ & $0.35 \pm 0.01$ & 35.18 & 35.15 & 22.43 & 22.23 & 1.84 & 1.95 & 1.72 & 1.91 & VF \\
\hline UGC 12110 & $18.03 \pm 0.02$ & $17.30 \pm 0.01$ & $0.73 \pm 0.02$ & $18.59 \pm 0.02$ & $17.93 \pm 0.01$ & $0.66 \pm 0.02$ & 36.12 & 36.24 & 10.35 & 10.78 & 2.40 & 2.60 & 2.46 & 2.69 & $? \mathrm{~F}$ \\
\hline NGC 7331 & $13.42 \pm 0.01$ & $12.72 \pm 0.01$ & $0.70 \pm 0.01$ & $13.47 \pm 0.01$ & $12.77 \pm 0.01$ & $0.69 \pm 0.01$ & 35.91 & 36.01 & 83.27 & 72.79 & 2.36 & 2.72 & 2.26 & 2.78 & $\mathrm{EF}$ \\
\hline NGC 7335 & $19.06 \pm 0.01$ & $17.86 \pm 0.04$ & $1.20 \pm 0.04$ & $\cdots$ & $17.90 \pm 0.03$ & $\cdots$ & 35.24 & 35.55 & $\cdots$ & $\cdots$ & $\cdots$ & $\cdots$ & $\cdots$ & $\cdots$ & VV \\
\hline NGC 7337 & $18.13 \pm 0.06$ & $17.29 \pm 0.01$ & $0.84 \pm 0.06$ & $18.25 \pm 0.05$ & $17.49 \pm 0.02$ & $0.76 \pm 0.06$ & 35.65 & 35.81 & 15.71 & 15.73 & $\cdots$ & $\cdots$ & $\cdots$ & $\cdots$ & EFn \\
\hline NGC 7343 & $16.55 \pm 0.02$ & $15.91 \pm 0.01$ & $0.64 \pm 0.03$ & $16.63 \pm 0.02$ & $15.99 \pm 0.01$ & $0.64 \pm 0.02$ & 36.39 & 36.47 & 13.01 & 12.24 & $\cdots$ & $\cdots$ & $\cdots$ & $\cdots$ & $\mathrm{EF}$ \\
\hline UGC 12134 & $16.42 \pm 0.01$ & $16.01 \pm 0.01$ & $0.41 \pm 0.01$ & $16.65 \pm 0.01$ & $16.25 \pm 0.01$ & $0.40 \pm 0.01$ & 36.42 & 36.41 & 19.71 & 19.18 & 2.13 & 2.32 & 2.10 & 2.34 & $? \mathrm{~F}$ \\
\hline NGC 7348 & $16.14 \pm 0.01$ & $15.80 \pm 0.01$ & $0.34 \pm 0.01$ & $16.25 \pm 0.01$ & $15.93 \pm 0.01$ & $0.32 \pm 0.01$ & 36.52 & 36.48 & 12.69 & 12.47 & $\ldots$ & $\ldots$ & $\cdots$ & $\cdots$ & ER \\
\hline IRAS 22491-1808 & $17.98 \pm 0.05$ & $17.63 \pm 0.01$ & $0.35 \pm 0.05$ & $18.02 \pm 0.03$ & $17.69 \pm 0.02$ & $0.32 \pm 0.04$ & 36.78 & 36.75 & $\cdots$ & $\cdots$ & $\cdots$ & $\cdots$ & $\cdots$ & $\cdots$ & $\cdots$ \\
\hline NGC 7396 & $18.48 \pm 0.15$ & $16.98 \pm 0.06$ & $1.50 \pm 0.16$ & $18.74 \pm 0.11$ & $17.30 \pm 0.02$ & $1.43 \pm 0.11$ & 35.25 & 35.67 & 24.14 & 23.48 & 3.01 & 3.35 & 3.04 & 3.29 & $\mathrm{EE}$ \\
\hline ESO 346-G006 & $16.76 \pm 0.01$ & $16.45 \pm 0.01$ & $0.30 \pm 0.01$ & $17.05 \pm 0.02$ & $16.70 \pm 0.01$ & $0.35 \pm 0.02$ & 36.51 & 36.45 & 15.06 & 13.17 & $\cdots$ & $\cdots$ & $\cdots$ & $\cdots$ & ER \\
\hline NGC 7398 & $17.32 \pm 0.04$ & $16.83 \pm 0.02$ & $0.48 \pm 0.04$ & $17.73 \pm 0.03$ & $17.24 \pm 0.02$ & $0.49 \pm 0.04$ & 35.67 & 35.68 & 22.92 & 22.21 & 1.60 & 1.77 & 1.31 & 1.62 & $\cdots$ \\
\hline UGC 12250 & $16.66 \pm 0.01$ & $16.19 \pm 0.01$ & $0.47 \pm 0.01$ & $16.70 \pm 0.02$ & $16.24 \pm 0.01$ & $0.46 \pm 0.02$ & 36.31 & 36.32 & 21.40 & 21.07 & 1.83 & 1.95 & 1.81 & 1.97 & $? \mathrm{~F}$ \\
\hline UGC 12253 & $19.09 \pm 0.03$ & $18.50 \pm 0.08$ & $0.59 \pm 0.08$ & $19.43 \pm 0.05$ & $18.92 \pm 0.02$ & $0.50 \pm 0.06$ & 35.40 & 35.46 & 11.53 & 12.33 & 2.53 & 2.72 & 2.55 & 2.76 & $\mathrm{EE}$ \\
\hline NGC 7418 & $13.76 \pm 0.01$ & $13.44 \pm 0.01$ & $0.32 \pm 0.01$ & $13.82 \pm 0.01$ & $13.50 \pm 0.01$ & $0.32 \pm 0.01$ & 35.95 & 35.91 & 45.61 & 44.75 & 2.16 & 2.22 & 2.00 & 2.09 & ?Fn \\
\hline NGC $7418 \mathrm{~A}$ & $14.52 \pm 0.01$ & $14.38 \pm 0.01$ & $0.14 \pm 0.02$ & $14.96 \pm 0.01$ & $14.81 \pm 0.01$ & $0.15 \pm 0.01$ & 36.01 & 35.89 & 47.16 & 45.76 & 4.50 & 4.80 & 4.29 & 4.46 & EEn \\
\hline ESO 534-G032 & $16.84 \pm 0.02$ & $16.54 \pm 0.01$ & $0.30 \pm 0.02$ & $17.04 \pm 0.02$ & $16.75 \pm 0.01$ & $0.29 \pm 0.02$ & 36.42 & 36.36 & 16.20 & 15.71 & 2.03 & 2.20 & 1.94 & 2.20 & $\mathrm{EF}$ \\
\hline IC 5264 & $16.84 \pm 0.02$ & $16.24 \pm 0.01$ & $0.60 \pm 0.03$ & $16.99 \pm 0.02$ & $16.43 \pm 0.70$ & $0.56 \pm 0.70$ & 35.01 & 35.08 & 18.05 & 17.93 & 2.23 & 2.37 & 2.23 & 2.37 & $\mathrm{EF}$ \\
\hline NGC 7421 & $14.94 \pm 0.01$ & $14.57 \pm 0.01$ & $0.37 \pm 0.01$ & $15.02 \pm 0.01$ & $14.66 \pm 0.01$ & $0.36 \pm 0.01$ & 35.71 & 35.68 & 33.54 & 32.55 & 1.71 & 1.81 & 1.49 & 1.62 & $\mathrm{ED}$ \\
\hline NGC 7432 & $19.45 \pm 0.16$ & $17.83 \pm 0.06$ & $1.62 \pm 0.17$ & $19.44 \pm 0.14$ & $18.22 \pm 0.04$ & $1.22 \pm 0.15$ & 35.24 & 35.71 & $\ldots$ & 21.28 & $\ldots$ & $\ldots$ & $\ldots$ & $\ldots$ & VV \\
\hline ARP 314 NED01 & $15.57 \pm 0.01$ & $15.03 \pm 0.01$ & $0.54 \pm 0.02$ & $15.69 \pm 0.02$ & $15.15 \pm 0.01$ & $0.54 \pm 0.02$ & 36.15 & 36.19 & $\cdots$ & $\cdots$ & $\ldots$ & $\cdots$ & $\cdots$ & $\cdots$ & ER \\
\hline ARP 314 NED03 & $16.46 \pm 0.06$ & $16.23 \pm 0.05$ & $0.23 \pm 0.08$ & $16.88 \pm 0.02$ & $16.76 \pm 0.01$ & $0.12 \pm 0.03$ & 35.79 & 35.71 & 20.95 & 22.73 & 2.67 & 2.89 & $\cdots$ & $\cdots$ & $\mathrm{EE}$ \\
\hline ARP 314 NED02 & $15.31 \pm 0.01$ & $14.90 \pm 0.01$ & $0.41 \pm 0.01$ & $15.40 \pm 0.02$ & $14.97 \pm 0.01$ & $0.43 \pm 0.02$ & 36.25 & 36.23 & $\cdots$ & $\cdots$ & $\cdots$ & $\cdots$ & $\cdots$ & $\cdots$ & VF \\
\hline UGC 12285 & $17.59 \pm 0.05$ & $16.89 \pm 0.02$ & $0.70 \pm 0.05$ & $17.71 \pm 0.05$ & $17.05 \pm 0.02$ & $0.66 \pm 0.05$ & 36.53 & 36.64 & 9.43 & 8.98 & $\ldots$ & $\cdots$ & $\cdots$ & $\ldots$ & $\mathrm{EE}$ \\
\hline ESO 406-G042 & $15.48 \pm 0.01$ & $15.30 \pm 0.01$ & $0.18 \pm 0.02$ & $15.68 \pm 0.01$ & $15.49 \pm 0.01$ & $0.19 \pm 0.01$ & 35.22 & 35.11 & 26.31 & 25.88 & 2.25 & 2.31 & 2.25 & 2.31 & EFn \\
\hline NGC 7469 & $14.32 \pm 0.02$ & $14.04 \pm 0.02$ & $0.29 \pm 0.02$ & $14.37 \pm 0.01$ & $14.09 \pm 0.01$ & $0.28 \pm 0.01$ & 36.91 & 36.84 & $\ldots$ & $\cdots$ & $\ldots$ & $\ldots$ & $\cdots$ & $\cdots$ & ?Fn \\
\hline NGC 7479 & $13.93 \pm 0.01$ & $13.46 \pm 0.01$ & $0.47 \pm 0.01$ & $14.00 \pm 0.01$ & $13.51 \pm 0.01$ & $0.48 \pm 0.01$ & 36.45 & 36.46 & 62.17 & 57.25 & 2.10 & 2.27 & 1.97 & 2.19 & $\mathrm{EF}$ \\
\hline UGC 12346 & $\cdots$ & $16.25 \pm 0.01$ & $\cdots$ & $\ldots$ & $16.44 \pm 0.01$ & $\cdots$ & 0.00 & 36.33 & $\cdots$ & 22.34 & $\ldots$ & $\ldots$ & $\cdots$ & $\cdots$ & $\mathrm{EF}$ \\
\hline UGC 12354 & $16.68 \pm 0.02$ & $15.84 \pm 0.01$ & $0.84 \pm 0.02$ & $16.90 \pm 0.04$ & $16.10 \pm 0.02$ & $0.80 \pm 0.05$ & 35.77 & 35.93 & 10.10 & 10.74 & $\ldots$ & $\ldots$ & $\ldots$ & $\ldots$ & $\mathrm{EE}$ \\
\hline ESO 469-G012 & $16.96 \pm 0.01$ & $16.58 \pm 0.01$ & $0.38 \pm 0.01$ & $17.41 \pm 0.03$ & $17.03 \pm 0.01$ & $0.38 \pm 0.03$ & 36.42 & 36.40 & 15.45 & 15.18 & 2.59 & 2.85 & $\ldots$ & $\ldots$ & $\mathrm{Ef}$ \\
\hline ESO 469-G015 & $16.08 \pm 0.04$ & $15.72 \pm 0.02$ & $0.37 \pm 0.05$ & $16.77 \pm 0.01$ & $16.38 \pm 0.01$ & $0.39 \pm 0.01$ & 35.16 & 35.12 & 16.39 & 16.12 & 3.85 & 3.67 & 3.57 & 3.51 & $\mathrm{ER}$ \\
\hline IC 5287 & $17.53 \pm 0.03$ & $17.09 \pm 0.01$ & $0.44 \pm 0.03$ & $17.59 \pm 0.03$ & $17.17 \pm 0.01$ & $0.43 \pm 0.03$ & 36.21 & 36.21 & 14.70 & 14.53 & $\ldots$ & $\ldots$ & $\ldots$ & $\ldots$ & ED \\
\hline
\end{tabular}


Table 3-Continued

\begin{tabular}{|c|c|c|c|c|c|c|c|c|c|c|c|c|c|c|c|}
\hline \multirow{2}{*}{$\begin{array}{c}\text { Object Name } \\
\text { (1) }\end{array}$} & \multicolumn{3}{|c|}{ Asymptotic magnitudes and color } & \multicolumn{3}{|c|}{ D25 magnitudes and color } & \multicolumn{2}{|c|}{$\log L$} & \multicolumn{2}{|c|}{ Effective Radii } & \multicolumn{2}{|c|}{ C31 } & \multicolumn{2}{|c|}{$\mathrm{C} 42$} & \multirow{2}{*}{$\begin{array}{c}\text { UV } \\
\text { profile } \\
(16)\end{array}$} \\
\hline & $\begin{array}{c}\text { FUV } \\
(\mathrm{mag}) \\
(2)\end{array}$ & $\begin{array}{c}\text { NUV } \\
(\mathrm{mag}) \\
(3)\end{array}$ & $\begin{array}{c}\text { FUV-NUV } \\
\text { (mag) } \\
(4)\end{array}$ & $\begin{array}{c}\text { FUV } \\
(\mathrm{mag}) \\
(5)\end{array}$ & $\begin{array}{c}\text { NUV } \\
(\mathrm{mag}) \\
(6)\end{array}$ & $\begin{array}{c}\text { FUV-NUV } \\
(\mathrm{mag}) \\
(7)\end{array}$ & $\begin{array}{c}\text { FUV } \\
(\mathrm{W}) \\
(8)\end{array}$ & $\begin{array}{c}\text { NUV } \\
(\mathrm{W}) \\
(9)\end{array}$ & $\begin{array}{c}\text { FUV } \\
(\operatorname{arcsec}) \\
(10)\end{array}$ & $\begin{array}{c}\text { NUV } \\
\text { (arcsec) } \\
(11)\end{array}$ & $\begin{array}{l}\text { FUV } \\
(12)\end{array}$ & $\begin{array}{l}\text { NUV } \\
(13)\end{array}$ & $\begin{array}{l}\text { FUV } \\
(14)\end{array}$ & $\begin{array}{l}\text { NUV } \\
(15)\end{array}$ & \\
\hline ESO 407-G007 & $.58 \pm 0.06$ & $16.57 \pm 0.04$ & $1.01 \pm$ & $.83 \pm 0.02$ & $17.00 \pm 0$ & 2 & 34.55 & 34 & 19. & 21 & 2.57 & 3.03 & 2.54 & 3.00 & $\mathrm{EE}$ \\
\hline NGC 7496 & 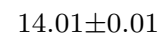 & $13.62 \pm 0.01$ & & $.08 \pm 0.01$ & $13.68=$ & 1 & 35.97 & & & & 2.26 & 2.49 & 2.55 & 3.00 & EFn \\
\hline ESO 291-G005 & $18.28 \pm 0.07$ & $18.06 \pm 0.02$ & $0.22 \pm 0$ & $8.89 \pm 0.06$ & $18.64 \pm 0.04$ & $0.25 \pm 0.07$ & 35.95 & 35.86 & 21.01 & 20.50 & 1.86 & 2.00 & 1.88 & 2.07 & ?Fn \\
\hline ESO 291-G006 & $21.33 \pm 0.64$ & $20.48 \pm 0.22$ & $0.85 \pm 0.68$ & $\ldots$ & $\ldots$ & $\ldots$ & 35.50 & 35.66 & $\ldots$ & 14.55 & $\ldots$ & $\ldots$ & $\ldots$ & $\ldots$ & $\ldots$ \\
\hline NGC $7496 \mathrm{~A}$ & $15.89 \pm 0.01$ & $15.69 \pm 0.01$ & $0.21 \pm 0.01$ & $16.05 \pm 0.01$ & $15.87 \pm 0.01$ & $0.18 \pm 0.02$ & 35.21 & 35.12 & 19.36 & 19.00 & 2.54 & $\ldots$ & $\ldots$ & $\ldots$ & $\mathrm{Ef}$ \\
\hline NGC 7511 & $17.68 \pm 0.14$ & $16.99 \pm 0.02$ & $0.69 \pm 0.14$ & $17.76 \pm 0.04$ & $17.06 \pm 0.01$ & $0.70 \pm 0.04$ & 35.56 & 35.66 & $\ldots$ & $\ldots$ & $\ldots$ & $\ldots$ & $\ldots$ & $\ldots$ & ?f \\
\hline ESO 407-G009 & $16.37 \pm 0.01$ & $16.00 \pm 0.01$ & $0.37 \pm 0.01$ & $16.70 \pm 0.02$ & $16.33 \pm 0.01$ & $0.37 \pm 0.02$ & 34.99 & 34.96 & 23.02 & 23.04 & 2.25 & 2.26 & 2.18 & 2.20 & ?d \\
\hline ESO 291-G009 & $18.81 \pm 0.02$ & $18.27 \pm 0.07$ & $0.54 \pm 0.08$ & $19.04 \pm 0.05$ & $18.60 \pm 0.03$ & $0.44 \pm 0.06$ & 36.17 & 36.21 & $\ldots$ & 13.42 & $\ldots$ & $\ldots$ & $\ldots$ & $\ldots$ & Er \\
\hline UGC 12434 & $16.50 \pm 0.01$ & $16.12 \pm 0.01$ & $0.39 \pm 0.02$ & $16.88 \pm 0.01$ & $16.53 \pm 0.01$ & $0.35 \pm 0.02$ & 35.52 & 35.49 & 7.60 & 7.90 & $\ldots$ & $\ldots$ & $\ldots$ & $\ldots$ & $\mathrm{EE}$ \\
\hline NGC 7535 & $16.02 \pm 0.05$ & $15.68 \pm 0.02$ & $0.34 \pm 0.05$ & $16.13 \pm 0.02$ & $15.78 \pm 0.01$ & $0.35 \pm 0.02$ & 36.17 & 36.13 & 25.36 & 24.31 & 2.04 & 2.10 & 1.93 & 2.01 & $? \mathrm{~F}$ \\
\hline NGC 7536 & $16.19 \pm 0.01$ & $15.66 \pm 0.01$ & $0.53 \pm 0.01$ & $16.39 \pm 0.01$ & $15.87 \pm 0.01$ & $0.51 \pm 0.02$ & 36.12 & 36.16 & 18.33 & 18.34 & 2.15 & 2.27 & 2.07 & 2.28 & $\mathrm{ED}$ \\
\hline NGC $7559 \mathrm{~B}$ & $\ldots$ & $17.83 \pm 0.18$ & & $\ldots$ & $18.35 \pm 0.04$ & & 0.00 & 35.27 & $\ldots$ & 20.15 & $\ldots$ & $\ldots$ & $\ldots$ & $\ldots$ & VV \\
\hline NGC 7563 & $.76 \pm 0.10$ & $18.06 \pm 0.04$ & $1.70 \pm 0.11$ & $\ldots$ & $18.09 \pm 0.04$ & & 34.59 & 35.10 & & & $\ldots$ & $\ldots$ & $\ldots$ & $\ldots$ & VV \\
\hline NGC 7552 & $14.18 \pm 0.01$ & $13.45 \pm 0.02$ & $0.73 \pm 0.02$ & $14.26 \pm 0.01$ & $13.53 \pm 0.01$ & $0.73 \pm 0.01$ & 35.96 & 36.08 & 36.25 & & 4.12 & $\ldots$ & $\ldots$ & $\ldots$ & EEn \\
\hline ESO 407-G014 & $15.07 \pm 0.01$ & $14.78 \pm 0.01$ & $0.28 \pm 0.01$ & $15.21 \pm 0.01$ & $14.92 \pm 0.01$ & $0.30 \pm 0.01$ & 36.04 & 35.98 & 14.61 & 13.83 & $\ldots$ & $\ldots$ & $\ldots$ & $\ldots$ & ER \\
\hline NGC 7589 & $17.74 \pm 0.05$ & $17.18 \pm 0.04$ & $0.56 \pm 0.06$ & $17.85 \pm 0.03$ & $17.37 \pm 0.02$ & $0.49 \pm 0.04$ & 36.05 & 36.10 & 13.69 & 13.53 & $\ldots$ & $\ldots$ & $\ldots$ & $\ldots$ & $\ldots$ \\
\hline NGC 7582 & $15.13 \pm 0.01$ & $14.26 \pm 0.01$ & $0.87 \pm 0.01$ & $15.20 \pm 0.01$ & $14.35 \pm 0.01$ & $0.86 \pm 0.01$ & 35.58 & 35.75 & 42.15 & 38.87 & 2.78 & 3.05 & 2.69 & 3.02 & EEn \\
\hline PGC 71025 & $20.18 \pm 0.21$ & $18.91 \pm 0.05$ & $1.26 \pm 0.21$ & $20.47 \pm 0.12$ & $19.69 \pm 0.05$ & $0.78 \pm 0.13$ & 35.09 & 35.42 & 9.31 & 14.54 & 2.10 & $\ldots$ & 2.13 & $\ldots$ & $\mathrm{EE}$ \\
\hline IC 5304 & $19.78 \pm 0.13$ & $18.21 \pm 0.09$ & $1.57 \pm 0.16$ & $19.78 \pm 0.15$ & $18.57 \pm 0.05$ & $1.21 \pm 0.16$ & 35.29 & 35.74 & $\ldots$ & 16.32 & $\ldots$ & $\ldots$ & $\ldots$ & $\ldots$ & VV \\
\hline NGC 7645 & $15.78 \pm 0.01$ & $15.33 \pm 0.01$ & $0.45 \pm 0.01$ & $16.06 \pm 0.01$ & $15.53 \pm 0.01$ & $0.53 \pm 0.02$ & 36.60 & 36.60 & 23.03 & 20.53 & $\ldots$ & $\ldots$ & $\ldots$ & $\ldots$ & $\mathrm{VF}$ \\
\hline UGC 12578 & $15.29 \pm 0.01$ & $15.21 \pm 0.01$ & $0.08 \pm 0.01$ & $15.42 \pm 0.01$ & $15.34 \pm 0.01$ & $0.07 \pm 0.02$ & 35.99 & 35.85 & 18.06 & & $\ldots$ & $\ldots$ & $\ldots$ & $\ldots$ & VFn \\
\hline UGC 12589 & $17.42 \pm 0.02$ & $17.03 \pm 0.01$ & $0.38 \pm 0$ & $17.59 \pm 0.03$ & $17.16 \pm 0.01$ & $0.44 \pm 0.03$ & 36.29 & & 11.84 & 11 & 2.18 & $\ldots$ & $\ldots$ & $\ldots$ & $\mathrm{EF}$ \\
\hline CGCG 406-109 & $17.88 \pm 0.03$ & $17.53 \pm 0$ & & $18.14 \pm 0.03$ & & & & & 7.40 & 7.32 & $\ldots$ & $\ldots$ & $\ldots$ & $\ldots$ & $\mathrm{Ef}$ \\
\hline NGC 7673 & $14.31 \pm$ & 14.0 & & $14.35 \pm 0.01$ & & $0.24 \pm 0$ & 36.61 & & $\ldots$ & $\ldots$ & $\ldots$ & $\ldots$ & $\ldots$ & $\ldots$ & $\mathrm{ER}$ \\
\hline NGC 7674 & $15.73 \pm 0.01$ & $15.37 \pm 0.01$ & $0.36 \pm 0.02$ & $15.82 \pm 0.01$ & $15.44 \pm 0.01$ & $0.38 \pm 0.02$ & 36.83 & 36.80 & $\ldots$ & $\ldots$ & $\ldots$ & $\ldots$ & $\ldots$ & $\ldots$ & $\mathrm{ER}$ \\
\hline NGC 7677 & $15.91 \pm 0.01$ & $15.58 \pm 0.01$ & $0.33 \pm 0.01$ & $16.21 \pm 0.01$ & $15.84 \pm 0.01$ & $0.37 \pm 0.02$ & 36.01 & 35.96 & 23.33 & 19.17 & 3.20 & $\ldots$ & $\ldots$ & $\ldots$ & $\mathrm{EE}$ \\
\hline IC 5325 & $14.02 \pm 0.01$ & $13.60 \pm 0.01$ & $0.43 \pm 0.01$ & $14.08 \pm 0.01$ & $13.65 \pm 0.01$ & $0.42 \pm 0.01$ & 35.87 & 35.86 & 27.64 & 26.64 & 2.74 & 2.79 & 2.69 & 2.77 & EEh \\
\hline UGC 12635 & $17.27 \pm 0.04$ & $16.93 \pm 0.02$ & $0.34 \pm 0.05$ & $17.29 \pm 0.03$ & $16.99 \pm 0.01$ & $0.30 \pm 0.03$ & 35.77 & 35.73 & 16.75 & 16.95 & $\ldots$ & $\ldots$ & $\ldots$ & $\ldots$ & $? \mathrm{~F}$ \\
\hline NGC 7684 & $19.76 \pm 0.14$ & $17.95 \pm 0.02$ & $1.81 \pm 0.14$ & $19.99 \pm 0.11$ & $18.18 \pm 0.02$ & $1.80 \pm 0.11$ & 34.76 & 35.31 & 9.62 & 10.13 & $\ldots$ & $\ldots$ & $\ldots$ & $\ldots$ & VV \\
\hline UGC 12685 & $16.76 \pm 0.01$ & $16.62 \pm 0.01$ & $0.14 \pm 0.01$ & $16.95 \pm 0.02$ & $16.81 \pm 0.01$ & $0.15 \pm 0.02$ & 35.99 & 35.87 & 20.74 & 19.61 & $\ldots$ & $\ldots$ & $\ldots$ & $\ldots$ & VF \\
\hline
\end{tabular}


Table 3 - Continued

\begin{tabular}{|c|c|c|c|c|c|c|c|c|c|c|c|c|c|c|c|}
\hline \multirow{2}{*}{$\begin{array}{c}\text { Object Name } \\
\text { (1) }\end{array}$} & \multicolumn{3}{|c|}{ Asymptotic magnitudes and color } & \multicolumn{3}{|c|}{ D25 magnitudes and color } & \multicolumn{2}{|c|}{$\log L$} & \multicolumn{2}{|c|}{ Effective Radii } & \multicolumn{2}{|c|}{ C31 } & \multicolumn{2}{|c|}{$\mathrm{C} 42$} & \multirow{2}{*}{$\begin{array}{l}\text { UV } \\
\text { profile } \\
(16)\end{array}$} \\
\hline & $\begin{array}{c}\text { FUV } \\
(\mathrm{mag}) \\
(2)\end{array}$ & $\begin{array}{c}\text { NUV } \\
(\mathrm{mag}) \\
(3)\end{array}$ & $\begin{array}{c}\text { FUV-NUV } \\
\text { (mag) } \\
(4)\end{array}$ & $\begin{array}{c}\text { FUV } \\
(\mathrm{mag}) \\
(5)\end{array}$ & $\begin{array}{l}\text { NUV } \\
(\mathrm{mag}) \\
(6)\end{array}$ & $\begin{array}{l}\text { FUV-NUV } \\
\text { (mag) } \\
(7)\end{array}$ & $\begin{array}{l}\text { FUV } \\
(\mathrm{W}) \\
(8)\end{array}$ & $\begin{array}{c}\text { NUV } \\
(\mathrm{W}) \\
(9)\end{array}$ & $\begin{array}{c}\text { FUV } \\
\text { (arcsec) } \\
(10)\end{array}$ & $\begin{array}{c}\text { NUV } \\
(\operatorname{arcsec}) \\
(11)\end{array}$ & $\begin{array}{l}\text { FUV } \\
(12)\end{array}$ & $\begin{array}{l}\text { NUV } \\
(13)\end{array}$ & $\begin{array}{l}\text { FUV } \\
(14)\end{array}$ & $\begin{array}{l}\text { NUV } \\
(15)\end{array}$ & \\
\hline IRAS $23365+3604$ & $86 \pm 0.02$ & $17.96 \pm 0.02$ & $0.90 \pm 0$ & $2 \pm 0.10$ & $18.22 \pm 0.03$ & $1.00 \pm 0$ & 36.28 & 36 . & & $\cdots$ & & & & & VV \\
\hline ARP 295A & $85 \pm 0.16$ & $18.13 \pm 0.09$ & $1.72 \pm 0.19$ & $.87 \pm 0.10$ & $18.73 \pm 0.03$ & $1.14 \pm 0.10$ & 34.94 & 35.45 & 11.35 & 18.07 & 2.31 & 2.99 & 2.16 & 2.93 & $\mathrm{EE}$ \\
\hline NGC 7735 & $87 \pm 0.32$ & $18.61 \pm 0.05$ & $1.25 \pm 0.32$ & $\cdots$ & $18.78 \pm 0.07$ & $\cdots$ & 35.27 & 35.60 & $\ldots$ & $\cdots$ & $\ldots$ & $\cdots$ & $\ldots$ & $\ldots$ & VV \\
\hline NGC 7741 & $13.44 \pm 0.01$ & $13.15 \pm 0.01$ & $0.29 \pm 0.01$ & $13.46 \pm 0.01$ & $13.17 \pm 0.01$ & $0.29 \pm 0.01$ & 35.74 & 35.68 & 47.92 & 47.74 & 2.10 & 2.17 & 2.25 & 2.36 & EFn \\
\hline NGC 7769 & $14.76 \pm 0.01$ & $14.34 \pm 0.01$ & $0.41 \pm 0.01$ & $14.79 \pm 0.01$ & $14.37 \pm 0.01$ & $0.41 \pm 0.01$ & 36.61 & 36.60 & 13.62 & 13.44 & $\ldots$ & $\ldots$ & $\ldots$ & $\cdots$ & $\mathrm{ER}$ \\
\hline NGC 7771 & $16.31 \pm 0.03$ & $15.50 \pm 0.01$ & $0.81 \pm 0.03$ & $16.50 \pm 0.02$ & $15.61 \pm 0.01$ & $0.89 \pm 0.02$ & 36.00 & 36.15 & 15.67 & 13.16 & $\ldots$ & $\ldots$ & $\ldots$ & $\ldots$ & VV \\
\hline CGCG 432-040 & $18.29 \pm 0.06$ & $17.77 \pm 0.04$ & $0.52 \pm 0.07$ & $18.78 \pm 0.05$ & $18.21 \pm 0.02$ & $0.58 \pm 0.05$ & 36.00 & 36.03 & 12.00 & 10.94 & 3.06 & $\cdots$ & $\ldots$ & $\cdots$ & $\mathrm{EE}$ \\
\hline NGC 7793 & $11.17 \pm 0.01$ & $11.00 \pm 0.01$ & $0.17 \pm 0.01$ & $11.23 \pm 0.01$ & $11.05 \pm 0.01$ & $0.18 \pm 0.01$ & 35.07 & 34.96 & 109.40 & 106.22 & 2.10 & 2.14 & 2.07 & 2.11 & EFn \\
\hline ESO 349-G014 & $18.50 \pm 0.02$ & $17.74 \pm 0.02$ & $0.76 \pm 0.03$ & $19.99 \pm 0.05$ & $18.99 \pm 0.02$ & $1.00 \pm 0.05$ & 36.01 & 36.14 & 16.10 & 14.62 & 2.17 & 2.61 & 2.16 & 2.64 & $\mathrm{EF}$ \\
\hline NGC 7798 & $14.87 \pm 0.01$ & $14.42 \pm 0.01$ & $0.46 \pm 0.01$ & $14.90 \pm 0.01$ & $14.44 \pm 0.01$ & $0.45 \pm 0.01$ & 36.09 & 36.09 & $\ldots$ & $\ldots$ & $\ldots$ & $\ldots$ & $\ldots$ & $\ldots$ & VFh \\
\hline
\end{tabular}

Note. - Global UV properties of the galaxies in the GALEX Atlas. Col. (1): Galaxy name. Col. (2): Asymptotic FUV magnitude in AB scale corrected for Galactic extinction. The errors quoted correspond to the error in the fit to the growth curve alone. The error in the FUV and NUV zero-point calibration is estimated to be $\sim 0.15 \mathrm{mag}$ in each band. Col. (3): The same for the NUV. Col. (4): Asymptotic (FUV-NUV) color computed as the difference between Col. (2) and Col. (3). Cols. (5,6,7): The same as Cols. $(2,3,4)$ for the aperture magnitudes within the D25 ellipse. Col. (8): Logarithm of the FUV luminosity in Watts. Col. (9): The same for the NUV. Col. (10); Effective radii [equivalent radius of the ellipse including half of the total (asymptotic) light of the galaxy] in the FUV band measured in arcsec. Col. (11): The same for the NUV. Col. (12): C31 concentration index in the FUV. Col. (13): The same for the NUV. Col. (14): C42 concentration index in the FUV. Col. (15): The same for the NUV. Col. (16): Morphological class of the UV surface brightness profile (see text for details). Note that we only computed the effective radius and concentration index C31 (C42) for those galaxies whose radii containing 50\% and 25\% (20\%), respectively, of the light were larger than 6 arcsec. 
Table 4. Corollary data

\begin{tabular}{|c|c|c|c|c|c|c|c|c|c|c|}
\hline \multirow{2}{*}{$\begin{array}{c}\text { Object Name } \\
(1) \\
\end{array}$} & \multicolumn{3}{|c|}{ Optical Photometry } & \multicolumn{3}{|c|}{ Near-Infrared Photometry } & \multicolumn{4}{|c|}{ IRAS fluxes } \\
\hline & $\begin{array}{c}U \\
(\mathrm{mag}) \\
(2)\end{array}$ & $\begin{array}{c}B \\
(\mathrm{mag}) \\
(3)\end{array}$ & $\begin{array}{c}V \\
(\mathrm{mag}) \\
(4)\end{array}$ & $\begin{array}{c}J \\
(\mathrm{mag}) \\
(5)\end{array}$ & $\begin{array}{c}H \\
(\mathrm{mag}) \\
(6)\end{array}$ & $\begin{array}{c}K \\
(\mathrm{mag}) \\
(7)\end{array}$ & $\begin{array}{c}12 \mu \mathrm{m} \\
(\mathrm{Jy}) \\
(8)\end{array}$ & $\begin{array}{c}25 \mu \mathrm{m} \\
(\mathrm{Jy}) \\
(9)\end{array}$ & $\begin{array}{c}60 \mu \mathrm{m} \\
(\mathrm{Jy}) \\
(10)\end{array}$ & $\begin{array}{c}100 \mu \mathrm{m} \\
(\mathrm{Jy}) \\
(11)\end{array}$ \\
\hline WLM & $\cdots$ & $11.03 \pm 0.08$ & $10.59 \pm 0.09$ & $\ldots$ & $\ldots$ & . & $<0.12$ & $<0.20$ & $0.32 \pm 0.08$ & $1.04 \pm 0.26$ \\
\hline NGC 7808 & $\ldots$ & $14.33 \pm 0.13$ & $13.48 \pm 0.13$ & $11.29 \pm 0.03$ & $10.65 \pm 0.03$ & $10.32 \pm 0.04$ & $0.11 \pm 0.03$ & $<0.21$ & $0.37 \pm 0.05$ & $1.58 \pm 0.21$ \\
\hline UGC 00017 & $14.78 \pm 0.23$ & $14.80 \pm 0.20$ & $14.19 \pm 0.22$ & $\ldots$ & $\ldots$ & $\ldots$ & $\ldots$ & $\ldots$ & $\ldots$ & $\ldots$ \\
\hline PGC 00282 & $\cdots$ & $\cdots$ & $\cdots$ & $13.36 \pm 0.06$ & $12.76 \pm 0.09$ & $12.56 \pm 0.13$ & $<0.08$ & $<0.18$ & $0.28 \pm 0.06$ & $0.66 \pm 0.15$ \\
\hline NGC 0024 & $12.12 \pm 0.13$ & $12.19 \pm 0.13$ & $11.61 \pm 0.13$ & $9.71 \pm 0.02$ & $9.11 \pm 0.03$ & $8.95 \pm 0.04$ & $<0.13$ & $0.16 \pm 0.04$ & $1.26 \pm 0.14$ & $3.59 \pm 0.40$ \\
\hline UGC 00128 & $\ldots$ & & $\ldots$ & $\ldots$ & $\ldots$ & $\ldots$ & $\ldots$ & $\ldots$ & $\ldots$ & $\ldots$ \\
\hline NGC 0055 & $8.54 \pm 0.06$ & $8.42 \pm 0.05$ & $7.87 \pm 0.06$ & $6.98 \pm 0.03$ & $6.55 \pm 0.04$ & $6.25 \pm 0.05$ & $1.34 \pm 0.20$ & $6.25 \pm 0.94$ & $77.00 \pm 11.60$ & $174.00 \pm 26.10$ \\
\hline ARP 256 NED02 & $\ldots$ & $\ldots$ & $13.60 \pm 0.30$ & $12.69 \pm 0.05$ & $12.35 \pm 0.09$ & $11.82 \pm 0.09$ & $\ldots$ & $\ldots$ & $\ldots$ & $\ldots$ \\
\hline ARP 256 NED01 & $\ldots$ & $14.81 \pm 0.13$ & $14.33 \pm 0.13$ & $12.44 \pm 0.03$ & $11.70 \pm 0.04$ & $11.34 \pm 0.05$ & $\ldots$ & $\ldots$ & $\ldots$ & $\ldots$ \\
\hline UGC 00226 & $\ldots$ & $14.81 \pm 0.18$ & $\ldots$ & $12.96 \pm 0.05$ & $12.26 \pm 0.08$ & $11.95 \pm 0.09$ & $<0.09$ & $<0.10$ & $0.41 \pm 0.05$ & $1.11 \pm 0.29$ \\
\hline NGC 0099 & $13.78 \pm 0.13$ & $13.99 \pm 0.13$ & $13.65 \pm 0.13$ & $12.26 \pm 0.03$ & $11.66 \pm 0.04$ & $11.76 \pm 0.10$ & $<0.10$ & $<0.20$ & $0.81 \pm 0.06$ & $1.49 \pm 0.30$ \\
\hline UGC 00247 & $\cdots$ & $\cdots$ & $\ldots$ & $14.07 \pm 0.08$ & $13.55 \pm 0.13$ & $13.18 \pm 0.13$ & $\cdots$ & $\cdots$ & $\ldots$ & $\cdots$ \\
\hline UGC 00249 & $\ldots$ & $\ldots$ & $\ldots$ & $12.83 \pm 0.04$ & $12.54 \pm 0.09$ & $12.17 \pm 0.09$ & $\ldots$ & $\ldots$ & $\ldots$ & $\ldots$ \\
\hline NGC 0115 & $\ldots$ & $13.71 \pm 0.21$ & $\cdots$ & $11.83 \pm 0.03$ & $11.19 \pm 0.05$ & $11.21 \pm 0.09$ & $<0.08$ & $<0.14$ & $0.38 \pm 0.07$ & $1.16 \pm 0.15$ \\
\hline NGC 0131 & $13.80 \pm 0.14$ & $13.78 \pm 0.13$ & $13.22 \pm 0.13$ & $11.29 \pm 0.02$ & $10.68 \pm 0.03$ & $10.49 \pm 0.05$ & $<0.13$ & $<0.17$ & $0.51 \pm 0.12$ & $<2.91$ \\
\hline PGC 01862 & $\ldots$ & $\ldots$ & $\ldots$ & $12.46 \pm 0.05$ & $11.78 \pm 0.07$ & $11.47 \pm 0.09$ & $<0.09$ & $<0.17$ & $0.29 \pm 0.06$ & $0.70 \pm 0.17$ \\
\hline UGC 00316 & $\ldots$ & $15.53 \pm 0.18$ & $\ldots$ & $12.66 \pm 0.05$ & $11.88 \pm 0.08$ & $11.38 \pm 0.06$ & $\ldots$ & $\ldots$ & $\ldots$ & $\ldots$ \\
\hline ESO $473-G 025$ & $\ldots$ & $14.64 \pm 0.21$ & $\ldots$ & $11.03 \pm 0.01$ & $10.12 \pm 0.01$ & $9.83 \pm 0.01$ & $<0.11$ & $<0.14$ & $0.39 \pm 0.05$ & $1.63 \pm 0.21$ \\
\hline IC 1554 & $\ldots$ & $13.59 \pm 0.21$ & $\ldots$ & $11.04 \pm 0.02$ & $10.39 \pm 0.02$ & $10.14 \pm 0.03$ & $0.25 \pm 0.05$ & $0.24 \pm 0.07$ & $2.32 \pm 0.28$ & $4.74 \pm 0.33$ \\
\hline UGC 00330 & $\ldots$ & $14.74 \pm 0.15$ & $\cdots$ & $11.49 \pm 0.03$ & $10.83 \pm 0.02$ & $10.61 \pm 0.03$ & $\ldots$ & $\ldots$ & $\ldots$ & $\ldots$ \\
\hline NGC 0151 & $12.44 \pm 0.13$ & $12.31 \pm 0.13$ & $11.59 \pm 0.13$ & $9.55 \pm 0.01$ & $8.95 \pm 0.02$ & $8.75 \pm 0.03$ & $0.22 \pm 0.04$ & $0.26 \pm 0.04$ & $1.53 \pm 0.11$ & $5.58 \pm 0.61$ \\
\hline NGC 0155 & $\ldots$ & $\ldots$ & $\ldots$ & $10.70 \pm 0.02$ & $10.01 \pm 0.03$ & $9.77 \pm 0.04$ & $\ldots$ & $\ldots$ & $\ldots$ & $\ldots$ \\
\hline UGC 00344 & $\ldots$ & $15.09 \pm 0.20$ & $\ldots$ & $\ldots$ & $\ldots$ & $\ldots$ & $\ldots$ & $\ldots$ & $\ldots$ & $\ldots$ \\
\hline NGC 0163 & $14.13 \pm 0.15$ & $13.64 \pm 0.14$ & $12.70 \pm 0.14$ & $10.70 \pm 0.02$ & $9.99 \pm 0.02$ & $9.80 \pm 0.04$ & $\ldots$ & $\ldots$ & $\ldots$ & $\ldots$ \\
\hline VV 548 & $\ldots$ & $\ldots$ & $\cdots$ & $12.82 \pm 0.05$ & $12.08 \pm 0.07$ & $12.27 \pm 0.16$ & $<0.14$ & $<0.15$ & $0.34 \pm 0.05$ & $0.75 \pm 0.14$ \\
\hline NGC 0165 & $14.12 \pm 0.15$ & $13.88 \pm 0.14$ & $13.08 \pm 0.14$ & $11.29 \pm 0.04$ & $10.35 \pm 0.04$ & $10.37 \pm 0.07$ & $<0.21$ & $<0.25$ & $0.81 \pm 0.06$ & $2.12 \pm 0.19$ \\
\hline UGC 00372 & $\ldots$ & $\ldots$ & $\ldots$ & $14.61 \pm 0.12$ & $13.49 \pm 0.11$ & $13.72 \pm 0.21$ & $\ldots$ & $\ldots$ & $\ldots$ & $\ldots$ \\
\hline Cartwheel & $\ldots$ & $\ldots$ & $\ldots$ & $13.12 \pm 0.05$ & $12.58 \pm 0.07$ & $12.60 \pm 0.13$ & $<0.10$ & $<0.13$ & $0.69 \pm 0.06$ & $1.57 \pm 0.16$ \\
\hline PGC 02269 & $\ldots$ & $\ldots$ & $\ldots$ & $12.12 \pm 0.04$ & $11.35 \pm 0.05$ & $11.60 \pm 0.12$ & $\ldots$ & $\ldots$ & $\ldots$ & $\ldots$ \\
\hline UGC 00394 & $\ldots$ & $15.10 \pm 0.29$ & $\ldots$ & $14.36 \pm 0.14$ & $14.05 \pm 0.27$ & $13.27 \pm 0.19$ & $\ldots$ & $\ldots$ & $\ldots$ & $\ldots$ \\
\hline NGC 0195 & $\ldots$ & $\ldots$ & $\ldots$ & $11.40 \pm 0.03$ & $10.70 \pm 0.03$ & $10.36 \pm 0.04$ & $<0.15$ & $<0.21$ & $1.28 \pm 0.10$ & $2.96 \pm 0.35$ \\
\hline NGC 0205 & $\ldots$ & $8.92 \pm 0.05$ & $8.07 \pm 0.07$ & $6.45 \pm 0.03$ & $5.86 \pm 0.04$ & $5.59 \pm 0.05$ & $0.15 \pm 0.02$ & $0.19 \pm 0.03$ & $0.60 \pm 0.09$ & $3.78 \pm 0.57$ \\
\hline
\end{tabular}


Table 4-Continued

\begin{tabular}{|c|c|c|c|c|c|c|c|c|c|c|}
\hline \multirow{2}{*}{$\begin{array}{l}\text { Object Name } \\
\text { (1) }\end{array}$} & \multicolumn{3}{|c|}{ Optical Photometry } & \multicolumn{3}{|c|}{ Near-Infrared Photometry } & \multicolumn{4}{|c|}{ IRAS fluxes } \\
\hline & $\begin{array}{c}U \\
(\mathrm{mag}) \\
(2)\end{array}$ & $\begin{array}{c}B \\
(\mathrm{mag}) \\
(3)\end{array}$ & $\begin{array}{c}V \\
(\mathrm{mag}) \\
(4)\end{array}$ & $\begin{array}{c}J \\
(\mathrm{mag}) \\
(5)\end{array}$ & $\begin{array}{c}H \\
(\mathrm{mag}) \\
(6)\end{array}$ & $\begin{array}{c}K \\
(\mathrm{mag}) \\
(7)\end{array}$ & $\begin{array}{c}12 \mu \mathrm{m} \\
(\mathrm{Jy}) \\
(8)\end{array}$ & $\begin{array}{c}25 \mu \mathrm{m} \\
(\mathrm{Jy}) \\
(9)\end{array}$ & $\begin{array}{c}60 \mu \mathrm{m} \\
(\mathrm{Jy}) \\
(10)\end{array}$ & $\begin{array}{c}100 \mu \mathrm{m} \\
(\mathrm{Jy}) \\
(11)\end{array}$ \\
\hline NGC 0213 & $\ldots$ & $14.23 \pm 0.28$ & $\ldots$ & $10.85 \pm 0.03$ & $10.17 \pm 0.03$ & $9.91 \pm 0.04$ & $<0.09$ & $<0.18$ & $0.25 \pm 0.05$ & $0.94 \pm 0.24$ \\
\hline NGC 0223 & $\ldots$ & $14.22 \pm 0.18$ & $\ldots$ & $11.29 \pm 0.02$ & $10.68 \pm 0.03$ & $10.35 \pm 0.04$ & $<0.15$ & $<0.24$ & $0.22 \pm 0.05$ & $<1.96$ \\
\hline MESSIER 032 & $9.51 \pm 0.05$ & $9.03 \pm 0.05$ & $8.08 \pm 0.05$ & $6.28 \pm 0.02$ & $5.37 \pm 0.02$ & $5.09 \pm 0.02$ & $\ldots$ & $\ldots$ & $<0.09$ & $<1.41$ \\
\hline MESSIER 031 & $4.86 \pm 0.03$ & $4.36 \pm 0.02$ & $3.44 \pm 0.03$ & $2.09 \pm 0.02$ & $1.28 \pm 0.02$ & $0.98 \pm 0.02$ & $163.00 \pm 24.50$ & $108.00 \pm 16.20$ & $536.00 \pm 80.40$ & $2930.00 \pm 439.00$ \\
\hline UGC 00484 & $\cdots$ & $13.86 \pm 0.19$ & $\cdots$ & $11.24 \pm 0.03$ & $10.55 \pm 0.03$ & $10.18 \pm 0.04$ & $<0.25$ & $<0.25$ & $0.91 \pm 0.10$ & $2.50 \pm 0.25$ \\
\hline NGC 0247 & $\ldots$ & $9.67 \pm 0.07$ & $9.11 \pm 0.10$ & $8.08 \pm 0.03$ & $7.67 \pm 0.05$ & $7.43 \pm 0.06$ & $<0.12$ & $<0.16$ & $7.93 \pm 1.98$ & $27.30 \pm 6.83$ \\
\hline NGC 0253 & $\ldots$ & $8.04 \pm 0.05$ & $\ldots$ & $4.81 \pm 0.02$ & $4.09 \pm 0.02$ & $3.77 \pm 0.02$ & $55.80 \pm 14.00$ & $156.00 \pm 38.90$ & $999.00 \pm 250.00$ & $1860.00 \pm 465.00$ \\
\hline NGC 0247B & $\cdots$ & $14.42 \pm 0.21$ & $\cdots$ & $11.93 \pm 0.03$ & $11.43 \pm 0.05$ & $11.21 \pm 0.06$ & $<0.23$ & $0.14 \pm 0.07$ & $1.15 \pm 0.08$ & $2.97 \pm 0.24$ \\
\hline ESO 540-G025 & $\ldots$ & $14.65 \pm 0.21$ & $\ldots$ & $13.18 \pm 0.05$ & $12.92 \pm 0.12$ & $12.52 \pm 0.13$ & $<0.10$ & $<0.16$ & $0.33 \pm 0.06$ & $<0.79$ \\
\hline NGC 0262 & $14.04 \pm 0.20$ & $13.90 \pm 0.19$ & $13.06 \pm 0.19$ & $11.24 \pm 0.03$ & $10.55 \pm 0.04$ & $10.10 \pm 0.05$ & $0.31 \pm 0.03$ & $0.84 \pm 0.02$ & $1.29 \pm 0.12$ & $1.55 \pm 0.20$ \\
\hline UGC 00507 & $\ldots$ & $14.99 \pm 0.18$ & $\ldots$ & $11.59 \pm 0.03$ & $10.70 \pm 0.03$ & $10.29 \pm 0.03$ & $<0.25$ & $<0.32$ & $0.93 \pm 0.10$ & $2.54 \pm 0.28$ \\
\hline NGC 0266 & $12.98 \pm 0.18$ & $12.54 \pm 0.17$ & $11.63 \pm 0.17$ & $9.64 \pm 0.02$ & $8.96 \pm 0.02$ & $8.67 \pm 0.02$ & $<0.15$ & $<0.16$ & $0.81 \pm 0.15$ & $3.29 \pm 0.26$ \\
\hline NGC 0270 & $\cdots$ & $\ldots$ & $\ldots$ & $10.56 \pm 0.02$ & $9.88 \pm 0.02$ & $9.59 \pm 0.03$ & $<0.11$ & $<0.10$ & $0.37 \pm 0.05$ & $1.11 \pm 0.28$ \\
\hline ESO 351-G011 & $\ldots$ & $14.65 \pm 0.21$ & $\ldots$ & $11.40 \pm 0.02$ & $10.66 \pm 0.03$ & $10.36 \pm 0.04$ & $\ldots$ & $\ldots$ & $\ldots$ & $\ldots$ \\
\hline NGC 0277 & $\ldots$ & $\ldots$ & $\ldots$ & $11.10 \pm 0.03$ & $10.44 \pm 0.03$ & $10.26 \pm 0.06$ & $\ldots$ & $\ldots$ & $\ldots$ & $\ldots$ \\
\hline PGC 03004 & $\ldots$ & $\ldots$ & $\ldots$ & $\cdots$ & $\ldots$ & $\ldots$ & $\ldots$ & $\ldots$ & $\ldots$ & $\cdots$ \\
\hline UGC 00533 & $\ldots$ & $14.95 \pm 0.22$ & $\ldots$ & $\ldots$ & $\ldots$ & $\ldots$ & $\ldots$ & $\ldots$ & $\ldots$ & $\ldots$ \\
\hline NGC 0291 & $\ldots$ & $\ldots$ & $\ldots$ & $11.86 \pm 0.05$ & $11.06 \pm 0.05$ & $10.59 \pm 0.05$ & $<0.31$ & $<0.53$ & $2.61 \pm 0.50$ & $3.80 \pm 0.46$ \\
\hline NGC 0300 & $8.83 \pm 0.06$ & $8.72 \pm 0.05$ & $8.13 \pm 0.06$ & $7.04 \pm 0.03$ & $6.57 \pm 0.04$ & $6.38 \pm 0.06$ & $0.53 \pm 0.13$ & $0.64 \pm 0.16$ & $23.10 \pm 5.77$ & $74.50 \pm 18.60$ \\
\hline UGC 00590 & $\ldots$ & $15.26 \pm 0.22$ & $\ldots$ & $13.10 \pm 0.06$ & $12.26 \pm 0.07$ & $11.90 \pm 0.08$ & $\ldots$ & $\ldots$ & $\ldots$ & $\ldots$ \\
\hline NGC 0311 & $14.57 \pm 0.13$ & $14.00 \pm 0.13$ & $13.00 \pm 0.13$ & $10.71 \pm 0.02$ & $10.02 \pm 0.03$ & $9.73 \pm 0.03$ & $<0.04$ & $<0.05$ & $<0.09$ & $<0.32$ \\
\hline NGC 0315 & $12.80 \pm 0.20$ & $12.20 \pm 0.20$ & $11.16 \pm 0.20$ & $8.95 \pm 0.01$ & $8.21 \pm 0.02$ & $7.95 \pm 0.02$ & $<0.08$ & $<0.15$ & $0.27 \pm 0.05$ & $<0.71$ \\
\hline ESO 351-G028 & $\ldots$ & $14.57 \pm 0.21$ & $\ldots$ & $13.15 \pm 0.05$ & $12.55 \pm 0.07$ & $12.68 \pm 0.16$ & $<0.08$ & $<0.12$ & $0.42 \pm 0.05$ & $0.93 \pm 0.15$ \\
\hline UGC 00619 & $\ldots$ & $\ldots$ & $\ldots$ & $13.32 \pm 0.06$ & $12.68 \pm 0.09$ & $12.34 \pm 0.09$ & $\ldots$ & $\ldots$ & $\ldots$ & $\ldots$ \\
\hline NGC 0337 & $11.97 \pm 0.13$ & $12.06 \pm 0.13$ & $11.61 \pm 0.13$ & $9.98 \pm 0.03$ & $9.39 \pm 0.03$ & $9.10 \pm 0.05$ & $0.40 \pm 0.06$ & $0.75 \pm 0.05$ & $9.33 \pm 0.04$ & $19.20 \pm 0.43$ \\
\hline PGC 03613 & $\ldots$ & $\ldots$ & $\ldots$ & $11.08 \pm 0.03$ & $10.41 \pm 0.04$ & $10.10 \pm 0.06$ & $\ldots$ & $\ldots$ & $\ldots$ & $\ldots$ \\
\hline UGC 00627 & $\ldots$ & $14.82 \pm 0.20$ & $\ldots$ & $13.25 \pm 0.07$ & $12.38 \pm 0.09$ & $12.19 \pm 0.11$ & $\ldots$ & $\ldots$ & $\ldots$ & $\ldots$ \\
\hline NGC 0337A & $12.57 \pm 0.31$ & $12.70 \pm 0.30$ & $12.17 \pm 0.31$ & $\ldots$ & $\ldots$ & $\ldots$ & $\ldots$ & $\ldots$ & $\ldots$ & $\ldots$ \\
\hline UGC 00652 & $\ldots$ & $\ldots$ & $\ldots$ & $\ldots$ & $\ldots$ & $\ldots$ & $\ldots$ & $\ldots$ & $\ldots$ & $\ldots$ \\
\hline ESO 352-G002 & $\ldots$ & $14.03 \pm 0.21$ & $\ldots$ & $\ldots$ & $\ldots$ & $\ldots$ & $<0.12$ & $<0.12$ & $0.55 \pm 0.05$ & $1.39 \pm 0.15$ \\
\hline IC 1613 & $\ldots$ & $9.88 \pm 0.09$ & $9.21 \pm 0.10$ & $\ldots$ & $\ldots$ & $\ldots$ & $<0.06$ & $<0.14$ & $1.42 \pm 0.21$ & $3.69 \pm 0.55$ \\
\hline IC 1616 & $\ldots$ & $13.33 \pm 0.21$ & $\ldots$ & $10.82 \pm 0.02$ & $10.14 \pm 0.03$ & $9.87 \pm 0.04$ & $0.11 \pm 0.03$ & $0.15 \pm 0.03$ & $0.65 \pm 0.06$ & $2.96 \pm 0.21$ \\
\hline
\end{tabular}


Table 4-Continued

\begin{tabular}{|c|c|c|c|c|c|c|c|c|c|c|}
\hline \multirow{2}{*}{$\begin{array}{l}\text { Object Name } \\
\text { (1) }\end{array}$} & \multicolumn{3}{|c|}{ Optical Photometry } & \multicolumn{3}{|c|}{ Near-Infrared Photometry } & \multicolumn{4}{|c|}{ IRAS fluxes } \\
\hline & $\begin{array}{c}U \\
(\mathrm{mag}) \\
(2)\end{array}$ & $\begin{array}{c}B \\
(\mathrm{mag}) \\
(3)\end{array}$ & $\begin{array}{c}V \\
(\mathrm{mag}) \\
(4)\end{array}$ & $\begin{array}{c}J \\
(\mathrm{mag}) \\
(5)\end{array}$ & $\begin{array}{c}H \\
(\mathrm{mag}) \\
(6)\end{array}$ & $\begin{array}{c}K \\
(\mathrm{mag}) \\
(7)\end{array}$ & $\begin{array}{c}12 \mu \mathrm{m} \\
(\mathrm{Jy}) \\
(8)\end{array}$ & $\begin{array}{c}25 \mu \mathrm{m} \\
(\mathrm{Jy}) \\
(9)\end{array}$ & $\begin{array}{c}60 \mu \mathrm{m} \\
(\mathrm{Jy}) \\
(10)\end{array}$ & $\begin{array}{c}100 \mu \mathrm{m} \\
(\mathrm{Jy}) \\
(11)\end{array}$ \\
\hline ESO 352-G007 & $\ldots$ & $14.76 \pm 0.21$ & $\cdots$ & $12.04 \pm 0.04$ & $11.29 \pm 0.05$ & $11.17 \pm 0.06$ & $<0.10$ & $<0.11$ & $0.25 \pm 0.04$ & $0.85 \pm 0.15$ \\
\hline NGC 0392 & $14.17 \pm 0.15$ & $13.68 \pm 0.13$ & $12.71 \pm 0.13$ & $10.48 \pm 0.02$ & $9.80 \pm 0.02$ & $9.56 \pm 0.03$ & $<0.03$ & $<0.04$ & $0.16 \pm 0.04$ & $0.38 \pm 0.11$ \\
\hline ESO 243-G041 & $\ldots$ & $14.43 \pm 0.21$ & $\ldots$ & $11.35 \pm 0.02$ & $10.67 \pm 0.02$ & $10.50 \pm 0.04$ & $\ldots$ & $\cdots$ & $\cdots$ & $\cdots$ \\
\hline ESO 296-G002 & $\ldots$ & $14.38 \pm 0.21$ & $\ldots$ & $11.30 \pm 0.03$ & $10.57 \pm 0.04$ & $10.50 \pm 0.06$ & $<0.11$ & $<0.09$ & $0.75 \pm 0.06$ & $1.65 \pm 0.15$ \\
\hline ESO 243-G045 & $\ldots$ & $13.39 \pm 0.21$ & $\ldots$ & $10.61 \pm 0.02$ & $9.93 \pm 0.02$ & $9.71 \pm 0.04$ & $\ldots$ & $\ldots$ & $\ldots$ & $\ldots$ \\
\hline NGC 0403 & $\ldots$ & $13.38 \pm 0.20$ & $\ldots$ & $10.23 \pm 0.01$ & $9.55 \pm 0.02$ & $9.30 \pm 0.03$ & $<0.03$ & $<0.03$ & $<0.04$ & $0.32 \pm 0.09$ \\
\hline IC 1633 & $\ldots$ & $12.60 \pm 0.20$ & $\ldots$ & $9.36 \pm 0.01$ & $8.66 \pm 0.01$ & $8.40 \pm 0.03$ & $<0.03$ & $<0.04$ & $<0.04$ & $<0.16$ \\
\hline UGC 00726 & $\cdots$ & $14.59 \pm 0.25$ & $\cdots$ & $\cdots$ & $\ldots$ & $\ldots$ & $\ldots$ & $\ldots$ & $\ldots$ & $\ldots$ \\
\hline NGC 0407 & $\ldots$ & $14.28 \pm 0.18$ & $\ldots$ & $10.74 \pm 0.03$ & $10.02 \pm 0.02$ & $9.80 \pm 0.03$ & $\ldots$ & $\ldots$ & $\ldots$ & $\ldots$ \\
\hline UGC 00732 & $\ldots$ & $14.64 \pm 0.18$ & $\ldots$ & $11.78 \pm 0.04$ & $10.93 \pm 0.05$ & $11.01 \pm 0.09$ & $\ldots$ & $\ldots$ & $\ldots$ & $\ldots$ \\
\hline UGC 00736 & $\ldots$ & $14.96 \pm 0.26$ & $\ldots$ & $13.20 \pm 0.06$ & $12.88 \pm 0.11$ & $12.42 \pm 0.12$ & $\ldots$ & $\ldots$ & $\ldots$ & $\ldots$ \\
\hline NGC 0410 & $13.08 \pm 0.13$ & $12.52 \pm 0.13$ & $11.48 \pm 0.13$ & $9.38 \pm 0.01$ & $8.66 \pm 0.02$ & $8.38 \pm 0.02$ & $<0.03$ & $<0.03$ & $<0.03$ & $<0.12$ \\
\hline ESO 243-G051 & $\cdots$ & $14.16 \pm 0.21$ & $\cdots$ & $11.03 \pm 0.02$ & $10.32 \pm 0.02$ & $10.02 \pm 0.03$ & $0.14 \pm 0.03$ & $<0.14$ & $0.60 \pm 0.07$ & $2.26 \pm 0.20$ \\
\hline ESO 243-G052 & $\ldots$ & $14.68 \pm 0.21$ & $\ldots$ & $11.68 \pm 0.03$ & $11.01 \pm 0.03$ & $10.78 \pm 0.04$ & $\ldots$ & $\ldots$ & $\ldots$ & $\ldots$ \\
\hline PGC 04663 & $\ldots$ & $\ldots$ & $\ldots$ & $\ldots$ & $\ldots$ & $\ldots$ & $\ldots$ & $\ldots$ & $\ldots$ & $\ldots$ \\
\hline NGC 0467 & $13.45 \pm 0.22$ & $12.90 \pm 0.20$ & $11.85 \pm 0.22$ & $9.97 \pm 0.02$ & $9.24 \pm 0.02$ & $9.01 \pm 0.03$ & $<0.04$ & $<0.06$ & $<0.05$ & $<0.21$ \\
\hline NGC 0470 & $12.63 \pm 0.14$ & $12.53 \pm 0.13$ & $11.78 \pm 0.13$ & $9.76 \pm 0.02$ & $9.10 \pm 0.02$ & $8.84 \pm 0.03$ & $0.41 \pm 0.04$ & $1.38 \pm 0.10$ & $7.09 \pm 0.04$ & $12.00 \pm 0.15$ \\
\hline NGC 0474 & $12.75 \pm 0.13$ & $12.37 \pm 0.13$ & $11.51 \pm 0.13$ & $9.49 \pm 0.03$ & $8.80 \pm 0.03$ & $8.56 \pm 0.04$ & $<0.03$ & $<0.06$ & $<0.03$ & $<0.10$ \\
\hline ESO 352-G047 & $\cdots$ & $15.36 \pm 0.21$ & $\cdots$ & $\ldots$ & $\ldots$ & $\ldots$ & $\ldots$ & $\ldots$ & $\ldots$ & $\ldots$ \\
\hline UGC 00885 & $\ldots$ & $\ldots$ & $\ldots$ & $\ldots$ & $\ldots$ & $\ldots$ & $\ldots$ & $\ldots$ & $\ldots$ & $\ldots$ \\
\hline ESO 352-G050 & $\ldots$ & $15.40 \pm 0.21$ & $\ldots$ & $\ldots$ & $\ldots$ & $\ldots$ & $\ldots$ & $\ldots$ & $\ldots$ & $\ldots$ \\
\hline NGC 0479 & $\ldots$ & $14.71 \pm 0.20$ & $\ldots$ & $12.84 \pm 0.06$ & $11.87 \pm 0.06$ & $11.82 \pm 0.10$ & $\ldots$ & $\ldots$ & $\ldots$ & $\ldots$ \\
\hline NGC 0491 & $13.22 \pm 0.15$ & $13.21 \pm 0.15$ & $12.54 \pm 0.15$ & $10.35 \pm 0.02$ & $9.70 \pm 0.02$ & $9.39 \pm 0.03$ & $0.27 \pm 0.03$ & $0.37 \pm 0.03$ & $2.84 \pm 0.17$ & $8.63 \pm 0.52$ \\
\hline UGC 00910 & $\ldots$ & $14.64 \pm 0.19$ & $\ldots$ & $12.56 \pm 0.05$ & $11.82 \pm 0.04$ & $12.18 \pm 0.13$ & $<0.21$ & $<0.07$ & $0.33 \pm 0.05$ & $<2.15$ \\
\hline ESO 352-G057 & $\ldots$ & $14.59 \pm 0.21$ & $\ldots$ & $11.71 \pm 0.03$ & $11.01 \pm 0.03$ & $10.79 \pm 0.05$ & $\ldots$ & $\ldots$ & $\ldots$ & $\ldots$ \\
\hline ESO 352-G062 & $\ldots$ & $15.23 \pm 0.21$ & $\ldots$ & $12.00 \pm 0.04$ & $11.21 \pm 0.04$ & $10.91 \pm 0.05$ & $\ldots$ & $\ldots$ & $\ldots$ & $\ldots$ \\
\hline ESO 352-G064 & $\ldots$ & $14.53 \pm 0.21$ & $\ldots$ & $11.80 \pm 0.04$ & $11.11 \pm 0.03$ & $10.86 \pm 0.05$ & $\ldots$ & $\ldots$ & $\ldots$ & $\ldots$ \\
\hline NGC 0527 & $\ldots$ & $14.04 \pm 0.21$ & $\cdots$ & $10.76 \pm 0.01$ & $10.04 \pm 0.01$ & $9.79 \pm 0.01$ & $\ldots$ & $\ldots$ & $\cdots$ & $\ldots$ \\
\hline NGC 0514 & $\ldots$ & $12.24 \pm 0.15$ & $11.65 \pm 0.15$ & $10.55 \pm 0.01$ & $9.91 \pm 0.02$ & $9.14 \pm 0.09$ & $0.15 \pm 0.04$ & $0.29 \pm 0.04$ & $0.97 \pm 0.09$ & $3.94 \pm 0.35$ \\
\hline ESO 352-G069 & $\ldots$ & $13.93 \pm 0.21$ & $\cdots$ & $11.61 \pm 0.03$ & $10.94 \pm 0.04$ & $10.59 \pm 0.05$ & $0.13 \pm 0.03$ & $0.22 \pm 0.02$ & $1.47 \pm 0.07$ & $3.17 \pm 0.22$ \\
\hline UGC 00957 & $\ldots$ & $\ldots$ & $\ldots$ & $\ldots$ & $\ldots$ & $\ldots$ & $\ldots$ & $\ldots$ & $\ldots$ & $\ldots$ \\
\hline NGC 0520 & $12.41 \pm 0.14$ & $12.24 \pm 0.13$ & $11.42 \pm 0.13$ & $9.43 \pm 0.02$ & $8.69 \pm 0.03$ & $8.37 \pm 0.03$ & $0.91 \pm 0.05$ & $3.04 \pm 0.04$ & $31.60 \pm 0.05$ & $46.60 \pm 0.16$ \\
\hline
\end{tabular}


Table 4-Continued

\begin{tabular}{|c|c|c|c|c|c|c|c|c|c|c|}
\hline \multirow{2}{*}{$\begin{array}{c}\text { Object Name } \\
\text { (1) }\end{array}$} & \multicolumn{3}{|c|}{ Optical Photometry } & \multicolumn{3}{|c|}{ Near-Infrared Photometry } & \multicolumn{4}{|c|}{ IRAS fluxes } \\
\hline & $\begin{array}{c}U \\
(\mathrm{mag}) \\
(2)\end{array}$ & $\begin{array}{c}B \\
(\mathrm{mag}) \\
(3)\end{array}$ & $\begin{array}{c}V \\
(\mathrm{mag}) \\
(4)\end{array}$ & $\begin{array}{c}J \\
(\mathrm{mag}) \\
(5)\end{array}$ & $\begin{array}{c}H \\
(\mathrm{mag}) \\
(6)\end{array}$ & $\begin{array}{c}K \\
(\mathrm{mag}) \\
(7)\end{array}$ & $\begin{array}{c}12 \mu \mathrm{m} \\
(\mathrm{Jy}) \\
(8)\end{array}$ & $\begin{array}{c}25 \mu \mathrm{m} \\
(\mathrm{Jy}) \\
(9)\end{array}$ & $\begin{array}{c}60 \mu \mathrm{m} \\
(\mathrm{Jy}) \\
(10)\end{array}$ & $\begin{array}{c}100 \mu \mathrm{m} \\
(\mathrm{Jy}) \\
(11)\end{array}$ \\
\hline NGC 0530 & $\ldots$ & $13.96 \pm 0.16$ & $\ldots$ & $10.68 \pm 0.02$ & $9.98 \pm 0.02$ & $9.72 \pm 0.03$ & $\ldots$ & $\ldots$ & $\ldots$ & $\cdots$ \\
\hline IC 0107 & $\ldots$ & $14.92 \pm 0.23$ & $\ldots$ & $12.92 \pm 0.07$ & $12.21 \pm 0.07$ & $11.98 \pm 0.16$ & $\ldots$ & $\ldots$ & $\ldots$ & $\ldots$ \\
\hline UGC 00984 & $\ldots$ & $14.62 \pm 0.15$ & $\ldots$ & $11.70 \pm 0.03$ & $11.08 \pm 0.04$ & $10.87 \pm 0.05$ & $\ldots$ & $\ldots$ & $\ldots$ & $\ldots$ \\
\hline IC 1698 & $\ldots$ & $14.43 \pm 0.18$ & $\ldots$ & $11.71 \pm 0.03$ & $11.00 \pm 0.04$ & $10.72 \pm 0.04$ & $\ldots$ & $\ldots$ & $\ldots$ & $\ldots$ \\
\hline UGC 00985 & $\cdots$ & $14.89 \pm 0.19$ & $\cdots$ & $11.70 \pm 0.03$ & $11.08 \pm 0.05$ & $10.64 \pm 0.05$ & $\cdots$ & $\cdots$ & $\cdots$ & $\cdots$ \\
\hline IC 1700 & $\ldots$ & $13.78 \pm 0.16$ & $\ldots$ & $10.79 \pm 0.03$ & $10.05 \pm 0.04$ & $9.81 \pm 0.04$ & $\ldots$ & $\ldots$ & $\ldots$ & $\ldots$ \\
\hline NGC 0538 & $\ldots$ & $14.58 \pm 0.18$ & $\ldots$ & $11.13 \pm 0.02$ & $10.46 \pm 0.03$ & $10.16 \pm 0.04$ & $\ldots$ & $\ldots$ & $\ldots$ & $\ldots$ \\
\hline NGC 0535 & $\ldots$ & $14.83 \pm 0.16$ & $\ldots$ & $11.56 \pm 0.02$ & $10.80 \pm 0.03$ & $10.48 \pm 0.03$ & $\ldots$ & $\ldots$ & $\ldots$ & $\ldots$ \\
\hline UGC 00999 & $\ldots$ & $\ldots$ & $\ldots$ & $13.28 \pm 0.08$ & $12.38 \pm 0.09$ & $12.19 \pm 0.10$ & $\ldots$ & $\ldots$ & $\ldots$ & $\ldots$ \\
\hline UGC 01003 & $\ldots$ & $14.88 \pm 0.16$ & $\ldots$ & $11.77 \pm 0.02$ & $11.08 \pm 0.03$ & $10.81 \pm 0.04$ & $\ldots$ & $\ldots$ & $\ldots$ & $\ldots$ \\
\hline NGC 0541 & $13.42 \pm 0.17$ & $13.03 \pm 0.15$ & $12.08 \pm 0.15$ & $10.28 \pm 0.02$ & $9.57 \pm 0.03$ & $9.30 \pm 0.03$ & $<0.02$ & $<0.06$ & $<0.05$ & $<0.13$ \\
\hline NGC 0545 & $\ldots$ & $13.21 \pm 0.18$ & $\ldots$ & $\ldots$ & $\ldots$ & $\ldots$ & $<0.03$ & $<0.06$ & $<0.03$ & $<0.22$ \\
\hline NGC 0547 & $\ldots$ & $13.16 \pm 0.15$ & $\ldots$ & $9.47 \pm 0.00$ & $8.76 \pm 0.01$ & $8.49 \pm 0.01$ & $<0.03$ & $<0.07$ & $<0.04$ & $<0.21$ \\
\hline NGC 0557 & $\ldots$ & $14.48 \pm 0.16$ & $\ldots$ & $11.12 \pm 0.03$ & $10.37 \pm 0.04$ & $10.34 \pm 0.06$ & $\ldots$ & $\ldots$ & $\ldots$ & $\ldots$ \\
\hline ESO 353-G002 & $\ldots$ & $15.27 \pm 0.21$ & $\ldots$ & $\ldots$ & $\ldots$ & $\ldots$ & $\ldots$ & $\ldots$ & $\ldots$ & $\ldots$ \\
\hline UGC 01026 & $\ldots$ & $\ldots$ & $\ldots$ & $\ldots$ & $\ldots$ & $\ldots$ & $\ldots$ & $\ldots$ & $\ldots$ & $\ldots$ \\
\hline UGC 01040 & $\ldots$ & $14.85 \pm 0.22$ & $\ldots$ & $11.72 \pm 0.02$ & $11.05 \pm 0.03$ & $10.87 \pm 0.04$ & $\ldots$ & $\ldots$ & $\ldots$ & $\ldots$ \\
\hline NGC 0568 & $14.09 \pm 0.17$ & $13.58 \pm 0.17$ & $12.59 \pm 0.17$ & $10.48 \pm 0.02$ & $9.81 \pm 0.03$ & $9.58 \pm 0.05$ & $\ldots$ & $\ldots$ & $\ldots$ & $\ldots$ \\
\hline UGC 01057 & $\ldots$ & $14.64 \pm 0.18$ & $\ldots$ & $12.26 \pm 0.04$ & $11.40 \pm 0.04$ & $11.21 \pm 0.05$ & $<0.08$ & $<0.13$ & $0.40 \pm 0.08$ & $1.12 \pm 0.21$ \\
\hline NGC 0574 & $\ldots$ & $14.14 \pm 0.21$ & $\ldots$ & $11.60 \pm 0.03$ & $10.91 \pm 0.04$ & $10.56 \pm 0.04$ & $0.17 \pm 0.04$ & $0.49 \pm 0.01$ & $3.43 \pm 0.21$ & $5.55 \pm 0.44$ \\
\hline IC 0127 & $\ldots$ & $\ldots$ & $\ldots$ & $10.94 \pm 0.06$ & $10.00 \pm 0.02$ & $9.79 \pm 0.06$ & $<0.17$ & $<0.12$ & $0.37 \pm 0.07$ & $1.27 \pm 0.18$ \\
\hline NGC 0584 & $11.93 \pm 0.13$ & $11.44 \pm 0.13$ & $10.48 \pm 0.13$ & $8.24 \pm 0.02$ & $7.48 \pm 0.02$ & $7.30 \pm 0.03$ & $<0.05$ & $<0.07$ & $<0.04$ & $0.59 \pm 0.10$ \\
\hline NGC 0586 & $14.32 \pm 0.21$ & $14.10 \pm 0.20$ & $13.16 \pm 0.21$ & $11.00 \pm 0.02$ & $10.31 \pm 0.03$ & $10.08 \pm 0.04$ & $\ldots$ & $\ldots$ & $\ldots$ & $\ldots$ \\
\hline MESSIER 033 & $6.17 \pm 0.04$ & $6.27 \pm 0.03$ & $5.72 \pm 0.04$ & $5.04 \pm 0.03$ & $4.35 \pm 0.04$ & $4.10 \pm 0.04$ & $32.70 \pm 4.90$ & $40.30 \pm 6.04$ & $420.00 \pm 62.90$ & $1260.00 \pm 188.00$ \\
\hline NGC 0628 & $\ldots$ & $9.95 \pm 0.10$ & $9.39 \pm 0.10$ & $7.63 \pm 0.03$ & $7.04 \pm 0.03$ & $6.84 \pm 0.05$ & $2.07 \pm 0.52$ & $1.90 \pm 0.48$ & $20.90 \pm 5.22$ & $65.60 \pm 16.40$ \\
\hline UGC 01181 & $\ldots$ & $\frac{1.00}{\ldots}$ & $\begin{array}{c}\ldots .00+0.10 \\
\ldots\end{array}$ & $\begin{array}{l}\ldots \\
\ldots\end{array}$ & $\begin{array}{l}1.0 \pm 0.00 \\
\ldots\end{array}$ & $\begin{array}{l}0.07 \pm 0 \\
\ldots\end{array}$ & $\begin{array}{l}2.01+0.02 \\
\ldots\end{array}$ & $\begin{array}{l}1.30+0.40 \\
\ldots\end{array}$ & $\begin{array}{l}20.001 .02 \\
\ldots\end{array}$ & $\frac{1}{\ldots}$ \\
\hline IC 0148 & $\ldots$ & $13.44 \pm 0.22$ & $\ldots$ & $\ldots$ & $\ldots$ & $\ldots$ & $\ldots$ & $\ldots$ & $\ldots$ & $\ldots$ \\
\hline UGC 01200 & $\ldots$ & $13.81 \pm 0.23$ & $\ldots$ & $\ldots$ & $\ldots$ & $\ldots$ & $<0.12$ & $<0.17$ & $0.29 \pm 0.05$ & $<0.90$ \\
\hline NGC 0660 & $\ldots$ & $12.02 \pm 0.10$ & $11.16 \pm 0.12$ & $8.53 \pm 0.02$ & $7.72 \pm 0.02$ & $7.34 \pm 0.02$ & $2.31 \pm 0.23$ & $7.05 \pm 0.70$ & $67.30 \pm 6.73$ & $105.00 \pm 10.50$ \\
\hline UGC 01211 & $\ldots$ & $\ldots$ & $\ldots$ & $\ldots$ & $\ldots$ & $\ldots$ & $\ldots$ & $\ldots$ & $\ldots$ & $\ldots$ \\
\hline IC 0159 & $\ldots$ & $\ldots$ & $\ldots$ & $12.32 \pm 0.06$ & $11.65 \pm 0.07$ & $11.51 \pm 0.11$ & $<0.13$ & $<0.22$ & $0.63 \pm 0.06$ & $1.61 \pm 0.19$ \\
\hline PGC 06504 & $\ldots$ & $\ldots$ & $\ldots$ & $\ldots$ & .. & $\ldots$ & $\ldots$ & $\ldots$ & $\ldots$ & $\ldots$ \\
\hline
\end{tabular}


Table 4-Continued

\begin{tabular}{|c|c|c|c|c|c|c|c|c|c|c|}
\hline \multirow{2}{*}{$\begin{array}{l}\text { Object Name } \\
\\
(1) \\
\end{array}$} & \multicolumn{3}{|c|}{ Optical Photometry } & \multicolumn{3}{|c|}{ Near-Infrared Photometry } & \multicolumn{4}{|c|}{ IRAS fluxes } \\
\hline & $\begin{array}{c}U \\
(\mathrm{mag}) \\
(2)\end{array}$ & $\begin{array}{c}B \\
(\mathrm{mag}) \\
(3)\end{array}$ & $\begin{array}{c}V \\
(\mathrm{mag}) \\
(4)\end{array}$ & $\begin{array}{c}J \\
(\mathrm{mag}) \\
(5)\end{array}$ & $\begin{array}{c}H \\
(\mathrm{mag}) \\
(6)\end{array}$ & $\begin{array}{c}K \\
(\mathrm{mag}) \\
(7)\end{array}$ & $\begin{array}{c}12 \mu \mathrm{m} \\
(\mathrm{Jy}) \\
(8)\end{array}$ & $\begin{array}{c}25 \mu \mathrm{m} \\
(\mathrm{Jy}) \\
(9)\end{array}$ & $\begin{array}{l}60 \mu \mathrm{m} \\
(\mathrm{Jy}) \\
(10)\end{array}$ & $\begin{array}{c}100 \mu \mathrm{m} \\
(\mathrm{Jy}) \\
(11)\end{array}$ \\
\hline NGC 0671 & $\cdots$ & $14.17 \pm 0.18$ & $\cdots$ & $11.44 \pm 0.01$ & $10.77 \pm 0.02$ & $10.43 \pm 0.03$ & $<0.22$ & $<0.14$ & $0.62 \pm 0.07$ & $1.54 \pm 0.25$ \\
\hline UGC 01261 & $\cdots$ & $15.21 \pm 0.18$ & $\cdots$ & $\cdots$ & $\cdots$ & $\cdots$ & $<0.14$ & $<0.18$ & $0.37 \pm 0.07$ & $1.12 \pm 0.26$ \\
\hline UGC 01262 & $\cdots$ & $\ldots$ & $\cdots$ & $\cdots$ & $\cdots$ & $\cdots$ & $\cdots$ & $\cdots$ & $\ldots$ & $\ldots$ \\
\hline UGC 01264 & $\cdots$ & $\cdots$ & $\cdots$ & $\cdots$ & $\ldots$ & $\ldots$ & $\cdots$ & $\ldots$ & $\ldots$ & $\ldots$ \\
\hline NGC 0676 & $\cdots$ & $\cdots$ & $\cdots$ & $\cdots$ & $\cdots$ & $\cdots$ & $<0.04$ & $<0.06$ & $0.27 \pm 0.05$ & $0.80 \pm 0.12$ \\
\hline UGC 01271 & $\cdots$ & $14.11 \pm 0.18$ & $\cdots$ & $11.11 \pm 0.01$ & $10.39 \pm 0.02$ & $10.17 \pm 0.03$ & $\ldots$ & $\ldots$ & $\ldots$ & $\ldots$ \\
\hline UGC 01274 & $\ldots$ & $14.85 \pm 0.19$ & $\ldots$ & $11.45 \pm 0.01$ & $10.72 \pm 0.02$ & $10.45 \pm 0.03$ & $\ldots$ & $\ldots$ & $\ldots$ & $\ldots$ \\
\hline UGC 01278 & $\ldots$ & $15.35 \pm 0.19$ & $\ldots$ & $12.92 \pm 0.05$ & $12.34 \pm 0.07$ & $11.79 \pm 0.10$ & $\ldots$ & $\ldots$ & $\ldots$ & $\ldots$ \\
\hline NGC 0693 & $13.39 \pm 0.14$ & $13.24 \pm 0.14$ & $12.44 \pm 0.14$ & $10.23 \pm 0.02$ & $9.70 \pm 0.03$ & $9.23 \pm 0.03$ & $0.28 \pm 0.05$ & $0.49 \pm 0.07$ & $6.86 \pm 0.04$ & $11.20 \pm 0.10$ \\
\hline UGC 01312 & $\ldots$ & $14.81 \pm 0.18$ & $\ldots$ & $12.45 \pm 0.04$ & $11.73 \pm 0.04$ & $11.45 \pm 0.06$ & $<0.15$ & $<0.21$ & $0.31 \pm 0.05$ & $1.14 \pm 0.26$ \\
\hline ESO 245-G007 & $\cdots$ & $13.07 \pm 0.21$ & $\cdots$ & $\ldots$ & $\ldots$ & $\ldots$ & $\ldots$ & $\ldots$ & $\ldots$ & $\ldots$ \\
\hline NGC 0707 & $\cdots$ & $\cdots$ & $\cdots$ & $11.29 \pm 0.02$ & $10.59 \pm 0.03$ & $10.40 \pm 0.04$ & $\cdots$ & $\cdots$ & $\cdots$ & $\cdots$ \\
\hline NGC 0706 & $13.24 \pm 0.13$ & $13.20 \pm 0.13$ & $12.50 \pm 0.13$ & $10.50 \pm 0.02$ & $9.82 \pm 0.02$ & $9.52 \pm 0.03$ & $0.28 \pm 0.04$ & $0.56 \pm 0.04$ & $3.41 \pm 0.24$ & $8.85 \pm 0.62$ \\
\hline UGC 01364 & $\ldots$ & $\ldots$ & $\ldots$ & $\ldots$ & $\ldots$ & $\ldots$ & $\ldots$ & $\ldots$ & $\ldots$ & $\ldots$ \\
\hline PGC 07064 & $\cdots$ & $\ldots$ & $\ldots$ & $11.40 \pm 0.04$ & $10.70 \pm 0.04$ & $10.36 \pm 0.06$ & $\cdots$ & $\cdots$ & $\cdots$ & $\cdots$ \\
\hline PGC 07210 & $\ldots$ & $\ldots$ & $\ldots$ & $12.62 \pm 0.05$ & $12.21 \pm 0.08$ & $11.65 \pm 0.09$ & $<0.13$ & $<0.15$ & $0.42 \pm 0.05$ & $0.77 \pm 0.14$ \\
\hline UGC 01408 & $\ldots$ & $15.32 \pm 0.20$ & $\ldots$ & $12.46 \pm 0.05$ & $11.66 \pm 0.04$ & $11.91 \pm 0.14$ & $\ldots$ & $\ldots$ & $\ldots$ & $\ldots$ \\
\hline IC 1755 & $\ldots$ & $14.70 \pm 0.18$ & $\ldots$ & $11.59 \pm 0.04$ & $10.85 \pm 0.04$ & $10.61 \pm 0.06$ & $\ldots$ & $\ldots$ & $\ldots$ & $\ldots$ \\
\hline UGC 01448 & $\cdots$ & $14.91 \pm 0.18$ & $\cdots$ & $12.10 \pm 0.03$ & $11.36 \pm 0.04$ & $11.15 \pm 0.05$ & $<0.13$ & $<0.21$ & $0.25 \pm 0.05$ & $0.78 \pm 0.14$ \\
\hline KUG 0156-084 & $\cdots$ & $13.60 \pm 0.40$ & $13.08 \pm 0.40$ & $13.14 \pm 0.04$ & $12.55 \pm 0.06$ & $12.22 \pm 0.09$ & $<0.11$ & $<0.14$ & $0.37 \pm 0.05$ & $1.03 \pm 0.14$ \\
\hline NGC 0770 & $\cdots$ & $13.91 \pm 0.15$ & $\ldots$ & $11.23 \pm 0.02$ & $10.48 \pm 0.02$ & $10.28 \pm 0.03$ & $\ldots$ & $\ldots$ & $\ldots$ & $\ldots$ \\
\hline NGC 0772 & $11.35 \pm 0.13$ & $11.09 \pm 0.13$ & $10.31 \pm 0.13$ & $8.17 \pm 0.03$ & $7.44 \pm 0.03$ & $7.20 \pm 0.04$ & $1.12 \pm 0.03$ & $0.91 \pm 0.05$ & $6.78 \pm 0.04$ & $24.10 \pm 0.15$ \\
\hline UGC 01468 & $\cdots$ & $\cdots$ & $\ldots$ & $13.69 \pm 0.09$ & $13.28 \pm 0.13$ & $12.82 \pm 0.18$ & $\cdots$ & $\ldots$ & $\cdots$ & $\cdots$ \\
\hline NGC 0774 & $\cdots$ & $13.97 \pm 0.16$ & $\ldots$ & $10.74 \pm 0.02$ & $10.08 \pm 0.02$ & $9.80 \pm 0.04$ & $\cdots$ & $\ldots$ & $\cdots$ & $\ldots$ \\
\hline NGC 0777 & $13.09 \pm 0.14$ & $12.49 \pm 0.14$ & $11.45 \pm 0.14$ & $9.39 \pm 0.01$ & $8.67 \pm 0.01$ & $8.37 \pm 0.02$ & $<0.03$ & $<0.04$ & $<0.04$ & $<0.09$ \\
\hline NGC 0778 & $\ldots$ & $14.22 \pm 0.16$ & $\ldots$ & $11.24 \pm 0.03$ & $10.56 \pm 0.03$ & $10.20 \pm 0.03$ & $\ldots$ & $\ldots$ & $\ldots$ & $\ldots$ \\
\hline NGC 0787 & $\cdots$ & $\ldots$ & $\ldots$ & $10.38 \pm 0.02$ & $9.72 \pm 0.03$ & $9.47 \pm 0.03$ & $<0.12$ & $<0.18$ & $0.29 \pm 0.05$ & $1.09 \pm 0.14$ \\
\hline PGC 07654 & $\ldots$ & $\ldots$ & $\ldots$ & $\ldots$ & $\ldots$ & $\ldots$ & $\ldots$ & $\ldots$ & $\ldots$ & $\ldots$ \\
\hline NGC 0783 & $\cdots$ & $12.84 \pm 0.19$ & $\ldots$ & $10.59 \pm 0.02$ & $9.83 \pm 0.03$ & $9.68 \pm 0.04$ & $0.17 \pm 0.03$ & $0.29 \pm 0.02$ & $2.02 \pm 0.14$ & $5.85 \pm 0.29$ \\
\hline UGCA 023 & $\ldots$ & $\cdots$ & $\ldots$ & $12.63 \pm 0.05$ & $11.85 \pm 0.05$ & $11.64 \pm 0.09$ & $<0.11$ & $<0.15$ & $0.34 \pm 0.05$ & $0.74 \pm 0.13$ \\
\hline NGC 0809 & $\ldots$ & $\ldots$ & $\ldots$ & $11.41 \pm 0.02$ & $10.72 \pm 0.02$ & $10.60 \pm 0.04$ & $\ldots$ & $\ldots$ & $\ldots$ & $\ldots$ \\
\hline UGC 01584 & $\ldots$ & $\ldots$ & $\ldots$ & $\ldots$ & $\ldots$ & $\ldots$ & $\ldots$ & $\ldots$ & $\cdots$ & $\ldots$ \\
\hline
\end{tabular}


Table 4-Continued

\begin{tabular}{|c|c|c|c|c|c|c|c|c|c|c|}
\hline \multirow{2}{*}{$\begin{array}{c}\text { Object Name } \\
\text { (1) }\end{array}$} & \multicolumn{3}{|c|}{ Optical Photometry } & \multicolumn{3}{|c|}{ Near-Infrared Photometry } & \multicolumn{4}{|c|}{ IRAS fluxes } \\
\hline & $\begin{array}{c}U \\
(\mathrm{mag}) \\
(2)\end{array}$ & $\begin{array}{c}B \\
(\mathrm{mag}) \\
(3)\end{array}$ & $\begin{array}{c}V \\
(\mathrm{mag}) \\
(4)\end{array}$ & $\begin{array}{c}J \\
(\mathrm{mag}) \\
(5)\end{array}$ & $\begin{array}{c}H \\
(\mathrm{mag}) \\
(6)\end{array}$ & $\begin{array}{c}K \\
(\mathrm{mag}) \\
(7)\end{array}$ & $\begin{array}{c}12 \mu \mathrm{m} \\
(\mathrm{Jy}) \\
(8)\end{array}$ & $\begin{array}{c}25 \mu \mathrm{m} \\
(\mathrm{Jy}) \\
(9)\end{array}$ & $\begin{array}{c}60 \mu \mathrm{m} \\
(\mathrm{Jy}) \\
(10)\end{array}$ & $\begin{array}{c}100 \mu \mathrm{m} \\
(\mathrm{Jy}) \\
(11)\end{array}$ \\
\hline NGC 0810 & $\ldots$ & $\ldots$ & $\ldots$ & $\cdots$ & $\cdots$ & $\ldots$ & $\ldots$ & $\cdots$ & $\ldots$ & $\ldots$ \\
\hline UGC 01593 & $\ldots$ & $15.13 \pm 0.23$ & $\ldots$ & $\ldots$ & $\ldots$ & $\ldots$ & $\ldots$ & $\ldots$ & $\ldots$ & $\ldots$ \\
\hline UGC 01603 & $\ldots$ & $15.01 \pm 0.28$ & $\ldots$ & $13.65 \pm 0.06$ & $12.94 \pm 0.07$ & $12.93 \pm 0.14$ & $\ldots$ & $\ldots$ & $\ldots$ & $\ldots$ \\
\hline NGC 0830 & $\ldots$ & $\begin{array}{l}10.01 \pm 0.20 \\
\ldots\end{array}$ & $\ldots$ & $10.76 \pm 0.02$ & $10.11 \pm 0.03$ & $9.87 \pm 0.03$ & $\ldots$ & $\ldots$ & $\ldots$ & $\ldots$ \\
\hline NGC 0842 & $14.10 \pm 0.16$ & $13.61 \pm 0.16$ & $12.66 \pm 0.16$ & $10.47 \pm 0.02$ & $9.80 \pm 0.02$ & $9.54 \pm 0.03$ & $\cdots$ & $\cdots$ & $\cdots$ & $\cdots$ \\
\hline NGC 0814 & $\ldots$ & $\ldots$ & $\ldots$ & $12.07 \pm 0.03$ & $11.35 \pm 0.04$ & $11.18 \pm 0.05$ & $<0.24$ & $0.90 \pm 0.02$ & $4.26 \pm 0.21$ & $3.83 \pm 0.31$ \\
\hline KUG 0210-078 & $\ldots$ & $\ldots$ & $\ldots$ & $11.88 \pm 0.01$ & $11.14 \pm 0.01$ & $10.91 \pm 0.02$ & $\ldots$ & $\ldots$ & $\ldots$ & $\ldots$ \\
\hline NGC 0855 & $13.33 \pm 0.14$ & $13.30 \pm 0.13$ & $12.59 \pm 0.13$ & $10.72 \pm 0.03$ & $10.07 \pm 0.03$ & $9.83 \pm 0.05$ & $<0.08$ & $<0.15$ & $1.15 \pm 0.08$ & $2.32 \pm 0.26$ \\
\hline ESO 415-G011 & $\ldots$ & $15.92 \pm 0.21$ & $\ldots$ & $\ldots$ & $\ldots$ & $\ldots$ & $\ldots$ & $\ldots$ & $\ldots$ & $\ldots$ \\
\hline KUG 0211-075 & $\ldots$ & $\ldots$ & $\ldots$ & $\ldots$ & $\ldots$ & $\ldots$ & $0.22 \pm 0.03$ & $0.33 \pm 0.03$ & $2.99 \pm 0.18$ & $7.15 \pm 0.50$ \\
\hline NGC 0871 & $\ldots$ & $14.20 \pm 0.20$ & $13.61 \pm 0.21$ & $11.23 \pm 0.02$ & $10.63 \pm 0.03$ & $10.27 \pm 0.04$ & $0.21 \pm 0.03$ & $0.31 \pm 0.03$ & $3.92 \pm 0.16$ & $6.79 \pm 0.41$ \\
\hline KUG 0214-057 & $\ldots$ & $\ldots$ & $\ldots$ & $13.71 \pm 0.08$ & $13.10 \pm 0.10$ & $12.90 \pm 0.16$ & $\ldots$ & $\ldots$ & $\ldots$ & $\ldots$ \\
\hline UGC 01761 & $\ldots$ & $\ldots$ & $\ldots$ & $\ldots$ & $\ldots$ & $\ldots$ & $\ldots$ & $\ldots$ & $\ldots$ & $\ldots$ \\
\hline NGC 0881 & $13.43 \pm 0.15$ & $13.23 \pm 0.15$ & $12.44 \pm 0.15$ & $10.30 \pm 0.02$ & $9.66 \pm 0.03$ & $9.37 \pm 0.04$ & $<0.11$ & $<0.22$ & $0.48 \pm 0.10$ & $<3.26$ \\
\hline NGC 0895 & $12.21 \pm 0.17$ & $12.26 \pm 0.17$ & $11.73 \pm 0.17$ & $10.15 \pm 0.02$ & $9.73 \pm 0.04$ & $9.40 \pm 0.05$ & $0.15 \pm 0.03$ & $<0.19$ & $1.31 \pm 0.09$ & $5.52 \pm 0.55$ \\
\hline NGC 0891 & $11.08 \pm 0.18$ & $10.81 \pm 0.18$ & $9.93 \pm 0.18$ & $7.26 \pm 0.02$ & $6.36 \pm 0.02$ & $5.94 \pm 0.02$ & $5.66 \pm 0.85$ & $7.78 \pm 1.17$ & $61.10 \pm 9.17$ & $199.00 \pm 29.80$ \\
\hline NGC 0898 & $\ldots$ & $13.84 \pm 0.13$ & $\ldots$ & $10.36 \pm 0.01$ & $9.57 \pm 0.02$ & $9.29 \pm 0.02$ & $\ldots$ & $\ldots$ & $\ldots$ & $\ldots$ \\
\hline UGC 01859 & $\ldots$ & $13.90 \pm 0.16$ & $\ldots$ & $10.81 \pm 0.02$ & $10.12 \pm 0.02$ & $9.78 \pm 0.02$ & $\ldots$ & $\ldots$ & $\ldots$ & $\ldots$ \\
\hline NGC 0906 & $\ldots$ & $13.76 \pm 0.16$ & $12.88 \pm 0.16$ & $10.91 \pm 0.03$ & $10.21 \pm 0.03$ & $9.92 \pm 0.04$ & $\ldots$ & $\ldots$ & $\ldots$ & $\ldots$ \\
\hline NGC 0925 & $\ldots$ & $10.69 \pm 0.11$ & $10.12 \pm 0.12$ & $8.74 \pm 0.03$ & $8.07 \pm 0.04$ & $7.87 \pm 0.05$ & $0.26 \pm 0.04$ & $0.66 \pm 0.10$ & $7.65 \pm 1.15$ & $26.70 \pm 4.00$ \\
\hline PGC 09333 & $\ldots$ & $\ldots$ & $\ldots$ & $12.66 \pm 0.04$ & $12.07 \pm 0.05$ & $11.83 \pm 0.09$ & $\ldots$ & $\ldots$ & $\ldots$ & $\ldots$ \\
\hline NGC 0934 & $\ldots$ & $14.00 \pm 0.20$ & $13.14 \pm 0.20$ & $11.37 \pm 0.03$ & $10.63 \pm 0.03$ & $10.45 \pm 0.05$ & $\ldots$ & $\ldots$ & $\ldots$ & $\ldots$ \\
\hline UGC 01949 & $\ldots$ & $\ldots$ & $\ldots$ & $\ldots$ & $\ldots$ & $\ldots$ & $\ldots$ & $\ldots$ & $\ldots$ & $\ldots$ \\
\hline UGC 01976 & $\ldots$ & $14.65 \pm 0.22$ & $\ldots$ & $11.94 \pm 0.03$ & $11.26 \pm 0.04$ & $10.98 \pm 0.05$ & $<0.05$ & $<0.12$ & $0.31 \pm 0.05$ & $0.90 \pm 0.21$ \\
\hline NGC 0955 & $13.29 \pm 0.13$ & $12.93 \pm 0.13$ & $11.97 \pm 0.13$ & $9.74 \pm 0.01$ & $9.04 \pm 0.01$ & $8.75 \pm 0.01$ & $<0.12$ & $<0.24$ & $0.25 \pm 0.04$ & $1.17 \pm 0.14$ \\
\hline UGC 02010 & $\ldots$ & $14.10 \pm 0.18$ & $\ldots$ & $11.63 \pm 0.04$ & $10.82 \pm 0.04$ & $10.80 \pm 0.07$ & $\ldots$ & $\ldots$ & $\ldots$ & $\ldots$ \\
\hline NGC 0959 & $12.87 \pm 0.14$ & $12.95 \pm 0.14$ & $12.38 \pm 0.14$ & $10.95 \pm 0.01$ & $10.24 \pm 0.02$ & $9.61 \pm 0.06$ & $<0.11$ & $0.11 \pm 0.03$ & $1.09 \pm 0.07$ & $2.71 \pm 0.24$ \\
\hline NGC 0986A & $\ldots$ & $14.73 \pm 0.10$ & $13.97 \pm 0.15$ & $\ldots$ & $\ldots$ & $\ldots$ & $<0.07$ & $<0.06$ & $0.16 \pm 0.04$ & $<0.60$ \\
\hline NGC 0986 & $11.72 \pm 0.13$ & $11.64 \pm 0.13$ & $10.91 \pm 0.13$ & $8.75 \pm 0.02$ & $8.02 \pm 0.02$ & $7.78 \pm 0.03$ & $0.85 \pm 0.04$ & $3.12 \pm 0.04$ & $22.90 \pm 1.15$ & $46.00 \pm 1.84$ \\
\hline KUG 0232-079 & $\ldots$ & $\ldots$ & $\ldots$ & $11.66 \pm 0.02$ & $11.03 \pm 0.03$ & $10.74 \pm 0.05$ & $0.10 \pm 0.03$ & $<0.18$ & $1.08 \pm 0.08$ & $2.94 \pm 0.18$ \\
\hline NGC 0991 & $\ldots$ & $12.36 \pm 0.28$ & $\ldots$ & $11.98 \pm 0.02$ & $11.45 \pm 0.03$ & $11.18 \pm 0.05$ & $0.13 \pm 0.03$ & $<0.17$ & $0.69 \pm 0.05$ & $2.89 \pm 0.17$ \\
\hline IC 0243 & $\ldots$ & $\ldots$ & $\ldots$ & $11.60 \pm 0.03$ & $10.91 \pm 0.03$ & $10.53 \pm 0.05$ & $<0.07$ & $<0.07$ & $0.29 \pm 0.04$ & $0.80 \pm 0.11$ \\
\hline
\end{tabular}


Table 4-Continued

\begin{tabular}{|c|c|c|c|c|c|c|c|c|c|c|}
\hline \multirow{2}{*}{$\begin{array}{l}\text { Object Name } \\
\text { (1) }\end{array}$} & \multicolumn{3}{|c|}{ Optical Photometry } & \multicolumn{3}{|c|}{ Near-Infrared Photometry } & \multicolumn{4}{|c|}{ IRAS fluxes } \\
\hline & $\begin{array}{c}U \\
(\mathrm{mag}) \\
(2)\end{array}$ & $\begin{array}{c}B \\
(\mathrm{mag}) \\
(3)\end{array}$ & $\begin{array}{c}V \\
(\mathrm{mag}) \\
(4)\end{array}$ & $\begin{array}{c}J \\
(\mathrm{mag}) \\
(5)\end{array}$ & $\begin{array}{c}H \\
(\mathrm{mag}) \\
(6)\end{array}$ & $\begin{array}{c}K \\
(\mathrm{mag}) \\
(7)\end{array}$ & $\begin{array}{c}12 \mu \mathrm{m} \\
(\mathrm{Jy}) \\
(8)\end{array}$ & $\begin{array}{c}25 \mu \mathrm{m} \\
(\mathrm{Jy}) \\
(9)\end{array}$ & $\begin{array}{c}60 \mu \mathrm{m} \\
(\mathrm{Jy}) \\
(10)\end{array}$ & $\begin{array}{l}100 \mu \mathrm{m} \\
(\mathrm{Jy}) \\
(11)\end{array}$ \\
\hline NGC 1022 & $12.33 \pm 0.13$ & $12.09 \pm 0.13$ & $11.34 \pm 0.13$ & $9.45 \pm 0.01$ & $8.79 \pm 0.01$ & $8.50 \pm 0.02$ & $0.73 \pm 0.03$ & $3.37 \pm 0.04$ & $19.60 \pm 0.03$ & $26.70 \pm 0.14$ \\
\hline NGC 1035 & $\ldots$ & $12.89 \pm 0.15$ & $\ldots$ & $10.12 \pm 0.01$ & $9.42 \pm 0.01$ & $9.13 \pm 0.01$ & $0.29 \pm 0.03$ & $0.38 \pm 0.03$ & $3.57 \pm 0.18$ & $11.10 \pm 0.78$ \\
\hline NGC 1033 & $\ldots$ & $\ldots$ & $\ldots$ & $11.65 \pm 0.05$ & $11.00 \pm 0.05$ & $10.76 \pm 0.08$ & $\ldots$ & & $\ldots$ & $\ldots$ \\
\hline NGC 1042 & $11.47 \pm 0.16$ & $11.56 \pm 0.11$ & $11.02 \pm 0.11$ & $10.29 \pm 0.05$ & $9.73 \pm 0.06$ & $8.85 \pm 0.05$ & $0.10 \pm 0.03$ & $0.22 \pm 0.02$ & $1.57 \pm 0.11$ & $5.89 \pm 0.47$ \\
\hline NGC 1023 & $10.91 \pm 0.06$ & $10.35 \pm 0.06$ & $9.35 \pm 0.06$ & $7.16 \pm 0.02$ & $6.49 \pm 0.02$ & $6.24 \pm 0.02$ & $0.16 \pm 0.02$ & $<0.09$ & $<0.13$ & $<0.30$ \\
\hline NGC 1047 & $\ldots$ & $\ldots$ & $\ldots$ & $11.76 \pm 0.04$ & $11.14 \pm 0.05$ & $11.00 \pm 0.08$ & $\cdots$ & $\ldots$ & $\ldots$ & $\ldots$ \\
\hline NGC $1023 \mathrm{~A}$ & $\ldots$ & $\ldots$ & $\ldots$ & $\ldots$ & $\ldots$ & $\ldots$ & $\ldots$ & $\ldots$ & $\ldots$ & $\ldots$ \\
\hline NGC 0961 & $\cdots$ & $\cdots$ & $\cdots$ & $\cdots$ & $\cdots$ & $\cdots$ & $<0.09$ & $<0.10$ & $0.33 \pm 0.04$ & $1.05 \pm 0.12$ \\
\hline NGC 1052 & $11.84 \pm 0.13$ & $11.41 \pm 0.13$ & $10.47 \pm 0.13$ & $8.37 \pm 0.01$ & $7.70 \pm 0.01$ & $7.45 \pm 0.01$ & $0.20 \pm 0.02$ & $0.49 \pm 0.02$ & $0.90 \pm 0.05$ & $1.52 \pm 0.14$ \\
\hline NGC 1055 & $11.59 \pm 0.10$ & $11.40 \pm 0.10$ & $10.59 \pm 0.10$ & $8.25 \pm 0.02$ & $7.49 \pm 0.03$ & $7.15 \pm 0.03$ & $2.20 \pm 0.04$ & $2.89 \pm 0.06$ & $23.30 \pm 0.06$ & $60.10 \pm 0.11$ \\
\hline PGC 10213 & $\ldots$ & $\ldots$ & $\ldots$ & $\ldots$ & $\ldots$ & $\ldots$ & $\ldots$ & $\ldots$ & $\ldots$ & $\ldots$ \\
\hline UGC 02174 & $\ldots$ & $14.50 \pm 0.30$ & $\ldots$ & $\ldots$ & $\ldots$ & $\ldots$ & $\ldots$ & $\ldots$ & $\ldots$ & $\ldots$ \\
\hline NGC 1068 & $9.70 \pm 0.10$ & $9.61 \pm 0.10$ & $8.87 \pm 0.10$ & $6.97 \pm 0.01$ & $6.26 \pm 0.01$ & $\cdots$ & $36.10 \pm 0.06$ & $84.20 \pm 0.19$ & $182.00 \pm 0.10$ & $236.00 \pm 0.22$ \\
\hline UGC 02182 & $\ldots$ & $\ldots$ & $\ldots$ & $\ldots$ & $\ldots$ & $\ldots$ & $\ldots$ & $\ldots$ & $\ldots$ & $\ldots$ \\
\hline NGC 1069 & $\ldots$ & $\ldots$ & $\ldots$ & $11.45 \pm 0.03$ & $10.77 \pm 0.03$ & $10.56 \pm 0.05$ & $<0.11$ & $<0.17$ & $0.57 \pm 0.06$ & $2.05 \pm 0.14$ \\
\hline NGC 1060 & $13.71 \pm 0.14$ & $13.00 \pm 0.14$ & $11.81 \pm 0.14$ & $9.24 \pm 0.01$ & $8.49 \pm 0.01$ & $8.20 \pm 0.02$ & $0.11 \pm 0.03$ & $0.25 \pm 0.04$ & $2.39 \pm 0.04$ & $4.13 \pm 0.56$ \\
\hline NGC 1072 & $\ldots$ & $14.16 \pm 0.18$ & $\ldots$ & $11.14 \pm 0.02$ & $10.39 \pm 0.03$ & $10.14 \pm 0.03$ & $<0.12$ & $<0.12$ & $0.44 \pm 0.04$ & $1.60 \pm 0.18$ \\
\hline PGC 10334 & $\ldots$ & $\ldots$ & $\ldots$ & $\ldots$ & $\ldots$ & $\ldots$ & $\ldots$ & $\ldots$ & $\ldots$ & $\ldots$ \\
\hline UGC 02201 & $\cdots$ & $15.45 \pm 0.19$ & $\ldots$ & $12.28 \pm 0.04$ & $11.57 \pm 0.05$ & $11.26 \pm 0.06$ & $0.13 \pm 0.03$ & $0.22 \pm 0.02$ & $1.51 \pm 0.11$ & $3.50 \pm 0.46$ \\
\hline NGC 1066 & $\ldots$ & $14.25 \pm 0.24$ & $\ldots$ & $9.88 \pm 0.02$ & $9.12 \pm 0.02$ & $8.89 \pm 0.03$ & $\ldots$ & $\ldots$ & $\ldots$ & $\ldots$ \\
\hline NGC 1067 & $\ldots$ & $14.55 \pm 0.15$ & $13.69 \pm 0.16$ & $11.88 \pm 0.05$ & $11.39 \pm 0.06$ & $11.04 \pm 0.07$ & $\ldots$ & $\ldots$ & $\ldots$ & $\ldots$ \\
\hline NGC 1084 & $11.22 \pm 0.13$ & $11.31 \pm 0.10$ & $10.73 \pm 0.10$ & $8.84 \pm 0.02$ & $8.20 \pm 0.02$ & $7.93 \pm 0.02$ & $1.96 \pm 0.02$ & $3.19 \pm 0.05$ & $29.30 \pm 0.04$ & $53.40 \pm 0.12$ \\
\hline NGC 1097 & $10.46 \pm 0.07$ & $10.23 \pm 0.07$ & $9.48 \pm 0.07$ & $7.18 \pm 0.02$ & $6.51 \pm 0.03$ & $6.25 \pm 0.03$ & $2.88 \pm 0.43$ & $7.70 \pm 1.16$ & $46.70 \pm 7.01$ & $116.00 \pm 17.50$ \\
\hline PGC 10766 & $\ldots$ & $\ldots$ & $\ldots$ & $11.65 \pm 0.03$ & $10.80 \pm 0.03$ & $10.43 \pm 0.04$ & $0.08 \pm 0.02$ & $0.12 \pm 0.02$ & $1.17 \pm 0.07$ & $2.96 \pm 0.18$ \\
\hline PGC 10794 & $\ldots$ & $\ldots$ & $\ldots$ & $12.66 \pm 0.04$ & $12.21 \pm 0.07$ & $11.76 \pm 0.09$ & $<0.09$ & $<0.10$ & $0.57 \pm 0.05$ & $1.25 \pm 0.14$ \\
\hline PGC 10875 & $\ldots$ & $\ldots$ & $\ldots$ & $11.57 \pm 0.02$ & $10.79 \pm 0.03$ & $10.54 \pm 0.05$ & $<0.07$ & $<0.15$ & $0.30 \pm 0.04$ & $0.64 \pm 0.13$ \\
\hline NGC 1140 & $12.41 \pm 0.14$ & $12.84 \pm 0.14$ & $12.49 \pm 0.14$ & $11.28 \pm 0.03$ & $10.63 \pm 0.03$ & $10.51 \pm 0.05$ & $0.09 \pm 0.03$ & $0.45 \pm 0.01$ & $3.36 \pm 0.20$ & $4.92 \pm 0.34$ \\
\hline NGC 1148 & $\ldots$ & $\ldots$ & $\ldots$ & $11.88 \pm 0.04$ & $11.30 \pm 0.05$ & $10.85 \pm 0.06$ & $<0.07$ & $<0.16$ & $0.22 \pm 0.03$ & $0.77 \pm 0.15$ \\
\hline UGC 02442 & $\ldots$ & $\ldots$ & $\ldots$ & $12.58 \pm 0.04$ & $11.88 \pm 0.05$ & $11.75 \pm 0.08$ & $\ldots$ & $\ldots$ & $\ldots$ & $\ldots$ \\
\hline NGC 1156 & $12.13 \pm 0.13$ & $12.32 \pm 0.13$ & $11.74 \pm 0.13$ & $10.36 \pm 0.01$ & $9.73 \pm 0.01$ & $9.55 \pm 0.02$ & $0.16 \pm 0.03$ & $0.52 \pm 0.01$ & $5.17 \pm 0.47$ & $9.20 \pm 0.55$ \\
\hline PGC 11767 & $\ldots$ & $\ldots$ & $\ldots$ & $11.77 \pm 0.04$ & $11.10 \pm 0.05$ & $10.80 \pm 0.06$ & $<0.08$ & $<0.11$ & $0.41 \pm 0.04$ & $1.42 \pm 0.24$ \\
\hline UGC 02519 & $\ldots$ & $14.30 \pm 0.18$ & $\ldots$ & $10.99 \pm 0.04$ & $10.31 \pm 0.05$ & $9.81 \pm 0.05$ & $0.21 \pm 0.02$ & $0.33 \pm 0.02$ & $2.82 \pm 0.17$ & $7.41 \pm 0.45$ \\
\hline
\end{tabular}


Table 4-Continued

\begin{tabular}{|c|c|c|c|c|c|c|c|c|c|c|}
\hline \multirow{2}{*}{$\begin{array}{l}\text { Object Name } \\
\text { (1) }\end{array}$} & \multicolumn{3}{|c|}{ Optical Photometry } & \multicolumn{3}{|c|}{ Near-Infrared Photometry } & \multicolumn{4}{|c|}{ IRAS fluxes } \\
\hline & $\begin{array}{c}U \\
(\mathrm{mag}) \\
(2)\end{array}$ & $\begin{array}{c}B \\
(\mathrm{mag}) \\
(3)\end{array}$ & $\begin{array}{c}V \\
(\mathrm{mag}) \\
(4)\end{array}$ & $\begin{array}{c}J \\
(\mathrm{mag}) \\
(5)\end{array}$ & $\begin{array}{c}H \\
(\mathrm{mag}) \\
(6)\end{array}$ & $\begin{array}{c}K \\
(\mathrm{mag}) \\
(7)\end{array}$ & $\begin{array}{c}12 \mu \mathrm{m} \\
(\mathrm{Jy}) \\
(8)\end{array}$ & $\begin{array}{c}25 \mu \mathrm{m} \\
(\mathrm{Jy}) \\
(9)\end{array}$ & $\begin{array}{c}60 \mu \mathrm{m} \\
(\mathrm{Jy}) \\
(10)\end{array}$ & $\begin{array}{l}100 \mu \mathrm{m} \\
(\mathrm{Jy}) \\
(11)\end{array}$ \\
\hline NGC 1241 & $12.07 \pm 0.14$ & $12.84 \pm 0.13$ & $11.99 \pm 0.13$ & $9.76 \pm 0.03$ & $8.96 \pm 0.03$ & $8.65 \pm 0.04$ & $\ldots$ & . & $\ldots$ & $\ldots$ \\
\hline NGC 1242 & $14.27 \pm 0.14$ & $14.32 \pm 0.13$ & $13.71 \pm 0.13$ & $12.08 \pm 0.04$ & $11.37 \pm 0.04$ & $11.29 \pm 0.08$ & $\ldots$ & $\ldots$ & $\ldots$ & $\ldots$ \\
\hline NGC 1266 & $\ldots$ & 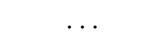 & $\ldots$ & $10.47 \pm 0.03$ & $9.88 \pm 0.04$ & $9.48 \pm 0.05$ & $0.17 \pm 0.03$ & $1.17 \pm 0.04$ & $12.80 \pm 0.04$ & $16.90 \pm 0.22$ \\
\hline NGC 1291 & $9.85 \pm 0.04$ & $9.39 \pm 0.04$ & $8.46 \pm 0.04$ & $6.52 \pm 0.02$ & $5.97 \pm 0.02$ & $5.66 \pm 0.02$ & $0.18 \pm 0.03$ & $0.17 \pm 0.03$ & $1.76 \pm 0.26$ & $10.10 \pm 1.52$ \\
\hline NGC 1285 & $\ldots$ & $\ldots$ & $\ldots$ & $11.02 \pm 0.02$ & $10.38 \pm 0.03$ & $10.15 \pm 0.05$ & $0.19 \pm 0.05$ & $0.26 \pm 0.02$ & $2.67 \pm 0.16$ & $6.15 \pm 0.37$ \\
\hline NGC 1299 & $\ldots$ & $\ldots$ & $\ldots$ & $11.04 \pm 0.02$ & $10.43 \pm 0.03$ & $10.11 \pm 0.04$ & $0.18 \pm 0.03$ & $0.20 \pm 0.03$ & $1.58 \pm 0.09$ & $3.92 \pm 0.28$ \\
\hline NGC 1310 & $12.43 \pm 0.19$ & $12.55 \pm 0.19$ & $12.08 \pm 0.19$ & $10.77 \pm 0.03$ & $10.19 \pm 0.04$ & $9.94 \pm 0.05$ & $0.14 \pm 0.02$ & $0.08 \pm 0.03$ & $0.88 \pm 0.04$ & $3.35 \pm 0.20$ \\
\hline KUG 0319-072 & $\cdots$ & $\cdots$ & $\cdots$ & $11.11 \pm 0.03$ & $10.42 \pm 0.03$ & $10.31 \pm 0.05$ & $0.09 \pm 0.03$ & $<0.16$ & $0.49 \pm 0.06$ & $1.04 \pm 0.24$ \\
\hline NGC 1316 & $9.81 \pm 0.09$ & $9.42 \pm 0.08$ & $8.53 \pm 0.08$ & $6.45 \pm 0.02$ & $5.87 \pm 0.02$ & $5.59 \pm 0.02$ & $0.32 \pm 0.02$ & $0.25 \pm 0.03$ & $2.98 \pm 0.15$ & $7.33 \pm 0.29$ \\
\hline NGC 1317 & $12.20 \pm 0.06$ & $11.91 \pm 0.06$ & $11.02 \pm 0.06$ & $8.65 \pm 0.02$ & $8.01 \pm 0.02$ & $7.74 \pm 0.02$ & $0.27 \pm 0.02$ & $0.31 \pm 0.02$ & $3.29 \pm 0.17$ & $9.48 \pm 0.38$ \\
\hline ESO 357-G025 & $\ldots$ & $14.93 \pm 0.21$ & $\ldots$ & $13.76 \pm 0.10$ & $12.80 \pm 0.09$ & $12.74 \pm 0.17$ & $\ldots$ & $\ldots$ & $\ldots$ & $\ldots$ \\
\hline PGC 12706 & $\ldots$ & $13.84 \pm 0.21$ & $\ldots$ & $12.41 \pm 0.03$ & $11.68 \pm 0.03$ & $11.50 \pm 0.05$ & $<0.09$ & $0.10 \pm 0.02$ & $0.57 \pm 0.10$ & $1.54 \pm 0.14$ \\
\hline NGC 1326 & $11.69 \pm 0.10$ & $11.41 \pm 0.10$ & $10.54 \pm 0.10$ & $8.36 \pm 0.02$ & $7.72 \pm 0.02$ & $7.45 \pm 0.02$ & $0.32 \pm 0.03$ & $0.78 \pm 0.02$ & $8.06 \pm 0.40$ & $13.30 \pm 0.67$ \\
\hline PGC 13005 & $\ldots$ & $\ldots$ & $\ldots$ & $12.10 \pm 0.03$ & $11.43 \pm 0.04$ & $11.24 \pm 0.07$ & $\ldots$ & $\ldots$ & $\ldots$ & $\ldots$ \\
\hline NGC 1346 & $\ldots$ & $\ldots$ & $\ldots$ & $10.81 \pm 0.02$ & $10.06 \pm 0.02$ & $9.79 \pm 0.03$ & $0.26 \pm 0.03$ & $0.28 \pm 0.03$ & $3.13 \pm 0.16$ & $6.27 \pm 0.38$ \\
\hline PGC 13058 & $\ldots$ & $14.60 \pm 0.10$ & $\ldots$ & $12.93 \pm 0.09$ & $12.30 \pm 0.11$ & $11.83 \pm 0.12$ & $\ldots$ & $\ldots$ & $\ldots$ & $\ldots$ \\
\hline ESO 418-G008 & $\ldots$ & $13.87 \pm 0.21$ & $\ldots$ & $12.77 \pm 0.05$ & $12.24 \pm 0.08$ & $12.18 \pm 0.13$ & $<0.12$ & $<0.08$ & $0.48 \pm 0.05$ & $1.22 \pm 0.12$ \\
\hline NGC 1365 & $10.48 \pm 0.08$ & $10.32 \pm 0.07$ & $9.63 \pm 0.07$ & $7.36 \pm 0.03$ & $6.74 \pm 0.03$ & $6.37 \pm 0.04$ & $4.42 \pm 0.66$ & $13.10 \pm 1.96$ & $84.20 \pm 12.60$ & $185.00 \pm 27.80$ \\
\hline PGC 13186 & $\ldots$ & $\ldots$ & $\ldots$ & $\ldots$ & $\ldots$ & $\ldots$ & $\ldots$ & $\ldots$ & $\ldots$ & $\ldots$ \\
\hline NGC 1361 & $\ldots$ & $\ldots$ & $\ldots$ & $11.18 \pm 0.03$ & $10.44 \pm 0.03$ & $10.36 \pm 0.04$ & $\ldots$ & $\ldots$ & $\ldots$ & $\ldots$ \\
\hline PGC 13230 & $\ldots$ & $14.50 \pm 0.10$ & $\ldots$ & $12.41 \pm 0.04$ & $11.99 \pm 0.08$ & $11.80 \pm 0.11$ & $\ldots$ & $\cdots$ & $\ldots$ & $\ldots$ \\
\hline NGC 1373 & $14.44 \pm 0.09$ & $14.12 \pm 0.08$ & $13.26 \pm 0.08$ & $11.43 \pm 0.02$ & $10.77 \pm 0.03$ & $10.59 \pm 0.05$ & $\ldots$ & $\ldots$ & $\ldots$ & $\ldots$ \\
\hline NGC 1374 & $12.46 \pm 0.08$ & $12.00 \pm 0.08$ & $11.08 \pm 0.08$ & $9.05 \pm 0.01$ & $8.36 \pm 0.01$ & $8.16 \pm 0.02$ & $<0.03$ & $<0.02$ & $<0.02$ & $<0.05$ \\
\hline NGC 1375 & $13.50 \pm 0.14$ & $13.18 \pm 0.13$ & $12.40 \pm 0.13$ & $10.52 \pm 0.02$ & $9.89 \pm 0.02$ & $9.61 \pm 0.03$ & $<0.03$ & $<0.02$ & $<0.03$ & $<0.09$ \\
\hline NGC 1379 & $12.17 \pm 0.10$ & $11.80 \pm 0.10$ & $10.91 \pm 0.10$ & $9.08 \pm 0.01$ & $8.45 \pm 0.01$ & $8.24 \pm 0.02$ & $<0.03$ & $<0.02$ & $<0.03$ & $0.14 \pm 0.05$ \\
\hline UGCA 080 & $\ldots$ & & $\ldots$ & & $\ldots$ & $\ldots$ & $\ldots$ & $\ldots$ & $\ldots$ & $\ldots$ \\
\hline NGC 1380 & $11.32 \pm 0.10$ & $10.87 \pm 0.10$ & $9.93 \pm 0.10$ & $7.77 \pm 0.02$ & $7.13 \pm 0.02$ & $6.87 \pm 0.02$ & $0.12 \pm 0.03$ & $<0.08$ & $1.08 \pm 0.07$ & $3.12 \pm 0.22$ \\
\hline NGC 1381 & $12.90 \pm 0.10$ & $12.44 \pm 0.10$ & $11.50 \pm 0.10$ & $9.33 \pm 0.02$ & $8.65 \pm 0.02$ & $8.42 \pm 0.02$ & $<0.02$ & $<0.02$ & $<0.02$ & $<0.07$ \\
\hline NGC 1386 & $12.42 \pm 0.10$ & $12.09 \pm 0.10$ & $11.23 \pm 0.10$ & $8.98 \pm 0.01$ & $8.32 \pm 0.01$ & $8.07 \pm 0.01$ & $0.49 \pm 0.03$ & $1.43 \pm 0.02$ & $5.40 \pm 0.38$ & $9.64 \pm 0.39$ \\
\hline NGC $1380 A$ & $13.63 \pm 0.13$ & $13.31 \pm 0.13$ & $12.41 \pm 0.13$ & $10.52 \pm 0.02$ & $9.84 \pm 0.02$ & $9.57 \pm 0.04$ & $\ldots$ & $\ldots$ & $\ldots$ & $\ldots$ \\
\hline PGC 13343 & $\ldots$ & $14.81 \pm 0.09$ & $\cdots$ & $12.37 \pm 0.03$ & $11.71 \pm 0.04$ & $11.61 \pm 0.08$ & $\ldots$ & $\ldots$ & $\ldots$ & $\ldots$ \\
\hline NGC 1387 & $12.18 \pm 0.10$ & $11.68 \pm 0.10$ & $10.69 \pm 0.10$ & $8.44 \pm 0.01$ & $7.76 \pm 0.01$ & $7.43 \pm 0.02$ & $0.16 \pm 0.04$ & $0.13 \pm 0.03$ & $2.16 \pm 0.13$ & $6.04 \pm 0.24$ \\
\hline
\end{tabular}


Table 4-Continued

\begin{tabular}{|c|c|c|c|c|c|c|c|c|c|c|}
\hline \multirow{2}{*}{$\begin{array}{c}\text { Object Name } \\
\text { (1) }\end{array}$} & \multicolumn{3}{|c|}{ Optical Photometry } & \multicolumn{3}{|c|}{ Near-Infrared Photometry } & \multicolumn{4}{|c|}{ IRAS fluxes } \\
\hline & $\begin{array}{c}U \\
(\mathrm{mag}) \\
(2)\end{array}$ & $\begin{array}{c}B \\
(\mathrm{mag}) \\
(3)\end{array}$ & $\begin{array}{c}V \\
(\mathrm{mag}) \\
(4)\end{array}$ & $\begin{array}{c}J \\
(\mathrm{mag}) \\
(5)\end{array}$ & $\begin{array}{c}H \\
(\mathrm{mag}) \\
(6)\end{array}$ & $\begin{array}{c}K \\
(\mathrm{mag}) \\
(7)\end{array}$ & $\begin{array}{c}12 \mu \mathrm{m} \\
(\mathrm{Jy}) \\
(8)\end{array}$ & $\begin{array}{c}25 \mu \mathrm{m} \\
(\mathrm{Jy}) \\
(9)\end{array}$ & $\begin{array}{l}60 \mu \mathrm{m} \\
(\mathrm{Jy}) \\
(10)\end{array}$ & $\begin{array}{l}100 \mu \mathrm{m} \\
(\mathrm{Jy}) \\
(11)\end{array}$ \\
\hline NGC $1380 \mathrm{~B}$ & $13.19 \pm 0.13$ & $13.87 \pm 0.13$ & $12.92 \pm 0.13$ & $10.89 \pm 0.02$ & $10.29 \pm 0.03$ & $10.04 \pm 0.04$ & $<0.02$ & $<0.02$ & $<0.01$ & $<0.12$ \\
\hline NGC 1389 & $12.80 \pm 0.13$ & $12.42 \pm 0.13$ & $11.50 \pm 0.13$ & $9.48 \pm 0.01$ & $8.86 \pm 0.01$ & $8.63 \pm 0.02$ & $<0.03$ & $<0.03$ & $<0.03$ & $<0.10$ \\
\hline NGC 1385 & $11.28 \pm 0.10$ & $11.45 \pm 0.10$ & $10.94 \pm 0.10$ & $9.46 \pm 0.02$ & $8.80 \pm 0.02$ & $8.57 \pm 0.03$ & $1.20 \pm 0.03$ & $2.03 \pm 0.03$ & $17.50 \pm 0.02$ & $35.00 \pm 0.08$ \\
\hline NGC 1383 & $13.81 \pm 0.14$ & $13.45 \pm 0.14$ & $12.47 \pm 0.14$ & $10.33 \pm 0.02$ & $9.62 \pm 0.02$ & $9.44 \pm 0.03$ & $\ldots$ & $\ldots$ & $\ldots$ & $\ldots$ \\
\hline NGC 1396 & $\ldots$ & $14.80 \pm 0.10$ & $\ldots$ & $12.74 \pm 0.07$ & $12.25 \pm 0.11$ & $11.72 \pm 0.12$ & $\cdots$ & $\cdots$ & $\cdots$ & $\cdots$ \\
\hline ESO 358-G042 & $\cdots$ & $15.40 \pm 0.10$ & $\cdots$ & $13.68 \pm 0.09$ & $13.32 \pm 0.14$ & $12.99 \pm 0.21$ & $\cdots$ & $\cdots$ & $\cdots$ & $\cdots$ \\
\hline NGC 1399 & $11.05 \pm 0.10$ & $10.55 \pm 0.10$ & $9.59 \pm 0.10$ & $7.21 \pm 0.02$ & $6.56 \pm 0.02$ & $6.31 \pm 0.03$ & $0.10 \pm 0.03$ & $<0.02$ & $<0.03$ & $0.30 \pm 0.08$ \\
\hline NGC 1393 & $13.41 \pm 0.13$ & $12.97 \pm 0.13$ & $12.02 \pm 0.13$ & $10.08 \pm 0.01$ & $9.38 \pm 0.02$ & $9.18 \pm 0.03$ & $\ldots$ & $\ldots$ & $\ldots$ & $\cdots$ \\
\hline NGC 1404 & $11.53 \pm 0.13$ & $10.97 \pm 0.13$ & $10.00 \pm 0.13$ & $7.77 \pm 0.02$ & $7.09 \pm 0.02$ & $6.82 \pm 0.02$ & $0.10 \pm 0.04$ & $<0.03$ & $<0.03$ & $0.27 \pm 0.06$ \\
\hline NGC 1391 & $14.79 \pm 0.14$ & $14.37 \pm 0.13$ & $13.34 \pm 0.13$ & $11.16 \pm 0.02$ & $10.45 \pm 0.02$ & $10.22 \pm 0.04$ & $\ldots$ & $\ldots$ & $\ldots$ & $\ldots$ \\
\hline NGC 1394 & $14.28 \pm 0.13$ & $13.82 \pm 0.13$ & $12.81 \pm 0.13$ & $10.64 \pm 0.02$ & $9.94 \pm 0.02$ & $9.71 \pm 0.03$ & $\ldots$ & $\ldots$ & $\ldots$ & $\ldots$ \\
\hline AM 0337-355 & $\cdots$ & $16.10 \pm 0.10$ & $\ldots$ & $\cdots$ & $\cdots$ & $\ldots$ & $\cdots$ & $\ldots$ & $\cdots$ & $\cdots$ \\
\hline NGC 1400 & $12.48 \pm 0.13$ & $11.92 \pm 0.13$ & $10.96 \pm 0.13$ & $8.75 \pm 0.01$ & $8.04 \pm 0.01$ & $7.81 \pm 0.02$ & $<0.09$ & $0.12 \pm 0.02$ & $0.72 \pm 0.06$ & $2.51 \pm 0.25$ \\
\hline IC 0343 & $\ldots$ & $14.10 \pm 0.14$ & $13.19 \pm 0.14$ & $11.31 \pm 0.03$ & $10.72 \pm 0.03$ & $10.50 \pm 0.06$ & $\ldots$ & $\ldots$ & $\cdots$ & $\cdots$ \\
\hline NGC $1427 \mathrm{~A}$ & $\ldots$ & $13.42 \pm 0.21$ & $\ldots$ & $\ldots$ & $\ldots$ & $\ldots$ & $<0.08$ & $<0.10$ & $0.21 \pm 0.03$ & $0.78 \pm 0.12$ \\
\hline NGC 1407 & $\ldots$ & $10.70 \pm 0.20$ & $9.67 \pm 0.20$ & $7.64 \pm 0.02$ & $6.99 \pm 0.02$ & $6.70 \pm 0.03$ & $0.12 \pm 0.03$ & $<0.03$ & $0.14 \pm 0.03$ & $0.48 \pm 0.07$ \\
\hline ESO 548-G068 & $\ldots$ & $14.02 \pm 0.21$ & $\ldots$ & $11.25 \pm 0.02$ & $10.56 \pm 0.03$ & $10.32 \pm 0.05$ & $\ldots$ & $\ldots$ & $\ldots$ & $\ldots$ \\
\hline PGC 13515 & $\ldots$ & $16.20 \pm 0.10$ & $\ldots$ & $\ldots$ & $\ldots$ & $\ldots$ & $\ldots$ & $\ldots$ & $\ldots$ & $\ldots$ \\
\hline PGC 13535 & $\ldots$ & $\ldots$ & $\ldots$ & $11.75 \pm 0.04$ & $11.23 \pm 0.05$ & $10.98 \pm 0.09$ & $<0.08$ & $<0.08$ & $0.22 \pm 0.04$ & $0.76 \pm 0.19$ \\
\hline PGC 13600 & $\ldots$ & $\ldots$ & $\ldots$ & $11.84 \pm 0.03$ & $11.15 \pm 0.03$ & $10.98 \pm 0.05$ & $\ldots$ & $\ldots$ & $\ldots$ & $\ldots$ \\
\hline IC 0334 & $13.14 \pm 0.13$ & $12.45 \pm 0.13$ & $11.33 \pm 0.13$ & $8.75 \pm 0.01$ & $7.95 \pm 0.02$ & $7.71 \pm 0.02$ & $\ldots$ & $\cdots$ & $\cdots$ & $\ldots$ \\
\hline PGC 13820 & $\ldots$ & $\ldots$ & $\ldots$ & $10.80 \pm 0.02$ & $9.94 \pm 0.02$ & $9.59 \pm 0.03$ & $<0.13$ & $0.10 \pm 0.04$ & $1.15 \pm 0.08$ & $4.41 \pm 0.44$ \\
\hline NGC 1481 & $\ldots$ & $14.40 \pm 0.21$ & $\ldots$ & $12.17 \pm 0.06$ & $11.41 \pm 0.06$ & $11.18 \pm 0.09$ & $<0.07$ & $<0.08$ & $0.36 \pm 0.04$ & $<4.25$ \\
\hline NGC 1482 & $13.15 \pm 0.15$ & $13.10 \pm 0.15$ & $12.15 \pm 0.15$ & $9.72 \pm 0.02$ & $8.91 \pm 0.02$ & $8.48 \pm 0.02$ & $1.55 \pm 0.03$ & $4.73 \pm 0.05$ & $35.30 \pm 0.07$ & $45.80 \pm 0.08$ \\
\hline PGC 14100 & $\ldots$ & $\ldots$ & $\ldots$ & $12.45 \pm 0.05$ & $11.95 \pm 0.08$ & $11.75 \pm 0.13$ & $\ldots$ & $\ldots$ & $\ldots$ & $\ldots$ \\
\hline NGC 1510 & $13.28 \pm 0.11$ & $13.47 \pm 0.11$ & $13.02 \pm 0.11$ & $11.28 \pm 0.04$ & $10.17 \pm 0.03$ & $10.36 \pm 0.06$ & $<0.40$ & $<0.25$ & $0.89 \pm 0.07$ & $1.13 \pm 0.17$ \\
\hline NGC 1512 & $11.30 \pm 0.10$ & $11.13 \pm 0.10$ & $10.32 \pm 0.10$ & $8.34 \pm 0.02$ & $7.76 \pm 0.03$ & $7.49 \pm 0.04$ & $0.22 \pm 0.02$ & $0.24 \pm 0.02$ & $3.14 \pm 0.16$ & $11.00 \pm 0.55$ \\
\hline UGC 02955 & $\ldots$ & $\ldots$ & $\ldots$ & $11.47 \pm 0.03$ & $10.82 \pm 0.04$ & $10.37 \pm 0.04$ & $<0.12$ & $0.14 \pm 0.02$ & $0.97 \pm 0.07$ & $2.44 \pm 0.64$ \\
\hline NGC 1546 & $12.15 \pm 0.11$ & $11.80 \pm 0.10$ & $10.92 \pm 0.10$ & $9.08 \pm 0.02$ & $8.33 \pm 0.02$ & $8.05 \pm 0.02$ & $0.58 \pm 0.03$ & $0.73 \pm 0.02$ & $6.53 \pm 0.33$ & $22.00 \pm 0.88$ \\
\hline NGC 1549 & $11.22 \pm 0.08$ & $10.72 \pm 0.08$ & $9.79 \pm 0.08$ & $7.68 \pm 0.02$ & $7.07 \pm 0.02$ & $6.78 \pm 0.02$ & $0.10 \pm 0.02$ & $0.06 \pm 0.02$ & $<0.02$ & $0.18 \pm 0.06$ \\
\hline NGC 1553 & $10.76 \pm 0.09$ & $10.28 \pm 0.08$ & $9.40 \pm 0.08$ & $7.18 \pm 0.02$ & $6.50 \pm 0.02$ & $6.28 \pm 0.02$ & $0.13 \pm 0.02$ & $0.10 \pm 0.02$ & $0.48 \pm 0.03$ & $0.95 \pm 0.11$ \\
\hline IC 2058 & $\ldots$ & $13.87 \pm 0.21$ & $\ldots$ & $11.95 \pm 0.03$ & $11.09 \pm 0.03$ & $10.99 \pm 0.04$ & $<0.05$ & $<0.07$ & $0.51 \pm 0.03$ & $1.38 \pm 0.12$ \\
\hline
\end{tabular}


Table 4-Continued

\begin{tabular}{|c|c|c|c|c|c|c|c|c|c|c|}
\hline \multirow{2}{*}{$\begin{array}{c}\text { Object Name } \\
\text { (1) }\end{array}$} & \multicolumn{3}{|c|}{ Optical Photometry } & \multicolumn{3}{|c|}{ Near-Infrared Photometry } & \multicolumn{4}{|c|}{ IRAS fluxes } \\
\hline & $\begin{array}{c}U \\
(\mathrm{mag}) \\
(2)\end{array}$ & $\begin{array}{c}B \\
(\mathrm{mag}) \\
(3)\end{array}$ & $\begin{array}{c}V \\
(\mathrm{mag}) \\
(4)\end{array}$ & $\begin{array}{c}J \\
(\mathrm{mag}) \\
(5)\end{array}$ & $\begin{array}{c}H \\
(\mathrm{mag}) \\
(6)\end{array}$ & $\begin{array}{c}K \\
(\mathrm{mag}) \\
(7)\end{array}$ & $\begin{array}{c}12 \mu \mathrm{m} \\
(\mathrm{Jy}) \\
(8)\end{array}$ & $\begin{array}{c}25 \mu \mathrm{m} \\
(\mathrm{Jy}) \\
(9)\end{array}$ & $\begin{array}{c}60 \mu \mathrm{m} \\
(\mathrm{Jy}) \\
(10)\end{array}$ & $\begin{array}{l}100 \mu \mathrm{m} \\
(\mathrm{Jy}) \\
(11)\end{array}$ \\
\hline NGC 1566 & $10.29 \pm 0.05$ & $10.33 \pm 0.03$ & $9.73 \pm 0.03$ & $7.76 \pm 0.02$ & $7.21 \pm 0.03$ & $6.89 \pm 0.03$ & $0.83 \pm 0.06$ & $1.22 \pm 0.05$ & $14.70 \pm 0.74$ & $46.40 \pm 1.85$ \\
\hline NGC 1569 & $11.72 \pm 0.09$ & $11.86 \pm 0.09$ & $11.03 \pm 0.09$ & $8.82 \pm 0.02$ & $8.18 \pm 0.02$ & $7.86 \pm 0.02$ & $0.79 \pm 0.05$ & $7.09 \pm 0.03$ & $45.40 \pm 1.82$ & $47.30 \pm 2.84$ \\
\hline NGC 1672 & $10.29 \pm 0.09$ & $10.28 \pm 0.08$ & $9.68 \pm 0.08$ & $7.90 \pm 0.02$ & $7.34 \pm 0.03$ & $7.02 \pm 0.03$ & $1.67 \pm 0.07$ & $4.03 \pm 0.07$ & $33.00 \pm 1.32$ & $69.90 \pm 2.80$ \\
\hline NGC 1705 & $12.32 \pm 0.13$ & $12.77 \pm 0.13$ & $12.39 \pm 0.13$ & $11.21 \pm 0.03$ & $10.76 \pm 0.05$ & $10.52 \pm 0.06$ & $<0.05$ & $<0.11$ & $0.87 \pm 0.06$ & $1.82 \pm 0.15$ \\
\hline ESO $422-G 027$ & $\cdots$ & $14.21 \pm 0.21$ & $\cdots$ & $\cdots$ & $\cdots$ & $\cdots$ & $<0.09$ & $<0.06$ & $0.28 \pm 0.03$ & $1.00 \pm 0.12$ \\
\hline NGC 1800 & $12.96 \pm 0.13$ & $13.13 \pm 0.13$ & $12.59 \pm 0.13$ & $11.13 \pm 0.04$ & $10.53 \pm 0.05$ & $10.21 \pm 0.06$ & $<0.08$ & $<0.08$ & $0.77 \pm 0.05$ & $1.82 \pm 0.13$ \\
\hline NGC 1808 & $11.05 \pm 0.10$ & $10.76 \pm 0.10$ & $9.94 \pm 0.10$ & $7.64 \pm 0.02$ & $6.98 \pm 0.02$ & $6.66 \pm 0.02$ & $4.44 \pm 0.18$ & $16.10 \pm 0.18$ & $87.80 \pm 3.51$ & $137.00 \pm 5.49$ \\
\hline IC 0411 & $\ldots$ & $14.09 \pm 0.21$ & $\cdots$ & $10.89 \pm 0.03$ & $10.18 \pm 0.03$ & $9.92 \pm 0.05$ & $\ldots$ & $\ldots$ & $\ldots$ & $\ldots$ \\
\hline ESO 204-G006 & $\ldots$ & $15.35 \pm 0.21$ & $\ldots$ & $11.80 \pm 0.04$ & $10.97 \pm 0.04$ & $10.69 \pm 0.05$ & $\ldots$ & $\ldots$ & $\ldots$ & $\ldots$ \\
\hline ESO 204-G007 & $\ldots$ & $15.84 \pm 0.21$ & $\ldots$ & $13.03 \pm 0.03$ & $12.39 \pm 0.04$ & $11.95 \pm 0.05$ & $\ldots$ & $\ldots$ & $\ldots$ & $\ldots$ \\
\hline ESO 033-G022 & $\ldots$ & $15.50 \pm 0.21$ & $\ldots$ & $14.94 \pm 0.22$ & $15.10 \pm 0.00$ & $13.60 \pm 0.25$ & $\ldots$ & $\ldots$ & $\ldots$ & $\ldots$ \\
\hline NGC 1964 & $11.79 \pm 0.13$ & $11.58 \pm 0.13$ & $10.81 \pm 0.13$ & $8.65 \pm 0.02$ & $7.93 \pm 0.03$ & $7.68 \pm 0.03$ & $0.49 \pm 0.03$ & $0.72 \pm 0.02$ & $7.98 \pm 0.40$ & $22.50 \pm 0.90$ \\
\hline NGC 1961 & $\ldots$ & $11.73 \pm 0.14$ & $10.99 \pm 0.15$ & $8.79 \pm 0.03$ & $8.01 \pm 0.04$ & $7.73 \pm 0.04$ & $0.55 \pm 0.04$ & $0.55 \pm 0.04$ & $6.38 \pm 0.38$ & $21.60 \pm 1.08$ \\
\hline UGC 03342 & $\ldots$ & $15.20 \pm 0.19$ & $\ldots$ & $11.79 \pm 0.04$ & $11.14 \pm 0.05$ & $10.79 \pm 0.07$ & $\ldots$ & $\ldots$ & $\ldots$ & $\ldots$ \\
\hline UGC 03344 & $\ldots$ & $14.20 \pm 0.30$ & $\ldots$ & $11.22 \pm 0.03$ & $10.46 \pm 0.03$ & $10.21 \pm 0.05$ & $<0.25$ & $<0.25$ & $0.58 \pm 0.05$ & $2.29 \pm 0.16$ \\
\hline NGC 2090 & $12.17 \pm 0.13$ & $11.99 \pm 0.13$ & $11.20 \pm 0.13$ & $8.92 \pm 0.02$ & $8.30 \pm 0.02$ & $8.05 \pm 0.02$ & $0.15 \pm 0.02$ & $0.21 \pm 0.02$ & $2.45 \pm 0.12$ & $11.20 \pm 0.56$ \\
\hline UGC 03403 & $\ldots$ & $14.30 \pm 0.20$ & $\ldots$ & $11.22 \pm 0.02$ & $10.47 \pm 0.02$ & $10.19 \pm 0.03$ & $0.10 \pm 0.02$ & $0.10 \pm 0.03$ & $1.04 \pm 0.07$ & $3.41 \pm 0.51$ \\
\hline UGC 03422 & $\ldots$ & $14.10 \pm 0.30$ & $\ldots$ & $11.07 \pm 0.03$ & $10.51 \pm 0.04$ & $10.32 \pm 0.04$ & $<0.07$ & $<0.10$ & $0.50 \pm 0.05$ & $<2.63$ \\
\hline Mrk 3 & $14.21 \pm 0.09$ & $14.03 \pm 0.08$ & $12.97 \pm 0.08$ & $10.03 \pm 0.01$ & $9.29 \pm 0.02$ & $8.97 \pm 0.02$ & $0.71 \pm 0.05$ & $2.90 \pm 0.04$ & $3.77 \pm 0.15$ & $3.36 \pm 0.44$ \\
\hline NGC 2207 & $\ldots$ & $11.63 \pm 0.16$ & $\ldots$ & $9.11 \pm 0.02$ & $8.49 \pm 0.03$ & $8.19 \pm 0.04$ & $0.88 \pm 0.06$ & $1.55 \pm 0.08$ & $14.60 \pm 1.02$ & $38.00 \pm 1.90$ \\
\hline IC 2163 & $\cdots$ & $12.55 \pm 0.21$ & $\cdots$ & $9.61 \pm 0.03$ & $8.96 \pm 0.03$ & $8.56 \pm 0.04$ & $\ldots$ & $\ldots$ & $\ldots$ & $\ldots$ \\
\hline UGC 03423 & $\ldots$ & $\ldots$ & $\ldots$ & $\ldots$ & $\ldots$ & $\ldots$ & $\ldots$ & $\ldots$ & $\ldots$ & $\ldots$ \\
\hline ESO 556-G012 & $\ldots$ & $14.91 \pm 0.21$ & $\ldots$ & $14.50 \pm 0.12$ & $13.84 \pm 0.13$ & $13.84 \pm 0.27$ & $<0.63$ & $<0.25$ & $0.37 \pm 0.04$ & $<1.00$ \\
\hline NGC 2146 & $11.67 \pm 0.14$ & $11.38 \pm 0.13$ & $10.59 \pm 0.13$ & $8.23 \pm 0.02$ & $7.42 \pm 0.02$ & $7.06 \pm 0.02$ & $6.23 \pm 0.25$ & $17.60 \pm 0.25$ & $131.00 \pm 5.24$ & $184.00 \pm 9.21$ \\
\hline NGC $2146 \mathrm{~A}$ & $13.52 \pm 0.21$ & $13.50 \pm 0.20$ & $12.87 \pm 0.20$ & $11.20 \pm 0.04$ & $10.49 \pm 0.04$ & $10.43 \pm 0.07$ & $<0.10$ & $0.07 \pm 0.03$ & $0.47 \pm 0.05$ & $1.79 \pm 0.25$ \\
\hline AM 0644-741 & $\ldots$ & $\ldots$ & $\ldots$ & $\ldots$ & $\ldots$ & $\ldots$ & $0.07 \pm 0.01$ & $0.15 \pm 0.01$ & $1.41 \pm 0.07$ & $3.89 \pm 0.31$ \\
\hline PGC 19480 & $15.60 \pm 0.23$ & $15.30 \pm 0.20$ & $14.17 \pm 0.22$ & $12.16 \pm 0.04$ & $11.44 \pm 0.06$ & $11.11 \pm 0.07$ & $\ldots$ & $\ldots$ & $\ldots$ & $\ldots$ \\
\hline PGC 19481 & $14.01 \pm 0.18$ & $13.82 \pm 0.17$ & $12.94 \pm 0.17$ & $10.42 \pm 0.02$ & $9.68 \pm 0.03$ & $9.43 \pm 0.04$ & $\ldots$ & $\ldots$ & $\ldots$ & $\ldots$ \\
\hline ESO 034-G013 & $\ldots$ & $14.65 \pm 0.21$ & $\ldots$ & $11.86 \pm 0.03$ & $11.39 \pm 0.06$ & $10.92 \pm 0.06$ & $0.08 \pm 0.02$ & $0.14 \pm 0.01$ & $0.91 \pm 0.05$ & $2.50 \pm 0.28$ \\
\hline NGC 2310 & $13.20 \pm 0.13$ & $12.74 \pm 0.13$ & $11.76 \pm 0.13$ & $9.39 \pm 0.02$ & $8.72 \pm 0.02$ & $8.48 \pm 0.03$ & $<0.02$ & $<0.02$ & $0.13 \pm 0.03$ & $0.40 \pm 0.19$ \\
\hline NGC 2366 & $\ldots$ & $11.43 \pm 0.10$ & $10.85 \pm 0.12$ & $11.29 \pm 0.07$ & $12.15 \pm 0.12$ & $10.62 \pm 0.13$ & $<0.12$ & $0.70 \pm 0.01$ & $3.51 \pm 0.18$ & $4.67 \pm 0.28$ \\
\hline Mrk 8 & $\ldots$ & $14.00 \pm 0.20$ & $\ldots$ & $\ldots$ & $\ldots$ & $\ldots$ & $0.09 \pm 0.02$ & $0.37 \pm 0.01$ & $2.46 \pm 0.15$ & $3.97 \pm 0.36$ \\
\hline
\end{tabular}


Table 4-Continued

\begin{tabular}{|c|c|c|c|c|c|c|c|c|c|c|}
\hline \multirow{2}{*}{$\begin{array}{c}\text { Object Name } \\
\text { (1) }\end{array}$} & \multicolumn{3}{|c|}{ Optical Photometry } & \multicolumn{3}{|c|}{ Near-Infrared Photometry } & \multicolumn{4}{|c|}{ IRAS fluxes } \\
\hline & $\begin{array}{c}U \\
(\mathrm{mag}) \\
(2)\end{array}$ & $\begin{array}{c}B \\
(\mathrm{mag}) \\
(3)\end{array}$ & $\begin{array}{c}V \\
(\mathrm{mag}) \\
(4)\end{array}$ & $\begin{array}{c}J \\
(\mathrm{mag}) \\
(5)\end{array}$ & $\begin{array}{c}H \\
(\mathrm{mag}) \\
(6)\end{array}$ & $\begin{array}{c}K \\
(\mathrm{mag}) \\
(7)\end{array}$ & $\begin{array}{c}12 \mu \mathrm{m} \\
(\mathrm{Jy}) \\
(8)\end{array}$ & $\begin{array}{c}25 \mu \mathrm{m} \\
(\mathrm{Jy}) \\
(9)\end{array}$ & $\begin{array}{c}60 \mu \mathrm{m} \\
(\mathrm{Jy}) \\
(10)\end{array}$ & $\begin{array}{l}100 \mu \mathrm{m} \\
(\mathrm{Jy}) \\
(11)\end{array}$ \\
\hline UGC 03864 & $\cdots$ & $14.80 \pm 0.19$ & $\cdots$ & $\cdots$ & $\cdots$ & $\cdots$ & $<0.11$ & $<0.09$ & $0.19 \pm 0.05$ & $0.68 \pm 0.19$ \\
\hline ESO 059-G006 & $\cdots$ & $15.13 \pm 0.21$ & $\cdots$ & $\cdots$ & $\cdots$ & $\cdots$ & $\cdots$ & $\cdots$ & $\cdots$ & $\ldots$ \\
\hline NGC 2434 & $12.82 \pm 0.14$ & $12.33 \pm 0.13$ & $11.26 \pm 0.13$ & $8.87 \pm 0.01$ & $8.14 \pm 0.02$ & $7.89 \pm 0.02$ & $<0.02$ & $<0.02$ & $<0.02$ & $<0.10$ \\
\hline ESO 059-G007 & $\cdots$ & $15.02 \pm 0.21$ & $\cdots$ & $11.61 \pm 0.05$ & $10.85 \pm 0.07$ & $10.83 \pm 0.10$ & $\cdots$ & $\cdots$ & $\cdots$ & $\cdots$ \\
\hline NGC 2442 & $11.47 \pm 0.14$ & $11.24 \pm 0.12$ & $10.42 \pm 0.12$ & $7.88 \pm 0.03$ & $7.21 \pm 0.03$ & $6.87 \pm 0.04$ & $0.36 \pm 0.04$ & $0.45 \pm 0.02$ & $8.14 \pm 0.41$ & $36.70 \pm 1.10$ \\
\hline NGC 2403 & $\cdots$ & $8.93 \pm 0.07$ & $8.46 \pm 0.08$ & $6.98 \pm 0.02$ & $6.42 \pm 0.03$ & $6.19 \pm 0.04$ & $3.34 \pm 0.50$ & $6.29 \pm 0.94$ & $51.60 \pm 7.73$ & $148.00 \pm 22.30$ \\
\hline ESO 059-G010 & $\cdots$ & $15.20 \pm 0.21$ & $\cdots$ & $12.02 \pm 0.04$ & $11.36 \pm 0.05$ & $10.98 \pm 0.06$ & $\cdots$ & $\cdots$ & $\cdots$ & $\cdots$ \\
\hline UGC 03942 & $\ldots$ & $15.54 \pm 0.19$ & $\cdots$ & $11.97 \pm 0.03$ & $11.25 \pm 0.02$ & $10.91 \pm 0.03$ & $\cdots$ & $\ldots$ & $\cdots$ & $\cdots$ \\
\hline ESO 059-G011 & $\ldots$ & $13.50 \pm 0.21$ & $\cdots$ & $10.06 \pm 0.02$ & $9.45 \pm 0.02$ & $9.10 \pm 0.03$ & $\ldots$ & $\ldots$ & $\ldots$ & $\ldots$ \\
\hline UGC 03995 & $\cdots$ & $13.28 \pm 0.18$ & $\cdots$ & $\cdots$ & $\ldots$ & $\ldots$ & $0.22 \pm 0.03$ & $0.31 \pm 0.03$ & $0.56 \pm 0.07$ & $1.99 \pm 0.18$ \\
\hline UGC 03997 & $\cdots$ & $\cdots$ & $\cdots$ & $13.92 \pm 0.11$ & $13.31 \pm 0.17$ & $12.68 \pm 0.14$ & $\ldots$ & $\ldots$ & $\ldots$ & $\ldots$ \\
\hline UGC 04056 & $\ldots$ & $14.81 \pm 0.18$ & $\ldots$ & $12.10 \pm 0.05$ & $11.55 \pm 0.05$ & $11.22 \pm 0.06$ & $<0.13$ & $0.11 \pm 0.04$ & $0.22 \pm 0.04$ & $1.11 \pm 0.18$ \\
\hline UGC 04136 & $\ldots$ & $14.96 \pm 0.18$ & $\ldots$ & $11.21 \pm 0.01$ & $10.44 \pm 0.02$ & $10.15 \pm 0.03$ & $\cdots$ & $\ldots$ & $\ldots$ & $\ldots$ \\
\hline UGC 04148 & $\ldots$ & $15.20 \pm 0.20$ & $\ldots$ & $\ldots$ & $\ldots$ & .. & $\ldots$ & $\ldots$ & $\ldots$ & $\ldots$ \\
\hline NGC 2500 & $11.98 \pm 0.15$ & $12.20 \pm 0.13$ & $11.62 \pm 0.13$ & $10.79 \pm 0.02$ & $10.16 \pm 0.03$ & $9.28 \pm 0.06$ & $<0.10$ & $0.12 \pm 0.03$ & $2.11 \pm 0.21$ & $6.00 \pm 0.36$ \\
\hline UGC 04176 & $\cdots$ & $14.90 \pm 0.20$ & $\cdots$ & $\cdots$ & $\cdots$ & $\cdots$ & $\cdots$ & $\ldots$ & $\ldots$ & $\ldots$ \\
\hline UGC 04188 & $\ldots$ & $14.52 \pm 0.15$ & $\ldots$ & $11.30 \pm 0.03$ & $10.56 \pm 0.03$ & $10.27 \pm 0.04$ & $\ldots$ & $\ldots$ & $\ldots$ & $\ldots$ \\
\hline NGC 2538 & $\ldots$ & $13.49 \pm 0.18$ & $\ldots$ & $10.61 \pm 0.02$ & $9.92 \pm 0.02$ & $9.63 \pm 0.03$ & $0.18 \pm 0.03$ & $0.57 \pm 0.02$ & $3.65 \pm 0.22$ & $5.96 \pm 0.30$ \\
\hline NGC 2543 & $\ldots$ & $12.70 \pm 0.18$ & $\ldots$ & $10.53 \pm 0.02$ & $9.74 \pm 0.03$ & $9.43 \pm 0.03$ & $0.16 \pm 0.03$ & $0.33 \pm 0.02$ & $2.77 \pm 0.14$ & $5.87 \pm 0.29$ \\
\hline NGC 2537 & $12.18 \pm 0.13$ & $12.32 \pm 0.13$ & $11.69 \pm 0.13$ & $9.95 \pm 0.02$ & $9.37 \pm 0.03$ & $9.13 \pm 0.03$ & $0.15 \pm 0.03$ & $0.33 \pm 0.02$ & $3.24 \pm 0.19$ & $6.45 \pm 0.39$ \\
\hline UGC4278 & $12.87 \pm 0.16$ & $13.07 \pm 0.15$ & $12.63 \pm 0.16$ & $11.46 \pm 0.03$ & $10.95 \pm 0.04$ & $10.75 \pm 0.06$ & $<0.11$ & $<0.11$ & $0.33 \pm 0.06$ & $0.95 \pm 0.21$ \\
\hline NGC 2541 & $12.03 \pm 0.15$ & $12.26 \pm 0.14$ & $11.80 \pm 0.14$ & $10.87 \pm 0.03$ & $10.20 \pm 0.04$ & $10.09 \pm 0.05$ & $<0.14$ & $<0.10$ & $1.38 \pm 0.10$ & $4.07 \pm 0.33$ \\
\hline NGC $2523 \mathrm{C}$ & $\ldots$ & $13.93 \pm 0.15$ & $\ldots$ & $11.40 \pm 0.03$ & $10.65 \pm 0.03$ & $10.53 \pm 0.04$ & $\ldots$ & $\ldots$ & $\ldots$ & $\ldots$ \\
\hline UGC 04311 & $\ldots$ & $\ldots$ & $\ldots$ & $13.03 \pm 0.05$ & $12.20 \pm 0.06$ & $11.85 \pm 0.07$ & $\cdots$ & $\ldots$ & $\cdots$ & $\cdots$ \\
\hline Holmberg II & $\cdots$ & $11.10 \pm 0.15$ & $10.66 \pm 0.17$ & $10.09 \pm 0.03$ & $8.92 \pm 0.03$ & $8.80 \pm 0.04$ & $<0.10$ & $<0.16$ & $1.15 \pm 0.13$ & $2.62 \pm 0.18$ \\
\hline NGC 2552 & $12.40 \pm 0.16$ & $12.56 \pm 0.15$ & $12.12 \pm 0.17$ & $14.52 \pm 0.09$ & $13.86 \pm 0.12$ & $13.61 \pm 0.15$ & $<0.11$ & $<0.16$ & $0.62 \pm 0.06$ & $1.53 \pm 0.20$ \\
\hline UGC 04387 & $\ldots$ & $\ldots$ & $\cdots$ & $12.54 \pm 0.04$ & $11.77 \pm 0.05$ & $11.35 \pm 0.06$ & $\cdots$ & $\cdots$ & $\ldots$ & $\ldots$ \\
\hline NGC 2551 & $\ldots$ & $13.10 \pm 0.20$ & $12.11 \pm 0.20$ & $10.27 \pm 0.01$ & $9.64 \pm 0.02$ & $9.37 \pm 0.02$ & $<0.12$ & $<0.07$ & $0.25 \pm 0.03$ & $1.01 \pm 0.15$ \\
\hline HS $0822+3542$ & $\ldots$ & $\ldots$ & $\ldots$ & $\ldots$ & $\ldots$ & $\ldots$ & $\ldots$ & $\ldots$ & $\ldots$ & $\ldots$ \\
\hline UGC 04393 & $\ldots$ & $13.35 \pm 0.20$ & $\ldots$ & $11.97 \pm 0.03$ & $11.41 \pm 0.05$ & $11.22 \pm 0.07$ & $<0.06$ & $<0.12$ & $0.87 \pm 0.06$ & $1.99 \pm 0.20$ \\
\hline UGC 04401 & $\ldots$ & $\ldots$ & $\ldots$ & $\ldots$ & $\ldots$ & $\ldots$ & $\ldots$ & $\ldots$ & $\ldots$ & $\ldots$ \\
\hline UGC 04390 & $\ldots$ & $14.90 \pm 0.50$ & $\ldots$ & $\cdots$ & $\ldots$ & $\ldots$ & $\ldots$ & $\cdots$ & $\ldots$ & $\ldots$ \\
\hline
\end{tabular}


Table 4-Continued

\begin{tabular}{|c|c|c|c|c|c|c|c|c|c|c|}
\hline \multirow{2}{*}{$\begin{array}{c}\text { Object Name } \\
\text { (1) }\end{array}$} & \multicolumn{3}{|c|}{ Optical Photometry } & \multicolumn{3}{|c|}{ Near-Infrared Photometry } & \multicolumn{4}{|c|}{ IRAS fluxes } \\
\hline & $\begin{array}{c}U \\
(\operatorname{mag}) \\
(2)\end{array}$ & $\begin{array}{c}B \\
(\mathrm{mag}) \\
(3)\end{array}$ & $\begin{array}{c}V \\
(\mathrm{mag}) \\
(4)\end{array}$ & $\begin{array}{c}J \\
(\mathrm{mag}) \\
(5)\end{array}$ & $\begin{array}{c}H \\
(\mathrm{mag}) \\
(6)\end{array}$ & $\begin{array}{c}K \\
(\mathrm{mag}) \\
(7)\end{array}$ & $\begin{array}{c}12 \mu \mathrm{m} \\
(\mathrm{Jy}) \\
(8)\end{array}$ & $\begin{array}{c}25 \mu \mathrm{m} \\
(\mathrm{Jy}) \\
(9)\end{array}$ & $\begin{array}{l}60 \mu \mathrm{m} \\
(\mathrm{Jy}) \\
(10)\end{array}$ & $\begin{array}{c}100 \mu \mathrm{m} \\
(\mathrm{Jy}) \\
(11)\end{array}$ \\
\hline NGC 2550A & $\cdots$ & $13.44 \pm 0.18$ & $\cdots$ & $10.95 \pm 0.05$ & $10.32 \pm 0.07$ & $10.05 \pm 0.08$ & $0.10 \pm 0.02$ & $0.10 \pm 0.02$ & $0.85 \pm 0.05$ & $3.18 \pm 0.19$ \\
\hline UGC 04436 & $\cdots$ & $15.38 \pm 0.18$ & $\cdots$ & $12.07 \pm 0.03$ & $11.39 \pm 0.03$ & $11.13 \pm 0.05$ & $<0.12$ & $0.11 \pm 0.03$ & $0.77 \pm 0.06$ & $2.24 \pm 0.18$ \\
\hline UGC 04461 & $\cdots$ & $14.32 \pm 0.18$ & $\cdots$ & $11.86 \pm 0.04$ & $11.17 \pm 0.05$ & $10.93 \pm 0.06$ & $<0.12$ & $0.14 \pm 0.02$ & $0.89 \pm 0.06$ & $2.06 \pm 0.21$ \\
\hline DDO 053 & $14.08 \pm 0.19$ & $14.48 \pm 0.17$ & $14.13 \pm 0.18$ & $\cdots$ & $\ldots$ & $\ldots$ & $<0.06$ & $<0.07$ & $0.28 \pm 0.06$ & $<0.98$ \\
\hline NGC 2600 & $\cdots$ & $15.00 \pm 0.18$ & $\cdots$ & $12.14 \pm 0.04$ & $11.41 \pm 0.05$ & $11.03 \pm 0.06$ & $<0.10$ & $<0.13$ & $0.30 \pm 0.04$ & $<0.91$ \\
\hline UGC 04499 & $\cdots$ & $13.50 \pm 0.30$ & $\cdots$ & $\ldots$ & $\ldots$ & $\ldots$ & $<0.07$ & $<0.13$ & $0.36 \pm 0.04$ & $1.00 \pm 0.17$ \\
\hline NGC 2623 & $14.10 \pm 0.16$ & $13.99 \pm 0.15$ & $13.36 \pm 0.15$ & $\ldots$ & $\ldots$ & $\ldots$ & $0.24 \pm 0.03$ & $1.85 \pm 0.06$ & $25.70 \pm 0.06$ & $27.40 \pm 0.12$ \\
\hline UGC 04514 & $\cdots$ & $13.87 \pm 0.19$ & $\ldots$ & $\cdots$ & $\cdots$ & $\cdots$ & $<0.07$ & $<0.09$ & $0.28 \pm 0.04$ & $0.80 \pm 0.17$ \\
\hline UGC 04515 & $\cdots$ & $14.38 \pm 0.18$ & $\cdots$ & $11.43 \pm 0.03$ & $10.74 \pm 0.03$ & $10.53 \pm 0.04$ & $<0.09$ & $<0.09$ & $0.22 \pm 0.04$ & $1.24 \pm 0.17$ \\
\hline UGC 04525 & $\ldots$ & $14.88 \pm 0.19$ & $\ldots$ & $13.05 \pm 0.05$ & $12.42 \pm 0.07$ & $12.40 \pm 0.11$ & $\ldots$ & $\ldots$ & $\ldots$ & $\ldots$ \\
\hline UGC 04529 & $\cdots$ & $\ldots$ & $\ldots$ & $13.40 \pm 0.05$ & $12.61 \pm 0.07$ & $12.28 \pm 0.07$ & $\ldots$ & $\ldots$ & $\ldots$ & $\ldots$ \\
\hline NGC 2639 & $12.90 \pm 0.14$ & $12.56 \pm 0.13$ & $11.65 \pm 0.13$ & $9.39 \pm 0.02$ & $8.68 \pm 0.02$ & $8.40 \pm 0.03$ & $0.16 \pm 0.03$ & $0.21 \pm 0.02$ & $1.99 \pm 0.08$ & $7.06 \pm 0.28$ \\
\hline UGC 04546 & $\cdots$ & $15.01 \pm 0.20$ & $\ldots$ & $11.54 \pm 0.05$ & $10.81 \pm 0.04$ & $10.50 \pm 0.03$ & $\ldots$ & $\cdots$ & $\ldots$ & $\ldots$ \\
\hline UGC 04551 & $13.87 \pm 0.20$ & $13.40 \pm 0.20$ & $12.46 \pm 0.20$ & $10.08 \pm 0.02$ & $9.43 \pm 0.01$ & $9.33 \pm 0.02$ & $<0.03$ & $<0.03$ & $<0.03$ & $<0.06$ \\
\hline UGC 04562 & $\ldots$ & $\ldots$ & $\ldots$ & $12.71 \pm 0.06$ & $11.85 \pm 0.07$ & $11.79 \pm 0.10$ & $0.09 \pm 0.02$ & $<0.13$ & $0.38 \pm 0.04$ & $0.85 \pm 0.14$ \\
\hline UGC 04560 & $\cdots$ & $15.55 \pm 0.18$ & $\ldots$ & $11.91 \pm 0.03$ & $11.23 \pm 0.04$ & $10.91 \pm 0.04$ & $\ldots$ & $\ldots$ & $\ldots$ & $\ldots$ \\
\hline VV 703 & $\cdots$ & $\ldots$ & $\ldots$ & $\ldots$ & $\ldots$ & $\ldots$ & $\ldots$ & $\ldots$ & $\ldots$ & $\ldots$ \\
\hline UGC 04628 & $\ldots$ & $15.27 \pm 0.19$ & $\ldots$ & $\ldots$ & $\ldots$ & $\ldots$ & $\ldots$ & $\ldots$ & $\ldots$ & $\ldots$ \\
\hline NGC 2675 & $14.84 \pm 0.20$ & $14.30 \pm 0.20$ & $13.26 \pm 0.20$ & $11.06 \pm 0.02$ & $10.40 \pm 0.03$ & $10.11 \pm 0.03$ & $\ldots$ & $\cdots$ & $\cdots$ & $\ldots$ \\
\hline NGC 2681 & $11.40 \pm 0.11$ & $11.09 \pm 0.10$ & $10.29 \pm 0.10$ & $8.37 \pm 0.02$ & $7.71 \pm 0.03$ & $7.43 \pm 0.03$ & $0.37 \pm 0.04$ & $0.59 \pm 0.03$ & $7.14 \pm 0.04$ & $11.90 \pm 0.09$ \\
\hline IC 0522 & $\ldots$ & $13.97 \pm 0.17$ & $\ldots$ & $10.95 \pm 0.02$ & $10.28 \pm 0.02$ & $9.97 \pm 0.03$ & $<0.03$ & $<0.02$ & $0.09 \pm 0.03$ & $0.55 \pm 0.09$ \\
\hline VV 761 & $\cdots$ & $\cdots$ & $\cdots$ & $\cdots$ & $\ldots$ & $\ldots$ & $<0.06$ & $<0.13$ & $0.51 \pm 0.05$ & $1.01 \pm 0.16$ \\
\hline UGC 04668 & $\cdots$ & $15.72 \pm 0.19$ & $\cdots$ & $12.52 \pm 0.04$ & $11.76 \pm 0.05$ & $11.48 \pm 0.05$ & $<0.07$ & $<0.10$ & $0.33 \pm 0.04$ & $0.59 \pm 0.15$ \\
\hline UGC 04684 & $\ldots$ & $14.00 \pm 0.20$ & $\cdots$ & $12.85 \pm 0.07$ & $12.44 \pm 0.09$ & $12.24 \pm 0.14$ & $<0.22$ & $<0.08$ & $0.35 \pm 0.04$ & $0.86 \pm 0.15$ \\
\hline UGC 04671 & $\cdots$ & $13.60 \pm 0.18$ & $\cdots$ & $11.26 \pm 0.04$ & $10.54 \pm 0.05$ & $10.26 \pm 0.06$ & $0.17 \pm 0.03$ & $0.23 \pm 0.03$ & $1.80 \pm 0.11$ & $4.62 \pm 0.32$ \\
\hline NGC 2692 & $\cdots$ & $14.18 \pm 0.19$ & $\cdots$ & $10.77 \pm 0.02$ & $10.13 \pm 0.03$ & $9.90 \pm 0.03$ & $\ldots$ & $\ldots$ & $\ldots$ & $\ldots$ \\
\hline NGC 2693 & $13.45 \pm 0.15$ & $12.84 \pm 0.15$ & $11.88 \pm 0.15$ & $9.59 \pm 0.01$ & $8.92 \pm 0.02$ & $8.60 \pm 0.02$ & $<0.03$ & $<0.03$ & $0.20 \pm 0.04$ & $0.89 \pm 0.13$ \\
\hline UGC 04676 & $\ldots$ & $\ldots$ & $\ldots$ & $14.46 \pm 0.11$ & $14.28 \pm 0.25$ & $13.82 \pm 0.24$ & $\ldots$ & $\ldots$ & $\ldots$ & $\ldots$ \\
\hline UGC 04679 & $\ldots$ & $\ldots$ & $\ldots$ & $13.23 \pm 0.05$ & $12.44 \pm 0.07$ & $12.40 \pm 0.09$ & $\ldots$ & $\ldots$ & $\ldots$ & $\ldots$ \\
\hline UGC 04690 & $\cdots$ & $14.75 \pm 0.18$ & $\cdots$ & $11.97 \pm 0.04$ & $11.25 \pm 0.05$ & $10.85 \pm 0.05$ & $\cdots$ & $\cdots$ & $\cdots$ & $\cdots$ \\
\hline UGC 04702 & $\cdots$ & $14.50 \pm 0.20$ & $\cdots$ & $11.36 \pm 0.03$ & $10.65 \pm 0.04$ & $10.32 \pm 0.04$ & $\cdots$ & $\cdots$ & $\cdots$ & $\cdots$ \\
\hline UGC 04704 & $\cdots$ & $15.00 \pm 0.30$ & $\cdots$ & $\ldots$ & $\ldots$ & $\ldots$ & $\cdots$ & $\cdots$ & $\cdots$ & $\cdots$ \\
\hline
\end{tabular}


Table 4-Continued

\begin{tabular}{|c|c|c|c|c|c|c|c|c|c|c|}
\hline \multirow{2}{*}{$\begin{array}{c}\text { Object Name } \\
\text { (1) }\end{array}$} & \multicolumn{3}{|c|}{ Optical Photometry } & \multicolumn{3}{|c|}{ Near-Infrared Photometry } & \multicolumn{4}{|c|}{ IRAS fluxes } \\
\hline & $\begin{array}{c}U \\
(\mathrm{mag}) \\
(2)\end{array}$ & $\begin{array}{c}B \\
(\mathrm{mag}) \\
(3)\end{array}$ & $\begin{array}{c}V \\
(\mathrm{mag}) \\
(4)\end{array}$ & $\begin{array}{c}J \\
(\mathrm{mag}) \\
(5)\end{array}$ & $\begin{array}{c}H \\
(\mathrm{mag}) \\
(6)\end{array}$ & $\begin{array}{c}K \\
(\mathrm{mag}) \\
(7)\end{array}$ & $\begin{array}{c}12 \mu \mathrm{m} \\
(\mathrm{Jy}) \\
(8)\end{array}$ & $\begin{array}{c}25 \mu \mathrm{m} \\
(\mathrm{Jy}) \\
(9)\end{array}$ & $\begin{array}{c}60 \mu \mathrm{m} \\
(\mathrm{Jy}) \\
(10)\end{array}$ & $\begin{array}{l}100 \mu \mathrm{m} \\
(\mathrm{Jy}) \\
(11)\end{array}$ \\
\hline NGC 2710 & $\cdots$ & $13.66 \pm 0.18$ & $\cdots$ & $11.68 \pm 0.06$ & $11.18 \pm 0.08$ & $10.45 \pm 0.06$ & $<0.10$ & $0.09 \pm 0.03$ & $0.42 \pm 0.05$ & $1.82 \pm 0.15$ \\
\hline UGC 04800 & $\cdots$ & $14.64 \pm 0.18$ & $\cdots$ & $\cdots$ & $\ldots$ & $\ldots$ & $\cdots$ & $\ldots$ & $\ldots$ & $\ldots$ \\
\hline UGC 04807 & $\cdots$ & $14.36 \pm 0.18$ & $\cdots$ & $12.73 \pm 0.08$ & $12.20 \pm 0.12$ & $11.75 \pm 0.12$ & $<0.12$ & $<0.10$ & $0.22 \pm 0.04$ & $0.66 \pm 0.14$ \\
\hline NGC 2768 & $11.30 \pm 0.10$ & $10.84 \pm 0.10$ & $9.87 \pm 0.10$ & $7.93 \pm 0.02$ & $7.23 \pm 0.03$ & $7.00 \pm 0.03$ & $0.09 \pm 0.02$ & $<0.08$ & $0.37 \pm 0.04$ & $1.16 \pm 0.15$ \\
\hline NGC 2784 & $12.02 \pm 0.13$ & $11.30 \pm 0.13$ & $10.16 \pm 0.13$ & $7.32 \pm 0.02$ & $6.59 \pm 0.02$ & $6.32 \pm 0.02$ & $0.13 \pm 0.03$ & $0.12 \pm 0.03$ & $0.18 \pm 0.05$ & $<1.96$ \\
\hline UGC 04844 & $\cdots$ & $13.94 \pm 0.18$ & $\cdots$ & $11.76 \pm 0.05$ & $11.08 \pm 0.08$ & $10.70 \pm 0.08$ & $0.09 \pm 0.02$ & $0.14 \pm 0.02$ & $0.54 \pm 0.05$ & $1.73 \pm 0.14$ \\
\hline UGC 04851 & $\cdots$ & $14.11 \pm 0.15$ & $\cdots$ & $11.18 \pm 0.02$ & $10.48 \pm 0.02$ & $10.18 \pm 0.03$ & $\cdots$ & $\cdots$ & $\cdots$ & $\cdots$ \\
\hline NGC 2782 & $12.29 \pm 0.13$ & $12.30 \pm 0.13$ & $11.63 \pm 0.13$ & $9.78 \pm 0.01$ & $9.12 \pm 0.01$ & $8.87 \pm 0.02$ & $0.71 \pm 0.04$ & $1.58 \pm 0.05$ & $9.60 \pm 0.05$ & $14.70 \pm 0.18$ \\
\hline UGC 04872 & $\cdots$ & $\cdots$ & $\cdots$ & $12.40 \pm 0.04$ & $11.53 \pm 0.04$ & $11.31 \pm 0.05$ & $<0.11$ & $<0.17$ & $0.26 \pm 0.04$ & $0.84 \pm 0.14$ \\
\hline NGC 2798 & $13.03 \pm 0.19$ & $13.04 \pm 0.16$ & $12.32 \pm 0.17$ & $10.10 \pm 0.02$ & $9.39 \pm 0.02$ & $9.03 \pm 0.03$ & $0.80 \pm 0.03$ & $3.23 \pm 0.03$ & $22.10 \pm 0.04$ & $31.40 \pm 0.18$ \\
\hline UGC 04915 & $\ldots$ & $15.20 \pm 0.20$ & $\ldots$ & $12.55 \pm 0.05$ & $11.80 \pm 0.06$ & $11.36 \pm 0.07$ & $<0.13$ & $<0.17$ & $0.76 \pm 0.05$ & $1.87 \pm 0.17$ \\
\hline NGC 2799 & $\cdots$ & $14.32 \pm 0.18$ & $\cdots$ & $11.92 \pm 0.03$ & $11.31 \pm 0.04$ & $11.15 \pm 0.07$ & $\ldots$ & $\ldots$ & $\ldots$ & $\ldots$ \\
\hline IC 0531 & $\ldots$ & $14.44 \pm 0.19$ & $\ldots$ & $11.91 \pm 0.05$ & $11.02 \pm 0.05$ & $10.67 \pm 0.06$ & $<0.14$ & $<0.27$ & $0.82 \pm 0.06$ & $1.61 \pm 0.16$ \\
\hline UGC 04921 & $\cdots$ & $\cdots$ & $\cdots$ & $\cdots$ & $\cdots$ & $\cdots$ & $\cdots$ & $\ldots$ & $\cdots$ & $\cdots$ \\
\hline NGC 2841 & $10.43 \pm 0.10$ & $10.09 \pm 0.10$ & $9.22 \pm 0.10$ & $7.01 \pm 0.02$ & $6.30 \pm 0.02$ & $6.06 \pm 0.02$ & $0.90 \pm 0.14$ & $0.83 \pm 0.12$ & $4.41 \pm 0.66$ & $24.20 \pm 3.63$ \\
\hline NGC 2854 & $\cdots$ & $13.82 \pm 0.18$ & $\cdots$ & $11.05 \pm 0.03$ & $10.33 \pm 0.03$ & $10.10 \pm 0.03$ & $0.15 \pm 0.02$ & $0.20 \pm 0.02$ & $2.01 \pm 0.10$ & $<7.99$ \\
\hline NGC 2856 & $\cdots$ & $14.10 \pm 0.20$ & $\cdots$ & $10.74 \pm 0.01$ & $10.01 \pm 0.02$ & $9.71 \pm 0.03$ & $0.34 \pm 0.04$ & $0.95 \pm 0.04$ & $6.15 \pm 0.03$ & $10.30 \pm 0.14$ \\
\hline NGC 2857 & $\ldots$ & $12.90 \pm 0.20$ & $12.27 \pm 0.21$ & $11.27 \pm 0.03$ & $10.57 \pm 0.05$ & $10.75 \pm 0.08$ & $0.09 \pm 0.03$ & $0.10 \pm 0.02$ & $0.58 \pm 0.06$ & $1.89 \pm 0.19$ \\
\hline NGC 2915 & $13.13 \pm 0.12$ & $13.25 \pm 0.12$ & $12.68 \pm 0.12$ & $10.57 \pm 0.03$ & $9.82 \pm 0.03$ & $9.83 \pm 0.06$ & $<0.25$ & $<0.25$ & $0.90 \pm 0.05$ & $1.67 \pm 0.15$ \\
\hline UGC 05013 & $\ldots$ & $15.10 \pm 0.30$ & $\ldots$ & $12.01 \pm 0.04$ & $11.48 \pm 0.05$ & $11.03 \pm 0.06$ & $\ldots$ & $\ldots$ & $\ldots$ & $\cdots$ \\
\hline UGC 05027 & $\cdots$ & $15.52 \pm 0.19$ & $\cdots$ & $13.48 \pm 0.08$ & $13.23 \pm 0.15$ & $12.90 \pm 0.18$ & $\cdots$ & $\cdots$ & $\cdots$ & $\cdots$ \\
\hline NGC 2870 & $\ldots$ & $13.83 \pm 0.18$ & $\ldots$ & $\ldots$ & $\ldots$ & $\ldots$ & $0.09 \pm 0.02$ & $0.12 \pm 0.02$ & $0.52 \pm 0.05$ & $2.07 \pm 0.17$ \\
\hline UGC 05053 & $\cdots$ & $\cdots$ & $\cdots$ & $\cdots$ & $\cdots$ & $\cdots$ & $\cdots$ & $\cdots$ & $\cdots$ & $\cdots$ \\
\hline NGC 2903 & $9.74 \pm 0.10$ & $9.68 \pm 0.10$ & $9.01 \pm 0.10$ & $6.95 \pm 0.02$ & $6.33 \pm 0.02$ & $6.04 \pm 0.02$ & $5.00 \pm 0.75$ & $7.64 \pm 1.15$ & $52.40 \pm 7.86$ & $147.00 \pm 22.10$ \\
\hline UGC 05077 & $\cdots$ & $14.90 \pm 0.20$ & $\cdots$ & $12.07 \pm 0.03$ & $11.27 \pm 0.03$ & $10.94 \pm 0.04$ & $<0.09$ & $0.13 \pm 0.02$ & $0.79 \pm 0.06$ & $1.74 \pm 0.16$ \\
\hline I Zw 18 & $15.33 \pm 0.17$ & $16.08 \pm 0.16$ & $15.98 \pm 0.16$ & $\cdots$ & $\cdots$ & $\ldots$ & $\cdots$ & $\ldots$ & $\cdots$ & $\cdots$ \\
\hline NGC 2916 & $12.81 \pm 0.14$ & $12.74 \pm 0.14$ & $12.05 \pm 0.14$ & $10.11 \pm 0.02$ & $9.43 \pm 0.02$ & $9.12 \pm 0.03$ & $<0.22$ & $<0.26$ & $1.19 \pm 0.12$ & $4.21 \pm 0.30$ \\
\hline UGC 05107 & $\cdots$ & $14.50 \pm 0.20$ & $\cdots$ & $\cdots$ & $\cdots$ & $\cdots$ & $\cdots$ & $\cdots$ & $\cdots$ & $\cdots$ \\
\hline UGC 05101 & $\cdots$ & $15.20 \pm 0.20$ & $\cdots$ & $12.47 \pm 0.03$ & $11.53 \pm 0.03$ & $10.76 \pm 0.03$ & $0.26 \pm 0.03$ & $1.08 \pm 0.03$ & $13.00 \pm 0.04$ & $21.20 \pm 0.18$ \\
\hline NGC 2936 & $13.92 \pm 0.21$ & $13.90 \pm 0.20$ & $13.06 \pm 0.20$ & $10.87 \pm 0.02$ & $10.14 \pm 0.03$ & $9.85 \pm 0.04$ & $\ldots$ & $\ldots$ & $\ldots$ & $\ldots$ \\
\hline NGC 2937 & $15.11 \pm 0.22$ & $14.60 \pm 0.20$ & $13.66 \pm 0.20$ & $11.25 \pm 0.03$ & $10.64 \pm 0.03$ & $10.20 \pm 0.05$ & $\cdots$ & $\cdots$ & $\cdots$ & $\cdots$ \\
\hline UGC 05147 & $\ldots$ & $\ldots$ & $\cdots$ & $13.78 \pm 0.08$ & $13.08 \pm 0.09$ & $13.01 \pm 0.14$ & $\cdots$ & $\cdots$ & $\cdots$ & $\cdots$ \\
\hline
\end{tabular}


Table 4-Continued

\begin{tabular}{|c|c|c|c|c|c|c|c|c|c|c|}
\hline \multirow{2}{*}{$\begin{array}{l}\text { Object Name } \\
\text { (1) }\end{array}$} & \multicolumn{3}{|c|}{ Optical Photometry } & \multicolumn{3}{|c|}{ Near-Infrared Photometry } & \multicolumn{4}{|c|}{ IRAS fluxes } \\
\hline & $\begin{array}{c}U \\
(\mathrm{mag}) \\
(2)\end{array}$ & $\begin{array}{c}B \\
(\mathrm{mag}) \\
(3)\end{array}$ & $\begin{array}{c}V \\
(\mathrm{mag}) \\
(4)\end{array}$ & $\begin{array}{c}J \\
(\mathrm{mag}) \\
(5)\end{array}$ & $\begin{array}{c}H \\
(\mathrm{mag}) \\
(6)\end{array}$ & $\begin{array}{c}K \\
(\mathrm{mag}) \\
(7)\end{array}$ & $\begin{array}{c}12 \mu \mathrm{m} \\
(\mathrm{Jy}) \\
(8)\end{array}$ & $\begin{array}{c}25 \mu \mathrm{m} \\
(\mathrm{Jy}) \\
(9)\end{array}$ & $\begin{array}{c}60 \mu \mathrm{m} \\
(\mathrm{Jy}) \\
(10)\end{array}$ & $\begin{array}{l}100 \mu \mathrm{m} \\
(\mathrm{Jy}) \\
(11)\end{array}$ \\
\hline UGC 05114 & $\cdots$ & $\cdots$ & $\cdots$ & $\cdots$ & $\cdots$ & $\cdots$ & $\cdots$ & $\cdots$ & $\cdots$ & $\cdots$ \\
\hline Holmberg I & $\cdots$ & $13.00 \pm 0.20$ & $\cdots$ & $\cdots$ & $\cdots$ & $\cdots$ & $\cdots$ & $\cdots$ & $\cdots$ & $\cdots$ \\
\hline UGC 05201 & $\cdots$ & $14.90 \pm 0.30$ & $\cdots$ & $13.70 \pm 0.06$ & $13.23 \pm 0.09$ & $13.19 \pm 0.15$ & $\cdots$ & $\cdots$ & $\cdots$ & $\cdots$ \\
\hline NGC 2992 & $13.54 \pm 0.13$ & $13.14 \pm 0.13$ & $12.18 \pm 0.13$ & $9.67 \pm 0.02$ & $8.93 \pm 0.02$ & $8.60 \pm 0.03$ & $\cdots$ & $\cdots$ & $\cdots$ & $\cdots$ \\
\hline NGC 2993 & $12.72 \pm 0.14$ & $13.11 \pm 0.13$ & $12.64 \pm 0.14$ & $11.04 \pm 0.02$ & $10.36 \pm 0.02$ & $10.13 \pm 0.04$ & $0.45 \pm 0.03$ & $1.66 \pm 0.03$ & $11.30 \pm 0.68$ & $17.90 \pm 1.43$ \\
\hline NGC 2976 & $10.82 \pm 0.13$ & $10.82 \pm 0.13$ & $10.16 \pm 0.13$ & $8.35 \pm 0.02$ & $7.72 \pm 0.02$ & $7.52 \pm 0.03$ & $0.90 \pm 0.03$ & $1.70 \pm 0.03$ & $12.70 \pm 0.04$ & $34.60 \pm 0.40$ \\
\hline UGC 05237 & $\ldots$ & $14.74 \pm 0.20$ & $\ldots$ & $13.52 \pm 0.07$ & $12.76 \pm 0.09$ & $12.82 \pm 0.13$ & $<0.08$ & $<0.11$ & $0.21 \pm 0.04$ & $0.66 \pm 0.14$ \\
\hline NGC 3018 & $\cdots$ & $14.13 \pm 0.18$ & $\cdots$ & $12.08 \pm 0.04$ & $11.74 \pm 0.06$ & $11.37 \pm 0.08$ & $\ldots$ & $\ldots$ & $\ldots$ & $\ldots$ \\
\hline NGC 3023 & $\ldots$ & $\ldots$ & $\cdots$ & $11.45 \pm 0.06$ & $10.69 \pm 0.06$ & $10.47 \pm 0.09$ & $0.09 \pm 0.02$ & $0.24 \pm 0.01$ & $2.27 \pm 0.14$ & $4.54 \pm 0.55$ \\
\hline UGC 05268 & $\cdots$ & $15.09 \pm 0.18$ & $\ldots$ & $12.00 \pm 0.03$ & $11.49 \pm 0.06$ & $11.09 \pm 0.05$ & $\ldots$ & $\ldots$ & $\ldots$ & $\cdots$ \\
\hline UGC 05314 & $\cdots$ & $16.00 \pm 0.20$ & $\cdots$ & $13.53 \pm 0.06$ & $12.97 \pm 0.08$ & $13.05 \pm 0.17$ & $\cdots$ & $\cdots$ & $\cdots$ & $\cdots$ \\
\hline NGC 3049 & $\ldots$ & $13.04 \pm 0.19$ & $\ldots$ & $10.93 \pm 0.04$ & $10.30 \pm 0.05$ & $9.96 \pm 0.06$ & $0.14 \pm 0.03$ & $0.43 \pm 0.02$ & $2.82 \pm 0.17$ & $4.24 \pm 0.30$ \\
\hline MESSIER 081 & $\cdots$ & $7.89 \pm 0.03$ & $6.94 \pm 0.03$ & $4.76 \pm 0.02$ & $4.09 \pm 0.02$ & $3.83 \pm 0.02$ & $5.86 \pm 0.88$ & $5.42 \pm 0.81$ & $44.70 \pm 6.71$ & $174.00 \pm 26.10$ \\
\hline MESSIER 082 & $9.61 \pm 0.09$ & $9.30 \pm 0.09$ & $8.41 \pm 0.09$ & $5.84 \pm 0.01$ & $5.07 \pm 0.01$ & $4.66 \pm 0.01$ & $66.60 \pm 9.99$ & $285.00 \pm 42.80$ & $1270.00 \pm 191.00$ & $1350.00 \pm 203.00$ \\
\hline Holmberg IX & $13.90 \pm 0.31$ & $14.30 \pm 0.30$ & $14.10 \pm 0.31$ & $\cdots$ & $\ldots$ & $\ldots$ & $\ldots$ & $\ldots$ & $\cdots$ & $\cdots$ \\
\hline ESO 435-G014 & $\cdots$ & $14.40 \pm 0.21$ & $\cdots$ & $11.40 \pm 0.03$ & $10.63 \pm 0.04$ & $10.40 \pm 0.05$ & $\cdots$ & $\cdots$ & $\cdots$ & $\cdots$ \\
\hline ESO 435-G016 & $\cdots$ & $13.41 \pm 0.21$ & $\cdots$ & $11.04 \pm 0.05$ & $10.40 \pm 0.05$ & $10.17 \pm 0.07$ & $\cdots$ & $\cdots$ & $\cdots$ & $\cdots$ \\
\hline Tol 2 & $\ldots$ & $14.38 \pm 0.21$ & $\cdots$ & $12.86 \pm 0.05$ & $12.46 \pm 0.08$ & $12.47 \pm 0.13$ & $\cdots$ & $\cdots$ & $\cdots$ & $\cdots$ \\
\hline NGC 3089 & $\ldots$ & $13.21 \pm 0.16$ & $\cdots$ & $10.31 \pm 0.03$ & $9.61 \pm 0.03$ & $9.33 \pm 0.04$ & $\cdots$ & $\cdots$ & $\ldots$ & $\cdots$ \\
\hline NGC 3073 & $14.12 \pm 0.14$ & $14.07 \pm 0.14$ & $13.40 \pm 0.14$ & $11.80 \pm 0.05$ & $11.01 \pm 0.06$ & $10.80 \pm 0.08$ & $<0.03$ & $\cdots$ & $\ldots$ & $<0.19$ \\
\hline NGC 3079 & $11.57 \pm 0.14$ & $11.54 \pm 0.14$ & $10.86 \pm 0.14$ & $8.44 \pm 0.02$ & $7.64 \pm 0.02$ & $7.26 \pm 0.02$ & $2.62 \pm 0.04$ & $3.58 \pm 0.03$ & $50.20 \pm 0.05$ & $103.00 \pm 0.15$ \\
\hline NGC 3109 & $\ldots$ & $10.39 \pm 0.07$ & $\ldots$ & $9.91 \pm 0.03$ & $9.66 \pm 0.06$ & $9.28 \pm 0.07$ & $<0.04$ & $0.07 \pm 0.01$ & $3.41 \pm 0.51$ & $7.97 \pm 1.20$ \\
\hline UGCA 196 & $\cdots$ & $13.30 \pm 0.21$ & $\cdots$ & $13.45 \pm 0.06$ & $12.91 \pm 0.08$ & $12.69 \pm 0.12$ & $\cdots$ & $\cdots$ & $\cdots$ & $\cdots$ \\
\hline IC 2537 & $12.86 \pm 0.21$ & $12.78 \pm 0.19$ & $12.09 \pm 0.20$ & $10.50 \pm 0.04$ & $9.81 \pm 0.04$ & $9.40 \pm 0.05$ & $\cdots$ & $\cdots$ & $\cdots$ & $\cdots$ \\
\hline UGC 05406 & $\cdots$ & $\cdots$ & $\cdots$ & $14.52 \pm 0.13$ & $13.67 \pm 0.15$ & $13.69 \pm 0.22$ & $\cdots$ & $\cdots$ & $\cdots$ & $\cdots$ \\
\hline Antlia Dwarf & $\cdots$ & $\cdots$ & $\cdots$ & $\cdots$ & $\cdots$ & $\cdots$ & $\cdots$ & $\cdots$ & $\cdots$ & $\cdots$ \\
\hline M81 Dwarf B & $\cdots$ & $15.19 \pm 0.18$ & $\cdots$ & $\cdots$ & $\cdots$ & $\cdots$ & $\cdots$ & $\cdots$ & $\cdots$ & $\cdots$ \\
\hline NGC 3125 & $13.03 \pm 0.13$ & $13.50 \pm 0.13$ & $13.00 \pm 0.13$ & $11.37 \pm 0.03$ & $10.84 \pm 0.04$ & $10.52 \pm 0.05$ & $0.20 \pm 0.03$ & $0.79 \pm 0.02$ & $5.04 \pm 0.30$ & $5.13 \pm 0.67$ \\
\hline UGC 05455 & $\ldots$ & $\ldots$ & $\ldots$ & $\ldots$ & $\ldots$ & $\ldots$ & $\ldots$ & $\ldots$ & $\ldots$ & $\ldots$ \\
\hline Sextans A & $\ldots$ & $11.86 \pm 0.07$ & $11.47 \pm 0.11$ & $\cdots$ & $\cdots$ & $\cdots$ & $<0.11$ & $<0.08$ & $0.26 \pm 0.04$ & $0.67 \pm 0.14$ \\
\hline UGC 05493 & $\cdots$ & $14.08 \pm 0.15$ & $13.34 \pm 0.16$ & $11.68 \pm 0.03$ & $11.06 \pm 0.04$ & $11.09 \pm 0.08$ & $<0.10$ & $<0.15$ & $0.32 \pm 0.04$ & $1.27 \pm 0.16$ \\
\hline UGC 05515 & $\cdots$ & $13.68 \pm 0.17$ & $\cdots$ & $10.83 \pm 0.03$ & $10.16 \pm 0.03$ & $9.85 \pm 0.05$ & $\cdots$ & $\cdots$ & $\cdots$ & $\cdots$ \\
\hline
\end{tabular}


Table 4-Continued

\begin{tabular}{|c|c|c|c|c|c|c|c|c|c|c|}
\hline \multirow{2}{*}{$\begin{array}{c}\text { Object Name } \\
\text { (1) }\end{array}$} & \multicolumn{3}{|c|}{ Optical Photometry } & \multicolumn{3}{|c|}{ Near-Infrared Photometry } & \multicolumn{4}{|c|}{ IRAS fluxes } \\
\hline & $\begin{array}{c}U \\
(\mathrm{mag}) \\
(2)\end{array}$ & $\begin{array}{c}B \\
(\mathrm{mag}) \\
(3)\end{array}$ & $\begin{array}{c}V \\
(\mathrm{mag}) \\
(4)\end{array}$ & $\begin{array}{c}J \\
(\mathrm{mag}) \\
(5)\end{array}$ & $\begin{array}{c}H \\
(\mathrm{mag}) \\
(6)\end{array}$ & $\begin{array}{c}K \\
(\mathrm{mag}) \\
(7)\end{array}$ & $\begin{array}{c}12 \mu \mathrm{m} \\
(\mathrm{Jy}) \\
(8)\end{array}$ & $\begin{array}{c}25 \mu \mathrm{m} \\
(\mathrm{Jy}) \\
(9)\end{array}$ & $\begin{array}{c}60 \mu \mathrm{m} \\
(\mathrm{Jy}) \\
(10)\end{array}$ & $\begin{array}{c}100 \mu \mathrm{m} \\
(\mathrm{Jy}) \\
(11)\end{array}$ \\
\hline UGC 05528 & $\ldots$ & $14.37 \pm 0.18$ & $\cdots$ & $11.87 \pm 0.03$ & $11.24 \pm 0.04$ & $10.85 \pm 0.07$ & . & & $\ldots$ & . \\
\hline NGC 3147 & $\cdots$ & $11.43 \pm 0.16$ & $10.61 \pm 0.16$ & $8.37 \pm 0.01$ & $7.76 \pm 0.02$ & $7.41 \pm 0.02$ & $0.93 \pm 0.03$ & $1.08 \pm 0.02$ & $8.40 \pm 0.04$ & $30.00 \pm 0.23$ \\
\hline NGC 3185 & $\cdots$ & $12.99 \pm 0.07$ & $12.17 \pm 0.09$ & $10.07 \pm 0.01$ & $9.41 \pm 0.02$ & $9.15 \pm 0.02$ & $0.15 \pm 0.04$ & $0.14 \pm 0.05$ & $1.43 \pm 0.09$ & $3.67 \pm 0.22$ \\
\hline NGC 3187 & $13.71 \pm 0.13$ & $13.91 \pm 0.11$ & $13.44 \pm 0.12$ & $12.15 \pm 0.02$ & $11.45 \pm 0.02$ & $10.85 \pm 0.07$ & $\ldots$ & $\ldots$ & $\ldots$ & $\ldots$ \\
\hline NGC 3190 & $12.60 \pm 0.10$ & $12.12 \pm 0.10$ & $11.15 \pm 0.10$ & $8.50 \pm 0.02$ & $7.77 \pm 0.02$ & $7.46 \pm 0.02$ & $0.32 \pm 0.03$ & $0.35 \pm 0.08$ & $3.19 \pm 0.35$ & $10.10 \pm 0.51$ \\
\hline UGC 05558 & $\cdots$ & $15.33 \pm 0.18$ & $\ldots$ & $12.13 \pm 0.04$ & $11.50 \pm 0.04$ & $11.14 \pm 0.05$ & $\ldots$ & $\ldots$ & $\ldots$ & $\cdots$ \\
\hline NGC 3193 & $12.29 \pm 0.04$ & $11.83 \pm 0.03$ & $10.88 \pm 0.03$ & $8.86 \pm 0.01$ & $8.19 \pm 0.01$ & $7.98 \pm 0.01$ & $<0.03$ & $<0.05$ & $<0.03$ & $<0.36$ \\
\hline NGC 3198 & $10.83 \pm 0.10$ & $10.87 \pm 0.10$ & $10.33 \pm 0.10$ & $8.73 \pm 0.03$ & $8.06 \pm 0.04$ & $7.78 \pm 0.04$ & $0.34 \pm 0.09$ & $0.61 \pm 0.15$ & $6.46 \pm 1.61$ & $17.70 \pm 4.42$ \\
\hline UGC 05570 & $\ldots$ & $\ldots$ & $\ldots$ & $13.93 \pm 0.08$ & $13.02 \pm 0.09$ & $12.84 \pm 0.11$ & $\ldots$ & $\ldots$ & $\ldots$ & $\ldots$ \\
\hline NGC 3183 & $\ldots$ & $12.68 \pm 0.11$ & $\ldots$ & $10.25 \pm 0.02$ & $9.51 \pm 0.02$ & $9.24 \pm 0.03$ & $0.27 \pm 0.03$ & $0.38 \pm 0.02$ & $3.35 \pm 0.20$ & $9.97 \pm 0.50$ \\
\hline ESO 317-G019 & $\ldots$ & $14.46 \pm 0.21$ & $\ldots$ & $11.68 \pm 0.05$ & $11.11 \pm 0.06$ & $10.77 \pm 0.09$ & $\ldots$ & $\ldots$ & $\ldots$ & $\ldots$ \\
\hline ESO 317-G023 & $\ldots$ & $13.93 \pm 0.21$ & $\ldots$ & $11.89 \pm 0.01$ & $10.99 \pm 0.01$ & $10.50 \pm 0.01$ & $0.35 \pm 0.03$ & $0.86 \pm 0.02$ & $12.60 \pm 0.63$ & $23.90 \pm 1.19$ \\
\hline ESO 263-G033 & $\ldots$ & $14.00 \pm 0.21$ & $\ldots$ & $10.65 \pm 0.02$ & $9.95 \pm 0.02$ & $9.73 \pm 0.03$ & $\ldots$ & $\cdots$ & $\ldots$ & $\ldots$ \\
\hline NGC 3225 & $\ldots$ & $13.30 \pm 0.18$ & $\ldots$ & $11.80 \pm 0.03$ & $11.42 \pm 0.06$ & $11.15 \pm 0.06$ & $<0.13$ & $<0.08$ & $0.61 \pm 0.05$ & $1.87 \pm 0.21$ \\
\hline NGC 3244 & $12.89 \pm 0.17$ & $12.89 \pm 0.15$ & $12.28 \pm 0.15$ & $10.58 \pm 0.04$ & $9.86 \pm 0.04$ & $9.61 \pm 0.05$ & $0.19 \pm 0.03$ & $0.17 \pm 0.02$ & $1.74 \pm 0.09$ & $5.61 \pm 0.45$ \\
\hline NGC $3256 \mathrm{~A}$ & $\ldots$ & $15.00 \pm 0.21$ & $\ldots$ & $13.44 \pm 0.07$ & $12.71 \pm 0.08$ & $12.90 \pm 0.17$ & $\ldots$ & $\ldots$ & $\ldots$ & $\ldots$ \\
\hline NGC 3238 & $\ldots$ & $13.90 \pm 0.16$ & $\ldots$ & $10.95 \pm 0.03$ & $10.23 \pm 0.03$ & $9.95 \pm 0.04$ & $\ldots$ & $\ldots$ & $\ldots$ & $\ldots$ \\
\hline IC 2574 & $\ldots$ & $10.80 \pm 0.19$ & $10.36 \pm 0.21$ & $11.48 \pm 0.05$ & $10.91 \pm 0.08$ & $10.72 \pm 0.10$ & $<0.05$ & $0.08 \pm 0.01$ & $2.41 \pm 0.36$ & $10.60 \pm 1.59$ \\
\hline NGC 3265 & $\ldots$ & $13.87 \pm 0.15$ & $\ldots$ & $11.37 \pm 0.02$ & $10.68 \pm 0.02$ & $10.44 \pm 0.03$ & $0.10 \pm 0.03$ & $0.36 \pm 0.02$ & $2.18 \pm 0.17$ & $3.39 \pm 0.20$ \\
\hline UGC 05715 & $\ldots$ & $14.20 \pm 0.18$ & $\ldots$ & $11.76 \pm 0.04$ & $11.03 \pm 0.05$ & $10.80 \pm 0.07$ & $\ldots$ & $\ldots$ & $\ldots$ & $\ldots$ \\
\hline UGC 05720 & $\cdots$ & $13.40 \pm 0.20$ & $\cdots$ & $11.41 \pm 0.03$ & $10.70 \pm 0.04$ & $10.43 \pm 0.04$ & $0.21 \pm 0.03$ & $0.95 \pm 0.01$ & $4.68 \pm 0.28$ & $5.32 \pm 0.32$ \\
\hline NGC 3277 & $12.74 \pm 0.13$ & $12.50 \pm 0.13$ & $11.68 \pm 0.13$ & $9.83 \pm 0.01$ & $9.16 \pm 0.01$ & $8.93 \pm 0.02$ & $0.12 \pm 0.03$ & $<0.11$ & $0.66 \pm 0.05$ & $1.97 \pm 0.16$ \\
\hline NGC 3288 & $\ldots$ & $14.79 \pm 0.20$ & $\ldots$ & $11.87 \pm 0.04$ & $11.08 \pm 0.05$ & $10.90 \pm 0.06$ & $<0.05$ & $<0.07$ & $0.18 \pm 0.04$ & $0.62 \pm 0.14$ \\
\hline UGC 05772 & $\ldots$ & $14.58 \pm 0.18$ & $\ldots$ & $11.98 \pm 0.05$ & $11.23 \pm 0.05$ & $10.94 \pm 0.07$ & $\ldots$ & $\ldots$ & $\ldots$ & $\ldots$ \\
\hline NGC 3319 & $\ldots$ & $11.48 \pm 0.17$ & $11.07 \pm 0.18$ & $10.85 \pm 0.03$ & $10.24 \pm 0.04$ & $10.07 \pm 0.05$ & $\ldots$ & $\ldots$ & $\ldots$ & $\ldots$ \\
\hline UGC 05818 & $\ldots$ & $14.60 \pm 0.20$ & $\ldots$ & $13.59 \pm 0.10$ & $13.04 \pm 0.13$ & $12.40 \pm 0.15$ & $\ldots$ & $\ldots$ & $\ldots$ & $\ldots$ \\
\hline UGC 05823 & $\ldots$ & $14.47 \pm 0.18$ & $\ldots$ & $13.94 \pm 0.11$ & $13.13 \pm 0.10$ & $13.07 \pm 0.21$ & $\ldots$ & $\ldots$ & $\ldots$ & $\ldots$ \\
\hline NGC 3344 & $10.38 \pm 0.13$ & $10.45 \pm 0.13$ & $9.86 \pm 0.13$ & $8.20 \pm 0.03$ & $7.65 \pm 0.04$ & $7.44 \pm 0.05$ & $0.96 \pm 0.03$ & $1.32 \pm 0.05$ & $9.27 \pm 0.07$ & $27.50 \pm 0.31$ \\
\hline MESSIER 095 & $10.71 \pm 0.10$ & $10.53 \pm 0.10$ & $9.73 \pm 0.10$ & $7.57 \pm 0.02$ & $6.95 \pm 0.03$ & $6.66 \pm 0.04$ & $1.07 \pm 0.04$ & $2.86 \pm 0.06$ & $19.90 \pm 0.07$ & $39.20 \pm 0.12$ \\
\hline UGC 05848 & $\ldots$ & $14.80 \pm 0.30$ & $\ldots$ & $\ldots$ & $\ldots$ & $\ldots$ & $\ldots$ & $\ldots$ & $\ldots$ & . \\
\hline UGC 05853 & $\cdots$ & $15.35 \pm 0.18$ & $\cdots$ & $12.57 \pm 0.05$ & $11.82 \pm 0.05$ & $11.35 \pm 0.06$ & $<0.07$ & $<0.08$ & $0.26 \pm 0.04$ & $0.78 \pm 0.14$ \\
\hline NGC 3353 & $12.90 \pm 0.13$ & $13.25 \pm 0.13$ & $12.79 \pm 0.13$ & $11.38 \pm 0.03$ & $10.71 \pm 0.03$ & $10.61 \pm 0.05$ & $0.27 \pm 0.05$ & $0.97 \pm 0.03$ & $5.54 \pm 0.05$ & $7.16 \pm 0.10$ \\
\hline
\end{tabular}


Table 4-Continued

\begin{tabular}{|c|c|c|c|c|c|c|c|c|c|c|}
\hline \multirow{2}{*}{$\begin{array}{l}\text { Object Name } \\
\text { (1) }\end{array}$} & \multicolumn{3}{|c|}{ Optical Photometry } & \multicolumn{3}{|c|}{ Near-Infrared Photometry } & \multicolumn{4}{|c|}{ IRAS fluxes } \\
\hline & $\begin{array}{c}U \\
(\mathrm{mag}) \\
(2)\end{array}$ & $\begin{array}{c}B \\
(\mathrm{mag}) \\
(3)\end{array}$ & $\begin{array}{c}V \\
(\mathrm{mag}) \\
(4)\end{array}$ & $\begin{array}{c}J \\
(\mathrm{mag}) \\
(5)\end{array}$ & $\begin{array}{c}H \\
(\mathrm{mag}) \\
(6)\end{array}$ & $\begin{array}{c}K \\
(\mathrm{mag}) \\
(7)\end{array}$ & $\begin{array}{c}12 \mu \mathrm{m} \\
(\mathrm{Jy}) \\
(8)\end{array}$ & $\begin{array}{c}25 \mu \mathrm{m} \\
(\mathrm{Jy}) \\
(9)\end{array}$ & $\begin{array}{c}60 \mu \mathrm{m} \\
(\mathrm{Jy}) \\
(10)\end{array}$ & $\begin{array}{c}100 \mu \mathrm{m} \\
(\mathrm{Jy}) \\
(11)\end{array}$ \\
\hline UGC 05869 & $\cdots$ & $14.30 \pm 0.20$ & $\ldots$ & $12.66 \pm 0.05$ & $11.95 \pm 0.06$ & $12.12 \pm 0.13$ & $0.14 \pm 0.04$ & $0.25 \pm 0.07$ & $0.32 \pm 0.10$ & $0.54 \pm 0.14$ \\
\hline NGC 3367 & $11.89 \pm 0.15$ & $12.05 \pm 0.14$ & $11.50 \pm 0.14$ & $9.76 \pm 0.02$ & $9.12 \pm 0.02$ & $8.76 \pm 0.03$ & $0.49 \pm 0.05$ & $1.46 \pm 0.07$ & $6.06 \pm 0.04$ & $12.50 \pm 0.09$ \\
\hline UGC 05876 & & $15.30 \pm 0.30$ & $\ldots$ & $14.04 \pm 0.06$ & $13.47 \pm 0.09$ & $13.45 \pm 0.14$ & $\ldots$ & $\ldots$ & $\ldots$ & $\ldots$ \\
\hline NGC 3359 & $10.83 \pm 0.06$ & $11.03 \pm 0.05$ & $10.57 \pm 0.05$ & $9.44 \pm 0.03$ & $8.73 \pm 0.04$ & $8.62 \pm 0.05$ & $0.43 \pm 0.03$ & $0.59 \pm 0.02$ & $6.27 \pm 0.03$ & $16.80 \pm 0.14$ \\
\hline MESSIER 096 & $10.42 \pm 0.13$ & $10.11 \pm 0.13$ & $9.25 \pm 0.13$ & $7.24 \pm 0.02$ & $6.57 \pm 0.02$ & $6.32 \pm 0.02$ & $0.99 \pm 0.04$ & $0.53 \pm 0.07$ & $10.70 \pm 0.07$ & $30.40 \pm 0.16$ \\
\hline UGC 05886 & & $14.33 \pm 0.18$ & $\ldots$ & $12.08 \pm 0.04$ & $11.49 \pm 0.06$ & $11.13 \pm 0.07$ & $\ldots$ & $\ldots$ & $\ldots$ & $\ldots$ \\
\hline NGC 3377A & $14.17 \pm 0.18$ & $14.22 \pm 0.16$ & $13.61 \pm 0.17$ & $\ldots$ & $\ldots$ & $\ldots$ & $\ldots$ & $\ldots$ & $\ldots$ & $\ldots$ \\
\hline UGC 05896 & $\ldots$ & $14.77 \pm 0.18$ & $\cdots$ & $11.99 \pm 0.04$ & $11.19 \pm 0.05$ & $10.96 \pm 0.07$ & $\ldots$ & $\ldots$ & $\ldots$ & $\ldots$ \\
\hline NGC 3377 & $11.55 \pm 0.10$ & $11.24 \pm 0.10$ & $10.38 \pm 0.10$ & $8.28 \pm 0.02$ & $7.63 \pm 0.02$ & $7.44 \pm 0.03$ & $0.11 \pm 0.03$ & $<0.07$ & $0.14 \pm 0.04$ & $0.35 \pm 0.07$ \\
\hline UGC 05888 & $\ldots$ & $14.80 \pm 0.30$ & $\ldots$ & $\ldots$ & $\ldots$ & $\ldots$ & $\ldots$ & $\ldots$ & $\ldots$ & $\ldots$ \\
\hline UGC 05904 & $\ldots$ & $\ldots$ & $\ldots$ & $10.94 \pm 0.02$ & $10.18 \pm 0.03$ & $9.81 \pm 0.03$ & $0.18 \pm 0.03$ & $0.15 \pm 0.03$ & $1.52 \pm 0.12$ & $4.83 \pm 0.29$ \\
\hline UGC 05907 & $\ldots$ & $\ldots$ & $\ldots$ & $\ldots$ & $\ldots$ & $\ldots$ & $\ldots$ & $\ldots$ & $\ldots$ & $\ldots$ \\
\hline UGC 05922 & $\ldots$ & $14.47 \pm 0.18$ & $\ldots$ & $12.44 \pm 0.07$ & $12.10 \pm 0.13$ & $11.48 \pm 0.13$ & $\ldots$ & $\ldots$ & $\ldots$ & $\ldots$ \\
\hline UGC 05929 & $\ldots$ & $14.83 \pm 0.18$ & $\ldots$ & $13.48 \pm 0.10$ & $12.87 \pm 0.12$ & $12.44 \pm 0.16$ & $\ldots$ & $\ldots$ & $\ldots$ & $\ldots$ \\
\hline UGC 05928 & $\ldots$ & $14.67 \pm 0.17$ & $\ldots$ & $11.57 \pm 0.03$ & $10.91 \pm 0.04$ & $10.59 \pm 0.05$ & $\ldots$ & $\ldots$ & $\ldots$ & $\ldots$ \\
\hline UGC 05943 & $\ldots$ & $14.01 \pm 0.16$ & $13.27 \pm 0.16$ & $12.90 \pm 0.07$ & $12.59 \pm 0.09$ & $12.01 \pm 0.11$ & $\ldots$ & $\ldots$ & $\ldots$ & $\ldots$ \\
\hline NGC 3394 & $\ldots$ & $13.09 \pm 0.18$ & $\ldots$ & $11.04 \pm 0.06$ & $10.46 \pm 0.07$ & $10.14 \pm 0.09$ & $<0.11$ & $0.11 \pm 0.02$ & $0.62 \pm 0.04$ & $2.19 \pm 0.17$ \\
\hline NGC 3412 & $11.84 \pm 0.13$ & $11.45 \pm 0.13$ & $10.54 \pm 0.13$ & $8.53 \pm 0.01$ & $7.86 \pm 0.01$ & $7.67 \pm 0.01$ & $\ldots$ & $\ldots$ & $\ldots$ & $\ldots$ \\
\hline NGC 3419 & $\ldots$ & $13.46 \pm 0.16$ & $\ldots$ & $11.26 \pm 0.02$ & $10.56 \pm 0.02$ & $10.28 \pm 0.03$ & $<0.05$ & $<0.13$ & $\ldots$ & $<0.24$ \\
\hline UGC 05974 & $\ldots$ & $14.00 \pm 0.20$ & $\ldots$ & $\ldots$ & $\ldots$ & $\ldots$ & $\ldots$ & $\ldots$ & $\ldots$ & $\ldots$ \\
\hline IC 0653 & $\ldots$ & $13.75 \pm 0.18$ & $\ldots$ & $10.90 \pm 0.02$ & $10.14 \pm 0.02$ & $9.91 \pm 0.04$ & $\ldots$ & $\ldots$ & $\ldots$ & $\ldots$ \\
\hline UGC 05971 & $\ldots$ & $15.39 \pm 0.18$ & $\ldots$ & $11.82 \pm 0.03$ & $11.06 \pm 0.03$ & $10.71 \pm 0.04$ & $\ldots$ & $\ldots$ & $\ldots$ & $\ldots$ \\
\hline UGC 06011 & $\ldots$ & $\ldots$ & $\ldots$ & $14.15 \pm 0.10$ & $13.20 \pm 0.09$ & $13.58 \pm 0.28$ & $\ldots$ & $\ldots$ & $\ldots$ & $\ldots$ \\
\hline NGC 3440 & $\cdots$ & $14.02 \pm 0.18$ & $\cdots$ & $12.32 \pm 0.04$ & $11.76 \pm 0.07$ & $11.68 \pm 0.08$ & $<0.06$ & $<0.08$ & $0.53 \pm 0.05$ & $1.22 \pm 0.15$ \\
\hline NGC 3445 & $12.61 \pm 0.22$ & $12.90 \pm 0.20$ & $12.55 \pm 0.20$ & $11.22 \pm 0.04$ & $10.67 \pm 0.06$ & $10.61 \pm 0.08$ & $0.09 \pm 0.02$ & $0.17 \pm 0.01$ & $2.09 \pm 0.13$ & $4.25 \pm 0.34$ \\
\hline NGC 3458 & $\ldots$ & $13.20 \pm 0.13$ & $\ldots$ & $10.23 \pm 0.01$ & $9.55 \pm 0.02$ & $9.33 \pm 0.02$ & $<0.02$ & $<0.04$ & $<0.03$ & $<0.09$ \\
\hline UGC 06039 & $\ldots$ & $\ldots$ & $\cdots$ & $\cdots$ & $\cdots$ & $\cdots$ & $\ldots$ & $\ldots$ & $\ldots$ & $\ldots$ \\
\hline NGC 3475 & $\ldots$ & $14.00 \pm 0.20$ & $\ldots$ & $10.92 \pm 0.01$ & $10.21 \pm 0.02$ & $9.89 \pm 0.02$ & $\ldots$ & $\ldots$ & $\ldots$ & $\ldots$ \\
\hline NGC 3470 & $\ldots$ & $14.10 \pm 0.19$ & $\ldots$ & $11.68 \pm 0.03$ & $10.84 \pm 0.04$ & $10.69 \pm 0.05$ & $\ldots$ & $\ldots$ & $\ldots$ & $\ldots$ \\
\hline NGC 3489 & $11.46 \pm 0.13$ & $11.12 \pm 0.13$ & $10.29 \pm 0.13$ & $8.27 \pm 0.00$ & $7.55 \pm 0.01$ & $7.37 \pm 0.01$ & $\ldots$ & $\ldots$ & $\ldots$ & $\ldots$ \\
\hline NGC 3486 & $10.89 \pm 0.10$ & $11.05 \pm 0.10$ & $10.53 \pm 0.10$ & $8.93 \pm 0.02$ & $8.16 \pm 0.03$ & $8.00 \pm 0.04$ & $0.62 \pm 0.04$ & $0.32 \pm 0.05$ & $6.24 \pm 0.04$ & $15.90 \pm 0.09$ \\
\hline UGC 06102 & $\ldots$ & $14.90 \pm 0.20$ & $\ldots$ & $\ldots$ & $\ldots$ & .. & $\ldots$ & $\ldots$ & $\ldots$ & $\ldots$ \\
\hline
\end{tabular}


Table 4-Continued

\begin{tabular}{|c|c|c|c|c|c|c|c|c|c|c|}
\hline \multirow{2}{*}{$\begin{array}{c}\text { Object Name } \\
\text { (1) }\end{array}$} & \multicolumn{3}{|c|}{ Optical Photometry } & \multicolumn{3}{|c|}{ Near-Infrared Photometry } & \multicolumn{4}{|c|}{ IRAS fluxes } \\
\hline & $\begin{array}{c}U \\
(\mathrm{mag}) \\
(2)\end{array}$ & $\begin{array}{c}B \\
(\mathrm{mag}) \\
(3)\end{array}$ & $\begin{array}{c}V \\
(\mathrm{mag}) \\
(4)\end{array}$ & $\begin{array}{c}J \\
(\mathrm{mag}) \\
(5)\end{array}$ & $\begin{array}{c}H \\
(\mathrm{mag}) \\
(6)\end{array}$ & $\begin{array}{c}K \\
(\mathrm{mag}) \\
(7)\end{array}$ & $\begin{array}{c}12 \mu \mathrm{m} \\
(\mathrm{Jy}) \\
(8)\end{array}$ & $\begin{array}{c}25 \mu \mathrm{m} \\
(\mathrm{Jy}) \\
(9)\end{array}$ & $\begin{array}{c}60 \mu \mathrm{m} \\
(\mathrm{Jy}) \\
(10)\end{array}$ & $\begin{array}{l}100 \mu \mathrm{m} \\
(\mathrm{Jy}) \\
(11)\end{array}$ \\
\hline NGC 3521 & $10.06 \pm 0.10$ & $9.83 \pm 0.10$ & $9.02 \pm 0.10$ & $6.74 \pm 0.02$ & $6.03 \pm 0.02$ & $5.78 \pm 0.02$ & $4.91 \pm 1.23$ & $4.36 \pm 1.09$ & $44.00 \pm 11.00$ & $125.00 \pm 31.20$ \\
\hline UGC 06151 & $14.52 \pm 0.22$ & $14.80 \pm 0.20$ & $14.39 \pm 0.22$ & $\ldots$ & $\ldots$ & $\ldots$ & $\ldots$ & $\ldots$ & $\ldots$ & $\cdots$ \\
\hline NGC 3522 & $\ldots$ & $14.13 \pm 0.15$ & $\ldots$ & $11.20 \pm 0.02$ & $10.56 \pm 0.03$ & $10.38 \pm 0.03$ & $\cdots$ & $\cdots$ & $\cdots$ & $\cdots$ \\
\hline IC 0671 & $\cdots$ & $14.10 \pm 0.20$ & $\cdots$ & $11.47 \pm 0.06$ & $10.88 \pm 0.07$ & $10.31 \pm 0.08$ & $\cdots$ & $\cdots$ & $\cdots$ & $\ldots$ \\
\hline UGC 06181 & $\cdots$ & $14.70 \pm 0.30$ & $\cdots$ & $\ldots$ & $\ldots$ & $\ldots$ & $\cdots$ & $\cdots$ & $\cdots$ & $\cdots$ \\
\hline NGC 3539 & $\cdots$ & $15.47 \pm 0.18$ & $\cdots$ & $15.74 \pm 0.21$ & $14.77 \pm 0.19$ & $14.45 \pm 0.27$ & $\ldots$ & $\cdots$ & $\cdots$ & $\cdots$ \\
\hline IC 0673 & $\ldots$ & $14.06 \pm 0.18$ & $\ldots$ & $11.82 \pm 0.03$ & $11.15 \pm 0.03$ & $10.99 \pm 0.05$ & $<0.09$ & $<0.12$ & $0.59 \pm 0.08$ & $1.90 \pm 0.29$ \\
\hline PGC 33931 & $\ldots$ & $14.37 \pm 0.19$ & $\ldots$ & $11.56 \pm 0.02$ & $10.79 \pm 0.02$ & $10.48 \pm 0.03$ & $\ldots$ & $\ldots$ & $\ldots$ & $\ldots$ \\
\hline NGC 3550 & $\cdots$ & $14.12 \pm 0.18$ & $\cdots$ & $\ldots$ & $\ldots$ & $\ldots$ & $\cdots$ & $\cdots$ & $\cdots$ & $\cdots$ \\
\hline NGC 3620 & $\ldots$ & $\ldots$ & $\ldots$ & $8.83 \pm 0.02$ & $7.93 \pm 0.02$ & $7.57 \pm 0.03$ & $1.10 \pm 0.06$ & $4.24 \pm 0.06$ & $42.80 \pm 2.57$ & $65.90 \pm 2.64$ \\
\hline NGC 3621 & $10.10 \pm 0.15$ & $10.18 \pm 0.15$ & $9.56 \pm 0.15$ & $7.46 \pm 0.02$ & $6.80 \pm 0.02$ & $6.60 \pm 0.04$ & $3.47 \pm 0.52$ & $5.09 \pm 0.76$ & $29.60 \pm 4.44$ & $90.10 \pm 13.50$ \\
\hline UGC 06329 & $\cdots$ & $14.50 \pm 0.20$ & $\cdots$ & $\ldots$ & $\cdots$ & $\cdots$ & $\cdots$ & $\ldots$ & $\cdots$ & $\cdots$ \\
\hline UGC 06331 & $\cdots$ & $15.06 \pm 0.18$ & $\cdots$ & $12.13 \pm 0.04$ & $11.34 \pm 0.04$ & $10.81 \pm 0.05$ & $<0.19$ & $<0.28$ & $1.62 \pm 0.13$ & $3.64 \pm 0.33$ \\
\hline NGC 3627 & $9.85 \pm 0.14$ & $9.65 \pm 0.13$ & $8.92 \pm 0.13$ & $6.84 \pm 0.02$ & $6.15 \pm 0.02$ & $5.88 \pm 0.02$ & $4.17 \pm 1.04$ & $7.72 \pm 1.93$ & $56.30 \pm 14.10$ & $145.00 \pm 36.20$ \\
\hline NGC 3630 & $\cdots$ & $12.91 \pm 0.13$ & $\ldots$ & $9.74 \pm 0.02$ & $9.06 \pm 0.02$ & $8.84 \pm 0.03$ & $<0.04$ & $<0.04$ & $<0.06$ & $<0.11$ \\
\hline NGC 3628 & $\ldots$ & $10.28 \pm 0.05$ & $9.48 \pm 0.07$ & $7.17 \pm 0.02$ & $6.38 \pm 0.02$ & $6.07 \pm 0.02$ & $3.08 \pm 0.77$ & $5.30 \pm 1.32$ & $48.50 \pm 12.10$ & $122.00 \pm 30.50$ \\
\hline NGC 3633 & $\ldots$ & $14.45 \pm 0.20$ & $\ldots$ & $10.97 \pm 0.02$ & $10.21 \pm 0.02$ & $9.87 \pm 0.03$ & $0.25 \pm 0.04$ & $0.61 \pm 0.03$ & $3.08 \pm 0.18$ & $5.34 \pm 0.37$ \\
\hline UGC 06359 & $\ldots$ & $\ldots$ & $\ldots$ & $11.34 \pm 0.02$ & $10.59 \pm 0.03$ & $10.42 \pm 0.04$ & $\ldots$ & $\ldots$ & $\ldots$ & $\ldots$ \\
\hline NGC 3640 & $11.89 \pm 0.13$ & $11.36 \pm 0.13$ & $10.44 \pm 0.13$ & $8.44 \pm 0.01$ & $7.79 \pm 0.01$ & $7.52 \pm 0.02$ & $<0.07$ & $<0.04$ & $<0.04$ & $<0.07$ \\
\hline NGC 3641 & $\ldots$ & $14.10 \pm 0.20$ & $13.20 \pm 0.20$ & $11.14 \pm 0.03$ & $10.45 \pm 0.03$ & $10.23 \pm 0.04$ & $<0.03$ & $<0.08$ & $<0.04$ & $<0.15$ \\
\hline NGC 3644 & $\ldots$ & $14.60 \pm 0.20$ & $\ldots$ & $11.99 \pm 0.03$ & $11.25 \pm 0.04$ & $10.87 \pm 0.05$ & $\ldots$ & $\ldots$ & $\ldots$ & $\ldots$ \\
\hline NGC 3646 & $11.76 \pm 0.14$ & $11.78 \pm 0.13$ & $11.13 \pm 0.13$ & $9.43 \pm 0.02$ & $8.84 \pm 0.02$ & $8.48 \pm 0.03$ & $\ldots$ & $\ldots$ & $\ldots$ & $\ldots$ \\
\hline NGC 3649 & $\ldots$ & $14.57 \pm 0.18$ & $\ldots$ & $11.44 \pm 0.02$ & $10.80 \pm 0.03$ & $10.40 \pm 0.03$ & $\ldots$ & $\ldots$ & $\ldots$ & $\ldots$ \\
\hline UGC 06387 & $\ldots$ & $\ldots$ & $\ldots$ & $\ldots$ & $\ldots$ & $\ldots$ & $\ldots$ & $\ldots$ & $\ldots$ & $\ldots$ \\
\hline NGC 3662 & $\ldots$ & $13.71 \pm 0.13$ & $\ldots$ & $\ldots$ & $\ldots$ & $\ldots$ & $0.14 \pm 0.04$ & $<0.15$ & $1.03 \pm 0.11$ & $3.55 \pm 0.39$ \\
\hline UGC 06435 & $\ldots$ & $13.88 \pm 0.15$ & $\ldots$ & $13.50 \pm 0.02$ & $10.37 \pm 0.03$ & $10.01 \pm 0.04$ & $\ldots$ & $\ldots$ & $\ldots$ & $\ldots$ \\
\hline VII Zw 403 & $\ldots$ & $14.50 \pm 0.19$ & $\ldots$ & $\ldots$ & $\ldots$ & $\ldots$ & $<0.08$ & $<0.05$ & $0.38 \pm 0.03$ & $<0.90$ \\
\hline NGC 3705 & $12.00 \pm 0.10$ & $11.86 \pm 0.10$ & $11.07 \pm 0.10$ & $8.82 \pm 0.02$ & $8.16 \pm 0.02$ & $7.92 \pm 0.03$ & $0.40 \pm 0.05$ & $0.31 \pm 0.11$ & $3.54 \pm 0.35$ & $10.20 \pm 0.92$ \\
\hline UGC 06519 & $\ldots$ & $15.68 \pm 0.19$ & $\ldots$ & $12.66 \pm 0.06$ & $12.04 \pm 0.07$ & $11.57 \pm 0.09$ & $\ldots$ & $\ldots$ & $\ldots$ & $\ldots$ \\
\hline IC 0716 & $\ldots$ & $14.80 \pm 0.18$ & $\ldots$ & $11.66 \pm 0.03$ & $10.86 \pm 0.03$ & $10.55 \pm 0.04$ & $\ldots$ & $\ldots$ & $\ldots$ & $\ldots$ \\
\hline NGC 3816 & $13.90 \pm 0.13$ & $13.46 \pm 0.13$ & $12.50 \pm 0.13$ & $10.52 \pm 0.02$ & $9.91 \pm 0.02$ & $9.60 \pm 0.03$ & $<0.04$ & $<0.04$ & $<0.04$ & $<0.08$ \\
\hline NGC 3821 & $13.93 \pm 0.20$ & $13.70 \pm 0.20$ & $12.88 \pm 0.20$ & $11.13 \pm 0.02$ & $10.40 \pm 0.02$ & $10.15 \pm 0.03$ & $\ldots$ & $\ldots$ & $\ldots$ & $\ldots$ \\
\hline
\end{tabular}


Table 4-Continued

\begin{tabular}{|c|c|c|c|c|c|c|c|c|c|c|}
\hline \multirow{2}{*}{$\begin{array}{c}\text { Object Name } \\
\text { (1) }\end{array}$} & \multicolumn{3}{|c|}{ Optical Photometry } & \multicolumn{3}{|c|}{ Near-Infrared Photometry } & \multicolumn{4}{|c|}{ IRAS fluxes } \\
\hline & $\begin{array}{c}U \\
(\mathrm{mag}) \\
(2)\end{array}$ & $\begin{array}{c}B \\
(\mathrm{mag}) \\
(3)\end{array}$ & $\begin{array}{c}V \\
(\mathrm{mag}) \\
(4)\end{array}$ & $\begin{array}{c}J \\
(\mathrm{mag}) \\
(5)\end{array}$ & $\begin{array}{c}H \\
(\mathrm{mag}) \\
(6)\end{array}$ & $\begin{array}{c}K \\
(\mathrm{mag}) \\
(7)\end{array}$ & $\begin{array}{c}12 \mu \mathrm{m} \\
(\mathrm{Jy}) \\
(8)\end{array}$ & $\begin{array}{c}25 \mu \mathrm{m} \\
(\mathrm{Jy}) \\
(9)\end{array}$ & $\begin{array}{c}60 \mu \mathrm{m} \\
(\mathrm{Jy}) \\
(10)\end{array}$ & $\begin{array}{l}100 \mu \mathrm{m} \\
(\mathrm{Jy}) \\
(11)\end{array}$ \\
\hline CGCG 097-068 & $14.59 \pm 0.11$ & $14.55 \pm 0.10$ & $13.84 \pm 0.10$ & $12.00 \pm 0.03$ & $11.27 \pm 0.03$ & $11.00 \pm 0.04$ & $0.15 \pm 0.03$ & $0.26 \pm 0.03$ & $1.87 \pm 0.13$ & $3.91 \pm 0.27$ \\
\hline UGC 06683 & $\ldots$ & $15.42 \pm 0.19$ & $\ldots$ & $11.96 \pm 0.03$ & $11.15 \pm 0.04$ & $10.92 \pm 0.04$ & $\ldots$ & $\ldots$ & $\ldots$ & $\ldots$ \\
\hline IC 2951 & $14.97 \pm 0.16$ & $14.46 \pm 0.16$ & $13.55 \pm 0.16$ & $11.23 \pm 0.03$ & $10.44 \pm 0.04$ & $10.23 \pm 0.05$ & $\ldots$ & $\cdots$ & $\ldots$ & $\cdots$ \\
\hline UGC 06697 & $13.76 \pm 0.10$ & $14.08 \pm 0.10$ & $13.59 \pm 0.10$ & $11.78 \pm 0.03$ & $11.01 \pm 0.03$ & $10.67 \pm 0.04$ & $0.13 \pm 0.04$ & $<0.20$ & $1.52 \pm 0.11$ & $2.88 \pm 0.26$ \\
\hline NGC 3840 & $14.55 \pm 0.10$ & $14.54 \pm 0.10$ & $13.84 \pm 0.10$ & $12.05 \pm 0.05$ & $11.18 \pm 0.04$ & $10.89 \pm 0.06$ & $<0.15$ & $<0.11$ & $0.82 \pm 0.07$ & $1.45 \pm 0.25$ \\
\hline NGC 3844 & $15.31 \pm 0.15$ & $14.85 \pm 0.14$ & $13.87 \pm 0.14$ & $11.56 \pm 0.04$ & $10.94 \pm 0.03$ & $10.80 \pm 0.04$ & $\ldots$ & $\ldots$ & $\ldots$ & $\ldots$ \\
\hline NGC 3842 & $13.27 \pm 0.13$ & $12.78 \pm 0.13$ & $11.82 \pm 0.13$ & $10.04 \pm 0.02$ & $9.39 \pm 0.02$ & $9.08 \pm 0.03$ & $0.09 \pm 0.03$ & $<0.10$ & $0.36 \pm 0.06$ & $1.49 \pm 0.19$ \\
\hline UGC 06719 & $14.72 \pm 0.13$ & $14.44 \pm 0.13$ & $13.59 \pm 0.13$ & $11.38 \pm 0.03$ & $10.71 \pm 0.03$ & $10.46 \pm 0.05$ & $\ldots$ & $\ldots$ & $\ldots$ & $\ldots$ \\
\hline NGC 3861 & $13.65 \pm 0.10$ & $13.47 \pm 0.10$ & $12.67 \pm 0.10$ & $10.92 \pm 0.03$ & $10.24 \pm 0.03$ & $9.97 \pm 0.04$ & $<0.10$ & $<0.15$ & $0.44 \pm 0.06$ & $1.66 \pm 0.23$ \\
\hline UGC 06725 & $\ldots$ & $13.86 \pm 0.19$ & $\ldots$ & $11.58 \pm 0.03$ & $10.90 \pm 0.04$ & $10.65 \pm 0.05$ & $\ldots$ & $\ldots$ & $\ldots$ & $\ldots$ \\
\hline ESO 440-G004 & $\ldots$ & $14.18 \pm 0.21$ & $\ldots$ & $\ldots$ & $\ldots$ & $\ldots$ & $\ldots$ & $\ldots$ & $\ldots$ & $\ldots$ \\
\hline UGC 06736 & $\ldots$ & $14.75 \pm 0.18$ & $\ldots$ & $12.80 \pm 0.06$ & $12.10 \pm 0.06$ & $11.98 \pm 0.11$ & $<0.11$ & $<0.26$ & $0.25 \pm 0.05$ & $<0.85$ \\
\hline NGC 3885 & $12.19 \pm 0.13$ & $12.84 \pm 0.13$ & $11.89 \pm 0.13$ & $9.44 \pm 0.01$ & $8.70 \pm 0.01$ & $8.38 \pm 0.02$ & $0.43 \pm 0.03$ & $1.64 \pm 0.05$ & $10.60 \pm 0.03$ & $14.60 \pm 0.17$ \\
\hline UGCA 247 & $\ldots$ & $12.97 \pm 0.21$ & $\ldots$ & $\ldots$ & $\ldots$ & $\ldots$ & $0.12 \pm 0.03$ & $<0.14$ & $0.85 \pm 0.08$ & $3.13 \pm 0.34$ \\
\hline NGC 3923 & $11.41 \pm 0.40$ & $10.80 \pm 0.40$ & $9.80 \pm 0.40$ & $7.42 \pm 0.02$ & $6.76 \pm 0.02$ & $6.50 \pm 0.03$ & $\ldots$ & $<0.03$ & $<0.03$ & $<0.14$ \\
\hline NGC 3938 & $10.80 \pm 0.10$ & $10.90 \pm 0.10$ & $10.38 \pm 0.10$ & $8.62 \pm 0.03$ & $8.17 \pm 0.05$ & $7.81 \pm 0.05$ & $0.90 \pm 0.03$ & $1.26 \pm 0.03$ & $9.24 \pm 0.03$ & $27.60 \pm 0.09$ \\
\hline UGC 06879 & $\ldots$ & $14.11 \pm 0.18$ & $\ldots$ & $12.20 \pm 0.03$ & $11.00 \pm 0.06$ & $10.92 \pm 0.09$ & $<0.12$ & $<0.14$ & $0.27 \pm 0.04$ & $0.90 \pm 0.14$ \\
\hline UGC 06934 & $\ldots$ & $14.91 \pm 0.18$ & $\ldots$ & $12.60 \pm 0.04$ & $11.85 \pm 0.05$ & $11.60 \pm 0.08$ & $<0.08$ & $<0.17$ & $0.26 \pm 0.06$ & $0.69 \pm 0.14$ \\
\hline UGC 06970 & $\ldots$ & $\ldots$ & $\ldots$ & $\ldots$ & $\ldots$ & $\ldots$ & $\ldots$ & $\ldots$ & $\ldots$ & $\ldots$ \\
\hline IC 0754 & $\ldots$ & $14.05 \pm 0.15$ & $\ldots$ & $10.91 \pm 0.01$ & $10.27 \pm 0.02$ & $10.02 \pm 0.03$ & $\ldots$ & $\ldots$ & $\ldots$ & $\ldots$ \\
\hline NGC 4030 & $\ldots$ & $11.42 \pm 0.17$ & $\ldots$ & $8.27 \pm 0.01$ & $7.60 \pm 0.01$ & $7.33 \pm 0.01$ & $1.44 \pm 0.06$ & $2.42 \pm 0.08$ & $18.30 \pm 0.05$ & $50.70 \pm 0.12$ \\
\hline UGC 07000 & $\ldots$ & $\ldots$ & $\ldots$ & $\ldots$ & $\ldots$ & $\ldots$ & $<0.09$ & $<0.28$ & $0.36 \pm 0.05$ & $0.95 \pm 0.15$ \\
\hline NGC 4038 & $\ldots$ & $10.91 \pm 0.12$ & $\ldots$ & $\ldots$ & $\ldots$ & $\ldots$ & $\ldots$ & $\ldots$ & $\ldots$ & $\ldots$ \\
\hline NGC 4039 & $\ldots$ & $11.08 \pm 0.21$ & $\ldots$ & $\ldots$ & $\ldots$ & $\ldots$ & $\ldots$ & $\ldots$ & $\ldots$ & $\ldots$ \\
\hline UGC 07011 & $\ldots$ & $15.37 \pm 0.19$ & $\ldots$ & $12.06 \pm 0.03$ & $11.38 \pm 0.03$ & $11.10 \pm 0.05$ & $\ldots$ & $\cdots$ & $\ldots$ & $\ldots$ \\
\hline NGC $4108 \mathrm{~A}$ & $\ldots$ & $14.63 \pm 0.18$ & $\ldots$ & $12.64 \pm 0.03$ & $12.18 \pm 0.05$ & $12.23 \pm 0.09$ & $\ldots$ & $\ldots$ & $\ldots$ & $\ldots$ \\
\hline UGC 07089 & $\ldots$ & $14.40 \pm 0.30$ & $\ldots$ & $\ldots$ & $\ldots$ & $\ldots$ & $\ldots$ & $\ldots$ & $\ldots$ & $\ldots$ \\
\hline NGC 4108 & $\ldots$ & $13.04 \pm 0.18$ & $\ldots$ & $10.72 \pm 0.02$ & $10.08 \pm 0.02$ & $9.81 \pm 0.03$ & $0.20 \pm 0.02$ & $0.22 \pm 0.02$ & $2.05 \pm 0.10$ & $5.26 \pm 0.21$ \\
\hline NGC 4109 & $\ldots$ & $14.84 \pm 0.20$ & $\ldots$ & $12.10 \pm 0.03$ & $11.42 \pm 0.05$ & $11.13 \pm 0.05$ & $\ldots$ & $\ldots$ & $\ldots$ & $\ldots$ \\
\hline NGC 4111 & $12.07 \pm 0.07$ & $11.63 \pm 0.07$ & $10.74 \pm 0.07$ & $8.48 \pm 0.02$ & $7.77 \pm 0.02$ & $7.55 \pm 0.02$ & $\ldots$ & $\ldots$ & $\ldots$ & $\ldots$ \\
\hline NGC $4108 B$ & $\ldots$ & $14.29 \pm 0.20$ & $\ldots$ & $13.48 \pm 0.06$ & $12.93 \pm 0.09$ & $12.87 \pm 0.13$ & $<0.06$ & $0.08 \pm 0.01$ & $0.41 \pm 0.04$ & $<0.62$ \\
\hline NGC 4116 & $\ldots$ & $12.41 \pm 0.06$ & $11.97 \pm 0.08$ & $11.04 \pm 0.04$ & $10.41 \pm 0.05$ & $10.27 \pm 0.08$ & $0.13 \pm 0.03$ & $<0.37$ & $1.91 \pm 0.13$ & $5.38 \pm 0.32$ \\
\hline
\end{tabular}


Table 4-Continued

\begin{tabular}{|c|c|c|c|c|c|c|c|c|c|c|}
\hline \multirow{2}{*}{$\begin{array}{c}\text { Object Name } \\
\text { (1) }\end{array}$} & \multicolumn{3}{|c|}{ Optical Photometry } & \multicolumn{3}{|c|}{ Near-Infrared Photometry } & \multicolumn{4}{|c|}{ IRAS fluxes } \\
\hline & $\begin{array}{c}U \\
(\mathrm{mag}) \\
(2)\end{array}$ & $\begin{array}{c}B \\
(\mathrm{mag}) \\
(3)\end{array}$ & $\begin{array}{c}V \\
(\mathrm{mag}) \\
(4)\end{array}$ & $\begin{array}{c}J \\
(\mathrm{mag}) \\
(5)\end{array}$ & $\begin{array}{c}H \\
(\mathrm{mag}) \\
(6)\end{array}$ & $\begin{array}{c}K \\
(\mathrm{mag}) \\
(7)\end{array}$ & $\begin{array}{c}12 \mu \mathrm{m} \\
(\mathrm{Jy}) \\
(8)\end{array}$ & $\begin{array}{c}25 \mu \mathrm{m} \\
(\mathrm{Jy}) \\
(9)\end{array}$ & $\begin{array}{l}60 \mu \mathrm{m} \\
(\mathrm{Jy}) \\
(10)\end{array}$ & $\begin{array}{c}100 \mu \mathrm{m} \\
(\mathrm{Jy}) \\
(11)\end{array}$ \\
\hline NGC 4117 & & $14.04 \pm 0.16$ & $\ldots$ & $10.93 \pm 0.02$ & $10.34 \pm 0.03$ & $10.05 \pm 0.03$ & & $\ldots$ & $\ldots$ & $\ldots$ \\
\hline NGC 4125 & $11.14 \pm 0.13$ & $10.65 \pm 0.13$ & $9.72 \pm 0.13$ & $7.77 \pm 0.02$ & $7.10 \pm 0.02$ & $6.86 \pm 0.02$ & $0.09 \pm 0.03$ & $<0.09$ & $0.60 \pm 0.05$ & $1.51 \pm 0.17$ \\
\hline NGC 4136 & $\ldots$ & $11.69 \pm 0.17$ & $\ldots$ & $10.31 \pm 0.04$ & $9.61 \pm 0.05$ & $9.31 \pm 0.06$ & $<0.07$ & $<0.24$ & $1.40 \pm 0.11$ & $4.32 \pm 0.34$ \\
\hline NGC 4138 & $12.46 \pm 0.16$ & $12.16 \pm 0.15$ & $11.32 \pm 0.16$ & $9.90 \pm 0.01$ & $9.20 \pm 0.01$ & $9.03 \pm 0.02$ & $\ldots$ & $\ldots$ & $\ldots$ & $\ldots$ \\
\hline UGC 07148 & $\ldots$ & $15.31 \pm 0.18$ & $\ldots$ & $12.52 \pm 0.05$ & $11.91 \pm 0.05$ & $11.69 \pm 0.08$ & $<0.31$ & $<0.29$ & $0.35 \pm 0.07$ & $0.78 \pm 0.14$ \\
\hline NGC 4150 & $12.72 \pm 0.13$ & $12.44 \pm 0.13$ & $11.64 \pm 0.13$ & $9.92 \pm 0.01$ & $9.25 \pm 0.01$ & $8.99 \pm 0.02$ & $<0.11$ & $<0.10$ & $1.21 \pm 0.10$ & $2.39 \pm 0.17$ \\
\hline VII Zw 173 & $\ldots$ & $\ldots$ & $\ldots$ & $\ldots$ & $\ldots$ & $\ldots$ & $<0.08$ & $<0.15$ & $0.49 \pm 0.08$ & $1.11 \pm 0.18$ \\
\hline UGC 07176 & $16.44 \pm 0.36$ & $16.60 \pm 0.20$ & $15.81 \pm 0.28$ & $\ldots$ & $\ldots$ & $\ldots$ & $\ldots$ & $\ldots$ & $\ldots$ & $\ldots$ \\
\hline UGC 07178 & $\ldots$ & $\ldots$ & $\ldots$ & $\ldots$ & $\ldots$ & $\cdots$ & $\ldots$ & $\cdots$ & $\ldots$ & $\ldots$ \\
\hline NGC 4157 & $12.48 \pm 0.10$ & $12.15 \pm 0.10$ & $11.35 \pm 0.10$ & $8.56 \pm 0.02$ & $7.59 \pm 0.02$ & $7.36 \pm 0.02$ & $1.75 \pm 0.04$ & $2.17 \pm 0.03$ & $17.70 \pm 0.05$ & $49.90 \pm 0.18$ \\
\hline IC 3033 & $\cdots$ & $14.92 \pm 0.15$ & $\cdots$ & $\cdots$ & $\ldots$ & $\ldots$ & $\ldots$ & $\ldots$ & $\ldots$ & $\ldots$ \\
\hline UGC 07184 & $\ldots$ & $14.33 \pm 0.20$ & $\ldots$ & $\ldots$ & $\ldots$ & $\ldots$ & $\ldots$ & $\ldots$ & $\ldots$ & $\ldots$ \\
\hline UGC 07196 & $\ldots$ & $15.09 \pm 0.18$ & $\ldots$ & $11.32 \pm 0.02$ & $10.63 \pm 0.03$ & $10.25 \pm 0.03$ & $\ldots$ & $\ldots$ & $\ldots$ & $\ldots$ \\
\hline NGC 4165 & $14.59 \pm 0.14$ & $14.38 \pm 0.11$ & $13.54 \pm 0.13$ & $11.03 \pm 0.03$ & $11.05 \pm 0.08$ & $10.50 \pm 0.07$ & $\ldots$ & $\ldots$ & $\ldots$ & $\ldots$ \\
\hline NGC 4168 & $12.58 \pm 0.05$ & $12.11 \pm 0.05$ & $11.18 \pm 0.05$ & $9.36 \pm 0.01$ & $8.69 \pm 0.02$ & $8.44 \pm 0.02$ & $<0.04$ & $<0.04$ & $<0.04$ & $0.66 \pm 0.16$ \\
\hline IC 3046 & $\ldots$ & $15.29 \pm 0.18$ & $\ldots$ & $12.33 \pm 0.03$ & $11.61 \pm 0.05$ & $11.30 \pm 0.05$ & $\ldots$ & $\ldots$ & $\ldots$ & $\ldots$ \\
\hline NGC 4192A & $\ldots$ & $\ldots$ & $\ldots$ & $\ldots$ & $\ldots$ & $\ldots$ & $\ldots$ & $\ldots$ & $\ldots$ & $\ldots$ \\
\hline NGC 4187 & $\ldots$ & $14.18 \pm 0.15$ & $\ldots$ & $10.79 \pm 0.02$ & $10.09 \pm 0.02$ & $9.77 \pm 0.03$ & $\ldots$ & $\ldots$ & $\ldots$ & $\ldots$ \\
\hline NGC 4189 & $\ldots$ & $12.51 \pm 0.06$ & $11.74 \pm 0.08$ & $9.95 \pm 0.03$ & $9.33 \pm 0.04$ & $8.91 \pm 0.04$ & $0.27 \pm 0.03$ & $0.40 \pm 0.03$ & $3.05 \pm 0.37$ & $8.93 \pm 0.62$ \\
\hline MESSIER 098 & $11.25 \pm 0.12$ & $10.95 \pm 0.08$ & $10.14 \pm 0.08$ & $7.82 \pm 0.02$ & $7.10 \pm 0.02$ & $6.89 \pm 0.02$ & $0.65 \pm 0.16$ & $0.36 \pm 0.09$ & $7.19 \pm 1.80$ & $23.20 \pm 5.79$ \\
\hline NGC 4193 & $\ldots$ & $13.18 \pm 0.07$ & $12.31 \pm 0.09$ & $10.31 \pm 0.02$ & $9.51 \pm 0.02$ & $9.32 \pm 0.03$ & $\ldots$ & $\ldots$ & $\ldots$ & $\ldots$ \\
\hline NGC 4186 & $\ldots$ & $14.65 \pm 0.19$ & $\ldots$ & $11.64 \pm 0.03$ & $10.96 \pm 0.05$ & $10.56 \pm 0.05$ & $\ldots$ & $\ldots$ & $\ldots$ & $\ldots$ \\
\hline UGC 07242 & $\cdots$ & $14.60 \pm 0.20$ & $\cdots$ & $\cdots$ & $\cdots$ & $\cdots$ & $\ldots$ & $\ldots$ & $\cdots$ & $\ldots$ \\
\hline UGC 07249 & $15.17 \pm 0.14$ & $15.43 \pm 0.11$ & $14.73 \pm 0.13$ & $\ldots$ & $\ldots$ & $\ldots$ & $\ldots$ & $\ldots$ & $\ldots$ & $\ldots$ \\
\hline IC 3059 & $14.78 \pm 0.25$ & $14.70 \pm 0.20$ & $14.19 \pm 0.23$ & $\cdots$ & $\cdots$ & $\cdots$ & $\cdots$ & $\cdots$ & $\cdots$ & $\cdots$ \\
\hline VCC 0132 & $\ldots$ & $\ldots$ & $\ldots$ & $\ldots$ & $\ldots$ & $\ldots$ & $\ldots$ & $\ldots$ & $\ldots$ & $\ldots$ \\
\hline IC 3066 & $\ldots$ & $15.58 \pm 0.13$ & $\ldots$ & $13.70 \pm 0.06$ & $13.54 \pm 0.14$ & $13.18 \pm 0.17$ & $\ldots$ & $\ldots$ & $\ldots$ & $\ldots$ \\
\hline NGC 4206 & $12.81 \pm 0.11$ & $12.82 \pm 0.10$ & $12.15 \pm 0.10$ & $10.32 \pm 0.03$ & $9.55 \pm 0.03$ & $9.39 \pm 0.05$ & $0.10 \pm 0.03$ & $<0.25$ & $0.63 \pm 0.06$ & $2.37 \pm 0.26$ \\
\hline IC 3073 & $\ldots$ & $15.19 \pm 0.20$ & $\ldots$ & $\ldots$ & $\ldots$ & .. & $\ldots$ & $\ldots$ & $\ldots$ & $\ldots$ \\
\hline NGC 4216 & $11.51 \pm 0.09$ & $10.99 \pm 0.08$ & $10.01 \pm 0.08$ & $7.53 \pm 0.02$ & $6.78 \pm 0.02$ & $6.52 \pm 0.02$ & $<0.12$ & $<0.20$ & $2.27 \pm 0.57$ & $12.80 \pm 3.20$ \\
\hline NGC 4222 & $\ldots$ & $13.86 \pm 0.15$ & $\ldots$ & $11.37 \pm 0.01$ & $10.64 \pm 0.02$ & $10.33 \pm 0.02$ & $<0.13$ & $<0.14$ & $0.99 \pm 0.08$ & $3.19 \pm 0.26$ \\
\hline NGC 4226 & $\ldots$ & $14.36 \pm 0.18$ & $\ldots$ & $11.18 \pm 0.02$ & $10.46 \pm 0.03$ & $10.11 \pm 0.03$ & $0.18 \pm 0.04$ & $0.37 \pm 0.03$ & $3.29 \pm 0.20$ & $<11.00$ \\
\hline
\end{tabular}


Table 4-Continued

\begin{tabular}{|c|c|c|c|c|c|c|c|c|c|c|}
\hline \multirow{2}{*}{$\begin{array}{l}\text { Object Name } \\
\text { (1) }\end{array}$} & \multicolumn{3}{|c|}{ Optical Photometry } & \multicolumn{3}{|c|}{ Near-Infrared Photometry } & \multicolumn{4}{|c|}{ IRAS fluxes } \\
\hline & $\begin{array}{c}U \\
(\mathrm{mag}) \\
(2)\end{array}$ & $\begin{array}{c}B \\
(\mathrm{mag}) \\
(3)\end{array}$ & $\begin{array}{c}V \\
(\mathrm{mag}) \\
(4)\end{array}$ & $\begin{array}{c}J \\
(\mathrm{mag}) \\
(5)\end{array}$ & $\begin{array}{c}H \\
(\mathrm{mag}) \\
(6)\end{array}$ & $\begin{array}{c}K \\
(\mathrm{mag}) \\
(7)\end{array}$ & $\begin{array}{c}12 \mu \mathrm{m} \\
(\mathrm{Jy}) \\
(8)\end{array}$ & $\begin{array}{c}25 \mu \mathrm{m} \\
(\mathrm{Jy}) \\
(9)\end{array}$ & $\begin{array}{c}60 \mu \mathrm{m} \\
(\mathrm{Jy}) \\
(10)\end{array}$ & $\begin{array}{l}100 \mu \mathrm{m} \\
(\mathrm{Jy}) \\
(11)\end{array}$ \\
\hline NGC 4236 & $\ldots$ & $10.05 \pm 0.17$ & $9.63 \pm 0.18$ & $9.91 \pm 0.03$ & $9.17 \pm 0.04$ & $9.01 \pm 0.05$ & $0.11 \pm 0.02$ & $0.57 \pm 0.09$ & $3.98 \pm 0.60$ & $10.00 \pm 1.50$ \\
\hline UGC 07301 & $\cdots$ & $15.49 \pm 0.19$ & $\ldots$ & $\cdots$ & $\cdots$ & $\cdots$ & $\ldots$ & $\cdots$ & $\cdots$ & $\ldots$ \\
\hline NGC 4231 & $\cdots$ & $14.27 \pm 0.16$ & $\cdots$ & $12.13 \pm 0.03$ & $11.33 \pm 0.04$ & $11.09 \pm 0.05$ & $\cdots$ & $\cdots$ & $\cdots$ & $\cdots$ \\
\hline NGC 4232 & $\cdots$ & $14.43 \pm 0.18$ & $\cdots$ & $11.57 \pm 0.03$ & $10.80 \pm 0.03$ & $10.78 \pm 0.05$ & $\cdots$ & $\cdots$ & $\cdots$ & $\cdots$ \\
\hline UGC 07325 & $\cdots$ & $15.11 \pm 0.18$ & $\cdots$ & $12.30 \pm 0.03$ & $11.55 \pm 0.04$ & $11.36 \pm 0.05$ & $\cdots$ & $\cdots$ & $\cdots$ & $\cdots$ \\
\hline NGC 4242 & $\cdots$ & $11.37 \pm 0.16$ & $10.83 \pm 0.17$ & $\ldots$ & $\ldots$ & $\ldots$ & $\cdots$ & $\cdots$ & $\cdots$ & $\ldots$ \\
\hline NGC 4248 & $\ldots$ & $13.12 \pm 0.08$ & $12.53 \pm 0.09$ & $11.45 \pm 0.02$ & $10.88 \pm 0.03$ & $10.59 \pm 0.04$ & $\ldots$ & $\ldots$ & $\ldots$ & $\ldots$ \\
\hline MESSIER 099 & $10.45 \pm 0.09$ & $10.44 \pm 0.08$ & $9.87 \pm 0.08$ & $7.89 \pm 0.02$ & $7.25 \pm 0.03$ & $6.93 \pm 0.03$ & $3.72 \pm 0.04$ & $4.48 \pm 0.05$ & $34.80 \pm 0.06$ & $92.80 \pm 0.18$ \\
\hline MESSIER 106 & $\cdots$ & $9.10 \pm 0.07$ & $8.41 \pm 0.08$ & $6.38 \pm 0.02$ & $5.72 \pm 0.02$ & $5.46 \pm 0.02$ & $2.25 \pm 0.34$ & $2.81 \pm 0.42$ & $21.60 \pm 3.24$ & $78.40 \pm 11.80$ \\
\hline NGC 4262 & $13.00 \pm 0.09$ & $12.49 \pm 0.09$ & $11.55 \pm 0.09$ & $9.28 \pm 0.01$ & $8.60 \pm 0.01$ & $8.36 \pm 0.02$ & $0.13 \pm 0.02$ & $<0.04$ & $0.18 \pm 0.03$ & $0.39 \pm 0.12$ \\
\hline NGC 4274 & $11.78 \pm 0.13$ & $11.34 \pm 0.13$ & $10.41 \pm 0.13$ & $8.02 \pm 0.02$ & $7.32 \pm 0.02$ & $7.03 \pm 0.02$ & $0.31 \pm 0.04$ & $0.43 \pm 0.04$ & $4.35 \pm 0.30$ & $13.30 \pm 1.86$ \\
\hline NGC 4278 & $11.54 \pm 0.13$ & $11.09 \pm 0.13$ & $10.16 \pm 0.13$ & $8.09 \pm 0.01$ & $7.41 \pm 0.01$ & $7.18 \pm 0.01$ & $<0.19$ & $<0.08$ & $0.56 \pm 0.11$ & $1.57 \pm 0.19$ \\
\hline UGC 07387 & $\ldots$ & $15.21 \pm 0.18$ & $\ldots$ & $11.81 \pm 0.04$ & $11.16 \pm 0.05$ & $10.74 \pm 0.06$ & $\ldots$ & $\ldots$ & $\ldots$ & $\cdots$ \\
\hline NGC 4283 & $13.47 \pm 0.13$ & $13.00 \pm 0.13$ & $12.07 \pm 0.13$ & $10.01 \pm 0.01$ & $9.34 \pm 0.02$ & $9.13 \pm 0.02$ & $<0.05$ & $<0.04$ & $<0.03$ & $<0.08$ \\
\hline NGC 4286 & $\ldots$ & $14.10 \pm 0.20$ & $\ldots$ & $11.77 \pm 0.05$ & $11.15 \pm 0.05$ & $10.82 \pm 0.06$ & $\ldots$ & $\ldots$ & $\ldots$ & $\ldots$ \\
\hline NGC 4292 & $13.49 \pm 0.15$ & $13.09 \pm 0.15$ & $12.21 \pm 0.15$ & $10.40 \pm 0.02$ & $9.75 \pm 0.02$ & $9.55 \pm 0.04$ & $\ldots$ & $\ldots$ & $\ldots$ & $\ldots$ \\
\hline NGC 4298 & $\ldots$ & $12.04 \pm 0.08$ & $11.34 \pm 0.09$ & $9.43 \pm 0.01$ & $8.66 \pm 0.01$ & $8.47 \pm 0.01$ & $0.97 \pm 0.05$ & $1.11 \pm 0.05$ & $6.69 \pm 0.04$ & $25.70 \pm 0.09$ \\
\hline UGC 07411 & $\ldots$ & $14.64 \pm 0.13$ & $\ldots$ & $12.08 \pm 0.04$ & $11.36 \pm 0.04$ & $11.20 \pm 0.07$ & $\ldots$ & $\ldots$ & $\ldots$ & $\ldots$ \\
\hline IC 0783 & $\ldots$ & $14.60 \pm 0.20$ & $\ldots$ & $11.82 \pm 0.05$ & $11.19 \pm 0.07$ & $11.06 \pm 0.09$ & $\ldots$ & $\ldots$ & $\ldots$ & $\ldots$ \\
\hline UGC 07425 & $\ldots$ & $\ldots$ & $\ldots$ & $13.63 \pm 0.08$ & $13.03 \pm 0.13$ & $12.83 \pm 0.14$ & $\ldots$ & $\ldots$ & $\ldots$ & $\ldots$ \\
\hline NGC 4303 & $10.07 \pm 0.11$ & $10.18 \pm 0.09$ & $9.65 \pm 0.09$ & $7.73 \pm 0.02$ & $7.09 \pm 0.02$ & $6.84 \pm 0.03$ & $3.21 \pm 0.05$ & $4.90 \pm 0.06$ & $37.50 \pm 0.08$ & $79.70 \pm 0.10$ \\
\hline VCC 0530 & $\ldots$ & $\ldots$ & $\ldots$ & $\ldots$ & $\ldots$ & $\ldots$ & $\ldots$ & $\ldots$ & $\ldots$ & $\ldots$ \\
\hline NGC 4310 & $\ldots$ & $13.22 \pm 0.15$ & $\ldots$ & $10.62 \pm 0.02$ & $10.01 \pm 0.03$ & $9.68 \pm 0.03$ & $0.11 \pm 0.03$ & $<0.23$ & $0.81 \pm 0.11$ & $2.49 \pm 0.20$ \\
\hline NGC 4301 & $\ldots$ & $13.40 \pm 0.08$ & $12.99 \pm 0.09$ & $12.14 \pm 0.06$ & $11.94 \pm 0.09$ & $11.20 \pm 0.10$ & $<0.18$ & $<0.35$ & $0.55 \pm 0.06$ & $1.05 \pm 0.15$ \\
\hline NGC 4312 & $\ldots$ & $12.53 \pm 0.10$ & $11.72 \pm 0.11$ & $9.69 \pm 0.03$ & $9.02 \pm 0.03$ & $8.79 \pm 0.04$ & $0.21 \pm 0.03$ & $0.27 \pm 0.04$ & $2.14 \pm 0.11$ & $6.44 \pm 0.45$ \\
\hline NGC 4314 & $11.72 \pm 0.16$ & $11.43 \pm 0.15$ & $10.58 \pm 0.15$ & $8.40 \pm 0.02$ & $7.72 \pm 0.02$ & $7.45 \pm 0.02$ & $0.17 \pm 0.05$ & $0.36 \pm 0.03$ & $3.79 \pm 0.27$ & $7.14 \pm 0.43$ \\
\hline NGC 4321 & $10.04 \pm 0.11$ & $10.05 \pm 0.08$ & $9.35 \pm 0.08$ & $7.46 \pm 0.03$ & $6.82 \pm 0.04$ & $6.59 \pm 0.04$ & $2.48 \pm 0.04$ & $3.22 \pm 0.05$ & $25.90 \pm 0.06$ & $69.20 \pm 0.14$ \\
\hline NGC 4323 & $\ldots$ & $14.81 \pm 0.11$ & $13.94 \pm 0.13$ & $12.88 \pm 0.05$ & $12.43 \pm 0.09$ & $12.14 \pm 0.10$ & $\ldots$ & $\ldots$ & $\ldots$ & $\ldots$ \\
\hline NGC 4328 & $\ldots$ & $14.04 \pm 0.13$ & $\ldots$ & $12.01 \pm 0.03$ & $11.45 \pm 0.05$ & $11.46 \pm 0.07$ & $\ldots$ & $\ldots$ & $\ldots$ & $\ldots$ \\
\hline NGC 4344 & $\ldots$ & $13.34 \pm 0.12$ & $\ldots$ & $11.35 \pm 0.01$ & $10.67 \pm 0.02$ & $10.48 \pm 0.02$ & $<0.17$ & $0.14 \pm 0.05$ & $.47 \pm 0.05$ & $1.89 \pm 0.17$ \\
\hline NGC 4371 & $12.35 \pm 0.09$ & $11.79 \pm 0.09$ & $10.81 \pm 0.09$ & $8.60 \pm 0.01$ & $7.95 \pm 0.02$ & $7.72 \pm 0.02$ & $<0.03$ & $<0.05$ & $<0.05$ & $<0.16$ \\
\hline MESSIER 084 & $10.62 \pm 0.05$ & $10.09 \pm 0.05$ & $9.11 \pm 0.05$ & $7.12 \pm 0.02$ & $6.47 \pm 0.02$ & $6.22 \pm 0.02$ & $0.17 \pm 0.03$ & $<0.27$ & $0.50 \pm 0.06$ & $0.98 \pm 0.22$ \\
\hline
\end{tabular}


Table 4-Continued

\begin{tabular}{|c|c|c|c|c|c|c|c|c|c|c|}
\hline \multirow{2}{*}{$\begin{array}{c}\text { Object Name } \\
\text { (1) }\end{array}$} & \multicolumn{3}{|c|}{ Optical Photometry } & \multicolumn{3}{|c|}{ Near-Infrared Photometry } & \multicolumn{4}{|c|}{ IRAS fluxes } \\
\hline & $\begin{array}{c}U \\
(\mathrm{mag}) \\
(2)\end{array}$ & $\begin{array}{c}B \\
(\mathrm{mag}) \\
(3)\end{array}$ & $\begin{array}{c}V \\
(\mathrm{mag}) \\
(4)\end{array}$ & $\begin{array}{c}J \\
(\mathrm{mag}) \\
(5)\end{array}$ & $\begin{array}{c}H \\
(\mathrm{mag}) \\
(6)\end{array}$ & $\begin{array}{c}K \\
(\mathrm{mag}) \\
(7)\end{array}$ & $\begin{array}{c}12 \mu \mathrm{m} \\
(\mathrm{Jy}) \\
(8)\end{array}$ & $\begin{array}{c}25 \mu \mathrm{m} \\
(\mathrm{Jy}) \\
(9)\end{array}$ & $\begin{array}{c}60 \mu \mathrm{m} \\
(\mathrm{Jy}) \\
(10)\end{array}$ & $\begin{array}{l}100 \mu \mathrm{m} \\
(\mathrm{Jy}) \\
(11)\end{array}$ \\
\hline IC 3305 & & $15.12 \pm 0.18$ & & $13.59 \pm 0.10$ & $13.11 \pm 0.15$ & $12.95 \pm 0.22$ & . & 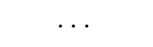 & & $\cdots$ \\
\hline NGC 4379 & $13.05 \pm 0.06$ & $12.63 \pm 0.06$ & $11.72 \pm 0.06$ & $9.65 \pm 0.01$ & $8.96 \pm 0.02$ & $8.77 \pm 0.02$ & $<0.03$ & $<0.04$ & $<0.05$ & $<0.13$ \\
\hline IC 0787 & $\cdots$ & $15.20 \pm 0.13$ & $\cdots$ & $11.51 \pm 0.03$ & $10.92 \pm 0.04$ & $10.56 \pm 0.04$ & $\cdots$ & $\cdots$ & $\cdots$ & $\cdots$ \\
\hline NGC 4383 & $12.41 \pm 0.10$ & $12.67 \pm 0.10$ & $12.12 \pm 0.10$ & $10.40 \pm 0.01$ & $9.77 \pm 0.02$ & $9.49 \pm 0.02$ & $0.32 \pm 0.05$ & $1.06 \pm 0.06$ & $8.40 \pm 0.04$ & $12.70 \pm 0.14$ \\
\hline IC 3311 & $\cdots$ & $14.87 \pm 0.12$ & $\cdots$ & $13.09 \pm 0.04$ & $11.81 \pm 0.05$ & $12.01 \pm 0.09$ & $\cdots$ & $\cdots$ & $\cdots$ & $\cdots$ \\
\hline CGCG 014-032 & $\cdots$ & $14.52 \pm 0.18$ & $\cdots$ & $11.84 \pm 0.03$ & $11.17 \pm 0.02$ & $11.15 \pm 0.06$ & $\cdots$ & $\cdots$ & $\cdots$ & $\cdots$ \\
\hline NGC 4387 & $13.42 \pm 0.05$ & $13.01 \pm 0.05$ & $12.12 \pm 0.05$ & $10.04 \pm 0.01$ & $9.34 \pm 0.02$ & $9.15 \pm 0.02$ & $<0.03$ & $<0.05$ & $<0.05$ & $<0.18$ \\
\hline Tol 65 & $\ldots$ & $\ldots$ & $\ldots$ & $\ldots$ & $\cdots$ & $\ldots$ & $\ldots$ & $\ldots$ & $\ldots$ & $\ldots$ \\
\hline NGC 4388 & $11.91 \pm 0.09$ & $11.76 \pm 0.09$ & $11.02 \pm 0.09$ & $8.98 \pm 0.02$ & $8.25 \pm 0.03$ & $8.00 \pm 0.03$ & $1.06 \pm 0.03$ & $3.42 \pm 0.07$ & $10.10 \pm 0.03$ & $17.40 \pm 0.18$ \\
\hline NGC 4395 & $\ldots$ & $10.64 \pm 0.15$ & $10.18 \pm 0.17$ & $10.66 \pm 0.03$ & $10.25 \pm 0.05$ & $9.98 \pm 0.06$ & $<0.11$ & $0.17 \pm 0.04$ & $4.21 \pm 1.05$ & $12.90 \pm 3.23$ \\
\hline IC 3330 & $\cdots$ & $14.90 \pm 0.18$ & $\cdots$ & $12.21 \pm 0.04$ & $11.56 \pm 0.04$ & $11.32 \pm 0.06$ & $<0.10$ & $<0.11$ & $0.27 \pm 0.05$ & $1.00 \pm 0.15$ \\
\hline NGC 4396 & $12.94 \pm 0.13$ & $13.06 \pm 0.11$ & $12.58 \pm 0.12$ & $11.06 \pm 0.05$ & $10.45 \pm 0.07$ & $10.34 \pm 0.08$ & $<0.10$ & $<0.22$ & $1.15 \pm 0.10$ & $3.90 \pm 0.27$ \\
\hline NGC 4405 & $\ldots$ & $13.03 \pm 0.13$ & $\ldots$ & $10.28 \pm 0.02$ & $9.58 \pm 0.02$ & $9.38 \pm 0.03$ & $0.12 \pm 0.03$ & $<0.16$ & $1.44 \pm 0.10$ & $4.56 \pm 0.27$ \\
\hline NGC 4402 & $\ldots$ & $12.55 \pm 0.05$ & $\ldots$ & $9.60 \pm 0.01$ & $8.62 \pm 0.01$ & $8.48 \pm 0.01$ & $0.79 \pm 0.05$ & $0.64 \pm 0.05$ & $5.43 \pm 0.04$ & $17.50 \pm 0.14$ \\
\hline MESSIER 086 & $10.32 \pm 0.05$ & $9.83 \pm 0.05$ & $8.90 \pm 0.05$ & $7.01 \pm 0.02$ & $6.33 \pm 0.03$ & $6.10 \pm 0.03$ & $0.16 \pm 0.04$ & $<0.06$ & $0.11 \pm 0.03$ & $0.33 \pm 0.07$ \\
\hline NGC 4414 & $11.12 \pm 0.13$ & $10.96 \pm 0.13$ & $10.12 \pm 0.13$ & $7.93 \pm 0.01$ & $7.23 \pm 0.01$ & $6.95 \pm 0.01$ & $3.00 \pm 0.06$ & $3.76 \pm 0.06$ & $30.10 \pm 0.10$ & $69.10 \pm 0.08$ \\
\hline NGC 4407 & $\cdots$ & $12.99 \pm 0.13$ & $12.25 \pm 0.16$ & $10.62 \pm 0.03$ & $10.11 \pm 0.04$ & $9.80 \pm 0.04$ & $<0.19$ & $<0.22$ & $1.04 \pm 0.08$ & $3.19 \pm 0.26$ \\
\hline IC 3356 & $\ldots$ & $15.19 \pm 0.15$ & $\ldots$ & $\ldots$ & $\cdots$ & $\ldots$ & $\ldots$ & $\cdots$ & $\ldots$ & $\ldots$ \\
\hline IC 3355 & $14.94 \pm 0.14$ & $15.18 \pm 0.10$ & $14.86 \pm 0.12$ & $\ldots$ & $\cdots$ & $\ldots$ & $\ldots$ & $\cdots$ & $\ldots$ & $\ldots$ \\
\hline IC 3358 & $\ldots$ & $14.28 \pm 0.13$ & $\ldots$ & $12.27 \pm 0.08$ & $11.50 \pm 0.10$ & $11.45 \pm 0.16$ & $\ldots$ & $\ldots$ & $\ldots$ & $\ldots$ \\
\hline ESO $380-G 029$ & $\ldots$ & $14.96 \pm 0.21$ & $\ldots$ & $13.35 \pm 0.08$ & $12.88 \pm 0.11$ & $12.69 \pm 0.21$ & $\ldots$ & $\ldots$ & $\ldots$ & $\ldots$ \\
\hline NGC 4419 & $12.42 \pm 0.10$ & $12.08 \pm 0.10$ & $11.16 \pm 0.10$ & $8.78 \pm 0.01$ & $8.04 \pm 0.01$ & $7.74 \pm 0.01$ & $0.72 \pm 0.03$ & $1.69 \pm 0.05$ & $7.67 \pm 0.04$ & $15.60 \pm 0.15$ \\
\hline NGC 4421 & $12.81 \pm 0.12$ & $12.47 \pm 0.11$ & $11.60 \pm 0.11$ & $9.71 \pm 0.01$ & $9.04 \pm 0.02$ & $8.80 \pm 0.02$ & $<0.04$ & $<0.04$ & $<0.04$ & $<0.07$ \\
\hline IC 3363 & $\ldots$ & $15.31 \pm 0.07$ & $\ldots$ & $13.64 \pm 0.08$ & $13.09 \pm 0.12$ & $12.75 \pm 0.13$ & $\ldots$ & $\ldots$ & $\ldots$ & $\ldots$ \\
\hline UGC 07553 & $\ldots$ & $\ldots$ & $\ldots$ & $14.09 \pm 0.08$ & $13.59 \pm 0.09$ & $13.44 \pm 0.20$ & $\cdots$ & $\ldots$ & $\ldots$ & $\ldots$ \\
\hline IC 0792 & $\cdots$ & $14.80 \pm 0.13$ & $\cdots$ & $12.24 \pm 0.04$ & $11.59 \pm 0.05$ & $11.26 \pm 0.05$ & $\cdots$ & $\cdots$ & $\ldots$ & $\cdots$ \\
\hline IC 3365 & $\ldots$ & $14.36 \pm 0.15$ & $\cdots$ & $\ldots$ & $\ldots$ & $\ldots$ & $\cdots$ & $\ldots$ & $\ldots$ & $\ldots$ \\
\hline NGC 4425 & $13.12 \pm 0.09$ & $12.73 \pm 0.08$ & $11.83 \pm 0.08$ & $9.88 \pm 0.01$ & $9.26 \pm 0.02$ & $9.01 \pm 0.03$ & $<0.03$ & $<0.05$ & $<0.06$ & $<0.17$ \\
\hline NGC 4431 & $14.17 \pm 0.09$ & $13.74 \pm 0.05$ & $12.89 \pm 0.07$ & $11.22 \pm 0.03$ & $10.54 \pm 0.04$ & $10.31 \pm 0.05$ & $\ldots$ & $\ldots$ & $\ldots$ & $\ldots$ \\
\hline NGC 4435 & $12.23 \pm 0.05$ & $11.74 \pm 0.05$ & $10.80 \pm 0.05$ & $8.42 \pm 0.01$ & $7.16 \pm 0.01$ & $7.30 \pm 0.02$ & $0.12 \pm 0.03$ & $<0.30$ & $1.85 \pm 0.28$ & $<7.50$ \\
\hline NGC 4436 & $14.20 \pm 0.09$ & $13.91 \pm 0.05$ & $12.98 \pm 0.07$ & $11.52 \pm 0.03$ & $10.86 \pm 0.03$ & $10.75 \pm 0.04$ & $\ldots$ & $\ldots$ & $\ldots$ & $\ldots$ \\
\hline NGC 4438 & $11.37 \pm 0.11$ & $11.02 \pm 0.07$ & $10.17 \pm 0.07$ & $8.25 \pm 0.02$ & $7.56 \pm 0.02$ & $7.26 \pm 0.02$ & $0.17 \pm 0.02$ & $<0.15$ & $4.28 \pm 0.43$ & $12.10 \pm 1.21$ \\
\hline
\end{tabular}


Table 4-Continued

\begin{tabular}{|c|c|c|c|c|c|c|c|c|c|c|}
\hline \multirow[b]{2}{*}{$\begin{array}{c}\text { Object Name } \\
\text { (1) }\end{array}$} & \multicolumn{3}{|c|}{ Optical Photometry } & \multicolumn{3}{|c|}{ Near-Infrared Photometry } & \multicolumn{4}{|c|}{ IRAS fluxes } \\
\hline & $\begin{array}{c}U \\
(\mathrm{mag}) \\
(2)\end{array}$ & $\begin{array}{c}B \\
(\mathrm{mag}) \\
(3)\end{array}$ & $\begin{array}{c}V \\
(\mathrm{mag}) \\
(4)\end{array}$ & $\begin{array}{c}J \\
(\mathrm{mag}) \\
(5)\end{array}$ & $\begin{array}{c}H \\
(\mathrm{mag}) \\
(6)\end{array}$ & $\begin{array}{c}K \\
(\mathrm{mag}) \\
(7)\end{array}$ & $\begin{array}{c}12 \mu \mathrm{m} \\
(\mathrm{Jy}) \\
(8)\end{array}$ & $\begin{array}{c}25 \mu \mathrm{m} \\
(\mathrm{Jy}) \\
(9) \\
\end{array}$ & $\begin{array}{c}60 \mu \mathrm{m} \\
(\mathrm{Jy}) \\
(10) \\
\end{array}$ & $\begin{array}{c}100 \mu \mathrm{m} \\
(\mathrm{Jy}) \\
(11) \\
\end{array}$ \\
\hline NGC 4440 & $\ldots$ & $12.70 \pm 0.08$ & $11.72 \pm 0.09$ & $9.82 \pm 0.01$ & $9.14 \pm 0.02$ & $8.91 \pm 0.02$ & $\ldots$ & $\ldots$ & $\ldots$ & $\ldots$ \\
\hline IC 0794 & $\ldots$ & $14.07 \pm 0.07$ & $\ldots$ & $11.69 \pm 0.07$ & $10.70 \pm 0.06$ & $10.76 \pm 0.12$ & $\ldots$ & $\ldots$ & $\ldots$ & $\ldots$ \\
\hline IC 3381 & $\ldots$ & $14.42 \pm 0.12$ & $\ldots$ & $11.67 \pm 0.05$ & $10.97 \pm 0.06$ & $11.05 \pm 0.12$ & $\ldots$ & $\ldots$ & $\ldots$ & $\ldots$ \\
\hline NGC 4450 & $\ldots$ & $10.90 \pm 0.08$ & $10.08 \pm 0.08$ & $7.94 \pm 0.02$ & $7.22 \pm 0.02$ & $7.05 \pm 0.03$ & $0.11 \pm 0.02$ & $<0.13$ & $1.34 \pm 0.09$ & $6.95 \pm 0.35$ \\
\hline UGC 07604 & $\ldots$ & $\ldots$ & $\ldots$ & $14.13 \pm 0.10$ & $13.02 \pm 0.09$ & $12.90 \pm 0.13$ & $\ldots$ & $\ldots$ & $\ldots$ & $\cdots$ \\
\hline IC 3393 & $\ldots$ & $14.85 \pm 0.08$ & $\ldots$ & $12.55 \pm 0.04$ & $11.85 \pm 0.05$ & $11.75 \pm 0.07$ & $\ldots$ & $\ldots$ & $\ldots$ & $\ldots$ \\
\hline NGC 4452 & $13.27 \pm 0.07$ & $12.87 \pm 0.06$ & $11.98 \pm 0.07$ & $9.95 \pm 0.02$ & $9.21 \pm 0.02$ & $9.07 \pm 0.03$ & $<0.03$ & $<0.04$ & $<0.03$ & $<0.10$ \\
\hline NGC 4454 & $12.95 \pm 0.16$ & $12.72 \pm 0.15$ & $11.87 \pm 0.15$ & $10.01 \pm 0.02$ & $9.33 \pm 0.02$ & $9.05 \pm 0.04$ & $<0.11$ & $<0.23$ & $0.30 \pm 0.07$ & $1.57 \pm 0.16$ \\
\hline NGC 4458 & $13.27 \pm 0.04$ & $12.93 \pm 0.04$ & $12.07 \pm 0.04$ & $10.22 \pm 0.01$ & $9.57 \pm 0.02$ & $9.31 \pm 0.02$ & $\ldots$ & $<0.06$ & $<0.03$ & $<0.14$ \\
\hline NGC 4461 & $12.56 \pm 0.05$ & $12.09 \pm 0.05$ & $11.19 \pm 0.05$ & $8.97 \pm 0.01$ & $8.24 \pm 0.01$ & $8.01 \pm 0.01$ & $\ldots$ & $<0.04$ & $<0.02$ & $<0.09$ \\
\hline IC 0796 & $\ldots$ & $14.07 \pm 0.13$ & $\ldots$ & $11.25 \pm 0.02$ & $10.60 \pm 0.03$ & $10.34 \pm 0.03$ & $<0.12$ & $<0.15$ & $0.59 \pm 0.05$ & $1.46 \pm 0.16$ \\
\hline IC 3418 & $\ldots$ & $\ldots$ & $\ldots$ & $\ldots$ & $\ldots$ & $\ldots$ & $\ldots$ & $\ldots$ & $\ldots$ & $\ldots$ \\
\hline NGC 4473 & $11.60 \pm 0.04$ & $11.16 \pm 0.04$ & $10.20 \pm 0.04$ & $8.04 \pm 0.02$ & $7.40 \pm 0.02$ & $7.16 \pm 0.02$ & $\ldots$ & $\ldots$ & $<0.06$ & $<0.11$ \\
\hline NGC 4476 & $13.28 \pm 0.04$ & $13.01 \pm 0.03$ & $12.19 \pm 0.03$ & $10.38 \pm 0.01$ & $9.72 \pm 0.02$ & $9.47 \pm 0.02$ & $<0.13$ & $<0.13$ & $0.55 \pm 0.06$ & $1.74 \pm 0.21$ \\
\hline NGC 4477 & $11.98 \pm 0.05$ & $11.38 \pm 0.05$ & $10.42 \pm 0.05$ & $8.28 \pm 0.01$ & $7.60 \pm 0.01$ & $7.35 \pm 0.01$ & $<0.16$ & $<0.19$ & $0.54 \pm 0.06$ & $1.18 \pm 0.19$ \\
\hline NGC 4478 & $12.82 \pm 0.03$ & $12.36 \pm 0.03$ & $11.45 \pm 0.03$ & $9.25 \pm 0.01$ & $8.56 \pm 0.01$ & $8.35 \pm 0.01$ & $<0.04$ & $<0.06$ & $<0.04$ & $<0.08$ \\
\hline NGC 4479 & $13.73 \pm 0.07$ & $13.40 \pm 0.06$ & $12.44 \pm 0.06$ & $10.64 \pm 0.02$ & $10.02 \pm 0.03$ & $9.77 \pm 0.03$ & $<0.03$ & $<0.06$ & $<0.03$ & $<0.15$ \\
\hline NGC 4485 & $12.10 \pm 0.07$ & $12.32 \pm 0.05$ & $11.93 \pm 0.07$ & $11.01 \pm 0.03$ & $10.44 \pm 0.04$ & $10.58 \pm 0.03$ & $\ldots$ & $\ldots$ & $\ldots$ & $\ldots$ \\
\hline NGC 4490 & $10.03 \pm 0.06$ & $10.22 \pm 0.06$ & $9.79 \pm 0.06$ & $8.21 \pm 0.02$ & $7.64 \pm 0.02$ & $7.35 \pm 0.03$ & $1.85 \pm 0.03$ & $4.95 \pm 0.05$ & $47.80 \pm 0.06$ & $85.90 \pm 0.19$ \\
\hline MESSIER 087 & $10.16 \pm 0.04$ & $9.59 \pm 0.04$ & $8.63 \pm 0.04$ & $6.72 \pm 0.02$ & $6.07 \pm 0.02$ & $5.81 \pm 0.02$ & $0.23 \pm 0.04$ & $<0.24$ & $0.39 \pm 0.05$ & $<1.02$ \\
\hline NGC 4491 & $\ldots$ & $13.50 \pm 0.20$ & $12.55 \pm 0.22$ & $10.69 \pm 0.03$ & $10.06 \pm 0.04$ & $9.88 \pm 0.05$ & $<0.12$ & $0.42 \pm 0.02$ & $2.77 \pm 0.17$ & $3.49 \pm 0.31$ \\
\hline CGCG 014-054 & $\ldots$ & $14.80 \pm 0.20$ & $\ldots$ & $\ldots$ & $\ldots$ & $\ldots$ & $\ldots$ & $\ldots$ & $\ldots$ & $\ldots$ \\
\hline IC 3446 & $\ldots$ & $15.63 \pm 0.11$ & $\ldots$ & $14.55 \pm 0.12$ & $14.13 \pm 0.19$ & $14.05 \pm 0.31$ & $\ldots$ & $\ldots$ & $\ldots$ & $\ldots$ \\
\hline NGC 4497 & $13.44 \pm 0.12$ & $13.19 \pm 0.10$ & $12.47 \pm 0.11$ & $10.61 \pm 0.03$ & $9.94 \pm 0.04$ & $9.74 \pm 0.05$ & $<0.03$ & $<0.05$ & $<0.02$ & $<0.35$ \\
\hline IC 3457 & $\ldots$ & $14.69 \pm 0.08$ & $\ldots$ & $\ldots$ & $\ldots$ & $\ldots$ & $\ldots$ & $\ldots$ & $\ldots$ & $\ldots$ \\
\hline IC 3459 & $\ldots$ & $14.83 \pm 0.08$ & $\ldots$ & $13.40 \pm 0.08$ & $13.19 \pm 0.09$ & $12.63 \pm 0.14$ & $\ldots$ & $\ldots$ & $\ldots$ & $\ldots$ \\
\hline NGC 4503 & $12.66 \pm 0.08$ & $12.05 \pm 0.08$ & $11.07 \pm 0.08$ & $8.82 \pm 0.01$ & $8.14 \pm 0.02$ & $7.89 \pm 0.02$ & $<0.03$ & $<0.03$ & $<0.04$ & $<0.19$ \\
\hline NGC 4506 & $\ldots$ & $13.63 \pm 0.13$ & $\ldots$ & $11.04 \pm 0.03$ & $10.52 \pm 0.04$ & $10.26 \pm 0.05$ & $\ldots$ & $\ldots$ & $\ldots$ & $\ldots$ \\
\hline IC 3467 & $\ldots$ & $15.28 \pm 0.13$ & $\ldots$ & $13.97 \pm 0.09$ & $13.50 \pm 0.13$ & $13.55 \pm 0.25$ & $\ldots$ & $\ldots$ & $\ldots$ & $\ldots$ \\
\hline UGC 07710 & $\ldots$ & $\ldots$ & $\ldots$ & $\ldots$ & $\ldots$ & $\ldots$ & $\ldots$ & $\ldots$ & $\ldots$ & $\ldots$ \\
\hline NGC 4528 & $13.39 \pm 0.09$ & $12.97 \pm 0.09$ & $12.06 \pm 0.09$ & $9.88 \pm 0.01$ & $9.14 \pm 0.01$ & $8.97 \pm 0.02$ & $<0.04$ & $<0.06$ & $<0.04$ & $<0.15$ \\
\hline NGC 4531 & $\ldots$ & $12.42 \pm 0.15$ & $\ldots$ & $9.59 \pm 0.02$ & $8.99 \pm 0.02$ & $8.76 \pm 0.03$ & $<0.14$ & $<0.20$ & $0.35 \pm 0.05$ & $1.75 \pm 0.24$ \\
\hline
\end{tabular}


Table 4-Continued

\begin{tabular}{|c|c|c|c|c|c|c|c|c|c|c|}
\hline \multirow{2}{*}{$\begin{array}{c}\text { Object Name } \\
\text { (1) }\end{array}$} & \multicolumn{3}{|c|}{ Optical Photometry } & \multicolumn{3}{|c|}{ Near-Infrared Photometry } & \multicolumn{4}{|c|}{ IRAS fluxes } \\
\hline & $\begin{array}{c}U \\
(\mathrm{mag}) \\
(2)\end{array}$ & $\begin{array}{c}B \\
(\mathrm{mag}) \\
(3)\end{array}$ & $\begin{array}{c}V \\
(\mathrm{mag}) \\
(4)\end{array}$ & $\begin{array}{c}J \\
(\mathrm{mag}) \\
(5)\end{array}$ & $\begin{array}{c}H \\
(\mathrm{mag}) \\
(6)\end{array}$ & $\begin{array}{c}K \\
(\mathrm{mag}) \\
(7)\end{array}$ & $\begin{array}{c}12 \mu \mathrm{m} \\
(\mathrm{Jy}) \\
(8)\end{array}$ & $\begin{array}{c}25 \mu \mathrm{m} \\
(\mathrm{Jy}) \\
(9)\end{array}$ & $\begin{array}{c}60 \mu \mathrm{m} \\
(\mathrm{Jy}) \\
(10)\end{array}$ & $\begin{array}{c}100 \mu \mathrm{m} \\
(\mathrm{Jy}) \\
(11)\end{array}$ \\
\hline NGC 4536 & $11.14 \pm 0.09$ & $11.16 \pm 0.08$ & $10.55 \pm 0.08$ & $8.50 \pm 0.02$ & $7.88 \pm 0.03$ & $7.52 \pm 0.03$ & $1.60 \pm 0.04$ & $3.90 \pm 0.07$ & $28.70 \pm 0.04$ & $44.60 \pm 0.21$ \\
\hline UGC 07748 & $\ldots$ & $15.30 \pm 0.20$ & $\ldots$ & $\ldots$ & $\ldots$ & $\ldots$ & $\ldots$ & $\ldots$ & $\ldots$ & $\cdots$ \\
\hline NGC 4546 & $11.89 \pm 0.13$ & $11.30 \pm 0.13$ & $10.32 \pm 0.13$ & $8.31 \pm 0.01$ & $7.64 \pm 0.01$ & $7.39 \pm 0.02$ & $<0.03$ & $0.14 \pm 0.07$ & $0.26 \pm 0.04$ & $0.89 \pm 0.22$ \\
\hline NGC 4550 & $12.97 \pm 0.05$ & $12.56 \pm 0.05$ & $11.68 \pm 0.05$ & $9.55 \pm 0.01$ & $8.87 \pm 0.01$ & $8.69 \pm 0.02$ & $<0.03$ & $<0.07$ & $0.14 \pm 0.03$ & $0.25 \pm 0.09$ \\
\hline NGC 4551 & $13.49 \pm 0.05$ & $12.97 \pm 0.05$ & $12.02 \pm 0.05$ & $9.78 \pm 0.01$ & $9.11 \pm 0.01$ & $8.87 \pm 0.02$ & $<0.03$ & $<0.07$ & $<0.04$ & $<0.15$ \\
\hline MESSIER 089 & $11.29 \pm 0.05$ & $10.73 \pm 0.05$ & $9.75 \pm 0.05$ & $7.62 \pm 0.02$ & $6.94 \pm 0.02$ & $6.73 \pm 0.02$ & $0.13 \pm 0.06$ & $<0.05$ & $0.16 \pm 0.05$ & $0.53 \pm 0.06$ \\
\hline NGC 4559 & $\cdots$ & $10.46 \pm 0.11$ & $10.01 \pm 0.12$ & $8.41 \pm 0.03$ & $7.83 \pm 0.05$ & $7.58 \pm 0.05$ & $0.49 \pm 0.12$ & $0.73 \pm 0.18$ & $9.69 \pm 2.42$ & $27.10 \pm 6.76$ \\
\hline PGC 42042 & $\ldots$ & $\ldots$ & $\ldots$ & $11.45 \pm 0.04$ & $10.82 \pm 0.05$ & $10.53 \pm 0.06$ & $<0.12$ & $0.35 \pm 0.03$ & $1.80 \pm 0.11$ & $3.01 \pm 0.30$ \\
\hline NGC 4564 & $12.51 \pm 0.05$ & $12.05 \pm 0.05$ & $11.12 \pm 0.05$ & $8.87 \pm 0.01$ & $8.09 \pm 0.01$ & $7.94 \pm 0.02$ & $<0.04$ & $<0.05$ & $<0.06$ & $<0.19$ \\
\hline NGC 4567 & $12.25 \pm 0.11$ & $12.06 \pm 0.07$ & $11.31 \pm 0.09$ & $9.19 \pm 0.02$ & $8.57 \pm 0.03$ & $8.30 \pm 0.03$ & $\ldots$ & $\ldots$ & $\ldots$ & $\ldots$ \\
\hline IC 3583 & $\ldots$ & $13.31 \pm 0.15$ & $\ldots$ & $\ldots$ & $\ldots$ & $\ldots$ & $<0.10$ & $<0.13$ & $0.49 \pm 0.06$ & $<3.87$ \\
\hline IC 3587 & $\cdots$ & $\cdots$ & $\cdots$ & $12.57 \pm 0.04$ & $11.68 \pm 0.04$ & $11.25 \pm 0.04$ & $<0.08$ & $<0.15$ & $0.48 \pm 0.05$ & $1.44 \pm 0.16$ \\
\hline NGC 4569 & $10.56 \pm 0.09$ & $10.26 \pm 0.08$ & $9.54 \pm 0.08$ & $7.50 \pm 0.02$ & $6.79 \pm 0.02$ & $6.58 \pm 0.03$ & $0.75 \pm 0.19$ & $1.28 \pm 0.32$ & $9.19 \pm 2.30$ & $27.30 \pm 6.83$ \\
\hline NGC $4559 \mathrm{~A}$ & $\ldots$ & $15.10 \pm 0.18$ & $\ldots$ & $12.20 \pm 0.03$ & $11.51 \pm 0.04$ & $11.15 \pm 0.04$ & $<0.17$ & $<0.22$ & $0.69 \pm 0.14$ & $1.58 \pm 0.29$ \\
\hline IC 3598 & $\ldots$ & $14.74 \pm 0.18$ & $\ldots$ & $11.68 \pm 0.02$ & $10.97 \pm 0.03$ & $10.64 \pm 0.03$ & $\ldots$ & $\ldots$ & $\ldots$ & $\ldots$ \\
\hline MESSIER 058 & $10.80 \pm 0.08$ & $10.48 \pm 0.08$ & $9.66 \pm 0.08$ & $7.37 \pm 0.02$ & $6.71 \pm 0.03$ & $6.49 \pm 0.03$ & $1.11 \pm 0.05$ & $0.76 \pm 0.06$ & $5.85 \pm 0.06$ & $20.90 \pm 0.27$ \\
\hline NGC 4584 & $\ldots$ & $13.77 \pm 0.15$ & $\ldots$ & $11.15 \pm 0.03$ & $10.56 \pm 0.04$ & $10.46 \pm 0.06$ & $\ldots$ & $\ldots$ & $\ldots$ & $\ldots$ \\
\hline NGC 4594 & $9.51 \pm 0.08$ & $8.98 \pm 0.06$ & $8.00 \pm 0.06$ & $5.89 \pm 0.01$ & $5.21 \pm 0.01$ & $4.96 \pm 0.02$ & $0.74 \pm 0.18$ & $0.50 \pm 0.12$ & $4.26 \pm 1.06$ & $22.90 \pm 5.71$ \\
\hline NGC 4612 & $\ldots$ & $11.90 \pm 0.20$ & .. & $9.42 \pm 0.01$ & $8.82 \pm 0.02$ & $8.56 \pm 0.03$ & $<0.03$ & $<0.04$ & $<0.04$ & $<0.09$ \\
\hline NGC 4618 & $11.03 \pm 0.04$ & $11.22 \pm 0.04$ & $10.78 \pm 0.04$ & $9.51 \pm 0.03$ & $8.79 \pm 0.04$ & $8.66 \pm 0.06$ & $0.40 \pm 0.03$ & $0.45 \pm 0.04$ & $4.92 \pm 0.04$ & $13.10 \pm 0.17$ \\
\hline NGC 4625 & $12.74 \pm 0.06$ & $12.92 \pm 0.04$ & $12.35 \pm 0.04$ & $10.65 \pm 0.03$ & $9.93 \pm 0.04$ & $9.74 \pm 0.05$ & $0.12 \pm 0.03$ & $0.19 \pm 0.02$ & $1.20 \pm 0.13$ & $3.58 \pm 0.25$ \\
\hline NGC 4627 & $13.16 \pm 0.15$ & $13.06 \pm 0.13$ & $12.43 \pm 0.13$ & $\ldots$ & $\ldots$ & $\ldots$ & $\ldots$ & $\ldots$ & $\ldots$ & $\ldots$ \\
\hline NGC 4631 & $\ldots$ & $9.75 \pm 0.16$ & $9.19 \pm 0.16$ & $7.53 \pm 0.02$ & $6.83 \pm 0.03$ & $6.47 \pm 0.02$ & $5.48 \pm 0.82$ & $9.65 \pm 1.45$ & $82.90 \pm 12.40$ & $209.00 \pm 31.30$ \\
\hline NGC 4623 & $\ldots$ & $13.24 \pm 0.15$ & $\ldots$ & $10.31 \pm 0.02$ & $9.64 \pm 0.02$ & $9.47 \pm 0.04$ & $<0.05$ & $<0.05$ & $<0.02$ & $<0.12$ \\
\hline NGC 4656 & $\cdots$ & $10.96 \pm 0.09$ & $10.52 \pm 0.10$ & $\ldots$ & $\cdots$ & $\cdots$ & $0.10 \pm 0.01$ & $0.30 \pm 0.04$ & $5.90 \pm 0.89$ & $11.50 \pm 1.72$ \\
\hline NGC 4665 & $\cdots$ & $11.50 \pm 0.11$ & $10.50 \pm 0.20$ & $8.34 \pm 0.01$ & $7.68 \pm 0.02$ & $7.43 \pm 0.03$ & $<0.02$ & $<0.05$ & $<0.03$ & $<0.05$ \\
\hline NGC 4691 & $11.59 \pm 0.14$ & $11.66 \pm 0.13$ & $11.08 \pm 0.13$ & $9.39 \pm 0.01$ & $8.75 \pm 0.02$ & $8.54 \pm 0.03$ & $0.83 \pm 0.04$ & $3.09 \pm 0.10$ & $14.40 \pm 0.05$ & $21.60 \pm 0.12$ \\
\hline DDO 149 & $\ldots$ & $\ldots$ & $\ldots$ & $\ldots$ & $\ldots$ & $\ldots$ & $\ldots$ & $\ldots$ & $\ldots$ & $\ldots$ \\
\hline UGC 07982 & $\ldots$ & $14.03 \pm 0.15$ & $\ldots$ & $11.28 \pm 0.02$ & $10.57 \pm 0.02$ & $10.17 \pm 0.05$ & $<0.12$ & $<0.28$ & $0.25 \pm 0.04$ & $1.08 \pm 0.16$ \\
\hline UGC 07991 & $\ldots$ & $14.79 \pm 0.18$ & $\ldots$ & $12.61 \pm 0.03$ & $11.64 \pm 0.05$ & $11.61 \pm 0.09$ & $<0.10$ & $<0.19$ & $0.24 \pm 0.04$ & $<0.58$ \\
\hline NGC 4736 & $9.15 \pm 0.13$ & $8.99 \pm 0.13$ & $8.24 \pm 0.13$ & $6.03 \pm 0.01$ & $5.35 \pm 0.02$ & $5.11 \pm 0.02$ & $4.77 \pm 0.72$ & $6.83 \pm 1.02$ & $62.40 \pm 9.36$ & $135.00 \pm 20.30$ \\
\hline NGC 4753 & $11.26 \pm 0.10$ & $10.85 \pm 0.10$ & $9.95 \pm 0.10$ & $7.65 \pm 0.02$ & $6.97 \pm 0.02$ & $6.72 \pm 0.03$ & $0.31 \pm 0.04$ & $0.29 \pm 0.06$ & $2.44 \pm 0.15$ & $9.01 \pm 0.63$ \\
\hline
\end{tabular}


Table 4-Continued

\begin{tabular}{|c|c|c|c|c|c|c|c|c|c|c|}
\hline \multirow{2}{*}{$\begin{array}{l}\text { Object Name } \\
\text { (1) }\end{array}$} & \multicolumn{3}{|c|}{ Optical Photometry } & \multicolumn{3}{|c|}{ Near-Infrared Photometry } & \multicolumn{4}{|c|}{ IRAS fluxes } \\
\hline & $\begin{array}{c}U \\
(\mathrm{mag}) \\
(2)\end{array}$ & $\begin{array}{c}B \\
(\mathrm{mag}) \\
(3)\end{array}$ & $\begin{array}{c}V \\
(\mathrm{mag}) \\
(4)\end{array}$ & $\begin{array}{c}J \\
(\mathrm{mag}) \\
(5)\end{array}$ & $\begin{array}{c}H \\
(\mathrm{mag}) \\
(6)\end{array}$ & $\begin{array}{c}K \\
(\mathrm{mag}) \\
(7)\end{array}$ & $\begin{array}{c}12 \mu \mathrm{m} \\
(\mathrm{Jy}) \\
(8)\end{array}$ & $\begin{array}{c}25 \mu \mathrm{m} \\
(\mathrm{Jy}) \\
(9)\end{array}$ & $\begin{array}{c}60 \mu \mathrm{m} \\
(\mathrm{Jy}) \\
(10)\end{array}$ & $\begin{array}{l}100 \mu \mathrm{m} \\
(\mathrm{Jy}) \\
(11)\end{array}$ \\
\hline UGC 08013 & $\cdots$ & $15.20 \pm 0.20$ & $\cdots$ & $12.92 \pm 0.05$ & $12.18 \pm 0.07$ & $12.16 \pm 0.10$ & $\cdots$ & $\cdots$ & $\cdots$ & $\cdots$ \\
\hline NGC 4771 & $\cdots$ & $12.92 \pm 0.15$ & $\cdots$ & $9.96 \pm 0.01$ & $9.28 \pm 0.01$ & $9.01 \pm 0.02$ & $0.13 \pm 0.03$ & $<0.15$ & $0.89 \pm 0.06$ & $3.88 \pm 0.23$ \\
\hline NGC 4772 & $12.29 \pm 0.15$ & $11.96 \pm 0.15$ & $11.04 \pm 0.15$ & $9.16 \pm 0.02$ & $8.53 \pm 0.02$ & $8.36 \pm 0.03$ & $\cdots$ & $\cdots$ & $\cdots$ & $\cdots$ \\
\hline DDO 154 & $13.67 \pm 0.19$ & $13.94 \pm 0.18$ & $13.61 \pm 0.19$ & $14.79 \pm 0.14$ & $14.33 \pm 0.22$ & $15.35 \pm 1.05$ & $\cdots$ & $\ldots$ & $\ldots$ & $\ldots$ \\
\hline NGC 4787 & $\cdots$ & $15.44 \pm 0.18$ & $\cdots$ & $12.19 \pm 0.04$ & $11.70 \pm 0.05$ & $11.40 \pm 0.05$ & $\ldots$ & $\cdots$ & $\ldots$ & $\cdots$ \\
\hline NGC 4789 & $\cdots$ & $13.12 \pm 0.15$ & $12.10 \pm 0.15$ & $10.00 \pm 0.02$ & $9.52 \pm 0.03$ & $9.24 \pm 0.03$ & $<0.04$ & $<0.03$ & $<0.04$ & $0.27 \pm 0.05$ \\
\hline NGC 4809 & $\cdots$ & $14.20 \pm 0.20$ & $\cdots$ & $\cdots$ & $\cdots$ & $\cdots$ & $\cdots$ & $\cdots$ & $\cdots$ & $\cdots$ \\
\hline NGC 4797 & $14.71 \pm 0.20$ & $14.20 \pm 0.20$ & $13.17 \pm 0.20$ & $10.92 \pm 0.02$ & $10.22 \pm 0.03$ & $9.89 \pm 0.03$ & $\cdots$ & $\cdots$ & $\cdots$ & $\cdots$ \\
\hline NGC 4799 & $\cdots$ & $14.32 \pm 0.18$ & $\cdots$ & $10.83 \pm 0.02$ & $10.16 \pm 0.02$ & $9.89 \pm 0.03$ & $0.13 \pm 0.03$ & $<0.38$ & $1.15 \pm 0.09$ & $2.76 \pm 0.22$ \\
\hline NGC 4807 & $\ldots$ & $14.50 \pm 0.20$ & $13.50 \pm 0.20$ & $12.65 \pm 0.05$ & $12.57 \pm 0.14$ & $11.83 \pm 0.10$ & $<0.04$ & $0.04 \pm 0.03$ & $<0.04$ & $0.38 \pm 0.10$ \\
\hline NGC 4816 & $14.25 \pm 0.21$ & $13.80 \pm 0.20$ & $12.84 \pm 0.20$ & $10.69 \pm 0.02$ & $10.01 \pm 0.04$ & $9.71 \pm 0.04$ & $<0.03$ & $<0.04$ & $<0.03$ & $<0.12$ \\
\hline NGC 4819 & $14.49 \pm 0.40$ & $14.10 \pm 0.40$ & $13.17 \pm 0.40$ & $11.05 \pm 0.02$ & $10.29 \pm 0.03$ & $10.12 \pm 0.03$ & $\cdots$ & $\ldots$ & $\cdots$ & $\ldots$ \\
\hline NGC 4827 & $\ldots$ & $13.90 \pm 0.20$ & $12.91 \pm 0.23$ & $10.98 \pm 0.02$ & $10.31 \pm 0.02$ & $10.04 \pm 0.03$ & $\cdots$ & $\ldots$ & $\cdots$ & $\ldots$ \\
\hline MESSIER 064 & $\cdots$ & $9.36 \pm 0.10$ & $8.52 \pm 0.10$ & $6.27 \pm 0.02$ & $5.58 \pm 0.02$ & $5.33 \pm 0.02$ & $1.71 \pm 0.17$ & $2.00 \pm 0.20$ & $33.90 \pm 3.39$ & $77.40 \pm 7.74$ \\
\hline NGC 4839 & $13.56 \pm 0.10$ & $13.02 \pm 0.10$ & $12.06 \pm 0.10$ & $10.09 \pm 0.03$ & $9.33 \pm 0.03$ & $9.20 \pm 0.04$ & $<0.06$ & $<0.03$ & $<0.02$ & $<0.09$ \\
\hline IC 3949 & $15.31 \pm 0.31$ & $15.10 \pm 0.30$ & $14.26 \pm 0.30$ & $11.98 \pm 0.03$ & $11.35 \pm 0.04$ & $11.01 \pm 0.04$ & $<0.10$ & $<0.06$ & $0.28 \pm 0.04$ & $1.01 \pm 0.18$ \\
\hline NGC 4861 & $\cdots$ & $12.90 \pm 0.30$ & $12.32 \pm 0.31$ & $12.44 \pm 0.05$ & $12.16 \pm 0.09$ & $11.77 \pm 0.11$ & $\cdots$ & $\cdots$ & $\cdots$ & $\ldots$ \\
\hline IC 0842 & $14.85 \pm 0.16$ & $14.72 \pm 0.15$ & $13.93 \pm 0.15$ & $11.78 \pm 0.04$ & $11.19 \pm 0.06$ & $10.76 \pm 0.05$ & $<0.08$ & $<0.12$ & $0.23 \pm 0.04$ & $0.84 \pm 0.14$ \\
\hline UGC 08127 & $\ldots$ & $14.73 \pm 0.20$ & $\ldots$ & $\ldots$ & $\ldots$ & $\ldots$ & $\ldots$ & $\ldots$ & $\ldots$ & $\ldots$ \\
\hline NGC 4922 & $\ldots$ & $\ldots$ & $\ldots$ & $\ldots$ & $\ldots$ & $\ldots$ & $0.23 \pm 0.04$ & $1.49 \pm 0.04$ & $6.20 \pm 0.05$ & $7.30 \pm 0.11$ \\
\hline IC 0843 & $\cdots$ & $14.62 \pm 0.15$ & $\cdots$ & $11.27 \pm 0.02$ & $10.55 \pm 0.02$ & $10.23 \pm 0.02$ & $\cdots$ & $\cdots$ & $\cdots$ & $\cdots$ \\
\hline IC 4088 & $15.01 \pm 0.21$ & $14.70 \pm 0.20$ & $13.82 \pm 0.20$ & $11.70 \pm 0.02$ & $10.99 \pm 0.03$ & $10.72 \pm 0.04$ & $<0.07$ & $<0.20$ & $0.44 \pm 0.05$ & $1.21 \pm 0.15$ \\
\hline NGC 4952 & $\cdots$ & $13.39 \pm 0.15$ & $\cdots$ & $10.70 \pm 0.02$ & $9.99 \pm 0.02$ & $9.76 \pm 0.02$ & $<0.04$ & $<0.04$ & $<0.05$ & $<0.05$ \\
\hline UGC 08195 & $\cdots$ & $\ldots$ & $\cdots$ & $\cdots$ & $\cdots$ & $\ldots$ & $\ldots$ & $\ldots$ & $\ldots$ & $\ldots$ \\
\hline DDO 165 & $12.64 \pm 0.22$ & $12.80 \pm 0.20$ & $12.46 \pm 0.21$ & $\cdots$ & $\cdots$ & $\cdots$ & $\cdots$ & $\cdots$ & $\cdots$ & $\cdots$ \\
\hline NGC 5004 & $\ldots$ & $13.89 \pm 0.16$ & $\ldots$ & $10.88 \pm 0.02$ & $10.20 \pm 0.03$ & $9.87 \pm 0.04$ & $\ldots$ & $\ldots$ & $\cdots$ & $\ldots$ \\
\hline NGC $5004 \mathrm{C}$ & $\cdots$ & $14.60 \pm 0.20$ & $\cdots$ & $11.61 \pm 0.04$ & $10.88 \pm 0.04$ & $10.72 \pm 0.07$ & $<0.18$ & $0.22 \pm 0.03$ & $2.04 \pm 0.27$ & $4.05 \pm 0.28$ \\
\hline UGC 08313 & $\cdots$ & $14.73 \pm 0.18$ & $\cdots$ & $12.86 \pm 0.06$ & $12.78 \pm 0.16$ & $11.92 \pm 0.10$ & $\cdots$ & $\cdots$ & $\cdots$ & $\cdots$ \\
\hline UGCA 342 & $\ldots$ & $\ldots$ & $\ldots$ & $\ldots$ & $\cdots$ & $\ldots$ & $\cdots$ & $\ldots$ & $\cdots$ & $\ldots$ \\
\hline NGC 5055 & $\ldots$ & $9.31 \pm 0.10$ & $8.59 \pm 0.10$ & $6.57 \pm 0.02$ & $5.83 \pm 0.02$ & $5.61 \pm 0.02$ & $5.56 \pm 0.83$ & $7.00 \pm 1.05$ & $40.00 \pm 6.00$ & $158.00 \pm 23.70$ \\
\hline UGC 08340 & $\cdots$ & $14.17 \pm 0.18$ & $\ldots$ & $11.73 \pm 0.03$ & $11.12 \pm 0.04$ & $11.00 \pm 0.08$ & $\ldots$ & $\ldots$ & $\ldots$ & $\ldots$ \\
\hline IC 4218 & $\ldots$ & $14.60 \pm 0.20$ & $\ldots$ & $11.74 \pm 0.04$ & $10.95 \pm 0.03$ & $10.58 \pm 0.05$ & $\ldots$ & $\ldots$ & $\ldots$ & $\ldots$ \\
\hline
\end{tabular}


Table 4-Continued

\begin{tabular}{|c|c|c|c|c|c|c|c|c|c|c|}
\hline \multirow[b]{2}{*}{$\begin{array}{l}\text { Object Name } \\
\text { (1) }\end{array}$} & \multicolumn{3}{|c|}{ Optical Photometry } & \multicolumn{3}{|c|}{ Near-Infrared Photometry } & \multicolumn{4}{|c|}{ IRAS fluxes } \\
\hline & $\begin{array}{c}U \\
(\mathrm{mag}) \\
(2)\end{array}$ & $\begin{array}{c}B \\
(\mathrm{mag}) \\
(3)\end{array}$ & $\begin{array}{c}V \\
(\mathrm{mag}) \\
(4)\end{array}$ & $\begin{array}{c}J \\
(\mathrm{mag}) \\
(5)\end{array}$ & $\begin{array}{c}H \\
(\mathrm{mag}) \\
(6)\end{array}$ & $\begin{array}{c}K \\
(\mathrm{mag}) \\
(7)\end{array}$ & $\begin{array}{c}12 \mu \mathrm{m} \\
(\mathrm{Jy}) \\
(8)\end{array}$ & $\begin{array}{c}25 \mu \mathrm{m} \\
(\mathrm{Jy}) \\
(9)\end{array}$ & $\begin{array}{c}60 \mu \mathrm{m} \\
(\mathrm{Jy}) \\
(10)\end{array}$ & $\begin{array}{c}100 \mu \mathrm{m} \\
(\mathrm{Jy}) \\
(11)\end{array}$ \\
\hline UGC 08365 & $14.04 \pm 0.22$ & $14.29 \pm 0.19$ & $13.83 \pm 0.21$ & $\cdots$ & $\cdots$ & $\cdots$ & $\cdots$ & $\ldots$ & $\ldots$ & $\ldots$ \\
\hline IC 4229 & $\ldots$ & $14.16 \pm 0.18$ & $\ldots$ & $11.82 \pm 0.03$ & $11.22 \pm 0.04$ & $10.83 \pm 0.05$ & $<0.25$ & $<0.33$ & $1.16 \pm 0.13$ & $2.20 \pm 0.24$ \\
\hline Centaurus A & $\ldots$ & $7.84 \pm 0.06$ & $6.84 \pm 0.08$ & $4.98 \pm 0.02$ & & $\ldots$ & $23.00 \pm 3.45$ & $30.70 \pm 4.61$ & $218.00 \pm 32.60$ & $501.00 \pm 75.20$ \\
\hline NGC 5169 & $\ldots$ & $14.30 \pm 0.20$ & $\ldots$ & $11.99 \pm 0.05$ & $11.34 \pm 0.06$ & $11.08 \pm 0.08$ & $<0.08$ & $<0.10$ & $0.40 \pm 0.06$ & $1.19 \pm 0.20$ \\
\hline IC 4263 & $\ldots$ & $15.10 \pm 0.20$ & $\cdots$ & $\cdots$ & $\ldots$ & $\ldots$ & $\cdots$ & $\ldots$ & $\ldots$ & $\ldots$ \\
\hline MESSIER 051a & $\cdots$ & $8.96 \pm 0.06$ & $8.36 \pm 0.06$ & $6.40 \pm 0.02$ & $5.65 \pm 0.02$ & $5.50 \pm 0.03$ & $11.00 \pm 0.00$ & $15.00 \pm 0.00$ & $98.80 \pm 0.00$ & $280.00 \pm 0.00$ \\
\hline MESSIER 051b & $10.76 \pm 0.07$ & $10.45 \pm 0.07$ & $9.55 \pm 0.07$ & $7.21 \pm 0.02$ & $6.46 \pm 0.02$ & $6.25 \pm 0.03$ & $\ldots$ & $\ldots$ & $\ldots$ & $<0.51$ \\
\hline NGC 5231 & $\ldots$ & $14.25 \pm 0.18$ & $\ldots$ & $10.99 \pm 0.03$ & $10.31 \pm 0.03$ & $10.14 \pm 0.05$ & $<0.22$ & $<0.31$ & $0.63 \pm 0.06$ & $1.27 \pm 0.14$ \\
\hline ESO 444-G077 & $\ldots$ & $15.20 \pm 0.21$ & $\ldots$ & $\ldots$ & $\ldots$ & $\ldots$ & $\ldots$ & $\ldots$ & $\ldots$ & $\ldots$ \\
\hline MESSIER 083 & $8.23 \pm 0.04$ & $8.20 \pm 0.03$ & $7.54 \pm 0.04$ & $5.54 \pm 0.02$ & $4.87 \pm 0.02$ & $4.62 \pm 0.03$ & $26.30 \pm 3.94$ & $47.70 \pm 7.16$ & $266.00 \pm 39.90$ & $639.00 \pm 95.80$ \\
\hline ESO 444-G087 & $\ldots$ & $14.10 \pm 0.21$ & $\ldots$ & $11.13 \pm 0.02$ & $10.46 \pm 0.02$ & $10.31 \pm 0.04$ & $\ldots$ & $\ldots$ & $\ldots$ & $\ldots$ \\
\hline NGC 5253 & $10.63 \pm 0.12$ & $10.87 \pm 0.12$ & $10.44 \pm 0.12$ & $9.06 \pm 0.02$ & $8.48 \pm 0.03$ & $8.25 \pm 0.04$ & $2.61 \pm 0.13$ & $12.00 \pm 0.13$ & $30.50 \pm 1.22$ & $29.40 \pm 1.76$ \\
\hline UGC 08650 & $\ldots$ & $14.65 \pm 0.18$ & $\ldots$ & $11.34 \pm 0.03$ & $10.63 \pm 0.03$ & $10.42 \pm 0.05$ & $\ldots$ & $\ldots$ & $\ldots$ & $\ldots$ \\
\hline UGC 08787 & $\ldots$ & $14.60 \pm 0.20$ & $\ldots$ & $12.08 \pm 0.04$ & $11.41 \pm 0.04$ & $11.08 \pm 0.06$ & $<0.12$ & $<0.16$ & $0.61 \pm 0.07$ & $1.55 \pm 0.17$ \\
\hline IC 0952 & $\ldots$ & $14.77 \pm 0.18$ & $\ldots$ & $11.92 \pm 0.04$ & $11.25 \pm 0.04$ & $11.06 \pm 0.07$ & $<0.10$ & $<0.25$ & $0.31 \pm 0.05$ & $1.24 \pm 0.15$ \\
\hline UGC 08816 & $\ldots$ & $14.98 \pm 0.18$ & $\ldots$ & $14.07 \pm 0.13$ & $13.50 \pm 0.15$ & $12.82 \pm 0.17$ & $\ldots$ & $\ldots$ & $\ldots$ & $\ldots$ \\
\hline NGC 5398 & $\cdots$ & $12.78 \pm 0.17$ & $\cdots$ & $11.10 \pm 0.04$ & $10.87 \pm 0.07$ & $10.34 \pm 0.07$ & $<0.11$ & $0.27 \pm 0.01$ & $1.56 \pm 0.08$ & $2.70 \pm 0.27$ \\
\hline MESSIER 101 & $\ldots$ & $8.31 \pm 0.09$ & $7.86 \pm 0.10$ & $6.52 \pm 0.03$ & $5.80 \pm 0.04$ & $5.51 \pm 0.05$ & $6.20 \pm 0.93$ & $11.80 \pm 1.77$ & $88.00 \pm 13.20$ & $253.00 \pm 37.90$ \\
\hline ESO $446-G 002$ & $\ldots$ & $14.69 \pm 0.21$ & $\ldots$ & $10.89 \pm 0.03$ & $10.06 \pm 0.03$ & $9.71 \pm 0.04$ & $0.19 \pm 0.03$ & $0.16 \pm 0.04$ & $1.69 \pm 0.08$ & $4.96 \pm 0.30$ \\
\hline UGC 08986 & $\ldots$ & $14.10 \pm 0.30$ & $\ldots$ & $12.23 \pm 0.09$ & $11.92 \pm 0.13$ & $11.35 \pm 0.15$ & $\ldots$ & $\ldots$ & $\ldots$ & $\ldots$ \\
\hline NGC 5474 & $\ldots$ & $11.28 \pm 0.15$ & $10.79 \pm 0.16$ & $10.23 \pm 0.02$ & $9.56 \pm 0.03$ & $9.48 \pm 0.05$ & $<0.09$ & $0.08 \pm 0.02$ & $1.33 \pm 0.07$ & $4.80 \pm 0.24$ \\
\hline NGC 5477 & $13.94 \pm 0.15$ & $14.36 \pm 0.14$ & $14.01 \pm 0.15$ & $\ldots$ & $\ldots$ & $\ldots$ & $<0.05$ & $<0.09$ & $0.29 \pm 0.03$ & $0.50 \pm 0.13$ \\
\hline UGC 09120 & $\ldots$ & $14.29 \pm 0.18$ & $\ldots$ & $12.48 \pm 0.06$ & $11.70 \pm 0.07$ & $11.77 \pm 0.13$ & $<0.08$ & $<0.15$ & $0.52 \pm 0.07$ & $1.33 \pm 0.15$ \\
\hline UGC 09140 & $\cdots$ & $\ldots$ & $\ldots$ & $13.51 \pm 0.06$ & $12.94 \pm 0.08$ & $12.48 \pm 0.10$ & $\ldots$ & $\ldots$ & $\ldots$ & $\ldots$ \\
\hline NGC 5560 & $13.38 \pm 0.20$ & $13.20 \pm 0.20$ & $12.37 \pm 0.20$ & $10.99 \pm 0.03$ & $10.21 \pm 0.03$ & $9.98 \pm 0.04$ & $0.23 \pm 0.04$ & $<0.31$ & $1.85 \pm 0.20$ & $<6.18$ \\
\hline NGC 5566 & $11.91 \pm 0.13$ & $11.46 \pm 0.13$ & $10.55 \pm 0.13$ & $8.35 \pm 0.02$ & $7.64 \pm 0.02$ & $7.39 \pm 0.03$ & $<0.25$ & $<0.28$ & $1.07 \pm 0.12$ & $5.61 \pm 0.67$ \\
\hline NGC 5569 & $\ldots$ & $13.90 \pm 0.30$ & $\ldots$ & $13.07 \pm 0.07$ & $13.01 \pm 0.08$ & $12.32 \pm 0.15$ & $\ldots$ & $\ldots$ & $\ldots$ & $\ldots$ \\
\hline NGC 5574 & $13.54 \pm 0.13$ & $13.23 \pm 0.13$ & $12.39 \pm 0.13$ & $10.45 \pm 0.02$ & $9.78 \pm 0.02$ & $9.54 \pm 0.03$ & $<0.04$ & $<0.04$ & $<0.04$ & $<0.50$ \\
\hline NGC 5576 & $12.25 \pm 0.13$ & $11.85 \pm 0.13$ & $10.96 \pm 0.13$ & $8.74 \pm 0.01$ & $7.97 \pm 0.01$ & $7.83 \pm 0.02$ & $0.07 \pm 0.04$ & $0.08 \pm 0.04$ & $0.09 \pm 0.03$ & $0.21 \pm 0.28$ \\
\hline
\end{tabular}


Table 4-Continued

\begin{tabular}{|c|c|c|c|c|c|c|c|c|c|c|}
\hline \multirow{2}{*}{$\begin{array}{c}\text { Object Name } \\
\text { (1) }\end{array}$} & \multicolumn{3}{|c|}{ Optical Photometry } & \multicolumn{3}{|c|}{ Near-Infrared Photometry } & \multicolumn{4}{|c|}{ IRAS fluxes } \\
\hline & $\begin{array}{c}U \\
(\mathrm{mag}) \\
(2)\end{array}$ & $\begin{array}{c}B \\
(\mathrm{mag}) \\
(3)\end{array}$ & $\begin{array}{c}V \\
(\mathrm{mag}) \\
(4)\end{array}$ & $\begin{array}{c}J \\
(\mathrm{mag}) \\
(5)\end{array}$ & $\begin{array}{c}H \\
(\mathrm{mag}) \\
(6)\end{array}$ & $\begin{array}{c}K \\
(\mathrm{mag}) \\
(7)\end{array}$ & $\begin{array}{c}12 \mu \mathrm{m} \\
(\mathrm{Jy}) \\
(8)\end{array}$ & $\begin{array}{c}25 \mu \mathrm{m} \\
(\mathrm{Jy}) \\
(9)\end{array}$ & $\begin{array}{c}60 \mu \mathrm{m} \\
(\mathrm{Jy}) \\
(10)\end{array}$ & $\begin{array}{c}100 \mu \mathrm{m} \\
(\mathrm{Jy}) \\
(11)\end{array}$ \\
\hline NGC 5577 & $\cdots$ & $13.05 \pm 0.19$ & $\cdots$ & $10.55 \pm 0.03$ & $9.98 \pm 0.04$ & $9.75 \pm 0.06$ & $<0.18$ & $<0.26$ & $0.58 \pm 0.05$ & $1.94 \pm 0.21$ \\
\hline UGC 09215 & $\ldots$ & $13.16 \pm 0.18$ & $\ldots$ & $11.54 \pm 0.06$ & $10.80 \pm 0.05$ & $10.54 \pm 0.09$ & $<0.14$ & $<0.17$ & $1.15 \pm 0.08$ & $2.57 \pm 0.23$ \\
\hline NGC 5619 & $\cdots$ & $13.40 \pm 0.20$ & $\cdots$ & $10.18 \pm 0.02$ & $9.47 \pm 0.02$ & $9.36 \pm 0.04$ & $<0.09$ & $<0.17$ & $0.46 \pm 0.04$ & $<2.83$ \\
\hline UGC 09277 & $\cdots$ & $14.99 \pm 0.18$ & $\cdots$ & $11.47 \pm 0.03$ & $10.73 \pm 0.03$ & $10.45 \pm 0.04$ & $\cdots$ & $\cdots$ & $\cdots$ & $\cdots$ \\
\hline UGC 09285 & $\ldots$ & $15.07 \pm 0.18$ & $\ldots$ & $13.69 \pm 0.07$ & $13.18 \pm 0.08$ & $13.40 \pm 0.22$ & $\ldots$ & $\ldots$ & $\ldots$ & $\ldots$ \\
\hline NGC 5646 & $\cdots$ & $14.99 \pm 0.19$ & $\cdots$ & $\cdots$ & $\cdots$ & $\cdots$ & $<0.12$ & $<0.09$ & $0.34 \pm 0.04$ & $1.02 \pm 0.13$ \\
\hline NGC 5636 & $\ldots$ & $13.70 \pm 0.20$ & $\ldots$ & $11.98 \pm 0.04$ & $11.32 \pm 0.04$ & $11.29 \pm 0.07$ & $\ldots$ & $\ldots$ & $\ldots$ & $\ldots$ \\
\hline NGC 5638 & $12.57 \pm 0.14$ & $12.14 \pm 0.14$ & $11.20 \pm 0.14$ & $9.16 \pm 0.01$ & $8.50 \pm 0.01$ & $8.25 \pm 0.02$ & $<0.03$ & $<0.04$ & $<0.03$ & $0.45 \pm 0.12$ \\
\hline UGC 09305 & $\ldots$ & $\ldots$ & $\ldots$ & $\ldots$ & $\ldots$ & $\ldots$ & $\ldots$ & $\ldots$ & $\ldots$ & $\ldots$ \\
\hline UGC 09310 & $\ldots$ & $14.50 \pm 0.20$ & $\ldots$ & $\ldots$ & $\ldots$ & $\ldots$ & $\ldots$ & $\ldots$ & $\ldots$ & $\ldots$ \\
\hline IC 1022 & $\cdots$ & $15.05 \pm 0.18$ & $\ldots$ & $12.78 \pm 0.07$ & $12.13 \pm 0.08$ & $11.70 \pm 0.11$ & $\cdots$ & $\ldots$ & $\ldots$ & $\ldots$ \\
\hline NGC 5656 & $\ldots$ & $12.73 \pm 0.18$ & $\ldots$ & $10.33 \pm 0.01$ & $9.64 \pm 0.02$ & $9.35 \pm 0.02$ & $0.27 \pm 0.03$ & $0.24 \pm 0.03$ & $2.61 \pm 0.13$ & $8.28 \pm 0.33$ \\
\hline UGC 09338 & $\ldots$ & $15.05 \pm 0.18$ & $\ldots$ & $12.72 \pm 0.05$ & $12.12 \pm 0.06$ & $11.76 \pm 0.09$ & $\ldots$ & $\ldots$ & $\ldots$ & $\ldots$ \\
\hline IC 1024 & $\ldots$ & $13.87 \pm 0.15$ & $\ldots$ & $11.07 \pm 0.03$ & $10.41 \pm 0.02$ & $10.07 \pm 0.03$ & $0.27 \pm 0.03$ & $0.41 \pm 0.03$ & $4.16 \pm 0.21$ & $7.20 \pm 0.36$ \\
\hline UGC 09380 & $\ldots$ & $14.20 \pm 0.30$ & $\ldots$ & $\ldots$ & $\ldots$ & $\ldots$ & $\ldots$ & $\ldots$ & $\ldots$ & $\ldots$ \\
\hline UGC 09382 & $\ldots$ & $15.33 \pm 0.20$ & $\ldots$ & $11.87 \pm 0.04$ & $11.17 \pm 0.04$ & $11.17 \pm 0.07$ & $\ldots$ & $\ldots$ & $\ldots$ & $\ldots$ \\
\hline UGC 09432 & $\ldots$ & $14.60 \pm 0.20$ & $\ldots$ & $\ldots$ & $\ldots$ & $\ldots$ & $\ldots$ & $\ldots$ & $\ldots$ & $\ldots$ \\
\hline NGC 5701 & $12.03 \pm 0.14$ & $11.76 \pm 0.14$ & $10.88 \pm 0.14$ & $9.06 \pm 0.02$ & $8.36 \pm 0.02$ & $8.14 \pm 0.03$ & $<0.08$ & $<0.14$ & $0.27 \pm 0.04$ & $1.36 \pm 0.15$ \\
\hline NGC 5705 & $\ldots$ & $13.30 \pm 0.30$ & $\ldots$ & $12.28 \pm 0.05$ & $11.65 \pm 0.07$ & $11.31 \pm 0.10$ & $<0.11$ & $<0.17$ & $0.52 \pm 0.04$ & $1.46 \pm 0.15$ \\
\hline NGC 5713 & $11.88 \pm 0.16$ & $11.84 \pm 0.15$ & $11.20 \pm 0.15$ & $9.22 \pm 0.02$ & $8.61 \pm 0.03$ & $8.33 \pm 0.04$ & $1.40 \pm 0.03$ & $2.87 \pm 0.04$ & $20.70 \pm 0.06$ & $36.30 \pm 0.10$ \\
\hline NGC 5727 & $\ldots$ & $14.20 \pm 0.30$ & $\ldots$ & $\ldots$ & $\ldots$ & $\ldots$ & $<0.08$ & $<0.09$ & $0.31 \pm 0.03$ & $0.78 \pm 0.11$ \\
\hline NGC 5719 & $\ldots$ & $13.10 \pm 0.20$ & $\ldots$ & $9.30 \pm 0.01$ & $8.54 \pm 0.01$ & $8.23 \pm 0.02$ & $0.52 \pm 0.03$ & $1.15 \pm 0.05$ & $8.05 \pm 0.04$ & $17.30 \pm 0.10$ \\
\hline UGC 09463 & $\ldots$ & $15.30 \pm 0.19$ & $\ldots$ & $11.90 \pm 0.03$ & $11.07 \pm 0.03$ & $10.82 \pm 0.05$ & $\ldots$ & $\ldots$ & $\ldots$ & $\ldots$ \\
\hline UGC 09479 & $\ldots$ & $14.77 \pm 0.18$ & $\ldots$ & $12.28 \pm 0.05$ & $11.45 \pm 0.05$ & $11.27 \pm 0.08$ & $\ldots$ & $\ldots$ & $\ldots$ & $\ldots$ \\
\hline UGC 09491 & $\ldots$ & $14.50 \pm 0.20$ & $\ldots$ & $11.57 \pm 0.06$ & $11.33 \pm 0.10$ & $10.77 \pm 0.10$ & $\cdots$ & $\ldots$ & $\ldots$ & $\ldots$ \\
\hline IC 1063 & $\ldots$ & $14.20 \pm 0.20$ & $\ldots$ & $11.91 \pm 0.04$ & $11.12 \pm 0.05$ & $10.83 \pm 0.06$ & $<0.11$ & $<0.17$ & $0.61 \pm 0.05$ & $1.58 \pm 0.16$ \\
\hline NGC 5770 & $13.49 \pm 0.13$ & $13.15 \pm 0.13$ & $12.26 \pm 0.13$ & $10.14 \pm 0.02$ & $9.46 \pm 0.02$ & $9.19 \pm 0.03$ & $<0.03$ & $<0.05$ & $<0.03$ & $<0.14$ \\
\hline IC 1071 & $\ldots$ & $14.22 \pm 0.15$ & $\ldots$ & $10.72 \pm 0.02$ & $10.01 \pm 0.02$ & $9.70 \pm 0.04$ & $\ldots$ & $\ldots$ & $\ldots$ & $\ldots$ \\
\hline UGC 09584 & $\ldots$ & $15.10 \pm 0.18$ & $\ldots$ & $12.69 \pm 0.05$ & $11.95 \pm 0.05$ & $11.71 \pm 0.09$ & $<0.11$ & $<0.14$ & $0.20 \pm 0.04$ & $0.63 \pm 0.16$ \\
\hline NGC 5832 & $\ldots$ & $12.90 \pm 0.30$ & $\ldots$ & $\ldots$ & $\ldots$ & $\ldots$ & $<0.07$ & $<0.06$ & $0.34 \pm 0.03$ & $1.53 \pm 0.14$ \\
\hline NGC 5806 & $\ldots$ & $12.40 \pm 0.20$ & $11.70 \pm 0.22$ & $9.42 \pm 0.01$ & $8.76 \pm 0.02$ & $8.45 \pm 0.02$ & $0.20 \pm 0.03$ & $<0.46$ & $2.69 \pm 0.19$ & $7.88 \pm 0.47$ \\
\hline NGC 5813 & $12.00 \pm 0.13$ & $11.45 \pm 0.13$ & $10.46 \pm 0.13$ & $8.34 \pm 0.02$ & $7.63 \pm 0.02$ & $7.41 \pm 0.03$ & $<0.03$ & $<0.03$ & $<0.02$ & $<0.10$ \\
\hline
\end{tabular}


Table 4-Continued

\begin{tabular}{|c|c|c|c|c|c|c|c|c|c|c|}
\hline \multirow{2}{*}{$\begin{array}{c}\text { Object Name } \\
\text { (1) }\end{array}$} & \multicolumn{3}{|c|}{ Optical Photometry } & \multicolumn{3}{|c|}{ Near-Infrared Photometry } & \multicolumn{4}{|c|}{ IRAS fluxes } \\
\hline & $\begin{array}{c}U \\
(\mathrm{mag}) \\
(2)\end{array}$ & $\begin{array}{c}B \\
(\mathrm{mag}) \\
(3)\end{array}$ & $\begin{array}{c}V \\
(\mathrm{mag}) \\
(4)\end{array}$ & $\begin{array}{c}J \\
(\mathrm{mag}) \\
(5)\end{array}$ & $\begin{array}{c}H \\
(\mathrm{mag}) \\
(6)\end{array}$ & $\begin{array}{c}K \\
(\mathrm{mag}) \\
(7)\end{array}$ & $\begin{array}{c}12 \mu \mathrm{m} \\
(\mathrm{Jy}) \\
(8)\end{array}$ & $\begin{array}{c}25 \mu \mathrm{m} \\
(\mathrm{Jy}) \\
(9)\end{array}$ & $\begin{array}{l}60 \mu \mathrm{m} \\
(\mathrm{Jy}) \\
(10)\end{array}$ & $\begin{array}{l}100 \mu \mathrm{m} \\
(\mathrm{Jy}) \\
(11)\end{array}$ \\
\hline UGC 09661 & $\cdots$ & $14.61 \pm 0.18$ & .. & $12.31 \pm 0.04$ & $11.76 \pm 0.05$ & $11.86 \pm 0.15$ & $<0.11$ & $<0.15$ & $0.24 \pm 0.04$ & $0.86 \pm 0.15$ \\
\hline NGC 5866 & $11.12 \pm 0.07$ & $10.74 \pm 0.07$ & $9.89 \pm 0.07$ & $7.83 \pm 0.02$ & $7.13 \pm 0.02$ & $6.87 \pm 0.02$ & $0.35 \pm 0.02$ & $0.36 \pm 0.02$ & $4.91 \pm 0.03$ & $16.80 \pm 0.08$ \\
\hline NGC 5826 & $\cdots$ & $14.89 \pm 0.19$ & $\cdots$ & $\cdots$ & $\cdots$ & $\cdots$ & $\cdots$ & $\cdots$ & $\cdots$ & $\cdots$ \\
\hline IC 1102 & $\cdots$ & $14.83 \pm 0.19$ & $\cdots$ & $11.89 \pm 0.03$ & $11.35 \pm 0.03$ & $11.09 \pm 0.06$ & $<0.09$ & $<0.10$ & $0.26 \pm 0.04$ & $<0.55$ \\
\hline NGC 5894 & $\cdots$ & $13.40 \pm 0.20$ & $\cdots$ & $10.11 \pm 0.01$ & $9.34 \pm 0.01$ & $9.05 \pm 0.01$ & $0.13 \pm 0.02$ & $0.14 \pm 0.02$ & $1.11 \pm 0.06$ & $4.96 \pm 0.20$ \\
\hline IRAS $15250+3609$ & $\cdots$ & $\cdots$ & $\cdots$ & $13.95 \pm 0.05$ & $13.12 \pm 0.06$ & $12.78 \pm 0.08$ & $0.12 \pm 0.03$ & $1.28 \pm 0.03$ & $7.20 \pm 0.04$ & $5.78 \pm 0.11$ \\
\hline UGC 09912 & $\cdots$ & $14.00 \pm 0.30$ & $\cdots$ & $\cdots$ & $\cdots$ & $\ldots$ & $<0.07$ & $<0.07$ & $0.22 \pm 0.04$ & $<0.88$ \\
\hline NGC 5962 & $12.04 \pm 0.13$ & $11.98 \pm 0.13$ & $11.34 \pm 0.13$ & $9.50 \pm 0.01$ & $8.81 \pm 0.01$ & $8.53 \pm 0.02$ & $0.72 \pm 0.02$ & $1.05 \pm 0.03$ & $8.99 \pm 0.03$ & $20.80 \pm 0.23$ \\
\hline UGC 09925 & $\cdots$ & $14.70 \pm 0.20$ & $\cdots$ & $\cdots$ & $\cdots$ & $\cdots$ & $\cdots$ & $\cdots$ & $\cdots$ & $\cdots$ \\
\hline NGC 5972 & $\cdots$ & $14.60 \pm 0.18$ & $\cdots$ & $11.71 \pm 0.03$ & $11.07 \pm 0.03$ & $10.69 \pm 0.04$ & $<0.12$ & $0.11 \pm 0.03$ & $0.25 \pm 0.07$ & $<0.76$ \\
\hline UGC 09953 & $\cdots$ & $14.46 \pm 0.19$ & $\cdots$ & $12.53 \pm 0.05$ & $11.82 \pm 0.05$ & $11.73 \pm 0.09$ & $\ldots$ & $\ldots$ & $\ldots$ & $\ldots$ \\
\hline UGC 10043 & $\cdots$ & $14.80 \pm 0.20$ & $\cdots$ & $11.76 \pm 0.03$ & $10.82 \pm 0.03$ & $10.41 \pm 0.03$ & $0.08 \pm 0.02$ & $0.10 \pm 0.02$ & $1.16 \pm 0.06$ & $3.45 \pm 0.24$ \\
\hline UGC 10109 & $\cdots$ & $14.67 \pm 0.19$ & $\cdots$ & $11.82 \pm 0.03$ & $11.22 \pm 0.03$ & $10.96 \pm 0.04$ & $<0.09$ & $<0.06$ & $0.25 \pm 0.03$ & $0.86 \pm 0.12$ \\
\hline UGC 10153 & $\cdots$ & $14.60 \pm 0.20$ & $\cdots$ & $12.10 \pm 0.04$ & $11.35 \pm 0.05$ & $10.97 \pm 0.06$ & $<0.07$ & $<0.06$ & $0.27 \pm 0.04$ & $1.12 \pm 0.26$ \\
\hline NGC 6036 & $\cdots$ & $14.40 \pm 0.30$ & $\cdots$ & $10.49 \pm 0.01$ & $9.74 \pm 0.01$ & $9.44 \pm 0.03$ & $<0.12$ & $<0.12$ & $0.33 \pm 0.05$ & $1.55 \pm 0.31$ \\
\hline NGC 6052 & $13.01 \pm 0.13$ & $13.44 \pm 0.13$ & $13.00 \pm 0.13$ & $\cdots$ & $\cdots$ & $\cdots$ & $0.26 \pm 0.03$ & $0.82 \pm 0.03$ & $6.46 \pm 0.04$ & $10.20 \pm 0.36$ \\
\hline UGC 10197 & $\cdots$ & $\cdots$ & $\cdots$ & $12.50 \pm 0.05$ & $12.10 \pm 0.09$ & $11.68 \pm 0.09$ & $\cdots$ & $\cdots$ & $\cdots$ & $\cdots$ \\
\hline UGC 10198 & $\cdots$ & $\cdots$ & $\cdots$ & $13.01 \pm 0.07$ & $12.19 \pm 0.09$ & $11.82 \pm 0.09$ & $\cdots$ & $\cdots$ & $\ldots$ & $\ldots$ \\
\hline UGC 10245 & $\cdots$ & $\cdots$ & $\ldots$ & $12.65 \pm 0.05$ & $11.72 \pm 0.05$ & $11.42 \pm 0.06$ & $<0.07$ & $<0.05$ & $0.17 \pm 0.03$ & $0.53 \pm 0.13$ \\
\hline CGCG 023-019 & $\cdots$ & $14.30 \pm 0.20$ & $\cdots$ & $11.78 \pm 0.04$ & $11.20 \pm 0.05$ & $10.92 \pm 0.07$ & $\cdots$ & $\cdots$ & $\ldots$ & $\cdots$ \\
\hline UGC 10261 & $\cdots$ & $15.20 \pm 0.20$ & $\cdots$ & $11.82 \pm 0.04$ & $11.05 \pm 0.05$ & $10.89 \pm 0.07$ & $\cdots$ & $\cdots$ & $\cdots$ & $\cdots$ \\
\hline NGC 6090 & $\cdots$ & $\ldots$ & $\cdots$ & $\ldots$ & $\ldots$ & $\ldots$ & $0.29 \pm 0.02$ & $1.22 \pm 0.03$ & $6.25 \pm 0.04$ & $9.34 \pm 0.10$ \\
\hline UGC 10278 & $\cdots$ & $14.79 \pm 0.18$ & $\cdots$ & $12.12 \pm 0.03$ & $11.58 \pm 0.04$ & $11.70 \pm 0.08$ & $0.08 \pm 0.02$ & $<0.06$ & $0.47 \pm 0.04$ & $1.17 \pm 0.13$ \\
\hline NGC 6100 & $\cdots$ & $13.90 \pm 0.20$ & $\cdots$ & $11.64 \pm 0.03$ & $10.95 \pm 0.03$ & $10.84 \pm 0.04$ & $\cdots$ & $\cdots$ & $\cdots$ & $\cdots$ \\
\hline IC 4595 & $\cdots$ & $\cdots$ & $\cdots$ & $10.05 \pm 0.02$ & $9.18 \pm 0.02$ & $8.84 \pm 0.02$ & $0.48 \pm 0.03$ & $0.68 \pm 0.02$ & $6.85 \pm 0.27$ & $17.90 \pm 0.89$ \\
\hline NGC 6154 & $\cdots$ & $13.60 \pm 0.20$ & $\cdots$ & $11.10 \pm 0.02$ & $10.40 \pm 0.03$ & $10.19 \pm 0.04$ & $\cdots$ & $\cdots$ & $\cdots$ & $\cdots$ \\
\hline NGC 6155 & $\ldots$ & $13.20 \pm 0.20$ & $\cdots$ & $10.76 \pm 0.02$ & $10.20 \pm 0.03$ & $9.97 \pm 0.04$ & $0.15 \pm 0.02$ & $0.22 \pm 0.01$ & $1.90 \pm 0.08$ & $5.45 \pm 0.22$ \\
\hline UGC 10404 & $\cdots$ & $15.00 \pm 0.30$ & $\ldots$ & $11.78 \pm 0.04$ & $11.19 \pm 0.06$ & $10.75 \pm 0.07$ & $\cdots$ & $\ldots$ & $\ldots$ & $\cdots$ \\
\hline NGC 6166 & $\cdots$ & $12.78 \pm 0.11$ & $11.78 \pm 0.12$ & $\ldots$ & $\ldots$ & $\ldots$ & $<0.02$ & $<0.02$ & $<0.10$ & $<0.45$ \\
\hline UGC 10420 & $14.43 \pm 0.16$ & $14.48 \pm 0.15$ & $13.78 \pm 0.16$ & $12.35 \pm 0.03$ & $11.85 \pm 0.05$ & $11.80 \pm 0.08$ & $\ldots$ & $\ldots$ & $\ldots$ & $\ldots$ \\
\hline UGC 10445 & $\cdots$ & $13.30 \pm 0.30$ & $\ldots$ & $13.24 \pm 0.05$ & $12.79 \pm 0.09$ & $12.77 \pm 0.12$ & $<0.06$ & $<0.05$ & $0.45 \pm 0.04$ & $1.38 \pm 0.21$ \\
\hline IC 1221 & $\ldots$ & $14.40 \pm 0.20$ & $\cdots$ & $11.94 \pm 0.07$ & $11.29 \pm 0.09$ & $10.99 \pm 0.12$ & $\ldots$ & $\cdots$ & $\ldots$ & $\cdots$ \\
\hline
\end{tabular}


Table 4-Continued

\begin{tabular}{|c|c|c|c|c|c|c|c|c|c|c|}
\hline \multirow{2}{*}{$\begin{array}{c}\text { Object Name } \\
\text { (1) }\end{array}$} & \multicolumn{3}{|c|}{ Optical Photometry } & \multicolumn{3}{|c|}{ Near-Infrared Photometry } & \multicolumn{4}{|c|}{ IRAS fluxes } \\
\hline & $\begin{array}{c}U \\
(\mathrm{mag}) \\
(2)\end{array}$ & $\begin{array}{c}B \\
(\mathrm{mag}) \\
(3)\end{array}$ & $\begin{array}{c}V \\
(\mathrm{mag}) \\
(4)\end{array}$ & $\begin{array}{c}J \\
(\mathrm{mag}) \\
(5)\end{array}$ & $\begin{array}{c}H \\
(\mathrm{mag}) \\
(6)\end{array}$ & $\begin{array}{c}K \\
(\mathrm{mag}) \\
(7)\end{array}$ & $\begin{array}{c}12 \mu \mathrm{m} \\
(\mathrm{Jy}) \\
(8)\end{array}$ & $\begin{array}{c}25 \mu \mathrm{m} \\
(\mathrm{Jy}) \\
(9)\end{array}$ & $\begin{array}{c}60 \mu \mathrm{m} \\
(\mathrm{Jy}) \\
(10)\end{array}$ & $\begin{array}{l}100 \mu \mathrm{m} \\
(\mathrm{Jy}) \\
(11)\end{array}$ \\
\hline IC 1222 & $14.22 \pm 0.14$ & $14.16 \pm 0.13$ & $13.45 \pm 0.13$ & $13.11 \pm 0.06$ & $12.36 \pm 0.08$ & $12.20 \pm 0.12$ & $<0.10$ & $0.17 \pm 0.01$ & $1.05 \pm 0.06$ & $2.42 \pm 0.29$ \\
\hline UGC 10468 & $\ldots$ & $\ldots$ & $\ldots$ & $12.19 \pm 0.03$ & $11.31 \pm 0.04$ & $10.86 \pm 0.04$ & $0.09 \pm 0.02$ & $0.11 \pm 0.01$ & $1.00 \pm 0.06$ & $2.75 \pm 0.17$ \\
\hline UGC 10491 & $15.19 \pm 0.23$ & $15.00 \pm 0.20$ & $14.17 \pm 0.21$ & $\ldots$ & $\ldots$ & $\ldots$ & $<0.07$ & $0.08 \pm 0.01$ & $0.46 \pm 0.04$ & $1.51 \pm 0.12$ \\
\hline NGC 6239 & $12.75 \pm 0.13$ & $12.94 \pm 0.13$ & $12.45 \pm 0.13$ & $10.94 \pm 0.02$ & $10.35 \pm 0.03$ & $10.05 \pm 0.04$ & $0.13 \pm 0.02$ & $0.36 \pm 0.01$ & $3.41 \pm 0.10$ & $6.17 \pm 0.25$ \\
\hline Mrk 501 & $14.09 \pm 0.14$ & $14.15 \pm 0.13$ & $13.29 \pm 0.13$ & $10.67 \pm 0.02$ & $9.87 \pm 0.02$ & $9.57 \pm 0.03$ & $0.08 \pm 0.02$ & $0.10 \pm 0.02$ & $0.17 \pm 0.04$ & $0.48 \pm 0.10$ \\
\hline UGC 10600 & $\ldots$ & $15.79 \pm 0.19$ & $\ldots$ & $12.29 \pm 0.03$ & $11.52 \pm 0.04$ & $11.10 \pm 0.04$ & $<0.12$ & $0.14 \pm 0.02$ & $1.40 \pm 0.08$ & $3.37 \pm 0.30$ \\
\hline NGC 6255 & $\ldots$ & $13.40 \pm 0.30$ & $\ldots$ & $13.23 \pm 0.06$ & $12.64 \pm 0.09$ & $12.85 \pm 0.18$ & $<0.08$ & $<0.12$ & $0.51 \pm 0.10$ & $1.23 \pm 0.15$ \\
\hline UGC 10651 & $\ldots$ & $15.20 \pm 0.30$ & $\ldots$ & $12.40 \pm 0.05$ & $12.00 \pm 0.08$ & $11.42 \pm 0.09$ & $\ldots$ & $\ldots$ & $\cdots$ & $\ldots$ \\
\hline UGC 10687 & $\ldots$ & $15.19 \pm 0.20$ & $\ldots$ & $\ldots$ & $\cdots$ & $\cdots$ & $<0.05$ & $<0.04$ & $0.18 \pm 0.03$ & $0.46 \pm 0.13$ \\
\hline UGC 10713 & $\ldots$ & $14.00 \pm 0.20$ & $\ldots$ & $11.55 \pm 0.03$ & $10.85 \pm 0.03$ & $10.59 \pm 0.04$ & $0.09 \pm 0.02$ & $0.11 \pm 0.01$ & $1.18 \pm 0.05$ & $3.00 \pm 0.21$ \\
\hline NGC 6306 & $14.42 \pm 0.21$ & $14.40 \pm 0.20$ & $13.74 \pm 0.20$ & $11.75 \pm 0.03$ & $11.02 \pm 0.04$ & $10.85 \pm 0.05$ & $0.15 \pm 0.01$ & $0.46 \pm 0.01$ & $3.01 \pm 0.12$ & $4.99 \pm 0.20$ \\
\hline NGC 6307 & $\ldots$ & $13.95 \pm 0.18$ & $\ldots$ & $10.40 \pm 0.02$ & $9.72 \pm 0.02$ & $9.48 \pm 0.03$ & $\ldots$ & $\ldots$ & & $\ldots$ \\
\hline UGC 10729 & $\ldots$ & $15.35 \pm 0.18$ & $\ldots$ & $13.32 \pm 0.05$ & $12.60 \pm 0.06$ & $12.39 \pm 0.08$ & $<0.08$ & $<0.11$ & $0.59 \pm 0.04$ & $1.26 \pm 0.21$ \\
\hline IC 1251 & $\ldots$ & $14.23 \pm 0.19$ & $\ldots$ & $12.86 \pm 0.06$ & $12.22 \pm 0.07$ & $12.07 \pm 0.11$ & $<0.04$ & $<0.05$ & $0.23 \pm 0.03$ & $<1.20$ \\
\hline NGC 6340 & $\ldots$ & $11.87 \pm 0.14$ & $11.01 \pm 0.15$ & $9.25 \pm 0.01$ & $8.55 \pm 0.01$ & $8.39 \pm 0.02$ & $<0.10$ & $<0.04$ & $0.20 \pm 0.03$ & $1.03 \pm 0.17$ \\
\hline IC 1254 & $15.55 \pm 0.24$ & $15.00 \pm 0.20$ & $13.84 \pm 0.23$ & $12.24 \pm 0.04$ & $11.61 \pm 0.05$ & $11.67 \pm 0.10$ & $\ldots$ & $\ldots$ & $\ldots$ & $\ldots$ \\
\hline IC 1248 & $\ldots$ & $14.30 \pm 0.20$ & $\ldots$ & $12.60 \pm 0.05$ & $12.05 \pm 0.08$ & $11.71 \pm 0.09$ & $<0.05$ & $<0.05$ & $0.29 \pm 0.03$ & $1.00 \pm 0.12$ \\
\hline UGC 10770 & $\ldots$ & $\ldots$ & $\ldots$ & $\ldots$ & $\ldots$ & $\ldots$ & $<0.06$ & $<0.06$ & $0.22 \pm 0.03$ & $<0.57$ \\
\hline UGC 10791 & $\ldots$ & $15.00 \pm 0.30$ & $\ldots$ & $\ldots$ & $\ldots$ & $\ldots$ & $\ldots$ & $\ldots$ & $\ldots$ & $\ldots$ \\
\hline NGC 6330 & $\ldots$ & $14.80 \pm 0.20$ & $\ldots$ & $11.90 \pm 0.03$ & $11.19 \pm 0.03$ & $10.87 \pm 0.04$ & $0.06 \pm 0.02$ & $0.12 \pm 0.01$ & $1.37 \pm 0.05$ & $2.77 \pm 0.30$ \\
\hline UGC 10783 & $\ldots$ & $15.16 \pm 0.20$ & $\ldots$ & $13.02 \pm 0.04$ & $12.40 \pm 0.05$ & $12.33 \pm 0.09$ & $\ldots$ & $\ldots$ & $\ldots$ & $\ldots$ \\
\hline UGC 10796 & $\ldots$ & $14.50 \pm 0.20$ & $\ldots$ & $13.06 \pm 0.06$ & $12.66 \pm 0.11$ & $12.15 \pm 0.12$ & $<0.05$ & $<0.05$ & $0.14 \pm 0.02$ & $0.45 \pm 0.11$ \\
\hline NGC 6359 & $14.08 \pm 0.14$ & $13.56 \pm 0.13$ & $12.64 \pm 0.13$ & $10.44 \pm 0.01$ & $9.77 \pm 0.02$ & $9.51 \pm 0.02$ & $<0.02$ & $<0.01$ & $<0.01$ & $0.31 \pm 0.11$ \\
\hline UGC 10795 & $\ldots$ & $\ldots$ & $\ldots$ & $\ldots$ & $\ldots$ & $\ldots$ & $\ldots$ & $\ldots$ & $\ldots$ & $\ldots$ \\
\hline NGC 6361 & $\ldots$ & $13.87 \pm 0.18$ & $\ldots$ & $10.32 \pm 0.02$ & $9.54 \pm 0.02$ & $9.10 \pm 0.02$ & $0.34 \pm 0.01$ & $0.45 \pm 0.01$ & $4.27 \pm 0.13$ & $13.60 \pm 0.41$ \\
\hline UGC 10811 & $\ldots$ & $14.93 \pm 0.19$ & $\ldots$ & $11.74 \pm 0.03$ & $11.04 \pm 0.04$ & $10.72 \pm 0.05$ & $\ldots$ & $\ldots$ & $\ldots$ & $\ldots$ \\
\hline NGC 6373 & $\ldots$ & $14.33 \pm 0.19$ & $\ldots$ & $13.32 \pm 0.07$ & $12.68 \pm 0.09$ & $12.52 \pm 0.12$ & $<0.04$ & $<0.04$ & $0.17 \pm 0.03$ & $0.76 \pm 0.13$ \\
\hline NGC 6364 & $\ldots$ & $13.87 \pm 0.17$ & $\ldots$ & $10.69 \pm 0.02$ & $9.98 \pm 0.02$ & $9.74 \pm 0.03$ & $\ldots$ & $\ldots$ & $\ldots$ & $\ldots$ \\
\hline UGC 10842 & $\ldots$ & $15.10 \pm 0.20$ & $\ldots$ & $12.36 \pm 0.04$ & $11.61 \pm 0.05$ & $11.34 \pm 0.05$ & $\ldots$ & $\ldots$ & $\ldots$ & $\ldots$ \\
\hline UGC 10872 & $\ldots$ & $\ldots$ & $\ldots$ & $\ldots$ & $\ldots$ & $\ldots$ & $\ldots$ & $\ldots$ & $\ldots$ & $\ldots$ \\
\hline UGC 10888 & $\ldots$ & $14.27 \pm 0.18$ & $\ldots$ & $11.62 \pm 0.03$ & $10.97 \pm 0.04$ & $10.68 \pm 0.05$ & $<0.06$ & $0.05 \pm 0.01$ & $0.32 \pm 0.03$ & $1.08 \pm 0.14$ \\
\hline NGC 6394 & $\ldots$ & $15.27 \pm 0.18$ & $\ldots$ & $11.86 \pm 0.04$ & $11.15 \pm 0.04$ & $10.76 \pm 0.04$ & $0.05 \pm 0.01$ & $0.12 \pm 0.01$ & $0.67 \pm 0.03$ & $1.84 \pm 0.15$ \\
\hline
\end{tabular}


Table 4-Continued

\begin{tabular}{|c|c|c|c|c|c|c|c|c|c|c|}
\hline \multirow{2}{*}{$\begin{array}{c}\text { Object Name } \\
\text { (1) }\end{array}$} & \multicolumn{3}{|c|}{ Optical Photometry } & \multicolumn{3}{|c|}{ Near-Infrared Photometry } & \multicolumn{4}{|c|}{ IRAS fluxes } \\
\hline & $\begin{array}{c}U \\
(\mathrm{mag}) \\
(2)\end{array}$ & $\begin{array}{c}B \\
(\mathrm{mag}) \\
(3)\end{array}$ & $\begin{array}{c}V \\
(\mathrm{mag}) \\
(4)\end{array}$ & $\begin{array}{c}J \\
(\mathrm{mag}) \\
(5)\end{array}$ & $\begin{array}{c}H \\
(\mathrm{mag}) \\
(6)\end{array}$ & $\begin{array}{c}K \\
(\mathrm{mag}) \\
(7)\end{array}$ & $\begin{array}{c}12 \mu \mathrm{m} \\
(\mathrm{Jy}) \\
(8)\end{array}$ & $\begin{array}{c}25 \mu \mathrm{m} \\
(\mathrm{Jy}) \\
(9)\end{array}$ & $\begin{array}{c}60 \mu \mathrm{m} \\
(\mathrm{Jy}) \\
(10)\end{array}$ & $\begin{array}{l}100 \mu \mathrm{m} \\
(\mathrm{Jy}) \\
(11)\end{array}$ \\
\hline UGC 10895 & $\ldots$ & $\ldots$ & $\ldots$ & $13.20 \pm 0.08$ & $12.42 \pm 0.09$ & $12.23 \pm 0.13$ & $\ldots$ & $\ldots$ & $\ldots$ & $\ldots$ \\
\hline UGC 10935 & $\ldots$ & $\ldots$ & $\ldots$ & $12.58 \pm 0.03$ & $11.94 \pm 0.05$ & $11.79 \pm 0.06$ & $\ldots$ & $\ldots$ & $\ldots$ & $\ldots$ \\
\hline UGC 10971 & $\ldots$ & $14.98 \pm 0.18$ & $\ldots$ & $11.52 \pm 0.03$ & $10.71 \pm 0.03$ & $10.41 \pm 0.03$ & $\ldots$ & $\ldots$ & $\ldots$ & $\ldots$ \\
\hline NGC 6482 & $12.71 \pm 0.13$ & $12.35 \pm 0.13$ & $11.45 \pm 0.13$ & $9.35 \pm 0.01$ & $8.61 \pm 0.01$ & $8.37 \pm 0.01$ & $\ldots$ & $<0.03$ & $<0.09$ & $<0.34$ \\
\hline IC 4836 & $13.28 \pm 0.14$ & $13.27 \pm 0.13$ & $12.65 \pm 0.13$ & $11.02 \pm 0.02$ & $10.27 \pm 0.03$ & $10.03 \pm 0.03$ & $0.22 \pm 0.02$ & $0.25 \pm 0.02$ & $2.13 \pm 0.09$ & $6.24 \pm 0.31$ \\
\hline NGC 6789 & $\ldots$ & $13.76 \pm 0.18$ & $\ldots$ & $12.87 \pm 0.04$ & $12.29 \pm 0.05$ & $12.24 \pm 0.09$ & $\ldots$ & $\ldots$ & $\ldots$ & $\ldots$ \\
\hline NGC 6769 & $\ldots$ & $12.55 \pm 0.08$ & $11.75 \pm 0.09$ & $9.43 \pm 0.02$ & $8.74 \pm 0.03$ & $8.48 \pm 0.04$ & $\ldots$ & $\ldots$ & $\ldots$ & $\ldots$ \\
\hline NGC 6770 & $13.17 \pm 0.12$ & $12.83 \pm 0.09$ & $11.94 \pm 0.09$ & $9.75 \pm 0.03$ & $9.12 \pm 0.03$ & $8.86 \pm 0.04$ & $\ldots$ & $\ldots$ & $\ldots$ & $\ldots$ \\
\hline NGC 6771 & $14.17 \pm 0.09$ & $13.57 \pm 0.08$ & $12.52 \pm 0.08$ & $9.98 \pm 0.02$ & $9.28 \pm 0.02$ & $9.00 \pm 0.03$ & $\ldots$ & $\ldots$ & $\ldots$ & $\ldots$ \\
\hline IC 4842 & $\ldots$ & $13.43 \pm 0.21$ & $\ldots$ & $10.28 \pm 0.02$ & $9.57 \pm 0.02$ & $9.37 \pm 0.03$ & $<0.03$ & $<0.02$ & $<0.03$ & $<0.09$ \\
\hline IC 4845 & $12.50 \pm 0.14$ & $12.34 \pm 0.14$ & $11.57 \pm 0.14$ & $9.86 \pm 0.01$ & $9.17 \pm 0.02$ & $8.92 \pm 0.02$ & $0.18 \pm 0.02$ & $0.19 \pm 0.02$ & $1.19 \pm 0.06$ & $4.51 \pm 0.27$ \\
\hline NGC 6782 & $\ldots$ & $12.63 \pm 0.15$ & $11.84 \pm 0.13$ & $9.82 \pm 0.01$ & $9.11 \pm 0.01$ & $8.87 \pm 0.02$ & $0.20 \pm 0.04$ & $0.22 \pm 0.04$ & $2.48 \pm 0.17$ & $6.13 \pm 0.37$ \\
\hline Superantena & $\ldots$ & $\ldots$ & $\ldots$ & $\ldots$ & $\ldots$ & $\ldots$ & $0.22 \pm 0.02$ & $1.24 \pm 0.01$ & $5.48 \pm 0.22$ & $5.79 \pm 0.46$ \\
\hline NGC $6845 \mathrm{~A}$ & $\ldots$ & $\ldots$ & $\ldots$ & $11.00 \pm 0.02$ & $10.27 \pm 0.03$ & $9.92 \pm 0.03$ & $\ldots$ & $\begin{array}{l}1.210 \\
\ldots\end{array}$ & $\ldots$ & $\ldots$ \\
\hline ESO 284-G009 & $\ldots$ & $14.06 \pm 0.21$ & $\ldots$ & $10.99 \pm 0.02$ & $10.33 \pm 0.03$ & $10.04 \pm 0.03$ & $\ldots$ & $\ldots$ & $\ldots$ & $\ldots$ \\
\hline NGC $6902 B$ & $13.95 \pm 0.20$ & $14.09 \pm 0.19$ & $13.63 \pm 0.19$ & $12.89 \pm 0.05$ & $12.28 \pm 0.06$ & $11.95 \pm 0.09$ & $<0.13$ & $<0.14$ & $0.27 \pm 0.05$ & $0.89 \pm 0.19$ \\
\hline IC 4946 & $12.87 \pm 0.13$ & $12.61 \pm 0.13$ & $11.79 \pm 0.13$ & $9.72 \pm 0.01$ & $8.99 \pm 0.01$ & $8.73 \pm 0.02$ & $0.22 \pm 0.03$ & $0.65 \pm 0.02$ & $5.18 \pm 0.26$ & $6.31 \pm 0.50$ \\
\hline NGC 6902 & $11.73 \pm 0.20$ & $11.64 \pm 0.18$ & $10.93 \pm 0.18$ & $9.40 \pm 0.03$ & $8.89 \pm 0.03$ & $8.61 \pm 0.04$ & $<0.21$ & $<0.17$ & $0.83 \pm 0.06$ & $3.92 \pm 0.28$ \\
\hline ESO 285-G009 & $\ldots$ & $15.37 \pm 0.21$ & $\ldots$ & $\ldots$ & $\ldots$ & $\ldots$ & $\ldots$ & $\ldots$ & $\ldots$ & $\ldots$ \\
\hline PGC 65022 & $\ldots$ & $\ldots$ & $\ldots$ & $11.51 \pm 0.02$ & $10.58 \pm 0.02$ & $10.16 \pm 0.02$ & $<0.12$ & $0.17 \pm 0.03$ & $0.69 \pm 0.06$ & $2.19 \pm 0.22$ \\
\hline NGC 6941 & $\ldots$ & $\ldots$ & $\ldots$ & $10.67 \pm 0.03$ & $9.98 \pm 0.03$ & $9.73 \pm 0.05$ & $<0.10$ & $<0.11$ & $0.39 \pm 0.05$ & $1.51 \pm 0.21$ \\
\hline NGC 6951 & $\ldots$ & $11.64 \pm 0.15$ & $10.65 \pm 0.16$ & $8.31 \pm 0.02$ & $7.47 \pm 0.02$ & $7.22 \pm 0.03$ & $0.62 \pm 0.02$ & $1.37 \pm 0.02$ & $13.20 \pm 0.53$ & $37.50 \pm 1.50$ \\
\hline NGC 6945 & $\ldots$ & $\ldots$ & $\ldots$ & $10.48 \pm 0.02$ & $9.81 \pm 0.03$ & $9.48 \pm 0.03$ & $\ldots$ & $\ldots$ & $\ldots$ & $\ldots$ \\
\hline PGC 65158 & $\ldots$ & $\ldots$ & $\ldots$ & $\ldots$ & $\ldots$ & $\ldots$ & $\ldots$ & $\ldots$ & $\ldots$ & $\ldots$ \\
\hline UGC 11612 & $\ldots$ & $15.52 \pm 0.18$ & $\ldots$ & $12.41 \pm 0.05$ & $11.70 \pm 0.05$ & $11.21 \pm 0.06$ & $<0.14$ & $<0.08$ & $0.22 \pm 0.04$ & $<1.65$ \\
\hline PGC 65328 & $\ldots$ & $\begin{array}{c}10.020 .10 \\
\ldots\end{array}$ & $\ldots$ & $12.23 \pm 0.06$ & $11.81 \pm 0.07$ & $11.19 \pm 0.07$ & $\ldots$ & $\ldots$ & $\begin{array}{c}0.22 \pm 0.04 \\
\ldots\end{array}$ & $\ldots$ \\
\hline ESO 341-G013 & $\ldots$ & $13.95 \pm 0.21$ & $\ldots$ & $11.03 \pm 0.03$ & $10.24 \pm 0.03$ & $10.01 \pm 0.04$ & $\ldots$ & $\ldots$ & $\ldots$ & $\ldots$ \\
\hline NGC 6962 & $13.38 \pm 0.23$ & $13.00 \pm 0.20$ & $12.14 \pm 0.20$ & $9.78 \pm 0.02$ & $9.06 \pm 0.03$ & $8.79 \pm 0.03$ & $<0.11$ & $<0.14$ & $0.34 \pm 0.05$ & $2.25 \pm 0.38$ \\
\hline NGC 6964 & $14.51 \pm 0.20$ & $14.00 \pm 0.20$ & $12.99 \pm 0.20$ & $10.47 \pm 0.02$ & $9.86 \pm 0.03$ & $9.55 \pm 0.03$ & $\ldots$ & $\ldots$ & $\ldots$ & $\ldots$ \\
\hline PGC 65420 & $\ldots$ & $\ldots$ & $\ldots$ & $14.60 \pm 0.12$ & $14.05 \pm 0.18$ & $13.83 \pm 0.24$ & $\ldots$ & $\ldots$ & $\ldots$ & $\ldots$ \\
\hline NGC 6958 & $12.79 \pm 0.13$ & $12.33 \pm 0.13$ & $11.42 \pm 0.13$ & $9.34 \pm 0.01$ & $8.65 \pm 0.01$ & $8.40 \pm 0.02$ & $<0.17$ & $0.16 \pm 0.04$ & $0.97 \pm 0.07$ & $1.91 \pm 0.23$ \\
\hline UGC 11646 & $\ldots$ & $15.01 \pm 0.18$ & $\ldots$ & $12.28 \pm 0.05$ & $11.49 \pm 0.05$ & $11.19 \pm 0.06$ & $\ldots$ & $\ldots$ & $\ldots$ & $\ldots$ \\
\hline
\end{tabular}


Table 4-Continued

\begin{tabular}{|c|c|c|c|c|c|c|c|c|c|c|}
\hline \multirow{2}{*}{$\begin{array}{c}\text { Object Name } \\
(1) \\
\end{array}$} & \multicolumn{3}{|c|}{ Optical Photometry } & \multicolumn{3}{|c|}{ Near-Infrared Photometry } & \multicolumn{4}{|c|}{ IRAS fluxes } \\
\hline & $\begin{array}{c}U \\
(\operatorname{mag}) \\
(2)\end{array}$ & $\begin{array}{c}B \\
(\mathrm{mag}) \\
(3)\end{array}$ & $\begin{array}{c}V \\
(\mathrm{mag}) \\
(4)\end{array}$ & $\begin{array}{c}J \\
(\mathrm{mag}) \\
(5)\end{array}$ & $\begin{array}{c}H \\
(\mathrm{mag}) \\
(6)\end{array}$ & $\begin{array}{c}K \\
(\mathrm{mag}) \\
(7)\end{array}$ & $\begin{array}{c}12 \mu \mathrm{m} \\
(\mathrm{Jy}) \\
(8)\end{array}$ & $\begin{array}{c}25 \mu \mathrm{m} \\
(\mathrm{Jy}) \\
(9)\end{array}$ & $\begin{array}{l}60 \mu \mathrm{m} \\
(\mathrm{Jy}) \\
(10)\end{array}$ & $\begin{array}{c}100 \mu \mathrm{m} \\
(\mathrm{Jy}) \\
(11)\end{array}$ \\
\hline PGC 66559 & $\cdots$ & & $\ldots$ & $15.89 \pm 0.13$ & $15.27 \pm 0.18$ & $15.11 \pm 0.26$ & $\cdots$ & .. & $\ldots$ & \\
\hline NGC 7080 & $13.25 \pm 0.18$ & $13.13 \pm 0.17$ & $12.33 \pm 0.17$ & $10.54 \pm 0.03$ & $9.79 \pm 0.04$ & $9.40 \pm 0.04$ & $0.20 \pm 0.03$ & $0.38 \pm 0.02$ & $3.16 \pm 0.22$ & $7.49 \pm 0.60$ \\
\hline UGC 11776 & $\cdots$ & $15.49 \pm 0.18$ & $\ldots$ & $12.44 \pm 0.04$ & $11.69 \pm 0.04$ & $11.38 \pm 0.06$ & $\ldots$ & $\cdots$ & $\ldots$ & $\cdots$ \\
\hline PGC 67153 & $\cdots$ & $15.10 \pm 0.29$ & $\cdots$ & $12.23 \pm 0.03$ & $11.46 \pm 0.04$ & $11.21 \pm 0.05$ & $<0.12$ & $<0.10$ & $0.46 \pm 0.06$ & $<2.49$ \\
\hline UGC 11789 & $\cdots$ & $14.67 \pm 0.18$ & $\cdots$ & $12.41 \pm 0.06$ & $12.28 \pm 0.13$ & $11.60 \pm 0.11$ & $\cdots$ & $\ldots$ & $\ldots$ & $\cdots$ \\
\hline Tol 2138-405 & $\cdots$ & $\ldots$ & $\cdots$ & $14.72 \pm 0.13$ & $14.03 \pm 0.15$ & $13.60 \pm 0.17$ & $<0.06$ & $<0.09$ & $0.18 \pm 0.04$ & $<0.57$ \\
\hline ESO 343-G018 & $\cdots$ & $14.62 \pm 0.21$ & $\ldots$ & $12.37 \pm 0.04$ & $11.75 \pm 0.06$ & $11.48 \pm 0.08$ & $<0.10$ & $<0.13$ & $0.29 \pm 0.04$ & $0.72 \pm 0.14$ \\
\hline UGC 11790 & $\ldots$ & $14.71 \pm 0.29$ & $\cdots$ & $12.70 \pm 0.04$ & $11.98 \pm 0.06$ & $11.39 \pm 0.10$ & $<0.08$ & $<0.12$ & $0.27 \pm 0.04$ & $1.09 \pm 0.23$ \\
\hline UGC 11794 & $\cdots$ & $15.19 \pm 0.19$ & $\cdots$ & $11.75 \pm 0.04$ & $10.99 \pm 0.03$ & $10.90 \pm 0.06$ & $<0.18$ & $<0.09$ & $0.29 \pm 0.05$ & $<2.25$ \\
\hline ESO 466-G001 & $\cdots$ & $14.63 \pm 0.21$ & $\cdots$ & $11.43 \pm 0.02$ & $10.61 \pm 0.02$ & $10.32 \pm 0.03$ & $<0.17$ & $<0.14$ & $0.29 \pm 0.04$ & $1.34 \pm 0.23$ \\
\hline ESO 466-G005 & $\ldots$ & $15.12 \pm 0.21$ & $\ldots$ & $13.12 \pm 0.05$ & $12.71 \pm 0.09$ & $12.41 \pm 0.11$ & $\ldots$ & $\ldots$ & $\ldots$ & $\ldots$ \\
\hline UGC 11816 & $\cdots$ & $14.27 \pm 0.25$ & $\cdots$ & $12.23 \pm 0.06$ & $11.54 \pm 0.08$ & $11.45 \pm 0.12$ & $\cdots$ & $\cdots$ & $\cdots$ & $\cdots$ \\
\hline NGC 7152 & $\cdots$ & $14.41 \pm 0.21$ & $\cdots$ & $11.88 \pm 0.04$ & $11.30 \pm 0.04$ & $10.85 \pm 0.05$ & $<0.08$ & $<0.12$ & $0.35 \pm 0.04$ & $1.55 \pm 0.15$ \\
\hline ESO 466-G014 & $\cdots$ & $15.29 \pm 0.21$ & $\cdots$ & $12.39 \pm 0.04$ & $11.69 \pm 0.04$ & $11.28 \pm 0.05$ & $\ldots$ & $\ldots$ & $\ldots$ & $\ldots$ \\
\hline UGC 11859 & $\cdots$ & $15.16 \pm 0.22$ & $\cdots$ & $12.55 \pm 0.04$ & $11.63 \pm 0.04$ & $11.43 \pm 0.06$ & $<0.19$ & $<0.13$ & $0.72 \pm 0.06$ & $1.55 \pm 0.22$ \\
\hline ESO 404-G015 & $\ldots$ & $14.50 \pm 0.21$ & $\ldots$ & $11.54 \pm 0.02$ & $10.87 \pm 0.03$ & $10.56 \pm 0.04$ & $<0.12$ & $<0.16$ & $0.42 \pm 0.05$ & $1.05 \pm 0.16$ \\
\hline NGC 7167 & $\ldots$ & $13.21 \pm 0.21$ & $\ldots$ & $11.01 \pm 0.05$ & $10.51 \pm 0.06$ & $9.94 \pm 0.06$ & $0.11 \pm 0.03$ & $<0.32$ & $1.31 \pm 0.08$ & $3.59 \pm 0.25$ \\
\hline ESO 404-G023 & $\ldots$ & $13.97 \pm 0.21$ & $\ldots$ & $11.57 \pm 0.05$ & $10.95 \pm 0.06$ & $10.61 \pm 0.08$ & $<0.07$ & $<0.22$ & $0.24 \pm 0.04$ & $<1.37$ \\
\hline IC 5156 & $13.12 \pm 0.13$ & $12.95 \pm 0.13$ & $12.17 \pm 0.13$ & $10.02 \pm 0.02$ & $9.33 \pm 0.02$ & $9.06 \pm 0.03$ & $0.13 \pm 0.04$ & $<0.19$ & $1.05 \pm 0.09$ & $4.45 \pm 0.36$ \\
\hline NGC 7215 & $\ldots$ & $14.90 \pm 0.15$ & $\ldots$ & $12.16 \pm 0.03$ & $11.41 \pm 0.05$ & $11.22 \pm 0.06$ & $<0.08$ & $<0.16$ & $0.53 \pm 0.05$ & $1.19 \pm 0.23$ \\
\hline NGC 7221 & $\cdots$ & $12.80 \pm 0.15$ & $12.13 \pm 0.15$ & $10.15 \pm 0.02$ & $9.32 \pm 0.02$ & $9.19 \pm 0.03$ & $<0.15$ & $<0.18$ & $0.65 \pm 0.06$ & $2.68 \pm 0.19$ \\
\hline CGCG 377-039 & $\cdots$ & $15.17 \pm 0.14$ & $14.23 \pm 0.15$ & $12.17 \pm 0.04$ & $11.47 \pm 0.05$ & $11.18 \pm 0.07$ & $\ldots$ & $\ldots$ & $\ldots$ & $\ldots$ \\
\hline NGC 7248 & $14.17 \pm 0.16$ & $13.50 \pm 0.15$ & $12.39 \pm 0.15$ & $10.14 \pm 0.04$ & $9.42 \pm 0.03$ & $9.12 \pm 0.01$ & $<0.03$ & $<0.04$ & $0.08 \pm 0.04$ & $1.19 \pm 0.30$ \\
\hline NGC 7250 & $13.18 \pm 0.14$ & $13.22 \pm 0.13$ & $12.58 \pm 0.14$ & $11.20 \pm 0.03$ & $10.58 \pm 0.04$ & $10.25 \pm 0.05$ & $0.12 \pm 0.03$ & $0.38 \pm 0.01$ & $3.40 \pm 0.24$ & $4.57 \pm 0.59$ \\
\hline NGC 7252 & $12.26 \pm 0.13$ & $12.72 \pm 0.13$ & $12.06 \pm 0.13$ & $10.26 \pm 0.02$ & $9.59 \pm 0.02$ & $9.31 \pm 0.03$ & $0.24 \pm 0.04$ & $0.43 \pm 0.02$ & $3.98 \pm 0.20$ & $7.02 \pm 0.42$ \\
\hline ESO 467-G058 & $\ldots$ & $14.81 \pm 0.21$ & $\ldots$ & $11.64 \pm 0.03$ & $10.81 \pm 0.03$ & $10.44 \pm 0.04$ & $\ldots$ & $\ldots$ & $\ldots$ & $\ldots$ \\
\hline ESO 345-G011 & $\cdots$ & $14.51 \pm 0.21$ & $\cdots$ & $11.49 \pm 0.02$ & $10.70 \pm 0.02$ & $10.39 \pm 0.03$ & $\cdots$ & $\ldots$ & $\cdots$ & $\cdots$ \\
\hline NGC 7279 & $14.28 \pm 0.15$ & $14.34 \pm 0.13$ & $13.78 \pm 0.13$ & $12.39 \pm 0.03$ & $11.64 \pm 0.04$ & $11.49 \pm 0.06$ & $<0.11$ & $0.21 \pm 0.03$ & $1.08 \pm 0.13$ & $2.56 \pm 0.21$ \\
\hline PKS 2225-308 & $\ldots$ & $\ldots$ & $\ldots$ & $11.59 \pm 0.06$ & $10.86 \pm 0.06$ & $10.56 \pm 0.07$ & $\ldots$ & $\ldots$ & $\ldots$ & $\cdots$ \\
\hline NGC 7289 & $\cdots$ & $14.17 \pm 0.21$ & $\cdots$ & $11.13 \pm 0.02$ & $10.44 \pm 0.03$ & $10.21 \pm 0.04$ & $\cdots$ & $\cdots$ & $\cdots$ & $\cdots$ \\
\hline ESO 468-G006 & $\cdots$ & $15.05 \pm 0.21$ & $\cdots$ & & $\ldots$ & & $\cdots$ & $\cdots$ & $\cdots$ & $\cdots$ \\
\hline NGC 7317 & $15.10 \pm 0.12$ & $14.57 \pm 0.11$ & $13.60 \pm 0.11$ & $11.59 \pm 0.03$ & $11.01 \pm 0.04$ & $10.60 \pm 0.04$ & $\cdots$ & $\cdots$ & $\cdots$ & $\ldots$ \\
\hline
\end{tabular}


Table 4-Continued

\begin{tabular}{|c|c|c|c|c|c|c|c|c|c|c|}
\hline \multirow{2}{*}{$\begin{array}{c}\text { Object Name } \\
\text { (1) }\end{array}$} & \multicolumn{3}{|c|}{ Optical Photometry } & \multicolumn{3}{|c|}{ Near-Infrared Photometry } & \multicolumn{4}{|c|}{ IRAS fluxes } \\
\hline & $\begin{array}{c}U \\
(\mathrm{mag}) \\
(2)\end{array}$ & $\begin{array}{c}B \\
(\mathrm{mag}) \\
(3)\end{array}$ & $\begin{array}{c}V \\
(\mathrm{mag}) \\
(4)\end{array}$ & $\begin{array}{c}J \\
(\mathrm{mag}) \\
(5)\end{array}$ & $\begin{array}{c}H \\
(\mathrm{mag}) \\
(6)\end{array}$ & $\begin{array}{c}K \\
(\mathrm{mag}) \\
(7)\end{array}$ & $\begin{array}{c}12 \mu \mathrm{m} \\
(\mathrm{Jy}) \\
(8)\end{array}$ & $\begin{array}{c}25 \mu \mathrm{m} \\
(\mathrm{Jy}) \\
(9)\end{array}$ & $\begin{array}{c}60 \mu \mathrm{m} \\
(\mathrm{Jy}) \\
(10)\end{array}$ & $\begin{array}{l}100 \mu \mathrm{m} \\
(\mathrm{Jy}) \\
(11)\end{array}$ \\
\hline NGC 7320 & $13.12 \pm 0.11$ & $13.23 \pm 0.10$ & $12.63 \pm 0.10$ & $11.25 \pm 0.04$ & $10.70 \pm 0.05$ & $10.52 \pm 0.07$ & $\cdots$ & $\cdots$ & 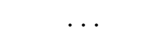 & $\cdots$ \\
\hline UGC 12110 & $\ldots$ & $15.33 \pm 0.19$ & $\ldots$ & $11.93 \pm 0.10$ & $11.11 \pm 0.04$ & $10.90 \pm 0.06$ & $<0.08$ & $<0.11$ & $0.35 \pm 0.05$ & $<1.83$ \\
\hline NGC 7331 & $10.65 \pm 0.10$ & $10.35 \pm 0.10$ & $9.48 \pm 0.10$ & $7.06 \pm 0.02$ & $6.29 \pm 0.02$ & $6.03 \pm 0.02$ & $3.36 \pm 0.84$ & $4.20 \pm 1.05$ & $35.30 \pm 8.82$ & $115.00 \pm 28.80$ \\
\hline NGC 7335 & $14.76 \pm 0.14$ & $14.44 \pm 0.13$ & $13.35 \pm 0.13$ & $10.16 \pm 0.01$ & $8.66 \pm 0.01$ & $9.24 \pm 0.03$ & $\cdots$ & $\cdots$ & $\cdots$ & $\cdots$ \\
\hline NGC 7337 & $\cdots$ & $15.24 \pm 0.26$ & $\cdots$ & $11.40 \pm 0.03$ & $10.65 \pm 0.03$ & $10.39 \pm 0.05$ & $\cdots$ & $\cdots$ & $\cdots$ & $\cdots$ \\
\hline NGC 7343 & $14.59 \pm 0.21$ & $14.40 \pm 0.20$ & $13.54 \pm 0.20$ & $11.46 \pm 0.02$ & $10.80 \pm 0.03$ & $10.47 \pm 0.04$ & $0.10 \pm 0.03$ & $0.19 \pm 0.01$ & $1.59 \pm 0.11$ & $2.98 \pm 0.30$ \\
\hline UGC 12134 & $14.22 \pm 0.16$ & $14.15 \pm 0.15$ & $13.51 \pm 0.15$ & $11.92 \pm 0.05$ & $11.21 \pm 0.06$ & $10.92 \pm 0.07$ & $<0.11$ & $<0.17$ & $0.24 \pm 0.05$ & $0.88 \pm 0.22$ \\
\hline NGC 7348 & $\ldots$ & $14.53 \pm 0.18$ & $\ldots$ & $13.02 \pm 0.08$ & $12.18 \pm 0.09$ & $11.83 \pm 0.10$ & $<0.11$ & $<0.14$ & $0.47 \pm 0.05$ & $1.00 \pm 0.21$ \\
\hline IRAS 22491-1808 & $\ldots$ & $\ldots$ & $\ldots$ & $\ldots$ & $\ldots$ & $\ldots$ & $\ldots$ & $0.56 \pm 0.07$ & $5.28 \pm 0.04$ & $4.58 \pm 0.10$ \\
\hline NGC 7396 & $\cdots$ & $13.75 \pm 0.19$ & $\cdots$ & $9.76 \pm 0.01$ & $9.02 \pm 0.02$ & $8.70 \pm 0.02$ & $0.12 \pm 0.03$ & $<0.19$ & $0.41 \pm 0.05$ & $2.46 \pm 0.32$ \\
\hline ESO 346-G006 & $\cdots$ & $14.65 \pm 0.21$ & $\cdots$ & $12.24 \pm 0.04$ & $11.77 \pm 0.05$ & $11.19 \pm 0.06$ & $<0.16$ & $<0.09$ & $0.32 \pm 0.05$ & $1.25 \pm 0.16$ \\
\hline NGC 7398 & $\cdots$ & $14.54 \pm 0.19$ & $\cdots$ & $11.53 \pm 0.03$ & $10.83 \pm 0.04$ & $10.51 \pm 0.06$ & $\cdots$ & $\cdots$ & $\cdots$ & $\cdots$ \\
\hline UGC 12250 & $\cdots$ & $14.06 \pm 0.19$ & $\cdots$ & $11.44 \pm 0.04$ & $10.69 \pm 0.04$ & $10.37 \pm 0.05$ & $<0.12$ & $<0.13$ & $0.29 \pm 0.05$ & $<2.16$ \\
\hline UGC 12253 & $\cdots$ & $\cdots$ & $\cdots$ & $12.25 \pm 0.04$ & $11.45 \pm 0.03$ & $11.02 \pm 0.04$ & $\cdots$ & $\cdots$ & $\cdots$ & $\cdots$ \\
\hline NGC 7418 & $\ldots$ & $11.65 \pm 0.17$ & $\cdots$ & $9.45 \pm 0.03$ & $8.77 \pm 0.04$ & $8.52 \pm 0.05$ & $0.29 \pm 0.04$ & $0.40 \pm 0.07$ & $4.34 \pm 0.30$ & $15.00 \pm 0.90$ \\
\hline NGC $7418 \mathrm{~A}$ & $\cdots$ & $13.82 \pm 0.21$ & $\cdots$ & $\ldots$ & $\ldots$ & $\ldots$ & $<0.22$ & $<0.14$ & $0.51 \pm 0.06$ & $1.08 \pm 0.21$ \\
\hline ESO 534-G032 & $\cdots$ & $14.70 \pm 0.21$ & $\cdots$ & $\cdots$ & $\cdots$ & $\cdots$ & $\cdots$ & $\cdots$ & $\cdots$ & $\cdots$ \\
\hline IC 5264 & $\cdots$ & $13.44 \pm 0.21$ & $\cdots$ & $10.33 \pm 0.01$ & $9.60 \pm 0.01$ & $9.36 \pm 0.01$ & $<0.11$ & $<0.15$ & $0.53 \pm 0.06$ & $1.90 \pm 0.29$ \\
\hline NGC 7421 & $12.61 \pm 0.09$ & $12.56 \pm 0.08$ & $11.93 \pm 0.08$ & $10.24 \pm 0.03$ & $9.61 \pm 0.04$ & $9.25 \pm 0.04$ & $\ldots$ & $\ldots$ & $\ldots$ & $\ldots$ \\
\hline NGC 7432 & $\ldots$ & $14.41 \pm 0.23$ & $\ldots$ & $10.89 \pm 0.03$ & $10.20 \pm 0.03$ & $9.91 \pm 0.04$ & $\ldots$ & $\cdots$ & $\ldots$ & $\cdots$ \\
\hline ARP 314 NED01 & $\cdots$ & $13.70 \pm 0.20$ & $13.21 \pm 0.20$ & $11.42 \pm 0.03$ & $10.69 \pm 0.04$ & $10.45 \pm 0.05$ & $\cdots$ & $\cdots$ & $\cdots$ & $\cdots$ \\
\hline ARP 314 NED03 & $\cdots$ & $\ldots$ & $\ldots$ & & & $\ldots$ & $\cdots$ & $\cdots$ & $\cdots$ & $\cdots$ \\
\hline ARP 314 NED02 & $\cdots$ & $13.81 \pm 0.13$ & $13.43 \pm 0.13$ & $12.05 \pm 0.07$ & $11.26 \pm 0.07$ & $10.91 \pm 0.09$ & $\cdots$ & $\cdots$ & $\cdots$ & $\cdots$ \\
\hline UGC 12285 & $\cdots$ & $15.25 \pm 0.19$ & $\cdots$ & $12.46 \pm 0.04$ & $11.75 \pm 0.05$ & $11.31 \pm 0.06$ & $<0.18$ & $<0.11$ & $0.33 \pm 0.07$ & $<2.19$ \\
\hline ESO 406-G042 & $\cdots$ & $14.43 \pm 0.21$ & $\cdots$ & $\cdots$ & $\cdots$ & $\cdots$ & $\cdots$ & $\cdots$ & $\cdots$ & $\cdots$ \\
\hline NGC 7469 & $12.60 \pm 0.20$ & $13.00 \pm 0.20$ & $12.34 \pm 0.20$ & $10.11 \pm 0.03$ & $\cdots$ & $\cdots$ & $1.60 \pm 0.05$ & $5.84 \pm 0.05$ & $27.70 \pm 0.04$ & $34.90 \pm 0.64$ \\
\hline NGC 7479 & $11.74 \pm 0.05$ & $11.60 \pm 0.05$ & $10.85 \pm 0.05$ & $9.19 \pm 0.01$ & $8.51 \pm 0.01$ & $8.20 \pm 0.02$ & $1.40 \pm 0.04$ & $3.92 \pm 0.07$ & $15.40 \pm 0.06$ & $24.60 \pm 0.31$ \\
\hline UGC 12346 & $\cdots$ & $15.00 \pm 0.29$ & $\cdots$ & $12.78 \pm 0.06$ & $12.20 \pm 0.09$ & $11.77 \pm 0.09$ & $\cdots$ & $\cdots$ & $\cdots$ & $\cdots$ \\
\hline UGC 12354 & $\cdots$ & $15.07 \pm 0.18$ & $\cdots$ & $12.59 \pm 0.07$ & $11.89 \pm 0.06$ & $11.61 \pm 0.10$ & $\ldots$ & $\cdots$ & $\cdots$ & $\cdots$ \\
\hline ESO 469-G012 & $\ldots$ & $15.22 \pm 0.21$ & $\ldots$ & $12.44 \pm 0.05$ & $11.94 \pm 0.08$ & $11.35 \pm 0.07$ & $\ldots$ & $\ldots$ & $\cdots$ & $\ldots$ \\
\hline ESO 469-G015 & $\cdots$ & $14.70 \pm 0.21$ & $\cdots$ & $12.14 \pm 0.04$ & $11.46 \pm 0.05$ & $11.11 \pm 0.05$ & $<0.10$ & $<0.14$ & $0.47 \pm 0.05$ & $1.32 \pm 0.16$ \\
\hline IC 5287 & $\cdots$ & $14.74 \pm 0.22$ & $\cdots$ & $11.86 \pm 0.04$ & $11.37 \pm 0.07$ & $10.84 \pm 0.06$ & $\cdots$ & $\ldots$ & $\cdots$ & $\cdots$ \\
\hline
\end{tabular}


Table 4-Continued

\begin{tabular}{|c|c|c|c|c|c|c|c|c|c|c|}
\hline \multirow{2}{*}{$\begin{array}{c}\text { Object Name } \\
(1) \\
\end{array}$} & \multicolumn{3}{|c|}{ Optical Photometry } & \multicolumn{3}{|c|}{ Near-Infrared Photometry } & \multicolumn{4}{|c|}{ IRAS fluxes } \\
\hline & $\begin{array}{c}U \\
(\mathrm{mag}) \\
(2)\end{array}$ & $\begin{array}{c}B \\
(\mathrm{mag}) \\
(3)\end{array}$ & $\begin{array}{c}V \\
(\mathrm{mag}) \\
(4)\end{array}$ & $\begin{array}{c}J \\
(\mathrm{mag}) \\
(5)\end{array}$ & $\begin{array}{c}H \\
(\mathrm{mag}) \\
(6)\end{array}$ & $\begin{array}{c}K \\
(\mathrm{mag}) \\
(7)\end{array}$ & $\begin{array}{c}12 \mu \mathrm{m} \\
(\mathrm{Jy}) \\
(8)\end{array}$ & $\begin{array}{c}25 \mu \mathrm{m} \\
(\mathrm{Jy}) \\
(9)\end{array}$ & $\begin{array}{c}60 \mu \mathrm{m} \\
(\mathrm{Jy}) \\
(10)\end{array}$ & $\begin{array}{l}100 \mu \mathrm{m} \\
(\mathrm{Jy}) \\
(11)\end{array}$ \\
\hline ESO 407-G007 & $\ldots$ & $13.78 \pm 0.21$ & $\ldots$ & $10.89 \pm 0.02$ & $10.11 \pm 0.02$ & $9.89 \pm 0.03$ & $0.14 \pm 0.03$ & $0.16 \pm 0.03$ & $1.30 \pm 0.08$ & $4.34 \pm 0.26$ \\
\hline NGC 7496 & $\ldots$ & $11.91 \pm 0.16$ & $\cdots$ & $9.49 \pm 0.02$ & $8.84 \pm 0.03$ & $8.65 \pm 0.04$ & $0.35 \pm 0.06$ & $1.60 \pm 0.02$ & $8.46 \pm 0.76$ & $15.60 \pm 0.78$ \\
\hline ESO 291-G005 & $\ldots$ & $15.26 \pm 0.21$ & $\cdots$ & $12.64 \pm 0.05$ & $11.91 \pm 0.06$ & $11.53 \pm 0.08$ & $\ldots$ & $\ldots$ & $\ldots$ & $\ldots$ \\
\hline ESO 291-G006 & $\cdots$ & $15.32 \pm 0.21$ & $\cdots$ & $13.56 \pm 0.06$ & $12.99 \pm 0.07$ & $12.59 \pm 0.10$ & $\cdots$ & $\cdots$ & $\cdots$ & $\cdots$ \\
\hline NGC 7496A & $\cdots$ & $14.56 \pm 0.21$ & $\cdots$ & $\cdots$ & $\cdots$ & $\cdots$ & $<0.09$ & $<0.07$ & $0.22 \pm 0.05$ & $<0.70$ \\
\hline NGC 7511 & $\ldots$ & $14.84 \pm 0.18$ & $\ldots$ & $11.92 \pm 0.03$ & $11.22 \pm 0.03$ & $10.87 \pm 0.05$ & $<0.11$ & $<0.20$ & $0.91 \pm 0.09$ & $2.18 \pm 0.33$ \\
\hline ESO 407-G009 & $\ldots$ & $14.06 \pm 0.21$ & $\ldots$ & $\ldots$ & $\ldots$ & $\cdots$ & $\cdots$ & $\ldots$ & $\ldots$ & $\cdots$ \\
\hline ESO 291-G009 & $\ldots$ & $14.64 \pm 0.21$ & $\ldots$ & $11.69 \pm 0.06$ & $10.89 \pm 0.06$ & $10.59 \pm 0.10$ & $\cdots$ & $\ldots$ & $\ldots$ & $\ldots$ \\
\hline UGC 12434 & $\cdots$ & $15.31 \pm 0.19$ & $\cdots$ & $12.84 \pm 0.06$ & $12.20 \pm 0.08$ & $11.90 \pm 0.12$ & $<0.13$ & $<0.12$ & $0.51 \pm 0.07$ & $<1.37$ \\
\hline NGC 7535 & $14.22 \pm 0.16$ & $14.24 \pm 0.15$ & $13.66 \pm 0.15$ & $\ldots$ & $\ldots$ & $\ldots$ & $\ldots$ & $\ldots$ & $\ldots$ & $\ldots$ \\
\hline NGC 7536 & $14.13 \pm 0.13$ & $14.07 \pm 0.13$ & $13.43 \pm 0.13$ & $11.39 \pm 0.03$ & $10.63 \pm 0.03$ & $10.35 \pm 0.05$ & $0.17 \pm 0.03$ & $<0.15$ & $1.12 \pm 0.09$ & $3.22 \pm 0.29$ \\
\hline NGC 7559B & $\ldots$ & $\cdots$ & $\cdots$ & $10.88 \pm 0.02$ & $10.17 \pm 0.02$ & $9.95 \pm 0.04$ & $\cdots$ & $\ldots$ & $\cdots$ & $\ldots$ \\
\hline NGC 7563 & $14.48 \pm 0.13$ & $13.87 \pm 0.13$ & $12.81 \pm 0.13$ & $10.44 \pm 0.01$ & $9.71 \pm 0.02$ & $9.45 \pm 0.03$ & $\cdots$ & $\ldots$ & $\ldots$ & $\cdots$ \\
\hline NGC 7552 & $11.34 \pm 0.13$ & $11.25 \pm 0.13$ & $10.57 \pm 0.13$ & $8.49 \pm 0.02$ & $7.84 \pm 0.02$ & $7.54 \pm 0.02$ & $2.95 \pm 0.21$ & $12.20 \pm 0.21$ & $72.00 \pm 4.32$ & $102.00 \pm 6.09$ \\
\hline NGC 7570 & $13.95 \pm 0.13$ & $13.89 \pm 0.13$ & $13.18 \pm 0.13$ & $10.86 \pm 0.03$ & $10.33 \pm 0.03$ & $10.13 \pm 0.05$ & $<0.12$ & $0.27 \pm 0.02$ & $1.32 \pm 0.09$ & $2.95 \pm 0.30$ \\
\hline UGC 12479 & $\ldots$ & $15.14 \pm 0.18$ & $\ldots$ & $12.16 \pm 0.04$ & $11.51 \pm 0.04$ & $11.31 \pm 0.06$ & $<0.10$ & $<0.37$ & $0.49 \pm 0.05$ & $1.09 \pm 0.15$ \\
\hline ESO 407-G014 & $\ldots$ & $13.46 \pm 0.21$ & $\ldots$ & $11.66 \pm 0.03$ & $11.00 \pm 0.03$ & $10.81 \pm 0.04$ & $<0.10$ & $0.13 \pm 0.03$ & $0.99 \pm 0.08$ & $2.77 \pm 0.28$ \\
\hline NGC 7589 & $\ldots$ & $15.01 \pm 0.20$ & $\ldots$ & $11.96 \pm 0.05$ & $11.20 \pm 0.04$ & $11.08 \pm 0.06$ & $\ldots$ & $\ldots$ & $\ldots$ & $\ldots$ \\
\hline NGC 7582 & $11.62 \pm 0.07$ & $11.37 \pm 0.07$ & $10.62 \pm 0.07$ & $8.35 \pm 0.02$ & $7.68 \pm 0.02$ & $7.32 \pm 0.02$ & $1.62 \pm 0.10$ & $6.44 \pm 0.08$ & $49.10 \pm 2.95$ & $72.90 \pm 5.10$ \\
\hline PGC 71025 & $\ldots$ & $\ldots$ & $\ldots$ & $12.02 \pm 0.04$ & $11.21 \pm 0.04$ & $10.83 \pm 0.05$ & $\ldots$ & $\ldots$ & $\ldots$ & $\ldots$ \\
\hline IC 5304 & $\ldots$ & $\cdots$ & $\cdots$ & $11.04 \pm 0.02$ & $10.34 \pm 0.02$ & $10.07 \pm 0.04$ & $\cdots$ & $\ldots$ & $\cdots$ & $\cdots$ \\
\hline NGC 7645 & $\ldots$ & $13.77 \pm 0.21$ & $\ldots$ & $11.24 \pm 0.03$ & $10.54 \pm 0.04$ & $10.25 \pm 0.04$ & $\ldots$ & $\ldots$ & $\ldots$ & $\ldots$ \\
\hline UGC 12578 & $\ldots$ & $14.54 \pm 0.26$ & $\ldots$ & $\ldots$ & $\ldots$ & $\ldots$ & $<0.08$ & $<0.23$ & $0.32 \pm 0.05$ & $<0.40$ \\
\hline UGC 12589 & $\ldots$ & $15.18 \pm 0.20$ & $\ldots$ & $\cdots$ & $\ldots$ & $\ldots$ & $<0.15$ & $<0.18$ & $1.19 \pm 0.08$ & $1.71 \pm 0.17$ \\
\hline CGCG 406-109 & $\ldots$ & $15.55 \pm 0.18$ & $\ldots$ & $13.56 \pm 0.07$ & $12.93 \pm 0.10$ & $12.60 \pm 0.11$ & $\ldots$ & $\ldots$ & $\ldots$ & $\ldots$ \\
\hline NGC 7673 & $12.84 \pm 0.13$ & $13.17 \pm 0.13$ & $12.76 \pm 0.13$ & $11.51 \pm 0.03$ & $10.90 \pm 0.04$ & $10.73 \pm 0.03$ & $0.14 \pm 0.05$ & $0.52 \pm 0.04$ & $4.98 \pm 0.05$ & $6.66 \pm 0.32$ \\
\hline NGC 7674 & $13.84 \pm 0.11$ & $13.92 \pm 0.10$ & $13.23 \pm 0.10$ & $10.92 \pm 0.03$ & $10.22 \pm 0.05$ & $9.79 \pm 0.05$ & $0.68 \pm 0.04$ & $1.88 \pm 0.04$ & $5.28 \pm 0.05$ & $7.91 \pm 0.17$ \\
\hline NGC 7677 & $13.93 \pm 0.13$ & $13.93 \pm 0.13$ & $13.19 \pm 0.13$ & $11.32 \pm 0.03$ & $10.62 \pm 0.03$ & $10.26 \pm 0.03$ & $0.23 \pm 0.03$ & $0.71 \pm 0.02$ & $3.96 \pm 0.20$ & $5.92 \pm 0.35$ \\
\hline IC 5325 & $11.80 \pm 0.13$ & $11.83 \pm 0.13$ & $11.27 \pm 0.13$ & $9.22 \pm 0.02$ & $8.60 \pm 0.02$ & $8.36 \pm 0.03$ & $0.39 \pm 0.09$ & $0.47 \pm 0.03$ & $4.53 \pm 0.32$ & $14.50 \pm 1.01$ \\
\hline UGC 12635 & $\ldots$ & $14.90 \pm 0.29$ & $\ldots$ & $13.82 \pm 0.06$ & $14.28 \pm 0.12$ & $13.68 \pm 0.19$ & $\ldots$ & $\ldots$ & $\ldots$ & $\ldots$ \\
\hline NGC 7684 & $\ldots$ & $14.57 \pm 0.15$ & $\ldots$ & $10.72 \pm 0.02$ & $10.04 \pm 0.02$ & $9.82 \pm 0.03$ & $\ldots$ & $\ldots$ & $\ldots$ & $\ldots$ \\
\hline UGC 12685 & $\ldots$ & $\ldots$ & $\ldots$ & $\ldots$ & $\ldots$ & $\ldots$ & $\ldots$ & $\ldots$ & $\ldots$ & $\ldots$ \\
\hline
\end{tabular}


Table 4-Continued

\begin{tabular}{|c|c|c|c|c|c|c|c|c|c|c|}
\hline \multirow[b]{2}{*}{$\begin{array}{c}\text { Object Name } \\
\text { (1) }\end{array}$} & \multicolumn{3}{|c|}{ Optical Photometry } & \multicolumn{3}{|c|}{ Near-Infrared Photometry } & \multicolumn{4}{|c|}{ IRAS fluxes } \\
\hline & $\begin{array}{c}U \\
(\mathrm{mag}) \\
(2)\end{array}$ & $\begin{array}{c}B \\
(\mathrm{mag}) \\
(3)\end{array}$ & $\begin{array}{c}V \\
(\mathrm{mag}) \\
(4)\end{array}$ & $\begin{array}{c}J \\
(\mathrm{mag}) \\
(5)\end{array}$ & $\begin{array}{c}H \\
(\mathrm{mag}) \\
(6)\end{array}$ & $\begin{array}{c}K \\
(\mathrm{mag}) \\
(7)\end{array}$ & $\begin{array}{c}12 \mu \mathrm{m} \\
(\mathrm{Jy}) \\
(8)\end{array}$ & $\begin{array}{c}25 \mu \mathrm{m} \\
(\mathrm{Jy}) \\
(9) \\
\end{array}$ & $\begin{array}{c}60 \mu \mathrm{m} \\
(\mathrm{Jy}) \\
(10) \\
\end{array}$ & $\begin{array}{l}100 \mu \mathrm{m} \\
(\mathrm{Jy}) \\
(11)\end{array}$ \\
\hline IRAS $23365+3604$ & $\ldots$ & $\ldots$ & $\ldots$ & $13.23 \pm 0.07$ & $12.54 \pm 0.10$ & $12.13 \pm 0.10$ & $<0.10$ & $0.81 \pm 0.01$ & $7.09 \pm 0.71$ & $8.36 \pm 0.50$ \\
\hline ARP 295A & $14.88 \pm 0.21$ & $14.50 \pm 0.20$ & $13.57 \pm 0.21$ & $11.23 \pm 0.02$ & $10.39 \pm 0.02$ & $10.02 \pm 0.03$ & $\ldots$ & $\cdots$ & $\ldots$ & $\ldots$ \\
\hline NGC 7735 & $\cdots$ & $14.56 \pm 0.16$ & $\cdots$ & $10.85 \pm 0.02$ & $10.20 \pm 0.03$ & $9.85 \pm 0.03$ & $\cdots$ & $\cdots$ & $\cdots$ & $\cdots$ \\
\hline NGC 7741 & $11.70 \pm 0.04$ & $11.84 \pm 0.04$ & $11.31 \pm 0.04$ & $10.34 \pm 0.03$ & $9.98 \pm 0.04$ & $9.63 \pm 0.04$ & $<0.17$ & $0.23 \pm 0.05$ & $2.27 \pm 0.34$ & $6.98 \pm 0.56$ \\
\hline NGC 7769 & $\cdots$ & $12.77 \pm 0.15$ & $\cdots$ & $9.90 \pm 0.01$ & $9.21 \pm 0.01$ & $8.93 \pm 0.02$ & $0.33 \pm 0.03$ & $0.58 \pm 0.03$ & $4.34 \pm 0.30$ & $<29.60$ \\
\hline NGC 7771 & $13.42 \pm 0.10$ & $13.08 \pm 0.05$ & $12.25 \pm 0.07$ & $9.47 \pm 0.01$ & $8.72 \pm 0.01$ & $8.35 \pm 0.02$ & $0.87 \pm 0.04$ & $2.18 \pm 0.05$ & $20.50 \pm 0.12$ & $37.40 \pm 0.86$ \\
\hline CGCG 432-040 & $\cdots$ & $15.30 \pm 0.18$ & $\ldots$ & $\ldots$ & $\ldots$ & $\ldots$ & $\cdots$ & $\cdots$ & $\ldots$ & $\ldots$ \\
\hline NGC 7793 & $\cdots$ & $9.63 \pm 0.05$ & $\ldots$ & $7.56 \pm 0.03$ & $7.02 \pm 0.04$ & $6.86 \pm 0.06$ & $1.54 \pm 0.23$ & $2.09 \pm 0.31$ & $19.60 \pm 2.94$ & $56.30 \pm 8.45$ \\
\hline ESO 349-G014 & $\cdots$ & $15.69 \pm 0.21$ & $\ldots$ & $12.40 \pm 0.04$ & $11.56 \pm 0.04$ & $11.27 \pm 0.06$ & $\ldots$ & $\cdots$ & $\cdots$ & $\cdots$ \\
\hline NGC 7798 & $12.83 \pm 0.13$ & $12.97 \pm 0.13$ & $12.37 \pm 0.13$ & $10.35 \pm 0.02$ & $9.65 \pm 0.02$ & $9.37 \pm 0.03$ & $0.36 \pm 0.04$ & $0.62 \pm 0.04$ & $4.87 \pm 0.34$ & $9.49 \pm 0.67$ \\
\hline
\end{tabular}

Note. - Corollary data for the GALEX Atlas sample. Col. (1): Galaxy name. Col. (2): Johnson- $U$ integrated magnitude (in the Vega scale) published as part of the RC3 catalog. Col. (3): The same for the Johnson- $B$ band. Col. (4): The same for the Johnson- $V$ band. Col. (5): 2MASS $J$-band total magnitude. Col. (6): The same for the $H$ band. Col. (7): The same for the $K$ band. Col. (8): Published $12 \mu \mathrm{m}$ IRAS fluxes (in Janskys) and upper limits. Col. (9): The same for the $25 \mu \mathrm{m}$ fluxes. Col. (10): The same for the $60 \mu \mathrm{m}$ fluxes. Col. (11): The same for the $100 \mu \mathrm{m}$ fluxes. 
Fig. Set 3. The GALEX Ultraviolet Atlas of Nearby Galaxies 

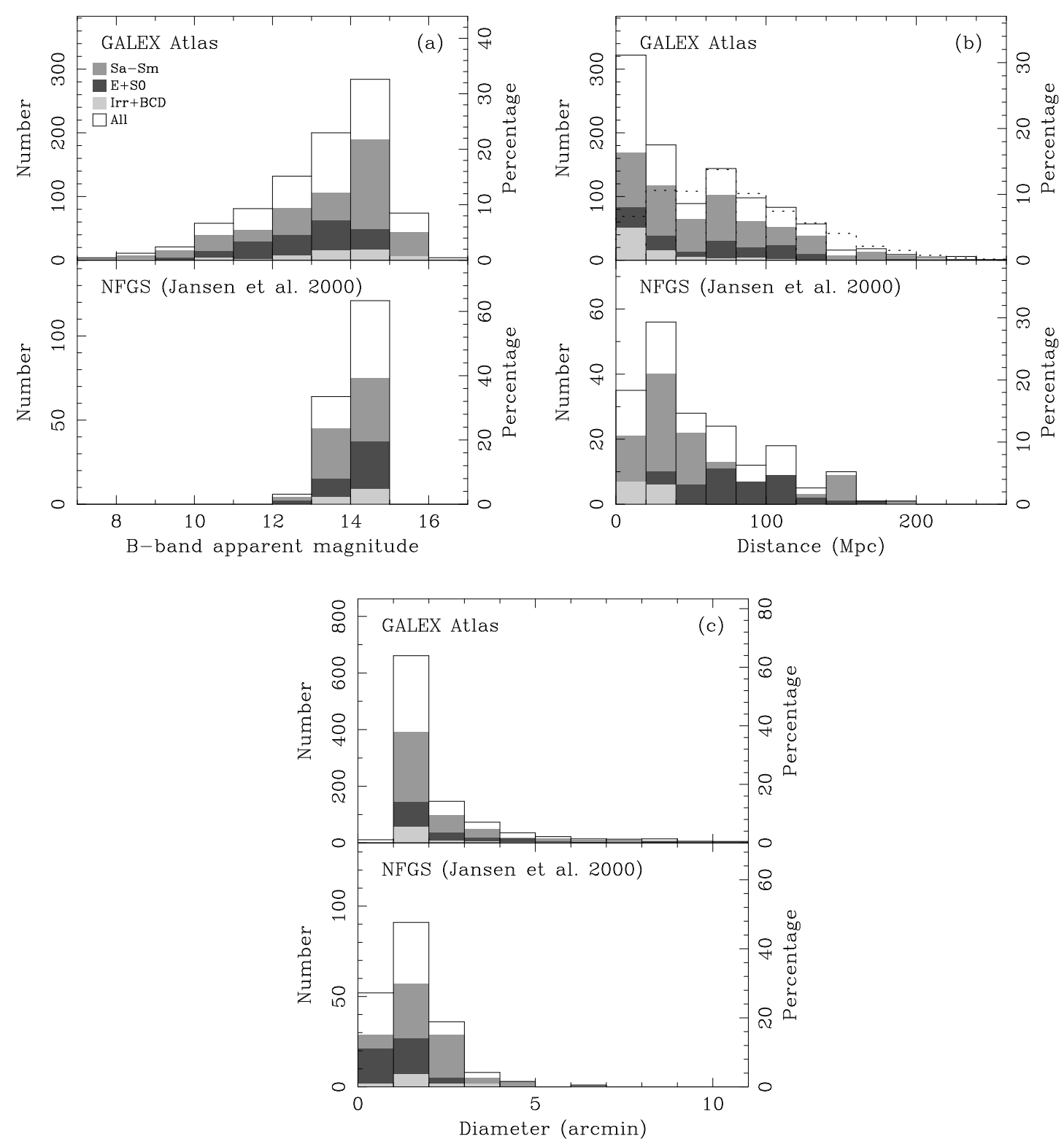

Fig. 1.- Comparison of the GALEX Atlas sample and the Nearby Field Galaxy Survey (NFGS) of Jansen et al. (2000). Galaxies classified as ellipticals/lenticulars are shown in dark grey, spiral galaxies in grey, and irregulars and Blue Compact Dwarf (BCD) galaxies in light grey. The outlined solid-line histogram represents the distribution for all galaxies. (a) Distribution in $B$-band apparent magnitude. Note that the sharp cutoff in magnitude for the NFGS is due to the fact that this survey was extracted from the magnitude-limited CfA survey sample (Huchra et al. 1983). (b) Distribution of distances in Mpc (see Section 2.2 for a detailed description of how the distances to galaxies in the GALEX Atlas were determined). The broken-line histogram represents the distribution of 10,663 galaxies with measured redshifts in RC3. (c) Distribution of major-axis diameters in arcmin. Note that the size-limit for the serendipitous part of the GALEX Atlas was set to $1 \mathrm{arcmin}$. 

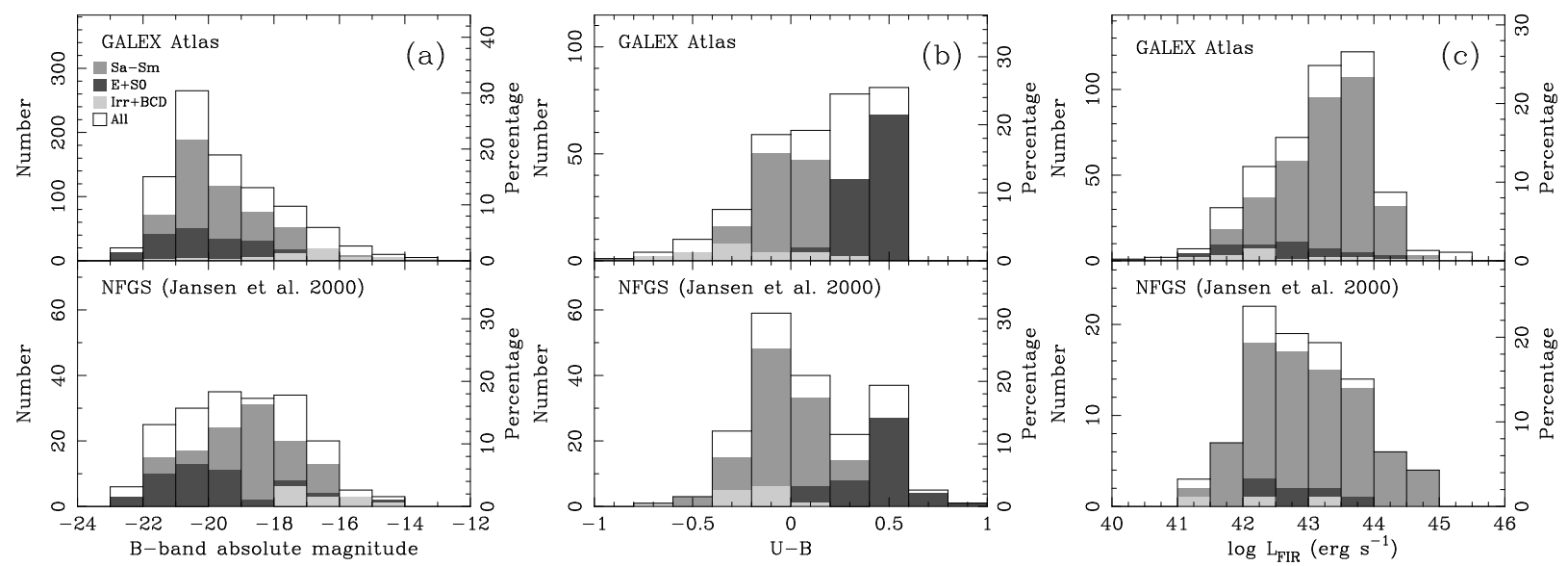

Fig. 2.- Comparison of the properties of the GALEX Atlas sample and the NFGS. Color coding is as in Figure 1. (a) B-band absolute magnitude. Note that the GALEX Atlas sample covers the full range of properties of the galaxies in the Local Universe as described by the magnitude-limited NFGS sample. The distribution is quite similar in the case of elliptical/lenticular and irregular galaxies with only a moderate excess of intrinsically bright spirals in the case of the GALEX Atlas sample compared with field galaxies. It is worth noting that there are still almost three times more low-luminosity spiral galaxies in the GALEX Atlas than in the NFGS. The relative fractions of elliptical/spiral/irregular galaxies is also very similar between the GALEX Atlas sample and field galaxies (see text for details). (b) $U-B$ color. (c) FIR luminosity obtained from the IRAS 60 and $100 \mu \mathrm{m}$ fluxes using the recipe of Londsdale et al. (1985). 

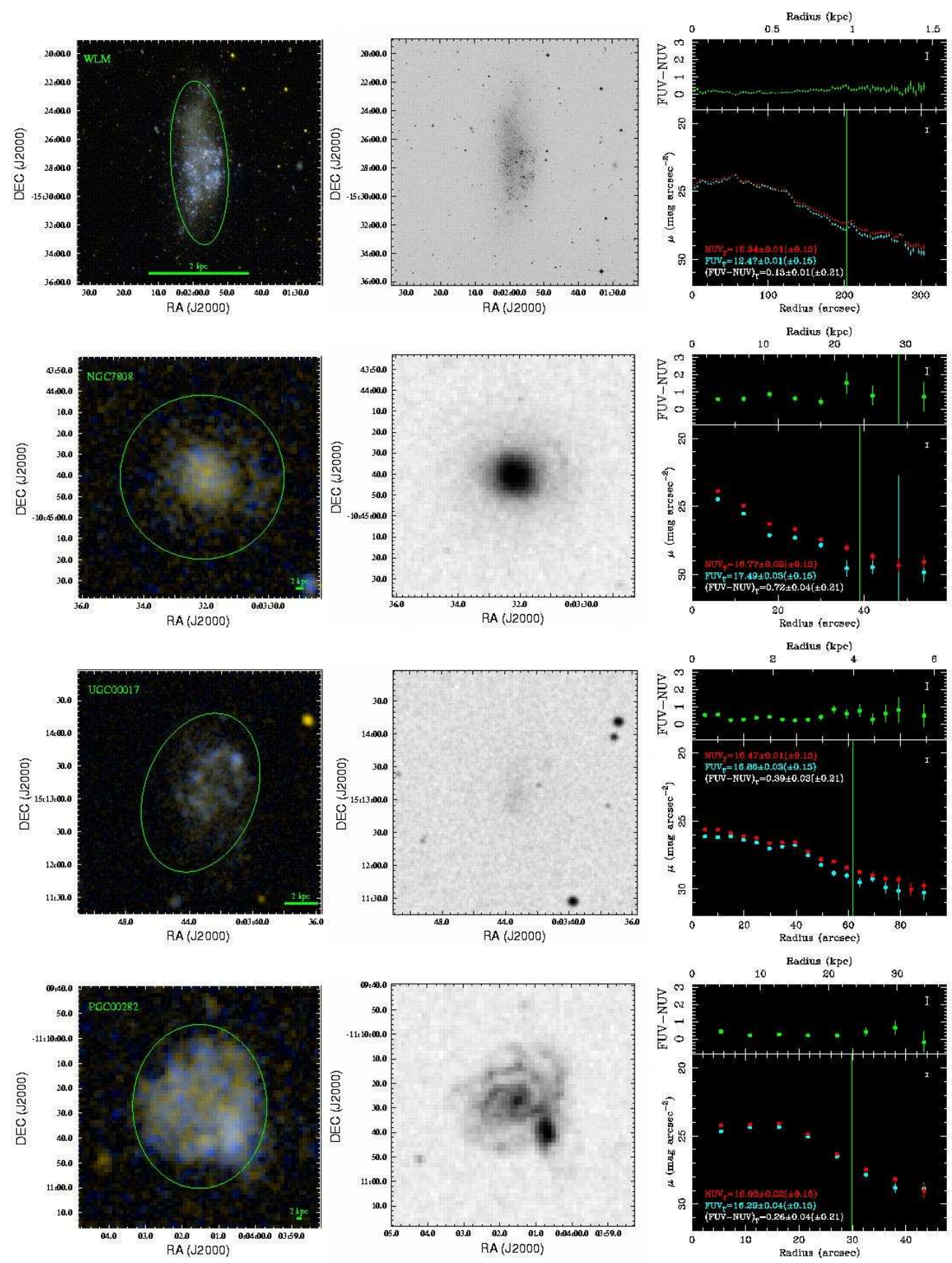

Fig. 3.- False-color GALEX images (left), DSS-1 images (center), surface brightness and color profiles (right) of the galaxies in the Atlas (see text for details). All panels are available online at http://nedwww.ipac.caltech.edu/level5/GALEX_Atlas/. 

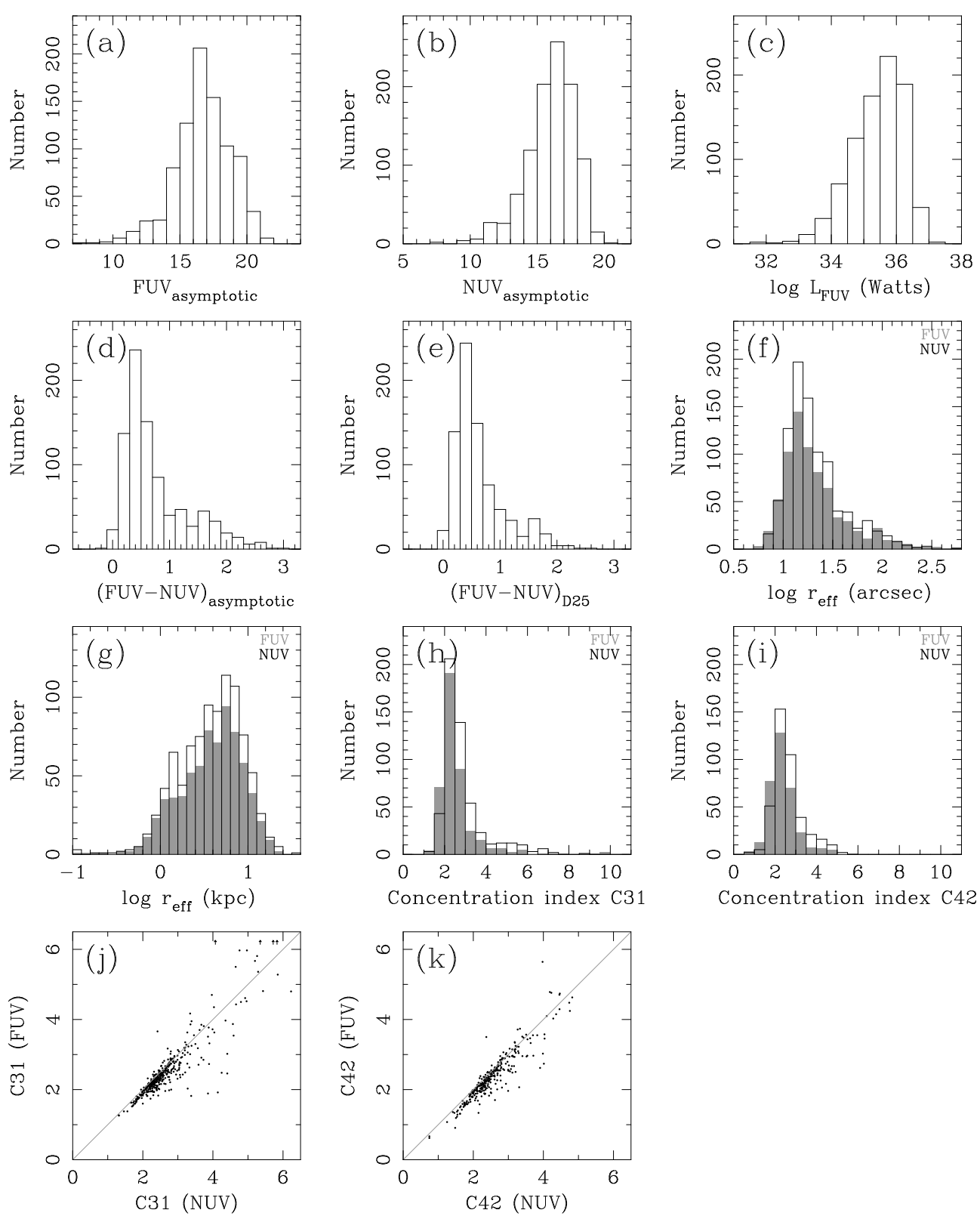
Fig. 4.- UV properties of the galaxies in the Atlas. a) Frequency histogram of apparent asymptotic FUV magnitudes (AB scale). b) Apparent asymptotic NUV magnitudes. c) FUV luminosity in Watts computed as $\nu \mathrm{F}_{\nu}$ (see Buat et al. 2005). d) (FUV-NUV) color. e) (FUV-NUV) color inside the D25 ellipse. f) Effective radius (in arcsec) of the galaxies in the FUV (gray-shaded histogram) and NUV (outlined histogram). g) The same with the radius in kpc. h) FUV (gray-shaded histogram) and NUV (outlined histogram) C31 concentration index. i) The same for the C42 concentration index. j) Comparison of the FUV and NUV C31 concentration indices. k) The same for the C42 concentration index. 

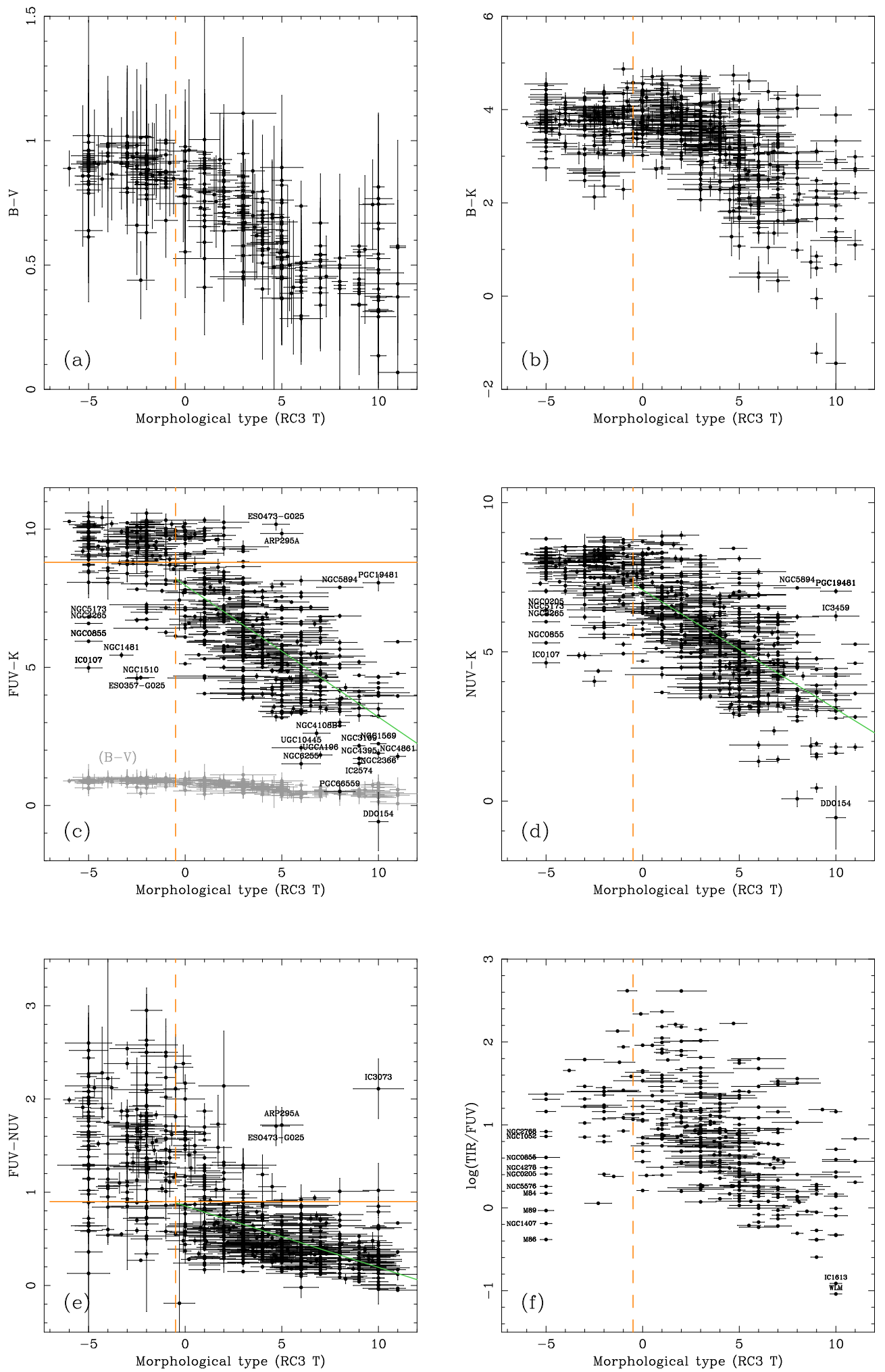
Fig. 5.- Variation in the observed colors and total-infrared (TIR) to FUV ratio of the galaxies in the Atlas with the morphological type (T). a) $(B-V)$ versus the morphological type for elliptical/lenticular $(\mathrm{T}<-0.5)$, spiral $(-0.5 \leq \mathrm{T}<9.5)$, and irregular/compact galaxies $(\mathrm{T} \geq 9.5)$. The separation between elliptical/lenticular and the rest is shown by a vertical dashed line. b) The same for $(B-K)$. Note the small segregation in color between the different types when the $(B-V)$ or $(B-K)$ colors are used. c) The same for $(\mathrm{FUV}-K)$. The segregation between ellipticals/lenticulars and spirals (horizontal solid line) and even between different kind of spiral galaxies is now remarkable. For comparison purposes we show (in the same scale) the range in $(B-V)$ color span by the galaxies in the sample (see panel a). d) The same for $(\mathrm{NUV}-K)$. e) The same for the (FUV-NUV) color. Note that FUV and NUV magnitudes are in AB scale and optical and NIR magnitudes are in the Vega system. Green lines represent the best linear fit to the data for types $\mathrm{T}=-0.5$ or later (i.e. spiral galaxies). f) The same for the TIR-to-FUV ratio (see Buat et al. 2005). 

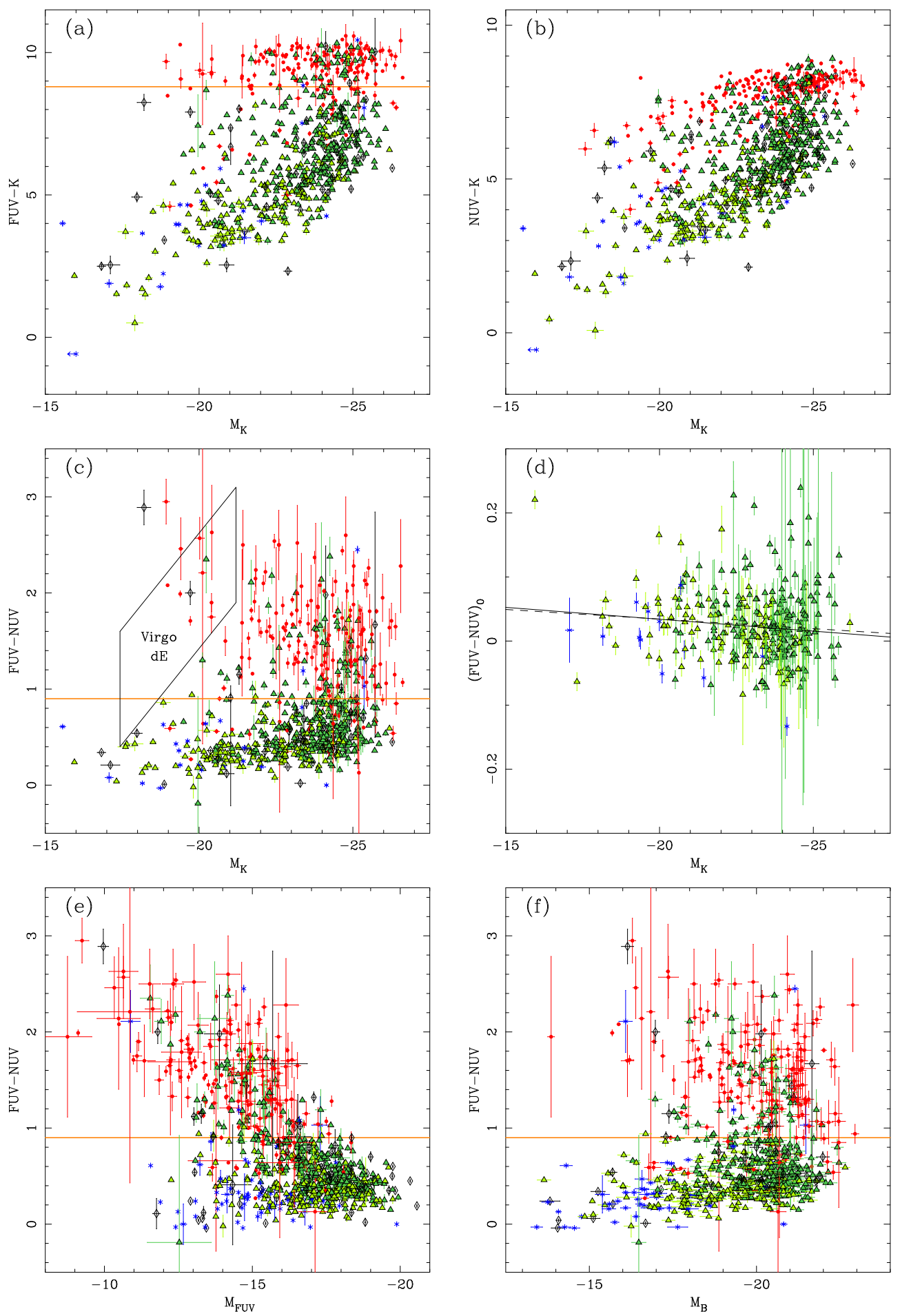
Fig. 6.- Color-magnitude diagrams (CMD) of the Atlas galaxies. Red dots are elliptical/lenticular galaxies, dark green triangles are early-type spirals $(\mathrm{T}<5)$, light green triangles are late-type spirals $(\mathrm{T} \geq 5)$, blue asterisks are irregular and compact galaxies, and black diamonds are galaxies currently lacking morphological classification. a) (FUV-K)- $\mathrm{M}_{K} \mathrm{CMD}$. Spiral and irregular galaxies show a systematic bluing as we move to galaxies of lower mass. Elliptical/lenticular galaxies, on the other hand, show a very small change in the (FUV $-K$ ) color with the $K$-band absolute magnitude (i.e. stellar mass) of the galaxy. b) (NUV-K)$\mathrm{M}_{K}$ CMD. In this case, however, lower mass ellipticals are also systematic bluer than more massive ones. c) (FUV-NUV)-M $\mathrm{M}_{K}$ CMD. This plot shows that the behavior observed in the elliptical galaxies in previous diagrams seems to be consequence of a much fainter UV upturn (best traced by the FUV-NUV color) in low-luminosity ellipticals than in massive ones. In this plot we show the position occupied by dwarf elliptical galaxies in Virgo (Boselli et al. 2005). Dwarf elliptical galaxies fainter than $\mathrm{M}_{K}<-21$ mag start to show the effects of recent star formation both on their (FUV-NUV) and UV-optical colors (see Boselli et al. 2005 for more details). d) (FUV-NUV $)_{0}-\mathrm{M}_{K}$ CMD. The (FUV-NUV) $)_{0}$ color is corrected for internal extinction using the relation between the total-infrared (TIR) to FUV ratio and the extinction in the FUV and NUV bands given by Buat et al. (2005). Only spiral and irregular/compact galaxies are used in this plot. Solid (dashed) line represents the best weighted (non-weighted) fit to the data. The narrow distribution in extinction-corrected UV slopes indicates that the tendency seen in the (FUV-NUV)- $\mathrm{M}_{K}$ CMD shown above for spiral galaxies is a direct consequence of the change in the amount of dust with the luminosity of the galaxy. e) (FUV-NUV)-M $\mathrm{MUV}_{\mathrm{FU}}$ CMD. f) (FUV-NUV)- $\mathrm{M}_{B}$ CMD. These two latter diagrams show a similar behavior to that shown in panel $\mathbf{c}$. The high-luminosity end of the sample in the FUV is clearly dominated by spiral galaxies with a very narrow distribution in observed (FUV-NUV) color. 

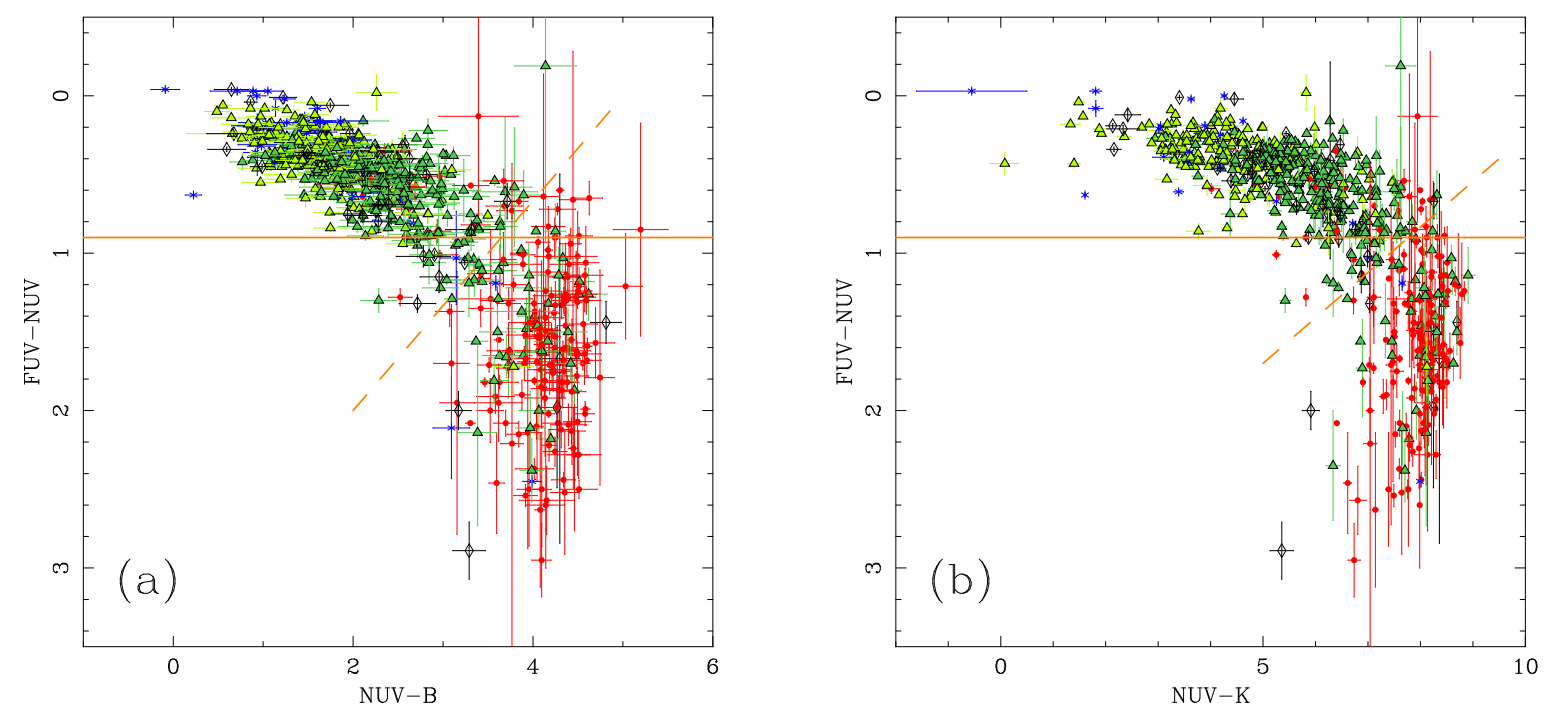

Fig. 7.- Color-color diagrams of the galaxies in the Atlas. a) (FUV-NUV)-(NUV-B) color-color diagram. b) (FUV-NUV)-(NUV-K) color-color diagram. Symbols have the same meaning as in Figure 6. Lines in this plot represent various criteria proposed to separate elliptical/lenticular galaxies from spirals (see text for details). Note that, in order to keep with the stellar convention, the (FUV-NUV) axis has been flipped and red (FUV-NUV) colors are now plotted at the bottom of the figure. 

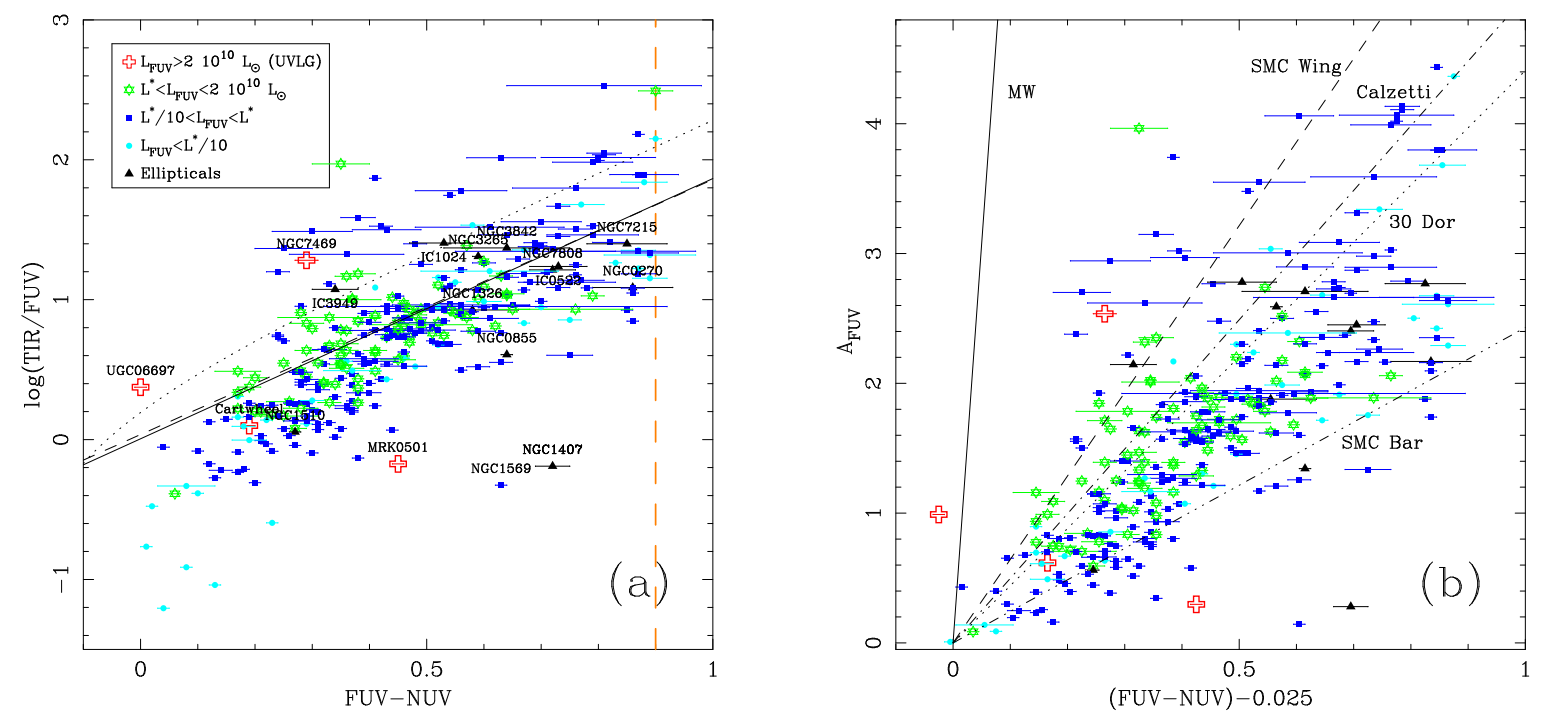

Fig. 8.- a) IRX-beta relation. The vertical long dashed-line represents the cutoff in (FUV-NUV) color used to select the sub-sample of galaxies used to study the relation between the TIR-to-FUV ratio and the slope of the UV. This selection criterion guarantees that in the galaxies considered both the infrared and the UV emission are in the most part associated with the presence of recent star formation activity. The dotted line represents the relation derived by using a sample of starburst galaxies (Kong et al. 2004; Meurer et al. 1999). The best fit to the whole set of data is shown by a solid line. The best fit obtained excluding the lowest luminosity galaxies (dots) is shown by a dashed line. Symbols are coded by UV luminosity. Galaxies with higher UV luminosities (green stars) seem to be located somewhat closer to the relation derived for starburst galaxies that fainter objects (blue squares). Triangles correspond to the elliptical galaxies in the sample. Note that most of the ellipticals with $(\mathrm{FUV}-\mathrm{NUV})<0.9$ are known to have some degree of residual star formation. b) $A_{F U V}$ versus (FUV-NUV)-0.025. The latter term is equivalent to $A_{F U V}-A_{N U V}$ if an intrinsic $(\mathrm{FUV}-\mathrm{NUV})=0.025 \mathrm{mag}$ is assumed for all star-forming galaxies in the sample (see Section 5.3). The lines drawn correspond to the total-to-selective extinction in the UV expected for different extinction laws (MW, solid line; LMC 30 Doradus, dotted line; SMC Wing, dashed line; SMC Bar, dot-dot-dot-dashed line) and the attenuation law of Calzetti et al. (1994, dot-dashed line). The $R_{V}$ values adopted for each of these laws are given in the text. Note that the inclusion of scattering would result in steeper relations between $\mathrm{A}_{\mathrm{FUV}}$ and $\mathrm{A}_{\mathrm{FUV}}-\mathrm{A}_{\mathrm{NUV}}$ than those shown here. Therefore, an attenuation law based on the SMC Bar extinction law seems to be favored by these results. High-UV-luminosity galaxies are still adequately represented by the Calzetti attenuation law. 


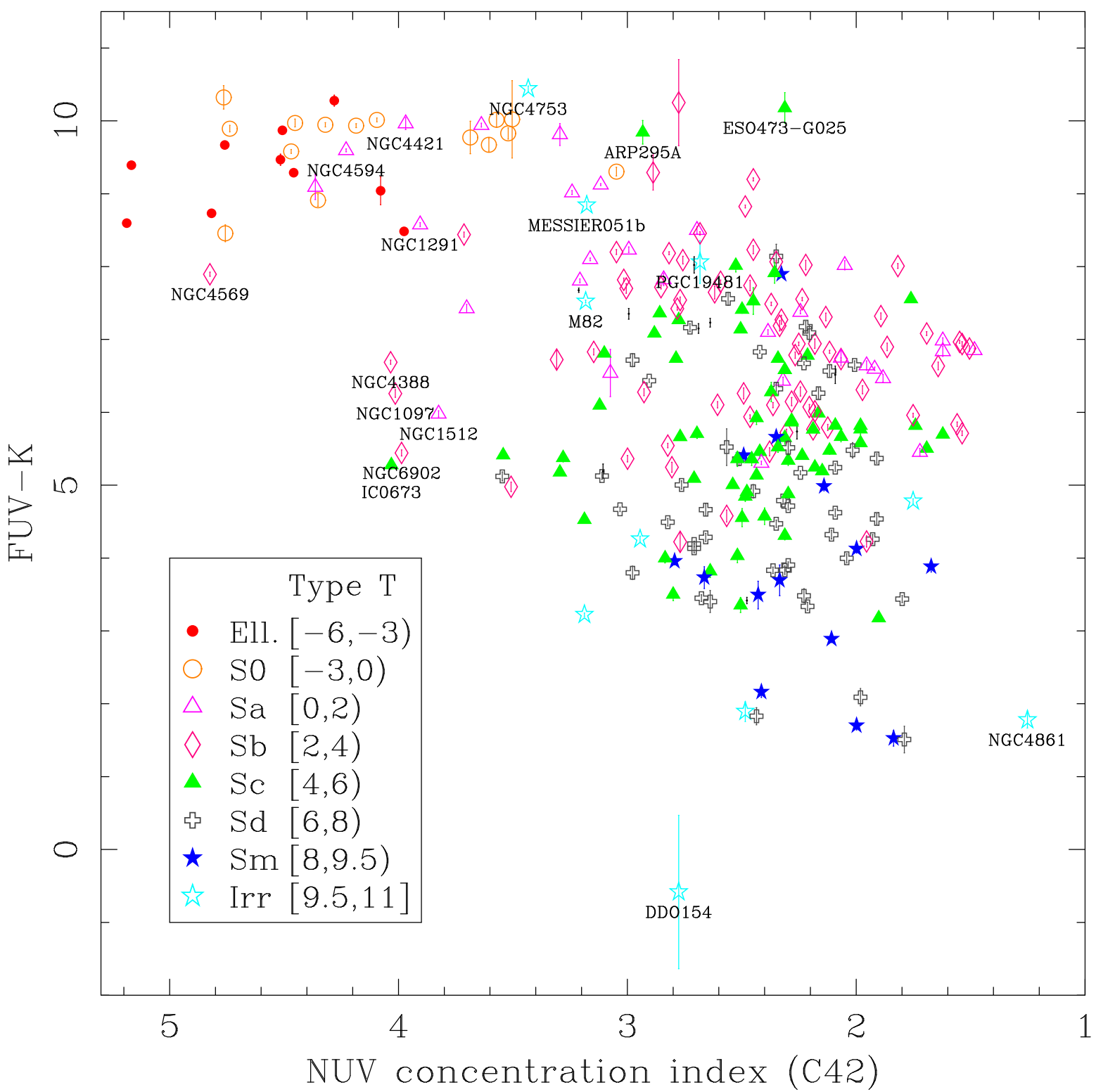

Fig. 9. - (FUV $-K)$ color versus the concentration index C42 in the NUV. Symbols are coded by morphological type. Although the galaxies are better segregated in (FUV-K) color than in concentration index, the value of $\mathrm{C} 42$ can be used improve to the discrimination between ellipticals and lenticulars and between these and some early-type spirals. 


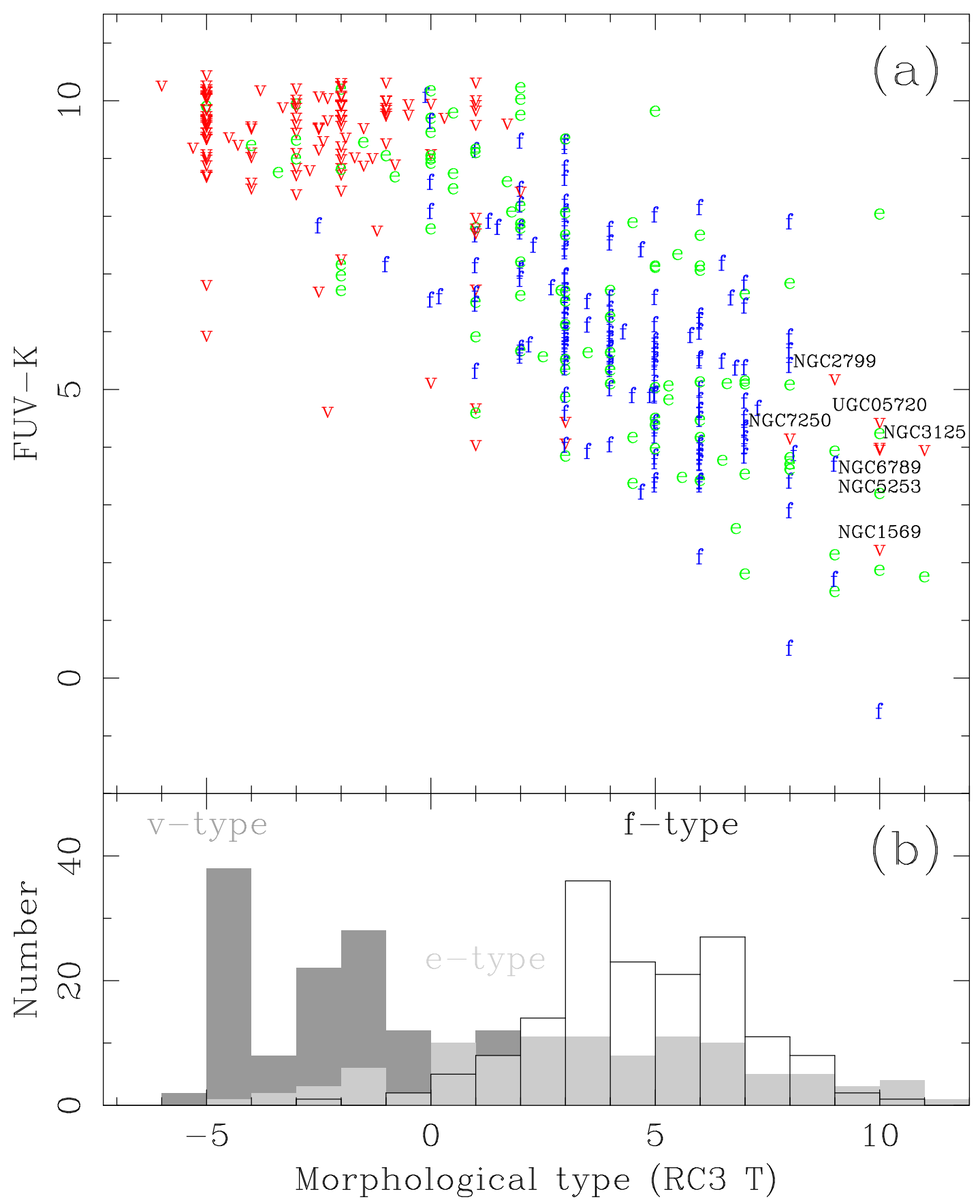

Fig. 10.- a) (FUV $-K)$ color versus the morphological type. The symbols are coded by letters that represent the morphology of their UV profiles: $\mathbf{v}$ for galaxies following a de Vaucouleurs $\mathrm{R}^{1 / 4}$ profile, $\mathbf{e}$ for galaxies with pure exponential profiles, and $\mathbf{f}$ for galaxies with exponential profiles in the outer regions and a flattened profile inside. b) Morphological-type distribution for each class of UV profile. 